\title{
Eco-Tourism and its Architecture: A Methodological Framework for Assessing Progress towards Sustainable Development
}

\author{
Abbas Mahravan \\ A thesis submitted to the Victoria University of Wellington in fulfil- \\ ment of the requirements for the degree of Doctor of Philosophy in \\ Architecture
}

Victoria University of Wellington 


\section{Acknowledgements}

First and foremost I offer my most sincere gratitude to my supervisors, Professor Brenda Vale and Professor Robert Vale, who have supported me throughout my thesis with their patience and knowledge, whilst allowing me the room to work in my own way. I attribute my $\mathrm{PhD}$ degree to their encouragement and effort and without them this thesis would not have been completed. I simply could not have wished for better or more friendly supervisors.

I would like to acknowledge the financial, academic and technical support of the Victoria University of Wellington, and the School of Architecture and Design, and its staff, particularly in the award of a Postgraduate Research Studentship.

I would like to thank my wife, Niloofar Maleki and my daughter Hasti Mahravan who were always there cheering me up and stood by me through good times and bad.

Finally I would like to thank all my friends who were always supporting me, and were most encouraging with their best wishes. 
Table of Contents

\begin{tabular}{|c|c|c|}
\hline \multicolumn{3}{|c|}{ Table of Contents } \\
\hline \multirow{2}{*}{\multicolumn{2}{|c|}{ 1. Chapter 1}} & $\begin{array}{c}\text { Page } \\
\text { Number }\end{array}$ \\
\hline & & 1 \\
\hline 1.1. & Thesis Abstract & 1 \\
\hline 1.2. & \begin{tabular}{|l|} 
Introduction \\
\end{tabular} & 3 \\
\hline 1.3. & Sustainability & 4 \\
\hline 1.4. & Ecotourism & 5 \\
\hline 1.5. & Architecture & 5 \\
\hline 1.6. & Knowledge Gap & 6 \\
\hline 1.7. & Key Research Question & 6 \\
\hline 1.8. & Comprehensive Framework & 6 \\
\hline 1.9. & Thesis Structure & 8 \\
\hline \multicolumn{2}{|r|}{ 2. Chapter 2} & 9 \\
\hline 2.1. & Sustainability & 9 \\
\hline 2.1.1. & Definition of Sustainability & 9 \\
\hline 2.1.2. & The Necessity for Sustainability & 10 \\
\hline 2.1.3. & Models of Sustainability & 11 \\
\hline 2.1.4. & Fundamental Characteristics of Sustainability & 13 \\
\hline 2.1.5. & Sustainable Development- Goals & 14 \\
\hline 2.1.6. & Sustainability- Principles & 14 \\
\hline 2.1.7. & Sustainability Frameworks and Indicators & 15 \\
\hline 2.1.7.1. & Ecological Footprint & 15 \\
\hline 2.1.7.2. & GDP and its Possible Adjustments & 18 \\
\hline 2.1.7.3. & Sustainability Assessment & 19 \\
\hline 2.1.7.4. & Genuine Saving (Sg) & 21 \\
\hline 2.2. & Ecotourism & 22 \\
\hline 2.2.1. & \begin{tabular}{|l|} 
Definition of Ecotourism \\
\end{tabular} & 22 \\
\hline 2.2.1.1. & General Views of a Definition of Ecotourism & 23 \\
\hline 2.2.1.2. & Location and Definition of Ecotourism & 23 \\
\hline 2.2.1.3. & Sustainability and Definition of Ecotourism & 24 \\
\hline 2.2.1.4. & Definition of Ecotourism as a Segment of Alternative Tourism & 25 \\
\hline 2.2.2. & Ecotourism and Environmental Heritage & 26 \\
\hline 2.2.2.1. & Case Study of Costa Rica & 28 \\
\hline 2.2.3. & Ecotourism and Local Economic Growth & 30 \\
\hline 2.2.3.1. & A Case Study of Thailand & 30 \\
\hline 2.2.3.2. & The Potential of Ecotourism & 33 \\
\hline 2.2.3.3. & Definition of Ecotourism in this Thesis & 34 \\
\hline 2.2.4. & Strategy for Sustainable Development of Tourism & 34 \\
\hline 2.2.4.1. & New Zealand's Tourism Strategy 2015 (2007) & 35 \\
\hline 2.2.4.2. & Discussion & 40 \\
\hline 2.3. & Sustainable Architecture & 40 \\
\hline 2.3.1. & Historic Perspective on Sustainable Architecture & 40 \\
\hline 2.3.2. & Learning from Vernacular Architecture & 42 \\
\hline 2.3.2.1. & The Clustered Villages of Nuristan- Afghanistan & 43 \\
\hline 2.3 .3$. & Sustainable or Green Architecture & 44 \\
\hline 2.3.4. & Sustainable Architecture and Environment & 46 \\
\hline 2.3.5. & Sustainable Architecture and Culture & 46 \\
\hline 2.3.6. & The Historical City of Kerman & 47 \\
\hline 2.3.6.1. & General View of Kerman City & 47 \\
\hline 2.3.6.2. & Kerman City - Structure and Elements & 49 \\
\hline 2.3.6.3. & Economy & 59 \\
\hline 2.3.6.4. & The Main Characteristics of Kerman’s Architecture & 60 \\
\hline 2.4. & Ecotourism and Its Architecture through Sustainable Development & 61 \\
\hline 2.5. & Knowledge Gap and Research Justification & 61 \\
\hline 2.5.1. & Shortage of Integrated Strategies & 62 \\
\hline
\end{tabular}




\begin{tabular}{|c|c|c|}
\hline 2.5.1.1. & Linkage between Anticipated Outcomes & 62 \\
\hline 2.5.1.2. & Linkage between Forecasted Priorities & 62 \\
\hline 2.5.1.3. & Linkage between the Indicators & 63 \\
\hline 2.5.1.4. & Deficit of an Integrated Method to view Ecotourism and Architecture & 63 \\
\hline 2.6. & Research Question & 64 \\
\hline 2.6.1. & Sub Research Questions & 65 \\
\hline \multirow[t]{2}{*}{ 2.6.2. } & Key Research Question & 65 \\
\hline & Chapter 2: Summary & 65 \\
\hline \multicolumn{2}{|r|}{ 3. Chapter 3: Methodology } & 68 \\
\hline 3.1. & Introduction & 68 \\
\hline 3.2. & Research Structure & 68 \\
\hline 3.3. & Justification of the Research Methodology & 70 \\
\hline 3.3.1. & Methodological Problems & 71 \\
\hline 3.3.2. & Research Case Studies & 71 \\
\hline 3.3.2.1. & OCRT & 71 \\
\hline 3.3.2.2. & Second Case Study: Naseby & 72 \\
\hline 3.3.2.3. & Third Case Study: Cromwell & 73 \\
\hline 3.4. & The Structure of Methodology & 73 \\
\hline 3.4.1. & $\begin{array}{l}\text { A Comprehensive Framework for Sustainable Development of Ecotourism } \\
\text { and its Architecture }\end{array}$ & 75 \\
\hline 3.4.1.1. & Framework for Sustainable Development of Ecotourism & 75 \\
\hline 3.4.1.2. & Framework for the Sustainable Development of Architecture & 76 \\
\hline 3.4.2. & Data Collection & 77 \\
\hline 3.4.2.1. & Visitor Data & 78 \\
\hline 3.4.2.2. & Data Related to Facilities, Services and Products & 78 \\
\hline 3.4.2.3. & Tourism Activities Data & 78 \\
\hline 3.4.3. & Data Analysis & 78 \\
\hline 3.4.3.1. & Analysis at the Regional Scale & 78 \\
\hline 3.4.3.2. & Analysis at the Site/Individual Building Scale & 84 \\
\hline 3.5. & Importance and Limitations & 85 \\
\hline 3.5.1. & Importance of the Methodology & 85 \\
\hline 3.5.1.1. & Comprehensive Approach & 85 \\
\hline 3.5.1.2. & The Integration of Environmental, Social and Economic Indicators & 85 \\
\hline 3.5.1.3. & Compatibility with Quantitative Methodologies & 85 \\
\hline 3.5.1.4. & Using Existing Information Systems and Available Technologies & 85 \\
\hline 3.5.1.5. & Indicating Required Data through Related Indicators & 85 \\
\hline 3.5.1.6. & Integrated Analysis & 86 \\
\hline 3.5.2. & Limitations. & 86 \\
\hline 3.5.2.1. & Shortcoming of Available Information Systems & 86 \\
\hline \multirow[t]{2}{*}{ 3.5.2.2. } & Accuracy & 86 \\
\hline & Chapter 3: Summary & 86 \\
\hline \multicolumn{2}{|c|}{$\begin{array}{c}\text { 4. Chapter 4: Comprehensive Framework for the Sustainable Devel- } \\
\text { opment of Ecotourism and Its Related Architecture }\end{array}$} & 89 \\
\hline 4.1. & A Framework for Assessing the Sustainable Development of Ecotourism & 89 \\
\hline 4.1.1. & Ecological Framework for the Sustainable Development of Ecotourism & 89 \\
\hline 4.1.1.1. & Environment and Framework for Development of Ecotourism & 90 \\
\hline 4.1.1.2. & Ecological Framework and Environmental Awareness & 91 \\
\hline 4.1.1.3. & Social- Ecological Goals for Ecotourism Development & 92 \\
\hline 4.1.1.4. & Definition of an Indicator & 93 \\
\hline 4.1.1.5. & EF by Types of Ecotourism Products and Activities & 95 \\
\hline 4.1.1.6. & Required data & 96 \\
\hline 4.1.1.7. & Discussion & 97 \\
\hline 4.1.2. & Cultural Framework for Sustainable Development of Ecotourism & 98 \\
\hline 4.1.2.1. & Culture & 99 \\
\hline 4.1.2.2. & Culture and Environment & 101 \\
\hline 4.1.2.3. & Culture and Development & 102 \\
\hline 4.1.2.4. & FCS- a Framework for Cultural Statistics & 103 \\
\hline 4.1.2.5. & Culture Cycle & 104 \\
\hline 4.1.2.6. & Cultural and Related Domains & 105 \\
\hline
\end{tabular}




\begin{tabular}{|c|c|c|}
\hline 4.1.2.7. & Tourism as a Related Domain & 107 \\
\hline 4.1.2.8. & Tourism and Transversal Domains & 109 \\
\hline 4.1.2.9. & G- Transversal Domains (Intangible Cultural Heritage) & 109 \\
\hline 4.1.2.10. & Education and Training & 110 \\
\hline 4.1.2.11. & Cultural Capital and Production of Goods and Services & 110 \\
\hline 4.1.2.12. & $\begin{array}{l}\text { Cultural frameworks and Indicators for the Evaluation of Tourism Products: } \\
\text { examples }\end{array}$ & 112 \\
\hline 4.1.2.13. & Cultural Framework for Sustainable Development of Ecotourism & 120 \\
\hline 4.1.3. & Economic Framework for Sustainable Development of Ecotourism & 137 \\
\hline 4.1.3.1. & Socio-Cultural Outcomes & 137 \\
\hline 4.1.3.2. & Environmental Outcome & 138 \\
\hline 4.1.3.3. & Economic Indicators & 139 \\
\hline 4.1.4. & Cultural Footprint of Ecotourism & 141 \\
\hline 4.1.4.1. & Definition & 141 \\
\hline 4.1.4.2. & CF Model & 143 \\
\hline 4.2. & Comprehensive Framework for Sustainable Development of Architecture & 148 \\
\hline 4.2.1. & $\begin{array}{l}\text { Environmental Framework for Sustainable Development of Ecotourism Ar- } \\
\text { chitecture }\end{array}$ & 149 \\
\hline 4.2.1.1. & Environmental Outcomes & 149 \\
\hline 4.2.1.2. & Ecological Indicators & 150 \\
\hline 4.2.1.3. & Required Data & 150 \\
\hline 4.2 .2 . & $\begin{array}{l}\text { Cultural Framework for Sustainable Architecture Used in Ecotourism Devel- } \\
\text { opment }\end{array}$ & 151 \\
\hline 4.2.2.1. & Anticipated Cultural Outcomes for Sustainable Architecture & 151 \\
\hline 4.2.2.2. & Cultural Indicators & 151 \\
\hline 4.2.2.3. & Required Data & 152 \\
\hline 4.2.3. & Economic Framework & 154 \\
\hline 4.2.3.1. & Environmental Outcomes and Activities & 154 \\
\hline 4.2.3.2. & Indicator & 155 \\
\hline 4.2.3.3. & Cultural Outcomes and Activities & 155 \\
\hline 4.2.3.4. & Indicators & 155 \\
\hline \multirow[t]{2}{*}{ 4.2.4. } & CF of Architecture & 155 \\
\hline & Chapter 4: Summary & 156 \\
\hline \multicolumn{2}{|c|}{$\begin{array}{c}\text { 5. Chapter 5: First Case Study - Otago Central Rail Trail (OCRT), } \\
\text { Otago, New Zealand }\end{array}$} & 162 \\
\hline 5.1. & Introduction & 162 \\
\hline 5.2 . & OCRT- Background & 162 \\
\hline 5.3. & OCRT-Visitors & 166 \\
\hline 5.3.1. & OCRT- Visitor Numbers & 166 \\
\hline 5.3.2. & OCRT- Average Visitor Nights & 167 \\
\hline 5.3.3. & OCRT Visitor Home Place & 168 \\
\hline 5.3.3.1. & OCRT International Visitors & 170 \\
\hline 5.3.3.2. & OCRT Domestic Visitors & 172 \\
\hline 5.4 . & OCRT Transportation & 173 \\
\hline 5.4.1. & OCRT- Types of International Transportation & 174 \\
\hline 5.4.2. & OCRT Types of Domestic Transportation & 175 \\
\hline 5.4.2.1. & OCRT Domestic Tourist-Kilometres (T-km) & 176 \\
\hline 5.4.3. & The Ecological Footprint (EF) of OCRT-Transportation & 177 \\
\hline 5.4.3.1. & Scenario 1 for Domestic Visitors & 177 \\
\hline 5.4.3.2. & Scenario 2 for Domestic Visitors & 178 \\
\hline 5.4.3.3. & OCRT- Transport EF & 178 \\
\hline 5.5. & OCRT: Food & 178 \\
\hline 5.5.1. & The EF of OCRT Consumed Food-First Scenario & 179 \\
\hline 5.5.2. & The EF of OCRT Consumed Food-Second Scenario & 180 \\
\hline 5.5.3. & Environmental Effects of Using Local Food & 183 \\
\hline 5.6. & OCRT Built up land: Accommodation Services & 183 \\
\hline 5.6.1. & OCRT: Types of Accommodation Service & 183 \\
\hline 5.6.2. & Quality of OCRT Accommodation Services & 184 \\
\hline 5.6.3. & OCRT Accommodation Services: Materials Used & 188 \\
\hline
\end{tabular}




\begin{tabular}{|c|c|c|}
\hline 5.6.4. & OCRT Accommodation Services: Energy, Facilities and Spaces & 191 \\
\hline 5.6.4.1. & $\begin{array}{l}\text { OCRT SC Accommodation Services: Energy Sources, Facilities and Open } \\
\text { Air Spaces }\end{array}$ & 191 \\
\hline 5.6.4.2. & $\begin{array}{l}\text { OCRT B\&B Accommodation Services: Energy Sources, Facilities and Open } \\
\text { Air Spaces }\end{array}$ & 193 \\
\hline 5.6.4.3. & $\begin{array}{l}\text { OCRT Bp Accommodation Services: Energy Sources, Facilities and Open } \\
\text { Air Spaces }\end{array}$ & 194 \\
\hline 5.6.4.4. & $\begin{array}{l}\text { OCRT Motel Accommodation Services: Energy Sources, Facilities and Open } \\
\text { Air Spaces }\end{array}$ & 194 \\
\hline 5.6.4.5. & $\begin{array}{l}\text { OCRT Homestead Accommodation Services: Energy Sources, Facilities and } \\
\text { Open Air Spaces }\end{array}$ & 195 \\
\hline 5.6.4.6. & $\begin{array}{l}\text { OCRT Hotel Accommodation Services: Energy Sources, Facilities and Open } \\
\text { Air Spaces }\end{array}$ & 196 \\
\hline 5.6.4.7. & $\begin{array}{l}\text { OCRT Camping Sites: Energy Sources, Facilities and Open Air Spaces } \\
\text { (2011) }\end{array}$ & 197 \\
\hline 5.6.4.8. & $\begin{array}{l}\text { All Types of OCRT Accommodation Services: Energy Sources, Facilities } \\
\text { and Open Air Spaces }\end{array}$ & 198 \\
\hline 5.6.4.9. & $\begin{array}{l}\text { OCRT Accommodation Services: Comparison between NB and RB Bed } \\
\text { spaces in terms of Energy Sources, Facilities and Open Air Spaces }\end{array}$ & 199 \\
\hline 5.6.5. & OCRT Accommodation Services: Occupancy Share & 200 \\
\hline 5.6.6. & OCRT Accommodation Services: Area & 203 \\
\hline 5.6.7. & OCRT Accommodation Services: Occupied Area & 205 \\
\hline 5.6.7.1. & Example: SC Occupied Area $\left(\mathrm{m}^{2}\right) /$ year & 205 \\
\hline 5.6.8. & The EF of OCRT Accommodation Services & 206 \\
\hline 5.6.8.1. & First Scenario: All Buildings are assumed to be New Buildings & 206 \\
\hline 5.6.8.2. & $\begin{array}{l}\text { The EF of OCRT Accommodation Services (Second Scenario: Reducing } \\
\text { Embodied Energy through the use of Refurbished Buildings) }\end{array}$ & 225 \\
\hline 5.6.8.3. & $\begin{array}{l}\text { EF of OCRT Accommodation Services (Third Scenario Including RB, NB } \\
\text { Buildings and All Types of Outdoor Sitting Area) }\end{array}$ & 226 \\
\hline 5.7. & OCRT Visitor Activities & 230 \\
\hline 5.7.1. & Indoor Activities (Frequency) & 230 \\
\hline 5.7 .2 & OCRT Outdoor Activities (Frequency) & 232 \\
\hline 5.7.3. & OCRT Other Visitor Opportunities by Accommodation Service & 233 \\
\hline 5.7.4. & OCRT Activities by Numbers of Visitors & 234 \\
\hline 5.7.5. & OCRT: EF of Visitor Activities & 236 \\
\hline 5.7.5.1. & EF of Indoor Activities (Curling at Naseby) & 236 \\
\hline 5.7.5.2. & EF of OCRT Outdoor Activities & 239 \\
\hline 5.7.5.3. & The Total EF of OCRT Visitor Activities & 250 \\
\hline 5.7.5.4. & Comparison between the EFs of Indoor and Outdoor Activities & 252 \\
\hline 5.8. & The Total EF of OCRT (2011) & 254 \\
\hline 5.9 . & OCRT: Overshoot Portion of EF & 256 \\
\hline 5.9.1. & The EF of Sustainable living & 256 \\
\hline 5.9 .2 . & The Overshoot Portion of OCRT EF Energy & 258 \\
\hline 5.9.3. & OCRT Overshoot Energy Use by Categories & 258 \\
\hline 5.10. & OCRT Economic Footprint & 259 \\
\hline 5.10.1. & OCRT GDP & 259 \\
\hline 5.10 .2 . & OCRT: Sustainable Portion of GDP (GDPs) & 260 \\
\hline 5.10.2.1. & Renewable Energy Cost & 260 \\
\hline 5.11. & OCRT Cultural Footprint (CF) & 263 \\
\hline 5.11 .1$. & OCRT CF: Food & 263 \\
\hline 5.11.1.1. & Sustainable Life: Food (Ideal Model) & 265 \\
\hline 5.11.2. & OCRT CF: Accommodation Services & 267 \\
\hline 5.11.2.1. & Sustainable living OCRT Accommodation Services (Ideal Model) & 271 \\
\hline 5.11.2.2. & Comparison between the Ideal Model, First and Second Scenarios & 271 \\
\hline 5.11.2.3. & Comparison between the Ideal Model, Second and Third Scenarios & 273 \\
\hline 5.11.3. & The CF of OCRT Visitor Activities & 275 \\
\hline 5.11.3.1. & Overshoot EF of OCRT Visitor Activities & 275 \\
\hline 5.11.3.2. & OCRT Activities: GDP & 275 \\
\hline
\end{tabular}

iv $\mid \mathrm{P}$ a g e 


\begin{tabular}{|c|c|c|}
\hline 5.11.3.3. & OCRT Activities: GDPs & 276 \\
\hline 5.11.3.4. & OCRT Visitor Activities: First Scenario & 276 \\
\hline 5.11.3.5. & OCRT Visitor Activities: Second Scenario & 277 \\
\hline 5.11.3.6. & Comparison between Ideal Model and Second Scenario & 278 \\
\hline 5.11.3.7. & OCRT Visitor Activities: Third Scenario & 278 \\
\hline \multirow[t]{2}{*}{ 5.11.3.8. } & Comparison between the Ideal Model and the Third Scenario & 279 \\
\hline & Chapter 5: Summary & 280 \\
\hline \multicolumn{2}{|c|}{$\begin{array}{l}\text { 6. Chapter 6: Second and Third Case Studies: Naseby and Cromwell } \\
\text {-OCRT, New Zealand }\end{array}$} & 282 \\
\hline \multicolumn{2}{|c|}{ 6.1. Case Study 2: Naseby } & 282 \\
\hline 6.1.1. & Introduction & 282 \\
\hline 6.1.2. & Background to Naseby & 283 \\
\hline 6.1.3. & Visitors to Naseby & 289 \\
\hline 6.1.3.1. & Visitor Numbers & 289 \\
\hline 6.1.3.2. & Visitors’ Home Place & 289 \\
\hline 6.1.4. & Transportation & 291 \\
\hline 6.1.4.1. & International Transportation & 291 \\
\hline 6.1.4.2. & Types of Domestic Transportation & 292 \\
\hline 6.1.4.3. & The Ecological Footprint (EF) of Naseby Transportation & 293 \\
\hline 6.1.5. & Food & 294 \\
\hline 6.1.5.1. & The EF of Naseby Food- First scenario & 294 \\
\hline 6.1.5.2. & The EF of Naseby Food- Second Scenario & 295 \\
\hline 6.1.5.3. & Environmental Effects of Using Local Food & 296 \\
\hline 6.1.6. & Naseby Accommodation Services & 297 \\
\hline 6.1.6.1. & Types of Accommodation Services & 297 \\
\hline 6.1.6.2. & Quality of Accommodation Services & 298 \\
\hline 6.1.6.3. & Materials Used for Accommodation Services & 300 \\
\hline 6.1.6.4. & Accommodation Energy Sources, Facilities and Spaces & 301 \\
\hline 6.1.6.5. & Occupancy Share & 306 \\
\hline 6.1.6.6. & Area of Naseby Accommodation Services & 307 \\
\hline 6.1.6.7. & The EF of Naseby Accommodation Services & 309 \\
\hline 6.1.7. & Naseby Tourism Products and Activities (Frequency) & 316 \\
\hline 6.1.7.1. & Local Produce & 316 \\
\hline 6.1.7.2. & Tourism Activities & 317 \\
\hline 6.1.8. & EF of Visitor Activities & 321 \\
\hline 6.1.8.1. & EF of Indoor Activities (Curling at Naseby) & 321 \\
\hline 6.1.8.2. & EF of Outdoor Activities & 322 \\
\hline 6.1.8.3. & Total EF of Naseby Visitor activities & 326 \\
\hline 6.1.8.4. & Comparison between the EFs of Indoor and Outdoor Activities & 328 \\
\hline 6.1.9. & The Total EF of Naseby (2011) & 330 \\
\hline 6.1.10. & Naseby Overshoot Portion of EF & 331 \\
\hline 6.1.11. & Overshoot Portion of Energy Used & 331 \\
\hline 6.1.11.1. & Overshoot Portion of Energy Use per Visitor & 331 \\
\hline 6.1.11.2. & Total Overshoot Portion of Energy Used & 331 \\
\hline 6.1.11.3. & Overshoot Portion of Energy Use by Categories & 332 \\
\hline 6.1.12. & Economic Footprint of Naseby Rail Trail Visitors & 332 \\
\hline 6.1.13. & Naseby Cultural Footprint (CF) & 334 \\
\hline 6.1.13.1. & Naseby CF- Food & 334 \\
\hline 6.1.13.2. & Naseby CF: Accommodation Services & 337 \\
\hline 6.1.13.3. & The CF of Naseby Visitor Activities & 343 \\
\hline \multicolumn{2}{|c|}{ 6.2. Case Study 3: Cromwell } & 349 \\
\hline 6.2.1. & Introduction & 349 \\
\hline 6.2.2. & Background to Cromwell & 349 \\
\hline 6.2.3. & Visitors to Cromwell & 354 \\
\hline 6.2.3.1. & Visitor Numbers & 354 \\
\hline 6.2.3.2. & Visitors Home Place & 354 \\
\hline 6.2.4. & Transportation & 356 \\
\hline
\end{tabular}




\begin{tabular}{|c|c|c|}
\hline 6.2.4.1. & International Transportation & 356 \\
\hline 6.2.4.2. & Types of Domestic Transportation & 357 \\
\hline 6.1.4.3. & The Ecological Footprint (EF) of Cromwell Transportation & 358 \\
\hline 6.2.5. & Food & 359 \\
\hline 6.2.5.1. & The EF of Cromwell Food (First Scenario) & 359 \\
\hline 6.2.5.2. & The EF of Cromwell Food (Second Scenario) & 360 \\
\hline 6.2.5.3. & Environmental Effects of using local Food & 361 \\
\hline 6.2.6. & Accommodation Services & 362 \\
\hline 6.2.6.1. & Types of Accommodation Services & 362 \\
\hline 6.2.6.2. & Cromwell - Quality of Accommodation Services & 363 \\
\hline 6.2.6.3. & Cromwell Accommodation Services - Materials & 363 \\
\hline 6.2.6.4. & Accommodation Services - Energy Sources, Facilities and Spaces & 364 \\
\hline 6.2.6.5. & Cromwell Accommodation Services - Occupancy Share & 368 \\
\hline 6.2.6.6. & Cromwell Accommodation Services - Area & 369 \\
\hline 6.1.6.7. & The EF of Cromwell's Accommodation Services & 370 \\
\hline 6.2.7. & Tourism Products and Activities (Frequency) & 376 \\
\hline 6.2.7.1. & Local Produce & 376 \\
\hline 6.2.7.2. & Tourism Activities & 376 \\
\hline 6.2.8. & EF of Visitor Activities & 380 \\
\hline 6.2.8.1. & EF of Indoor Activities (Curling at Naseby) & 380 \\
\hline 6.2.8.2. & EF of Outdoor Activities & 380 \\
\hline 6.2.8.3. & Total EF of Cromwell Visitor Activities & 385 \\
\hline 6.2.8.4. & Comparison between the EFs of Indoor and Outdoor Activities & 387 \\
\hline 6.2.9. & The Total EF of Cromwell (2011) & 389 \\
\hline 6.2.10. & Cromwell Overshoot Portion of EF & 390 \\
\hline 6.2.11. & Overshoot portion as Energy & 390 \\
\hline 6.2.11.1. & Overshoot Portion of Energy Use per Visitor & 390 \\
\hline 6.2.11.2. & Total Overshoot Portion of Energy Used & 390 \\
\hline 6.2.11.3. & Overshoot Portion of Energy Use by Categories & 391 \\
\hline 6.2.12. & Economic Footprint of Cromwell Rail Trail Visitors & 391 \\
\hline 6.2.13. & Cromwell Cultural Footprint (CF) & 393 \\
\hline 6.2.13.1. & Cromwell CF -Food & 393 \\
\hline 6.2.13.2. & The CF of Cromwell Accommodation Services & 397 \\
\hline 6.2.13.3. & The CF of Cromwell Visitor Activities & 400 \\
\hline 6.2.14. & Comparison between the CFs of Naseby and Cromwell & 406 \\
\hline 6.2.14.1. & Food & 406 \\
\hline 6.2.14.2. & Accommodation Services & 409 \\
\hline \multirow[t]{2}{*}{ 6.2.14.3. } & Visitor Activities & 412 \\
\hline & Chapter 6: Summary & 414 \\
\hline \multicolumn{2}{|r|}{ 7. Chapter 7: Conclusion } & 416 \\
\hline \multicolumn{2}{|r|}{ Bibliography } & 425 \\
\hline \multicolumn{2}{|r|}{ Appendices } & 434 \\
\hline
\end{tabular}




\begin{tabular}{|c|c|}
\hline List of Tables & Page \\
\hline Table 2.1: Advantages and disadva & 17 \\
\hline $\begin{array}{l}\text { Table 2.2: Anticipated outcomes for sustainable development of tourism in New Zealand- } 2015 . \\
\text { (NZST,2015:6-7) }\end{array}$ & 35 \\
\hline Table 2.3: NZTS 2015- priorities for delivering a world-class visitor experience (outcome 1) & 36 \\
\hline Table 2.4: NZTS 2015- priorities for a prosperous tourism sector (outcome2) & 37 \\
\hline $\begin{array}{l}\text { Table 2.5: NZTS 2015- priorities for protecting and enhancing New Zealand's environment } \\
\text { (outcome 3) }\end{array}$ & 38 \\
\hline $\begin{array}{l}\text { Table 2.6: NZTS 2015- priorities for the tourism sector and communities to work together (out- } \\
\text { come 4) }\end{array}$ & 39 \\
\hline Table 4.1: Operating principles for 'mainstream' sustainable development & 90 \\
\hline Table 4.2: Ecological outcomes for development of sustainable Ecotourism & 93 \\
\hline $\begin{array}{l}\text { Table 4.3: Ecological Indicators for Evaluation of the Environmental Impacts of Ecotourism } \\
\text { Development on the host destinations }\end{array}$ & 95 \\
\hline Table 4.4: Tourist EF and its Sub-Categories & 96 \\
\hline Table 4.5: EF categories and Required Data & 97 \\
\hline Table 4.6: The process of Min-Max Definition & 99 \\
\hline Table 4.7: ‘Culture’: Min-Max Definitions & 100 \\
\hline Table 4.8: Framework for Cultural Statistics Domains-related domains (Tourism) & 108 \\
\hline Table 4.9: Indicators for the cultural dimension & 113 \\
\hline $\begin{array}{l}\text { Table 4.10: Cultural outcomes framework and related Indicators for sustainable cultural devel- } \\
\text { opment in New Zealand }\end{array}$ & 115 \\
\hline Table 4.11: Cultural indicators for New Zealand (SNZ, 2009:6-76) & 116 \\
\hline Table 4.12: Cultural outcomes for sustainable development of Tourism & 121 \\
\hline Table 4.13:Attributes of a good [cultural] indicator & 130 \\
\hline $\begin{array}{l}\text { Table 4.14: Proposed Cultural Indicators for development of Ecotourism based on Cultural Ed- } \\
\text { ucation }\end{array}$ & 134 \\
\hline Table 4.15: Required Data related to anticipated Cultural Indicators & 135 \\
\hline Table 4.16: The framework for sustainable architecture (outcomes and activities) & 149 \\
\hline Table 4.17: Social-Ecological indicators & 150 \\
\hline $\begin{array}{l}\text { Table 4.18: Forecasted cultural outcomes for sustainable architecture and its related productive } \\
\text { activities }\end{array}$ & 151 \\
\hline Table 4.19: Cultural indicators to evaluate culturally appropriate architecture & 152 \\
\hline Table 4.20: Required data for evaluation of the CF of architecture & 153 \\
\hline $\begin{array}{l}\text { Table 4.21: Environmental outcome and related productive activities for economic develop- } \\
\text { ment of architecture }\end{array}$ & 154 \\
\hline Table 5.1: OCRT- International Visitors 2009-2011 & 171 \\
\hline Table 5.2: OCRT- International Visitors -2011 & 171 \\
\hline Table 5.3: OCRT- Number of domestic visitors by home place & 173 \\
\hline Table 5.4: OCRT- International Tourist-km & 174 \\
\hline Table 5.5: Types of transportation used by New Zealanders for recreational travel & 176 \\
\hline Table 5.6: OCRT- car based domestic T-km & 176 \\
\hline Table 5.7a: OCRT- domestic transport based on Mathews & 177 \\
\hline Table 5.7b: OCRT- domestic transport scenario 1 & 177 \\
\hline Table 5.7c: OCRT- domestic transport scenario 2 & 178 \\
\hline Table 5.8: OCRT- transport EF & 178 \\
\hline Table 5.9: EF of food consumed by OCRT visitors (2011) & 179 \\
\hline Table 5.10: OCRT- local produce (summary) & 181 \\
\hline Table 5.11: The effect on footprint of consuming food from $100 \%$ organic sources & 182 \\
\hline $\begin{array}{l}\text { Table 5.12: Ecological footprint of home cooked and conventional foods consumed by OCRT } \\
\text { visitors (2011) }\end{array}$ & 182 \\
\hline $\begin{array}{l}\text { Table 5.13: The effect of consuming home cooked food on ecological footprint of food con- } \\
\text { sumed by OCRT visitors (2011) }\end{array}$ & 183 \\
\hline Table 5.14: OCRT types of accommodation service (2011) & 184 \\
\hline $\begin{array}{l}\text { Table 5.15: OCRT- Refurbished (RB) and new buildings (NB) used as accommodation services } \\
\text { and their capacity }\end{array}$ & 185 \\
\hline $\begin{array}{l}\text { Table 5.16: OCRT- accommodation services- materials used by types of accommodation, } \\
\text { summary, (2011) }\end{array}$ & 188 \\
\hline Table 5.17: Central Otago Accommodation Variables by accommodation type and year & 201 \\
\hline Table 5.18: OCRT- Occupancy share by type of accommodation service & 201 \\
\hline
\end{tabular}


Table 5.19: OCRT- accommodation services- occupancy shares (2011)

Table 5.20: OCRT- Average area per bed by type of accommodation (2011)

Table 5.21: OCRT- Area of accommodation services by type and quality

Table 5.22: OCRT- Area of accommodation services by quality

Table 5.23: OCRT- Accommodation services- Total area and Occupied area (2011)

Table 5.24: Embodied energy coefficients of New Zealand building materials

Table 5.25: Initial embodied energy intensities for BIAC standard house

Table 5.26: Initial embodied energy intensities for OCRT- accommodation services by type of construction

Table 5.27: Embodied energy of OCRT- Types of construction (2011)

Table 5.28: Mean energy intensities for various accommodation categories

Table 5.29: OCRT- Life cycle energy use of SC accommodation services (2011)

Table 5.30: OCRT- Life cycle energy use of B\&B accommodation services (2011)

Table 5.31: OCRT- Life cycle energy use of Bp accommodation services (2011)

Table 5.32: OCRT- Life cycle energy use of Motel accommodation services (2011)

Table 5.33: OCRT- Life cycle energy use of Homestead accommodation services (2011)

Table 5.34: OCRT- Life cycle energy use of Hotel accommodation services (2011)

Table 5.35: OCRT- Life cycle energy use of Camping (powered sites) accommodation services (2011)

Table 5.36: OCRT- EF of accommodation services (2011)

Table 5.37: OCRT- accommodation services- Life cycle energy of new and refurbished buildings (second scenario)

Table 5.38: Reduced EF of OCRT accommodation services influenced by the use of RB as

tourism accommodation

Table 5.39: OCRT- Outdoor sitting used as part of accommodation services

Table 5.40: OCRT- Outdoor sitting used as part of accommodation services (summary)

Table 5.41: OCRT- accommodation services-initial embodied energy intensities for ve-

randa/balcony

Table 5.42: OCRT-accommodation services, life cycle energy and EF of outdoor sitting space

(veranda/balcony)

Table 5.43: Reduced EF of OCRT-accommodation serviced influenced by the use of ve-

randa/balcony

Table 5.44: OCRT- Classification of the indoor activities offered by accommodation services to visitors (2011)

Table 5.45: OCRT- Indoor activities offered by accommodation services to visitors - (summary)

Table 5.46: OCRT- Frequency of outdoor activities offered by accommodation services to visitors

Table 5.47: OCRT- accommodation services- opportunities for visits

Table 5.48: OCRT activities conducted by visitors $2011(\mathrm{n}=160)$

Table 5.49: OCRT activities conducted by 11,788 ${ }^{1}$ visitors -2011

Table 5.50: Naseby Indoor Curling Centre, embodied energy

Table 5.51: Oliver Curling Club- Operating energy use per square metre per year

Table 5.52: Naseby Curling Club- Operating energy use per square metre per year

Table 5.53: Total ecological footprint ${ }^{1}$ of Naseby Curling Centre

Table 5.54: Naseby Curling Club - Annual operating energy use per visitor

Table 5.55: Naseby Curling Centre - working time

Table 5.56: OCRT- Visitors- Curling EF (gha) (2011)

Table 5.57: Range of energy values relating to different aspects of travel, and range of total values $(\mathrm{MJ} / \mathrm{km})$

Table 5.58: Ecological footprint of OCRT- visitors (walking)

Table 5.59: Total impact per player of land and equipment associated with golf

Table 5.60: EF of OCRT visitors - golf (2011)

Table 5.61: Classification of tourism attractions and activities

Table 5.62: Energy use per annum for the three categories

Table 5.63: Energy use per tourist for the three categories

Table 5.64: Energy use per tourist of key operator types

Table 5.65: Classification of OCRT Tourism attractions and activities

Table 5.66: EF of OCRT visitors -biking (2011)

Table 5.67: EF of OCRT visitors - mountain biking (2011) 


\begin{tabular}{|c|c|}
\hline Table 5.68: EF of OCRT visitors - fishing (2011) & 245 \\
\hline Table 5.69: EF of OCRT visitors - swimming at Ophir (2011) & 246 \\
\hline Table 5.70: EF of OCRT visitors - wineries (2011) & 246 \\
\hline Table 5.71: EF of OCRT visitors - sightseeing (2011) & 246 \\
\hline Table 5.72: EF of OCRT visitors - Hayes engineering (2011) & 247 \\
\hline Table 5.73: EF of OCRT visitors - Ophir high country farm (2011) & 247 \\
\hline Table 5.74: EF of OCRT visitors - Taieri Gorge Railway (2011) & 247 \\
\hline Table 5.75: EF of OCRT visitors - Gold mining towns (2011) & 248 \\
\hline Table 5.76: EF of OCRT visitors - Old Cromwell town (2011) & 248 \\
\hline Table 5.77: EF of OCRT visitors - Homestead (2011) & 249 \\
\hline Table 5.78: EF of OCRT visitors - visiting friends and relatives (2011) & 249 \\
\hline Table 5.79: EF of OCRT visitors - Clyde dam tour (2011) & 250 \\
\hline Table 5.80: EF of OCRT visitors - Golden Progress mine (2011) & 250 \\
\hline Table 5.81: EF of OCRT visitors - fruit picking along trail (2011) & 250 \\
\hline Table 5.82: Total EF of OCRT- visitor activities & 251 \\
\hline Table 5.83: Comparison between EFs of OCRT visitors - indoor and outdoor activities (2011) & 252 \\
\hline Table 5.84: Total EF of OCRT (2011) & 255 \\
\hline Table 5.85: The ecological footprint of sustainable living & 256 \\
\hline Table 5.86: The ecological footprint of OCRT- sustainable tourism (2011) & 256 \\
\hline Table 5.87: The energy use of OCRT sustainable tourism (2011) & 258 \\
\hline Table 5.88: The energy use of OCRT- sustainable tourism (2011) & 258 \\
\hline Table 5.89: OCRT overshoot energy use by categories (2011) & 259 \\
\hline Table 5.90: The economic impact of the OCRT on the Central Otago/Otago economy & 259 \\
\hline Table 5.91: Economic impact of OCRT- by categories & 260 \\
\hline $\begin{array}{l}\text { Table 5.92: Approximate fully annualized generation and conventional transmission cost for } \\
\text { WWs power. }\end{array}$ & 261 \\
\hline Table 5.93: New Zealand energy costs & 261 \\
\hline Table 5.94: Average energy cost (wind-solar) & 261 \\
\hline Table 5.95: OCRT- Sustainable Portion of total GDP (GDPs) & 262 \\
\hline Table 5.96: OCRT- Local product (Food) - contribution to GDPs & 265 \\
\hline Table 5.97: OCRT- Accommodation services- CF (2011) & 270 \\
\hline Table 5.98: OCRT- visitor activities - EF and GDPs & 276 \\
\hline Table 5.99: The CF of OCRT visitor activities - first and second scenarios & 277 \\
\hline Table 5.100: The CF of OCRT visitor activities - first and third scenarios & 279 \\
\hline Table 6.1: Naseby visitors - based on CODC survey (2011) & 289 \\
\hline Table 6.2: Naseby international and domestic visitors (2011) & 289 \\
\hline Table 6.3: Naseby - international visitors -2011 & 290 \\
\hline Table 6.4: Naseby - number of domestic visitors by home place & 290 \\
\hline Table 6.5: Naseby - international Tourism-km & 291 \\
\hline Table 6.6: OCRT- domestic T-km & 292 \\
\hline Table 6.7: Types of transport used by OCRT domestic visitors & 293 \\
\hline Table 6.8: EF of Naseby transportation (2011) & 293 \\
\hline Table 6.9: EF of food consumed by Naseby visitors (2011) & 295 \\
\hline Table 6.10: Naseby- local produce (summary) & 295 \\
\hline $\begin{array}{l}\text { Table 6.11: Ecological footprint of home cooked and conventional foods consumed by Naseby } \\
\text { visitors (2011) }\end{array}$ & 296 \\
\hline $\begin{array}{l}\text { Table 6.12: The effect of consuming home prepared food on the EF of food consumed by } \\
\text { Naseby visitors (2011) }\end{array}$ & 296 \\
\hline Table 6.13: Naseby- types of accommodation service & 297 \\
\hline $\begin{array}{l}\text { Table 6.14: Naseby- accommodation services, refurbished (RB) and new buildings (NB) used } \\
\text { as accommodation services and their capacity }\end{array}$ & 298 \\
\hline Table 6. 15: Naseby - materials used by type of accommodation (2011) & 300 \\
\hline Table 6.16: Naseby accommodation services- occupancy share (2011) & 307 \\
\hline Table 6.17: Naseby accommodation services - total area and occupied area (2011) & 308 \\
\hline Table 6.18: Naseby- area of accommodation services by type and quality & 308 \\
\hline Table 6.19: Naseby- area of accommodation services by quality & 308 \\
\hline $\begin{array}{l}\text { Table 6.20: Naseby- Life cycle energy use and the EF of self contained accommodation ser- } \\
\text { vices (2011) }\end{array}$ & 310 \\
\hline Table 6.21: Naseby- Life cycle energy use of B\&B accommodation services (2011) & 311 \\
\hline Table 6.22: Naseby - Life cycle energy use of Hotel ac & 312 \\
\hline
\end{tabular}


Table 6.23: Naseby - Life cycle energy use of Camping (powered sites) accommodation services (2011)

Table 6.24: The total EF of Naseby accommodation services (First scenario)

Table 6.25: Naseby accommodation services- life cycle energy of new and refurbished buildings

Table 6.26: Reduced EF of Naseby accommodation services by use of RB as tourism accommodation

Table 6.27: Naseby- Outdoor areas used as part of accommodation services

Table 6.28: Naseby accommodation services, life cycle energy and EF of outdoor sitting space (veranda/ balcony)

Table 6.29: EF of Naseby accommodation services influenced by the use of veranda/balcony

Table 6.30: Naseby local produce (summary)

Table 6.31: Naseby indoor activities offered by accommodation services to visitors

Table 6.32: Naseby- Frequency of outdoor activities offered by accommodation services to visitors

Table 6.33: Naseby - Frequency of subjects to visit offered by accommodation services to visitors (2011)

Table 6.34: Naseby - activities conducted by 4,645 visitors (2011)

Table 6.35: Naseby - visitors curling EF (gha) (2011)

Table 6.36: Ecological footprint of Naseby visitors (walking)

Table 6.37: Ecological footprint of Naseby visitors (golf)

Table 6.38: Ecological footprint of Naseby visitors (mountain biking).

Table 6.39: Ecological footprint of Naseby visitors (fishing).

Table 6.40: Ecological footprint of Naseby visitors (swimming)

Table 6.41: Ecological footprint of Naseby visitors (wineries)

Table 6.42: Ecological footprint of Naseby visitors (sightseeing)

Table 6.43: Ecological footprint of Naseby visitors (Hayes Engineering)

Table 6.44: Ecological footprint of Naseby visitors (Ophir High Country Farm)

Table 6.45: Ecological footprint of Naseby visitors (Taieri)

Table 6.46: Ecological footprint of Naseby visitors (Gold mining towns)

Table 6.47: Ecological footprint of Naseby visitors (Old Cromwell town)

Table 6.48: Ecological footprint of Naseby visitors (visiting friends and relatives)

Table 6.49: Ecological footprint of Naseby visitors (Clyde Dam Tour)

Table 6.50: Ecological footprint of Naseby visitors (Golden Progress Mine)

Table 6.51: Ecological footprint of Naseby visitors (fruit picking along trail)

Table 6.52: Ecological footprint of Naseby visitors (biking)

Table 6.53: Total EF of Naseby visitor activities

Table 6.54: Comparison between EFs of Naseby- visitors- indoor and outdoor activities

Table 6.55: Total EF of Naseby (2011)

Table 6.56: Overshoot portion of Naseby visitors- EF (gha/visitor/year) (2011)

Table 6.57: Overshoot portion of Naseby visitor energy use (GJ/visitor/year) (2011)

Table 6.58: Total overshoot portion of Naseby visitor energy use (GJ/ year) (2011)

Table 6.59: Naseby overshoot portion of energy used by categories (2011)

Table 6.60: Naseby GDP (2011)

Table 6.61: Naseby Sustainable Portion of GDP (GDPs)

Table 6.62: Naseby- Local product (Food) contribution to GDPs

Table 6.63: Naseby Accommodation services CF (2011)

Table 6.64: Naseby visitor activities- EF and GDPs

Table 6.65: The CF of Naseby visitor activities - first and second scenarios

Table 6.66: The CF of Naseby visitor activities - first and third scenarios

Table 6.67: Cromwell visitors based on CODC survey (2011)

Table 6.68: Cromwell international and domestic visitors (2011)

Table 6.69: Cromwell international visitors (2011)

Table 6.70: Cromwell domestic visitors by home place

Table 6.71: Cromwell international T-km

Table 6.72: Cromwell domestic T-km

Table 6.73: Types of transport used by Cromwell domestic visitors

Table 6.74: EF of Cromwell Transportation (2011)

Table 6.75: EF of food consumed by Cromwell visitors (2011)

Table 6.76: Cromwell local produce (summary) 


\begin{tabular}{|c|c|}
\hline $\begin{array}{l}\text { Table 6.77: Ecological footprint of home prepared and conventional foods consumed by } \\
\text { Cromwell visitors (2011) }\end{array}$ & 361 \\
\hline $\begin{array}{l}\text { Table 6.78: The effect of consuming home prepared food on the EF of food consumed by } \\
\text { Cromwell visitors (2011) }\end{array}$ & 361 \\
\hline Table 6.79: Cromwell accommodation services (2011) & 362 \\
\hline Table 6.80: Cromwell accommodation services - materials used by type of accommodation & 363 \\
\hline Table 6.81: All Cromwell accommodation services by type - energy sources and facilities & 368 \\
\hline Table 6.82: Cromwell accommodation services - occupancy shares (2011) & 369 \\
\hline Table 6.83: Cromwell Accommodation services - Total area and Occupied area (2011) & 370 \\
\hline Table 6.84: Cromwell - Life cycle energy of SC accommodation services (2011) & 371 \\
\hline Table 6.85:Cromwell - Life cycle energy of B\&B accommodation services (2011) & 372 \\
\hline Table 6.86: Cromwell - Life cycle energy use of Motel accommodation services (2011) & 373 \\
\hline Table 6.87: Cromwell camping sites - available bed spaces and visitor nights & 373 \\
\hline $\begin{array}{l}\text { Table 6.88: Cromwell - Life cycle energy use of camping (powered sites) accommodation ser- } \\
\text { vices (2011) }\end{array}$ & 373 \\
\hline Table 6.89: Cromwell - EF of accommodation services (2011) & 374 \\
\hline Table 6.90: Cromwell - Outdoor sitting space used as part of accommodation services & 374 \\
\hline $\begin{array}{l}\text { Table 6.91: Cromwell accommodation services, life cycle energy and EF of outdoor sitting } \\
\text { spaces (veranda/balcony) }\end{array}$ & 375 \\
\hline $\begin{array}{l}\text { Table 6.92: Reduced EF of Cromwell accommodation services influenced by the use of ve- } \\
\text { randa/balcony }\end{array}$ & 376 \\
\hline Table 6.93: Cromwell - Indoor activities & 377 \\
\hline $\begin{array}{l}\text { Table 6.94: Cromwell - Frequency of outdoor activities offered by accommodation services to } \\
\text { visitors }\end{array}$ & 377 \\
\hline $\begin{array}{l}\text { Table 6.95: Cromwell - frequency of subjects to visit offered by accommodation services to } \\
\text { visitors (2011) }\end{array}$ & 378 \\
\hline Table 6.96: Cromwell activities conducted by $2,652^{1}$ visitors (2011) & 379 \\
\hline Table 6.97: Cromwell visitors - Curling EF (gha) (2011) & 380 \\
\hline Table 6.98: Ecological footprint of Cromwell visitors (walking). & 380 \\
\hline Table 6.99: Ecological footprint of Cromwell visitors (golf) & 381 \\
\hline Table 6.100: Ecological footprint of Cromwell visitors (mountain biking) & 381 \\
\hline Table 6.101: Ecological footprint of Cromwell visitors (fishing) & 381 \\
\hline Table 6.102: Ecological footprint of Cromwell visitors (swimming) & 382 \\
\hline Table 6.103: Ecological footprint of Cromwell visitors (wineries) & 382 \\
\hline Table 6.104: Ecological footprint of Cromwell visitors (sightseeing) & 382 \\
\hline Table 6.105: Ecological footprint of Cromwell visitors (Hayes Engineering) & 383 \\
\hline Table 6.106: Ecological footprint of Cromwell visitors (Ophir High Country Farm) & 383 \\
\hline Table 6.107: Ecological footprint of Cromwell visitors (Taieri) & 383 \\
\hline Table 6.108: Ecological footprint of Cromwell visitors (Gold mining towns) & 383 \\
\hline Table 6.109: Ecological footprint of Cromwell visitors (Old Cromwell town) & 384 \\
\hline Table 6.110: Ecological footprint of Cromwell visitors (visiting friends and relatives) & 384 \\
\hline Table 6.111: Ecological footprint of Cromwell visitors (Clyde Dam Tour) & 384 \\
\hline Table 6.112: Ecological footprint of Cromwell visitors (Golden Progress Mine) & 384 \\
\hline Table 6.113: Ecological footprint of Cromwell visitors (fruit picking along trail) & 385 \\
\hline Table 6.114: Ecological footprint of Cromwell visitors (biking) & 385 \\
\hline Table 6.115: Total EF of Cromwell visitor activities & 386 \\
\hline Table 6.115: Total EF of Cromwell visitor activities & 387 \\
\hline Table 6.116: Comparison between EFs of Cromwell & 389 \\
\hline Table 6.117: Total EF of Cromwell (2011) & 390 \\
\hline Table 6.118: Overshoot portion of Cromwell visitors EF (gha/visitor/year) (2011) & 390 \\
\hline Table 6.119: Overshoot portion of Cromwell visitor energy use (GJ/visitor/year) (2011) & 391 \\
\hline Table 6.121: Cromwell overshoot portion of energy used by categories (2011) & 391 \\
\hline Table 6.122: Cromwell GDP (2011) & 391 \\
\hline Table 6.123: Cromwell Sustainable Portion of GDP (GDPs) & 392 \\
\hline Table 6.124: Cromwell - Local product (Food) contribution to GDPs & 394 \\
\hline Table 6.125: Cromwell Accommodation services - CF (2011) & 398 \\
\hline Table 6.126: Cromwell visitor & 402 \\
\hline Table 6.127: The CF of Cromwell visitor activities - first and & 403 \\
\hline Table 6.128: The & 5 \\
\hline
\end{tabular}




\begin{tabular}{|c|c|}
\hline List of Figures & Page \\
\hline Figure 2.1: Weak model of sustainability & 11 \\
\hline Figure 2.2: Strong model of sustainability & 12 \\
\hline Figure 2.3: Framework for sustainability assessment tools & 20 \\
\hline $\begin{array}{l}\text { Figure 2.4: Perceived relationship between sustainability, mass tourism, alternative tourism } \\
\text { (AT), and ecotourism }\end{array}$ & 25 \\
\hline $\begin{array}{l}\text { Figure 2.5: Nuristan village, Afghanistan } \\
\text { http://viaterra.net/photos/pakistan/pakistan_nuristani2.jpg viewed December } 2010\end{array}$ & 43 \\
\hline $\begin{array}{l}\text { Figure 2.6: Kerman city and Kerman province, Iran } \\
\text { http://geology.com/world/iran-satellite-image.shtml viewed August } 2012\end{array}$ & 48 \\
\hline $\begin{array}{l}\text { Figure 2.7: Historical districts of Kerman city which are linked by the Bazaar } \\
\text { Google Earth viewed September } 2011\end{array}$ & 49 \\
\hline $\begin{array}{l}\text { Figure 2.8: Bazaarl and Chahar Sogh }{ }^{2} \\
\text { 1. http://www.flickr.com/photos/lfphotos/1248475691/viewed September } 2011 \\
\text { 2. http://www.avayeettehad.ir/farsi/tour/kerman/255428 orig.jpg viewed September } 2011\end{array}$ & 50 \\
\hline $\begin{array}{l}\text { Figure 2.9: The dome of Chahar Sogh }{ }^{1} \text { and the roof of the Bazaarr } \\
\text { 1. http://farm2.static.flickr.com/1261/4725357313_4e813a189f.jpg viewed September } 2011 \\
\text { 2. http://i1.trekearth.com/photos/38865/coppersmith_bazaar.jpg viewed September } 2011\end{array}$ & 51 \\
\hline $\begin{array}{l}\text { Figure 2.10: Top view }{ }^{1} \text { and inside }{ }^{2} \text { of Qanat } \\
\text { 1. http://thma02.yimg.com/nimage/75d2a55357471914 viewed October } 2011 \\
\text { 2. http://www.mondellolido.it/pagine/cenni\%20storici/lo\%20cascio/immagini/qanat02.gif } \\
\text { viewed October } 2011\end{array}$ & 52 \\
\hline $\begin{array}{l}\text { Figure 2.11: Cross section of a Qanat } \\
\text { http://diyscholar.files.wordpress.com/2010/09/image-110.jpg?w=330\&h=204 viewed October } \\
2011\end{array}$ & 52 \\
\hline $\begin{array}{l}\text { Figure 2.12: Courtyards with different functions in the historical part of Kerman } \\
\text { Google Earth viewed October } 2011\end{array}$ & 54 \\
\hline $\begin{array}{l}\text { Figure 2.13: Arg Square }{ }^{1} \text { and Gang Ali Khan Square }{ }^{2} \text {,Kerman } \\
\text { 1. http://innocent.persiangig.com/image/Kerman\%20Ghadim/Saeed017.jpg viewed December } \\
\text { 2011 } \\
\text { 2. http://farm2.staticflickr.com/1383/4725402177_c052e0c31b_z.jpg viewed December } 2011\end{array}$ & 54 \\
\hline $\begin{array}{l}\text { Figure 2.14: The open space (white areas) and built areas in the historical part of Kerman } \\
\text { The open space (white areas) and built areas in the historical part of Kerman (Herdeg, 1990:37) }\end{array}$ & 55 \\
\hline $\begin{array}{l}\text { Figure 2.15: Courtyard } \\
\text { http://nazaronline.ir/files/fa/news/1389/7/3/1309_325.jpg viewed October } 2011 \\
\text { http://images.travelpod.com/tw_slides/ta00/9d9/eca/kashan-traditional-house-kashan.jpg } \\
\text { viewed October } 2011\end{array}$ & 56 \\
\hline $\begin{array}{l}\text { Figure 2.16: Dome } \\
\text { http://lajourd.com/HTMLs/dome.htm viewed October } 2011\end{array}$ & 57 \\
\hline Figure 2.17: Conceptual cross section of an Iranian Islamic city & 58 \\
\hline $\begin{array}{l}\text { Figure 2.18: Friday Mosque of Kerman, main entrance } \\
\text { http://www.destinationiran.com/gallery/wp-content/uploads/2010/05/kerman-friday- } \\
\text { mosque.jpg viewed October } 2011\end{array}$ & 59 \\
\hline $\begin{array}{l}\text { Figure 2.19: Friday Mosque of Kerman, decorative coverage of walls } \\
\text { http://cascolytravel.com/images/28122.jpg viewed October } 2011\end{array}$ & 59 \\
\hline Figure 3.1: Thesis structure & 69 \\
\hline Figure 3.2: Research methodology structure & 74 \\
\hline Figure 3.3: A sample model to show the CF of local products & 83 \\
\hline Figure 4.1: Flows of energies and materials in an organism & 101 \\
\hline Figure 4.2: Culture cycle (UIS, 2010:20) & 105 \\
\hline Figure 4.3: Framework for Cultural Statistics Domains & 107 \\
\hline Figure 4.4: The interaction between forms of capital and the protection of welfare & 111 \\
\hline Figure 4.5: Integration of FCS cultural domains and indicators & 118 \\
\hline Figure 4.6: Tourism, FCS cultural frameworks, and SNZ themes and indicators & 119 \\
\hline Figure 4.7: Community tourism planning model (Reid et al, 2004:625) & 121 \\
\hline Figure 4.8: Comprehensive framework for sustainability and its segments & 136 \\
\hline $\begin{array}{l}\text { Figure 4.9: Relationship between ecotourism, culture of the host society, environment and eco- } \\
\text { nomic system }\end{array}$ & 142 \\
\hline $\begin{array}{l}\text { Figure 4.10: A model to evaluate interaction between cultural, environmental and economic } \\
\text { changes caused by ecotourism development }\end{array}$ & 143 \\
\hline
\end{tabular}




\begin{tabular}{|c|c|}
\hline Figure 4.11: Cultural footprint (CF) model & 144 \\
\hline $\begin{array}{l}\text { Figure 4.12: CF model used for comparison between the CFs of two different Ecotourism ac- } \\
\text { tivities }\end{array}$ & 147 \\
\hline Figure 4.13: Structure of the comprehensive framework proposed in Chapter 4 & 157 \\
\hline $\begin{array}{l}\text { Figure 5.1: OCRT, Otago region, New Zealand } \\
\text { http://www.linz.govt.nz/sites/default/files/survey-titles/land-registration/land- } \\
\text { districts/images/land-districts.jpg viewed August } 2012\end{array}$ & 163 \\
\hline $\begin{array}{l}\text { Figure 5.2: OCRT, Otago, South Island, New Zealand } \\
\text { http://www.centralotagonz.com/rail-trail-map viewed August } 2012\end{array}$ & 164 \\
\hline $\begin{array}{l}\text { Figure 5.3: OCRT, Naseby and Cromwell, Otago, New Zealand } \\
\text { http://www.centralotagonz.com/rail-trail-map viewed August } 2012\end{array}$ & 165 \\
\hline $\begin{array}{l}\text { Figure 5.4: Clyde, Otago, New Zealand } \\
\text { http://upload.wikimedia.org/wikipedia/commons/thumb/8/84/Panorama_of_Clyde,_Otago,_Ne } \\
\text { wZealand.jpg/640px-Panorama_of_Clyde,_Otago,_New_Zealand.jpg viewed September } 2012\end{array}$ & 165 \\
\hline $\begin{array}{l}\text { Figure 5.5: Middlemarch, Otago, New Zealand } \\
\text { http://www.otagorailtrail.co.nz/index.php?option=com_content\&task=view\&id=26\&Itemid=57 } \\
\text { \#photos1 viewed August } 2012\end{array}$ & 166 \\
\hline Figure 5.6: OCRT visitor numbers (2009-2011) & 167 \\
\hline Figure 5.7: OCRT- average visitor nights (2009-2011) & 167 \\
\hline Figure 5.8: OCRT visitor home place -2009 & 168 \\
\hline Figure 5.9: OCRT- visitor home place- 2010 & 169 \\
\hline Figure 5.10: OCRT visitor home place- 2011 & 169 \\
\hline Figure 5.11: OCRT- visitors (2009-2011) & 170 \\
\hline Figure 5.12: OCRT- domestic visitors (2009-2011) & 172 \\
\hline Figure 5.13: OCRT- domestic and international visitors (2011) & 173 \\
\hline $\begin{array}{l}\text { Figure 5.14: OCRT- Frequency of local produce offered by accommodation services to visitors } \\
\text { (2011) }\end{array}$ & 180 \\
\hline $\begin{array}{l}\text { Figure 5.15: OCRT- Percentage of type and available bed spaces by type of accommodation } \\
\text { service }\end{array}$ & 184 \\
\hline Figure 5.16: OCRT- RB and NB buildings used as accommodation services & 186 \\
\hline Figure 5.17: OCRT- RB and NB accommodation services - available bed spaces (2011) & 186 \\
\hline Figure 5 18: OCRT- number of RB and NB accommodation services & 187 \\
\hline $\begin{array}{l}\text { Figure 5.19: OCRT- capacity of RB and NB accommodation services - total capacity } \\
=5,543 \text { bed spaces (2011) }\end{array}$ & 187 \\
\hline Figure 5.20: OCRT- accommodation - bed spaces by type of materials used & 189 \\
\hline Figure 5.21: OCRT- accommodation services- materials used by type of accommodation (2011) & 190 \\
\hline Figure 5.22: OCRT- SC accommodation services- energy source, facilities and spaces (2011) & 192 \\
\hline $\begin{array}{l}\text { Figure 5.23: OCRT- B\&B accommodation services- energy sources, facilities and spaces } \\
\text { (2011) }\end{array}$ & 193 \\
\hline Figure 5.24: OCRT- Bp accommodation services- energy sources and facilities (2011) & 194 \\
\hline Figure 5.25: OCRT- Motel accommodation services- energy sources and facilities (2011) & 195 \\
\hline Figure 5.26: OCRT- Homestead accommodation services- energy sources and facilities (2011) & 196 \\
\hline Figure 5.27: OCRT- Hotel accommodation services- energy sources and facilities (2011) & 197 \\
\hline $\begin{array}{l}\text { Figure 5.28: OCRT- Camping site accommodation services- energy sources and facilities } \\
\text { (2011) }\end{array}$ & 198 \\
\hline $\begin{array}{l}\text { Figure 5.29: OCRT- All types of accommodation services- energy sources, facilities and open } \\
\text { air spaces (2011) }\end{array}$ & 199 \\
\hline $\begin{array}{l}\text { Figure 5.30: Comparison between OCRT- NB and RB accommodation bed spaces in terms of } \\
\text { energy sources, facilities and open air spaces }\end{array}$ & 200 \\
\hline Figure 5.30.a: Embodied energy of OCRT- types of construction (2011) & 214 \\
\hline Figure 5.31: The annual EF/visitor night of OCRT- accommodation services & 225 \\
\hline $\begin{array}{l}\text { Figure 5.32: OCRT- frequency of indoor activities offered by accommodation services to visi- } \\
\text { tors }\end{array}$ & 232 \\
\hline $\begin{array}{l}\text { Figure 5.33: Percentage frequencies of OCRT outdoor activities offered by accommodation } \\
\text { services to visitors (2011) }\end{array}$ & 233 \\
\hline $\begin{array}{l}\text { Figure 5.34: Frequency of the subjects to visit offered by OCRT accommodation services to } \\
\text { visitors (2011) }\end{array}$ & 234 \\
\hline Figure 5.35: OCRT- the EF of visitor activities (2011) & 251 \\
\hline Figure 5.36: OCRT- the EF per visitor of activities (2011) & 252 \\
\hline Figure 5.37: comparison between the EF of OCRT visitor & 253 \\
\hline
\end{tabular}




\begin{tabular}{|c|c|}
\hline $\begin{array}{l}\text { Figure 5.38: Comparison between the EFs of golf, curling, walking and other OCRT visitor } \\
\text { activities (2011) }\end{array}$ & 253 \\
\hline $\begin{array}{l}\text { Figure 5.39: Comparison between the numbers of OCRT visitors who engage in golf, curling, } \\
\text { walking and other activities (2011) }\end{array}$ & 254 \\
\hline Figure 5.40: The EF (gha/visitor) of golf, curling, walking and other activities & 254 \\
\hline $\begin{array}{l}\text { Figure 5.41: Comparison between the EF categories of OCRT- transportation, food, accommo- } \\
\text { dation services and visitors activities (2011) }\end{array}$ & 255 \\
\hline $\begin{array}{l}\text { Figure 5.41.a: The average EF of OCRT- visitors and global tourism, and the goal for sustain- } \\
\text { able tourism }\end{array}$ & 256 \\
\hline Figure 5.42: OCRT- GDP and GDPs & 263 \\
\hline Figure 5.43: The cultural footprint (CF) of OCRT visitors' food & 267 \\
\hline $\begin{array}{l}\text { Figure 5.44: The CF of OCRT accommodation services- using RB buildings and V/B as parts } \\
\text { of accommodation services }\end{array}$ & 274 \\
\hline Figure 5.45: The CF of OCRT visitor activities & 278 \\
\hline $\begin{array}{l}\text { Figure 6.1: Naseby-Central Otago- New Zealand } \\
\text { http://www.no1guide.co.nz/images/map/Map_of_Central_Otago.jpg viewed August } 2012\end{array}$ & 284 \\
\hline $\begin{array}{l}\text { Figure 6.2: A view of Naseby } \\
\text { http://www.odt.co.nz/files/story/2010/04/naseby_township_nestled_close_to_the_naseby_fore } \\
\text { s1659 772314.JPG viewed August } 2012\end{array}$ & 285 \\
\hline $\begin{array}{l}\text { Figure 6.3: Ancient Briton Hotel, Naseby } \\
\text { http://www.otagorailtrail.co.nz/images/stories/ancientbritonold.jpg viewed August } 2012\end{array}$ & 286 \\
\hline Figure 6.4: Ancient Briton Hotel, location in Naseby & 286 \\
\hline $\begin{array}{l}\text { Figure 6.5: Ancient Briton Hotel, Naseby- } 2012 \\
\text { http://www.historic.org.nz/corporate/registersearch/Register/data/3214a_lg.jpg viewed August } \\
2012\end{array}$ & 287 \\
\hline $\begin{array}{l}\text { Figure 6.6: Ladies' curling rink of the Mount Ida Curling Club }{ }^{1} \text {, Naseby, built in } 1878^{2} \\
\text { 1. http://nzetc.victoria.ac.nz/etexts/Cyc04Cycl/Cyc04Cycl0606a(h280).jpg viewed August } \\
2012 \\
\text { 2. http://www.ancientbriton.co.nz/curling.htm viewed August } 2012\end{array}$ & 287 \\
\hline $\begin{array}{l}\text { Figure 6.7: Naseby’s indoor curling rink } \\
\text { http://4.bp.blogspot.com/_HwUk2QL7wc/TDrrWj5dZpI/AAAAAAAABZc/Y9ytVNHfe0/s400 } \\
\text { curling wide.jpg viewed August } 2012\end{array}$ & 288 \\
\hline $\begin{array}{l}\text { Figure 6.8: Naseby outdoor curling } \\
\text { http://www.odt.co.nz/files/story/2010/07/competition_for_the_baxter_cup_was_hot_yesterday_ } \\
\text { d4c399d2244.JPG viewed August } 2012\end{array}$ & 288 \\
\hline $\begin{array}{l}\text { Figure 6.9: Naseby - cycling track } \\
\text { http://www.maniototo.co.nz/wpcontent/uploads/2009/06/Biking-outlook-from-rear-landscape- } \\
\text { comp.jpg viewed August } 2012\end{array}$ & 288 \\
\hline $\begin{array}{l}\text { Figure 6.10: Naseby - walking track } \\
\text { http://www.lugenz.com/photos/Theres-so-much-to-do-in-Naseby/Walk\%20the\%20forest.jpg viewed } \\
\text { August } 2012\end{array}$ & 288 \\
\hline $\begin{array}{l}\text { Figure 6.11: Naseby- percentage of type and available bed spaces by type of accommodation } \\
\text { service }\end{array}$ & 297 \\
\hline Figure 6.12: Naseby- Refurbishe & 299 \\
\hline vices (2011) & 299 \\
\hline Figure 6.14: Naseby - bed spaces by type of materials used (2011) & 300 \\
\hline Figure 6.15: Naseby- used materials by types of accommodation services (2011) & 301 \\
\hline Figure 6.16: Naseby SC accommodation services - energy sources, facilities and spaces (2011) & 302 \\
\hline $\begin{array}{l}\text { Figure 6.17: Naseby B\&B accommodation services - energy sources, facilities and spaces } \\
\text { (2011) }\end{array}$ & 303 \\
\hline $\begin{array}{l}\text { Figure 6.18: Naseby hotel accommodation services - energy sources, facilities and spaces } \\
\text { (2011) }\end{array}$ & 304 \\
\hline $\begin{array}{l}\text { Figure 6.20: All types of Naseby accommodation services - energy sources, facilities and } \\
\text { spaces }\end{array}$ & 305 \\
\hline $\begin{array}{l}\text { Figure 6.21: Comparison between NB and RB bed spaces in terms of energy sources, facilities } \\
\text { and open air spaces (2011) }\end{array}$ & 306 \\
\hline $\begin{array}{l}\text { Figure 6.22: Naseby - frequency of indoor activities offered by accommodation services to visi- } \\
\text { tors (2011) }\end{array}$ & 318 \\
\hline $\begin{array}{l}\text { Figure 6.23: The frequency of outdoor activities offered by Naseby accommodation services to } \\
\text { visitors }\end{array}$ & 319 \\
\hline Figure 6.24: Naseby- frequency of subjects to visit offered by accommodation services & 320 \\
\hline
\end{tabular}




\begin{tabular}{|c|c|}
\hline Figure 6.25: Naseby- EF of visitor activities (2011) & 327 \\
\hline Figure 6.26: Comparison between EFs of Naseby indoor and outdoor activities & 328 \\
\hline $\begin{array}{l}\text { Figure 6.27: Naseby- Comparison between EFs of golf, curling, walking, and other activities } \\
\text { excluding these three }\end{array}$ & 329 \\
\hline $\begin{array}{l}\text { Figure 6.28: Naseby- Comparison between number of visitor activities for golf, curling, walk- } \\
\text { ing, and other activities }\end{array}$ & 329 \\
\hline $\begin{array}{l}\text { Figure 6.29: Comparison between EFs of Naseby for transportation, food, accommodation ser- } \\
\text { vices, and visitor activities (2011) }\end{array}$ & 330 \\
\hline Figure 6.30: Naseby- comparison between GDP and GDPs & 333 \\
\hline Figure 6.31: The cultural footprint (CF) of Naseby related to food & 337 \\
\hline $\begin{array}{l}\text { Figure 6.32: The CF of Naseby accommodation services using RB buildings and V/B as part of } \\
\text { accommodation services }\end{array}$ & 342 \\
\hline Figure 6.33: The CF of Naseby visitor activities & 348 \\
\hline $\begin{array}{l}\text { Figure 6.34: Cromwell, Central Otago, New Zealand } \\
\text { http://www.centralotagonz.com/PicsHotel/CentralOtagoRTO/Images/Discovery/RailTrail/Hots } \\
\text { potMap.jpg viewed August } 2012\end{array}$ & 350 \\
\hline $\begin{array}{l}\text { Figure 6.35: A view of Cromwell } \\
\text { http://cromwellsports.files.wordpress.com/2010/05/cromwell.jpg viewed August } 2012\end{array}$ & 350 \\
\hline $\begin{array}{l}\text { Figure 6.36: Cromwell Chinese miners } \\
\text { http://www.cromwell.org.nz/gfx/popup/chinese.jpg viewed August } 2012\end{array}$ & 351 \\
\hline $\begin{array}{l}\text { Figure 6.37: The Clyde Dam } \\
\text { http://www.niwa.co.nz/sites/default/files/imported/attachments/92967/Clyde-Dam.jpg viewed } \\
\text { August } 2012\end{array}$ & 352 \\
\hline $\begin{array}{l}\text { Figure 6.38: Old Cromwell Town } \\
\text { http://oldcromwelltown.files.wordpress.com/2012/02/old-cromwell-cavalcade.jpg viewed Au- } \\
\text { gust } 2012\end{array}$ & 352 \\
\hline $\begin{array}{l}\text { Figure 6.39: Old Cromwell Town } \\
\text { http://farm3.staticflickr.com/2449/4065341439_ce1516df36_z.jpg?zz=1 viewed August } 2012\end{array}$ & 353 \\
\hline $\begin{array}{l}\text { Figure 6.40: Cromwell- fruit picking } \\
\text { http://img.scoop.co.nz/stories/images/0903/a3c9ec856fb8af54f8b9.jpeg viewed August } 2012\end{array}$ & 353 \\
\hline Figure 6.41: Percentage and available bed spaces by type of accommodation service (2011) & 362 \\
\hline Figure 6.42: Cromwell - bed spaces by type of materials used (2011) & 363 \\
\hline Figure 6.43: Cromwell - materials used by type of accommodation service (2011) & 364 \\
\hline $\begin{array}{l}\text { Figure 6.44: Cromwell SC accommodation services - energy sources, facilities and spaces } \\
\text { (2011) }\end{array}$ & 365 \\
\hline $\begin{array}{l}\text { Figure 6.45: Cromwell B\&B accommodation services - energy source, facilities and spaces } \\
\text { (2011) }\end{array}$ & 366 \\
\hline $\begin{array}{l}\text { Figure 6.46: Cromwell Motel accommodation services - energy sources, facilities and spaces } \\
\text { (2011) }\end{array}$ & 366 \\
\hline Figure 6.47: Cromwell camping sites - energy sources, facilities and spaces (2011) & 367 \\
\hline Figure 6.48: Cromwell accommodation services - energy sources, facilities and spaces (2011) & 368 \\
\hline $\begin{array}{l}\text { Figure 6.49: The frequency of outdoor activities offered by Cromwell accommodation services } \\
\text { to its visitors }\end{array}$ & 378 \\
\hline $\begin{array}{l}\text { Figure 6.50: The frequency of subjects offered by Cromwell accommodation services to visit } \\
\text { (2011) }\end{array}$ & 379 \\
\hline Figure 6.51: Cromwell - EF of visitor activities (2011) & 386 \\
\hline Figure 6.52: Comparison between EFs of Cromwell indoor and outdoor activities & 387 \\
\hline $\begin{array}{l}\text { Figure 6.53: Cromwell - Comparison between EFs of golf, curling, walking, and other activities } \\
\text { excluding these three }\end{array}$ & 388 \\
\hline $\begin{array}{l}\text { Figure 6.54: Cromwell - comparison between number of visitor activities for golf, curling, } \\
\text { walking, and other activities }\end{array}$ & 388 \\
\hline $\begin{array}{l}\text { Figure 6.55: Comparison between EFs of Cromwell for transportation, food, accommodation } \\
\text { services, and visitor activities (2011) }\end{array}$ & 389 \\
\hline Figure 6.56: Cromwell - comparison between GDP and GDPs & 392 \\
\hline Figure 6.57: The cultural footprint (CF) of Cromwell visitor food & 396 \\
\hline Figure 6.58: The CF of Cromwell accommodation services with V/B as part of the used spaces & 400 \\
\hline Figure 6.59: The CF of Cromwell visitor activities & 406 \\
\hline Figure 6.60: The CF of Naseby and Cromwell related to Food & 408 \\
\hline Figure 6.61: The CFs of Naseby and Cromwell related to accommodation services & 411 \\
\hline Figure 6.47: The CFs of Naseby and Cromwell related to visitor activities & 413 \\
\hline
\end{tabular}




\begin{tabular}{|c|c|}
\hline List of Appendices & Page \\
\hline $\begin{array}{l}\text { Appendix 1: NZTS 2015: forecasted activity necessary to achieve anticipated priorities (cited } \\
\text { in Table 2.2) }\end{array}$ & 434 \\
\hline $\begin{array}{l}\text { Appendix 2: NZTS 2015: forecasted activity necessary to achieve anticipated priorities (cited } \\
\text { in Table 2.3) }\end{array}$ & 435 \\
\hline $\begin{array}{l}\text { Appendix 3: NZTS 2015: forecasted activity necessary to achieve anticipated priorities (cited } \\
\text { in Table 2.4) }\end{array}$ & 436 \\
\hline $\begin{array}{l}\text { Appendix 4: NZTS 2015: forecasted activity necessary to achieve anticipated priorities (cited } \\
\text { in Table 2.5) }\end{array}$ & 438 \\
\hline Appendix 5: OCRT- accommodation facilities, area and materials & 439 \\
\hline Appendix 6: OCRT- types of accommodation services (2011) & 491 \\
\hline Appendix 7: OCRT- cultural products and activities (2011) & 508 \\
\hline $\begin{array}{l}\text { Appendix 8: OCRT- accommodation services -materials used by type of accommodation } \\
\text { service }\end{array}$ & 511 \\
\hline $\begin{array}{l}\text { Appendix 9: OCRT- SC (self contained) accommodation services, energy sources and facili- } \\
\text { tates }\end{array}$ & 513 \\
\hline Appendix 10: OCRT- B\&B accommodation services, energy sources and facilities & 514 \\
\hline Appendix 11: OCRT- Bp accommodation services, energy sources and facilities & 515 \\
\hline Appendix 12: OCRT- motel accommodation services, energy sources and facilities & 516 \\
\hline Appendix 13: OCRT- homestead accommodation services, energy sources and facilities & 516 \\
\hline Appendix 14: OCRT- hotel accommodation services, energy sources and facilities & 517 \\
\hline Appendix 15: OCRT- camping accommodation services, energy sources and facilities & 518 \\
\hline Appendix 16: OCRT- all types of accommodation services, energy sources and facilities & 519 \\
\hline Appendix 17: OCRT- all accommodation services by type - energy sources and facilities & 520 \\
\hline Appendix 18: OCRT- accommodation services- area per bed space (2011) & 520 \\
\hline Appendix 19: OCRT- accommodation services-average area of outdoor sitting spaces & 522 \\
\hline Appendix 20: OCRT- types of outdoor activities and their frequency & 523 \\
\hline Appendix 21: Visits offered by OCRT- accommodation services to visitors (2011) & 524 \\
\hline Appendix 22: Naseby- all types of accommodation services - energy sources and facilities & 526 \\
\hline Appendix 23: Naseby- self contained accommodation services, energy sources and facilities & 526 \\
\hline Appendix 24: Naseby- B\&B accommodation services, energy sources and facilities & 527 \\
\hline Appendix 25: Naseby- hotel accommodation services, energy sources and facilities & 527 \\
\hline Appendix 26: Naseby- camping accommodation services, energy sources and facilities & 527 \\
\hline Appendix 27: Naseby- all accommodation services by type- energy sources and facilities & 527 \\
\hline Appendix 28: Naseby- cultural products and activities & 528 \\
\hline $\begin{array}{l}\text { Appendix 29: Cromwell- self-contained accommodation services, energy sources and facili- } \\
\text { ties }\end{array}$ & 529 \\
\hline Appendix 30: Cromwell- B\&B accommodation services, energy sources and facilities & 529 \\
\hline Appendix 31: Cromwell- motel accommodation services, energy sources and facilities & 530 \\
\hline Appendix 32: Cromwell- camping accommodation services, energy sources and facilities & 530 \\
\hline Appendix 33: Cromwell- all types of accommodation services, energy sources and facilities & 531 \\
\hline Appendix 34: Cromwell- cultural products and activities & 532 \\
\hline Appendix 35: Cromwell- outdoor activities & 533 \\
\hline Appendix 36: Cromwell- subjects offered to visit and their fre & 533 \\
\hline
\end{tabular}




\begin{tabular}{|c|c|}
\hline \multicolumn{2}{|r|}{ Glossary } \\
\hline AT: & Alternative Tourism \\
\hline B\&B: & Bed and Breakfast \\
\hline BBQ: & Barbecue \\
\hline BC: & Bio Capacity \\
\hline Bp: & Backpacker \\
\hline C: & Capacity (number of bed spaces) \\
\hline cap: & Capita \\
\hline CBET: & Community Based Ecotourism of Thailand \\
\hline CF: & Cultural Footprint \\
\hline CODC: & Central Otago District Council \\
\hline CPC: & Central Product Classification \\
\hline CTD: & Community Tourism Development \\
\hline DOC: & Department Of Conservation \\
\hline eaNNp: & Environmental Adjusted Net National Income \\
\hline ECF: & Economic Footprint \\
\hline EF: & Ecological Footprint \\
\hline EL: & Electricity \\
\hline FCS: & The Framework for Cultural Statistics \\
\hline GDP: & Gross Domestic Products \\
\hline GDPs: & Sustainable Portion of GDP \\
\hline gha: & Global Hectare \\
\hline GJ: & Gigajoule \\
\hline GNP: & Gross National Product \\
\hline CSP: & Tidal and Concentrating Solar Power \\
\hline GST: & Goods, Services Tax \\
\hline HS: & Harmonized Commodity Description and Coding System \\
\hline ICATUS: & International Classification of Activities for Time-use Statistics \\
\hline ICH: & Intangible Cultural Heritage \\
\hline IFACCA: & The International Federation of Arts Councils and Culture Agencies \\
\hline Int: & Internet \\
\hline ISCO: & International Standard Classification of Occupations \\
\hline ISIC: & International Standard Industrial Classification \\
\hline kWh: & Kilowatt Hour \\
\hline MCMS: & The Ministry of Culture, Madrid, Spain \\
\hline MJ: & Megajoule \\
\hline NB: & New Building \\
\hline NGO: & Non Governmental Organisation \\
\hline NI: & North Island \\
\hline NV: & Number of Visitors \\
\hline NRC.U.S: & National Research Council (U.S.) \\
\hline NZCTU: & New Zealand Council of Trade Unions \\
\hline NZTS: & New Zealand Tourism Strategy \\
\hline OCRT: & Otago Central Rail Trail \\
\hline PV: & Solar photovoltaic panel \\
\hline OECD: & Organisation for Economic Development and Co-operation \\
\hline QCPA: & Quantity of Cultural Product \\
\hline RB: & Refurbished Building \\
\hline SC: & Self-Contained \\
\hline SCT: & Sustainable Community Tourism \\
\hline Sg: & Genuine Saving \\
\hline SI: & South Island \\
\hline SNZ: & Statistics New Zealand \\
\hline So: & Solar Water Heating System \\
\hline SPC: & Secretariat of the Pacific Community \\
\hline TAT: & Tourism Authority of Thailand \\
\hline TIES: & The International Ecotourism society \\
\hline T-km: & Tourism-kilometre \\
\hline UIS: & The United Nations Education Scientific and Cultural Organisation \\
\hline
\end{tabular}




\begin{tabular}{|l|l|}
\hline UN: & United Nations \\
\hline UNCSD: & United Nations Commission on Sustainable Development \\
\hline UNDP: & United Nations Development Programme \\
\hline UNEP: & United Nations Environment Programme \\
\hline V/B: & Veranda/ Balcony \\
\hline WCED: & World Commission on Environment and Development \\
\hline WCOCAD: & The World Commission on Culture and Development \\
\hline WWF: & World Wildlife Fund \\
\hline
\end{tabular}




\section{Chapter 1: Abstract and Introduction}

\subsection{Thesis Abstract}

This thesis proposes a composite framework for the evaluation of the environmental impact of tourism development on host destinations. In this study, the environmental impact of tourism is considered as a social-ecological phenomenon that can be categorized into the two aspects of natural and social-cultural impacts. Ecotourism is introduced as a type of sustainable tourism in that its policies and principles for development are based on conservation of environmental heritage (including natural and cultural heritage) via engagement of local people and communities in the tourism development process.

The proposed composite framework arises from the integration of an ecological and a cultural framework for the sustainable development of tourism. This framework places the conservation of natural resources as the main ecological outcome for eco-tourism. The proposed framework uses the ecological footprint (EF) of the main tourism activities and services (including transportation, food and accommodation services) as the ecological indicator for evaluation of ecotourism development to ensure it is ecologically compatible.

Ecological footprint (EF) is a way of measuring environmental impact. It assumes that everything needed for living, including all energy, goods and services can be obtained from land, and that any wastes produced can be absorbed by land (Wackernagel and Rees, 1996:9). Because land productivity varies considerably around the world the average global productivity is the normal measure, and this is called global hectares (gha). The ecological footprint of a product or activity produced/ consumed and conducted by a defined group of people can be measured through the following equation:

EF (gha) = Lifecycle energy use of a given group of people (product and activity produced or conducted) Gigajoules (GJ) / carrying capacity of the Earth (GJ/gha)

The above equation determines the area required to produce resources and to absorb pollutants like carbon dioxide generated in the lifecycle energy use of the product or activity through using fossil fuels (coal, oil and natural gas).

Through using an ecological perspective, this thesis views culture as a system that links a group of people or a community to their surroundings through their use of local materials and energies, and the production and consumption of products from these. The proposed framework determines the main cultural outcomes for ecotourism development such as conservation of heritage and making the host people aware of their cultural values. In addition, it introduces related activities such as social events, conservation of heritage and producing and consuming local products as contributive activities for achieving the outcomes stated above. Furthermore it investigates and refines a number of indicators that can be used as tools for evaluation of the cultural footprint of the de- 
velopment of ecotourism. Within these, local gross domestic product (GDP) as a socialeconomic indicator arising from tourism activities is also considered.

This thesis concludes by presenting a case study of 'The Otago Central Rail Trail' (OCRT) as an example of ecotourism. The OCRT was introduced in the New Zealand Tourism Strategy (NZTS) for 2015 as a successful model for community-based development of sustainable tourism. The investigation reviews the impacts of the OCRT on natural and cultural heritage by using the framework developed in the thesis and its related ecological and cultural indicators.

Through using the proposed holistic framework and the model for sustainable development of ecotourism, this thesis finds that, influenced by the development of the OCRT, many cultural heritage items including 60 bridges, 3 tunnels, and 78 buildings (used as OCRT accommodation services) have been refurbished. The restoration of this heritage can be considered as a policy that contributes to awareness of OCRT residents of their cultural heritage and identity. Likewise, as a part of the educational process related to ecotourism, this also makes an opportunity for OCRT visitors to learn about the cultural values of their host people.

As shown in Table 5.84, in 2011, the total EF of 11,788 OCRT visitors including the EFs of the four categories of transportation, food, accommodation and activities is 1,617 gha (0.138 gha /visitor). In comparison with the EF of $0.03 \mathrm{gha} /$ visitor for sustainable tourism (see Table 5.86), the overshoot portion of the EF of the OCRT is equivalent to $(0.138-0.03) 0.108$ gha/ visitor. Likewise the total overshoot EF of 11,788 OCRT visitors is (11,788 visitors $\times 0.108$ gha) 1,273 gha (see Table 5.86$)$. This overshoot EF of the OCRT indicates that although the project has already been considered as a successful sustainable tourism project, still needs to reduce its total EF by 1,273 gha to be environmentally sustainable.

As determined in Chapter 5 (see Table 5.88), the overshoot portion of the OCRT is calculated as 127,310.4 GJ/year. Since the costs to generate $1 \mathrm{GJ}$ of overshoot energy use through using renewable energy sources (in this case wind + solar) is NZ\$ 19.8, consequently the total cost to generate the overshoot energy use of the OCRT through using the latter systems (wind + solar) is equivalent to $(127,310.4 \mathrm{GJ} \times \mathrm{NZ \$ 19.8)} \mathrm{NZ \$}$ $2,720,746$.

Table 5.90 shows that in 2011, the total GDP of the OCRT is NZ\$ 6,245,289 This means the total cost that must be spent to generate the overshoot portion of the OCRT energy use in a sustainable way (NZ\$ 2,720,746) is $43.6 \%$ of its total GDP (NZ\$ $6,245,289)$. Consequently the sustainable portion of the GDP (GDPs) of the OCRT is (NZ\$ 6,245,289 - NZ\$2, 720,746) NZ\$3,524,543.

The results of this thesis demonstrate that using local products (e.g. home prepared foods) as a sustainable cultural behavior not only contributes to the ability of the host destinations located along the OCRT (such as Naseby and Cromwell, used as further case studies) to present their cultural products, but also contributes to reduce the environmental impacts and increase the economic outcomes of the OCRT. For example, producing $17 \%(13,346.4 \mathrm{~kg})$ of the total of $65165.05 \mathrm{~kg}$ consumed food as home prepared food reduces by $5.5 \%$ the ecological footprint related to the food consumed by OCRT visitors. As determined in Chapters 5 and 6, in three case studies, producing 
home prepared food contributes in a sustainable way to the increasing of the economic outcomes by about NZ\$3.5 / kg of food produced.

This thesis indicates that in all three case studies, using refurbished buildings as accommodation services, which is considered as an activity that contributes to preserving the cultural identity of the host destinations also contributes to reducing of environmental impacts and increasing the economic outcomes related to OCRT. For example in OCRT using $12.9 \%$ of bed space as refurbished buildings reduces by14.5\% (7.3gha) the total 50.5 gha ecological footprint of accommodation services compared with when all accommodation buildings are assumed as new buildings. Chapters 5 and 6 determine that in OCRT using 21,378 $\mathrm{m}^{2}$ of refurbished buildings as accommodation services (12.9\% of total bed spaces) increases by $5 \%$ the sustainable portion of GDP related to accommodation services. In Naseby also using refurbished buildings as accommodation services has the same sustainable ecological, cultural and economic outcomes as for the OCRT as a whole.

The framework indicates the use of open air areas as being a cultural-ecological indicator for evaluation of architecture as being sustainable. Through using the framework, it is determined that using open air areas including balconies and verandas as part of OCRT accommodation buildings exerts environmentally friendly influences on the host destination. For instance in OCRT using 387 square meters of veranda and balcony as part of the $80,356 \mathrm{~m}^{2}$ accommodation buildings decreases by $1.5 \%$ the total ecological footprint (50.5gha) of buildings used as accommodation services in OCRT.

The thesis results demonstrate that an architecture that will contribute to sustainable development through ecotourism has a cultural footprint area in which the architecture contributes to the host societies knowing and preserving their cultural identity, capitals and heritages. Simultaneously, it contributes to reducing the environmental impacts and increasing the economic outcomes of the host societies through ecotourism. This thesis determines that since all ecological, cultural and economic characteristics of sustainable architecture are linked together, a strategy to develop sustainable architecture is successful when it considers the linkage between all of these profiles and influences that they exert on each other.

\subsection{Introduction}

This thesis proposes a comprehensive framework and related indicators that can be used as tools to explore and evaluate the main environmental, cultural and economic characteristics of an architecture that contributes to sustainable development through ecotourism. The need for such a comprehensive framework arises from the experiences of the author who was involved with planning tourism projects in his home country between 1995 and 2007. This work revealed that the lack of a holistic strategy for tourism development meant the environmental, cultural and economic heritage and capitals of the host destinations were impacted by tourism. Likewise, one of the main goals of tourism 
development and its architecture, the goal of human well-being, can be ignored due to the lack of a comprehensive approach to tourism and its development.

This thesis involves three main subjects: sustainability, ecotourism and architecture. Since sustainability is considered as an umbrella that covers all human activities and products, in this thesis sustainability and its policies and principles for sustainable development are considered as factors that make a link between ecotourism and its related architecture.

In addition, the holistic framework for sustainable development of ecotourism and architecture arises from the dominant polices and principles of sustainability. This study explores the current examples of the strategies (for example the New Zealand Strategy for the Sustainable Development of Tourism) and the proposed models for sustainable development of tourism, and determines the knowledge gap in this field that must be bridged for sustainable development of ecotourism and its related architecture. In Chapter 2, sustainability and its related policies and principles for development and strategies for the sustainable development of ecotourism and architecture are investigated. The literature review in Chapter 2 indicates lack of a comprehensive framework as the main knowledge gap for the sustainable development of ecotourism and architecture.

\subsubsection{Sustainability}

Sustainability is introduced as a multi-dimensional phenomenon that involves the three categories of: environment, society and economy. In sustainable development these three categories overlap to preserve environmental resources, promote and conserve socio-cultural capitals and heritage and develop the economic system in a way that responds to the needs of present and future generations.

Sustainable development is considered as a process in which all components of a given society are engaged in environmental, cultural and economic development through a social and educational process. Arising from this perspective the main anticipated outcomes for sustainable development are categorised into the three categories of: environmental, socio-cultural and economic outcomes.

The conservation of natural resources is viewed as the main environmental outcome for sustainable development that is considered a social-ecological phenomenon rather than 
merely a technologically based process. Likewise achieving the environmental outcome for sustainable development is linked with other aspects of social and economic policies operated for development. In this thesis the main cultural outcomes for sustainable development are considered as awareness of local and indigenous people about their environmental and cultural capitals and heritage, and engagement of these culturally educated people in planning, management and monitoring of the sustainable development process. The main economic outcomes for sustainable development are introduced as equal distribution of natural, cultural and economic capitals among all components of a given society and engagement of them in a horizontal economic development.

In this thesis all of the mentioned above outcomes for sustainable development are linked together and contribute to each other during the course of operation.

\subsubsection{Ecotourism}

In this thesis ecotourism is considered as a sustainable part of mass tourism that follows the main principles of sustainable development. The main anticipated cultural outcome for the sustainable development of ecotourism is explained as awareness of people about their environmental and cultural capitals and heritage. Ecotourism is introduced as a set of purposeful nature based activities that has the ability to change the attitude of its host society to environmental, social and economic problems caused by development of capitalism. This can be viewed as influences exerted by ecotourism on its host destination towards being more sustainable. In this thesis these influences are defined as the cultural footprint of ecotourism and its products and activities on the host destination.

\subsubsection{Architecture}

Architecture is viewed as one of the ecotourism products that can contribute to sustainable development through ecotourism. In this thesis a given host destination or community is explained as an organism that has spiritual and physical interaction with its surrounding environment. In addition, architecture is explained as part of the social products that contribute to the interaction between society and its surrounding environment towards being sustainable through using spaces, materials and renewable source of energy in a sustainable way. The main cultural outcomes for architecture used as one of the ecotourism products are the same as the anticipated cultural outcomes for sustainable development of ecotourism. These outcomes include awareness of people about their cultural and environmental capitals and heritage and engagement of local people in 
the sustainable development process through architecture used in ecotourism development.

\subsubsection{Knowledge Gap}

This thesis investigates the shortage of a comprehensive framework for assessing the sustainable development of ecotourism and its related architecture that can be used as a tool to determine the main ecological, cultural and economic characteristics of these two sustainable phenomena. On the other hand the shortage of a holistic strategy for ecotourism development and its architecture that has the ability to make a link between the main characteristics of both ecotourism and architecture as being sustainable is introduced as the main knowledge gap in these fields.

\subsubsection{Key Research Question}

Since the author of this thesis has already been involved with planning and development of rural and urban tourism and ecotourism in his home country, Iran, from 1988 to 2007, this has revealed how the negative impacts of uncontrolled tourism development and its modern products, such as architecture, can change the lifestyle of the host destination in a unsustainable way. It has also been determined that the top down approach to the planning, management and monitoring of a process like tourism development is not efficient when the major roles of local and indigenous people are ignored in whole process.

Moreover, it has been determined that the sustainable development of tourism and its architecture needs a holistic strategy beyond the economic and political goals arising from the development of capitalism. As some of the currently used modernised tourism products exert negative impacts on environmental resources, traditional lifestyle and cultural heritage of the host societies in Iran, the main key question has been formed as:

\section{What are the main characteristics of an architecture that will contribute to sus- tainable development through ecotourism?}

\subsubsection{Comprehensive Framework}

The comprehensive framework proposed for sustainable development of ecotourism and its products and activities is separated into the two sections:

1) a framework for ecotourism development 
2) a framework for sustainable development of architecture (which here contains accommodation services) used in ecotourism development.

The comprehensive framework indicates the main environmental, cultural and economic outcomes for ecotourism and its architecture, and proposes the productive activities that can contribute to achieving these outcomes. The comprehensive framework is conceptualised in a holistic model that has the ability to make a link between the social behaviours of the host societies and visitors, their environmental impacts and economic footprint. On the other hand the holistic model proposed for sustainable development of ecotourism and its products (including architecture as a part of the used built up land) determines an area introduced as their cultural footprint area formed by the socialcultural behaviours of the ecotourism participants, and their ecological and economic footprints.

This thesis uses three case studies in Otago, New Zealand: the Otago Central Rail Trail (at regional scale), and the settlements of Naseby and Cromwell (at urban scale) that are considered host destinations for rural and urban New Zealand ecotourism (soft ecotourism). The proposed framework and holistic model for sustainable development of ecotourism and architecture will be tested in these three case studies to determine the linkage between ecological, cultural and economic characteristics of ecotourism and architecture in terms of sustainability.

In the 2009 provisional proposal for this thesis, Iran and New Zealand were proposed as the case studies for comparison of the sustainable development of tourism. The main reason for choosing these two countries was the differences between their environmental circumstances, cultural background, heritage and capitals, and economic systems. Both countries attended the 1992 Rio summit and approved Agenda 21 as their main policy for development. Consequently it was anticipated the outcomes for tourism development in both countries would follow a similar strategy. However, as the environmental, cultural and economic activities in the two countries are different, the productive activities contributing to achieving sustainable development of tourism could be different. For example, in New Zealand development of wineries could be seen as an activity that contributes to achieving conservation of cultural heritage and development of cultural capital, while in Iran, development of the Iranian rug (as a handcraft) or production of saffron (in north-east Iran) could play a similar role. Consequently the main 
anticipated outcomes for both Iran and New Zealand could be same but the setting, priorities and productive activities to achieve the forecasted outcomes would be different.

Over a year after the research was started the Iranian government issued a statement that no overseas thesis could include an Iranian case study. There was a consequent urgent need to select a second case study. In addition to the original New Zealand OCRT case study, Naseby and Cromwell were selected as two locations within the OCRT for separate investigation, and together these formed the second case study. These two places were chosen because they have different cultural heritage, environmental circumstances and attractions for visitors (as explained in the thesis). The differences between Naseby and Cromwell in terms of these aspects allow the proposed framework for sustainable development of ecotourism and its related architecture to be tested against the OCRT as a whole.

\section{I.2.7. Thesis Structure}

This thesis contains seven Chapters comprising introduction (Chapter 1), literature review (Chapter 2), research methodology (Chapter 3), comprehensive framework (Chapter 4), first case study (Otago Central Rail Trail Chapter 5), second and third case studies (Naseby and Cromwell Chapter 6) and conclusion (Chapter 7).

The next Chapter investigates the three areas of sustainability ecotourism and architecture, the relationship between these areas, and the knowledge gap in terms of sustainable development of ecotourism and its architecture. The final section of Chapter 2 introduces the key research questions of this thesis which are integrated in its main key research question briefly explained in section 1.2.5. 


\section{Chapter 2: Literature Review}

This chapter reviews literature on sustainability, ecotourism and architecture as the three main components considered in this thesis. The aim here is to identify: the relationship between these areas the factors that exert influence on this interaction, and uncover the knowledge gap in relevant policies and strategies for the development of ecotourism and its related architecture through sustainability. The discussion begins with a definition of sustainability, this is followed by explanation of its necessity, and its models, characteristics, goals, principles, framework and related indicators.

The literature review continues with a definition of ecotourism, introducing its environmental, social and economic effects on the host areas. It continues with an explanation of strategies for the sustainable development of tourism and its subsectors, such as ecotourism, by using the New Zealand Tourism Strategy 2015 (NZTS 2015) as an example. The next part of this literature review discusses sustainable architecture and its mutual relationship with environmental, societal and economic systems. Examples are given through use of the clustered villages of Nuristan in Afghanistan and the historic city of Kerman in Iran as examples of vernacular architectures and traditional urban fabrics. At the end, this literature review explains the main factors that make a link between ecotourism and its related architecture; it discusses the shortage of the writings that focus on ecotourism's architecture, and presents the key question of this thesis.

\subsection{Sustainability}

This thesis views sustainability as a key factor that plays an important role in defining ecotourism and architecture and in determining the main policies and strategies for their development. The reasons for this are discussed below.

\subsubsection{Definition of Sustainability}

Appleby (2011:7) argues that the roots of modern sustainability and related energy policy lie in the scenario proposed by the 18th century political economist Thomas Malthus, commonly known as the Malthus Catastrophe or Nightmare. In this scenario Mal- 
thus forecasted that "population growth would outpace agricultural production" (Appleby, 2011:7). Since the onset of the industrial revolution in the latter parts of the $18^{\text {th }}$ century and the subsequent development of capitalism, numerous catastrophic events have happened in the world (for instance famines and conflict in African countries such as Ethiopia and the Sudan) that suggest the truth of the 'Malthus Catastrophe or Nightmare’ as people struggle for control of insufficient resources.

The World Commission on Environment and Development (WCED) has put forth the definition of sustainability as "meeting the needs of the present without compromising the ability of future generations to meet their needs” (Kim and Rigdon, 1998:6). This concept of sustainable development, which was established in the Brundtland Report (WCED, 1987), attempts to support continuous development of human societies for the present and future generations. "The concept also emphasizes balance of growth in social, economic and environmental aspects which are regarded as the key attributes that support the development of a sustainable society.” (Robin and Poon, 2009:3616). However, this very definition raises questions, as 'growth' in environmental aspects within a finite system like planet earth is itself not a sustainable activity.

As the Board on Sustainable Development of the U.S. National Academy of Science identifies, through sustainable development "what is to be sustained fall into three major areas of nature, life support system and community” (NRC.U.S, 1999:23). They also identify intermediate categories for each such as earth, environment and cultures. Likewise, "what is to be developed [through sustainable development], also fall within three major areas of people, economy and society” (NRC.U.S, 1999:25).

\subsubsection{The Necessity for Sustainability}

Nowadays, humans are faced with different issues like uncontrolled growth of population, dramatically expanding urban areas and, consequently, increasing needs to use more energy resources, as well as degradation of the environmental heritage and unequal distribution of capital.

Due to the industrialisation and the expansion of modernisation, urban areas throughout the world have increased in size and population. According to Omer (2008:2272), about $50 \%$ of the world's population are urban dwellers. In addition, he (2008:2272) reveals 
that all population growth expected between 2000 and 2030 will be concentrated in urban areas because of the rural-to-urban shift of populations. Furthermore, with an expected annual growth of $1.8 \%$, the world's urban population will double in 38 years. In terms of resource consumption, at present $2 \%$ of the land surfaces in the world are covered by cities, yet the people living in them consume $75 \%$ of the resources consumed by humankind. Omer (2008: 2272) points out that the ecological footprint of cities is many times larger than the areas they physically occupy. Continued growth of the world's population has caused serious need to use all sources of energy, particularly fossil fuels like oil and gas. As many researchers such as Appleby (2011:7) point out if everyone in the world lived like the average North American "we would need a planet five time bigger that the earth to live on” (Appleby, 2011:7). This is obviously an unsustainable situation.

\subsubsection{Models of Sustainability}

Vale and Vale (2009:10) discuss the two models for portraying the meaning of sustainability; the 'weak model' and the 'strong model' of sustainability. According to Vale and Vale (2009:10) in the weak model of sustainability, the three main components of sustainable development, the environment, human society and the economy are represented by three intersecting circles of equal size and values (Figure 2.1).

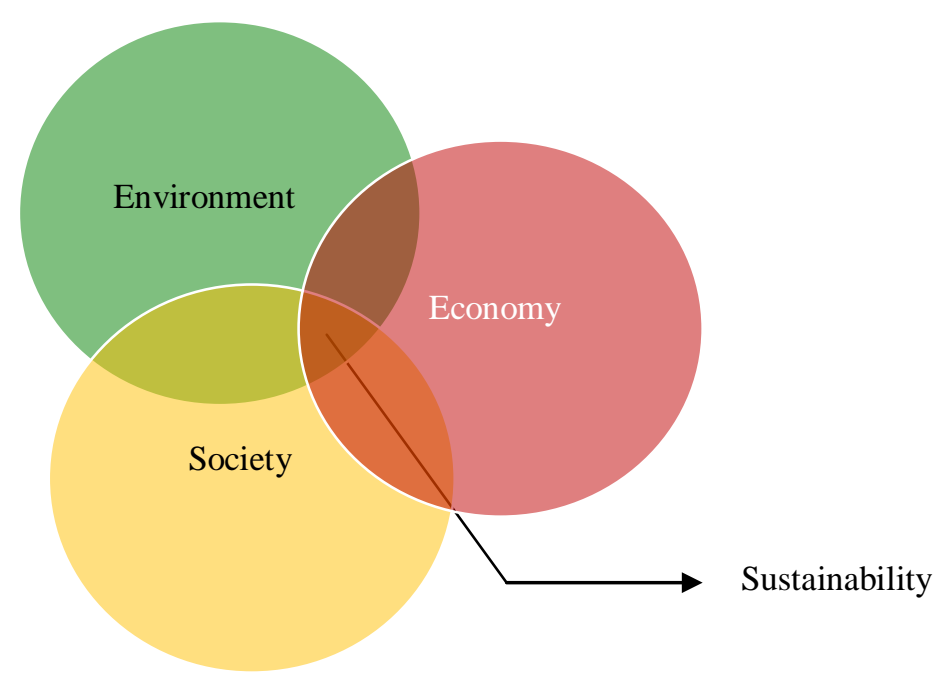

Figure2.1: Weak model of sustainability

In the weak model of sustainability, the three main components are portrayed as competing interests so that degradation of one can be compensated for by improvement in 
another. In the strong sustainability model, as explained by Vale and Vale (2009:10) the environment is considered as a circle in which society (as a base for human activities) is set (Figure 2.2). In this model, the environment is considered as the main foundation, which strongly exerts influences on society, culture and all their subsectors such as the economy, architecture, politics, art etc. (Figure2.2).

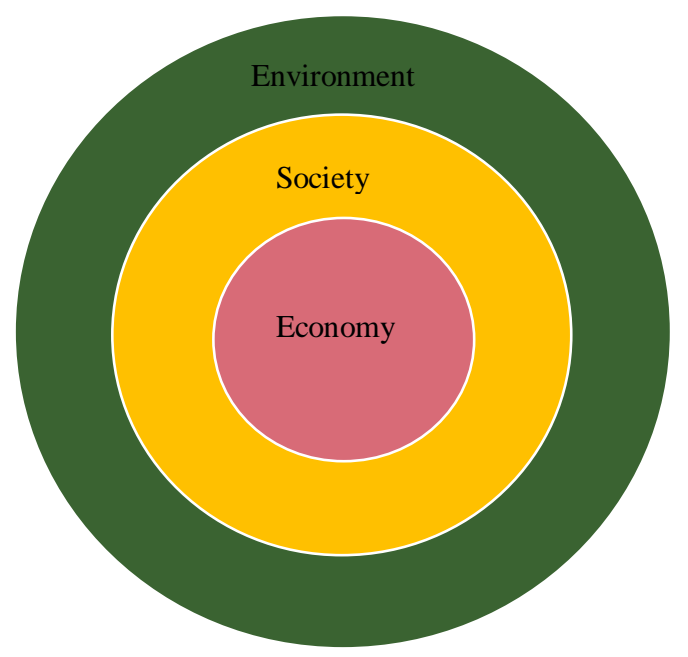

Figure 2.2: Strong model of sustainability

As a reason to make an effort to move towards environmental conservation through sustainable development as a priority policy, it could be conjectured that "if we destroy the environment, which sustains us and provides our air, water and food, then we will destroy human society; if we destroy human society; we will certainly destroy the economy” (Vale and Vale, 2009:11).

Although the main anticipated outcome for sustainable development is environmental conservation, its process must be started from economic development in a sustainable way which is conducted by educated societies. Natural capital, physical or produced capital, human capital and social capital are four factors that determine sustainable development. "Sustainability or the capability of future generations to meet their needs is ensured when the total stock of these assets remains constant or is increased in the production process” (Veron, 2001:602). As Graff et al (1995:205) reveal, in sustainable development, economic, cultural and social developments are interrelated. They (1995:205) argue that in sustainable development environmental problems cannot be isolated from others such as poverty and social disintegration. Likewise, economic growth makes poverty alleviation easier and cultural development can change the pat- 
tern of the consumption of natural resources in order to ensure their protection. Furthermore, social education and development can also exert influences on economic growth in an economically sustainable way. For example, decentralisation and democratization in developed societies may lead local economic systems toward more equal distribution of capital among the local participants.

Environmental sustainability exerts positive impacts on development of human capital because all humans are part of nature. Thus, the environment plays an important role in ensuring human survival, health, social life, sustainable energy production, food production and access to clean drinking water and clean air. Generally, sustainability is viewed as a phenomenon that is a common area between environmental conservation, cultural restoration and promotion and socio-environmental economic adaptation. Using a social perspective, sustainability can be viewed as a way in which an educated group of people or societies can choose to live in an effort to conserve their environmental and cultural heritage and capitals and develop their economic system. It strives to present a comprehensive strategy which covers all human activities and products and adapt them with sustainable patterns of consuming the natural and cultural resources in the development process.

\subsubsection{Fundamental Characteristics of Sustainability}

Culture, environment and economy, as categories that are subject to conservation and development in the sustainability process, can be changed in terms of their relative positions depending on different places and times. Consequently, sustainability is an inherently dynamic, indefinite and contested concept. "Sustainable development must, therefore, be seen as an unending process-defined not by fixed goals or the specific means of achieving them, but by an approach to creating change through continuous learning and adaptation” (Mog, 2004: 2139). Therefore, flexibility and adaptability in terms of different cultural, environmental and economic situations are the main factors, which can be used as frameworks to evaluate sustainability. A sustainable development project will be successful if its products (including cultural, natural and economic outputs) are culturally appropriate, economically viable and environmentally sensitive.

To achieve sustainable development, indigenous people and their needs, natural resources and the development of conservation policies to protect them, and local eco- 
nomic systems and their shift toward sustainability must all be viewed as the main components of progressive strategies. If a development project ignores some of these components during its programming, management and implementation, it cannot be called a sustainable project. For example, Mog (2004: 2143) argues that a project that reduces land degradation but at the same time increases poverty or inequality, or achieves its goals through the establishment of rigid institutions (e.g. forcing all farmers off a parcel of land and establishing a strictly exclusionary protected area) would not be considered successful in promoting sustainable development, no matter how great the improvement in environmental quality. On the other hand a project which enhances environmental quality in a culturally sensitive manner (e.g. through extension of appropriate conservation farming techniques) without having any positive impact on poverty, is also grossly insufficient. As a result, projects which create significant positive change in many realms while generating little or no negative impacts can be viewed as sustainable activities.

\subsubsection{Sustainable Development - Goals}

One of the main aims for sustainable development is to reduce the use of non-renewable energy resources. The World Summit on Sustainable Development in Johannesburg in 2002 committed itself to supporting and encouraging the expansion of renewable energy resources to accelerate the shift towards sustainable consumption and production. It could be argued that the main goal for the latter summit was to make productivity independent from resource use. According to Omer (2008: 2270), breaking the linkage between resource use and productivity can be achieved by trying to ensure economic growth does not cause environmental pollution. He (2008: 2270) argues that the main policies for achieving the goal of a pristine environment are; improving resource efficiency, examining the whole life cycle of the product, enabling consumers to receive more information on products and services, and examining taxes, voluntary agreements, subsidies, regulation and information campaigns regarding the best stimulation to innovation. However, this approach still fails to recognise the fundamental problem that there must be a limit to growth within a finite system.

\subsubsection{Sustainability- Principles}

One of the basic strategies to achieve the goals associated with sustainability is to make societies aware of the components and structure of sustainability, its policies and bene- 
fits. In 1991, the World Conservation Strategy (cited in Robin and Poon, 2009:3616) stated the principles of a sustainable society include; respect and care for the community; improvement in the quality of human life; conserving the earth's vitality and diversity; minimising the depletion of non-renewable resources; keeping within the earth's carrying capacity; changing attitudes and practices; enabling communities to care for their own environments; providing a national framework for integrating development and conservation; and creating a global alliance. According to the latter principles, change of attitudes and practices can be viewed as a focal point for cultural shift toward sustainability. Robin and Poon (2009:3616) point out that change of attitudes and practices means a transformation of mind-set and actions. These criteria demand changes in the innate basic assumptions, values, beliefs and behaviour that are present in the established culture of society.

\subsubsection{Sustainability Frameworks and Indicators}

Sustainable development is viewed as a multi-complex phenomenon that rests on three pillars: "economic growth, social progress and protection of the environment and natural resources” (Hueting and Reijnders, 2004:250). Such a complex phenomenon cannot be evaluated without the existence of a comprehensive framework that covers all the components mentioned above. Although different frameworks and indicators like "ecological footprint” (Wackernagel and Rees, 1996) and “Genuine Saving (Sg)” (Pearce et al, 2001) in terms of sustainable development have already been suggested, most of them do not cover all levels of production (global, national or local), environmental conservation or social development. To explain more about indicators, their strengths and weaknesses, some examples are given below.

\subsubsection{Ecological Footprint}

One of the alternative measurement mechanisms which emphasises environmental conservation is the ecological footprint. "The ecological footprint is an accounting tool that estimates the resource consumption and waste assimilation requirements of a given population or economy in terms of corresponding area” (Ness et al, 2007: 502). According to Ness et al (2007:502), ecological footprint is a multi-stage process which attempts to estimate the average person's annual consumption of food, housing, transport, consumer goods and services in terms of the productive land needed to generate it. Munda (2006:87) views the ecological footprint index as an indicator related to the idea 
of urban carrying capacity. In this view, ecological footprint solves some of the problems of traditional carrying capacity simply by inverting the usual carrying capacity proportions. Munda (2006:87) argues that ecological footprint computes the land area required per person (or population) instead of presenting population per unit area.

The main core of the ecological footprint index is that every category of energy, material consumption and waste discharged needs the productive or absorptive capacity of a finite area of land or water. In fact, in terms of ecological footprint, if the land requirements for all categories of consumption and waste discharge by a defined population are summed up, the total area represents that population's ecological footprint. Of course, this area can cover a region many times larger than the home region of the same population. According to Munda (2006:87), the ecological footprint of a specified population or economy is the area that would be required for providing all the energy and material resources consumed, and absorbing all the waste discharged by a given population in a given area.

\section{a. Strengths and weaknesses of the Ecological Footprint}

The Ecological Footprint (EF) index is an efficient way of showing the balance between the quantity of products and environmental carrying capacity in a given area. In some cases it will show how the ecological footprint of a given population can exceed the political frontiers of a country. It means, in the latter cases, economic development, conservation of natural resources and social promotion in a given area and population depend on other countries, with their different economic systems, socio-cultural structures and conservation policies.

Ecological Footprint can also look at components of a lifestyle. Looking at the land used to produce, say, the current diet could cause intensive development of agriculture production systems in an effort to lower the EF. Munda (2006:88) states that intensive agricultural systems can reduce the horizontal expansion of a city but at the same time will significantly increase energy consumption (in terms of intensive agricultural development) and degradation of biodiversity, due to the use of fertilisers and pesticides and the introduction of exotic species. Calculating the EF will reduce all these effects to a single number for comparison with other food producing strategies. 
Table 2.1 sets out the advantages and disadvantages of ecological footprints (EF) as determined by Mayer (2008:286) and Walsh et al (2006:4). As demonstrated in Table 2.1, the EF is a single unit ecological indicator that can be applied to different human activities and products, from food to transportation, to water consumption, to recreational activities and built up land. Since the EF has the ability for continual updating, it can be viewed as an efficient indicator for monitoring and managing the process of development. The EF can be used to raise alarm about the shortcomings of policies related to the conservation of finite natural capitals. It can also be used to make people aware of environmental problems. As shown in this thesis, the EF has the ability to be combined with other social and economic indicators, such as GDP. Its combination with such social economic indicators results in environmentally sensitive economic indicators such as 'Sustainable Portion of GDP’ (GDPs) (See Chapter 4- section 4.1.3.3).

Table 2.1 shows the accuracy and clarity of the EF and its results can also be viewed as its dominant weaknesses. Since the measurement of the EF of a product and activity relies on using related data, most of the shortcomings of the EF arise from the deficiency of the information systems used for producing, managing, collecting and analysing data at regional, national and global scales. On the other hand these weaknesses cannot be considered as innate, but rather explained as weaknesses exerted on the EF by the deficiencies of other factors, such current information systems.

\begin{tabular}{|c|c|}
\hline Advantage of EF & Disadvantage of EF \\
\hline $\begin{array}{l}\text { Single unit indicator that allows disaggrega- } \\
\text { tion. }\end{array}$ & Mostly hypothetical land uses \\
\hline Widely applicable to activities & Can oversimplify resource use \\
\hline Top down or bottom up & $\begin{array}{l}\text { Detailed studies incur uncertainty, particularly at } \\
\text { sub-national level. }\end{array}$ \\
\hline Useful communication tool & Double counting is always a risk. \\
\hline Continually being updated and improved & Mostly snapshots of consumption. \\
\hline $\begin{array}{l}\text { Demonstrates the finite nature of natural capi- } \\
\text { tals }\end{array}$ & $\begin{array}{l}\text { Provides no clear policy guidance except to con- } \\
\text { sume less or create additional productive land. }\end{array}$ \\
\hline Growing credibility as a policy tool & Based on sometimes doubtful assumptions \\
\hline $\begin{array}{l}\text { Measurability and comparability for systems } \\
\text { of any scale, from bio fuel production systems } \\
\text { to urban areas, to nations and the human spe- } \\
\text { cies. }\end{array}$ & $\begin{array}{l}\text { Can be difficult to reconcile annual consumption } \\
\text { with the necessarily much slower provision of } \\
\text { bio-capacity. }\end{array}$ \\
\hline $\begin{array}{l}\text { Addressing intergenerational equity and in- } \\
\text { ternational fairness. }\end{array}$ & $\begin{array}{l}\text { May not be a completely accurate representation } \\
\text { of strong sustainability given some methodologi- } \\
\text { cal shortcomings. }\end{array}$ \\
\hline $\begin{array}{l}\text { Can be used to monitor a system over time if } \\
\text { the index is calculated repeatedly with new } \\
\text { data. }\end{array}$ & \\
\hline
\end{tabular}




\subsubsection{GDP and its Possible Adjustments}

\section{a. GDP}

GDP is a very common macroeconomic indicator, having been used in the United States since 1963 (Bagstad and Shammin, 2012:313). It is a measure of the monetary value of the annual production of goods and services in a domestic location, so covers the natural and human created capital in a particular place, such as a nation. It can be calculated in nominal or real values (Lawn, 2003:106). Lawn further argues that if GDP is measured in nominal values, it is measured in terms of the prices at the time of production and if in real values, in terms of the prices of all goods in a particular year, which is often referred to as the base year. Consequently, differences in the quantity of goods and services produced from year to year can be reflected by annual changes in real GDP.

In 1992, the United Nations Conference on Environment and Development in Rio de Janeiro underlined the limitations of gross domestic product (GDP) as a measure of sustainable development for a country. Agenda21 (1992: paragraph 40.4) found GDP to be an example of common indicators that do not assess the sustainability of an economic system. Rennings and Wiggering (1997:26) suggest that measuring the economic and environmental aspects of sustainable development need to be complementary, while Barrass et al (1997:101) go further by stating that growth in GDP will only align with sustainable development if the environment and the resources it supplies are valued in the same way. The same authors go on to say that conventional ways of assessing GDP tend to undervalue the vital natural resources supplied by the environment, since no account is taken of their replacement times.

Bagstad and Shammin (2012:331) identify five common criticisms of GDP: (1) it consists of simple addition of all expenditure, even expenditure that does not improve social welfare, such as that spent on defence; (2) it excludes the value of unpaid household labour and volunteer work in the community; (3) it fails to account for the value of nonrenewable resource depletion while total resource stocks decline over time; (4) it fails to account for income distribution, poverty, and the costs of inequality; (5) it lacks inclusion of the positive contributions from natural, human and social capital types. 


\section{b. Adjustments to GDP}

Given the shortcomings of GDP, economists have proposed adjustments to it to make it a better measure of social wellbeing. One of the notable works is Daly and Cobb's (1989) index of Sustainable Economic Welfare (ISEW) which was later revised as the Genuine Progress Indicator (GPI). ISEW/ GPI is a composite indicator which includes economic, social and environmental indicators. Examples of the former are "Personal consumption per capita” and "Value of household labour”, social indicators include "Cost of crime" and "Loss of leisure time" and environmental indicators are based on measurables such as "Cost of air pollution" and "Loss of forest cover" (Bagstad and Shammin, 2012:331). GPI calculation includes the cost of environmental degradation as well as factors for natural capital regeneration but is still arbitrary in what is included and excluded (Pulselli et al, 2006:273).

In addition Pulselli et al argue that since ISEW/GPI is composed of a list of items which indicate economic welfare or sustainability, ISEW/ GPI cannot be used as an indicator of the real level of economic welfare at the same time as being an indicator of sustainability. As explained by Pulselli et al (2006:273) ISEW/GPI does not really achieve its anticipated outcomes as it was based on the strong sustainability model within which it is not possible to substitute human capital for natural capital. Furthermore the authors go on to say they believe ISEW/GPI does not make a clear distinction between national and natural capitals or between the two forms of natural resources (non-renewable and renewable). Nourry (2008:447) also points out that in ISEW/GPI, the incorporation of the cost of environmental degradation is not sufficient to determine sustainability or otherwise, so it cannot be strictly considered as an indicator of sustainable economic welfare.

\subsubsection{Sustainability Assessment}

Ness et al (2007:500) have suggested another sustainability assessment tool that consists of three umbrellas or general categorisation areas (Figure 2.3). These areas are indicators and indices, which are further broken down into non-integrated and integrated product-related assessment tools. In the non-integrated assessment the focus is on the material and/or energy flows of a product or service from a life cycle perspective, while the integrated assessment is a collection of tools usually focused on policy change or project implementation. 


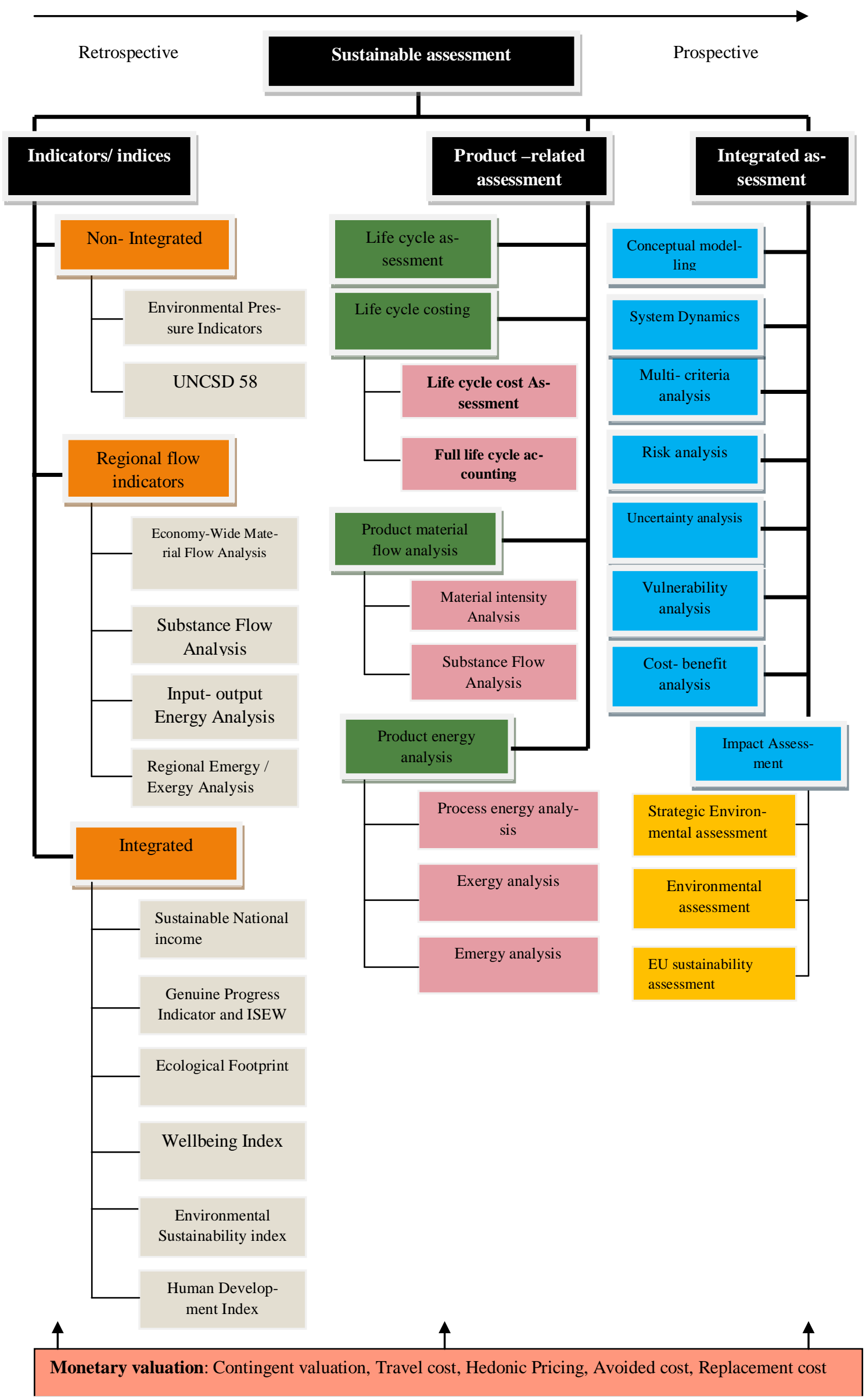

Figure 2.3: Framework for sustainability assessment tools (Ness et al, 2007:500) 
There is also the overarching category at the bottom of the figure used when market values are needed in the three categories. In Figure 2.3, the proposed assessment tool framework is based on the temporal focus of the tool along with its objective focus. The arrow at the top of the framework shows the temporal focus, which is either retrospective (indicators/indices), prospective (integrated assessment) or both (product-related assessment).

The objective focus of the tools is either spatial, referring to a proposed change in policy (indicators/indices and integrated assessment), or at the product level (product-related assessment). The monetary valuation tools on the bottom are used when monetary valuations are needed in the above tools. "Thick lines around the boxes mean that these tools are capable of integrating nature-society systems into a single evaluation” (Ness et al, 2007:500).

\subsubsection{Genuine Saving (Sg)}

Genuine saving ( $\mathrm{Sg}$ ) is a proposed economic indicator for sustainability which is defined by Pearce et al (2001), (cited in Hueting and Reijnders, 2004:258). The term is a version of environmentally adjusted net national income (eaNNP) which includes adjustment for damages, compensation and depletion. The general equation is given below.

eaNNp $=\mathrm{C}+\mathrm{Sg}$

This is analogous to gross savings ' $S$ ' in the definition equation of gross national product $(\mathrm{GNP}=\mathrm{C}+\mathrm{S})$ and also analogous to net savings $\mathrm{Sn}(=\mathrm{S}-\mathrm{dK})$ in net national product $(\mathrm{NNP}=\mathrm{C}+\mathrm{Sn})$. In these expressions, $\mathrm{C}=$ consumption, $\mathrm{S}=$ gross saving and $d K=$ depreciation of produced assets. Consequently, $S g=S-d K-r(R-G)-p(E-A)$, where $\mathrm{r}=$ unit resource rent (defined as the difference between the price obtained for a unit of extracted or harvested resource and its marginal costs of extraction or harvesting); $R=$ resource extraction or harvest; $G=$ natural growth rate of the resource (zero for nonrenewable); $\mathrm{p}=$ marginal social damages from pollution; $\mathrm{E}=$ emissions; $\mathrm{A}=$ natural assimilation (i.e., dissipation) of pollutants; and $r(R-G)$ and $p(E-A)$ are the values for depreciation of natural resources and the value of net pollution damage, respectively. 
Hueting and Reijnders (2004:258) argue that welfare depends on total stocks of produced, natural and human assets. Moreover, they argue that the combination of labour (technology) and elements from physical surroundings (the environment) give produced capital. Consequently this system is dependent on only two factors, human beings and the environment. As a result, a production level that guarantees conservation of vital environmental functions with available technology is an unavoidable policy for environmentally sustainable development.

Hueting and Reijnders (2004:258) have suggested some conditions for the calculation of the Sg indicator and for versions of the related eaNNp. Some examples of these conditions are summarised here. First, any increase in human assets must be used exclusively for environmental protection and/or for growth of production that does not (further) damage the environment. Secondly, likewise increases in stocks of produced assets must be exclusively for the purpose of environmental protection or 'clean' growth. Thirdly, resource revenues must be invested in environmental protection or 'clean' growth. These conditions are hard to satisfy because, for instance in relation to the first and second conditions, "expenditures on environmental protection check production growth" (Hueting and Reijnders, 2004:258) and "it is precisely the most environmentally damaging sectors of the economy that account for the bulk of production growth" (Hueting and Reijnders, 2004:258).

A sustainable development framework should be viewed as a multi-dimensional umbrella in which all sectors are represented by efficient indicators for evaluating economic, social and environmental development. If there are any conditions attached to using these indicators, these conditions must be practical in terms of using the available technologies and should not neglect the fact that sustainability is based on multidirectional development. Thus, a multi-dimensional framework is of paramount importance for a correct indicator framework for sustainability.

\subsection{Ecotourism}

\subsubsection{Definition of Ecotourism}

Like sustainability, ecotourism is a concept that seems easy to recognise as a broad idea but is difficult to define in detail. 


\subsubsection{General Views of a Definition Ecotourism}

At present there is no consensus on the definition of the term ecotourism, and its identification and usage remain associated with a great deal of confusion and disagreement. Buckley (2009:2) states that the earliest known use of the term 'eco-tour', undefined, seems to have been by Parks Canada in the 1960s. Wearing (2008:6) states that ecotourism is recognised as one of the segments of the tourism industry which is growing three times faster than other parts. This definition only puts stress on the speed of growth as a main difference between ecotourism and the other main sector of mass-tourism. Because of this, this definition cannot clarify and cover all the different dimensions of ecotourism such as its relationship with the economic system, environment and people.

According to Blangy and Mehta (2006:233), the International Ecotourism Society (TIES) views ecotourism as responsible travel to natural destinations which protects the environmental resources and develops the well-being of the indigenous people. This definition is complex as it brings together the idea of both 'untouched' landscape and an 'untouched' people within it, recognising that both may be changed by ecotourism.

In 1980, Ceballos Lascurain (cited in Weaver, 2009:3) introduced ecotourism as tourism that involves travelling to relatively undisturbed or uncontaminated areas with the specific objective of studying, admiring, and enjoying the scenery and its wild plants and animals, as well as any existing cultural manifestations (both past and present) found in these areas. This definition only deals with a pristine landscape, ignoring to a large extent those who live in it. In addition, areas which have already been developed for other purposes are omitted from ecotourism development in this definition.

\subsubsection{Location and Definition of Ecotourism}

The location where ecotourism occurs is one of the factors which give rise to different opinions about its definition. For instance, in contrast with Lascurain whose definition emphasizes undisturbed or uncontaminated areas as locations where ecotourism can happen, Weaver (1999:795) argues that while such environments (for example protected areas like national parks) for obvious reasons clearly constitute a desirable context for appreciation of natural attractions, there is no a priori basis for excluding modified landscapes attractive to certain types of wildlife, such as reservoirs, managed forests, communal or private lands adjacent to parks, and some types of agriculture lands as ecotour- 
ism destinations. According to this view, even the remaining "changed" land and water areas have the ability to accommodate demand for ecotourism and other recreational pursuits. Furthermore, once a pristine landscape becomes an eco-tourist destination it is no longer pristine, and must be changed. This suggests that ecotourism cannot just rely on undisturbed landscape.

\subsubsection{Sustainability and Definition of Ecotourism}

Sustainability is the essential characteristic of ecotourism which basically becomes its main difference with regard to other segments of the tourism industry. More recent definitions have proclaimed some particularity for ecotourism such that it "must be economically viable, ecologically sensitive, and culturally appropriate” (Wall, 1997, p.483). According to this definition, if ecotourism and its development cause negative impacts on the environmental resources, visitors, host communities and their economic systems, it cannot be defined as “ecotourism”.

Perhaps the most restrictive definition is given by Butler (cited in Weaver,1999:794) who insists that ecotourism must simultaneously satisfy eight descriptive criteria, including consistency with a positive environmental ethos, biocentrism, enhancement of the resource base, attainment of educational benefits, and conveyance of high informational and emotional dimensions. Although Butler has introduced these rigorous parameters to clarify the definition of ecotourism, it is clear that, among all segments of mass-tourism, very few activities would qualify as “ecotourism” within his criteria.

Against this idea that sustainability is an umbrella that covers all ecotourism products and policies, several qualifications have been suggested as the basis for introducing a liberal ecotourism model. For example, Weaver (1999:794) argues that any insistence that ecotourism must not negatively affect the environment or host society is unrealistic, since it is impossible to ensure, categorically, that a particular visit or resort will not result in any significant long-term negative consequences. An emphasis on sustainability in intention is thus more realistic than an insistence on sustainability in outcome. But, in contrast to this view, Khan (2003:111) considers ecotourism as purposeful time spent in an environment in a sustainable way that attempts to learn other culture and help economically its host communities that work toward conservation of ecosystems. 


\subsubsection{Definition of Ecotourism as a Segment of Alternative Tourism}

Ecotourism has come to be closely associated, and is even sometimes synonymous, with equally controversial and much debated terms such as sustainable tourism and alternative tourism. Buckley (2009:18) defines ecotourism as a part of a much broader tourism product sector, which includes nature, wild life, adventure, cultural and perhaps also farm or rural tourism. Ecotourism is viewed by Weaver (1999:797) as a segment of alternative tourism. Weaver (1999:797) also argues that alternative tourism (AT) is commonly defined, at least in its ideal configuration, as a locally controlled sector adhering to vernacular architectural norms and attracting a more allo-centric or community minded type of client. Figure 2.4 depicts an interpretation of the popularly perceived relationships among the various types of tourism.

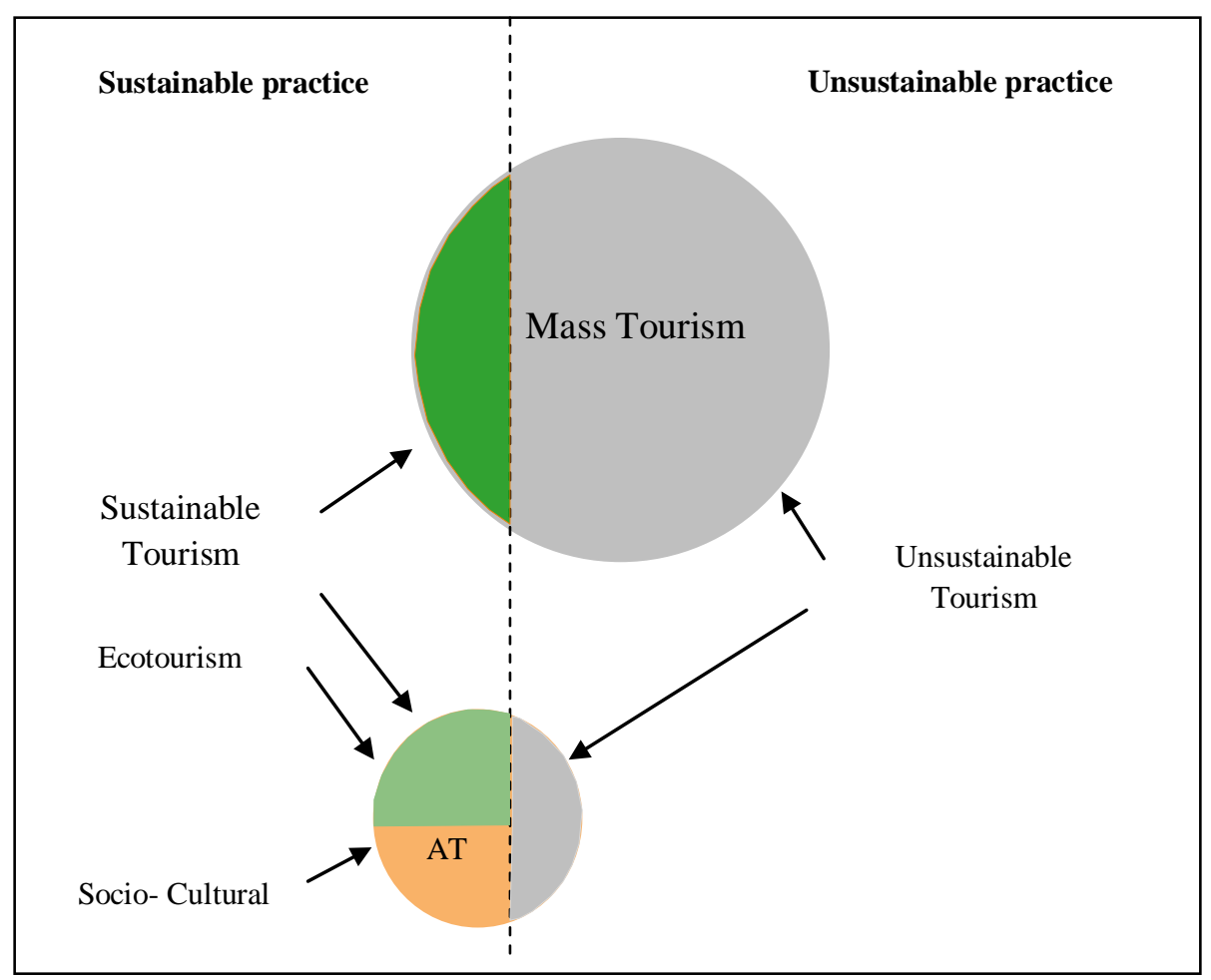

Figure 2.4: Perceived relationship between sustainability, mass tourism, alternative tourism (AT), and ecotourism

According to Weaver (1999:798), internally alternative tourism may be separated into nature oriented and socio-cultural components, depending on whether the stress is on natural or "authentic" cultural attractions. However, since the above perspective of ecotourism requires coherence with sustainability, then only those instances of naturebased AT that are situated on the sustainable side of Figure 2.4 can be classified as ecotourism. 
In contrast to the latter classification, according to several recent definitions (Blangy and Mehta, 2006:233; Wall, 1997, p.483; and Weaver and Lawton, 2007:170), ecotourism must be viewed overall as a sustainable activity which directly attempts to protect and develop both environmental potential and socio-cultural structure in the host areas. Consequently, ecotourism is similar to sustainable AT and has an ability to cover other sustainable segments of mass tourism.

\subsubsection{Ecotourism and Environmental Heritage}

The relationship between tourism and the environment and the environmental implication of the development of tourism can be polarized into both negative and positive aspects. For example, on one side, “conservationists see both [tourism] developments and tourists themselves as threats to survival of wildlife and of treasured natural resources" (Coppock, 1982:270). Pigram (1980:559) points out that even some researchers such as Cohen (1978), who conceded the existence of some beneficial effects from tourism, particularly in the economic, political and cultural spheres, appear convinced that, "in the long run, tourism like any other industry, contributes to environmental destruction” (Cohen 1978: 220).

On the other side, researchers such as Weaver and Lawton (1999:16) believe that alternative tourism as one part of mass tourism can be considered to be the legitimate form of tourism, and the only one that could be considered sustainable. They (1999:16) argue that special mention should be made of ecotourism, which is widely defined as a variant of alternative tourism that puts primary emphasis on the natural environment to be used and protected as the basis for product attraction. In addition, ecotourism "posits that the basis of interaction with the natural environment is one of inherent appreciation and/ or educational interests, and not merely as a suitable setting for a hedonistic or thrill seeking experience (as in sunbathing and white-water rafting, respectively)” (Weaver and Lawton, 1999:16).

There is no debate over the fact that uncontrolled development of tourism can exert negative influences on natural conservation. Coppock (1982:272) categorizes these impacts into five categories comprising loss of habitat; damage caused by the pressure of human feet or vehicles on soil and vegetation; damage to flora and fauna by fire; damage to, or destruction of, flora and fauna by pollution; and disturbance of fauna, espe- 
cially birds and mammals. In addition, some other negative impacts such as increasing the ecological footprint of the host destination as influenced by food consumed, transportation used, an increase in built up land, water consumed and the sewage generated resulting from tourism can be added to these other categories.

May (1991:113), in a general view, separates the impacts of tourism into two types, these being those which are associated with structures (hotels, roads, aircraft etc.) and those resulting from tourists themselves. Likewise, Coppock (1982:272) reveals that the impacts of tourism on nature conservation are the products of conscious actions taken by developers, promoters, planners or visitors and that some are the unintended and often unconscious by-products of tourism.

Many researchers, such as Ferrell and Runyan (1991:26) and Marcouiller et al (2004:1034), view the natural environment as a crucial potential for the attractiveness of almost all travel destinations and recreation areas. May (1991:114) reveals that much tourism occurs in locations which are particularly sensitive to change, e.g. rivers, forests and lakes. According to Ferrell and Runyan (1991:30) it is characteristics of most natural features that they are physically altered by use. They (1991:30) suggest that positive change should be the goal and negative change, which even in small amounts can be critically damaging, should be omitted or reduced.

To clarify the relationship between ecotourism and environmental conservation, Buckley (2009:149) views minimizing impacts on the natural environment as a key criterion for its evaluation. On the other hand, one of the main goals for developing sustainable ecotourism is to protect ecosystems which provide the services essential for human activity. In terms of ecotourism, an ecosystem "in short can be described as supporting life, supplying materials and energy, and absorbing waste products” (Gössling, 1999:303).

Although it is clear that human life seriously depends on conservation of natural resources, currently, particularly in developing and industrialized countries, natural resources are decreasing dramatically as the result of human activities. For example, according to Gössling (1999:310), tropical rainforests, which contain more than half of all species in the entire world and provide a range of essential services, are the biome in 
greatest danger, declining by rather more than 150,000 km2 per year. Moreover, current protective policies and programmes cannot cover all valuable natural resources.

Gössling (1999:310) states that in 1993, less than 5\% of tropical forests is protected within parks and reserves. Furthermore, exploitation of environmental heritage, consumerism, and extremely high per capita demand for resources also support the loss of biodiversity. Gössling (1999:304) further argues that the latter problems are caused by tourist activities, like air travel which consumes fossil fuels and leads to significant emissions, a lack of successful economic systems, and weaknesses of the present policies to value nature and its profits. Indeed, the root cause of the exploitation of natural resources is substantially a multi-dimensional problem and needs a comprehensive solution.

Sustainable ecotourism as a multi-dimensional phenomenon could be viewed as a means of mitigating the socio-economic situation at both local and international scale, and supporting environmental conservation. Development of ecotourism and other segments of mass-tourism, without effective policies to manage and monitor the process of development, causes issues such as loss of biodiversity, and expansion of poverty in the adjacent and other areas which are not covered by development programmes. To explore and introduce some of the latter issues, it is useful to look at the example of Costa Rica, a country which is widely cited as a popular ecotourism destination.

\subsubsection{Case Study of Costa Rica}

Costa Rica is a small country which has $51,100 \mathrm{~km}^{2}$ of land surface, being only $0.03 \%$ of the earth's surface. It is an ideal reference point for tropical ecology in the world. Costa Rica has abundant majestic wet and dry forests, impenetrable coastal and inland wetlands, wild rivers, productive estuaries, fog and rain-shrouded mountains, and active volcanic landscapes that support some of the most impressive and dense biodiversity found on the planet. "Costa Rica ranks about 20th among nations of the world in terms of its biodiversity. Costa Rica has 28.2 species of terrestrial vertebrates per $1000 \mathrm{~km}^{2}$. By contrast, Ecuador, which has the richest biodiversity in the world in absolute species number, has only 9.2 species of vertebrates per 1000 km²” (Kohlmann et al, 2008:255). Because of its fortuitous tropical setting, Costa Rica has also served as an important lo- 
cation for agricultural production, including crops such as coffee, bananas, pineapples, wood and wood products.

In terms of its protected areas, nearly $23 \%$ of Costa Rica's land area is under some sort of public park system. In 2007, the Costa Rican Tourism Institute reported that "because of its incredible ecological systems, Costa Rica has also attracted more ecotourists and adventure travellers per square kilometre than other countries in the world (22.5 international eco-tourists/ $\mathrm{km}^{2}$ )” (Kohlmann et al, 2008:256). In fact, Costa Rica’s biodiversity has been a major reason for tourists and eco-tourists to visit this country and has contributed to making ecotourism the primary source for foreign currency generation. Kohlmann et al. (2008:256) states that during the last decade, ecotourism has surpassed coffee, bananas and beef production, which have been three traditional export products of Costa Rica. In 2007, tourism generated US\$ 1,895 million, while coffee generated US\$ 255 million and banana production generated US\$ 674 million in foreign currency.

In Costa Rica, natural resources have been used as the basic tools for development, but use of these alone will not contribute to complete conservation policies in the protected areas. On the other hand, a number of inefficient policies for development of ecotourism and conservation of natural resources have caused some problems which now have to be solved by Costa Rica's government and related organizations. In Costa Rica, unequal distribution of services is one of the problems in the protected areas. According to Weaver (1999:800), of approximately 23,000 to 25,000 total accommodation rooms, about $40 \%$ are located in San Jose and its environs, 30\% in the Pacific province of Puntarenas (mostly as beach hotels) and 15\% in the North-Western province of Guanacaste (also mainly as beach hotels). Most of the remainder are small facilities located within other provinces. Furthermore, the number of specialized “ecolodge” type rooms is around 500 , or about $2 \%$ of the total inventory. These statistics expose that the output of the system in Costa Rica is likely to be depletion of capital and destruction of natural resources in one place and the exclusion of other places from ecotourism development.

In Costa Rica, partly as a result of financial constraints and the lack of compatible alternatives to traditional economic activities, there are several conflicts with the local population in the protected areas. For instance, Bernard et al. (2009:175) reveals that with the 
change of the Rio Macho Reserve into a National Park in 2000, local activities such as coffee growing, livestock keeping, sheep and goat breeding, firewood collection, and harvest of mosses became illegal. The alternatives like the cultivation and harvest of wild blackberries, and the cultivation of colder climate fruits or cattle breeding are considered very unsatisfactory to the local people and they continue some of the illegal activities which damage the natural resources.

\subsubsection{Ecotourism and Local Economic Growth}

Sustainable ecotourism development depends on a particular economic system that is based on conservation of natural and cultural heritage and the contribution of local communities so they gain benefits from their sustainable activities. As GLOBE 90 (1990:2) reveal, one of the main goals of sustainable development of tourism and its related subsectors such as ecotourism is to develop greater awareness and understanding of the significant contributions that tourism can make to the environment and economy.

In some societies, particularly among the less developed countries, the shift from traditional to industrial strategies for economic development has caused destruction of the natural and cultural resources. Since post-industrialization, against the governing of people through technocracy, sustainability is viewed as a way of avoiding resource degradation and cultural decadence. In the following section, Thailand is used as a specific example as this is a country which has experienced traditional, industrial and postindustrial modes of economic development.

\subsubsection{A Case Study of Thailand}

Thailand is a country whose economy probably depends on the development of ecotourism. According to Wang (2004:1), 6\% of the Thai GDP is derived from tourism and almost $30 \%$ of the population is employed in tourism and related industries. Furthermore, in Thailand, natural resources are considered the main attractive potentials which can contribute to development of tourism and an environmentally based economic system. Wang (2004:2) argues that there are eight categories of protected areas in Thailand which include: national parks, forest parks, wild life sanctuaries, non hunting areas, national forest reserves, botanical gardens, arboretums, and biosphere reserves. Pipithvanichtham (1997:2) points out that since the establishment of Thailand's first national park in 1961, the number of national parks in Thailand has slowly increased. For exam- 
ple, there were only 16 parks which covered 9,357 km2 in 1979, and this had grown to 45 parks covering 24,222 km2 in 1982.

Between 1950 and 1980, Thailand underwent a massive transformation fuelled by overseas development aid and technical assistance, all facilitated by having a development oriented government. Wester and Yongvanit (2005:746) reveal that this transformation caused an ecological transition in some areas such as Dong Mun that was associated with rapid population growth, expanding demand for resources, increased connectivity and control by external forces and technologies that enlarged expectation, and environmental degradation.

Wester and Yongvanit (2005:738) also point out that until 1965 Thailand was in a preindustrial mode. It had a common property regime which allowed local communities with small populations and limited technology to extract food, fodder, fuel and medicines from the land. According to Wester and Yongvanit (2005:738-739) after that date, forest management shifted to an industrial mode and exploitation of forests was part of the overall plan for regional economic development. This changed policy caused development of industrialised agriculture and infrastructures such as dams and roads and degradation of natural resources like the forests.

According to Wester and Yongvanit (2005:746), in Thailand the industrial mode happened first in the form of licensed timber concessionaires, who extracted the high value old growth wood, and later in the form of a plantation system based on single purpose, low value woods grown on land legally under the jurisdiction of the Royal Forest Department to the exclusion of all others. As a result of this policy, after 1970, severe reduction in the area of designated forest reserves occurred. Wester and Yongvanit further reveal (2005:736) that in 1961 approximately 53\% of the land area was forested. Influenced by industrial development and unsustainable extraction of these resources, by 1986 the forested area had been reduced to $25 \%$ of total land.

After exploitation of natural resources during the industrialization period, the Thai government and related organizations attempted to find efficient strategies to recover and restore the lost environmental heritage while keeping a healthy economic system. 
Since 1976, tourism development has been considered a significant solution to solving the latter issues. Pipithvanichtham (1997:1) points out that between 1976 and 1986 tourism in Thailand became the country's leading industry because of its generated revenue. However, as tourism became increasingly important, several areas which were subject to its development faced resource degradation and cultural decadence. Wang (2004:2) explains that although economic benefits as an output of tourism development have been claimed, outside investors reap the biggest benefits at the expense of the local communities.

The negative impacts of tourism development on the natural resources, local economic systems and socio-cultural structure led the Thai government and other organizations such as the Tourism Authority of Thailand (TAT) to shift their policy towards more sustainable tourism development, focusing on conservation and benefits to local communities. In the new strategy, local communities and indigenous people play an important role. In fact, as Wang (2004:4) suggested, the key to assessing tourism potential for improving nature conservation and community well-being is the direct involvement of local communities and improvement of the linkage between conservation and enterprise development. Consequently, this kind of tourism can be viewed as sustainable ecotourism.

Community Based Eco-tourism of Thailand (CBET) was introduced by Wang (2004) as a strategy whose development is based on the participation of indigenous people. One of the main goals for this system is the contribution to local well-being of the environmental economic development. He further states (2004:3) that the CBET approach is a response to the rising criticism of the negative impact of mass tourism and the abuse of the term ecotourism. CBET has attempted to establish a sustainable method of tourism in natural reserves while providing a source of community-building or development for indigenous participants without exploitation of their natural habitats and cultural structures. Wang (2004:3) points out that there is a difference between community-based ecotourism and other types of tourism because the former focuses on community development and the participation of the marginalized sectors rather than on national or regional development. 
For more explanation about the CBET and its impacts on local societies, Wang (2004:6) chooses to describe two hill-tribe villages from Mai Hong Son Province, Northern Thailand. Mae Huay Hee village has been involved in CBET for more than 5 years and the degree of participation in tourism is high. In Mae Lana, approximately $80 \mathrm{~km}$ away from Mae Huay Hee, the CBET initiative started only one year before the reporting, although the locals had been involved in tourism for almost 10 years. A comparative study between these two villages that were receiving different degrees of tourist activity and experiencing different levels of participation was conducted to judge the impact caused by CBET. The positive impact of CBET which is described (Wang, 2004:7) can be attributed to the low number of tourist activities and the fact it is a controlled activity. In addition, participation of local people in conservative projects and development of an environmental economic system which "is based on the idea that environmental goods and services are scarce” (Graaf et al, 1996:208), are other positive impacts of CBET.

In comparison with other types of tourism, ecotourism and its related activities which have particular characteristics need specific management and consideration to be correctly implemented. Wang (2004:10) reveals that in comparison with other projects, ecotourism projects are hardly likely to succeed immediately and yield immediate profits. Even one successful project may need a lot of time to adapt to a new situation. When there is conflict between the efforts to keep the number of visitors low to minimize cultural and environmental intrusion and the local people's desire to increase their income, alternative forms of livelihood should be encouraged.

\subsubsection{The Potential of Ecotourism}

In conclusion, ecotourism projects should not be viewed as an enterprise that will solve all issues. Consequently, it is necessary for related communities to integrate ecotourism with other alternative productive options to reduce the expectations placed on having one activity alone, and to reduce the tensions that arise from unmet expectations. Wang (2004:12) argues that community-based ecotourism is a "multi-dimensional productive and cultural/social system" which is not an isolated industry from other economic activities. This system will lead to stability in the economy at the national, regional, or community levels. 
Ecotourism is not a 'magic bullet' that will suddenly result in conservation of natural resources at the same time as development and increased foreign revenue. It can skew development within a country, such as the development of a few places within Costa Rica for housing and servicing tourists. At best, ecotourism that gives at least part of its benefits back to the local communities that support it can have positive outcomes but these may be slow to arrive and may not meet the expectations of those involved. In some ways the problems of ecotourism establishment can be seen as a mirror at a small scale of the problems of trying to move the whole world economy on to a much more sustainable basis. Both will only succeed if those involved expect less, rather than more. For the eco-tourist, less might mean less comfortable travel and accommodation and for the local people involved, less might mean a lower level of life style improvement. However, the big benefit of ecotourism is that local natural resources are conserved to a much greater extent, which is the basis for all sustainability.

\subsubsection{Definition of Soft Ecotourism in this Thesis}

Arising from the environmental and economic characteristics of ecotourism discussed above, soft ecotourism can be defined as a part of sustainable tourism that happens in rural and urban places and their adjacent areas. Soft ecotourism aims to make local and indigenous people aware of their environmental and cultural heritage and capital and engage them in the sustainable development process. This process includes conservation of natural resources and social-cultural heritage, production and development of environmental and the social capitals, and development of an ecological-social economic system in a sustainable way.

\subsubsection{Strategy for Sustainable Development of Tourism}

The anticipated goals and outcomes of tourism plans will inevitably determine their role for environmental protection, social and economic development. According to Fennell and Dowling (2003:8), at the beginning of the last two decades, some researchers such as Murphy (1983) and Getz (1986) argued that most tourism goals and planning were oriented towards business interests, economic growth and development. However, influenced by some phenomena such as global warming, degradation of environmental resources, and evolution of sustainability polices, the strategies for tourism development have been oriented to being more comprehensive to cover the multi dimensions of the sustainability of tourism. This thesis uses the New Zealand Tourism Strategy 2015 
(2007), as an example of the recent frameworks, here suggested by the government of New Zealand, for the sustainable development of tourism.

\subsubsection{New Zealand's Tourism Strategy 2015 (2007)}

During the last two decades, New Zealand's attitude to defining the best kind of tourism for the country has changed. "Twenty years ago, the debate about what kind of tourism would be the best for New Zealand largely centered on how many international visitors would be appropriate” (New Zealand Tourism Strategy 2015, 2007:14). Now tourism is viewed as a much more complex phenomenon. For New Zealand's government and related organizations, there are many factors which must be considered to achieve their goal of becoming a sustainable nation and promote the value of tourism to New Zealand's visitors, economy, environment and communities. According to the New Zealand Tourism Strategy 2015 (2007:14) these factors include the money that visitors expend whilst they are in New Zealand, the time of year they come, and the sort of experiences they expect during their travel. In order for the tourism sector to prosper, the New Zealand Tourism Strategy 2015 suggests increasing the amount that each visitor spends during their visit, finding strategies to reduce seasonal fluctuations in visitor arrivals, and providing products that are appropriate for visitors who are concerned about their environmental footprint. The structure of the NZTS 2015 sets out four outcomes needed to achieve these goals, as shown in Table 2.2.

\begin{tabular}{|l|l|}
\hline \multicolumn{2}{|c|}{ Table 2.2: Anticipated outcomes for sustainable development of tourism in New Zealand's Tourism } \\
Strategy 2015
\end{tabular}

Together these four outcomes (Table 2.2) shape a suggested framework for the development of sustainable tourism in New Zealand by 2015. As demonstrated in Table 2.2, the anticipated outcomes for sustainable development of tourism can be categorized into the four categories of promotion of the quality of services and products, economic development in a sustainable way, environmental conservation and engagement of the local communities and related organisations in the development process. In the next part of this Chapter, the four outcomes and their related contributive activities as forecast by 
the New Zealand government for the sustainable development of tourism are explained as an example of a sustainable framework and strategy for tourism.

\section{a. Outcome1: New Zealand Delivers a World-Class Visitor Experience}

World class experiences are authentic, unique and delivered with superb service. To achieve this goal the New Zealand government suggests a range of priorities for its delivery, which are shown in Table 2.3. These priorities can be conceptualised as increasing the quality of planning, management and monitoring of the offered services, facilities and products, and the economic and cultural engagement of local people and communities in the development process (Table 2.3).

\section{Table 2.3: NZTS 2015- setting priorities to deliver a world-class visitor experience (Outcome 1)}

\begin{tabular}{|c|l|}
\hline No. & \multicolumn{1}{|c|}{ Priorities for delivery } \\
\hline 1 & New Zealand is seen by visitors as a desirable, high-quality destination \\
\hline 2 & The New Zealand tourism industry offers booking services that are easy to use \\
\hline 3 & $\begin{array}{l}\text { The I-Site network is recognized by visitors as being a source of high-quality and objective visi- } \\
\text { tor information }\end{array}$ \\
\hline 4 & Qualmark is recognised by consumers as a mark of quality \\
\hline 5 & The tourism sector is committed to monitoring visitor satisfaction and perceptions of quality \\
\hline 6 & New Zealand's infrastructure supports a quality visitor experience at all stages of the journey \\
\hline 7 & Domestic travelers help create a strong demand for visitor products and services \\
\hline 8 & Maori actively participate and invest in the tourism sector \\
\hline
\end{tabular}

The New Zealand government proposes a list of necessary activities related to each priority (cited in Table 2.3) which must be done to achieve that priority. These activities can be summarised as development of investment in tourism at global and domestic scales; promotion of quality and accuracy of researches related to both domestic and international tourism markets; using appropriate technology for each activity, service and product; monitoring the quality of all related activities, facilities and products used; managing and monitoring of the engagement of local and indigenous people (Maori) in the development process, and encouraging them to present their cultural product at word-class level (see Appendix1).

\section{b. Outcome 2: New Zealand's Tourism Sector Is Prosperous and Attracts Ongoing} Investment

Outcome 2 proposes an economic aspect of sustainable development policies for the tourism market in New Zealand. Based on this economic perspective, NZTS 2015 assumes that "the success of New Zealand's tourism sector is ultimately dependent on the performance of the businesses within it. If they are successful, the sector will keep 
growing, which will deliver significant benefits to businesses themselves, to their communities, and to the New Zealand economy as a whole” (NZTS 2015, 2007:30). The New Zealand government also offers five priorities for a successful tourism sector and these are shown in Table 2.4.

\begin{tabular}{|c|l|}
\hline No. & Table 2.4: NZTS 2015- setting priorities for a prosperous tourism sector (Outcome2) \\
\hline 1 & $\begin{array}{l}\text { Tourism businesses identify and put in place strategies that will consistently increase their returns } \\
\text { on investments. }\end{array}$ \\
\hline 2 & Business delivers products and experiences that meet the expectations of high-value visitors. \\
\hline 3 & Tourism attracts and retains an appropriately skilled workforce. \\
\hline 4 & $\begin{array}{l}\text { The tourism sector improves demand during the off-season. } \\
\text { New Zealand's tourism research is accessible, timely, high quality, and relevant to the decisions } \\
\text { that need to be made. }\end{array}$ \\
\hline
\end{tabular}

The main objectives of the proposed economic priorities (Table 2.4) can be considered as a contribution to increasing returns on investments; increasing the quality of products compatible with the expectation of consumers; and increasing the demand for conducting researches in the context of the sustainable tourism strategy.

NZTS 2015 introduces the main actions that must be conducted to achieve the priorities for a prosperous tourism sector. These activities can be summarised as community based actions that aim to improve resource efficiency; work force management; tourism services, products and facilities; and access to researches and information systems. In addition, training and education of the workforces involved is considered to be one of the pillar activities to make tourism economically successful in a sustainable way (see Appendix 2).

Likewise, NZTS 2015 values both international and domestic tourism at the same level and aims to develop them both to improve the economic benefits of tourism in New Zealand. For instance, NZTS 2015 proposes investment in marketing initiatives that target domestic and international visitors who are more likely to travel in the shoulder and off-peak seasons (see Appendix 2). As a result, increasing the number of visitors, both domestic and international, can be viewed as one of the main goals and activities to support the economic development of tourism in New Zealand up to 2015. 


\section{c. Outcome 3: The Tourism Sector Takes a Leading Role In Protecting and En- hancing New Zealand's Environment}

The third anticipated outcome for the sustainable development of tourism demonstrates an ecological aspect of the tourism strategy in New Zealand (Table 2.2). NZTS 2015 views tourism as a phenomenon that can contribute to environmental conservation through its sustainable development. To achieve this goal by 2015, the New Zealand government suggests eight priorities for tourism development in terms of environmental conservation to be followed by the tourism sector and related organisations (Table 2.5). These priorities consist of maintaining environmental resources for the next generations; producing environmentally friendly products through the development process; reducing the carbon emission of the products, services and activities of tourism; using renewable energies in the tourism sector; and management of produced waste in a sustainable way through interaction with other related sectors (Table 2.5).

\begin{tabular}{|c|c|}
\hline No. & Priorities for delivery \\
\hline 1 & $\begin{array}{l}\text { The tourism sector contributes to a whole-of-New Zealand approach to ensure that New Zea- } \\
\text { land's environment will continue to be enjoyed by future generations and visitors, in the spirit of } \\
\text { kaitiakitanga (guardianship) }\end{array}$ \\
\hline 2 & $\begin{array}{l}\text { The expectations of our visitors are clearly understood, and visitors are able to identify and select } \\
\text { products that deliver good environmental performance easily }\end{array}$ \\
\hline 3 & $\begin{array}{l}\text { The tourism sector leads the way in introducing initiatives that will reduce carbon emissions and } \\
\text { increase the energy efficiency of transport within and en route to New Zealand }\end{array}$ \\
\hline 4 & $\begin{array}{l}\text { The tourism sector improves its energy efficiency, energy conservation, and use of renewable } \\
\text { energy at all levels }\end{array}$ \\
\hline 5 & $\begin{array}{l}\text { The tourism sector takes active steps to reduce and manage its waste, encourages other sectors to } \\
\text { do the same, and engages with the local, regional, and national agencies that provide waste man- } \\
\text { agement standards and facilities }\end{array}$ \\
\hline 6 & $\begin{array}{l}\text { Tourism businesses have the capability and knowledge to take a leading role in protecting and } \\
\text { enhancing the environment }\end{array}$ \\
\hline 7 & $\begin{array}{l}\text { The tourism sector, DOC, and other key agencies continue to work together to make sure that } \\
\text { conservation values are enhanced }\end{array}$ \\
\hline 8 & $\begin{array}{l}\text { Visitors' tourism experiences give them the opportunity to learn about and contribute to New } \\
\text { Zealand's environmental and conservation goals }\end{array}$ \\
\hline
\end{tabular}

NZTS 2015 determines necessary activities to make tourism development an ecologically friendly phenomenon. These suggested activities contain some key points such as providing a national environmental plan to be followed by New Zealand and its tourism sectors; participation in global forums that attempt to manage and monitor environmental issues; contribution to stronger sustainable environmental policies, regulations and standards; and development of ecological indicators that can be used as tools to measure environmental impacts of tourism (see Appendix 3). 
Furthermore, NZTS 2015 proposes use of products, services and facilities such as transportation with fewer carbon emissions and involving less non-renewable energy. Renewable resources are seen as the main sources of energy to be used through the sustainable development process at both international and domestic levels (see Appendix $3)$.

\section{d. Outcome 4: The Tourism Sector and Communities Work Together for Mutual Benefits}

NZTS 2015 views sustainable development of tourism as a community-based activity that attempts to engage local communities and participants in the whole development process including its planning, management and monitoring (Table 2.6).The forecasted priorities by NZTS 2015 (Table 2.6) also focus on making opportunities for local participants to produce and present their cultural products and conserve their cultural heritage through the sustainable development of tourism. This policy is linked with local economic development in a sustainable way (Table 2.6).

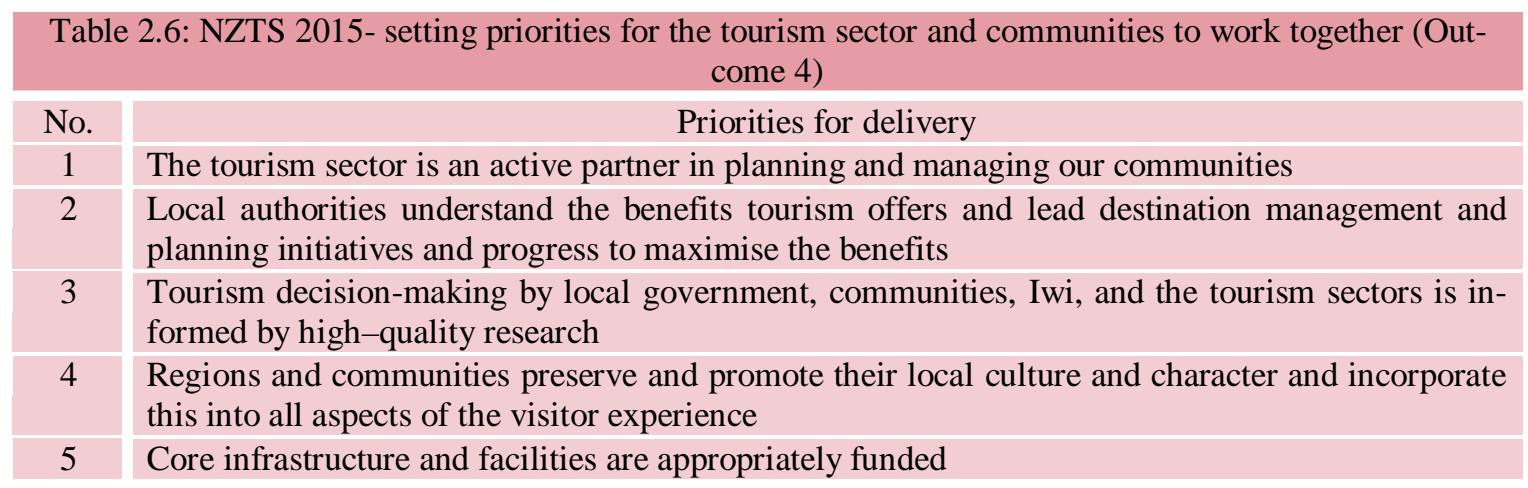

Appendix 4 shows a range of community based activities suggested by NZTS 2015 which can support New Zealand tourism sectors to be more socially sustainable through engagement with local participants and communities. The latter community based activities cover the social dimension of the sustainable development of tourism and, in some aspects, are linked with the environmental and economic dimensions of sustainability. For instance "making sure that high-quality research is available to local communities to inform decision-making by local government and the tourism sector, including information on the social, economic, environmental, and cultural benefits of tourism to communities” (NZTS, 2015) can be considered as an educational policy and activity that attempts to make local people aware about the linkage between the environmental, social and economic dimensions of sustainable development. 
Some of the suggested activities that can contribute to the New Zealand tourism sector becoming a socially sustainable activity, for example "assess core needs, and investigate and consider funding models to improve the standard and provision of appropriate infrastructure” (NZTS, 2015), also benefit this sector in the achievement of other forecasted outcomes, such as "deliver[ing] a world-class visitor experience" as cited in Outcome1 (Table 2.2).

\subsubsection{Discussion}

NZTS -2015 attempts to propose a framework that has the ability to cover all the different dimensions of sustainability through development of tourism. As discussed above, its four anticipated outcomes (Table 2.2) have the ability to lead tourism sectors and their related services, products, facilities and activities to develop in a sustainable way with positive social and economic effects and lower negative environmental impacts. This strategy can be viewed as a bottom up approach to a democratised development process that in turn will be supported by the New Zealand government.

However, some of the forecasted priorities for the sustainable development of tourism (in particular economic and environmental priorities) might cancel each other out. For example "invest in marketing initiatives that target those domestic and international visitors who are more likely to travel in the shoulder and off-peak seasons” (Appendix 2), can increase the number of international visitors that in turn increases the ecological footprint of tourism in New Zealand, itself a national and global environmental issue. Although the development of ecological indicators is suggested by NZTS 2015 as an environmental strategy for New Zealand tourism (Appendix 3), can support the tourism sectors to do more accurate planning, management and monitoring of their services, activities and products as being sustainable.

\subsection{Sustainable Architecture}

\subsubsection{Historic Perspective on Sustainable Architecture}

Architecture is one of humanity's products. Its historic roots lie in the connections between the environment and its inherent potential to provide comfort and security for the inhabitants. Williams (2007:103) argues that architecture up until 100 years ago had to be ingenious in providing comfort by integrating passive elements of the natural place 
into a design solution. Williams (2007:103) encapsulates these passive elements, including warm air rising, prevailing breezes, ventilation chimneys, floor plans proportioned and oriented to provide daylight and fresh air to all users, unique methods of construction, virtual elimination of waste, a symbiotic relationship between the structure and the materials needed to build it, and the reuse or return to the earth of the materials after they are used. The use of these elements was the very foundation of an architectural and planning profession which was based on sustainable principles before sustainability was defined.

Sustainability and sustainable architecture can be considered as a phenomena rising from the traditions as officially defined in the modern style. Steele (2005:12) reveals that in the spirit of its Latin origin, 'tradere' means 'to carry forward'. In this context tradition essentially represents the accumulated knowledge of past generations in relation to effective ways of dealing with the environment. In turn this led to place-specific techniques that historically have been used to control microclimates by the people that live in them.

There are many examples where traditions and technology have met each other to create an ecological or sustainable architecture. Steele (2005:15) argues that since there are traditions of technology in dealing with the environment, and as tradition itself is based on making, which is the essence of technology, to set tradition and technology against each other is to establish a false dialectic. Steele (2005:15) suggests that a more accurate approach might be to try to discover where tradition and technology concur or overlap and how this may be applied to environmental problems.

As an example of this approach, Gissen (2009:125) cites Hassan Fati's [also Fathy] work in Egypt. Beginning with designs in 1973 and after several experimental developments that were completed years later, Fati developed mud construction techniques (as a compatible technique for the environment and traditions) for the sake of helping impoverished rural populations. Gissen (2009:125) states that, significantly, Fati’s work provided a material-based construction language for a postcolonial approach to architecture. Fati's mud architecture was characterized by its use of validated construction techniques to make an ecological linkage between the materials and energies resident in a place, architecture, and people as consumers. On the other hand, Fati's architecture 
which is characterized by being environmentally sensitive, culturally appropriate and economically viable, can be considered as an effort based on the combination of traditions and techniques, to create a sustainable architecture and to shift a society toward sustainability.

As will be explained in the next section, the characteristics of architecture discussed above also appear in vernacular and traditional architectures that have historic backgrounds and that are still used as places for living.

\subsubsection{Learning from Vernacular Architecture}

Humanity is in a period of luxury development, which has been raised by the needs of capitalism to create new markets, and the technological advancement that exerts influence on every aspect of human lives. Because of this it may be unfashionable or impossible to regress in time and place to live in vernacular buildings in a vernacular manner. However, the dominant principles in vernacular architecture can be used to make frameworks and guidelines to create an architecture which is correspondent with nature, social-cultural values and local economic systems. Oliver (cited in Kazimee, 2008:4) states that almost $90 \%$ of the world's housing shortages will be met with self-help means, using locally available resources and technologies and not through high-tech and specialized methods. Kazimee (2008:4) explains that in applying the properties of vernacular architecture to current times and seeing in the vernacular a solution for modern urban problems, it is the notions of flexibility and working in traditional ways that are important.

Vernacular cultures generated buildings and spaces that are accommodating of time and place. Creating multifunctional and flexible spaces to be used at different times for different activities (which Kazimee introduces as a characteristic of vernacular architecture), has been suggested by Vale and Vale (2009:129) as an option for optimizing the use of space where it is limited. Vale and Vale (2009:129) use a caravan as an example of timetabling various uses in a multi-functional space. In a caravan "the same space is used for eating and later, when the table and benches have been converted into a bed, also for sleeping. Where movement or transport is involved, timetabled use of space is common" (Vale and Vale, 2009:129). 


\subsubsection{The Clustered Villages of Nuristan- Afghanistan}

Kazimee (2008) describes the villages of the Nuristan region, located in the rugged geographical region of northeast Afghanistan, as fine examples of sustainable communities. This is because they are adapted to the harsh cold climate zone of the southern slopes of the Hindu Kush Mountains and residents depend on local resources for building their homes and villages. He (2008:6) explains that these villages are clustered on the steep slopes of the mountain sides because there is a limited amount of arable land available in the Hindu Kush, so the lower valley and flat land is saved for agriculture and grazing. Kazimee (2008:6) points out that the roofs of the dwellings are constructed above each other, so that one household's flat roof serves as the patio for the neighbor above (Figure 2.5).

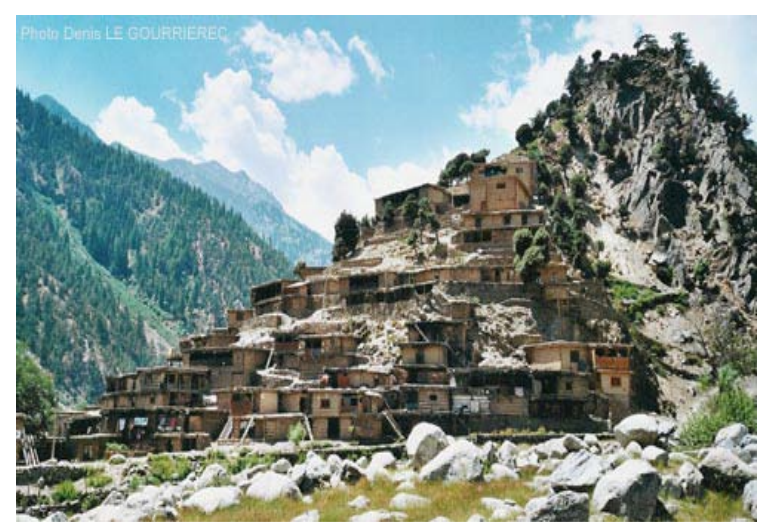

Figure 2.5: Nuristan village, Afghanistan

http://viaterra.net/photos/pakistan/pakistan_nuristani2.jpg viewed December 2010

According to Kazimee (2008:6) these stepped roof areas with their verandahs provide the necessary flat exterior space or patios for many domestic and social tasks. In addition, as Kazimee (2008:6) points out, the patios are connected to each other with notched logs used as ladders that serve as pathways for the vertical movement of people through the village. This using of roofs as patios and pedestrian pathways can be viewed as an example of the creation of multi-functional spaces to avoid wasting arable land.

Kazimee (2008:7) further states that in the villages of Nuristan, people use local materials such as wood, soil and stone to construct their homes. The main stages of construction are conducted by the landowner with the help of neighbors if necessary. Participation of landowners and neighbors makes the construction process become a community based activity. According to Kazimee (2008:7) the south facing terraced houses on the hill respond logically to the climate and solar orientation, taking full advantage of the 
opportunities to save energy through clustering, be heated by solar energy, and therefore create a more comfortable environment for the residents.

Nuristan's villages represent a participatory paradigm of construction using the local and available materials and energies. These villages can be used as an example of an optimized pattern of using an area of poor land for construction to save other natural resources. Agriculture is the main economic activity in these villages and is conducted by traditional methods to provide food for the residents. In this way, these villages are behaving like organisms which are linked with and adapted to nature. The principles that are dominant in the construction process, social-cultural structure and economic system of Nuristan's villages can also be considered as guidelines and principles for architecture created through sustainable development.

\subsubsection{Sustainable or Green Architecture}

Sustainable architecture is a comprehensive phenomenon in which many different subjects such as community based activities, design and planning (at different scales), resource consumption (materials and energies), cost, and nature, are interwoven in the interest of creating functional spaces and structures which meet the principles of the strong model of sustainable development. Wanlass (2005:1) proposes that sustainable architecture can be looked at as two different branches of thought that affect one another. Wanlass (2005:1) explains that an evaluation of the impact of current building materials and use of energy in buildings, and finding better socio-ecological solutions (green building) is one branch and, more importantly, rethinking the way in which architecture is conceived from the beginning, using the metaphor of "architecture as an organism" forms the other branch.

Williams (2007:15) points out the main characteristics of sustainable design and hence sustainable architecture. He (2007:15) states that sustainable designs function using available sustainable energy supplies. Furthermore, "sustainable designs last; they are flexible; they are loved and cherished; they endure; they function when they are tethered to [non renewable resources] and also when the [non renewable resources] are unavailable. Sustainable architecture can function in a blackout or a drought or natural disaster or on a beautiful day without any input from non renewable resources" (Williams, 2007:15). This idea that sustainable architecture must function independently of non 
renewable materials and energies makes a boundary between the modernist idea that architecture is a mechanism or machine and the other idea that view that architecture is an organism. Wanlass (2005:1) points out that if architecture is imagined as an organism instead of a machine, a new idea is created about how it will interact with its environment. He (2005:1) argues that while architecture as a machine is thought to function independently of its environment (which it does not), an organism has a balanced existence with its surrounding nature, responding to changing conditions, and going through a predictable life cycle.

If sustainable architecture is defined as an organism compatible with its environment, then green architecture can be viewed as an element of this definition. Williams (2007:16) reveals that green buildings and communities that integrate the local conditions and natural resources, create healthy interior spaces with natural light, and have complete recycling and reuse of materials are critical to the development of a sustainable future. He (2007:16) argues that although green buildings that efficiently use grid based non renewable energy slow the energy and pollution crisis, if the energy sources powering these buildings are unsustainable, the design is not sustainable.

The main characteristics of a sustainable architecture that make it different from a green architecture include continuing, surviving, thriving, and adapting. According to Williams (2007:16), green design incorporates ecologically sensitive materials and creates healthy buildings and processes that do not negatively affect the environment before, during, or after manufacture, construction, and deconstruction. It also incorporates efficient mechanical systems and high performance technologies. However, it still functions primarily through the use of fossil fuels. In sustainable architecture, buildings are viewed as a part of an organism instead of being a machine (even a very efficient machine) for living in. Its inputs are natural and renewable energies and materials and its outputs are the used materials which can be easily recycled or absorbed by nature or reused to build new buildings and infrastructure. This implies that the people using the buildings must also be part of this organism and that their behavior has to follow the same rules as set out for the building. This suggests a significant change to current globalised consumer culture. 


\subsubsection{Sustainable Architecture and Environment}

Sustainable architecture as an organism has an ecological interaction with its environment. Together the sites and regions play a significant role as they supply the renewable energies and materials used in the buildings. As a result sustainable design has responsibility for protecting its natural environment by optimization of the use of spaces and areas. This responsibility can be conducted by using local renewable materials for construction and manufacture with low embodied energy and less ecological footprint (EF). As can be seen in the Nuristan example above, using open spaces as part of architecture with individual, common or multi functions can contribute to the extension of indooroutdoor interaction and optimize the use of occupied areas. For instance, according to Vale and Vale (2009:176), a local open-air market which can be conducted in a multi functional open-air space, with an EF of 0.8 gha per year has the lowest ecological footprint among other types of places for shopping, including a neighborhood supermarket, low energy supermarket (energy efficient) and low energy supermarket (energygenerating).

Sustainable architecture can be defined as a community based phenomenon that needs to be designed, managed and monitored at the scale of the building, site and region. As a building has a close relationship and interaction with its surroundings (as an element of an organism), its designer(s), constructor(s) and user(s) must have interaction and communication with other participants of the community where the building is situated.

\subsubsection{Sustainable Architecture and Culture}

As a cultural product, architecture can make manifest the different cultural dimensions of a given society. Likewise, both the negative and positive reactions of a culture towards the environment can exert influence on its architecture (as a component of the cultural system) to being compatible or incompatible with the environment. Torres and Sakamoto (2004:19) point out that the built environment with architecture as one of its components is influenced by the society and its cultural system that produces it. On the other hand the cultural system of a given society can make an architectural framework that determines the materials and energy sources used, the size and function of spaces, the interaction between a building and its neighbors, the maintenance of privacy and security, and the form of spaces and their elements. As Torres and Sakamoto (2004:19) 
state, buildings also function as a link between the material world of nature and the symbolic world of human cultures.

To take these ideas further, this next section is included to demonstrate the mutual relationship between architecture and a given society (in this case Kerman). In this section, architecture is considered as a product that can exert influence on and be influenced by the ecological ethics of a given society, its social (spiritual and physical) behaviors and economic system. The section demonstrates the main characteristics of an architecture that could contribute to the social, environmental and economic development of a society.

These examples have been selected because they are more self-contained and demonstrate these relationships much more clearly than the architecture of the west, such as that of New Zealand, which has been influenced by global cultural values. Kerman city, which is 1500 years old, clearly shows the link between culture and architecture, as the forms and materials of its streets, buildings and even its services have been influenced by and evolved in parallel with, the culture of its inhabitants over a long period of time.

\subsubsection{The Historical City of Kerman}

The author has previously worked on the Comprehensive Plan for Restoration of the Historic Parts of Kerman City - Iran (1989-1990). Kerman is a city with a rich historic heritage that demonstrates a compatibility between architecture and environment that has been sustained for more than one thousand years. This is a practical example of the main characteristics of sustainable architecture as determined by researchers such as Williams (2007) and Steele (2005). This section introduces the different ecological, cultural and economic dimensions of architecture and their interaction with each other that must be considered for development of soft ecotourism and its architecture.

\subsubsection{General View of Kerman City}

Kerman is one of Iran's oldest cities and has always been an important centre on the trans-Asian trade routes. It is believed to have been founded in the early $3^{\text {rd }}$ century AD by Ardashir I, founder of the Sassanian dynasty.

Kerman is located on a high margin of Kavir-e Lut (Lut Desert) in the central south of Iran (Figure 2.6). 


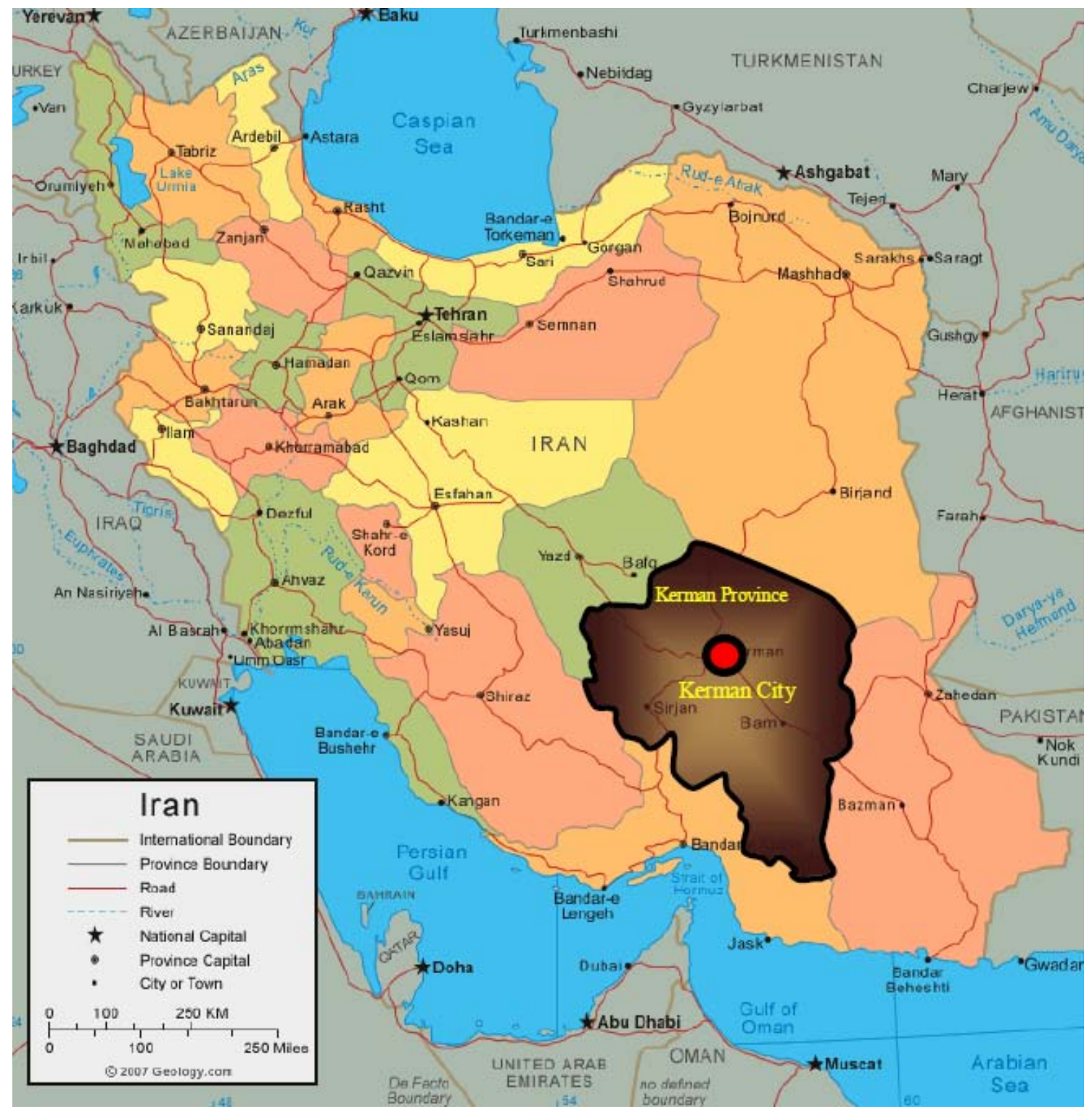

Figure 2.6: Kerman city and Kerman province, Iran http://geology.com/world/iran-satellite-image.shtml viewed August 2012

The climate in the province varies in different regions depending on the relief of the land. The north, northwest and central areas experience a dry and moderate climate, whereas in the south and southeast, the weather is warm and relatively humid. The desert trading city of Kerman has long been a staging point for people passing between Persia and the Indian subcontinent, and for tourists today it remains the best place from which to explore the southeastern region of the country. The city's many districts are surrounded by mountains which bring variety to Kerman's year round weather pattern, thus the northern part of the city is located in an arid desert area, while the highland of the southern part of the city enjoys a more moderate climate. 
The city of Kerman (with the mean elevation of $1755 \mathrm{~m}$ above sea level) and the surrounding regions have a semi-moderate and dry climate, with maximum and minimum temperatures of $39.6^{\circ} \mathrm{C}$, and $-7^{\circ} \mathrm{C}$ respectively. Kerman city has a moderate climate and the average annual rainfall is $135 \mathrm{~mm}$. Because it is located close to the Kavir-e Lut, Kerman has hot summers and in the spring it often has violent sand storms. The average monthly temperatures during the March - June period have been recorded as ranging from $20^{\circ}-25^{\circ} \mathrm{C}$.

Kerman city can be divided into the two historical and modern parts. The historical part is located in the center of the city and surrounded by districts of modernized development. The area surrounded by the red line in Figure 2.7 shows the core of the historical part of Kerman.

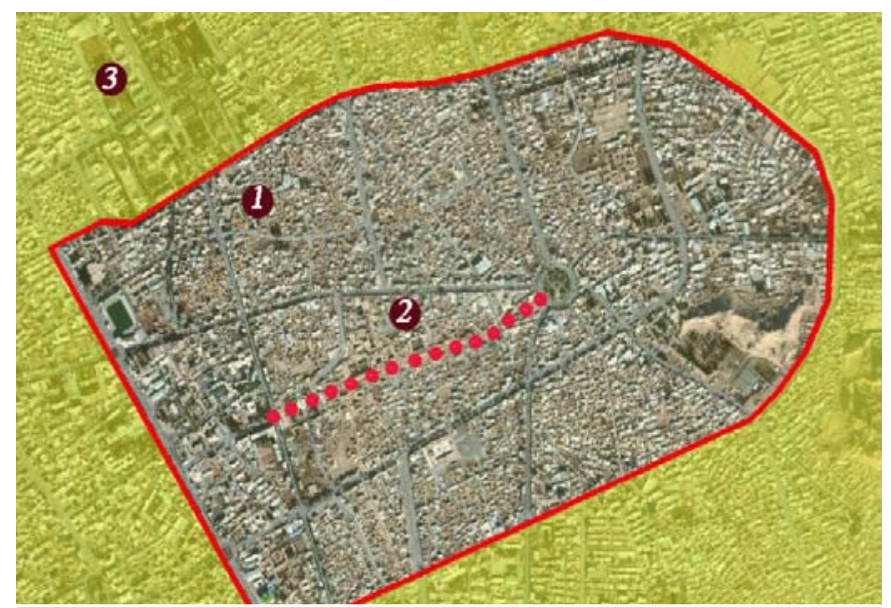

1. Historic part

2. Bazaar

3. Modernized part

Figure 2.7: Historical districts of Kerman city which are linked by the Bazaar

Google Earth viewed September 2011

\subsubsection{Kerman City - Structure and Elements}

\section{a. Bazaar}

The traditional bazaar of Kerman, as a backbone for the city, plays an important role in shaping and organizing the other public and residential areas of Kerman. In particular, it reflects the values of the Safaviyid Dynasty (1502-1736 AD). In Kerman (as an example of the Islamic cities of Iran) the bazaar is a multi-functional element. It functions as the economic heart of the city and, synchronously it functions to connect together the 
mosques, baths, caravanserai, and other common services in an attempt to enclose all areas of life in a harmonized architecture.

The bazaar is used as a covered pedestrian pathway during the cold and hot seasons for access to the different parts of the city (Figure 2.8, left). Every Friday when people want to attend Friday prayer which is held in the Friday Mosque (the biggest mosque of the city called "Masjed-e Jame”) they use the bazaar as the main access to it. Consequently, even on Fridays which are public holidays when the commercial buildings are closed, the bazaar still lives. Kerman's bazaar has two main branches (orders) that intersect each other at a four way stopping point. This intersectional place is called "Chahar Sogh” (four-leads) (Figure 2.8, right).

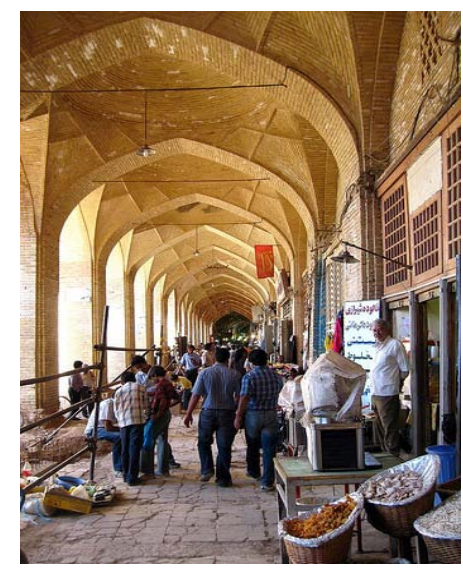

Figure 2.8: Left, Bazaar, http://www.flickr.com/photos/lfphotos/ 1248475691/viewed September 2011

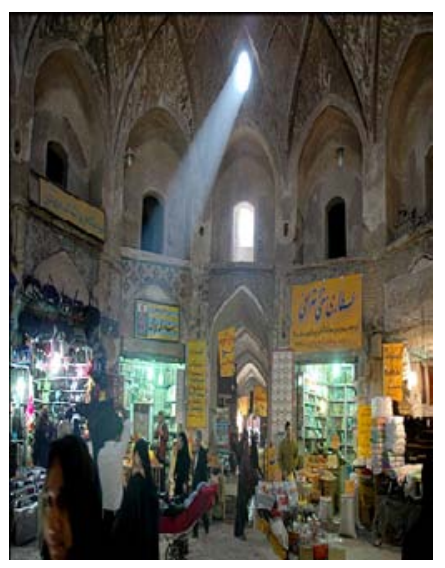

Right, Chahar Sogh

http://www.avayeettehad.ir/farsi /tour/kerman/255428_orig.jpg viewed September 2011

In comparison with other elements of the bazaar, because of its function as the junction between the two main branches of the bazaar, Chahar Sogh occupies a bigger and higher space. The roof of the bazaar is covered by a series of domes structured using brick (Figure 2.9). The dome which covers the Chahar Sogh area also has a bigger diameter and size than others in the bazaar (Figure 2.9, left). 


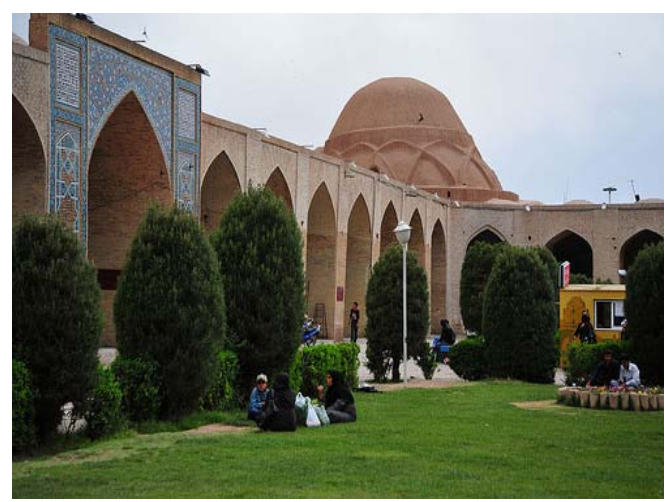

Figure 2.9: Left, the dome of Chahar Sogh http://farm2.static.flickr.com/1261/472535731 3_4e813a189f.jpg viewed September 2011

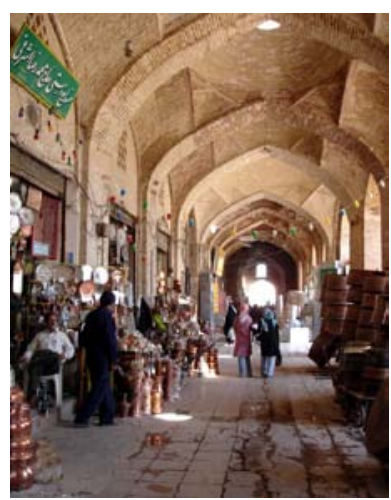

Right, bottom up view of the roof of Bazaar http://i1.trekearth.com/photos/38865/co ppersmith bazaar.jpg viewed September 2011

\section{b. Materials}

Building construction in Kerman was influenced by the available natural materials and in particular the soil and water used to produce brick and sun-dried brick. These materials are environmentally compatible with the surrounding climate and desert terrain. Using these materials contributes to reducing climate extremes, such as temperature difference between indoor and outdoor spaces during the hot and cool seasons.

In addition mud is the main material used to produce enamelled tiles. However, different metal oxides and natural resins must be applied to create the enamel tiles and enamelled bricks that are used as finishing and decorative elements. Furthermore, all other materials such as chalk (gatch), lime, sand and natural pigments have been produced locally by using natural resources. Based on using natural resources for construction, the traditional architecture and urbanism created can be viewed as a part of the environmental materials and energy flows in the city.

\section{c. Qanat (Subterranean Channel)}

In the early part of the first millennium B.C., Persians started constructing elaborate tunnel systems called Qanats for extracting groundwater in the dry mountain basins of present-day Iran (Figure 2.10). 


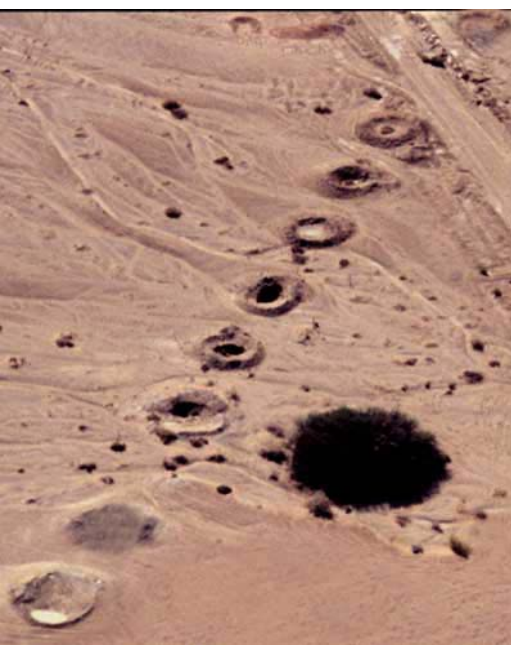

Figure 2.10: Left, top view of Qanat http://thma02.yimg.com/nimage/75d2a553574 $\underline{71914}$ viewed October 2011

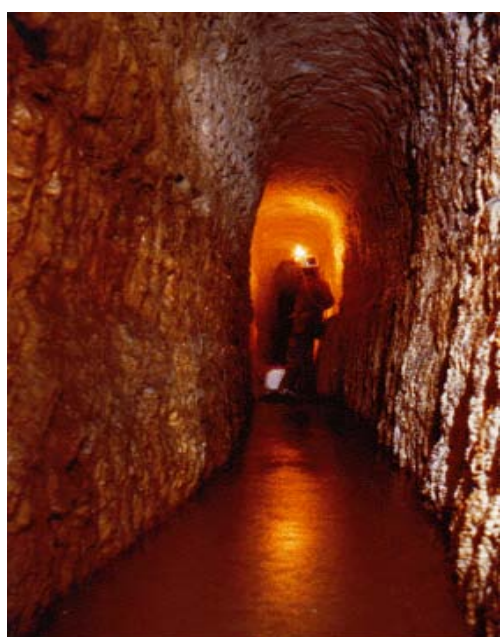

Right: Inside of Qanat http://www.mondellolido.it/pagine/cenni \%20storici/lo\%20cascio/immagini/qanat 02.gif viewed October 2011

These Qanat tunnels were hand-dug, and consequently some are just large enough to fit the person doing the digging. Along the length of a Qanat, which can be several kilometres, vertical shafts were sunk at intervals of 20 to 30 metres to remove excavated material and to provide ventilation and access for repairs (Figure 2.11).

The main Qanat tunnel sloped gently down from pre-mountainous alluvial fans to an outlet in a village or a city. From there, canals would distribute water to fields for irrigation or to residential places for consumption. These amazing structures allowed Persian farmers to succeed despite long dry periods when there was no surface water to be had. Many Qanats are still in use, the technology stretching from China in the east to Morocco in the west, and even to the Americas.

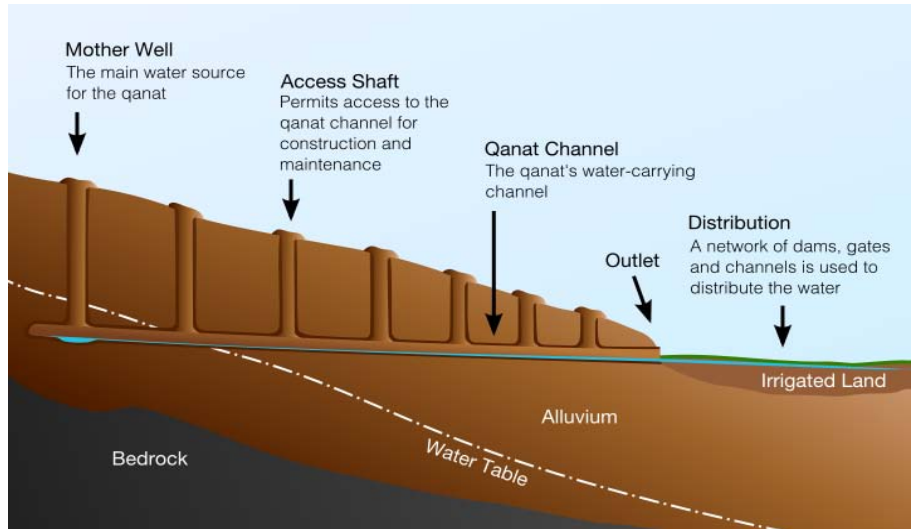

Figure 2.11, Cross section of a Qanat http://diyscholar.files.wordpress.com/2010/09/image-110.jpg?w=330\&h=204 viewed October 2011 
There are significant advantages to a Qanat water delivery system including the following:

1. Putting the majority of the channel underground reduces water loss from seepage and evaporation;

2. Since the system is fed entirely by gravity, the need for pumps is eliminated; and

3. It exploits groundwater as a renewable resource.

The third benefit warrants additional discussion. In Kerman, water in Qanats flows in tunnels beneath residential areas and surfaces near the cultivated area. Staircases from the surface reach down to these streams. The first access is usually at a public cistern where drinking water is available to the entire community. Sometimes these cisterns are sizable vaults as much as 10 metres across and 15 or more metres deep with spiral stairs leading down to small platforms at water level.

In the cities such as Kerman, these cisterns are ancient constructions encased in tile. Other more modest urban access points are found along major streets, and even in some alleys, a factor that probably played an important role in the social and physical layout of the town. The Qanat can be viewed as an element of Kerman which makes a sustainable linkage between the city as an organism and the environment as the main source for the supply of clean water.

\subsubsection{Form and Structure}

Herdeg (1990:37) reveals that three fundamental layers of spaces and activities make up the Islamic cities of Iran. "First above the datum plane of the roofs, [are] the sculptural objects including domes, wind towers, etc. (Figure 2.9, left), second below that datum, courtyards of every kind and size, (Figure 2.12), third, spaces on the ground usually denoting circulation (Figure 2.9, right)” (Herdeg, 1990: 37).

\section{a. Courtyard}

In Kerman, the open air space of the courtyard can be seen as one of the dominant forms of the urban structure, being used at the scale of both urban form and architecture. As shown in Figures 2.12 and 2.13, the unity of the city fabric is reinforced by the use of courtyards with different scales and functions but similar geometry, form and materials. 
According to the figure ground (Figure 2.14), the city fabric can be separated into the three morphological objects of, courtyards as public and individual open air areas (white coloured rectangles in Figure 2.14), built area as parts of individual or public buildings (black coloured areas in Figure 2.14), and streets (white lines in Figure 2.14). The latter are not merely streets but also appear as gaps between buildings with organic form.

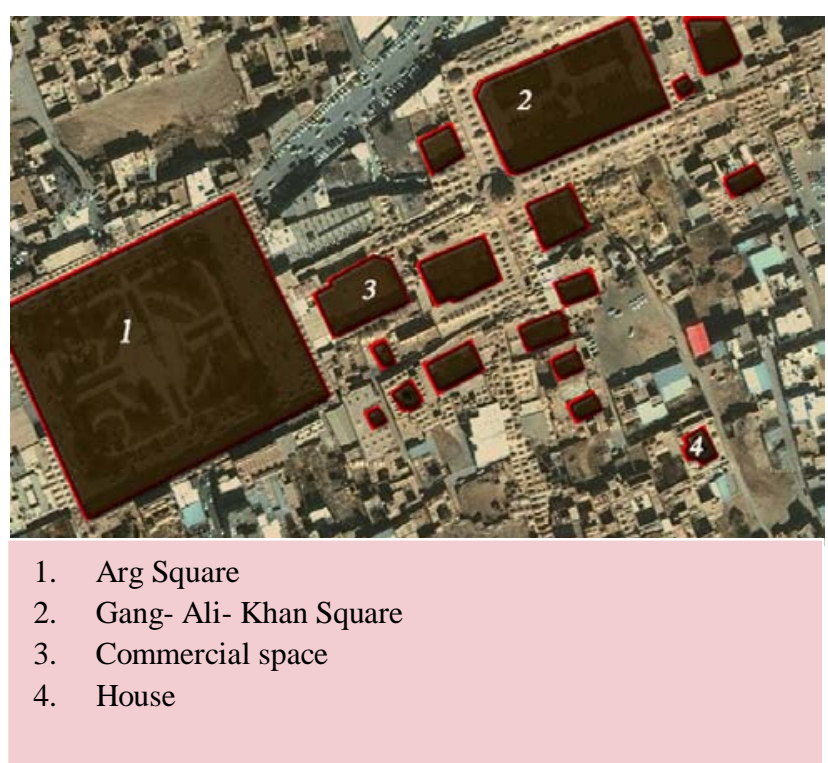

Figure 2.12: The courtyards (dark areas) with different functions in the historical part of Kerman. Google Earth viewed October 2011

At the urban scale, courtyards form the public spaces such as Arg Square (Figure 2.12, No.1) and Gang Ali Khan Square (Figure 2.12, No.2) which connect other urban spaces and services to each other. Courtyards also form the central open spaces of commercial buildings and collections such as caravansaries, and the central open spaces of mosques called "Sahn". Courtyards as public spaces play a role in the holding of some religious and public customs (Figure 2.13).
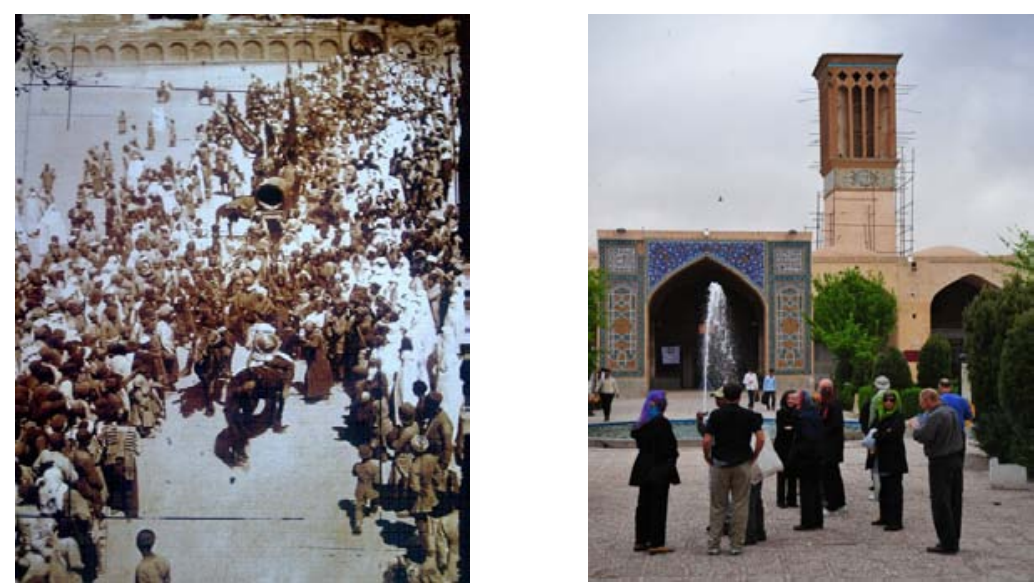

Figure 2.13:

Left: Arg Square, Ashora Funeral Custom.

http://innocent.persiangig.com/i mage/Kerman\%20Ghadim/Saee d017.jpg viewed December 2011

Right: Gang Ali Khan Square http://farm2.staticflickr.com/13 83/4725402177 c052e0c31b z. jpg viewed December 2011 


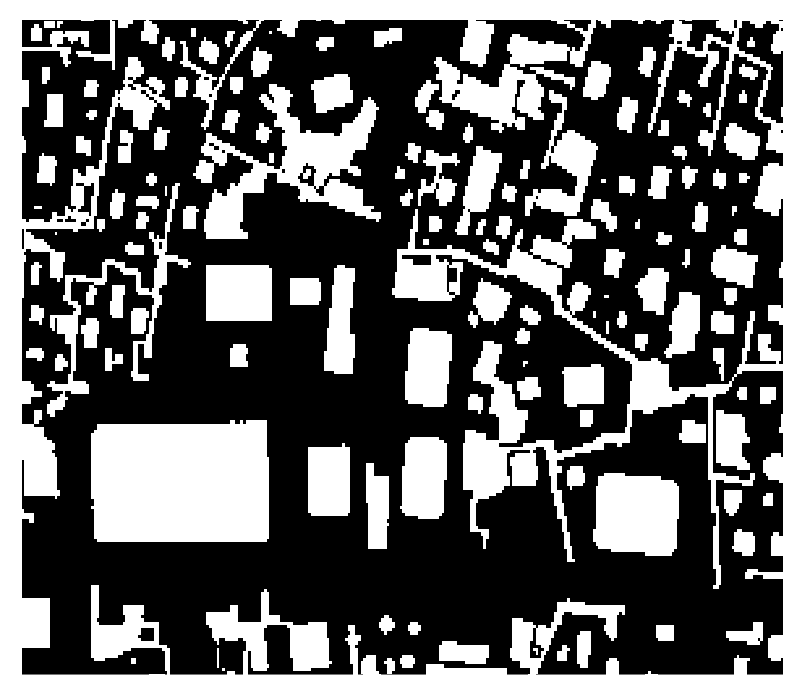

Figure 2.14: The open space (white areas) and built areas in the historical part of Kerman (Herdeg, 1990:37)

At the scale of architecture, the courtyard is an open air area located in the centre of houses (Figure 2.15). It connects the northern and southern parts of houses which are used seasonally. Northern areas (Shah Neshin) are used during the hot seasons (Figure 2.15, right) and southern spaces (Figure 2.15, left), which receive the sunlight during the cool seasons, are appropriate to live in during autumn and winter. Furthermore, the courtyard plays an important role in dividing private spaces from semi-common spaces in a house. Indeed the relationship between the courtyard and other elements of the circulation system, such as the vestibule (Hashti) and corridors, forms a hierarchical circulation system in a vernacular house to protect the privacy of the residents and contribute to the facility of access to different spaces.

As shown in Figure 2.15 (left) during the hot seasons, people use the courtyard as a place to sleep at night. The existence of a pool in the centre of the courtyard contributes to reducing temperature during summer. In addition, people use the water from the pool for washing and cleaning and children use it as a place for swimming during summer. 


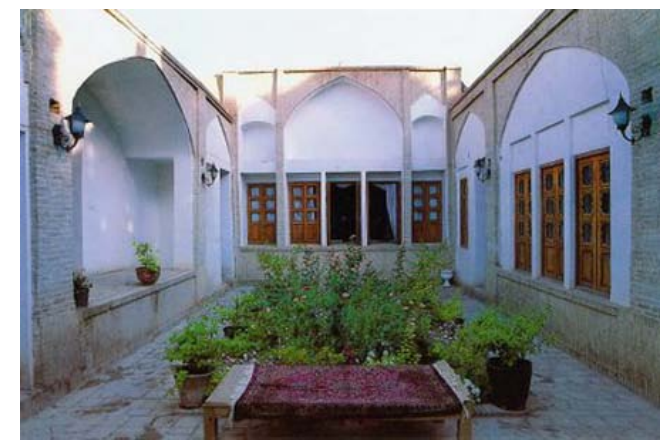

Figure 2.15: Left, courtyard used in summer as open air bedroom http://nazaronline.ir/files/fa/news/1389/7/3/1309 325.jpg viewed October 2011

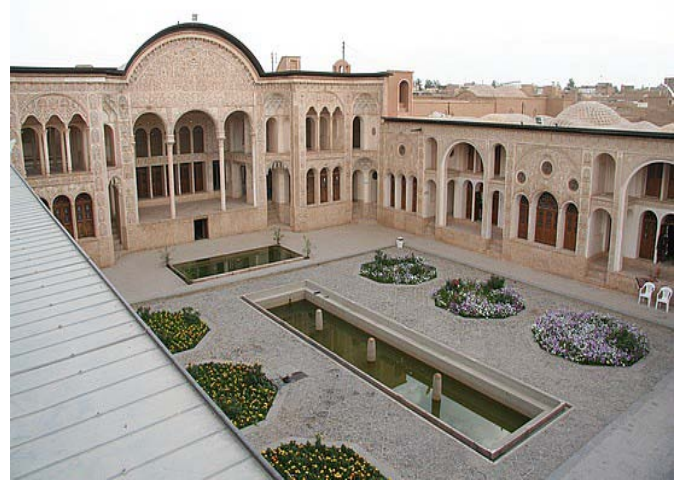

Right, courtyard connects all internal spaces to each other

http://images.travelpod.com/tw_slides/ta00/9d 9/eca/kashan-traditional-house-kashan.jpg viewed October 2011

The courtyard is also used as a safe and private space by the female residents for communication with female neighbours. Likewise, some customs such as wedding and birthday celebrations or funeral customs and "Rohozi" (traditional performance art which is held on the pool surface temporarily covered by wood to form a platform) are held in the courtyard. In Kerman, the courtyard can be viewed as a multi-functional space that is the heart of traditional houses. It can be seen as an environmentally appropriate element which functions as a social-cultural and commercial space at the scale of urbanism and as the main space for conducting daily and family activities at the scale of individual buildings.

\section{b. Dome}

The dome can be considered as an element which makes a visual relationship between the different components of the city of Kerman. The use of domes as the roof of bazaars, commercial and spiritual buildings, water storage structures, and traditional ice boxes called "Yakhchal", points out the compatibility of the dome with a variety of functions and spaces. Many researchers such as Brill (1974) and Herdage (1990) have described the dome as one of the main symbolic characteristic structures used in Islamic cities. However, the use of this curved form by traditional architects arises from the essential characteristics of materials such as brick and stone when they have to form the roof covering. Brick is a material that can naturally resist compression forces and is weak against bending and shear forces. The curved profile of the dome contributes to reducing the shear and bending forces exerted on its elements. In Islamic architecture, 
domes can be classified by the number of their layers. A traditional dome can have a single, two or three layers (Figure 2.16).

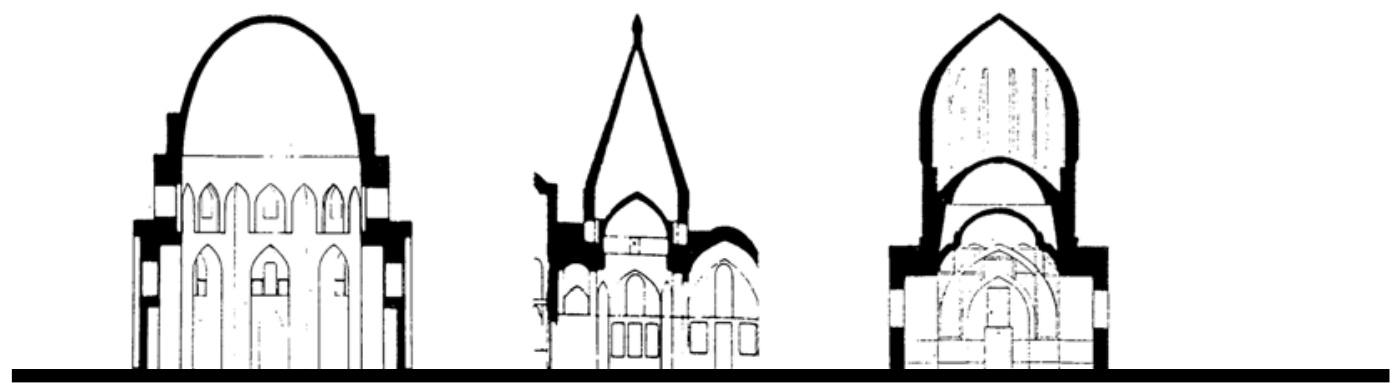

Figure 2.16: Left, single layer dome, middle, double layer dome, right, three layer dome http://lajourd.com/HTMLs/dome.htm viewed October 2011

The spaces created between two or three layers can be considered as natural insulation to reduce the flow of energy between indoor and outdoor spaces through the roof. Moreover, because of the curved form of the dome, the height from floor to soffit is large and therefore vertical natural ventilation can be conducted. Since warm air is lighter and rises to the top and cooler air will replace it drawn in through the multiple openings around the spaces, hot air is removed from the top vents and a natural flow of air from the bottom up is established to provide comfort in hot seasons.

\section{c. The Dome as an Islamic Symbol}

Many researchers such as Brill (1974) discuss the mosque and its related elements, such as the minaret and dome, as the main symbolic forms for Islamic cities. Brill (1974:1) explains that the mosque forms the centre of social life for all Muslim communities throughout their history. Herdage (1990:21) points out that in Islamic societies, a mosque is not only a house of worship, but also a public building serving a multiplicity of uses. He (1990:21) describes the mosque as a gathering place for prayers five times a day, an Islamic college, a community centre with its present day Western associations, and an emergency shelter for travellers.

Although Muslim prayer may be performed anywhere, traditionally, praying together has been held in higher esteem and is obligatory for the Friday noon service which is usually accompanied by a speech from the religious leader called "Khutba". Friday mosques are places which need to have larger spaces in comparison with other small mosques, to contain all the males in the city who attend at Friday noon to pray. In Is- 
lamic cities, the Friday mosque has been made larger and higher than other buildings, influenced by its social and physical functions and spiritual value.

In most of the Iranian cities in which brick or mud are used as the dominant materials for construction, the Friday mosques are almost always covered by tiles of different colours to be distinctive among other buildings and places. In terms of its spiritual value, the "Iranian mosque, and in particular the Friday Mosque with its resplendent ceramic sheath of the paradisal colours of blue, green and yellow, sought actually to evoke the image of the Muslim paradise, with the sapphire of its water, the emerald of its foliage and the gold of its fruits and vines” (Pereira, 1994:15).

In terms of its physical form and scale, particularly in desert areas, where Caravans travelled at night to avoid the hot temperature of the desert in the day, in comparison with other buildings and places in Islamic cities, Mosques have been made of a size such that their elements including domes, minarets (lit by fire) or entrances could be seen by Caravans for navigation. As a result, the size of the Friday mosque can be viewed as a social and physical factor or indicator to control the vertical development of most Islamic cities in Iran. Figure 2.17shows a conceptual cross section of an Islamic city in which the Friday Mosque and its main elements, including the dome and minarets, are depicted as the vertical benchmarks of the city.

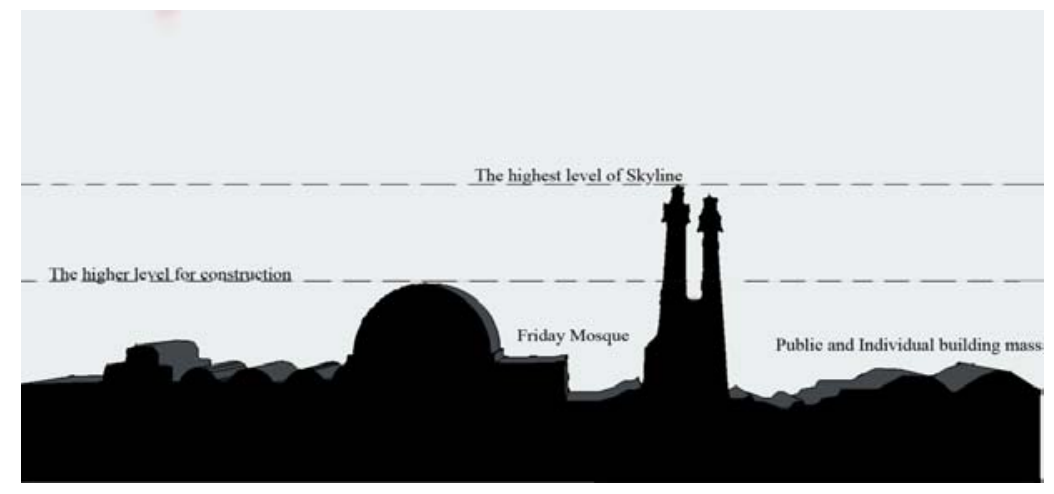

Figure 2.17: Conceptual cross section of an Iranian Islamic city

Figures 2.18 and 2.19 show the scale of the Friday Mosque, and how its decorative but also functional coverage by tiles and their colours makes a difference between this space and other urban spaces with ordinary functions. 


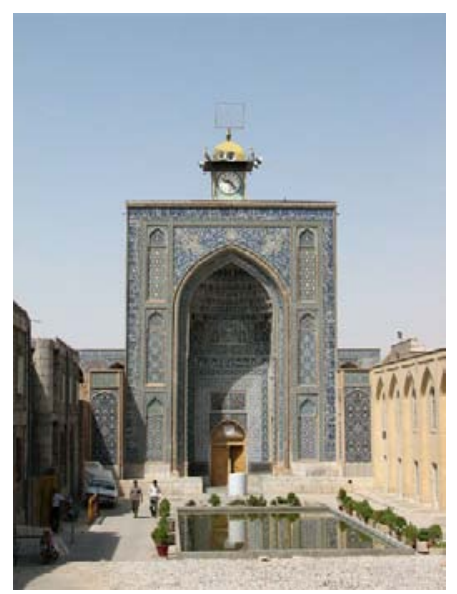

Figure 2.18: Friday Mosque of Kerman, main entrance http://www.destinationiran.com/gallery/ wp-content/uploads/2010/05/kermanfriday-mosque.jpg viewed October 2011

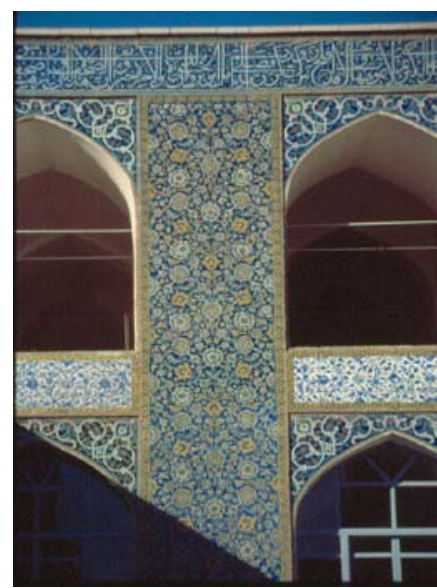

Figure 2.19: Friday Mosque of Kerman, decorative coverage of walls http://cascolytravel.com/images/281 22.jpg viewed October 2011

\subsubsection{Economy}

Rizvi (1991:2) believes that Islam is a complete way of life. He (1991:2) argues that Islam is not only concerned with the spiritual uplifting of human beings, but is also equally concerned about their material and physical well-being. As a result, Islam guides its followers in financial and economic matters, in social and political affairs, and also in the moral and personal spheres of human life. In Islamic cities and societies, the economic system and its activities are influenced by religious economic laws such as "Khums". "Khums" literally means "one-fifth or 20\%". In Islamic legal terminology, it means "one-fifth of certain items which a person acquires as wealth, and which must be paid as an Islamic tax". The Qur'an mentions it in the following verse: "Know that whatever of a thing you acquire, a fifth of it is for Allah, for the Messenger, for the near relative, and the orphans, the needy, and the wayfarer...” (8:41).

Rizvi (1991:2) states that as Khums are an important pillar of the Islamic economic system, it is appropriate to briefly describe the system itself. However, it must always be remembered that the Islamic economic system is not in itself complete; it is a part of the over-all system of life. Islam is a compact system of life in which all its aspects (religious, ideological, social, political and ethical) are well synchronized. Muslims will succeed only if they make the whole system work, and not just pick and choose from it according to their likes and dislikes. 
The Islamic economic framework and its related activities such as paying Khums can be viewed as a cultural-economic phenomenon that contributes to the horizontal development of a local economic system through more equal distribution of capital among the people of Muslim communities.

\subsubsection{The Main Characteristics of Kerman's Architecture}

The main characteristics of vernacular architecture in Kerman can be summarised as: a. Using local natural material and renewable energies (e.g. producing sun dried mud brick through the use of sunlight) with low ecological foot print.

b. Using available technologies for construction at the scale of urbanism and architecture.

c. Compatibility between architectural spaces and elements (in size, form, materials used and function) and cultural identity.

d. Using open air areas (as multi-functional spaces) for cultural activities that contribute to being culturally sustainable.

e. Protection of individual and social privacy by using a hierarchical structure to link spaces to each other at the scale of urbanism and architecture.

f. Horizontal development of built up land rising from natural, cultural and economic factors.

Kerman city can be seen as an example of an organism in that its cultural structure has a mutual relationship with the surrounding environment (the basis of the strong model of sustainability) and its economic system (as the central factor of the strong sustainability model). Kerman reveals practical examples of the main cultural characteristics of sustainable architecture as determined by Torres and Sakamoto (2004). Each of its structural dimensions complements the others to achieve an environmentally and culturally appropriate and economically viable living. In Kerman, the combination of elements such as the bazaar (an economic symbol), the Friday mosque (a spiritual and social symbol), the Qanat (a technical symbol for the use of natural resources) and the overall environmental circumstances present a pattern for living.

Comparison between the main characteristics of Kerman's architecture and sustainable architecture (Torres and Sakamoto, 2004; Williams, 2007, and Steele, 2005) indicates this city has the potential to be approached as an example of an area for living sustaina- 
bly. However, the sustainability of Kerman city still needs to be evaluated through use of a comprehensive framework and related indicators to give a true picture. This is an area for further research once such a framework has been developed.

\subsection{Ecotourism and Its Architecture through Sustainable Development}

Sustainability and 'being sustainable' can be viewed as the main factors that make a strong link between ecotourism and its related architecture. Sustainable ecotourism and sustainable architecture each attempt to respond to the societal needs of a given group of people or a society in an environmentally compatible, culturally appropriate and economically viable way. Arising from the literature presented above, ecotourism can be explained as a reaction to (and part of) mass tourism that aims to reduce tourism's constant destruction of various parts of the world, specifically developing world countries. Since sustainability seeks to preserve the environmental, cultural and economic capitals and heritage for the present and next generations of humankind, both ecotourism and its related architecture aim to make a balance point between human needs, environmental conservation policies, cultural development principles and economic development strategies in a sustainable way.

The relationship between architecture and ecotourism is not yet defined and clarified. There are no direct works written on architecture's relationship to ecotourism, even though some focus separately on both categories through discussion of sustainability. There are many examples of ecotourism resorts, or what are claimed to be ecotourism resorts, throughout the world. The truth is that true ecotourism resorts are very few and far between. Even though there are many destinations in the world that claim to have eco-friendly accommodation for ecotourists, there are still no criteria that define what architecture has to be for consideration as part of ecotourism.

\subsection{Knowledge Gap and Research Justification}

This section has summarised the literature on sustainability, ecotourism, and architecture and the dominant strategies and principles for their development. This summary suggests that a knowledge gap exists in terms of frameworks and strategies for sustain- 
able development of ecotourism and its related architecture. This knowledge gap can be further described as a lack of a comprehensive framework that has the ability to integrally consider the ecological, cultural and economic influences exerted by ecotourism and its architecture on a given host destination. Furthermore, there is a deficient link between anticipated activities and each forecasted outcome and an absence of efficient cultural and economic indicators that can be used to evaluate ecotourism and architecture as being culturally and economically sustainable. This is discussed in more detail below.

\subsubsection{Shortage of Integrated Strategies}

\subsubsection{Linkage between Anticipated Outcomes}

The literature discussed above shows that the current strategies for sustainable development (for example NZTS 2015) aim to propose separately the main environmental, cultural and economic outcomes for sustainable development of tourism (and ecotourism as a segment of sustainable tourism). But there is no policy to make a logical link between these outcomes. Furthermore, influences that are exerted through achieving an outcome (for example an economic outcome) on the other areas such as cultural structure or the local economic system of the host destinations are almost ignored.

For instance, as discussed above, in NZTS 2015 there is a need to clarify how economic development in a host society through increasing the number of international visitors, as an economic priority for sustainable development of tourism, influences the environmental resources and cultural structure of that society. Likewise as another example, there is a need to clarify how the policy of production and consumption of local products (as cultural capitals) or restoration of cultural heritage of a host society (as sustainable social outcomes) can contribute to conservation of environmental resources and development of the local economy in a sustainable way.

\subsubsection{Linkage between Forecasted Priorities}

The priorities that are proposed to contribute to achieving the related outcomes must be ecologically, culturally and economically compatible with other priorities related to the same or other outcome(s). For example, in NZTS 2015, the relationship between 'improving demand during the off-season by the tourism sector' (proposed in Table 2.4) and 'protecting and enhancing the environmental resources' (proposed in Table 2.5) 
must be clarified. This is because the second priority, for example, may cause an increase in the number of visitors that in turn increases the ecological footprint of tourism.

\subsubsection{Linkage between the Indicators}

Absence of efficient cultural and economic indicators is one of the main issues in the current strategies that exist for the sustainable development of tourism and its architecture. On the other hand, a comprehensive sustainable development strategy for tourism needs to use these indicators to evaluate and measure the cultural and economic as well as the ecological impacts of tourism on the host destinations. These impacts can be caused by the products, activities, and facilities proposed through tourism strategies.

Using environmental indicators such as ecological footprint can contribute to approving a phenomenon such as tourism as being environmentally compatible or not. However, reducing the ecological footprint of an activity or a product is not merely reliant on technical or ecological policy, but, can be considered as a social-ecological strategy. As evidence, Vale and Vale (2009) in their book, 'Time to Eat the Dog?”, use the ecological footprint of each activity or product as an ecological indicator to show the environmental impacts of different patterns of resource consumption, activities and lifestyles. However, to reduce these physical impacts, they propose social-ecological policies that rely on changing the cultural behaviour in terms of maintenance, production and consumption of environmental resources. A sustainable tourism strategy should rely on the use of cultural and economic as well as ecological indicators for evaluation of the proposed activities or products (such as architecture) as being environmentally sensitive, culturally appropriate and economically viable.

\subsubsection{Deficit of an Integrated Method to view Ecotourism and Architecture}

As shown through the examples above (Nuristan villages and Kerman city), the main characteristics of an architecture that can be categorised into the three ecological, cultural and economic domains are linked together. As a result, each of the separate characteristics of ecotourism and architecture can be explored and identified through explanation of its links with the other characteristics. For instance the cultural characteristic of an ecotourism project or its proposed architecture can be conceptualised as influences that are exerted on the surrounding environment and local economic system through encouraging people to follow a particular social behaviour such as walking, cycling, or 
using restored buildings as accommodation services (as cultural heritage). Exploring the linkage between the different characteristics of ecotourism and its products, such as architecture, needs to have a comprehensive system that can quantitatively evaluate and measure the effects of each characteristic on the others.

\subsection{Research Question}

This section determines seven sub research questions arising from the knowledge gap discussed above. These sub research questions are then integrated into the key research question in section 2.6.2.

\subsubsection{Sub Research Questions}

This thesis considers strong sustainability as the starting point, such that its strategies can form a framework for the sustainable development of ecotourism and its architecture. Through the following questions, the environmental, cultural and economic dimensions of this dominant strategy will be clarified. Sustainable strategies for development are also used to indicate the main outcomes for ecotourism and its architecture, and the relationships between these outcomes. The research questions also aim to investigate the main priorities and productive activities that contribute to achieving the forecasted outcomes, and the interaction between the outcomes and activities and measurement of the influences exerted by the proposed priorities on each other.

The sub research questions are:

2.6.1.1. What are the main characteristics of an efficient strategy for the sustainable development of ecotourism and its products, such as architecture?

2.6.1.2. What are the relationships between the anticipated outcomes for ecotourism and its architecture and how are they linked to each other?

2.6.1.3. What are the main priorities for the development of ecotourism and its related architecture that can contribute to achieving their forecasted outcomes in a sustainable way?

2.6.1.4. How do the priorities for the sustainable development of ecotourism and its products influence each other?

2.6.1.5. What are the main environmental, cultural and economic characteristics of the activities and products proposed for the sustainable development process of ecotourism? 
2.6.1.6. How will these products and activities influence the environmental, cultural and economic profiles of the host destinations?

2.6.1.7. How can these influences can be evaluated and measured through the use of an integrated method?

The comprehensive framework for the sustainable development of ecotourism and its architecture are proposed in Chapter 4 and tested in Chapters 5 and 6, with the aim of answering the researches questions. The sub questions above are first answered in Chapters 4, 5 and 6 and in the conclusion (Chapter 7) before the research key question is answered.

\subsubsection{Key Research Question}

The seven sub research questions are summarised in the key research question (answered in Chapter 7) of this thesis:

\section{What are the main characteristics of an architecture that can contribute to sus- tainable development through ecotourism?}

This thesis intends to discover new knowledge about the integrated cultural, ecological and economic characteristics of ecotourism and its architecture. It can be considered as the first time research has proposed an integrated method that sets the ecological, cultural and economic characteristics of a phenomenon (such as ecotourism and architecture) in a holistic frame to measure their influences on each other through sustainable development.

\subsection{Chapter 2: Summary}

Chapter 2 explores the three areas of sustainability, ecotourism and architecture and aims to investigate the literature on these areas and their interactions with each other. It introduces sustainability as an umbrella that covers all human activities and products including ecotourism and architecture. Sustainability is viewed as a solution for problems such as uncontrolled population growth, degradation of environmental resources and unequal distribution of capital. 
Chapter 2 introduces the weak and strong models of sustainability as two different strategies for development. As discussed, in the weak model of sustainability, environmental, social and economic capitals have equal value and can be substituted for each other. In the strong model of sustainability, environment and conservation of natural resources is considered fundamental for social and economic development. The chapter argues that the strong model of sustainability that has more potential than the weak model to solve humanity’s issues.

The strong model of sustainability shows the main characteristics and goals of sustainability and sustainable development fall into the three categories of environmental, cultural and economic characteristics and goals. One of the main environmental goals for sustainable development is awareness of local and indigenous people about their environmental heritage and capitals through an educational process. Another environmental goal for sustainable development is engagement of educated people in an environmental conservation process.

Chapter 2 argues for the conservation of social - cultural heritage and capitals and development of cultural products and capitals as being the most important social goals for sustainable development. These goals can also be achieved through an educational process in which local and indigenous people are made aware of their cultural heritage and capitals and are engaged in the social development process. Sustainable economic development is determined as a social- ecological economic phenomenon that aims for equal distribution of capitals among all components of a given society in an environmental and social sustainable way. Through using the main characteristics of sustainability and its ecological, social and economic policies and strategies, ecotourism is defined as part of sustainable tourism with the same environmental, cultural and economic outcomes and goals as sustainability itself.

Ecotourism can be divided into the two types of hard and soft ecotourism. Since, hard ecotourism occurs in untouched areas with restrictive access, the soft ecotourism that occurs in rural and urban places and their adjacent areas has more opportunity for engaging people in the development process, and so is focused on in this thesis. 
Two case studies of Thailand and Costa Rica are used to determine the influences exerted on these destinations by ecotourism development. Both show that the sustainable development of ecotourism can be a strategy to reduce environmental degradation, improve social wellbeing and develop local economic systems in a sustainable way.

The literature reviewed in this chapter indicates the dominant strategies for the sustainable development of architecture related to ecotourism must follow the same goals as the development of ecotourism. On the other hand, as ecotourism, its architecture must aim to conserve the environment and social cultural system of its host society. Likewise architecture must relate to development of the local economic system in an ecologically and socially compatible way.

Through assessment of strategies and indicators, such as NZTS 2015, ISEW, GPI, EF and GDP, proposed for sustainable development of tourism, there is no holistic framework for the sustainable development of ecotourism and its architecture. This is the identified knowledge gap, leading to the key research question:

What are the main characteristics of an architecture that can contribute to sustainable development through ecotourism?

Chapter 3 outlines a quantitative methodology that can be used to answer the research questions. 


\section{Chapter 3: Research Methodology}

\subsection{Introduction}

This chapter outlines a quantitative methodology that will be developed and tested in this thesis. In particular, this methodology will be used to answer the sub-research and key research questions. To achieve this goal, the research structure is planned to clarify the information gained from the literature review on the three areas of sustainability, ecotourism and its architecture and their interaction with each other, the knowledge gap, and the research questions. The structure also shows how these steps relate to the proposed comprehensive framework for ecotourism and its architecture (Chapter 4), testing the framework in the three case studies (Chapters 5 and 6), and the general conclusion, which include discussion of the research shortcomings and limitations (Chapter 7).

\subsection{Research Structure}

The research framework is shown in Figure 3.1 and summarised as follows:

The research begins with a literature review that introduces sustainability, ecotourism as a sustainable type of tourism, and sustainable architecture as one of ecotourism's products (Figure 3.1). The literature explains the main principles that dominate sustainability and how these might be used as objectives to make a framework for the development of ecotourism and its related architecture. 


\section{Literature Review}

\begin{tabular}{|c|c|c|}
\hline $\begin{array}{ll}\text { Sustainability: } \\
\text { - } & \text { Definition } \\
\text { - } & \text { Necessity } \\
\text { - } & \text { Models } \\
\text { - } & \text { Characteristics } \\
\text { - } & \text { Goals- Principles } \\
\text { - } & \text { Framework } \\
\text { - } & \text { Indicators }\end{array}$ & $\begin{array}{ll}\text { Ecotourism: } \\
-\quad \text { Definition } \\
- & \text { Ecotourism and envi- } \\
& \text { ronment } \\
- & \text { Ecotourism and society } \\
- & \text { Ecotourism and econ- } \\
& \text { omy } \\
- & \text { Strategy for sustainable } \\
& \text { development of tourism }\end{array}$ & $\begin{array}{ll}\text { Architecture: } \\
\text { - } \quad \text { Historic perspective of sustainable } \\
\text { architecture } \\
\text { - } \quad \text { Vernacular architecture and sustain- } \\
\text { ability } \\
\text { - } \quad \text { Sustainable or green architecture? } \\
\text { - } \quad \begin{array}{l}\text { Relationship between culture, envi- } \\
\text { ronment, economy and architecture }\end{array}\end{array}$ \\
\hline
\end{tabular}

\section{Knowledge Gap}

Lack of an integrated strategy for sustainable development of and its products (architecture) that makes a link between:

- $\quad$ Anticipated outcomes,

- $\quad$ Forecasted priorities,

- $\quad$ Indicators used.

Lack of a holistic method to set environmental, cultural and economic characteristics of ecotourism and its products (architecture) in a framework for exploring their influences on each other.

\section{Research Question}

o What are the main characteristics of an architecture that can contribute to sustainable development through ecotourism?

Factors considered:

Holistic strategy for sustainable development of ecotourism and its architecture that determines:

o Environmental, cultural and economic outcomes and their related priorities, products, activities and indicators.

o Environmental, cultural, economic influences exerted by the development of ecotourism and its related products and activities on the host destinations.

o Influences that are exerted by environmental, cultural and economic characteristics of ecotourism and its related architecture on each other.

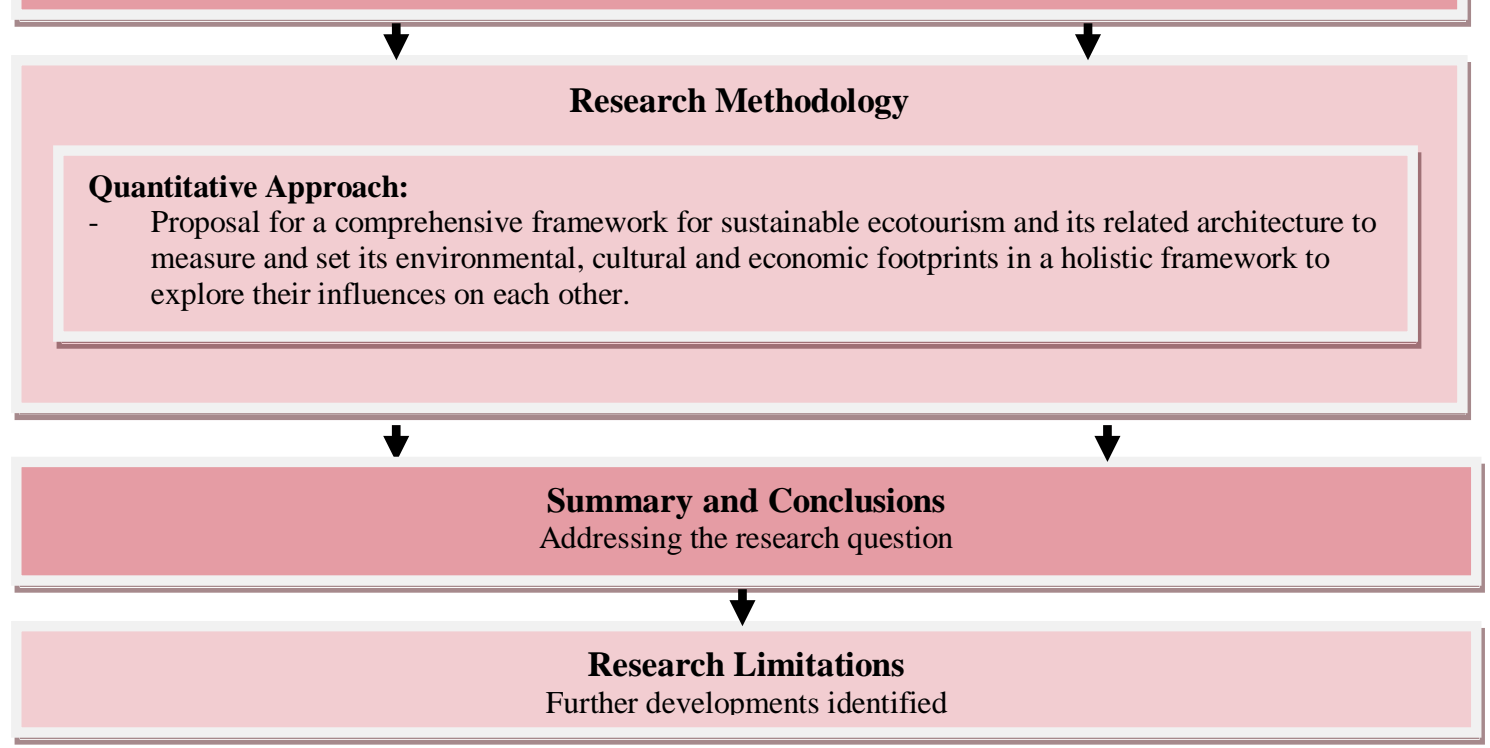

Figure 3.1: Thesis structure 
The strategy for this work is to determine the main characteristics of ecotourism and architecture through determining their environmental, cultural and economic outcomes and related priorities, productive activities and indicators.

This research identifies the lack of a comprehensive strategy that has the ability to set all the environmental, cultural and economic characteristics of ecotourism and its related activities and products, such as architecture, in a holistic framework to evaluate these characteristics as being sustainable. This is the identified knowledge gap in this thesis. This gap is then expressed as a research question:

\section{"What are the main characteristics of an architecture that can contribute to sus- tainable development through ecotourism?"}

To answer the research question, a quantitative method is used. The quantitative approach involves evaluation and measurement of the environmental, cultural and economic footprints of ecotourism and its architecture and their interaction with each other. This is conducted through using the proposed comprehensive framework for sustainable development of ecotourism and its architecture. The quantitative method developed here has the ability to be combined with a qualitative approach in future research. This combination would allow researchers to measure how far apart the beliefs of people are about a given subject when compared with their acts. The results of this research are summarised and conclusions drawn, research limitations revealed, and aspects requiring further development are discussed.

\subsection{Justification of the Research Methodology}

The research methodology is intended to answer the primary research question about the main characteristics of an architecture that can contribute to sustainable development through ecotourism. The methodology requires a quantitative approach. This research is explorative is setting out to see if it is possible to design a method that links together assessment of the cultural/economic/environmental aspects of sustainability. It is also explorative in testing the method through using selected case studies and reflecting on the results of the investigation through a comparative approach. The proposed methodology is faced with ten problems explained in the next section that must be solved. 


\subsubsection{Methodological Problems}

The problems that the quantitative methodology faces can be represented by the following questions:

3.3.1.1. What are the main elements of a comprehensive framework that comprises the environmental, cultural and economic characteristics of ecotourism and its architecture within an integrated approach?

3.3.1.2. What are the main environmental, cultural and economic outcomes for the sustainable development of ecotourism and its architecture?

3.3.1.3. What are the priorities for achieving the anticipated outcomes of the sustainable development of ecotourism and its architecture?

3.3.1.4. What indicators can be used to evaluate ecotourism and its architecture as being sustainable?

3.3.1.5. How can the environmental, cultural and economic influences of ecotourism and its products and activities (including architecture) on a given host destination be measured and evaluated?

3.3.1.6. How can the differences between the existing circumstances of an ecotourism project and its architecture and the goal of being sustainable be measured?

3.3.1.7. How can the ecological, cultural and economic indicators used for the evaluation of ecotourism and its architecture be linked to each other in a holistic method?

3.3.1.8. What data are required?

3.3.1.9. How can the required data be collected?

3.3.1.10. How can the collected data be analysed?

\subsubsection{Research Case Studies}

The three case studies of the Otago Central Rail Trail (OCRT) (regional scale), Naseby and Cromwell (site scale) have been chosen for evaluation through using the proposed framework, model and indicators. The main reasons for choosing these three case studies are explained in following sections.

\subsubsection{OCRT}

This section introduces the main characteristics of the OCRT as an appropriate case study for this thesis. 


\section{a. OCRT as a Community Based Soft Ecotourism}

The OCRT is a community based soft ecotourism project that is managed and monitored by the OCRT trust. This characteristic gives the opportunity for local people to participate in its development. The OCRT and its community are linked to related organisations such as the Otago Regional Council and the Department of Conservation, which also have an interest in its sustainable development.

\section{b. Rural and Urban Destinations along the OCRT}

The OCRT makes a linkage between many rural and urban destinations and their adjacent areas. This characteristic contributes to its visitor experience of various host destinations with different environmental, cultural and economic particularities. Through using the OCRT as a case study, the proposed framework and model can be used to investigate how soft ecotourism influences its host societies and how far its cultural footprint is from an ideal sustainable model for ecotourism.

\section{c. The OCRT as a Successful Tourism Project}

The OCRT has been claimed as a successful tourism project by the New Zealand government (New Zealand Tourism strategy 2015, 2007:62). This makes it an ideal case study to explore the proposed framework, the model and the indicators in New Zealand as a developed country.

\subsubsection{Second Case Study: Naseby}

Naseby and Cromwell are two of the host destinations for OCRT visitors according to the OCRT trust, which describes them as attractive places for their visitors. In Naseby 6 of 26 accommodation buildings (excluding 72 camping sites) are refurbished buildings and 20 are new buildings. Conservation of the cultural heritage of Naseby in terms of its historical buildings used as accommodation services makes it distinctive in the ORCT.

In 2011, 4,350 of the total 11,788 OCRT visitors played curling at Naseby, making this activity another reason for using Naseby as one of the most important host destinations for OCRT visitors. The variety and frequency of other activities, products and services offered by Naseby accommodation services is another reason to choose it as a case study (see appendix 28). 


\subsubsection{Third Case Study: Cromwell}

Unlike Naseby and as shown in Table 6.79 all accommodation services in Cromwell are newly constructed buildings. Comparison between the cultural footprints of Naseby and Cromwell related to their accommodation services through using the framework, the model and the indicators, will determine how using refurbished building as cultural heritage contributes to ecotourism and its architecture having a more sustainable cultural footprint.

As shown in Table 5.48, in 2011, 22.5\% of all OCRT visitors came to old Cromwell town. Appendix 34 determines the variety of social and cultural products and activities offered by Cromwell's accommodation services to OCRT visitors. This is another reason to choose this host destination as a case study. Comparison between the cultural footprint (CF) of products and activities produced/ consumed and conducted by Naseby and Cromwell visitors will demonstrate how the framework, model and indicators can be used to compare two host destinations in terms of delivering sustainable tourism. Using the three case studies of the OCRT, Naseby and Cromwell to test the framework will also determine its strengths and limitations.

\subsection{The Structure of the Methodology}

Figure 3.2 shows the structure of the methodology used in this thesis included three main sections of: the comprehensive framework for ecotourism and its architecture; data collection and data analysing (Figure 3.2). 


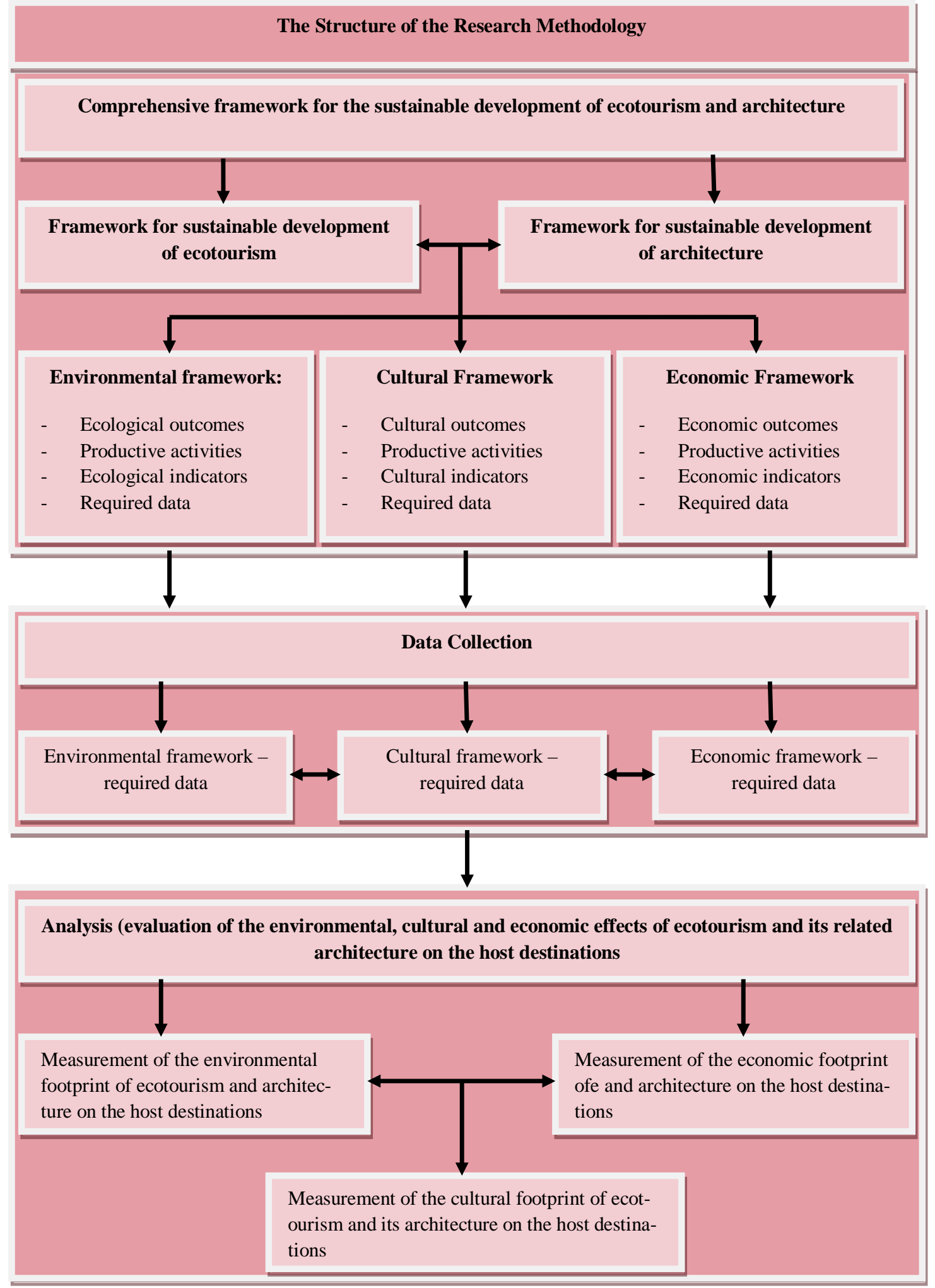

Figure 3.2: Research methodology structure

In this methodology, the comprehensive framework is the core of the methodology the influences the other sections of data collection and data analysis. The main sections of the methodology are explained below and the relationship between these sections determined. 


\subsubsection{A Comprehensive Framework for Sustainable Development of Ecotourism and its Architecture}

The comprehensive framework proposed in this thesis is divided into two frameworks; the first for the sustainable development of ecotourism and the second for its architecture. One of the main aims of the proposed framework is answering the issues explained as methodological problems in section 3.3.1.

\subsubsection{Framework for Sustainable Development of Ecotourism}

As shown in Figure 3.2, the proposed framework for the sustainable development of ecotourism contains three subsectors: the environmental, cultural and economic frameworks. Each of these frameworks aims to determine the main outcomes for the sustainable development of ecotourism; productive activities that can contribute to achieving these outcomes; indicators that can be used to evaluate ecotourism products and activities as being sustainable; and the required data that can be used for this evaluation.

The following sections introduce the main components of the comprehensive framework for ecotourism.

\section{a. Environmental Framework for Sustainable Development of Ecotourism}

The environmental framework for ecotourism determines the main ecological outcomes for its development (such as conservation of environmental resources), the activities that can contribute to reducing the ecological impacts of ecotourism through its development, and environmental indicators (such as ecological footprint) that can be used to measure and monitor these impacts. In the framework and the model, the EF is the main environmental indicator used for evaluation of ecotourism and its architecture as being environmentally sustainable.

\section{b. Cultural Framework for the Sustainable Development of Ecotourism}

This thesis aims to present a theory-based cultural framework for the sustainable development of ecotourism that directs it and its products and activities in a way that makes it culturally appropriate and sustainable. This thesis attempts to conceptualise the main characteristics of a culture and its meaning through the use of an ecological perspective to make a link between the main social structure of the host societies of ecotourism and the surrounding environment. It can contribute anticipation of the cultural outcomes for ecotourism in a way that not only is compatible with the cultural system of the host societies, but is also harmonised with the surrounding environment. The cultural frame- 
work proposes a range of cultural productive activities that aim to change and direct the socio-cultural behaviours of the host societies in a way that supports achievement of the anticipated outcomes for the sustainable development of ecotourism.

This thesis presents a new definition of the cultural footprint of an activity such as ecotourism, and, for the first time in this area of research, proposes a new method to calculate it quantitatively (Chapter 4). In this thesis, the cultural footprint of ecotourism is used as one of the main cultural indicators to evaluate it as being culturally sustainable. Likewise, this indicator is considered a main factor that can be used to explore the linkage between different environmental, social and economic characteristics of an activity or product through a sustainable strategy for its development.

\section{c. Economic Framework for the Sustainable Development of Ecotourism}

The economic framework for the sustainable development of ecotourism determines the main economic outcomes of this development process. However these anticipated outcomes (such as equal distribution of capitals among all participants who are engaged in the process) are not merely economic goals but can also be viewed as social outcomes. In addition, the economic framework for the sustainable development of ecotourism proposes productive activities (such as employment of local people through the process of development) which have both economic and social profiles.

The economic framework for the sustainable development of ecotourism introduces related economic indicators that can be used as tools to evaluate ecotourism development as being economically sustainable. Based on inability of efficient economic indicators and weakness of some methods (cited in Chapter 2) to offer such tools, this thesis proposes a new method to calculate the sustainable portion of GDP which is here called “GDPs” (see Chapter 4). This can then be used as an economic indicator for evaluation of activities and products such as ecotourism and architecture as being economically sustainable.

\subsubsection{Framework for the Sustainable Development of Architecture}

Figure 3.2 demonstrates a framework for the sustainable development of architecture related to ecotourism as a part of the comprehensive framework. This framework follows the same strategy and structure as the ecotourism framework. In this framework, the main anticipated environmental, cultural and economic outcomes for sustainable 
development of architecture are similar to those forecasted for the development of ecotourism. However, the anticipated productive activities (for example using refurbished buildings as accommodation services) that must be conducted to achieve the outcomes contain the activities (such as having refurbished buildings as accommodation services) that are related to architecture.

The environmental and cultural indicators for the sustainable development of architecture rely on the quantity and quality of spaces, products, materials, facilities, and activities that are used by or offered to visitors through the use of architecture. Likewise, the economic indicators that are proposed for the sustainable development of architecture are similar to those used for the economic evaluation of ecotourism development, but in relation to architecture.

The framework for the sustainable development of architecture determines the data required in relation to each of the environmental, cultural and economic indicators. In a general view these data are raised from the quantity, quality and types of the spaces, facilities and products used by visitors; services and activities offered to visitors; and visitor data such as the number of users per year and average visitor nights.

\subsubsection{Data Collection}

Development of appropriate information systems was set as a priority in the New Zealand Tourism Strategy 2015 for the sustainable development of tourism. To test the effectiveness of this, in this thesis, the existing information systems used by the OCRT, New Zealand Statistics and related organisations, and research related to the OCRT are used as the basic sources for the required data. This strategy for data collection provides an opportunity to explore the strengths and the weakness of the information systems and, if necessary, propose guidelines for their development.

The data required by the proposed framework can be classified into three types. These are visitor data (such as the number of visitors and visitor nights); data related to facilities, services and products used (for instance types of transportation and accommodation services); and tourism activities data (for example the number of visitors who play golf or cycling). The following explains the methods used in this research to collect each type of data. 


\subsubsection{Visitor Data}

The visitor data contains information such as: the numbers of visitors per year that visit each of the case study destinations; nationality of the visitors; and average visitor nights per year. These data are collected using Statistics New Zealand (tourism), official tourism statistics, and reports published by the Ministry of Tourism, Department of Conservation, regional councils, and related communities. These data are available through using the related web sites or contacting the related organisations or communities.

\subsubsection{Data Related to Facilities, Services and Products}

These data are collected through the use of official websites of related communities and organisations; official surveys related to the case studies; information published by tourism accommodation services as found in their websites; using websites such as Google Earth, Google Maps and Map Tool 2 (software that can measure distances and areas shown in Google maps and satellite maps - see http://www.zonums.com).

\subsubsection{Tourism Activities Data}

Data on tourism activities cover activities that visitors have done or that have been offered by the tourism sector over a year. These data are collected through the use of the official websites of the related tourism sectors and communities, and official surveys that have already been done for the selected case studies.

\subsubsection{Data Analysis}

The aim of the analysis is to explore the interaction between the ecological, cultural and economic characteristics of ecotourism and its related products and activities such as architecture through sustainable development. This analysis is conducted at two scales: regional and site scale.

\subsubsection{Analysis at the Regional Scale}

At the regional scale, this research analyses the ecological, economic and cultural influences of an ecotourism project, the New Zealand Otago Central Rail Trail (OCRT) in the host destination. This is conducted through calculation of the environmental, economic and cultural footprint of areas related to ecotourism including: transportation used; products (such as food and beverages); built up land (accommodation services); and tourism activities such as cycling, walking, golf and curling. 


\section{a. Ecological Analysis}

This research analyses and evaluates the environmental impacts of the development of ecotourism in the host destination (at the regional scale) through the use of related indicators proposed in its environmental framework. To achieve this goal, this study calculates the ecological footprints (EFs) of:

a. 1 . The types of transportation used

a. 2. The production and consumption of local and conventional foods

a. 3.The refurbished and new buildings used as accommodation services by types of accommodation

a. 4.Tourism activities

The calculated EF (the sum of the above EFs) is then compared with a target sustainable EF of holidays as the goal to be achieved through the sustainable development of the OCRT. The present EF is compared with the target EF to calculate the overshoot portion of the current EF (this process is explained in detail in Chapter 5, section 5.9.1). This portion of ecological footprint can then be used as an indicator to evaluate the development of ecotourism towards being environmentally sustainable.

\section{b. Economic Analysis}

The economic footprint of ecotourism in the host destination is calculated by using the economic indicators (such as employment and GDPs) as proposed in the economic frameworks for the sustainable development of ecotourism and its architecture.

\section{b. 1. GDPs}

In this analysis, GDPs (see Chapter 4) is considered a fundamental economic indicator that clarifies how much of the GDP earned through development of ecotourism must be spent to restore its environmental damages.

The total GDPs of an ecotourism project (OCRT) is calculated through measurement of:

- GDPs earned through transportation.

- GDPs earned through local products (food).

- GDPs earned through accommodation services.

- GDPs earned through tourism activities and related services. 


\section{b. 2. Employment}

The number of staff employed in the OCRT is used as an economic indicator for the development of ecotourism and architecture.

\section{c. Cultural Analysis}

This thesis proposes a quantitative method to calculate the Cultural Footprint (CF) (defined in Chapter 4) of an activity or a product such as ecotourism or architecture that, in turn, can be used as a cultural indicator to evaluate its development as being culturally sustainable. This thesis uses the following steps to calculate the CF of ecotourism and its accommodation services at a regional scale:

\section{c. 1. Quantities of Ecotourism Products and Activities}

Calculation of the CF needs to use quantitative data including:

- The quantities of conventional and local products used such as foodstuffs (kg). It should be remembered that the quantities of local products (cultural products) are introduced as cultural indicators for the sustainable development of ecotourism in its related cultural framework.

- Calculation of the quantities of refurbished and new buildings used as accommodation services ( $\mathrm{m}^{2}$ or number of bed spaces). The measured factors include: areas of open and indoor spaces; number of bed spaces; volume of materials used; facilities used; and energy used, for example, all by types of accommodation services.

- Calculation of the number of visitors per tourism activity. Activities include: sports and recreational activities; visiting historical sites and buildings; and art activities (such as landscape photography). In this thesis, the activities mentioned are introduced as part of the socio-cultural behaviour of visitors and host people.

\section{c. 2. The Reduced/ Increased Portion of EF}

The portion of the EF of ecotourism and architecture which is reduced or increased is influenced by using cultural products, and is calculated through using two scenarios for the measurement of EF.

In the first scenario, the EF of the products used is calculated as the sum of:

- The EF of conventional products used (for example conventional foods), and that of cultural products used (for example local and organic foodstuffs). 
- The EFs of the new and refurbished buildings (including open air spaces such as balconies and verandas) used as accommodation services.

- The result of this scenario (EF1) is the same as the result of the ecological analysis for products used (3.2.4.1-a).

In the second scenario, the ecological footprint of the products used (EF2) is calculated based on the assumption that:

- All the products used are conventional (such as conventional foods).

- All accommodation services are new buildings without open air spaces (verandas and balconies).

The difference between the results of these two scenarios (EF1-EF2) shows the reduced or increased portion of EF influenced by the use of:

- Local products (which are considered to be cultural products).

- Refurbished buildings and open air spaces.

\section{c. 3. Increased / Reduced Portion of GDPs}

This research calculates the increased and reduced portion of GDPs influenced by the use of local products and refurbished buildings as accommodation services using the two following scenarios:

- $\quad$ Measurement of GDPs based on EF1 = (GDPs1)

- $\quad$ Measurement of GDPs based on EF2 = (GDPs2)

Difference between GDPs1 and GDPs2 (GDPs1-GDPs2) is equivalent to the portion of GDPs that is reduced or increased by using local products, refurbished buildings as accommodation services, and using open air areas as parts of the related architecture.

\section{c. 4. The CF of Local Products and Accommodation Services}

The CF of using local products can be shown through using a triangular framework in which the quantity of cultural products used, the reduced/ increased portion of the EF of ecotourism, and the reduced/ increased portion of GDPs as influenced by the product(s), are set on each apex of the framework (Figure 3.3). 
In Figure 3.3 the present CF of local products is shown as the area defined by Q 2, EF2 and GDPs2. In this figure Q 2 is the present quantity of product that contains Q 3 (the present quantity of conventional product) and Q 4 (the quantity of local products).

$$
\mathrm{Q} 2=\mathrm{Q} 3+\mathrm{Q} 4
$$

In Figure 3.3, Q is the quantity of the Ideal model for sustainable living. In the Ideal model for sustainable living the EF of producing and consuming Q is the sustainable ecological foot print.

Shown in Figure 3.3, Q 1 is the quantity of products assumed as $100 \%$ conventional products.

EF 1 in Figure 3.3 presents the EF of consuming and producing 100\% conventional products (Q 1).

Demonstrated in Figure 3.3, the EF2 is the present EF of producing Q 2 (including Q3 and Q 4). In addition in this figure, Ideal EF is the sustainable ecological footprint of products.

In Figure 3.3, the GDP is the total GDP related to products. In the Ideal model of sustainability, since the overshoot portion of the EF is zero, the total GDP= GDPs. Shown in Figure 3.3, GDPs1 is the sustainable portion of GDP when $100 \%$ of products are conventional. Moreover in this figure, GDPs2 is the present GDPs related to producing Q 2 that contains Q 3 and Q 4. As illustrated in Figure 3.3 the difference between EF 1 and EF2 shown as (R) EF 1 is the reduced portion of the EF 1 influenced by producing and consuming Q 4. Moreover comparison between the Ideal EF and other EFs (EF 1 and EF2) indicates the overshoot portion of each EF. Furthermore, in Figure 3.3, the difference between GDPs1 and GDPs2 shown as (I) GDPs1 indicates increased portion of total GDPs influenced by producing Q 3.

The model illustrated in Figure 3.3 has the ability to show the present CF of the products, also it can be used to determine how much of the total GDP must be spent to change the present EF of the products to fit with its ideal ecological footprint. Figure 3.3 illustrates a model that can be used to show the CF area of other cultural products and activities. Also this model can be used to compare the CFs of different activities or products with each other at the different scales of the region, community, site and build- 
ing. In addition, this model can be used as a tool for planning, management and mentoring of the process of development as being sustainable.

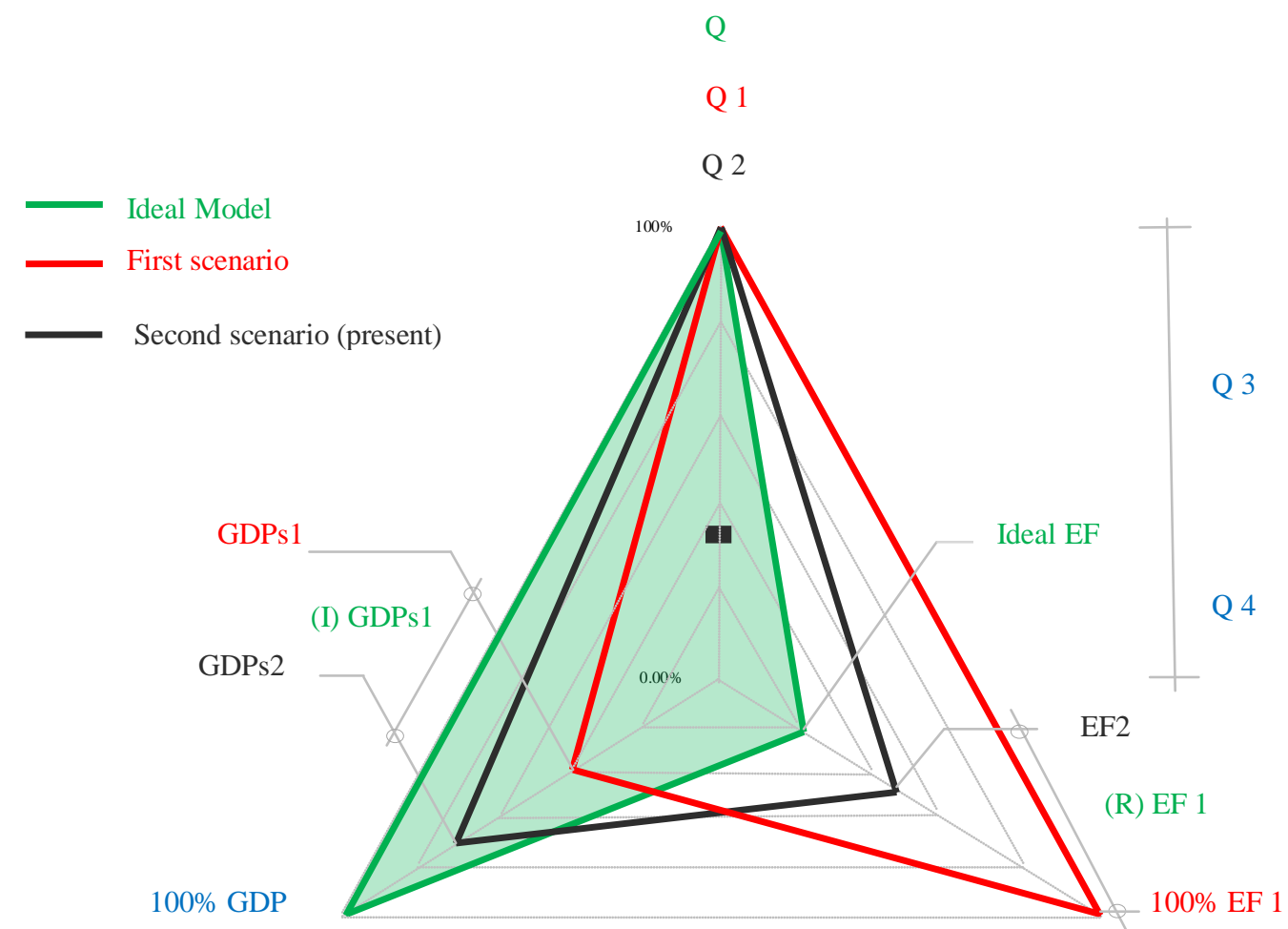

Figure 3.3: A sample model to show the CF of local products

\section{d. Calculation of the CF of Tourism Activities}

The CF of tourism activities is done through the following steps:

d. 1. Calculation of the number of visitors for each activity.

d. 2. Calculation of the EF of each visitor's activities.

d. 3. Calculation of the GDPs per visitor for each activity.

The results determine the environmental and economic footprints of each tourism activity which is considered to be a part of visitors' cultural behaviour that are contributing through ecotourism to development. Setting these results in a common framework shows a part of the CF of ecotourism in the host destination. This study makes a comparison between the numbers of visitors, EF and GDPs of each type of tourism activities (such as walking, golfing and curling) through setting them in the holistic framework mentioned. At the regional scale, it allows this research to explore which types of tourism activities (as proposed cultural behaviours) are more culturally appropriate, envi- 
ronmentally sensitive, and economically viable. The CF of these activities is considered a part of the total CF of ecotourism development.

\section{e. Total CF}

The total CF of ecotourism development determines the influences exerted by the total cultural products and activities on the EF and GDPs of ecotourism. In Figure 3.3, quantities of all cultural products and activities (QCPA) can be set on the top apex of the Figure Also the reduced/increased portions of GDPs and EF can be set on the left and right bottom vertices of the model.

\subsubsection{Analysis at the Site/Individual Building Scale}

At the site/individual building scale, this thesis analyses the ecological, cultural and economic footprint of ecotourism and its related architecture (as used as accommodation services in the OCRT) in the host destinations through the use of Naseby and Cromwell as second and third case studies. Both these case studies are settlements located along and influenced by the OCRT. The factors for which their footprints are analysed include: transportation used; products (such as food and beverages); built up land (accommodation services); and tourism activities and services such as cycling, walking, golf and curling. The analysis at the site/architecture scale for Naseby and Cromwell follows the same method explained above for the regional scale of the OCRT.

This thesis makes a comparison between the results of the analysis for Naseby and Cromwell to find how an ecotourism project can exert different ecological, cultural and economic influences on these two areas. These belong to different bioregions, with different cultural heritages, capitals and behaviours, but that are linked together through a community based ecotourism project.

The comparison between the environmental, cultural and economic characteristics of the accommodation services used allows this thesis to test the proposed holistic frameworks for ecotourism and architecture, which are here used to determine the ecological, cultural and economic characteristics of an architecture that contributes to sustainable development through ecotourism. 


\subsection{Importance and Limitations}

This section discusses the strengths and weaknesses of the proposed methodology.

\subsubsection{Importance of the Methodology}

\subsubsection{Comprehensive Approach}

The methodology aims at a comprehensive approach to the sustainable development of ecotourism and its architecture. For a first time this thesis presents an original methodology that can make a linkage between the surrounding environment, culture and economic system in a given host destination for ecotourism (and its architecture) in order to measure its level of sustainability. This also relies on the comprehensive framework included as the main part of this methodology. The comprehensive framework, its importance and limitations will be discussed in detail in the next chapter (Chapter 4).

\subsubsection{The Integration of Environmental, Social and Economic Indicators}

Integration of ecological, social and economic indicators in a quantitative method makes an opportunity for this thesis to explore the environmental, social-cultural and economic influences exerted by ecotourism and its architecture on a given host society. Likewise this relies on using the proposed holistic framework and model.

\subsubsection{Compatibility with Quantitative Methodologies}

The quantitative methodology can be used as complementary method to any qualitative methodology for assessment of the sustainable development of ecotourism and its architecture.

\subsubsection{Using Existing Information Systems and Available Technologies}

This methodology uses existing information systems, and available research results to show how these can be used for this research. The reason for doing this is to explore the limitations of the existing information systems, data and available research. The study was made without site visits; so as to ascertain if it were possible to draw meaningful conclusions from publicly- available data. If this could be shown to be the case it would increase the value of the methodology, as it would be able carried out remotely, and at lower cost, increasing its applicability. 


\subsubsection{Indicating Required Data through Related Indicators}

The methodology indicates the required data needed from the proposed environmental, cultural and economic indicators. This allows determination of the data linked to the sustainable development of ecotourism and its architecture, thus avoiding collecting irrelevant data.

\subsubsection{Integrated Analysis}

The methodology proposes an method in which the ecological, social and economic data are analysed in a way that indicates the interaction between them. It allows the thesis to cover all dimensions of sustainability related to the development of ecotourism and its related products and activities.

\subsubsection{Limitations}

\subsubsection{Shortcoming of Available Information Systems}

One of the main problems of the approach is relying on other peoples' data and results, with no means of checking their accuracy. However, development of the method and testing it will be done by attempting to collect data from the same type of sources, in this case information published on official OCRT accommodation websites, and research in the public domain related to the OCRT.

\subsubsection{Accuracy}

Since the methodology relies on using existing information systems with their shortcomings, the quantitative results cannot be completely accurate, although comparisons between results produced by the research will still be relevant. However the intention of thesis is not to come out with accurate results but rather to make people aware of the problem and hence improve data collection for more accurate results.

\subsection{Chapter 3: Summary}

This chapter describes the quantitative methodology used in this thesis for answering the research questions in Chapter 2. From the literature review, the three areas of sustainability, ecotourism and architecture and their relationship with each emerged as the point of focus for the research. Sustainability and its policies and principles are the factors that link ecotourism and its architecture to each other as being sustainable. This the- 
sis aims to propose a methodological framework for sustainable development of both ecotourism and architecture.

The comprehensive framework for ecotourism and its architecture needs to be able to:

- Comprise all environmental, social and economic characteristics of ecotourism and its architecture such that these dimensions can be linked to each other.

- Measure the ecological, cultural and economic influences exerted by ecotourism and its architecture on a given host society.

- Use efficient indicators for evaluation of ecotourism and its architecture through a holistic approach to sustainable development.

- Be used as a comprehensive tool for evaluating of ecotourism and its architecture through whole process of sustainable development.

Figure 3. 1. shows the research structure. The research is explorative and aims to design a method that links together assessment of the environmental/ cultural/ economic aspects of ecotourism and its architecture through sustainability. The method will be tested on selected case studies through taking a comparative approach.

Problems with this process are lack of first hand data will mean relying on secondary data sources. However, this is also an opportunity to test such sources and make recommendations about data collection improvements.

In this thesis the EF is the main ecological indicator for evaluation of ecotourism and architecture. To solve shortcomings identified with this indicator earlier, the thesis proposes a comparative method to use the EF and other indicators for evaluation of ecotourism and its products and activities as being sustainable. As shown in Figure 3.2, the comprehensive framework uses the quantity of the social products and activities (for example the quantity of food consumed by visitors, or the number of visitors who play golf) (also see Chapter 4). These quantities are then converted to EFs. GDPs forms the main ecological-social economic indicator for evaluation of ecotourism and its architecture as being economically sustainable.

The methodology proposes an original method for analysing the data related to each of the environmental, social and economic indicators through use of an holistic model that is called the Cultural Footprint model (CF model) (Figure 3.3). Using this model allows 
exploration of the ecological, social and economic influences exerted by development of ecotourism and its architecture on a given host society. Furthermore the CF model has the ability to be used as a tool to compare the ecological, social and economic influences of ecotourism and its products and activities on different host destinations.

One of the strengths of the methodology is its comprehensive approach to the sustainable development of ecotourism and its architecture. Using the CF model allows the thesis to analyse the ecological, social and economic footprint of ecotourism and its architecture in relation to each other through a holistic approach to sustainable development. The main limitation of the methodology is having to rely on existing information systems which will affect the accuracy of the results. However, using the comparative methodology will help to reduce the negative impacts of this shortcoming on the results.

As discussed above proposing the comprehensive framework is one key goal of this thesis. Chapter 4 introduces the framework in detail. 


\section{Chapter 4: Comprehensive Framework for the Sus- tainable Development of Ecotourism and Its Related Architecture}

This Chapter presents a comprehensive framework for assessing the sustainable development of ecotourism and its related products and activities. This framework is divided into two segments. The first segment contains a holistic framework for the sustainable development of ecotourism and the second section introduces a multi-dimensional framework for the sustainable development of architecture through ecotourism.

Each of the frameworks contains three subsectors, these being the cultural, environmental and economic frameworks. Each of the three is further considered in terms of their related outcomes; productive activities; and indicators and required data. This thesis attempts to conceptualise the results of these holistic frameworks as a simplified cultural footprint model (CF-model). This model has the ability to put all ecological, cultural and economic characteristics of an activity, such as ecotourism, or a product, such as architecture, in a holistic framework so that its sustainability can be evaluated.

Moreover to construct the CF-model, this chapter proposes a new method to measure the sustainable portion of GDP (GDPs) to be used as an economic indicator for sustainability that has ecological and social characteristics.

\subsection{A Framework for Assessing the Sustainable Development of Ecotourism}

\subsubsection{Ecological Framework for the Sustainable Development of Ecotourism}

This study presents a social-ecological framework that portrays the main ecological goals which can be used as a pivotal part of an integrated set of principles for the sustainable development of ecotourism. In addition, ecological indicators and their required data are presented as the main output of this part of the thesis, in order to evaluate the compatibility of ecotourism products, services and activities with the environment. 


\subsubsection{Environment and Framework for Development of Ecotourism}

Any discussion of the sustainable development of ecotourism and its framework should reference its environmental impacts because, according to the definition of sustainability as a multi-dimensional phenomenon, these form a significant part of a framework for sustainability through development of ecotourism. In this view, as explained by Pigram (1980:557):

[The] environment is not just a constraint, it is a resource; it is not just a problem, it is an opportunity. That mankind has advanced as far as he has is as much due to the abundance of the natural environment as to his own skills in exploiting those resources. Properly safeguarded, the environment can continue to nurture his economic, social and cultural progress. The need therefore is to understand the characteristics of the environment within which development is to take place and the extent to which it can be manipulated to serve mankind's needs.

Anticipation of what an ecological framework for ecotourism development might be is related to the different approaches to mainstream thinking about sustainable development. Weaver and Lawton (1999:12) introduce a set of moderate operating principles for 'mainstream' sustainable development shown in Table 4.1.

Table 4.1: Operating principles for 'mainstream’ sustainable development.

Operating principle (Weaver and Lawton 1999:12, Modified from Murphy, 1994).

Category

Establishing ecological limits and more equitable standards of consumption

Ecological

Redistribution of economic activity and re-allocation of resources to fulfill principles Economic of equity

Control of human population level

Preservation of basic resources that support the earth's essential support systems

Socio-cultural

More equitable access to resources and increased technological effort to use them more effectively

Attention to carrying capacity and sustainable yield

Retention of renewable resources

Maintenance of biodiversity and cultural diversity

Minimization of adverse impacts on air, water and other natural elements

Local community control

Planning and policy at a broad national and international level

Economic viability

Maintenance of environmental quality

Environmental auditing as a dominant monitoring procedure

Ecological

Ecological

Ecological

Ecological

Socio-cultural

Ecological

Social

Political

Economic

Ecological

Ecological 
The proposed operating principles (Table 4.1) entail a combination of ideologies presented by resource conservationists and resource preservationists. In this approach the 'resource conservationists' address those who are supportive of economic growth, but only under conditions which do not threaten the natural resource base. Likewise the 'resource preservationists' address those who are supportive of ecosystem integrity, but do not advocate a drastic de-population of the earth, or radical reduction in material lifestyle.

Table 4.1 shows a composite framework for sustainable development in which the components are interwoven with each other. This study sorts these operating principles into the four categories of ecological, economic, socio-cultural and political principles (Table 4.1-right column). Even for “mainstream” sustainable development, as shown in Table 4.1, ecological principles play a crucial role in achieving the forecasted outcomes for sustainable development.

\subsubsection{Ecological Framework and Environmental Awareness}

Environmental conservation should not be considered as a merely technological or economic activity; rather it should be viewed as a social-ecological phenomenon. May (1991:118) argues that if tourism is to play a conservative role in the maintenance and enhancement of the environment in the developing world, where many of the worst excesses of the so-called developed world have not yet been seen, it needs to consider the values which developers, host communities and visitors alike place on the environment. He (1991:118) introduces the idea of having a "better understanding of the values attached to people's environments" as the first step to bringing humanity closer to the goal of sustainability. Thus, awareness about environmental and natural values, and efficient strategies to conserve the natural heritage and its economic and social impacts on the host destinations could be pivotal parts of an ecological framework for the sustainable development of tourism.

Environmental awareness, as one of the main social-ecological outcomes for the sustainable development of tourism, can be conducted through an educational process which makes host communities, local and indigenous people, stakeholders and developers ready to be engaged in the environmental conservation process. Furthermore, this environmental awareness plays an important role to "move visitor experiences beyond 
passive enjoyment to an active role that promotes positive environmental ethics and fosters preferred behavior” (Fallon and Kriwoken, 2003: 289).

\subsubsection{Social-Ecological Goals for Ecotourism Development}

As Murphy (1983:181) points out, all planning (including planning for the sustainable development of tourism) needs goals to supply a frame of reference for detailed physical planning and for the constant management decisions that follow. He (1983:183) argues that these goals are abstract and continuous concepts intended to prepare a general direction rather than specific guidelines, but that they must have general support and be feasible to be effective.

This study views the environmental impacts of ecotourism development on the host destinations as a social-ecological issue caused by an unsustainable pattern of resource consumption, lack of social and cultural sensitivity to natural heritage, and lack of environmental awareness, plus expansion of the ecological footprint (EF) of tourism activities, products and services through the development process.

Table 4.2 shows the anticipated ecological outcomes which can be achieved from the sustainable development of ecotourism. By introducing these goals, this thesis attempts to progress the attitude of the tourism industry towards the environment from one of being merely economically exploitative to one of stewardship. The latter is something required for natural and environmental conservation.

Environmental awareness together with engagement of local and indigenous people in the natural conservation process are considered goals which can be achieved through a community approach to tourism development. In this view, these two prime goals play a pivotal role as the basic principles for the realization of the other forecasted ecological outcomes (Table 4.2). Furthermore, in this approach, education as potentially a main tourism activity can be explained as a transversal domain which has a close and effective relationship with all the other ecotourism activities shown in Table 4.2. 
Table 4.2: Ecological outcomes for development of sustainable ecotourism

Ecological outcomes

Environmental awareness

Engagement of local and indigenous people in the conservation process.

Attention to carrying capacity and sustainable yields

Protection of environmental resources and maintenance of biodiversity

Contribution through using renewable resources to generate energy used by tourism products and services.

\section{Productive activities}

Engagement of local and indigenous people as well as all related organizations (individuals, governmental and NGOs) in environmental education.

Employment of local and indigenous participants in the development process as employed or volunteer staff.

Making a balance between carrying capacity, resource consumption and the ecological footprint of tourism activities, products and services.

Using policies to reduce the ecological footprint of tourism activities, products and services

Enhancing use of green technologies based on renewable resource consumption and decreasing demands for fossil fuels to generate consumed energy.

\subsubsection{Definition of an Indicator}

An indicator "can be an objective measurement, such as a number, a specific rate or ration; it can however, also refer to an opinion, or to a perception that points to a specific condition or situation, and can be used to measure changes in that condition or situation over time” (Secretariat of the Pacific Community -SPC, 2010:1). In other words these pointers can be used to look closely at the resultant outcomes of initiatives and actions or interventions. In addition, indicators provide the benchmarks needed to monitor and measure promotion of a particular goal. The first section introduces the ecological, cultural and economic indicators used in this thesis for evaluation of ecotourism and its architecture, and the following, the proposed ecological indicators for sustainable development of ecotourism.

\section{a. Ecological Indicators}

"Ecological indicators are mainly used to assess the condition of the environment, as early-warning signals of ecological problems, and as barometers for trends in ecological resources” (Niemi and MacDonald, 2004: 89). Esther et al (2005:218) consider ecological indicators necessary for evaluating the effect of human activity on nature and the environment. As Christian et al (1996:89) reveal, most sets of ecological indicators proposed so far have focused on the state of the environment rather than on the interaction between society and ecosystems. Current environmental issues are caused not only by using technologies dependent on fossil fuels but also by social-cultural patterns of consumption of environmental resources. As a result, the solutions for these problems must be considered as social cultural policies. Thus, ecological indicators can be introduced 
as phenomena arising from socio-ecological principles. According to Christian et al (1996:89), the principles that form the basis of the socio-ecological indicators, focus on the societal activities and interactions between nature and society through the use of materials and energies.

\section{a. 1. The EF}

One of main aims of this thesis is to explore the current environmental impacts of tourism and propose guidelines for reducing these impacts. As discussed in Chapter 3, due to its strengths, the EF is used as a basic ecological indicator to evaluate ecotourism and its architecture as being environmentally sustainable.

The EF was used in master's study (O’Connor, 2009) to assess the impact of international tourists in New Zealand. The EFs were calculated of five categories of activities and services undertaken by international visitors to New Zealand: food; accommodation; transport; and goods, using the 2003 version 3,2 calculator (O’Connor, 2009:103).

According to O’Connor (2009:103) a survey was conducted in September 2008, from which 236 responses were received, resulting in a 33\% response rate. O’Connor (2009:92) calculates the EF of New Zealand international tourists as 8.26 gha/capita, which is 2.36 gha/capita more that the EF of a New Zealand resident (5.9 gha). She argues that the EF for tourists still sits at 55\% of New Zealand biocapacity.

O’Connor (2009:104) points out that the main tourist energy consumption is for transport, goods and purchases, and activities and attractions. She (2009:104) argues that cropland, as the second largest land area of the EF, contributed to food having the largest overall land area in the tourist EF.

O’Connor (2009) suggests lack of information; financial issues and time for research are the main limitations for calculating the EF of New Zealand tourists. She also determines approximation of the results arising from shortcomings in available information as another limitation of her research. This parallels discussions of the limitations of the EF in Chapter 3 Both studies indicate development of information systems as one of the necessities for sustainable development of tourism. 
Table 4.3 determines the proposed social-ecological indicators for evaluating the environmental impacts of ecotourism development on the host destinations and achieving the ecological goals of ecotourism shown in Table 4.2. Indicators ' $1 a$ ' and ' $1 b$ ' (Table 4.3), which indicate the number of participants in environmental education and the conservation process are also introduced as social cultural indicators. These indicators can also be viewed as complementary indicators to the EF to evaluate the success of the social dimension of environmental conservation principles.

\begin{tabular}{|c|c|c|}
\hline Ecological outcome & Indicator & Definition \\
\hline Environmental awareness & $1 \mathrm{a}$ & $\begin{array}{l}\text { The number of local people who participate in the } \\
\text { educational process }\end{array}$ \\
\hline $\begin{array}{l}\text { Engagement of local and indige- } \\
\text { nous people in the conservation } \\
\text { process. }\end{array}$ & $1 b$ & $\begin{array}{l}\text { The number of people who engage in the environ- } \\
\text { mental conservation process including volunteer or } \\
\text { employed participants }\end{array}$ \\
\hline $\begin{array}{l}\text { Attention to carrying capacity and } \\
\text { sustainable yield }\end{array}$ & 1e & $\begin{array}{l}\text { Ecological footprint of tourism activities, products } \\
\text { and services }\end{array}$ \\
\hline $\begin{array}{l}\text { Protection of environmental re- } \\
\text { sources and maintenance of biodi- } \\
\text { versity }\end{array}$ & 1e & $\begin{array}{l}\text { Ecological footprint of tourism activities, products } \\
\text { and services }\end{array}$ \\
\hline $\begin{array}{l}\text { Contribution through use of renew- } \\
\text { able resources to generate energy } \\
\text { used by tourism products and ser- } \\
\text { vices. }\end{array}$ & 1e & $\begin{array}{l}\text { Ecological footprint of tourism activities, products } \\
\text { and services }\end{array}$ \\
\hline
\end{tabular}

\subsubsection{EF by Types of Ecotourism Products and Activities}

This thesis attempts to calculate the pressure on natural resources exerted by visitors in their host destinations. As mentioned above, the EF is used as an indicator to evaluate tourism activities, products and services as being environmentally compatible. As a first attempt to achieve this goal, the EF of a visitor is divided into the three categories of; food consumption, accommodation services (as the most important additional infrastructural factor related to tourism expansion) and transportation (Table 4.4).

Mahravan and Vale (2010) calculated the ecological footprint of food, transportation, consumed water and accommodation services for the Otago Central Rail Trail (OCRT) visitors in 2009. Their study (2010:1393) shows that the total OCRT- EF is equivalent to 7,281gha, and the consumed water EF is equivalent to 116 gha, or just $1.6 \%$ of the total EF for OCRT visitors in 2009. This earlier study (2010:1393) shows that in comparison with food EF (2,570 gha), transportation EF (4,356 gha) and accommodation EF (239 gha), the consumed water EF is so small that, to simplify the list of ecological in- 
dicators used in this thesis, it can be ignored. In addition, as O’Connor (2009) reveals, the EF of waste related to New Zealand tourism accounts for 5\% of the total EF of New Zealand international tourism. Consequently, this thesis focuses on the four categories of transportation, food, accommodation and tourism activities and ignores other factors with small EFs.

\section{Table 4.4: Tourist EF and its sub-categories}

\begin{tabular}{|l|l|}
\hline \multicolumn{1}{|c|}{ Category } & \multicolumn{1}{c}{ Sub-categories } \\
\hline & Transportation EF \\
\hline Visitors' ecological footprint & Food EF \\
\hline Accommodation services EF \\
\hline Visitor activities \\
\hline
\end{tabular}

\subsubsection{Required Data}

Since the EF of tourism products and activity is calculated yearly, the required data must relate to a year, which is the baseline year of the EF. Table 4.5 shows the required data related to each EF category. This thesis aims to use the data related to 2011 to calculate the EF of tourism in the three case studies. Since some of the required data related to 2011 are not available, data related to the previous years are used to calculate the EF and where this happens will be addressed throughout the thesis.

As shown in Table 4.5, the data required to measure the EF of the transportation used by visitors are divided into two categories: required data related to transportation used by international visitors and data related to travel by domestic tourists. The differences between these two sets of data can be explained as the differences between the types of transportation used and their different EFs and the total tourism-km travelled (international visitors travel a lot further).

In this thesis, visitors travelling along the Otago Rail Trail, whether by foot or on bicycles are viewed as participating in visitor activities and EF is separately calculated separately. Thus to avoid double counting in the EF of visitors activities related to travelling, in Table 4.5 transportation used while in Otago is excluded. In Table 4.5, transportation comprises the types of transportation used to access the OCRT.

In Table 4.5 it is assumed both international and domestic visitors effectively eat the same amount of food and use the same types of accommodation services in a host desti- 
nation. Thus, as shown in Table 4.5, to calculate the EFs of food and accommodation services consumed and used by visitors, the total number of visitors (international and domestic) is used. Likewise, some data such as ecological carrying capacity and visitor nights are common between all relevant EF categories.

\begin{tabular}{|c|c|c|}
\hline \multicolumn{3}{|c|}{ Table 4.5: EF categories and required data } \\
\hline \multicolumn{2}{|c|}{ EF Category } & Required Data \\
\hline \multirow{12}{*}{ Transportation } & \multirow{8}{*}{$\begin{array}{l}\text { International } \\
\text { Transportation }\end{array}$} & Number of international visitors by home place \\
\hline & & Total number of international visitors \\
\hline & & $\begin{array}{l}\text { Distance between international visitor home places and host } \\
\text { destination by types of transportation used }\end{array}$ \\
\hline & & Total international tourism- km \\
\hline & & International T-km by types of transportation used \\
\hline & & Energy use MJ/ Passenger-km by types of transportation used \\
\hline & & Total Energy use MJ \\
\hline & & Ecological Carrying Capacity \\
\hline & \multirow{4}{*}{$\begin{array}{c}\text { Domestic } \\
\text { Transportation }^{1}\end{array}$} & Number of domestic visitors by visitor home place \\
\hline & & Domestic tourism- km by type of transportation used \\
\hline & & $\begin{array}{l}\text { Energy use MJ/ (domestic) Passenger-km by types of transporta- } \\
\text { tion used }\end{array}$ \\
\hline & & Total Energy use MJ (for domestic visitors) \\
\hline \multirow{6}{*}{\multicolumn{2}{|c|}{ Food }} & Number of visitors \\
\hline & & Average number of nights visitors stay during their visit \\
\hline & & Food eaten out per visitor \\
\hline & & Energy use MJ per kg food \\
\hline & & Total energy use (for food consumed by visitors) \\
\hline & & Ecological carrying capacity \\
\hline \multirow{6}{*}{\multicolumn{2}{|c|}{ Accommodation }} & $\begin{array}{l}\text { Average number of visitor nights by types of accommodation } \\
\text { used }\end{array}$ \\
\hline & & Number of visitors \\
\hline & & Total visitor nights by types of accommodation used \\
\hline & & Energy use per visitor night by types of accommodation used \\
\hline & & Total energy use by types of accommodation used \\
\hline & & Ecological carrying capacity ${ }^{2}$ \\
\hline \multicolumn{3}{|c|}{$\begin{array}{l}\text { 1. Domestic transportation comprises the access to the OCRT and travelling along the OCRT is excluded. } \\
\text { 2. Ecological carrying capacity is used to calculate the EF of the products (e.g. accommodation services) and ac- } \\
\text { tivities (e.g. walking and golfing) through following equation: } \\
\text { EF = Total lifecycle energy use / Ecological carrying capacity. }\end{array}$} \\
\hline
\end{tabular}

\subsubsection{Discussion}

This section considers the process of environmental conservation through the development of sustainable ecotourism as a social-ecological process based on a community approach to tourism. Forecasted ecological outcomes for the sustainable development of tourism tend to engage local and indigenous participants (who achieve environmental awareness through taking part in an educational process) in the environmental conservation procedure. Likewise, reducing the negative impacts of ecotourism on natural resources, which can be achieved by decreasing the EF of ecotourism products, services 
and activities, forms a crucial part of the anticipated ecological goals for tourism in this study.

The three categories of transportation, food and accommodation are introduced as the important representative products and services of ecotourism because they have the highest energy use and the biggest EF. Consequently, in this thesis, the EFs of these three categories are used as ecological indicators to evaluate the environmental compatibility of ecotourism development in the host destinations. This study, therefore, also presents a list of the required data which can be used to measure two things:

a. The level of environmental awareness among local and indigenous people and the number of participants in environmental conservation through tourism development.

b. The ecological footprint of tourism's products, services and activities.

This ecological framework, related indicators and required data are viewed as a subsector of an integrated framework for the sustainability of ecotourism development.

\subsubsection{Cultural Framework for the Sustainable Development of Ecotourism}

To attempt to achieve sustainability through the development of ecotourism needs constant monitoring and this could be done through the evaluation of the cultural footprint of tourism as well as its ecological and economic impacts on the host destinations. This study attempts to introduce an efficient cultural framework for this, together with its related indicators and required data. This can then be used to evaluate the CF of ecotourism through sustainable development in a host destination.

This study introduces a theoretical base for the creation of a cultural framework which includes definition of pivotal factors (such as culture, development, and cultural dimension of development) that play important roles in structuring a cultural framework. To support the latter theory, this study presents some examples of existing frameworks for cultural statistics, and cultural frameworks for sustainable tourism. One of the main outcomes of this study will be a cultural framework for ecotourism and development of sufficient cultural indicators with their required data. These can then be used as a tool for evaluating the CF of ecotourism in the host destinations. This cultural framework is considered as complementary to the ecological and economic frameworks to create an overall comprehensive framework which has the ability to evaluate sustainability through development of ecotourism. 


\subsubsection{Culture}

As defined by The Mexico Declaration on Cultural Polices in 1982, "culture is the whole of distinctive traits - spiritual and material, intellectual and affective characterizing a society or a social group” (Breis, 1992:2). It is the sum total of the ways through which a group builds up a pattern for living that is transmitted from one generation to another. Cochrane (2006:322) argues that culture consists of patterns, explicit and implicit, of and for behavior acquired and passed on by symbols, constituting the distinctive achievement of human groups, including their embodiments in artifacts. The essential core of culture consists of traditional (i.e. historically derived and selected) ideas and especially their attached values; cultural systems may, on the one hand, be considered as products of action, and on the other as conditioning elements of further action.

Gerring and Barresi (2003) have suggested a 'min-max' strategy to produce a core definition of the concept of culture. They (2003:205) break down this min-max strategy into three steps (as shown in Table 4.6), comprising sampling usage, typologizing attributes and constructing minimal and ideal-type definitions.

Table 4.6: The process of Min-Max Definition (Gerring and Barresi, 2003:205)

1 Sample (sample representative usages and definitions within a linguistic context)

2 Typologize (arrange non-idiosyncratic attributes in a single typology).

3 Define

(a) Minimal (identify those few attributes that all non-idiosyncratic uses of the term have in common).

(b) Ideal-type (identify those attributes that define a term in its purest, most 'ideal', form).

"Minimal definitions identify the bare essentials of a concept, sufficient to [bind] it extensionally while maintaining all non-idiosyncratic meanings associated with the term" (Gerring and Barresi, 2003:207). They (2003:207) point out that minimal definitions embody all definitional attributes that are necessary and therefore, are always present. Gerring and Barresi (2003:208) state that in contrast, ideal-type definitions aim for a collection of attributes that is 'maximal' in that it includes all non-idiosyncratic characteristics that together define the concept in its purest, most 'ideal' form. As Cochrane (2006: 322) argues, the ideal definition is 'fuzzier' but the minimal definition has relatively clear borders. Table 4.7 shows attributes of minimal and ideal-type definitions of culture. 


\begin{tabular}{|c|c|}
\hline \multicolumn{2}{|c|}{ Table 4.7: ‘Culture’: Min-Max Definitions (Gerring and Barresi, 2003:210). } \\
\hline $\begin{array}{l}\text { Minimal Attributes: } \\
\text { Production and Transmission: } \\
\text { Characteristics: }\end{array}$ & $\begin{array}{l}\text { Social } \\
\text { Ideational or symbolic } \\
\text { Patterned } \\
\text { Shared }\end{array}$ \\
\hline $\begin{array}{l}\text { Ideal-Type Attributes: } \\
\text { Production and Transmission: } \\
\text { Characteristics: }\end{array}$ & $\begin{array}{l}\text { Social } \\
\text { Human } \\
\text { Ideational } \\
\text { Patterned } \\
\text { Shared } \\
\text { Enduring } \\
\text { Cumulative } \\
\text { Coherent } \\
\text { Differentiated } \\
\text { Comprehensive } \\
\text { Holistic } \\
\text { Non-interest-based } \\
\text { Implicit }\end{array}$ \\
\hline Functions: & $\begin{array}{l}\text { Casual } \\
\text { Constitutive }\end{array}$ \\
\hline
\end{tabular}

Cochrane (2006:322) and Gerring and Barresi (2003:211) when taken together reveal that culture is a set of beliefs or behaviors in its minimal form which are produced, transmitted and learned socially and often spoken of as a heritage or tradition. Cochrane (2006:322) states that only formal rules and behaviors which signify something other than themselves can be viewed as cultural. Cultures are also captured by social scientists by being recognized as phenomena having the near synonyms such as 'ordered' or 'organized'. Gerring and Barresi (2003:211) use this as an attribute by saying that cultures are patterned. Cochrane (2006:322) believes that there is a degree of pattern to a culture (although it is not always obvious), as humans are born into a culture, rather than randomly producing one. In addition, in terms of both minimal and ideal-type attributes, a culture is shared by a social group such as a nation, an ethnic group, or tribe (see Table 4.7).

A culture, in its ideal type, as well as having the characteristics mentioned above, is enduring in nature (being slow to change), coherent and interconnected, unique to a particular group, and comprehensive and holistic in the range of beliefs and practices covered by it (rather than being one dimensional and related to a single issue). Likewise, beliefs and practices related to culture are general instead of being merely a function of self-interest and formulized. The functions of culture are both casual and constitutive. In fact, culture constrains and influences human actions and influences people and their experiences by imparting a certain recognizable essence. 


\subsubsection{Culture and Environment}

This part of the study presents an ecological view of a society and its cultural structure to explore the relationship between culture and environment. It begins with introducing the interaction between an ecological organism and its components with their surrounding environment.

Williams (2007:2) explains that the biosphere (as the livable part of the environment for plants, animals and humans) is composed of the earth plus the sliver of thin air extending out six miles from the earth's surface and that all life in this zone relies on the sun's energy. "The biosphere has specific bioclimatic zones called biomes, which are tailored to their climate, soil, physical features, and plant and animal life” (Williams, 2007:2). Lawrence (2003:31) argues that ecology is defined as a science that deals with the interrelationship between organisms and their surroundings.

Humans are part of the environment, and all changes in nature and their habitat affects them. It is important to know what is being affected and how. Odum (cited in Williams, 2007:3) devised an ecological model which illustrates the relationship between flows of energy and materials, between system components and between producers and consumers (Figure 4.1).

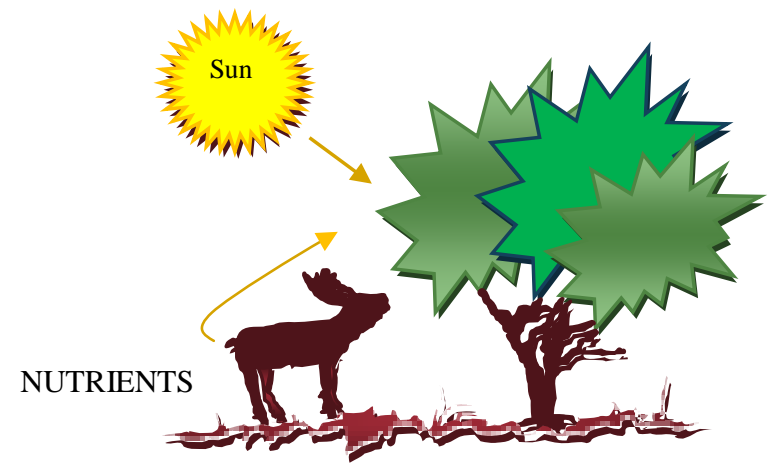

Figure 4.1: Flows of energies and materials in an organism

Williams (2007:3) remarks that all of life functions in the way shown in Figure 4.1. The relationship between the components can be changed by changing the connections and flows between the components and some of these changes can be caused by humans. For example, construction of a dam to increase production of electricity or to contribute to the development of agriculture can change the covering of plants and increase the expansion of desert in the adjacent areas located on the lower levels. This land use change 
results in a gain to an organic community (people), but also the loss of contributions from nature, such as failure to access clean water.

Odum's model illustrates the simple and essential relationship between natural energies and renewable resources (Figure 4.1). In this view, becoming a sustainable organism is achieved by using natural energies and renewable resources. On the other hand, an organism is sustainable when all components, micro-organisms and communities are adapted to fit with the natural laws that are dominant in the whole process of energy and material cycles in nature. Humans are not exempted from being compatible with natural laws. In this they are like all other natural organisms.

Using an ecological perspective, culture is a system which as Rapoport (1969) demonstrates has an interconnection with the environment. Culture can be manifested through the religious beliefs, intellectual and spiritual engagement, materials used, and products (such as art, architecture, food, and textiles) of a given group or society. If a society is viewed as an organism, culture can be defined as the way in which physically and spiritually it makes a linkage between the given society and its surroundings.

\subsubsection{Culture and Development}

Development is defined in the Final Report of Mondiacult (cited in Breis, 1992:2) as a complex, global and multi-dimensional process, going beyond mere economic growth to include all dimensions of life and all the energies of a community. In addition all members of a society must take part in the effort to achieve the economic and social transformation of its welfare.

The World Commission on Culture and Development (WCOCAD) (1996) analyzed development in two different ways. Firstly, "according to the standard view, development equals economic growth, sometimes qualified by insistence on a wide spread of the benefits of growth" (WCOCAD, 1996:14). Secondly, as advocated by UNESCO, UNDP's annual Human Development Report and many distinguished thinkers, (cited in WCOCAD, 1996:14), development is a process that enhances the effective freedom of the people involved to pursue whatever they have reason to value. WCOCAD (1996: 14) indicates that in this view, not only the lack of essential goods and services, but also a shortage of opportunities to choose a fuller, more satisfying, more valuable and valued existence cause poverty of life. However, this raises the question of whose values form 
the benchmark - those of the existing community that may well relate to what the community can have based on sustainable use of the natural resources available, or of those, such as UN thinkers based in the west, doing the assessment?

The cultural dimension of development can be viewed at global, national and regional scales. One of the main aims for such development is promotion of cultural cooperation. Such co-operation between peoples with widely different cultural backgrounds and interests can only be achieved when they all share certain principles. WCOCAD (1996:17) defines five ethical "pillars” as tools for achieving this goal, comprising: human rights and responsibility; democracy and the elements of civil society; the protection of minorities; commitment to peaceful conflict resolution and negotiation; and intergenerational equity. WCOCAD (1996) suggests a top-down approach for cultural development based on these five pillars. This approach emphasizes that "it is incumbent upon all governments to give effect to such principles. But the implementation of a global ethics requires other factors as well: transnational co-operation, international organizations, and the global civil society” (WCOCAD, 1996:17).

\subsubsection{FCS—a Framework for Cultural Statistics}

As explained by The Institute for Statistics of the United Nations Education Scientific and Cultural Organization (UIS) (2010:9), the Framework for Cultural Statistics (FCS) is viewed as a tool for organizing cultural statistics both nationally and internationally. This framework is based on a conceptual foundation and a common understanding of culture that will enable the measurement of a wide range of cultural expression irrespective of the particular economic and social mode of its production. One of the main aims for this framework is the production of internationally comparable data related to cultural activities and products.

According to UIS (2010:9) because of the greater availability of economic data and existence of the many international classification systems, in comparison with the social dimension of culture, the measurement of its economic dimension is more fully developed in FCS. This framework as a classification instrument attempts to incorporate the use of available international classification systems such as the International Standard Industrial Classification (ISIC) for cultural production activities, the Central Product Classification (CPC) for cultural goods and services, the International Standard Classification of Occupations (ISCO) for cultural employment, the Harmonized Commodity 
Description and Coding System (HS) for international flows of cultural goods, and the UN Trial International Classification of Activities for Time-use Statistics (ICATUS) for cultural participation.

The outcome of FCS is a tool and methodology which can be used at the international and national levels as a foundation for organizing the collection and dissemination of cultural data based on using the available economic data. According to UIS (2010:9) FCS makes efforts to reflect the widest range possible of activities related to the production, distribution and use of culture.

One of the main issues related to using FCS as a framework with its related indicators to evaluate products as being culturally appropriate is a shortage of data and information that can cover the social dimension of culture, including cultural participation and intangible cultural heritage. As Horn (1983:25) points out, the scope of culture obviously goes beyond the economic aspects, despite the fact some cultural activities can be fitted into an economic schema of provision of goods and services.

\subsubsection{Culture Cycle}

A framework for cultural statistics contains information and data raised from different sectors of the culture cycle. Thus, exploration and clarification of the culture cycle can portray the areas from which the framework, indicators and data come.

UNESCO (2001) has suggested that "culture should be regarded as the set of distinctive spiritual, material, intellectual and emotional features of society or a social group, and that it encompasses, in addition to art and literature, lifestyles, ways of living together, value systems, traditions and beliefs” (UIS, 2010:18). Based on this, the culture cycle has been explained by UIS (2010) as shown in Figure 4.2.

The culture cycle covers all of the different phases of the creation, production, and dissemination of culture (Figure 4.2). In this view, culture can be approached as resulting from a cognate set of processes. These processes capture institutionalized and noninstitutionalized activities. In addition, these cultural activities may or may not be governed by the state. The culture cycle in the diagram is divided into five stages: creation, production, dissemination, exhibition/ reception/transmission, and consumption/ participation (Figure 4.2). According to UIS (2010:19) these stages are presented in a cyclical 
instead of hierarchical model to emphasis the idea that relationships between the latter stages can be complex and occur more as a network than in a linear form.

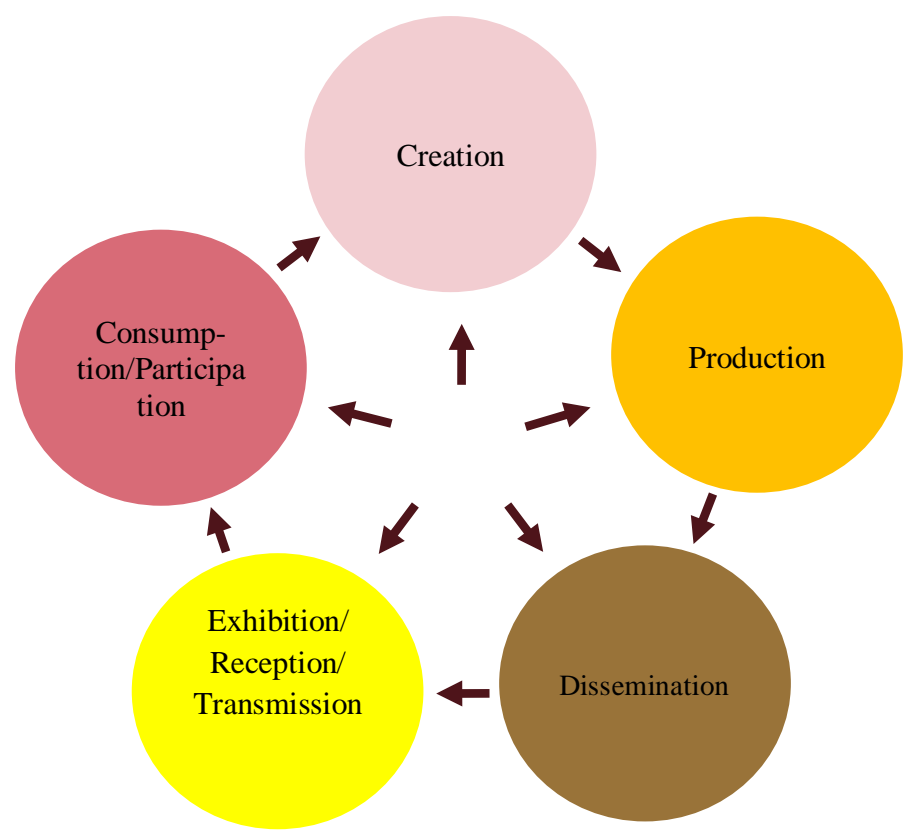

Figure 4.2: Culture cycle (UIS, 2010: 20)

The UIS (2010:20) further explains that the term culture cycle includes the interconnection across cultural activities, including the feedback processes by which activities (consumption) inspire the creation of new cultural products and artifacts. For some cultural activities, the process can be started at any stage of the culture cycle. For instance in terms of cultural heritage, the stages of creation and production have occurred in the past and culture cycle processes related to heritage include the other three stages.

As introduced by the UIS (2010:20), what is important for the culture cycle is to understand and be able to track the totality of activities and necessary resources that are required to transform ideas into cultural goods and products. These, in turn, have to reach consumers, participants or users. This approach is more than just being concerned with making judgments on how 'cultural' any specific aspects of the cycle are.

\subsubsection{Cultural and Related Domains}

According to the UIS (2010:22) the FCS uses a pragmatic definition of culture which is based on the representation of culture by domains for which the aim is to calculate cul- 
tural activities, goods and services that are produced by industrial or non-industrial processes.

Cultural goods and services capture artistic, aesthetic, symbolic and spiritual values. Throsby (2001) (cited in UIS 2010:22) points out that there are differences between the characteristics of cultural goods and services and other products. Throsby (2001) argues that these differences arise from the system of assigning values to the former, which includes a characteristic of being irreproducible, and which is linked to their appreciation or the pleasure they give. Cultural goods play an important role in conveying ideas, symbols and way of life and cultural services facilitate the production and distribution of these goods. UIS 2010 sets out the FCS cultural domains as shown in Figure 4.3.

Figure 4.3 reveals a set of common culturally productive industries, activities and practices which are represented as domains in the FCS including:
A: Cultural and natural heritage.
B: Performance and celebration.
C: Visual arts and crafts.
D: Books and press.
E: Audio-visual and interactive media.
F: Design and creative services.
G: Transversal domains (UNESCO 2006 and 2009).

FCS considers these domains to be cultural. According to UIS (2010:23) these domains represent the minimum set of core cultural domains for which UNESCO would encourage countries to collect comparative data. Moreover, four other transversal domains are included: intangible cultural heritage, education and training, archiving and presentation, and equipment and supporting materials, because of their important influences on the culture cycle in terms of production and transmission of culture. These transversal domains can be applied to all of the cultural and related domains (Figure 4.3). As shown in Figure 4.3, related domains are separated into the two categories of: ' $\mathrm{H}$ '- tourism and 'I'- sports and recreation. 


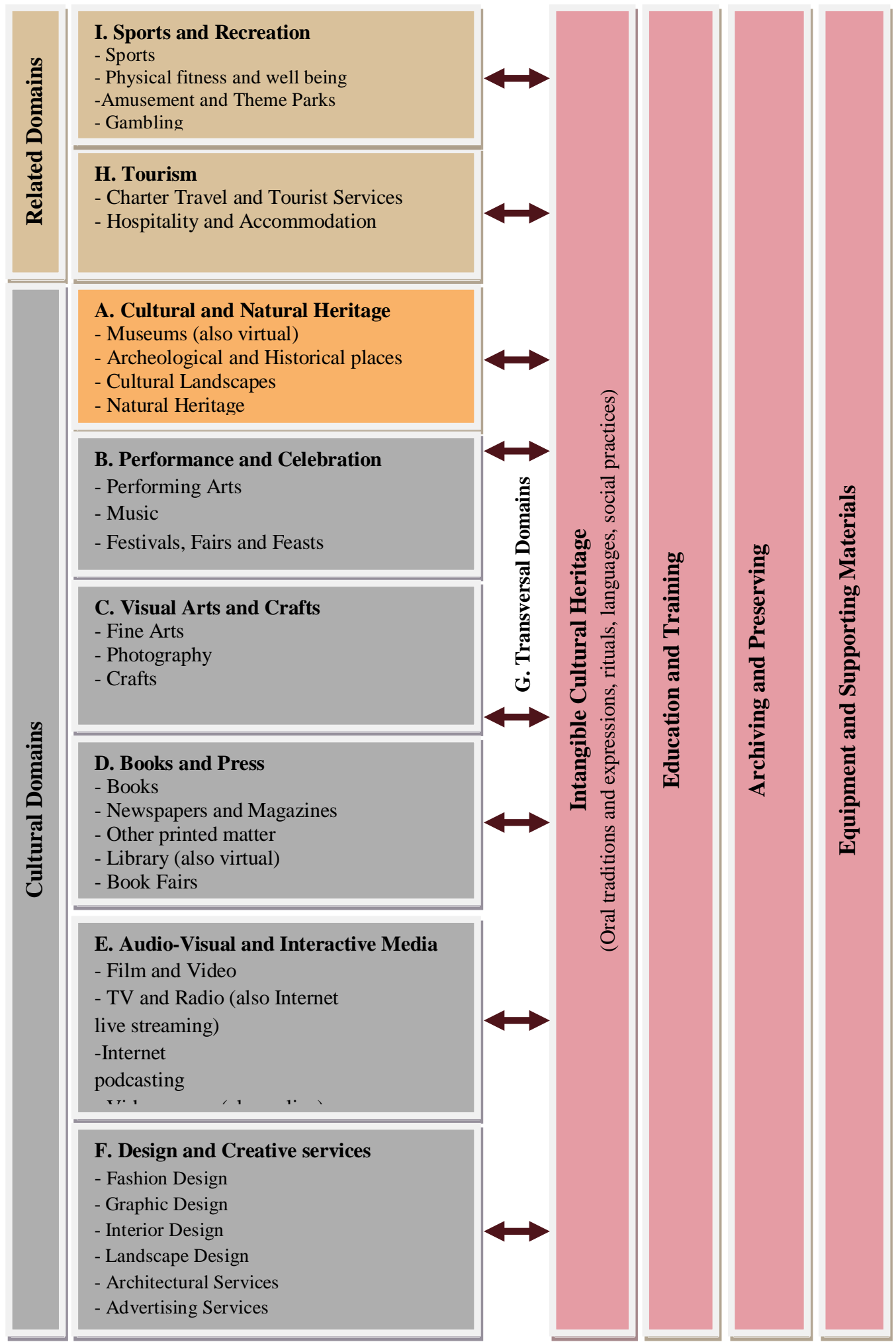

Figure 4.3: Framework for Cultural Statistics Domains (UIS, 2010:24).

\subsubsection{Tourism as a Related Domain}

The UIS (2010:30) views tourism as a domain which is qualitatively different from the other cultural domains, as it cannot be readily classified as a sector in the traditional sense. For instance, it cannot be measured by either a particular market or industrial 
output. Rather tourism should be considered as an activity which is intimately related with all other cultural and transversal domains.

The UIS (2010:58) also identifies the most important productive activities and their related products (goods and services) which can be classified in the tourism domain (Table 4.8). Overall these productive activities can be classified into three categories comprising: types of transportation, types of accommodation (short-term accommodation and camping grounds as examples), and travel activities (travel agency, tour operators and reservation activities) (Table 4.8). Moreover the suggested goods and services which are viewed as tourism products are limited to these productive activities.

Table 4.8: Framework for cultural statistics domains—related domains (tourism). (UIS, 2010:58)

\begin{tabular}{|c|c|c|c|c|}
\hline \multicolumn{5}{|c|}{ RELATED DOMAINS } \\
\hline Domain & & Productive activities & & Goods and services \\
\hline \multirow{7}{*}{$\begin{array}{l}\text { H. Tour- } \\
\text { ism }\end{array}$} & ISIC $^{1} 4$ & Description & $\mathrm{CPC}^{2} 2$ & Description \\
\hline & $\begin{array}{l}4911 \\
4922 \\
5011 \\
5110\end{array}$ & $\begin{array}{l}\text { Passenger rail transport, Inter } \\
\text { urban } \\
\text { Other passenger land transport } \\
\text { Sea and costal passenger water } \\
\text { transport } \\
\text { Passenger air transport }\end{array}$ & $\begin{array}{l}64131 \\
64132 \\
64133 \\
64134\end{array}$ & $\begin{array}{l}\text { Sightseeing services by rail } \\
\text { Sightseeing services by land, except rail } \\
\text { Sightseeing services by water } \\
\text { Sightseeing services by air }\end{array}$ \\
\hline & 5510 & $\begin{array}{l}\text { Short-term accommodation activi- } \\
\text { ties }\end{array}$ & $\begin{array}{l}63111 \\
63112 \\
63113 \\
63114\end{array}$ & $\begin{array}{l}\text { Room or unit accommodation services for } \\
\text { visitors with daily housekeeping services } \\
\text { Room or unit accommodation services for } \\
\text { visitors without daily housekeeping ser- } \\
\text { vices } \\
\text { Room or unit accommodation services for } \\
\text { visitors in time-share properties } \\
\text { Room or unit accommodation services for } \\
\text { visitors in rooms for multiple occupancy }\end{array}$ \\
\hline & 5520 & $\begin{array}{l}\text { Camping grounds, recreational } \\
\text { vehicle parks and trailer parks }\end{array}$ & $\begin{array}{l}63120 \\
63130\end{array}$ & $\begin{array}{l}\text { Camp site services } \\
\text { Recreational vacation camp services }\end{array}$ \\
\hline & 7911 & Travel agency activities & $\begin{array}{l}85511 \\
85521 \\
85523 \\
85524\end{array}$ & $\begin{array}{l}\text { Reservation services for air transportation } \\
\text { Reservation services for accommodation } \\
\text { Reservation services for cruises } \\
\text { Reservation services for package tours }\end{array}$ \\
\hline & 7912 & Tour operator activities & 85540 & Tour operator services \\
\hline & 7990 & $\begin{array}{l}\text { Other reservation services and } \\
\text { related activities }\end{array}$ & $\begin{array}{l}85512 \\
85513 \\
85514 \\
85519 \\
\\
85539 \\
\\
85550 \\
85561 \\
85562\end{array}$ & $\begin{array}{l}\text { Reservation services for rail transportation } \\
\text { Reservation services for bus transportation } \\
\text { Reservation services for vehicle rental } \\
\text { Other transportation arrangement and res- } \\
\text { ervation services } \\
\text { Reservation services for event tickets, } \\
\text { entertainment and recreational services and } \\
\text { other reservation services } \\
\text { Tourist guide services } \\
\text { Tourism promotion services } \\
\text { Visitor information services }\end{array}$ \\
\hline
\end{tabular}

Overall then these products can be divided into the two categories of: transportation and accommodation, within which both categories have their related reservation services 
(Table 4.8). The mutual relationship between tourism and other cultural domains through sustainable development of the former obligates consideration of tourism in a wider area, because its domain covers all other cultural productive activities and their produced goods and services. On the other hand, a cultural framework that is aimed to be used as a foundation for cultural indicators in order to evaluate the CF of tourism must contain all the tools used and activities conducted during the development process.

\subsubsection{Tourism and Transversal Domains}

The UIS (2010:28) considers “transversal domains” (used by UNESCO 2006 and 2009) as domains that are viewed transversely because they can be applied across the other cultural domains, although these domains can stand alone as independent domains (Figure 4.3). These transversal domains are discussed in detail below.

\subsubsection{G- Intangible Cultural Heritage}

UNESCO (2003) defines Intangible Cultural Heritage (ICH) as the "practices, representations, expressions, knowledge, skills - as well as the instruments, objects, artifacts and cultural spaces associated therewith - that communities, groups and, in some cases, individuals recognize as part of their cultural heritage” (UNESCO, 2003:2). These are wide ranging definitions and suggest that a heritage which is transmitted from generation to generation and created by communities and groups in response to their environment, their interaction with nature and their history, will have the ability to reflect its identification. Furthermore a unique feature which is defined for ICH by UIS (2010) is that it can be defined as such when a community recognizes this as a part of its heritage.

An intimate link between sustainable tourism and ICH can be manifested such that the cultural framework and indicators related to tourism must contain an expanded range of cultural activities and products that are accepted as heritage by given communities and groups. This means that a top-down approach to the creation of cultural frameworks and indicators at international or national level may ignore local cultural activities or products because, according to their features, these heritage activities can be different from one place to another or different communities at the local scale may have differing cultural heritages. 


\subsubsection{Education and Training}

The education and training domain as part of the ICH domain can play an important role in the expansion of the tourism domain and its related activities and products. According to UIS (2010:31) FCS refers to the cultural dimension of tourism, embracing cultural tourism, spiritual tourism and ecotourism activities. In this view, FCS defines cultural tourism as a "customized excursion into other cultures and places to learn about their people, lifestyle, heritage and arts in an informed way that genuinely represents their values and historical context including the experiencing of difference” (UIS, 2010:31). Accordingly this definition can also cover spiritual tourism or ecological tourism (ecotourism).

This definition shows that learning about culture is a core activity for cultural tourism and all other cultural activities and related goods and services can be used in the process of learning or education through the development of tourism. On the other hand education can be conducted in different ways, such as learning about culture by having face to face contact with local people, visiting museums, direct interaction with architectural spaces, or by using photography as a tool to record cultural events. These characteristics of education allow viewing it as a part of comprehensive framework which has the ability to define cultural indicators and to evaluate the CF of sustainable tourism and its sub-segments such as ecotourism.

\subsubsection{Cultural Capital and Production of Goods and Services}

One of the important goals for the sustainable development of ecotourism is the production of welfare, goods and services for host communities and visitors. Cochrane (2006:321) presents a model to introduce the role of interaction between cultural capital and other forms of capital, such as natural capital, human-made capital and cultivated natural capital, to produce welfare, goods and services for users, as shown in Figure 4.4.

In this model human-made capital is produced by the use of natural capital through its provision of raw materials and environmental services. Sinks for human produced waste products are also a part of natural capital (Figure 4.4). 


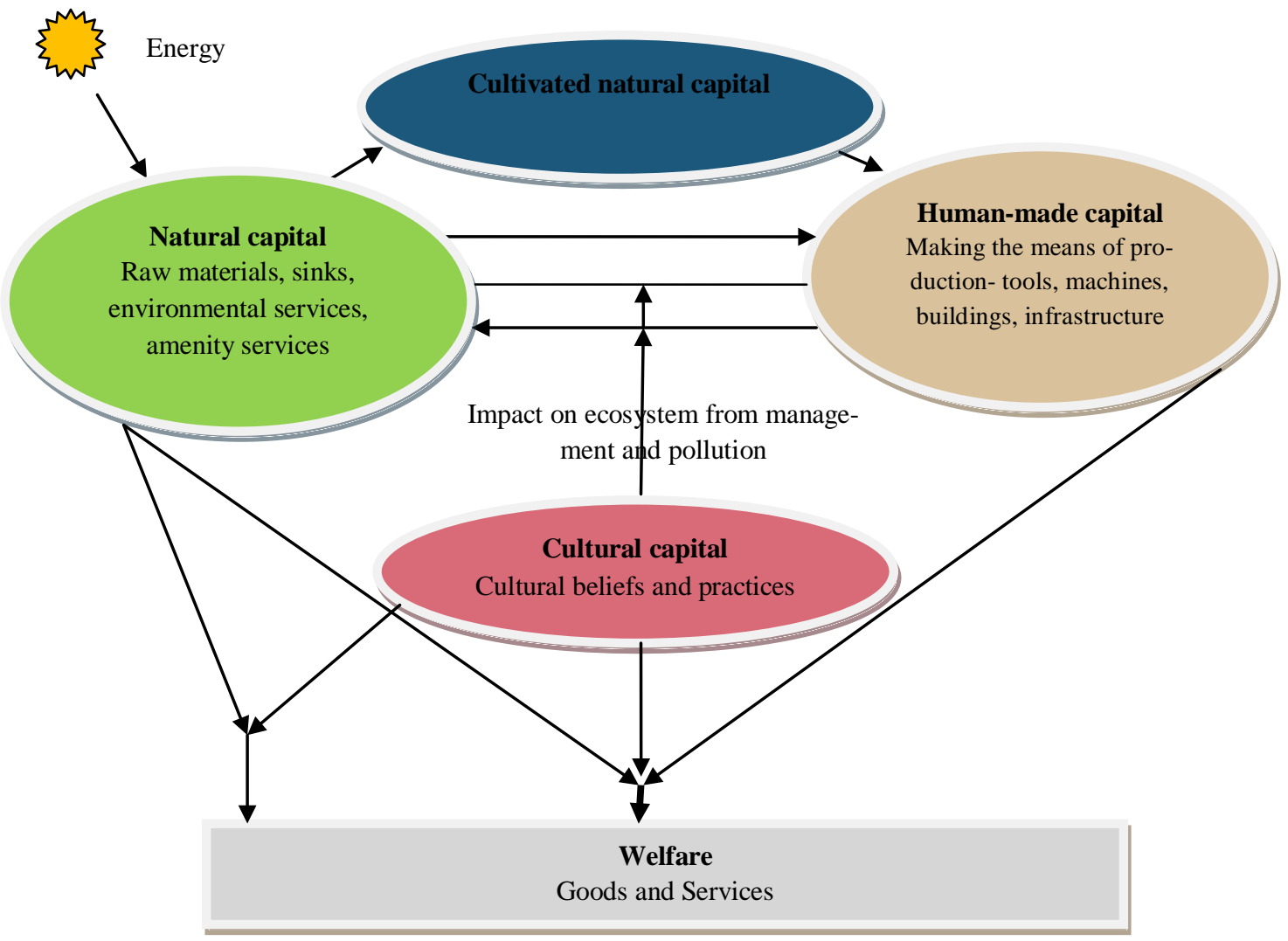

Figure 4.4: The interaction between forms of capital and the protection of welfare (Cochrane, 2006:321)

Although the processes described above will deplete natural capital stocks, this depletion can be reduced by replacing what is taken with renewable natural resources, for instance by planting trees or composting organic wastes. Berkers and Folke (1994, cited in Cochrane, 2006:321) argue that cultural capital is positioned at the interface of natural capital and human-made capital and plays an important role in mediating and controlling natural capital and human made capital interactions. Berkers and Folke (cited in Cochrane, 2006:321) also explain the fundamental and co-evolutionary relationship between the different types of capital. In this example, three forms of capital combine to produce final products (welfare, goods and services). Indeed, these products are dictated by the nature of the latter capitals. This again illustrates the complex nature of culture (society), environment and economy. In fact the idea that they can be simply separated, as in the weak sustainability model, may be impossible. This idea will be explored further in this part of the thesis. 


\subsubsection{Cultural Frameworks and Indicators for the Evaluation of Tourism}

\section{Products: examples}

\section{a. Choi and Sirakaya Model (2006)}

A 2006 study by Choi and Sirakaya presented sustainability indicators for the measurement of community tourism development (CTD) within a sustainable framework. In order to develop such objective indicators their study employed a modified Delphi technique. The Delphi technique is "a method for structuring a group communication process so that the process is effective in allowing a group of individuals, as a whole, to deal with a complex problem” (Linstone \& Turoff page, 1975: 3). According to Linstone and Turoff (1975) and Wechsler and Wolfgang (1978) in the Delphi method, the experts answer questionnaires in two or more rounds. The earlier answers of the experts are revised in light of the feedback from other members of their panel. Through this process the range of answers will decrease and the group will converge towards a single answer. Finally, the process is stopped after a pre-defined stop criterion (e.g. number of rounds,) and the final results are indicated as the mean or median scores of the final rounds.

According to Choi and Sirakaya (2006:1274), based on the Delphi technique, a panel of 38 academic researchers in tourism, after three rounds of discussion, reached consensus on the set of 125 indicators which were divided into six categories: political (32), social (28), ecological (25), economic (24), technological (3) and cultural dimensions (13) for CTD.

Choi and Sirakaya (2006:1274) further reveal that if the changes wrought by tourism upon all aspects of community life are to be effectively tracked, the latter indicators must be based on policy relevance, analytical soundness, and measurability. They (2006:1275) point out those indicators with these characteristics can be used in various settings and particularly at the local level where it matters the most.

Choi and Sirakaya (2006) suggest that sustainable community tourism (SCT) is comprised of ecological, social, economic, institutional/political, cultural and technological dimensions at the international, national, regional and local community levels, and within agriculture, tourism, political science, economic and ecology. However much of the literature on sustainable tourism has focused on the traditional dimensions (economic, social, cultural and ecological dimensions), and the other dimensions (political and technological) have been viewed as sub-segments of the main traditional dimensions. 
As a result, in comparison with Choi and Sirakaya's (2006) framework with its greater number of dimensions, the traditional view suggests simpler frameworks and indicator sets that could be used to evaluate sustainability through tourism.

According to the latter view, Choi and Sirakaya (2005:1281) have suggested some indicators for evaluation of the cultural dimension of sustainability through the development of tourism (Table 4.9). As shown in Table 4.9, the cultural framework is divided into the four domains of: building and architecture, cultural (site) management, sociocultural fabric, and cultural education. According to the basic definitions of culture which have been presented in this section, all the cultural domains (Table 4.9) are interwoven together and in some cases the indicators which are suggested for a particular domain could be used as indicators in the other domains. For instance, types of building material and décor could be used as an indicator to evaluate goods and services which are produced in relation to cultural education or cultural (site) management.

Table 4.9: Indicators for the cultural dimension (Choi and Sirakaya, 2005:1282)

\begin{tabular}{|c|c|}
\hline Key themes & Indicators/Issues \\
\hline \multirow[t]{2}{*}{ Building/architecture } & Comparability of new construction with local vernacular \\
\hline & Types of building material and décor \\
\hline \multirow{4}{*}{$\begin{array}{l}\text { Cultural (site) manage- } \\
\text { ment }\end{array}$} & Cultural sites maintenance level \\
\hline & Availability of cultural site maintenance funds and resources \\
\hline & Commodification \\
\hline & Number of officially designated sites and their management \\
\hline \multirow[t]{4}{*}{ Socio-cultural fabric } & Retention of local customs and language \\
\hline & Shift in level of pride in local cultural heritage \\
\hline & Percent satisfied with cultural integrity/sense of security \\
\hline & Loss of authenticity and becoming impersonal \\
\hline \multirow[t]{3}{*}{ Cultural education } & Type and amount of training given to tourism employees (guides) \\
\hline & $\begin{array}{l}\text { Type of information given to visitors before and during site visits (e.g. } \\
\text { tourist in-flight video or public awareness material) }\end{array}$ \\
\hline & Level of sensitivity of interpretive material and activities pursued \\
\hline
\end{tabular}

One of the important policy actions to further simplify cultural frameworks and indicators and the evaluation of the CF of ecotourism, is to distinguish the cultural domain(s) that have the ability to cover other domains, indicators, cultural goods and services. This can be conducted by using the Delphi methodology. This method can reduce the number of frameworks and make linkages between indicators which are currently viewed as being separate. 


\section{b. Cultural Framework for New Zealand}

In 2006 the New Zealand government put forward a cultural outcomes framework which by 2009 had been justified and improved (Tables 4.10 and 4.11). According to Statistics New Zealand (SNZ) (2006:1), the government has recognized that a sense of nationhood and identity depends to a significant extent on actively supporting New Zealand's arts, culture and heritage. Likewise, it has admitted that economic growth, social cohesion, the acceptance and encouragement of diversity, and creative thinking in a range of fields can be contributed to by confidence in that culture, an appreciation of its unique aspects, and a strong sense of cultural identity.

The cultural sector is considered by government as a factor which is driven by an infinitely renewable resource - human creativity - that, when given nourishment and contribution, provides the potential for job creation through the generation and exploitation of human intellectual property. Because of these characteristics, the cultural sector plays a pivotal role in the New Zealand government's goal of achieving sustainable development.

The New Zealand government has proposed a number of themes to structure the development of cultural indicators. These themes are divided into the five domains of: engagement, identity, diversity, social cohesion, and economic development (Table 4.10). A number of desired outcomes are set under each theme. The total 10 outcomes that have been proposed are set out in Table 4.10. These outcomes attempt to create a significant environment for the engagement of New Zealanders in the cultural dimension of the sustainable development process as participants, consumers, creators, and providers or as decision-makers, all with equal access to cultural capital. In this cultural framework, the cultural structure of New Zealand is viewed as an integration of the cultures of Maori and Pakeha in which both cultures are respected and valued by New Zealanders. This outcome can also foster a tolerant and inclusive society. In addition, it is anticipated that the cultural outcomes will enhance community-based development by the involvement of New Zealanders in sustainable development through cultural activities.

Culture-based economic development is another proposed outcome in Table 4.10. Although, as indicated in Table 4.10, the arts, culture and heritage make a growing contribution to the economy, sustainable development of the economic system ultimately has 
a relationship with the other outcomes. For example, participation of local people in economic activities and equal distribution of capital are both recognised pillars of sustainable economic development. The related indicators are described in Table 4.11.

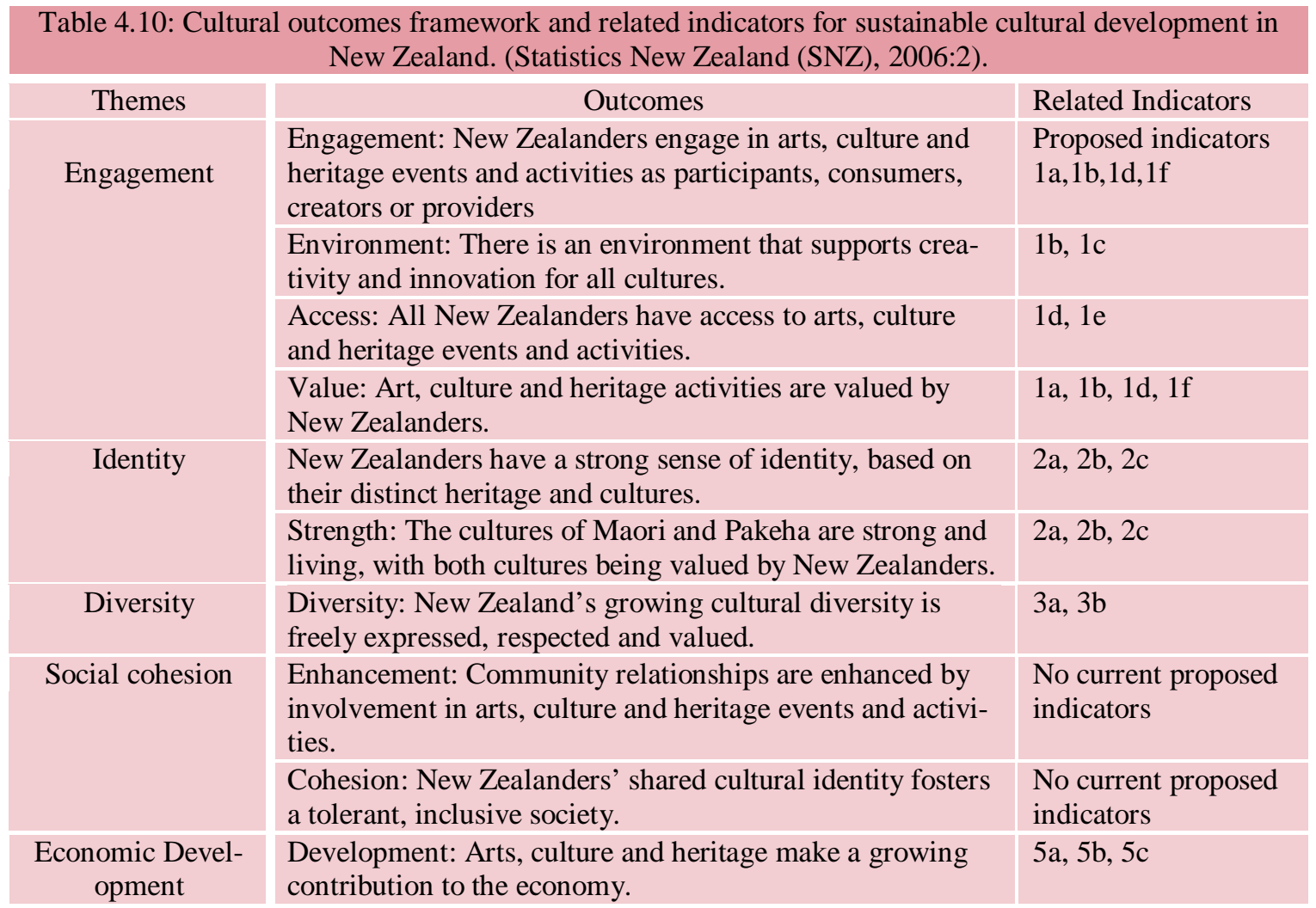

\section{b. 1. Cultural Indicators for New Zealand}

The proposed cultural indicators for New Zealand (2009) form a justified model for cultural indicators, which have been developed by Statistics New Zealand (SNZ) from those of 2006. As shown in Table 4.11, indicator 1d which is described in SNZ (2006: 18) as "How often people experience cultural activities, on average", has been changed in SNZ 2009 to "cultural experiences” (Table 4.11). Furthermore, the five indicators 1g, 1h, 2d, 2e and 3c have been added to the first set of cultural indicators in 2009 (Table 4.11).

Table 4.11 shows that a total of 19 cultural indicators are proposed for New Zealand in 2009. According to their definitions (Table 4.11) eight of them (1a, 1b, 1c, 1f, 3a, 5a, 5b and 5c) are cultural-economic indicators and 11 of them can be viewed as indicators which particularly focus on cultural experiences, events and heritage. Although economic indicators cover $42 \%$ (8 out of 19) of total cultural indicators for New Zealand, 
as Table 4.10 shows, $52 \%$ (12 out of 23 ) of the indicators which are set out in the outcomes are economic based indicators.

Table 4.11: Cultural indicators for New Zealand (SNZ, 2009:6-76)

\begin{tabular}{|c|c|c|c|}
\hline $\mathrm{T}^{1}$ & Ind $^{2}$ & Title & Definition \\
\hline \multirow{8}{*}{ 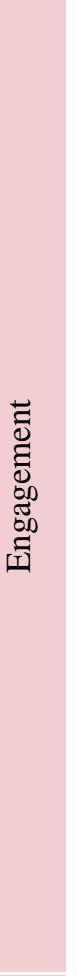 } & $1 \mathrm{a}$ & Cultural employment & $\begin{array}{l}\text { The number of people in cultural employment as a percent- } \\
\text { age of total employment. }\end{array}$ \\
\hline & $1 b$ & $\begin{array}{l}\text { Employment in creative } \\
\text { occupations }\end{array}$ & $\begin{array}{l}\text { The number of people employed in creative occupations as a } \\
\text { percentage of total employment. }\end{array}$ \\
\hline & 1c & $\begin{array}{l}\text { Median incomes from } \\
\text { creative occupations }\end{array}$ & $\begin{array}{l}\text { The median income received by people in creative occupa- } \\
\text { tions }{ }^{1} \text { as a percentage of the median income of all employed } \\
\text { people. }\end{array}$ \\
\hline & $1 d$ & Cultural experiences & $\begin{array}{l}\text { This indicator is expressed as the average (per adult) fre- } \\
\text { quency of experiencing cultural activities. }\end{array}$ \\
\hline & 1e & $\begin{array}{l}\text { Barriers to cultural expe- } \\
\text { riences }\end{array}$ & $\begin{array}{l}\text { This indicator is expressed as the proportion of adults en- } \\
\text { countering barriers which prevent them from experiencing } \\
\text { particular cultural activities. }\end{array}$ \\
\hline & 1f & $\begin{array}{l}\text { Household spending on } \\
\text { cultural items }\end{array}$ & $\begin{array}{l}\text { This indicator shows the value of household spending on } \\
\text { cultural goods and services as a proportion of all household } \\
\text { expenditure. }\end{array}$ \\
\hline & $1 g$ & Heritage protection & $\begin{array}{l}\text { The proportion of those sites either registered with the New } \\
\text { Zealand Historical Places Trust, or listed as places with his- } \\
\text { torical value in territorial authority District Plans that have } \\
\text { been destroyed, relocated or partly removed during the peri- } \\
\text { od. }\end{array}$ \\
\hline & $1 \mathrm{~h}$ & $\begin{array}{l}\text { Access to arts, culture } \\
\text { and heritage activities } \\
\text { and events }\end{array}$ & $\begin{array}{l}\text { The proportion of shows, performances and exhibitions at a } \\
\text { sample of venues outside the five main centers. }\end{array}$ \\
\hline \multirow{5}{*}{ 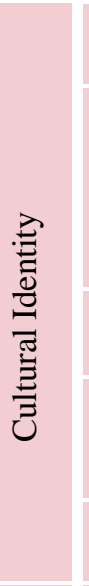 } & $2 a$ & Speakers of te reo Maori & $\begin{array}{l}\text { This indicator is expressed as the proportion of Maori able } \\
\text { to hold an everyday conversation in Maori. }\end{array}$ \\
\hline & $2 b$ & $\begin{array}{l}\text { Local content on televi- } \\
\text { sion }\end{array}$ & $\begin{array}{l}\text { This indicator is expressed as the first run hours of local } \\
\text { content, as a proportion of the total television schedule. Lo- } \\
\text { cal content is generally defined as material that is both pre- } \\
\text { dominantly made in New Zealand and reflects New Zealand } \\
\text { identity and culture. }\end{array}$ \\
\hline & 2c & Maori TV ratings & $\begin{array}{l}\text { This indicator is expressed as the viewer ratings for Maori } \\
\text { TV by Maori, non-Maori and all viewers of this channel. }\end{array}$ \\
\hline & $2 d$ & $\begin{array}{l}\text { The importance of cul- } \\
\text { ture to national identity }\end{array}$ & $\begin{array}{l}\text { The proportion of New Zealanders who believe that culture } \\
\text { and cultural activities are important to New Zealand's sense } \\
\text { of national identity. }\end{array}$ \\
\hline & $2 \mathrm{e}$ & New Zealand events & $\begin{array}{l}\text { Proportion of New Zealand shows, performances and exhi- } \\
\text { bitions at a national sample of venues. }\end{array}$ \\
\hline \multirow{3}{*}{ 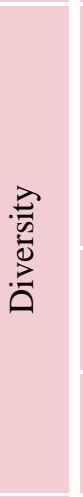 } & За & $\begin{array}{l}\text { Grants to minority ethnic } \\
\text { cultural groups }\end{array}$ & $\begin{array}{l}\text { This indicator is an index of the percentage of grants made } \\
\text { by the New Zealand Lottery Grants Board (LGB) for arts, } \\
\text { culture and heritage purposes to non-Maori and non-New } \\
\text { Zealand European ethnic organizations and individuals, rela- } \\
\text { tive to those ethnic groups' percentage in the population of } \\
\text { New Zealand. }\end{array}$ \\
\hline & $3 b$ & $\begin{array}{l}\text { Attendance /participation } \\
\text { at/in ethnic cultural ac- } \\
\text { tivities }\end{array}$ & $\begin{array}{l}\text { This indicator is expressed as the percentage of the popula- } \\
\text { tion aged } 15 \text { years and over attending/participating in at least } \\
\text { one cultural activity in the year before survey. }\end{array}$ \\
\hline & 3c & $\begin{array}{l}\text { Minority culture activi- } \\
\text { ties }\end{array}$ & $\begin{array}{l}\text { The proportion of arts, culture and heritage events and activ- } \\
\text { ities produced by minority cultures, taking place at a nation- } \\
\text { al sample of venues. }\end{array}$ \\
\hline \multirow{2}{*}{ 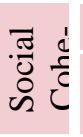 } & - & No indicator & \\
\hline & - & $\begin{array}{l}\text { Non-Maori attendance at } \\
\text { Maori cultural events. }\end{array}$ & $\begin{array}{l}\text { Proportion of non-Maori attending a sample of Maori cul- } \\
\text { tural events }\end{array}$ \\
\hline
\end{tabular}




\begin{tabular}{|c|c|c|c|}
\hline & - & $\begin{array}{l}\text { Other ethnicities attend- } \\
\text { ance }\end{array}$ & $\begin{array}{l}\text { Involvement in community arts, cultural and heritage groups } \\
\text { which are not Maori or New Zealand European /Pakeha. }\end{array}$ \\
\hline & - & $\begin{array}{l}\text { Community cultural ex- } \\
\text { periences }\end{array}$ & $\begin{array}{l}\text { The percentage of people taking part in religious activities, } \\
\text { ethnic cultural activities, and other secular activities } \\
\text { (NZFCS sub categories 811, } 821 \text { and 822), and the frequen- } \\
\text { cy of participation. }\end{array}$ \\
\hline & $5 a$ & $\begin{array}{l}\text { Income of the cultural } \\
\text { industries }\end{array}$ & $\begin{array}{l}\text { This indicator is expressed as the value of the sales of goods } \\
\text { and services and other income of the cultural industries in } \\
\text { constant prices. }\end{array}$ \\
\hline$\Xi$ & $5 b$ & $\begin{array}{l}\text { Value added contributed } \\
\text { by the creative industries }\end{array}$ & $\begin{array}{l}\text { This indicator is expressed as the value added by the crea- } \\
\text { tive industries in year } 2005 \text { dollars expressed as an index } \\
\text { ( } 2005 \text { equals } 100 \text { on the index). }\end{array}$ \\
\hline$[\mathrm{I}]$ & $5 c$ & $\begin{array}{l}\text { The creative industries' } \\
\text { proportion of total indus- } \\
\text { try value-added }\end{array}$ & $\begin{array}{l}\text { This indicator is expressed as the proportion of total industry } \\
\text { value added produced by the creative industries. It can be } \\
\text { read in conjunction with the previous indicator (5b), which } \\
\text { measured changes in value added in the creative industries. } \\
\text { Total industry value added is gross domestic product less } \\
\text { unallocated indirect taxes (i.e. less GST, import duties and } \\
\text { stamp duty). }\end{array}$ \\
\hline
\end{tabular}

\section{c. Integrated Cultural Frameworks and Indicators for New Zealand}

Figure 4.5 proposes a model for the integration of the FCS and the cultural framework and indicators for New Zealand. This model shows that there are mutual relationships between the themes proposed by SNZ (2009) and the cultural domains suggested by UNESCO, 2009 (cited in UIS, 2010) (Figure 4.5).

The model shows that all cultural domains and related domains can be set under each of the themes (Figure 4.5). On the other hand, the cultural domains, their productive activities and their related cultural goods and services can be viewed as factors in which each theme must be involved in order to achieve its complete anticipated outcomes. For example, in terms of the engagement of New Zealanders in cultural activities, this can be placed into the 8 categories of: engagement in cultural heritage; performance and celebration; visual arts and crafts; books and press; audio-visual and interactive media; design and creative services; tourism; and sports and recreation (Figure 4.5). 


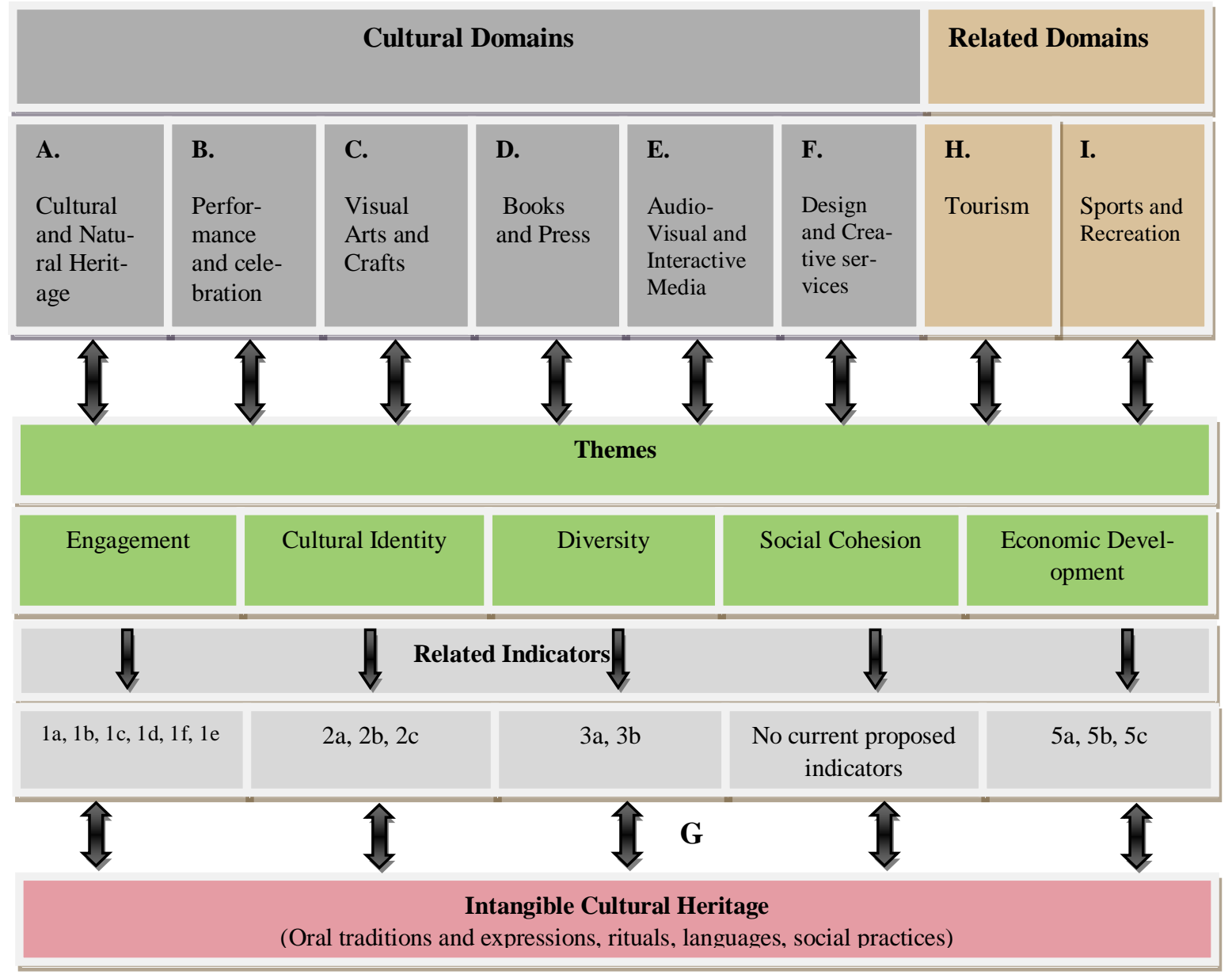

Education and Training

Archiving and Preserving

Equipment and Supporting Materials

Figure 4.5: Integration of FCS cultural domains and indicators

Tourism can also be considered as a related domain in which most of the proposed themes are involved (Figure 4.6). On the other hand, the anticipated cultural outcomes for sustainable development of tourism can be categorized as subsets of the themes that are introduced in Figure 4.6.

The cultural outcomes of tourism and the goods and services produced for it can be considered as pivotal and supporting tools which are set under the transverse cultural domains and in particular the education and training domain (Figure 4.6). These outcomes in terms of goods and services can be used as educational tools to make a host society into a place for cultural education. 


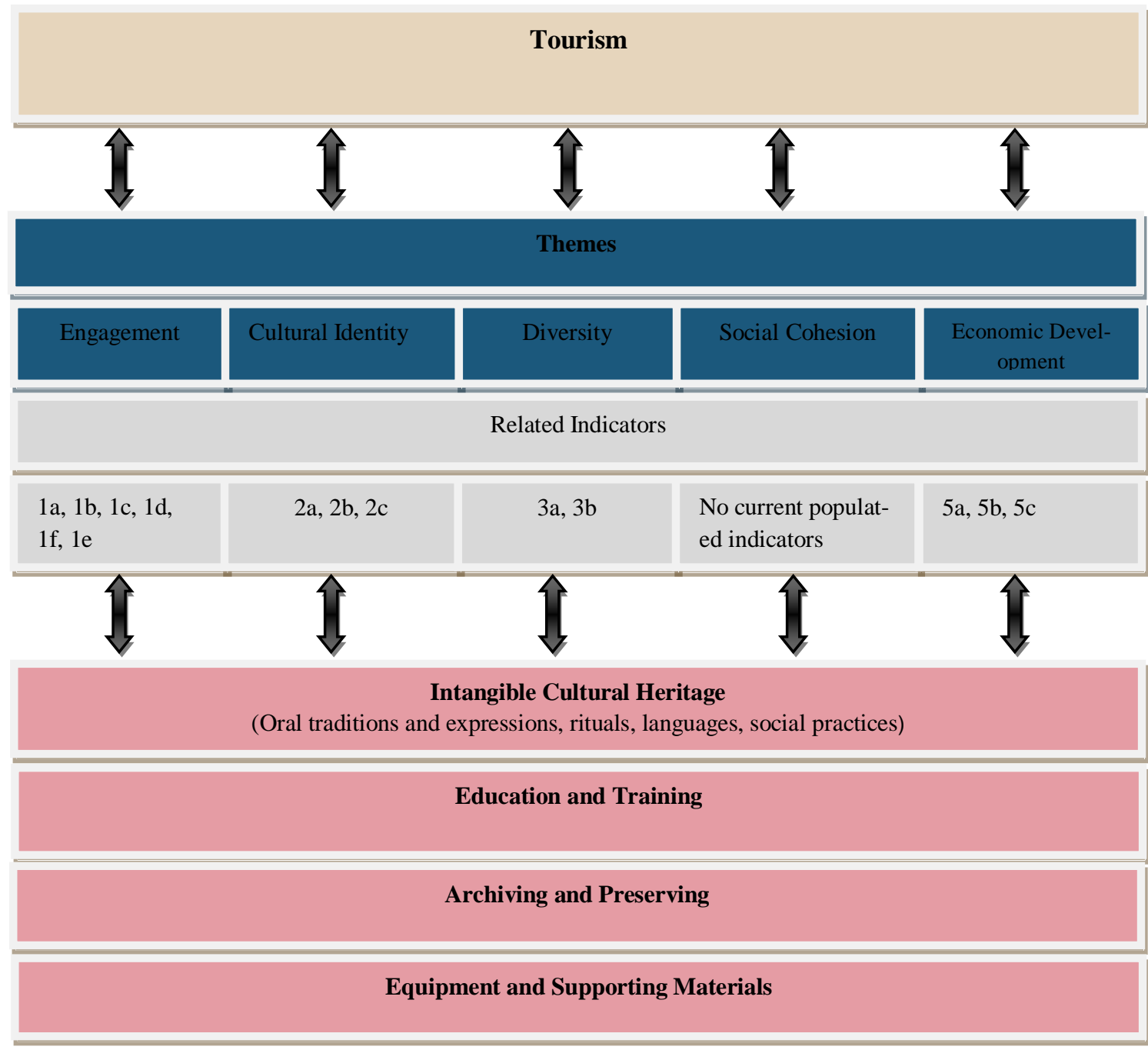

Figure 4.6: Tourism, FCS cultural frameworks, and SNZ themes and indicators

Schianetz et al (2007:1485) reveal that sustainability must be conceived of as a transition and learning process, and as a “moving” rather than a static goal. They (2007:1485) state that these findings are particularly important with respect to the tourism industry, which is an inherently non-linear, complex and dynamic system that has to be managed adaptively.

Schianetz et al (2007:1485) explain that adaptive management approaches are based on continuous and collective learning concepts that acknowledge uncertainties, and allow for timely adjustment of planning and management strategies. This implies that in order to advance sustainability in the tourism industry, approaches are needed that promote learning at an organizational as well as a place related destination or regional level. In this view sustainable tourism with its social-cultural essence can be considered as a potential form of education (learning). 
Minnaert et al (2009:320) reveal another approach in which the focus is on ideas, in terms of experiential learning that is linked to experience in a non-institutionalized setting, such as going on holiday. According to Minnaert et al (2009:321) learning which is rooted in experience was introduced at the end of the 1970s and during the 1980s, and it represented an alternative to the more traditional, cognitive learning theories. It can be explained as education that "begins with the experience followed by reflection, analysis and evaluation of the experience” (Boydell, 1976, cited in Minnaert et al, 2009:321). From this perspective, tourism activities can offer participants the chance to envisage new situations, meet different social interaction situations, and compare these to their behavior patterns. For instance, a holiday can create an opportunity for visitors to explore a new environment, engage in new activities, and meet exotic societies and cultures. It can provide the encounters participants need to start their cultural education, even if this education is not anticipated.

Cultural education and learning through participation in tourism can be explained as a comprehensive social activity which "reintroduces people to their cultural roots and reinvigorates people’s interest in [their] history and culture” (McKercher et al, 2005:539), and which is largely rooted in participating in community-based practices carried out by the host communities and related organizations. In this view, the forecasted indicators for each theme can be used as indicators to evaluate the cultural footprint of tourism on both the host communities and visitors through development of this educational process.

\subsubsection{Cultural Framework for Sustainable Development of Ecotourism}

\section{a. Cultural Outcomes}

Based on the educational approach described above, the cultural outcomes for sustainable development of tourism can be put into the eight categories shown in Table 4.12. 
Table 4.12: Cultural outcomes for sustainable development of tourism (based on cultural education).

Development of Tourism based on Cultural Education

\section{Cultural Outcomes}

Awareness of local participants about their existing cultural heritage and the methods to protect this through the educational process.

Participation of local people in the cultural development process (programming, management and monitoring for the production, consumption and presentation of cultural products)

Democratized environment for participation of people in cultural development with equal access to the cultural sources.

Culture-based development of economic systems that guarantees equal distribution of cultural capital and income among the local people.

Combination of ordinary tourism activities with compatible cultural activities

Protection and restoration of tangible cultural heritage

Protection and restoration of intangible cultural heritage

(oral traditions and expressions, rituals, languages, social practices)

Tourist experience of authentic culture (as the better alternative) or staged authentic cultural heritage of the host destinations.

\section{a. 1. Awareness}

One of the main stages in the cultural development process is the awareness of local people and communities about what they have in terms of their cultural capital and heritage. Reid et al (2004:626) introduce 'Community Awareness Raising and Value Identification' as one of the important components of the model for community tourism (and ecotourism) development planning offered by him (Figure 4.7.). This model provides a macro framework for community tourism development planning focused at the local level.

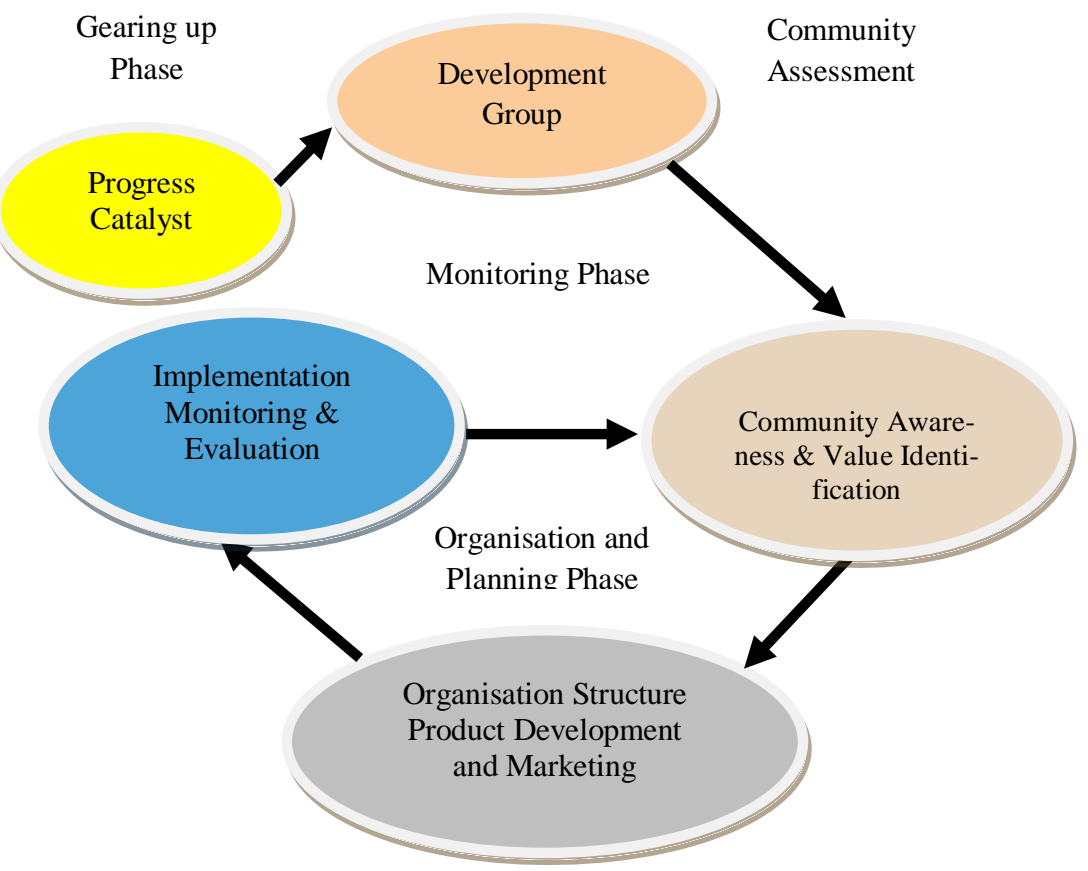

Figure 4.7: Community tourism planning model (Reid et al, 2004:625) 
As shown in Figure 4.7, the model's third step concentrates on raising community awareness about tourism. This awareness can be introduced in terms of the tourism development process, local cultural, environmental and economic potentials and values, market, products, and impacts of tourism on the host destination.

As a first stage in the engagement of local and indigenous people in the development process, they should know about their cultural values that may have been ignored or hidden behind new lifestyles. This outcome can be achieved by education of the host people through an educational process that needs particular programming, management and a supportive environment and necessary equipment. The number of people who participate in the educational process and the time that they spend in learning, as well as the places and tools which are allocated for education, can be considered as indicators for the cultural footprint of tourism. Without this initial education, ecotourism as cultural tourism that engages with the local community will not be possible. In addition, how well this engagement occurs and how much it benefits the local people is also dependent upon the type and level of education.

\section{a. 2. The Participation of People in the Development Process}

Local and indigenous participants who belong to the host culture and have significant knowledge about their cultural capital can play an important role in terms of programming, management and monitoring for the production, consumption and presentation of cultural products (Table 4.12). However, the necessity for the existence of related communities and NGOs to organize this process is unavoidable. Furthermore, another role for these NGOs and communities is to harmonize the local development policies with national strategies for development. As a result, sustainable development of ecotourism, based on cultural education, can be considered a community based activity within the wider national goals.

\section{a. 3. A Culturally Sensitive Economic System}

The culture based development of economic systems is another anticipated outcome for sustainable development linked to ecotourism. From this viewpoint, as some convergence theorists have argued "culture changes with each stage of economic development” (Frederking, 2002:107). As host communities and groups progress through the various stages of development, a convergence of the cultural values will be created. This view emphasizes the influences of economic development (as a dominant theme) 
on cultural systems. In contrast others have argued that "culturalists maintain that the groups preserve their unique traits” (Frederking, 2002:107). According to this view, “different cultures create and perpetuate models of development which may be similarly economically successful and simultaneously rich in the norms and values which underlie those activities” (Frederking, 2002:107). This has a relationship with the earlier discussion of Kerman in Iran. Although the city has developed in a modern way, the historic area at the centre of the city, normally the most expensive land in any city, is so linked to the cultural and religious values of the residents that it has been retained, virtually unchanged. This is a group preserving its unique traits.

Frederking (2002) presents a comparative study to explain different empirical examples in terms of the existence of an endogenous relationship between culture and economic development. The Punjabi community in Southall, London (which is commonly referred to as "Little India" in London) is introduced as his first case study. Frederking (2002) portrays "Little India" as a community which has created an ethnic enclave and, by separating the community geographically, one whose culture appears to be a consistent and integral part of their economic activities. According to Frederking (2002:108) in Southall, the main characteristic of "Little India" is the production and consumption of Indian cultural products such as the Indian language, food, and books. In addition, most of the businesses and services and productive activities are managed or owned by Punjabis. As a result of the social-cultural and economic dominations of Punjabis in Southall, according to Frederking (2002:108), a very profound image of Southall emerges, which from even a brief encounter with the area is the image of a self-contained, self-sufficient community.

The Gujarati community in Wembley, London is the second area which Frederking (2002) uses to discuss cultural and economic mechanisms. Frederking (2009:109) argues that in Wembley, at the same time that Indians appear to be the majority group in the neighborhood, in contrast with Southall, the community is far from being an exclusively Indian area. He points to the multi-ethnic face of Wembley, with much more diversity in terms of individuals and business activities. Evidence comes from the local offering of western variations of Indian products, thus creating a difference between Wembley and Southall. 
The Punjabi and Gujarati communities present two different aspects in terms of the relationship between economic mechanisms and culture in an area. The Punjabi community attempts to conserve its cultural heritage by isolation of the community from foreign cultures, including that of the host country, whereas the Gujaratis have created a multiethnic economic system which leads to close relationships and interactions between various cultures. Although the Gujarati community mechanism may have negative impacts on the authenticity of the cultural products, it creates an environment for engagement of different social fields in the development process. However the main cultural structures and behaviors are changed in Wembley through being influenced by consuming western products, so that an equal opportunity for presentation of cultural products is available for the different ethnic groups.

A culture based economic system for the sustainable development of tourism could be created by the combination of the positive dimensions of the two latter economic systems. According to the Punjabi pattern, a tourism economic system must be managed and developed by local or indigenous participants and communities. In addition, production and consumption of the host cultural products must have a priority over imported foreign products. However, according to the Gujarati pattern, if the host area is part of a multicultural community, all participants with different cultures should have an equal opportunity to present their cultural products and conserve their heritage. Therefore, a culture based economic system related to both mono-cultural and multi-cultural communities must contribute to an equal distribution of cultural income streams between the local participants.

These discussions suggest that employment of local people in culture based economic activities, gross domestic cultural products, and moves to restore and protect cultural heritage can be used as indicators to evaluate the economic outcomes of ecotourism that is culturally appropriate.

\section{a. 4. Combination of Ordinary Tourism Activities with Compatible Cultural Activ- ities}

Localized foreign tourism activities as a result of tourists originally coming from other areas with different cultural and behavioral patterns can be considered as one of the main outcomes for tourism development. This outcome can be achieved by the combination of some ordinary tourism activities (such as hunting, fishing, boating and horse 
riding) with compatible host cultural activities. For example, in New Zealand hunting, as an example of an ordinary tourism sporting activity, can be combined with learning about the methods and equipment which have been used by Maori people for hunting, as these are part of their historical and cultural activity and products. In this case, an ordinary tourism recreational activity substantially shifts to being a cultural-educational activity managed and presented by Maori participants. The number of visitors who participate in these culturally justified activities can be used as a cultural indicator for the development of tourism (and ecotourism) as being culturally appropriate.

\section{a. 5. Protection and Restoration of Tangible Cultural Heritage}

Tangible cultural heritage, which covers the wide domain of physical cultural resources, includes "movable or immovable objects, sites, structures, group of structures, and natural features and landscapes that have archeological, paleontological, historical, architectural, religious, aesthetic, or other cultural significance” (World Bank, 2010:173). The World Bank (2006, cited in the World Bank 2010:173) recognizes that physical cultural resources are important as sources of valuable scientific and historical information, as assets for economic and social development, and as integral parts of a people's cultural identity and practices.

These physical cultural resources are subjects that could be restored and protected through the development of sustainable ecotourism. These heritages can be viewed as having the potential to attract visitors to the host areas and also as part of the participation of visitors and indigenous peoples in the cultural-educational process. The level of restored physical cultural resources can be used as a measurable indicator for ecotourism development. However the quality of restoration and protection must be also qualified according to the relevant standards and restoration must be conducted and approved by appropriate processes.

\section{a. 6. Protection and Restoration of Intangible Cultural Heritage}

According to UNESCO (2003:2), and as quoted above, the intangible cultural heritage is a combination of cultural knowledge and skills and the artifacts these produce that a community of individuals would regard as part of their cultural heritage. This intangible cultural heritage is transmitted from generation to generation and is constantly recreated by communities and groups in response to their environment, their interaction with nature and their history. UNESCO (2003:2) sorts intangible cultural heritage into five cat- 
egories: oral traditions and expressions, including language as a vehicle of intangible cultural heritage; performing arts; social practices, rituals and festive events; knowledge and practices concerning nature and the universe; and traditional craftsmanship.

Preservation and restoration of the intangible cultural heritage, as one of the cultural outcomes for the sustainable development of tourism, can be achieved through the development of community based cultural activities and events such as traditional celebrations, festivals and concerts. Likewise, cultural goods and services such as local foods, some types of accommodation services, art, crafts, and vernacular sports can be considered as tools which have the ability to make explicit intangible cultural values for visitors. For example, some types of accommodation services that create an opportunity for visitors to stay with the local or indigenous people, contribute to them learning about the intangible cultural heritage of their hosts through the consumption of traditional products such as local foods, language, and through using vernacular equipment and spaces.

The proposed indicators for protection and restoration of intangible cultural heritage cover the cultural products, goods and services that contribute both to the way host communities can present their intangible cultural heritage, and to visitors who "come to feel rather than to gaze” (Poria et al, 2003:238) so they can become familiar with the deeper features of the host culture.

\section{a. 7. Authentic Culture or Staged Authenticity at the Host Destinations}

Development of ecotourism based on cultural education focuses on the concept of authenticity as one of its outcomes. Cohen (1988:373) argues that authenticity is an eminently modern value whose emergence is closely related to the impact of modernity upon the unity of social existences. In pre-modern society, identity has been defined primarily by social place and the individual has been identified and constituted in and through the certainty of his or her social role.

Cohen (1988:373) further argues that as institutions become "weightless" and lose their reality, the individual within them is said to turn into himself. Since modern society is inauthentic, the modern man who desires to overcome the contrast between the authenticity seeking self and society has to seek elsewhere for an authentic life. 
The opposition between self and society which has now reached its maximum could be reduced by the sustainable development of ecotourism. As a solution, the quest for authenticity must be considered as one of the prominent motifs of sustainable ecotourism. Ecotourism can give an opportunity to visitors who desire to obtain experiences and products that are authentic, by the production and presentation of original and local products that are not contaminated by being fake or impure. These authentic cultural products can be used as indicators to evaluate the footprint of ecotourism related to the authenticity of the host community's life and their products.

\section{b. Cultural Indicators for Development of Tourism based on Cultural Education}

This section discusses the main characteristics of cultural indicators and proposes indictors that can be used to evaluate ecotourism and its related products and activities as being culturally sustainable.

\section{b. 1. The Main Characteristics of Cultural Indicators}

Madden (2005:221) introduces a cultural indicator as a statistic that can be used to make sense of, monitor, or evaluate some aspect of a culture, such as its architecture, or cultural policies, programmes, and activities. The USAID defines an indicator as "a variable [whose] purpose it is to measure changes in a phenomenon or progress" (MDF Training and Consultancy (MDF), 2005:1).

Cultural indicators can be categorised into the two types of quantitative and qualitative indicators. "Quantitative indicators measure change through numerical or statistical facts or physical outputs" (Chapman, 2000:2) and “qualitative indicators are languagebased descriptions of cultural phenomena” (Madden: 2005:220). MDF (2005:1) reveals that an indicator is a quantitative or qualitative factor or variable that provides a simple and reliable means to measure achievement, to reflect changes connected to an intervention, or to help assess the performance of a development actor.

A cultural indicator as defined by SPC (2010:2) is a measure that points out something about the state or situation of a culture in a country. SPC (2010:2) explains that these indicators can be narrowed down within the regional and local scale to measure more specific areas of culture such as oral culture (as intangible culture constructed / expressed in local language), traditional practice (skills/ values), arts, crafts, vernacular architecture and cultural sites. 
The Ministry of Culture, Madrid, Spain (MCMS) (1985:68) has defined a cultural indicator as a direct and valid statistical measurement that makes it possible to observe the level of a fundamental cultural concern and its variations over a period of time. Arising from this definition, this thesis proposes two fundamental properties for a cultural indicator:

- First, "it must be a direct measurement, that is, it must refer to the very essence of the cultural concern and not to the instruments or agencies used to satisfy that concern” (MCMS, 1985:68).

- Second, "it must be valid in the sense that changes in the indicator must correspond to changes in the cultural element being measured, it being assumed that there is no variation in these remaining elements” (MCMS, 1985:68).

In addition to these two fundamental properties, MCMS (1985:68) suggests further requirements for a cultural indicator including:

- Capacity for aggregation and disaggregation

- Comparability in space or time

- Intelligibility

- Comprehensiveness

- Capacity for co-ordination

- Reliability

- $\quad$ Timeliness

- Viability

One of the main purposes of using cultural indicators is to calculate the level of particular cultural concerns of individuals. Consequently, it is necessary that the cultural indicators should allow aggregation or disaggregation at different levels (for instance national, regional or community scale) for the purpose of analysis.

A cultural indicator should have the ability to be used for the same group or society at different points in time to measure and judge whether anticipated cultural outcomes in a particular framework have been promoted or have worsened. Furthermore, the cultural indicator should allow comparison between different groups, communities or societies that belong to different places (geographical regions or countries). 
Simplicity is also a characteristic of a cultural indicator that allows its interpretation in a particular time or space. However, it does not mean that the basic theory behind it and the technique of its elaboration must be simple.

MCMS (1985:68) also suggest that as cultural indicators should be kept to a minimum, they should be devised adequately, so that the greatest quantity of information possible about the cultural concern to be measured can be interpreted. MCMS (1985:69) points out that cultural indicators should form a co-ordinated, consistent and interrelated whole and consequently be drawn up with a soundly constructed framework so that different data series may be associated and interrelated.

Cultural statistics are the basis for the elaboration of the cultural indicators arising from a cultural framework. As Pattanaik (1997:11) states, the evaluative framework based on functioning rather than commodities, can be used to abstract information, at least conceptually, from a large number of details to focus on a related small number of ends (their functioning). He (1997:11) argues that in practice, if the exercise is to remain tractable, attention must be restricted to the small number of functionings that are considered to be crucial and that can be captured through suitable indicators.

According to MCMS (1985:70) and Pfenniger (2004, cited in Madden, 2005:228), cultural indicators and the required data for their assessment should be available at the right moment to guide, evaluate and monitor cultural policy effectively. These cultural indicators should also be operational and must be stated in a realistic way instead of being ideal but unrealisable.

The International Federation of Arts Councils and Culture Agencies (IFACCA) (2005:10) has summarised and ranked the main attributes of a good [cultural] indicator based on the number of times they appear in the literature consulted (Table 4.13). 
Table 4.13: Attributes of a good [cultural] indicator. (IFACCA, 2005:10).

\begin{tabular}{|c|c|}
\hline Attribute & No. of citations \\
\hline Grounded in theory & 9 \\
\hline Relevant (serve a practical or valued purpose) & 6 \\
\hline Grounded in and/or linked to policy practice & 5 \\
\hline Comparable across regions & 5 \\
\hline Comparable across time periods & 5 \\
\hline Measurable (able to be measured, and data available). & 4 \\
\hline Easily understood & 4 \\
\hline Unambiguous/clear & 4 \\
\hline Able to be disaggregated by population subgroups & 4 \\
\hline Consistent with purpose & 4 \\
\hline Timely (up-to-date) & 3 \\
\hline Measurable over time & 3 \\
\hline Universal & 2 \\
\hline Able to be benchmarked & 2 \\
\hline Contextualised (presented with additional contextual information) & 2 \\
\hline Revisable & 2 \\
\hline Methodologically defensible & 2 \\
\hline Reliable & 1 \\
\hline Sensitive to cultural diversity & 1 \\
\hline Realistic & 1 \\
\hline Capture the essence of an issue & 1 \\
\hline Designed through consultation & 1 \\
\hline Trusted & 1 \\
\hline \multicolumn{2}{|c|}{$\begin{array}{l}\text { - Sources: Belgian Government (2001), Brown and Corbett (1997), Chapman (2000), Cobb anc } \\
\text { Rixford (1998), Duxbury (2003:8-9), Fukuda-Parr (2001:2-3), Innes and Booher (2000) } \\
\text { Lievesley (2001:377), Mercer (2004), Morton (1996:120), Pfnniger (2004:4), Piganatarc } \\
\text { (2003), Sawicki (2002:25), Schuster (2001:5), Sharpe(1999:44), UNRISD and UNESCC } \\
\text { (1997:8). (IFACCA, 2005:10) }\end{array}$} \\
\hline
\end{tabular}

IFACCA (2005:10) points out that the attributes are not necessarily mutually exclusive, and some rudimentary interpretation and grouping can be undertaken. Consequently, although according to the IFACCA (2005:10) the list cannot be taken as a scientific survey of opinion, this study uses this list as both providing a quick summary of recommendations made by indicator developers, and as highlighting the priorities perceived by these developers.

\section{b. 2. Classification of Cultural Indicators}

Madden (2005:224) proposes two main types of uses for cultural indicators: monitoring (observing cultural phenomena) and evaluation (measuring the efficacy of cultural policies and programmes). Brown and Corbett (1997, cited in Madden, 2005:224) categorise cultural indicators into a five-part typology of basic uses of [cultural] social indicators in policy:

- Description, for the sake of knowledge about society [and culture]. 
- Monitoring, to track outcomes that may require policy intervention.

- Setting goals, to establish quantifiable thresholds to be met within specific timeframes.

- Outcomes-based accountability, to hold managers, agencies, government and communities responsible for improving social well being and for meeting established goals.

- Evaluation, to determine which programmes and policies are effective (or destructive) and why.

As Madden (2005:227) points out, cultural indicators can be classified hierarchically based on the scale of detail at which they are applied. He (2005:227) classifies cultural indicators into the three categories of macro, meso and micro indicators. Madden (2007:227) describes macro indicators as indicators used for monitoring and evaluation, e.g. cultural indicators of development, and indicators of cultural rights. He (2005:227) considers meso indicators for use for regional or cross-agency policy monitoring and evaluation, e.g. indicators that measure outcomes of an arts council policy. Micro indicators are suggested for "agency programme monitoring and evaluation, e.g. indicators that measure outcomes of an arts event” (Madden, 2005:227).

\section{b. 3. Ecotourism Related Cultural Indicators}

Anticipated cultural outcomes as a framework for the development of ecotourism based on cultural education need to be evaluated by efficient cultural indicators with particular characteristics such as "measurability, data availability, data quality “(Duxbury, 2003:8) and "International comparability" (Breis, 1992:11). As Breis (1992:6) points out, the sequence for the creation of cultural indicators should be based on some type of constructed theory as a first attempt, and the subsequent search for the required data whether quantitative or non-quantitative.

According to Duxbury (2003:9), throughout the entire process of producing cultural indicators, all actions and decisions must be permeated by the question of intent and meaningfulness. Likewise, the main purpose and necessity for indicators must be clarified and the relevant subjects which need to be assessed must be explained through the constructed theory behind the process. Although cultural indicators must be comprehensive in order to consider all the different dimensions of cultural outcomes, these can be limited by some factors such as limitation of resources to do assessment and gaps be- 
tween indicator statements/topics and data available to address or measure the cultural footprint of a phenomenon, such as ecotourism development.

As Pearce (cited in Travis, 1982:259) points out, "the social [cultural] impact of tourism will vary according to the differences between the visitors and the visited, whether in terms of numbers, race, culture, or social outlook.” Particularly in multi-cultural destinations, as Travis (1982:259) reveals, there may be several host cultures as well as several tourist cultures represented in one place, at one point in time, so it is not possible to see cultural impacts simply in the monolithic terms of host culture and visitor culture. As a result, in this case integration of the anticipated cultural indicators must be able to cover all valuable cultural productive activities and related goods and services. The latter are defined as items that are worthy of being presented at national or global level.

Furthermore, influenced by the existence of different cultures or communities (with various social structures in one destination), the suggested cultural framework and related indicators can be considered as parts of a policy to make a linkage between these various cultural systems and communities. However, to achieve this goal, some incompatible existing cultural heritage or social behavior might need to be adjusted, modified or completely ignored, particularly when these social-cultural behaviors are against human rights or cause conflict between communities.

\section{b. 4. Cultural Indicators and Tourism Resources}

Tourism resources are placed by Jafari and Ritchie (1981:17) into the three categories of natural, man-made and socio-cultural resources. To explain the man-made resources they (1981:17) argue that the term "sight-seeing” commonly refers to the observation of some man-made creation or building such as museums, churches, tall buildings, or other architectural masterpieces. In this view, the resources just refer to attractive buildings and associated landscapes as man-made tourism resources and other man-made resources such as food, textiles and handcrafts, which can be introduced as tangible cultural heritage, are neglected in this definition.

In terms of the socio-cultural resources of tourism, Jafari and Ritchie (1981:17) state that this category of resources represents the cultural heritage and social fabric of the peoples of the world, and includes both tangible (e.g. the man-made resources listed above) and intangible manifestations (e.g. cultural events and festivals). They (1981:17) introduce socio-cultural resources as the primary reasons for traveling. As a result of the 
discussion above, tourism resources can be placed into the two categories of natural and social-cultural resources. In this view, social-cultural resources cover all man-made or tangible resources as well as intangible cultural activities and products.

This categorization of tourism resources can exert an influence on decision making related to the cultural framework and indicators for ecotourism development. Otherwise, the themes which are viewed as the cultural outcomes of ecotourism, being influenced by categorization of tourism resources as shown above, can be further sorted into the two areas of protection and development of intangible cultural heritage, and production, and consumption and conservation of tangible cultural capital. Following this, related cultural indicators can be classified into these two common groups. The first group can be used to qualify protection, development and presentation of the intangible cultural heritage (such as traditional festivals, concerts, and other celebrations) through participation of local and indigenous people in the development process. The second group of cultural indicators is viewed as the tools to assess qualitatively the production, consumption and protection of tangible cultural capital and products (such as vernacular architecture, handcrafts, food and textiles).

Ecotourism development based on cultural education aims to engage local and indigenous people in the protection of cultural heritage and in decision making related to the sustainable development process. As Reid et al (2004:625) reveal, it is important that deliberate measures such as cultural productive activities are carefully introduced to enable participants to take advantage of the opportunities brought by ecotourism. Without the careful implementation of such measures, which are viewed as part of the cultural activities related to ecotourism, the ecotourism industry might gradually lose the host communities' support. This, in turn, might threaten the sustainability of development in the future.

\section{b. 5. Anticipated Cultural Indicators}

This thesis attempts to present cultural indicators for the development of sustainable ecotourism (as a subsector of sustainable tourism) based on cultural education. According to the above discussion, data availability, measurability and international comparability are viewed as factors that form a framework for choosing these indicators. 
As shown in Table 4.14, suggested cultural indicators can be categorized into three main types:

- The number of local and indigenous participants who participate in the development process including education, management, monitoring, protection of cultural heritage and economic activities (1a, 2a, 3a) (Table 4.14).

- The quality of tools, goods, services and places which are used or protected during the development process (1b, 1c, 3c, 4a, 5a, 6a, 7a) (Table 4.14).

- The economic benefits which participants earn from tourism development based on cultural education (3b) (Table 4.14).

Table 4.14: Proposed cultural indicators for development of ecotourism based on cultural education

\begin{tabular}{|c|c|c|c|}
\hline Cultural Outcome & Ind & Title & Definition \\
\hline \multirow{3}{*}{$\begin{array}{l}\text { Awareness of the local participants of } \\
\text { their existing cultural heritage and the } \\
\text { methods to protect this through the educa- } \\
\text { tional process }\end{array}$} & 1a & Cultural education & $\begin{array}{l}\text { The number of local people who } \\
\text { participate in the educational pro- } \\
\text { cess }\end{array}$ \\
\hline & $1 b$ & Educational places & $\begin{array}{l}\text { The areas, buildings and related } \\
\text { infrastructure which are used for } \\
\text { education }\end{array}$ \\
\hline & 1c & $\begin{array}{l}\text { Educational tools and } \\
\text { equipment }\end{array}$ & $\begin{array}{l}\text { The tools and equipment that are } \\
\text { used for education }\end{array}$ \\
\hline $\begin{array}{l}\text { Participation of local people in the cultur- } \\
\text { al development process (programming, } \\
\text { management and monitoring for the pro- } \\
\text { duction, consumption and presentation of } \\
\text { cultural products) }\end{array}$ & $2 a$ & $\begin{array}{l}\text { Participation in cultural } \\
\text { development process }\end{array}$ & $\begin{array}{l}\text { The number of people who engage } \\
\text { in the cultural development process } \\
\text { including volunteer or employed } \\
\text { participants }\end{array}$ \\
\hline \multirow{3}{*}{$\begin{array}{l}\text { Culture-based development of economic } \\
\text { systems that guarantee equal distribution } \\
\text { of cultural capital and income among the } \\
\text { local people }\end{array}$} & За & $\begin{array}{l}\text { Employment of local } \\
\text { people }\end{array}$ & $\begin{array}{l}\text { The number of local people em- } \\
\text { ployed in the culture-based eco- } \\
\text { nomic system }\end{array}$ \\
\hline & $3 b$ & GDPs $^{1}$ & Sustainable portion of GDP \\
\hline & 3c & $\begin{array}{l}\text { Restored and protected } \\
\text { cultural heritage (this } \\
\text { indicator covers indica- } \\
\text { tors } 5 \mathrm{a} \text { and } 5 \mathrm{a} \text { ) }\end{array}$ & $\begin{array}{l}\text { The quantity of the intangible and } \\
\text { tangible cultural heritage that is } \\
\text { protected or restored through de- } \\
\text { velopment of tourism }\end{array}$ \\
\hline $\begin{array}{l}\text { Combination of ordinary tourism activi- } \\
\text { ties with compatible cultural activities }\end{array}$ & $4 a$ & $\begin{array}{l}\text { Local-national or inter- } \\
\text { national cultural prod- } \\
\text { ucts }\end{array}$ & $\begin{array}{l}\text { The quantity of local products } \\
\text { which are combined with national } \\
\text { or international products }\end{array}$ \\
\hline $\begin{array}{l}\text { Protection and restoration of tangible } \\
\text { cultural heritage }\end{array}$ & $5 a$ & $\begin{array}{l}\text { Restored tangible cul- } \\
\text { tural heritage }\end{array}$ & $\begin{array}{l}\text { The quantity of tangible cultural } \\
\text { heritage that is protected or re- } \\
\text { stored through development of } \\
\text { tourism }\end{array}$ \\
\hline $\begin{array}{l}\text { Protection and restoration of intangible } \\
\text { cultural heritage }\end{array}$ & $6 a$ & $\begin{array}{l}\text { Products related to in- } \\
\text { tangible cultural herit- } \\
\text { age }\end{array}$ & $\begin{array}{l}\text { Cultural goods and services that } \\
\text { contribute to restoration of intangi- } \\
\text { ble heritage }\end{array}$ \\
\hline Tourist experience of authentic culture & $7 a$ & Authenticity & $\begin{array}{l}\text { Original cultural products that are } \\
\text { labeled as local products }\end{array}$ \\
\hline
\end{tabular}

As demonstrated in Table 4.14, the anticipated cultural indicators attempt to assess quantitatively the engagement of local and indigenous people in the sustainable development process through assessing cultural activities and products related to cultural education. The integrated findings of these cultural indicators create a base for evaluating 
policies and practices to be culturally appropriate through collecting and analyzing data related to each indicator.

\section{c. Required Data Related to the Cultural Indicators}

Table 4.15 shows a list of data which can be used to measure the cultural footprint of the sustainable development of tourism (in particular cultural education tourism which is viewed as the cultural dimension of ecotourism).

\begin{tabular}{|c|c|c|}
\hline \multicolumn{3}{|c|}{ Table 4.15: Required data related to anticipated cultural indicators } \\
\hline Indicator & Title & Required Data \\
\hline \multirow{3}{*}{$1 \mathrm{a}$} & \multirow{3}{*}{ Cultural education } & $\begin{array}{l}\text { D1. Creators } \\
\text { In any one year, for people undertaking both paid and unpaid activity, the } \\
\text { number, location, sex, age , ethnicity, cultural occupation/ activity type, } \\
\text { hours worked, income, qualifications and training, plus whether this activ- } \\
\text { ity is a primary or secondary occupation ( and if secondary, then primary } \\
\text { occupation/ activity also). }\end{array}$ \\
\hline & & $\begin{array}{l}\text { D2. Organizations } \\
\text { In any financial year, by location: } \\
\text { The number of organizations which are related to cultural education. } \\
\text { Number of paid workers, by sex, age, and ethnicity, by type. }\end{array}$ \\
\hline & & $\begin{array}{l}\text { D3. Participants } \\
\text { The number of local and indigenous people who participate in cultural } \\
\text { education process, by sex, age, ethnicity, by type of anticipated activity } \\
\text { related to tourism development. }\end{array}$ \\
\hline $1 \mathrm{~b}$ & Educational place(s) & $\begin{array}{l}\text { D4. In any one year, the number of buildings and landscapes by area which } \\
\text { are used for educational activities and services. }\end{array}$ \\
\hline 1c & $\begin{array}{l}\text { Educational tools and } \\
\text { equipment }\end{array}$ & $\begin{array}{l}\text { D5. In any one year, the quantity of tools and equipment used in education } \\
\text { process by type (magazines, books, media, Internet } \\
\text {, radio). }\end{array}$ \\
\hline $2 \mathrm{a}$ & $\begin{array}{l}\text { Participation in cultural } \\
\text { development process }\end{array}$ & $\begin{array}{l}\text { D6. In any one year, the number of local and indigenous people who en- } \\
\text { gage in the educational development of tourism by sex, age, ethnicity, type } \\
\text { of activity, level of education. }\end{array}$ \\
\hline За & $\begin{array}{l}\text { Employment of the local } \\
\text { people }\end{array}$ & $\begin{array}{l}\text { In any financial year, by location, sex, age, ethnicity, level of education, } \\
\text { activity: } \\
\text { D7. The number of employed local and indigenous people by type of em- } \\
\text { ployment (full or part time). } \\
\text { D8. The number of volunteers. } \\
\text { D9. Annual income ( } \$ 000) \text { by type of employment (full or part time). }\end{array}$ \\
\hline $3 b$ & GDPs & $\begin{array}{l}\text { D10. In any financial year, a part of GDPs which is generated by produc- } \\
\text { tion and presentation of cultural products. }\end{array}$ \\
\hline 3c & $\begin{array}{l}\text { Restored and protected } \\
\text { tangible cultural heritage }\end{array}$ & $\begin{array}{l}\text { D 11. In any one year, the quantity (area/number) of restored tangible cul- } \\
\text { tural heritage by type (architecture, handcraft, etc.). }\end{array}$ \\
\hline $4 a$ & $\begin{array}{l}\text { Local-national or Inter- } \\
\text { national cultural prod- } \\
\text { ucts }\end{array}$ & $\begin{array}{l}\text { D12. In any one year, the number of produced cultural products which are } \\
\text { combined with national or international products, by type (cultural events } \\
\text { such as festivals, concerts; architecture; publications, sport tournaments, } \\
\text { etc.), and by level (local, national or international). }\end{array}$ \\
\hline $6 a$ & $\begin{array}{l}\text { Products related to in- } \\
\text { tangible cultural heritage }\end{array}$ & $\begin{array}{l}\text { D13. In any one year, the number of products related to intangible cultural } \\
\text { heritage by type (publication, music, performance arts, radio programmes). }\end{array}$ \\
\hline $7 a$ & Authenticity & $\begin{array}{l}\text { D14. In any one year, the quantity of cultural products which are locally } \\
\text { produced and consumed, purchased or visited by visitors, by type (food, } \\
\text { architecture, music, cultural events, etc.). }\end{array}$ \\
\hline
\end{tabular}

O’Mahony et al (2009:1135) introduce the achieving of sustainable tourism as an iterative process based on constant monitoring of impacts and the introduction of required preventive and / or corrective measures whenever necessary. As a result, they 
(2009:1135) reveal that the role of data and information is central to both the implementation and monitoring of sustainable tourism development.

In order to appreciate and understand fully the major factors influencing the performance of cultural education through the sustainable development of ecotourism, this thesis offers a range of data to measure or to explain the various benefits or aspects of cultural education. These range from the education and awareness of the local and indigenous participants about their cultural capital and its potential, protection of cultural heritage, and strengthening of identity and development of cultural products related to the various economic, commercial and artistic opportunities (Table 4.15).

Table 4.15 shows data relating to all facets of cultural education, for instance, the creators (D1), related organizations (D2), participants in the development process (D3 and D6), cultural products (D13 and D14), required places, tools and equipment to be used for cultural education (D4 and D5), the activities that support progressing cultural education and development of tourism (D11 and D13) and the factors affecting cultural products to be improved, modified, justified or combined with imported cultural products (D12).

Integration of the data shown in Table 4.15 can be used to evaluate the progress towards achieving the anticipated cultural outcomes for the sustainable development of ecotourism. However, this cultural framework, its cultural outcomes, indicators and required data must be integrated with the ecological and economic frameworks and related indicators and data to create a comprehensive framework (Figure 4.8) which will have the ability to evaluate all dimensions of sustainability through development of tourism and its sub-sectors, such as ecotourism. 


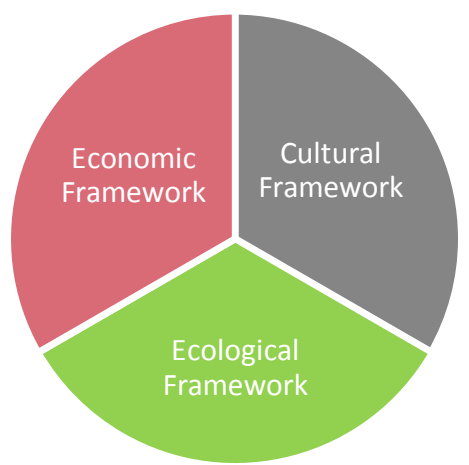

Figure 4.8: Comprehensive framework for sustainability and its segments

However, it is possible that in the integration of the cultural, ecological and economic frameworks, some of the indicators and related data will overlap each other. This can contribute to the creation of a smaller framework for the sustainable development of tourism and this process will be explored in the case study section of this thesis.

\subsubsection{Economic Framework for Sustainable Development of Ecotourism}

As previously shown in the model of sustainability (Chapter 2), the economy should be an area which has a social and ecological interaction with society and its surrounding environment. This model considers such an economy to be a multi-dimensional activity which is sensitive about the social-ecological footprint of its product and outcomes in terms of being socially appropriate and environmentally friendly. Arising from this social-ecological perspective, UNEP (2011:01) defines a green economy as one that results in improved human well-being and social equity, while significantly reducing environmental risks and ecological scarcities. However in this definition, the prefix of 'green' does not merely put emphasis on being ecologically friendly and it can be assumed to be the same as the prefix 'sustainable' for an economy.

Relying on the above definition of a green or sustainable economy and as discussed in the literature on sustainability and its proposed strategies for development (Chapter 2), the main outcomes of sustainable economic development can be categorised into the three areas of socio-cultural, ecological and economic outcomes which are linked together in a sustainable way.

\subsubsection{Socio-Cultural Outcomes}

The main social outcomes of sustainable economic development as proposed in the above cultural framework for ecotourism in this chapter, and as pointed out by research- 
ers such as Costanza (2009:20), are opportunities for the involvement of local participants in activities that achieve economic growth and equal distribution of capitals among all components of a society. This can be viewed as a strategy that contributes to horizontal economic development through ecotourism.

The second cultural outcome for sustainable economic development relies on the strategy that results from the conservation of cultural heritage and that makes an opportunity for the people engaged to present their cultural products and capitals. This has been conceptualised in Table 4.10 as "development: arts, culture and heritage make a growing contribution to the economy".

\subsubsection{Environmental Outcome}

Sustainable economic strategy relies on the idea that the conventional economic systems are the cause of environmental issues for human life through degradation of natural resources. Furthermore, these issues can be solved through changing social behaviours in terms of patterns of resource consumption.

As Organisation for Economic Development and Co-operation (OECD) (2008) reveals, two points are essential for achieving sustainable economic development. First: "economic growth alone is not enough to solve the world's problem” (OEDC, 2008: 2). Second: "the economic, social and environmental aspects of any action are interconnected" (OEDC, 2008: 2). As a result, considering only one of these aspects at a time leads to ignoring other aspects of the sustainable environmental outcomes of an economic system. For example, if the environmental issues are assumed to be merely technical problems, the technical progress that attempts to reduce environmental impacts and increase human well being can cause other unrelated issues. For instance, as OECD (2008:3) argues, cars are now much more fuel-efficient than before, but air pollution is getting worse because so many more people have cars.

The main anticipated environmental outcome for sustainable economic development can be introduced as conservation of environmental resources through a socialeconomic educational process. 


\subsubsection{Economic Indicators}

The shortage of efficient economic indicators can be seen as one of the critical issues for measuring the economic footprint of an activity (such as ecotourism) or a product (such as architecture) as being economically sustainable. As many researchers and related organisations such as NZCTU (2010:22) and UNEP (2011:26) have shown, the use of conventional economic indicators, such as GDP and other macroeconomic aggregates, can lead to a distorted picture of economic performance, particularly since such measures do not reflect the extent to which production and consumption activities may be drawing down natural capital.

As previously discussed in Chapter 2, many attempts have also been conducted to introduce economic indicators that have the ability to cover the social and ecological dimensions of sustainable economic development but the results contain potential weaknesses that must be justified. For instance, NZCTU (2010) proposes taxation as a strategy to monitor and control the environmental degradation caused by economic development. NZCTU (2010:21) suggests that polluters should face taxes on their emissions, including greenhouse gas emissions with the aim of them paying the costs of all significant "externalities" (side effects such as pollution and global warming). But, how the level of environmental pollution or degradation should be measured is a question still to be answered.

\section{a. Measurement of Sustainable Portion of GDP}

This thesis aims to help develop economic indicators that have the ability to calculate economic progress and can be comprehensive measures of well-being and environmentally sustainability. To achieve this aim, an economic indicator is needed that is as clear and appealing as GDP but more inclusive of other dimensions of progress, in particular the environmental and social aspects.

As discussed above, development of local and domestic products can be viewed as fundamental social-cultural priorities in a sustainable development strategy. This progress can clearly be economically measured using GDP. However, to measure the environmental impacts of these products by GDP, it is essential to integrate both gross domestic product and the cost that must be spent to conserve or restore the environment which is damaged during the process of development. 


\section{a. 1. Equation}

This research proposes a method to calculate the sustainable portion of GDP (called here GDPs) that can be introduced as an economic indicator which measures both the social-economic benefits of producing local and domestic products and their environmental impacts in an integrated concept.

The method to measure GDPs involves five factors which are listed below, and then explained in detail:

- $\quad$ EF (gha): Ecological footprint of an activity or a product.

- GDP (\$): Earned GDP from this activity or product.

- $\quad$ EF1 (gha): Overshoot portion of EF of the product or activity relative to the available bio capacity

- $\quad$ E1 (GJ): Overshoot portion of the life cycle energy use of the product or activity relevant to the energy equivalent of bio capacity.

- CE1 (\$): The cost to generate the overshoot portion of the life cycle energy used through the use of renewable resources.

\section{a. 2. Ecological Footprint (EF)}

The ecological footprint (EF) measures the extent to which humanity is using nature's resources faster than they can regenerate. EFs are usually presented together with bio capacities (BCs), which measure the bio-productive supply. The EF of a given product or an activity is equal to the area (gha) needed to absorb the $\mathrm{CO}_{2}$ emissions generated through using fossil fuel to produce its life cycle energy use (GJ).

\section{- EF1}

If an EF is larger than the available BC for a selected time period the EF/BC resource accounting results in a deficit or overshoot (EF1). A deficit occurs in the case where human resource extraction and waste generation exceed an ecosystem's ability to regenerate the extracted resources and to absorb the generated waste.

\section{- $\quad$ E1 (GJ)}

Overshoot portion of the life cycle energy use of the product or activity such that its $\mathrm{CO}_{2}$ emissions are more than the related BC. 
- CE1 (\$)

CE1 is assumed equal to the money needed to generate the overshoot portion of the life cycle energy used (E1) through the use of renewable resources. Its value depends on the level, type and cost of the available technology and renewable resources. As a result, CE1 can be different from one location to another or from region to region or country to country.

The following equation can be used to evaluate the sustainable portion of GDP (GDPs):

$$
\text { GDPs }=\text { GDP }- \text { CE1 }
$$

\section{b. The Main Characteristics of GDPs}

GDPs is introduced here as a multi-dimensional indicator that has the ability to measure and evaluate the socio-economic and environmental success of human products and activities as being sustainable.

\section{b. 1. Social-Cultural Characteristics}

Making opportunities for local and indigenous people to produce and present their cultural capitals and products is one of the fundamental cultural outcomes for the sustainable development of an activity such as ecotourism. Since GDPs emphasises the development of local and domestic products, it can be determined as a social-economic indicator that contributes to the evaluation of success in achieving this cultural outcome.

\section{b. 2. Environmental Characteristics}

GDPs is an economic indicator that is sensitive to the environmental impacts of human products and activities. Integration of EF and economic development in GDPs allows it to be considered as a social-economic indicator as well as an ecological indicator.

GDPs addresses the amount of GDP that must be spent to conserve and restore damaged environmental resources. It can contribute to the more accurate accounting of other economic indicators such as Goods, Services Tax (GST).

\subsubsection{Cultural Footprint of Ecotourism}

\subsubsection{Definition}

The thesis introduces ecotourism as a sustainable phenomenon that aims to conserve the natural and cultural heritage of its host societies and engage them in social-economic 
development through an educational process. The anticipated outcomes of ecotourism are linked together, influenced by the strategy for sustainable development in a way so that achieving each of them contributes to the others, all being set under the umbrella of sustainability.

Figure 4.9 shows the relationship between the cultural changes to the host society caused by the development of ecotourism and its following environmental and economic changes. Based on its anticipated cultural outcomes, the cultural footprint (CF) of ecotourism can be conceptualized as the influences exerted by ecotourism on a given host society that change its attitudes to existing cultural and natural heritage and capital, and that develops cultural communication between it and its visitors.

Since the economic development of ecotourism is introduced as a social-economic activity that is environmentally sensitive as well, the cultural changes of the host society can direct the economic system toward sustainable economic strategies.

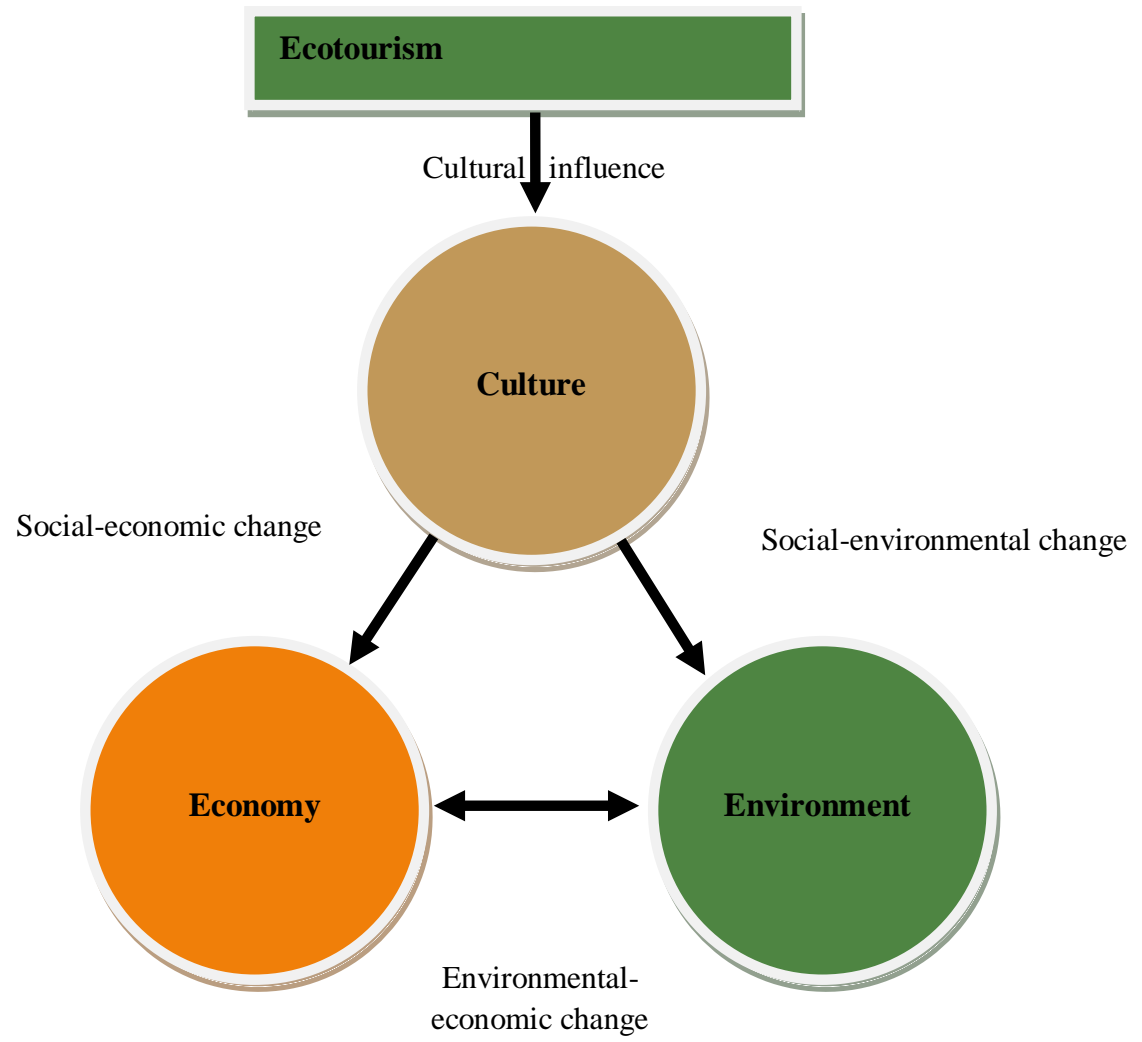

Figure 4.9: Relationship between ecotourism, culture of the host society, environment and economic system 


\subsubsection{CF-Model}

Figure 4.10 shows a proposed model that can be used to explore the quantitative interaction between the cultural, environmental and economic changes caused by ecotourism in its host societies. In this model (Figure 4.10), each of the cultural, environmental and economic indicators (proposed in the comprehensive framework) is set on one of the apexes. In this model, cultural indicators determine and measure the quantity of the cultural products and activities (for example producing local foodstuffs) that are anticipated as cultural productive activities in the cultural framework. These cultural indicators determine a part of the CF of ecotourism development.

In this model, the environmental impacts of the cultural products and activities are calculated by using the related ecological indicators such as EF. The EF of the cultural products and activities contributed by ecotourism, determines whether these cultural products and activities are environmentally sustainable or not. Evaluation of the EF can be conducted through comparison between the measured EF and fair EF (Ideal EF for sustainable living) for each product and activity. Likewise two different products (for example organic and conventional foods) or activities (for example walking and golfing) that are offered by ecotourism can be ecologically compared with each other by making comparison between their EFs.

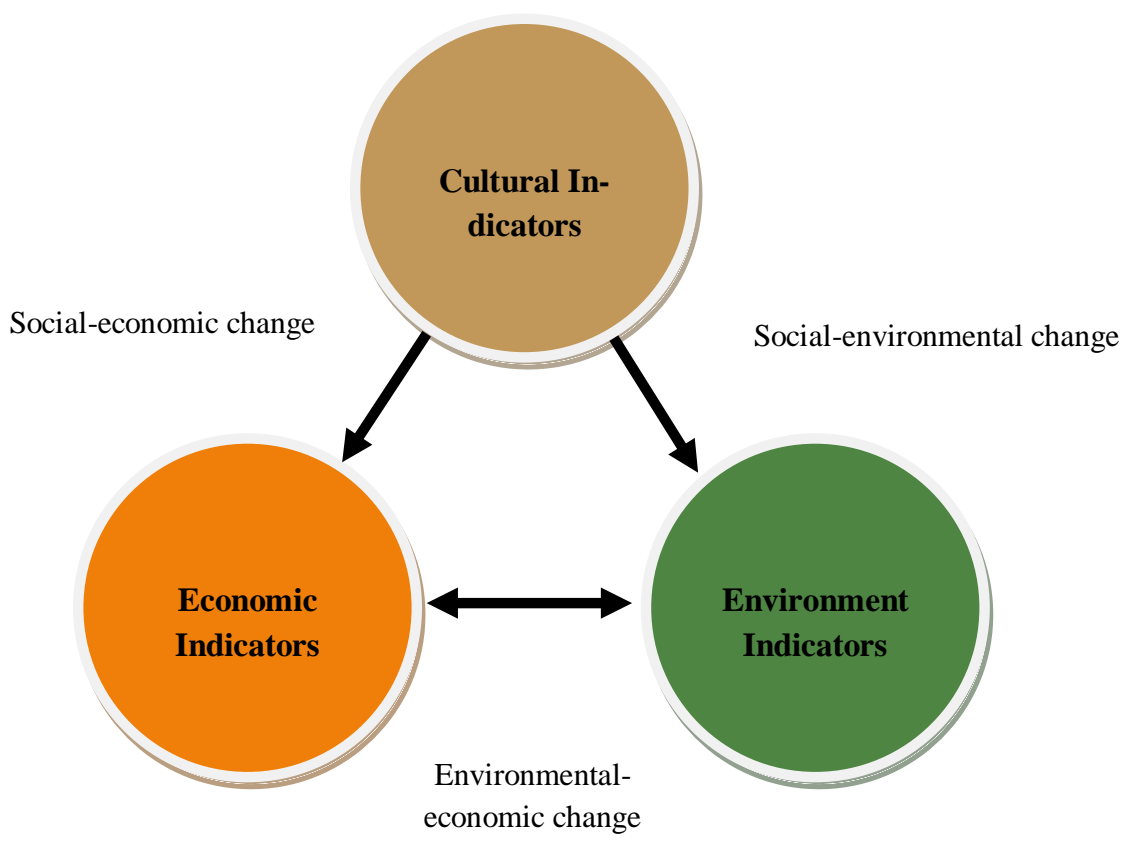

Figure 4.10: A model to evaluate interaction between cultural, environmental and economic changes caused by ecotourism development. 
The social-economic footprint of the cultural products and activities can be measured by using economic indicators such as GDPs. Since the GDPs of each product or activity is influenced by its EF, if a cultural product or an activity contributes to reducing the EF of ecotourism, this activity contributes to increasing GDPs.

Figure 4.11 illustrates an area the boundaries of which are determined by: the quantity of the cultural products and activities developed through ecotourism; the reduced or increased (R/I) portion of EF influenced by these products and activities; and (R/I) portion of GDPs caused by them. This area can be considered as the cultural footprint (CF) of these products or activities. Thus, the total CF of ecotourism development can be illustrated as an area in which its apexes are the total quantity, and the reduced or increased portions of EF and GDPs caused by its cultural products and activities (Figure 4.11).

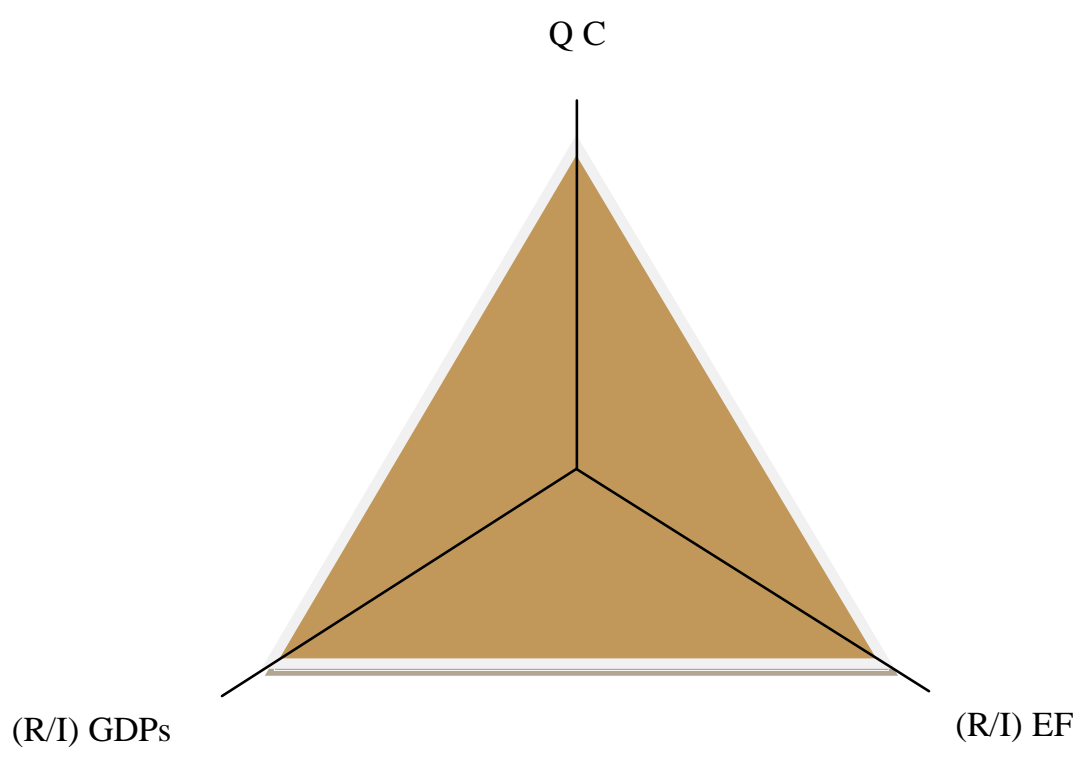

Figure 4.11: Cultural footprint (CF) model

In Figure 4.11:

Q C: Quantity of the cultural products or activities.

(R/I) EF: Reduced or increased portion of EF influenced by Q C.

(R/I) GDPs: Reduced or increased portion of GDPs influenced by Q C.

\section{a. Measurement of the (R/I) EF of Cultural Products}

The calculation of (R/I) EF of cultural products is based on the following factors: 
EF: Total ecological footprint of all cultural and conventional products. The EF determines the present ecological footprint of all cultural and conventional products.

EF1: the ecological footprint of ecotourism products with the assumption that all are conventional.

The difference between EF1 and EF is equal to the portion of EF that is reduced or increased by using cultural products.

$$
(\mathrm{R} / \mathrm{I}) \mathrm{EF}=\mathrm{EF} 1-\mathrm{EF}
$$

The (R/I) GDPs of ecotourism products can be measured by using the same method explained above.

\section{b. Measurement of the (R/I) EF of Activities}

In this study the EFs of ecotourism activities are compared with the fair EF of sustainable living related to each type of activity. According to WWF Living Planet Report (2012:12), WWF Living Planet Report (2010:74), and Vale and Vale (2009:358), in 2007, the total fair EF of sustainable living is $1.85 \mathrm{gha} /$ capita that covers all human life, activities and products. In this thesis the fair EF of sustainable living related to each category of ecotourism products and activities is calculated as a portion of the total fair EF of sustainable living (1.85 gha/ capita) proposed by WWF Living Planet Report (2010 and 2012).

Using the fair EF for sustainable living as a benchmark, ecotourism activities can be categorized by their EF into two types of activities with large (Type 1) and small EFs (Type 2). Measurement of (R/I) EF as influenced by ecotourism activities involves the following factors:

- $\quad E F=$ Ecological footprint of ecotourism activities including activities with large and small EFs

- EF1= Ecological footprint of ecotourism activities with the assumption that all activities are Type 2 .

$$
\mathrm{R} / \mathrm{I} \text { EF }=\text { EF- EF1 }
$$


The result of this equation addresses the ecotourism activities that should be considered and those that must be justified or ignored. The difference between the present EF of an activity or product and ideal (fair) EF indicates the overshoot portion of the present ecological footprint.

EF (present/ assumed) - Ideal EF = Overshoot portion of the EF (present/ assumed)

\section{c. Measurement of the (R/I) GDPs of Ecotourism Activities}

The (R/I) GDPs of ecotourism activities can be measured using the same method as mentioned above through the following equation:

(R/ I) GDPs= GDPs1- GDPs

In this equation:

- (R/I) GDPs: Reduced or increased portion of GDPs influenced by Type 2 activities

- GDPs = sustainable portion of GDP earned by ecotourism activities including Type 1 and 2 activities.

- GDPs1= Sustainable portion of GDP activities with the assumption that all activities are Type 2.

The result of the equation shows the reduced portion of GDPs as influenced by Type 1 activities.

\section{d. Example}

The CF model can be used for comparing the CFs of different projects, products or activities by setting their CFs in one model as shown in Figure 4.12. Figure 4.12 shows an example of using the CF model to compare the CFs of two different ecotourism activities (for instance walking and golfing). 


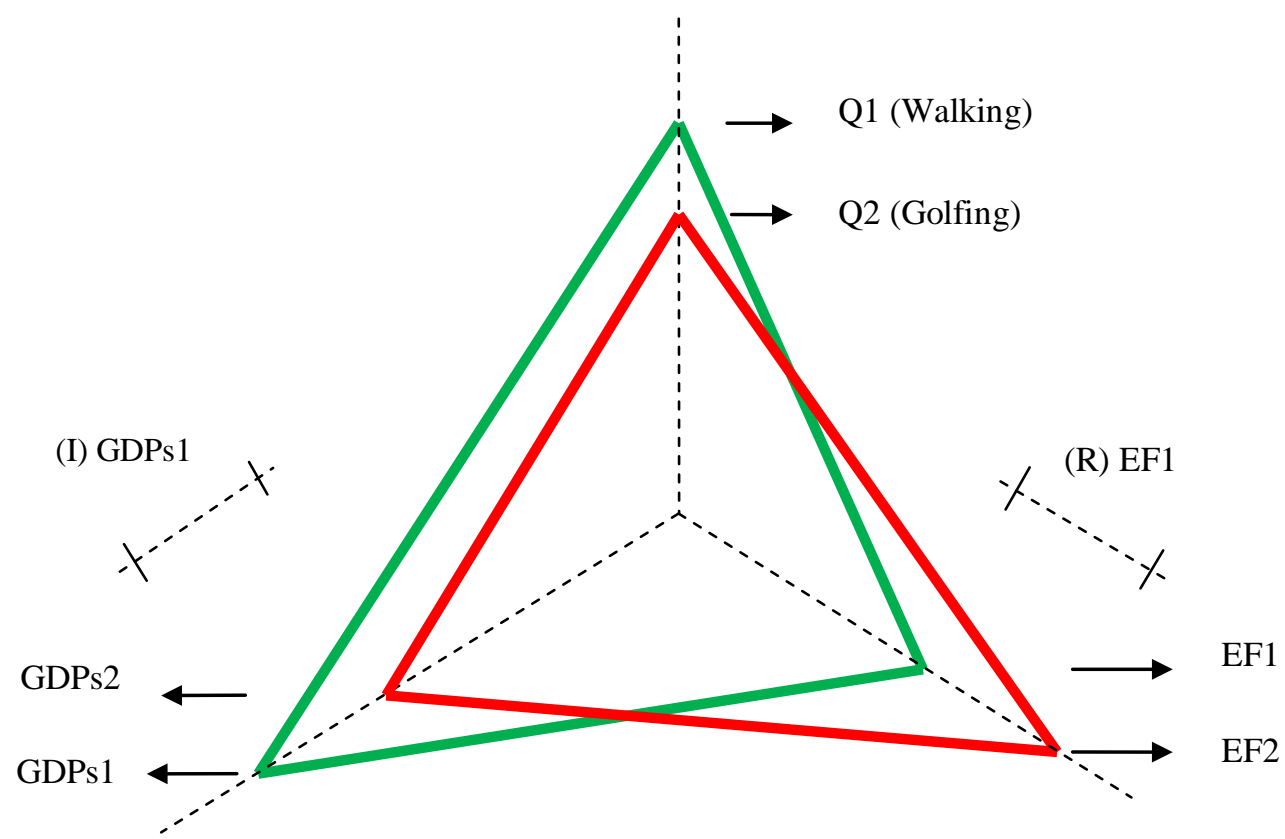

Figure 4.12: CF model used for comparison between the CFs of two different Ecotourism activities

In this model (Figure 4.12):

- Q1: Quantity of walking (number of visitors who walk).

- Q2: Quantity of golfing (number of visitors who play golf).

- EF1: Ecological footprint of walking/visitor.

- EF2: Ecological footprint of golfing /visitor.

- GDPs1: Portion of GDPs earned by walking

- GDPs: Portion of GDPs earned by golfing

- (I) GDPs1: Increase in portion of GDPs by walking as an activity.

- (R) EF1: Reduced portion of total EF influenced by walking as an activity.

- Red area: CF area of golfing

- Green area: CF area of walking.

As illustrated in Figure 4.12 the number of visitors who choose walking as one of their activities (Q1) is more than the number of visitor who play golf (Q2). In addition as shown in Figure4.12 the total EF of visitors related to walking is smaller than the total EF of visitors who play golf.

Furthermore the GDPs related to walking is more than the GDPs related to golf. Thus in this example in comparison with the CF of golfing, walking as a social behavior attracts more visitors, has less EF and more GDPs. Consequently, comparison between the CFs of walking and golfing results in demonstrating that walking is more culturally appro- 
priate, because more people do it, ( Q1 > Q2), it is more ecologically sensitive (EF1< EF2) and more economically viable (GDPs1 > GDPs2).

This research uses the CF model as a tool to make a link between the social-cultural behaviors that are offered to visitors through ecotourism and their environmental and economic footprints. The CF model can help in making the decision as to which products and activities must be developed, further justified or ignored through ecotourism development. The CF model can be considered as a holistic framework in which the environmental, cultural and economic frameworks for the development of ecotourism are set.

\subsection{Comprehensive Framework for Sustainable Development of Architecture}

This section presents a multi-dimensional framework to determine the main environmental, cultural and economic outcomes for a sustainable architecture (in particular accommodation services) through the sustainable development of ecotourism. This study introduces some productive activities that can contribute to achieving the anticipated outcomes for sustainable architecture.

In this research, as explained in Chapter 2, sustainable architecture is explained as being part of a natural organism which has an ecological relationship with its surroundings that in turn exert an influence on the supply of renewable materials and energies for construction and other requirements, such as heating, cooling and lighting.

Architecture is one of the ecotourism products and its main strategy for development is as a segment of the holistic framework for ecotourism as explained in this Chapter In addition, the main anticipated outcomes for sustainable architecture can be similar to the ecotourism outcomes explained in the framework, but particular activities to achieve these outcomes are related to the field of architecture.

Arising from the literature on sustainability, ecotourism and its framework for development, and the main profiles of sustainable architecture (Chapter 2), the thesis suggests a range of environmental, cultural and economic outcomes, their related produc- 
tive activities, indicators and required data which can be used as a tool to evaluate the sustainability of ecotourism architecture.

\subsubsection{Environmental Framework for Sustainable Development of Ecotourism Ar- chitecture}

The following part of this section explains the main components of the proposed environmental framework for sustainable architecture used in the development of ecotourism. It contains the anticipated environmental outcomes, related productive activities, ecological indicators and required data.

\subsubsection{Environmental Outcomes}

Based on the role of environment in the strong model of sustainability (Chapter 2), the fundamental forecasted environmental outcome for sustainable architecture related to ecotourism can be thought of as conservation of environmental resources (Table 4.16).

\begin{tabular}{|c|c|}
\hline \multicolumn{2}{|c|}{ Anticipated outcomes for ecotourism architecture ( accommodation services) } \\
\hline Environmental outcomes & Productive activities \\
\hline \multirow{8}{*}{ Conservation of natural resources } & $\begin{array}{l}\text { Using minimal resources and optimizing size of } \\
\text { spaces used for accommodation services }\end{array}$ \\
\hline & $\begin{array}{l}\text { Using some parts of open areas to generate food, } \\
\text { energy and some required materials and presenting } \\
\text { these activities to ecotourists }\end{array}$ \\
\hline & Horizontal distribution of accommodation services \\
\hline & $\begin{array}{l}\text { Development of accommodation services at the } \\
\text { local level }\end{array}$ \\
\hline & $\begin{array}{l}\text { Use existing buildings for accommodation services } \\
\text { - these might be repaired or made to match with } \\
\text { sustainable patterns of architecture }\end{array}$ \\
\hline & Using renewable resources for heating and cooling \\
\hline & $\begin{array}{l}\text { Using local and durable materials for construction } \\
\text { with lowest EF }\end{array}$ \\
\hline & $\begin{array}{l}\text { Using local manufacturers and equipment made } \\
\text { from natural products with low EF in interior spac- } \\
\text { es }\end{array}$ \\
\hline
\end{tabular}

Table 4.16 shows a range of productive activities that can contribute to architecture to achieve this outcome. Since environmental conservation has been explained as a socialecological activity that can be conducted through the use of available technologies, the anticipated activities shown in Table 4.16 have social-ecological and technicalecological characteristics. All these activities can be conducted in a community based process that attempts to engage local and indigenous people (as components of the organism) in the conservation process. This will thereby make them aware of the impact 
of their behavior on the environment and the benefits of using renewable resources to reduce these impacts through an educational process.

Indeed, when this approach is applied to the sustainable development of architecture, as Ding (2008:463) reveals, it involves the efficient allocation of resources, minimum energy consumption, low embodied energy intensity in building materials and construction processes, reuse and recycling, using renewable resources of energy and materials and changing unsustainable socio-cultural patterns of materials, energies and spaces consumption.

\subsubsection{Ecological Indicators}

Table 4.17 shows a range of proposed social-ecological indicators that can be divided into two types of indicators focused on the pattern of using spaces at different scales ( $E$ 1-4), and indicators focused on use of materials and energy resources (E 5-7). In Table 4.17, the EFs of materials and manufactures (E 5-7) are used as separate ecological indicators from the other indicators (E 1-4). However, it should be remembered that indicators E 1-4 arise from policies aimed at reducing the EF of all human activities and products, such as the patterns of use of spaces, materials and energy resources.

\begin{tabular}{|c|c|c|}
\hline \multicolumn{3}{|c|}{ Table 4.17: Social-ecological indicators } \\
\hline Indicator & Description & Unit \\
\hline E 1 & The area used as accommodation services & $\mathrm{m}^{2}$,visitor \\
\hline E 2 & $\begin{array}{l}\text { Public open air area used for generation of food, energy and } \\
\text { some required materials. }\end{array}$ & $\mathrm{m}^{2}$,visitor \\
\hline E 3 & Distance between accommodation services. & $\mathrm{km}$ \\
\hline E 4 & Modified or repaired buildings used for accommodation services & $\mathrm{m}^{2}$, visitor \\
\hline E 5 & Renewable resources used to generate energy & GJ \\
\hline E 6 & EF of local materials used to build accommodation services & GJ/gha \\
\hline E 7 & EF of manufactured components produced locally & GJ/gha \\
\hline
\end{tabular}

\subsubsection{Required Data}

The quantity and quality (refurbished and new) of architectural spaces at the different scales of region, site and building can be considered as forming the required data related to indicators E 1-4. Moreover the quantity and types of materials and manufactures used form the required data to measure EFs related to indicators E 5-7. 


\subsubsection{Cultural Framework for Sustainable Architecture Used in Ecotourism De- velopment}

\subsubsection{Anticipated Cultural Outcomes for Sustainable Architecture}

As shown in Table 4.18, this study anticipates some cultural outcomes for architecture that can be used as tools to explore its cultural footprint on host destinations through the sustainable development of ecotourism. The main cultural outcomes can be introduced as engagement of educated local and indigenous people in protection and presentation of their cultural capitals, and development of cultural interaction between visitors and the host communities (Table 4.18).

Table 4.18: Forecasted cultural outcomes for sustainable architecture and its related productive activities.

\section{Cultural outcomes}

- Changing attitudes of local and indigenous people to their cultural capitals including vernacular architecture. Making valuable local products which are also environmentally compatible and can be used as goods and services by visitors

- Development of cultural relationships between host communities and visitors

- Opportunity for visitors to be familiar with a part of host culture through using its architecture and related products

- Opportunity for host people to present their intangible cultural products including architecture and its related components.

\section{Productive activities}

Modifying and justifying the existing buildings to avoid unsustainable and unnecessary development of services

Development of types of accommodation services which contribute to close relationships between host people and visitors

Equal distribution of accommodation services in any project to avoid massing of capitals in a particular place and depression of other areas. Avoiding luxuries and fashions and giving more focus and attention to local values in architecture. Using local and available technologies for construction.

Engagement of local communities, organizations and people in the processes related to accommodation services (planning, management, investment, and construction etc).

Table 4.18shows a range of productive activities that can contribute to achieving to the latter cultural outcomes. However, some of the productive activities introduced in Table 4.16 can also be viewed as social-cultural productive activities.

\subsubsection{Cultural Indicators}

This section proposes cultural indicators which are classified into the two types of macro and micro indicators to evaluate the sustainability of architecture (in particular accommodation services) through the sustainable development of ecotourism. This classification arises from the essential characteristics of the cultural indicators explained above.

Table4.19 determines the cultural indicators to be used for the evaluation of architecture related activities (shown in Table 4.18) as being culturally sustainable. 


\begin{tabular}{|c|c|c|c|}
\hline $\begin{array}{l}\text { Cultural } \\
\text { Indicator }\end{array}$ & Description & Unit & Level/Scale \\
\hline $\mathrm{C} 1$ & $\begin{array}{l}\text { Modified or new buildings used for accommoda- } \\
\text { tion services. }\end{array}$ & $\mathrm{m}^{2} /$ visitor & Region \\
\hline $\mathrm{C} 2$ & Farm stay/ home stay accommodation services & $\mathrm{m}^{2} /$ visitor & Region \\
\hline C3 & Distances between accommodation services & $\mathrm{km}$ & Region \\
\hline $\mathrm{C} 4$ & $\begin{array}{l}\text { Proportion of built up land (accommodation) to } \\
\text { public open air areas. }\end{array}$ & $\%$ & Site \\
\hline C5 & $\begin{array}{l}\text { Public open air areas as multi-functional spaces for } \\
\text { cultural events }\end{array}$ & $\mathrm{m}^{2} /$ visitor & $\begin{array}{l}\text { Architecture/ } \\
\text { site/region }\end{array}$ \\
\hline C6 & $\begin{array}{l}\text { Locally constructed building(s) used as accommo- } \\
\text { dation services. }\end{array}$ & $\mathrm{m}^{2} /$ visitor & $\begin{array}{l}\text { Architecture/ } \\
\text { Site/region }\end{array}$ \\
\hline C7 & Types of materials used for construction & $\begin{array}{l}\mathrm{m}^{3} / \text { visitor of } \\
\text { different types }\end{array}$ & $\begin{array}{l}\text { Architecture/ } \\
\text { Site/region }\end{array}$ \\
\hline C8 & $\begin{array}{l}\text { The number of local communities, organizations } \\
\text { and people engaged in the processes related to ac- } \\
\text { commodation services (planning, management, } \\
\text { investment, and construction). }\end{array}$ & $\begin{array}{l}\text { number in dif- } \\
\text { ferent categories }\end{array}$ & $\begin{array}{l}\text { Architecture/ } \\
\text { site/region }\end{array}$ \\
\hline
\end{tabular}

As shown in Table 4.19, these objective cultural indicators can be classified, according to the level of detail at which they are applied, into three scale types: macro (regional$\mathrm{C} 1, \mathrm{C} 2, \mathrm{C} 3$ ), micro (one specific site-C4), and macro - micro (composite indicators-C5, C6, C7).

This thesis attempts to use objective cultural indicators which are measurable. Moreover, these indicators must be comparable for use in different places with different cultural and environmental circumstances. It should be remembered that most of the environmental indicators which are used to evaluate architecture as being environmentally sensitive can also be viewed as cultural indicators to evaluate its cultural footprint.

\subsubsection{Required Data}

Table 4.20 shows a range of required data to be used for evaluation of the CF of architecture (in particular accommodation services) through the sustainable development of ecotourism. 


\begin{tabular}{|c|c|c|c|}
\hline $\begin{array}{l}\text { Required } \\
\text { Data }\end{array}$ & Description & unit & Level \\
\hline C-D1 & $\begin{array}{l}\text { The area of modified or new buildings used as accommo- } \\
\text { dation services at the scale of region or site }\end{array}$ & $\mathrm{m}^{2}$ & Macro \\
\hline C-D2 & $\begin{array}{l}\text { The number of visitors per year at the scale of region or } \\
\text { site }\end{array}$ & Visitor/year & Macro \\
\hline C-D3 & The number of visitors per year at the scale architecture & Visitor/year & Micro \\
\hline C-D4 & $\begin{array}{l}\text { Types of accommodation services at the scale of region or } \\
\text { site or community. }\end{array}$ & $\mathrm{m}^{2} /$ type & Macro \\
\hline C-D5 & $\begin{array}{l}\text { Distance between accommodation services at the scale of } \\
\text { region or site or community }\end{array}$ & $\mathrm{km}$ & Macro \\
\hline C-D6 & $\begin{array}{l}\text { Multi-functional spaces (closed and open air) used for cul- } \\
\text { tural events at the scale of region or site or community }\end{array}$ & $\mathrm{m}^{2}$ & Macro \\
\hline C-D7 & $\begin{array}{l}\text { Multi-functional spaces (closed and open air) used for cul- } \\
\text { tural events at the scale of architecture }\end{array}$ & $\mathrm{m}^{2}$ & Micro \\
\hline C-D8 & $\begin{array}{l}\text { Buildings which are constructed by using local technolo- } \\
\text { gies at the scale of region or site or community }\end{array}$ & $\mathrm{m}^{2}$ & Macro \\
\hline C-D9 & $\begin{array}{l}\text { Buildings which are constructed by using local technolo- } \\
\text { gies at the scale of architecture. }\end{array}$ & $\mathrm{m}^{2}$ & Macro \\
\hline C-D10 & $\begin{array}{l}\text { Types of main materials used for construction at the scale } \\
\text { of region or site or community }\end{array}$ & $\mathrm{m}^{3} /$ type & Micro \\
\hline C-D10 & $\begin{array}{l}\text { Types of the main materials used for construction at the } \\
\text { scale of architecture. }\end{array}$ & $\mathrm{m}^{3} /$ type & Micro \\
\hline C-D11 & $\begin{array}{l}\text { Number of local communities, organizations and people } \\
\text { engaged in the processes related to accommodation ser- } \\
\text { vices (planning, management, investment, and construc- } \\
\text { tion) at the scale of region or site or community. }\end{array}$ & $\begin{array}{l}\text { Number/ } \\
\text { category }\end{array}$ & Micro \\
\hline C-D12 & $\begin{array}{l}\text { The number of local communities, organizations and peo- } \\
\text { ple engaged in the processes related to accommodation } \\
\text { services (planning, management, investment, and construc- } \\
\text { tion) at the scale of architecture. }\end{array}$ & $\begin{array}{l}\text { Number/ } \\
\text { category }\end{array}$ & Macro \\
\hline
\end{tabular}

As shown in Table 4.20, the required data can be categorised into the two types of macro and micro scale data. The macro scale data refers to the cultural footprint of the development policies at the scale of region or community or site, and micro scale data refers to the cultural footprint of a building at the scale of an individual unit. Since regional dimensions of the cultural footprint refer to the participation of local people (with similar cultural backgrounds) in the sustainable development process through engagement in forecasted cultural activities (cited in Table 4.18), macro scale data can be used to measure the quantity of this engagement at the scale of a region, a community or a site.

The cultural footprint of a building refers to the interaction between architecture as a micro-organism and its surrounding environment, in particular through using materials and sources of energy as influenced by the dominant cultural system. Micro scale data can be used to measure the cultural footprint of architecture at the scale of a building. 
As a result, the combination of the required macro and micro data can be considered as a tool for the comprehensive measurement of the cultural footprint of architecture.

\subsubsection{Economic Framework}

This section presents an economic framework for architecture as used in the sustainable development of ecotourism. Since architecture is one of the ecotourism products, its economic policies and principles are dominated by the social-economic strategies of ecotourism. Furthermore, in this case, the main environmental and social outcomes for the economic development of architecture can be viewed as the same as those anticipated for the economic development of ecotourism, but in an area limited to architecture and its profiles.

\subsubsection{Environmental Outcomes and Activities}

The main environmental outcome for the economic development of architecture is introduced as being the same as that anticipated for ecotourism, as demonstrated in section 4.1.3.2. As shown in Table 4.21, the economic productive activities that must be accomplished in the field of architecture can be an economic contribution to the environmental activities forecasted for architecture in Table 4.16.

Table 4.21: Environmental outcome and related productive activities for economic development of architecture

\begin{tabular}{|l|l|}
\hline Environmental outcome & Productive activities \\
& Economic contribution to: \\
& Using minimal resources and optimizing size of \\
& spaces used for accommodation services; \\
& Using some parts of open areas to generate food, \\
energy and some required materials and presenting & these activities to Eco-tourists; \\
& Horizontal distribution of accommodation services; \\
Development of accommodation services at the local \\
level; \\
Conservation of the environmental resources \\
these might be repaired or made to match with sus- \\
tainable patterns of architecture; \\
Using renewable resources for heating and cooling; \\
Using local and durable materials for construction \\
with lowest EF; \\
Using local manufacturers and equipment which is \\
made from natural products with low EF in interior \\
spaces.
\end{tabular}




\subsubsection{Indicator}

Since GDPs has been introduced as an economic indicator that is involved in the ecological and social dimensions of development, this research suggests using GDPs as a fundamental economic indicator for the evaluation of the economic-ecological impacts of architecture used in ecotourism development.

\subsubsection{Cultural Outcome and Activities}

The main forecasted social-cultural outcome for the economic development of architecture is the same as the outcomes introduced in Table 4.18. Moreover its forecasted productive activities can be explained as an economic contribution to the anticipated activities, as explained in Table 18.

\subsubsection{Indicators}

GDPs and employment of local people are the two main economic indicators that are proposed for evaluating the social dimension of the economic development of architecture through ecotourism. However, GDPs also has the ability to cover the second indicator (employment), because contribution to the development of local products also makes opportunities for local people to be engaged in the economic development process.

\subsubsection{CF of Architecture}

The CF of architecture at the different scales of region, site and building can be evaluated and determined though using the CF model introduced in this chapter the CF model allows this research to make a link between the cultural, ecological and economic profiles of an architecture that can contribute to sustainable development through ecotourism.

The description of the comprehensive framework and its derivation has unavoidably been quite complex and highly theoretical. Chapters 5 and 6 will demonstrate its use for evaluation of an objective case study. This will clarify how the framework operates in practice. 


\subsection{Chapter 4: Summary}

Chapter 4 presents the comprehensive framework for the sustainable development of ecotourism and its architecture that is a pivotal part of the methodology as explained in Chapter 3. The aim of the framework is to introduce a method that can solve the methodological problems discussed in Chapter 3 and contribute to answering the research questions in Chapter 2.

The comprehensive framework is in two sections: the framework for ecotourism; and the framework for architecture (Figure 4.13). In response to the methodological problems discussed, the comprehensive framework integrates the three sub-sections of the ecological, cultural and economic frameworks for both ecotourism and architecture in the related cultural footprint (CF) model (Figure 4.13).

The framework indicates five ecological outcomes for the development of ecotourism (Table 4.2). The outcomes emphasize awareness of local and indigenous people about their environmental heritage and capital through an educational process, and subsequent engagement of such people in the development of ecotourism (Table 4.2). Furthermore, environmental conservation through using renewable resources to generate the energy used by tourism is another anticipated goal for the sustainable development of ecotourism. 


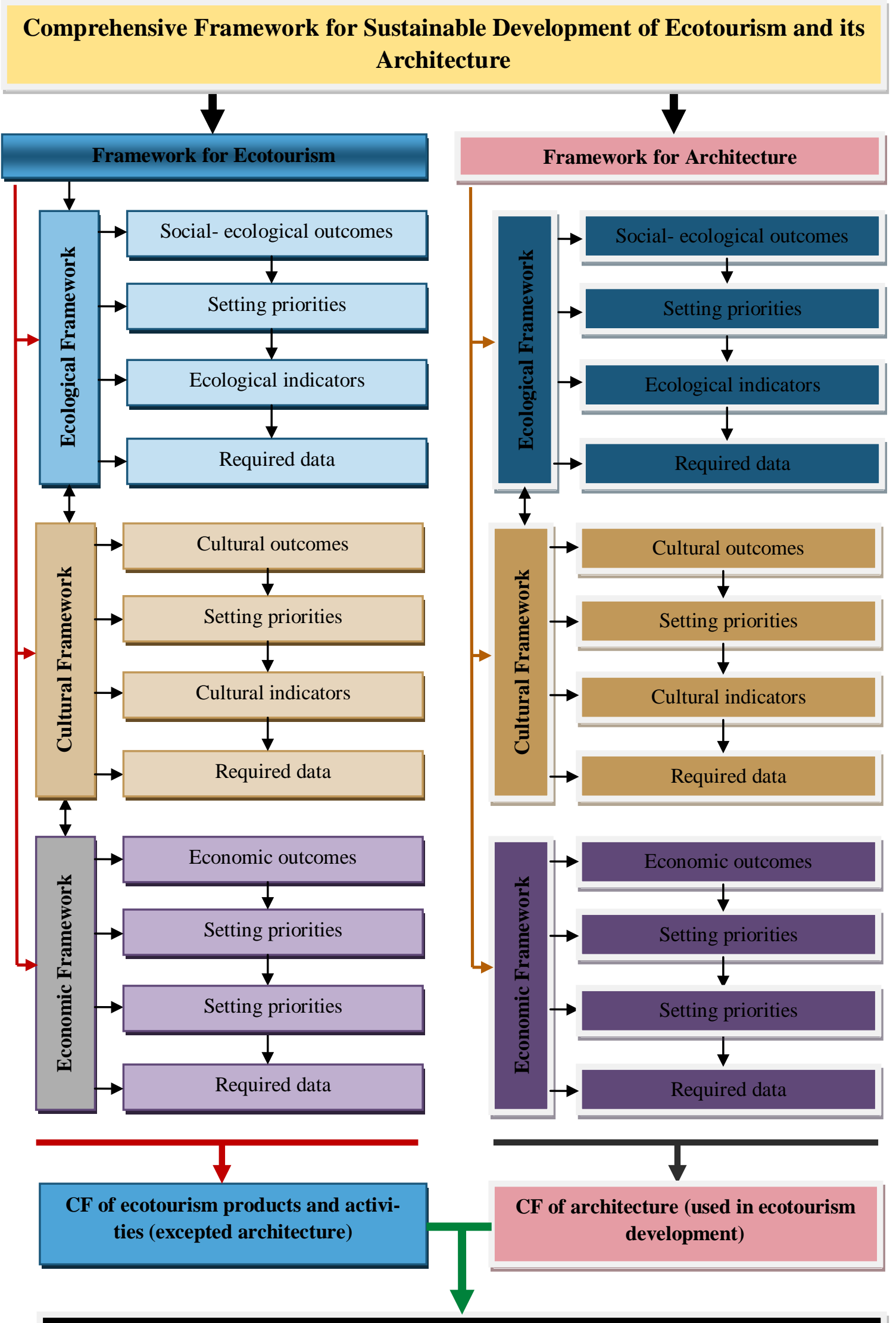

\section{Total CF of ecotourism and its architecture}

Figure 4.13: Structure of the compressive framework proposed through Chapter 4. 
Table 4.12 shows the eight anticipated cultural outcomes for the sustainable development of tourism that can be also applied to ecotourism and its architecture. These outcomes can be conceptualised as engagement of the host society in the conservation and development process of their cultural heritage and capitals through an educational process that also economically benefits them and ecologically protects their environment.

The comprehensive framework considers the economic development of ecotourism and its architecture as a social-ecological economic phenomenon. In this approach, the main policies for economic development rely on sustainability principles and polices. The framework indicates the social-cultural outcomes for the sustainable economic development of ecotourism and architecture as making opportunity for the engagement of the host societies in horizontal economic development, while the development process follows the sustainability strategies.

As demonstrated in Table 4.2, five priorities are proposed for achieving the ecological outcomes for the sustainable development of ecotourism. These include social ecological policies (e.g. engagement of people as well as all related organizations in environmental education) and social-economic strategies (e.g. employment of local and indigenous participants in the development process as employed or volunteer staff). These priorities aim to inform and benefit people through development of ecotourism as being ecologically sustainable (Table 4.2).

The framework introduces seven productive activities for achieving the forecasted ecological outcomes for architecture used in ecotourism development (Table 4.16). Conceptually, these are strategies for using materials, energy resources and spaces created, and management and organisation of services related to ecotourism architecture at regional and local scales (Table 4.16).

In this chapter the cultural priorities are viewed as the development of products and activities that contribute to achieving the forecasted cultural outcomes for the sustainable development of ecotourism. These can be summarised as:

- Development of cultural education and its related educational tools, equipment and spaces; 
- Restoration of cultural heritage and development of cultural capitals and products in a way that ecologically and economically benefits the host society ;

- Protection of host authenticity.

The six cultural priorities proposed for the development of architecture through ecotourism (Table 4.18) can be summarised as using types of architecture and related services that contribute to:

- $\quad$ Restoring the cultural heritage;

- Cultural interaction between host society and visitors;

- Avoiding luxuries and fusions;

- Using available technologies;

- Democratic engagement of local people in the development process.

Economic priorities proposed for ecotourism and its architecture are considered the same as the ecological and cultural productive activities that economically benefit the host destinations of ecotourism in a sustainable way.

Chapter 4 presents the ecological indicators for the development of ecotourism (Table 4.3) and its architecture (Table 4.17). In terms of ecotourism, the ecological indicators include: the number of people who engage in the sustainable development of ecotourism through an educational process; and, the EF of products and activities produced/ consumed / conducted in relation to ecotourism (Table 4.3).

The ecological indicators for evaluation of architecture can be categorised into the three categories of quality (e.g. using refurbished buildings), quantity (e.g. the area used as accommodation) and the EF of the architecture involved (Table 4.17).

Mahravan and Vale (2010) and O’Connor (2009) reveal that the EFs of some tourism products and activities (such as waste and water consumption) are small, whereas the EFs of transportation, food, accommodation services and tourism activities form the major part of the overall EF. Consequently these four categories have been chosen for evaluation using the comprehensive framework. The EFs of these categories are also used as the main ecological indicators to evaluate the three case studies, introduced in previous chapters, as being ecologically sustainable. 
The cultural indicators for development of ecotourism (Table 4.14) and its architecture (4.19) can be summarised as the priorities proposed for both ecotourism and architecture. However, as will be discussed in the conclusion, some indicators can be covered by other indicators to shorten the list and to solve shortcomings in the related data.

Due to a deficit of efficient economic indicators for the sustainable development of ecotourism and its products, this thesis introduces GDPs as a new economic indicator. The GDPs aims to connect the economic development of ecotourism and architecture to the ecological and the social indicators proposed for these categories as being economically sustainable.

The framework proposes the CF model as an original system to analyse the cultural footprint of ecotourism and its products and activities. The CF model indicates the environmental influences exerted by ecotourism and its products and activities on a given host destination. The model can also be a tool to compare the current ecological, social and economic circumstances of an ecotourism project and its sustainable pattern (ideal model). In addition the CF model can be used to compare different ecotourism projects in terms of being sustainable. Furthermore, it can be used to compare the cultural footprint of a project at different times.

The framework thus answers the research question "What are the main characteristics of an efficient strategy for the sustainable development of ecotourism and its products, such as architecture?” (Chapter 2, section 2.6.1.1) as:

- A holistic approach to sustainable development that covers all ecological, cultural and economic dimensions of ecotourism and its architecture as being sustainable.

- Proposing ecological, cultural, economic outcomes, priorities, related indicators and required data for evaluation of ecotourism and its architecture as being sustainable.

In response to the research question "What are the relationships between the anticipated outcomes for ecotourism and its architecture and how are they linked to each other?” (Chapter 2, section 2.6.1.2) it could be said that:

The anticipated outcomes are linked together through sustainable policies and strategies. This means that all the anticipated outcomes simultaneously must be ecologically sensitive, culturally appropriate and economically sustainable. 
The framework answers the research question "what are the main priorities for the development of ecotourism and its related architecture that can contribute to achieving their forecasted outcomes in a sustainable way?” through proposing the priorities discussed in this Chapter. Other research questions will be answered through testing the CF model and the results (Chapters 6 and 7). 


\section{Chapter 5: First Case Study - Otago Central Rail Trail (OCRT), Otago, New Zealand}

\subsection{Introduction}

The thesis results are presented in Chapters 5 and 6. This chapter describes the case study of the 'The Otago Central Rail Trail (OCRT)' introduced in the New Zealand Tourism Strategy (NZTS) 2015 as a successful example of sustainable tourism development. Since the OCRT crosses many rural and urban places and their adjacent areas with different environmental, cultural and economic characteristics, it can be considered as the integration of both rural and urban ecotourism projects. This chapter investigates the impacts of the OCRT as a tourism project (managed by the OCRT community and other related organizations) and its related architecture on cultural heritage, local economic systems and natural resources at regional scale. The investigation is conducted through using the comprehensive framework proposed for the sustainable development of ecotourism and its products (including architecture) and activities (see Chapter 4).

This chapter responds to four research questions (Questions 2.6.1.4 to 2.6.1.7) introduced in Chapter 2. Through answering these how the proposed priorities for the sustainable development of ecotourism and its architecture influence each other and the host society at the regional scale ecologically, socially and economically, will be clarified. Chapter 5 also sets out how these influences can be measured and evaluated through using the comprehensive framework introduced in Chapter 4.

\subsection{Otago Central Rail Trail (OCRT) - Background}

Originally the Otago Central Railway that is located in the South Island of New Zealand (Figure 5.1) was constructed to transport gold out from the booming Central Otago goldfields of the late 1800s and into the bustling city of Dunedin (Graha, 2009:3). In the forty years it took to complete the railway, over 60 bridges and 3 tunnels were built as part of the railway between Middlemarch and Clyde. According to Burns and Corbet (2007:62) the railway closed in 1990, at a time when the rural community was in a state 
of stagnation. Graham (2009:4) points out that through the early 1990s, towns in Central Otago as in other rural areas in New Zealand lost many of their services and suffered from a reducing population, as banks and post offices closed and many businesses moved away. However, as explained by Burns and Corbet (2007:62) some local people viewed the closed railway line as an opportunity and potential attraction for developing the tourism industry in Central Otago.

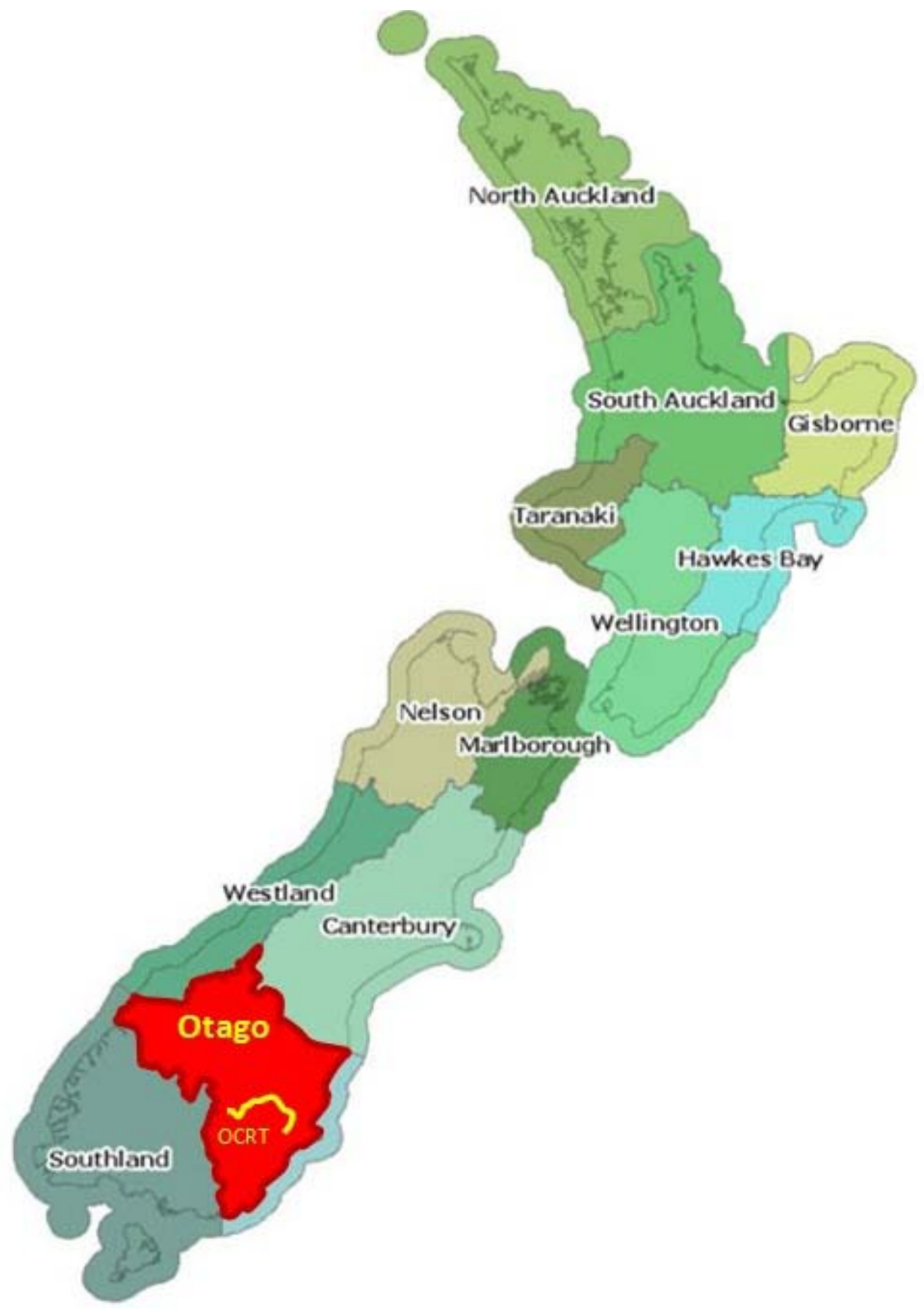

Figure 5.1: OCRT, Otago region, New Zealand

http://www.linz.govt.nz/sites/default/files/survey-titles/land-registration/land-districts/images/land-districts.jpg viewed August 2012 
The Department of Conservation (DOC), which had bought the land in 1993, was persuaded to consider the potential for outdoor recreation that the rail land offered. DOC spent six years and more than $\$ 850,000$ preparing, upgrading and restoring the closed railway and related historical heritage and infrastructure, which are now viewed as one of the main attractions for OCRT visitors (Burns and Corbet, 2007:62). Jellum and Reis (2008:7) point out that the OCRT was established in February 2000 through a cooperative effort between DOC and the Otago Central Rail Trail Trust, which had been formed in 1994. Since that time, the positive economic and environmental impacts of the OCRT have changed local attitudes and created greater community pride because of its cultural impacts. "Many of those who first opposed the trail now provide services such as farm stays, farm visits, cafes, and accommodation” (Burns and Corbet, 2007:62).

The $150 \mathrm{~km}$ recreational rail trail runs west from Middlemarch, travels along the Taieri River valley and through the Maniototo Plains, crosses the Rock and Pillar, North Rough, and Raggedy Ridge ranges, and passes through Ida Valley and Poolburn Gorge along the Manuherikia River (Figures 5.2 and 5.3).

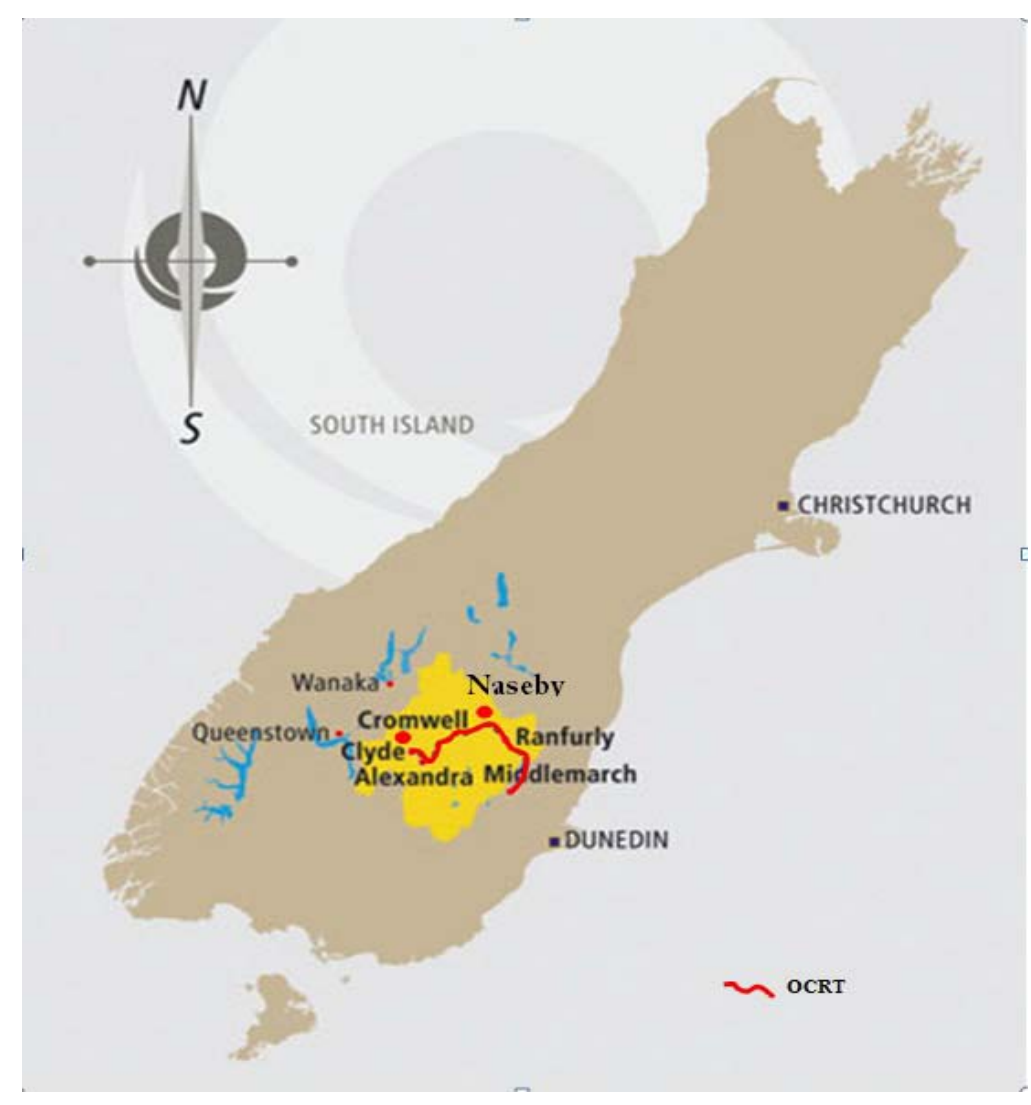

Figure 5.2: OCRT, Otago, South Island, New Zealand http://www.centralotagonz.com/rail-trail-map viewed August 2012 


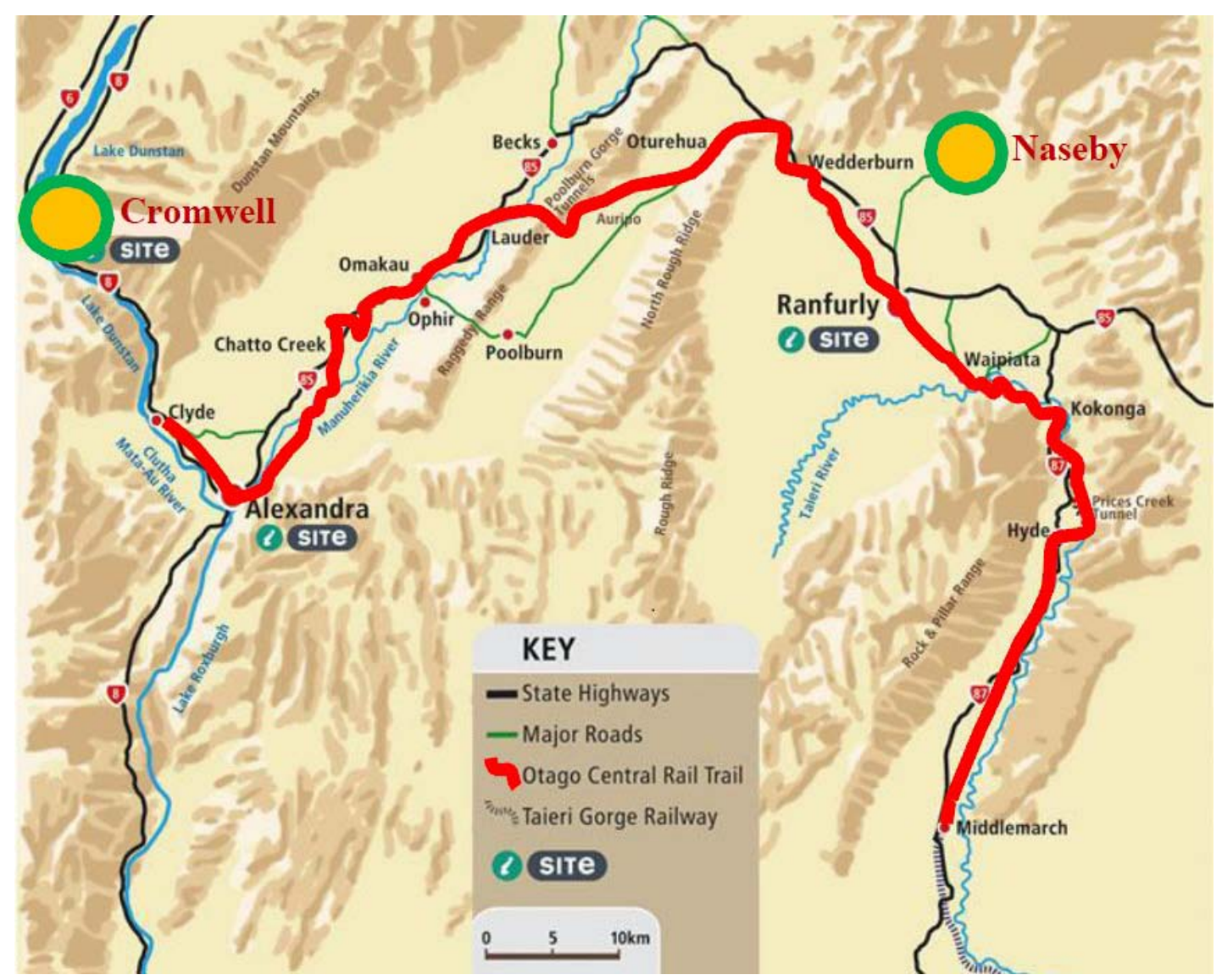

Figure 5.3: OCRT, Naseby and Cromwell, Otago, New Zealand http://www.centralotagonz.com/rail-trail-map viewed August 2012

A trip along the entire $150 \mathrm{~km}$ length of the Otago Central Rail Trail may begin at either end, at Clyde (see Figures 5.3 and 5.4) or Middlemarch (see Figures 5.3 and 5.5), and can be cycled in approximately 4 days, walked in approximately 6 days, or experienced by any number of shorter station to station trips.

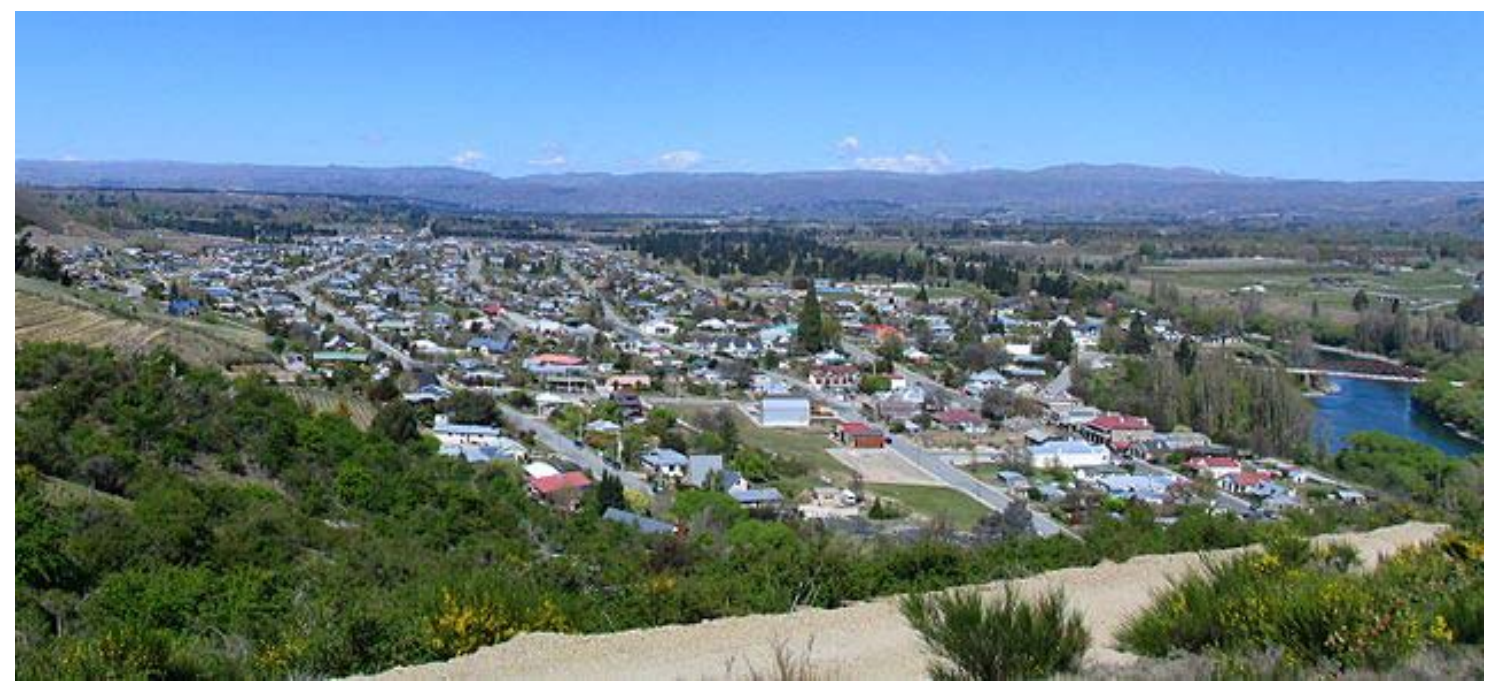

Figure 5.4: Clyde, Otago, New Zealand

http://upload.wikimedia.org/wikipedia/commons/thumb/8/84/Panorama_of_Clyde,_Otago,_New_Zeala nd.jpg/640px-Panorama_of_Clyde,_Otago,_New_Zealand.jpg viewed September 2012 


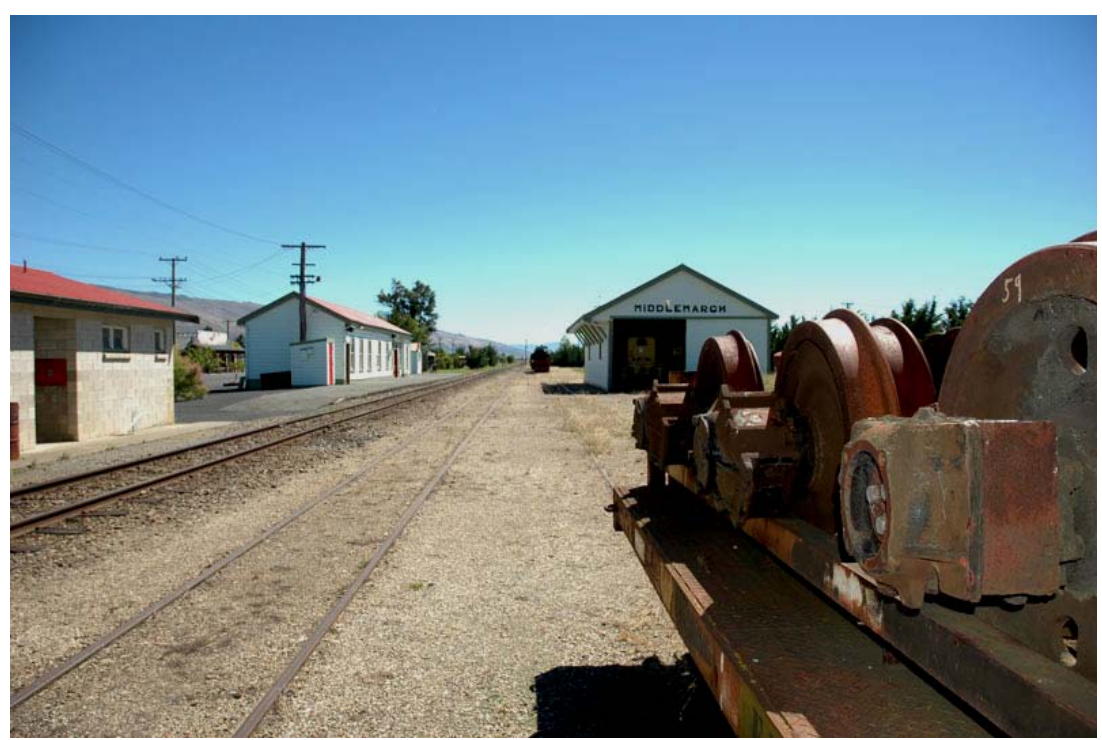

Figure 5.5: Middlemarch, Otago, New Zealand

http://www.otagorailtrail.co.nz/index.php?option=com_content\&task=view

\&id=26\&Itemid=57\#photos1 viewed August 2012

The OCRT's highest point is near the township of Wedderburn, $618 \mathrm{~m}$ above sea level, with gentle descents in either direction. Whether visitors are engaged in a multi-day excursion or a one day trip, a large selection of accommodation providers, restaurants, and other businesses support these rail trail visitors both in communities adjacent to the rail trail and at nearby off-trail locations.

\subsection{OCRT- Visitors}

In this study OCRT visitors are classified into the two groups of international and domestic visitors. In this section information related to each group of OCRT visitors including the number of visitors, OCRT visitor nights, and the home place of visitors by their types is presented.

\subsubsection{OCRT Visitor Numbers}

As shown in Figure 5.6, in 2009, 10,058 visitors visited the OCTR. This number increased to 12,157 visitors in 2010 (Figure 5.6). In 2011, in comparison with the year 2010, the number of OCRT visitors decreased to 11,788 visitors (Figure 5.6). 


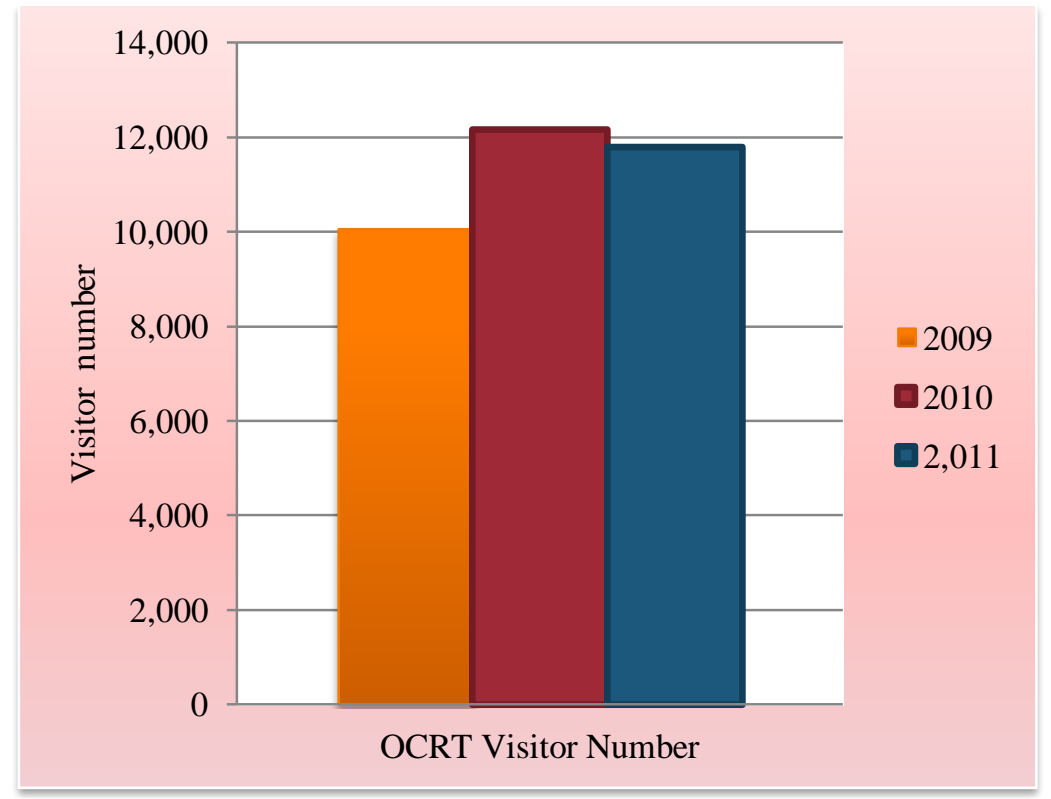

Figure 5.6: OCRT visitor numbers (2009- 2011)

\subsubsection{OCRT Average Visitor Nights}

Figure 5.7 indicates that in 2009 the OCRT average visitor nights were 3.8. As shown this increased to 4.2 nights in 2010 and dropped to 3.6 in 2011. In comparison with 2009, there were more visitors in 2011 but on average each stayed for less time.

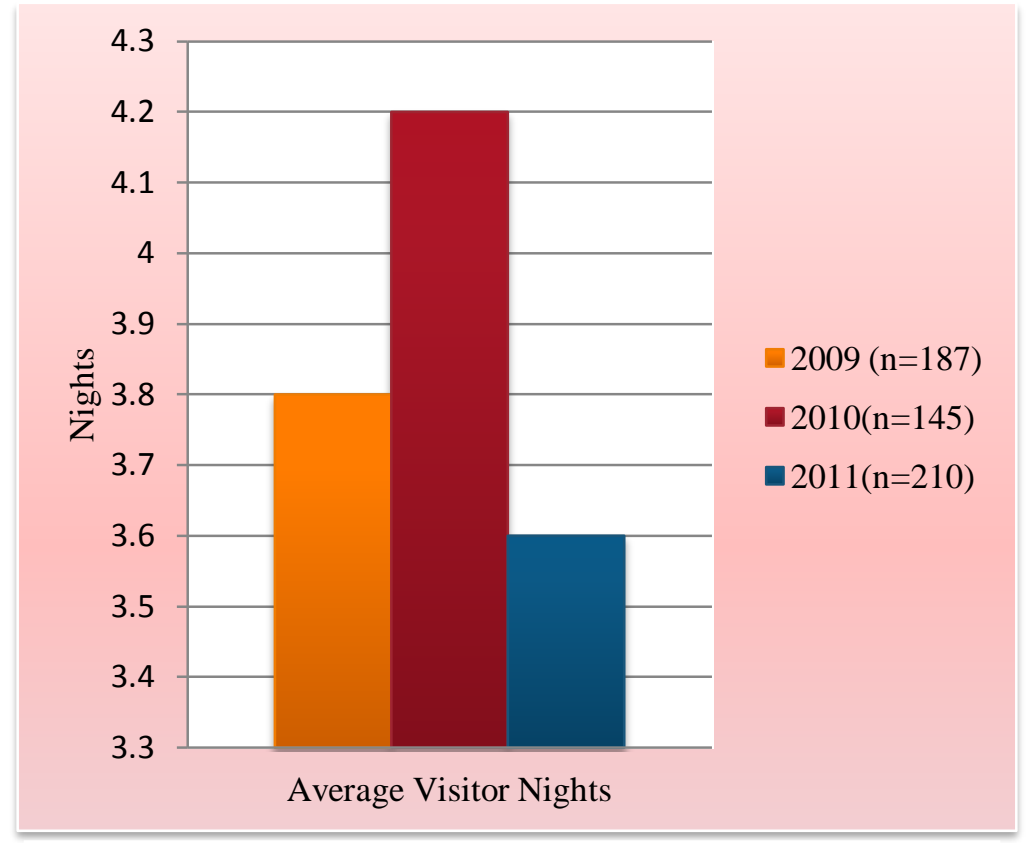

Figure 5.7: OCRT- average visitor nights (2009-2011) 


\subsubsection{OCRT Visitor Home Place}

Three OCRT surveys have been conducted respectively by Central Otago District Council (CODC) (2009); Reis et al (2010); and CODC (2011). These are used in this thesis as the basic data for assessing the home place of OCRT visitors. Figure 5.8 shows that in 2009, 31.2\% of the total 602 OCRT visitors sampled came from Auckland and Canterbury (15.6\% from each) followed in terms of numbers by $14.3 \%$ other North Island, 11.30\% Otago, 7\% Nelson and Marlborough, 6.5\% South Island, 5\% local, 4.7\% Wellington, 3.5\% Australia, 3.2\% Europe, 2.3\% UK and Ireland and 2.1\% North America. Moreover, in 2009, 7.1\% of OCRT visitors sampled did not state their home place (Figure 5.8).

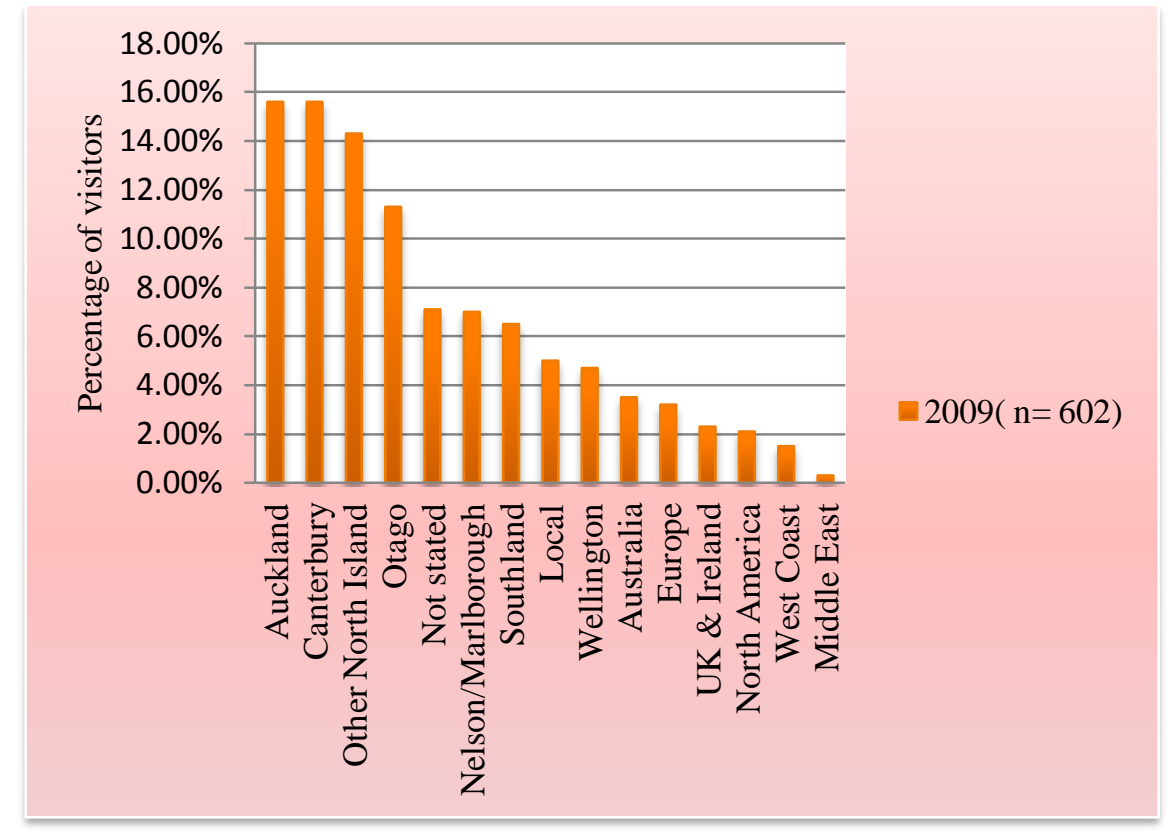

Figure 5.8: OCRT visitor home place - 2009 (CODC, 2009:9)

As shown in Figure 5.9, in 2010, of 154 OCRT visitors sampled 27.9\% of them came from the North Island other than its three main cities, followed by $21.4 \%$ Auckland, 10.4\% South Island other than Christchurch and Dunedin, 14.40\% Australia and Wellington (7.20\% each), 13\% Dunedin and Christchurch (6.5\% each), 4.5\% Hamilton, $3.2 \%$ other international and $5.20 \%$ UK and USA (2.60\% each). 


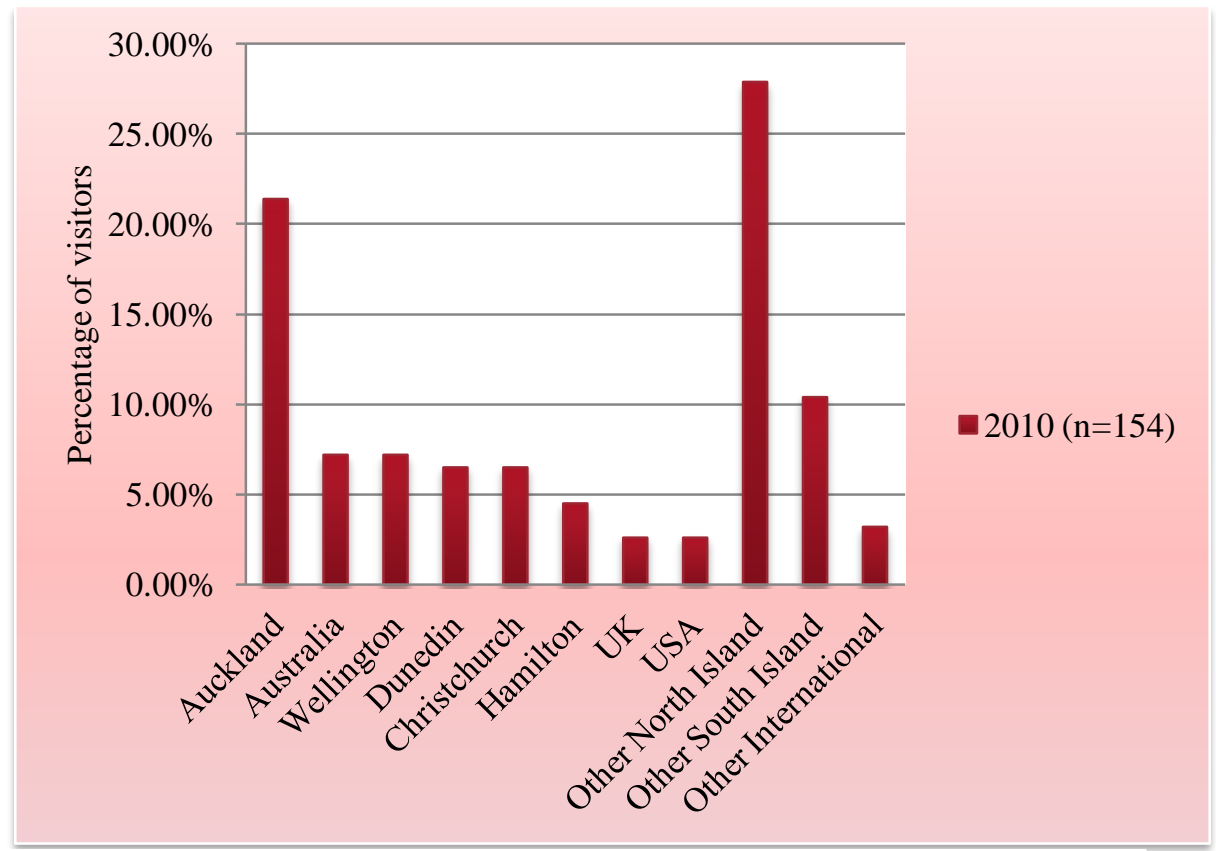

Figure 5.9: OCRT- visitor home place- 2010 (Reis et al, 2010:7)

In 2011, the majority of the 648 OCRT visitors sampled (40\%) came from the North Island, closely followed by 38\% from the South Island and 22\% international (Figure 5.10).

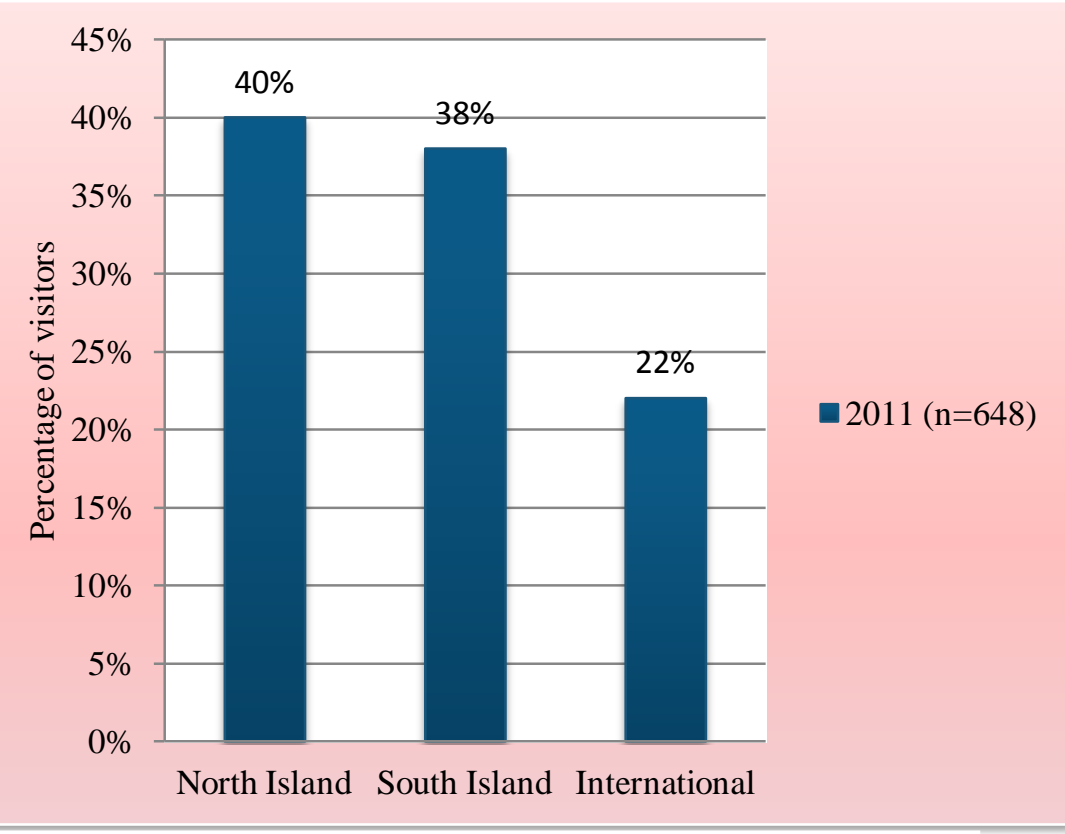

Figure 5.10: OCRT visitor home place- 2011 (CODC, 2011:12)

Since the home place of OCRT visitors is presented in different local, regional, national and international scales by the three surveys (CODS, 2009; Reis et al, 2010; CODC,2011), the results cannot be set out in a figure or table to be compared with each other. As a result, to make a link between OCRT visitor data arising from the three sur- 
veys, this study uses the CODC (2011) data as a base format. The OCRT visitor data from CODC (2009) and Reis (2010) are then converted to match the format used in CODC (2011) as shown in Figure 5.11.

Figure 5.11 shows that in 2009, of 602 OCRT visitors sampled, $45.5 \%$ came from the South Island followed by $35.9 \%$ from the North Island and $11.5 \%$ from overseas. In 2009, 7\% of OCRT visitors did not state their home place (Figure 5.11). In 2010, the majority or $61 \%$ of 154 OCRT visitors sampled came from the North Island followed by $23.4 \%$ from the South Island and 15.6\% international (Figure 5.11). In 2011, the majority or $40 \%$ of 648 OCRT visitors sampled came from the South Island followed by $38 \%$ from the North Island and 22\% international (Figure 5.11). What this figure shows is that domestic visitors were relatively stable in total, though their home place varied, but international visitor numbers rose.

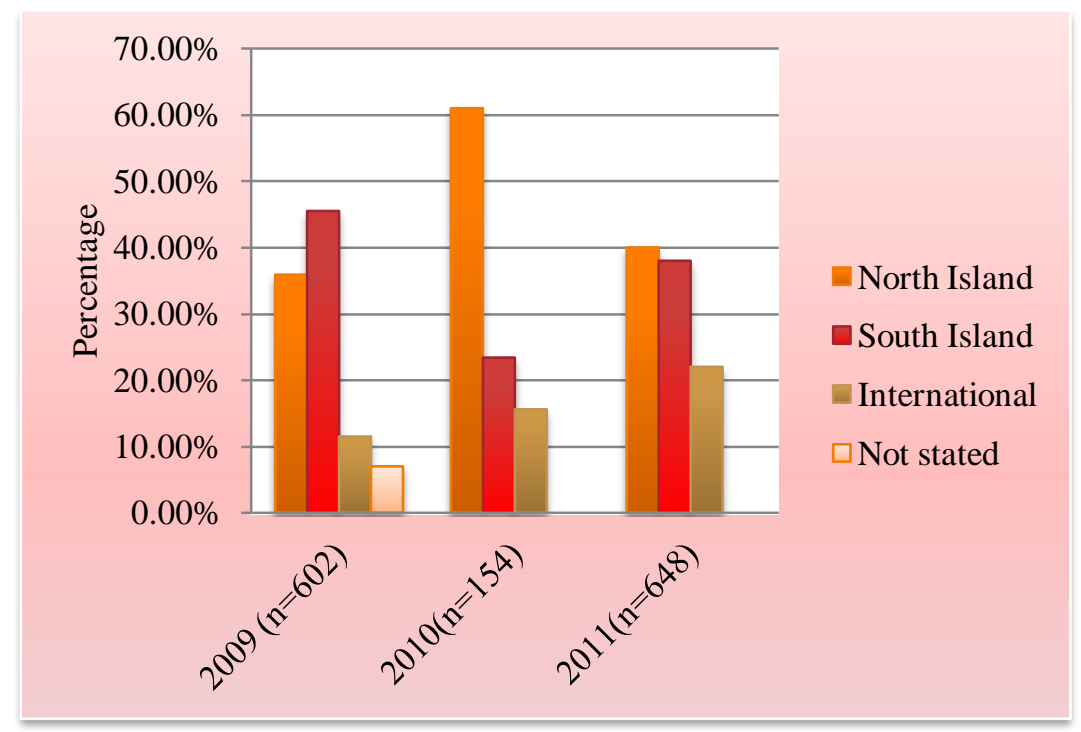

Figure 5.11: OCRT- visitors (2009- 2011)

\subsubsection{OCRT International Visitors}

Table 5.1 shows that in 2009, 11.5\% (69 visitors) of all 602 OCRT visitors sampled came from overseas. Of these in 2009, the majority or 3.5\% of total sample were from Australia followed by 3.2\% Europe, 2.3\% UK, 2.2\% North America and 0.3\% Middle East. As shown in Table 5.1, in 2010, the majority of overseas visitors still came from Australia but had doubled, being $7.1 \%$ of the total 154 OCRT visitors sampled, while those from the UK and North America were very slightly up, with 5.2\% UK and North America (2.6\% each), leaving $3.2 \%$ of total as other international. However, this changed in 2011. Table 5.1 indicates that of 648 OCRT visitors sampled in 2011, the 
majority or $42.7 \%$ were now from Europe (except UK) followed by $32.9 \%$ Australia, 12.6\% UK, 9\% North America and 1.4\% each from the Middle East and South Africa.

Table 5.1. OCRT- International visitors (2009-2011)

\begin{tabular}{|c|c|c|c|c|c|c|}
\hline \multirow[t]{2}{*}{ Home place } & \multicolumn{2}{|c|}{$2009(n=602)$} & \multicolumn{2}{|c|}{$2010(n=154)$} & \multicolumn{2}{|c|}{$2011(n=648)$} \\
\hline & $\mathrm{NV}^{3}$ & $\%$ & $\mathrm{NV}^{3}$ & $\%$ & $\mathrm{NV}^{3}$ & $\%$ \\
\hline Europe & 19 & 3.2 & - & - & 61 & 42.7 \\
\hline Australia & 21 & 3.5 & 11 & 7.1 & 47 & 32.9 \\
\hline UK & 14 & $2.3^{1}$ & 4 & 2.6 & 18 & 12.6 \\
\hline North America & 13 & 2.2 & $4^{2}$ & 2.6 & 13 & 9 \\
\hline Middle East & 2 & 0.3 & - & - & 2 & 1.4 \\
\hline South Africa & - & - & - & - & 2 & 1.4 \\
\hline Other International & - & - & 5 & 3.2 & - & - \\
\hline Total & 69 & 11.5 & 24 & 15.5 & 143 & 22 \\
\hline
\end{tabular}

As shown in Figure 5.6 the total number of OCRT visitors in 2011 was 11,788. This study uses the percentages of OCRT visitor home place (cited in Table 5.1) that are based on OCDC (2011:12) $(n=648)$ to calculate the total numbers of OCRT international visitors by their home place (Table 5.2).

In 2011, of 11,788 OCRT visitors coming from overseas the majority or 1,107 (42.7\%) were from Europe followed by 853 (32.9\%) Australia, 327 (12.6\%) UK, 234 (9\%) North America, and 72 (2.8\%) Middle East and South Africa (1.4\% each) (Table 5.2). In total this was $22 \%$ of all visitors.

\begin{tabular}{|c|c|c|}
\hline \multicolumn{3}{|c|}{ Table 5.2: OCRT- International visitors (2011) } \\
\hline Home place & $\mathrm{NV}^{3}$ & $\%$ \\
\hline Europe & 1,107 & 42.7 \\
\hline Australia & 853 & 32.9 \\
\hline UK & 327 & 12.6 \\
\hline North America & 234 & 9 \\
\hline Middle East & 36 & 1.4 \\
\hline South Africa & 36 & 1.4 \\
\hline $\begin{array}{l}\text { Other Interna- } \\
\text { tional }\end{array}$ & - & - \\
\hline Total & 2,593 & 22 \\
\hline $\begin{array}{l}\text { 1. NV: Number o } \\
\text { 2. Total visitor nu } \\
\text { 3. Percentage of I }\end{array}$ & Itor & \\
\hline
\end{tabular}




\subsubsection{OCRT Domestic Visitors}

Figure 5.12 shows that in 2009, 30\% of the 602 OCRT domestic visitors sampled were from the upper North Island (NI) followed by 27.5\% upper South Island (SI), 18.40\% Central SI (Otago), 9\% Lower NI, 7.30\% Lower SI, and 7\% did not state their home place.

In 2010, although the majority (56.54\%) of 154 OCRT visitors sampled came from Upper NI, other relative positions had changed with $15.76 \%$ from Lower NI, 12.30\% Central SI (Otago) and 15.4\% Upper SI and Lower SI (each 7.7\%) (Figure 5.12).In 2011, the majority (35.8\%) of 648 OCR visitors sampled were still from the Upper NI followed by $22.75 \%$ central SI, 21.20\% Upper SI, 14.90\% Lower NI and 5.35\% Lower SI (Figure 5.12).

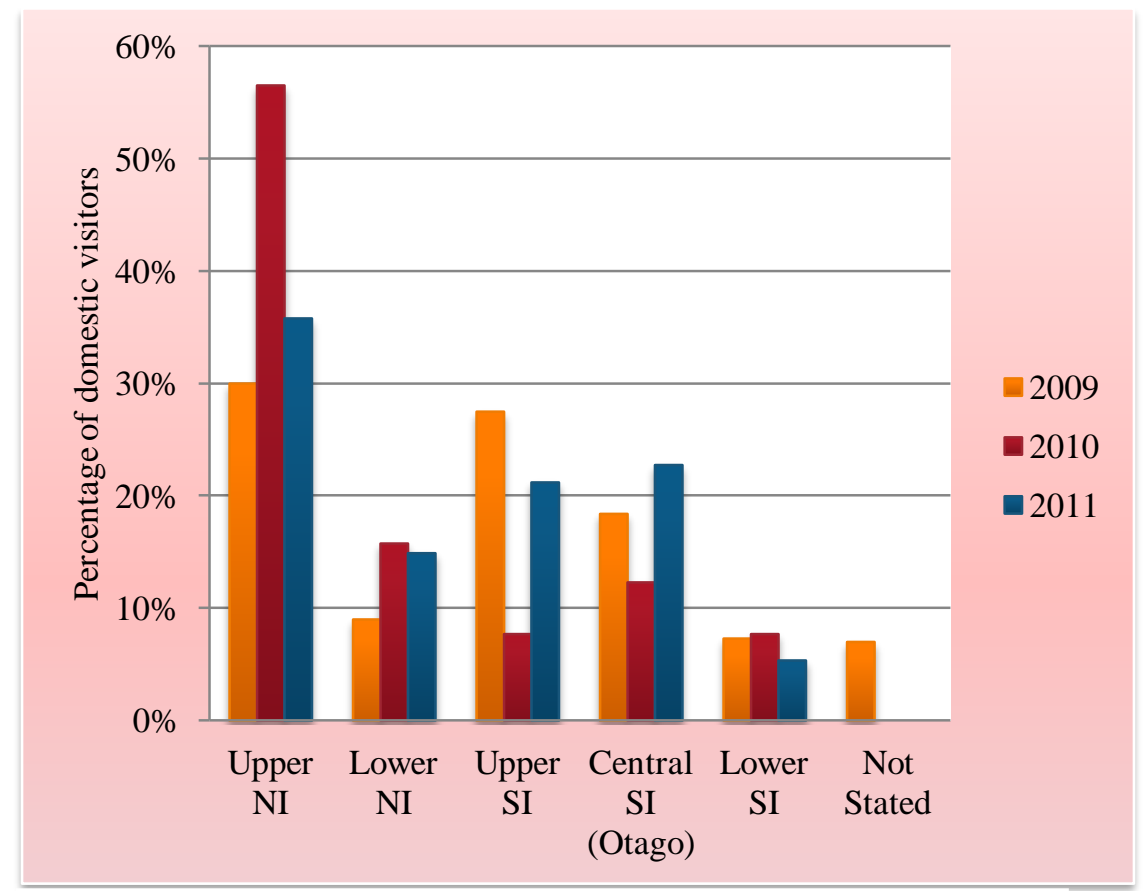

Figure 5.12: OCRT- domestic visitors (2009-2011)

This study calculates the numbers of OCRT domestic visitors in 2011 by their home place (Table 5.3), based on the percentages of OCRT domestic visitors from the OCDC survey (2011) shown in Figure 5.12. According to CODC (2011) 78\% of the total 648 OCRT visitors sampled are domestic visitors (Figure 5.8). Using this portion as a base, 9,195 (78\%) of 11,788 (total number of OCRT visitors in 2011, shown in Figure 5.6) can be considered domestic visitors (Table 5.3). 
From the discussion above, in 2011 most domestic visitors came from Upper NI, the estimated number being 3,292 (35.8\%) of 9,195 (100\%). This is followed by 2,092 (22.75\%) from central SI (Otago), 1,949 (21.2\%) Upper SI, 1,370 (14.9\%) Lower NI and 492 (5.35\%) Lower SI (Table 5.3). In 2011, as Figure 5.13 illustrates, of 11,788 (100\%) OCRT visitors, 9,195 (78\%) were domestic and 2,593 (22\%) were international.

\begin{tabular}{|l|c|c|}
\hline \multicolumn{3}{|c|}{ Table 5.3: OCRT- Number of domestic visitors by home place } \\
\hline Region/ city & Number of visitors & $\%$ \\
\hline Upper NI & 3,292 & 35.8 \\
\hline Lower NI & 1,370 & 14.9 \\
\hline Upper SI & 1,949 & 21.2 \\
\hline Central SI (Otago) & 2,092 & 22.75 \\
\hline Lower SI & 492 & 5.35 \\
\hline Total & 9,195 & 100 \\
\hline
\end{tabular}

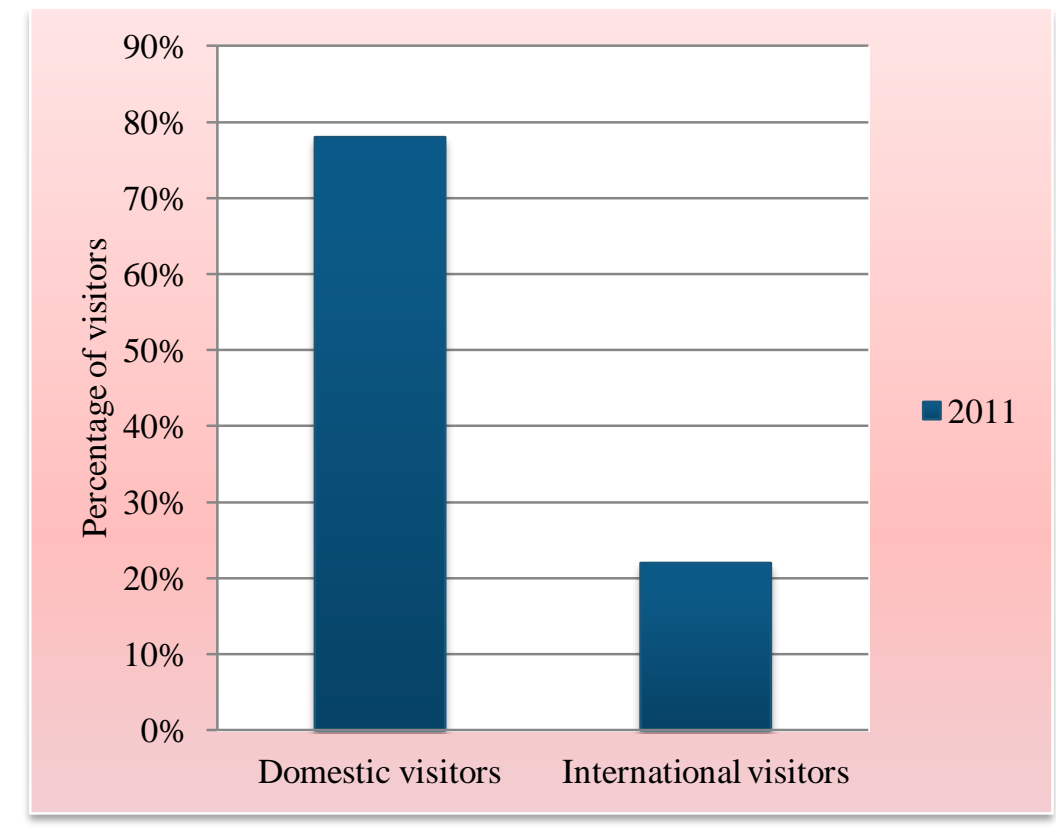

Figure 5.13: OCRT- domestic and international visitors (2011)

\subsection{OCRT Transportation}

This section explores types of international and domestic transportation used by OCRT visitors and calculates their Ecological Footprints (EFs). The total EF of OCRT transportation is calculated through integration of the EFs of international and domestic transportation. 


\subsubsection{OCRT- Types of International Transportation}

Since travelling from overseas to New Zealand by car, bus and train is not possible because of its geographical location, and because of the long travel times involved using ships, in this study of international transportation only airlines are considered.

\subsubsection{OCRT International Tourist-kilometres (T-Km)}

Table 5.4: shows that 2,593 OCRT visitors (22\%) are international tourists and the total distance between their home places and Auckland's international airport is equivalent to 32,041,486 Tourist-km (T-km). International distances are calculated here as a one way trip because their ecological footprint is assumed to be related to where the plane is refueled and so is divided between New Zealand and the home country. It could equally be argued that the full international trip should be counted, as belonging to the individual tourist's holiday that included the OCRT, but this has not been done in this thesis. Due to international tourists having to travel from Auckland to Dunedin and back this part of the journey is a domestic return flight and is equivalent to 5,590,508T-km, which is shown in Table s.5.4 and 5.7 as 'Air domestic international visitors'.

\begin{tabular}{|c|c|c|c|c|c|c|c|}
\hline \multirow{2}{*}{$\begin{array}{l}\text { Home } \\
\text { place }\end{array}$} & \multirow[b]{2}{*}{$\begin{array}{l}\text { Assumed } \\
\text { city of } \\
\text { origin } \\
\text { (central in } \\
\text { country of } \\
\text { origin) }\end{array}$} & \multirow[b]{2}{*}{$\begin{array}{c}\text { Distance } \\
\text { between } \\
\text { central } \\
\text { cities and } \\
\text { Auckland } \\
\text { (km). }\end{array}$} & \multicolumn{2}{|c|}{ Visitors } & \multirow{2}{*}{$\begin{array}{c}\text { Total In- } \\
\text { ternational } \\
\text { T-km }\end{array}$} & \multirow{2}{*}{$\begin{array}{l}\text { Auckland to } \\
\text { Dunedin } \\
\text { (km, return) }\end{array}$} & \multirow[b]{2}{*}{$\begin{array}{c}\text { Air domestic } \\
\text { International } \\
\text { visitors T-km } \\
\text { from Auckland } \\
\text { to Dunedin (re- } \\
\text { turn) }\end{array}$} \\
\hline & & & No & $\%$ & & & \\
\hline Australia & $\begin{array}{l}\text { Sydney } \\
\text { and Mel- } \\
\text { bourne }\end{array}$ & 2,512 & 853 & 32.9 & $2,142,736$ & 2,156 & $1,839,068$ \\
\hline Europe & Frankfurt & 18,180 & 1,107 & 42.7 & $20,125,260$ & 2,156 & $2,386,692$ \\
\hline UK & London & 18,334 & 327 & 12.6 & $5,995,218$ & 2,156 & 705,012 \\
\hline $\begin{array}{c}\text { North } \\
\text { America }\end{array}$ & $\begin{array}{l}\text { Montana } \\
\text { Billings }\end{array}$ & 11,950 & 234 & 9 & $2,796,300$ & 2,156 & 504,504 \\
\hline $\begin{array}{c}\text { Middle } \\
\text { East }\end{array}$ & Tehran & 15,005 & 36 & 1.4 & 540,180 & 2,156 & 77,616 \\
\hline $\begin{array}{l}\text { South } \\
\text { Africa }\end{array}$ & Pretoria & 12,272 & 36 & 1.4 & 441,792 & 2,156 & 77,616 \\
\hline Total & - & - & 2593 & 100 & $32,041,486$ & - & $5,590,508$ \\
\hline
\end{tabular}

As demonstrated in Table 5.4, to calculate the international T-km, the distance between the capital city of the home country of each visitor (except Australia) and Auckland was calculated and used as the international T-km. Since in Australia, just over half the population live in Sydney and Melbourne (Australian Government, 2010), the average distances between these cities and Auckland has been calculated and used as the interna- 
tional visitor distance from Australia. The flight distances between the international visitors home countries and Auckland have been calculated using a Flight Distance Calculator (http://www.travelmath.com/flying-distance).

In Table 5.4, total international T-km for each visitor home country is calculated through the following equation:

Distance between central city of the home country and Auckland $(\mathrm{km}) \times$ visitor numbers from the home country $=\mathrm{T}-\mathrm{km}$

In Table 5.4, total international T-km is the sum of all identified international visitor Tkm. Likewise in Table 5.4 air domestic international visitor T-km from Auckland to Dunedin (return) is calculated as the number of international visitors $\times$ distance between Auckland and Dunedin (2,156 km return).

\subsubsection{OCRT Types of Domestic Transportation}

The types and percentages of transport used for holidays in New Zealand as shown in Table 5.5 (Matthews, 2009:13) are used as a pattern for transport used to access the OCRT. Data from Matthews (2009) is used as the basis of the transport calculations as it is the most recently available study on New Zealand domestic tourist transportation. Table 5.5 indicates that in 2009, the majority or $87 \%$ of New Zealanders, used car/van (driver and passenger) for recreational travelling followed by 5\% pedestrian, 5\% bus/train/ferry, and 2\% bike. In Table 5.5, air domestic travel is included in 'motorcyclists and other' that are less than 1 percent of the total types of transportation used.

Since Matthews (2009) does not include data on domestic tourist air travel, this category is not included in Table 5.5. Due to the lack of data related to domestic international tourist travel and the domestic tourist air travel the following two assumptions are made in this thesis:

First: All International OCRT visitors use domestic air travel (Auckland to Dunedin return) to access the OCRT (Table 5.4).

Second: All domestic OCRT visitors use land travel to access the OCRT (Table 5.5). 
Table 5.5: Types of transportation used by New Zealanders for recreational travelling

\begin{tabular}{|l|c|c|c|c|c|c|}
\hline Activity & $\begin{array}{l}\text { Car/van } \\
\text { driver }\end{array}$ & $\begin{array}{l}\text { Car/van } \\
\text { passenger }\end{array}$ & Pedestrian & Cyclist & $\begin{array}{l}\text { Bus/ } \\
\text { train/ferry }\end{array}$ & $\begin{array}{l}\text { Total (incl mo- } \\
\text { torcyclists and } \\
\text { other) }\end{array}$ \\
\hline $\begin{array}{l}\text { Recreational } \\
\text { - Reference: (Matthews, 2009:13) }\end{array}$ & $42 \%$ & $45 \%$ & $5 \%$ & $2 \%$ & $5 \%$ & $100 \%$ \\
\hline
\end{tabular}

\subsubsection{OCRT Domestic Tourist-Kilometres (T-km)}

This thesis uses Auckland as being the central city for the Upper NI, with Wellington (Lower NI), Nelson /Marlborough (Upper SI), Otago (central SI) and Invercargill (Lower SI) to calculate the distance between domestic visitor home places and the OCRT (Table 5.6). Moreover, to measure the distance between the OCRT and the home places of visitors from the Upper SI, the distances between Nelson-OCRT and Marlborough-OCRT are calculated and averaged (Table 5.6). Since the OCRT is in Otago these distances to Middlemarch (the OCRT starting place) are considered separately.

\begin{tabular}{|c|c|c|c|c|c|c|c|c|}
\hline \multicolumn{8}{|c|}{ Table 5.6: OCRT- car based domestic T-km } & \multirow[b]{2}{*}{$\begin{array}{l}\text { Domes- } \\
\text { tic ferry } \\
\text { T-km }\end{array}$} \\
\hline $\begin{array}{l}\text { Re- } \\
\text { gion }\end{array}$ & Central city & Distan & to Middle & rch (km-re & & $\begin{array}{l}\text { Num- } \\
\text { ber of } \\
\text { Visi- } \\
\text { tors }\end{array}$ & $\begin{array}{l}\text { Domestic } \\
\text { road T- } \\
\mathrm{km}\end{array}$ & \\
\hline \multirow[t]{2}{*}{$\begin{array}{l}\text { Upper } \\
\text { NI }\end{array}$} & \multirow[t]{2}{*}{ Auckland } & $\begin{array}{l}\text { Auckland } \\
\text { to Wel- } \\
\text { lington }\end{array}$ & $\begin{array}{l}\text { Welling- } \\
\text { ton to } \\
\text { Picton }\end{array}$ & $\begin{array}{l}\text { Picton to } \\
\text { Middle- } \\
\text { march }\end{array}$ & Total & & & \\
\hline & & 1,318 & 204 & 1,480 & 3,002 & 3,295 & $9,219,410$ & 672,180 \\
\hline $\begin{array}{l}\text { Lower } \\
\text { NI }\end{array}$ & Wellington & - & 204 & 1,480 & 1,684 & 1,370 & $2,027,600$ & 279,480 \\
\hline $\begin{array}{l}\text { Upper } \\
\text { SI }\end{array}$ & $\begin{array}{l}\text { Nelson/ } \\
\text { Marlbor- } \\
\text { ough }\end{array}$ & & 1,686 & & 1,686 & 1,949 & $3,286,014$ & \\
\hline $\begin{array}{l}\text { Cen- } \\
\text { tral SI }\end{array}$ & Otago & & 125 & & 125 & 2,092 & 261,500 & \\
\hline $\begin{array}{l}\text { Lower } \\
\text { SI }\end{array}$ & $\begin{array}{l}\text { Invercar- } \\
\text { gill, South- } \\
\text { land }\end{array}$ & & 488 & & 488 & 492 & 240,096 & \\
\hline Total & - & & - & & - & 9,195 & $\begin{array}{c}15,034,62 \\
0\end{array}$ & 951,660 \\
\hline \multicolumn{9}{|c|}{$\begin{array}{l}\text { - Distance between Upper SI and Middlemarch is calculated as the average of distances between Nelson and } \\
\text { Middlemarch and Marlborough and Middlemarch. } \\
\text { - Average distance between Central Otago cities and OCRT. }\end{array}$} \\
\hline
\end{tabular}

OCRT visitors who come by car/van from Upper NI (Auckland) and Lower NI (Wellington) (grey areas in Table 5.6) need to use the ferry to cross to the South Island. Distances between these places and Middlemarch are separated into three parts; home to Wellington (except Wellington); Wellington to Picton by ferry; and Picton to Middlemarch (Table 5.6). Car visitors are here assumed to be $87 \%$ of total $(8,000$ out of 9,195 domestic visitors. The first scenario assumes that all OCRT visitors (9,195 total) come by car (Table 5.6).However, according to Matthews only $87 \%$ of recreational travel is 
by car and in New Zealand 1\% of people going on holiday are pedestrians (Matthews, 2009:13). In this study pedestrians have been ignored as the OCRT is far from the places of origin of visitors. Using Matthews' break down of recreational travel in Table 5.5 as a basis, the second scenario assumes the remaining $13 \%$ of visitors are split so that $10 \%$ reach the OCRT trail by bus and the remaining 3\% use domestic air travel (Table 5.7. a). All bus travel is assumed to be in the SI and the distances are calculated based on the split shown for car travel in Table 5.6. Similarly, air travel is split proportionally Auckland to Dunedin and Wellington to Dunedin.

\begin{tabular}{|c|c|c|c|c|}
\hline $\begin{array}{l}\text { Type of } \\
\text { transport }\end{array}$ & $\begin{array}{c}\text { Car/van/driver/passenger } \\
\text { road travel }\end{array}$ & $\begin{array}{c}\text { Car/van/driver/passenger } \\
\text { ferry travel }\end{array}$ & Bus & Air domestic \\
\hline$\%$ of total & 87 & & 10 & 3 \\
\hline Tourist-km & $13,080,119$ & 827,944 & 768,133 & 400,178 \\
\hline $\begin{array}{l}\text { Number of } \\
\text { visitors }\end{array}$ & 8000 & & $\begin{array}{c}919 \text { (395 Up- } \\
\text { per SI; } 423 \\
\text { Otago; } 101 \\
\text { Invercargill) }\end{array}$ & $\begin{array}{l}276 \text { (177 from } \\
\text { Auckland, } \\
2120 \mathrm{~km} \text {; } 99 \\
\text { from Welling- } \\
\text { ton, } 1262 \mathrm{~km} \text { ) }\end{array}$ \\
\hline
\end{tabular}

These two scenarios will be used to work out the EF of domestic travel, to see the effect of the assumptions made.

\subsubsection{The Ecological Footprint (EF) of OCRT-Transportation}

Table 5.8 shows that international and domestic transportation energy use of OCRT international visitors (2011) is equivalent to $61,743,029 \mathrm{MJ}$ or $61,743 \mathrm{GJ}$. The land to energy conversion capacity of New Zealand is assumed to be equivalent to $100 \mathrm{GJ} / \mathrm{gha}$ (Field, 2011:11). As demonstrated in Table 5.8 the EF of OCRT transportation for international visitors is equivalent to $61,743 \mathrm{GJ} / 100 \mathrm{GJ} / \mathrm{gha}=617$ gha.

\subsubsection{Scenario 1 for Domestic Visitors}

Using the same method, the EF of domestic visitors assuming that all travel is by car is 552 gha (Table 5.7.b)

Table 5.7.b: OCRT- domestic transport scenario 1

\begin{tabular}{|l|c|c|c|}
\hline Transport mode & Tourist-km & MJ/passenger-km & $\begin{array}{c}\text { Total energy use per yr } \\
\text { by OCRT visitors (MJ) }\end{array}$ \\
\hline Car/van driver and passenger & $15,034,620$ & 3.1 (average) & $46,607,322$ \\
\hline Car-ferry & 951,660 & 9.06 & $8,622,040$ \\
\hline Total & & & $55,229,362$ \\
\hline EF for domestic travel & & & 552 gha \\
\hline
\end{tabular}




\subsubsection{Scenario 2 for Domestic Visitors}

The EF of domestic visitors assuming that $87 \%$ come by car and the others use bus and air travel, is 504 gha (Table 5.7.c)

Table 5.7.c: OCRT- domestic transport scenario 2

\begin{tabular}{|l|c|c|c|}
\hline \multicolumn{1}{|c|}{ Transport mode } & Tourist-km & MJ/passenger-km & $\begin{array}{c}\text { Total energy use per yr } \\
\text { by OCRT visitors (MJ) }\end{array}$ \\
\hline Car/van driver and passenger & $13,080,119$ & 3.1 (average) & $40,548,369$ \\
\hline Car-ferry & 827,944 & 9.06 & $7,501,173$ \\
\hline Bus & 768,133 & 1.01 & 775,814 \\
\hline Air travel & 400,178 & 3.88 & $1,552,691$ \\
\hline Total & & & $50,378,047$ \\
\hline EF for domestic travel & & & 504 gha \\
\hline
\end{tabular}

Taking the larger result from the two domestic transport scenarios and adding it to the international air travel gives the following result, as shown in Table 5.8. The total transportation EF for international and domestic visitors is 1,169gha.

\subsubsection{OCRT- Transport EF}

Table 5.8: OCRT- transport EF

\begin{tabular}{|l|c|c|c|}
\hline Transport mode & Tourist-km & $\begin{array}{c}\text { Energy use } \\
\text { MJ/passenger-km }\end{array}$ & $\begin{array}{c}\text { Total energy use per yr } \\
\text { by OCRT visitors (MJ) }\end{array}$ \\
\hline Air international & $32,041,486$ & 1.25 (Boeing 747) & $40,051,858$ \\
\hline $\begin{array}{l}\text { Air domestic } \\
\text { (International visitors } \\
\text { from Auckland to Dun- } \\
\text { edin) }\end{array}$ & $5,590,508$ & 3.88 & $21,691,171$ \\
\hline Sub total & & & \\
\hline EF international travel & & & $61,743,029$ \\
\hline EF domestic travel & & & 617 gha \\
\hline Total transport EF & & & 552gha \\
\hline
\end{tabular}

What this demonstrates is $22 \%$ of OCRT visitors because they are international account for $53 \%$ of the transportation $\mathrm{EF}$, a not unexpected result.

\subsection{OCRT: Food}

The food EF of OCRT visitors is equal to the area which is needed to produce the food consumed by tourists during their use of the OCRT. The four factors involved in this assessment are: the number of OCRT visitors, average number of nights they stay during their visit, food eaten out per visitor, and the EF of the consumed food per visitor. 
As shown in Figure 5.6, 11,788 visitors used the OCRT in 2011 and as Figure 5.7 shows, each tourist stayed an average of 3.6 nights. Thus, for this calculation the total visitor nights for tourists coming to the OCRT are 42,437 visitor nights. Because of the lack of local information, the food consumption data presented by Collins et al (2005) are used to calculate the EF of food consumption for OCRT visitors.

This thesis uses two scenarios to calculate the EF of food consumed by OCRT visitors in 2011. In the first method, the EF is calculated based on the assumption $100 \%$ of food consumed is conventional food eaten out. In the second method, the total amount of food eaten by OCRT visitors is separated into the two areas of local (home prepared) food and conventional food. Comparison between the results of the two methods will indicate the influence exerted by the use of home cooked food (as a cultural product) on the EF and the Economic Footprint (ECF) of the OCRT.

\subsubsection{The EF of OCRT Consumed Food-First Scenario}

In the first scenario, the total amount of food eaten by OCRT visitors has been assumed to have a footprint equivalent to that of food consumed outside the home by Cardiff residents. This totals $67.04 \mathrm{~kg}$ per resident/yr (Collinset al, 2005:25) with a footprint of $0.429 \mathrm{gha} /$ resident (Collins et al, 2005:32). This means $1 \mathrm{~kg}$ of food eaten out has an EF of $0.429 / 67.04 \mathrm{gha} / \mathrm{kg}=0.006 \mathrm{gha} / \mathrm{kg}$. The average amount of food eaten by a Cardiff resident each day is $1.85 \mathrm{~kg}$, and this value has been used for visitors to OCRT. The results are shown in Table 5.9. The EF of tourist food is equivalent to $1.85 \times 0.006=0.01$ gha/visitor night (Table 5.9). The total ecological footprint of food consumed by OCRT visitors is equivalent to $42,437 \times 0.01=424.4$ gha and the EF of food consumed by ORCT visitors/visitor is equivalent to $424.4 / 11,788=0.036$ gha (Table 5.9)

\begin{tabular}{|l|c|c|}
\hline \multicolumn{2}{|c|}{ Table 5.9: EF of food consumed by OCRT visitors (2011) } \\
\hline \multicolumn{1}{|c|}{ Item } & Data & Reference \\
\hline $\begin{array}{l}\text { OCRT visitor numbers (2011) } \\
\text { Average visitor nights/ visitor }\end{array}$ & 11,788 & \\
\hline Total visitor nights & 3.6 & Collins et al, 2005:32 \\
\hline EF of food eaten out gha/cap & 0.429 & \\
\hline $\begin{array}{l}\text { EF of tourist food gha/visitor } \\
\text { night }\end{array}$ & 0.01 \\
\hline $\begin{array}{l}\text { Total EF of food consumed by } \\
\text { OCRT visitors (gha) }\end{array}$ & 424.4 (gha) \\
\hline $\begin{array}{l}\text { EF of food consumed by OCRT } \\
\text { visitors/visitor(gha) }\end{array}$ & 0.036 (gha/visitor) \\
\hline
\end{tabular}




\subsubsection{The EF of OCRT Consumed Food-Second Scenario}

This thesis explores the local products (such as food, beverages, and wine) offered to OCRT visitors by the 783 separate accommodation services available with their 5,543 bed spaces. These accommodation services are located along the OCRT from Middlemarch to Clyde (Appendix5).This information has been collected through the use of the official website of the OCRT and by referring to the 201 websites belonging to each accommodation service, which are accessed through the OCRT website (Appendix5). The information related to OCRT products and activities arising from Appendix5 is summarised and set out in Appendix7. This appendix is used as the base to develop the related figures and Table s about OCRT locally produced food used in the following part of this section.

Figure 5.14 and Table 5.10 demonstrate that in 2011, 234 separate OCRT accommodation services offered local produce in four identifiable categories. Of these, $56.40 \%$ offered home baking and local food and beverages to their visitors, followed by $39.3 \%$ offering locally produced alcoholic drinks, 3.4\% local grazing (for horses) and $0.9 \%$ local art works and crafts.

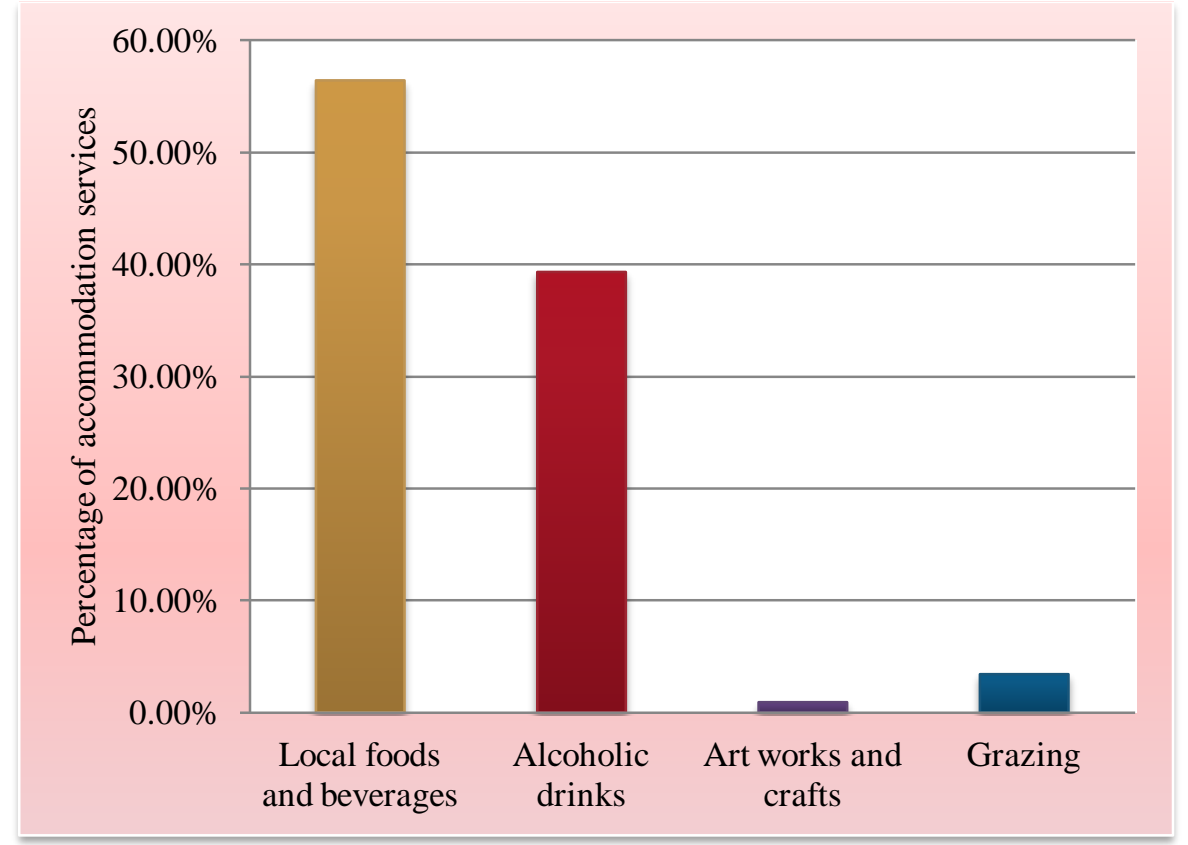

Figure 5.14: OCRT- Frequency of local produce offered by accommodation services to visitors (2011).

Table 5.10 shows that 132 OCRT accommodation services (16.9\% of the total 783 OCRT accommodation services) make available local and home baked foods. As a result an equivalent 937 (16.9\% of 5,543) bed spaces serve local food to their users. As 
discussed above, in 2011 total visitor nights were equivalent to 11,788 (OCRT visitors $\times$ 3.6 (average visitor nights/visitor - see Figure 5.7) $=42,437$. As the number of total available bed spaces of the OCRT is 5,543 bed spaces, consequently, the average number of visitor nights per bed space is 42,437 (total visitor nights) / 5,543 (total bed spaces) $=7.7$ (visitor nights / bed space) .

It should be remembered that 3.6 (average visitor nights/visitor) is a number that involves two factors, the number of OCRT visitors and the number of nights that they stay in OCRT. But 7.7 (visitor nights/bed space) is a number that indicates the time that each of the bed spaces is used based on total visitor nights. If the total numbers of bed spaces were equal with the total number of visitors, both average visitor nights/visitor and average visitor nights/ bed space would be equal, but that does not happen in OCRT. As a result, the total visitor nights that visitors use local foods are 937 (bed spaces available in establishments which offer local food) $\times 7.7$ (nights per bed space) $=7,215$.

\begin{tabular}{|c|c|c|c|c|}
\hline \multicolumn{5}{|c|}{ Table 5.10: OCRT- local produce (summary) } \\
\hline Produce & $\mathrm{F}$ & $\begin{array}{c}\% \text { of } 783 \text { accommodations ser- } \\
\text { vices }\end{array}$ & Bed spaces & Visitor nights $^{1}$ \\
\hline Local food and beverage & 132 & 16.9 & 937 & 7,215 \\
\hline Alcoholic drinks & 92 & 11.7 & 649 & 4,997 \\
\hline Art works and crafts & 2 & 0.3 & 17 & 131 \\
\hline $\begin{array}{l}\text { Grazing (for those doing } \\
\text { OCRT on horseback) }\end{array}$ & 8 & 1 & 55 & 424 \\
\hline Total & 234 & 29.9 & 1,658 & 12,767 \\
\hline
\end{tabular}

As shown in Appendix 5, all OCRT accommodation services that produce local and home cooked foods use productive gardens and local farm produce as their main sources when preparing such food. Assuming that OCRT local foods are the equivalent of organic food, it is possible to compare the EF of local and conventional food consumed by OCRT visitors.

The difference between The EFs of conventional and organic foods arises from the energy used to produce and process these types of food. Collins and Fairchild (2009:11) argue that the ecological footprint for food and drink consists of two main components: 'real land' requirements (i.e. crop, pasture, sea) and 'energy land'. “The former relates to the area of land required to rear and grow food produce, the latter relates to the embodied energy required for food production and its processing.” (Collins and Fairchild, 2009:11). Due to a lack of information, Collins and Fairchild (2009) assume the real 
land for conventional and organic foods is equal. They (2009:15) argue that since the production of conventional food is more energy intensive than the production of organic food it consequently has a bigger EF than organic food.

Table 5.11 indicates that in comparison with conventional food, organic food has a 40 \% (0.52gha) lower EF.

Table 5.11: The effect on footprint of consuming food from $100 \%$ organic sources.

Ecological footprint of food (gha/person)

Ecological footprint of transporting food (gha/person)

Total ecological footprint

- Reference: Collins et al, 2005: 36
$100 \%$ conventional

1.315

0.022

1.337
$100 \%$ organic

0.799

0.022

0.817

Consequently, this study uses $60 \%$ of the EF of conventional food (0.429gha/cap) as the EF of local and home baked food (0.254gha/cap) to calculate the whole EF of OCRT food in 2011 (Table 5.12). The EF of $1 \mathrm{~kg}$ home cooked food is equivalent to 0.254/ 67.04 (eaten out food/resident/year in Cardiff) = 0.0037 gha/kg (Table 5.12). Table 5.10 shows that 132 OCRT accommodation services with the capacity of 937 bed spaces offered local food to their visitors. The total visitor nights those OCRT visitors consumed local food is 7,215 (Table 5.10). The EF of OCRT local food is equivalent to 0.0068 gha/ visitor night $(0.0037 \mathrm{gha} / \mathrm{kg} \times 1.85 \mathrm{~kg})$ and the total EF of OCRT local food per year is equivalent to $(7,215$ visitor nights $\times 0.0068$ gha/ visitor night) or 49 gha (Table 5.12). The number of visitor nights when OCRT visitors consumed conventional food is 35,222 visitor nights (42,437 total OCRT visitor nights $-7,215$ visitor nights when local food is consumed). The EF of OCRT conventional food is 352.2 gha (Table 5.12).The total EF of OCRT local and conventional food as shown in Table 5.12 is 401.2 gha.

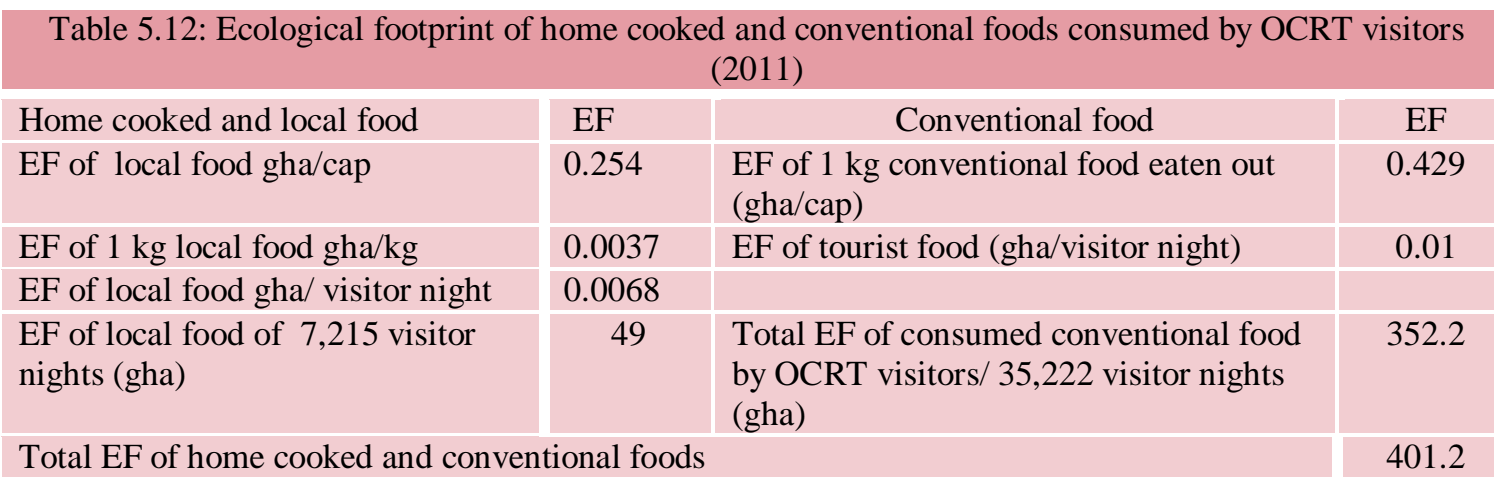




\subsubsection{Environmental Effects of Using Local Food}

Comparing the results of the two scenarios shows that producing $17 \%$ of OCRT food locally reduces the total EF of food by 23.2 gha (5.5\% of 424.4 gha) (Table 5.13).

\begin{tabular}{|c|c|c|}
\hline & $\begin{array}{l}100 \% \text { conventional eaten } \\
\text { out food }\end{array}$ & $\begin{array}{l}83 \% \text { conventional eaten out food and } \\
17 \% \text { home cooked (organic) food }\end{array}$ \\
\hline OCRT EF of food & $424.4^{1}$ & $401.2^{2}$ \\
\hline $\begin{array}{l}\text { Reduction in EF of food con- } \\
\text { sumed by OCRT visitors (gha) }\end{array}$ & & $23.2^{3}$ \\
\hline $\begin{array}{l}\text { 1. } 424.4 \mathrm{gha} / 11,788 \text { OCRT visitor } \\
\text { 2. } 401.2 \mathrm{gha} / 11,788 \text { OCRT visitor } \\
\text { 3.23.2 gha / } 11,788 \text { OCRT visitors }\end{array}$ & $\begin{array}{l}0.036 \text { gha/visitor } \\
.034 \text { gha/visitor } \\
.002 \text { gha/visitor }\end{array}$ & \\
\hline
\end{tabular}

\section{6. OCRT Built up land: Accommodation Services}

\subsubsection{OCRT: Types of Accommodation Service}

This research categorises the total number of 783 OCRT accommodation services into seven categories; self-contained (SC), bed and breakfast (B\&B), backpacker (Bp), motel, homestead, hotel and camping. Again categories have been derived through using the official web site of OCRT and 210 related accommodation services websites (Appendix 5 and 6). Table 5.14 summarises the parts of Appendix 5 that address available bed spaces by types of accommodation service.

As shown in Table 5.14, in 2011, of the total 783 separate accommodation services the majority or $71.1 \%$ are camping sites followed by $11.8 \%$ SC and $8.5 \%$ B\&B. There are small numbers of other types of OCRT accommodation services including Bp, motel, homestead, and hotel in the range of 1.5- 4.2\% of total (Table 5.14).As shown in Table 5.14 the majority of available bed spaces are in camping sites (59.8\%) followed by $12 \%$ motel, 9.8\% B\&B and 9.2\% SC. Other types of OCRT accommodation services including, Bp, homestead and hotel hold between $0.5-6.2 \%$ of available bed spaces (Table 5.14 and Figure 5.15). 
Table 5.14. OCRT types of accommodation services (2011)

\begin{tabular}{|l|c|c|c|c|}
\hline \multicolumn{1}{|c|}{ Type } & $\begin{array}{c}\text { Number of } \\
\text { type }\end{array}$ & $\%$ & Available bed spaces & $\%$ \\
\hline $\mathrm{SC}^{1,4}$ & 92 & 11.8 & 510 & 9.2 \\
\hline${\mathrm{B} \& \mathrm{~B}^{2}}^{2}$ & 66 & 8.5 & 541 & 9.8 \\
\hline $\mathrm{Bp}^{3}$ & 12 & 1.5 & 136 & 2.5 \\
\hline $\mathrm{Motel}^{5}$ & 33 & 4.2 & 665 & 12 \\
\hline Homestead $^{6}$ & 4 & 0.5 & 28 & 0.5 \\
\hline Hotel $^{6}$ & 19 & 2.4 & 346 & 6.2 \\
\hline Camping $^{6}$ & 557 & 71.1 & 3,317 & 59.8 \\
\hline Total & 783 & 100 & 5,543 & 100 \\
\hline $\begin{array}{l}\text { 1. SC: Self contained } \\
\text { 2. B\&B: Bed and breakfast }\end{array}$ & & & \\
3. Bp: Backpacker & & & \\
4. Capacity of 1 unknown SC accommodation service is calculated as average bed spaces of all SC = 6 \\
$\begin{array}{l}\text { 5. Capacity of } 1 \text { unknown motel is calculated as average bed spaces of all motels = 20 } \\
\text { 6. Capacity of } 1 \text { unknown hotel is calculated as average bed spaces of all hotels =18 }\end{array}$
\end{tabular}

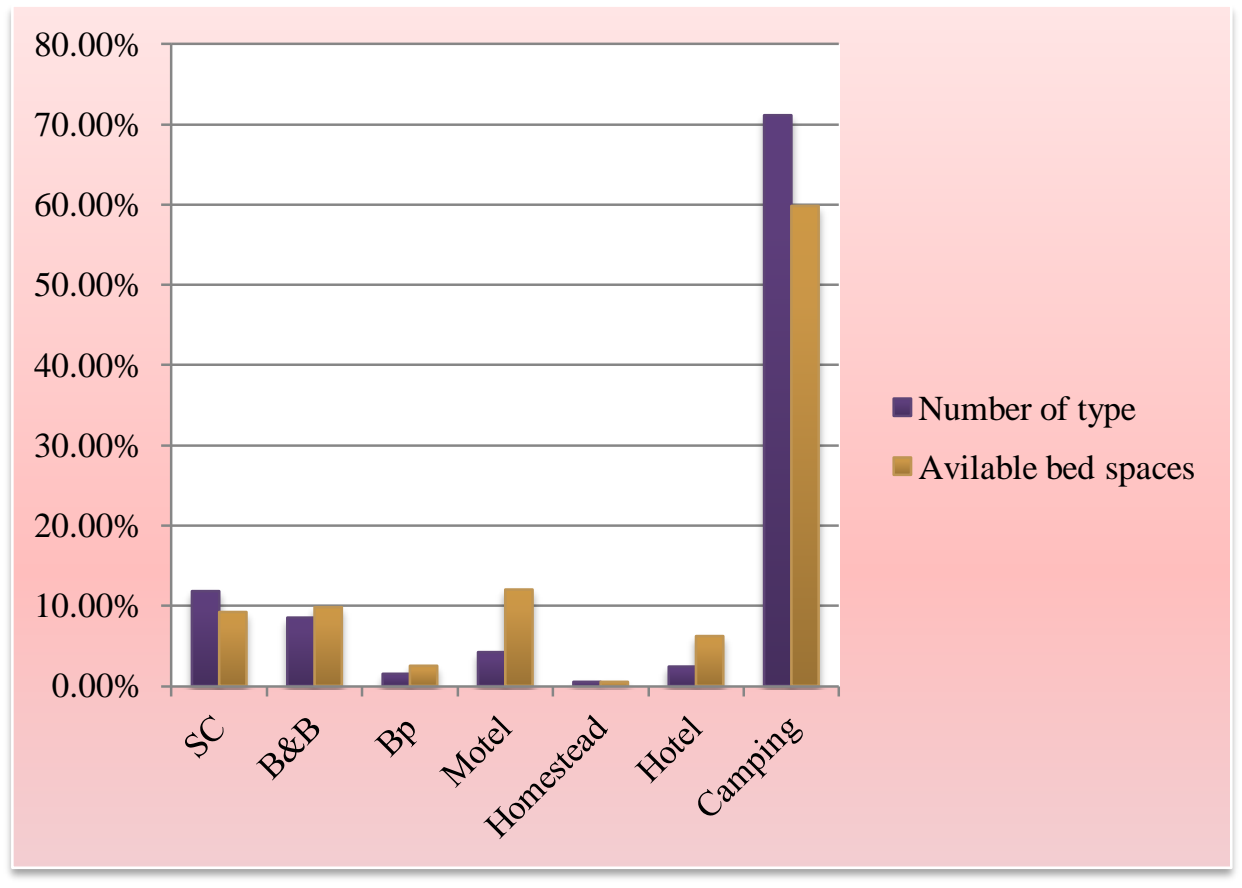

Figure 5.15: OCRT- Percentage of type and available bed spaces by types of accommodation services.

\subsubsection{Quality of OCRT Accommodation Services}

According to the information shown in Appendix5 and summarised in Table 5.15 this study qualitatively classifies OCRT accommodation services into the two types of new (NB) and refurbished (RB) buildings. 


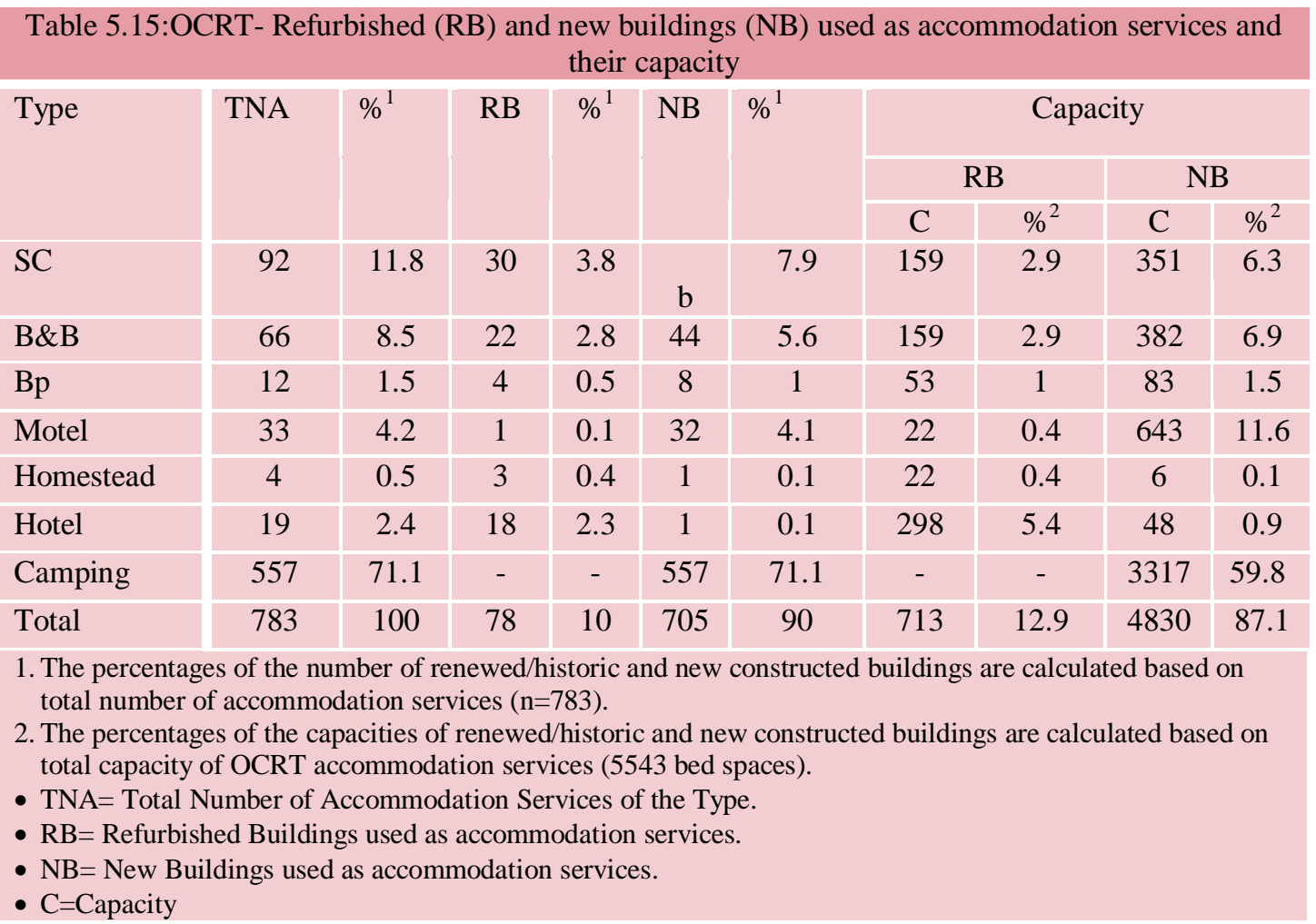

In OCRT, RB accommodation services cover a range of historical and restored buildings with different original functions, but currently all of them are used for visitor accommodation. Table 5.15 shows the number and percentage of NB and RB buildings used as accommodation services in the OCRT. Moreover, this table demonstrates the number and percentage of available bed spaces of NB and RB OCRT accommodation services by type. As shown in Table 5.15 and Figure 5.16, of 781 OCRT accommodation services, only $10 \%$ are $\mathrm{RB}$ and $90 \%$ are NB.

Table 5.15 and Figure 5.17 show that of all OCRT available bed spaces $12.9 \%$ are RB and $87.1 \%$ are NB. 


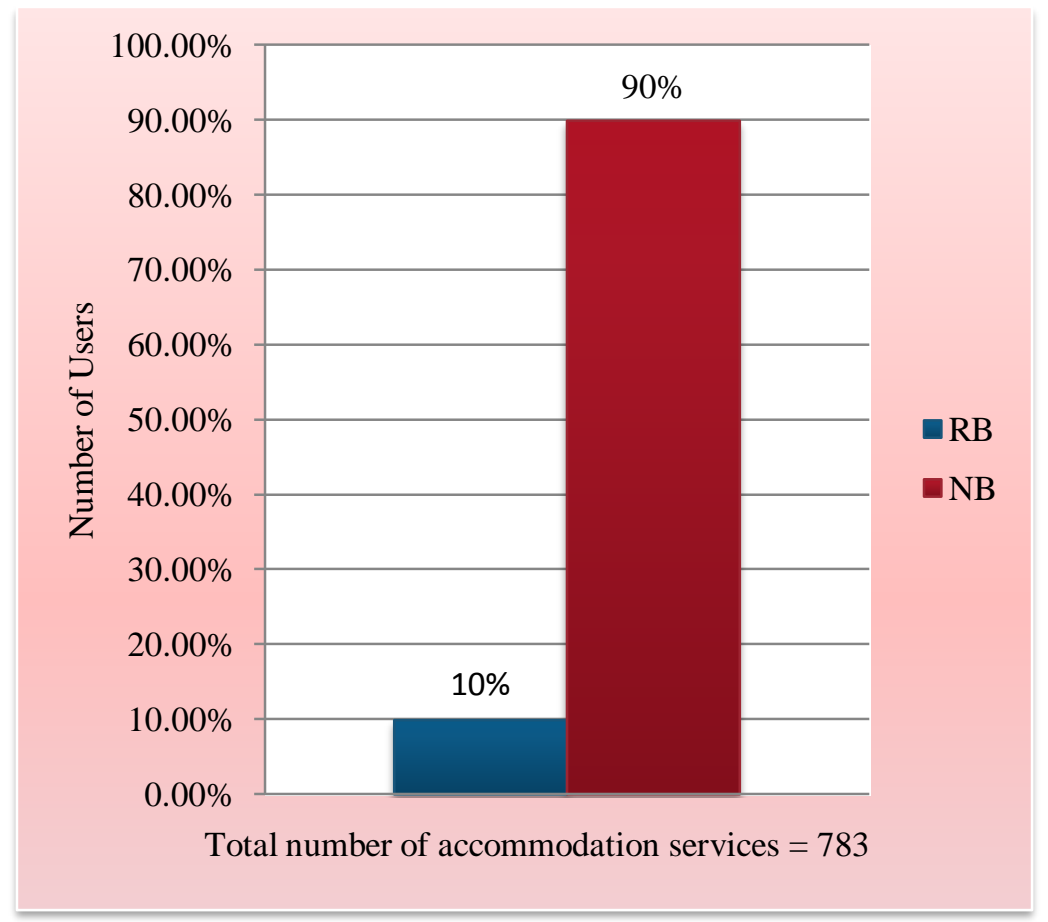

Figure 5.16: OCRT- RB and NB buildings used as accommodation services

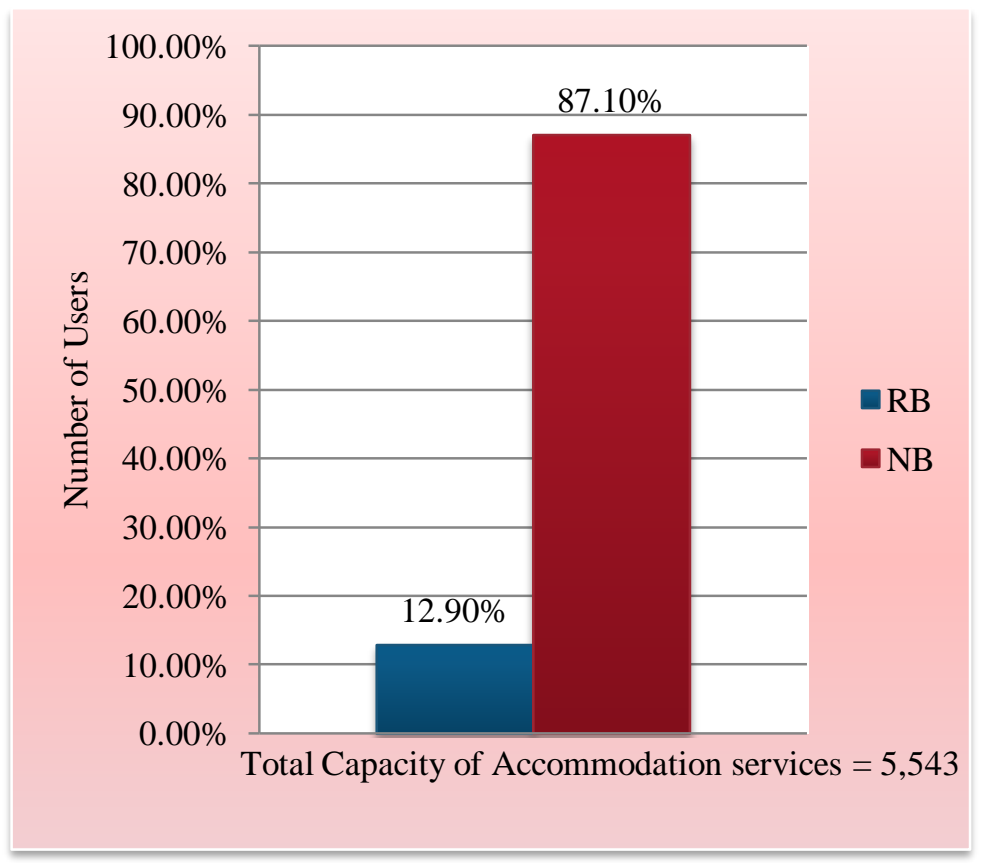

Figure 5.17: OCRT- RB and NB accommodation services - available bed spaces (2011)

Figure 5.18 and Table 5.15 indicate that of the 10\% (78) RB accommodation services, the majority or $3.8 \%$ of total (30) are SC, followed by $2.8 \%$ (22) B\&B, and $2.3 \%$ (18) hotel. Other RB types of accommodation including Bp, motel, homestead and camping only make up $0-0.5 \%$ of total. Moreover as shown in Figure 5.18 and Table 5.15, of $90 \%$ of total (705) NB accommodation services, the majority or $71.1 \%$ (557) are camp- 
ing sites (powered and non-powered) followed by 7.9\% (62) SC, 5.6\% (44) B\&B and 4.1\% (32) motel. Other NB types of accommodation services including B\&B, Bp and hotel only make up $0.1-1 \%$ of total (Figure 5.18 and Table 5.15).

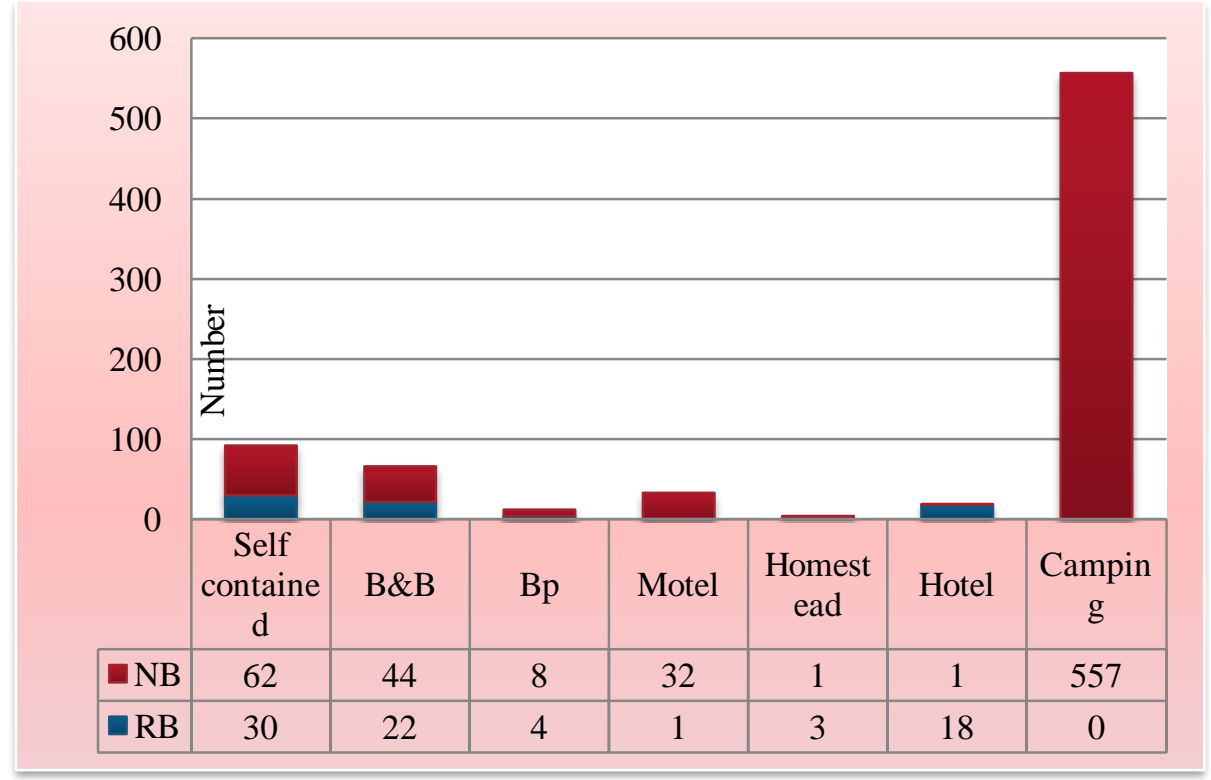

Figure 5 18: OCRT- number of RB and NB accommodation services

Table 5.15 and Figure 5.19 indicate that of the $12.9 \%$ (713 out of 5,543) RB available bed spaces, the majority $5.4 \%$ are found in the hotel category, while $5.8 \%$ of total are equally divided between SC and B\&B (2.9\% each type). Other RB bed spaces including Bp, homestead, motel and camping sites only make up $0-1 \%$ of total (Table 5.15 and Figure 5.19).

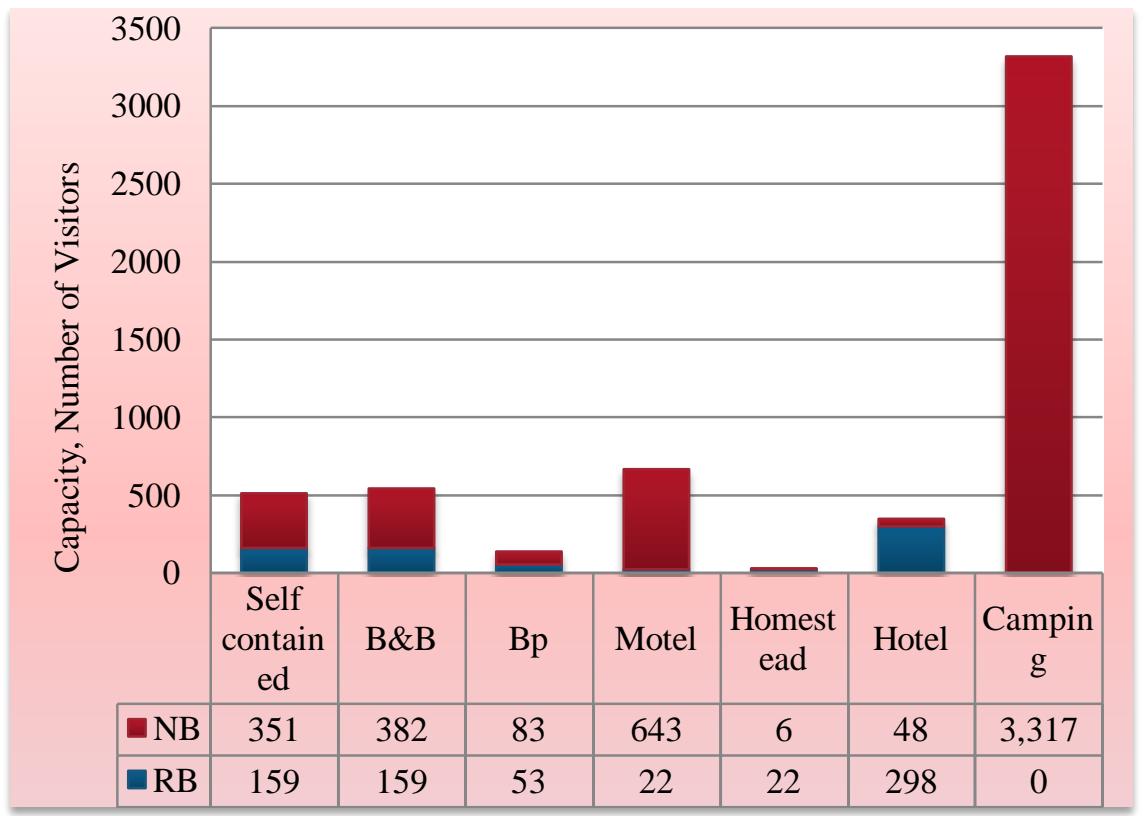

Figure 5.19: OCRT- capacity of RB and NB accommodation services - total capacity $=5,543$ bed spaces $(2011)$ 
Furthermore, Table 5.15 and Figure 5.19 show that the majority (68.7\%) 3,317 of 4,830 NB bed spaces (59.8\% of 5,543 total bed spaces) are camping sites (powered and nonpowered) followed by 13.3\% (643) motel, 7.9\% (382) B\&B, and 7.3\% (351) SC. Other types of NB bed spaces including Bp, homestead, and hotel are in a range $0.9-1.5 \%$ of total NB bed spaces.

\subsubsection{OCRT Accommodation Services: Materials Used}

Since the materials used in the construction of architecture are considered one of the main factors that makes a link between the building (and its users) and the surrounding environment, this research explores the types of materials used for constructing OCRT accommodation services. This exploration is conducted through using the related information and documents published in 210 official websites of OCRT accommodation services (Appendices 5 and 8). The main materials used for constructing each type of accommodation services (except camping sites) are set out in Appendix 5 and the summarised information shown in Appendix 8. As shown in Appendix 8, this information has been categorised into materials used by type, number and capacity of OCRT accommodation services in 2011.

\begin{tabular}{|c|c|c|c|c|c|c|c|c|c|c|c|c|c|c|}
\hline \multirow[t]{3}{*}{ material(s) } & \multirow{3}{*}{$\begin{array}{l}\text { Total } \\
\text { bed } \\
\text { spaces }\end{array}$} & \multirow[t]{3}{*}{$\%$} & \multicolumn{12}{|c|}{ Types of accommodation services } \\
\hline & & & \multicolumn{2}{|c|}{ SC } & \multicolumn{2}{|c|}{$\mathrm{B} \& \mathrm{~B}$} & \multicolumn{2}{|c|}{$\mathrm{Bp}$} & \multicolumn{2}{|c|}{ Motel } & \multicolumn{2}{|c|}{ Homestead } & \multicolumn{2}{|c|}{ Hotel } \\
\hline & & & No & $\mathrm{C}$ & No & $\mathrm{C}$ & No & $\mathrm{C}$ & No & $\mathrm{C}$ & No & $\mathrm{C}$ & No & $\mathrm{C}$ \\
\hline Timber & 1094 & 49.1 & 72 & 388 & 29 & 225 & 6 & 54 & 26 & 357 & 1 & 6 & 4 & 64 \\
\hline $\begin{array}{l}\text { Timber } \\
\text { and ma- } \\
\text { sonry }\end{array}$ & 628 & 28.3 & 8 & 55 & 14 & 169 & 3 & 54 & 3 & 163 & - & - & 7 & 187 \\
\hline $\begin{array}{l}\text { Mud brick } \\
\text { (mixed } \\
\text { with other } \\
\text { materials) }\end{array}$ & 79 & 3.5 & 5 & 33 & 4 & 26 & 1 & 6 & - & - & 1 & 6 & 1 & 8 \\
\hline $\begin{array}{l}\text { Brick }^{1} \\
\text { (mixed } \\
\text { with other } \\
\text { materials) }\end{array}$ & 126 & 5.7 & 1 & 6 & 7 & 50 & 1 & 18 & 1 & 44 & - & - & 1 & 8 \\
\hline Stone $^{2}$ & 178 & 8 & 4 & 20 & 8 & 37 & 1 & 4 & 1 & 22 & 2 & 16 & 6 & 79 \\
\hline Concrete & 113 & 5 & - & - & 4 & 34 & - & - & 2 & 79 & - & - & - & - \\
\hline Container & 4 & 0.2 & 1 & 4 & - & - & - & - & - & - & - & - & - & - \\
\hline Caravan & 4 & 0.2 & 1 & 4 & - & - & - & - & - & - & - & - & - & - \\
\hline Total & 2,226 & 100 & 92 & 510 & 66 & 541 & 12 & 136 & 33 & 665 & 4 & 28 & 19 & 346 \\
\hline $\begin{array}{ll}\text { 1. } & \text { Brick } \\
\text { 2. } & \text { Stone } \\
\text { - } & \mathrm{No}=\mathrm{N} \\
\text { - } & \mathrm{C}=\mathrm{Ca}\end{array}$ & $\begin{array}{l}\text { cludes: } \mathrm{r} \\
\text { cludes: } \mathrm{s} \\
\text { mber of } \\
\text { acity (nu }\end{array}$ & $\begin{array}{l}\text { d brick } \\
\text { hist st } \\
\text { ccomm } \\
\text { aber of }\end{array}$ & $\begin{array}{l}\text { clay } \\
\text { he, st } \\
\text { datic }\end{array}$ & $\begin{array}{l}\text { ick ar } \\
\text { e and } \\
\text { servi } \\
\text { ces). }\end{array}$ & $\begin{array}{l}\text { brick } \\
\text { ock. }\end{array}$ & & & & & & & & & \\
\hline
\end{tabular}


This thesis classifies the types of materials used into the eight types of timber, timber and masonry, mud brick (mixed with other materials), brick (mixed with other materials), stone, concrete, container, and caravan (Table 5.16).

As shown in Table 5.16 and Figure 5.20, of the total 2,226 building based OCRT accommodation bed spaces the majority $49.1 \%(1,094)$ of bed spaces are in timber buildings followed by 28.3\% (628) timber and masonry, 8\% (178) stone, 5.7\% (126) brick (mixed with other materials) and 5\% (113) concrete. Other materials used including mud brick (mixed with other materials), container and caravan make up $0.2 \%-3.5 \%$ of total (Table 5.16 and Figure 5.20).

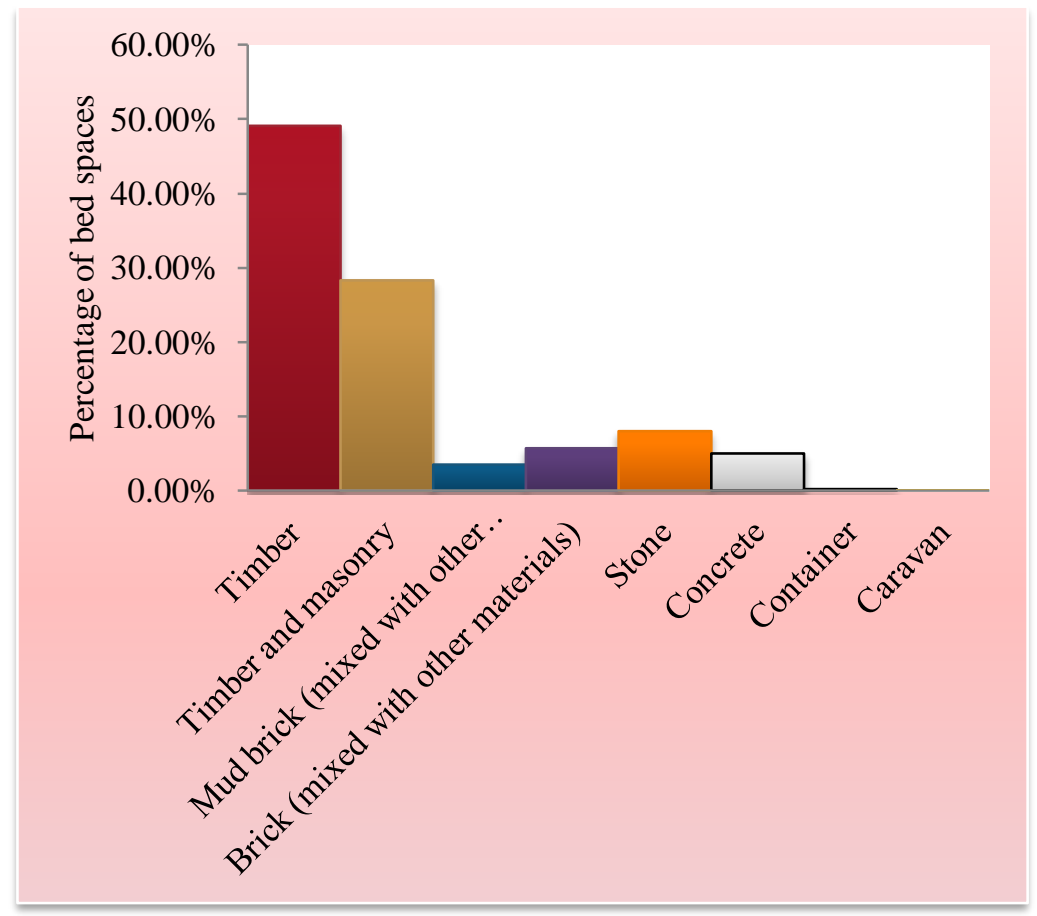

Figure 5.20: OCRT- accommodation - bed spaces by type of materials used (2011)

Table 5.16 and Figure 5.21 indicate that the SC accommodation services comprise 388 (76\%) bed spaces using timber as the main construction material followed by 55 (10.8\%) timber and masonry and 33 (6.5\%) mud brick mixed with other materials. Bed spaces that are in buildings of other materials including stone, brick, concrete and caravan/container fall in the range of 4 (1.2\%) - 20 (3.9\%) out of total (Table 5.16 and Figure 5.21)

Table 5.16 and Figure 5.21 indicate that of 541 B\&B bed spaces the majority or 225 use timber as main construction material, followed by 169 timber and masonry and 50 brick 
(mixed with other materials). The numbers of $B \& B$ bed spaces of other materials including mud brick (mixed with other materials) and stone are between $26-37$ of the total bed spaces. As shown in Table 5.16 and Figure 5.21, 108 (79.4\%) of 136 Bp bed spaces are constructed from timber and timber (mixed with other materials). The other $28 \mathrm{Bp}$ bed spaces used materials including mud brick (mixed with other material, brick (mixed with other materials), stone and concrete, forming between 4 (3\%) - 18 (13.20\%) of total in this category (Table 5.16 and Figure 5.21).

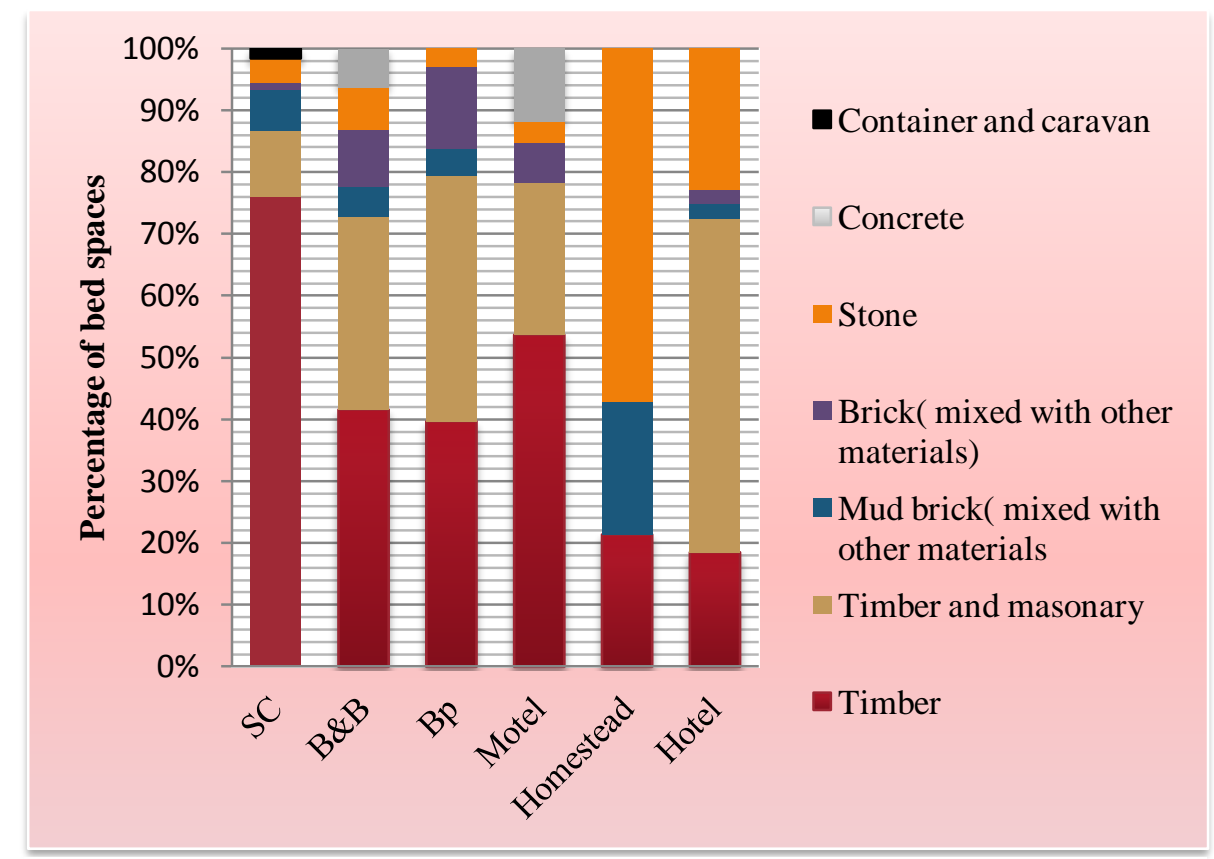

Figure 5.21: OCRT- accommodation services- materials used by type of accommodation (2011)

The majority of 357 (53.7\%) motel bed spaces were built in timber followed by 163 (24.5\%) timber and masonry, 79 (11.9\% ) concrete, 44 (6.6\%) brick (mixed with other materials), and 22 (3.3\%) stone (Table 5.16 and Figure 5.21). In contrast, of 28 homestead bed spaces, the majority of 16 (57.2\%) were in stone followed by equal numbers of timber, and mud brick (mixed with other materials), at 6 (21.4\%) each (Table 5.16 and Figure 5.21).

OCRT hotels comprise 346 bed spaces of which 187 (54\%) are built of timber and masonry, 79 (22.8\%) stone, and 64 (18.5\%) timber (Table 5.16 and Figure 5.21). Other hotel bed spaces are equal in number between mud brick and brick mixed with other materials at 8 (2.3\%) each (Table 5.16 and Figure 5.21). 


\subsubsection{OCRT Accommodation Services: Energy, Facilities and Spaces}

As shown in Appendix 5 this research explores the energy sources and facilities used in 783 OCRT accommodation services through using information published on these accommodation websites. The sources of energy used are categorised into conventional and solar. The first category (heating and hot water) comprises electricity (EL), logs and gas and the second category contains solar systems, almost always used for water heating (Appendix 5). The facilities provided in OCRT accommodation services include a wide range of equipment (e.g. kitchen equipment, BBQ, TV, Internet, laundry, electric blanket, hair dryer, and log burner) as shown in Appendix 5. Likewise Appendix 5 contains data related to the available spaces (e.g. lounge, laundry, kitchen and kitchenette, veranda/balcony, outdoor sitting, bedroom, bathroom, and pool) in OCRT accommodation services.

This study classifies related information into the energy sources used, and facilities and spaces provided shown in Appendix 5 by types of OCRT accommodation services as shown in Appendices 9-16. In this research, all types of energy systems (EL, log, gas and solar) are explored in relation to types of accommodation services. However, for facilities and equipment, only the TV and Internet are chosen for analysis, as using these facilities can be considered as indicative of a modernised lifestyle (Appendices 916). Since using open air areas is proposed here as one of the environmental and cultural indicators for evaluating architecture as being sustainable because of the cultural link in New Zealand between indoor and outdoor areas and because these areas generally have low energy footprints in use, the veranda/balcony and outdoor seating are selected for further investigation in this study. The following section presents data classified by types of accommodation service as shown in Appendix16 and summarises Appendices 9-15.

\subsubsection{OCRT SC Accommodation Services: Energy Sources, Facilities and Open}

\section{Air Spaces (2011)}

Figure 5.22 and Appendix16 demonstrate that of 510 SC bed spaces 422 (82.7\%) use electricity for space or water heating. As shown in Figure 5.22 and Appendix16, 279 (58.2\%) of these 422 bed spaces are NB and 125 (24.5\%) are RB. In addition, 146 (28.6\%) SC bed spaces including 107 (21\%) RB and 39 (7.5\%) NB use logs, and 106 SC bed spaces comprising 54 (10.6\%) RB and 52 (5.92\%) NB use gas for heating. So 
wood is more likely to be used in older buildings, as many of these may have come with chimneys and fireplaces.

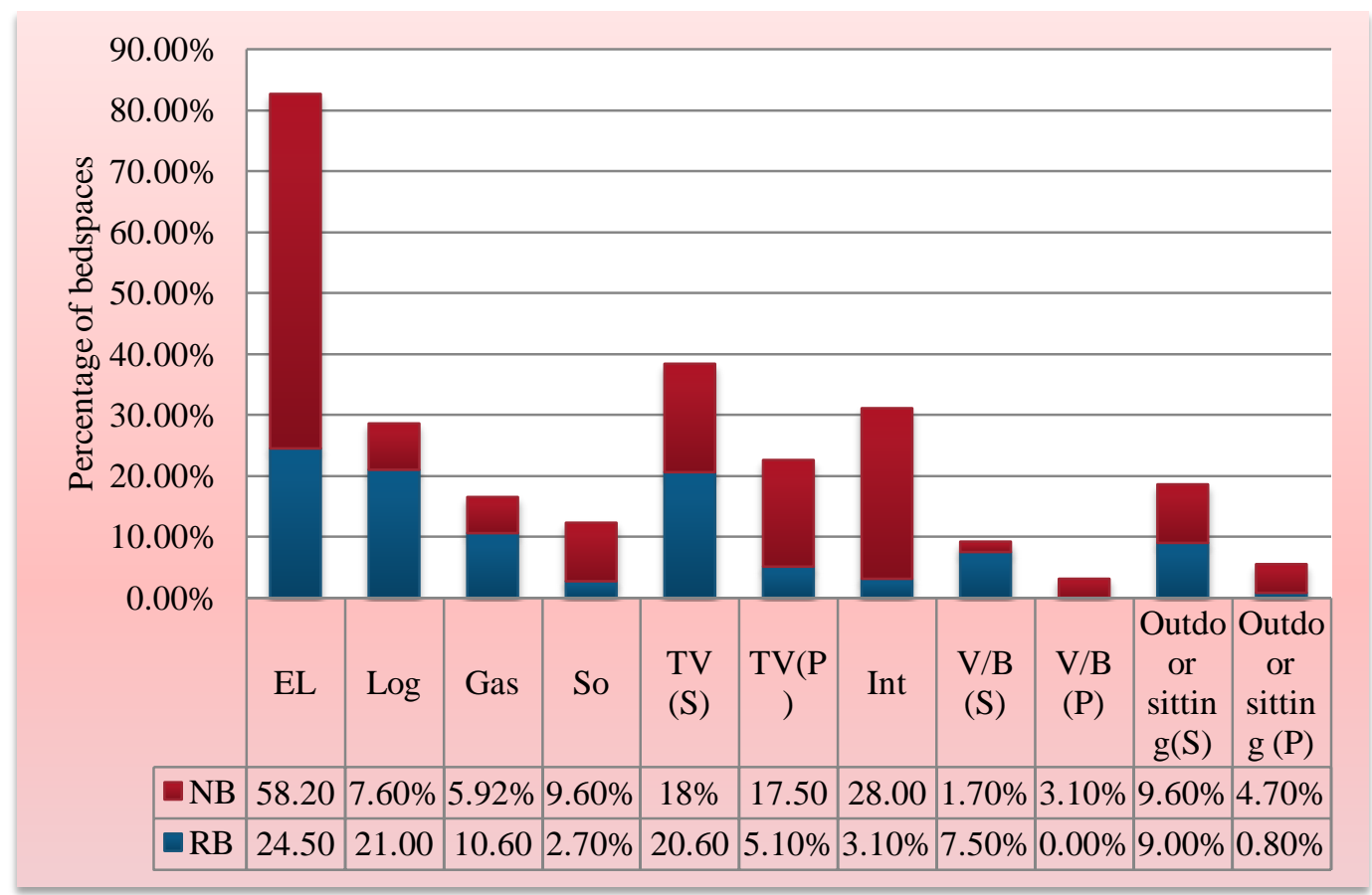

Figure 5.22: OCRT- SC accommodation services- energy source, facilities and spaces (2011)

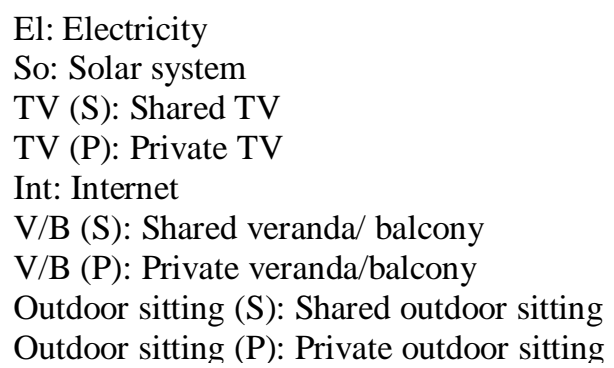

Figure 5.22 and Appendix 16 indicate that 63 (12.3\%) of 510 SC bed spaces including 14 (2.7\%) RB and 49 (9.6\%) NB use a solar system, generally for water heating, showing that such systems can be fitted to existing buildings In terms of equipment, 311 (61\%) of 510 SC bed spaces have TV (shared and private). Furthermore, of the 311 (61\%) SC bed spaces that have TV, 131 (25.7\%) are RB and 180 (35.3\%) are NB (Figure 5.22 and Appendix16). Moreover as shown in Figure5.22 and Appendix16, 159 (31.1\%) SC bed spaces provide the Internet including 16 (3.1\%) RB and 143 (28\%) NB bed spaces. So in refurbished accommodation the TV is more likely to be provided than the Internet. In terms of outdoor space, 60 (11.76\%) OCRT SC bed spaces have access to 16 verandas or balconies (both shared and private). These bed spaces are separated into 38 RB and 22 NB (Figure 5.22 and Appendix16). Likewise, 123 (24.1\%) SC bed 
spaces including 50 (9.8\%) RB and 73 (14.3\%) NB have access to shared and private outdoor sitting areas (Figure 5.22 and Appendix16).

\subsubsection{OCRT B\&B Accommodation Services: Energy Sources, Facilities and Open Air Spaces (2011)}

Figure 5.23 and Appendix16 demonstrate that of 541 B\&B bed spaces 499 (82.7\%) use electricity for heating. As shown in Figure 5.23 and Appendix16, 356 (65.8\%) of these 499 bed spaces are NB and 143 (26.4\%) are RB. In addition, of 136 (25.1\%) B\&B bed spaces 66 (12.2\%) RB and 70 (12.9\%) NB use logs, again showing refurbished buildings are more likely to use wood as an energy source. Of the remaining $37 \mathrm{~B} \& \mathrm{~B}$ bed spaces 5 (0.9\%) RB and 32 (5.9\%) NB use gas for heating.

Figure 5.23 and Appendix 16 indicate that 12 (2.2\%) NB of 541 B\&B bed spaces have a solar water heating system. For equipment, 353 (65.2\%) of 541 B\&B bed spaces have TV (shared and private) made up of 85 (15.7\%) RB and 268 (49.5\%) NB bed spaces (Figure 5.23 and Appendix 16).

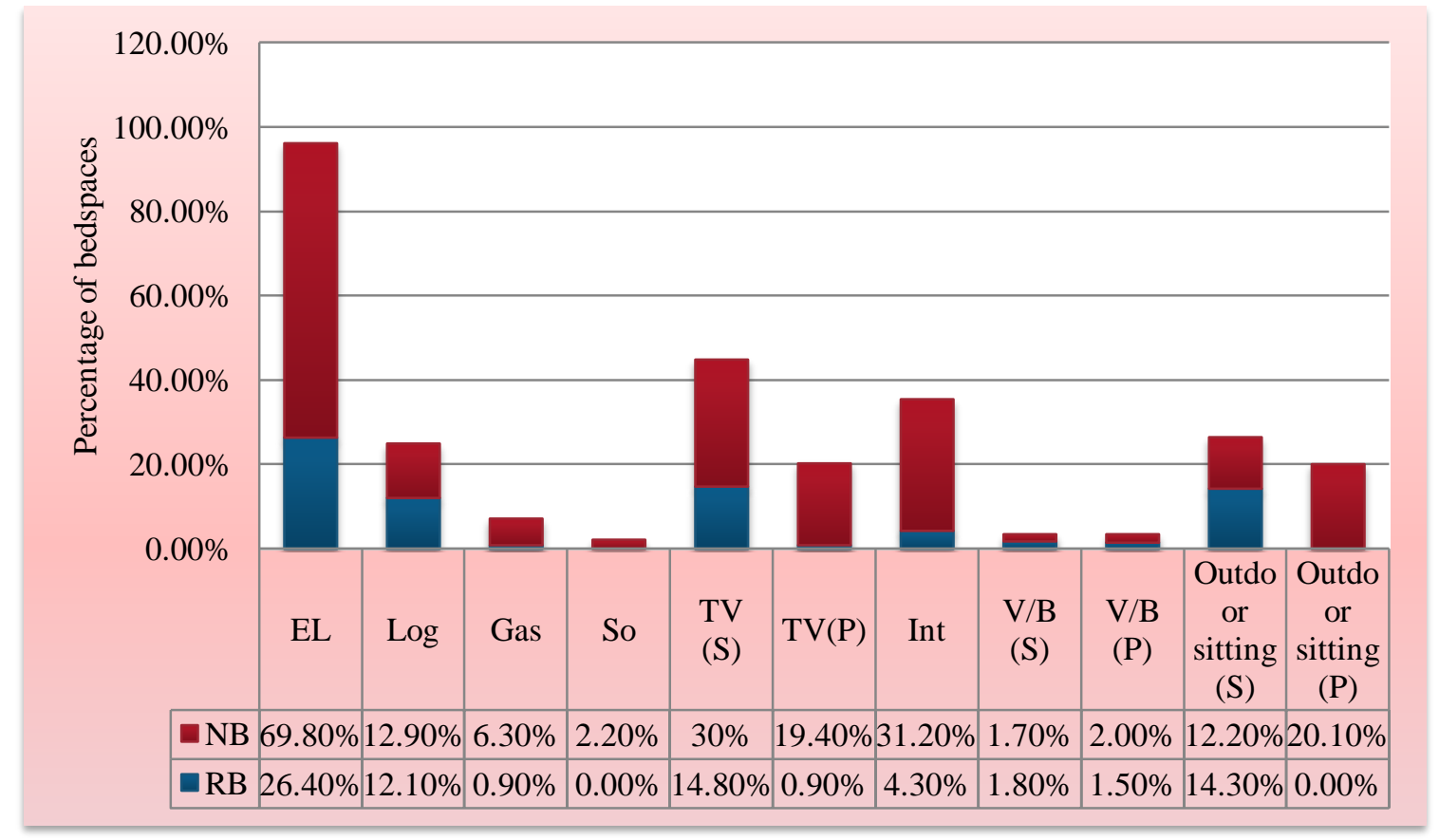

Figure 5.23: OCRT- B\&B accommodation services- Energy sources, facilities and spaces (2011)

As demonstrated in Figure 5.23 and Appendix16, 182 (33.6\%) B\&B bed spaces are equipped with the Internet including 23 (4.2\%) RB and 159 (29.4\%) NB, again showing refurbished buildings are less likely to have the Internet. For outdoor space, 38 (7.2\%) OCRT B\&B bed spaces comprising18 RB and 20 NB have 6 verandas and balconies 
(both shared and private) (Figure 5.23 and Appendix16). Furthermore, some 248 (24.1\%) of B\&B bed spaces comprising 73 (13.5\%) RB and 175 (32.3\%) NB have access to shared or private outdoor sitting areas (Figure 5.23 and Appendix 16).

\subsubsection{OCRT Bp Accommodation Services: Energy Sources, Facilities and Open Air Spaces (2011)}

Figure 5.20 and Appendix16 demonstrate that of 136 Bp bed spaces 75 (55.1\%) use electricity for heating. As shown in Figure 5.24 and Appendix 16, 65 (47.80\%) of these 136 bed spaces are NB and 10 (7.30\%) are RB. In addition, 6 (4.4\%) Bp bed spaces that all are NB use logs and 18 (13.2\%) NB Bp bed spaces use gas for heating. Figure 5.24 and Appendix16 indicate that 18 (13.2\%) NB of 136 Bp bed spaces use solar water heating. For equipment 33 (24.3\%) of 136 BP bed spaces have TV (shared and private) including 8 (5.9\%) RB and 25 (18.4\%) NB bed spaces (Figure 5.24 and Appendix 16). Furthermore, as demonstrated in Figure 5.24 and Appendices 16, 18, 13.2\% of Bp beds spaces provide the Internet and are NB.

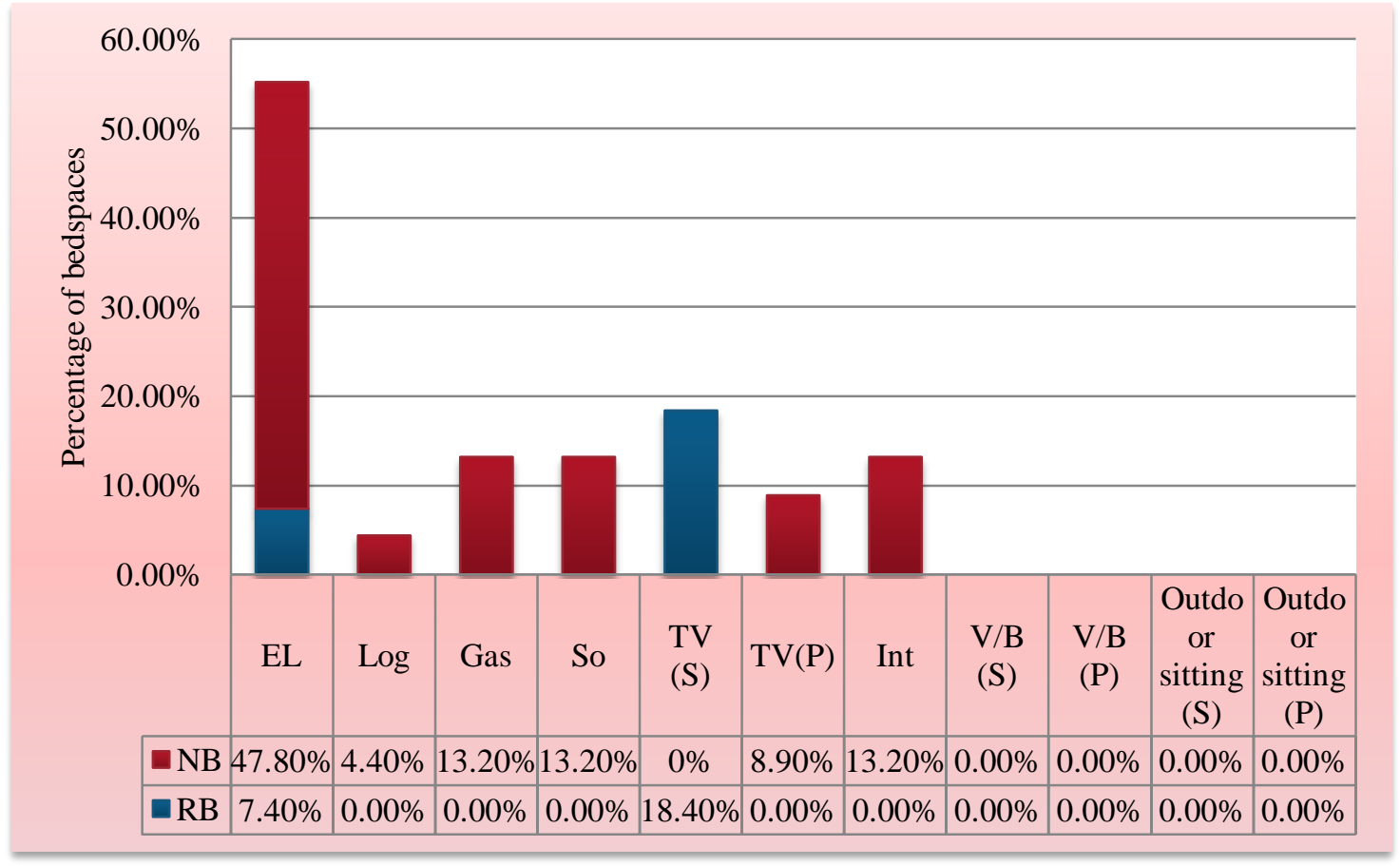

Figure 5.24: OCRT- Bp accommodation services- energy sources and facilities (2011) 


\subsubsection{OCRT Motel Accommodation Services: Energy Sources, Facilities and Open Air Spaces (2011)}

Figure 5.25 and Appendix16 indicate that of 665 motel bed spaces 508 (76.4\%) made up of 22 (3.3\%) RB and 486 (73.1\%) NB use electricity for heating. For equipment, 611 (91.9\%) of 665 motel bed spaces have TV (shared and private) and all are NB (Figure 5.25 and Appendix 16). Moreover as shown in Figure 5.25 and Appendix 16, 582(87.5\%) motel bed spaces have access to the Internet and all are NB. motels are dominated by NB.

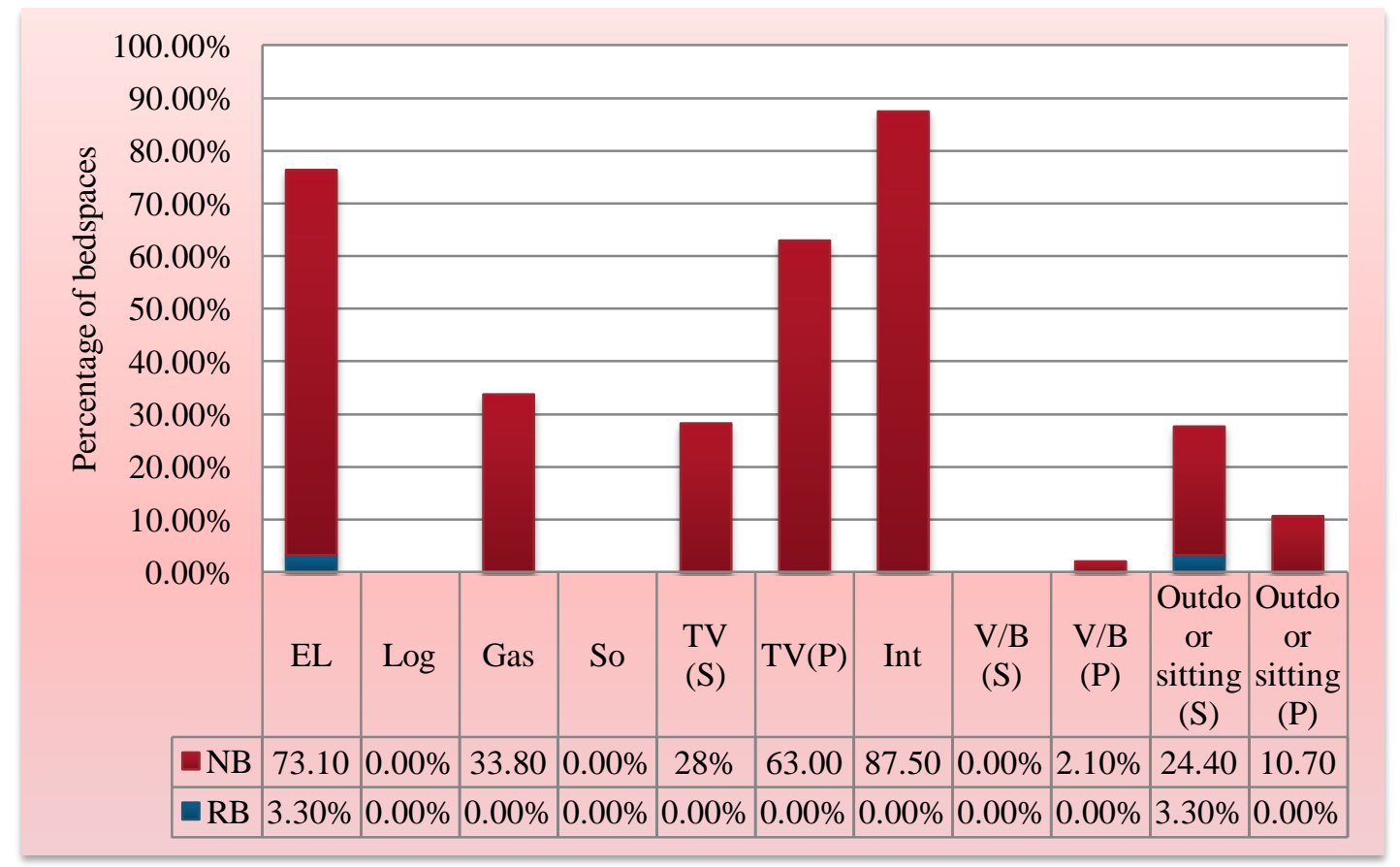

Figure 5.25: OCRT- Motel accommodation services- energy sources and facilities (2011)

As shown in Figure 5.25 and Appendix16, only 14 (2.1\%) NB OCRT motel bed spaces can access 2 private verandas. However, 255 (39\%) of motel bed spaces comprising 22 (3.4\%) RB and 233 (35.6 \%) NB have access to shared and private outdoor sitting areas (Figure 5.25 and Appendix16).

\subsubsection{OCRT Homestead Accommodation Services: Energy Sources, Facilities and Open Air Spaces (2011)}

As demonstrated in Figure 5.26 and Appendix 16, all 28 homestead bed spaces made up of 22 (78.6\%) RB and 6 (21.4\%) NB use electricity for heating. In addition these figures show that 4 (14.3\%) RB homestead bed spaces also use logs to heat part of the accommodation services, in particular common spaces such as living rooms and guest lounge 
areas. This again underlines that log burning is more likely to be associated with refurbished accommodation.

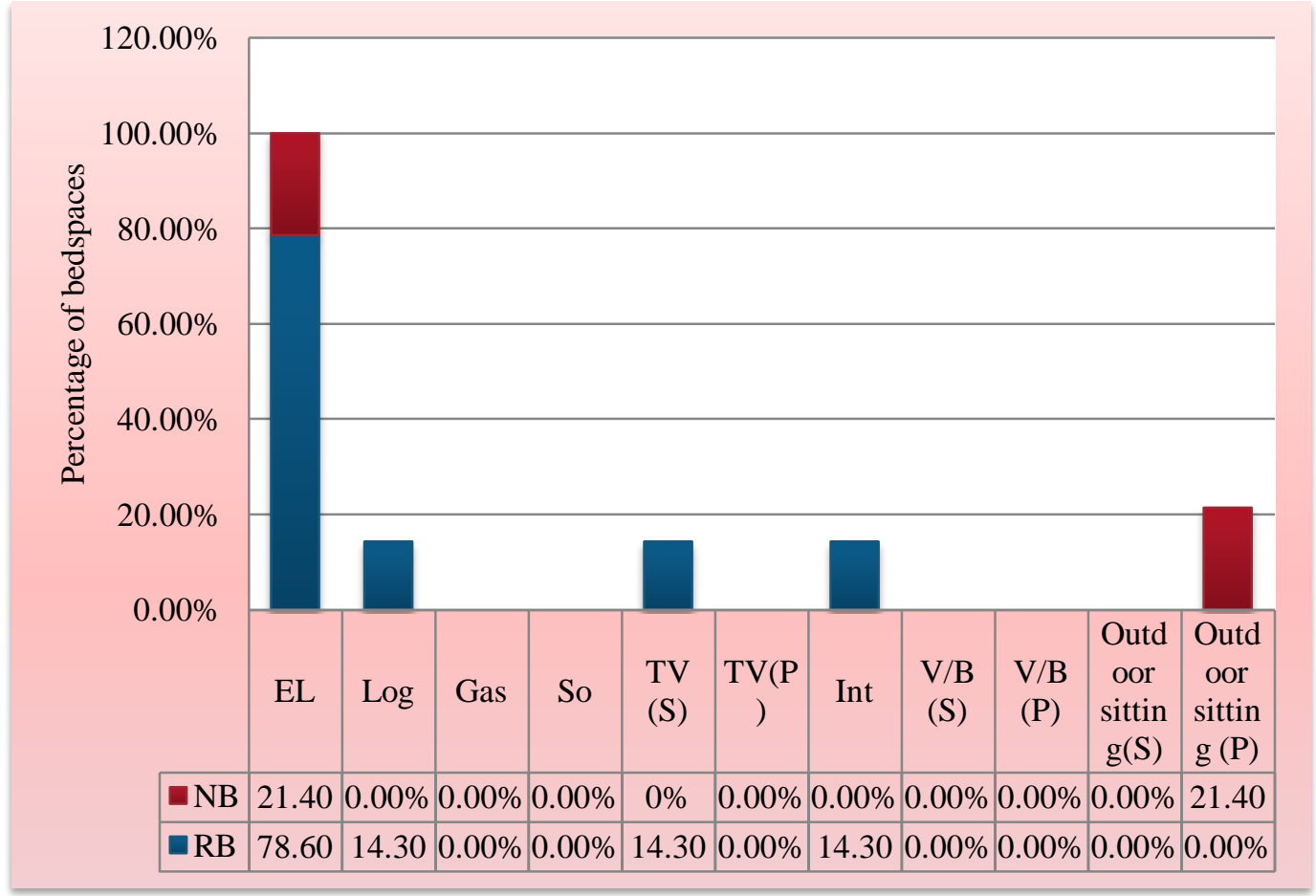

Figure 5.26: OCRT- Homestead accommodation services- energy sources and facilities (2011)

Figure 5.26 and Appendix16 show that 4 (14.3\%) out of 28 homestead bed spaces have a shared TV and all are RB. Likewise as shown in Figure 5.26 and Appendix16, 4 (14.3\%) of RB homestead bed spaces have access to the Internet and 6 (21.4\%) of NB bed spaces have use of a private outdoor sitting area.

\subsubsection{OCRT Hotel Accommodation Services: Energy Sources, Facilities and Open Air Spaces (2011)}

As shown in Figure 5.27 and Appendix16, 303 (87.6\%) out of 346 OCRT hotel bed spaces that are RB use electricity for heating. Furthermore, 90 (26\%) hotel bed spaces including 84 (24.3\%) RB and 6 (1.7\%) NB have log burning facilities and 29 (8.4\%) RB bed spaces use gas for heating.

As indicated in Figure 5.27 and Appendix16, 158 (45\%) RB hotel bed spaces have access to 8 shared TVs and 81 (23.4\%) RB bed spaces have access to the Internet. Moreover as shown in Figure 5.27 and Appendix16, 43 (12.4\%) of RB bed spaces have use of 3 shared verandas. Also, of 73 (21.1\%) RB bed spaces, 44 have access to 3 shared outdoor sitting areas and 29 can use 6 private outdoor sitting spaces (note, this is 
bed spaces, and there is usually more than one bed space per room) (Figure 5.27 and Appendix 16). It seems the refurbished hotels are continuing the New Zealand tradition of providing indoor and outdoor spaces.

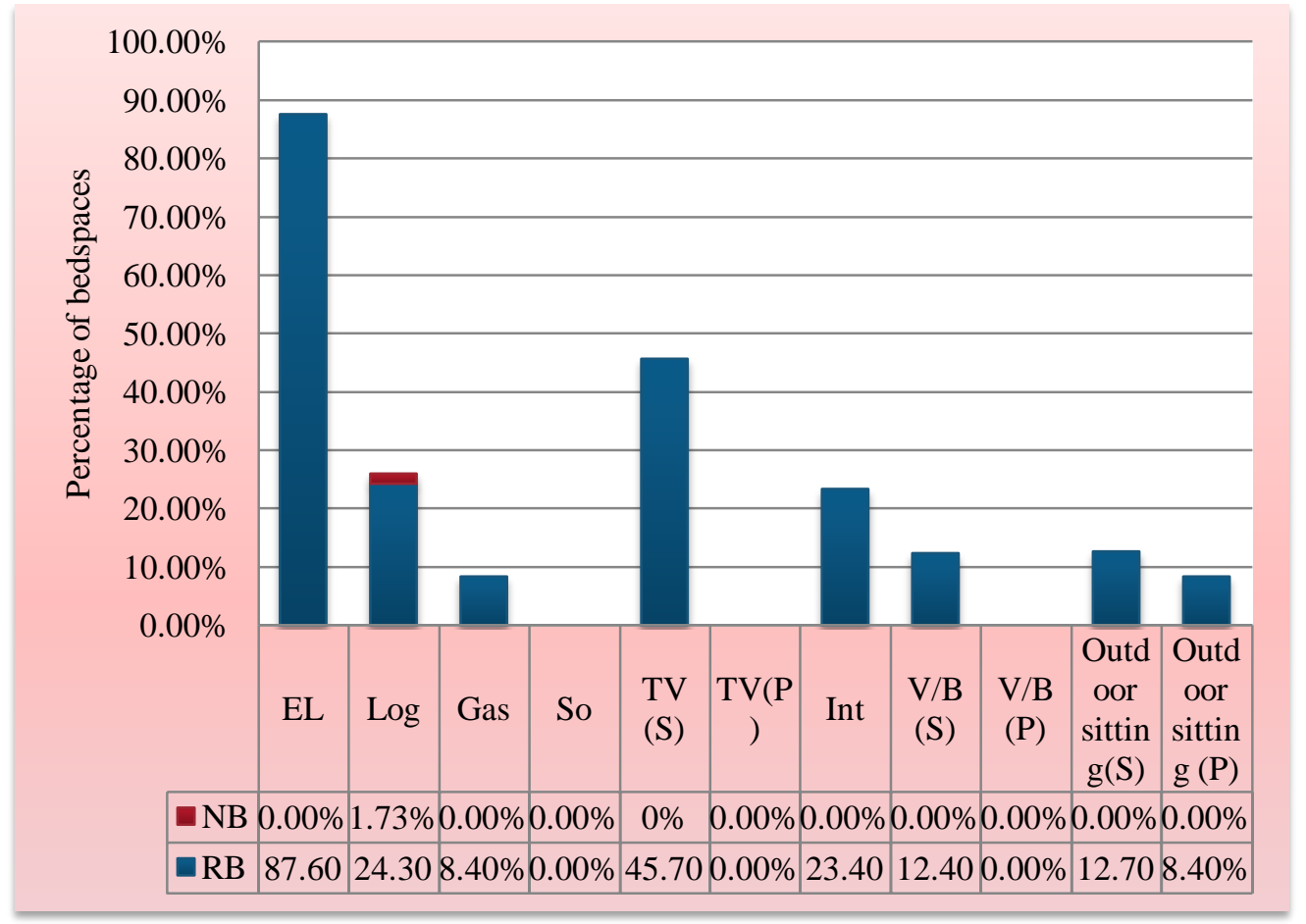

Figure 5.27: OCRT- Hotel accommodation services- Energy sources and facilities (2011)

\subsubsection{OCRT Camping Sites: Energy Sources, Facilities and Open Air Spaces} (2011)

As shown in Figure 5.28 and Appendix16, of 3,317 OCRT camping sites, 2,048 (61.7\%) are powered sites with electricity and a surprising 3,114 (93.9\%) have access to the Internet (almost all wireless). The old New Zealand tradition of the simple family camping holiday seems to have changed. 


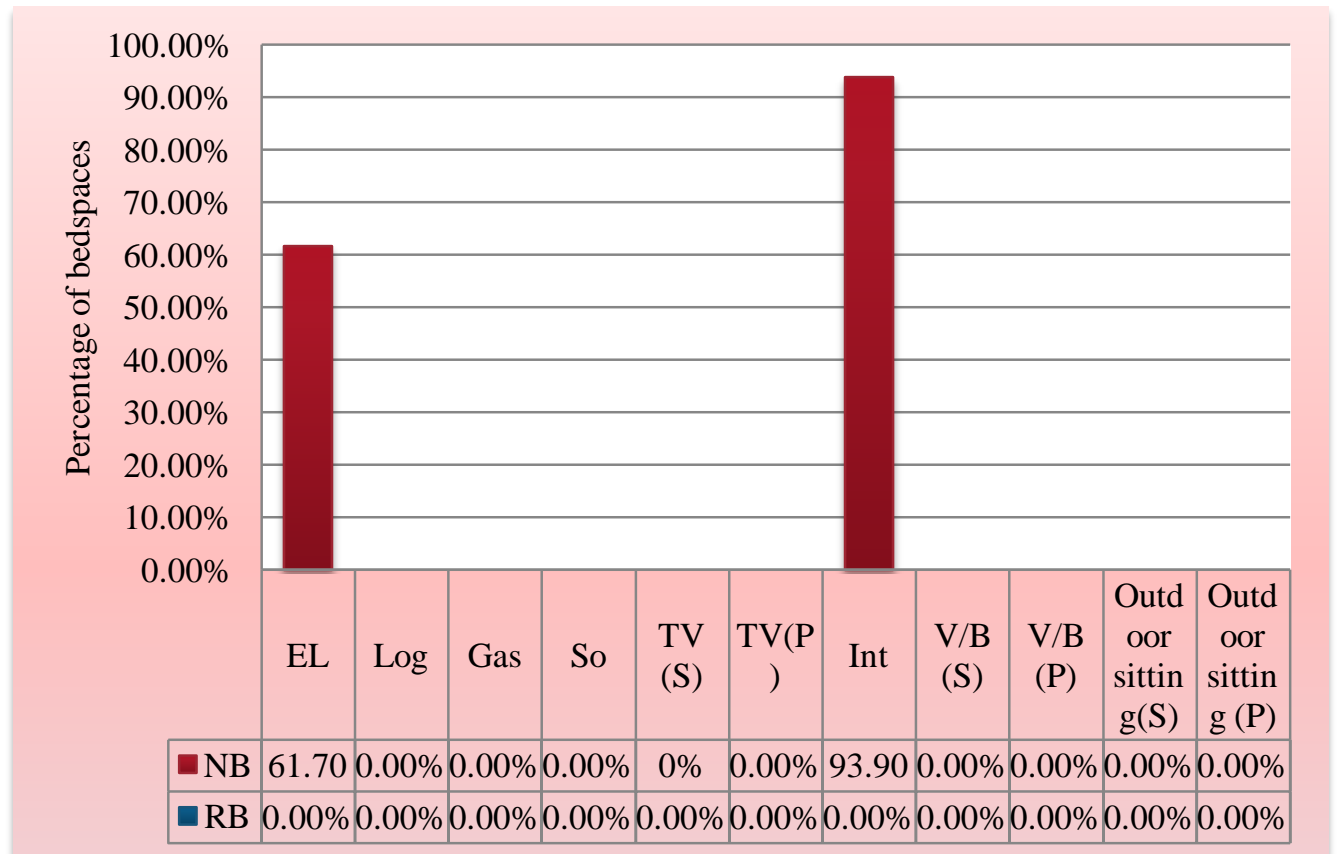

Figure 5.28: OCRT- camping site accommodation services- energy sources and facilities (2011)

\subsubsection{All Types of OCRT Accommodation Services: Energy Sources, Facilities and Open Air Spaces (2011)}

Figure 5.29 indicates the energy resources used, facilities and open air spaces in all types of OCRT accommodation services. It shows of 5,496 OCRT bed spaces, 3,233 (58.80\%) NB and 621 (11.30\%) RB use electricity for heating, also 121 (2.2\%) NB and 258 (4.7\%) RB use logs for heating, 324 (5.9\%) NB and 88 (1.6\%) RB use gas, and 77 (1.4\%) NB and 16 (0.3\%) RB use a solar system for heating water.

When it comes to equipment, Figure 5.29 shows that of 5,496 OCRT bed spaces, 401 (7.3\%) RB and 687 (12.5\%) NB bed spaces have TV (shared and private) and only 121 (2.2\%) RB have access to the Internet compared with 3,985 (72.5\%) NB bed spaces. As indicated in Figure 5.29, 278 (5.60\%) RB and 539 (9.80\%) NB bed spaces use shared and private outdoor sitting areas (including verandas, balconies and open air sitting spaces). 


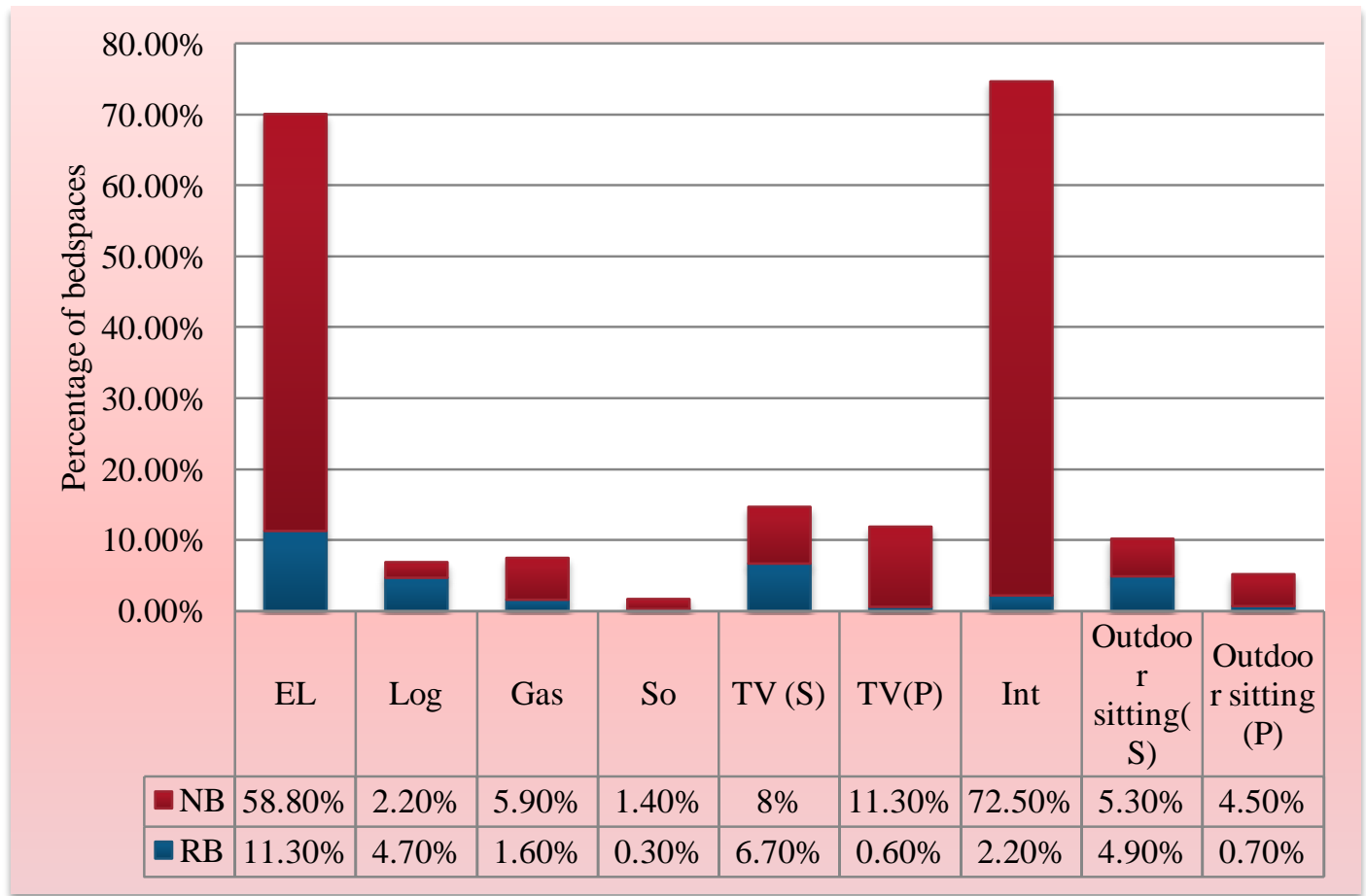

Figure 5.29: OCRT- all types of accommodation services- energy sources, facilities and open air spaces (2011)

\subsubsection{OCRT Accommodation Services: Comparison between NB and RB Bed spaces in terms of Energy Sources, Facilities and Open Air Spaces (2011)}

Figure 5.30 compares OCRT NB and RB bed spaces for use of energy resources, facilities and outdoor sitting. As demonstrated, $66.5 \%$ of NB and $87 \%$ of RB bed spaces use electricity for heating. However, only $2.5 \%$ of NB have facilities for log burning compared to $36.20 \%$ of RB bed spaces. Use of gas is more even with $6.70 \%$ of NB and $12.30 \%$ of RB bed spaces using it for heating. Figure 5.30 also indicates that $1.60 \%$ of NB and $2.40 \%$ of RB bed spaces use a solar system for heating water, showing that both types of buildings can accommodate more sustainable technologies.

Figure 5.30 also shows that $14.22 \%$ of NB and $56 \%$ of RB bed spaces provide a TV, but it should be remembered that the NB bed spaces include 3,317 camp bed spaces that do not provide TV. In terms of using the Internet, $82.50 \%$ of NB and only $17 \%$ of RB bed spaces have access to the web (Figure 5.30). In contrast, when it comes to outdoor spaces only $11.15 \%$ of NB can access outdoor sitting areas compared to $39 \%$ of RB OCRT bed spaces. There are some clear differences in what visitors can expect to find in the different types of accommodation. 


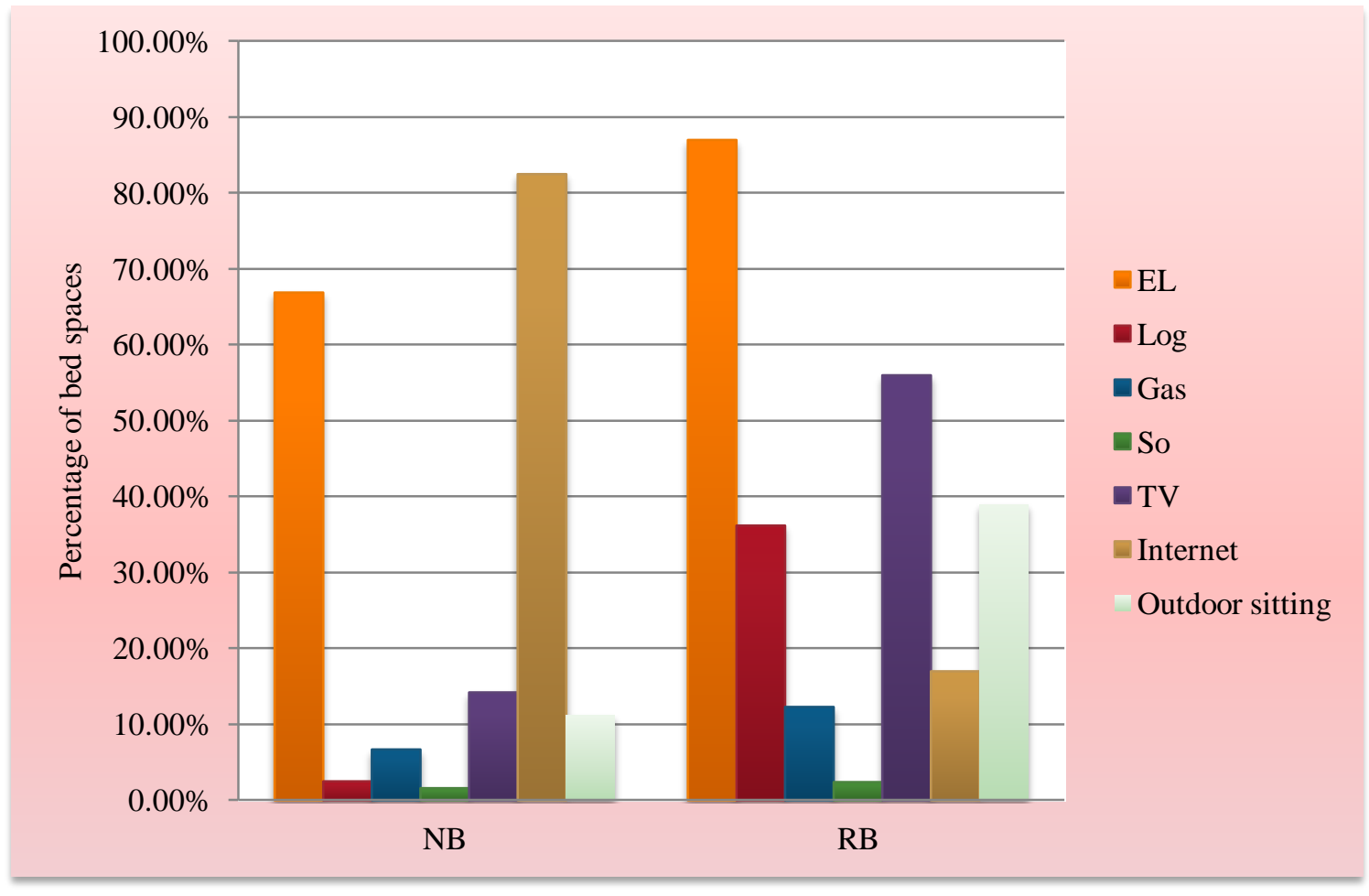

Figure 5.30: Comparison between OCRT- NB and RB accommodation bed spaces in terms of energy sources, facilities and open air spaces

\subsubsection{OCRT Accommodation Services: Occupancy Share}

Table 5.17 presents the calculated occupancy rates of Central Otago accommodation services (2010 and 2011) as published by New Zealand Statistics (2011:5). This table determines the occupancy rate of motels/apartments at $43.1 \%$ and holiday parks at $10.1 \%$ in 2010. Moreover, the overall occupancy rate of accommodation services including holiday parks in 2010 is estimated to be $14.9 \%$. In addition total occupancy rate of types of accommodation services excluding holiday parks is estimated to be $31 \%$. (Table 5.17).The "occupancy rates excluding holiday parks are included because the capacity of holiday parks can distort total occupancy rates”. (New Zealand Statistics, 2011:5).

The occupancy rate of Central Otago motels dropped to 41.1\% in 2011. In addition, as shown in Table 5.17, the occupancy rate of Central Otago holiday parks also dropped from $10.1 \%$ to $9.4 \%$ in 2011 . The overall occupancy rates of Otago Central accommodation including holiday parks and excluding holiday parks dropped to $14.3 \%$ and 30.4\% respectively in 2011 (Table 5.17). 
Table 5.17: Central Otago accommodation variables by accommodation type and year

\begin{tabular}{|c|c|c|}
\hline \multicolumn{3}{|c|}{ Occupancy rates ${ }^{(1)}(\%)$} \\
\hline \multirow[t]{3}{*}{ Type of accommodation } & \multirow{2}{*}{\multicolumn{2}{|c|}{ Annual }} \\
\hline & Year ended & \\
\hline & September 2010 & September 2011 \\
\hline Hotels $^{2}$ & $\mathrm{C}$ & $\mathrm{C}$ \\
\hline $\operatorname{Motel}^{2} /$ apartments & 43.1 & 41.1 \\
\hline Backpackers $^{2}$ & $\mathrm{C}$ & $\mathrm{C}$ \\
\hline Holiday parks² & 10.1 & 9.4 \\
\hline Total $^{2}$ & 14.9 & 14.3 \\
\hline $\begin{array}{l}\text { Total excluding holiday } \\
\text { parks }^{2} \text {. }\end{array}$ & 31 & 30.4 \\
\hline \multicolumn{3}{|c|}{$\begin{array}{l}\text { 1. Occupancy rates are calculated as the ratio of visitor occupied unit nights to monthly capacity } \\
\text { 2. Hotels: includes both hostels and resorts } \\
\text { 3. Motels: includes motor inns, apartments and motels. } \\
\text { 4. Backpackers: includes Backpackers/hostels } \\
\text { 4. Holiday parks: includes Caravan parks/camping grounds } \\
\text { C to be calculated } \\
\text { - Reference: Statistics New Zealand, Central Otago, September 2011:5 }\end{array}$} \\
\hline
\end{tabular}

Since the occupancy rates of Central Otago hotels, backpacker, self-contained and homestead accommodation are not available in New Zealand Statistics (2011), to obviate this shortage of information, this study uses the occupancy share of OCRT accommodation services in 2005, from Department of Conservation (DOC) (June 2011) as shown in Table 5.18.

Table 5.18 indicates that the occupancy share of hotel/ motel in OCRT is 32\% followed by B\&B (27\%), motor home/ camping (24\%), backpackers (14\%) and own home/ holiday home/ friends and relatives (3\%).

\begin{tabular}{|l|c|}
\hline \multicolumn{2}{|c|}{ Table 5.18: OCRT- Occupancy share by type of accommodation service } \\
\hline Types & \% of visitor nights $(2005)^{1}$ \\
\hline Hotel/Motel & 32 \\
\hline B\&B & 27 \\
\hline Motor home/Camping & 24 \\
\hline Backpackers & 14 \\
\hline Own home/Holiday home/friends and relatives & 3 \\
\hline Total & 100 \\
\hline 1. Reference: OCRT, 2005:10 & \\
- Total visitor numbers in 2005= 8,923 (Department of Conservation, June 2011) \\
\hline
\end{tabular}

This thesis uses the occupancy share cited in Table 5.18 to measure the occupancy of all OCRT accommodation types in 2011. The occupancy share of hotel/motel which is $32 \%$ in 2005 (Table 5.18) is separated into the two occupancy shares of hotel (10.9\%) and motel (21.1\%) in 2011(Table 5.19) based on the following discussion. 
As indicated in Table 5.14, in 2011 the OCRT motels and hotels contain 665 and 346 bed spaces respectively, making 1011 in total. In 2011, the 665 OCRT motel bed spaces comprise 65\% of total (1011) motel/hotel bed spaces. In addition, 346 OCRT hotel bed spaces make up 34.2\% of total (1011) motel/hotel-bed spaces in 2011. This study uses these proportions to divide the 32\% occupancy of motel/hotel (cited in Table 5.18) into the two occupancy shares of $21.1 \%$ (65\% of 32) motel and $10.9 \%$ (34.2\% of 32) hotel (Table 5.19).

\begin{tabular}{|l|c|c|c|c|}
\hline \multicolumn{5}{|c|}{ Table 5.19: OCRT- accommodation services- occupancy shares (2011) } \\
\hline Type of accommodation service & $\begin{array}{c}\text { \% of visitor } \\
\text { nights } \\
(2005)\end{array}$ & $\begin{array}{c}\text { Visitor nights } \\
(2011)\end{array}$ & $\begin{array}{c}\text { Available } \\
\text { bed spaces }\end{array}$ & $\begin{array}{c}100 \% \text { occu- } \\
\text { pancy per year }\end{array}$ \\
\hline SC & 13.1 & 5,559 & 510 & 186,150 \\
\hline B\&B & 13.9 & 5,899 & 541 & 197,465 \\
\hline Bp & 14 & 5,941 & 136 & 49,640 \\
\hline Motel & 21.1 & 8,954 & 665 & 24,2725 \\
\hline Homestead & 3 & 1,273 & 28 & 10,220 \\
\hline Hotel & 10.9 & 4,626 & 346 & 126,290 \\
\hline Camping & 24 & 10,185 & 3,317 & $1,210,705$ \\
\hline Total & 100 & 42,437 & 5,543 & $2,023,195$ \\
\hline $\begin{array}{l}\text { 1. 100\% occupancy rate of each type of accommodation service / year= number of } \\
\text { (days) }\end{array}$ & & & & \\
\hline
\end{tabular}

Since this thesis uses the occupancy shares of OCRT accommodation services proposed by Department of Conservation (2011- see Table 5.18) as basic data in which the occupancy share of the OCRT self-contained accommodation services is not included, the occupancy share of SC accommodation service in 2011 is calculated through the following discussion.

As demonstrated in Table 5.18, the occupancy share of B\&B accommodation services is $27 \%$ in 2005. This research makes this assumption that in 2005 B\&B occupancy share contains both occupancy shares of B\&B and SC accommodation services. Based on this assumption, the $27 \%$ occupancy of B\&B accommodation in 2005 (see Table 5.18) is divided into the two portions of B\&B and SC occupancy shares according to the percentage of their available bed spaces in 2011 through the following explanation.

As shown in Table 5.14, OCRT SC and B\&B accommodation services comprise respectively $510(48.5 \%)$ and 541(51.5\%) of the total $1051(510+541)$ SC/B\&B bed spaces in 2011. This thesis divides the $27 \%$ occupancy share of B\&B accommodation calculated 
in 2005 (see Table 5.18) into the two portions of $13.1 \%$ (48.5\% of 27 ) SC and $13.9 \%$ (51.5\% of 27) B\&B accommodation (Table 5.19).

Furthermore, the occupancy share of OCRT homestead accommodation in 2011(3\%) is assumed as equal to own home/holiday home/friends and relatives occupancy share calculated in 2005 (Tables 5.18 and 5.19). The total of OCRT visitor nights $(42,437)$ is multiplied by the occupancy share of each type of accommodation services to calculate their related visitor nights (Table 19).

\subsubsection{OCRT Accommodation Services: Area}

This chapter calculates the areas of a representative sample of 41 of the OCRT accommodation services buildings (21RB and 20NB) made up of 10 SCs, 13 B\&Bs, 4Bps, 5 motels, 2 homesteads and 7 hotels and their area per bed space $\left(\mathrm{m}^{2}\right)$ to calculate an average area per bed space for each type of accommodation service used in OCRT (Appendix18). The area of each of the 41 accommodation services is calculated using Map Tool2 for floor area calculation and scaled elevation based on information published in the websites and satellite Google Maps.

The resulting information shown in Appendix 18 is summarised in the following Table 5.20 determines the capacity (C), floor area and area per bed space of the chosen types of OCRT accommodation and their numbers. As shown in Table 5.20, the largest area of building per bed space is in B\&B at $40 \mathrm{~m}^{2}$ followed by a similar group of just under $30 \mathrm{~m}^{2} /$ bed $\left(29.5 \mathrm{~m}^{2} /\right.$ bed SC, $29.4 \mathrm{~m}^{2} /$ bed homestead, and $29.1 \mathrm{~m}^{2} /$ bed hotel), with a smaller area of $16.9 \mathrm{~m}^{2} /$ bed for motel and, as might be expected, the lowest area of 12 $\mathrm{m}^{2} /$ bed for Bp.

\begin{tabular}{|l|c|c|c|c|}
\hline \multicolumn{4}{|c|}{ Table 5.20: OCRT- average area per bed by type of accommodation (2011) } \\
\hline Category & No. & C & Floor space ${ }^{2}$ & Area per Bed ${ }^{2}$ \\
\hline SC & 10 & 61 & 1,817 & 29.5 \\
\hline B\&B & 13 & 93 & 3,436 & 40 \\
\hline Bp & 4 & 58 & 697 & 12 \\
\hline Motel & 5 & 166 & 2,812 & 16.9 \\
\hline Homestead & 2 & 353 & 12 & 29.4 \\
\hline Hotel & 7 & 156 & 4,538 & 29.1 \\
\hline Camping & - & - & - & 6 \\
\hline Total & 41 & 887 & 13,312 & - \\
\hline 1. The average area of camping sites is assumed & $6 \mathrm{~m}^{2}$ per bed space & & \\
\hline
\end{tabular}


Table 5.21 shows the average area, number of bed spaces and total area of each type of OCRT accommodation service and whether they are NB or RB (called here quality or Q). In this table the area of each type of accommodation services is calculated as its average area/bed space multiplied by the number of bed spaces.

Comparing the areas of types of NB OCRT accommodation services, camping sites comprise the majority or 19,902 $\mathrm{m}^{2}$ followed by B\&B $\left(15,280 \mathrm{~m}^{2}\right)$, motel $\left(10,866.7 \mathrm{~m}^{2}\right)$ and SC $\left(10,354.5 \mathrm{~m}^{2}\right)$ (Table 5.21). Other types of NB accommodation services including Bp, hotel and homestead contain areas in a range of 176.4-1,396.8 $\mathrm{m}^{2}$ (Table 5.21). In addition, $\mathrm{RB}$ hotels contain the largest $\mathrm{RB}$ area of $8,671 \mathrm{~m}^{2}$ followed by $\mathrm{B} \& \mathrm{~B}(6,360$ $\left.\mathrm{m}^{2}\right)$ and SC $\left(4,690.5 \mathrm{~m}^{2}\right)$. The areas of other refurbished accommodation services including Bp, hotel and homestead are between 371.8- 646.8 $\mathrm{m}^{2}$ (Table 5.21).

\begin{tabular}{|c|c|c|c|c|}
\hline Category & $\mathrm{Q}$ & $\begin{array}{l}\text { Average area/bed } \\
\text { space }\left(\mathrm{m}^{2}\right)\end{array}$ & Bed space & Area $\left(\mathrm{m}^{2}\right)$ \\
\hline \multirow[t]{2}{*}{ SC } & NB & 29.5 & 351 & $10,354.5$ \\
\hline & $\mathrm{RB}$ & 29.5 & 159 & $4,690.5$ \\
\hline \multirow[t]{2}{*}{ B\&B } & NB & 40 & 382 & 15,280 \\
\hline & $\mathrm{RB}$ & 40 & 159 & 6,360 \\
\hline \multirow[t]{2}{*}{ Bp } & NB & 12 & 83 & 996 \\
\hline & $\mathrm{RB}$ & 12 & 53 & 636 \\
\hline \multirow[t]{2}{*}{ Motel } & NB & 16.9 & 643 & $10,866.7$ \\
\hline & $\mathrm{RB}$ & 16.9 & 22 & 371.8 \\
\hline \multirow[t]{2}{*}{ Homestead } & NB & 29.4 & 6 & 176.4 \\
\hline & $\mathrm{RB}$ & 29.4 & 22 & 646.8 \\
\hline \multirow[t]{2}{*}{ Hotel } & NB & 29.1 & 48 & $1,396.8$ \\
\hline & $\mathrm{RB}$ & 29.1 & 298 & $8,671.8$ \\
\hline \multirow[t]{2}{*}{ Camping site } & NB & 6 & 3,317 & 19,902 \\
\hline & $\mathrm{RB}$ & - & 0.00 & - \\
\hline Total & - & - & 5,543 & 80,350 \\
\hline
\end{tabular}

The total area of OCRT NB accommodation services is 58,972 $\mathrm{m}^{2}$ and RB accommodation is $21,378 \mathrm{~m}^{2}$, making a total of $80,350 \mathrm{~m}^{2}$ (Table 5.22 .).

Table 5.22: OCRT- area of accommodation services by quality

\begin{tabular}{|c|c|} 
Quality of buildings & Area $\left(\mathrm{m}^{2}\right)$ \\
\hline NB buildings & 58,972 \\
\hline RB buildings & 21378 \\
\hline Total & 80,350 \\
\hline
\end{tabular}




\subsubsection{OCRT Accommodation Services: Occupied Area}

Table 5.23 demonstrates that OCRT B\&B accommodation services have the largest occupied area of 235,960 $\mathrm{m}^{2}$, followed by SC (163,990 $\left.\mathrm{m}^{2}\right)$, motel $\left(151,322.6 \mathrm{~m}^{2}\right)$ and hotel 134,616.6 $\mathrm{m}^{2}$. Bp and homestead contain smaller areas at 71,292 $\mathrm{m}^{2}$ and 37,426.2 $\mathrm{m}^{2}$ respectively (Table 5.23).The occupied area $\left(\mathrm{m}^{2} /\right.$ year) of each type of accommodation as shown in Table 5.23 is calculated through the following steps:

Occupancy share of each type $\times$ Total OCRT visitor nights $=$ Visitor nights of each type Visitor nights/year $\times$ Area/bed $\left(\mathrm{m}^{2}\right)=$ Occupied area $\left(\mathrm{m}^{2}\right) /$ year

\subsubsection{Example: SC Occupied Area $\left(\mathrm{m}^{2}\right) /$ year (2011)}

13.1\% (occupancy share) $\times$ 42,437 (total OCRT visitor nights) $=5$,559 (SC visitor nights)

5,559 (SC visitor nights $) \times 29.5\left(\mathrm{~m}^{2} /\right.$ bed $)=163,990.5 \mathrm{~m}^{2} /$ year Occupied Area (2011)

\begin{tabular}{|l|c|c|c|c|c|c|c|}
\hline \multicolumn{5}{|c|}{ Table 5.23: OCRT- Accommodation services- Total area and Occupied area (2011) } \\
\hline Type & No. & C & $\begin{array}{c}\text { Area per bed } \\
\mathrm{m}^{2}\end{array}$ & $\begin{array}{c}\text { Total } \\
\text { Area }\end{array}$ & $\begin{array}{c}\text { Occupancy } \\
\text { share (\%) }\end{array}$ & $\begin{array}{c}\text { Visitor } \\
\text { nights }\end{array}$ & $\begin{array}{c}\text { Occupied Area } \\
\mathrm{m}^{2} / \text { year } \\
(2011)\end{array}$ \\
\hline SC & 92 & 510 & 29.5 & 15,045 & 13.1 & 5,559 & $163,990.5$ \\
\hline B\&B & 66 & 541 & 40 & 21,640 & 13.9 & 5,899 & 235,960 \\
\hline Bp & 12 & 136 & 12 & 1,632 & 14 & 5,941 & 71,292 \\
\hline Motel & 33 & 665 & 16.9 & $11,238.5$ & 21.1 & 8,954 & $151,322.6$ \\
\hline Homestead & 4 & 28 & 29.4 & 823.2 & 3 & 1,273 & $37,426.2$ \\
\hline Hotel & 19 & 346 & 29.1 & $10,068.6$ & 10.9 & 4,626 & $134,616.6$ \\
\hline Camping & 557 & 3,317 & 6 & 19,909 & 24 & 10,185 & - \\
\hline Total & 783 & 5543 & & 80,350 & 100 & 42,437 & 794,608 \\
\hline
\end{tabular}




\subsubsection{The EF of OCRT Accommodation Services}

This section explores the EF of OCRT accommodation services through using three scenarios. In the first scenario, all buildings are assumed to be new buildings (NB) and in the second scenario the buildings include both NB and RB accommodation as currently found in the ORCT. However, in the first and second scenario all areas of buildings (indoor and outdoor spaces including verandas and balconies) are assumed to have the footprint of indoor spaces. In the third scenario that reflects the current position of OCRT accommodation services, the buildings include NB and RB accommodation and spaces that are both indoor and open air (veranda and balcony) areas.

In the first scenario (5.6.8.1) the calculation of the EF of OCRT accommodation is conducted through measuring: the embodied energy of each type of (a1 to a9); the operation energy use of each type of OCRT accommodation services (b); and, the lifecycle energy use of the accommodation (c). Section 5.6.8.2-d calculates the total EF of OCRT accommodation services based on the first scenario discussed above. Sections 5.6.8.2 and 5.6.8.3 calculate the EF of OCRT accommodation services based on the second and third scenarios.

Comparison between the EFs from the first and second scenarios can indicate the influence exerted on the EF of OCRT accommodation services by using refurbished buildings. In addition, comparison between the EFs of the second and third scenarios determines the influence of using open air spaces on the EF of OCRT accommodation services.

\subsubsection{First Scenario: All Buildings are assumed to be New Buildings}

\section{a. Embodied Energy}

Table 5.24 comprises the calculated embodied energy of New Zealand building materials including ceramics, ready mix concrete and stone, as calculated by Alcorn (1996 and 2003) and Alcorn and Wood (1998). 


\begin{tabular}{|c|c|c|c|c|c|c|c|}
\hline \multirow{2}{*}{\multicolumn{2}{|c|}{ Material }} & \multirow{2}{*}{\multicolumn{2}{|c|}{ Alcorn 1996}} & \multirow{2}{*}{\multicolumn{2}{|c|}{ Alcorn and Wood 1998}} & \multirow{2}{*}{\multicolumn{2}{|c|}{ Alcorn 2003}} \\
\hline & & & & & & & \\
\hline \multirow[t]{2}{*}{ Ceramic } & $\begin{array}{l}\text { Brick, new } \\
\text { technology }\end{array}$ & $\begin{array}{c}\mathrm{MJ} / \mathrm{kg} \\
2.5\end{array}$ & $\begin{array}{r}\mathrm{MJ} / \mathrm{m}^{3} \\
5.170\end{array}$ & $\begin{array}{c}\mathrm{MJ} / \mathrm{kg} \\
2.5\end{array}$ & $\begin{array}{c}\mathrm{MJ} / \mathrm{m}^{3} \\
5,170\end{array}$ & $\begin{array}{c}\mathrm{MJ} / \mathrm{kg} \\
2.7\end{array}$ & $\begin{array}{r}\mathrm{MJ} / \mathrm{m}^{3} \\
5,310\end{array}$ \\
\hline & $\begin{array}{l}\text { Brick, old } \\
\text { technology }\end{array}$ & - & - & 7.7 & 1,580 & 6.7 & 13,188 \\
\hline $\begin{array}{l}\text { Ready mix } \\
\text { Concrete }\end{array}$ & $17.5 \mathrm{MP} \mathrm{a}$ & 1 & 2,350 & 1 & 2,350 & 0.9 & 2,019 \\
\hline \multirow[t]{2}{*}{ Stone } & Local & 0.79 & 1,890 & 0.79 & 1,890 & - & - \\
\hline & imported & 6.8 & 17,610 & 6.8 & 17,610 & - & - \\
\hline
\end{tabular}

Table 5.25 classifies NZ residential building construction into the two types of light and heavy constructions and gives the embodied energy of the building elements used in each type of structure. According to Mithraratne et al (2007: 159) the embodied energy of walls (see Table 5.25) used in two types of light and heavy construction are calculated based on this assumption (which arises from their research) that $30 \%$ of the areas of external walls are covered by windows. This research uses this assumption (30\% of external walls covered by windows) to calculate the embodied energy of walls with mud brick, brick, stone and concrete in OCRT accommodation.

As most of OCRT refurbished buildings with mud brick, brick, stone and timber structure (see Appendix 5) dominantly use timber frame windows, for calculation of the embodied energy uses of these types of construction, the windows are assumed with timber frame. Moreover, as most of the NB buildings with concrete construction dominantly use windows with aluminium frame (see Appendix 5), the embodied energy use of this type of construction is calculated based on this assumption that $30 \%$ of the area of external walls are covered by windows with aluminium frames.

This study uses the information in Tables 5.23 and 5.24 to calculate the embodied energy of OCRT accommodation services by type of construction as shown in Table 5.25. 
Table 5.25: Initial embodied energy intensities for BIAC standard house

\begin{tabular}{|c|c|c|c|c|}
\hline \multirow{3}{*}{$\begin{array}{l}\text { Building } \\
\text { element } \\
\text { Foundation }\end{array}$} & \multicolumn{4}{|c|}{ Embodied energy intensity $\left(\mathrm{MJ} / \mathrm{m}^{2}\right)$} \\
\hline & \multicolumn{2}{|c|}{ Light construction } & \multicolumn{2}{|c|}{ Heavy construction } \\
\hline & 30 & $2 \%$ & 80 & $3 \%$ \\
\hline Floor & 220 & $12 \%$ & 740 & $28 \%$ \\
\hline Walls $^{1}$ & 450 & $25 \%$ & 820 & $31 \%$ \\
\hline Roof & 400 & $22 \%$ & 340 & $13 \%$ \\
\hline Joinery & 230 & $13 \%$ & 230 & $9 \%$ \\
\hline $\begin{array}{l}\text { Electrical } \\
\text { work }\end{array}$ & 100 & $5 \%$ & 100 & $4 \%$ \\
\hline Plumbing & 170 & $9 \%$ & 170 & $6 \%$ \\
\hline Finishes & 210 & $12 \%$ & 160 & $6 \%$ \\
\hline Total & 1,810 & $100 \%$ & 2,640 & $100 \%$ \\
\hline
\end{tabular}

Table 5.26 contains the calculated embodied energy of OCRT accommodation services by types of construction through the measurement of the embodied energy of wall, roof, floor and foundation as used in each type of construction.

\section{a. 1. OCRT Accommodation Services with Timber Structure: Embodied Energy}

As shown in Table 5.26, the embodied energy of walls (30\% covered by windows), roofs, floors and foundations used in an OCRT timber structure are assumed to have equal $\mathrm{GJ} / \mathrm{m}^{2}$ (and $\mathrm{MJ} / \mathrm{m}^{2}$ embodied energies) to the light construction in Table 5.25.

\section{a. 2. OCRT Accommodation Services with Timber and Masonry Structure: Em- bodied Energy}

Table 5.26 determines that OCRT timber and masonry type of construction uses timber frame, brick veneer for walls, timber frame for roofs and floors, and light foundations. The embodied energy for OCRT timber frame and brick veneer wall (Table 5.26) is assumed to be equal with the embodied energy for heavy construction walls (30\% covered by windows) cited in Table $5.25\left(0.82 \mathrm{GJ} / \mathrm{m}^{2}\right)$. Furthermore, the embodied energy for roofs and floors (Table 5.26) are assumed to be equal with the embodied energy for heavy construction roofs and floors in Table 5.25. $\left(0.34 \mathrm{GJ} / \mathrm{m}^{2}\right.$ and $0.74 \mathrm{GJ} / \mathrm{m}^{2}$ respectively). 
Table 5.26: Initial embodied energy intensities for OCRT- accommodation services by type of construction.

\begin{tabular}{|c|c|c|c|c|c|c|c|c|}
\hline $\begin{array}{l}\text { Type of con- } \\
\text { struction }\end{array}$ & Wall & $\begin{array}{l}\text { GJ per } \\
\mathrm{m}^{2}\end{array}$ & Roof & $\begin{array}{l}\text { GJ per } \\
\mathrm{m}^{2}\end{array}$ & Floor & $\begin{array}{l}\text { GJ } \\
\text { per } \\
\mathrm{m}^{2}\end{array}$ & Foundation & $\begin{array}{c}\text { GJ per } \\
\mathrm{m}^{2}\end{array}$ \\
\hline Timber & Timber & 0.45 & Timber frame & 0.4 & $\begin{array}{l}\text { Timber } \\
\text { Frame }\end{array}$ & 0.22 & $\begin{array}{l}\text { (Light con- } \\
\text { struction) }\end{array}$ & 0.03 \\
\hline $\begin{array}{l}\text { Timber and } \\
\text { masonry }\end{array}$ & $\begin{array}{l}\text { Timber frame, } \\
\text { brick veneer }\end{array}$ & 0.82 & $\begin{array}{l}\text { Timber frame } \\
\text { (light con- } \\
\text { struction) }\end{array}$ & 0.4 & Timber & 0.74 & $\begin{array}{l}\text { Heavy con- } \\
\text { struction }\end{array}$ & $0.08^{1}$ \\
\hline $\begin{array}{l}\text { Mud brick } \\
\text { (mixed with } \\
\text { other materials) }\end{array}$ & Mud brick*1 & 0.18 & $\begin{array}{l}\text { Timber frame } \\
\text { (light con- } \\
\text { struction) }\end{array}$ & 0.4 & $\begin{array}{l}\text { Heavy } \\
\text { con- } \\
\text { struction }\end{array}$ & 0.74 & $\begin{array}{l}\text { Heavy con- } \\
\text { struction }\end{array}$ & $0.08^{1}$ \\
\hline $\begin{array}{l}\text { Brick mixed } \\
\text { with other ma- } \\
\text { terials (old } \\
\text { technology) }\end{array}$ & Brick $^{2}$ & 3.9 & $\begin{array}{l}\text { Timber frame } \\
\text { (light con- } \\
\text { struction) }\end{array}$ & 0.4 & $\begin{array}{l}\text { Heavy } \\
\text { con- } \\
\text { struction }\end{array}$ & 0.74 & $\begin{array}{l}\text { Heavy con- } \\
\text { struction. }\end{array}$ & 0.08 \\
\hline $\begin{array}{l}\text { Brick mixed } \\
\text { with other ma- } \\
\text { terials (new } \\
\text { technology) }\end{array}$ & $\begin{array}{l}\text { Brick }(0.35 \mathrm{~m} \\
\text { wide } \times 3 \mathrm{~m} \\
\text { high })\end{array}$ & 1.8 & $\begin{array}{l}\text { Timber frame } \\
\text { (light con- } \\
\text { struction) }\end{array}$ & 0.4 & $\begin{array}{l}\text { Heavy } \\
\text { con- } \\
\text { struction }\end{array}$ & 0.74 & $\begin{array}{l}\text { Heavy con- } \\
\text { struction. }\end{array}$ & 0.08 \\
\hline Stone $^{3}$ (local) & $\begin{array}{l}\text { Stone }(0.4 \mathrm{~m} \\
\text { wide } \times 3 \mathrm{~m} \\
\text { high) }\end{array}$ & 0.36 & $\begin{array}{l}\text { Timber frame } \\
\text { (light con- } \\
\text { struction) }\end{array}$ & 0.4 & $\begin{array}{l}\text { Heavy } \\
\text { con- } \\
\text { struction }\end{array}$ & 0.74 & $\begin{array}{l}\text { Heavy con- } \\
\text { struction. }\end{array}$ & 0.08 \\
\hline $\begin{array}{l}\text { Stone }^{3} \text { (im- } \\
\text { ported) }\end{array}$ & $\begin{array}{l}\text { Stone }(0.4 \mathrm{~m} \\
\text { wide } \times 3 \mathrm{~m} \\
\text { high) }\end{array}$ & 5.75 & $\begin{array}{l}\text { Timber frame } \\
\text { (light con- } \\
\text { struction) }\end{array}$ & 0.4 & $\begin{array}{l}\text { Heavy } \\
\text { con- } \\
\text { struction }\end{array}$ & 0.74 & $\begin{array}{l}\text { Heavy con- } \\
\text { struction. }\end{array}$ & 0.08 \\
\hline Concrete $^{4}$ & $\begin{array}{l}\text { Ready mixed } \\
\text { concrete ( } \\
0.2 \mathrm{~m} \\
\text { wide } \times 3 \mathrm{~m} \text { high }\end{array}$ & 1.93 & $\begin{array}{l}\text { Timber frame } \\
\text { (light con- } \\
\text { struction) }\end{array}$ & 0.4 & $\begin{array}{l}\text { Heavy } \\
\text { con- } \\
\text { struction }\end{array}$ & 0.74 & $\begin{array}{l}\text { Heavy con- } \\
\text { struction. }\end{array}$ & 0.08 \\
\hline \multicolumn{9}{|c|}{$\begin{array}{l}\text { 1. Mud brick wall } 0.35 \mathrm{~m} \text { wide and } 3 \mathrm{~m} \text { high in } 100 \mathrm{~m}^{2} \text { building (only exterior walls calculated) }=40.5 \mathrm{~m}^{3}=0.4 \\
\mathrm{~m}^{3} / \mathrm{m}^{2} \\
\text { 2. Brick wall } 0.35 \mathrm{~m} \text { wide and } 3 \mathrm{~m} \text { high in } 100 \mathrm{~m}^{2} \text { building (only exterior walls calculated) }=40.5 \mathrm{~m}^{3}=0.4 \mathrm{~m}^{3} / \mathrm{m}^{2} \\
\text { 3. Stone wall } 0.4 \mathrm{~m} \text { wide and } 3 \mathrm{~m} \text { high in } 100 \mathrm{~m}^{2} \text { building (only exterior walls calculated) }=46.1 \mathrm{~m}^{3}=0.46 \mathrm{~m}^{3} / \mathrm{m}^{2} \\
\text { 4. Concrete wall } 0.2 \mathrm{~m} \text { wide and } 3 \mathrm{~m} \text { high in } 100 \mathrm{~m}^{2} \text { building (only exterior walls calculated) }=23.5 \mathrm{~m}^{3}=0.2 \mathrm{~m}^{3} / \mathrm{m}^{2} \\
\text { * Reference: Australia's guide to environmentally sustainable homes. www.yourhome.gov.au/technical/fs52.html }\end{array}$} \\
\hline
\end{tabular}

\section{a. 3. OCRT Accommodation Services with Mud Brick Structure: Embodied En- ergy}

As shown in Table 5.26, the type mud brick (mixed with other materials) uses mud brick walls the embodied energy of which is calculated through the following steps:

1. Embodied energy of mud $=0.0016 \mathrm{MJ} / \mathrm{kg}$ (Chel and Tiwari, 2009:1968)

2. The weight of $1 \mathrm{~m}^{3}$ mud packed $=1,906 \mathrm{~kg}$ (www. simetric.co.UK)

3. Embodied energy of $1 \mathrm{~m}^{3}$ mud packed $=1,906 \times 0.0016=3.05 \mathrm{MJ} / \mathrm{m}^{3}=0.003 \mathrm{GJ} / \mathrm{m}^{3}$

4. Mud brick wall $0.35 \mathrm{~m}$ wide and $3 \mathrm{~m}$ high in $100 \mathrm{~m}^{2}$ building (exterior walls only) and assuming windows occupy 30\% of total area of exterior walls (36 $\mathrm{m}^{2}$ of 120 $\left.\mathrm{m}^{2}\right)=27.9 \mathrm{~m}^{3}=0.28 \mathrm{~m}^{3} / \mathrm{m}^{2}$

5. Embodied energy of mud brick wall $0.35 \mathrm{~m}$ wide and $3 \mathrm{~m}$ high in $100 \mathrm{~m}^{2}$ building assuming windows occupy $30 \%$ of total area of exterior walls $\left(36 \mathrm{~m}^{2}\right.$ of $\left.120 \mathrm{~m}^{2}\right)=$ $27.9 \mathrm{~m}^{3}$ (mud brick) $\times 0.003 \mathrm{GJ} / \mathrm{m}^{3}=0.084 \mathrm{GJ} / 100 \mathrm{~m}^{2}=0.00084 \mathrm{GJ} / \mathrm{m}^{2}$ 
6. Embodied energy of timber frame window $\left(1.2 \mathrm{~m}^{2} \times 1.2 \mathrm{~m}^{2}\right)=738 \mathrm{MJ}=0.74 \mathrm{GJ}$

7. Embodied energy of $1 \mathrm{~m}^{2}$ timber frame window $=512.5 \mathrm{MJ} / \mathrm{m}^{2}=0.51 \mathrm{GJ} / \mathrm{m}^{2}$

8. Embodied energy of $36 \mathrm{~m}^{2}$ (30\% exterior wall area) timber frame window/100 $\mathrm{m}^{2}$ (building area) $=18.36 \mathrm{GJ} / 100 \mathrm{~m}^{2}=0.18 \mathrm{GJ} / \mathrm{m}^{2}$

9. Embodied energy mud brick wall $0.35 \mathrm{~m}$ wide and $3 \mathrm{~m}$ high in $100 \mathrm{~m}^{2}$ building including windows $=0.18 \mathrm{GJ} / \mathrm{m}^{2}+0.00084 \mathrm{GJ} / \mathrm{m}^{2}=0.1808 \mathrm{GJ} / \mathrm{m}^{2} \sim 0.18 \mathrm{GJ} / \mathrm{m}^{2}$

In Table 5.26, the embodied energy of roofs in OCRT mud brick construction is assumed equal with the embodied energy of light construction roofs $\left(0.4 \mathrm{GJ} / \mathrm{m}^{2}\right)$ in Table 5.25. Moreover, in this type of OCRT construction, the embodied energy for floors and foundations are assumed equal with the embodied energy for heavy construction floor and foundation $\left(0.74 \mathrm{GJ} / \mathrm{m}^{2}\right.$ and $0.08 \mathrm{GJ} / \mathrm{m}^{2}$ respectively) as shown in Table 5.25.

\section{a. 4. Brick (Old Technology) Mixed with Other Material}

Since most of the OCRT accommodation buildings with brick construction are RB and historic buildings and used old technology to produce brick (see Appendix5) this study considers brick (old technology) as the dominant used material for brick construction. Moreover, as most of the buildings with brick construction use brick wall as bearing wall through using old technology the average width of this type of wall is considered to be $0.35 \mathrm{~m}$.

In Table 5.26, the embodied energy of a brick wall (old technology) is calculated through the following steps:

1. Embodied energy of brick (old technology) $=13,188 \mathrm{MJ} / \mathrm{m}^{3}=13.188 \mathrm{GJ} / \mathrm{m}^{3}$ (reference: Mithraratne et al, 2007: 215)

2. Brick wall $0.35 \mathrm{~m}$ wide and $3 \mathrm{~m}$ high in $100 \mathrm{~m}^{2}$ building for exterior walls assuming windows occupy $30 \%\left(36 \mathrm{~m}^{2}\right.$ of $\left.120 \mathrm{~m}^{2}\right)$ the area of exterior walls $=27.9 \mathrm{~m}^{3}=$ $0.28 \mathrm{~m}^{3} / \mathrm{m}^{2}$ (house area)

3. Embodied energy of a brick wall $0.35 \mathrm{~m}$ wide and $3 \mathrm{~m}$ high in $100 \mathrm{~m}^{2}$ building excluding the windows $=27.9 \mathrm{~m}^{3}$ (brick) $\times 13.188 \mathrm{GJ} / \mathrm{m}^{3}=368 \mathrm{GJ} / 100 \mathrm{~m}^{2}=3.68 \mathrm{GJ} / \mathrm{m}^{2}$

4. Embodied energy of $36 \mathrm{~m}^{2}$ timber frame windows (as above) $=18.36 \mathrm{GJ} / 100 \mathrm{~m}^{2}=$ $0.18 \mathrm{GJ} / \mathrm{m}^{2}$

5. Total embodied energy of a brick (old technology) wall $0.35 \mathrm{~m}$ wide and $3 \mathrm{~m}$ high in $100 \mathrm{~m}^{2}$ building with windows $=0.18 \mathrm{GJ} / \mathrm{m}^{2}+3.68 \mathrm{GJ} / \mathrm{m}^{2}=3.86 \mathrm{GJ} / \mathrm{m}^{2} \sim 3.9 \mathrm{GJ} / \mathrm{m}^{2}$ 
In Table 5.26, the embodied energy of roofs in OCRT brick (old technology) construction is assumed equal to the embodied energy of light construction roofs $\left(0.4 \mathrm{GJ} / \mathrm{m}^{2}\right)$ in Table 5.25. In addition, in this type of OCRT construction, the embodied energy for floors and foundations are assumed equal with the embodied energy for heavy construction floor and foundations $\left(0.74 \mathrm{GJ} / \mathrm{m}^{2}\right.$ and $0.08 \mathrm{GJ} / \mathrm{m}^{2}$ respectively) as shown in Table 5.25 .

\section{a. 5. Brick (New Technology) Mixed with Other Material}

Although in OCRT accommodation services with brick construction, the buildings are RB and historic buildings (see Appendix 5) and use brick (old technology), this thesis also calculates embodied energy of brick (new technology) building to be compared with the embodied energy of brick (old technology).

In Table 5.26, the embodied energy of a brick wall (new technology) is calculated through the following steps:

1. Embodied energy of brick (new technology) $=5,310 \mathrm{MJ} / \mathrm{m}^{3}=5.31 \mathrm{GJ} / \mathrm{m}^{3}$ (reference: Mithraratne et al, 2007: 215)

2. A brick wall $0.35 \mathrm{~m}$ wide and $3 \mathrm{~m}$ high in $100 \mathrm{~m}^{2}$ building assuming the windows occupy $30 \%\left(36 \mathrm{~m}^{2}\right.$ of $\left.120 \mathrm{~m}^{2}\right)$ of the exterior walls $=27.9 \mathrm{~m}^{3}=0.28 \mathrm{~m}^{3} / \mathrm{m}^{2}$ (house area)

3. The embodied energy of a brick wall $0.35 \mathrm{~m}$ wide and $3 \mathrm{~m}$ high in $100 \mathrm{~m}^{2}$ building excluding windows that cover $30 \%\left(36 \mathrm{~m}^{2}\right.$ of $\left.120 \mathrm{~m}^{2}\right)$ of the exterior walls = $27.9 \mathrm{~m}^{3}$ (brick) $\times 5.310 \mathrm{GJ} / \mathrm{m}^{3}=157.7 \mathrm{GJ} / 100 \mathrm{~m}^{2}=1.58 \mathrm{GJ} / \mathrm{m}^{2}$

4. Embodied energy of $36 \mathrm{~m}^{2}$ (30\% exterior wall area) timber frame windows in 100 $\mathrm{m}^{2}$ building $=18.36 \mathrm{GJ} / 100 \mathrm{~m}^{2}=0.18 \mathrm{GJ} / \mathrm{m}^{2}$

5. Embodied energy of brick (new technology) wall $0.35 \mathrm{~m}$ wide and $3 \mathrm{~m}$ high in 100 $\mathrm{m}^{2}$ building including windows $=0.18 \mathrm{GJ} / \mathrm{m}^{2}+1.58 \mathrm{GJ} / \mathrm{m}^{2}=1.78 \mathrm{GJ} / \mathrm{m}^{2} \sim 1.8 \mathrm{GJ} / \mathrm{m}^{2}$

In Table 5.26, the embodied energy of roofs in OCRT brick (new technology) construction is assumed equal with the embodied energy of light construction roofs $\left(0.4 \mathrm{GJ} / \mathrm{m}^{2}\right)$ in Table 5.25. Likewise, in this type of OCRT construction, the embodied energy for floors and foundations are assumed equal with the embodied energy for heavy construction floors and foundations $\left(0.74 \mathrm{GJ} / \mathrm{m}^{2}\right.$ and $0.08 \mathrm{GJ} / \mathrm{m}^{2}$ respectively), as in Table 5.25. 


\section{a. 6. Local Stone}

In Table 5.26, the embodied energy of a local stone wall is calculated through the following steps:

1. Embodied energy of local stone $=1,890 \mathrm{MJ} / \mathrm{m}^{3}=1.89 \mathrm{GJ} / \mathrm{m}^{3}$ (reference: Mithraratne et al, 2007: 218)

2. The volume of a stone wall $0.4 \mathrm{~m}$ wide and $3 \mathrm{~m}$ high in $100 \mathrm{~m}^{2}$ building, assuming the windows occupy $30 \%\left(36 \mathrm{~m}^{2}\right.$ of $\left.120 \mathrm{~m}^{2}\right)$ of the exterior walls $=31.7 \mathrm{~m}^{3} / 100 \mathrm{~m}^{2}$ $=0.31 \mathrm{~m}^{3} / \mathrm{m}^{2}$

3. Embodied energy of a local stone wall $0.4 \mathrm{~m}$ wide and $3 \mathrm{~m}$ high in $100 \mathrm{~m}^{2}$ building excluding the windows as described above $=31.7 \mathrm{~m}^{3}$ (stone) $\times 1.89 \mathrm{GJ} / \mathrm{m}^{3}=59.9$ $\mathrm{GJ} / 100 \mathrm{~m}^{2}=0.59 \mathrm{GJ} / \mathrm{m}^{2}$

4. Embodied energy of $36 \mathrm{~m}^{2}$ (30\% exterior wall area) timber frame windows $=18.36$ $\mathrm{GJ} / 100 \mathrm{~m}^{2}=0.18 \mathrm{GJ} / \mathrm{m}^{2}$

5. Total embodied energy of local stone wall $0.4 \mathrm{~m}$ wide and $3 \mathrm{~m}$ high in $100 \mathrm{~m}^{2}$ building including windows $=0.18 \mathrm{GJ} / \mathrm{m}^{2}+0.18 \mathrm{GJ} / \mathrm{m}^{2}=0.36 \mathrm{GJ} / \mathrm{m}^{2}$

In Table 5.26, the embodied energy of roofs in OCRT local stone construction is assumed equal with the embodied energy of light construction roofs $\left(0.4 \mathrm{GJ} / \mathrm{m}^{2}\right)$ in Table 5.25. Moreover, in this type of OCRT construction, the embodied energy for floors and foundations are assumed equal with the embodied energy for heavy construction floors and foundations $\left(0.74 \mathrm{GJ} / \mathrm{m}^{2}\right.$ and $0.08 \mathrm{GJ} / \mathrm{m}^{2}$ respectively) in Table 5.25.

\section{a.7 . Imported Stone}

In Table 5.26, the embodied energy of an imported stone wall is calculated in exactly the same way as for a local stone wall above, but using the following embodied energy coefficient.

Embodied energy of stone (imported) $=17,610 \mathrm{MJ} / \mathrm{m}^{3}=17.61 \mathrm{GJ} / \mathrm{m}^{3}$ (reference: Mithraratne et al, 2007: 218)

The embodied energy of an imported stone wall $0.4 \mathrm{~m}$ wide and $3 \mathrm{~m}$ high in $100 \mathrm{~m}^{2}$ building with windows thus $=0.18 \mathrm{GJ} / \mathrm{m}^{2}+5.58 \mathrm{GJ} / \mathrm{m}^{2}=5.75 \mathrm{GJ} / \mathrm{m}^{2}$

In Table 5.26, the embodied energy of roofs in OCRT imported stone construction is assumed equal with the embodied energy of light construction roofs $\left(0.4 \mathrm{GJ} / \mathrm{m}^{2}\right)$ in Ta- 
ble 5.25. In addition, in this type of OCRT construction, the embodied energy for floors and foundations are assumed equal with the embodied energy for heavy construction floor and foundation $\left(0.74 \mathrm{GJ} / \mathrm{m}^{2}\right.$ and $\left.0.08 \mathrm{GJ} / \mathrm{m}^{2}\right)$ in Table 5.25.

\section{a. 8. Concrete}

In Table 5.26, embodied energy of concrete wall with aluminium framed windows is calculated through the following steps:

1. Embodied energy of concrete (ready $\operatorname{mix} 30 \mathrm{MPa}$ ) $=2,762 \mathrm{MJ} / \mathrm{m}^{3}=2.8 \mathrm{GJ} / \mathrm{m}^{3}$ (reference: Mithraratne et al, 2007: 218)

2. Volume of a concrete wall $0.2 \mathrm{~m}$ wide and $3 \mathrm{~m}$ high in $100 \mathrm{~m}^{2}$ building excluding windows that occupy $30 \%\left(36 \mathrm{~m}^{2}\right.$ of $\left.120 \mathrm{~m}^{2}\right)$ of the exterior walls $=16.3 \mathrm{~m}^{3} / 100 \mathrm{~m}^{2}$ $=0.16 \mathrm{~m}^{3} / \mathrm{m}^{2}$

3. Embodied energy of a concrete wall $0.2 \mathrm{~m}$ wide and $3 \mathrm{~m}$ high in $100 \mathrm{~m}^{2}$ building excluding windows $=16.3 \mathrm{~m}^{3}($ concrete $) \times 2.8 \mathrm{GJ} / \mathrm{m}^{3}=45.6 \mathrm{GJ} / 100 \mathrm{~m}^{2}=0.46 \mathrm{GJ} / \mathrm{m}^{2}$

4. Embodied energy of aluminium framed window $\left(1.2 \mathrm{~m}^{2} \times 1.2 \mathrm{~m}^{2}\right)=5,978 \mathrm{MJ} \sim 6$ GJ (reference: Wolf, 2011:5)

5. Embodied energy of $1 \mathrm{~m}^{2}$ aluminium framed window $=4,151 \mathrm{MJ} / \mathrm{m}^{2}=4.15 \mathrm{GJ} / \mathrm{m}^{2}$

6. Embodied energy of $36 \mathrm{~m}^{2}$ (30\% exterior walls area) aluminium framed window in $100 \mathrm{~m} 2$ (building area) $=147.6 \mathrm{GJ} / 100 \mathrm{~m}^{2}=1.47 \mathrm{GJ} / \mathrm{m}^{2}$

7. Embodied energy of a concrete wall $0.2 \mathrm{~m}$ wide and $3 \mathrm{~m}$ high in $100 \mathrm{~m}^{2}$ building including windows, therefore $=1.47 \mathrm{GJ} / \mathrm{m}^{2}+0.46 \mathrm{GJ} / \mathrm{m}^{2}=1.93 \mathrm{GJ} / \mathrm{m}^{2}$

In Table 5.26, the embodied energy of roofs in OCRT concrete construction is assumed equal with the embodied energy of light construction roofs $\left(0.4 \mathrm{GJ} / \mathrm{m}^{2}\right)$ in Table 5.25. Furthermore, in this type of OCRT construction, the embodied energy for floors and foundations are assumed equal with the embodied energy for heavy construction floor and foundation $\left(0.74 \mathrm{GJ} / \mathrm{m}^{2}\right.$ and $\left.0.08 \mathrm{GJ} / \mathrm{m}^{2}\right)$ in Table 5.25 .

\section{a. 9. OCRT Accommodation Services: Embodied Energy Summary}

Using the results from Table 5.26, the integrated embodied energy of wall, roof, floor and foundations used in each type of OCRT accommodation is presented in Table 5.27 and Figure 5.30.a. As revealed, imported stone construction has the largest embodied energy of $6.97 \mathrm{GJ} / \mathrm{m}^{2}$ and a timber structure has the lowest embodied energy at 1.1 
$\mathrm{GJ} / \mathrm{m}^{2}$. The embodied energy of other types of OCRT constructions are in the range of $1.4-5.12 \mathrm{MJ} / \mathrm{m}^{2}$.

Table 5.27. Embodied energy of OCRT- types of construction (2011)

Type of building structure

Timber

Timber and masonry

Mud brick (mixed with other materials)

Brick $^{1}$ mixed with other materials (old technology)

Brick $^{1}$ mixed with other materials (new technol-

ogy)

Stone ${ }^{2}$ (local)

Stone $^{2}$ (imported)

Concrete
Embodied energy use $\left(\mathrm{GJ} / \mathrm{m}^{2}\right)$

1.1

2.04

1.4

5.12

3.02

1.58

6.97

3.15

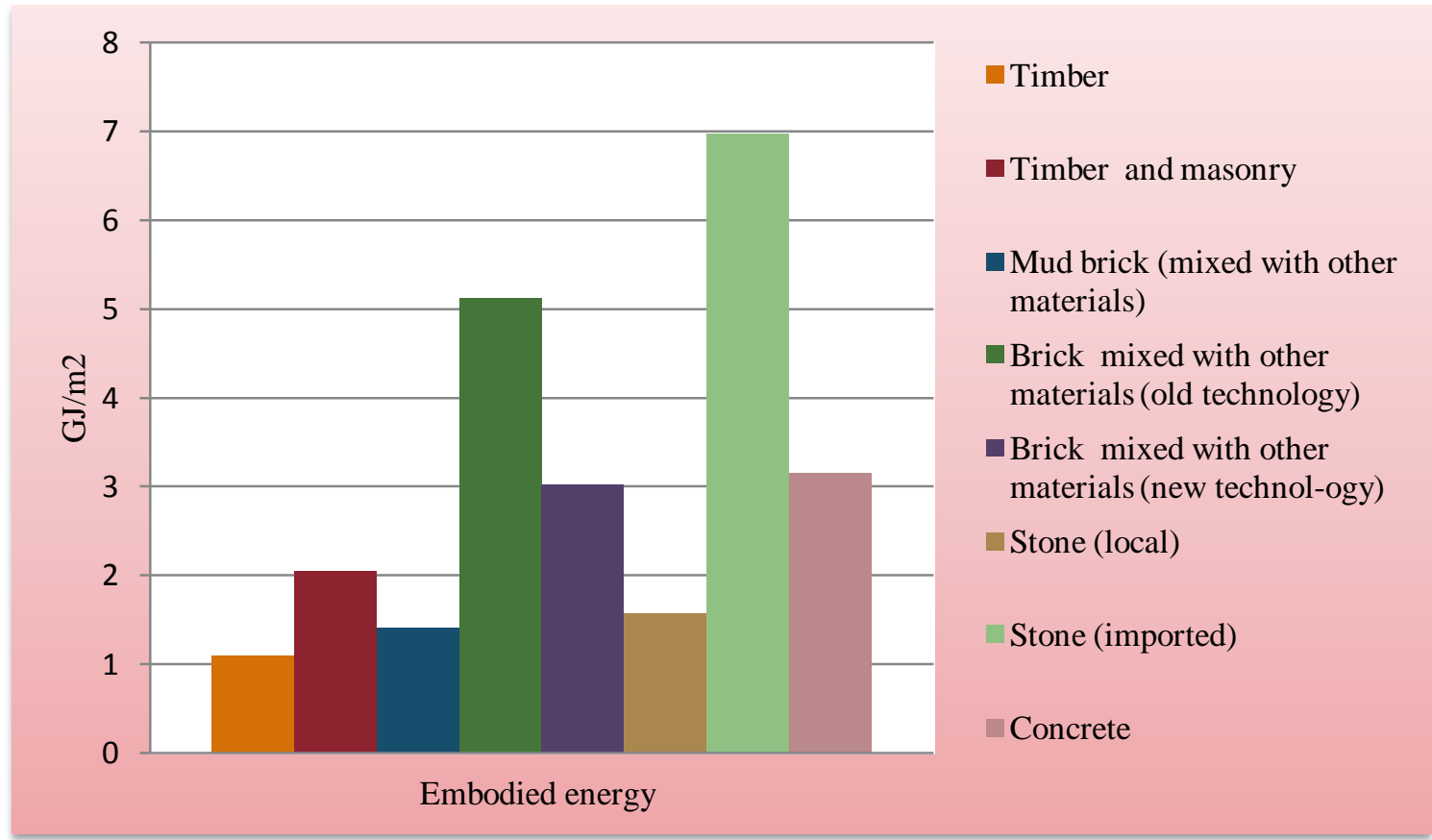

Figure 5.30.a: Embodied energy of OCRT- types of construction (2011)

\section{b. OCRT Accommodation Services: Operating Energy}

As shown in Figure 5.30 OCRT accommodation services (NB and RB) use conventional sources of energy and facilities so that operational energy for heating and lighting in both NB and RB types should be similar. Based on this assumption, this thesis uses the operating energy for each type of New Zealand accommodation service proposed by Becken et al (2001:376) (Table 5.28). As indicated in Table 5.28, in New Zealand, the hotel category has the largest operational energy at $155 \mathrm{MJ} /$ visitor night followed by B\&B (110 MJ/visitor night), Backpacker (39 MJ/visitor night), motel (32 MJ/visitor night) and camping grounds (25 MJ/visitor night). 


\begin{tabular}{|l|c|c|}
\hline Category & Energy use (MJ/m²/year) & $\begin{array}{c}\text { Energy use per visitor night } \\
\text { (MJ/visitor) }\end{array}$ \\
\hline Hotel & 571 & 155 \\
\hline B\&B & 300 & 110 \\
\hline Motel & 250 & 32 \\
\hline Backpacker & 617 & 39 \\
\hline Camping ground & N/A & 25 \\
\hline - Reference: Becken et al, 2001: 376 & & \\
\hline
\end{tabular}

\section{c. OCRT Accommodation Services: Life Cycle Energy Use and EF}

The life cycle energy use of each type of OCRT accommodation is calculated as the sum of the embodied and operational energy over an assumed life of the building, here taken as 50 years. In Tables 5.29-5.35 the embodied energy use of each type of construction is calculated through the following equation:

Embodied energy (GJ/year) of each type of construction $=$ Total area $\left(\mathrm{m}^{2}\right)$ of the type of construction (see Table 5.23) $\times$ Embodied energy over a 50 year life cycle $\left(\mathrm{GJ} / \mathrm{m}^{2}\right) \div 50$

In this equation 'total area $\left(\mathrm{m}^{2}\right)$ of the type of construction' is calculated as follows: Total area $\left(\mathrm{m}^{2}\right)$ of the type of construction = Capacity of the type of construction (see Table 5.14) $\times$ average area/bed of the type of accommodation service (see Table 5.20) In Tables 5.28-5.34 the operational energy use (GJ/year) of each type of construction is equivalent to the area occupied $\times$ operational energy use $\left(\mathrm{GJ} / \mathrm{m}^{2}\right)$ of the type of construction

In the above equation the area occupied of each type of construction is equivalent to the percentage occupancy rate of the type of accommodation $\times$ total area $\left(\mathrm{m}^{2}\right)$ of the type of construction. Note, this is not the same as the overall occupied area shown in Table 5.23 , which is the total space occupied in a year.

In Table s 5.29- 5.35:

1. Life cycle energy use (GJ/year) of each type of construction = Operational energy (GJ/year) + embodied energy (GJ/year)

2. EF (gha/year) of each type of construction = life cycle energy $(\mathrm{GJ} /$ year $) \div 100$ (GJ/ha) (global average carrying capacity) 
3. The total EF of each type of OCRT accommodation services is equivalent to the sum of its types of construction EFs (Tables 5.29-5.35).

\section{c.1 . SC Accommodation Service: Life Cycle Energy Use and EF}

As shown in Table 5.29, SC timber construction with an area of $11,466 \mathrm{~m}^{2}$ accounts for the largest embodied energy value of $252.2 \mathrm{GJ} /$ year followed by $1622.5 \mathrm{~m}^{2}$ timber and masonry (66.2 GJ/year). Other SC types of construction including mud brick mixed with other materials $\left(973.5 \mathrm{~m}^{2}\right)$, brick mixed with other materials $\left(177 \mathrm{~m}^{2}\right)$ and local stone $\left(590 \mathrm{~m}^{2}\right)$ use embodied energy in the range of 18.1- 27.3 GJ/year (Table 5.29).

In Table 5.29, SC operational energy is assumed to be $0.3 \mathrm{GJ} / \mathrm{m}^{2} /$ year, equal to $\mathrm{B} \& \mathrm{~B}$ operational energy use (see Table 5.28). As determined in Table 5.29, SC timber construction accounts for the largest total operational energy $450.6 \mathrm{GJ} /$ year and other types of SC construction operational energy use are in the rage of 6.9- $63.7 \mathrm{GJ} /$ year.

In addition, the $11,466 \mathrm{~m}^{2}$ of SC timber construction as expected account for the largest total life cycle energy of $702.8 \mathrm{GJ} /$ year and other types of construction have life cycle energy between 25-129.9 GJ/year. Influenced by its life cycle energy use, as shown in Table 5.29, SC timber construction has the biggest EF (7.03gha), much larger than other SC construction types which have EFs between 0.42-1.29 gha. Also from Table 5.29, timber construction with the lowest embodied energy over a 50 year life cycle $\left(1.1 \mathrm{GJ} / \mathrm{m}^{2}\right)$ can be considered the dominant type of SC construction. Table 5.29 shows that the total EF of OCRT SC accommodation services is 9.65 gha. 


\begin{tabular}{|c|c|c|c|c|c|c|c|c|}
\hline Material & $\begin{array}{l}\text { Embodied } \\
\text { energy } \\
\text { Over a 50- } \\
\text { year life } \\
\text { cycle } \\
\left(\mathrm{GJ} / \mathrm{m}^{2}\right)\end{array}$ & $\begin{array}{l}\text { Total } \\
\text { Area } \\
\left(\mathrm{m}^{2}\right)\end{array}$ & $\begin{array}{l}\text { Embodied } \\
\text { energy } \\
\text { (GJ/year) }\end{array}$ & $\begin{array}{l}\text { Operation } \\
\text { energy } \\
(\mathrm{GJ} / \\
\mathrm{m}^{2} / \text { year) }\end{array}$ & $\begin{array}{l}\text { Occupied } \\
\text { area }\left(\mathrm{m}^{2}\right)^{1}\end{array}$ & $\begin{array}{l}\text { Operating } \\
\text { energy } \\
\text { (GJ/year) }\end{array}$ & $\begin{array}{l}\text { Life- } \\
\text { cycle } \\
\text { energy } \\
\text { use (GJ/ } \\
\text { year) }\end{array}$ & $\begin{array}{l}\text { EF } \\
\text { gha/yea } \\
\text { r }\end{array}$ \\
\hline Timber & 1.1 & 11,466 & 252.2 & 0.3 & 1,502 & 450.6 & 702.8 & 7.03 \\
\hline $\begin{array}{l}\text { Timber and } \\
\text { masonry }\end{array}$ & 2.04 & 1622.5 & 66.2 & 0.3 & 212.5 & 63.7 & 129.9 & 1.29 \\
\hline $\begin{array}{l}\text { Mud brick } \\
\text { (mixed with } \\
\text { other mate- } \\
\text { rials) }\end{array}$ & 1.4 & 973.5 & 27.3 & 0.3 & 127.5 & 38.2 & 65.5 & 0.66 \\
\hline $\begin{array}{l}\text { Brick mixed } \\
\text { with other } \\
\text { materials } \\
\text { (old tech- } \\
\text { nology) }\end{array}$ & 5.12 & 177 & 18.1 & 0.3 & 23 & 6.9 & 25 & 0.25 \\
\hline Stone (local) & 1.58 & 590 & 18.6 & 0.3 & 77 & 23.1 & 41.7 & 0.42 \\
\hline Concrete & - & - & - & - & - & - & - & - \\
\hline Total & & & 382.4 & & & 582.5 & 964.9 & 9.65 \\
\hline
\end{tabular}

\section{c. 2. B\&B Accommodation Services}

Table 5.30 shows B\&B timber construction with the lowest embodied energy over a 50 year life cycle $\left(1.1 \mathrm{GJ} / \mathrm{m}^{2}\right)$ accounts for the majority area of $9,000 \mathrm{~m}^{2}$ followed by timber and masonry $\left(6,760 \mathrm{~m}^{2}\right)$ and brick mixed with other materials $\left(2,000 \mathrm{~m}^{2}\right)$. Other types of construction including mud brick (mixed with other materials), local stone and concrete account for areas between 1,040-1,480 $\mathrm{m}^{2}$.

Table 5.30 demonstrates that $\mathrm{B} \& \mathrm{~B}$ timber and masonry construction with an area of $6,760 \mathrm{~m}^{2}$ accounts for the largest single embodied energy value of 275GJ/year, followed by brick mixed with other materials $\left(2,000 \mathrm{~m}^{2}\right.$ area, $204.7 \mathrm{GJ} /$ year $)$ and timber $(9,000$ $\mathrm{m}^{2}$ area, 198GJ/year). Other types of B\&B construction including mud brick (mixed with other materials), concrete and local stone with areas between 1,040-1,480 $\mathrm{m}^{2}$ have total embodied energies in the range of 29.12-85.7 GJ/year.

When it comes to operational energy, calculated as before based on occupancy, as shown in Table 5.30 the largest occupied area of $1,251 \mathrm{~m}^{2}$ in OCRT B\&B accommodation is timber construction, followed by $940 \mathrm{~m}^{2}$ timber and masonry, and $278 \mathrm{~m}^{2}$ brick (mixed with other materials). The occupied areas of other types of B\&B construction including mud brick (mixed with other materials), concrete and local stone are in the range of 144.6-206 $\mathrm{m}^{2}$. Table 5.30 indicates that B\&B timber construction accounts for 
the largest operating energy use of $375.3 \mathrm{GJ} /$ year and $573.3 \mathrm{GJ} /$ year life cycle energy (based on a 50 year life) followed by timber and masonry (282 and 557.8 GJ/year) and brick mixed with other materials (83.4 and 288.2 GJ/year). Other types of B\&B construction have operational and life cycle energy between (43.7 and 72.5 GJ/year) - (61.8 and 108.6 GJ/year).

Comparing the EF of B\&B construction types, timber has the largest EF (5.73gha) followed by timber and masonry (5.6gha) and brick mixed with other materials (2.9gha) (Table 5.30). The annual EF of other types of B\&B construction including mud brick (mixed with other materials), concrete and local stone are between 0.73 and 1.4 gha. The total life cycle energy and EF of OCRT B\&B accommodation services are 1,743 GJ/year and 17 gha /year (Table 5.30).

\begin{tabular}{|c|c|c|c|c|c|c|c|c|}
\hline Material & $\begin{array}{l}\text { Embod- } \\
\text { ied en- } \\
\text { ergy over } \\
\text { a } 50 \text { year } \\
\text { life cycle } \\
\left(\mathrm{GJ} / \mathrm{m}^{2}\right)\end{array}$ & $\begin{array}{l}\text { Total } \\
\text { Area } \\
\left(\mathrm{m}^{2}\right)^{3}\end{array}$ & $\begin{array}{l}\text { Em- } \\
\text { bodied } \\
\text { energy } \\
\text { (GJ/yea } \\
\text { r) }\end{array}$ & $\begin{array}{l}\text { Operation } \\
\text { energy } \\
(\mathrm{GJ} / \\
\left.\mathrm{m}^{2} / \text { year }\right)^{1}\end{array}$ & $\begin{array}{l}\text { Occupied } \\
\text { area }\left(\mathrm{m}^{2}\right)^{2}\end{array}$ & $\begin{array}{l}\text { Operat- } \\
\text { ing en- } \\
\text { ergy } \\
\text { (GJ/year) }\end{array}$ & $\begin{array}{l}\text { Life- } \\
\text { cycle } \\
\text { energy } \\
\text { use }(\mathrm{GJ} / \\
\text { year) }\end{array}$ & $\begin{array}{l}\text { EF } \\
\text { gha/y } \\
\text { ear }\end{array}$ \\
\hline Timber & 1.1 & 9,000 & 198 & 0.3 & 1,251 & 375.3 & 573.3 & 5.73 \\
\hline $\begin{array}{l}\text { Timber and } \\
\text { masonry }\end{array}$ & 2.04 & 6,760 & 275.8 & 0.3 & 940 & 282 & 557.8 & 5.6 \\
\hline $\begin{array}{l}\text { Mud brick } \\
\text { (mixed with } \\
\text { other mate- } \\
\text { rials) }\end{array}$ & 1.4 & 1040 & 29.12 & 0.3 & 144.6 & 43.4 & 72.5 & 0.73 \\
\hline $\begin{array}{l}\text { Brick mixed } \\
\text { with other } \\
\text { materials } \\
\text { (old tech- } \\
\text { nology) }\end{array}$ & 5.12 & 2000 & 204.8 & 0.3 & 278 & 83.4 & 288.2 & 2.9 \\
\hline Stone (local) & 1.58 & 1480 & 46.8 & 0.3 & 206 & 61.8 & 108.6 & 1.1 \\
\hline Concrete & 3.15 & 1360 & 85.7 & 0.3 & 189 & 56.7 & 142.4 & 1.4 \\
\hline Total & & & 840.2 & & & 859.2 & 1,743 & 17 \\
\hline \multicolumn{9}{|c|}{$\begin{array}{l}\text { 1. See Table } 5.24 \\
\text { 2. Occupancy rate of OCRT B\&B }=13.9 \% \text { (see Table 5.17) } \\
\left.\text { 3. Area per bed space }=40 \mathrm{~m}^{2} \text { (see Table } 5.18\right)\end{array}$} \\
\hline
\end{tabular}

\section{c. 3. BP Accommodation Services: Life Cycle Energy Use and EF}

As shown in Table $5.31 \mathrm{Bp}$ timber and timber mixed with masonry construction equally account for the largest floor area of $1,296 \mathrm{~m}^{2}$ (648 $\mathrm{m}^{2}$ each) followed by brick mixed with other materials $\left(216 \mathrm{~m}^{2}\right)$. Other type of constructions including mud brick (mixed with other materials) and local stone occupy smaller areas of 48 and $72 \mathrm{~m}^{2}$ respectively (Table 5.31). 
Table 5.31 indicates that the $648 \mathrm{~m}^{2}$ of Bp timber and masonry construction have the largest embodied energy of $26.4 \mathrm{GJ} /$ year, followed by brick mixed with other materials (22.1GJ/year) and timber (14.3GJ/year). Other types of B\&B constructions including mud brick (mixed with other materials) and local stone account for 2GJ/year and 1.5GJ/year respectively (Table 5.31).

As shown in Table 5.31, in comparison with other types of Bp construction, timber and timber mixed with masonry have the largest occupied area of $182 \mathrm{~m}^{2}$ ( $91 \mathrm{~m}^{2}$ each). Other types are smaller with brick mixed with other materials $\left(30 \mathrm{~m}^{2}\right)$, mud brick mixed with other materials $\left(10 \mathrm{~m}^{2}\right)$ and local stone $\left(7 \mathrm{~m}^{2}\right)$. Likewise, Bp timber and timber mixed with masonry constructions account for the largest value of operating energy at 111 GJ/year (each uses 55.5 GJ/year). Other types of Bp construction including mud brick (mixed with other materials), brick (mixed with other materials) and local stone have operational energy values of between 4.27 and 18.3 GJ/year (Table 5.31).

In comparison with other types of Bp construction, the timber and masonry type has the largest life cycle energy of $81.6 \mathrm{GJ} /$ year, followed by timber (69.8 GJ/year), brick mixed with other materials (40.4 GJ/year), mud brick mixed with other materials (8.1 GJ/year) and local stone (5.77 GJ/year) (Table 5.31).

Comparing the EFs of Bp constructions, timber and masonry has the largest EF (0.8 gha) followed by timber ( 0.7 gha) and brick mixed with other materials ( 0.4 gha), mud brick mixed with other materials (0.008 gha) and local stone (0.06gha). The total life cycle energy and EF of OCRT Bp accommodation services are 205 GJ/year and 2.05 gha /year respectively (Table 5.31). 


\begin{tabular}{|c|c|c|c|c|c|c|c|c|}
\hline \multicolumn{9}{|c|}{ Table 5.31: OCRT- life cycle energy use of Bp accommodation services (2011) } \\
\hline Material & $\begin{array}{l}\text { Embodied } \\
\text { energy } \\
\text { Over a } \\
50 \text {-year } \\
\text { life cycle } \\
\left(\mathrm{GJ} / \mathrm{m}^{2}\right)\end{array}$ & $\begin{array}{l}\text { Total } \\
\text { Area } \\
\left(\mathrm{m}^{2}\right)\end{array}$ & $\begin{array}{l}\text { Embodied } \\
\text { energy } \\
\text { (GJ/year) }\end{array}$ & $\begin{array}{l}\text { Operation } \\
\text { energy } \\
\text { (GJ/ } \\
\mathrm{m}^{2} / \text { year) }\end{array}$ & $\begin{array}{l}\text { Occupied } \\
\text { area }\left(\mathrm{m}^{2}\right)\end{array}$ & $\begin{array}{l}\text { Operating } \\
\text { energy } \\
\text { (GJ/year) }\end{array}$ & $\begin{array}{l}\text { Life- } \\
\text { cycle } \\
\text { energy } \\
\text { use } \\
\text { (GJ/ } \\
\text { year) }\end{array}$ & $\begin{array}{l}\text { EF } \\
\text { gha/year }\end{array}$ \\
\hline Timber & 1.1 & 648 & 14.3 & 0.61 & 91 & 55.5 & 69.8 & 0.7 \\
\hline $\begin{array}{l}\text { Timber } \\
\text { and ma- } \\
\text { sonry }\end{array}$ & 2.04 & 648 & 26.4 & 0.61 & 91 & 55.5 & 81.6 & 0.8 \\
\hline $\begin{array}{l}\text { Mud brick } \\
\text { (mixed } \\
\text { with other } \\
\text { materials) }\end{array}$ & 1.4 & 72 & 2 & 0.61 & 10 & 6.1 & 8.1 & 0.08 \\
\hline $\begin{array}{l}\text { Brick }^{1} \\
\text { mixed with } \\
\text { other mate- } \\
\text { rials (old } \\
\text { technology) }\end{array}$ & 5.12 & 216 & 22.1 & 0.61 & 30 & 18.3 & 40.4 & 0.4 \\
\hline $\begin{array}{l}\text { Stone (lo- } \\
\text { cal) }\end{array}$ & 1.58 & 48 & 1.5 & 0.61 & 7 & 4.27 & 5.77 & 0.06 \\
\hline Concrete & 3.15 & - & - & 0.61 & - & - & - & - \\
\hline Total & & & 66.3 & & & 139.67 & 205 & 2.05 \\
\hline
\end{tabular}

\section{c. 4. OCRT Motels: Life Cycle Energy Use and EF}

As shown in Table 5.32 timber construction forms the largest single area of motels at $6,033 \mathrm{~m}^{2}$ followed by timber and masonry $\left(2,755 \mathrm{~m}^{2}\right)$, and concrete $\left(1,335 \mathrm{~m}^{2}\right)$. Other types of construction are brick (mixed with other materials) and local stone and these occupy areas of 744 and $372 \mathrm{~m}^{2}$ (Table 5.32).

Table 5.32 indicates that motel timber construction accounts for the largest embodied energy value of $132.7 \mathrm{GJ} /$ year $\left(6,033 \mathrm{~m}^{2} \times 1.1 \mathrm{GJ} / \mathrm{m}^{2} / 50\right.$ years/ 50$)$, followed by timber and masonry $\left(2,755 \mathrm{~m}^{2} \times 2.04 \mathrm{GJ} / \mathrm{m}^{2} / 50\right.$ years/ $50=112 \mathrm{GJ} /$ year $)$, concrete $(1,335$ $\mathrm{m}^{2} \times 3.15 \mathrm{GJ} / 50$ years $/ 50=84.1 \mathrm{GJ} /$ year $)$, brick mixed with other materials $\left(744 \mathrm{~m}^{2} \times\right.$ $5.12 \mathrm{GJ} / \mathrm{m}^{2} / 50$ years $/ 50=76.2 \mathrm{GJ} /$ year $)$ and local stone $\left(372 \mathrm{~m}^{2} \times 1.58 \mathrm{GJ} / \mathrm{m}^{2} / 50\right.$ years $/$ $50=11.8 \mathrm{GJ} /$ year $)$.

As shown in Table 5.32, in comparison with other types of motel construction, timber comprises the majority of occupied area at $1,273 \mathrm{~m}^{2}$ followed by timber and masonry $\left(581 \mathrm{~m}^{2}\right)$, concrete $\left(282 \mathrm{~m}^{2}\right)$, brick mixed with other materials $\left(157 \mathrm{~m}^{2}\right)$, and local stone $\left(78 \mathrm{~m}^{2}\right)$. 
In Table 5.32 the operating energy for different constructions is calculated through following equation:

Operating energy $(\mathrm{GJ} /$ year $)=$ Occupied area $\left(\mathrm{m}^{2}\right) \times$ Operation energy $\left(\mathrm{m}^{2} /\right.$ year $)$

Table 5.32 indicates that motel timber constructions account for the largest value of operating energy at 318GJ/year and life cycle energy at 450.9GJ/year, followed by timber mixed with masonry (145.2GJ/year and 257.6 GJ/year). Operational and life cycle energies of other types of construction including concrete, brick mixed with other materials and local stone are in a range of (19.5GJ/year and 31.3GJ/year) to (70.5GJ/year and 154.6GJ/year) (Table 5.32).

In terms of EF for motels, timber motels account for the largest annual EF (4.5 gha) followed by timber and masonry (2.6 gha) (Table 5.32). The annual EF of brick mixed with other materials, concrete and local stone are in the range of 0.31-1.5 gha. The total EF of OCRT motel accommodation services is 10.1 gha (Table 5.32).

\begin{tabular}{|c|c|c|c|c|c|c|c|c|}
\hline \multicolumn{9}{|c|}{ Table 5.32: OCRT- Life cycle energy use of motel accommodation services (2011) } \\
\hline Material & $\begin{array}{l}\text { Embodied } \\
\text { energy } \\
\text { Over a } \\
50 \text {-year } \\
\text { life cycle } \\
\left(\mathrm{GJ} / \mathrm{m}^{2}\right)\end{array}$ & $\begin{array}{l}\text { Total } \\
\text { Area } \\
\left(\mathrm{m}^{2}\right)\end{array}$ & $\begin{array}{l}\text { Embodied } \\
\text { energy } \\
\text { (GJ/year) }\end{array}$ & 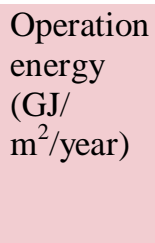 & $\begin{array}{l}\text { Occupied } \\
\text { area } \\
\left(\mathrm{m}^{2}\right)^{1}\end{array}$ & $\begin{array}{l}\text { Operating } \\
\text { energy } \\
\text { (GJ/year) }\end{array}$ & $\begin{array}{l}\text { Life- } \\
\text { cycle } \\
\text { energy } \\
\text { use } \\
\text { (GJ/ } \\
\text { year) }\end{array}$ & $\begin{array}{l}\text { EF } \\
\text { gha/year }\end{array}$ \\
\hline Timber & 1.1 & 6,033 & 132.7 & 0.25 & 1,273 & 318.2 & 450.9 & 4.5 \\
\hline $\begin{array}{l}\text { Timber } \\
\text { and ma- } \\
\text { sonry }\end{array}$ & 2.04 & 2,755 & 112.4 & 0.25 & 581 & 145.2 & 257.6 & 2.6 \\
\hline $\begin{array}{l}\text { Mud brick } \\
\text { (mixed } \\
\text { with other } \\
\text { materials) }\end{array}$ & - & - & - & - & - & - & - & - \\
\hline $\begin{array}{l}\text { Brick }^{1} \\
\text { mixed with } \\
\text { other mate- } \\
\text { rials (old } \\
\text { technology) }\end{array}$ & 5.12 & 744 & 76.2 & 0.25 & 157 & 39.2 & 115.4 & 1.2 \\
\hline $\begin{array}{l}\text { Stone }^{2} \text { (lo- } \\
\text { cal) }\end{array}$ & 1.58 & 372 & 11.8 & 0.25 & 78 & 19.5 & 31.3 & 0.31 \\
\hline Concrete & 3.15 & 1,335 & 84.1 & 0.25 & 282 & 70.5 & 154.6 & 1.5 \\
\hline Total & & & 417.2 & & & 592.6 & & 10.1 \\
\hline
\end{tabular}

\section{c. 5. OCRT Homestead: Life Cycle Energy Use and EFAs shown in Table 5.33}

homestead accommodation in local stone construction contains the largest area of 470 $\mathrm{m}^{2}$ followed by timber and mud brick (mixed with other materials) each with $179 \mathrm{~m}^{2}$. 
Table 5.33 shows that homestead stone construction accounts for the largest embodied energy value of $14.9 \mathrm{GJ} /$ year. Mud brick and timber have lower embodied energy values of $4.9 \mathrm{GJ} /$ year and 3.9 GJ/year (Table 5.33).

As shown in Table 5.33, homesteads of stone contain the largest occupied area of 470 $\mathrm{m}^{2}$. They also account for the largest operational energy (3.5 GJ/year) and life cycle energy (18.4GJ/year). Timber is lower at $1.25 \mathrm{GJ} /$ year and $5.12 \mathrm{GJ} /$ year, almost the same as mud brick at $1.25 \mathrm{GJ} /$ year and 6.15GJ/year (Table 5.33). This means for the homestead category, stone construction accounts for the largest annual EF (0.18 gha) followed by mud brick (mixed with other materials) (0.06 gha) and timber (0.05gha) (Table 5.33).

\begin{tabular}{|c|c|c|c|c|c|c|c|c|}
\hline Material & $\begin{array}{l}\text { Embodied } \\
\text { energy } \\
\text { Over a } \\
50 \text {-year } \\
\text { life cycle } \\
\left(\mathrm{GJ} / \mathrm{m}^{2}\right)\end{array}$ & $\begin{array}{l}\text { Total } \\
\text { Area }\left(m^{2}\right)^{2}\end{array}$ & $\begin{array}{l}\text { Embodied } \\
\text { energy } \\
\text { (GJ/year) }\end{array}$ & $\begin{array}{l}\text { Operation } \\
\text { energy } \\
(\mathrm{GJ} / \\
\left.\mathrm{m}^{2} / \text { year }\right)^{3}\end{array}$ & $\begin{array}{l}\text { Occupied } \\
\text { area }\left(\mathrm{m}^{2}\right)^{1}\end{array}$ & $\begin{array}{l}\text { Operating } \\
\text { energy } \\
\text { (GJ/year) }\end{array}$ & $\begin{array}{l}\text { Life- } \\
\text { cycle } \\
\text { energy } \\
\text { use (GJ/ } \\
\text { year) }\end{array}$ & $\begin{array}{l}\text { EF } \\
\text { gha/year }\end{array}$ \\
\hline Timber & 1.1 & 176 & 3.9 & 0.25 & 5 & 1.25 & 5.12 & 0.05 \\
\hline $\begin{array}{l}\text { Timber and } \\
\text { masonry }\end{array}$ & - & - & - & - & - & - & - & - \\
\hline $\begin{array}{l}\text { Mud brick } \\
\text { (mixed with } \\
\text { other mate- } \\
\text { rials) }\end{array}$ & 1.4 & 176 & 4.9 & 0.25 & 5 & 1.25 & 6.15 & 0.06 \\
\hline $\begin{array}{l}\text { Brick } \\
\text { mixed with } \\
\text { other mate- } \\
\text { rials (old } \\
\text { technology) }\end{array}$ & - & - & - & - & - & - & - & - \\
\hline Stone (local) & 1.58 & 470 & 14.9 & 0.25 & 14 & 3.5 & 18.4 & 0.18 \\
\hline Concrete & - & - & - & - & - & - & - & - \\
\hline Total & & & 23.7 & & & 6 & & 0.3 \\
\hline $\begin{array}{l}\text { 1. Occupancy } \\
\text { 2. Area per be } \\
\text { 3. Operation } \\
\text { motel. }\end{array}$ & $\begin{array}{l}\text { rate of OCR } \\
d \text { space }=2 c \\
\text { nergy }(G J / n\end{array}$ & $\begin{array}{l}\text {-homestead } \\
4 \mathrm{~m}^{2} \text { (see Ta } \\
\text { /year) of hor }\end{array}$ & $\begin{array}{l}=3 \%(\text { see } \mathrm{Ta} \\
\text { ble } 5.18) \\
\text { nestead acco }\end{array}$ & $\begin{array}{l}\text { le 5.17) } \\
\text { modation }\end{array}$ & sumed to & he same & eration & gy of \\
\hline
\end{tabular}

\section{c. 6.OCRT Hotels: Life Cycle Energy Use and EF}

As shown in Table 5.34, timber and masonry hotel construction accounts for the largest area in this category of $5,442 \mathrm{~m}^{2}$ area followed by local stone $\left(2,299 \mathrm{~m}^{2}\right)$, timber $(1,862$ $\mathrm{m}^{2}$ ), and brick mixed with other materials and mud brick (mixed with other materials) with equal areas of $233 \mathrm{~m}^{2}$ (Table 5.34). In addition, the timber and masonry type accounts for the largest embodied energy value of 222GJ/year, followed by stone (72.6 GJ/year) (Table 5.34). The types of mud brick (mixed with other materials) and brick 
(mixed with other construction) have embodied energy of 6.5 and 23.9 GJ/year (Table $5.34)$.

As determined in Table 5.34, hotel timber and masonry construction contains the biggest occupied area $\left(593 \mathrm{~m}^{2}\right)$ and has the largest annual operating energy of 338 GJ/year). Other types of hotel construction including timber, mud brick, brick and stone contain occupied areas between $25-251 \mathrm{~m}^{2}$ and have operational energy use in the range 14-143 GJ/year (Table 5.34).

Moreover, the same hotel category of timber and masonry construction accounts for the largest life cycle energy of 560 GJ/year and has the biggest EF (5.6 gha) (Table 5.34). The life cycle energy uses of other types of construction are between 20.5-215.5 GJ/year and their EFs are in the range of 0.2-2.2 gha. (Table 5.34).The total life cycle energy use of OCRT hotels is $481.1 \mathrm{GJ} /$ year and its total annual EF is 9.8 gha (Table 5.34).

\begin{tabular}{|c|c|c|c|c|c|c|c|c|}
\hline Material & $\begin{array}{l}\text { Embodied } \\
\text { energy } \\
\text { Over a } \\
50 \text {-year } \\
\text { life cycle } \\
\left(\mathrm{GJ} / \mathrm{m}^{2}\right)\end{array}$ & $\begin{array}{l}\text { Total } \\
\text { Area }\left(\mathrm{m}^{2}\right)^{2}\end{array}$ & $\begin{array}{l}\text { Embodied } \\
\text { energy } \\
\text { (GJ/year) }\end{array}$ & $\begin{array}{l}\text { Operation } \\
\text { energy } \\
(\mathrm{GJ} / \\
\left.\mathrm{m}^{2} / \text { year }\right)^{3}\end{array}$ & $\begin{array}{l}\text { Occupied } \\
\text { area }\left(\mathrm{m}^{2}\right)^{1}\end{array}$ & $\begin{array}{l}\text { Operating } \\
\text { energy } \\
\text { (GJ/year) }\end{array}$ & $\begin{array}{l}\text { Life- } \\
\text { cycle } \\
\text { energy } \\
\text { use (GJ/ } \\
\text { year) }\end{array}$ & $\begin{array}{l}\text { EF } \\
\text { gha/year }\end{array}$ \\
\hline Timber & 1.1 & 1,862 & 41 & 0.57 & 188 & 107 & 148 & 1.48 \\
\hline $\begin{array}{l}\text { Timber and } \\
\text { masonry }\end{array}$ & 2.04 & 5,442 & 222 & 0.57 & 593 & 338 & 560 & 5.6 \\
\hline $\begin{array}{l}\text { Mud brick } \\
\text { (mixed with } \\
\text { other mate- } \\
\text { rials) }\end{array}$ & 1.4 & 233 & 6.5 & 0.57 & 25 & 14 & 20.5 & 0.2 \\
\hline $\begin{array}{l}\text { Brick } \\
\text { mixed with } \\
\text { other mate- } \\
\text { rials (old } \\
\text { technology) }\end{array}$ & 5.12 & 233 & 23.9 & 0.57 & 25 & 14 & 37.9 & 0.4 \\
\hline Stone (local) & 1.58 & 2,299 & 72.6 & 0.57 & 251 & 143 & 215.5 & 2.2 \\
\hline Concrete & - & - & - & - & - & - & - & - \\
\hline Total & & & 366 & & & 616 & 981.9 & 9.8 \\
\hline
\end{tabular}

\section{c.7 . OCRT Camping Sites: Life Cycle Energy Use and EF}

As determined in Table 5.35 the embodied energy of camping sites is not available and this study considers the operational energy use of this type of accommodation to be equal to its life cycle energy use. Table 5.35 indicates that the total annual EF of 2,048 
OCRT- powered sites with 6,248 visitor nights and 0.025 GJ/visitor night operational energy use is equivalent to 1.6 gha.

Table 5.35: OCRT- life cycle energy use of camping (powered sites) accommodation services (2011)

\begin{tabular}{|c|c|c|c|c|c|c|c|}
\hline Material & $\begin{array}{l}\text { Embodied } \\
\text { energy } \\
\text { Over a } 50- \\
\text { year life } \\
\text { cycle } \\
\left(\mathrm{GJ} / \mathrm{m}^{2}\right)\end{array}$ & $\begin{array}{l}\text { Total } \\
\text { visitor } \\
\text { nights }\end{array}$ & $\begin{array}{l}\text { Embodied } \\
\text { energy } \\
\text { (GJ/year) }\end{array}$ & $\begin{array}{l}\text { Operation } \\
\text { energy (GJ/ } \\
\text { visitor } \\
\text { night) }\end{array}$ & $\begin{array}{l}\text { Operating } \\
\text { energy } \\
\text { (GJ/year) }\end{array}$ & $\begin{array}{l}\text { Life-cycle } \\
\text { energy use } \\
\text { (GJ/ year) }\end{array}$ & EF gha/year \\
\hline $\begin{array}{l}\text { Assumed } \\
\text { negligible }\end{array}$ & N/A & 6,248 & N/A & 0.025 & 156.2 & 156.2 & 1.6 \\
\hline Total & & & & & & & \\
\hline
\end{tabular}

\section{d. The Total EF of OCRT Accommodation Services (First Scenario)}

The total EF of 788 OCRT accommodation services (based on the assumption that all are NB) is 50.5gha/year in 2011 (Table 5.36). As shown in Table 5.36, OCRT motels comprise the majority of $22.6 \%$ of visitor nights followed by camping (powered sites) (18.6\%), Bps (15\%), B\&Bs (14.9\%), SCs (14\%), hotels (11.7\%) and homestead (3.2\%). Likewise, as demonstrated in Table 5.36, in comparison with other types of OCRT accommodation services, motels have the biggest EF (10.1 gha) and homestead accommodation has the smallest EF (0.3gha). Other types of OCRT accommodation services have an annual EF in a range of 1.6-9.8 gha.

Table 5.36: OCRT- EF of accommodation services (2011)

\begin{tabular}{l} 
SC \\
B\&B \\
Bp \\
Motel \\
\hline Homestead \\
\hline Hotel \\
\hline Camping (powered sites) \\
\hline Total
\end{tabular}

\begin{tabular}{|c|c|c|c|}
\hline Visitor nights & $\%$ & EF (gha/year) & $\%$ \\
\hline 5,559 & 14 & 9.65 & 19.1 \\
\hline 5,899 & 14.9 & 17 & 33.7 \\
\hline 5,941 & 15 & 2.05 & 4 \\
\hline 8,954 & 22.6 & 10.1 & 20 \\
\hline 1,273 & 3.2 & 0.3 & 0.6 \\
\hline 4,626 & 11.7 & 9.8 & 19.4 \\
\hline 7,373 & 18.6 & 1.6 & 3.2 \\
\hline 39,625 & 100 & 50.5 & 100 \\
\hline
\end{tabular}

Figure 5.27 demonstrates that OCRT B\&B accommodation services have the biggest annual EF/visitor night (0.003gha/visitor night) and homesteads and powered camp sites have the smallest annual EF/visitor night (0.0002gha). Other OCRT accommodation services have annual EF/visitor night in a range of 0.0003- 0.002gha (Figure 5.31). 


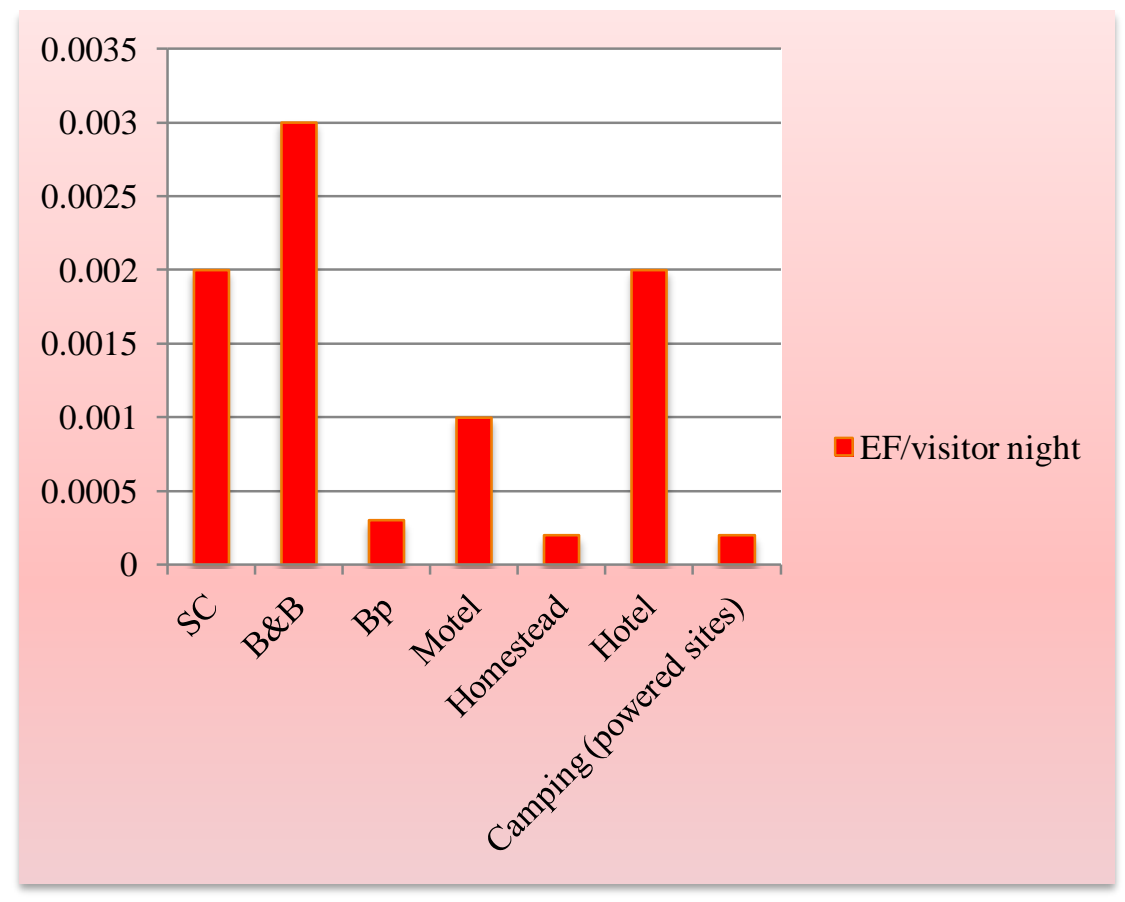

Figure 5.31: The annual EF/visitor night of OCRT- accommodation services

\subsubsection{The EF of OCRT Accommodation Services (Second Scenario: Reducing Embodied Energy through the use of Refurbished Buildings)}

Throughout the second scenario the embodied energies of RB buildings are subtracted from the whole life cycle energy use of OCRT accommodation services. Consequently, the life cycle energy use of OCRT accommodation services in the second scenario includes the embodied energy of NB buildings plus the total operating energy of both RB and NB buildings. As RB accommodation services use similar energy resources and facilities as those used in NB buildings, their operation energy use is assumed to be the same as that of NB accommodation, and is included in the whole life cycle energy use of OCRT accommodation services (Table 5.37).

Table 5.37 demonstrates that in the second scenario the total life cycle energy of OCRT accommodation services is equal to 4,318.2 GJ/year. Moreover, the EF of OCRT accommodation services is equivalent to $4,318.2 \mathrm{GJ} /$ year $\div 100(\mathrm{GJ} / \mathrm{ha})=43.2$ gha/year (Table 5.37). 


\begin{tabular}{|l|c|c|c|c|c|c|c|}
\hline \multicolumn{7}{|c|}{ Table 5.37: OCRT- accommodation services- life cycle energy of new and refurbished buildings (sec- } \\
ond scenario) \\
\hline Type & $\begin{array}{c}\text { Total em- } \\
\text { bodied en- } \\
\text { ergy } \\
\text { (GJ/year) }\end{array}$ & $\begin{array}{c}\text { NB } \\
(\%)\end{array}$ & $\begin{array}{c}\text { RB } \\
(\%)\end{array}$ & $\begin{array}{c}\text { Embodied } \\
\text { energy of NB } \\
\text { (GJ/year) }\end{array}$ & $\begin{array}{c}\text { Total oper- } \\
\text { ating en- } \\
\text { ergy (GJ/ } \\
\text { year) }\end{array}$ & $\begin{array}{c}\text { Life- } \\
\text { cycle } \\
\text { energy } \\
\text { (GJ/year) }\end{array}$ & EF (gha/year) \\
\hline SC & 382.4 & 68.8 & 31.2 & 263 & 582.5 & 846 & 8.5 \\
\hline B\&B & 840.2 & 70.6 & 29.4 & 593.2 & 859.2 & 1452.4 & 14.5 \\
\hline Bp & 66.3 & 61 & 39 & 40.4 & 139.67 & 180.1 & 1.8 \\
\hline Motel & 417.2 & 96.7 & 3.3 & 403.4 & 592.6 & 996 & 10 \\
\hline Homestead & 23.7 & 21.4 & 78.6 & 5.1 & 6 & 11.1 & 0.11 \\
\hline Hotel & 366 & 13.9 & 86.1 & 50.9 & 616 & 667 & 6.7 \\
\hline Camping & N/A & 100 & 0.00 & N/A & 156.2 & 156.2 & 1.6 \\
\hline Total & $2,095.8$ & - & - & 1,356 & $2,952.17$ & $4,318.2$ & 43.2 \\
\hline
\end{tabular}

Table 5.38 compares the EF of OCRT accommodation services from the two scenarios. As shown in Table 5.38, the influence of using RB buildings as accommodation means the annual EF of OCRT accommodation is decreased by 14.5\% (7.3 gha) from 50.5 gha to 43.2gha. The main reason for the reduced EF of OCRT accommodation services in the second scenario is the reduction in embodied energy from the use of RB buildings over the whole life cycle energy of accommodation services. This is based on the assumption that they are more than 50 years old, as the embodied energy of NB buildings is calculated over a 50 year assumed life.

$\begin{aligned} & \text { Table 5.38: Reduced EF of OCRT accommodation services influenced by the use of RB as tourism } \\
& \text { accommodation }\end{aligned}$
\begin{tabular}{|l|l} 
EF1 (all buildings are assumed to be new buildings) & Present EF \\
\hline 50.5 gha/year & 43.2 gha/year \\
\hline
\end{tabular} - Reduced EF of OCRT influenced by the use of RB (12.9\% of total bed spaces) as a part of accommodation
services $=7.3$ gha/year= $14.5 \%$ of EF1

\subsubsection{EF of OCRT Accommodation Services (Third Scenario Including RB, NB Buildings and All Types of Outdoor Sitting Area)}

This section attempts to determine the influence of using open air spaces including verandas and balconies (V/B) on the EF of OCRT accommodation buildings. In the second scenario, calculation of the EF of OCRT accommodation services has been conducted based on the assumption that all NB and RB spaces are indoor spaces. In this section, OCRT accommodation services are assumed to contain both indoor and outdoor spaces and their EFs also comprise the EFs of both types of space. The difference between the results of the second and third scenario for measurement of EF indicates the influence of using open air areas on the EF of OCRT accommodation services.

This thesis explores the number of V/B and outdoor sitting areas by their types (shared and private) through using OCRT accommodation websites in which related documents, 
pictures and videos are published (see Appendix 5). Table 5.39 contains summarised information about the numbers and types of V/Bs and outdoor sitting areas by type of accommodation, as explained in Appendix 5.

Table 5.39 shows that 106 bed spaces have access to 13 shared V/Bs and 49 bed spaces use 14 private V/Bs. As shown in Table 5.39 the largest access comes from the 44 SC bed spaces that use 8 shared V/Bs followed by 43 hotel bed spaces and 19 B\&B bed spaces that use 3 and 2 shared V/Bs respectively. Table 5.39 also shows most private access occurs in $19 \mathrm{~B} \& \mathrm{~B}$ bed spaces that can use 4 private $\mathrm{V} / \mathrm{Bs}$ (there is more than one bed space per room), followed by 16 SC bed spaces and 14 motel bed spaces that use 8 and 2 private $\mathrm{V} / \mathrm{Bs}$ respectively.

Also as shown in Table 5.39, 462 OCRT bed spaces have access to 44 shared outdoor sitting areas and 29 bed spaces (more than one bed space per room) can use 6 private outdoor sitting areas. The largest shared access is in the motel category with 184 motel bed spaces with access to 6 shared outdoor sitting spaces, followed by 139 B\&B bed spaces (17), 95 SC bed spaces (16), and 44 hotel bed spaces (3) (Table 5.39). Furthermore, of 243 OCRT-bed spaces that use 73 private outdoor sitting areas, the majority occur in the 109 B\&B bed spaces that use 52 private outdoor sitting areas (Table 5.39). The other types of accommodation bed spaces have lower overall access to private facilities, with 28 SC bed spaces accessing 10 such sitting areas. Other categories have limited access to such facilities (Table 3.39).

\begin{tabular}{|c|c|c|c|c|c|c|c|c|}
\hline \multirow[t]{2}{*}{ Туре } & \multicolumn{4}{|c|}{$\mathrm{V}^{1} / \mathrm{B}^{2}$} & \multicolumn{4}{|c|}{ Outdoor sitting } \\
\hline & $\mathrm{S}^{3}$ & $\mathrm{NV}^{4}$ & $\mathrm{P}^{5}$ & NV & $\mathrm{S}$ & $\mathrm{No}^{6}$ & $\mathrm{P}$ & No \\
\hline SC & 44 & 8 & 16 & 8 & 95 & 16 & 28 & 10 \\
\hline B\&B & 19 & 2 & 19 & 4 & 139 & 17 & 109 & 52 \\
\hline Bp & 0 & 0 & 0 & 0 & 0 & 0 & 0 & 0 \\
\hline Motel & 0 & 0 & 14 & 2 & 184 & 6 & 71 & 4 \\
\hline Homestead & 0 & 0 & 0 & 0 & 0 & 0 & 6 & $1^{7}$ \\
\hline Hotel & 43 & 3 & 0 & 0 & 44 & 3 & 29 & 6 \\
\hline Total & 106 & 13 & 49 & 14 & 462 & 42 & 243 & 73 \\
\hline \multicolumn{9}{|c|}{$\begin{array}{l}\text { 1: Veranda } \\
\text { 2: Balcony } \\
\text { 3: Number of bed spaces sharing area } \\
\text { 4: Number of veranda/ balcony } \\
\text { 5: Number of bed spaces using private area } \\
\text { 6: Number of outdoor sitting spaces. } \\
\text { 7. Outdoor sitting with timber pergola roof }\end{array}$} \\
\hline
\end{tabular}


This thesis calculates the areas of 12 selected V/Bs (6 NB and $6 \mathrm{RB}$ ) found in OCRT accommodation services to measure the average area $\left(\mathrm{m}^{2}\right)$ of all V/Bs used in OCRT accommodation services (Appendix19). This calculation is conducted through using the roof plans, and scaled views and elevations (Appendix19), published in websites and from satellite maps. The calculation of the areas is conducted by using Map tool 2 and a scaled ruler.

As shown in Appendix19, in OCRT accommodation services, the average area per V/B is $14.3 \mathrm{~m}^{2}$. Table 5.40 includes the areas of the number of V/Bs used in each type of OCRT accommodation service. As shown in Table 5.40, of $27 \mathrm{~V} / \mathrm{Bs}$ with a total area of $387 \mathrm{~m}^{2}$, the majority are used in SC accommodation services (16 V/Bs with an area of $\left.229 \mathrm{~m}^{2}\right)$, followed by 6 in B\&B $\left(86 \mathrm{~m}^{2}\right), 3$ in hotels $\left(43 \mathrm{~m}^{2}\right)$ and 2 in motels $\left(29 \mathrm{~m}^{2}\right)$.

\begin{tabular}{|l|c|c|}
\hline \multicolumn{4}{|c|}{ Table 5.40: OCRT- outdoor sitting used as part of accommodation services (summary) } \\
\hline Type & No. $^{1}$ & V/B \\
\hline SC & 16 & Area $\left(\mathrm{m}^{2}\right)^{2}$ \\
\hline B\&B & 6 & 229 \\
\hline Bp & 0 & 86 \\
\hline Motel & 2 & 0 \\
\hline Homestead & 0 & 29 \\
\hline Hotel & 3 & 0 \\
\hline Total & 27 & 43 \\
\hline 1. Number of V/B & & 387 \\
\hline 2. Average area $\left(\mathrm{m}^{2}\right)$ per veranda/balcony $=14.3$ & \\
\hline
\end{tabular}

Table 5.41 sets out the initial embodied energy of a V/B element based on its floor and roof. Since the embodied energy of V/B foundation has already been calculated and included in embodied energy of indoor spaces, it is ignored in Table 5.41. As the embodied energy of partition walls between a veranda/balcony and indoor spaces has already been calculated and included in embodied energy of indoor spaces, it is not calculated in Table 5.41. As result, in Table 5.41, the total embodied energy for a V/B over a 50 year life is $620 \mathrm{MJ} / \mathrm{m}^{2}$ or $0.62 \mathrm{GJ} / \mathrm{m}^{2}$. Consequently the annual embodied energy intensity for a V/B is equivalent to $0.62 \mathrm{GJ} / \mathrm{m}^{2} \div 50=0.012 \mathrm{GJ} / \mathrm{m}^{2} /$ year. 


\begin{tabular}{|c|c|c|}
\hline \multirow{2}{*}{$\begin{array}{l}\text { Building } \\
\text { element } \\
\text { Foundation }^{1}\end{array}$} & \multicolumn{2}{|c|}{$\begin{array}{l}\text { Embodied energy intensity over a } 50 \text { year life }\left(\mathrm{MJ} / \mathrm{m}^{2}\right. \\
\text { Light construction }\end{array}$} \\
\hline & - & - \\
\hline Floor & 220 & $35.5 \%$ \\
\hline Walls $^{2}$ & - & - \\
\hline Roof & 400 & $64.5 \%$ \\
\hline Total & 620 & $100 \%$ \\
\hline
\end{tabular}

Table 5.42 shows that the embodied energy of $387 \mathrm{~m}^{2}$ OCRT accommodation service V/Bs is $4.6 \mathrm{GJ} /$ year. Since V/Bs are open areas and do not use energy in operation (in particular for heating), their operating energy is assumed to be zero. Thus as shown in Table 5.42, the life cycle energy use of $387 \mathrm{~m}^{2}$ is equal to its embodied energy (4.6 $\mathrm{GJ} / \mathrm{year}$ ) and its annual EF is 0.046 gha.

\begin{tabular}{|l|l|l|l|l|l|l|}
\hline \multicolumn{3}{|c|}{ Table 5.42: OCRT-accommodation services, life cycle energy and EF of outdoor sitting space (ve- } \\
randa/balcony)
\end{tabular}

In Table 5.43 the total area of OCRT accommodation services is separated into the two areas of 79,978 $\mathrm{m}^{2}$ indoor spaces and $378 \mathrm{~m}^{2} \mathrm{~V} / \mathrm{B}$. The annual EF of OCRT accommodation services (indoor spaces) as shown in Table 5.43 is $0.00053 \mathrm{gha} / \mathrm{m}^{2}$ (43.1gha $\div$ $80,356 \mathrm{~m}^{2}$ ). Thus the annual EF of $79,978 \mathrm{~m}^{2}$ indoor spaces is equivalent to $79,978 \mathrm{~m}^{2} \times$ $0.00053 \mathrm{gha} / \mathrm{m}^{2}=42.3$ gha (Table 5.43). Likewise the annual EF of V/Bs with the area of $378 \mathrm{~m}^{2}$ area is equivalent to 0.046 gha (see Tables 5.42 and 43 ). As a result the total annual EF of 80,356 $\mathrm{m}^{2}$ OCRT accommodation services comprising 79,978 $\mathrm{m}^{2}$ indoor spaces and $378 \mathrm{~m}^{2} \mathrm{~V} / \mathrm{B}$ is equivalent to the sum of 0.046 gha and 42.3 gha $=42.4$ gha (Table 5.43).

As shown in Tables.5.37 and 5.38, the results from the second scenario in which all OCRT accommodation services are assumed to be indoor spaces give a total annual EF of 43.2 gha. In addition, in the third scenario, in which outdoor spaces are considered, the annual EF reduces to 42.4gha. This is a small 1.9\% reduction in annual EF. Alternatively, thinking of the EF as a land area, 0.8 gha $\left(8000 \mathrm{~m}^{2}\right) \div 387 \mathrm{~m}^{2}=20.7 \mathrm{~m}^{2}$, so $1 \mathrm{~m}^{2}$ V/B reduces the EF of OCRT accommodation services by $20.7 \mathrm{~m}^{2}$. 
Table 5.43: Reduced EF of OCRT-accommodation serviced influenced by the use of veranda/balcony

\begin{tabular}{|c|c|c|c|c|c|}
\hline \multicolumn{3}{|c|}{ Indoor spaces } & \multicolumn{3}{|c|}{ Outdoor sitting (veranda/balcony) } \\
\hline Area $\left(m^{2}\right)^{1}$ & $\mathrm{EF}\left(\mathrm{gha} / \mathrm{m}^{2}\right)$ & EF (gha) & Area $\left(\mathrm{m}^{2}\right)$ & $\mathrm{EF}\left(\mathrm{gha} / \mathrm{m}^{2}\right)$ & EF (gha) \\
\hline 79,978 & 0.00053 & 42.3 & 387 & 0.00012 & 0.046 \\
\hline $\begin{array}{l}\text { 1. Total area o } \\
\text { - Total EF of } \\
\text { - Total EF of } \\
\text { - Reduction ir }\end{array}$ & $\begin{array}{l}\Gamma \text { accommodat } \\
\text { accommodatic } \\
\Gamma \text { accommodat } \\
\Gamma \text { - EF influenc }\end{array}$ & ices $\left(\mathrm{m}^{2}\right.$ & $\begin{array}{l}\text { (see Table } \\
\text { ) gha= 43. } \\
+ \text { Veranda/ }\end{array}$ & $\begin{array}{l}\text { ble } 5.34 \text { ) } \\
\text { gha }=42.4 \\
.48 \% \text { of } 80,\end{array}$ & \\
\hline
\end{tabular}

\subsection{OCRT Visitor Activities}

This section investigates the environmental impacts of OCRT visitor activities (as part of their social-cultural behaviours) through calculation of their annual EF. This study explores the frequency of activities offered by OCRT accommodation services to visitors through using the official websites of the OCRT community and 201websites of 783 OCRT accommodation services. The offered activities are explained in Appendix 5 and they are classified into indoor and outdoor activities in Appendix 7.

In addition the frequency of the offered activities shown in Appendix 7 and summarised in Tables 5.44 and 5.45 can be considered as an indicator of the attitude of OCRT participants and members (in this case accommodation owners) to the types of sustainable tourism activities. Comparison between the EFs of the conducted activities and their frequency also can indicate a social-ecological perspective of the attitude of tourism participants to being sustainable.

\subsubsection{Indoor Activities (Frequency)}

Table 5.44 sets out OCRT indoor activities offered by accommodation services to visitors. These activities are classified into seven types: curling at Naseby, indoor sports (except curling at Naseby), indoor water sports, education, art activities, cultural/spiritual activities, and games (Table 5.44). According to CODC, 2011:18, the curling at Naseby is considered as the only indoor curling sport and attracts about $36.6 \%$ (see Table 5.48) of OCRT visitors in 2011, while curling at Alexandra is included in other activities. Since this study attempts to evaluate the environmental, cultural and economic footprint of curling at Naseby on OCRT (at regional scale) and on Naseby (at urban scale) its frequency is separated from curling at Alexandra in Table 5.44. This makes an opportunity to explore the relationship between frequency of curling at Naseby and its environmental, cultural and economic impacts. 
Table 5.44: OCRT- Classification of the indoor activities offered by accommodation services to visitors (2011)

\begin{tabular}{|c|c|c|c|c|c|c|c|c|c|c|c|c|c|}
\hline $\begin{array}{l}\text { Indoor } \\
\text { sports }\end{array}$ & $\mathrm{F}^{1}$ & $\begin{array}{l}\text { Indoor } \\
\text { sports } \\
\text { (except } \\
\text { curling at } \\
\text { Naseby) }\end{array}$ & $\mathrm{F}^{1}$ & $\begin{array}{l}\text { Indoor } \\
\text { water } \\
\text { sports }\end{array}$ & $\mathrm{F}^{1}$ & Education & $F^{1}$ & Art & $\mathrm{F}^{1}$ & $\begin{array}{l}\text { Cul- } \\
\text { tural/spi } \\
\text { ritual } \\
\text { activi- } \\
\text { ties }\end{array}$ & $\begin{array}{l}\mathrm{F} \\
1\end{array}$ & Games & $\begin{array}{l}\mathrm{F} \\
1\end{array}$ \\
\hline $\begin{array}{l}\text { Curling } \\
\text { at } \\
\text { Naseby }\end{array}$ & 46 & $\begin{array}{l}\text { Curling at } \\
\text { Alexandra }\end{array}$ & 2 & $\begin{array}{l}\text { Swim- } \\
\text { ming pool }\end{array}$ & 7 & $\begin{array}{l}\text { Reading } \\
\text { materials }\end{array}$ & 46 & $\begin{array}{l}\mathrm{Mu}- \\
\text { sic }\end{array}$ & 1 & $\begin{array}{l}\text { Catholic } \\
\text { Church }\end{array}$ & 1 & Games & 4 \\
\hline- & - & Bowling & 2 & $\begin{array}{l}\text { Aquatic } \\
\text { centre }\end{array}$ & 1 & $\begin{array}{l}\text { Maps and } \\
\text { local in- } \\
\text { formation }\end{array}$ & 1 & - & - & $\begin{array}{l}\text { Cafe } \\
\text { Culture }\end{array}$ & 2 & - & - \\
\hline- & - & Gun club & 1 & - & - & $\begin{array}{l}\text { Photogra- } \\
\text { phy work- } \\
\text { shop }\end{array}$ & 2 & - & - & - & - & - & - \\
\hline Total & 46 & & 5 & & 8 & & 49 & & 1 & & 3 & & 4 \\
\hline
\end{tabular}

Table 5.45 presents a summary of OCRT indoor activities and their frequency (F) cited in Table 5.44. As determined in Table 5.45 and Figure 5.32, of the total 116 (100\%) indoor activities offered to OCRT visitors, education has the majority share of 49 (42.2\%) followed by curling at Naseby at 46 (39.7\%). The frequency of other indoor activities including indoor sport (except curling at Naseby), indoor water sports, art, cultural/spiritual activities and games occur in the F range of 1(0.9\%)-8(6.9\%).

Table 5.45: OCRT- Indoor activities offered by accommodation services to visitors - (summary).

\begin{tabular}{|l|c|c|}
\hline \multicolumn{1}{|c|}{ Activities } & F & \% \\
\hline Indoor sports (curling at Naseby) & 46 & 39.7 \\
\hline Indoor sports (except curling at Naseby) & 5 & 4.3 \\
\hline Indoor water sports & 8 & 6.9 \\
\hline Education & 49 & 42.2 \\
\hline Art & 1 & 0.9 \\
\hline Cultural/spiritual activities & 3 & 2.6 \\
\hline Games & 4 & 3.4 \\
\hline Total & 116 & 100 \\
\hline
\end{tabular}




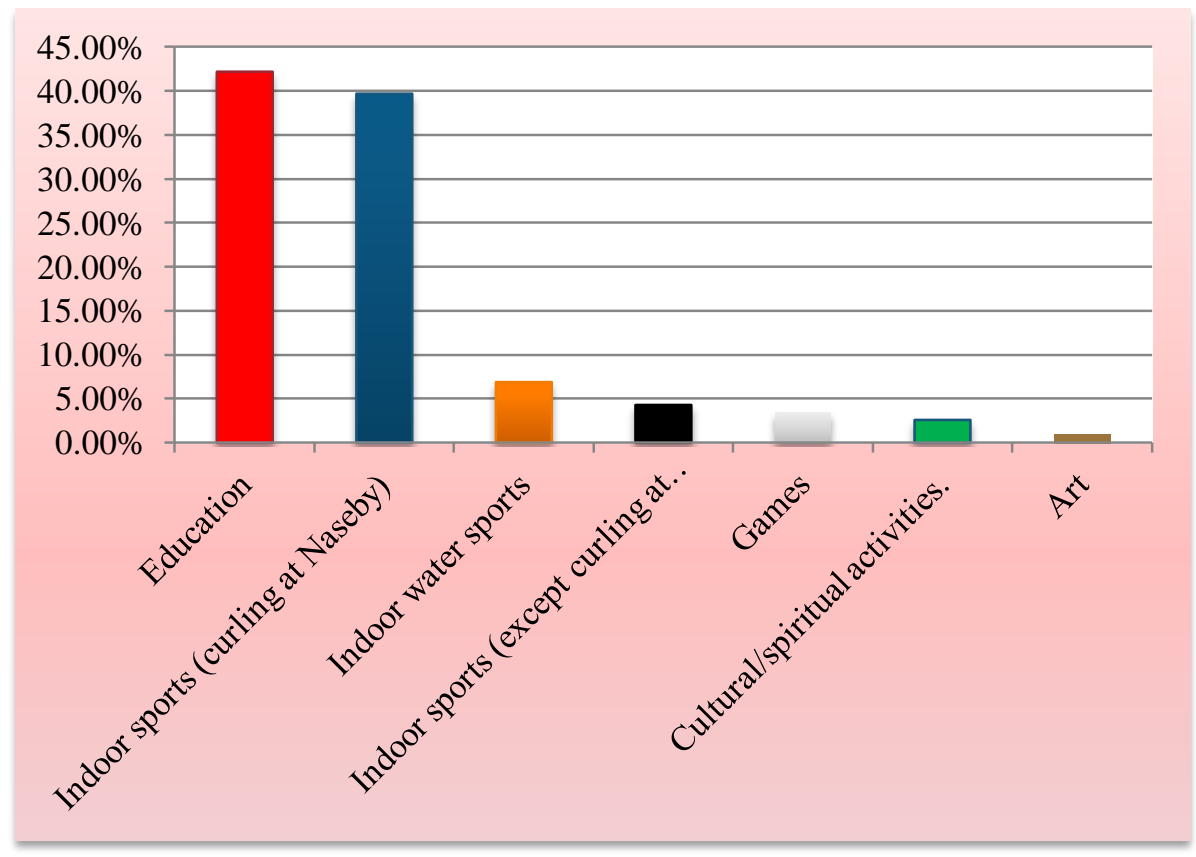

Figure 5.32: OCRT- frequency of indoor activities offered by accommodation services to visitors

\subsubsection{OCRT Outdoor Activities (Frequency)}

This study explores 47 types of outdoor activity offered by OCRT accommodation services to visitors in 2011 as shown in Appendix 7, and based on access to information about outdoor activities through using 201 OCRT accommodation websites. The 47 offered outdoor activities are explained by their related accommodation service in Appendix 7. This Thesis classifies the 47 types of OCRT outdoor activities into seven types: sports (at a playground), motorised sports and activities, water sports, winter sports, cycling, sports on horseback, recreational activities, art activities, farm activities, walking and climbing (See Appendix 20 and its summary, Table 5.46).

Table 5.46 shows that of total 492 OCRT outdoor activities, water sports form the largest category at 122 occurrences, followed by sports at a playground (86), winter sports (54), walking (52), and cycling (42). The frequencies of other OCRT outdoor activities offered are between 1 and 38 (Table 5.46). 
Table 5.46: OCRT- Frequency of the outdoor activities offered by accommodation services to visitors.

Outdoor activity
Sports (at a playground)
Motorised sports and activities
Water sports
Winter sports
Cycling
Sports on horseback
Recreational activities
Art activities
Farm activities
Walking
Climbing
Total

\begin{tabular}{|c|c|}
\hline$F$ & $\%$ \\
\hline 86 & 17.5 \\
\hline 31 & 6.3 \\
\hline 122 & 24.8 \\
\hline 54 & 11 \\
\hline 42 & 8.5 \\
\hline 12 & 2.4 \\
\hline 38 & 7.7 \\
\hline 22 & 6.5 \\
\hline 52 & 4.5 \\
\hline 1 & 10.6 \\
\hline 492 & 0.2 \\
\hline
\end{tabular}

Figure 5.33 illustrates the types of OCRT outdoor activities by their percentage frequencies as cited in Table 5.46 (right column).

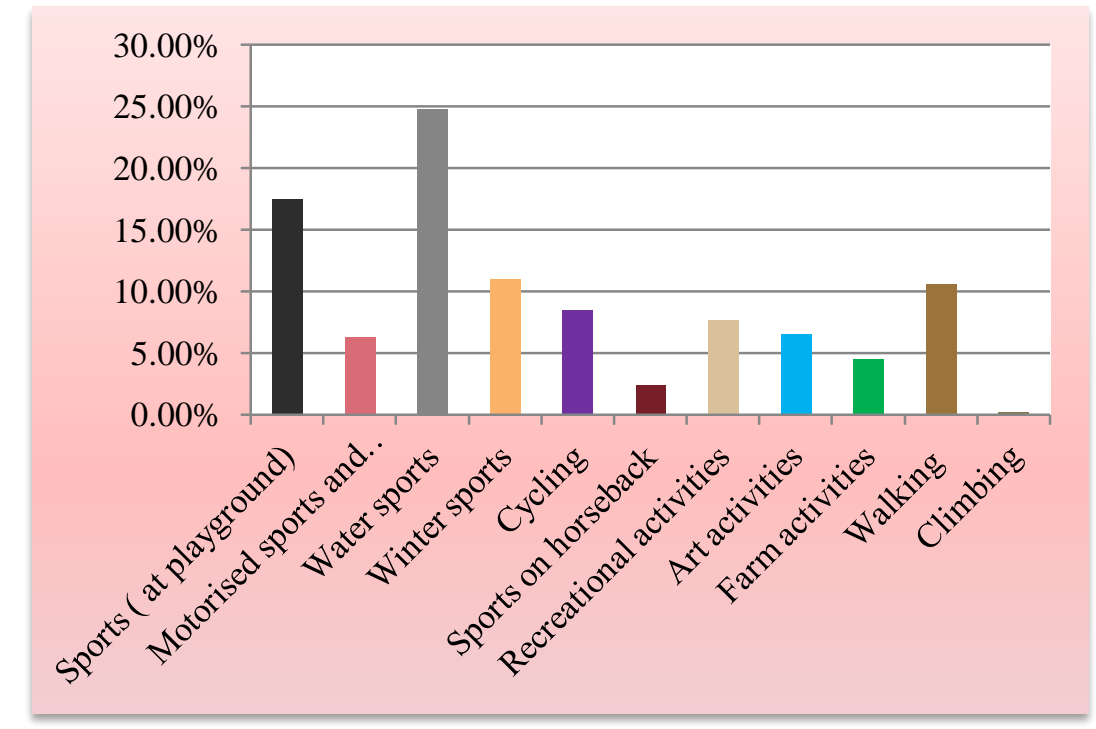

Figure 5.33: Percentage frequencies of OCRT outdoor activities offered by accommodation services to visitors (2011)

Total Frequency of OCRT outdoor activities = 492 (see Table 5.43)

\subsubsection{OCRT Other Visitor Opportunities by Accommodation Service}

Apart from the indoor and outdoor activities discussed above, other suggestions as to things visitors can do are offered by ORTC accommodation services. Appendix 21 contains summarised information about these and their frequency of occurrence. Here, these opportunities for tourist visits are categorised into five groups: cities and villages of note, historic sites and buildings, industrial archaeology sites, scenery, garden/farm/park, and museum/art gallery (Appendix 21). Table 5.47 offers summarised information related to OCRT visiting opportunities offered as cited in Appendix 21. 
Table 5.47 and Figure 5.34 determine that in comparison with other subjects, visits to scenery are suggested most frequently with 132 occurrences (24.1\% of a total of 548), followed by historic sites and buildings (118-21.5\%) and garden/farm/park (10018.3\%). The frequency of other subjects to visit including cities and villages, industrial archaeological sites, and museum/art gallery are in the range of 40 (7.3\%) - 97(17.7\%), see Table 5.47 and Figure 5.34.

\begin{tabular}{|l|c|c|}
\hline \multicolumn{3}{|c|}{ Table 5.47: OCRT- accommodation services- opportunities for visits } \\
\hline \multicolumn{1}{|c|}{ Subjects } & F & $\%$ \\
\hline Cities and villages & 97 & 17.7 \\
\hline Historic sites and buildings & 118 & 21.5 \\
\hline Industrial archaeological sites & 61 & 11.1 \\
\hline Scenery & 132 & 24.1 \\
\hline Garden/ farm/park & 100 & 18.3 \\
\hline Museum/art gallery & 40 & 7.3 \\
\hline Total & 548 & 100 \\
\hline
\end{tabular}

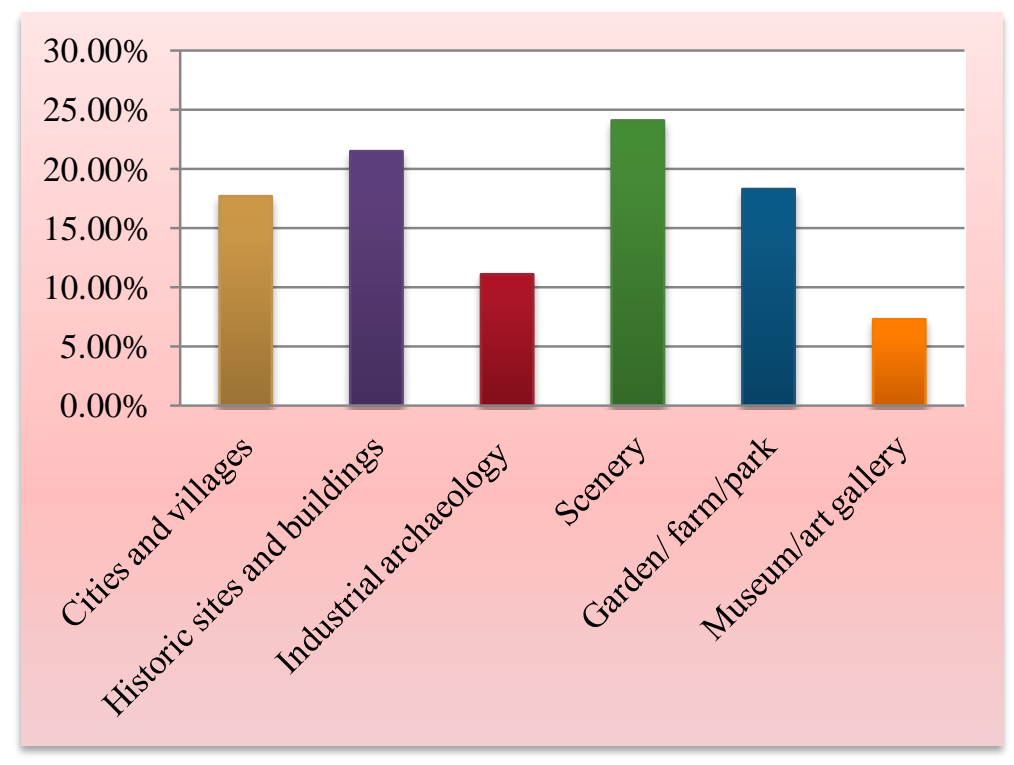

Figure 5.34: Frequency of the subjects to visit offered by OCRT accommodation services to visitors (2011)

\subsubsection{OCRT Activities by Numbers of Visitors}

This Thesis uses the CODC research (2011) as a base to calculate the number of OCRT visitors per activity in 2011. According to CODC (2011:18) in 2011, 160 OCRT visitors nominated a total of 354 activities that they did or were going to undertake while in Central Otago. These nominated activities are aggregated in Table 5.48, and show that of the 160 OCRT visitors the most visited site was the Taieri Gorge Railway 74 visitors (46.3\%), followed by curling 59 visitors (36.9\%), visits to gold mining towns 56 visitors 
(35\%), sightseeing tours 49 visitors (30.6\%) and visits to old Cromwell town 36 visitors (22.5\%). Other activities as shown in Table 5.48 fell in the range of 1 visitor (0.6\%) -27 visitors (16.9\%).

\begin{tabular}{|l|c|c|}
\hline \multicolumn{2}{|c|}{ Table 5.48: OCRT activities conducted by visitors 2011 (n=160) } \\
\hline Activities & Count & \% of visitors \\
\hline Taieri Gorge Railway & 74 & 46.3 \\
\hline Curling & 59 & 36.9 \\
\hline Gold mining towns & 56 & 35 \\
\hline Sightseeing tours & 49 & 30.6 \\
\hline Old Cromwell town & 36 & 22.5 \\
\hline Wineries & 27 & 16.9 \\
\hline Visiting friends and relatives & 24 & 15 \\
\hline Hayes engineering & 13 & 8.1 \\
\hline Mountain biking Naseby & 4 & 2.5 \\
\hline fishing & 3 & 1.9 \\
\hline Golf & 2 & 1.3 \\
\hline Swim at Ophir and Alexandra & 1 & 0.6 \\
\hline Clyde dam Tour & 1 & 0.6 \\
\hline Golden Progress mine & 1 & 0.6 \\
\hline Walking & 1 & 0.6 \\
\hline Ophir- high country farm & 1 & 0.6 \\
\hline Biking & 1 & 0.6 \\
\hline Fruit picking along trail & 1 & 0.6 \\
\hline Other & 354 & - \\
\hline - Reference: CODC, 2011:18 & & \\
\hline
\end{tabular}

This chapter calculates the number of OCRT visitors per activity through using the percentage proportional split of the 160 visitors (cited in Table 5.48) and applying it to the total 11,788 OCRT visitors in 2011 (Table 5.49). This calculated split for the number of visitors is then used as the basis to calculate the EF of each OCRT tourism activity. 
Table 5.49: OCRT activities conducted by $11,788^{1}$ visitors -2011

\begin{tabular}{|l|c|c|}
\hline Activities & \% of visitors & Number of Visitors \\
\hline Taieri Gorge Railway & 46.3 & 5,458 \\
\hline Curling & 36.9 & 4,350 \\
\hline Gold mining towns & 35 & 4,126 \\
\hline Sightseeing tours & 30.6 & 3,607 \\
\hline Old Cromwell town & 22.5 & 2,652 \\
\hline Wineries & 16.9 & 1,992 \\
\hline Visiting friends and relatives & 15 & 1,768 \\
\hline Hayes engineering & 8.1 & 955 \\
\hline Mountain biking Naseby & 2.5 & 295 \\
\hline fishing & 1.9 & 224 \\
\hline Golf & 1.3 & 153 \\
\hline Swim at Ophir and Alexandra & 0.6 & 71 \\
\hline Clyde dam Tour & 0.6 & 71 \\
\hline Golden Progress mine & 0.6 & 71 \\
\hline Walking & 0.6 & 71 \\
\hline Ophir- high country farm & 0.6 & 71 \\
\hline Biking & 0.6 & 71 \\
\hline Fruit picking along trail & 0.6 & 71 \\
\hline 1. See Figure 5.2. & & \\
\hline 2. \% split of 11,788 visitor assumed same as for 160 visitors cited in Table 5.45. & \\
\hline - Reference: CODC, 2011:18 & & \\
\hline
\end{tabular}

\subsubsection{OCRT: EF of Visitor Activities}

\subsubsection{EF of Indoor Activities (Curling at Naseby)}

\section{a. Embodied Energy}

Table 5.50 shows that the total area of the Naseby Indoor Curling Centre is $1,300 \mathrm{~m}^{2}$. To calculate the embodied energy of the Naseby Indoor Curling Centre, its structure is assumed to involve materials similar to the super insulated construction (see Table 5.25) set out in Mithraratne et al (2007:159). Table 5.50 determines that the embodied energy intensity of the Naseby Indoor Curling Centre is 1,248,000 MJ over a 50 year life, which is equivalent to $(1,248,000 / 50) 40,040 \mathrm{MJ} /$ year (40GJ/year).

\begin{tabular}{|c|c|c|c|c|}
\hline \multirow[b]{2}{*}{$\begin{array}{l}\text { Total area } \\
\left(\mathrm{m}^{2}\right)\end{array}$} & \multicolumn{4}{|c|}{ Super insulated construction } \\
\hline & Building element & $\begin{array}{l}\text { Embodied energy } \\
\text { intensity over } 50 \\
\text { years }\left(\mathrm{MJ} / \mathrm{m}^{2}\right)^{1}\end{array}$ & $\begin{array}{l}\text { Embodied en- } \\
\text { ergy intensity } \\
\text { over } 50 \text { years } \\
\text { (MJ) }\end{array}$ & $\begin{array}{l}\text { Embodied energy inten- } \\
\text { sity (MJ/year) }\end{array}$ \\
\hline \multirow{5}{*}{1,300} & Foundation & 30 & 39,000 & 780 \\
\hline & Floor & 370 & 481,000 & 9,620 \\
\hline & Walls & 580 & 754,00 & 15,080 \\
\hline & Roof & 560 & 728,000 & 14,560 \\
\hline & Total & - & $1,248,000$ & 40,040 \\
\hline
\end{tabular}




\section{b. Operational Energy Use}

Due to the shortage of information about the operating energy of Naseby Curling Club, this thesis assumes its annual operational energy use is similar to the "Oliver Curling Club, British Colombia” (Renewable Energy magazine (RE)-2000) and uses this to calculate the total life cycle energy use of Naseby Curling Club.

\section{b. 1. Oliver Curling Club}

According to RE (2000:2) the Oliver Curling Club is a two-storey, wood frame building constructed in 1974. As pointed out by RE (2000:2) the Club includes approximately $167 \mathrm{~m}^{2}$ of floor space, in addition to the $836 \mathrm{~m}^{2}$ four-sheet ice surface and its walls are insulated to R12 $\mathrm{ft}^{2 \circ} \mathrm{Fh} / \mathrm{Btu}$ (approximately $2.1 \mathrm{~m}^{20} \mathrm{C} / \mathrm{W}$ ) and the ceiling to $\mathrm{R} 20$ $\mathrm{ft}^{2 \circ} \mathrm{Fh} / \mathrm{Btu}$ (approximately $3.5 \mathrm{~m}^{20} \mathrm{C} / \mathrm{W}$ ), which are about the same as required in the New Zealand Building Code. The Oliver Curling Club (RE, 2000:2) has an ammonia refrigeration system to make ice, and electric heaters warm the viewing area and locker rooms, second floor lounge and the ice area.

\section{b. 2. Oliver Curling Club: Operating Energy}

As shown in Table 5.51 the average energy consumption of the Oliver Curling Club is $41,800 \mathrm{kWh} /$ year, which is equivalent to $150,480 \mathrm{MJ} /$ year. Since the total area of the Oliver Curling Club is $1,170 \mathrm{~m}^{2}$, its annual average energy consumption $=(150,480 \mathrm{MJ}$ $\div 1,170 \mathrm{~m}^{2}$ ) $=128.6 \mathrm{MJ} / \mathrm{m}^{2}$ or $0.35 \mathrm{MJ} / \mathrm{m}^{2}$ per day (Table 5.51 ).

\begin{tabular}{|c|c|c|c|c|c|}
\hline \multicolumn{6}{|c|}{ Table 5.51: Oliver Curling Club- Operating energy use per square metre per year } \\
\hline Area $\left(\mathrm{m}^{2}\right)^{1}$ & $\begin{array}{l}\text { Average en- } \\
\text { ergy consump- } \\
\text { tion (kWh in } 4 \\
\text { years) }\end{array}$ & $\begin{array}{l}\text { Average en- } \\
\text { ergy consump- } \\
\text { tion } \\
\text { (kWh/year) }\end{array}$ & $\begin{array}{l}\text { Average en- } \\
\text { ergy consump- } \\
\text { tion (MJ /year) }\end{array}$ & $\begin{array}{l}\text { Average en- } \\
\text { ergy consump- } \\
\text { tion } \\
\left(\mathrm{MJ} / \mathrm{m}^{2} / \text { year }\right)\end{array}$ & $\begin{array}{l}\text { Average en- } \\
\text { ergy consump- } \\
\text { tion } \\
\left(\mathrm{MJ} / \mathrm{m}^{2} / \text { day) }\right.\end{array}$ \\
\hline 1,170 & 167,200 & 41,800 & 150,480 & 128.6 & 0.35 \\
\hline
\end{tabular}

\section{b. 3. Naseby Curling Club: Operating Energy}

As shown in Table 5.52 the area of Naseby Curling Club $\left(1300 \mathrm{~m}^{2}\right)$ includes the $980 \mathrm{~m}^{2}$ four-sheet ice surface and a $320 \mathrm{~m}^{2}$ two storey building. Thus the average energy consumption of Naseby Curling Club is $167,180 \mathrm{MJ} /$ Year $\left(1,300 \mathrm{~m}^{2} \times 128.6 \mathrm{MJ} / \mathrm{m}^{2} /\right.$ year $)$ (Table 5.52). 
Table 5.52: Naseby Curling Club- Operating energy use per square metre per year

\begin{tabular}{|c|c|c|c|}
\hline Area $\left(m^{2}\right)^{1-2}$ & $\begin{array}{l}\text { Average energy con- } \\
\text { sumption (MJ/year) }\end{array}$ & $\begin{array}{l}\text { Average energy consump- } \\
\text { tion }\left(\mathrm{MJ} / \mathrm{m}^{2} / \text { year }\right)\end{array}$ & $\begin{array}{l}\text { Average energy consumption } \\
\left(\mathrm{MJ} / \mathrm{m}^{2} / \text { day) }\right.\end{array}$ \\
\hline 1,300 & 167,180 & $128.6^{3}$ & 0.35 \\
\hline
\end{tabular}

\section{c. EF of Naseby Curling Centre}

The life cycle energy of Naseby Curling centre, which is the sum of its total embodied energy (see Table 5.50) and operating energy (see Table 5.52) is equivalent to (40.04 GJ/year + 167 GJ/year) = 207.2 GJ/year (Table 5.53). The annual EF (gha) of Naseby Curling Centre as shown in Table 5.53 is 207.2 GJ/year (life cycle energy use)/100 (global average carrying capacity) $=2.07$ gha

\begin{tabular}{|c|c|c|c|c|}
\hline \multicolumn{5}{|c|}{ Table 5.53: Total ecological footprint ${ }^{1}$ of } \\
\hline Area $\left(\mathrm{m}^{2}\right)$ & $\begin{array}{c}\text { Embodied energy } \\
\text { (GJ/year) }\end{array}$ & $\begin{array}{c}\text { Average energy } \\
\text { consumption } \\
\text { (GJ/year) }\end{array}$ & $\begin{array}{c}\text { Life-cycle energy } \\
\text { (GJ/year) }\end{array}$ & EF (gha) \\
\hline 1,300 & 40.04 & 167.2 & 207.2 \\
\hline
\end{tabular}

\section{d. EF of Naseby Curling Centre per Visitor}

As demonstrated in Table 5.54, in 2008, 13,000 New Zealand visitors (including 4,350 OCRT visitors- see Table 5.56) used the Naseby Indoor Curling Centre. The average time that visitors book the sheet ice is 1.5 hours. As a result, the average energy consumption of Naseby Curling Centre per visitor can be calculated through the following equation:

Average energy consumption MJ/visitor/year $=13,000$ (visitors) $\div 167,180 \mathrm{MJ} /$ year (average energy consumption of Naseby Curling Centre $)=11.6 \mathrm{MJ} /$ visitor/year $($ Table 5.54).

Table 5.54: Naseby Curling Club - annual operating energy use per visitor

\begin{tabular}{|c|c|c|c|}
\hline Area $\left(\mathrm{m}^{2}\right)$ & $\begin{array}{c}\text { Average energy con- } \\
\text { sumption (MJ /year) }\end{array}$ & Visitors per year (2008) $^{1}$ & $\begin{array}{c}\text { Average energy consumption } \\
\text { (MJ/visitor/ year) }\end{array}$ \\
\hline 1,300 & 167,180 & 13,000 & 11.6 \\
\hline 1.Reference: www.curling.co.nz & & \\
\hline
\end{tabular}


As shown in Table 5.55, the Naseby Curling Centre is open seven hours a day throughout the year. Its total working time per year is thus ( 7 hours/day $\times 365$ days) 2,555 hours (Table 5.55). In Naseby indoor curling there are four sheets of ice and at any time a maximum of 32 people can curl in the indoor rink. (www.curling.co.nz) and minimum time to book a sheet is one hour (www.curling.co.nz) (Table 5.55).

Table .5.55: Naseby Curling Centre - working time

Naseby Curling Centre working time (hours per day) ${ }^{1}$

Numbers of ice sheets

Maximum number of people who can curl in one time

Naseby Curling Centre working time (hours per year)

Maximum number of player (1 hour per player) per year

Max time that each visitor $(13,000)$ can play curling (hours/per year)

Min time that a visitor can book to play indoor curling ${ }^{1}$

average time that a visitor could play indoor curling (hours/visitor)

1. Reference: www.curling.co.nz

As shown in Table 5.56, in 2011, 4,350 OCRT visitors who play curling at Naseby cover $33.5 \%$ of its whole visitors (13,000 visitors). Thus the annual EF of the 4,350 OCRT visitors is equal to $33.5 \%$ of the EF of 13,000 visitors (2.07gha-see Table 5.53) = 0.7 gha (Table 5.56), consequently the annual EF of an OCRT visitor playing curling is equivalent to 0.7 (gha) $\div 4,350$ visitors $=0.000016$ gha. The annual EF of the Naseby Curling Centre is 0.00016 gha /visitor.

\section{Table 5.56. OCRT- Visitors- curling EF (gha) (2011)}

\begin{tabular}{|c|c|c|c|c|}
\hline $\begin{array}{l}\text { Total num- } \\
\text { ber of } \\
\text { OCRT visi- } \\
\text { tors }\end{array}$ & $\begin{array}{l}\% \text { of OCRT visi- } \\
\text { tors who play in- } \\
\text { door curling at } \\
\text { Naseby Curling } \\
\text { Centre }\end{array}$ & $\begin{array}{l}\text { Total number of } \\
\text { OCRT- visitors } \\
\text { who play curling } \\
\text { at Naseby curl- } \\
\text { ing centre }\end{array}$ & $\begin{array}{l}\text { EF of } 13,000 \text { visi- } \\
\text { tors of Curling cen- } \\
\text { tre (gha) }\end{array}$ & $\begin{array}{l}\text { EF of } 4,350 \text { OCRT visi- } \\
\text { tors ( } 33.5 \% \text { of } 13,000 \\
\text { Naseby indoor curling } \\
\text { centre visitors) gha }\end{array}$ \\
\hline 11,788 & $36.9 \%$ & 4,350 & $2.07^{3}$ & 0.7 (33.5 \% of 2.07$)$ \\
\hline
\end{tabular}

\subsubsection{EF of OCRT Outdoor Activities}

\section{a. OCRT: the EF of Walking}

As indicated in Table 5.57, the total energy of walking is in a range 0.37-2.21 MJ/km. Table 5.57 shows that of 11,788 OCRT visitors $0.6 \%$ (71) walk. 
Table 5.57. Range of energy values relating to different aspects of travel, and range of total values (MJ/ $\mathrm{km})$

\begin{tabular}{l|c|c|c|c|}
\hline Mode & Embodied energy & Food/fuel & shower & Total \\
\hline Walking & 0.25 & $0.12-1.96$ & No shower & $0.37-2.21$ \\
\hline Cycling & $0.03-0.08$ & $0.06-1.42$ & No shower & $0.09-1.50$ \\
\hline Cycling(shower & $0.03-0.08$ & $0.06-1.42$ & $0.57-2.52$ & $0.60-4.02$ \\
\hline $\begin{array}{l}\text { Driving (average) } \\
\text { * The value for average driving is included as a comparison }\end{array}$ \\
$\begin{array}{l}\text { - Reference: Vale and Vale, 2009:109 } \\
\text { R }\end{array}$
\end{tabular}

Since the distance that a person can walk (easy walking) in an hour is equal to $5 \mathrm{~km}$ (Browning et al, 2005:390), the total distance that 71 OCRT visitors can walk in an hour is $355 \mathrm{~km}$.(Table 5.58). As a result, the distance that 71 OCRT visitors can walk in a typical assumed walk of 3 hours duration is $1,065 \mathrm{~km}(355 \times 3)$ (Table 5.58$)$.

Since the EF of food consumed by OCRT visitors has already been calculated in food category, the minimum value of $0.37 \mathrm{MJ} / \mathrm{km}$ (see Table 5.57) is used to calculate the $\mathrm{EF}$ of OCRT visitors- walking.

As shown in Table 5.58, the energy use (MJ) of 71 OCRT visitors who walk for 3 hours $(1,065 \mathrm{~km})$ is equivalent to $394.05 \mathrm{MJ}(0.394 \mathrm{GJ})$ and their EF is equivalent to $0.394 \mathrm{GJ}$ (energy use) $\div 100$ (global average carrying capacity) $=0.0039$ gha. In addition, the EF of an OCRT visitor who walks 3 hours is 0.00005 gha /visitor (Table 5.58).

Table 5.58: Ecological footprint of OCRT- visitors (walking)

Total number of OCRT visitors

$\%$ of visitors who walk (see Table 5.45)

Total number of OCRT visitors who walk (see Table 5.49)

Distance that a person can walk (easy walking) (km/hour)

Distance that 71 OCRT visitors can walk an hour (km)

Distance that 71 OCRT visitors can walk in 3 hours (km)

Energy use of walking (MJ/km) (see Table 5.57)

Energy use (MJ) of 71 OCRT visitors who walk 3 hours $(1,065 \mathrm{~km})$

EF of 71 OCRT visitors who walk 3 hours (gha)

EF (gha) of an OCRT visitor who walks 3 hours

1. Reference: (Browning et al, 2005:390)

2. See Table 5.57

3. $394.05(\mathrm{MJ})=0.394(\mathrm{GJ})$

\section{b. EF of Golf}

As demonstrated in Table 5.59, golf is classified into two types; the walking golfer and the driving golfer. The integration of each golfer's share of the land and the embodied energy of equipment used in each type of golf determines its total impact (MJ/participant/year). As shown in Table 5.59, the total impact of a walking golfer and 
driving golfer who play 38 rounds per year (Vale and Vale, 2009: 269) are 2,393 and 5,621 MJ/participant/year respectively. Since the average of OCRT visitor nights is 3.6 (see Figure 5.7), this study makes this assumption that each OCRT visitor who on average stays in OCRT 3.6 nights, plays 3 rounds of golf per 3.6 nights.

As shown in Table 5.59 the average impacts of each player who play 38 rounds is 4007(MJ/participants/38 rounds/year). Consequently the average impact of golfing per visitor per round is equal to 4007 (MJ/participants/38 rounds/ year) $\div 38$ (rounds) $=105$ $\mathrm{MJ} /$ participants/round/year). As a result, the average impact of each OCRT visitor who plays 3 rounds $\mathrm{f}$ golf is equal to 105 (MJ/participants/rounds/year) $\times 3=315$ $\mathrm{MJ} /$ participant $=0.315 \mathrm{GJ} /$ participant

Table 5.59: Total impact per player of land and equipment associated with Golf

\begin{tabular}{|c|c|c|c|c|}
\hline Туре & $\begin{array}{c}\text { Land } \\
\text { area }\end{array}$ & $\begin{array}{c}\text { Energy equivalent of } \\
\text { land (MJ/38 rounds/year) }\end{array}$ & $\begin{array}{l}\text { Embodied energy of } \\
\text { equipment (MJ/ year) }\end{array}$ & $\begin{array}{c}\text { Total impact(MJ/ partici- } \\
\text { pant/38 rounds/year) }\end{array}$ \\
\hline $\begin{array}{l}\text { Golf (walking } \\
\text { golfer) }\end{array}$ & 170 & 2,295 & 98 & 2,393 \\
\hline $\begin{array}{l}\text { Golf (driving } \\
\text { golfer) }\end{array}$ & 170 & 2,295 & 3,326 & 5,621 \\
\hline \multicolumn{5}{|c|}{$\begin{array}{l}\text { 1. Average impact of both Golf (walking and driving) }=4007 \mathrm{MJ} / \text { participant } / 38 \text { rounds } / \text { year } \\
\text { 2. Average impact of both Golf (walking and driving) }=105 \mathrm{MJ} / \text { participant } / \text { round } / \text { year } \\
\text { 3. Average impact of both Golf (walking and driving) }=315 \mathrm{MJ} / \text { participant } / 3 \text { rounds } / \text { year }\end{array}$} \\
\hline
\end{tabular}

Table 5.60 determines that of 11,788 OCRT visitors only $1.3 \%$ (153) play golf (Table 5.40 ). Table 5.60 shows that the total impact of the 153 OCRT visitors who play 3 rounds of golf is equivalent to $153 \times 315 \mathrm{MJ} /$ participant/year (See Table 5.59) = 48,195 $\mathrm{MJ} /$ participant/year. As a result the annual EF of these 153 visitors is equivalent to 48.195 GJ/year/100 = 0.48 gha. In addition, the EF of an OCRT visitor who plays golf is equivalent to the total EF of 153 visitors (0.48gha) $\div$ total number of OCRT visitors who play golf $(153)=0.003$ gha/participant/year .

\section{Table 5.60: EF of OCRT visitors - golf (2011)}

\begin{tabular}{|c|c|c|c|c|c|}
\hline $\begin{array}{l}\text { Total Numbers } \\
\text { of OCRT visitors }\end{array}$ & $\begin{array}{l}\% \text { of OCRT } \\
\text { visitors who } \\
\text { play golf }\end{array}$ & $\begin{array}{l}\text { Numbers of } \\
\text { OCRT visitors } \\
\text { who play golf. }\end{array}$ & $\begin{array}{l}\text { Total impact } \\
\text { (MJ/ partici- } \\
\text { pant/3 } \\
\text { rounds/year) }{ }^{1}\end{array}$ & $\begin{array}{l}\text { Total impact of } \\
\text { OCRT visitors } \\
\text { who play golf } \\
\text { (MJ/year) }\end{array}$ & $\begin{array}{c}\mathrm{EF} \\
(\mathrm{gha})^{2}\end{array}$ \\
\hline 11,788 & 1.3 & 153 & 315 & 48,195 & 0.48 \\
\hline
\end{tabular}




\section{c. EF of Other Activities (except curling, walking and golf)}

Becken (2001) proposes a method for the classification of tourism attractions and activities as shown in Table 5.61. According to Becken (2001:2) these can be put into the three categories of attraction, entertainment, and activity (Table 5.61). These three main categories comprise related sub-categories as shown in Table 5.61.

\section{Table 5.61: Classification of tourism attractions and activities.}

\begin{tabular}{|c|c|c|}
\hline Generic category & Sub-category & $\begin{array}{l}\text { Types of operation/ establish- } \\
\text { ments }\end{array}$ \\
\hline \multirow{5}{*}{ Attractions } & Building & $\begin{array}{l}\text { Art gallery, Historic building, } \\
\text { Museum, Visitor centre. }\end{array}$ \\
\hline & Park & $\begin{array}{l}\text { Aquarium, Botanical garden, } \\
\text { Wildlife park, Zoo. }\end{array}$ \\
\hline & Amusement & $\begin{array}{l}\text { Experience centre, Gondola, } \\
\text { Sporting complex, Theme park. }\end{array}$ \\
\hline & Industry & $\begin{array}{l}\text { Brewery, Farm show, Wine trail } \\
\text { and tasting. }\end{array}$ \\
\hline & Natural attraction & $\begin{array}{l}\text { Geothermal attractions (hot } \\
\text { pools), Glow worm caves. }\end{array}$ \\
\hline Entertainment & Performance, Other & $\begin{array}{l}\text { Cinema, live theatre or concert, } \\
\text { Maori culture performance, bar/ } \\
\text { night club, casino, shopping. }\end{array}$ \\
\hline \multirow{4}{*}{ Activity } & Air activity & $\begin{array}{l}\text { Scenic flight, Air sports (skydiv- } \\
\text { ing, ballooning, parapenting), } \\
\text { Whale watching by air, Heliski- } \\
\text { ing. }\end{array}$ \\
\hline & Sea activity & $\begin{array}{l}\text { Diving, Dolphins/Whale watch- } \\
\text { ing, Jet boating, Sailing, Scenic } \\
\text { boat cruise, Sea/coastal fishing. }\end{array}$ \\
\hline & Adventure activity & $\begin{array}{l}\text { Adventure (bungy and others), } \\
\text { Kayaking, Mountain biking, } \\
\text { Mountain, rock climbing/caving, } \\
\text { Rafting, Skiing, Surfing. }\end{array}$ \\
\hline & Nature recreation & $\begin{array}{l}\text { Cycling, Golf, Horse riding, } \\
\text { Lake/river fishing, Tramping, } \\
\text { Viewing wildlife in natural set- } \\
\text { ting, Walking (guided), sightsee- } \\
\text { ing. }\end{array}$ \\
\hline
\end{tabular}

Table 5.62 contains the average, minimum and maximum energy use suggested by Becken (2001:3) for each of the three categories of tourism activities and attractions. As shown in Table 5.62, the total annual energy use of the three categories varies considerably from a minimum energy use of 9GJ to the maximum of 77,020GJ (Table 5.62). 
Table 5.62: Energy use per annum for the three categories.

\begin{tabular}{|l|c|c|c|}
\hline Energy use & Attraction & Entertainment & Activity \\
\hline Average (GJ) & 411 & 1,599 & 495 \\
\hline Min (GJ) & 4 & 36 & 9 \\
\hline Max (GJ) & 53,223 & 77,020 & 29,208 \\
\hline
\end{tabular}

The energy use per visitor for the three categories of attraction, entertainment and activity are also calculated by Becken (2001) and presented in Table 5.63. Although the minimum energy use/visitor is relatively constant across the three categories, the maximums vary considerably, with activities being clearly the most energy consuming.

\begin{tabular}{|l|c|c|c|}
\hline \multicolumn{4}{|c|}{ Table 5.63: Energy use per tourist for the three categories. } \\
\hline & Attraction & Entertainment & Activity \\
\hline Average (MJ/ tourist) & 6.2 & 9.4 & 95.6 \\
\hline Min (MJ/ tourist) & 0.6 & 0.8 & 0.6 \\
\hline Max (MJ/ tourist) & 174.0 & 38.5 & 2902.8 \\
\hline
\end{tabular}

Becken (2001:5) also sets out the energy use per visitor of key operator types as shown in Table 5.64. In this analysis, the maximum energy is used by diving (800MJ/tourist) and the minimum by a farm show. Operator types that involve transport have the higher values in Table 5.64.

Table 5.64: Energy use per tourist of key operator types.

\begin{tabular}{|l|c|}
\hline Operator type & Energy (MJ/tourist) \\
\hline Farm show & 7 \\
\hline Museum & 10 \\
\hline Zoo/wildlife park & 16 \\
\hline Experience centre & 29 \\
\hline Rafting & 36 \\
\hline Guided walks (including transport to sites) & 110 \\
\hline Scenic boat cruise & 165 \\
\hline Scenic flight & 340 \\
\hline Diving & 800 \\
\hline - Reference: Becken, 2001:5 & \\
\hline
\end{tabular}

This thesis uses the categorisation of tourism activities and attractions proposed by Becken (2001) as discussed above to classify OCRT tourism activities in the following table. 


\begin{tabular}{|c|c|c|c|}
\hline Generic category & Sub-category & Types of operation/establishment & $\begin{array}{l}\text { Average } \\
\text { MJ/tourist }\end{array}$ \\
\hline \multirow[t]{5}{*}{ Attractions } & Building & $\begin{array}{l}\text { Hayes engineering, Gold mining } \\
\text { towns, Old Cromwell town, Taieri, } \\
\text { Golden progress mine }\end{array}$ & 6.2 \\
\hline & Park & - & - \\
\hline & Amusement & - & - \\
\hline & Industry & $\begin{array}{l}\text { Wineries, Ophir high country farm, } \\
\text { fruit picking along trail }\end{array}$ & 6.2 \\
\hline & Natural attraction & - & \\
\hline Entertainment & Performance, Other & - & \\
\hline \multirow{4}{*}{ Activity } & Air activity & - & - \\
\hline & Sea activity & - & - \\
\hline & Adventure activity & Mountain biking at Naseby & 95.6 \\
\hline & Nature recreation & $\begin{array}{l}\text { Fishing, swim at Ophir, Sightseeing, } \\
\text { Clyde dam tour, biking. }\end{array}$ & 95.6 \\
\hline
\end{tabular}

Likewise, the average energy use of each type of operation/establishment as calculated by Becken (2001:3) (Table 5.63) is considered here to represent the energy use of OCRT types of operation/establishment (Table 5.65).

\section{c. 1. EF of Biking (excluding mountain biking, Table 5.49)}

As shown in Table 5.66, in 2011, 71 visitors (0.6 \% of 11,788 OCRT visitors) engaged in biking (mountain biking is a separate category, see Table 5.49). The total impact of a participant who bikes is $95.6 \mathrm{MJ} /$ year (see Table 5.65) thus the total impact of 71 OCRT visitors is $71 \times 95.6 \mathrm{MJ} /$ visitor/year $=6,788 \mathrm{MJ} /$ year $=6.8 \mathrm{GJ} /$ year (Table 5.66). As a result, the EF of 71 OCRT visitors who bike is $6.8 \mathrm{GJ} /$ year $\div 100$ (average global carrying capacity) $=0.068$ gha/year (Table 5.66). Moreover the EF of an OCRT visitor who bikes is $0.068 \div 71=0.0009$ gha/visitor/year.

\section{Table 5.66: EF of OCRT visitors - biking (2011)}

\begin{tabular}{|c|c|c|c|c|c|}
\hline $\begin{array}{l}\text { Total Num- } \\
\text { bers of } \\
\text { OCRT visi- } \\
\text { tors }\end{array}$ & $\begin{array}{l}\text { \% of OCRT visi- } \\
\text { tors who play } \\
\text { biking }\end{array}$ & $\begin{array}{l}\text { Numbers of } \\
\text { OCRT visitors } \\
\text { who play bik- } \\
\text { ing. }\end{array}$ & $\begin{array}{c}\text { Total impact } \\
\text { (MJ/ partici- } \\
\text { pant })^{1}\end{array}$ & $\begin{array}{l}\text { Total impact of } \\
71 \text { OCRT visi- } \\
\text { tors who play } \\
\text { biking (MJ) }\end{array}$ & $\begin{array}{c}\mathrm{EF} \\
\text { (gha) }\end{array}$ \\
\hline 11,788 & 0.6 & 71 & 95.6 & $6,788^{2}$ & 0.068 \\
\hline \multicolumn{6}{|c|}{$\begin{array}{l}\text { 1. (See Table } 5.65 \text { ) } \\
\text { 2. } 6,788 \mathrm{MJ} / \text { year = } 6.8 \mathrm{GJ} / \text { year } \\
\text { 3. EF (gha/ visitor/ year) of an } \mathrm{O}\end{array}$} \\
\hline
\end{tabular}




\section{c. 2. The EF of OCRT Visitors: Mountain Biking at Naseby}

Table 5.67 shows 295 (2.5\% of 11,788 OCRT) visitors go mountain biking at Naseby (see Table 5.49). The total impact of a participant who does this is $95.6 \mathrm{MJ} / \mathrm{year}$ (see Table 5.65). Consequently the total impact of 295 OCRT visitors is $295 \times 95.6 \mathrm{MJ} / \mathrm{visi}$ tor/year $=28,202 \mathrm{MJ} /$ year $=28.2 \mathrm{GJ} /$ year (Table 5.67). As a result, the EF of 295 OCRT visitors who go mountain biking is $28.2 \mathrm{GJ} /$ year $\div 100$ (global average carrying capacity) $=0.28$ gha (Table 5.67). In addition the EF of an OCRT visitor who goes mountain biking is $0.28 \div 295=0.0009$ gha/visitor/year (Table 5.67).

\begin{tabular}{|c|c|c|c|c|c|}
\hline \multicolumn{6}{|c|}{ Table 5.67: EF of OCRT visitors - mountain biking (2011) } \\
\hline $\begin{array}{l}\text { Total Numbers } \\
\text { of OCRT visi- } \\
\text { tors }\end{array}$ & $\begin{array}{l}\% \text { of OCRT } \\
\text { visitors } \\
\text { mountain } \\
\text { biking }\end{array}$ & $\begin{array}{l}\text { Numbers of } \\
\text { OCRT visitors } \\
\text { mountain biking }\end{array}$ & 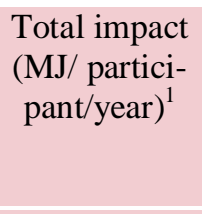 & $\begin{array}{l}\text { Total impact } \\
\text { of } 295 \text { OCRT } \\
\text { visitors moun- } \\
\text { tain biking } \\
\text { (MJ /year) }\end{array}$ & $\begin{array}{c}\mathrm{EF} \\
(\mathrm{gha})^{3}\end{array}$ \\
\hline 11,788 & 2.5 & 295 & 95.6 & $28,202^{2}$ & 0.28 \\
\hline \multicolumn{6}{|c|}{$\begin{array}{l}\text { 1. Reference: Becken, } 2001: 2 \\
\text { 2. } 28,202 \mathrm{MJ} / \text { year = } 28.2 \mathrm{GJ} / \text { year } \\
\text { 3. EF (gha/visitor/year) of OCRT visitor mountain biking = } 0.0009\end{array}$} \\
\hline
\end{tabular}

\section{c. 3. EF of OCRT Visitors: Fishing}

Table 5.68 indicates that 224 (1.9\% of 11,788 OCRT visitors) go fishing (see Table 5.49). The total impact of a participant going fishing, assuming they need transport to get themselves and the gear to the fishing ground, is 95.6 MJ/year (see Table 5.65). In addition the total impact of 224 OCRT visitors going fishing is $224 \times 95.6 \mathrm{MJ} /$ visitor/year $=21,414 \mathrm{MJ} /$ year $=21.4 \mathrm{GJ} /$ year (Table 5.68). As a result, the EF of 224 OCRT visitors who go fishing is equivalent to $21.4 \mathrm{GJ} /$ year $\div 100$ (global average carrying capacity) $=0.21$ gha (Table 5.68). Moreover the EF of an OCRT visitor who goes fishing is $0.21 \div 224=0.0009$ gha /visitor/year (Table 5.68).

\section{Table 5.68: EF of OCRT visitors - fishing (2011)}

\begin{tabular}{|c|c|c|c|c|c|}
\hline $\begin{array}{l}\text { Total Numbers } \\
\text { of OCRT visitors }\end{array}$ & $\begin{array}{l}\% \text { of OCRT } \\
\text { visitors fish- } \\
\text { ing }\end{array}$ & $\begin{array}{l}\text { Numbers of } \\
\text { OCRT visitors } \\
\text { fishing }\end{array}$ & 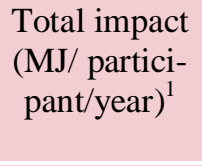 & $\begin{array}{l}\text { Total impact of } \\
224 \text { OCRT visi- } \\
\text { tors fishing } \\
\text { (MJ/year) }\end{array}$ & $\begin{array}{c}\mathrm{EF} \\
(\mathrm{gha})^{3}\end{array}$ \\
\hline 11,788 & 1.9 & 224 & 95.6 & 21,414 & 0.21 \\
\hline
\end{tabular}




\section{c. 4. The EF of OCRT Visitors: Swimming at Ophir}

Table 5.69 shows that 71 (0.6 \% of 11,788 OCRT) visitors swim at Ophir (see Table 5.49). Using the values in Table 5.65 and the same method as before, the EF per visitor for this activity is set out in Table 5.69.

\section{Table 5.69: EF of OCRT visitors - swimming at Ophir (2011)}

\begin{tabular}{|c|c|c|c|c|c|}
\hline $\begin{array}{l}\text { Total Numbers } \\
\text { of OCRT visitors }\end{array}$ & $\begin{array}{l}\text { \% of OCRT } \\
\text { visitors } \\
\text { swimming }\end{array}$ & $\begin{array}{l}\text { Numbers of } \\
\text { OCRT visitors } \\
\text { swimming }\end{array}$ & $\begin{array}{c}\text { Total impact } \\
\text { (MJ/ partici- } \\
\text { pant/year) }^{1}\end{array}$ & $\begin{array}{l}\text { Total impact of } \\
71 \text { OCRT visi- } \\
\text { tors swimming } \\
\text { (MJ/year) }\end{array}$ & $\begin{array}{c}\mathrm{EF} \\
\text { (gha) }\end{array}$ \\
\hline 11,788 & 0.6 & 71 & 95.6 & $6,788^{2}$ & 0.068 \\
\hline \multicolumn{6}{|c|}{$\begin{array}{l}\text { 1.Reference: Becken, 2001:2 } \\
\text { 2. } 6,788 \mathrm{MJ} / \text { year = } 6.8 \mathrm{GJ} / \text { year }\end{array}$} \\
\hline
\end{tabular}

\section{c. 5. The EF of OCRT Visitors: Wineries}

Using the impact factor of 6.2 MJ/year (see Table 5.65) and the method above the EF of visitors to wineries is shown Table 5.70 .

Table 5.70: EF of OCRT visitors - wineries (2011)

\begin{tabular}{|c|c|c|c|c|c|}
\hline $\begin{array}{l}\text { Total Numbers of } \\
\text { OCRT visitors }\end{array}$ & $\begin{array}{c}\% \text { of OCRT } \\
\text { visitors to } \\
\text { wineries }\end{array}$ & $\begin{array}{l}\text { Numbers of } \\
\text { OCRT visitors to } \\
\text { wineries }\end{array}$ & $\begin{array}{l}\text { Total impact } \\
\text { (MJ/ partici- } \\
\text { pant/year) }^{1}\end{array}$ & $\begin{array}{l}\text { Total impact of } \\
\text { 1,992 OCRT } \\
\text { visitors to win- } \\
\text { eries (MJ/year) }\end{array}$ & $\begin{array}{c}\mathrm{EF} \\
(\mathrm{gha})^{3}\end{array}$ \\
\hline 11,788 & 16.9 & 1,992 & 6.2 & $12,350.4^{2}$ & 0.12 \\
\hline \multicolumn{6}{|c|}{$\begin{array}{l}\text { 1. Reference: Becken, 2001:2 } \\
\text { 2. } 12,350.4 \mathrm{MJ} / \text { year = } 12.3 \mathrm{GJ} / \text { year }\end{array}$} \\
\hline
\end{tabular}

\section{c. 6. The EF of OCRT Visitors: Sightseeing}

Table 5.71 shows that 3,607 (30.6 \% of 11,788 OCRT visitors) look at the scenery (see Table 5.49). Assuming that some form of transport is involved the total impact of a participant who visits scenery is $95.6 \mathrm{MJ} /$ year (see Table 5.65). The impact per visitor is calculated as before and the results are shown in Table 5.71 .

\begin{tabular}{|c|c|c|c|c|c|}
\hline \multicolumn{6}{|c|}{ Table 5.71: EF of OCRT visitors -sightseeing (2011) } \\
\hline $\begin{array}{l}\text { Total Numbers } \\
\text { of OCRT visitors }\end{array}$ & $\begin{array}{l}\% \text { of OCRT } \\
\text { visitors sight- } \\
\text { seeing }\end{array}$ & $\begin{array}{l}\text { Numbers of } \\
\text { OCRT visitors } \\
\text { sightseeing }\end{array}$ & $\begin{array}{l}\text { Total impact } \\
\text { (MJ/ partici- } \\
\text { pant/year) }^{1}\end{array}$ & $\begin{array}{c}\text { Total impact of } \\
3,607 \text { OCRT } \\
\text { visitors sight- } \\
\text { seeing } \\
\text { (MJ/year) }\end{array}$ & $\begin{array}{c}\mathrm{EF} \\
(\mathrm{gha})^{3}\end{array}$ \\
\hline 11,788 & 30.6 & 3,607 & 95.6 & $344,829.2$ & 3.4 \\
\hline \multicolumn{6}{|c|}{$\begin{array}{l}\text { 1. Reference: Becken, } 2001: 2 \\
\text { 2. } 344,829.2 \mathrm{MJ} / \text { year = } 344.8 \mathrm{GJ} / \text { year } \\
\text { 3. EF (gha/ visitor/ year) of OCRT visitor sightseeing = } 0.0009\end{array}$} \\
\hline
\end{tabular}




\section{c. 7. The EF of OCRT Visitors: visit to Hayes Engineering}

The total impact of a participant who visits this heritage site is $6.2 \mathrm{MJ} /$ year (see Table 5.65) on the assumption that unlike sightseeing, travel to the attraction is not using a lot of energy. The overall impact of this activity is calculated as before and set out in Table 5.72 .

\begin{tabular}{|c|c|c|c|c|c|}
\hline $\begin{array}{c}\text { Total Numbers of } \\
\text { OCRT visitors }\end{array}$ & $\begin{array}{l}\% \text { of OCRT } \\
\text { visitors to } \\
\text { Hayes engi- } \\
\text { neering }\end{array}$ & $\begin{array}{c}\text { Numbers of } \\
\text { OCRT visitors to } \\
\text { Hayes engineer- } \\
\text { ing }\end{array}$ & $\begin{array}{c}\text { Total impact } \\
\text { (MJ/participant } \\
\text { /year) }\end{array}$ & $\begin{array}{l}\text { Total impact of } \\
955 \text { OCRT visi- } \\
\text { tors to Hayes } \\
\text { engineering } \\
\text { (MJ/year) }\end{array}$ & $\begin{array}{c}\mathrm{EF} \\
(\mathrm{gha})^{3}\end{array}$ \\
\hline 11,788 & 8.1 & 955 & 6.2 & 5,921 & 0.06 \\
\hline $\begin{array}{l}\text { 1. Reference: Becke } \\
\text { 2. 5,921 MJ/year = } \\
\text { 3. EF (gha/ visitor/ }\end{array}$ & $\begin{array}{l}\text { 2001:2 } \\
\text { GJ/ year }\end{array}$ & & 006 & & \\
\hline
\end{tabular}

\section{c. 8. The EF of OCRT Visitors: Ophir High Country Farm}

Using the energy factor from Table 5.65 and the method established above, the impact of visitors to this activity is shown in Table 5.73 .

\begin{tabular}{|l|c|c|c|c|c|}
\hline \multicolumn{5}{|c|}{ Table 5.73: EF of OCRT visitors -Ophir high country farm (2011) } \\
\hline $\begin{array}{c}\text { Total Numbers of } \\
\text { OCRT visitors }\end{array}$ & $\begin{array}{c}\text { \% of OCRT } \\
\text { visitors to } \\
\text { Ophir high } \\
\text { country farm }\end{array}$ & $\begin{array}{c}\text { Numbers of } \\
\text { OCRT visitors to } \\
\text { Ophir high coun- } \\
\text { try farm }\end{array}$ & $\begin{array}{c}\text { Total impact } \\
\text { (MJ/participant } \\
\text { /year) }\end{array}$ & $\begin{array}{c}\text { Total impact of } \\
\text { 71 OCRT visi- } \\
\text { tors to Ophir } \\
\text { high country } \\
\text { farm (MJ/year) }\end{array}$ & $\begin{array}{c}\text { EF } \\
\text { (gha) }\end{array}$ \\
\hline $\begin{array}{c}11,788 \\
\text { 1. Reference: Becken, 2001:2 }\end{array}$ & 71 & 6.2 & 440.2 & 0.004 \\
\hline $\begin{array}{l}\text { 2. } 440.2 \mathrm{MJ} / \text { year = 0.440 GJ/ year } \\
\text { 3. EF (gha/ visitor/ year) of OCRT visitor to Ophir high country farm = } 0.00006\end{array}$ & & & \\
\hline
\end{tabular}

\section{c. 9. The EF of OCRT Visitors; Taieri Gorge Railway}

Based on the established method, the impact of visitors to this activity is shown in Table 5.74 .

\begin{tabular}{|c|c|c|c|c|c|}
\hline \multicolumn{6}{|c|}{ Table 5.74: EF of OCRT visitors - Taieri Gorge Railway (2011) } \\
\hline $\begin{array}{l}\text { Total Numbers } \\
\text { of OCRT visitors }\end{array}$ & $\begin{array}{c}\% \text { of OCRT } \\
\text { visitors to } \\
\text { Taieri }\end{array}$ & $\begin{array}{c}\text { Numbers of } \\
\text { OCRT visitors to } \\
\text { Taieri }\end{array}$ & $\begin{array}{c}\text { Total impact } \\
\text { (MJ/participant } \\
\text { /year) }^{1}\end{array}$ & $\begin{array}{c}\text { Total impact of } \\
5,458 \text { OCRT } \\
\text { visitors to } \\
\text { Taieri } \\
\text { (MJ/year) }\end{array}$ & $\begin{array}{c}\mathrm{EF} \\
(\mathrm{gha})^{3}\end{array}$ \\
\hline 11,788 & 46.3 & 5,458 & 6.2 & $33,839.6$ & 0.33 \\
\hline $\begin{array}{l}\text { 1. Reference: Bec } \\
\text { 2.33839.6 MJ/yea } \\
\text { 3.EF (gha/ visitor }\end{array}$ & $\begin{array}{l}2001: 2 \\
33.8 \mathrm{GJ} / \mathrm{y}\end{array}$ & , & & & \\
\hline
\end{tabular}




\section{c. 10. The EF of OCRT Visitors: Gold Mining Towns}

Based on the established method, the impact of visitors to this activity is shown in Table 5.75 .

\begin{tabular}{|c|c|c|c|c|c|}
\hline \multicolumn{6}{|c|}{ Table 5.75: EF of OCRT visitors - Gold mining towns (2011) } \\
\hline $\begin{array}{c}\text { Total Numbers of } \\
\text { OCRT visitors }\end{array}$ & $\begin{array}{l}\% \text { of OCRT } \\
\text { visitors to } \\
\text { Gold mining } \\
\text { towns }\end{array}$ & $\begin{array}{l}\text { Numbers of } \\
\text { OCRT visitors to } \\
\text { Gold mining } \\
\text { towns }\end{array}$ & 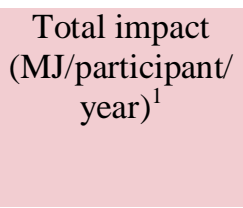 & $\begin{array}{c}\text { Total impact of } \\
4,126 \text { OCRT } \\
\text { visitors to Gold } \\
\text { mining towns } \\
(\mathrm{MJ} / \text { year })\end{array}$ & $\mathrm{EF}$ (gha) ${ }^{3}$ \\
\hline 11,788 & 35 & 4,126 & 6.2 & $25,581.2^{2}$ & 0.26 \\
\hline $\begin{array}{l}\text { 1. Reference: Beck } \\
\text { 2. 25,581.2 MJ/yea } \\
\text { 3. EF (gha/ visitor/ }\end{array}$ & $\begin{array}{l}\text { 2001:2 } \\
\text { 25.6 GJ/ year } \\
\text { ) of OCRT v }\end{array}$ & & 0.00006 & & \\
\hline
\end{tabular}

\section{c. 11. The EF of OCRT Visitors: Old Cromwell Town}

Based on the established method, the impact of visitors to this activity is shown in Table 5.76 .

\begin{tabular}{|c|c|c|c|c|c|}
\hline \multicolumn{6}{|c|}{ Table 5.76: EF of OCRT visitors - Old Cromwell town (2011) } \\
\hline $\begin{array}{c}\text { Total Numbers of } \\
\text { OCRT visitors }\end{array}$ & $\begin{array}{l}\% \text { of OCRT } \\
\text { visitors to } \\
\text { Old Crom- } \\
\text { well town }\end{array}$ & $\begin{array}{c}\text { Numbers of } \\
\text { OCRT visitors to } \\
\text { Old Cromwell } \\
\text { town }\end{array}$ & $\begin{array}{c}\text { Total impact } \\
\text { (MJ/participant } \\
\text { /year) }^{1}\end{array}$ & $\begin{array}{c}\text { Total impact of } \\
2,652 \text { OCRT } \\
\text { visitors to Old } \\
\text { Cromwell town } \\
\text { (MJ/year) }\end{array}$ & $\begin{array}{c}\mathrm{EF} \\
(\mathrm{gha})^{3}\end{array}$ \\
\hline 11,788 & 22.5 & 2,652 & 6.2 & $16,442^{2}$ & 0.16 \\
\hline $\begin{array}{l}\text { 1. Reference: Becke } \\
\text { 2. 16,442 MJ/year = } \\
\text { 3. EF (gha/ visitor/ }\end{array}$ & $\begin{array}{l}001: 2 \\
\text { GJ/ year }\end{array}$ & 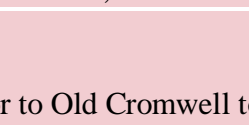 & $=0.00006$ & & \\
\hline
\end{tabular}

\section{c. 12. The EF of OCRT Visitors: Visiting Friends and Relatives}

Since the energy use and the EF of visiting friends and relatives is not available, this study assumes them to be the same as the energy use and EF of homestead visitors. As a first attempt to calculate the EF of visiting friends and relatives, this study measures the energy use and EF of homestead visitors as shown in the following table

Table 5.77 shows that the total homestead visitor nights are 1, 273 (see Table 5.19) and the average OCRT accommodation services stay is equivalent 3.6 nights. Consequently, the total number of visitors who use OCRT homestead accommodation is $1,273 \div 3.6=$ 354 (Table 5.77). As shown in Table 5.37, the total EF of OCRT homestead accommodation is 0.11(gha). But it should be remembered that in Table 5.37 this EF does not consider the influence of using a veranda on the total EF of homestead accommodation services. 
As indicated in Table 5.39, OCRT homestead accommodation services include 1 veranda (outdoor sitting area with a timber frame roof) with an (average) area of $14.3 \mathrm{~m}^{2}$. Furthermore, as explained in Section 5.6.8.3 using $1 \mathrm{~m}^{2}$ V/B can reduce the EF of accommodation services by $20.7 \mathrm{~m}^{2}$. Thus the total EF of OCRT homestead accommodation services including $14.3 \mathrm{~m}^{2} \mathrm{~V} / \mathrm{B}$ is equivalent to $1100 \mathrm{~m}^{2}$ (0.11 gha) - (14.3 m $\times$ $\left.18.1 \mathrm{~m}^{2}\right)=803.99 \mathrm{~m}^{2}=0.08$ gha (Table 5.77).

Table 5.77: EF of OCRT visitors - homestead (2011)

\begin{tabular}{|c|c|c|c|c|}
\hline $\begin{array}{l}\text { Total homestead } \\
\text { visitor nights }^{1}\end{array}$ & $\begin{array}{c}\text { Average OCRT } \\
\text { accommodation } \\
\text { services visitor } \\
\text { nights }^{2}\end{array}$ & $\begin{array}{l}\text { Number of OCRT } \\
\text { homestead visitors } \\
\frac{3}{3}\end{array}$ & $\begin{array}{l}\text { Total EF of OCRT } \\
\text { homesteads }^{4}\end{array}$ & $\begin{array}{c}\text { EF of OCRT- } \\
\text { homestead/visitor }\end{array}$ \\
\hline 1,273 & 3.6 & 354 & 0.08 & 0.0002 \\
\hline $\begin{array}{l}\text { 1. Reference: Table } \\
\text { 2. Reference: Figur } \\
\text { 3. } 1,273 / 3.6=354 \\
\text { 4. Reference: Table } \\
\text { 5. } 0.08 \text { gha/354 visi }\end{array}$ & $.0002 \mathrm{gha} / \mathrm{vis}$ & & & \\
\hline
\end{tabular}

In 2011, 15\% (1,768) of 11,788 OCRT visitors came to OCRT to visit their friends and relatives (Table 5.78). As discussed above, the EF of this type of visitor is assumed to be the same as the EF of visitors who stay in OCRT homestead accommodation services. Thus the EF (gha) of 1,768 people visiting friends and relatives $=1,768 \times 0.0002$ (gha/visitor) $=0.35$ gha

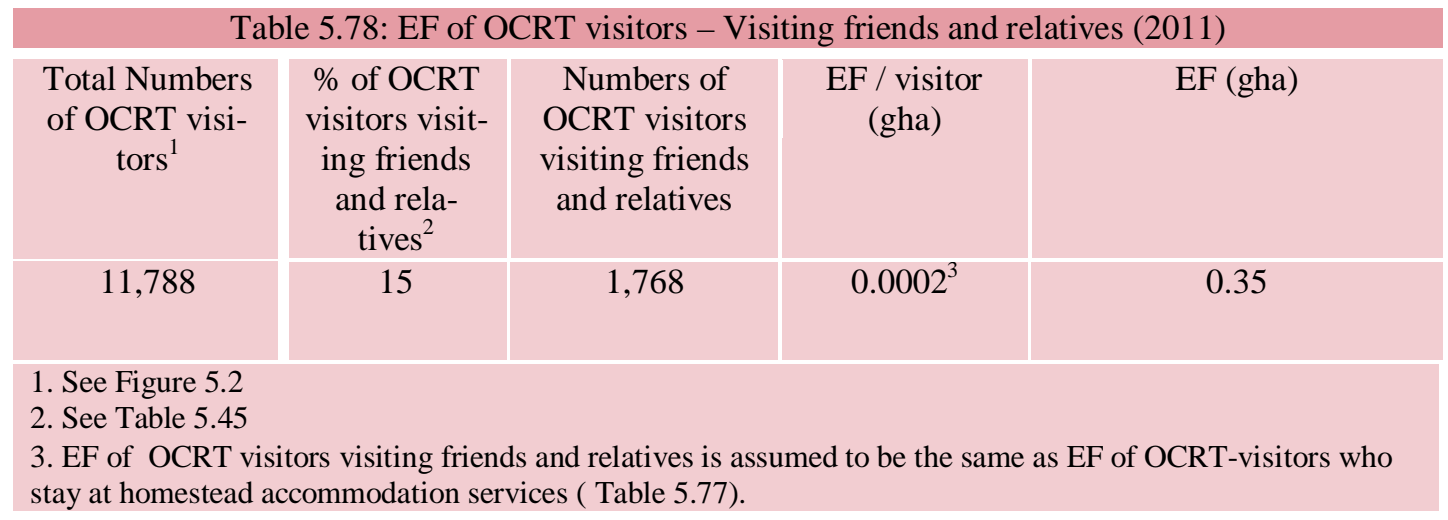

\section{c. 13. The EF of OCRT Visitors: Clyde Dam Tour}

Based on the method in Section c-12 and earlier, the impact of visitors taking the Clyde Dam Tour is set out in Table 5.79. 


\begin{tabular}{|c|c|c|c|c|c|}
\hline \multicolumn{6}{|c|}{ Table 5.79: EF of OCRT visitors - Clyde dam tour (2011) } \\
\hline $\begin{array}{l}\text { Total Numbers } \\
\text { of OCRT visi- } \\
\text { tors }\end{array}$ & $\begin{array}{c}\% \text { of OCRT } \\
\text { visitors on } \\
\text { Clyde dam } \\
\text { tour }\end{array}$ & $\begin{array}{c}\text { Numbers of } \\
\text { OCRT visitors } \\
\text { on Clyde dam } \\
\text { tour }\end{array}$ & $\begin{array}{c}\text { Total impact } \\
\text { (MJ/participant } \\
\text { /year) }^{1}\end{array}$ & $\begin{array}{c}\text { Total impact of } \\
71 \text { OCRT visi- } \\
\text { tors on Clyde } \\
\text { dam tour } \\
\text { (MJ/year) }\end{array}$ & $\begin{array}{c}\mathrm{EF} \\
\text { (gha) }\end{array}$ \\
\hline 11,788 & 0.6 & 71 & 95.6 & $6,787.6$ & 0.068 \\
\hline $\begin{array}{l}\text { 1. Reference: Bec } \\
\text { 2. 6,787.6 MJ/yea } \\
\text { 3. EF (gha/ visito }\end{array}$ & $\begin{array}{l}\text { 1, 2001:2 } \\
6.8 \mathrm{GJ} / \text { year } \\
\text { ear) of OCRT }\end{array}$ & & & & \\
\hline
\end{tabular}

\section{c. 14. The EF of OCRT Visitors: Golden Progress Mine}

Based on the method above, the impact of visitors taking the Golden Progress Mine Tour is set out in Table 5.80.

\begin{tabular}{|c|c|c|c|c|c|}
\hline \multicolumn{6}{|c|}{ Table 5.80: EF of OCRT visitors - Golden Progress mine (2011) } \\
\hline $\begin{array}{l}\text { Total Numbers } \\
\text { of OCRT visitors }\end{array}$ & $\begin{array}{l}\% \text { of OCRT } \\
\text { visitors to } \\
\text { Golden Pro- } \\
\text { gress mine }\end{array}$ & $\begin{array}{c}\text { Numbers of } \\
\text { OCRT visitors to } \\
\text { Golden Progress } \\
\text { mine }\end{array}$ & $\begin{array}{c}\text { Total impact } \\
\text { (MJ/participant } \\
\text { /year) }^{1}\end{array}$ & $\begin{array}{l}\text { Total impact of } \\
71 \text { OCRT visi- } \\
\text { tors to Golden } \\
\text { Progress mine } \\
\text { (MJ /year) }\end{array}$ & $\begin{array}{c}\mathrm{EF} \\
(\mathrm{gha})^{3}\end{array}$ \\
\hline 11,788 & 0.6 & 71 & 6.2 & 404.4 & 0.004 \\
\hline $\begin{array}{l}\text { 1. Reference: Beck } \\
\text { 2. } 404.4 \mathrm{MJ} / \text { year }= \\
\text { 3.EF (gha/ visitor/ }\end{array}$ & $\begin{array}{l}2001: 2 \\
4 \mathrm{GJ} / \text { year } \\
\text { ar) of OCR }\end{array}$ & & e & & \\
\hline
\end{tabular}

\section{c. 15. The EF of OCRT Visitors: Fruit Picking Along Trail}

Based on the method above, the impact of fruit picking visitors (the same as that of visitors to the Golden Progress mine) is set out in Table 5.81.

\begin{tabular}{|c|c|c|c|c|c|}
\hline \multicolumn{6}{|c|}{ Table 5.81: EF of OCRT visitors - fruit picking along trail (2011) } \\
\hline $\begin{array}{l}\text { Total Numbers of } \\
\text { OCRT visitors }\end{array}$ & $\begin{array}{c}\% \text { of OCRT } \\
\text { visitors fruit } \\
\text { picking along } \\
\text { trail }\end{array}$ & $\begin{array}{l}\text { Numbers of } \\
\text { OCRT visitors } \\
\text { fruit picking } \\
\text { along trail }\end{array}$ & $\begin{array}{c}\text { Total impact } \\
\text { (MJ/participant } \\
\text { /year) }^{1}\end{array}$ & $\begin{array}{l}\text { Total impact of } \\
71 \text { OCRT visi- } \\
\text { tors fruit pick- } \\
\text { ing along trail } \\
\text { (MJ/year) }\end{array}$ & $\begin{array}{c}\mathrm{EF} \\
(\mathrm{gha})^{3}\end{array}$ \\
\hline 11,788 & 0.6 & 71 & 6.2 & 404.4 & 0.004 \\
\hline $\begin{array}{l}\text { 1. Reference: Becke } \\
\text { 2. } 404.4 \mathrm{MJ} / \text { year = } \\
\text { 3. EF (gha/ visitor/ }\end{array}$ & $\begin{array}{l}2001: 2 \\
4 \mathrm{GJ} / \text { year } \\
\text { ar) of OCR }\end{array}$ & - & $=0.00006$ & & \\
\hline
\end{tabular}

\subsubsection{The Total EF of OCRT Visitor Activities}

The total annual EF of OCRT visitor activities as shown in Table 5.82 is 6.57 gha. Table 5.82 and Figure 5.35 demonstrate that the three largest visitor activity EFs are those of sightseeing (3.4 gha), curling at Naseby (0.7 gha) and golf (0.48 gha). Moreover, as presented in Table 5.82 and Figure 5.35, fruit picking along the trail, visiting the Golden progress mine, and walking are the three types of OCRT visitor activities with the 
smallest EFs (0.004 gha). Furthermore, the EFs of other OCRT visitor activities are in the range of 0.06-0.35 gha (Table 5.82 and Figure 5.35).

\begin{tabular}{|c|c|c|c|c|c|}
\hline \multicolumn{6}{|c|}{ Table 5.82: Total EF of OCRT- visitor activities } \\
\hline Type of activity & Activities & $\begin{array}{l}\text { Numbers } \\
\text { of visitor }^{1}\end{array}$ & $\begin{array}{c}\% \text { of total } 11,788 \\
\text { OCRT visitors }\end{array}$ & $\begin{array}{c}\mathrm{EF} \\
\text { (gha/visitor) }^{3}\end{array}$ & $\begin{array}{c}\mathrm{EF} \\
(\mathrm{gha})^{2}\end{array}$ \\
\hline Indoor activity & Curling & 4,350 & 36.9 & 0.00016 & 0.7 \\
\hline \multirow{18}{*}{ Outdoor activities } & Walking & 71 & 0.6 & 0.00006 & 0.004 \\
\hline & Golf & 153 & 1.3 & 0.003 & 0.48 \\
\hline & Taieri & 5,458 & 46.3 & 0.00006 & 0.33 \\
\hline & Gold mining towns & 4,126 & 35 & 0.00006 & 0.26 \\
\hline & Sightseeing & 3,607 & 30.6 & 0.0009 & 3.4 \\
\hline & Old Cromwell town & 2,652 & 22.5 & 0.0006 & 0.16 \\
\hline & Wineries & 1,992 & 16.9 & 0.00006 & 0.12 \\
\hline & $\begin{array}{l}\text { Visiting friends and } \\
\text { relatives }\end{array}$ & 1,768 & 15 & 0.0002 & 0.35 \\
\hline & Hayes engineering & 955 & 8.1 & 0.00006 & 0.06 \\
\hline & $\begin{array}{l}\text { Mountain biking at } \\
\text { Naseby }\end{array}$ & 295 & 2.5 & 0.0009 & 0.28 \\
\hline & Fishing & 224 & 1.9 & 0.0009 & 0.21 \\
\hline & Swim at Ophir & 71 & 0.6 & 0.0009 & 0.068 \\
\hline & Clyde dam tour & 71 & 0.6 & 0.0009 & 0.068 \\
\hline & $\begin{array}{l}\text { Golden progress } \\
\text { mine }\end{array}$ & 71 & 0.6 & 0.00006 & 0.004 \\
\hline & $\begin{array}{l}\text { Ophir high country } \\
\text { farm }\end{array}$ & 71 & 0.6 & 0.00006 & 0.004 \\
\hline & Biking & 71 & 0.6 & 0.0009 & 0.068 \\
\hline & $\begin{array}{l}\text { Fruit picking along } \\
\text { trail }\end{array}$ & 71 & 0.6 & 0.00006 & 0.004 \\
\hline & Total & 26,077 & 100 & - & 6.57 \\
\hline
\end{tabular}

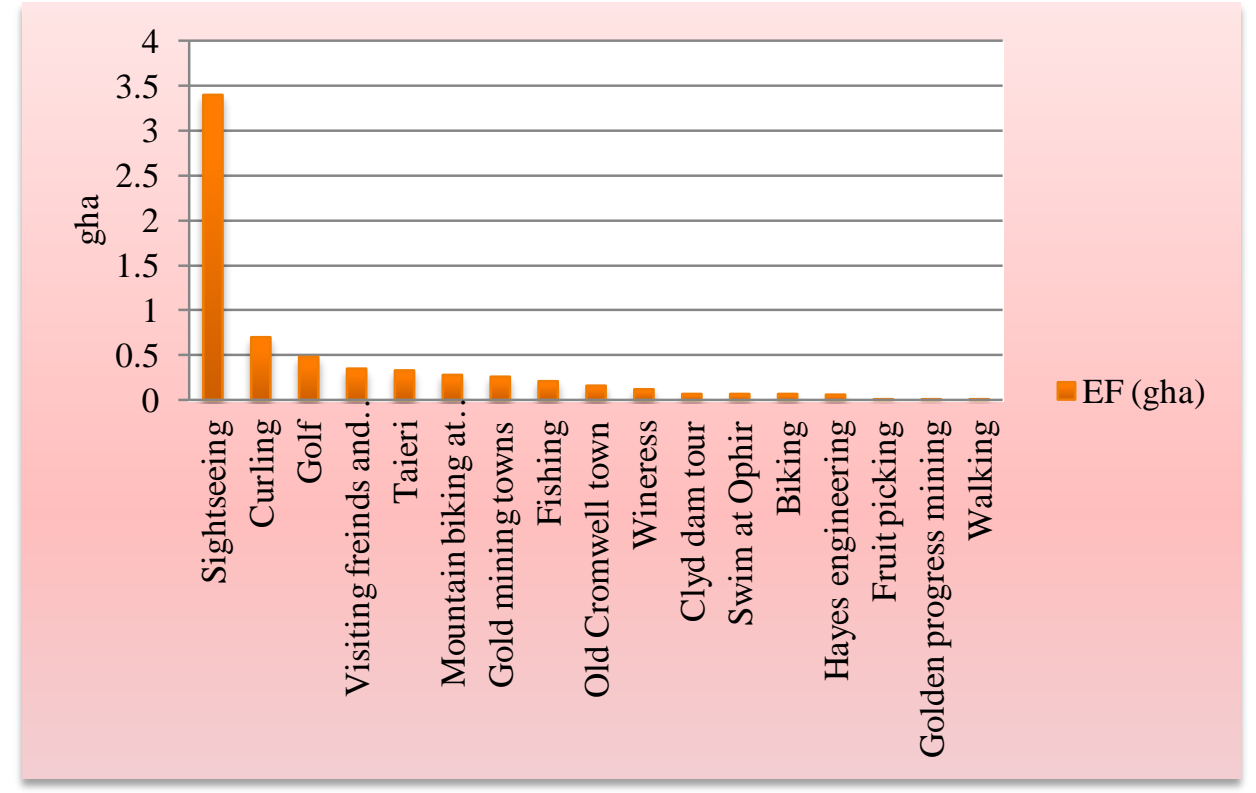

Figure 5.35: OCRT- the EF of visitor activities (2011) 
Figure 5.36 compares the types of OCRT tourism activities by their EF per visitor. This figure shows a distinct difference between the EF of golfing (0.003 gha /visitor) and all other types of tourist activity.

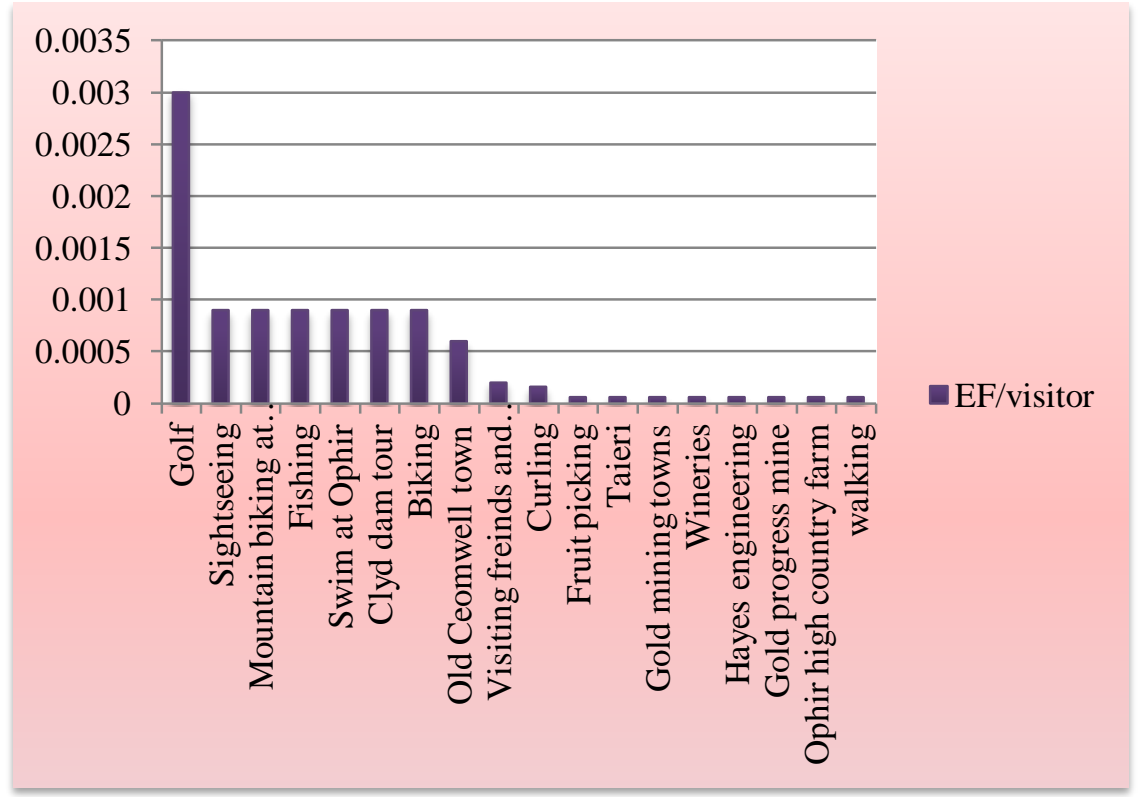

Figure 5.36: OCRT- the EF per visitor of activities (2011)

\subsubsection{Comparison between the EFs of Indoor and Outdoor Activities}

As shown in Table 5.83 and Figure 5.37 the EF of one OCRT indoor activity (curling at Naseby) is equivalent to $10.7 \%$ ( 0.7 gha) of the Total EF of OCRT visitor activities (6.57 gha). Likewise, the total EF of outdoor activities (5.85) accounts for 89.3\% of the total EF of OCRT visitor activities (6.57gha). However, it should be noted that $8.1 \%$ (0.48) of the EF of outdoor activities (5.87) is the EF of golfing (0.48gha) which is conducted by just 1.3\% (153) of total OCRT visitors (See Table 5.82 and Figure 5.35).

\begin{tabular}{|c|c|c|c|c|c|c|c|c|c|}
\hline \multicolumn{5}{|c|}{ Indoor activity (curling at Naseby) } & \multicolumn{5}{|c|}{ Outdoor activities } \\
\hline $\begin{array}{l}\text { Numbers } \\
\text { of visi- } \\
\text { tors }\end{array}$ & $\begin{array}{c}\% \text { of } \\
\text { total } \\
26,077 \\
\text { visitor } \\
\text { activities }\end{array}$ & $\begin{array}{c}\text { EF } \\
\text { (gha) }\end{array}$ & $\%^{1}$ & $\begin{array}{c}\text { EF } \\
\text { (gha/visit } \\
\text { or) }\end{array}$ & $\begin{array}{l}\text { Num- } \\
\text { bers of } \\
\text { visitors }\end{array}$ & $\begin{array}{c}\text { \% of total } \\
26,077 \\
\text { visitor } \\
\text { activities }\end{array}$ & $\begin{array}{c}\text { EF } \\
\text { (gha) }\end{array}$ & $\%^{1}$ & $\begin{array}{c}\text { EF } \\
\text { (gha/visito } \\
\text { r) }\end{array}$ \\
\hline 4,350 & 16.7 & 0.7 & 10.7 & 0.000016 & 21,727 & 83.3 & 5.87 & 89.3 & 0.0004 \\
\hline
\end{tabular}




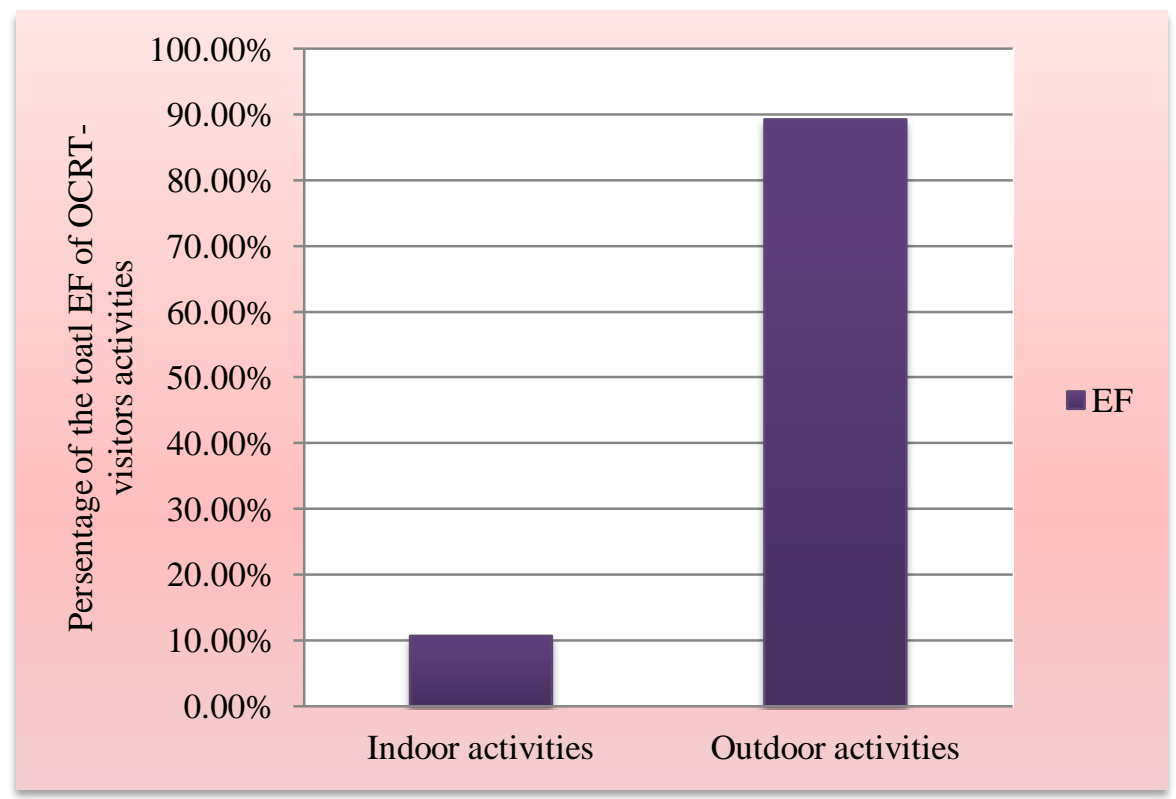

Figure 5.37: comparison between the EF of OCRT visitors indoor and outdoor activities.

This Thesis chooses three activities, curling at Naseby as an indoor activity, and golf as outdoor activity with the biggest EF/visitor, and walking with its low EF for further comparison. As Figure 5.38 illustrates, other activities (5.39 gha) has the biggest EF followed by, curling (0.7 gha), golf (0.48gha) and walking (0.004 gha).

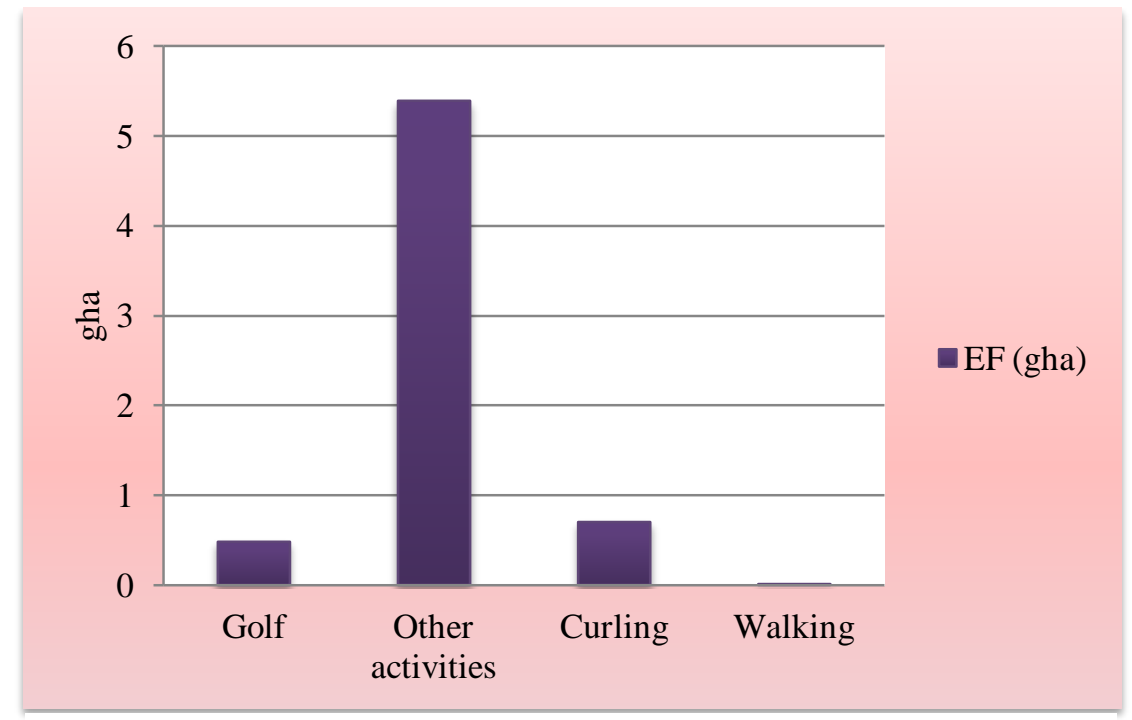

Figure 5.38: Comparison between the EFs of golf, curling, walking and other OCRT visitor activities (2011).

As illustrated in Figure 5.39, the majority of the 21,503 OCRT visitor activities fall into the other activities (except golf, curling and walking) category, followed by curling (4,350), golf (153) and walking (71). 


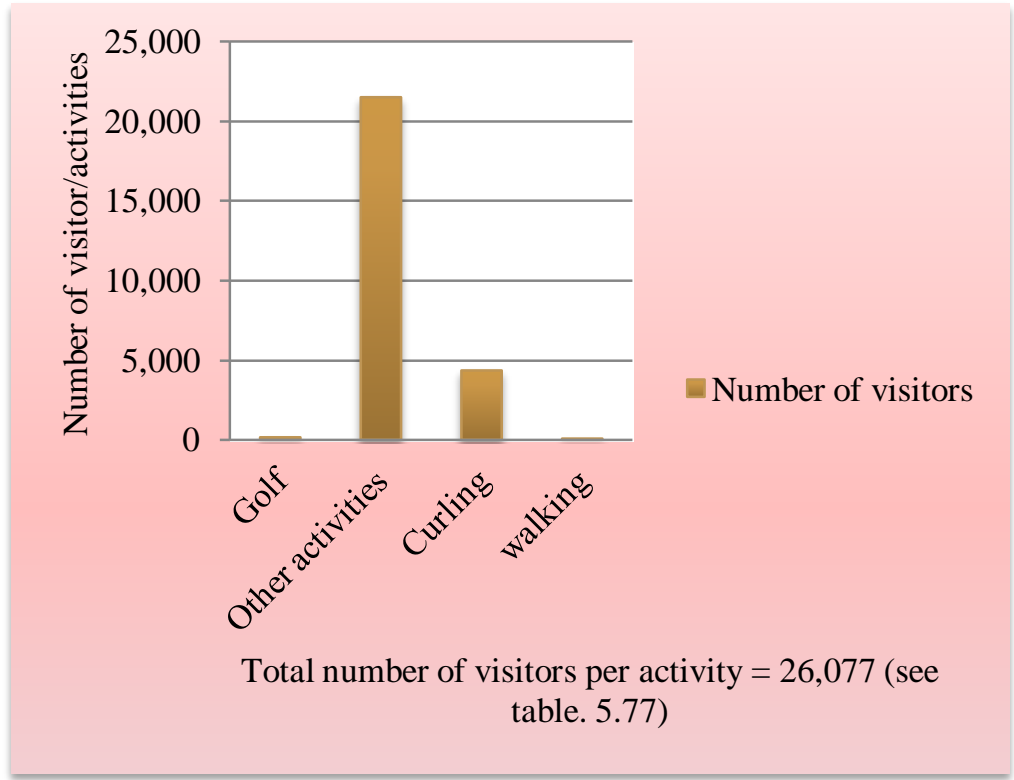

Figure 5.39: Comparison between the numbers of OCRT visitors who engage in golf, curling, walking and other activities (2011)

Figure 5.40 compares the EFs of these same four categories. Golf now dominates showing how a small number of people can have a large effect on overall EF.

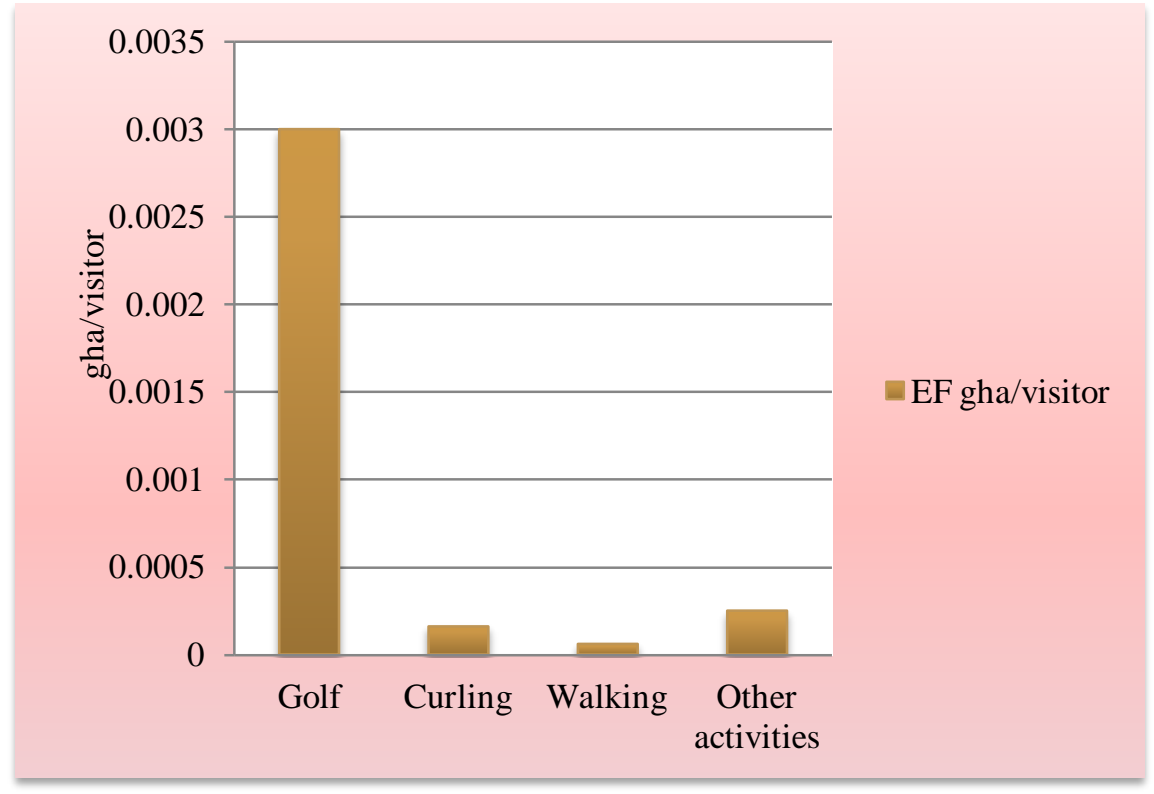

Figure 5.40: The EF (gha/visitor) of golf, curling, walking and other activities

\subsection{The Total EF of OCRT (2011)}

Table 5.84 demonstrates that the total EF of the OCRT is equivalent to 1,617 gha, which derives from the integration of the EFs of transportation, food, accommodation 
services and activities used and engaged in by 11,788 OCRT visitors in 2011. As shown in Table 5.84 and Figure 5.41 transportation has the largest footprint of 1,167gha (72.2\%) of the total EF, followed by 401.2 gha (24.8\%) food, 42.24 gha (2.6\%) accommodation services and 6.57 gha $(0.4 \%)$ visitor activities. However, the total EF of transportation can be divided into 38.2\% of total EF for international transportation and 34\% for domestic transportation (Table 5.84). This shows the importance of getting to a tourist destination for overall environmental impact.

\begin{tabular}{|c|c|c|c|}
\hline \multicolumn{4}{|c|}{ Table 5.84: Total EF of OCRT (2011) } \\
\hline Category & EF (gha) & $\%$ & EF (gha/visitor ${ }^{\mathrm{b}}$ ) \\
\hline Transportation & $1,167^{1}$ & $72.2^{5}$ & 0.1 \\
\hline Food & $401.2^{2}$ & 24.8 & 0.034 \\
\hline Accommodation & $42.4^{3}$ & 2.6 & 0.0036 \\
\hline Activities & $6.57^{4}$ & 0.4 & 0.00056 \\
\hline Total & 1,617 & 100 & 0.138 \\
\hline \multicolumn{4}{|c|}{$\begin{array}{l}\text { 1. See Table } 5.7 \\
\text { 2. See Table } 5.11 \\
\text { 3. See Table } 5.40 \\
\text { 4. See Table } 5.82 \\
\text { 5. } 72.2 \% \text { includes } 38.2 \%(617.4 \text { gha) EF International transportation }+34 \% \text { ( } 549.3 \mathrm{gha}) \text { EF of domestic } \\
\text { transportation) (see Table 5.8) } \\
\text { 5. Total OCRT visitor numbers }=11,788 \text { (see Figure } 5.6)\end{array}$} \\
\hline
\end{tabular}

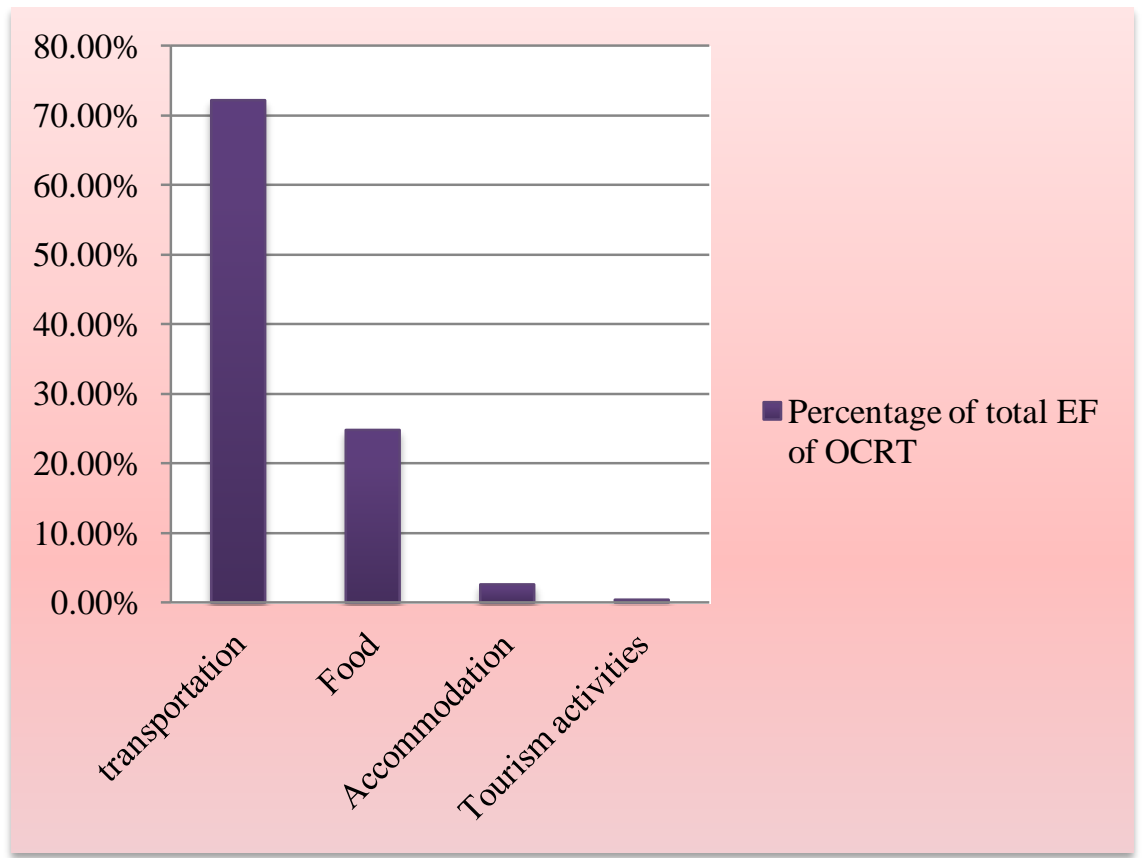

Figure 5.41: Comparison between the EF categories of OCRT- transportation, food, accommodation services and visitors activities (2011) 
Figure 5.41.a compares the EF of OCRT visitors (0.138 gha/ capita), the present global average EF of holidays (0.3 gha/ capita) (Vale and Vale, 2009:358) and the sustainable EF (goal) for holidays (0.1 gha/ capita) (Vale and Vale, 2009:358). As illustrated, the EF of the OCRT is $66 \%$ smaller than the global average EF of tourism but its EF still needs to be reduced by $38 \%$ to be ecologically sustainable. According to O'Connor (2009:92) in 2008 the EF of New Zealanders was 5.9 gha/ capita. As a result the EF of OCRT visitors ( 0.136 gha) is equivalent to $2.3 \%$ of the EF of New Zealanders.

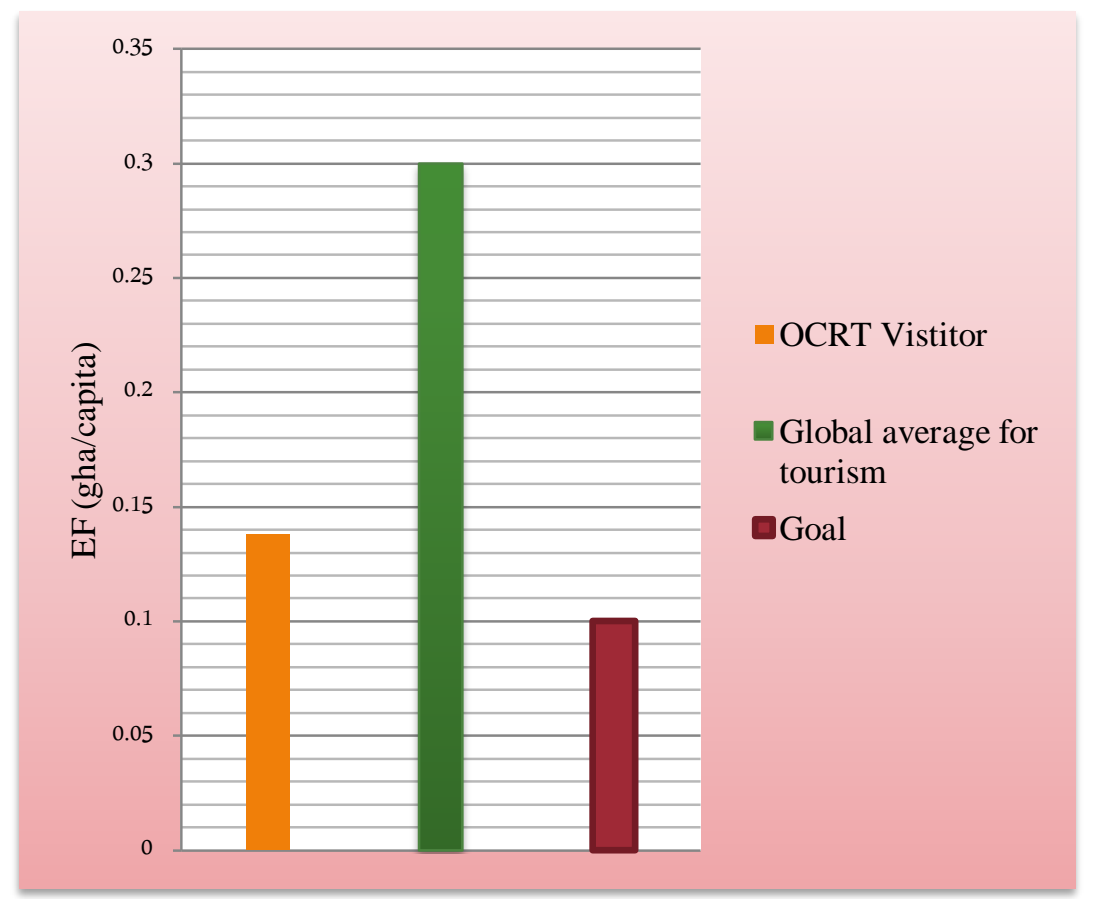

Figure 5.41.a: The average EF of OCRT- visitors and global tourism, and the goal for sustainable tourism (gha/ visitor)

\subsection{OCRT: Overshoot Portion of EF}

\subsubsection{The EF of Sustainable living}

Vale and Vale (2009:358) using data from the Cardiff Report (2001:97), propose the EFs of sustainable living for the ten categories of life activities and products shown in Table 5.85 and compare these proposed EFs with their present amounts. Vale and Vale (2009:358) classify the ten categories into the two areas of individual and collective products and activities (Table 5.85). The individual products and activities including; food and drink, domestic energy, domestic travel, stuff, housing, and holidays, are proposed to have EFs in the range of 0.03-0.43 gha/capita for being sustainable (Table 5.85). Moreover the sustainable EFs of the collective categories, including infrastructure, government, and services, are calculated to be in the range of 0.24-0.47 gha/capita 
(Table 5.85). As shown in Table 5.85, the total EF of sustainable living (as the goal) is equivalent to 1.85 gha/capita and the total EF at present (2009) is 6.41gha/capita.

As presented in Table 5.85, the sustainable EF of holidays is calculated to be 0.03 gha /capita and, according to the Cardiff report (2001:97), this includes the EFs of transportation, food, accommodation and waste water that are consumed/used/produced by Cardiff visitors. This study uses the sustainable EF of holidays (Table 5.85) as the goal to be achieved through the sustainable development of the OCRT and compares its present EF with its target EF to calculate the overshoot portion of the current EF.

\begin{tabular}{|l|c|c|}
\hline \multicolumn{2}{|c|}{ Table 5.85: The ecological footprint of sustainable living } \\
\hline Individual & Now & Goal \\
\hline Food and drink & 1.33 & 0.43 \\
\hline Domestic energy & 0.99 & 0.33 \\
\hline Domestic travel & 0.99 & 0.33 \\
\hline Stuff & 0.64 & 0.21 \\
\hline Housing & 0.16 & 0.05 \\
\hline Holidays & 0.10 & 0.03 \\
\hline Individual total & 5.00 & 1.38 \\
\hline Collective & & \\
\hline Infrastructure & 0.74 & 0.24 \\
\hline Government & 0.41 & 0.14 \\
\hline Services & 0.26 & 0.09 \\
\hline Collective total & 1.41 & 0.47 \\
\hline Total revised footprint & 6.41 & 1.85 \\
\hline$\bullet$ Reference: Vale and Vale, 2009:358 & & \\
\hline
\end{tabular}

Table 5.86 compares the present EF (0.138 gha/visitor) of the OCRT (see Table 5.84) and its goal (0.03 gha/visitor) for being environmentally sustainable. The difference between these two EFs (0.138 gha/visitor - 0.03 gha/visitor) shows the overshoot portion of the OCRT EF, which is 0.108 gha/visitor (Table 5.86).

Table 5.86: The ecological footprint of OCRT- sustainable tourism (2011)

\begin{tabular}{|c|c|c|} 
& Overshoot portion of OCRT visitors- EF (gha/visitor/year) \\
\hline Now & Goal & Overshoot EF(gha/visitor/year) \\
\hline 0.138 & 0.03 & 0.108 \\
\hline
\end{tabular}

- Total overshoot EF (gha) of 11,788 OCRT visitors = 1,273 


\subsubsection{The Overshoot Portion of OCRT EF Energy}

As EF (gha) = energy used $\div 100$ (carrying capacity of land)

Consequently

Energy used $=$ EF $($ gha $) \times 100$ (carrying capacity of land $)$

As shown in Table 5.82 the EF of OCRT is 0.138 , so its energy use is equivalent to:

$0.138($ gha/visitor $) \times 100($ carrying capacity of land $)=13.8(\mathrm{GJ} /$ visitor $)$

Moreover its sustainable energy use is:

$0.03($ gha/visitor $) \times 100($ carrying capacity of land $)=3.0(\mathrm{GJ} /$ visitor $)$

The overshoot portion of OCRT energy use is:

13.8 GJ/visitor $-3.0 \mathrm{GJ} /$ visitor = 10.8 GJ/visitor (Table 5.87).

As a result the overshoot energy use of 11,788 OCRT visitors is equivalent to:

10.8 (overshoot energy use GJ/visitor/year) × 11,788 (visitors) = 127,310.4 GJ/year (Table 5.88).

Table 5.88: The energy use of OCRT- sustainable tourism (2011)

Total overshoot portion of OCRT visitors energy use (GJ/year)

Number of visitors

Overshoot energy use

(GJ/visitor/year)

10.8

11,788

\section{Table 5.87: The energy use of OCRT sustainable tourism (2011)}

Overshoot energy use

(GJ/visitor/year)

10.8

\subsubsection{OCRT Overshoot Energy Use by Categories}

The overshoot portion of each category (transportation, food, accommodation and visitor activities) is calculated through the following equation:

Overshoot portion of energy use per category $(\mathrm{GJ} /$ year) $=\%$ of its EF from total OCRT EF (gha) $\times$ total overshoot energy use of OCRT $(127,310.4$ GJ/year)

For example using the above equation the overshoot portion of transportation is equivalent to $72.2 \%$ of $127,310.4 \mathrm{GJ} /$ year, which is 91,918.1 GJ/year (Table 5.89). As shown in Table 5.89 transportation causes the largest overshoot energy us of 91,918.1GJ/year, followed by food (31445.7 GJ/year), accommodation services (3310.1 GJ/year), and OCRT visitor activities (1018.4 GJ/year) (Table 5.89). 


\begin{tabular}{|l|c|c|c|}
\hline & \multicolumn{3}{|c|}{ Table 5.89: OCRT overshoot energy use by categories (2011) } \\
\hline Category & EF (gha) & $\%$ & Overshoot portion of energy use (GJ/year) \\
\hline Transportation & 1,167 & 72.2 & $91,918.1$ \\
\hline Food & 401.2 & 24.8 & 31,573 \\
\hline Accommodation & 42.4 & 2.6 & $3,310.1$ \\
\hline Activities & 6.57 & 0.4 & 509.2 \\
\hline Total & 1,617 & 100 & $127,310.4$ \\
\hline
\end{tabular}

\subsection{OCRT Economic Footprint}

\subsubsection{OCRT GDP}

According to CODC (2011: 23) in 2011, the direct output of the OCRT was equivalent to $\$ 8,159,042$ and its total output was $\$ 12,277,024$ (Table 5.90). Likewise, in 2011 the OCRT direct contribution to GDP was $\$ 4,213,793$ and its total contribution to GDP was $\$ 6,245,289$ (Table 5.90). Table 5.90 also shows that in 2011 a total of 121 full time staffs were in employment related to the OCRT.

\begin{tabular}{|c|c|c|}
\hline Impact summary & Direct & Total \\
\hline Output & $\$ 8,159,042$ & $\$ 12,277,024$ \\
\hline GDP & $\$ 4,213,793$ & $\$ 6,245,289$ \\
\hline Employment (FTE’s) & 96.0 & 121.3 \\
\hline
\end{tabular}

In 2011 of the total \$6,245,289 OCRT contribution, accommodation formed the majority of $\$ 2,837,412$ of total OCRT GDP followed by vehicles and equipment hire $(\$ 975,119)$, and business administration and managerial services $(\$ 900,602)$ (Table 5.91). Other categories included food and consumables, road passenger transport, retail, and entertainment, with GDP contributions in the range of \$61,925- \$782,795 (Table 5.91). Moreover, in comparison with other the categories, OCRT accommodation had the largest total output of $\$ 5,301,246$, followed by food and consumables $(\$ 1,840,428)$, business administration and managerial services $(\$ 1,736,101)$, and vehicles and equipment hire $(\$ 1.735,894)$ (Table 5.91). Other categories including retail, road passenger transport, and entertainment had total outputs between \$452,305 and \$107,016 (Table 5.91). 


\begin{tabular}{|l|c|c|c|c|}
\hline \multicolumn{5}{|c}{ Table 5.91: Economic impact of OCRT- by categories } \\
\hline Category & Output & Total output & GDP & Total GDP \\
\hline Accommodation & $\$ 3,557,833$ & $\$ 5,301,246$ & $\$ 1,956,837$ & $\$ 2,837,412$ \\
\hline Food and consumables & $\$ 1,226,952$ & $\$ 1,840,428$ & $\$ 539,859$ & $\$ 782,795$ \\
\hline Retail & $\$ 297,569$ & $\$ 452,305$ & $\$ 157,712$ & $\$ 241,299$ \\
\hline Road Passenger Transport & $\$ 665,081$ & $\$ 1,104,034$ & $\$ 259,382$ & $\$ 446,136$ \\
\hline Vehicle and equipment hire & $\$ 1,257,894$ & $\$ 1.735,894$ & $\$ 717,000$ & $\$ 975,119$ \\
\hline $\begin{array}{l}\text { Business administration and } \\
\text { managerial services }\end{array}$ & $\$ 1,085,063$ & $\$ 1,736,101$ & $\$ 542,532$ & $\$ 900,602$ \\
\hline Entertainment & $\$ 68,600$ & $\$ 107,016$ & $\$ 40,474$ & $\$ 61,925$ \\
\hline Total & $\$ 8,159,042$ & $\$ 12,277,024$ & $\$ 4,213,793$ & $\$ 6,245,289$ \\
\hline
\end{tabular}

\subsubsection{OCRT: Sustainable Portion of GDP (GDPs)}

As explained in Chapter 4 this thesis uses the sustainable portion of GDP (GDPs) as an economic indicator to evaluate OCRT economic development as being sustainable. Calculation of the GDPs of the OCRT involves the following four factors:

1. Total cost to generate $1 \mathrm{GJ}$ energy through using renewable resources in New Zealand

2. Total overshoot portion of OCRT energy use/year

3. Total cost to generate overshoot portion of OCRT energy use through using renewable resources

4. Total OCRT contribution to GDP

\subsubsection{Renewable Energy Cost}

Table 5.92 comprises the annualized cost to generate energy through using different technologies in the USA. As shown in this Table, in comparison with other energy technologies, using Solar photovoltaics (PV) costs the most at $>\$ 0.20 / \mathrm{kWh}$ in 20052010, followed by wind offshore (\$0.10-0.17/ kWh), and wave, tidal and concentrating solar power (CSP) at about the same cost $(\$ 0.11-0.1 / \mathrm{kWh})$. Interestingly, even at present geothermal, onshore wind and hydro-electricity (all classed as renewables) can work out cheaper than conventional energy sources (Table 5.92). 
Table 5.92: Approximate fully annualized generation and conventional transmission cost for WWs power.

Energy technology
Wind onshore
Wind offshore
Wave
Geothermal
Hydro-electricity
CSP (Concentrating Solar Power)
Solar PV
Tidal
Conventional (mainly fossil) generation in
US
- Reference: Delucchi and Jacobson, 2011:1175

Annualized cost ( 2007 US\$/kWh-delivered)

Present (2005-2010)

\$ 0.04-0.07

$\$ 0.10-0.17$

$\geq \mathrm{US} \$ 0.11$

\$ 0.04-0.07

US\$0.04

$\$ 0.11-0.1$

$>\$ 0.20$

$>\$ 0.11$

\$0.07 (social cost: \$0.12)
Future $(2020+)$ $\leq \$ 0.04$

$\$ 0.08-0.13$

$\$ 0.04$

\$0.04-0.07

$\$ 0.04$

$\$ 0.08$

$\$ 0.10$

$\$ 0.05-0.07$

\$0.08 (social cost: \$0.14)

This thesis uses the average cost of using wind and solar technology in New Zealand to calculate the cost of generating $1 \mathrm{GJ}$ energy through using renewable energy resources. Table 5.93 shows New Zealand energy costs of using wind and solar systems. This table assumes that the average cost of using wind technology is NZ\$100/MWh (NZ\$0.1/kWh) and solar is \$NZ 42.5/MWh ( $\$ N Z 0.042 / \mathrm{kWh})$. The cost for solar is based on the cost of $\$ 350$ to $\$ 500$ per MWh in New Zealand given by EECA (http://www.energywise.govt.nz/how-to-be-energy-efficient/generating-renewableenergy-at-home/solar-electricity-generation\#costs) but reduced by a factor of 10 based on decreasing PV cost data from the National Renewable Energy Laboratory in the United States.(US Department of Energy (2011) 2010 Solar Technologies Market Report).

Table 5.93: New Zealand energy costs

\begin{tabular}{|c|c|c|}
\hline Energy technology & Cost ( NZ\$/MWh) & Average cost (NZ\$/MWh) \\
\hline Wind & $80-120^{1}$ & 100 \\
\hline Solar system & $35-50^{2}$ & 42.5 \\
\hline \multicolumn{2}{|c|}{ Average cost of energy generated by wind-solar systems (NZ\$/MWh) } & 71.25 \\
\hline
\end{tabular}

As presented in Table 5.94, the average cost of using wind and solar systems in New Zealand is assumed here to be NZ\$71.25/MWh or NZ\$19.8/ GJ.

\begin{tabular}{|c|c|}
\hline \multicolumn{2}{|c|}{ Table 5.94: Average energy cost (wind-solar) } \\
\hline \multicolumn{2}{|c|}{$1 \mathrm{MWh}$} \\
\hline $\mathrm{NZ} \$ 71.25$ & $\mathrm{GJ}^{1}$ \\
\hline & $\mathrm{NZ} \$ 19.8$ \\
\hline
\end{tabular}


As shown in Table 5.95, in 2011 the total OCRT GDP was \$6,245,289 and its overshoot energy use is equivalent to $127,310.4 \mathrm{GJ} /$ year. Since the average cost of generating $1 \mathrm{GJ}$ of energy through using wind and solar systems is NZ\$19.8 (Table 5.95), the consequent cost of generating the total OCRT overshoot energy use is equivalent to $(127,310.4$ GJ/year $\times$ NZ\$19.8) $=$ NZ\$2,720,746 (Table 5.95). The OCRT GDPs is equivalent to its total GDP $(\$ 6,245,289)$ less the total cost to generate the overshoot portion of its energy use by wind-solar systems $(\mathrm{NZ} \$ 2,720,746)=\mathrm{NZ} \$ 3,524,543$ (Table 5.95).

\begin{tabular}{|c|c|}
\hline Total OCRT GDP (NZ\$) & $6,245,289^{1}$ \\
\hline EF (gha) & $1,617^{2}$ \\
\hline Overshoot EF (gha) & $1,273^{3}$ \\
\hline Overshoot energy use (GJ) & $127,310.4^{4}$ \\
\hline Energy cost (NZ\$/GJ) wind-solar & $19.8^{5}$ \\
\hline $\begin{array}{l}\text { Total cost (NZ\$) to generate the overshoot portion } \\
\text { of OCRT energy use by wind-solar systems }\end{array}$ & $2,720,746$ \\
\hline GDPs $(N Z \$)^{6}$ & $3,524,543$ \\
\hline $\begin{array}{l}\text { 1.See Table } 5.91 \\
\text { 2.See Table } 5.89 \\
\text { 3.See Table } 5.89 \\
\text { 4.See Table } 5.89 \\
\text { 5.See Table } 5.94 \\
\text { 6.GDPs= Total GDP - Total cost to generate the overshoc } \\
\text { resources }\end{array}$ & se through the use of renewable \\
\hline
\end{tabular}

Figure 5.42 makes a comparison between OCRT GDP and its GDPs. As illustrated OCRT GDPs is NZ\$2,720746 (43.6\%) less than OCRT GDP (NZ\$6,245,289). On the other hand, $43.6 \%$ of total OCRT GDP must be spent to reduce its environmental impacts to the level of sustainable living as it relates to the EF of holidays, as cited in Table 5.87. As explained above, the calculation of GDPs is based on the assumption of the reducing cost of solar energy following historic cost trends. 


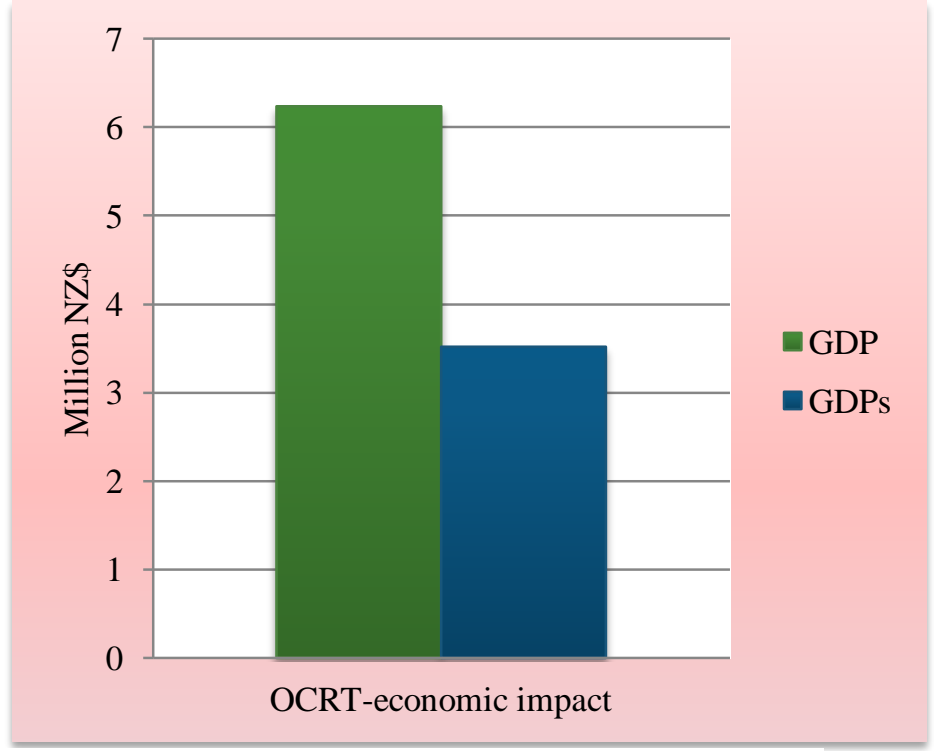

Figure 5.42: OCRT- GDP and GDPs

\subsection{OCRT Cultural Footprint (CF)}

This Thesis uses the CF model explained in Chapter 4 to explore the cultural footprint of the OCRT on the host destination. In this case, study of the CF of the OCRT is related to the three categories of the CFs of food, accommodation, and visitor activities . Since there is no suggestion about using sustainable transportation on any OCRT websites and all current transportation is of conventional type, the CF of this category is not included in this research. Moving to sustainable transport is considered here to be a national rather than a local issue when it comes to tourism. Nevertheless, it must still be recognised that the largest component of the tourism EF relates to visitor transportation. Local sustainable transport as it relates to tourism is an area for further research.

\subsubsection{OCRT CF: Food}

Table 5.96 contains summarised results of two proposed scenarios for OCRT food production (see Chapter 5-5.5.1 and 5.5.2). As shown in Table 5.96 the total conventional food consumed in the first scenario is 78,508.45kg (100\% of consumed food) and in the second scenario this same total is made up of $65165.05 \mathrm{~kg}$ (83\%) conventional food and $13346.4 \mathrm{~kg}$ (17\%) home generated (organic food).

The total EF of OCRT food in the first scenario is $424.4 \mathrm{gha} /$ year and in the second scenario this reduces by $5.5 \%$ to 401 gha/year. Obviously more use of local food would not 
just be an advertising advantage for OCRT tourism but would also reduce its environmental impact.

As shown in Table 5.96, in the second scenario the overshoot portion of the energy related to the food consumed is $31,573 \mathrm{GJ} /$ year. This is calculated by working out the EF of providing the same food in a sustainable way. As shown in Table 5.86, in the overall OCRT, overshoot EF is 0.108gha/visitor, and the total overshoot in 2011 was 1,273gha.

Since the EF of consumed food in the first scenario (EF1) is 23.2 gha bigger than the EF of food in the second scenario (EF2), the overshoot portion of the energy related to food consumed in the first scenario is calculated through the following steps:

\section{$\mathrm{EF} 1-\mathrm{EF} 2=23.2$ gha}

Overshoot energy of the first compared to the second scenario $=23.2$ gha $\times 100$ (average global carrying capacity) $=2,320 \mathrm{GJ} /$ year

Since the overshoot portion of energy use related to the current situation with regard to food eaten by visitors to the OCRT has already been calculated (see Table 5.89), it is used as a base to calculate the overshoot energy related to the first scenario through the following steps.

Overshoot energy consumed food (second scenario) $=31,573(\mathrm{GJ} /$ year $)$

Overshoot portion of energy used of the first scenario $=31,573 \mathrm{GJ} /$ year (overshoot energy second scenario) $+2,320 \mathrm{GJ} /$ year (difference between first and the second scenarios expressed as energy) = 33,893 GJ/year (Table 5.96).

Moreover the overshoot portions of EF2 and EF1 are calculated by dividing their overshoot energy into 100 (average carrying capacity of the land) (Table 5.96). Consequently the overshoot portion of EF2 is 31,573 $\div 100=315.73$ gha/year (see Table 5.96) and the overshoot portion of EF1 is 33,893 $\div 100=338.93$ gha/year (see Table 5.96). As the cost to generate 1GJ energy through using wind-solar systems is NZ\$19.8, the cost to generate the overshoot energy of food in the first scenario is 33,893 GJ $\times$ NZ\$ $19.8=$ NZ\$671,081 (see Table 5.96). Likewise the total cost to generate the overshoot energy through using wind-solar systems in the second scenario is NZ\$19.8 $\times 31,573 \mathrm{GJ} /$ year $=$ NZ\$625,145 (Table 5.96). 
As shown in Table 5.96, the food GDP in both scenarios is NZ\$782,795. As a result GDPs1=NZ\$782,795 (GDP) - NZ\$671,081 = NZ\$111,417

and GDPs2 = NZ\$782,795(GDP) - NZ\$625,145 = NZ\$157,650

The difference between GDPs1 and GDPs2 gives the NZ\$45,936 of contribution to GDPs through producing 13,346.4kg home generated (organic) food by OCRT accommodation services. In addition it can be considered that producing $1 \mathrm{~kg}$ home generated (organic) food increases GDPs by NZ\$3.44 (NZ\$45,936 $\div 13,346.4 \mathrm{~kg}$ ) (Table 5.96).

\begin{tabular}{|c|c|c|c|}
\hline \multicolumn{4}{|c|}{ Table 5.96: OCRT- Local product (food) - contribution to GDPs } \\
\hline \multicolumn{2}{|c|}{ First scenario (100\% conventional food) } & \multicolumn{2}{|c|}{$\begin{array}{c}\text { Second scenario (83\% conventional food and 17\% } \\
\text { home generated (Organic) food }\end{array}$} \\
\hline Total conventional food (kg) & $78,508.45$ & Total conventional food (kg) & 65165.05 \\
\hline $\begin{array}{l}\text { Total home generated food } \\
\text { (kg) }\end{array}$ & 0.00 & $\begin{array}{l}\text { Total home generated food } \\
(\mathrm{kg})\end{array}$ & $13,346.4$ \\
\hline $\begin{array}{l}\text { Total EF of consumed food } \\
\text { gha/year - (EF1) }\end{array}$ & 424.4 & $\begin{array}{l}\text { Total EF of consumed food } \\
\text { gha (EF2) }\end{array}$ & 401.2 \\
\hline $\begin{array}{l}\text { Overshoot portion of energy } \\
\text { of consumed food (GJ/year) }\end{array}$ & 33,893 & $\begin{array}{l}\text { Overshoot portion of energy } \\
\text { of consumed food (GJ/year) }\end{array}$ & $31,573^{1}$ \\
\hline $\begin{array}{l}\text { Overshoot portion of EF1 } \\
\text { (gha/year) }\end{array}$ & 338.93 & $\begin{array}{l}\text { Overshoot portion of EF2 } \\
\text { (gha/year) }\end{array}$ & 315.73 \\
\hline $\begin{array}{l}\text { Cost to generate } 1 \mathrm{GJ} \text { energy } \\
\text { through using wind-solar sys- } \\
\text { tems (NZ\$) }\end{array}$ & 19.8 & $\begin{array}{l}\text { Cost to generate } 1 \mathrm{GJ} \text { energy } \\
\text { through using wind-solar } \\
\text { systems (NZ\$) }\end{array}$ & 19.8 \\
\hline $\begin{array}{l}\text { Total cost to generate over- } \\
\text { shoot energy through wind- } \\
\text { solar systems (NZ\$) }\end{array}$ & 671,081 & $\begin{array}{l}\text { Total cost to generate over- } \\
\text { shoot energy through wind- } \\
\text { solar systems (NZ\$) }\end{array}$ & 625,145 \\
\hline Total food GDP (NZ\$) & 782,795 & Total food GDP (NZ\$) & 782,795 \\
\hline Total food GDPs1 & 111,714 & Total food GDPs 2 & 157,650 \\
\hline
\end{tabular}

\subsubsection{Sustainable Life: Food (Ideal Model)}

Table 5.96 shows that the present EF of the 78,508.45kg OCRT food consumed (second scenario) is 401.2 gha and its overshoot portion of EF2 is 315.73gha. As a result the sustainable EF of OCRT food consumed can be determined through the following equation:

a. 401.2 gha $(\mathrm{EF} 2)$ - 315.73gha (overshoot portion of EF2) $=$ 85.47gha = sustainable life EF of OCRT food

In the ideal model for OCRT food consumed, since the overshoot portion of energy use is equal to zero, the GDPs is equal to the total GDP of OCRT food (NZ\$782,795, see Table 5.96). This study uses the ideal model of OCRT food consumed by visitors to 
compare the two scenarios shown in Table 5.96 with the sustainable life model for food consumption.

As shown in Figure 5.43, the area of the triangle described by 'Q 1, EF 1, and GDPs1' illustrates ordinary life, or the area in which all OCRT visitors consume 100\% $(78,508.45 \mathrm{~kg})$ conventional food (first scenario). In this area, the development of the OCRT does not exert any influence on its host destination and visitors to produce and consume local food. In Figure 5.43 the EF of using 100\% conventional food (EF 1) is $424.4 \mathrm{gha} /$ year and this figure is used to represent 100\% of the EF of OCRT food consumed by visitors. In the ordinary life area, GDPs1 is NZ\$111,714 (see Table 5.96).

Comparison between the first scenario and the ideal model of OCRT food consumption indicates that in the first scenario EF 1 (424.4 gha) is 79.9\% bigger than the ideal EF for food consumption (85.47gha-see 5.11.1.1.a) (Figure 5.43 and Table 5.96). Moreover, as shown in Figure 5.43 in the first scenario, GDPs1 is equal to $14.3 \%$ of the total GDP of OCRT food. On the other hand, $85.7 \%$ of the total GDP of food must be spent to change EF 1 to the ideal EF through generating the overshoot portion of food energy use by using wind-solar systems.

In Figure 5.43, the area of the triangle 'Q 2, EF2, and GDPs2'determines the area of the OCRT CF through producing and consuming home generated (organic) food. As demonstrated in Figure 5.43 in the CF, total food consumed by OCRT visitors Q 2 $(78,508.45 \mathrm{~kg})$ is divided into the two portions of $17 \%(13,346.4)$ home cooked food (Q 3) and 83\% (65,165.05 kg) conventional food (Q 4). The GDPs2 in the area of the CF is NZ\$157,650, which is $5.8 \%$ (NZ\$45,936) more than GDPs1(NZ\$111,714) in the ordinary life area (Table 5.96).

Figure 5.43 and Table 5.95 indicate that using 17\% home generated (organic) food reduces the total EF (EF 1) of OCRT food consumed by 5.5\% (shown in Figure 5.43 as REF1). Moreover, as illustrated in Figure 5.43, producing $17 \%$ local food increases GDPs1 by 5.8\% (NZ\$ 45,936), shown in the figure as (I) GDPs1.

Comparison between the ideal model and present pattern of OCRT food consumption shows that EF2 (401.2 gha) is 74.4\% (315.7 gha) more than the ideal EF (85.47gha) and 
$79.9 \%(\mathrm{NZ} \$ 625,145)$ of the GDP $(\mathrm{NZ} \$ 782,795)$ of food must be spent to reduce this overshoot portion of EF2 to the ideal EF (Table 5.96 and Figure 5.43).

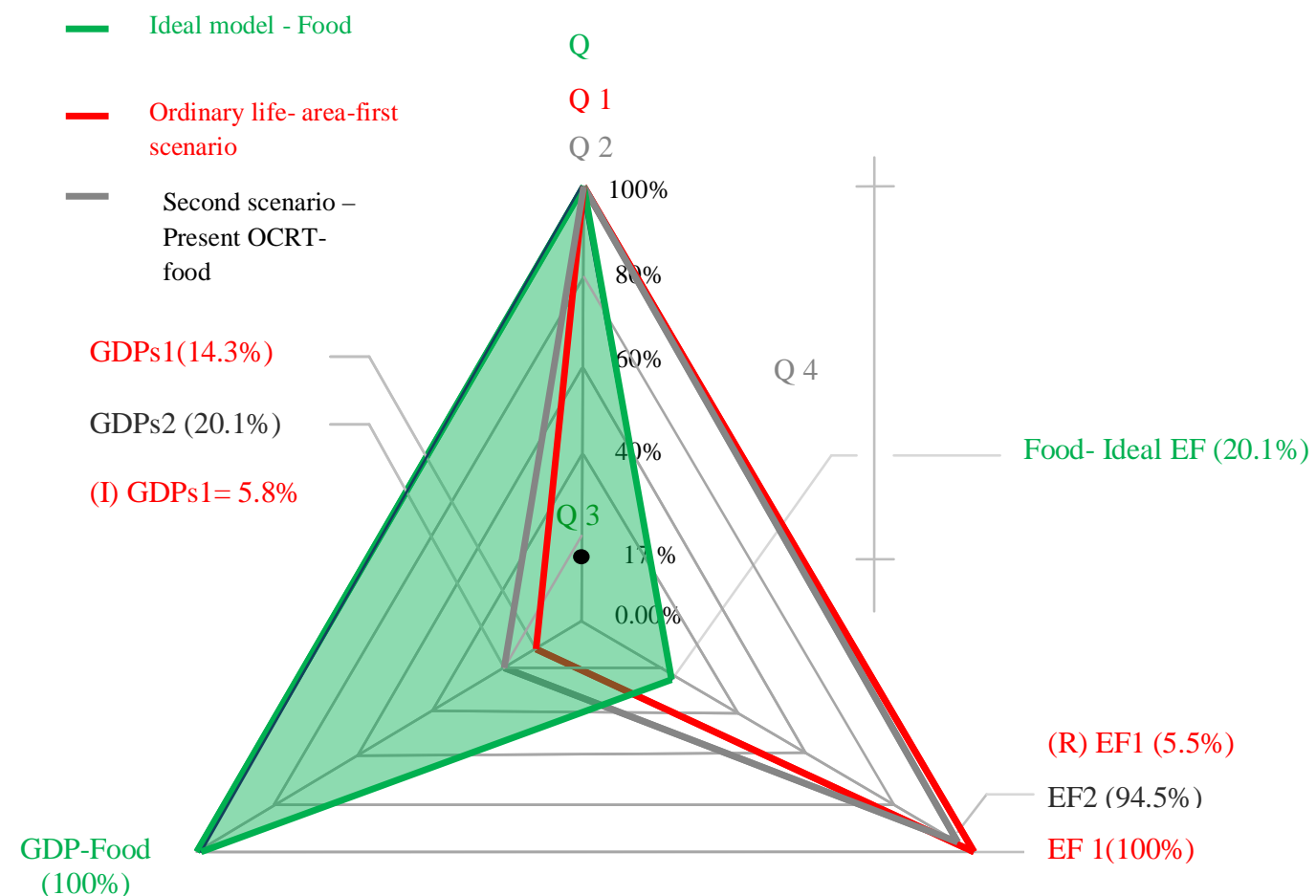

Figure 5.39: The cultural footprint (CF) of OCRT visitors' food

- Q: $100 \%$ local food in ideal model

- Q 1: $100 \%$ conventional food - first scenario

- $\quad$ Q 2: $83 \%$ conventional food $+17 \%$ home generated food - second scenario

- $\quad$ Q 3: $17 \%$ home generated food (second scenario)

- Q 4: 83\% conventional food (second scenario)

- GDPs1: sustainable portion of GDP - first scenario

- GDPs2: Sustainable portion of GDP - second scenario

- (I) GDPs1: increased portion of GDPs1

- EF 1: ecological footprint of food- first scenario

- EF2: ecological footprint of food- second scenario

- R- EF 1: reduced portion of total EF 1 of consumed food

\subsubsection{OCRT CF: Accommodation Services}

This study explores the CF of the 788 OCRT accommodation services through using three scenarios. In the first scenario, all buildings used as accommodation services are assumed to be newly constructed buildings (NB) without any open air areas (veranda and balcony). In the second scenario 10\% of OCRT accommodation buildings are RB and $90 \%$ are NB. In addition, in the second scenario all areas of buildings including indoor and outdoor (V/B) spaces are calculated as indoor spaces. In the third scenario $90 \%\left(58,972 \mathrm{~m}^{2}\right)$ of buildings are NB, $10 \%\left(21,378 \mathrm{~m}^{2}\right)$ are RB and $387 \mathrm{~m}^{2}$ of total building area is outdoor sitting space (V/B) (Table 5.97). 
The third scenario determines the present quantity, quality, EF and GDPs of the OCRT accommodation services. Comparison between the EFs and GDPs of the first and the second scenario indicates the ecological and economic effects of using RB buildings (as cultural heritage and capital) on a host destination. Likewise, comparison between the second and third scenario suggests the environmental and economic footprint of using open air areas (V/B) in accommodation services as an element of architecture. In addition, comparison between the first and third scenario reflects the total environmental and economic effects of using RB buildings as accommodation services and V/B as a part of architecture.

Table 5.97 shows that in the first scenario the total EF of using 100\% (80,356 $\left.\mathrm{m}^{2}\right) \mathrm{NB}$ as accommodation services is 50.5 gha, and the total GDP of OCRT accommodation services is equivalent to NZ\$2,837,412 (see Table 5.91). Since the overshoot portion of energy use related to the third scenario (current situation) has already been calculated (see Table 5.89), it is used as a base to calculate the overshoot energy related to the first and second scenario through the following steps:

Overshoot energy use (third scenario) $=3,310.1 \mathrm{GJ} /$ year

EF 1: Ecological footprint of accommodation services (first scenario) $=50.5$ gha/year

EF 3: Ecological footprint of accommodation services (third scenario) $=42.4$ gha/year Increased in EF 1 relative to EF 3= $($ EF 1) $-($ EF 3) $=50.5-42.4=8.1$ gha/year Increased portion of overshoot energy for first scenario relative to third scenario $=8.1$ gha $\times 100 \mathrm{GJ} /$ gha (global average carrying capacity of land) $=810 \mathrm{GJ}$ The overshoot energy of the first scenario $=810 \mathrm{GJ}+3,310.1 \mathrm{GJ}$ (overshoot energy of the third scenario) $=4,120.1$ (Table 5.97).

As the cost to generate $1 \mathrm{GJ}$ of energy using wind-solar systems is NZ\$19.8, consequently the total cost to generate the overshoot energy of the first scenario is: NZ\$19.8× $4,120.1 \mathrm{GJ}=\mathrm{NZ} \$ 81,578$.

GDP earned through OCRT accommodation services in all three scenarios is equivalent to NZ\$2,837,412. The sustainable portion of GDP in the first scenario (GDPs1) is equivalent to: NZ\$2,837,412 (total OCRT accommodation GDP) - NZ\$ 81,578 (cost to generate overshoot energy of the first scenario) $=$ NZ\$2,755,834. 
In the second scenario, the area of OCRT accommodation buildings $\left(80,356 \mathrm{~m}^{2}\right)$ is divided into the two parts of NB buildings $\left(58,972 \mathrm{~m}^{2}\right)$ and RB buildings $\left(21,378 \mathrm{~m}^{2}\right)$ (Table 5.96). In the second scenario the total EF of OCRT accommodation services ( EF2) is 43.2 (gha) and total accommodation GDP is NZ\$2,837,412, as for GDP in the first scenario.

The overshoot energy use for the third scenario is used as a base to calculate the overshoot energy use of OCRT accommodation services in the second scenario through the following steps:

Overshoot energy use (third scenario) = 3,310.1 GJ/ year

EF2: Ecological footprint of accommodation services (second scenario) $=43.2$ gha/year EF 3: Ecological footprint of accommodation services (third scenario) $=42.4$ gha/year Increased portion of EF2 over EF $3=(\mathrm{EF} 2)-(\mathrm{EF} 3)=43.2-42.4=0.8$ gha Increased overshoot energy use of second scenario relative to third scenario $=0.8$ gha $\times$ $100 \mathrm{GJ} /$ gha (global average carrying capacity of land) = $80 \mathrm{GJ}$

The overshoot energy use of the second scenario $=80$ GJ $+3,310.1$ GJ (overshoot energy use of the third scenario) $=3,390.1$ GJ (Table 5.97)

As the cost to generate $1 \mathrm{GJ}$ of energy through using wind-solar systems is NZ\$19.8, the total cost to generate the overshoot energy of the second scenario is: NZ\$19.8 $\times$ 3,390.1 GJ = NZ\$67,124 (Table 5.97). The sustainable portion of GDP in the second scenario (GDPs2) is equivalent to: NZ\$2,837,412 (total OCRT accommodation GDP) NZ\$67,124 (cost to generate overshoot energy of the second scenario) $=\mathrm{NZ} \$ 2,770,288$ (Table 5.97).

In the third scenario, the area of OCRT accommodation buildings $\left(80,356 \mathrm{~m}^{2}\right)$ is divided into the two areas of NB buildings $\left(58,972 \mathrm{~m}^{2}\right)$ and $\mathrm{RB}$ buildings $\left(21,378 \mathrm{~m}^{2}\right)$ (Table 5.97). In this scenario the total area $\left(80,356 \mathrm{~m}^{2}\right)$ of $\mathrm{NB}$ and $\mathrm{RB}$ is made up of 378 $\mathrm{m}^{2} \mathrm{~V} / \mathrm{B}$ and 79,978 $\mathrm{m}^{2}$ indoor spaces.

In the third scenario the total EF of OCRT accommodation services (EF 3) is equivalent to 42.4 (gha) and total accommodation GDP is NZ\$2,837,412, the same as GDP in the first and second scenarios (Table 5.97). EF 3 that contains the EFs of NB and RB build- 
ings and open air spaces (V/B) used as part of the accommodation services is the present EF of OCRT accommodation services (Table 5.97).

As shown in Table 5.97, the total overshoot energy use in the third scenario (the present overshoot energy of OCRT accommodation services) is 3,310.1 GJ. Since the cost to generate 1 GJ of energy through using wind-solar systems is NZ\$19.8, then the total cost to generate 3,310.1 GJ through using wind-solar systems is: NZ\$19.8 × 3310.1(GJ) $=$ NZ\$65,540 (Table 5.97).

\begin{tabular}{|c|c|c|c|c|c|}
\hline \multicolumn{6}{|c|}{ Table 5.97: OCRT- Accommodation services- CF (2011) } \\
\hline \multirow{2}{*}{\multicolumn{2}{|c|}{$\begin{array}{l}\text { First scenario } \\
\Gamma \text { accommodation build- } \\
\text { ings are NB }\end{array}$}} & \multirow{2}{*}{\multicolumn{2}{|c|}{$\begin{array}{c}\text { Second scenario } \\
90 \% \text { of } 788 \text { OCRT accommoda- } \\
\text { tion buildings are NB and } 10 \% \\
\text { RB (V/B not considered) }\end{array}$}} & \multirow{2}{*}{\multicolumn{2}{|c|}{$\begin{array}{l}\text { Third scenario } \\
90 \% \text { of } 788 \text { accommodations } \\
\text { (including camp sites and build- } \\
\text { ings) are NB and } 10 \% \text { are RB } \\
\text { buildings including } 387 \mathrm{~m}^{2} \mathrm{~V} / \mathrm{B}\end{array}$}} \\
\hline & & & & & \\
\hline $\begin{array}{l}\text { Total number of } \\
\text { accommodation ser- } \\
\text { vices }\end{array}$ & $788^{1}$ & $\begin{array}{l}\text { Total number of ac- } \\
\text { commodation ser- } \\
\text { vices }\end{array}$ & 788 & $\begin{array}{l}\text { Total number of } \\
\text { accommodation } \\
\text { services }\end{array}$ & 788 \\
\hline $\begin{array}{l}\text { NB accommodation } \\
\text { area }\left(\mathrm{m}^{2}\right)\end{array}$ & $80,356^{2}$ & $\begin{array}{l}\text { NB accommodation } \\
\text { area }\left(\mathrm{m}^{2}\right)\end{array}$ & $58,972^{2}$ & $\begin{array}{l}\text { NB accommoda- } \\
\text { tion area }\left(\mathrm{m}^{2}\right)\end{array}$ & $58,972^{2}$ \\
\hline $\begin{array}{l}\text { RB accommodation } \\
\text { area }\left(\mathrm{m}^{2}\right)\end{array}$ & 0.00 & $\begin{array}{l}\text { RB accommodation } \\
\text { area }\left(\mathrm{m}^{2}\right)\end{array}$ & $21,378^{2}$ & $\begin{array}{l}\text { RB accommoda- } \\
\text { tion area }\end{array}$ & $21,378^{2}$ \\
\hline $\begin{array}{l}\text { Total EF (EF 1) } \\
\text { (gha) }\end{array}$ & $50.5^{3}$ & Total EF (EF2) (gha) & $43.2^{4}$ & $\begin{array}{l}\text { Total EF (EF 3) } \\
\text { (gha) }\end{array}$ & 42.4 \\
\hline GDP (NZ\$) & $2,837,412$ & GDP (NZ\$) & $2,837,412$ & GDP (NZ\$) & $2,837,412$ \\
\hline $\begin{array}{l}\text { Overshoot energy } \\
\text { use (GJ/year) }\end{array}$ & $4,120.1$ & $\begin{array}{l}\text { Overshoot energy use } \\
\text { (GJ/year) }\end{array}$ & 3,390.1 & $\begin{array}{l}\text { Overshoot energy } \\
\text { use (GJ/year) }\end{array}$ & 3,310.1 \\
\hline $\begin{array}{l}\text { Cost to generate } 1 \\
\text { GJ energy using } \\
\text { wind- solar systems } \\
\text { (NZ\$) }\end{array}$ & 19.8 & $\begin{array}{l}\text { Cost to generate } 1 \mathrm{GJ} \\
\text { energy using wind- } \\
\text { solar systems (NZ\$) }\end{array}$ & 19.8 & $\begin{array}{l}\text { Cost to generate } 1 \\
\text { GJ energy using } \\
\text { wind- solar sys- } \\
\text { tems (NZ\$) }\end{array}$ & 19.8 \\
\hline $\begin{array}{l}\text { Cost to generate } \\
4,120.1 \text { GJ energy } \\
\text { using wind-solar } \\
\text { systems (NZ\$) }\end{array}$ & 81,578 & $\begin{array}{l}\text { Cost to generate } \\
3390.1 \mathrm{GJ} \text { energy } \\
\text { using wind-solar } \\
\text { systems (NZ\$) }\end{array}$ & 67,124 & $\begin{array}{l}\text { Cost to generate } \\
3,310.1 \text { energy } \\
\text { using wind-solar } \\
\text { systems (NZ\$) }\end{array}$ & 65,540 \\
\hline GDPs1 & $2,755,834$ & GDPs2 & $2,770,288$ & GDPs3 & $2,771,872$ \\
\hline \multicolumn{6}{|c|}{$\begin{array}{l}\text { 1. See Table } 5.15 \\
\text { 2. See Table } 5.23 \text { (the sum of areas include } 378 \mathrm{~m}^{2} \mathrm{~V} / \mathrm{B} \text { ) } \\
\text { 3. See Table } 5.36 \\
\text { 4. See Table } 5.37\end{array}$} \\
\hline
\end{tabular}

The GDPs of the third scenario GDPs 3 (the present GDPs of OCRT accommodation services) is calculated through the following equation:

GDPs 3 = NZ\$2,837,412 (total GDP of OCRT accommodation services) - NZ\$65,540

(cost to generate 3,310.1 GJ energy through using wind-solar systems) $=\mathrm{NZ} \$ 2,771,872$

(Table 5.97). 


\subsubsection{Sustainable living OCRT Accommodation Services (Ideal Model)}

This section determines an ideal model for OCRT accommodation services such that the EF is in an environmentally sustainable range. The GDPs for this model is then calculated. This ideal model is used to compare each of the three scenarios for OCRT accommodation services (Table 5.96) with an environmentally and economically sustainable version to explore how using RB buildings as accommodation and V/B as parts of architecture (as social behaviours) can contribute to achieving this ideal model.

\section{a. Sustainable EF}

As shown in Table 5.97 the total overshoot energy of OCRT accommodation services at present is 3,310.1 GJ/year. As a result the overshoot portion of the EF can be calculated through the following steps:

Overshoot portion of the EF of OCRT accommodation services = 3,310.1 GJ (overshoot energy use) $\div 100$ (carrying capacity of land) $=33.1$ gha

Table 5.96 shows that EF 1 (present EF of OCRT accommodation services) is 42.4 gha So the sustainable EF of OCRT accommodation services is equivalent to:

42.4 gha (present EF of OCRT accommodation services) - 33.1gha (overshoot portion of the EF of OCRT accommodation services) $=9.3$ gha

\section{b. Sustainable Living GDPs}

Since for sustainable living the EF of accommodation services is not in overshoot, consequently, in this position both the overshoot energy use and the cost to generate this through using wind-solar systems are zero. Consequently: assuming that the Ideal model has the same GDPs as the current model, GDPs of sustainable accommodation services $=$ Total GDP of accommodation services -0.00 (total cost to generate overshoot energy of accommodation services) $=$ Total GDP of accommodation services = NZ\$2,837,412 (see Table 5.97).

\subsubsection{Comparison between the Ideal Model, First and Second Scenarios}

Figure 5.44 comprises the quantities, EF and GDPs of OCRT accommodation services for the ideal model and first and second scenarios as discussed above. This figure gives the opportunity to explore the ecological and economic influences exerted by using RB buildings as accommodation on the host destination. 
As shown in Figure 5.44, in the ideal model of accommodation services, Q is $100 \%$ of the OCRT accommodation area $\left(80,356 \mathrm{~m}^{2}\right)$ with an EF of 9.3 gha EF and a GDPs of NZ\$2,837,412, which is equal to $100 \%$ of the GDP of OCRT accommodation services. In the ideal model as discussed above the overshoot portion of EF and energy use are zero. In the first scenario, Q1 is the total area of OCRT accommodation services $\left(80,356 \mathrm{~m}^{2}\right)$ for which $100 \%$ of accommodation services are assumed to be new buildings (NB) (Figure 5.44). In the second scenario as illustrated in Figure 5.44, Q 2 is the OCRT accommodation area $\left(80,356 \mathrm{~m}^{2}\right)$ divided into the two parts; $73.4 \%\left(58,972 \mathrm{~m}^{2}\right.$ see Table 5.97) NB and 26.6\% (21,378 $\mathrm{m}^{2}$-see Table 5.97) RB.

In Figure 5.44, EF 1 is the biggest EF in comparison with the EFs of the other scenarios and it is considered as the $100 \%$ EF benchmark. Moreover, in Figure 5.44, EF2 and ideal EF are compared with EF 1 in percentage terms.

As shown in Figure 5.44, in the ideal model of OCRT accommodation services the acceptable EF is 9.3 gha or $18.4 \%$ of EF 1 (EF 1 is 50.5gha-see Table 5.96). Alternatively, in the first scenario in which all accommodation is assumed as NB, the EF (EF 1= $50.5 \mathrm{gha}$ ) is $82 \%$ bigger than the sustainable EF (ideal EF= 9.3gha).

As illustrated in Figure 5.44, the EF of OCRT accommodation services in the second scenario (EF2= 43.2gha- see Table 5.97) is $85.4 \%$ of EF $1(50.5)$. Consequently in this scenario (R) EF 1 (reduced portion of EF 1) is equal to 14.6\% of EF 1= 7.3 gha.

As shown in Figure 5.40, the total GDPs1 (GDPs of the first scenario $=$ NZ $\$ 2,755,834$ ) is equal to $97.1 \%$ of total GDP of OCRT accommodation services (NZ\$2,837,412). On the other hand, in the first scenario 2.9\% (NZ\$82,285) of the total GDP of OCRT accommodation services must be spent to generate the overshoot portion of accommodation energy use through wind-solar systems to change EF 1 to the ideal EF.

Figure 5.44 determines that GDPs2 (NZ\$2,770,288-GDPs of second scenario) is equal to $97.6 \%$ of total OCRT accommodation GDP (NZ\$2,837,412). Indeed in the second scenario $2.4 \%(\mathrm{NZ} \$ 68,098)$ of the total GDP of OCRT accommodation services must be spent to generate the overshoot portion of OCRT accommodation energy use through wind-solar systems to reduce EF2 to an ideal EF. Likewise, as determined in Figure 5.44, (I) GDPs1 (increased portion of GDPs1) is equal to $0.5 \%$ of total OCRT accom- 
modation GDP. This means using 21,378 $\mathrm{m}^{2}$ (26.6\% of total OCRT accommodation services area) of accommodation buildings as RBs contributes to an increase of $0.5 \%$ in GDPs1.

\subsubsection{Comparison between the Ideal Model, Second and Third Scenarios}

As shown in Figure 5.44, in the third scenario Q 3 is the total area of OCRT accommodation services in which $73.4 \%\left(58,972 \mathrm{~m}^{2}\right)$ of the area is NB and $26.6 \%\left(21,378 \mathrm{~m}^{2}\right)$ is RB. Furthermore, in the third scenario of total Q $3\left(80,356 \mathrm{~m}^{2}\right) 387 \mathrm{~m}^{2}(0.5 \%)$ is V/B and 79,996 $\mathrm{m}^{2}(99.5 \%)$ is indoor spaces (Figure 5.44).

In the third scenario, the total EF of OCRT accommodation services (EF 3) is 42.4 gha (see Table 5.97) which is equal to 84\% of EF 1 (Figure 5.44). As shown in Figure 5.44, EF 3 (84\% of EF 1) is 1.5\% smaller than EF2 (85.5\% of EF 1). On the other hand (R) EF2 is equivalent to $(\mathrm{EF} 2)-(\mathrm{EF} 3)=1.5 \%$ of EF 1 (EF of the first scenario that is used as a base or $100 \%$ EF of OCRT accommodation services). EF 3 is the present EF of OCRT accommodation services, which is still 65.6\% (84\%-18.4\%) bigger than the ideal $\mathrm{EF}$, and is shown in Figure 5.44.

Figure 5.44 and Table 5.97 show that the GDPs of the third scenario (GDPs 3) which is the present GDPs of OCRT accommodation services is 1\% (NZ\$28,374 of NZ\$2,837,412) more than GDPs2. Furthermore, GDPs 3 is 2.3\% (NZ\$ 65,261) less than the total GDP $(\mathrm{NZ} \$ 2,837,412)$ of OCRT accommodation services (Figure 5.44 and Table 5.97). This means (I) GDPs2 is influenced by using $387 \mathrm{~m}^{2} \mathrm{~V} / \mathrm{B}$ at a value equal with $1 \%$ of total GDP of OCRT accommodation services. In addition, Figure 5.40 shows that in the third scenario (present circumstance of OCRT accommodation services) $2.3 \%(\mathrm{NZ} \$ 65,260)$ of its GDP must be spent to generate its overshoot portion of energy use through using wind-solar systems to reduce its present EF to the ideal EF. 


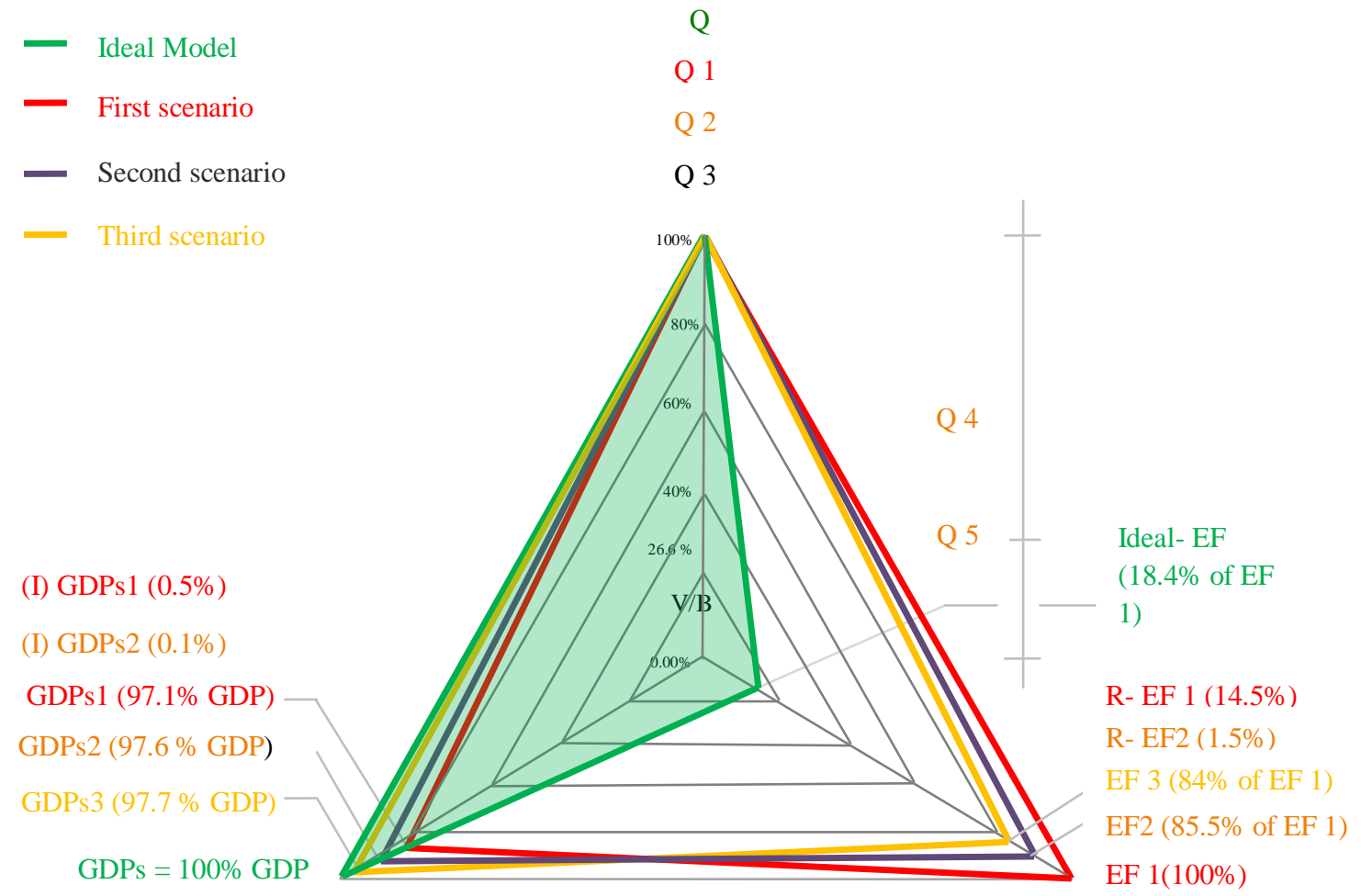

Figure 5.44: The CF of OCRT accommodation services- using RB buildings and $\mathrm{V} / \mathrm{B}$ as parts of accommodation services

- Q: (100\%) area of 788 OCRT accommodation services $\left(80,356 \mathrm{~m}^{2}\right)$ - ideal model

- GDP: Total NZ\$2,837,412 GDP of OCRT accommodation services (see Table 5.95)

- GDPs: Total sustainable portion of OCRT accommodation services - ideal model

- Q 1: Total (100\%) area of 788 OCRT accommodation services $\left(80,356 \mathrm{~m}^{2}\right)-100 \%$ NB accommodation services - first scenario

- $\quad$ EF 1: Total EF of OCRT accommodation services - first scenario

- (R) EF 1: Reduction of OCRT EF 1 influenced by using RB buildings as OCRT accommodation services

- GDPs1: Total GDPs of OCRT accommodation services - first scenario

- (I) GDPs1: Increased portion of GDPs1 from using RB buildings as part of OCRT accommodation services

- Q 2: Total (100\%) area of 788 OCRT accommodation services (80,356m²); 73.4\% (58,972m²) NB and 26.6\% (21,378m²) RB - second scenario

- $\quad$ Q 3: Total (100\%) area of 788 OCRT accommodation services $\left(80,356 \mathrm{~m}^{2}\right) ; 73.4 \%\left(58,972 \mathrm{~m}^{2}\right)$ NB and $26.6 \%\left(21,378 \mathrm{~m}^{2}\right)$ RB. Of total Q $3\left(80,356 \mathrm{~m}^{2}\right) 387 \mathrm{~m}^{2}(0.5 \%)$ is V/B and $79,996 \mathrm{~m}^{2}$ is indoor spaces - third scenario

- $\quad$ Q 4 (NB area): $73.4 \%\left(58,972 \mathrm{~m}^{2}\right)$ of total OCRT accommodation area $\left(80,356 \mathrm{~m}^{2}\right)$ - second scenario

- $\quad$ Q 5 (RB area): $26.6 \%\left(21,378 \mathrm{~m}^{2}\right)$ of total OCRT accommodation area $\left(80,356 \mathrm{~m}^{2}\right)$ - second scenario

- $\quad$ EF2: EF of 80,356m² OCRT accommodation services (Q 2) including EFs of Q 3 and Q 4

- GDPs2: Total GDPs of OCRT accommodation services - second scenario 


\subsubsection{The CF of OCRT Visitor Activities}

\subsubsection{Overshoot EF of OCRT Visitor Activities}

As shown in Table 5.98, other activities (excepting curling, golf and walking) have the largest share at 82\% (5.39 gha) of the total EF of OCRT activities (6.57gha), followed by curling (10.65\%), golf (7.3\%) and walking (0.06 \%). Table 5.98 shows that the total overshoot of OCRT activities is equal to 10.18 gha (see also Table 5.88). The overshoot portion of each of the above EF categories (Other activities, curling, walking and golf) is calculated by the following equation:

Overshoot of the EF of each category = the percentage of the EF of activity $\times$ total overshoot EF of OCRT activities (10.18gha)

For example:

Overshoot EF of golf $=7.3 \% \times 10.18($ gha $)=0.74$ gha $($ see Table 5.98$)$.

Furthermore the overshoot energy use of each category is calculated as its overshoot EF $\times 100$ (global average carrying capacity) (Table 5.98).

As shown in Table 5.98, in comparison with the other categories, other activities have the largest overshoot energy at 835 GJ, followed by, curling (108GJ), golf (74) and walking (0.6 GJ).

\subsubsection{OCRT Activities: GDP}

This thesis proposes that the sum of the monies for vehicle-equipment hire (NZ\$975,119), road passenger transport $(\mathrm{NZ \$ 446,136)}$ and entertainment $(\$ 61,925)$, as shown in Table 5.91, are used to represent the total GDP of OCRT visitor activities $(\mathrm{NZ} \$ 1,483,200)$ (Table 5.98). As the total number of OCRT annual visitor activities is 26,077 (see Table 5.82) then the GDP/visitor activity can be calculated as follows:

NZ\$1,483,200 $\div$ 26,077 = NZ\$56.88/visitor activity (Table 5.98).

In Table 5.98 the GDP of each category is calculated as the number of visitor activities $\times$ NZ\$57/visitor activity. Table 5.98 shows that for the total GDP of OCRT activities, the major share of $\mathrm{NZ} \$ 1,223,100$ is the GDP of other visitors-activities, followed by NZ\$247,400 (curling), NZ\$ 8,700 (golf) and NZ\$4,000 walking. 


\subsubsection{OCRT Activities: GDPs}

Table 5.98 contains the total cost to generate the overshoot energy use of each type of OCRT activity through using wind-solar systems. It shows that other activities have the largest cost at NZ\$16,533 and walking the minimum cost for generating overshoot energy in a sustainable way.

As shown in Table 5.98 the GDPs of the four categories of other activities, curling, golf and walking fall between NZ\$3,988 - NZ\$1,206,567. In addition the total GDPs of OCRT visitor activities is $\mathrm{NZ} \$ 1,463,052$, equivalent to $\mathrm{NZ} \$ 1,483,200$ - NZ\$20,149 (see Table 5.98).

\begin{tabular}{|c|c|c|c|c|c|c|c|c|}
\hline \multicolumn{9}{|c|}{ Table 5.98: OCRT- visitor activities - EF and GDPs } \\
\hline Category & $\begin{array}{l}\text { Number of } \\
\text { visitor ac- } \\
\text { tivities }\end{array}$ & $\begin{array}{l}\mathrm{EF}^{1} \\
\text { (gha) }\end{array}$ & $\%$ & $\begin{array}{l}\text { Overshoot } \\
\text { EF(gha) }\end{array}$ & $\begin{array}{l}\text { Overshoot } \\
\text { energy use } \\
\text { (GJ) }\end{array}$ & $\begin{array}{l}\text { GDP } \\
(\mathrm{NZ} \$)\end{array}$ & $\begin{array}{l}\text { Cost of } \\
\text { over- } \\
\text { shoot } \\
\text { energy }\end{array}$ & $\begin{array}{l}\text { GDPs } \\
(\mathrm{NZ} \$)\end{array}$ \\
\hline Golf & 153 & 0.48 & 7.3 & 0.37 & 37 & 8,700 & 733 & 7,967 \\
\hline Curling & 4,350 & 0.7 & 10.65 & 0.54 & 54 & 247,400 & 1,069 & 246,331 \\
\hline Walking & 71 & 0.004 & 0.06 & 0.003 & 0.3 & 4,000 & 6 & 3,994 \\
\hline Other & 21,503 & 5.39 & 82 & 4.17 & 417 & $1,223,100$ & 8,257 & $1,214,843$ \\
\hline Total & 26,077 & 6.57 & 100 & $5.09^{2}$ & 509 & $1,483,200$ & 10,065 & $1,473,135$ \\
\hline \multicolumn{9}{|c|}{$\begin{array}{l}\text { 1. See Figure } 5.34 \text { and Table } 5.82 \\
\text { 2. See Table } 5.89\end{array}$} \\
\hline
\end{tabular}

Figure 5.45 illustrates three scenarios for the CF area of OCRT visitor activities and compares these areas with each other and the sustainable (ideal model) area of these activities (in the figure shown as the green area).

\subsubsection{OCRT Visitor Activities: First Scenario}

The present CF of OCRT visitor activities is shown as the first scenario in Figure 5. 45. In the first scenario the total number of visitor activities $(26,077)$ comprises 21,503 visitors (other activities), 4,350 visitors (curling), 153 visitors (golf) and 71 visitors (walking) (see Table 5.99).

The total EF of OCRT visitor activities (EF 1) in the first scenario is 6.57 gha (see Table 5.98). In comparison with the EFs of other scenarios and the ideal model, the EF 1 is considered as $100 \%$ of the EF of visitor activities. As shown in Figure 5.45 and Table 5.98 in the first scenario GDPs1 is $99.3 \%(\mathrm{NZ} \$ 1,473,135)$ of the total GDP (NZ\$1,483,200) of OCRT visitor activities. 


\subsubsection{OCRT Visitor Activities: Second Scenario}

The second scenario is proposed based on the assumption that the 153 visitors who play golf in the first scenario (see Table 5.98) choose walking as their activity instead of golf (see Table 5.99). In the second scenario, walking is considered as an example of other outdoor activities with same EF/visitor (0.00025-0.00006 gha/visitor- see Table 5.82).

\begin{tabular}{|c|c|c|c|c|c|c|c|}
\hline \multicolumn{4}{|c|}{ First scenario } & \multicolumn{4}{|c|}{ Second scenario } \\
\hline Category & $\mathrm{NV}^{4}$ & EF/visitor & EF 1 & Category & NV & $\begin{array}{c}\text { EF } \\
\text { (gha/visitor) }\end{array}$ & EF2 (gha) \\
\hline Golf & 153 & $0.003^{1}$ & 0.48 & - & - & - & - \\
\hline Curling & 4,350 & $0.00016^{2}$ & 0.7 & Curling & 4,350 & $0.00016^{2}$ & 0.7 \\
\hline Walking & 71 & $0.00005^{3}$ & 0.004 & Walking & 224 & $0.00005^{3}$ & 0.1 \\
\hline Other & 21,503 & 0.00025 & 5.39 & Other & 21,503 & 0.00025 & 5.3 \\
\hline Total & 26,077 & - & 6.57 & Total & 26,077 & - & 6.1 \\
\hline $\begin{array}{l}\text { 1. See Tabl } \\
\text { 2. See Tabl } \\
\text { 3. See Tabl } \\
\text { 4. NV: Nun } \\
\text { - (EF 1)-( }\end{array}$ & $\begin{array}{l}50 \\
54 \\
56 \\
\text { of visitol } \\
=6.57-\end{array}$ & $\begin{array}{l}\text { ivities } \\
=0.47 \text { gha }\end{array}$ & & & & & \\
\hline
\end{tabular}

As shown in Table 5.99 and Figure 5.45, compared with the first scenario, in the second scenario EF2 is 92.8\% (6.1gha) of EF 1(6.57gha) influenced by the 153 visitors who change their activity from golfing to walking. In addition, the overshoot portion of the EF of OCRT activities that in the first scenario is 5.09 gha (see Table 5.98) is reduced in the second to 4.62gha (5.09 - 0.47gha) for the same reason. As a result the overshoot energy use of OCRT activities in the second scenario is equal to 4.62gha $\times 100$ (carrying capacity) $=462$ GJ.

The total cost to generate the overshoot energy of OCRT visitor activities in the second scenario is equal to:

462 GJ (overshoot portion of energy use) $\times$ NZ\$19.8 (the cost to generate 1GJ energy through using wind-solar systems) $=\mathrm{NZ} \$ 9,148$.

Since the total GDP of OCRT visitor activities is NZ\$1,483,200 (Table 5.98) the GDPs of the second scenario is equal to NZ\$1,483,200 (GDP) - NZ\$ NZ\$9,148 (the total cost of generating the overshoot energy of OCRT visitor activities in the second scenario) = NZ\$1,474,052 (GDPs2). As shown in Figure 5.45, GDPs2 is 99.4\% of the total GDP of OCRT visitor activities and (I) GDPs2 is equal to GDPs2 - GDPs1 $=0.1 \%$ of GDP $(\mathrm{NZ} \$ 1,483,200)=\mathrm{NZ} \$ 1,483$. 


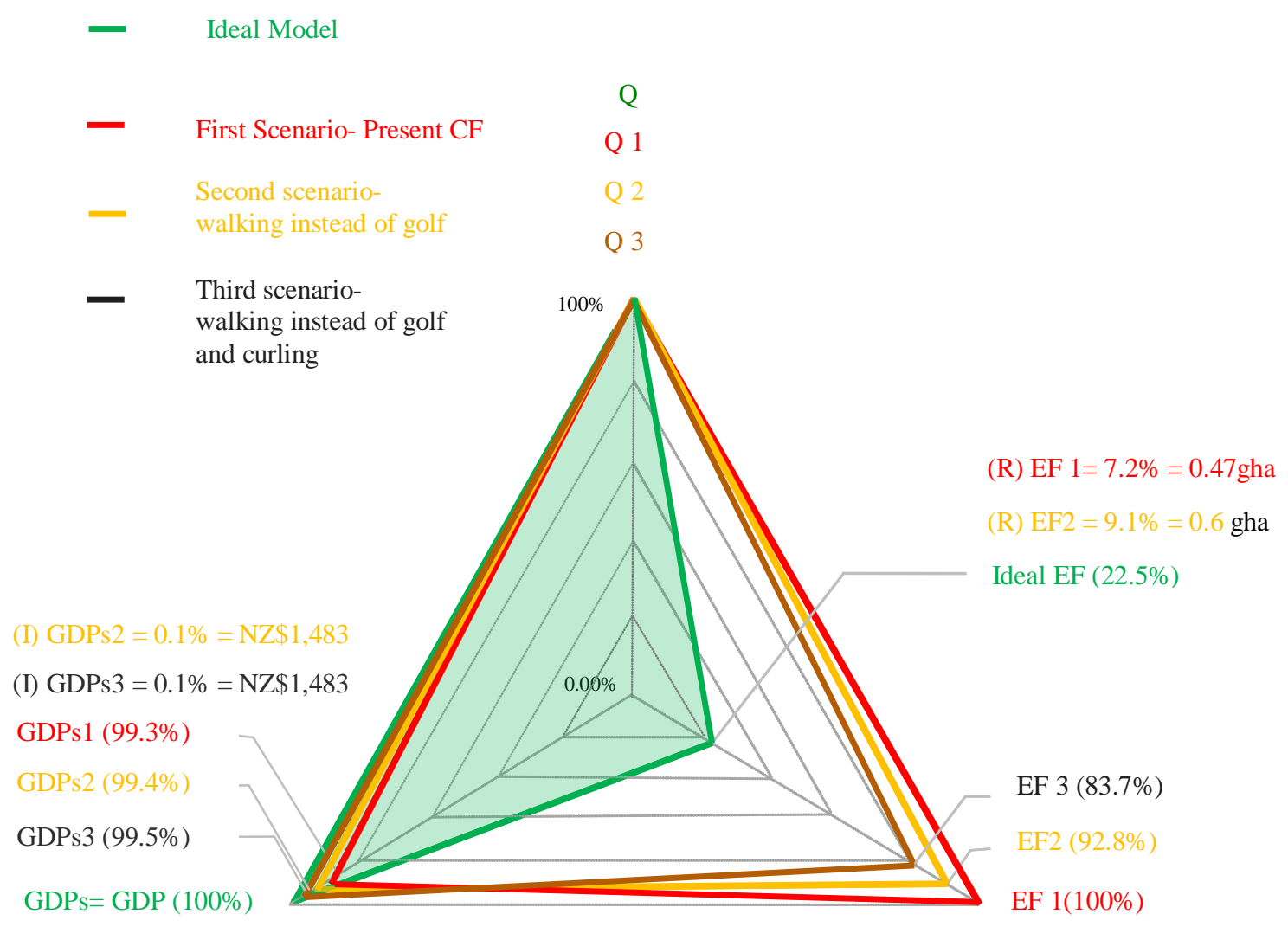

Figure 5.45: The CF of OCRT visitor activities

\subsubsection{Comparison between Ideal Model and Second Scenario}

As illustrated in Figure 5.45, in the ideal model (the green area in the figure) the environmentally sustainable EF for OCRT visitor activities is equal to:

6.57 gha total EF (see Table 5.98) - 5.09 gha overshoot portion of EF (see Table 5.98) = 1.48 gha.

In comparison with the EFs of other scenarios (EF 1 and EF2), the EF of the ideal model (1.48gha) is 22.53\% of EF 1 and $24.26 \%$ of EF2 (6.1gha, see Table 5.99) Figure 5.45 indicates that for changing the EF of OCRT visitor activities in the second scenario to the ideal model (from EF2 to sustainable 1.48gha EF), 0.1\% (NZ\$ 1,483) of the total GDP of OCRT activities (NZ\$1,483,200) must be spent to generate its overshoot portion of energy use.

\subsubsection{OCRT Visitor Activities: Third Scenario}

The third scenario is proposed based on the assumption that 153 visitors who play golf and 4,350 visitors who play curling in the first scenario (see Table 5.98) choose walking as their activity instead (see Table 5.100). In the third scenario, walking is considered as 
an example of other outdoor activities with the EF/visitor of 0.00006 gha/visitor - see Table 5.82.

\begin{tabular}{|c|c|c|c|c|c|c|c|}
\hline \multicolumn{8}{|c|}{ Table 5.100: The CF of OCRT visitor activities - first and third scenarios } \\
\hline \multicolumn{4}{|c|}{ First scenario } & \multicolumn{4}{|c|}{ Third scenario } \\
\hline Category & $\mathrm{NV}^{4}$ & EF/visitor & EF 1 & Category & NV & $\begin{array}{c}\text { EF } \\
\text { (gha/visitor) }\end{array}$ & $\begin{array}{l}\text { EF } 3 \\
\text { (gha) }\end{array}$ \\
\hline Golf & 153 & $0.003^{1}$ & 0.48 & - & - & - & - \\
\hline Curling & 4,350 & $0.00016^{2}$ & 0.7 & - & - & - & - \\
\hline Walking & 71 & $0.00006^{3}$ & 0.004 & Walking & 4,574 & $0.00006^{3}$ & 0.2 \\
\hline Other & 21,503 & 0.00025 & 5.39 & Other & 21,503 & 0.00025 & 5.3 \\
\hline Total & 26,077 & - & 6.57 & & 26,077 & - & 5.5 \\
\hline $\begin{array}{l}\text { 1. See Table } \\
\text { 2. See Table } \\
\text { 3. See Table } \\
\text { 4. NV: Num }\end{array}$ & 8 & & & & & & \\
\hline
\end{tabular}

As shown in Table 5.100 and Figure 5.45 in comparison with the first scenario, in the third scenario the EF 3 is reduced by 16.28 \% (1.07 gha) when compared with EF 1 because of the change in activities. In addition, the overshoot portion of the EF of OCRT activities that in the first scenario is 5.09 gha (see Table 5.98) in the third scenario is reduced to 4.02 gha (5.09gha $-1.07 \mathrm{gha})$. As a result the overshoot energy use of OCRT activities in the third scenario is equal to 4.02gha $\times 100$ (carrying capacity of land) $=$ 402 GJ. In addition, the total cost to generate the overshoot energy use of OCRT visitor activities in the third scenario is equal to:

402 GJ (overshoot portion of energy use) $\times$ NZ\$19.8 (the cost to generate 1GJ energy through using wind-solar systems) $=$ NZ\$7,959.

Since the total GDP of OCRT visitor activities is NZ\$1,483,200 (Table 5.98), the GDPs of the third scenario is equal to NZ\$1,483,200 (GDP) - NZ\$7,959 (the total cost to generate overshoot energy use of OCRT visitor activities in the third scenario) $=$ NZ\$ 1,475,241 (GDPs 3). As shown in Figure 5.45, GDPs 3 is equal to 99.5\% of the total GDP of OCRT visitor activities and (I) GDPs 3 is equal to:

GDPs $3-$ GDPs2 $=0.1 \%$ of GDP $(\mathrm{NZ} \$ 1,483,200)=\mathrm{NZ} \$ 1,483$.

\subsubsection{Comparison between the Ideal Model and the Third Scenario}

As illustrated in Figure 5.45, in the ideal model (the green area in the figure) the environmentally sustainable EF for OCRT visitor activities is 22.5\% of EF 1(1.48 gha- see above discussion). 
Figure 5.45 indicates that to change the EF of OCRT visitor activities in the third scenario (EF 3 to sustainable 1.48 gha EF), 0.1\% (NZ\$1,483) of total GDP of OCRT activities $(\mathrm{NZ} \$ 1,483,200)$ must be spent to generate its overshoot portion of energy use.

\section{Chapter 5: Summary}

This chapter uses the comprehensive framework for the sustainable development of ecotourism and its architecture to evaluate the ecological, social and economic influences exerted by the OCRT on its host society. As Mahravan and Vale (2010) and O’Connor (2009) reveal, in New Zealand, the largest portions of the tourism EF are the four categories of transportation, food, accommodation services and tourism activities. Therefore this thesis uses these four categories as the main factors to be evaluated using the comprehensive framework and the model.

This chapter demonstrates that the 2011 EF of the OCRT is equivalent to 1,617 gha (0.138 gha/visitor) which derives from the integration of the calculated EFs of transportation, food, accommodation services and activities used by 11,788 OCRT visitors that year (see Table 5.84). As shown in Table 5.84 transportation has by far the largest footprint of 1,169 gha (72.2\%) of the total EF, followed by 401.2 gha (24.8\%) for food, 42.4 gha (2.6\%) accommodation services and 6.6 gha (0.4\%) visitor activities. The total EF of transportation can be further divided, giving 38.2\% (617 gha) of total EF for international and 34\% (552gha) for domestic transportation. This shows the importance of getting to a tourist destination in its overall environmental impact.

This chapter uses a comparative method by using different scenarios to investigate the current ecological, social and economic influences of the OCRT and its architecture on its host society. Using the scenarios allows this thesis to determine how far the OCRT is from its sustainable goal. For instance, this chapter demonstrates that the total overshoot EF of the OCRT is equivalent to 1,273 gha (Table 5, 95). This means the existing EF of the OCRT is 1,273 gha more than its sustainable EF of 388 gha (1,617- 1273) (Table 5.95).

This chapter indicates that $43.6 \%$ of OCRT total GDP must be spent to reduce its EF to its sustainable EF of (1,617- 1273) 388 gha (Table 5.95). 
In response to the research question: "How do the priorities for the sustainable development of ecotourism and its products influence each other?” (2.6.1) this chapter determines that changing the social behaviours of the host society and visitors can influence their ecological and economic footprints. For example, consuming organic food instead of conventional food, and using refurbished buildings as accommodation instead of constructing new buildings, not only contribute to conserving cultural identity in the host society, but also contribute to reducing their ecological footprint and consequently increasing their economic footprint.

Furthermore in response to the research question: "What are the main environmental, cultural and economic characteristics of the activities and products proposed for the sustainable development process of ecotourism?” (2.6.1), Chapter 5 indicates that the products and activities which simultaneously contribute to the cultural, environmental and economic development of the host society of an ecotourism project can be considered as having the sustainable cultural footprint as their main characteristic.

This chapter uses the framework and the model through a comparative method to show how the environmental, social and economic influences of ecotourism products and activities can be evaluated as being sustainable. Using the CF model and its results can thus be seen as the answer to question 2.6.1.7.

In the next chapter the comprehensive framework and the model will be used to explore and compare the cultural footprints of ecotourism and its architecture in two different host societies (Naseby and Cromwell) as the second and third case studies. 


\section{Chapter 6: Second and Third Case Studies: Naseby and Cromwell -OCRT, New Zealand}

This chapter uses the proposed comprehensive framework for the sustainable development of ecotourism and its architecture at the scale of the site and individual building. The results provide further answers to research questions at these different scales. In addition this chapter shows how the framework and model can be used to compare different ecotourism host destinations as being sustainable. To achieve this goal, two case studies of Naseby and Cromwell, as the host destinations of soft ecotourism (OCRT) with different environmental, cultural, economic heritage and capitals, are used. Also Naseby and Cromwell are shown to be two host destinations with different attractions that arise from their particular social ecological characteristics. The outcome of this chapter will demonstrate the influences exerted by ecotourism and its architecture on the two case studies from the viewpoint of sustainable development.

\subsection{Case Study 2: Naseby}

\subsubsection{Introduction}

This thesis is based on Naseby as a host destination of OCRT visitors with a rich environmental and cultural heritage and associated capitals. As explained in Chapter 3 the main reasons for choosing Naseby as a case study are its use of refurbished buildings as accommodation services, existence of the curling club visited by 4,350 OCRT visitors in 2011, and the variety of products and activities offered to visitors at this place (see 3.3.2.2).

This research attempts to explore the ecological, cultural and economic footprints of the OCRT as a soft ecotourism project at this destination, and their interaction with each other. This exploration is conducted through using the proposed holistic framework for the development of ecotourism and its architecture as proposed earlier in this thesis. 
This section aims to investigate the influences exerted by ecotourism on the sociocultural behaviours of visitors and host communities that in turn could change their environmental and economic footprint to being sustainable.

The investigation of the influences of the OCRT and its architecture on Naseby is conducted through measurement of the quantity of the cultural products and activities produced and consumed (numbers of visitors who participate in these activities), and calculation of their ecological and economic footprints. The results are compared with the quantities of these factors in a sustainable scenario, as proposed for the development of ecotourism and its products.

\subsection{2: Background to Naseby}

Naseby is a town located at elevation of $627 \mathrm{~m}, 45^{\circ} \mathrm{S}$ and $170^{\circ} \mathrm{E}$, about 15 kilometres from Ranfurly in Central Otago, New Zealand (Figure 6.1). Naseby is set in a valley surrounded by hills and 25 square kilometres of forest (Figure 6.2). As pointed out by Sorrel (1999-cited in Naseby Vision, 2012: 2), in more recent times, Naseby has become an important forestry centre with the initial forest having been planted as early as 1900. Naseby Community Program (NCP) (2010: 8) introduces the landscape and heritage aspects of Naseby as fundamental factors that contribute to the perception of this area as a special place. 


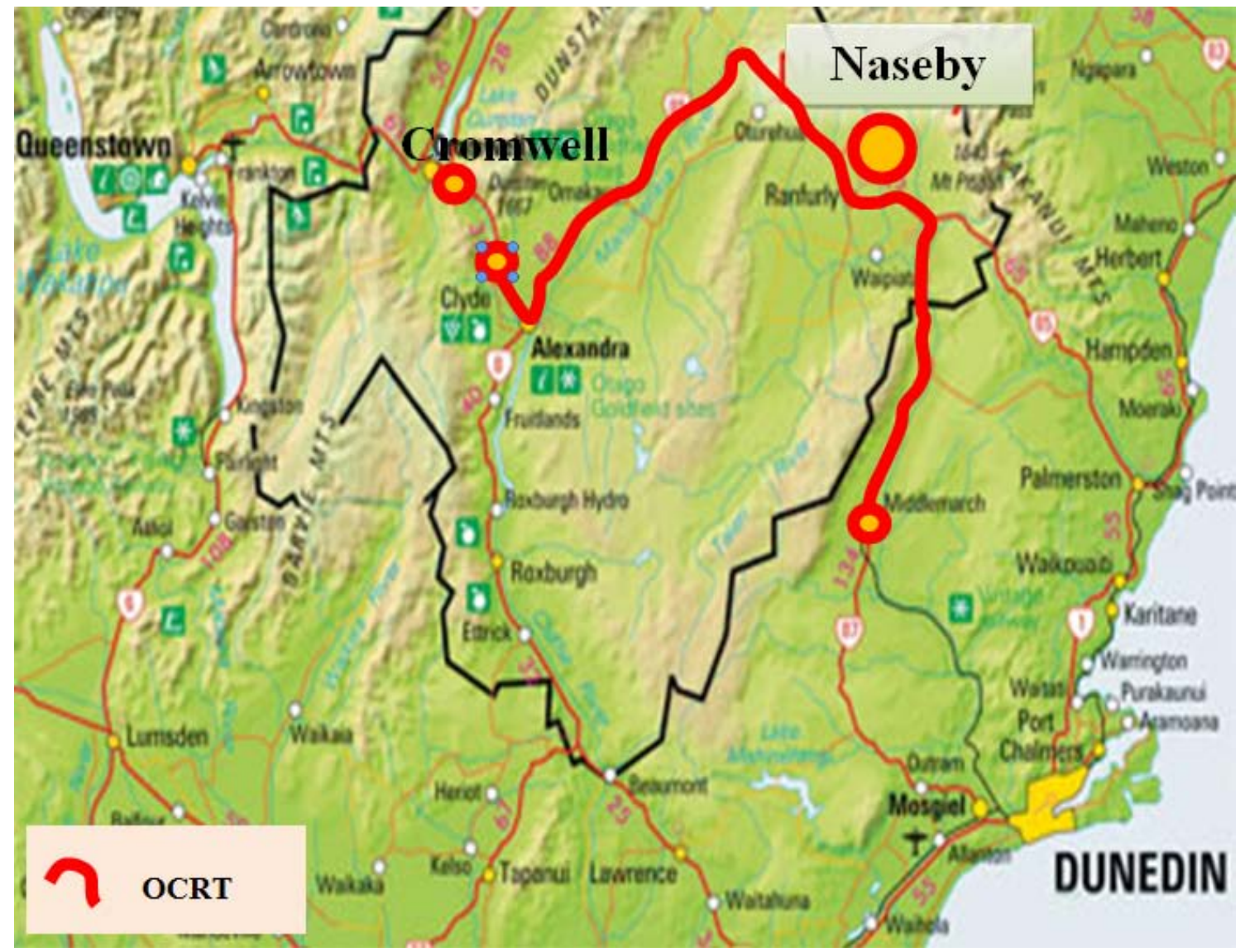

Figure 6.1: Naseby-Central Otago- New Zealand

http://www.no1guide.co.nz/images/map/Map_of_Central_Otago.jpg viewed August 2012 


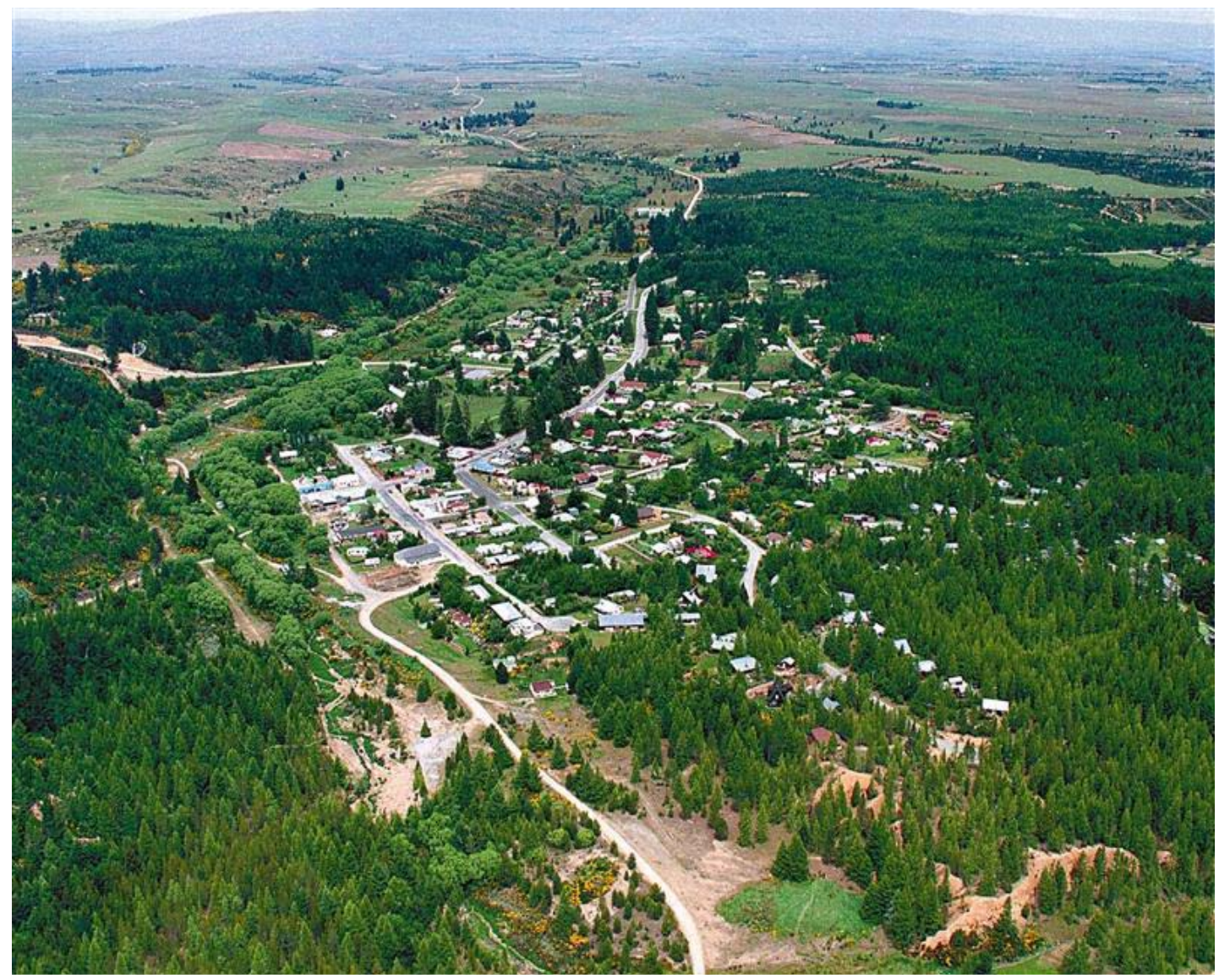

Figure 6.2: A view of Naseby

http://www.odt.co.nz/files/story/2010/04/naseby township_nestled_close to the naseby fores_1659 772314.JPG viewed August 2012

NCP (2010:7) reveals that Naseby came into existence in 1863 when gold was discovered close to where it stands now and how Naseby was moved to get at the rich ground beneath it. As noted in the Ancient Briton Hotel, Naseby website (2011:1) the town's first buildings were of a wooden frame with a calico (cloth) construction and twenty canvas shops lined each side of a muddy main street. The Ancient Briton Hotel (built in 1863) was the first hotel in Naseby (see Appendix 5 and Figures 6.3, 6.4 and 6.5).

When the town grew, the buildings become more permanent with timber frame construction and with walls and roof covered with corrugated iron sheets. Some buildings were made of flat schist and glacial moraine stone with a corrugated iron roof, and others were constructed from adobe (sun dried mud brick) (see http://www. ancientbriton.co.nz). As shown in Appendix 5, many of the original miners' cottages and other historic buildings and sites still remain in use today in Naseby. NCP (2010:36-37) introduces a list of 23 such buildings, places, sites and objects that are registered as part of the natural and cultural heritage of New Zealand. 


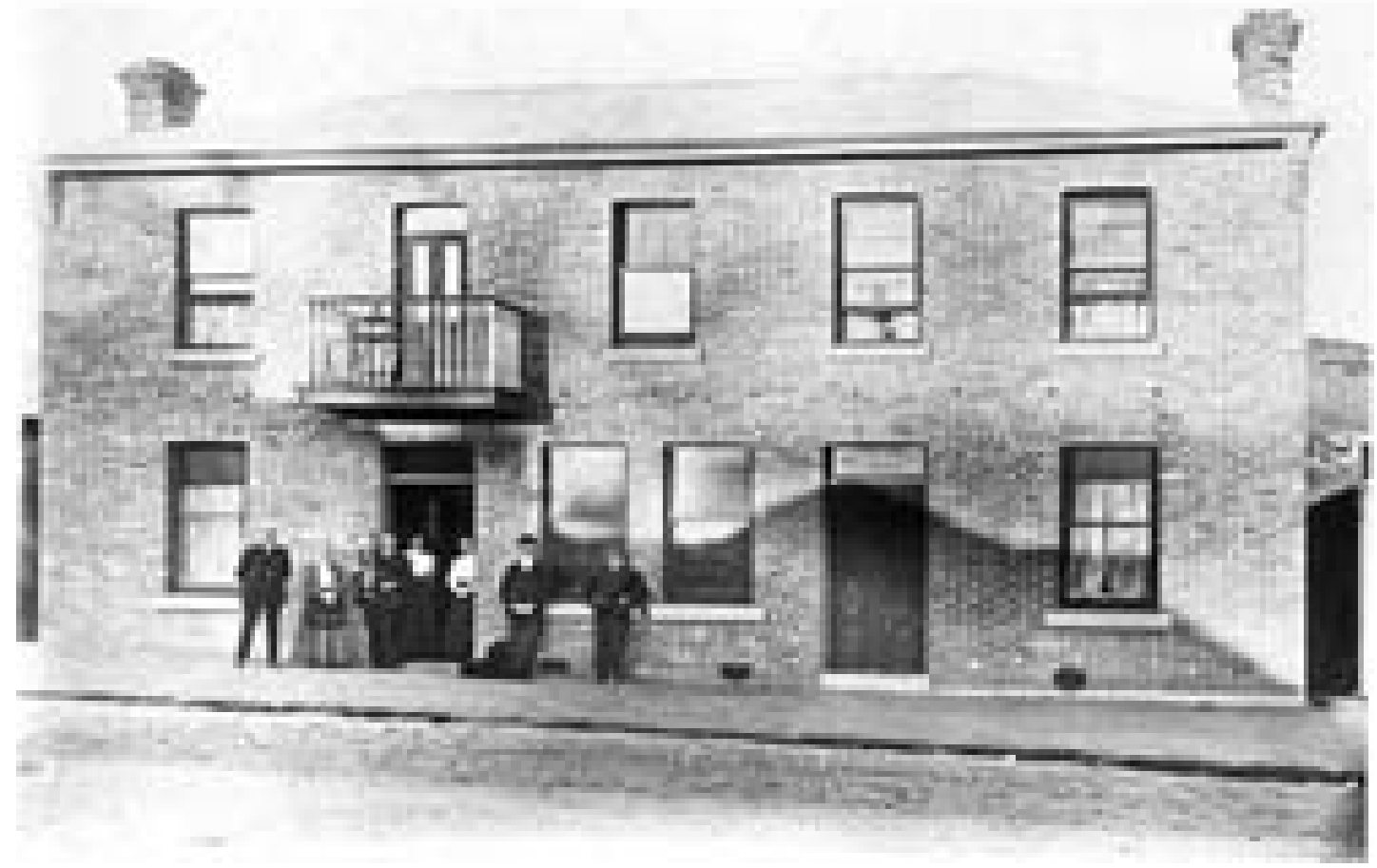

Figure 6.3: Ancient Briton Hotel, Naseby, Built in 1863

httb://www.otagorailtrail.co.nz/images/stories/ancientbritonold.ipg viewed August 2012

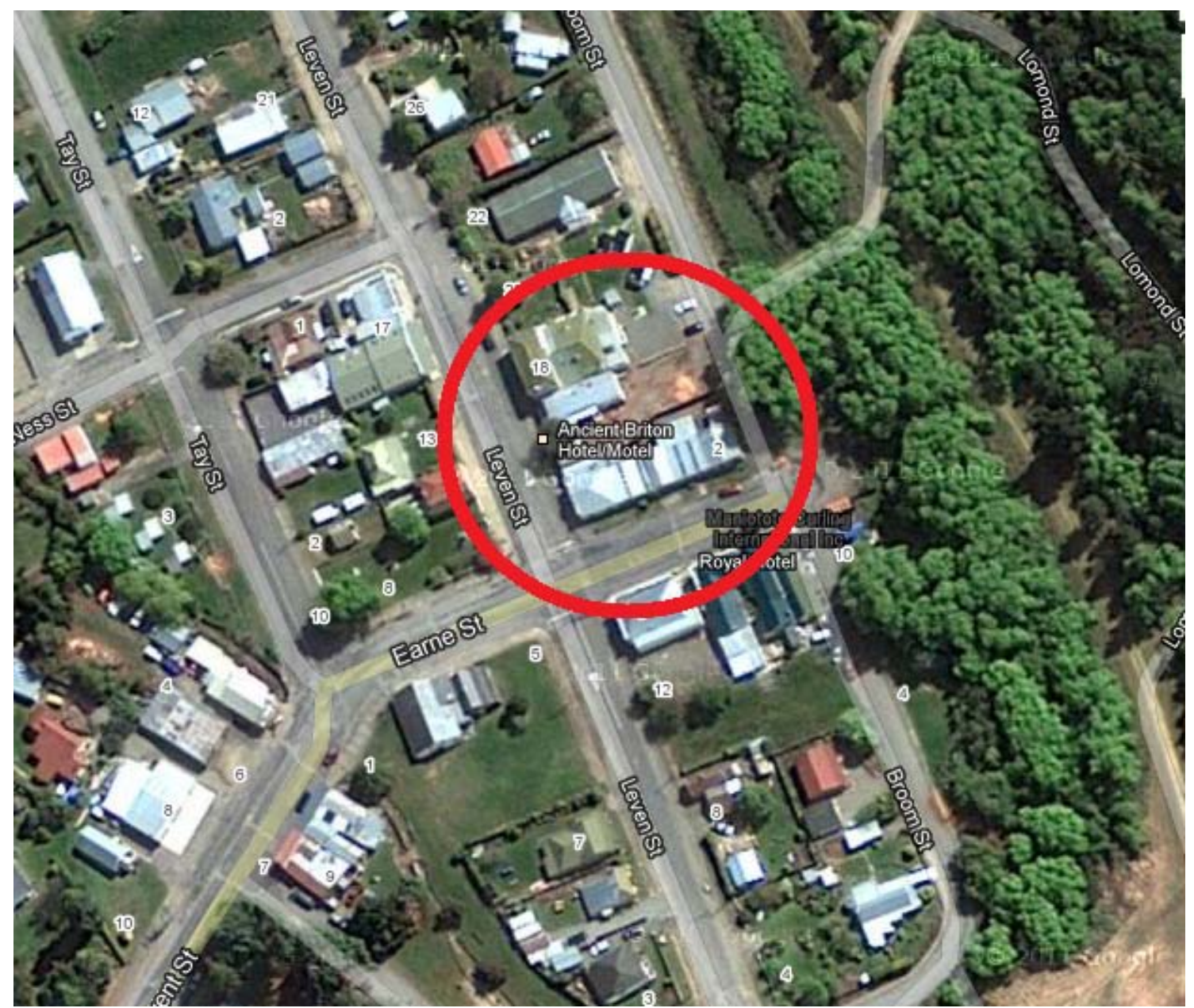

Figure 6.4: Ancient Briton Hotel, location in Naseby

Google map viewed 2012 


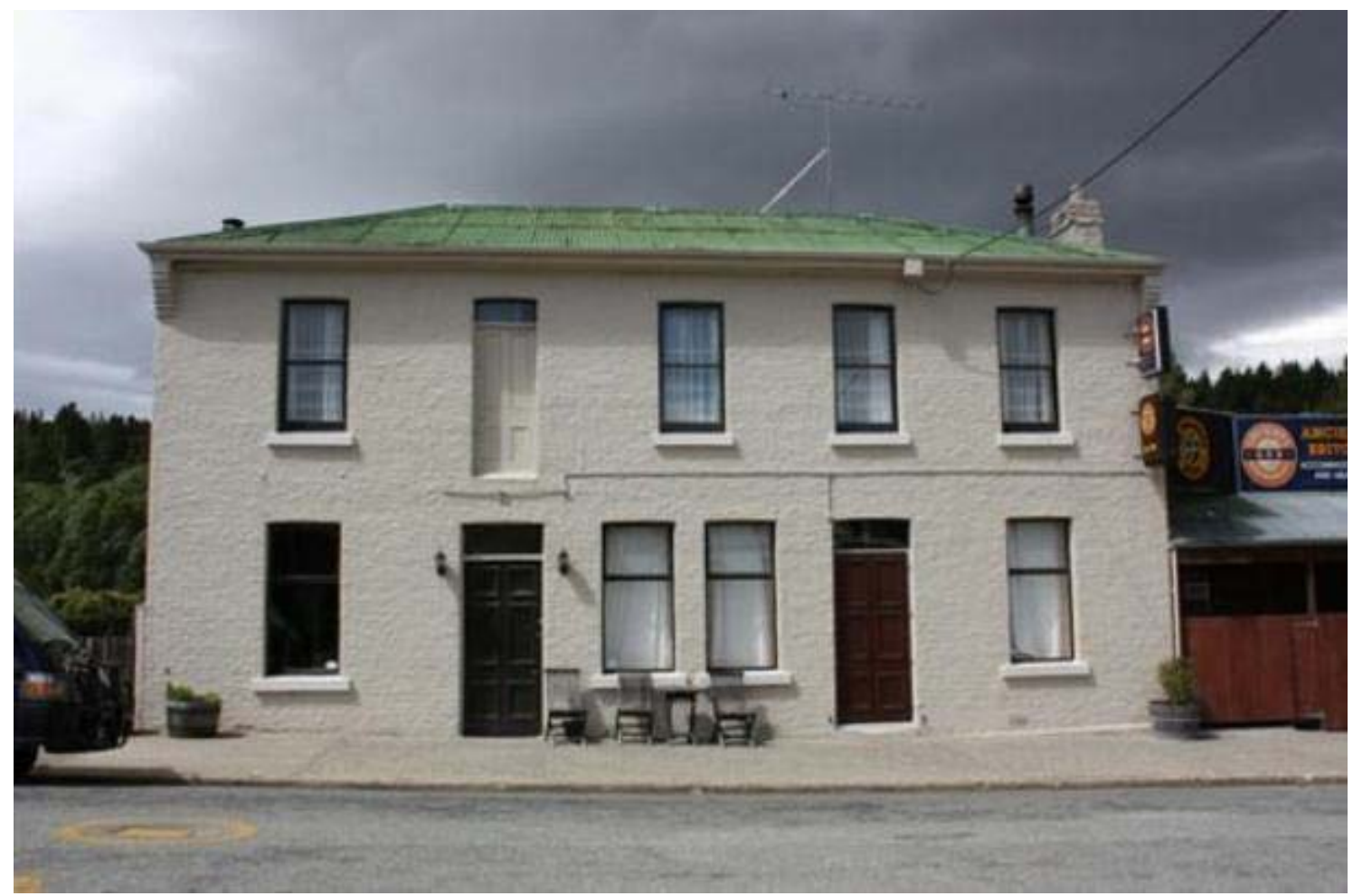

Figure 6.5: Ancient Briton Hotel, Naseby- 2012

http://www.historic.org.nz/corporate/registersearch/Register/data/3214a_lg.jpg viewed August 2012

Tourism is a major component of the local economy at Naseby, as for other places located along the OCRT. As explained by the NCP (2010:19) many people visit Naseby for recreation and sport. The former includes forest walks, heritage, township and goldfield remnants and the ambience of peace and quiet, while the latter include the more active pursuits of curling, ice skating, luge, and cycling/mountain biking . The growth of the OCRT is reflected in an increased number of visitors passing through Naseby. The town has traditionally been a holiday spot at regional scale for domestic visitors; especially those coming to Naseby for curling and mountain biking at this place (see Figures 6.6, 6.7 and 6.8).

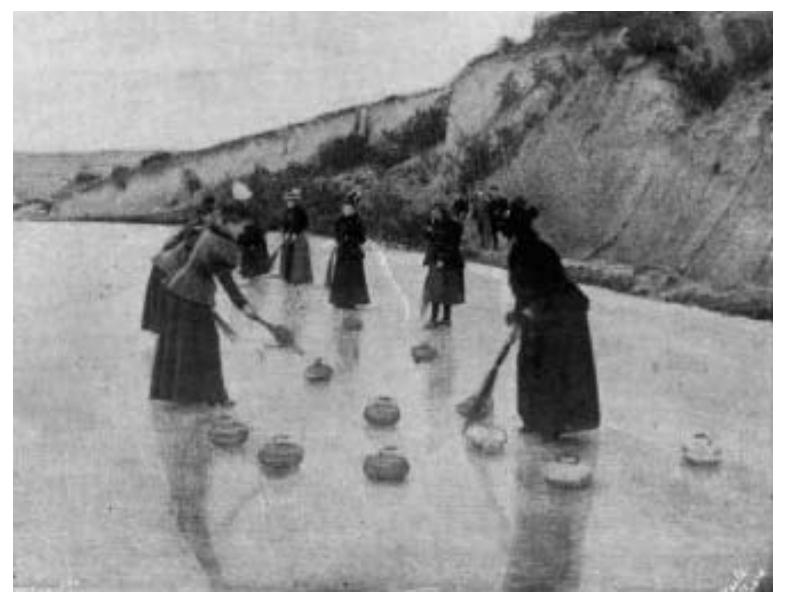

Figure 6.6: Ladies' curling rink of the Mount Ida Curling Club, Naseby, built in 1878

1. http://nzetc.victoria.ac.nz/etexts/Cyc04Cyc 1/Cyc04Cycl0606a(h280).jpg viewed August 2012

2. http://www.ancientbriton.co.nz/curlin g.htm viewed August 2012 


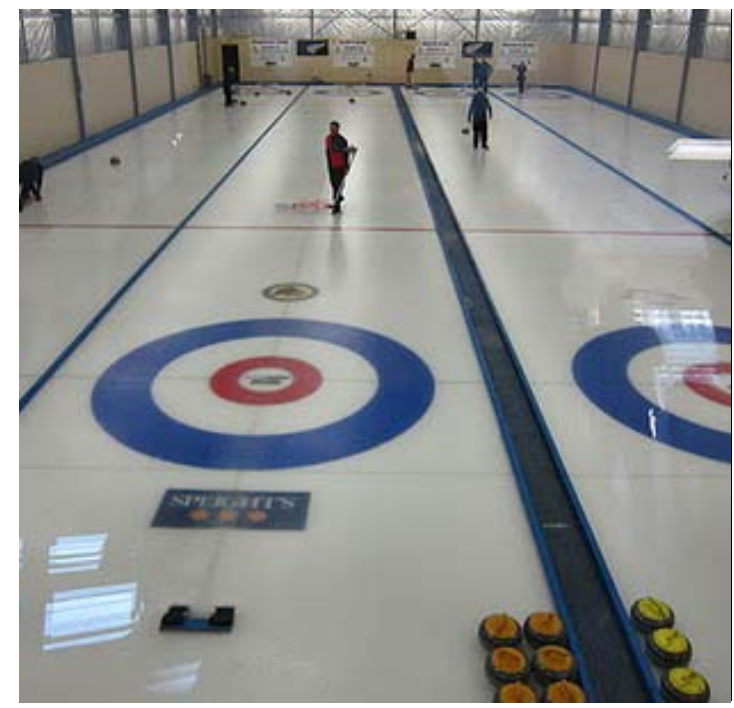

Figure 6.7: Naseby's indoor curling rink http://4.bp.blogspot.com/_HwUk2QL7wc/TDrrWj5 dZpI/AAAAAAAABZc/Y9ytVNHfe0/s400/curling _wide.jpg viewed August 2012

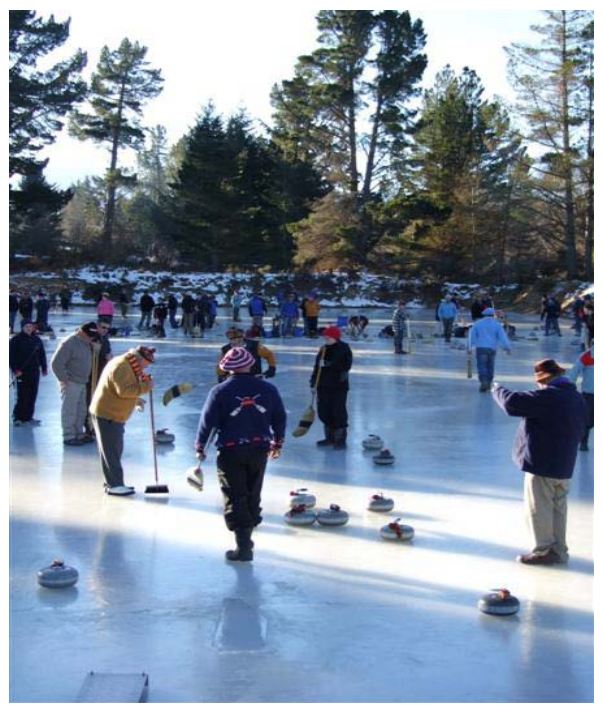

Figure 6.8: Naseby outdoor curling competition (2010) http://www.odt.co.nz/files/story/2010/07/competi tion_for_the baxter_cup_was hot_yesterday d 44 c399d2244.JPG viewed August 2012

Moreover as NCP (2010:28) points out, Naseby has access to the adjacent forest including a 500ha recreation area of mostly exotic trees, 50km of walking/cycling tracks (see Figures 6.9 and 6.10), picnic areas, a children's playground, historic gold mining sites and two dams for fishing and swimming. Due to Naseby's attractions, such as ambience, heritage and recreation, this place is offered by the OCRT to its visitors as a distinctive destination along the trail to stay for relaxing times, recreational activities, enjoying scenery and study of a part of New Zealand's history.

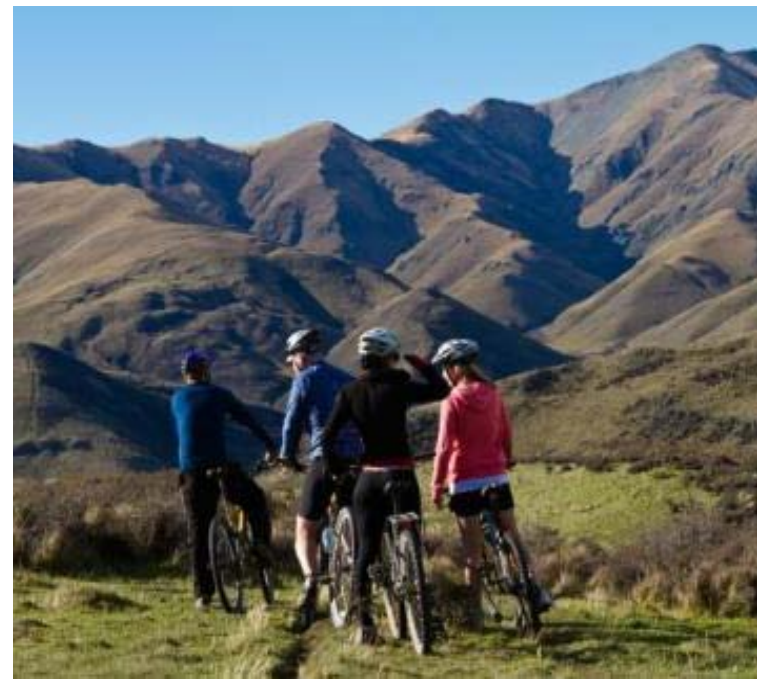

Figure 6.9: Naseby - cycling track http://www.maniototo.co.nz/wpcontent/uploads/20 09/06/Biking-outlook-from-rear-landscapecomp.jpg viewed August 2012

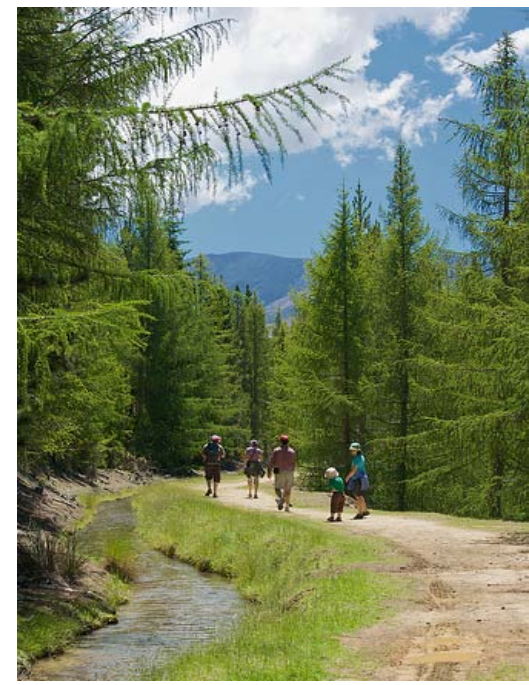

Figure 6.10: Naseby - walking track http://www.lugenz.com/photos/Theres-so-muchto-do-in-Naseby/Walk\%20the\%20forest.jpg viewed August 2012 
However, as NCP (2010:19) points out, an important question is how to best take advantage of the tourism market without negating the natural and cultural values of Naseby itself.

\subsection{3: Visitors to Naseby}

In this thesis Naseby's visitors are classified into the two groups of international and domestic visitors. This section (6.1.3) presents information related to each group, including the number of visitors, visitor nights and their home places.

\subsubsection{1: Visitor Numbers}

As shown in Table 5.49 (Chapter 5- 5.7.4) in 2011 of 11,788 OCRT visitors 36.9\% $(4,350)$ played curling at Naseby and 2.5\% (295) came to Naseby for mountain biking. In the absence of any other data, this study assumes these 4,645 visitors are the total number of visitors who stopped at Naseby in 2011, as shown in Table 6.1. Moreover, as shown in Figure 5.7 (Chapter 5) in 2011 the average stay of OCRT visitors is 3.6 nights. So the total visitor nights at Naseby based on its 4,645 visitors and 3.6 average visitor nights is assumed here to be 16,722 (4,645 visitors $\times 3.6$ visitor nights) (Table 6.1).

\begin{tabular}{|l|l|l|l|l|l|l|l|}
\hline \multicolumn{7}{|c|}{ Table 6.1: Naseby visitors - based on CODC survey (2011) } \\
\hline $\begin{array}{l}\text { Total num- } \\
\text { bers of } \\
\text { OCRT visi- } \\
\text { tors }\end{array}$ & $\begin{array}{l}\text { \% of OCRT } \\
\text { visitors who } \\
\text { play curling } \\
\text { at Naseby }\end{array}$ & $\begin{array}{l}\text { Number } \\
\text { OCRT visi- } \\
\text { tors who } \\
\text { play curl- } \\
\text { ing at } \\
\text { Naseby }\end{array}$ & $\begin{array}{l}\text { visitors } \\
\text { who go } \\
\text { mountain } \\
\text { biking at } \\
\text { Naseby }\end{array}$ & $\begin{array}{l}\text { Number } \\
\text { OCRT visi- } \\
\text { tors who go } \\
\text { mountain } \\
\text { biking at } \\
\text { Naseby }\end{array}$ & $\begin{array}{l}\text { Total } \\
\text { Naseby } \\
\text { visitors }\end{array}$ & $\begin{array}{l}\text { Average } \\
\text { visitor } \\
\text { nights }\end{array}$ & $\begin{array}{l}\text { Total } \\
\text { visitor } \\
\text { nights }\end{array}$ \\
\hline 11,788 & 36.9 & 4,350 & 2.5 & 295 & 4,645 & 3.6 & 16,722 \\
\hline
\end{tabular}

\subsubsection{2: Visitors' Home Place}

As illustrated in Figure 5.13 (Chapter 5: 5.3.3) 78\% of the total 11,788 OCRT visitors are domestic and $22 \%$ are international. This thesis uses these proportions to calculate the number of Naseby international and domestic visitors as shown in Table 6.2.

Table 6.2: Naseby international and domestic visitors (2011)

\begin{tabular}{|c|c|c|c|}
\hline \multicolumn{3}{|c|}{ Domestic visitors } & \multicolumn{2}{c|}{ International visitors } \\
\hline Number of visitors & $\begin{array}{l}\text { \% of total number of visi- } \\
\text { tors }\end{array}$ & Number of visitors & $\begin{array}{l}\% \text { of total number of visi- } \\
\text { tors }\end{array}$ \\
\hline 3,623 & \multicolumn{1}{|c|}{78} & 1,022 & 22 \\
\hline - Naseby total visitors $(2011)=4,645$ (see Table 6.1)
\end{tabular}




\section{a. International Visitors}

This thesis uses the percentage of OCRT international visitors cited in Table 5.1 (Chapter 5: section 5.3.3.1) and the right hand column in Table 6.3 as a base to calculate the number of international visitors to Naseby in 2011. As presented in Table 6.3, of the 1,022 international visitors, the majority of 436 visitors came from Europe (excluding the UK) followed by 336 (32.9\%) Australia and 129 (12.6\%) UK. The numbers of other international visitors including those from North America, Middle East and Africa are in the range of $14(1.4 \%)$ - 92 (9\%) (Table 6.3).

\begin{tabular}{|l|c|c|}
\hline \multicolumn{3}{|c|}{ Table 6.3: Naseby - International Visitors -2011 } \\
\hline Home place & NV$^{1}$ & $\%$ \\
\hline Europe & 436 & 42.7 \\
\hline Australia & 336 & 32.9 \\
\hline UK & 129 & 12.6 \\
\hline North America & 92 & 9 \\
\hline Middle East & 14 & 1.4 \\
\hline South Africa & 14 & 1.4 \\
\hline Total & $1,021 \sim 1,022^{2}$ & 100 \\
\hline $\begin{array}{l}\text { 1. NV: Number of Visitors. } \\
\text { 2. NVs are rounded } \\
\text { • Total visitor numbers (2011) = 4,645 (See Table 6.2) } \\
\text { • Percentage of International visitors (2011)=22\% (See Table 6.2) } \\
\text { • Total number of Naseby international visitors=1,022 (See Table 6.2) }\end{array}$ \\
\hline
\end{tabular}

\section{b. Domestic Visitors}

This thesis uses the percentages of OCRT domestic visitors cited in Table 5.3 (Chapter 5: section 5.3.3.2) and Table 6.4 (right hand column) to calculate the number of domestic visitors in 2011. As shown in Table 6.4, of Naseby’s 3,623 domestic visitors, the majority of 1,297 (35.8\%) are from upper NI, followed by 824 (22.75\%) central SI, 768 (21.2\%) upper SI, 540 (14.9\%) lower NI, and 194 (5.35\%) lower SI.

\begin{tabular}{|l|c|c|}
\hline \multicolumn{2}{|c|}{ Table 6.4: Naseby- Number of domestic visitors by home place } \\
\hline Region/ city & Number of visitors & $\%$ \\
\hline Upper NI & 1,297 & 35.8 \\
\hline Lower NI & 540 & 14.9 \\
\hline Upper SI & 768 & 21.2 \\
\hline Central SI (Otago) & 824 & 22.75 \\
\hline Lower SI & 194 & 5.35 \\
\hline Total & 3,623 & 100 \\
\hline
\end{tabular}




\subsubsection{Transportation}

This section explores the types of international and domestic transportation used by Naseby's visitors and calculates their EFs. The total EF of Naseby transportation is calculated through integration of the EFs of international and domestic visitors.

\subsubsection{International Transportation}

\section{a. Types of International Transportation Used}

Since travelling from overseas to New Zealand by car, bus and train is not possible because of its geographical location and using a ship takes a long time, all international travel is here assumed to be by air.

\section{b. International Tourism-kilometres (T-km)}

Table 6.5 shows that 1,022 Naseby visitors (22\%) are international tourists and the total distance between their home places and Auckland's international airport is equivalent to 12,616,876 (T-km). International distances are calculated as a one way trip because the assumption is made here that their ecological footprint is related to where the plane is refueled and hence is divided between New Zealand and the home country. Because international tourists have to travel from Auckland to Dunedin and back, this part of the journey is a domestic flight and is equivalent to 2,201,246 T-km which is shown in Table 6.5 as air domestic international visitors.

\begin{tabular}{|c|c|c|c|c|c|c|c|}
\hline \multirow{3}{*}{$\begin{array}{l}\text { Home } \\
\text { place }\end{array}$} & \multirow[b]{3}{*}{$\begin{array}{l}\text { Assumed } \\
\text { city of } \\
\text { origin } \\
\text { (central in } \\
\text { country of } \\
\text { origin) }\end{array}$} & \multirow[b]{3}{*}{$\begin{array}{c}\text { Distance } \\
\text { between } \\
\text { central } \\
\text { cities and } \\
\text { Auckland } \\
(\mathrm{km}) .\end{array}$} & \multirow{2}{*}{\multicolumn{2}{|c|}{ Visitors }} & & \multirow{3}{*}{$\begin{array}{l}\text { Auckland to } \\
\text { Dunedin } \\
\text { (km, return) }\end{array}$} & \multirow[b]{3}{*}{$\begin{array}{c}\text { Air domestic } \\
\text { international } \\
\text { visitors T-km } \\
\text { from Auckland } \\
\text { to Dunedin (re- } \\
\text { turn) }\end{array}$} \\
\hline & & & & & \multirow{2}{*}{$\begin{array}{l}\text { Total in- } \\
\text { ternational } \\
\text { T-km }\end{array}$} & & \\
\hline & & & No & $\%$ & & & \\
\hline Australia & $\begin{array}{l}\text { Sydney } \\
\text { and Mel- } \\
\text { bourne }\end{array}$ & 2,512 & 336 & 32.9 & 844,032 & 2,156 & 724,416 \\
\hline Europe & Frankfurt & 18,180 & 436 & 42.7 & $7,926,480$ & 2,156 & 940,016 \\
\hline UK & London & 18,334 & 129 & 12.6 & $2,365,086$ & 2,156 & 278,124 \\
\hline $\begin{array}{c}\text { North } \\
\text { America }\end{array}$ & $\begin{array}{c}\text { Montana } \\
\text { Billings }\end{array}$ & 11,950 & 92 & 9 & $1,099,400$ & 2,156 & 198,352 \\
\hline $\begin{array}{c}\text { Middle } \\
\text { East }\end{array}$ & Tehran & 15,005 & 14 & 1.4 & 210,070 & 2,156 & 30,184 \\
\hline $\begin{array}{l}\text { South } \\
\text { Africa }\end{array}$ & Pretoria & 12,272 & 14 & 1.4 & 171,808 & 2,156 & 30,154 \\
\hline Total & - & - & 1,022 & 100 & $12,616,876$ & - & $2,201,246$ \\
\hline
\end{tabular}




\subsubsection{Types of Domestic Transportation}

The types and percentages of transport used for holidays in New Zealand as cited in Chapter 5 and shown in Table 5.5 (Matthews, 2009:13) are used as a pattern for transport used by domestic visitors to access Naseby.

\section{a. Domestic Tourism Kilometres (T-km)}

This study uses Auckland as the central city for the upper NI and, respectively, Wellington (lower NI), Nelson/Marlborough (upper SI), Otago (central SI) and Southland (lower SI), to calculate the distances between domestic visitors' home place and Naseby (Table 6.6).In addition, to measure the distance between home places of visitors who came from the upper SI, the average distance between Nelson and Naseby and Marlborough and Naseby is used (Table 6.6).

Naseby's visitors who travel by car/van from the upper NI (Auckland) and lower NI (Wellington) (grey areas in Table 6.6) need to use the ferry to cross to the South Island. Distances between these places and Middlemarch are separated into three parts; home to Wellington (except Wellington); Wellington to Picton by ferry; and Picton to Middlemarch (Table 6.6).

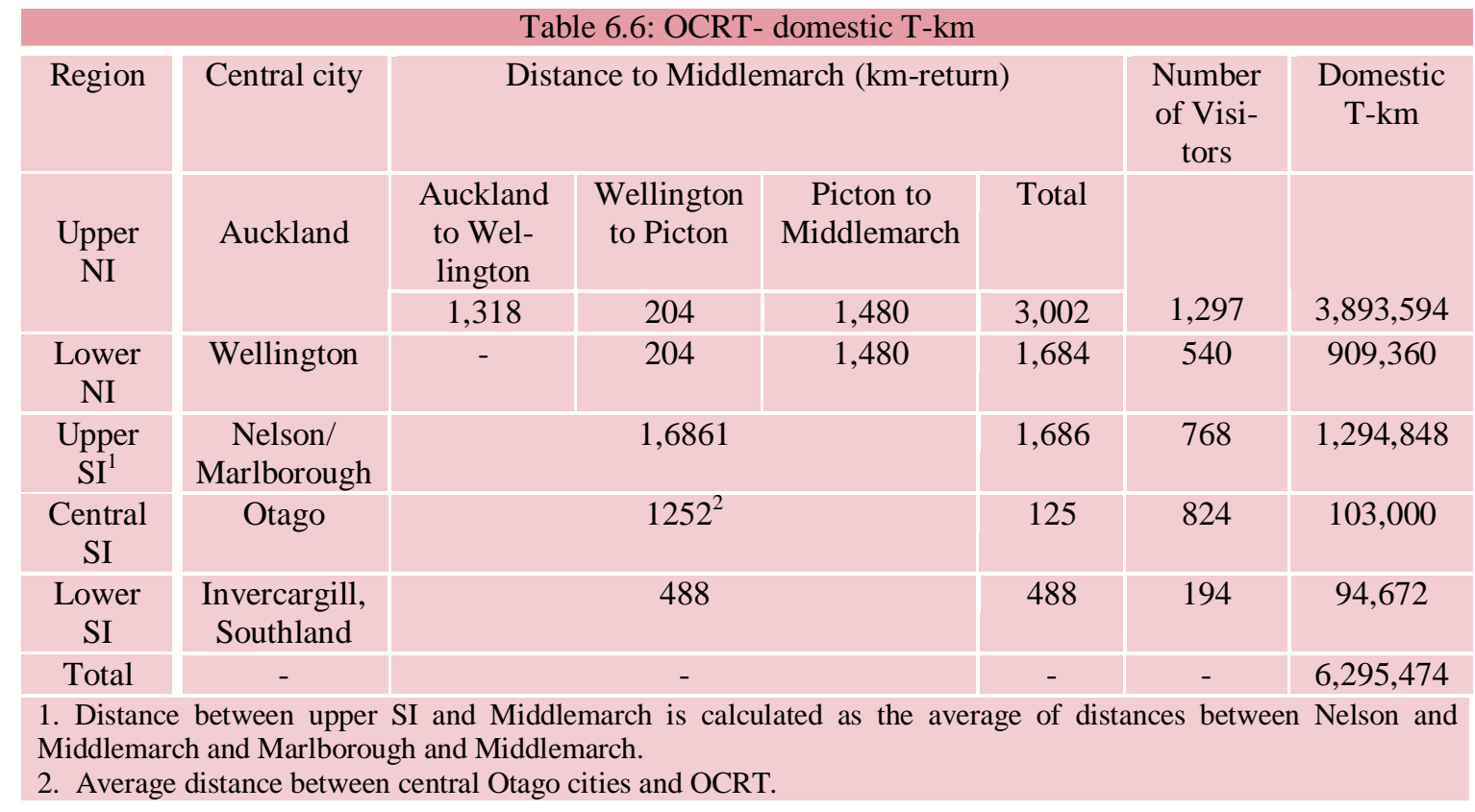

According to Matthews (2009:13), in New Zealand 1\% of people going on holiday are pedestrians but in this study these have been ignored. The remaining $3 \%$ of visitors is assumed to be domestic air travel in Table 6.7. In addition, the ferry T-km of 377,728 
(Table 6.7) is subtracted from the total car/van T-km of 5,980,700 to give the road T$\mathrm{km}$.

Table 6.7: Types of transport used by OCRT domestic visitors (based on Matthews, 2009:13)

\begin{tabular}{|c|c|c|c|c|}
\hline Type of Transport & Car/van driver/passenger (\%) & $\begin{array}{c}\text { Bus } \\
(\%)\end{array}$ & $\begin{array}{c}\text { Car-Ferry } \\
(\%)\end{array}$ & Air domestic (\%) \\
\hline $\begin{array}{c}\text { percent } \\
\text { Type of Transport }\end{array}$ & $\begin{array}{c}89 \\
2\end{array}$ & 6 & 3 \\
\hline Tourist-km & $5,602,972$ & $\begin{array}{c}\text { Bus } \\
(\mathrm{km})\end{array}$ & $\begin{array}{c}\text { Ferry } \\
(\mathrm{km})\end{array}$ & Air domestic (km) \\
\hline $\begin{array}{c}\text { Total domestic T- } \\
\text { km }\end{array}$ & 125,909 & 377,728 & 188,864 \\
\hline - Numbers are rounded & $6,295,474$ & & \\
\hline
\end{tabular}

\subsubsection{The Ecological Footprint (EF) of Naseby Transportation}

Table 6.8 shows that total transportation energy used related to Naseby (2011) is equivalent to 45,963,318 MJ (45,963.318GJ). The global average land energy conversion capacity is equivalent to $100 \mathrm{GJ} / \mathrm{gha}$. As demonstrated in Table 6.8 the total EF of Naseby transportation is equivalent to 45,963.318 GJ/ $100 \mathrm{GJ} / \mathrm{gha}=459.6$ gha.

\begin{tabular}{|c|c|c|c|}
\hline \multicolumn{4}{|c|}{ Table 6.8: EF of Naseby Transportation (2011) } \\
\hline Transport Mode & Tourist-km & $\begin{array}{l}\text { Energy use } \\
\text { MJ/passenger-km }\end{array}$ & $\begin{array}{l}\text { Total energy use per yr } \\
\text { by OCRT visitors (MJ) }\end{array}$ \\
\hline Air International & $12,616,876$ & 1.25 (Boeing 747) & $15,771,095$ \\
\hline $\begin{array}{c}\text { Air domestic } \\
\text { (International visitors from } \\
\text { Auckland to Dunedin) }\end{array}$ & $2,201,246$ & 3.88 & $8,540,834$ \\
\hline Air domestic & 188,864 & 3.88 & 732,792 \\
\hline Car/van driver and passenger & $5,602,972$ & 3.1 (average) & 17369213 \\
\hline Bus & 125,909 & 1.01 & 127,168 \\
\hline Car-ferry & 377,728 & 9.06 & 3422216 \\
\hline Total & & $\begin{array}{c}\text { Reference: Vale \& Vale, } \\
2009 .\end{array}$ & $45,963,318$ \\
\hline \multicolumn{3}{|c|}{ EF for all types of transportation used by Naseby visitors } & 459.6 gha $^{1}$ \\
\hline \multicolumn{4}{|c|}{$\begin{array}{l}\text { 1. Total EF of Naseby transportation includes: } 243.1 \text { gha ( EF of international transportation) }+216.5 \text { gha (EF of } \\
\text { domestic transportation) }\end{array}$} \\
\hline
\end{tabular}

Table 6.8 shows the total energy use of Naseby transportation (45,963.318GJ) that includes 24,311.929 GJ for air international and air domestic (international visitors from Auckland to Dunedin) and 21,651.389 GJ for domestic transportations. As a result, the total EF of Naseby transportation (459.6gha) is comprised of $52.9 \%$ (243.1 gha) international and 47.1\% (216.5 gha) domestic transportation. 


\subsubsection{Food}

As shown in Table 6.1, 4,645 visitors came to Naseby in 2011 and the mean number of nights that tourists stayed is assumed to be 3.6. Thus the total visitor nights for tourists coming to Naseby/yr are 16,722 visitor nights. Due to the lack of local information, the food consumption data presented by Collins et al (2005) are used to calculate the EF of food for Naseby's visitors.

This thesis uses two scenarios to calculate the EF of food consumed by Naseby visitors in 2011. In the first scenario, the EF of food is calculated based on the assumption that $100 \%$ of food consumed is conventional food eaten out. In the second scenario, the total food eaten by Naseby visitors is separated into the two areas of local (home prepared) food (11.8\% of total consumed food) and conventional food (88.2\% of total consumed food).

Comparison between the results of the two scenarios will indicate the influence exerted by the use of home prepared food (as a cultural product) on the EF and the economic footprint (ECF) of the OCRT.

\subsubsection{The EF of Naseby Food- First scenario}

In the first scenario, the total amount of food eaten by Naseby visitors has been assumed to have a footprint equivalent to that of food consumed outside the home by Cardiff residents. This totals $67.04 \mathrm{~kg}$ per resident/yr (Collins et al, 2005:25) with a footprint of 0.429gha/resident (Collins et al, 2005:32). This means $1 \mathrm{~kg}$ of food eaten out has an EF of $0.429 / 67.04 \mathrm{gha} / \mathrm{kg}=0.006 \mathrm{gha} / \mathrm{kg}$. The average amount of food eaten by a Cardiff resident each day is $1.85 \mathrm{~kg}$, and this value has been used for visitors to Naseby. The results are shown in Table 6.9. The EF of tourist food is equivalent to $1.85 \times 0.006=0.01$ gha/visitor night (Table 6.9). The total EF of food consumed by Naseby's visitors is equivalent to $16,722 \times 0.01=167.22$ gha and the EF of food consumed by Nasby's visitors/visitor is equivalent to $167.22 / 4,645=0.036$ gha (Table 6.9). 
Table 6.9: EF of food consumed by Naseby visitors (2011)

\begin{tabular}{|l|c|c|}
\hline \multicolumn{1}{|c|}{ Item } & Data & Reference \\
\hline Naseby visitor numbers (2011) & 4,645 (See Table 6.2) \\
\hline Average visitor nights/ visitor & 3.6 & \\
\hline Total visitor night & 16,722 & Collins et al , 2005:32 \\
\hline $\begin{array}{l}\text { EF of food eaten out gha/cap } \\
\text { EF of tourist food gha/visitor } \\
\text { night }\end{array}$ & 0.429 & \\
\hline $\begin{array}{l}\text { Total EF of food consumed by } \\
\text { Naseby visitors (gha) }\end{array}$ & 0.01 & \\
\hline $\begin{array}{l}\text { EF of food consumed by Naseby } \\
\text { visitors/visitor(gha) }\end{array}$ & 0.036 (gha/visitor) \\
\hline
\end{tabular}

\subsubsection{The EF of Naseby Food- Second Scenario}

This thesis explores the local products (such as food, beverages and wine) offered to Naseby visitors by 98 separate accommodation services with 611 available bed spaces. (Appendix 5- Rows 75-90, Naseby). The information related to Naseby products arising from Appendix 5, are summarised and set out in Table 6.10.

Table 6.10 demonstrates that in 2011, of the 98 separate Naseby accommodation services with 611 bed spaces that offered local produce, 10.2\% (10) with 72 bed spaces offered home baking and local food and beverages to their visitors and 2\% (2) with 27 bed spaces were offering locally produced wine. As shown in Figure 5.3, the OCRT average visitor nights is 3.6 visitor nights. Likewise, the total Naseby visitors are 4,645 visitors. Thus the total visitor nights of Naseby is (3.6 visitor nights $\times 4,645$ visitors) 16,722. Since the total Naseby bed spaces are 611, the average visitor nights per bed space is equivalent to 16,722 (visitor nights) $\div 611$ (bed spaces) $=27.4$ (visitor nights/ bed space).Consequently as shown in Table 6.10 the total visitor nights that Naseby visitors consume local food is equivalent to $72 \times 27.5=1,973$ visitor nights or $11.8 \%$ of the total 16,722 Naseby visitor nights (Table 6.10).

\begin{tabular}{|c|c|c|c|c|c|}
\hline \multicolumn{6}{|c|}{ Table 6.10. Naseby- local produce (summary) } \\
\hline Produce & $\mathrm{F}$ & $\%$ & $\begin{array}{c}\% \text { of } 98 \text { accommoda- } \\
\text { tions services }\end{array}$ & Bed spaces & Visitor nights $^{1}$ \\
\hline Local food and beverage & 10 & 83 & 10.2 & 72 & $1,973^{2}$ \\
\hline Wine & 2 & 17 & 2 & 27 & 740 \\
\hline Total & 12 & 100 & 12.2 & & \\
\hline
\end{tabular}

This thesis uses the EFs of home cooked and conventional foods (per capita, per kg and per visitor night) as discussed in Chapter 5 (section.5.5.2 and Tables 5.11 and 5.12) to 
calculate the EFs of home cooked and conventional foods consumed by Naseby's visitors as cited in the following Table

Table 6.11 indicates that the EF of local food consumed by Naseby visitors in 2011 is equivalent to $(1,973$ visitor nights $\times 0.0068$ gha $) 13.4$ gha. As the total Naseby visitor nights is 16,722 (see Table 6.1) and total visitor nights that visitors consume home cooked food is 1,973 , consequently the total visitor nights that Naseby's visitors consume conventional food is equivalent to $16,722-1,973=14,749$ visitor nights (see Table 6.11). Moreover the EF of consumed conventional food by Naseby visitors in 2011 is equivalent to 14,749 visitor nights $\times 0.01$ (EF of tourist conventional food- gha/visitor night $)=147.5$ gha $($ Table 6.11). The total EF of consumed food in the second scenario (present circumstance) including EFs of conventional and home prepared foods is 13.4 gha +147.5 gha $=160.9$ gha $($ Table 6.11).

Table 6.11: Ecological footprint of home cooked and conventional foods consumed by Naseby visitors (2011)

\begin{tabular}{|c|c|c|c|}
\hline Home cooked and local food & $\mathrm{EF}$ & Conventional food & $\mathrm{EF}$ \\
\hline EF of local food gha/cap & 0.254 & $\begin{array}{l}\text { EF of } 1 \mathrm{~kg} \text { conventional food eaten out } \\
\text { (gha/cap) }\end{array}$ & 0.429 \\
\hline EF of $1 \mathrm{~kg}$ local food gha/kg & 0.0037 & EF of tourist food (gha/visitor night) & 0.01 \\
\hline EF of local food gha/ visitor night & 0.0068 & & \\
\hline $\begin{array}{l}\text { EF of local food of } 1,973 \text { visitor } \\
\text { nights (gha) }\end{array}$ & 13.4 & $\begin{array}{l}\text { Total EF of conventional food consumed } \\
\text { by Naseby visitors } / 14,749^{1} \text { visitor nights } \\
\text { (gha) }\end{array}$ & 147.5 \\
\hline \multicolumn{3}{|c|}{ Total EF of home prepared and conventional foods (gha) } & 160.9 \\
\hline
\end{tabular}

\subsubsection{Environmental Effects of Using Local Food}

Comparing the results of the two scenarios shows that producing $11.8 \%(3,650 \mathrm{~kg})$ of food locally reduces the total EF of food by 6.32 gha (3.8\% of 167.22 gha) (Table 6.12).

\begin{tabular}{|c|c|c|}
\hline & $\begin{array}{l}100 \% \text { ( } 30,936 \mathrm{~kg} \text { ) con- } \\
\text { ventional eaten out food }\end{array}$ & $\begin{array}{c}88.2 \%(27,286 \mathrm{~kg}) \text { conventional eaten } \\
\text { out food and } 11.8 \%(3,650 \mathrm{~kg}) \text { home } \\
\text { prepared (organic) food }\end{array}$ \\
\hline Naseby EF of food & 167.22 & 160.9 \\
\hline $\begin{array}{l}\text { Reduction in EF of food con- } \\
\text { sumed by OCRT visitors (gha) }\end{array}$ & & 6.32 \\
\hline
\end{tabular}




\subsubsection{Naseby Accommodation Services}

\subsubsection{Types of Accommodation Services}

This thesis categorises the 98 Naseby accommodation services into the four categories of self-contained (SC), bed and breakfast $(\mathrm{B} \& \mathrm{~B})$, hotel, and camping based on the information collected through using the official web site of the OCRT and 15 related websites for Naseby accommodation services (Appendix 5 and 6-Rows:75-90). Table 6.13 summarises parts of Appendix 5 that address available bed spaces by types of Naseby accommodation service.

As shown in Table 6.13 and Figure 6.11, in 2011, of the 98 separate Naseby accommodation services the majority of $73.5 \%$ are camping sites followed by $18.4 \%$ SCs, $6.1 \%$ B\&Bs, and 2\% hotels, as shown in Table 6.13 and Figure 6.11. This table also shows how the percentages change when bed space numbers are considered by accommodation type. Camping still has the highest percentage of bed spaces followed by SC, as before, but the relative positions of hotels and $B \& B$ have changed.

\begin{tabular}{|l|c|c|c|c|}
\hline \multicolumn{1}{|c|}{ Type } & \multicolumn{2}{|c|}{ Table 6.13: Naseby- types of accommodation services } & Available bed spaces \\
\hline Sumber of & $\%$ & 93 & 15.2 \\
\hline BC & 18 & 18.4 & 28 & 4.6 \\
\hline Hotel & 6 & 6.1 & 58 & 9.5 \\
\hline Camping & 2 & 2 & 432 & 70.7 \\
\hline Total & 72 & 73.5 & 611 & \\
\hline
\end{tabular}

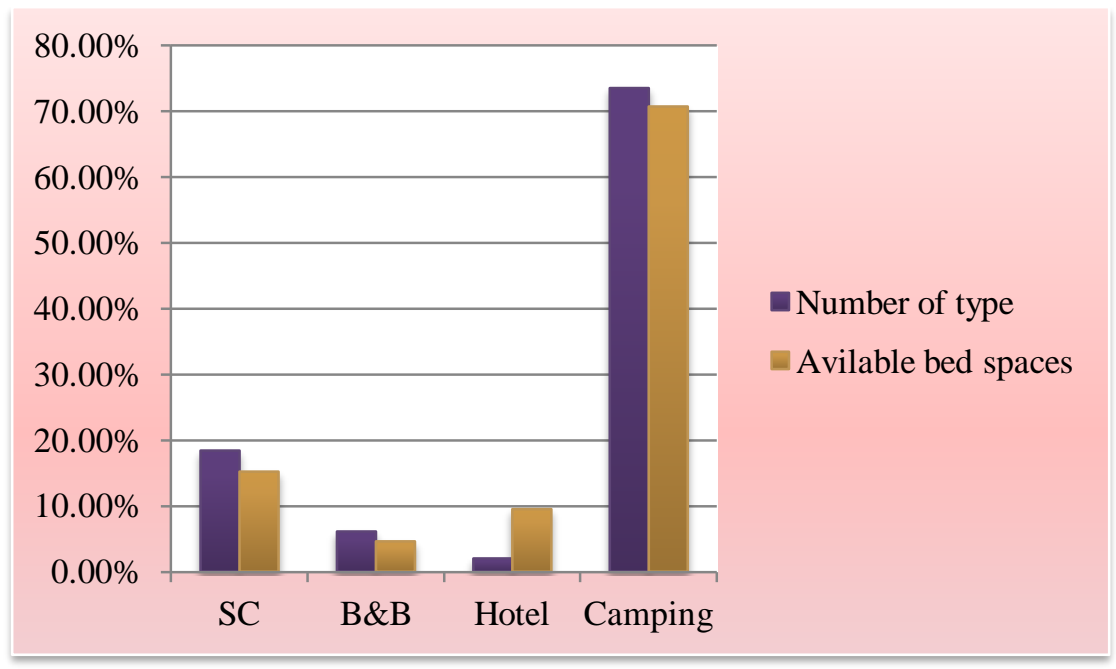

Figure 6.11: Naseby- percentage of type and available bed spaces by types of accommodation services (2011) 


\subsubsection{Quality of Accommodation Services}

According to the information shown in Appendix 5 and summarised in Table 6.14 this study qualitatively classifies Naseby's accommodation services into the two types of new (NB) and refurbished (RB) buildings. In Naseby, RB accommodation services cover a range of historical and restored buildings with different original functions, but currently all of them are used as accommodation services. Likewise new buildings here are those that have been constructed after the establishment of the OCRT and are used as accommodation services.

\begin{tabular}{|c|c|c|c|c|c|c|c|c|c|c|}
\hline \multirow[t]{3}{*}{ Type } & \multirow[t]{3}{*}{ TNA } & \multirow[t]{3}{*}{$\%^{1}$} & \multirow[t]{3}{*}{$\mathrm{RB}$} & \multirow[t]{3}{*}{$\%^{1}$} & \multirow[t]{3}{*}{ NB } & \multirow[t]{3}{*}{$\%$} & \multicolumn{4}{|c|}{ Capacity } \\
\hline & & & & & & & \multicolumn{2}{|c|}{$\mathrm{RB}$} & \multicolumn{2}{|c|}{ NB } \\
\hline & & & & & & & $\mathrm{C}$ & $\%^{2}$ & $\mathrm{C}$ & $\%^{2}$ \\
\hline SC & 18 & 18.4 & 3 & 3.1 & 15 & 15.3 & 10 & 1.6 & 83 & 13.6 \\
\hline B\&B & 6 & 6.1 & 1 & 1 & 5 & 5.1 & 5 & 0.8 & 23 & 3.8 \\
\hline Hotel & 2 & 2 & 2 & 2 & - & - & 58 & 9.5 & - & - \\
\hline Camping & 72 & 73.5 & - & & 72 & 73.5 & - & - & 432 & 70.7 \\
\hline Total & 98 & 100 & 6 & 6.1 & 92 & 93.9 & 73 & 11.9 & 538 & 88.1 \\
\hline \multicolumn{11}{|c|}{$\begin{array}{l}\text { 1. The percentages of the number of renewed/historic and new constructed buildings are calculated based on total num- } \\
\text { ber of Naseby accommodation services ( } \mathrm{n}=98) \text {. } \\
\text { 2. The percentages of the capacities of renewed/historic and new constructed buildings are calculated based on total ca- } \\
\text { pacity of Naseby accommodation services ( } 611 \text { bed spaces). } \\
\text { - TNA= Total Number of Accommodation Services Types. } \\
\text { - RB= Refurbished Buildings used as accommodation services. } \\
\text { - NB= New Buildings used as accommodation services. } \\
\text { - } \mathrm{C}=\text { Capacity }\end{array}$} \\
\hline
\end{tabular}

Table 6.14 and Figure 6.12 show that, of 98 Naseby accommodation services, $6.1 \%$ (6) including 3.1\% (3) SC, 1\% (1) B\&B and 2\% (2) hotels are RB and $93.9 \%$ including 15.3\% (15) SCs, 5.1\% (5) B\&Bs and 73.5\% (72) camping sites are NB. 


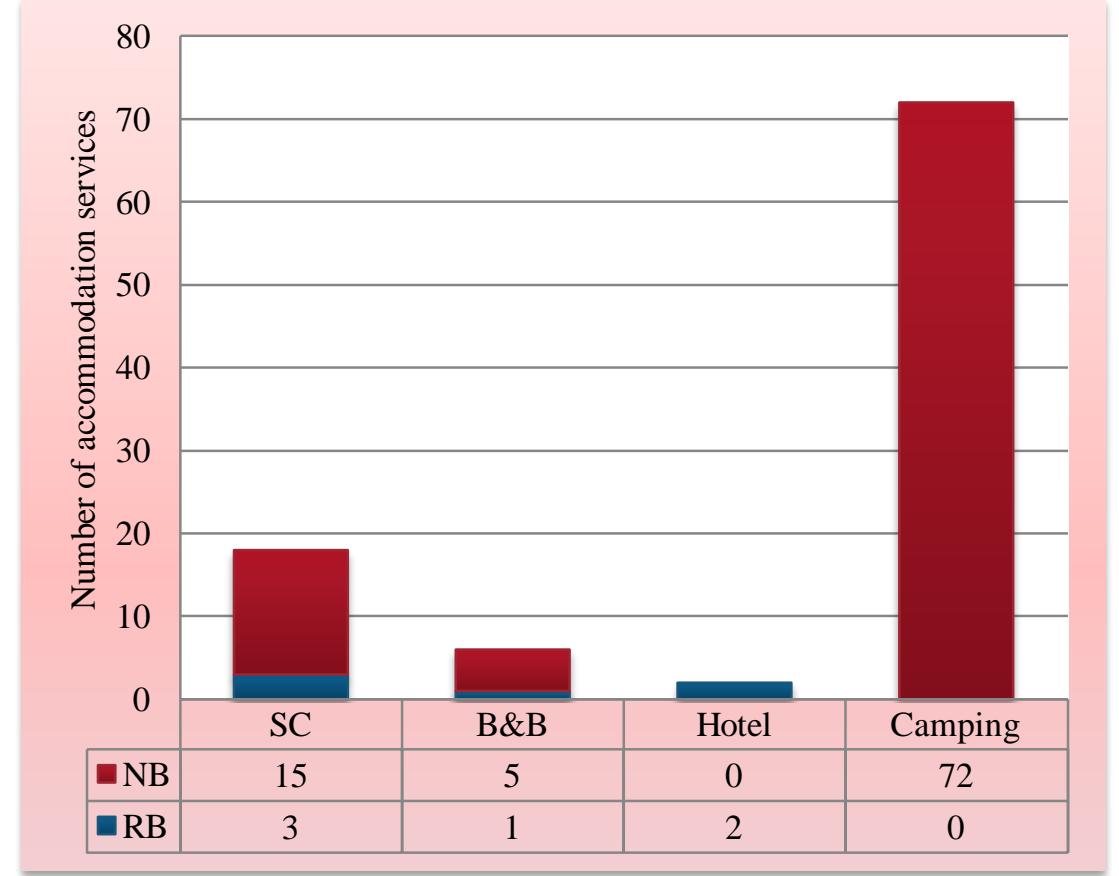

Figure 6.12: Naseby- Refurbished (RB) and new (NB) accommodation services (2011)

Table 6.14 and Figure 6.13 determine that $11.9 \%$ of 611 available bed spaces including 1.6\% (10) SCs, 0.8\% (5) B\&Bs and (58) 9.5\% hotels are RB and 88.1\% including 13.6\% (83) SCs, 3.8\% (23) B\&Bs and 70.7\% (432) camping sites are NB.

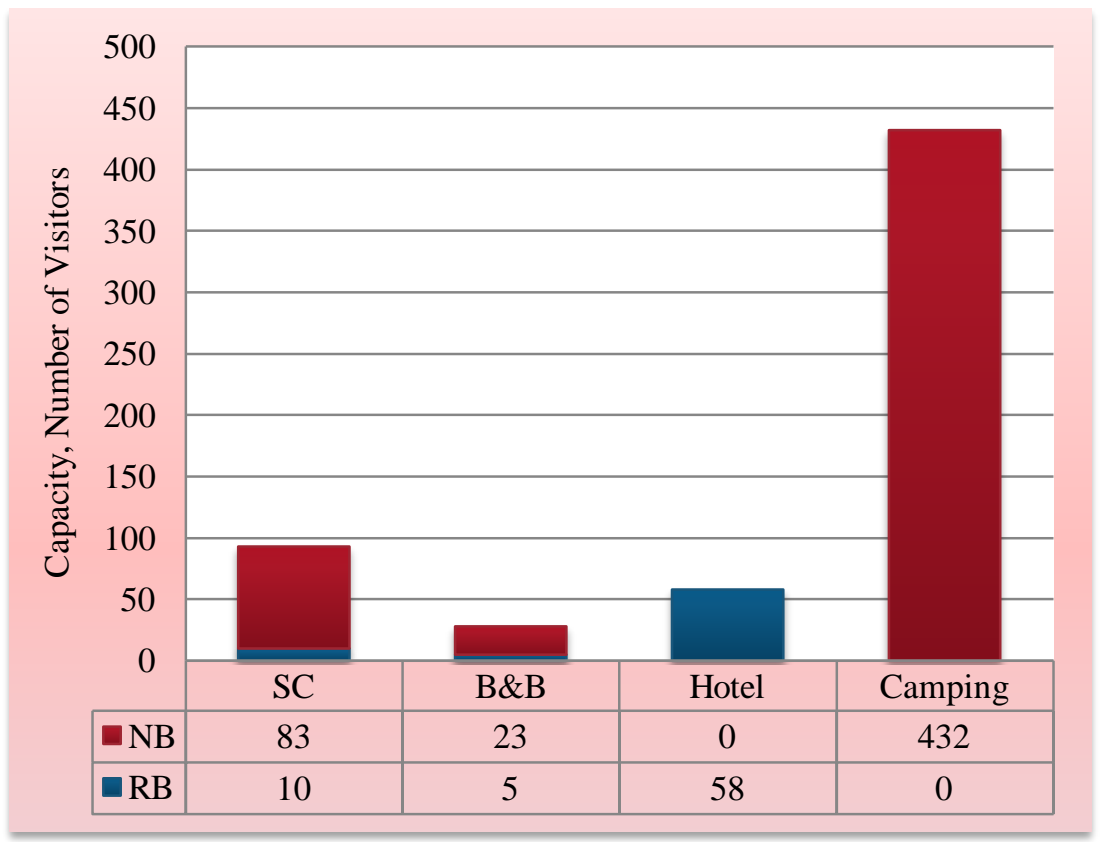

Figure 6.13: Naseby- capacity of RB and NB accommodation services 2011 (total bed spaces $=611$ ) 


\subsubsection{Materials Used for Accommodation Services}

In this thesis the materials used for Naseby accommodation services are explored through using relevant information published in the 15 official websites of Naseby OCRT accommodation services (Appendix 5). The main materials used for constructing each type of accommodation service in 2011 (except camping sites) are set out in Appendix 5 and the summarised information is shown in Table 6.15, and Figures 6.14 and 6.15 .

\begin{tabular}{|c|c|c|c|c|c|c|c|c|}
\hline \multirow[b]{3}{*}{ material(s) } & \multirow[t]{3}{*}{ ied spaces } & \multirow[t]{3}{*}{ ́ } & \multicolumn{6}{|c|}{ Type of accommodation service } \\
\hline & & & \multicolumn{2}{|l|}{ SC } & \multicolumn{2}{|l|}{ B\&B } & \multicolumn{2}{|l|}{ Iotel } \\
\hline & & & No & $\mathrm{C}$ & No & $\mathrm{C}$ & No & $\mathrm{C}$ \\
\hline Timber & 114 & 63.7 & 16 & 73 & 11 & 23 & 1 & 18 \\
\hline Mud brick & 8 & 4.5 & 1 & 8 & & & & \\
\hline Mixed brick ${ }^{2}$ and timber & 45 & 25.1 & - & - & 1 & 5 & 1 & 40 \\
\hline $\begin{array}{l}\text { Mixed timber, aluminium fa- } \\
\text { cade and wood joinery }\end{array}$ & 12 & 6.7 & 1 & 12 & - & - & - & - \\
\hline Total bed spaces & 179 & 100 & 18 & 93 & 12 & 28 & 2 & 58 \\
\hline
\end{tabular}

This thesis classifies the types of materials used into the four types of timber, mud brick, mixed brick and timber and mixed timber, aluminium facade and wood joinery (Table 6.15). As shown in this Table and Figure 6.14, of the total 179 Naseby accommodation bed spaces the majority of 114 bed spaces (63.7\%) are timber followed by 45 mixed brick and timber (25.1\%), 12 mixed timber, aluminium facade and wood joinery (6.7\%) and 8 mud brick (4.5\%).

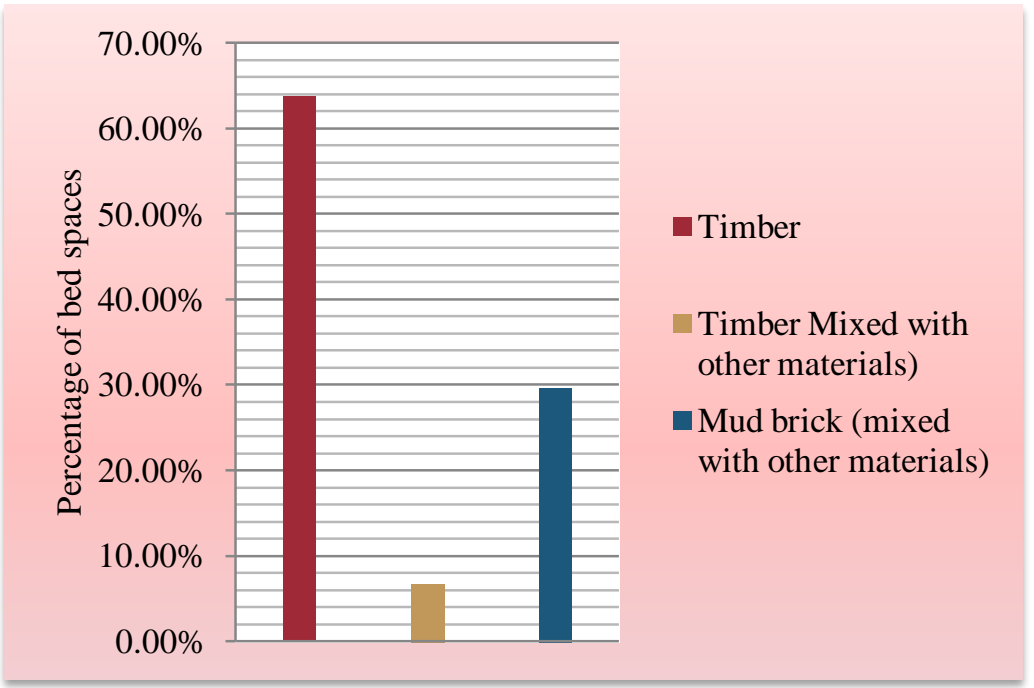

Figure 6.14: Naseby - bed spaces by type of materials used (2011) 
Table 6.15 and Figure 6.15 indicate that the 93 Naseby SC bed spaces comprise 73 (79\%) that use timber as the main construction material followed by 12 (12.9\%) mixed timber with other materials and 8 (8.6\%) mud brick. Furthermore, of 28 Naseby B\&B bed spaces the majority of 23 (82.1\%) use timber as the main material for construction and 5 (17.9 \%) use mud brick and timber. Similarly 40 (69\%) out of 58 Naseby hotel bed spaces are mixed mud brick and 18 (31\%) are timber as the main construction material.

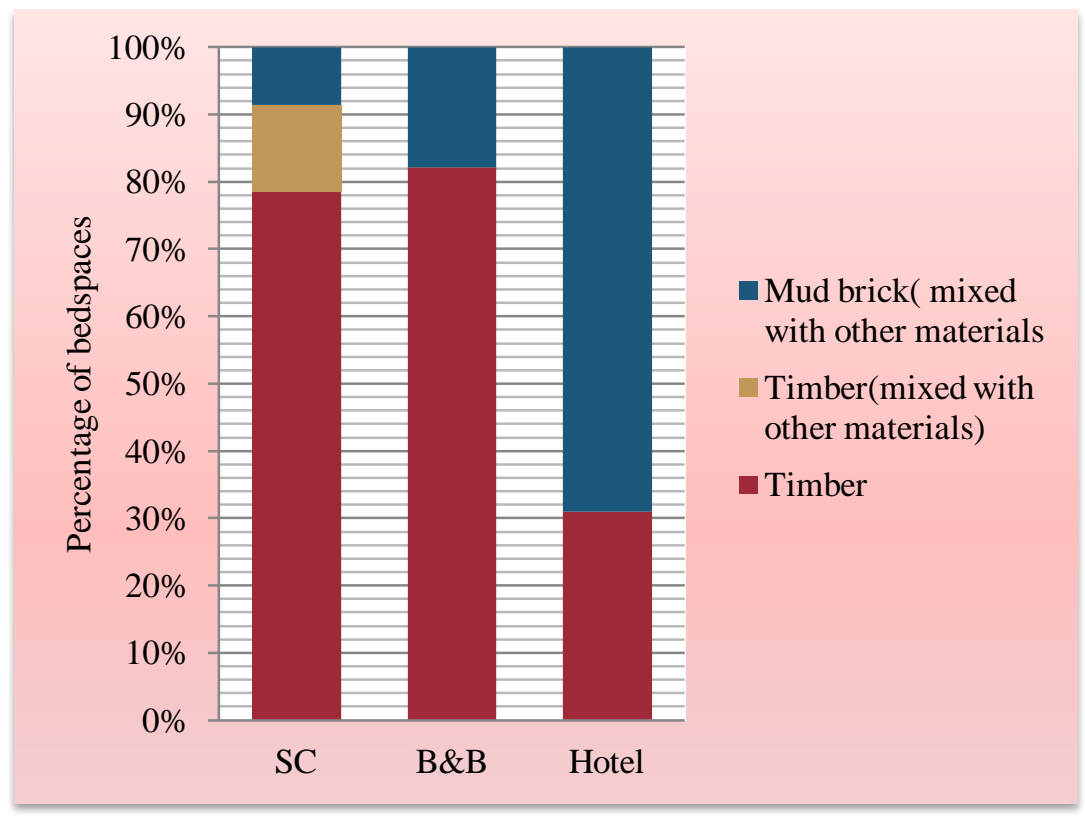

Figure 6.15: Naseby- used materials by types of accommodation services (2011)

\subsubsection{Accommodation Energy Sources, Facilities and Spaces}

As shown in Appendix 5 (Rows 75- 90) this thesis explores the energy sources and facilities used in 98 Naseby accommodation services through using related information published in these accommodation websites. This thesis classifies this information by types of Naseby's accommodation services as shown in Appendix 5 and summarised in Appendices 22-27. In this thesis, all heating systems (electricity, log, gas and solar) are explored by type of accommodation service. Of possible facilities and equipment used, $\mathrm{TV}$, and internet are chosen to be explored, as using these facilities can be considered as indicator for a modernised lifestyle (Appendices 22-27). Since using open air areas has been introduced in this thesis as one of the environmental and cultural indicators for evaluating architecture as being sustainable, verandas, balconies and outdoor seating are also investigated. The following part presents this data classified by type of accommodation service, shown in Appendix16 which summarise Appendices 9-15. 


\section{a. SC Energy Sources, Facilities and Open Air Spaces (2011)}

Figure 6.16 and Appendices 22 and 23 demonstrate that, of 93 SC bed spaces 54 (58.1\%), including 48 (51.6\%) NB and 6 (6.5\%) RB use electricity for heating. In addition, 23 (24.7\%) SC bed spaces including 6 (6.5\%) RB and 17 (18.3\%) NB use logs, and 30 (32.3\%) including 4 (4.3\%) RB and 26 (28\%) NB use gas for heating (Figure 6.16 and Appendix 23).

Likewise, of 93 Naseby SC bed spaces, 49 (52.7\%) use a solar system for heating (water-heating) (Figure 6.16 and Appendix 23). As illustrated in Figure 6.16and shown in Appendix 23, 35 (37.5\%) of 93 Naseby SC bed spaces including 6 (6.5\%) RB and 29 (31\%) NB bed spaces have a shared TV. In addition, 79 (85\%) of Naseby SC bed spaces including10 (10.8\%) RB and 69 (74.2\%) NB have access to the Internet (Figure 6.16and Appendix 23). Furthermore, 6 (6.5\%) Naseby SC bed spaces (NB) can access a shared V/B and 12 (12.9\%) NB SC bed spaces have a private V/B (Figure 6.16 and Appendix 23).

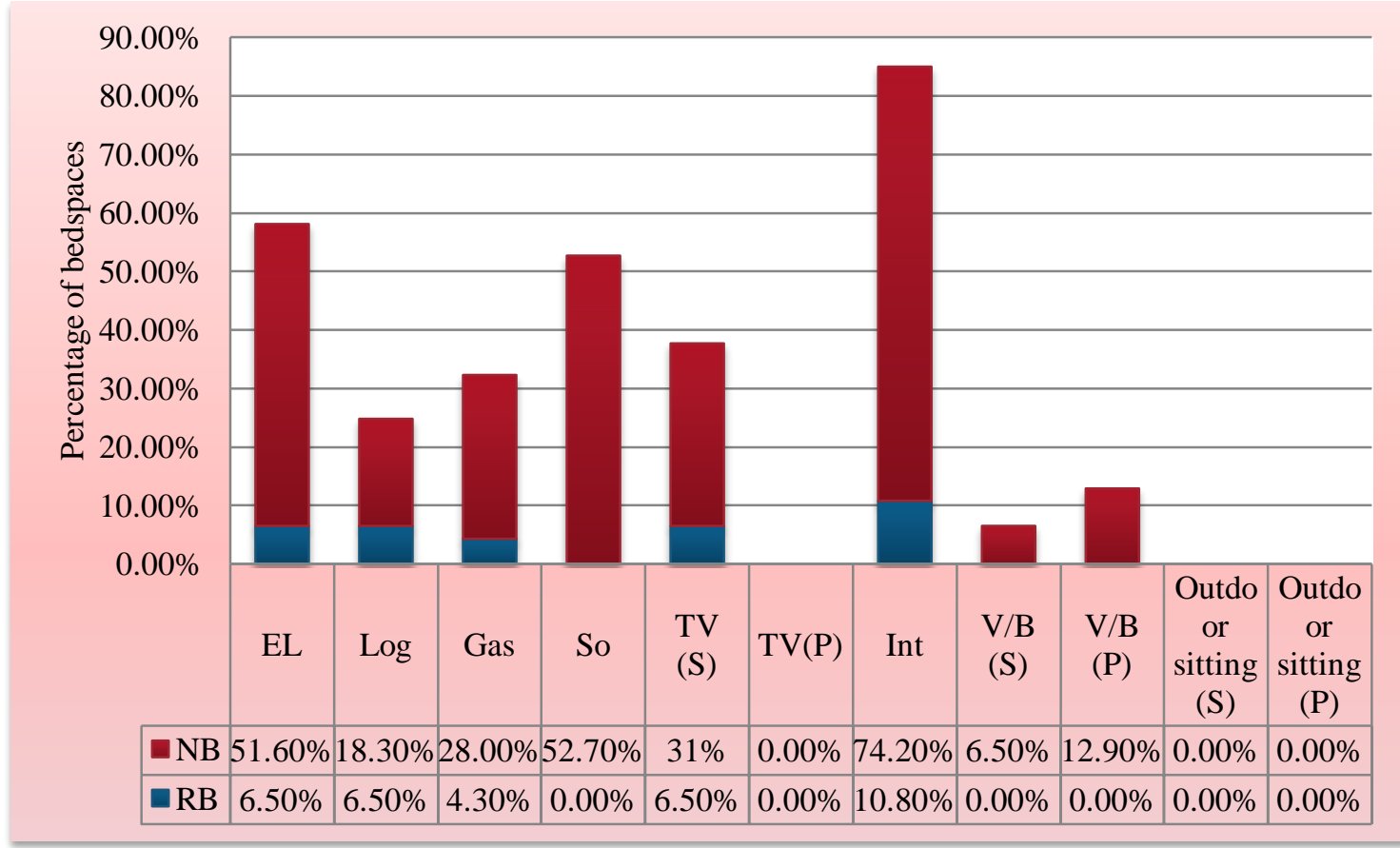

Figure 6.16: Naseby SC accommodation services - energy sources, facilities and spaces (2011)

El: Electricity

So: Solar system

TV (S): Share TV

TV (P): Private TV

Int: Internet

V/B (S): Share veranda/ balcony

V/B (P): Private veranda/balcony

Outdoor sitting (S): Share outdoor sitting

Outdoor sitting (P): Private outdoor sitting 


\section{b. B\&B Energy Sources, Facilities and Open air Spaces (2011)}

Figure 6.17 and Appendices 22 and 24 demonstrate that of 28 B\&B bed spaces 23 (82.1\%) NB and 5 (17.9\%) RB use electricity for heating. In addition, 12 (24.7\%) NB B\&B bed spaces have a solar system for heating (water-heating) (Figure 6.17 and Appendix 24).

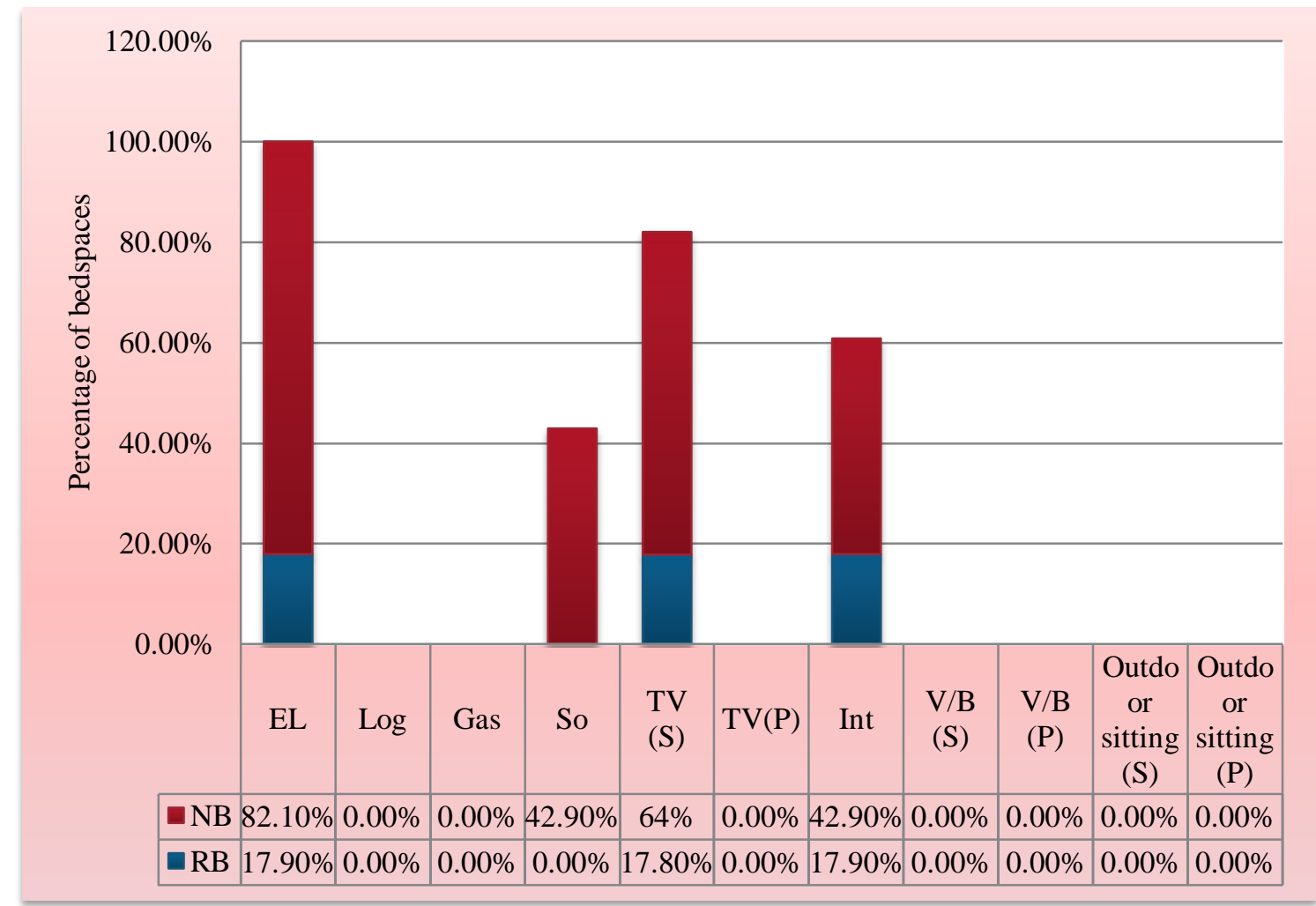

Figure 6.17: Naseby B\&B accommodation services - energy sources, facilities and spaces (2011)

As illustrated in Figure 6.17 and shown in Appendix 24, 23 (82.1 \%) out of 28 NasebyB\&B bed spaces including 5 (17.8\%) RB and 18 (64.3\%) NB bed spaces have a shared TV. Likewise, 17 (60.8\%) of 28 Naseby B\&B bed spaces including 5 (17.85\%) RB and 12 (42.85\%) NB have access to the Internet (Figure 6.17 and Appendix 24).

\section{c. Hotel Energy Sources, Facilities and Open Air Spaces (2011)}

As illustrated in Figure 6.18 and shown in Appendices 22 and 25, all 58 (100\%) Naseby-hotel accommodation services are refurbished buildings and use electricity as the main source for heating. Moreover, 40 (69\%) of these bed spaces also use logs (Figure 6.18 and Appendix 25). Furthermore, 18 (31\%) of 58 hotel bed spaces have a shared TV and 18 (31\%) have outdoor sitting areas (Figure 6.18 and Appendix 25). 


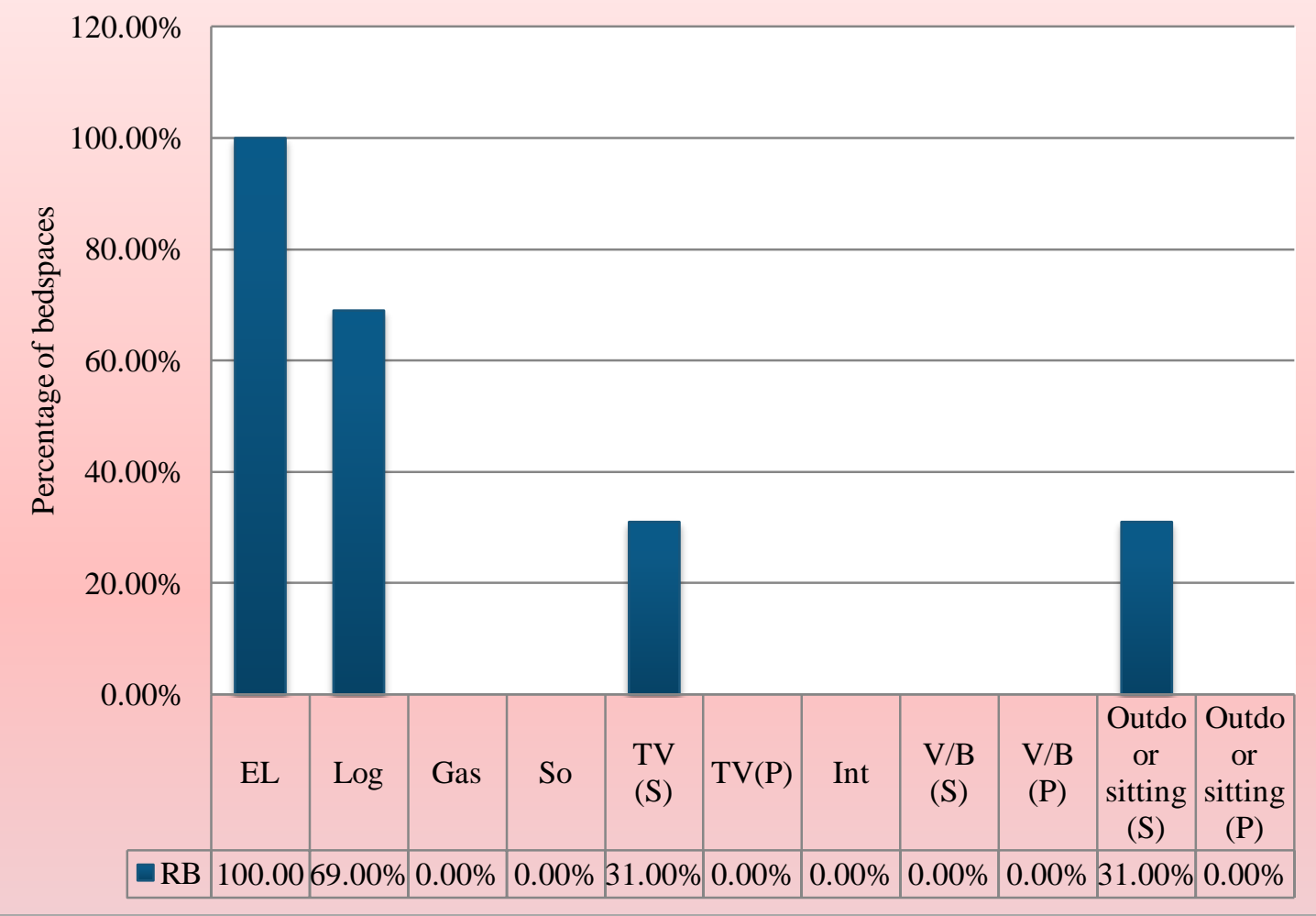

Figure 6.18: Naseby hotel accommodation services - Energy sources, facilities and spaces (2011)

\section{d. Camping Energy Sources, Facilities and Open Air Spaces (2011)}

Figure 6.19and Appendix 26 show that of 432 Naseby camping sites (powered and nonpowered), 288 (66.7\%) powered sites use electricity for heating cabins and caravans, lighting and other services such as a BBQ and also have access to the Internet.

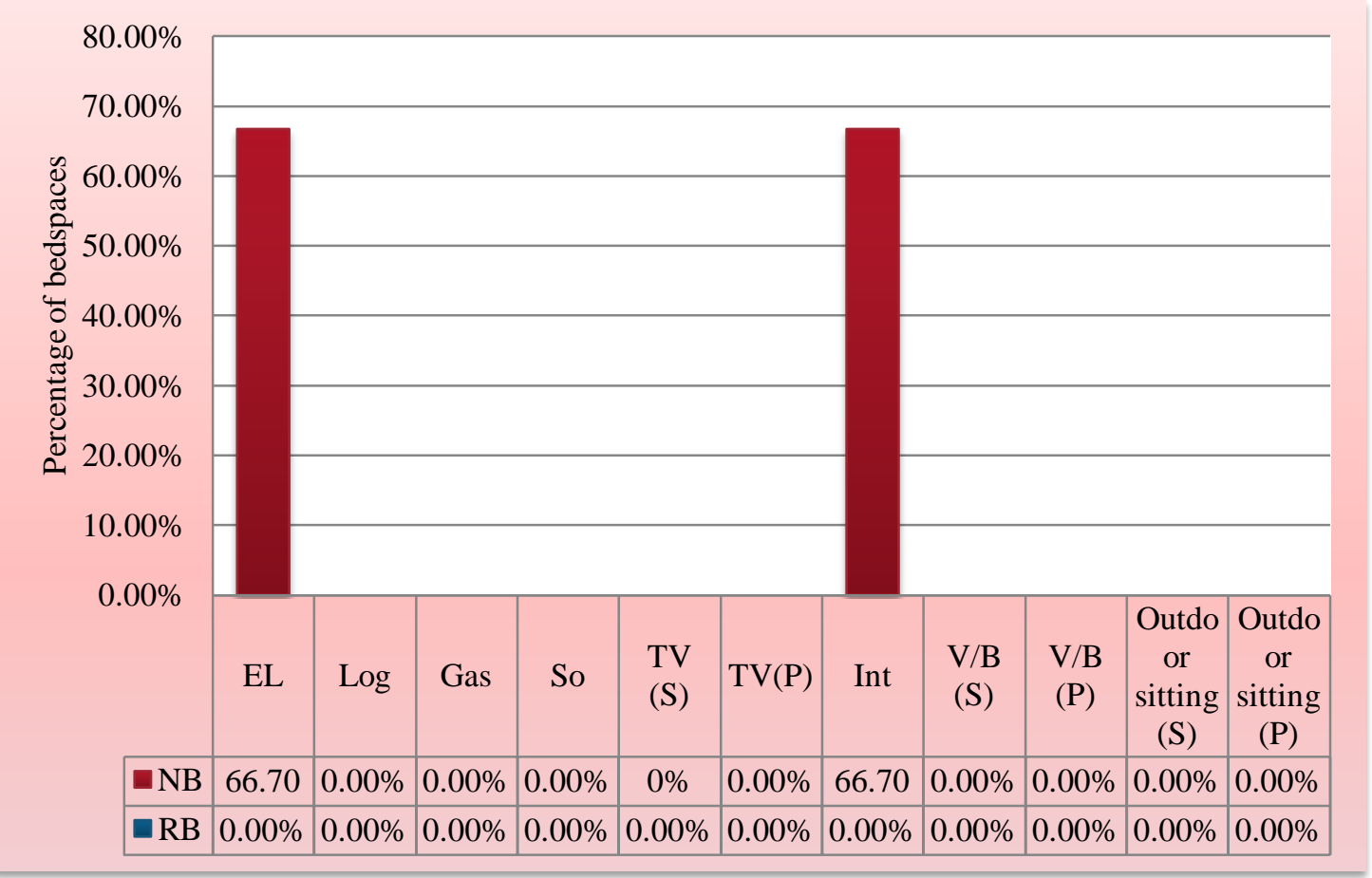

Figure 6.19: Naseby camping accommodation services - energy sources, facilities and spaces (2011) 


\section{e. All Types of Naseby Accommodation Services Energy Sources, Facilities and Open Air Spaces (2011)}

Figure 6.20 and Appendix 27 indicate the energy sources, facilities and open air spaces in all types of Naseby accommodation services. As shown, of 611 Naseby bed spaces 428 bed spaces including 359 (58.70\%) NB and 69 (11.30\%) RB bed spaces use electricity for heating. In addition, of the same 611 Naseby bed spaces, 63 including 17 (2.8\%) NB and 46 (7.5\%) RB use logs (Figure 6.20 and Appendix 27). Furthermore, as shown in Figure 6.20 and Appendix 27, of the 611 Naseby bed spaces 30 (4.9\%) bed spaces including 26 (4.3\%) NB and 4 (0.6 \%) RB use gas, and 61(10\%) NB bed spaces have a solar system for heating water.

Figure 6.20 and Appendix 27 show that 76 (12.4\%) of 611 Naseby bed spaces including 47 (7.7\%) NB and 29 (4.7\%) RB bed spaces have access to a shared TV and 384 (62.8\%) including 369 (60.4\%) NB and 15 (2.4\%) RB rooms have access to the internet. Similarly, of the 611 Naseby bed spaces, 24 including 6 (0.9\%) NB and 18 (3\%) RB have access to a shared outdoor space and 12 (2\%) have a private outdoor area (including veranda, balcony and open air seating).

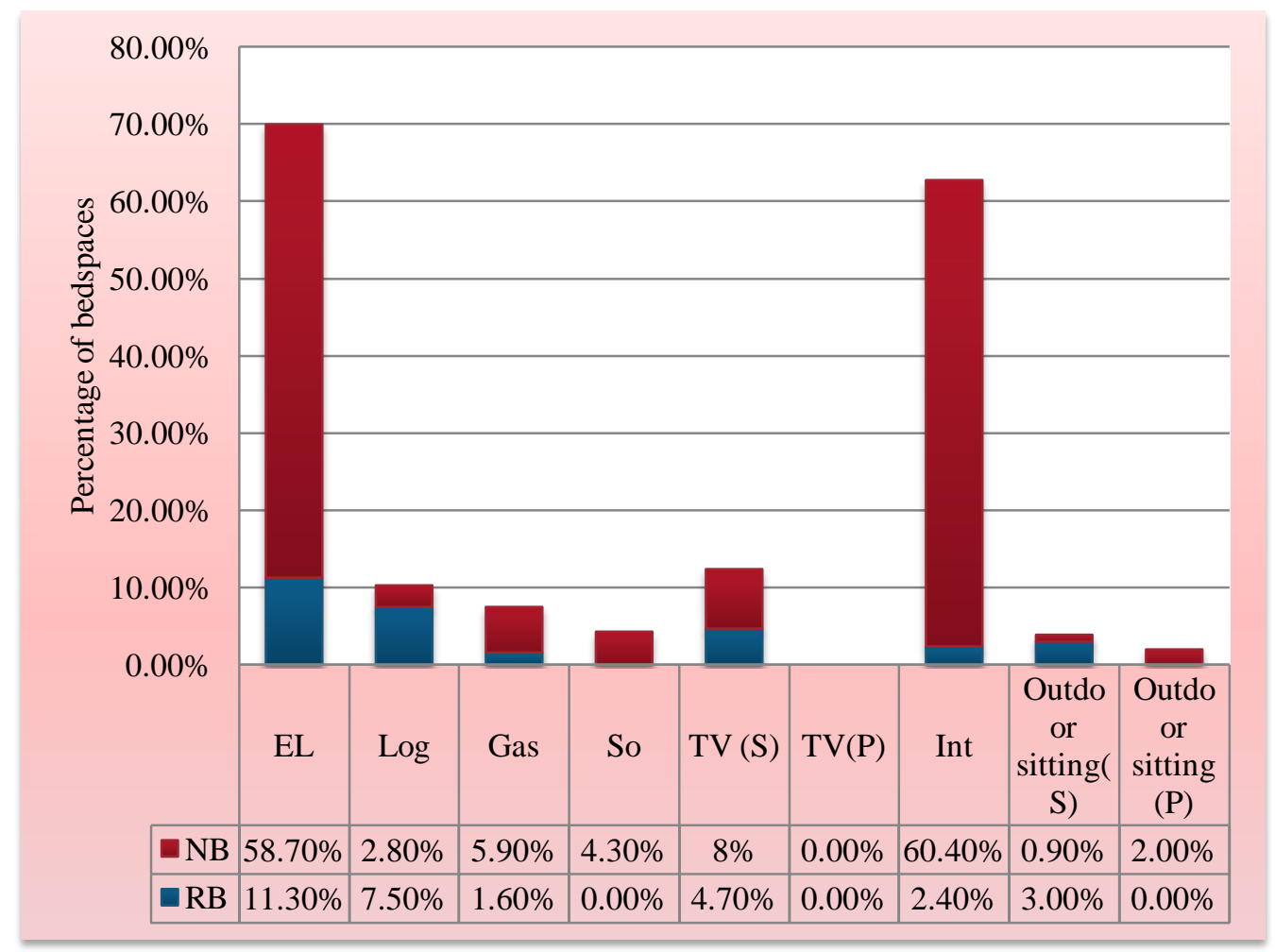

Figure 6.20: All types of Naseby accommodation services - energy sources, facilities and spaces 


\section{f. Comparison between NB and RB Bed Spaces in terms of Energy sources, Facili- ties and Open air spaces (2011)}

Figure 6.21 makes a comparison between 538 (100\%) NB (see Table 6.14) and 73 (100\%) RB (see Table 6.14) bed spaces in terms of energy sources, facilities and open air spaces. As shown, $66.7 \%$ of NB and $94.5 \%$ of RB bed spaces use electricity for heating and 3.1\% of NB and 67.1\% RB bed spaces use logs. Likewise $4.8 \%$ of 538 NB and 5.5\% of $73 \mathrm{RB}$ bed spaces use gas for heating. Figure 6.21 illustrates that the solar heating is used by only $11.3 \%$ of NB bed spaces and RB bed spaces do not use this type of system (Figure 6.21). Furthermore, as shown in Figure 6.21, 8.7\% of NB and 39.7\% of RB bed spaces have a TV and $68.6 \%$ of NB and $20.5 \%$ of RB bed spaces have access to the Internet. Moreover, Figure 6.21 indicates that 3.3\% of NB bed spaces use open air spaces, including veranda/balcony and outdoor seating.

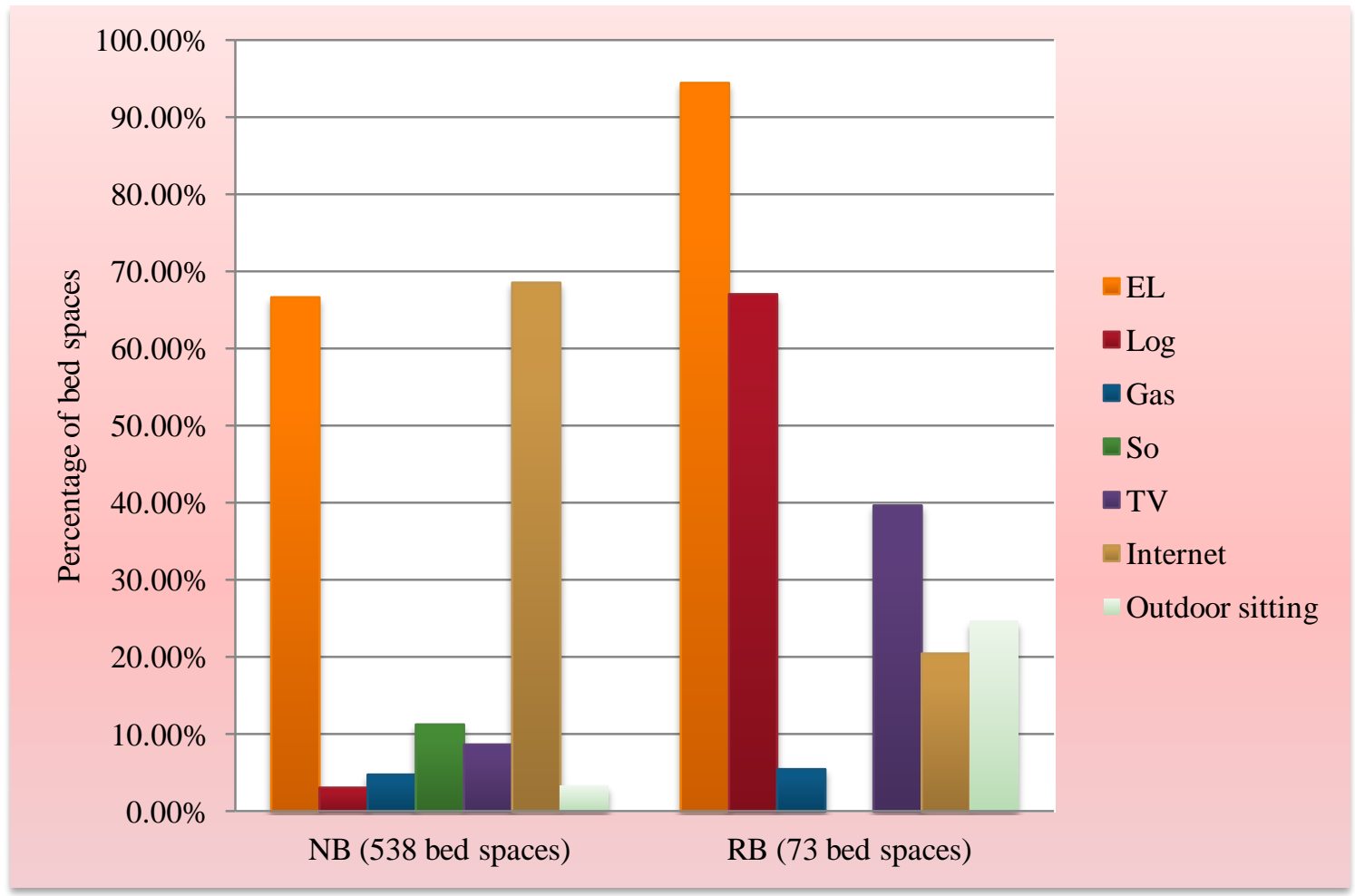

Figure 6.21: Comparison between NB and RB bed spaces in terms of energy sources, facilities and open air spaces (2011)

\subsubsection{Occupancy Share}

This thesis calculates the occupancy share of Naseby accommodation services based on the occupancy share of OCRT accommodation services cited in Table 5.19 (Chapter 5section 5.6.5). Since the types of Bp and homestead accommodation are not found in Naseby, $50 \%$ of the sum of their occupancy share (cited in Table 5.19) has been in- 
cluded in the occupancy share of Naseby SC and the other 50\% has been included in the occupancy rate of Naseby B\&B accommodation. Consequently, the occupancy share of Naseby SC is $13.1 \%$ (occupancy share of OCRT SC accommodation services cited in Table $5.19+8.5 \%$ (50\% of total occupancy share of OCRT Bp and homestead cited in Table 5.19$)=21.6 \%$ (see Table 6.16). Similarly the occupancy of Naseby B\&B is 22.4\% (see Table 6.16).

Since there are no motels at Naseby, its occupancy share as cited in Table 5.19 has been added to the occupancy of Naseby hotels. As a result, the occupancy share of Naseby hotels is $21.1 \%$ (occupancy share of OCRT motels-) $+10.9 \%$ (occupancy share of OCRT-hotels cited in Table 5.19) = 32\% (see Table 6.16).

Table 6.16 comprises the visitor nights of each type of Nasby accommodation service, which are calculated as: occupancy share of each type $\times$ total Naseby visitor nights (16,722 see Table 6.1). As shown in Table 6.16, Naseby hotels have the most visitor nights at 5,351 followed by camping sites $(4,013)$, B\&B $(3,746)$ and SC $(3,612)$.

Table 6.16: Naseby accommodation services- occupancy share (2011)

\begin{tabular}{|c|c|c|}
\hline Type of accommodation service & $\%$ of visitor nights (2005) & Visitor nights (2011) \\
\hline SC & $21.6^{1}$ & 3,612 \\
\hline $\mathrm{B} \& \mathrm{~B}$ & $22.4^{2}$ & 3,746 \\
\hline Hotel & $32^{3}$ & 5,351 \\
\hline Camping & 24 & 4,013 \\
\hline Total & 100 & $16,722^{4}$ \\
\hline \multicolumn{3}{|c|}{$\begin{array}{l}\text { 1. SC } \% \text { of visitor nights }=8.5 \%(50 \% \text { of total } \mathrm{Bp} \text { and homestead })+13.1 \% \text { (\% of visitor nights of SC in } 2005) \text {. } \\
\text { 2. } \mathrm{B} \& \mathrm{~B} \% \text { of visitor nights }=8.5 \%(50 \% \text { of total } \mathrm{Bp} \text { and homestead })+13.9 \% \text { (\% of visitor nights of } \mathrm{B} \& \mathrm{~B} \text { in } \\
\text { 2005). } \\
\text { 3. Hotel } \% \text { of visitor nights }=10.9 \%+21.1 \%\end{array}$} \\
\hline
\end{tabular}

\subsubsection{Area of Naseby Accommodation Services}

This thesis uses the average area per bed space of OCRT accommodation services (Table 5.20) as the average area per bed space of Naseby accommodation services. Table 6.17 indicates that the total area of 98 Naseby accommodation services is $8,144 \mathrm{~m}^{2}$, including 2,744 $\mathrm{m}^{2}$ SC, 2,592 $\mathrm{m}^{2}$ camping, 1,688 $\mathrm{m}^{2}$ hotel and 1,120 $\mathrm{m}^{2} \mathrm{~B} \& \mathrm{~B}$.

Table 6.17 shows the annual occupied area of each type of Naseby accommodation services, calculated as its total area multiplied by its percentage occupancy rate. The total annual occupied area is 2,006 $\mathrm{m}^{2}$, including $662 \mathrm{~m}^{2}$ camping, $593 \mathrm{~m}^{2} \mathrm{SC}, 540 \mathrm{~m}^{2}$ hotel and $251 \mathrm{~m}^{2} \mathrm{~B} \& \mathrm{~B}$. 
Table 6.17: Naseby accommodation services - total area and occupied area (2011)

\begin{tabular}{|c|c|c|c|c|c|c|c|}
\hline Type & No. & C & $\begin{array}{c}\text { Area } \\
\text { per bed } \\
\left(\mathrm{m}^{2}\right)^{1}\end{array}$ & $\begin{array}{c}\text { Total Area } \\
\left(\mathrm{m}^{2}\right)\end{array}$ & $\begin{array}{c}\text { Occupancy } \\
(\%)\end{array}$ & $\begin{array}{l}\text { Visitor } \\
\text { nights }\end{array}$ & $\begin{array}{l}\text { Occupied } \\
\text { Area }\left(\mathrm{m}^{2}\right)\end{array}$ \\
\hline SC & 18 & 93 & 29.5 & 2,744 & $21.6^{1}$ & 3,612 & 593 \\
\hline B\&B & 6 & 28 & 40 & 1,120 & $22.4^{2}$ & 3,746 & 251 \\
\hline Hotel & 2 & 58 & 29.1 & 1,688 & $32^{3}$ & 5,351 & 540 \\
\hline Camping & 72 & 432 & 6 & 2,592 & 24 & 4,013 & 622 \\
\hline Total & 98 & 611 & - & 8,144 & - & 16,722 & 2,006 \\
\hline
\end{tabular}

Table 6.18 contains the average area, number of bed spaces and total area of each type of Naseby accommodation service by their quality. In this table the area of each type of accommodation services is calculated as its average area/bed space multiplied by the number of bed spaces.

In comparison with other types of NB Naseby accommodation services, camping sites comprise the majority area of 2,592 $\mathrm{m}^{2}$ followed by SC $\left(2,449 \mathrm{~m}^{2}\right)$ and B\&B $\left(920 \mathrm{~m}^{2}\right)$ (Table 6.18). In addition, RB hotels contain the majority area of $1,688 \mathrm{~m}^{2}$ followed by SC (295 $\mathrm{m}^{2)}$ and B\&B (200m²) (Table 6.18).

\begin{tabular}{|c|c|c|c|c|}
\hline \multicolumn{5}{|c|}{ Table 6.18: Naseby- area of accommodation services by type and quality } \\
\hline Category & Q & $\begin{array}{c}\text { Average area/bed } \\
\text { space }\left(\mathrm{m}^{2}\right)\end{array}$ & Bed space & Area $\left(\mathrm{m}^{2}\right)$ \\
\hline \multirow[t]{2}{*}{ SC } & NB & 29.5 & 83 & 2,449 \\
\hline & RB & 29.5 & 10 & 295 \\
\hline \multirow[t]{2}{*}{$\mathrm{B} \& \mathrm{~B}$} & NB & 40 & 23 & 920 \\
\hline & RB & 40 & 5 & 200 \\
\hline \multirow[t]{2}{*}{ Hotel } & NB & 29.1 & - & - \\
\hline & RB & 29.1 & 58 & 1,688 \\
\hline \multirow[t]{2}{*}{ Camping site } & NB & 6 & 432 & 2,592 \\
\hline & RB & - & - & - \\
\hline \multirow[t]{2}{*}{ Total } & NB & - & 538 & 5,961 \\
\hline & RB & & 73 & 2,183 \\
\hline
\end{tabular}

Total area of Naseby NB accommodation services is $5,961 \mathrm{~m}^{2}$ and RB accommodation is $2,183 \mathrm{~m}^{2}$ (Table 6.19), making a total of $8,144 \mathrm{~m}^{2}$.

\begin{tabular}{|c|c|}
\hline Table 6.19: Naseby- area of accommodation services by quality \\
\hline Quality of buildings & Area $\left(\mathrm{m}^{2}\right)$ \\
\hline NB buildings & 5,961 \\
\hline RB buildings & 2,183 \\
\hline Total & 8,144 \\
\hline
\end{tabular}




\subsubsection{The EF of Naseby Accommodation Services}

This chapter explores the EF of Naseby accommodation services through using three scenarios. In the first scenario, all buildings are assumed to be newly constructed (NB) and in the second scenario these buildings are split between NB and RB accommodation as found in 2011. However, in the first and second scenarios all areas of buildings (indoor and outdoor spaces including verandas and balconies) are assumed to be indoor spaces. In the third scenario that reflects the current position of Naseby accommodation services the buildings include the current NB and RB split and considers both indoor and open air (veranda and balcony) areas.

Comparison between the EFs of the first and the second scenarios can indicate the influence exerted on the EF of Naseby accommodation services by using refurbished buildings. In addition, comparison between the EFs of the second and the third scenarios determines the influence of using open air spaces on this EF.

\section{a. First Scenario: All Buildings Are Assumed To Be New Buildings}

\section{a.1. The EF of SC Accommodation Services}

As shown in Table 6.20, SC timber constructions with an area of 2,153 $\mathrm{m}^{2}$ account for the largest portion of embodied energy of $47.4 \mathrm{GJ} /$ year, followed by $354 \mathrm{~m}^{2}$ timber and masonry (14.4 GJ/year) and 236m² mud brick mixed with other materials (6.6 GJ/year).

In Table 6.20, SC operational energy is assumed to be $0.3\left(\mathrm{GJ} / \mathrm{m}^{2} /\right.$ year $)$, equal with B\&B operational energy use (Table 5.28). As determined in Table 6.20, SC timber construction accounts for the largest operating energy of $139.5 \mathrm{GJ} /$ year, followed by timber and masonry (15.3 GJ/year) and mud brick mixed with other materials (15.3 GJ/year).

Table 6.20 also shows SC timber construction with an area of 2,153 $\mathrm{m}^{2}$ has the largest life cycle energy of $186.9 \mathrm{GJ} /$ year and other types of construction including timber mixed with masonry and mud brick mixed with other materials use 37.2 and 21.9 GJ/year respectively. Consequently, the total EF of Naseby SC accommodation is 2.4 gha including 1.8 gha as the EF of timber construction, 0.37 gha for timber and masonry construction, and 0.22 gha mud brick mixed with other materials. 


\begin{tabular}{|c|c|c|c|c|c|c|c|c|}
\hline Material & $\begin{array}{c}\text { Embodied } \\
\text { energy } \\
\text { over 50- } \\
\text { year life } \\
\text { cycle } \\
\left(\mathrm{GJ} / \mathrm{m}^{2}\right)^{1}\end{array}$ & $\begin{array}{l}\text { Total } \\
\text { Area } \\
\left(\mathrm{m}^{2}\right)^{2}\end{array}$ & $\begin{array}{c}\text { Embodied } \\
\text { energy } \\
\text { (GJ/year) }\end{array}$ & $\begin{array}{c}\text { Opera- } \\
\text { tional } \\
\text { energy } \\
\text { (GJ/ } \\
\mathrm{m}^{2} / \text { year) }\end{array}$ & $\begin{array}{c}\text { Occupied } \\
\text { area } \\
\left(\mathrm{m}^{2}\right)^{4}\end{array}$ & $\begin{array}{l}\text { Operating } \\
\text { energy } \\
\text { (GJ/year) }\end{array}$ & $\begin{array}{c}\text { Life- } \\
\text { cycle } \\
\text { energy } \\
\text { (GJ/ } \\
\text { year) }\end{array}$ & $\begin{array}{c}\text { EF } \\
\text { gha/y } \\
\text { ear }\end{array}$ \\
\hline Timber & 1.1 & 2,153 & 47.4 & 0.3 & 465 & 139.5 & 186.9 & 1.87 \\
\hline $\begin{array}{l}\text { Mud brick } \\
\text { (mixed } \\
\text { with other } \\
\text { materials) }\end{array}$ & 1.4 & 236 & 6.6 & 0.3 & 51 & 15.3 & 21.9 & 0.22 \\
\hline $\begin{array}{l}\text { Timber } \\
\text { and ma- } \\
\text { sonry }\end{array}$ & 2.04 & 354 & 14.4 & 0.3 & 76 & 22.8 & 37.2 & 0.37 \\
\hline Total & - & - & 68.4 & - & - & 177.6 & 246 & 2.46 \\
\hline \multicolumn{9}{|c|}{$\begin{array}{l}\text { 1. See Table } 5.27 \text { (Chapter } 5 \text { - section 5.6.8.1-a-8) } \\
\text { 2. Total area of each type of SC construction = capacity of the type of construction (See Table } 6.15) \times \text { SC aver- } \\
\text { age area per bed }\left(29.5 \mathrm{~m}^{2}\right) \\
\text { 3. See Table } 5.28 \text { (Chapter 5- section 5.6.8.1- c-1) and Chapter 5- section 5.6.8.1-c-1 }\end{array}$} \\
\hline
\end{tabular}

\section{a.2. EF of B\&B Accommodation Services}

Table 6.21 demonstrates that $920 \mathrm{~m}^{2}$ of Naseby B\&B accommodation services of timber construction account for $20.4 \mathrm{GJ} /$ year embodied energy and $354 \mathrm{~m}^{2}$ Naseby B\&B of mud brick construction use $14.4 \mathrm{GJ} /$ year embodied energy.

In Table 6.21, B\&B operating energy is assumed to be the same as that of the OCRT at $0.3 \mathrm{GJ} / \mathrm{m}^{2} /$ year (Table 5.28). As determined in Table 6.21, Naseby B\&B accommodation services of timber construction use $61.8 \mathrm{GJ} /$ year operating energy and B\&B mud brick constructions use $13.5 \mathrm{GJ} /$ year. Also, $920 \mathrm{~m}^{2}$ of Naseby B\&B accommodation of timber construction accounts for the largest life cycle energy of $82.2 \mathrm{GJ} /$ year and B\&B accommodation of mud brick construction is $19.1 \mathrm{GJ} /$ year.

The total EF of Naseby B\&B accommodation services as shown in Table 6.21 is 1.01 gha including 0.82gha for the EF of timber construction and 0.19 gha for the EF of mud brick mixed with other materials. 


\begin{tabular}{|c|c|c|c|c|c|c|c|c|}
\hline \multicolumn{9}{|c|}{ Table 6.21:Naseby- life cycle energy use of B\&B accommodation services (2011) } \\
\hline Material & $\begin{array}{l}\text { Embod- } \\
\text { ied en- } \\
\text { ergy over } \\
50 \text {-year } \\
\text { life cycle } \\
\left(\mathrm{GJ} / \mathrm{m}^{2}\right)^{1}\end{array}$ & $\begin{array}{l}\text { Total } \\
\text { Area } \\
(\mathrm{m})^{2}\end{array}$ & $\begin{array}{l}\text { Embod- } \\
\text { ied en- } \\
\text { ergy } \\
\text { (GJ/year) }\end{array}$ & $\begin{array}{l}\text { Opera- } \\
\text { tion en- } \\
\text { ergy (GJ/ } \\
\text { m²/year) }^{3}\end{array}$ & $\begin{array}{l}\text { Occu- } \\
\text { pied area } \\
\left(\mathrm{m}^{2}\right)^{4}\end{array}$ & $\begin{array}{l}\text { Operat- } \\
\text { ing en- } \\
\text { ergy } \\
\text { (GJ/year) }\end{array}$ & $\begin{array}{l}\text { Life- } \\
\text { cycle } \\
\text { en- } \\
\text { ergy } \\
\text { use } \\
\text { (GJ/ } \\
\text { year) }\end{array}$ & $\begin{array}{l}\text { EF } \\
\text { gha/ } \\
\text { year }\end{array}$ \\
\hline Timber & 1.1 & 920 & 20.4 & 0.3 & 206 & 61.8 & 82.2 & 0.82 \\
\hline $\begin{array}{l}\text { Mud } \\
\text { brick } \\
\text { (with } \\
\text { other } \\
\text { materi- } \\
\text { als) }\end{array}$ & 1.4 & 200 & 5.6 & 0.3 & 45 & 13.5 & 19.1 & 0.19 \\
\hline Total & & 1,120 & 26 & & & 75.3 & 101.3 & 1.01 \\
\hline
\end{tabular}

\section{a. 3. EF of Hotel Accommodation Services}

Naseby hotel accommodation services comprise $524 \mathrm{~m}^{2}$ timber and 1,164 $\mathrm{m}^{2}$ mud brick (mixed with other materials) and account for 11.5 and $32.6 \mathrm{GJ} /$ year respectively of embodied energy (Table 6.22).

Hotel operating energy as shown in Table 6.22 is assumed to be $0.57 \mathrm{GJ} / \mathrm{m}^{2} /$ year, equal to OCRT hotel operating energy use as cited in Table 5.28. As determined in Table 6.22, in Naseby, the annual operating energy of timber hotels is $95.8 \mathrm{GJ} /$ year and mud brick is 212 GJ/year. In addition total life cycle energy use of Naseby hotels is 307.8 GJ/year this being the sum of the life cycle energy of timber (95.8 GJ/ year) and mud brick (212 GJ/year) hotels.

As shown in Table 6.22 the total EF of Naseby hotel accommodation services is 3.47 gha, made up of the EF of timber hotels (1.07gha) and mud brick mixed with other materials hotels (2.4gha). 
Table 6.22:Naseby - Life cycle energy use of Hotel accommodation services (2011)

\begin{tabular}{|c|c|c|c|c|c|c|c|c|}
\hline Material & $\begin{array}{l}\text { Embod- } \\
\text { ied en- } \\
\text { ergy over } \\
50 \text {-year } \\
\text { life cycle } \\
\left(\mathrm{GJ} / \mathrm{m}^{2}\right)^{1}\end{array}$ & $\begin{array}{l}\text { Total } \\
\text { Area } \\
\left(\mathrm{m}^{2}\right)\end{array}$ & $\begin{array}{l}\text { Embod- } \\
\text { ied en- } \\
\text { ergy } \\
\text { (GJ/year) }\end{array}$ & $\begin{array}{l}\text { Opera- } \\
\text { tion en- } \\
\text { ergy (GJ/ } \\
\text { m²/year) }^{3}\end{array}$ & $\begin{array}{l}\text { Occu- } \\
\text { pied area } \\
\left(\mathrm{m}^{2}\right)^{4}\end{array}$ & $\begin{array}{l}\text { Operat- } \\
\text { ing en- } \\
\text { ergy } \\
\text { (GJ/year) }\end{array}$ & $\begin{array}{l}\text { Life- } \\
\text { cycle } \\
\text { en- } \\
\text { ergy } \\
\text { use } \\
\text { (GJ/ } \\
\text { year) }\end{array}$ & $\begin{array}{l}\text { EF } \\
\text { gha/ } \\
\text { year }\end{array}$ \\
\hline Timber & 1.1 & 524 & 11.5 & 0.57 & 168 & 95.8 & 107.3 & 1.07 \\
\hline $\begin{array}{l}\text { Mud } \\
\text { brick } \\
\text { (mixed } \\
\text { with } \\
\text { other } \\
\text { materi- } \\
\text { als) }\end{array}$ & 1.4 & 1,164 & 32.6 & 0.57 & 372 & 212 & 244.6 & 2.4 \\
\hline Total & - & - & 44.1 & - & - & 307.8 & 366.3 & 3.47 \\
\hline \multicolumn{9}{|c|}{$\begin{array}{l}\text { 1. See Table } 5.27 \text { (Chapter 5: section 5.6.8.1-a-8) } \\
\text { 2. Total area of each type of construction = capacity of the type of construction (see Table } 6.15) \times \text { hotel average } \\
\text { area per bed }\left(29.1 \mathrm{~m}^{2}-\text { see Table } 5.20 \text { ) }\right. \\
\text { 3. See Table } 5.28 \text { (Chapter 5: section 5.6.8.1- c-1) and Chapter 5: section 5.6.8.1-c-1 } \\
\text { 4. Occupancy rate of Naseby Hotels }=32 \% \text { (see Table 6.16) }\end{array}$} \\
\hline
\end{tabular}

\section{a. 4. EF of Camping Accommodation Services}

As determined in Table 6.23 the embodied energy of Naseby camping sites is not available and this study considers the operating energy of this type of accommodation service to be equal to its life cycle energy. Table 6.23 indicates that the total EF of 288 Naseby powered sites with 2,689 visitor nights and 0.025 GJ/visitor night operating energy use is equivalent to 0.67 gha

Table 6.23: Naseby- life cycle energy use of Camping (powered sites) accommodation services (2011)

\begin{tabular}{|c|c|c|c|c|c|c|c|}
\hline Material & $\begin{array}{c}\text { Embodied } \\
\text { energy } \\
\text { over 50- } \\
\text { year life } \\
\text { cycle } \\
\left(\mathrm{GJ} / \mathrm{m}^{2}\right)\end{array}$ & $\begin{array}{c}\text { Total } \\
\text { visitor } \\
\text { nights }\end{array}$ & $\begin{array}{c}\text { Embodied } \\
\text { energy } \\
\text { (GJ/year) }\end{array}$ & $\begin{array}{l}\text { Operation } \\
\text { energy } \\
\text { (GJ/ visi- } \\
\text { tor night) }\end{array}$ & $\begin{array}{c}\text { Operating } \\
\text { energy } \\
\text { (GJ/year) }\end{array}$ & $\begin{array}{c}\text { Life- } \\
\text { cycle } \\
\text { energy } \\
\text { use (GJ/ } \\
\text { year) }\end{array}$ & $\begin{array}{c}\text { EF } \\
\text { gha/year }\end{array}$ \\
\hline $\begin{array}{l}\text { Assumed } \\
\text { negligible }\end{array}$ & N/A & $2,689^{1}$ & N/A & 0.025 & 67.2 & 67.2 & 0.67 \\
\hline \multicolumn{8}{|c|}{$\begin{array}{l}\text { 1. } 2,689 \text { visitor nights }=66.7 \% \text { (portion of camping bed spaces that are powered) of total camping (powered and } \\
\text { non powered camp sites) visitor nights ( } 4,013 \text { visitor nights). }\end{array}$} \\
\hline
\end{tabular}

\section{a. 5. EF of Naseby Accommodation Services (First Scenario)}

The total 2011 EF of 98 Naseby accommodation services based on the assumption that all are NB is 7.6gha/year (Table 6.24). As determined in Table 6.24, Naseby hotel accommodation services have the largest EF of 3.47 gha followed by SC (2.46 gha), B\&B (1.01gha) and camping (powered sites) 0.67 gha. 
Table 6.24: The total EF of Naseby accommodation services (first scenario)

\begin{tabular}{l} 
Types of accommodation \\
\hline SC \\
\hline B\&B \\
\hline Hotel \\
\hline Camping \\
Total
\end{tabular}

EF (gha/year)

2.46

1.01

3.47

0.67

7.6

\section{b. EF of Naseby Accommodation Services (Second Scenario)}

In the second scenario the embodied energies of RB buildings are subtracted from the whole life cycle energy use of Naseby accommodation services. Consequently, the life cycle energy of Naseby accommodation services in the second scenario includes the embodied energy of NB plus the total operating energy of both RB and NB buildings. As RB accommodation services use the same energy sources and have the same facilities as NB buildings, their operational energy use is assumed to the same as that of NB (Table 6.25). In Table 6.25, the embodied energies of NB and RB accommodation services are calculated through the following equation:

Embodied energy of NB/RB accommodation services $=$ total embodied energy $\times$ percentage of NB/RB bed spaces

For example in Table 6.25, the total embodied energy of SC accommodation services is 68.4 GJ/year. NB SC bed spaces contain 89.2\% (83 out of 93) of the total 93 SC bed spaces (see Figure 6.13). Consequently, the embodied energy of NB SC accommodation services is equivalent to: $68.4 \mathrm{GJ} /$ year $\times 89.2 \%=61.1 \mathrm{GJ} /$ year $($ Table 6.25$)$.

Table 6.25 demonstrates that in the second scenario, the total life cycle energy use of Naseby accommodation services is $710.3 \mathrm{GJ} /$ year (Table 6.25). Likewise the EF of Naseby accommodation services is $710.3 \mathrm{GJ} /$ year $\div 100 \mathrm{GJ} / \mathrm{ha}$ (global average carrying capacity of land $)=7.1$ gha $($ Table 6.25$)$. 


\section{Table 6.25:Naseby accommodation services- life cycle energy of new and refurbished buildings}

\begin{tabular}{|c|c|c|c|c|c|c|c|}
\hline Туре & $\begin{array}{l}\text { Total em- } \\
\text { bodied } \\
\text { energy } \\
\text { (GJ/year) }\end{array}$ & $\begin{array}{c}\text { NB- } \\
\text { Bed } \\
\text { space } \\
(\%)\end{array}$ & $\begin{array}{c}\text { RB- } \\
\text { Bed } \\
\text { space } \\
(\%)\end{array}$ & $\begin{array}{l}\text { Embodied } \\
\text { energy of NB } \\
\text { (GJ/year) }\end{array}$ & $\begin{array}{l}\text { Total oper- } \\
\text { ating en- } \\
\text { ergy (GJ/ } \\
\text { year) }\end{array}$ & $\begin{array}{c}\text { Life-cycle } \\
\text { energy } \\
\text { (GJ/year) }\end{array}$ & $\begin{array}{c}\text { EF } \\
\text { (gha/year) }\end{array}$ \\
\hline SC & 68.4 & 89.2 & 10.8 & 61.1 & 177.6 & 238.7 & 2.38 \\
\hline$B \& B$ & 26 & 82.1 & 17.9 & 21.3 & 75.3 & 96.6 & 0.96 \\
\hline Hotel & 44.1 & 0.00 & 100 & 0.00 & 307.8 & 307.8 & 3.08 \\
\hline Camping & N/A & 100 & 0.00 & N/A & 67.2 & 67.2 & 0.67 \\
\hline Total & 157.88 & - & - & 86.6 & 589.45 & 710.3 & 7.1 \\
\hline
\end{tabular}

Table 6.26 compares the EF of Naseby accommodation services arising from the two scenarios (EF1 and EF2). It shows the influence of using RB buildings as accommodation means the EF is decreased by $6.6 \%$ (0.5 gha) from 7.6 gha to 7.1 gha. As the total area of Naseby RB accommodation services is $2,183 \mathrm{~m}^{2}$, the EF reduction per $\mathrm{m}^{2}$ is: 0.5 gha $\left(5,000 \mathrm{~m}^{2}\right) \div 2,183 \mathrm{~m}^{2}=2.3 \mathrm{~m}^{2}$. This means using $1 \mathrm{~m}^{2} \mathrm{RB}$ buildings as accommodation services can reduce the EF of Naseby accommodation services by $2.3 \mathrm{~m}^{2}$.

Table 6.26: Reduced EF of Naseby accommodation services by use of RB as tourism accommodation EF1 (all buildings are assumed to be new) EF2 (Accommodation services include NB and $\mathrm{RB})$

7.6 gha/year 7.1 gha/year

- Reduced EF of Naseby influenced by the use of RB (11.9\% of total bed spaces) as a part of accommodation services $=0.5$ gha/year $=6.6 \%$ of $\mathrm{EF} 1$

\section{c. EF of Naseby Accommodation Services (Third Scenario)}

This section attempts to determine the influence of using open air spaces including verandas and balconies (V/B) on the EF of Naseby accommodation buildings. In the second scenario, calculation of the EF of Naseby accommodation services has been conducted based on the assumption that all NB and RB spaces are indoor spaces. In this scenario both indoor and outdoor spaces and their EFs are considered. The difference between the results of the second and third scenario indicates the influence of using open air areas on EF of Naseby accommodation services.

The number of V/B and outdoor sitting areas by type (shared and private) were found through using Naseby accommodation websites (see Appendix 5- rows 75-90). Table 6.27 contains summarised information about the numbers and types of V/B and outdoor sitting areas by type of accommodation, as explained in Appendix 5 (rows-75-90). Table 6.27 shows that 6 Naseby SC bed spaces have access to 1 shared V/B and 12 bed 
spaces can use 6 private V/Bs. Furthermore, as shown in Table 6.27, 18 Naseby hotel bed spaces have access to 1 shared outdoor sitting area.

\begin{tabular}{|c|c|c|c|c|c|c|c|c|}
\hline \multirow[t]{2}{*}{ Туре } & \multicolumn{4}{|c|}{$\mathrm{V}^{1} / \mathrm{B}^{2}$} & \multicolumn{4}{|c|}{ Outdoor sitting } \\
\hline & $S^{3}$ & $\mathrm{NV}^{4}$ & $\mathrm{P}^{5}$ & NV & $\mathrm{S}$ & $\mathrm{No}^{6}$ & $\mathrm{P}$ & No \\
\hline SC & 6 & 1 & 12 & 6 & - & - & - & - \\
\hline$B \& B$ & - & - & - & - & - & - & - & - \\
\hline Hotel & 0 & 0 & 0 & 0 & 18 & 1 & - & - \\
\hline \multicolumn{9}{|c|}{$\begin{array}{l}\text { 1. V: Veranda } \\
\text { 2. B: Balcony } \\
\text { 3. S: Number of bedrooms sharing area } \\
\text { 4. NV: Number of veranda/ balcony } \\
\text { 5. P: Number of bedrooms using private area } \\
\text { 6. No: Number of outdoor sitting spaces. }\end{array}$} \\
\hline
\end{tabular}

Table 6.28 shows that the embodied energy of $100.1 \mathrm{~m}^{2}$ Naseby V/Bs is $1.2 \mathrm{GJ} /$ year. Since V/Bs are open areas and do not use operating energy (in particular for heating), their operating energy is assumed to be zero. As a result, the life cycle energy use of $100.1 \mathrm{~m}^{2} \mathrm{~V} / \mathrm{B}$ is equal to its embodied energy (1.2 GJ/year) and its EF is $1.2 \mathrm{GJ} /$ year (life cycle energy use) $\div 100$ (global average carrying capacity of land) $=0.012$ gha (Table 6.28).

\begin{tabular}{|c|c|c|c|c|c|c|}
\hline Type & $\begin{array}{l}\text { Embodied } \\
\text { energy } \\
\left(\mathrm{GJ} / \mathrm{m}^{2} \text { per }\right. \\
\text { year })\end{array}$ & $\begin{array}{c}\text { Area } \\
\left(m^{2}\right)^{1-2}\end{array}$ & $\begin{array}{l}\text { Embodied en- } \\
\text { ergy (GJ/year) }\end{array}$ & $\begin{array}{l}\text { Operating } \\
\text { energy }\end{array}$ & $\begin{array}{l}\text { Life-cycle } \\
\text { energy } \\
\text { (GJ/year) }\end{array}$ & EF (gha) \\
\hline Veranda/balcony & $0.012^{3}$ & 100.1 & 1.2 & 0 & 1.2 & 0.012 \\
\hline \multicolumn{7}{|c|}{$\begin{array}{l}\text { 1. Total number of verandas/ balconies }=7(\text { See Table } 6.27) \\
\text { 2. Average area }\left(\mathrm{m}^{2}\right) \text { of veranda/balcony }=14.3(\text { Table } 5.40) \\
\text { 3. Embodied energy of veranda/ balcony }\left(\mathrm{GJ} / \mathrm{m}^{2} / \text { year }\right)=0.012(\text { Table } 5.42)\end{array}$} \\
\hline
\end{tabular}

In Table 6.29 the total 8,144 $\mathrm{m}^{2}$ of Naseby-accommodation services (see Table 6.17) is separated into the two areas of 8,043.9 $\mathrm{m}^{2}$ indoor spaces and $100.1 \mathrm{~m}^{2} \mathrm{~V} / \mathrm{B}$. The EF of Naseby accommodation services (indoor spaces) as shown in Table 6.25 is $0.00087 \mathrm{gha} /$ $\mathrm{m}^{2}$ (7.1gha $\div 8,144 \mathrm{~m}^{2}$ ). Thus the EF of $8,043.9 \mathrm{~m}^{2}$ indoor spaces is $8,043.9 \mathrm{~m}^{2} \times$ $0.00087 \mathrm{gha} / \mathrm{m}^{2}=7$ gha (Table 6.29). Likewise the EF of $100.1 \mathrm{~m}^{2} \mathrm{~V} / \mathrm{B}$ is 0.012 gha (see Table 6.28). As a result the total EF of 8,144 $\mathrm{m}^{2}$ Naseby accommodation services including $8,043.9 \mathrm{~m}^{2}$ indoor spaces and $100.1 \mathrm{~m}^{2} \mathrm{~V} / \mathrm{B}$ is the sum of 7 and 0.012 gha $=$ 7.012gha (Table 6.29). 
As shown in Table 6.26, the EF from the second scenario in which all Naseby accommodation services are assumed to be indoor spaces (EF2) is 7.1 gha. Moreover, in third scenario where outdoor spaces are the total EF (EF 3) is 7.012 gha. Thus 0.09 gha as the difference between the two EFs (EF2 and EF 3) can be considered as the reduction in Naseby EF from the use of $100.1 \mathrm{~m}^{2}$ verandas and balconies (Table 6.29). On the other hand, as 0.09 gha $\left(900 \mathrm{~m}^{2}\right) \div 100.1 \mathrm{~m}^{2}=8.9 \mathrm{~m}^{2}$, it can be considered that using $1 \mathrm{~m}^{2} \mathrm{~V} / \mathrm{B}$ reduces the EF of Naseby accommodation services by $8.9 \mathrm{~m}^{2}$.

Table 6.29: EF of Naseby accommodation services influenced by the use of veranda/balcony Indoor spaces and powered camping sites Outdoor sitting (Veranda/balcony)
Area $\left(\mathrm{m}^{2}\right)$
$\mathrm{EF}\left(\mathrm{gha} / \mathrm{m}^{2}\right)$
EF (gha)
Area $\left(\mathrm{m}^{2}\right)$
$\mathrm{EF}\left(\mathrm{gha} / \mathrm{m}^{2}\right)$
EF (gha)

8043.9

0.00087

7

100.1

0.00012

0.012

- Total area of Naseby accommodation services $\left(\mathrm{m}^{2}\right)=8,144$ (see Table 6.19).

- Total EF of Naseby accommodation services (second scenario) (gha)= 7.1 (see Table 6.25)

- Total EF of Naseby accommodation services (indoor space + veranda/balcony) (gha) $=7.012$

- Reduction in Naseby EF by use of veranda and balcony $\left(1.2 \%\right.$ of 8,043.9 $\left.\mathrm{m}^{2}\right)=0.09$ gha $=900 \mathrm{~m}^{2}$

- Reduction in Naseby EF by use of $1 \mathrm{~m}^{2}$ veranda and balcony $=8.9 \mathrm{~m}^{2}$

\subsubsection{Naseby Tourism Products and Activities (Frequency)}

This section investigates the environmental impacts of Naseby visitor activities (as a part of their social cultural behaviour) through calculation of their EF. The frequency of activities offered by Naseby accommodation services to visitors is found through official websites of the OCRT and 15 websites of 93 Naseby accommodation services. The offered activities are explained in Appendix 5 (rows 75-90) and they are classified into indoor and outdoor activities in Appendix 28.

In addition the frequency of the activities offered, shown in Appendix 28 and summarised in Tables 6.31 and 6.32, can be considered as an indicator of the attitude of Naseby participants and members (in this case accommodation owners) to sustainable tourism activities. Comparison between EFs of the conducted activities and their frequency also can indicate a social-ecological perspective of the attitude of Tourism participants to being sustainable.

\subsubsection{Local Produce}

Table 6.30 indicates that $12.2 \%$ (12) of 98 Naseby accommodation services offer local products to their visitors. As demonstrated in Table 6.30, of these 10 (83\%) offer home 
cooked food (local food) and 2 (17\%) offer wine to their visitors. The environmental effects of using home cooked foods have been discussed in section 6.1.5.1.

\begin{tabular}{|c|c|c|}
\hline \multicolumn{3}{|c|}{ Table 6.30: Naseby local produce (summary) } \\
\hline Produce & $\mathrm{F}$ & $\%$ \\
\hline Local food and beverage & 10 & 83 \\
\hline Wine & 2 & 17 \\
\hline Total & 12 & 100 \\
\hline
\end{tabular}

\subsubsection{Tourism Activities}

This section investigates the environmental impacts of Naseby visitor activities (as a part of their social-cultural behaviour) through calculation of their EF.

\section{a. Tourism Indoor Activities (Frequency)}

Table 6.31 summarises the indoor activities offered by Naseby accommodation services to their visitors as cited in Appendices 5 and 28. These activities are classified into four types; indoor sport (curling at Naseby), education, music, and cultural/spiritual activities (Table 6.31).

\begin{tabular}{|l|c|c|}
\hline \multicolumn{2}{|c|}{ Table 6.31: Naseby indoor activities offered by accommodation services to visitors } \\
\hline Activities & $\mathrm{F}^{1}$ & $\%^{2}$ \\
\hline Indoor sports (curling at Naseby). & 16 & 84 \\
\hline Education. & 1 & 5 \\
\hline Music & 1 & 5 \\
\hline Cultural/spiritual activities. & 1 & 5 \\
\hline Total & 19 & 100 \\
\hline 1. Frequency & & \\
\hline 2. Percentages have been rounded & & \\
\hline
\end{tabular}

Figure 6.22 and Table 6.31 determine that indoor visitor activities are dominated by sports (curling) at 16 offerings (84\%) followed by other indoor activities with 1 offering each (5\%). 


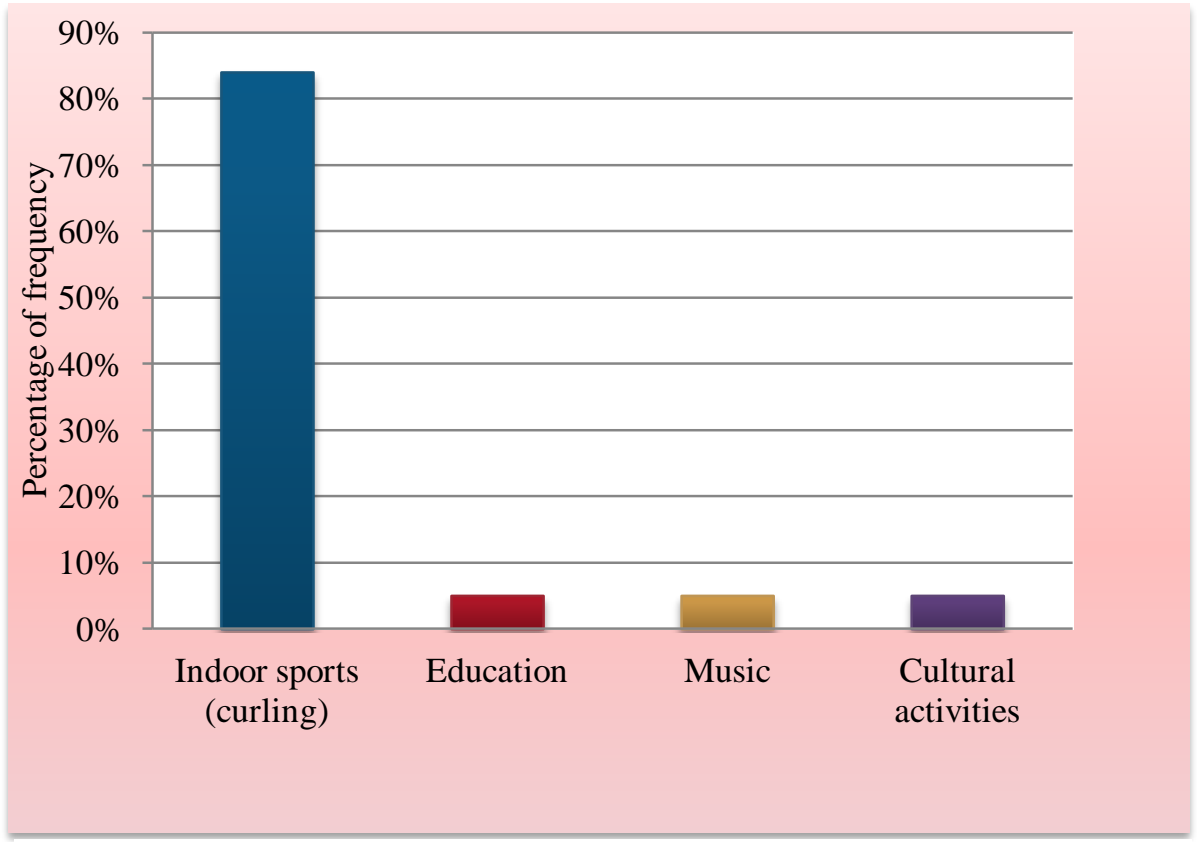

Figure 6.22: Naseby - frequency of indoor activities offered by accommodation services to visitors (2011)

\section{b. Outdoor Activities (Frequency)}

This study explores 11 types of outdoor activity offered by Naseby accommodation services to visitors in 2011 as shown in Appendix 28. Theses outdoor activities are explained by their related accommodation service in Appendix 5. This study classifies the 11 types of Naseby outdoor activities into the 8 types of activity shown in Table 6.32.

\begin{tabular}{|l|c|c|}
\hline $\begin{array}{l}\text { Table 6.32: Naseby- frequency of outdoor activities offered by accommodation services to visi- } \\
\text { tors. }\end{array}$ & F & $\%^{1}$ \\
\hline \multicolumn{1}{|c|}{ Outdoor activity } & 19 & 18.6 \\
\hline Water sports & 15 & 14.7 \\
\hline Walking & 14 & 13.7 \\
\hline Winter sports & 14 & 13.7 \\
\hline Cycling & 13 & 12.7 \\
\hline Motorised sports and activities & 11 & 10.8 \\
\hline Recreational activity & 9 & 8.8 \\
\hline Sports (at playground) & 7 & 6.9 \\
\hline Sports on horseback & 102 & 100 \\
\hline Total & & \\
\hline 1. Percentages have been rounded & & \\
\hline
\end{tabular}

Figure 6.23 and Table 6.32 illustrate that the activity most offered is water sports with 19 mentions (18.6\%), followed by walking 15 (14.7\%), and winter sports and cycling $14(13.7 \%)$ each. The frequency of offering other Naseby outdoor activities falls in a range of 7-13 times (Figure 6.23 and Table 6.32). 


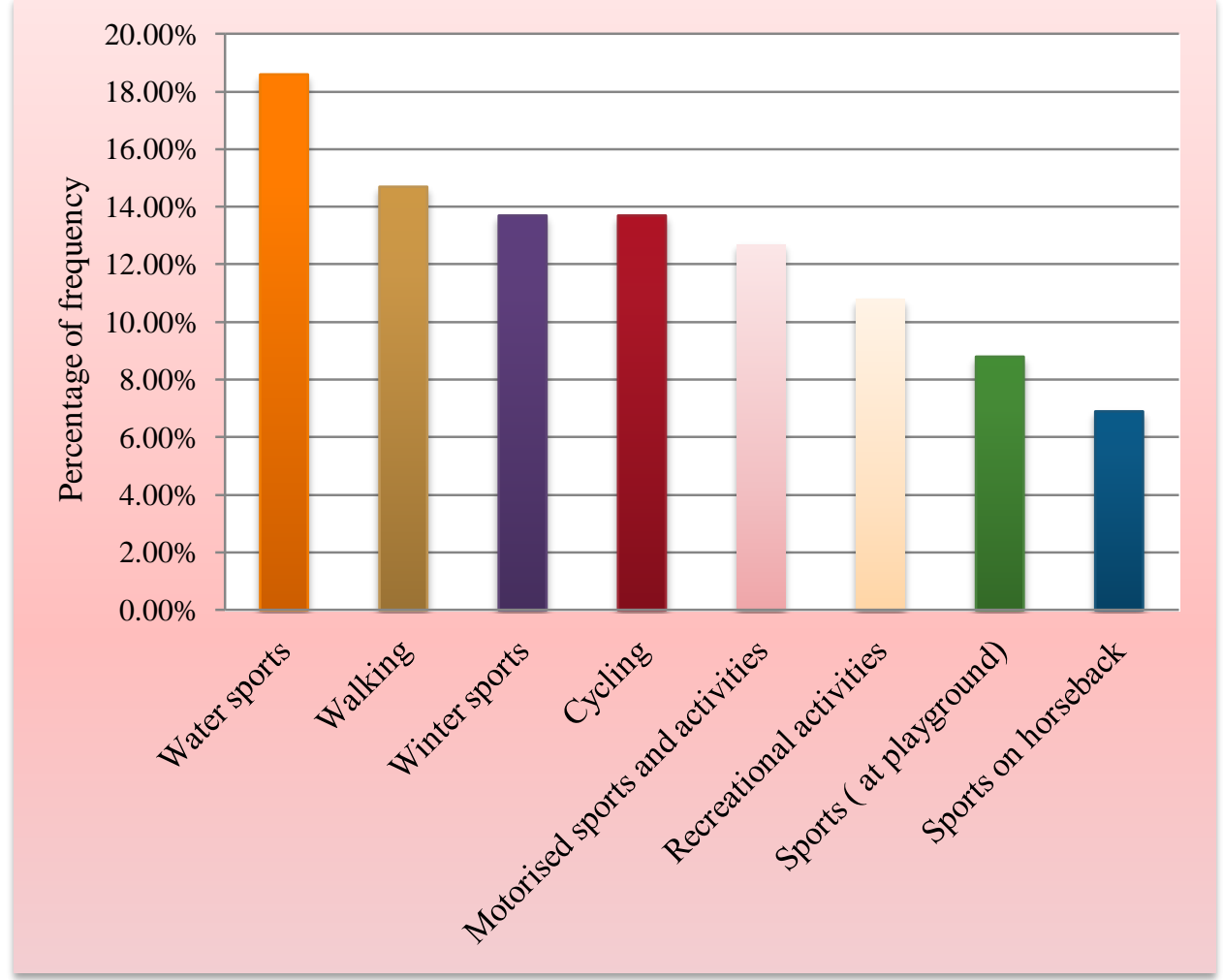

Figure 6.23: The frequency of outdoor activities offered by Naseby accommodation services to visitors

\section{c. Frequency of the Subjects to Visit Offered by Accommodation Services}

As shown in Appendix 5, this study looks at what is offered by way of places to visit by Naseby accommodation services in 2011. Appendix 28 contains summarised information about Naseby visiting activities and their frequency is shown in Appendix 5. Naseby subjects to visit are here put into seven categories; Naseby itself, historical sites and buildings, scenery, museum, gold mining heritage, Naseby town garden, and art, sculpture, and gardens (Appendix 28). Table 6.33 and Figure 6.24 further classify these seven categories into five; cities and villages, historical sites and buildings, scenery, museum, and town garden. As illustrated in Figure 6.24 and Table 6.33, cities and villages, and historic sites and buildings occur at a similar frequency of 13 mentions (33.3\%). In addition, scenery and museum have the same frequency of 6 (15.4\%) and the town garden is only suggested once (2.6\%) (Figure 6.24 and Table 6.33).

\begin{tabular}{|l|c|c|}
\hline \multicolumn{2}{|c|}{ Table 6.33: Naseby - frequency of subjects to visit offered by accommodation services to } \\
visitors (2011)
\end{tabular}




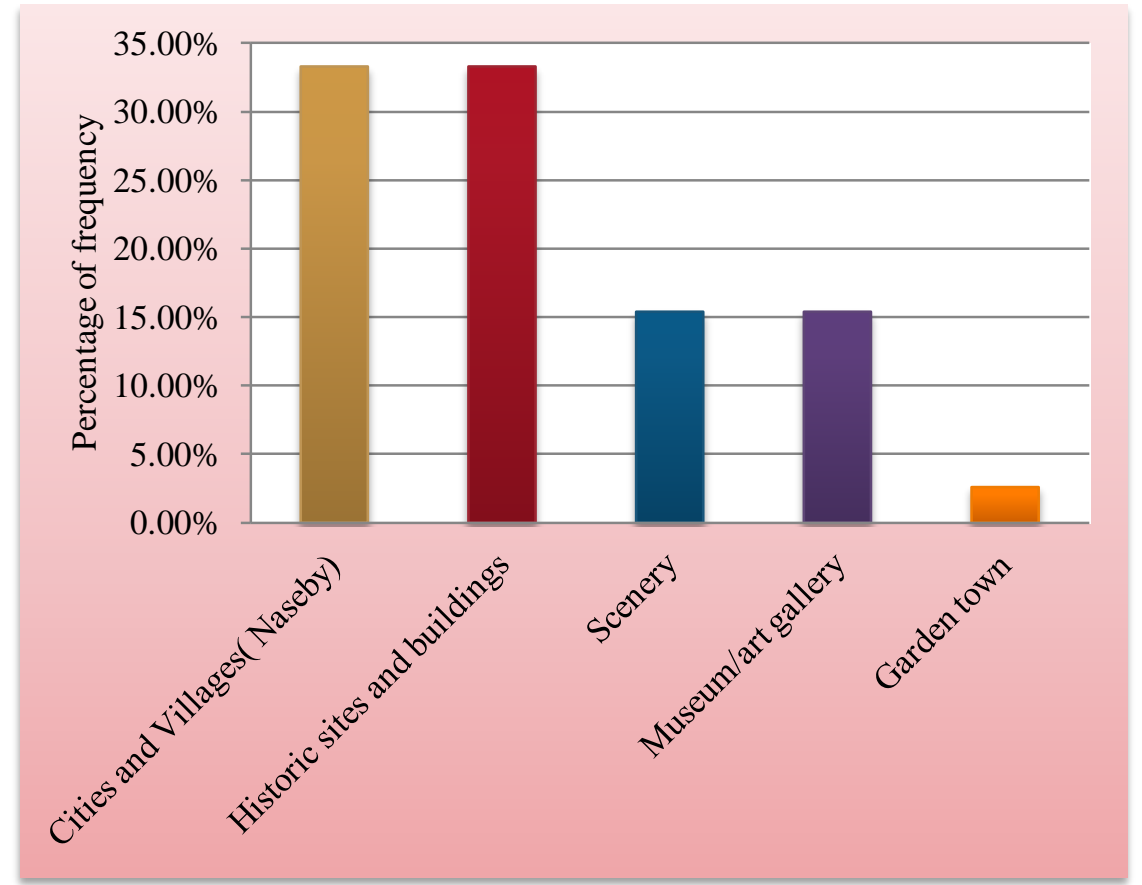

Figure 6.24: Naseby- frequency of subjects to visit offered by accommodation services

\section{c. Activities per Visitor}

This thesis calculates the number of Naseby visitors per activity (except curling and mountain biking at Naseby) through using the percentage split of 160 visitors Table 5.48) and applying this to the 4,645 Naseby visitors in 2011 (Table 6.34). The assumption is that the visitors who come for curling and mountain bike riding will also do other activities. The numbers of OCRT visitors who play curling and go mountain biking at Naseby is already known (see Table 4.45). Table 6.34 shows that in comparison with other Naseby activities, the majority visitor activity is curling $(4,350)$, followed by Taieri Gorge Railway (2,151), visiting gold mining towns $(1,626)$, sightseeing tours $(1,421)$ and visiting old Cromwell town $(1,045)$. The numbers of Naseby visitors who do other types of activity are between 28 and 758 . 


\begin{tabular}{|c|c|c|}
\hline \multicolumn{3}{|c|}{ Table 6.34: Naseby - activities conducted by 4,645 ${ }^{1}$ visitors (2011) } \\
\hline Activities & $\%$ of 4,645 visitors ${ }^{2}$ & Number of Visitor activities \\
\hline Curling (at Naseby) & 93.6 & 4,350 \\
\hline Taieri Gorge Railway & 46.3 & 2,151 \\
\hline Gold mining towns & 35 & 1,626 \\
\hline Sightseeing tours & 30.6 & 1,421 \\
\hline Old Cromwell town & 22.5 & 1,045 \\
\hline Wineries & 16.9 & 785 \\
\hline Visiting friends and relatives & 15 & 697 \\
\hline Hayes engineering & 8.1 & 376 \\
\hline Mountain biking Naseby & 6.4 & 295 \\
\hline fishing & 1.9 & 88 \\
\hline Golf & 1.3 & 60 \\
\hline Swim at Ophir and Alexandra & 0.6 & 28 \\
\hline Clyde dam Tour & 0.6 & 28 \\
\hline Golden Progress mine & 0.6 & 28 \\
\hline Walking & 0.6 & 28 \\
\hline Ophir- high country farm & 0.6 & 28 \\
\hline Biking & 0.6 & 28 \\
\hline Fruit picking along trail & 0.6 & 28 \\
\hline \multicolumn{3}{|c|}{$\begin{array}{l}\text { 1. See Table } 6.1 \\
\text { 2. \% of } 4,645 \text { visitor assumed same as \% of } 160 \text { visitors cited in Table } 5.45 \text { (excluding curling and } \\
\text { mountain biking at Naseby). } \\
\text { - Reference: CODC, } 2011: 18\end{array}$} \\
\hline
\end{tabular}

\subsubsection{EF of Visitor Activities}

\subsubsection{EF of Indoor Activities (Curling at Naseby)}

As indicated in Table 5.56 (see Chapter 5, section 5.7.5.1-d), the total EF of 4,350 OCRT visitors who play curling at Naseby is 0.7 gha which is equivalent to 0.00016 gha/ visitor (Table 6.35).

Table 6.35: Naseby- visitors curling EF (gha) (2011)

\begin{tabular}{|c|c|c|c|c|}
\hline $\begin{array}{l}\text { Total num- } \\
\text { ber of } \\
\text { Naseby visi- } \\
\text { tors }\end{array}$ & $\begin{array}{l}\text { \% of Naseby visi- } \\
\text { tors who play in- } \\
\text { door curling at } \\
\text { Naseby Curling }_{\text {Centre }^{1}}\end{array}$ & $\begin{array}{l}\text { Total number of } \\
\text { Naseby visitors } \\
\text { who play curl- } \\
\text { ing }\end{array}$ & $\begin{array}{l}\text { EF of } 13,000 \text { visi- } \\
\text { tors of Curling cen- } \\
\text { tre (gha) }\end{array}$ & $\begin{array}{l}\text { EF of } 4,350 \text { Naseby visi- } \\
\text { tors ( } 33.5 \% \text { of } 13,000 \\
\text { Naseby curling visitors) } \\
\text { gha }^{2}\end{array}$ \\
\hline 4,645 & 93.6 & 4,350 & $2.07^{3}$ & 0.7 \\
\hline \multicolumn{5}{|c|}{$\begin{array}{l}\text { 1. See Table } 6.34 \\
\text { 2. EF of Naseby Curling Centre (indoor curling) per visitor per } 3 \text { hours playing/ year }=(0.7 \text { gha } \div 4,350 \text { visitors }) \\
0.00016 \text { (gha) } \\
\text { 3. See Table } 5.56\end{array}$} \\
\hline
\end{tabular}




\subsubsection{EF of Outdoor Activities}

\section{a. EF of Walking}

Tables 6.34 and 6.36 show that 28 Naseby visitors chose walking as a tourist activity. As shown in Chapter 5 (Table 5.58) the EF of a visitor who walks 3 hours is 0.00005 gha. Thus the EF of 28 Naseby visitors who walk 3 hours is equivalent to: 0.00005 (gha/visitor) $\times 28$ (number of visitors) $=0.0014$ (gha) $($ Table 6.36)

Table 6.36: Ecological footprint of Naseby visitors (walking).

Number of Naseby visitors who walk.

EF (gha) of Naseby visitor who walks 3 hours

EF of 28 Naseby visitors who walk 3 hours (gha)

1. See Table 6.34

2. See Table 5.58

\section{b. The EF of Golf}

Tables 6.34 and 6.37 show 60 Naseby visitors played golf in 2011. Tables 5.60 and 6.37 indicate that the EF of a visitor who plays golf is $0.003 \mathrm{gha} /$ visitor. Consequently the EF of 60 Naseby visitors playing golf is $60 \times 0.003$ gha/visitor $=018$ gha (Table 6.37 ).

Table 6.37: Ecological footprint of Naseby visitors (golf)

Number of Naseby visitors who play golf

EF (gha) of Naseby visitor who plays golf

EF of 60 Naseby visitors who play golf (gha)

$28^{1}$

$0.00005^{2}$

0.0014

1. See Table 6.34

2. See Table 5.60

\section{c. EF of Mountain Biking}

As shown in Table s 6.34 and 6.38, 295 Naseby visitors went mountain biking at Naseby in 2011. Tables 5.67 and 6.38 indicate that the EF of a visitor who goes mountain biking is 0.0009 gha/visitor. As a result the EF of 295 mountain biking Naseby visitors is $295 \times 0.0009$ gha/visitor $=0.27$ gha $($ Table 6.38$)$.

Table 6.38: Ecological footprint of Naseby visitors (mountain biking)

Number of Naseby visitors who go mountain biking

EF (gha)of Naseby visitor who goes mountain biking

EF of 295 Naseby visitors who go mountain biking (gha)

1. See Table 6.34

2. See Table 5.67

\section{$60^{1}$}

$0.003^{2}$

0.18 


\section{d. EF of Fishing}

In 2011, 88 Naseby visitors chose fishing as a leisure activity (Tables 6.34 and 6.39). Since the EF of a visitor fishing is 0.0009 gha (see Tables 5.68 and 6.39), the EF of 88 Naseby visitors fishing is 0.0009 (gha/visitor) $\times 88$ (visitors) $=0.08$ gha (Table 6.39).

\begin{tabular}{l|c|}
\multicolumn{1}{|c|}{ Table 6.39: Ecological footprint of Naseby visitors (fishing) } \\
\hline Number of Naseby visitors who fish & $88^{1}$ \\
\hline EF (gha) of Naseby visitor who fishes & $0.0009^{2}$ \\
\hline EF of 88 Naseby visitors fishing (gha) & 0.08 \\
\hline $\begin{array}{l}\text { 1. See Table } 6.34 \\
\text { 2. See Table } 5.68\end{array}$
\end{tabular}

\section{e. EF of Swimming at Ophir and Alexandra}

Tables 6.34 and 6.40 show 28 Naseby visitors went swimming at Ophir and Alexandra in 2011. Tables 5.69 and 6.38 determine that the EF of a visitor who swims is 0.0009 gha. As a result the EF of 28 Naseby visitors going swimming is $28 \times 0.0009$ gha/visitor $=0.025$ gha (Table 6.40).

\begin{tabular}{l|c|}
\multicolumn{2}{|c|}{ Table 6.40: Ecological footprint of Naseby visitors (swimming) } \\
\hline Number of Naseby visitors who swim & $28^{1}$ \\
\hline EF (gha) of Naseby visitor swimming & $0.0009^{2}$ \\
\hline EF of 28 Naseby visitors swimming (gha) & 0.025 \\
\hline 1. See Table 6.34 & \\
2. See Table 5.69 & \\
\hline
\end{tabular}

\section{f. EF of Wineries}

Tables 6.34 and 6.41 show that 785 Naseby visitors went to wineries. Tables 5.70 and 6.41 determine that the EF of a visitor going to a winery is 0.00006 gha/visitor. As a result the EF of 785 Naseby visitors who go to wineries is $785 \times 0.00006$ gha/visitor $=$ 0.047 gha (Table 6.41).

\begin{tabular}{l|c|}
\multicolumn{2}{|c|}{ Table 6.41: Ecological footprint of Naseby visitors (wineries) } \\
\hline Number of Naseby visitors who visit wineries & $785^{1}$ \\
\hline EF (gha) of Naseby visitor to winery & $0.00006^{2}$ \\
\hline EF of 785 Naseby visitors to wineries (gha) & 0.047 \\
\hline 1. See Table 6.34 & \\
2. See Table 5.70 &
\end{tabular}

\section{g. EF of Sightseeing}

Tables 6.42 and 6.34 indicate that 1,421 visitors chose sightseeing as one of their activities at Naseby. As explained in Table 5.71 (Chapter 5) the EF of a visitor sightseeing is 
0.0009 gha. Consequently, the EF of 1,421 Naseby visitors is 1,421 x 0.0009 gha/ visitor $=1.3$ gha (Table 6.42).

Table 6.42: Ecological footprint of Naseby visitors (sightseeing)

\begin{tabular}{l|c|}
\hline Number of Naseby visitors sightseeing & $1,421^{1}$ \\
\hline EF (gha) of Naseby visitor sightseeing & $0.0009^{2}$ \\
\hline EF of 1,421 Naseby visitors sightseeing (gha) & 1.3 \\
\hline $\begin{array}{l}\text { 1. See Table } 6.34 \\
\text { 2. See Table } 5.71\end{array}$ & \\
\hline
\end{tabular}

\section{h. EF of Hayes Engineering}

Hayes Engineering heritage was visited by 378 Naseby visitors as shown in Tables 6.34 and 6.43. Since the EF of a visitor in this case is 0.00006 gha (see Table 5.72) the EF of the latter visitors is equivalent to 376 (number of visitors) $\times 0.00006$ gha/visitor $=0.02$ gha (Table 6.43).

\begin{tabular}{|c|c|}
\hline \multicolumn{2}{|c|}{ Table 6.43: Ecological footprint of Naseby visitors (Hayes Engineering) } \\
\hline Number of Naseby visitors to Hayes Engineering) & $376^{1}$ \\
\hline EF (gha) of a Naseby visitor to Hayes Engineering & $0.00006^{2}$ \\
\hline EF of 376 Naseby visitors to Hayes Engineering (gha) & 0.02 \\
\hline $\begin{array}{l}\text { 1. See Table } 6.34 \\
\text { 2. See Table } 5.72\end{array}$ & \\
\hline
\end{tabular}

\section{i. EF of Ophir High Country Farm}

The total EF of 28 Naseby visitors who go to Ophir High Country Farm Tour is calculated as for the examples above. As shown in Table 6.44 it is $0.0017 \mathrm{gha}$.

Table 6.44: Ecological footprint of Naseby visitors (Ophir High Country Farm)

Number of Naseby visitors to Ophir High Country Farm

EF (gha) of Naseby visitor to Ophir High Country Farm

EF of 28 Naseby visitors to Ophir High Country Farm (gha)

1. See Table 6.34

2. See Table 5.73

\section{j. EF of Visitors to Taieri Gorge Railway}

Using the same method as before the total EF of 2,151 Naseby visitors to the Taieri Gorge Railway is 0.13 gha (Table 6.45). 
Table 6.45: Ecological footprint of Naseby visitors (Taieri)

Number of Naseby visitors to Taieri GR

EF (gha) of Naseby visitor to Taieri GR

EF of 2,151 Naseby visitors to Taieri GR

1. See Table 6.34

2. See Table 5.74
$2,151^{1}$

$0.00006^{2}$

0.13

\section{k. EF of Visitors to Gold Mining Towns}

The total EF of 1,626 Naseby visitors who visit gold mining towns is 0.01 gha (Table 6.46).

Table 6.46: Ecological footprint of Naseby visitors (gold mining towns)

Number of Naseby visitors to gold mining towns

EF (gha) of Naseby visitor to gold mining towns

EF of 1,626 Naseby visitors to gold mining towns

$1,626^{1}$

$0.00006^{2}$

1. See Table 6.34

2. See Table 5.75

\section{l. EF of Visitors to Old Cromwell Town}

The EF of a Naseby visitor to old Cromwell town is 0.063 gha (Table 6.47).

Table 6.47: Ecological footprint of Naseby- visitors (Old Cromwell town)

\begin{tabular}{|l|c|}
\hline Number of Naseby visitors to old Cromwell town & $1,045^{1}$ \\
\hline EF (gha) of Naseby visitor to old Cromwell town & $0.00006^{2}$ \\
\hline EF of 1,045 Naseby visitors to old Cromwell town & 0.063 \\
\hline 1. See Table 6.34 & \\
\hline 2. See Table 5.76 & \\
\hline
\end{tabular}

\section{m. EF of Visitors to Friends and Relatives}

The EF of a Naseby visitor who visits friends and relatives is 0.14 gha (Table 6.48).

Table 6.48: Ecological footprint of Naseby visitors (visiting friends and relatives)

\begin{tabular}{|l|c|}
\hline Number of Naseby visitors to friends and relatives & $697^{1}$ \\
\hline EF (gha) of Naseby visitor to friends and relatives & $0.0002^{2}$ \\
\hline EF of 697 Naseby visitors to friends and relatives & 0.14 \\
\hline 1. See Table 6.34 & \\
\hline 2. See Table 5.78 & \\
\hline
\end{tabular}

\section{n. EF of Visitors on Clyde Dam Tour}

The 28 Naseby visitors who participate in a Clyde Dam Tour have an EF of 0.025 gha (Table 6.49). 
Table 6.49: Ecological footprint of Naseby visitors (Clyde Dam Tour)

Number of Naseby visitors on Clyde Dam Tour

EF (gha) of Naseby visitor on Clyde Dam Tour

$28^{1}$

EF of 28 Naseby visitors on Clyde Dam Tour

$0.0009^{2}$

1. See Table 6.34

0.025

2. See Table 5.79

\section{o. EF of Visitors to Golden Progress Mine}

The EF of visitors to the Golden Progress Mine is 0.0017 gha (Table 6.50).

\begin{tabular}{l|c|}
\hline \multicolumn{2}{|c|}{ Table 6.50: Ecological footprint of Naseby visitors (Golden Progress Mine) } \\
\hline Number of Naseby visitors to Golden Progress Mine & $28^{1}$ \\
\hline EF (gha) of Naseby visitor to Golden Progress Mine & $0.00006^{2}$ \\
\hline EF of 28 Naseby visitors to Golden Progress Mine & 0.0017 \\
\hline 1. See Table 6.34 \\
2. See Table 5.80
\end{tabular}

\section{p. EF of Visitors Fruit Picking Along Trail}

The EF of 28 visitors fruit picking along the trail is 0.0017 gha (Table 6.51).

Table 6.51: Ecological footprint of Naseby visitors (fruit picking along trail)

\begin{tabular}{|l|c|}
\hline Number of Naseby visitors fruit picking along trail & $28^{1}$ \\
\hline EF (gha) of Naseby visitor fruit picking along trail & $0.00006^{2}$ \\
\hline EF of 28 Naseby visitors fruit picking along trail & 0.0017 \\
\hline
\end{tabular}

1. See Table 6.34

2. See Table 5.81

\section{q. EF of Biking}

The EF of 28 visitors biking as a tourist activity is 0.025 gha (Table 6.52).

Table 6.52: Ecological footprint of Naseby visitors (biking)

$$
\begin{aligned}
& \text { Number of Naseby visitors biking } \\
& \text { EF (gha) of Naseby visitor biking } \\
& \text { EF } 28 \text { Naseby visitors biking } \\
& \text { 1. See Table } 6.34 \\
& \text { 2. See Table } 5.66
\end{aligned}
$$

\subsubsection{Total EF of Naseby Visitor activities}

The total EF of Naseby visitor activities as shown in Table 6.53 is 3.02 gha. Table 6.53 and Figure 6.25 demonstrate that the four largest EFs are the EF of sightseeing (1.3gha) curling (0.7) at Naseby, mountain biking (0.27) and golf (0.18 gha). Furthermore, walking as a Naseby visitor activity has the smallest EF of 0.0014 gha (Table 6.53 and Figure 6.25). 


\begin{tabular}{|c|c|c|c|c|c|}
\hline \multicolumn{6}{|c|}{ Table 6.53: Total EF of Naseby visitor activities } \\
\hline $\begin{array}{l}\text { Type of activ- } \\
\text { ity }\end{array}$ & Activities & $\begin{array}{l}\text { Numbers } \\
\text { of visi- } \\
\text { tors per } \\
\text { activity }\end{array}$ & $\begin{array}{l}\% \text { of total } \\
4,645 \text { Naseby } \\
\text { visitors }\end{array}$ & $\begin{array}{c}\text { EF } \\
\text { (gha/visitor) }\end{array}$ & EF (gha) \\
\hline Indoor activity & Curling at Naseby ${ }^{1}$ & 4,350 & 93.7 & 0.00016 & 0.7 \\
\hline \multirow{18}{*}{$\begin{array}{c}\text { Outdoor activi- } \\
\text { ties }\end{array}$} & Walking & 28 & 0.6 & 0.00005 & 0.0014 \\
\hline & Golf & 60 & 1.3 & 0.04 & 0.18 \\
\hline & Taieri & 2,151 & 46.3 & 0.00006 & 0.13 \\
\hline & Gold mining towns & 1,625 & 35 & 0.00006 & 0.01 \\
\hline & Sightseeing & 1,421 & 30.6 & 0.0009 & 1.3 \\
\hline & Old Cromwell town & 1,045 & 22.5 & 0.00006 & 0.063 \\
\hline & Wineries & 785 & 16.9 & 0.00006 & 0.047 \\
\hline & $\begin{array}{l}\text { Visiting friends and } \\
\text { relatives }\end{array}$ & 697 & 15 & 0.0002 & 0.14 \\
\hline & Hayes engineering & 375 & 8.1 & 0.00006 & 0.02 \\
\hline & $\begin{array}{l}\text { Mountain biking at } \\
\text { Naseby }^{1}\end{array}$ & 295 & 6.4 & 0.0009 & 0.27 \\
\hline & Fishing & 88 & 1.9 & 0.0009 & 0.08 \\
\hline & $\begin{array}{l}\text { Swim at Ophir and Al- } \\
\text { exandra }\end{array}$ & 28 & 0.6 & 0.0009 & 0.025 \\
\hline & Clyde dam Tour & 28 & 0.6 & 0.0009 & 0.025 \\
\hline & Golden Progress mine & 28 & 0.6 & 0.00006 & 0.0017 \\
\hline & Ophir high country farm & 28 & 0.6 & 0.00006 & 0.0017 \\
\hline & Biking & 28 & 0.6 & 0.0009 & 0.025 \\
\hline & Fruit picking along trail & 28 & 0.6 & 0.00006 & 0.0017 \\
\hline & Total & 13,088 & - & - & 3.02 \\
\hline
\end{tabular}

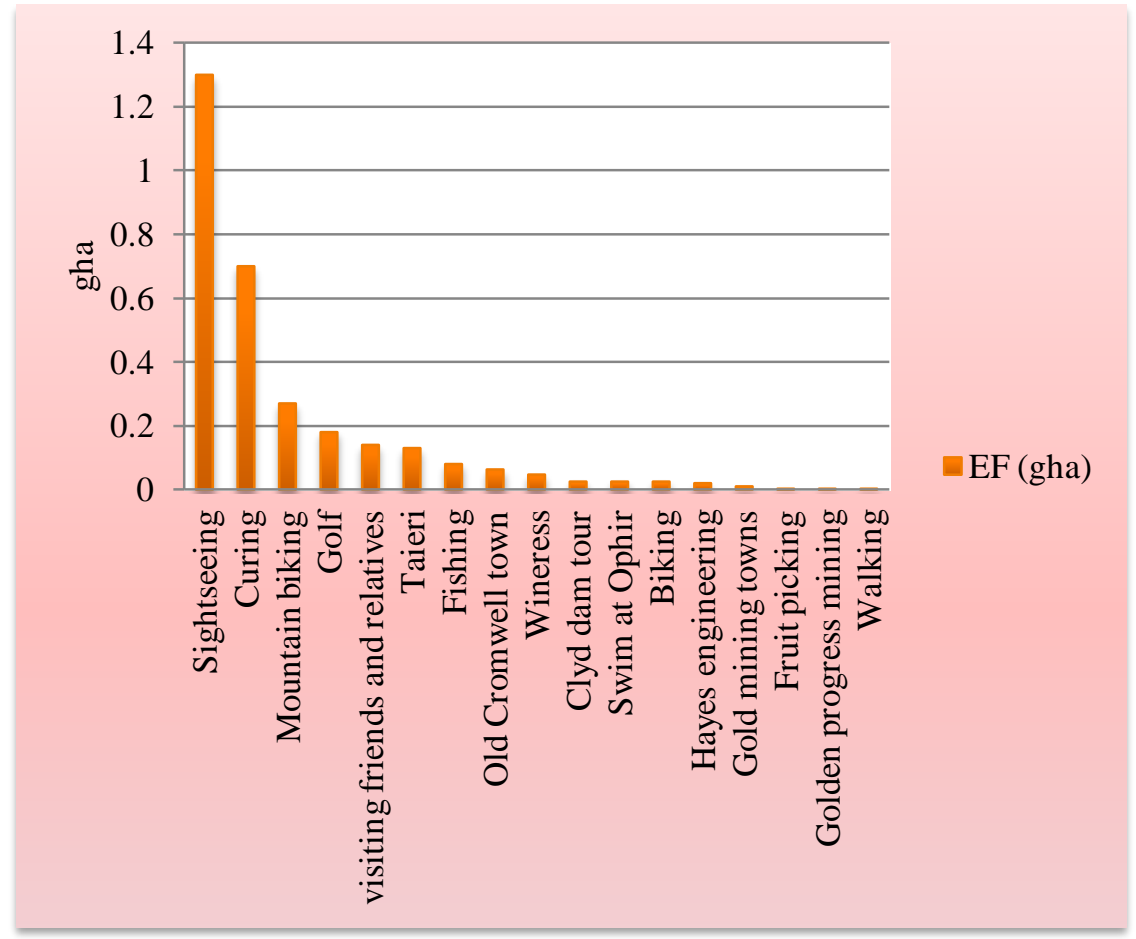

Figure 6.25: Naseby- EF of visitor activities (2011) 


\subsubsection{Comparison between the EFs of Indoor and Outdoor Activities}

Table 5.54 shows that 4,645 Naseby visitors participate in 13,088 visitor activities. As a result the average number of activities per visitor is 2.8 activities, so each Naseby visitor engages in 2.8 activities proposed by the OCRT.

As shown in Table 6.54 and Figure 6.26, the EF of 4,350 Naseby visitors (33.2\% of 13,088 visitors activities) who participate in indoor activity (curling at Naseby) is 0.7 gha that accounts for $23.12 \%$ of the total EF of Naseby activities (indoor and outdoor). Furthermore, Table 6.54 and Figure 6.26 indicate that the total EF of 8,738 Nasebyoutdoor visitor activities (66.8\% of 13,088 Naseby visitor activities) is 2.32 gha, or $76.20 \%$ of the total EF of Naseby visitor activities (3.02gha).

\begin{tabular}{|c|c|c|c|c|c|c|c|c|c|}
\hline \multicolumn{5}{|c|}{ Indoor activity } & \multicolumn{5}{|c|}{ Outdoor activities } \\
\hline $\begin{array}{l}\text { Numbers } \\
\text { of visi- } \\
\text { tors- } \\
\text { activities }\end{array}$ & $\begin{array}{l}\% \text { of } \\
13,088 \\
\text { Naseby } \\
\text { visitors- } \\
\text { activities }\end{array}$ & $\begin{array}{c}\text { EF } \\
\text { (gha) }\end{array}$ & $\begin{array}{c}\% \\
\text { total } \\
\mathrm{EF}^{1}\end{array}$ & $\begin{array}{c}\text { EF } \\
\text { (gha/vi } \\
\text { sitor) }\end{array}$ & $\begin{array}{l}\text { Numbers } \\
\text { of visi- } \\
\text { tors- } \\
\text { activities }\end{array}$ & $\begin{array}{c}\text { \% of total } \\
\text { Naseby } \\
\text { visitors }\end{array}$ & $\begin{array}{c}\text { EF } \\
\text { (gha) }\end{array}$ & $\begin{array}{c}\% \\
\text { total } \\
\mathrm{EF}^{1}\end{array}$ & $\begin{array}{c}\mathrm{EF} \\
\text { (gha/visit } \\
\text { or) }{ }^{2}\end{array}$ \\
\hline 4,350 & 33.2 & 0.7 & 13.4 & $\begin{array}{c}0.0001 \\
6\end{array}$ & 8,738 & 66.8 & 2.32 & 86.6 & 0.00026 \\
\hline \multicolumn{10}{|c|}{$\begin{array}{l}\text { 1. Percentage of the total 3.02gha EF of 13,088 Naseby visitor activities } \\
\text { - Total number of Naseby visitor }=4,645 \text { (see Table } 6.2 \text { ) } \\
\text { - Total number of Naseby Visitor activities }=13,088 \text { (see Table } 6.51 \text { ) } \\
\text { - Average number of activities per visitor }=13,088 \div 4,645=2.8\end{array}$} \\
\hline
\end{tabular}

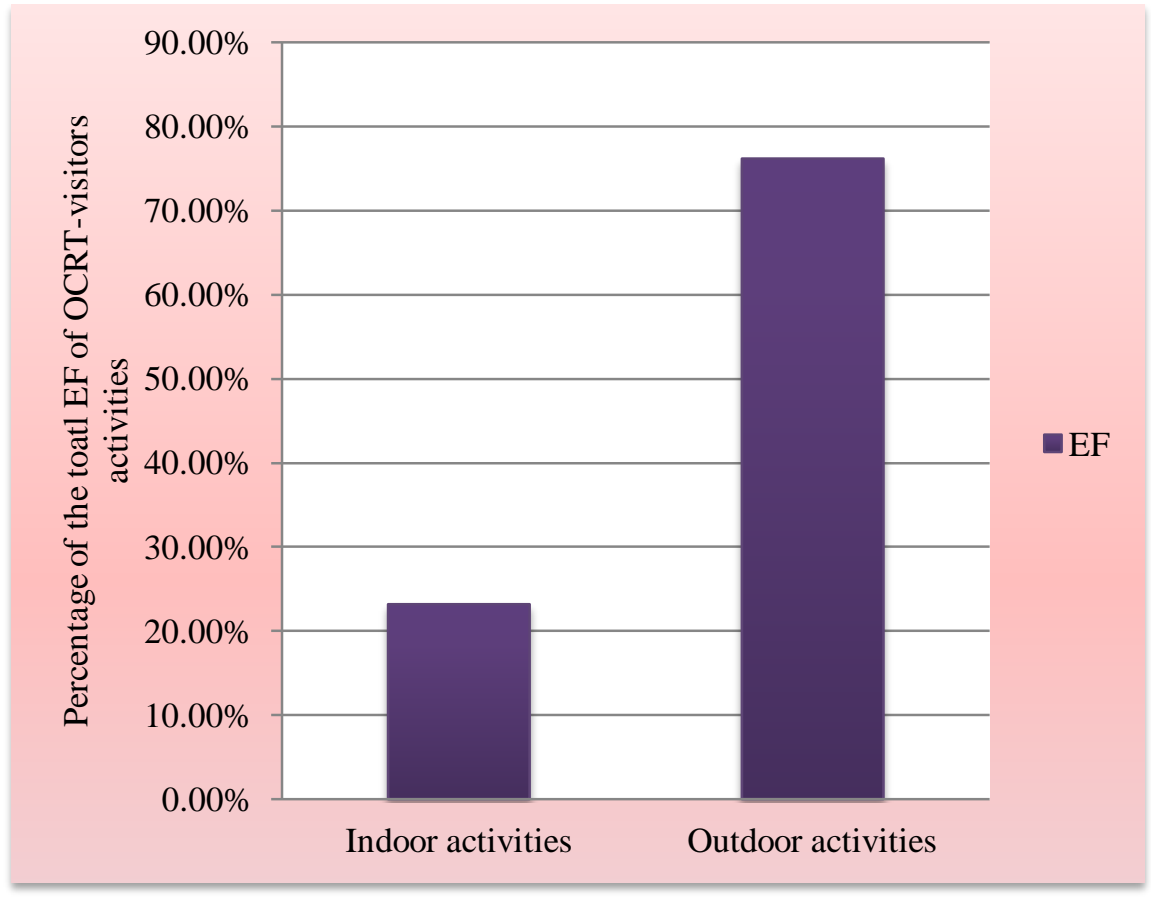

Figure 6.26: Comparison between EFs of Naseby indoor and outdoor activities 
This Thesis selects curling as an indoor activity and golf as an outdoor activity with the biggest EF/visitor in their categories to be compared with the EF of walking as the outdoor activity with the smallest (Figure 6.27).

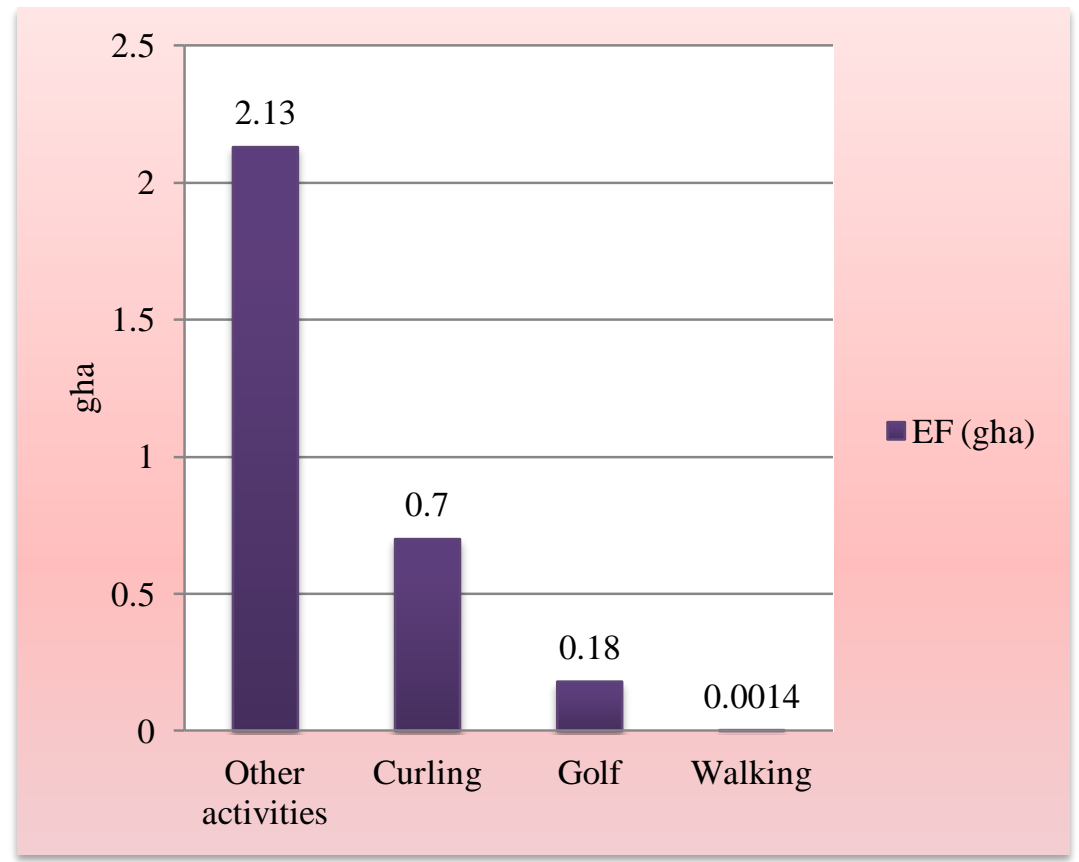

Figure 6.27: Naseby- Comparison between EFs of golf, curling, walking, and other activities excluding these three

Figure 6.28 determines that the majority of 8,650 Naseby visitor activities are within the 'other activity' category (excluding golfing, curling and walking) followed by curling (4,350), golfing (60) and walking (28).

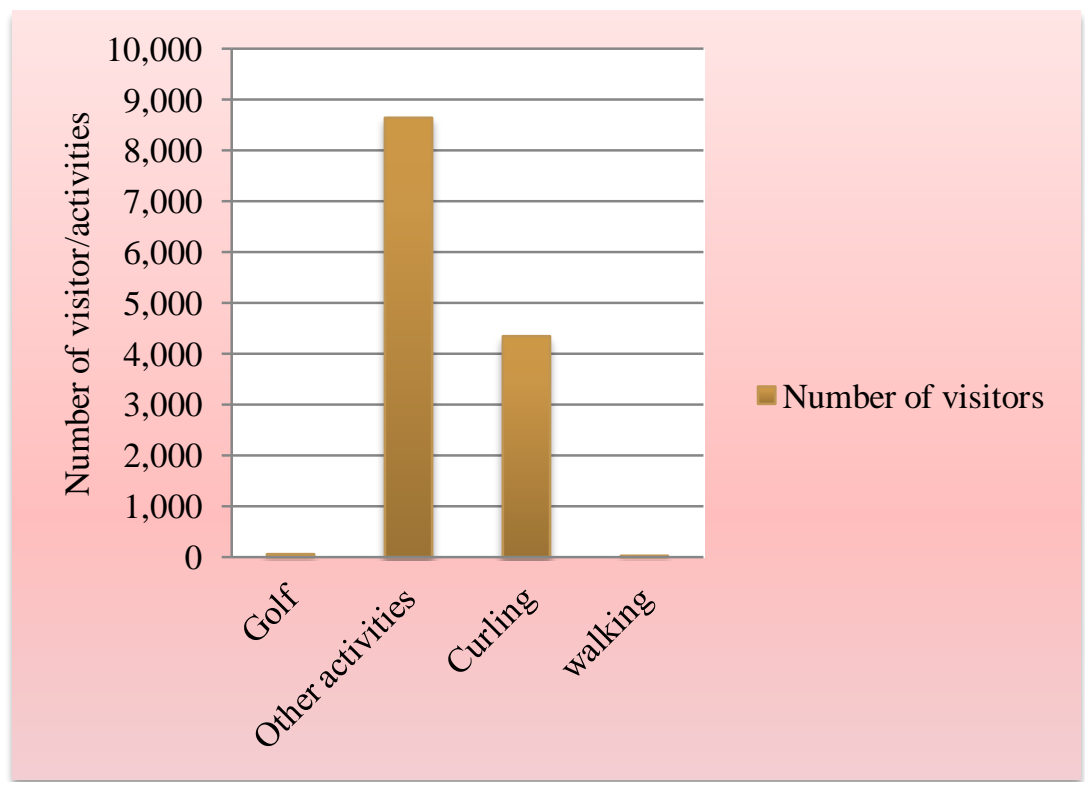

Figure 6.28: Naseby- Comparison between number of visitor activities for golf, curling, walking, and other activities 
Since this study uses the same EF/visitor of activities as explained in Chapter 5, comparison between the EF/visitor of the four categories in Figure 6.28 can be conducted through using Figure 5.36 (Chapter 5- section 5.7.5.4) with the same results.

\subsubsection{The Total EF of Naseby (2011)}

Table 6.55 demonstrates that in 2011 the total EF of Naseby visitors is 632.75 gha. As shown in Table 6.55 and Figure 6.29 transportation accounts for the largest share of the total EF at 459.6gha (72.9\%), followed by food at 160.9 gha (25.5\%), accommodation services at 7.012 gha (1.1\%) and visitors' activities at 3.02 gha (0.5\%). However, the transportation EF that forms 72.9 \% of the total EF of Naseby can be divided into 38.6 \% of international transportation and 34.3\% domestic transportation (see Chapter 6section 6.4.3 and Table 6.8).

\begin{tabular}{|c|c|c|}
\hline \multicolumn{3}{|c|}{ Table 6.55: Total EF of Naseby (2011) } \\
\hline Category & EF (gha) & $\%$ \\
\hline Transportation & $459.6^{1}$ & 72.9 \\
\hline Food (second scenario) & $160.9^{2}$ & 25.5 \\
\hline Accommodation (third scenario) & $7.012^{3}$ & 1.1 \\
\hline Activities & $3.02^{4}$ & 0.5 \\
\hline Total & 630.53 & 100 \\
\hline $\begin{array}{l}\text { 1. See Table } 6.8 \\
\text { 2. See Table } 6.12 \\
\text { 3. See Table } 6.29 \\
\text { 4. See Table } 6.52 \\
\text { - Total number of Naseby visitors }( \\
\text { - EF (gha/visitor) of Naseby }=630 .\end{array}$ & visitors) & \\
\hline
\end{tabular}

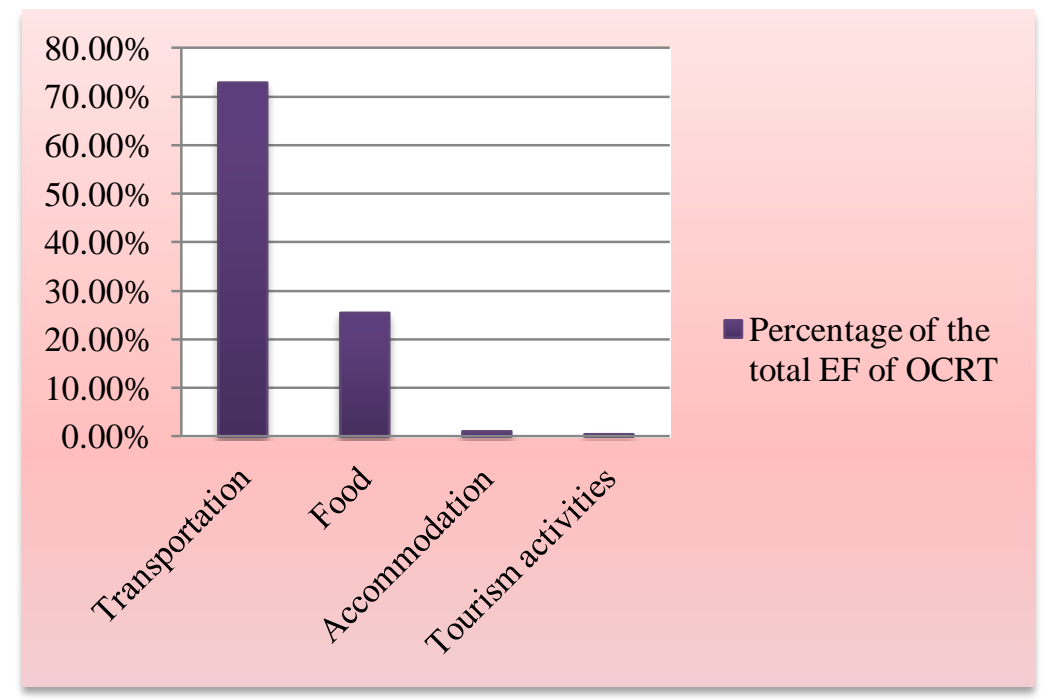

Figure 6.29: Comparison between EFs of Naseby for transportation, food, accommodation services, and visitor activities (2011) 


\subsubsection{Naseby Overshoot Portion of EF}

Table 6.56 compares the present EF of Naseby per visitor and the target EF (0.03gha) investigated in Chapter 5- section 5.9.1. As shown in this table the present EF of Naseby at $0.14 \mathrm{gha} /$ visitor (see Table 6.55 ) is $0.11 \mathrm{gha} /$ visitor more than the target EF for sustainable living. Furthermore, the overshoot portion of the total EF of 4,645 Naseby visitors as shown in Table 6.56 is $(4,645 \times 0.11)=511$ gha.

\subsubsection{Overshoot Portion of Energy Used}

\subsubsection{Overshoot Portion of Energy Use per Visitor}

This chapter calculates the overshoot portion of Naseby visitors through using the method explained in Chapter 5- section 5.9.2. As shown in Table 6.56 the EF of Naseby is.0.14 gha/visitor and as a result energy use is equivalent to:

0.14 (gha/visitor) $\times 100($ carrying capacity of land $)=14(\mathrm{GJ} /$ visitor $)$

Moreover sustainable energy use per visitor is:

0.03 (gha/visitor) $\times 100$ (carrying capacity of land $)=3(\mathrm{GJ} /$ visitor $)$

The overshoot portion of Naseby energy use is:

$14 \mathrm{GJ} /$ visitor $-3 \mathrm{GJ} /$ visitor $=11 \mathrm{GJ} /$ visitor $($ Table 6 .57).

\begin{tabular}{|c|c|c|}
\hline \multicolumn{3}{|c|}{ Table 6.57: Overshoot portion of Naseby visitor energy use (GJ/visitor/year) (2011) } \\
\hline Now & Goal & $\begin{array}{l}\text { Overshoot energy use } \\
\text { (GJ/visitor/year) }\end{array}$ \\
\hline 14 & 3 & 11 \\
\hline
\end{tabular}

\subsubsection{Total Overshoot Portion of Energy Used}

The total overshoot energy of Naseby visitors can be calculated as the total number of visitors $(4,645)$ multiplied by the overshoot energy use per visitors (11 GJ/visitor/year), which is 51,095 GJ/year (Table 6.58). 
Table 6. 58. Total overshoot portion of Naseby visitor energy use (GJ/ year) (2011)

Number of visitors

4,645
Overshoot energy use

(GJ/visitor/year)

11
Total overshoot energy use

(GJ/year)

51,095

\section{1.11.3. Overshoot Portion of Energy Use by Categories}

In this section, the overshoot portion of each category (transportation, food, accommodation and visitor activities) is calculated through using the equation explained in Chapter 5- section 5.9.3. As shown in Table 6.59, transportation uses the majority share of 37,248 GJ/year overshoot energy, followed by food (13,029GJ/year), accommodation services (562GJ/year) and Naseby visitor activities (255 GJ/year).

\begin{tabular}{|l|c|c|c|}
\hline \multicolumn{4}{|c}{ Table 6.59: Naseby overshoot portion of energy used by categories (2011) } \\
\hline Category & EF (gha) & $\begin{array}{c}\text { \% of total } \\
\text { EF }^{1}\end{array}$ & \begin{tabular}{c} 
Overshoot portion of energy use (GJ/year) \\
\hline Transportation
\end{tabular} \\
\hline Food & 459.6 & 72.89 & 37,243 \\
\hline Accommodation & 160.9 & 25.5 & 13,029 \\
\hline Activities & 7.012 & 1.11 & 567 \\
\hline Total & 3.02 & 0.5 & 255 \\
\hline 1. See Table 6.55 & 630.53 & 100 & $51,095^{2}$ \\
\hline 2. See Table 6.58 & & & \\
\hline
\end{tabular}

\subsubsection{Economic Footprint of Naseby Rail Trail Visitors}

In 2011, the total GDP of the OCRT was NZ\$6,245,289 (see Table 5.90) and GDP per visitor was NZ\$530 (NZ\$6,245,289 $\div$ 11,788 OCRT visitors) (Table 6.60). This chapter uses this visitor GDP figure to calculate the GDP of Naseby visitors in 2011. Table 6.60 determines that the total GDP of Naseby rail trail visitors is NZ\$530 (GDP/visitor) $\times$ 4,645 (total number of Naseby visitors) $=$ NZ\$2,461,850.

\begin{tabular}{|c|c|c|c|c|}
\hline \multicolumn{5}{|c|}{ Table 6.60: Naseby GDP (2011) } \\
\hline $\begin{array}{l}\text { OCRT total GDP } \\
\text { ( NZ\$) }\end{array}$ & $\begin{array}{l}\text { OCRT number of } \\
\text { visitors }\end{array}$ & $\begin{array}{l}\text { OCRT GDP } \\
\text { (NZ\$/visitor) }\end{array}$ & $\begin{array}{l}\text { Naseby number } \\
\text { of visitors }\end{array}$ & $\begin{array}{l}\text { Naseby total } \\
\text { GDP }(N Z \$)^{3}\end{array}$ \\
\hline $6,245,289^{1}$ & $11,788^{2}$ & 530 & 4.645 & $2,461,850$ \\
\hline $\begin{array}{l}\text { 1. See Table } 5.90 \\
\text { 2. See Figure } 5.2 \\
3.4,645 \times \mathrm{NZ} \$ 530\end{array}$ & or $=\mathrm{NZ} \$ 2,4$ & & & \\
\hline
\end{tabular}

Table 6.61 shows that the total overshoot energy of Naseby visitors is 51,095 GJ/year. Since the cost to generate $1 \mathrm{GJ}$ through using renewable resources is NZ\$19.8 (see Ta- 
ble 5.94), then the total cost that must be paid to generate 51,095 GJ/year is NZ\$1,011,681 (51,095 × NZ\$19.8) (Table 6.61). This means the sustainable portion of Naseby GDP is NZ\$2,461,850 (GDP) - NZ\$1,011,681 = NZ\$1,450,169 = 58.9\% of the total Naseby GDP (Table 6.61 and Figure 6 .30).

On the other hand the GDPs of Naseby as an ecological-economic indicator indicates that $41.1 \%$ (100\%-58.9\%) of Naseby GDP must be paid to restore its environmental impacts to being ecologically sustainable. In addition, this GDPs as a social-economic indicator evaluates the contribution of the Rail Trail to local economic development, through engagement of local participants and organizations (in this case accommodation services) in the development process.

\begin{tabular}{|c|c|}
\hline \multicolumn{2}{|c|}{ Table 6.61: Naseby sustainable portion of GDP (GDPs) } \\
\hline Total Naseby GDP (NZ\$) & $2,461,850^{1}$ \\
\hline Overshoot energy use (GJ/year) & $51,095^{2}$ \\
\hline Energy cost (NZ\$/GJ) wind-solar & $19.8^{3}$ \\
\hline $\begin{array}{l}\text { Total cost(NZ\$) to generate overshoot portion of } \\
\text { Naseby energy by wind-solar systems }\end{array}$ & $1,011,681$ \\
\hline GDPs $(N Z \$)^{1}$ & $1,450,169$ \\
\hline $\begin{array}{l}\text { 1. See Table } 6.60 \\
\text { 2. See Table } 6.58 \\
\text { 3. See Table } 5.94 \\
\text { - GDPs= Total GDP - total cost to generate the oversl } \\
\text { (see Chapter 5- section.5.10.2). }\end{array}$ & e through renewable resources \\
\hline
\end{tabular}

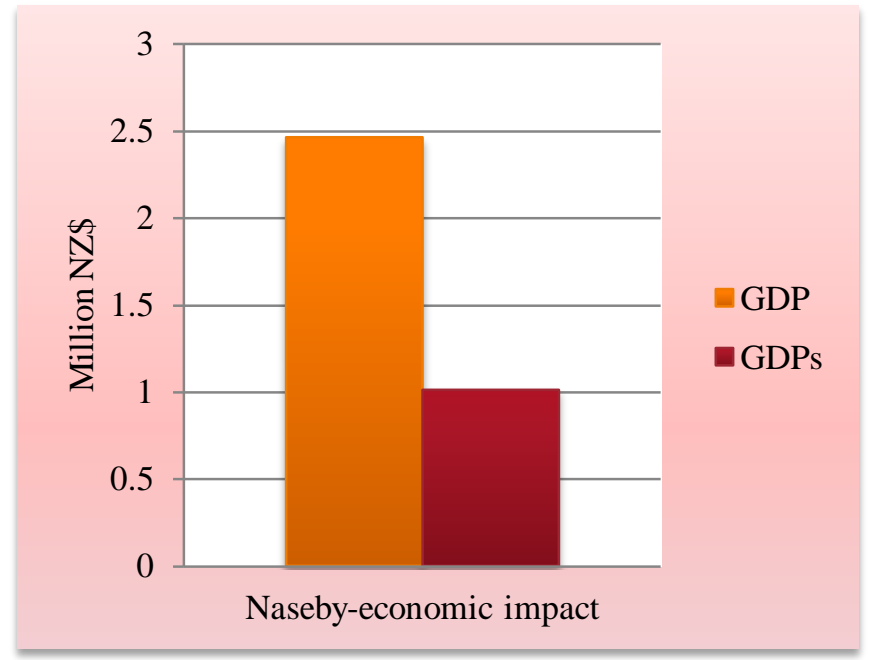

Figure 6.30: Naseby- comparison between GDP and GDPs 


\subsubsection{Naseby Cultural Footprint (CF)}

This thesis uses the CF model explained in Chapter 4 to explore the cultural footprint of the OCRT on Naseby as one of its host destinations. In this case study the CF of the Rail Trail in Naseby is categorised into the three areas of food, accommodation and visitor activities and, as previously explained in Chapter 5 (section 5.11), the CF of transportation is not included in this research.

\subsubsection{Naseby CF- Food}

Table 6.62 contains the summarised results of two proposed scenarios for Naseby food (sections 6.5.1 and 6.5.2). As shown in Table 6.62, the total conventional eaten out food in the first scenario is $30,936 \mathrm{~kg}$ ( $100 \%$ of food consumed) and in the second scenario it is $30,936 \mathrm{~kg}$ made up of $27286 \mathrm{~kg}$ (88.2\%) conventional food and 3,650 $\mathrm{kg}(11.8 \%)$ home prepared (organic food). As a result, the total EF of Naseby food in the first scenario is 167.22gha /year and in the second scenario this reduces to 160.9gha/ year (Table 6.62).

Table 6.62 indicates that in the second scenario the overshoot portion of the energy value of the food consumed is equivalent to 13,029 GJ/year. Since the EF of food in the first scenario (EF1) is 6.32 gha bigger than that of the second scenario (EF2), the overshoot energy value of food consumed in the first scenario is 632 GJ (6.32 gha $\times 100-$ global average carrying capacity of land). This is bigger than the overshoot portion of the energy value of food in the second scenario (13,029 GJ/year). Consequently the overshoot energy value of food in the first scenario is $13,029 \mathrm{GJ}+632 \mathrm{GJ}=$ 13,661GJ/year (Table 6.62).

In this section, the overshoot portions of EF1 of 136.61 gha (see Table 6.62) and EF2 of 130.29 gha (see Table 6.62) are calculated using the method explained in Chapter 5section 5.11.1. As the cost to generate 1GJ energy using wind-solar systems is NZ\$19.8, the cost to generate 13,661 GJ/year (overshoot portion of energy value of food in the first scenario) is $13,661 \mathrm{GJ} \times \mathrm{NZ} \$ 19.8=\mathrm{NZ} \$ 270,488$ (see Table 6.62). Likewise the total cost to generate the overshoot energy using wind-solar systems in the second scenario is NZ\$19.8× 13,029 GJ/year = NZ\$257,974 (Table 6.62). 
The total GDP of Naseby food is calculated using the GDP/kg food of OCRT multiplied by total Naseby food consumed (kg). As the total GDP for food related to the OCRT is NZ\$782,795 (see Table 5.91) and total produced/consumed food is 78,508.45 kg, the GDP of food related to OCRT per kilogram is equal NZ\$782,795 $\div 78,508.45 \mathrm{~kg}=9.97$ $\sim \mathrm{NZ} \$ 10 / \mathrm{kg}$.

In Naseby the total amount of food consumed is 30,936 kg (see Table 6.62). As a result the GDP of Naseby food is equivalent to $30,936(\mathrm{~kg}) \times \mathrm{NZ} \$ 10 / \mathrm{kg}$ of food $=$ NZ\$309,360 (Table 6.62).

Table 6.62 shows the GDP associated with food for both scenarios is NZ\$309,360. As a result:

GDPs1= NZ\$309,360 (GDP) - NZ\$270,488 (renewable energy cost) = NZ\$38,872;

GDPs2 = NZ\$309,360 (GDP) - NZ\$257,974 (renewable energy cost) = NZ\$ 51,386.

The difference between GDPs1 and GDPs2 shows the NZ\$12,514 contribution to GDPs from producing 3,650 kg home prepared (organic) food by Naseby accommodation services. In addition, it can be considered that producing $1 \mathrm{~kg}$ home prepared (organic) food contributes NZ\$3.43 to GDPs (Table 6.62).

\begin{tabular}{|c|c|c|c|}
\hline \multicolumn{4}{|c|}{ Table 6.62: Naseby- local product (Food) contribution to GDPs } \\
\hline \multicolumn{2}{|c|}{ First scenario (100\% conventional food) } & \multicolumn{2}{|c|}{$\begin{array}{l}\text { Second scenario (88.2\% conventional food and } \\
11.8 \% \text { home prepared (organic) food }\end{array}$} \\
\hline $\begin{array}{l}\text { Total conventional eaten out } \\
\text { food }(\mathrm{kg})\end{array}$ & $30,936^{1}$ & $\begin{array}{l}\text { Total conventional eaten out } \\
\text { food }(\mathrm{kg})\end{array}$ & $27,286^{1}$ \\
\hline $\begin{array}{l}\text { Total home prepared food } \\
\text { (kg) }\end{array}$ & 0.00 & $\begin{array}{l}\text { Total home prepared food } \\
\text { (kg) }\end{array}$ & $3,650^{1}$ \\
\hline $\begin{array}{l}\text { Total EF of consumed food } \\
\text { gha/year - (EF1) }\end{array}$ & $167.22^{1}$ & $\begin{array}{l}\text { Total EF of consumed food } \\
\text { gha - (EF2) }\end{array}$ & $160.9^{1}$ \\
\hline $\begin{array}{l}\text { Overshoot portion of energy } \\
\text { related to food (GJ/year) }\end{array}$ & 13,661 & $\begin{array}{l}\text { Overshoot portion of energy } \\
\text { related to food (GJ/year) }\end{array}$ & $13,029^{2}$ \\
\hline $\begin{array}{l}\text { Overshoot portion of EF1 } \\
\text { (gha/year) }\end{array}$ & 136.61 & $\begin{array}{l}\text { Overshoot portion of EF2 } \\
\text { (gha/year) }\end{array}$ & 130.29 \\
\hline $\begin{array}{l}\text { Cost to generate } 1 \mathrm{GJ} \text { energy } \\
\text { using wind-solar systems } \\
\text { (NZ\$) }\end{array}$ & 19.8 & $\begin{array}{l}\text { Cost to generate } 1 \mathrm{GJ} \text { energy } \\
\text { using wind-solar systems } \\
(\mathrm{NZ \$ )}\end{array}$ & 19.8 \\
\hline $\begin{array}{l}\text { Total cost to generate over- } \\
\text { shoot energy using wind-solar } \\
\text { systems (NZ\$) }\end{array}$ & 270,488 & $\begin{array}{l}\text { Total cost to generate over- } \\
\text { shoot energy using wind- } \\
\text { solar systems (NZ\$) }\end{array}$ & 257,974 \\
\hline Total food GDP (NZ\$) & 309,360 & Total food GDP (NZ\$) & 309,360 \\
\hline Total food GDPs1 & 38,872 & Total food GDPs2 & 51,386 \\
\hline $\begin{array}{l}\text { 1. See Table } 6.12 \\
\text { 2. See Table } 6.59 \\
\text { - Increased portion }\end{array}$ & 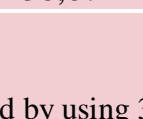 & & \\
\hline
\end{tabular}




\section{a. Ideal Sustainable Life Model: Food}

Table 6.62 shows that the present EF2 of Naseby food (second scenario) is 160.9 gha and the overshoot portion of EF2 is 130.29 gha. As a result the sustainable EF of Naseby food can be determined through the following equation:

160.9 gha $($ EF2, present EF) - 130.29 gha (overshoot portion of EF2) = 30.61gha In the ideal model for Naseby tourist related food, since the overshoot portion of energy use is zero, the GDPs is equal to the total GDP of Naseby food (NZ\$309,360, see Table 6.62).This study uses the ideal model of Naseby food and compares it with the two scenarios shown in Table 6.62.

In Figure 6.31, the area of 'Q1, EF1, and GDPs1' illustrates ordinary life; this is the area in which all Naseby visitors consume 100\% (30,936 kg) conventional eaten out food (first scenario). In this area, development of the Rail Trail (OCRT) does not exert any influence on Naseby (as one of the host destinations) and its visitors to produce and consume local foods. In Figure 6.31 the EF of using 100\% conventional food (EF1) is 167.22 gha / year and this represents the total food EF. In the ordinary life area, GDPs1 is equal to NZ\$38,872 (see Table 6.62).

Comparison between the first scenario and the ideal model of Naseby food consumption indicates that in the first scenario the EF1 (167.22 gha) is 81.7 \% bigger than the ideal EF (30.61 gha) (Figure 6.31 and Table 6.62). Moreover, as shown in Figure 6.31, in the first scenario, GDPs1 is equal to $12.56 \%$ of the total GDP of Naseby food. On the other hand, $87.44 \%$ (100\%- 12.56\%) of total GDP related to the food must be spent to change EF1 to the ideal EF through generating the overshoot portion of food energy by using wind-solar systems.

In Figure 6.31, the area of 'Q2, EF2, and GDPs2' determines the CF area of Naseby for producing and consuming home prepared (organic) foods. As demonstrated in Figure 6.31 total Q2 in the CF for food consumed by Naseby visitors (30,936 kg) is divided into the two portions of $11.8 \%(3,650 \mathrm{~kg})$ home prepared food (Q3) and $88.2 \%$ $(27,286 \mathrm{~kg})$ conventional eaten out food (Q 4). The EF in the CF (EF2) contains the EF of using $11.8 \%(3,650 \mathrm{~kg})$ home cooked and $88.2 \%(27,286 \mathrm{~kg})$ conventional eaten out foods. The GDPs2 in the CF is equal to NZ\$51,386 which is $4 \%(\mathrm{NZ} \$ 12,514)$ more than GDPs1 (NZ\$38,872) (Table 6.62). 
Figure 6.31 and Table 6.62 indicate that using 11.8\% home prepared (organic) food reduces total Naseby food EF by 3.8\% (shown in Figure 6.31 as R-EF1). Likewise, as shown in this figure, producing $11.8 \%$ local food increases GDPs1 by $4 \%$, shown in the figure as (I) GDPs1.

Comparison between the ideal model and present pattern of Naseby food consumption demonstrates that EF2 (160.7gha) is 77.9 \% (130.29 gha) more than ideal EF (30.61 gha), and $83.4 \%(\mathrm{NZ} \$ 257,974)$ of the GDP $(\mathrm{NZ} \$ 309,360)$ of food must be spent to reduce this overshoot portion of EF2 to the ideal EF (Table 6.62 and Figure 6.31).

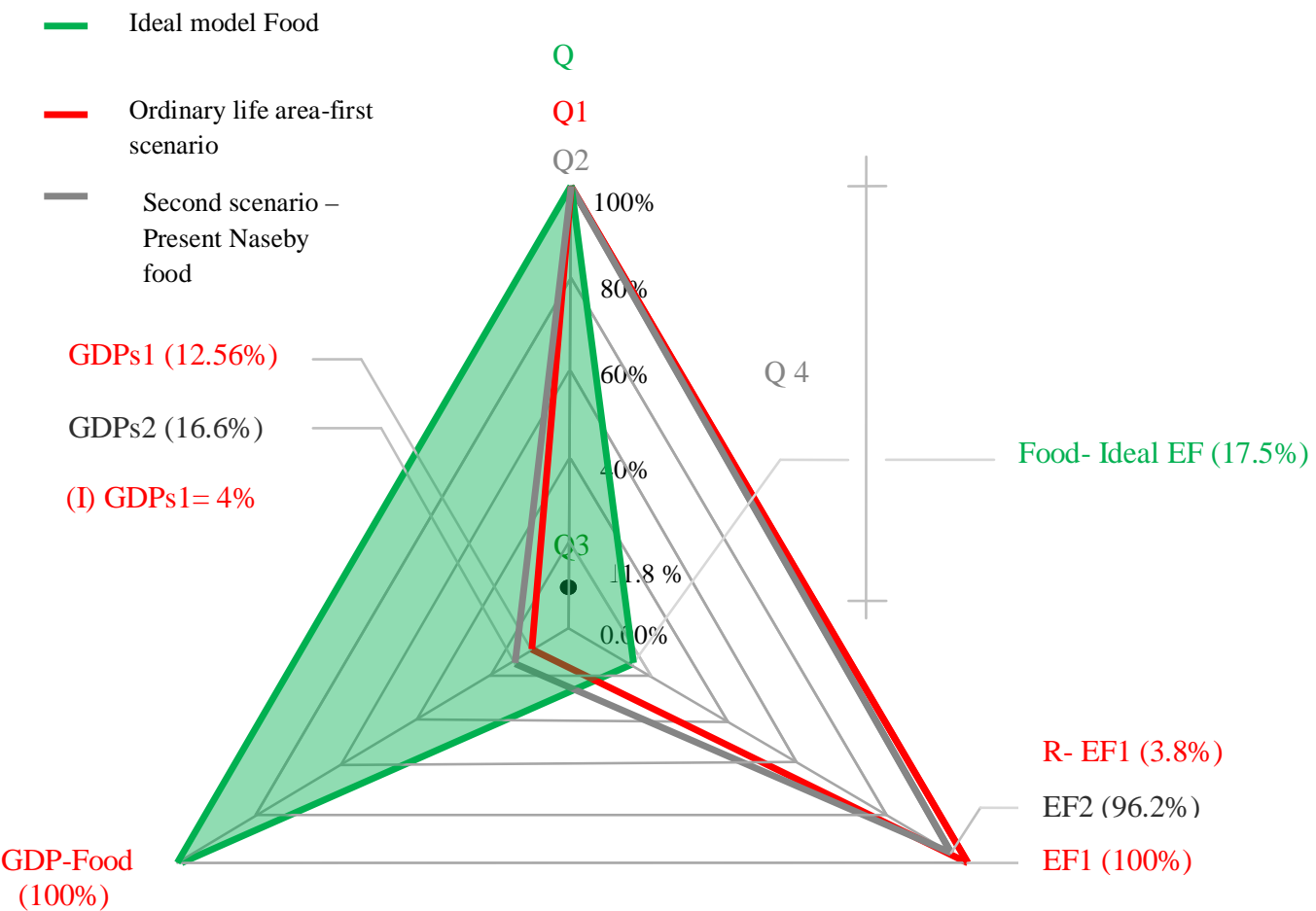

Figure 6.31: The cultural footprint (CF) of Naseby related to food

- Q: $100 \%$ food in Ideal model

- Q1: $100 \%$ conventional eaten out food - first scenario

- Q2: $88.2 \%$ conventional eaten out food $+11.8 \%$ home prepared food - second scenario

- Q3: $11.8 \%$ home prepared food (second scenario)

- Q4: 88.2\% conventional eaten out food (second scenario)

- GDPs1: sustainable portion of GDP - first scenario

- GDPs2: sustainable portion of GDP - second scenario

- (I) GDPs1: increased portion of GDPs1

- EF1: ecological footprint of consumed food - first scenario

- EF2: ecological footprint of consumed food - second scenario

- R- EF1: reduced portion of total EF1 of consumed food

\subsubsection{Naseby CF: Accommodation Services}

This thesis explores the CF of 98 Naseby accommodation services through three scenarios based on different quantities and qualities of accommodation services, their EFs and 
related GDPs values. In the first scenario, all buildings used as accommodation services are assumed to be newly constructed (NB) without open air areas (veranda and balcony). In the second scenario 6.1\% (6) of 98 Naseby accommodation buildings with an

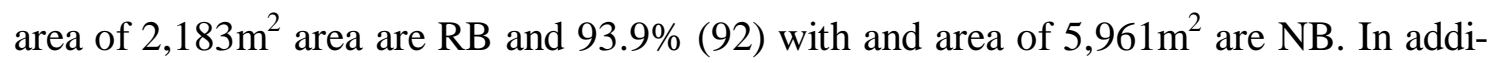
tion in the second scenario all areas of buildings including indoor and outdoor (V/B) spaces are calculated as indoor spaces. In the third scenario $93.9 \%$ of 98 buildings are $\mathrm{NB}, 6.1 \%$ of buildings are $\mathrm{RB}$ and $100.1 \mathrm{~m}^{2}$ of total building area is outdoor sitting space (V/B) (Table 6.64).

Table 6.63 shows that in the first scenario the total EF of using 100\% NB buildings as accommodation services is 7.6 gha. In this Chapter, the GDP of Naseby's accommodation services is calculated through the following equations:

GDP of OCRT/visitor nights $=2,837,412$ (total GDP of OCRT, see Table 5.91) $\div$ 42,437 (total OCRT visitor nights, see Table 5.9) = NZ\$ 66.86 per visitor night

GDP of Naseby accommodation services $=$ NZ\$66.86/visitor night $\times 16,722$ (Naseby visitor nights, see Table 6.9) = NZ\$1,118,033 (Table 6.63).

Since the overshoot portion of energy use in the third scenario has already been calculated as 567 GJ (see Tables 6.63 and 6.59), it is used here as a base to calculate the overshoot energy of the first and second scenarios through the method which has been explained in Chapter 5-section-5.11.2. Table 6.64 shows that the overshoot portions of energy for accommodation services in the first and the second scenarios are 625.8 GJ and $575.8 \mathrm{GJ}$ respectively.

Table 6.63 contains the total costs to generate the overshoot energy related to each of the three scenarios. These costs are calculated through using the method explained in Chapter 5-section.5.11.2. As shown in Table 6.63, the costs to generate the overshoot energy of the first, second and third scenarios in an environmentally sustainable way are NZ\$12,391, NZ\$11,401 and NZ\$11,227 respectively.

In this section, the GDPs of the three scenarios cited in Table 6.63 are calculated based on the method used in Chapter 5-section.5.11.2 for the GDPs of OCRT accommodation 
services. Table 6.63 determines that the GDPs of the third scenario (GDPs3) is the largest at NZ\$1,106,806 followed by NZ\$1,106,632 (GDPs2) and NZ\$1,105,642 (GDPs1).

\begin{tabular}{|c|c|c|c|c|c|}
\hline \multicolumn{6}{|c|}{ Table 6.63: Naseby Accommodation services CF (2011) } \\
\hline \multicolumn{2}{|c|}{ First scenario } & \multicolumn{2}{|c|}{ Second scenario } & \multicolumn{2}{|c|}{ Third scenario } \\
\hline \multicolumn{2}{|c|}{$\begin{array}{l}\text { All Naseby accommodation } \\
\text { buildings are NB }\end{array}$} & \multicolumn{2}{|c|}{$\begin{array}{l}93.9 \% \text { of } 98 \text { Naseby accommo- } \\
\text { dation buildings are NB and } 6.1 \\
\% \text { are RB (V/B not considered) }\end{array}$} & \multicolumn{2}{|c|}{$\begin{array}{c}93.9 \% \text { of 98buildings are NB } \\
\text { and } 6.1 \% \text { are RB including } \\
100.1 \mathrm{~m}^{2} \mathrm{~V} / \mathrm{B}\end{array}$} \\
\hline $\begin{array}{l}\text { Total number of } \\
\text { accommodation ser- } \\
\text { vices }\end{array}$ & $98^{1}$ & $\begin{array}{l}\text { Total number of ac- } \\
\text { commodation ser- } \\
\text { vices }\end{array}$ & 98 & $\begin{array}{l}\text { Total number of } \\
\text { accommodation } \\
\text { services }\end{array}$ & 98 \\
\hline $\begin{array}{l}\text { NB accommodation } \\
\text { area }\left(\mathrm{m}^{2}\right)\end{array}$ & $8,144^{2}$ & $\begin{array}{l}\text { NB accommodation } \\
\text { area }\left(\mathrm{m}^{2}\right)\end{array}$ & $5,961^{2}$ & $\begin{array}{l}\text { NB accommoda- } \\
\text { tion area }\left(\mathrm{m}^{2}\right)\end{array}$ & $5,961^{2}$ \\
\hline $\begin{array}{l}\text { RB accommodation } \\
\text { area }\left(\mathrm{m}^{2}\right)\end{array}$ & 0.00 & $\begin{array}{l}\text { RB accommodation } \\
\text { area }\left(\mathrm{m}^{2}\right)\end{array}$ & $2,592^{2}$ & $\begin{array}{l}\text { RB accommoda- } \\
\text { tion area }\left(\mathrm{m}^{2}\right)\end{array}$ & $2,592^{2}$ \\
\hline Total EF (EF1) (gha) & $7.6^{3}$ & Total EF (EF2) (gha) & $7.1^{4}$ & $\begin{array}{l}\text { Total EF (EF3) } \\
\text { (gha) }\end{array}$ & 7.012 \\
\hline GDP (NZ\$) & $1,118,033$ & GDP (NZ\$) & $1,118,033$ & GDP (NZ\$) & $1,118,033$ \\
\hline $\begin{array}{l}\text { Overshoot energy } \\
\text { (GJ/year) }\end{array}$ & 625.8 & $\begin{array}{l}\text { Overshoot energy } \\
\text { (GJ/year) }\end{array}$ & 575.8 & $\begin{array}{l}\text { Overshoot energy } \\
\text { (GJ/year) }\end{array}$ & 567 \\
\hline $\begin{array}{l}\text { Cost to generate } 1 \\
\text { GJ energy using } \\
\text { wind-solar systems } \\
\text { (NZ\$) }\end{array}$ & 19.8 & $\begin{array}{l}\text { Cost to generate } 1 \mathrm{GJ} \\
\text { energy using wind- } \\
\text { solar systems (NZ\$) }\end{array}$ & 19.8 & $\begin{array}{l}\text { Cost to generate } 1 \\
\text { GJ energy using } \\
\text { wind-solar systems } \\
\text { (NZ\$) }\end{array}$ & 19.8 \\
\hline $\begin{array}{l}\text { Cost to generate } \\
625.8 \text { GJ energy } \\
\text { using wind-solar } \\
\text { systems (NZ\$) }\end{array}$ & 12,391 & $\begin{array}{l}\text { Cost to generate } \\
575.8 \text { GJ energy us- } \\
\text { ing wind-solar sys- } \\
\text { tems (NZ\$) }\end{array}$ & 11,401 & $\begin{array}{l}\text { Cost to generate } \\
567 \mathrm{GJ} \text { energy } \\
\text { using wind-solar } \\
\text { systems (NZ\$) }\end{array}$ & 11,227 \\
\hline GDPs1 & $1,105,642$ & GDPs2 & $1,106,632$ & GDPs3 & $1,106,806$ \\
\hline $\begin{array}{l}\text { 1. See Table } 5.15 \\
\text { 2. See Table } 5.23 \\
\text { 3. See Table } 5.36 \\
\text { 4. See Table } 5.37\end{array}$ & & & & & \\
\hline
\end{tabular}

\section{a. Sustainable living OCRT Accommodation Services (Ideal Model)}

This section determines an ideal model for Naseby accommodation services in which its EF is considered environmentally sustainable and its GDPs is equal to the total GDP of Naseby accommodation services. This model is then used to compare each of the three scenarios for Naseby accommodation services (cited in Table 6.63) with an environmentally and economically sustainable pattern of accommodation.

\section{a.1. Sustainable EF}

As shown in Table 6.63 the total overshoot energy of Naseby accommodation services at present is $567 \mathrm{GJ} /$ year. As a result the overshoot portion of its $\mathrm{EF}$ is $567 / 100=5.67$ gha. As indicated in Table 6.63, the present EF of Naseby is 7.012 gha (EF3). Consequently the environmentally sustainable EF can be considered as 7.012 gha $-5.67=$ 1.34 gha. 


\section{a.2 . Sustainable Living: GDPs}

Since for sustainable living the EF of accommodation services is not in overshoot, consequently in this position both the overshoot energy and the cost to generate this through using wind-solar systems are zero. Consequently the GDPs of the Ideal Model is equal to the total GDP of Naseby accommodation services (NZ\$1,118,033, see Table 6.63).

\section{b. Comparison between the Ideal Model, First and Second Scenarios}

Figure 6.32 comprises the quantities, EFs and GDPs(s) of Naseby accommodation services arising from the Ideal model, first, second, and third scenarios discussed above. This figure makes this an opportunity to explore the ecological and economic influences exerted by using $2,183 \mathrm{~m}^{2}$ (26.8\% of the total $8,144 \mathrm{~m}^{2}$, see Table 6.19 ) of accommodation area as refurbished (RB) buildings on the host destination.

As shown in Figure 6.32, in the Ideal model of accommodation services, Q is the $100 \%$ Naseby accommodation services area $\left(8,144 \mathrm{~m}^{2}\right)$ with an EF of 1.34 gha and GDPs of NZ\$1,118,033 GDPs. In the ideal model as discussed above, the overshoot portion of the EF and energy use are equal to zero. In first scenario, Q1 is the total area of Naseby accommodation services $\left(8,144 \mathrm{~m}^{2}\right)$ where $100 \%$ are assumed to be new buildings (NB) (Figure 6.23). In the second scenario as illustrated in Figure 6.32, Q2 represents the Naseby accommodation area $\left(8,144 \mathrm{~m}^{2}\right)$ divided into two portions of $73.2 \%\left(5,961 \mathrm{~m}^{2}\right.$, see Table 6.19) NB and 26.8\% (2,183 $\mathrm{m}^{2}$, see Table 6.19) RB buildings.

In Figure 6.32, EF1 is the biggest EF in comparison with the EFs of the other scenarios and is here considered to be the $100 \%$ value. In addition, in this figure, the percentages of other EFs including EF2 and Ideal- EF are compared with EF1 as the 100\% benchmark.

As shown in Figure 6.32, in the ideal model of Naseby accommodation services, the acceptable EF of these services is 17.6\% of EF1 (7.6gha, see Table 6.63).On the other hand, in the first scenario in which all accommodation is assumed to be NB, the EF (EF1= 7.6gha) is 82.4\% (6.26gha) larger than the sustainable EF (1.34gha) (Figure 6.32). 
As illustrated in Figure 6.32, the EF of Naseby accommodation services in the second scenario (EF2 = 7.1 gha, see Table 6.64) is 93.4\% of EF1 (7.6gha, see Table 6.63). Consequently (R) EF1 is equal to $6.6 \%$ of EF1= 0.5 gha (Figure 6.23).

GDPs1 (GDPs of the first scenario $=$ NZ\$ 1,105,642) is equal to $98.9 \%$ of total GDP of Naseby -accommodation services (NZ\$1,118,033) (Figure 6.32). On the other hand, in the first scenario $1.1 \%(\mathrm{NZ} \$ 12,298)$ of the total GDP of Naseby accommodation services must be spent to generate the overshoot portion of accommodation energy use through wind-solar systems to change EF1 to the ideal EF.

As shown in Figure 6.32, GDPs2 (NZ\$1,106,632, the GDPs of the second scenario) is $99 \%$ of the total GDP of Naseby accommodation (NZ\$1,118,033). On the other hand in the second scenario 1\% (NZ\$11,180) of the total GDP of Naseby accommodation services must be spent to generate the overshoot portion of associated energy use through using wind-solar systems to reduce EF2 to the ideal EF. Likewise, as determined in Figure 6.32, (I) GDPs1 is equal to $0.1 \%$ of the total GDP of Naseby accommodation services. This means using 2,183 $\mathrm{m}^{2} \mathrm{RB}$ (26.8\% of total Naseby accommodation areas) contributes to an increase of $0.1 \%$ of GDPs1.

\section{c. Comparison between the Ideal Model, Second and Third Scenarios}

In third scenario Q3 is total area of Naseby accommodation services in which 73.2\% $\left(5,961 \mathrm{~m}^{2}\right)$ is NB and $26.8 \%\left(2,183 \mathrm{~m}^{2}\right)$ is RB (Figure 6.32 and Table 6.63). Furthermore, in the third scenario, of total Q3 $\left(8,144 \mathrm{~m}^{2}\right) 100.1 \mathrm{~m}^{2}(1.2 \%)$ is $\mathrm{V} / \mathrm{B}$ and $8,043.9 \mathrm{~m}^{2}$ (98.8\%) is indoor spaces (Figure 6.32).

In the third scenario, the total EF of Naseby accommodation services (EF3) is 7.012 gha (see Table 6.63), which is $92.3 \%$ of EF1 (Figure 6.32). In addition, as shown in Figure 6.32, EF3 (92.3 \% of EF1) is 1.1\% less than EF2 (93.4\% of EF1). On the other hand (R) $\mathrm{EF} 2$ is equivalent to $(\mathrm{EF} 2)-(\mathrm{EF} 3)=1.1 \%$ of $\mathrm{EF} 1(\mathrm{EF}$ of the first scenario that is used as a base, representing the $100 \%$ EF of Naseby accommodation services). Thus EF3 is the present EF of Naseby accommodation services, which is $74.94 \%$ (92.3\%- $17.36 \%)$ bigger than the Ideal EF shown in Figure 6.32.

Figure 6.32 and Table 6.63 show that the GDPs of the third scenario (GDPs3), which is the present GDPs of Naseby accommodation services, is 0.01\% more than GDPs2. Fur- 
thermore, GDPs3 is 1.01\% (NZ\$11,292) less than the total GDP $(\mathrm{NZ} \$ 1,118,033)$ of Naseby accommodation services (Figure 6.32 and Table 6.63). This means (I) GDPs2 which is influenced by using $100.1 \mathrm{~m}^{2} \mathrm{~V} / \mathrm{B}$ is $0.01 \%$ of the total GDP of Naseby accommodation services. In addition, Figure 6.23 shows that in the third scenario (present circumstance of Naseby accommodation services), 1.01\% (NZ\$11,292) of the GDP of Naseby accommodation services must be spent to generate its overshoot portion of energy use through using wind-solar systems to reduce its present EF to the ideal EF.

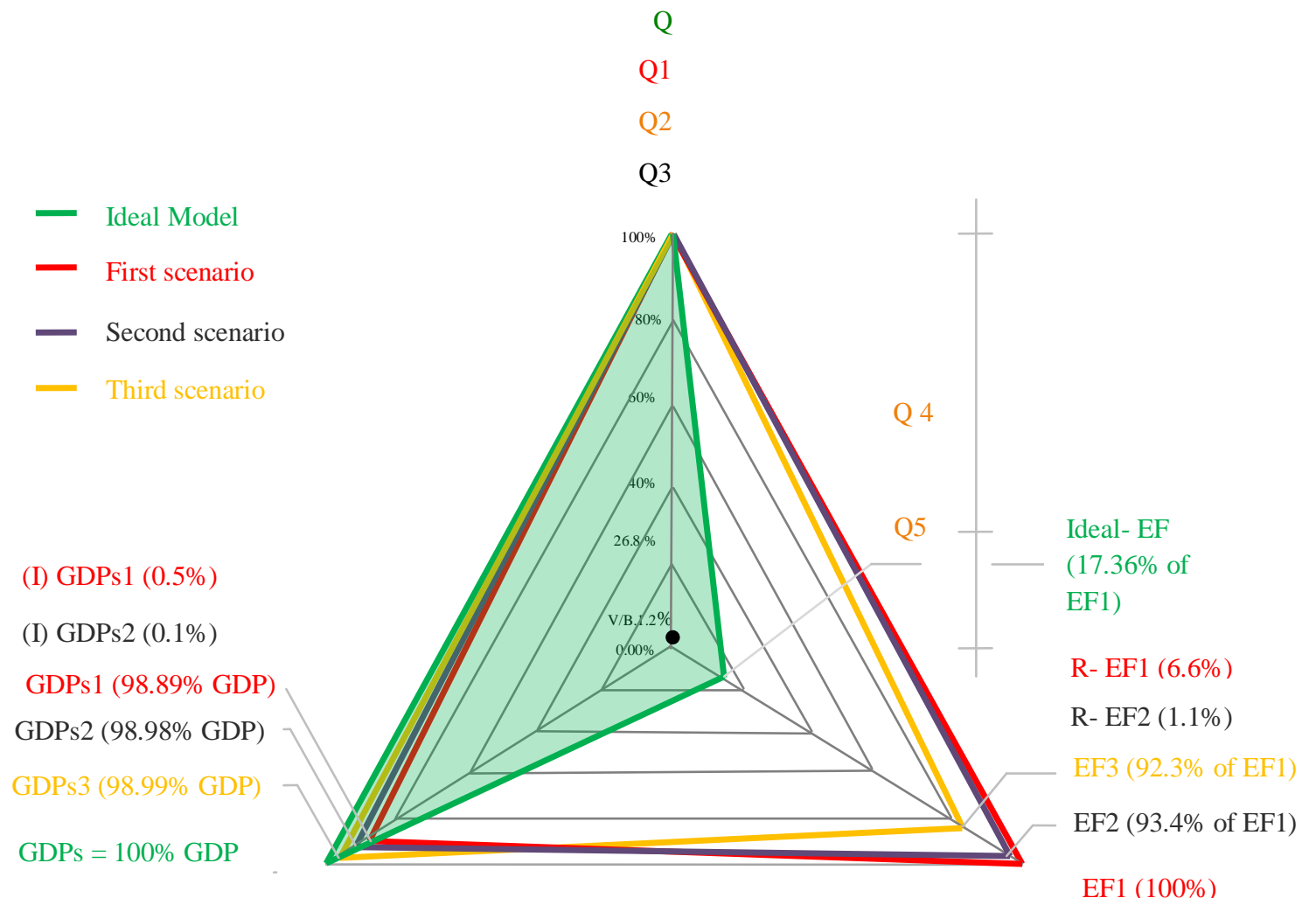

Figure 6.32: The CF of Naseby accommodation services using RB buildings and $\mathrm{V} / \mathrm{B}$ as part of accommodation services

- Q: (100\%) area of 98 Naseby accommodation services $(8,144 \mathrm{~m} 2)$ - Ideal model

- GDP: GDP of Naseby-accommodation services NZ\$1,118,033 (see Table 6.62)

- GDPs: Total sustainable portion of Naseby accommodation services - Ideal model

- Q1: Total (100\%) area of 98 Naseby accommodation services $\left(8,144 \mathrm{~m}^{2}\right)$ - all assumed as NB (first scenario)

- EF1: Total EF of Naseby accommodation services (first scenario)

- (R) EF1: Reduced portion of Naseby EF1 influenced by using RB buildings as accommodation services.

- GDPs1: Total GDPs of Naseby accommodation services (first scenario)

- (I) GDPs1: Increased portion of GDPs1 influenced by using RB as part of Naseby accommodation services

- Q2: Area 98 Naseby accommodation services $\left(8,1446 \mathrm{~m}^{2}\right)$ including; $73.2 \%\left(5,961 \mathrm{~m}^{2}\right) \mathrm{NB}$ and $26.8 \%\left(2,183 \mathrm{~m}^{2}\right) \mathrm{RB}$ (second scenario)

- Q3: Area of 98 Naseby accommodation services $\left(8,144 \mathrm{~m}^{2}\right)$ including; $73.2 \%\left(5,961 \mathrm{~m}^{2}\right) \mathrm{NB}$ and $26.8 \%\left(2,183 \mathrm{~m}^{2}\right) \mathrm{RB}$. Of total Q3 $\left(8,144 \mathrm{~m}^{2}\right), 100.1 \mathrm{~m}^{2}(1.2 \%)$ is V/B and $8,043.9 \mathrm{~m}^{2}$ is indoor spaces (third scenario) 
- $\quad$ Q 4 (NB area): $73.2 \%\left(5,961 \mathrm{~m}^{2}\right)$ of total Naseby accommodation area $\left(8,144 \mathrm{~m}^{2}\right)$ (second scenario)

- $\quad$ Q5 (RB area): $26.8 \%\left(2,183 \mathrm{~m}^{2}\right)$ of total Naseby accommodation area $\left(8,144 \mathrm{~m}^{2}\right)$ (second scenario)

- $\quad$ EF2: EF of 8,144 $\mathrm{m}^{2}$ Naseby accommodation services (Q2) including EFs of Q3 and Q 4

- (R) EF2: Reduced portion of Naseby EF2 influenced by using V/B

- GDPs2: Total GDPs of Naseby accommodation services (second scenario)

- EF3: EF of Naseby accommodation services (third scenario) (present EF of accommodation services)

\subsubsection{The CF of Naseby Visitor Activities}

\section{a. Overshoot EF of Naseby Visitor Activities}

As shown in Table 6.64, the category of other activities has the largest portion at $71 \%$ (2.13gha) of the total EF of Naseby visitor activities (3.02 gha), followed by curling (23\%), golf $(0.18 \%)$ and walking $(0.05 \%)$.Table 6.64 shows that the total overshoot portion of the EF of Naseby activities is 2.55 gha (see also Table 6.59). The overshoot portions of the EFs of the four activity groups above are calculated as follows:

Overshoot portion of the EF of each activity category = the activity percentage of the EF $\times$ total overshoot EF of Naseby activities (2.55 gha)

For example:

Overshoot $\mathrm{EF}$ of golf $=6 \% \times 2.55($ gha $)=0.15$ gha $($ see Table 6.64$)$.

In addition the overshoot energy use of each category is calculated as its overshoot EF $\times$ 100 (global average carrying capacity) (Table 6.64). As shown in Table 6.64, the category of other activities has the largest overshoot energy of 180 GJ, followed by 59 GJ curling, 15 golf and $0.1 \mathrm{GJ}$ walking.

\section{b. Naseby Activities GDP}

This chapter uses the average GDP of OCRT activities per visitor (NZ\$56.88) cited in Chapter 5- Table 5.98 as Naseby GDP per visitor activity (Table 6.64). As shown in Table 6.64 the GDP of each category is calculated as the number of visitor activities $\times$ NZ\$56.88/visitor activity. Table 6.64 demonstrates that of the NZ\$744,446 GDP of Naseby activities (13,088 visitor activities $\times$ NZ\$56.88 GDP/visitor activity), the largest portion of NZ $\$ 492,012$ is the GDP of other visitor activities, followed by NZ $\$ 247,428$ (curling), NZ\$ 3,413 (golf) an NZ\$1,593 walking.

\section{c. Naseby Activities GDPs}

As demonstrated in Table 6.64 the overshoot energy use of each category is calculated as its overshoot EF multiplied by $100 \mathrm{GJ} /$ gha (global average carrying capacity of land). 
Also as shown, the category of other activities the largest overshoot of $180 \mathrm{GJ}$, followed by curling (59 GJ), golf (15) and walking (0.1GJ).

This chapter calculates the cost to generate the overshoot portion of the energy of each category of Naseby activities through using the method explained in Chapter 5- section 5.10.2. Table 6.64 contains the total cost that must be paid to generate the overshoot energy of each type of Naseby activity through using wind-solar systems. As presented in Table 6.64, the category of other activities has the largest cost of NZ\$ 3,510 and walking the minimum cost ( $\$ N Z 2 \sim 0.00$ ) to generate the overshoot energy in a sustainable way. The total cost of Naseby activities overshoot energy as shown in Table 6.64 is \$NZ4, 948.

The GDPs of each category of Naseby visitor activities (GDP - cost of overshoot energy used) are set out in Table 6.64. As shown, the GDPs of the category of activities has the largest GDPs of NZ\$488,502 and other activities including curling, golf and walking have the GDPs in a range of NZ\$1,591 - NZ\$246,277. In addition, the total GDPs of Naseby visitor activities is NZ\$739,498, equivalent to $99.33 \%$ of its total GDP (\$NZ744, 446) (Table 6.64).

\begin{tabular}{|c|c|c|c|c|c|c|c|c|}
\hline \multicolumn{9}{|c|}{ Table 6.64: Naseby visitor activities- EF and GDPs } \\
\hline Category & $\begin{array}{l}\text { Number } \\
\text { of visi- } \\
\text { tors/acti } \\
\text { vity }\end{array}$ & $\begin{array}{l}\mathrm{EF}^{1} \\
\text { (gha) }\end{array}$ & $\%^{4}$ & $\begin{array}{l}\text { Overshoot } \\
\text { EF (gha) }\end{array}$ & $\begin{array}{c}\text { Overshoot } \\
\text { energy }(G J)^{2}\end{array}$ & $\begin{array}{c}\text { GDP } \\
(\mathrm{NZ \$})^{2}\end{array}$ & $\begin{array}{l}\text { Cost of } \\
\text { over- } \\
\text { shoot } \\
\text { energy } \\
(\mathrm{NZ} \$)^{2}\end{array}$ & $\begin{array}{l}\text { GDPs } \\
(\mathrm{NZ \$ )}\end{array}$ \\
\hline Golf & 60 & 0.18 & 6 & 0.15 & 15 & 3,413 & 285 & 3128 \\
\hline Curling & 4,350 & 0.7 & 23 & 0.59 & 59 & 247,428 & 1,151 & 246,277 \\
\hline Walking & 28 & 0.0014 & 0.05 & 0.001 & 0.1 & 1,593 & 2 & 1,591 \\
\hline Other & 8,650 & 2.13 & 71 & 1.8 & 180 & 492,012 & 3,510 & 488,502 \\
\hline Total & 13,088 & 3.02 & 100 & $2.55^{3}$ & 255 & 744,446 & 4,948 & 739,498 \\
\hline \multicolumn{9}{|c|}{$\begin{array}{l}\text { 1. See Figure } 6.27 \text { and Table } 6.55 \\
\text { 2. Numbers are rounded } \\
\text { 3. See Table } 6.59 \\
\text { 4. percentages are rounded } \\
\text { - Average GDP of OCRT visitor activity = Total GDP of OCRT activities/visitor activities }=1483,180 \div 26,077 \\
=\text { NZ } \$ 56.88 \text { (see Chapter } 5 \text { : Table } 5.98 \text { ). }\end{array}$} \\
\hline
\end{tabular}

\section{d. CF: Ideal Model for Sustainable Activities and Three Scenarios}

This chapter proposes three scenarios for the CF of Naseby visitor activities (illustrated in Figure 6.33) and compares the areas generated with each other and the sustainable area (Ideal model) of these activities (shown as the green area in Figure 6.33). 


\section{d.1. Naseby Visitor Activities (Ideal model)}

This thesis considered the sustainable area for ecotourism activities to be the area in which the overshoot portion of the EF of activities is zero and consequently the GDPs of this area is equal with the total GDP arising from these activities under that constraint.

As shown in Table 6.64, the present EF of Naseby visitor activities is 3.02 gha and its overshoot EF is 2.55 gha. The EF of the Ideal model for Naseby visitors activities (zero overshoot EF) is 3.02gha -2.55 gha $=0.47$ gha. This means the ecologically sustainable EF of Naseby visitors activities (0.47 gha) is $15.6 \%$ of its present EF (EF1= 3.02gha). Moreover, in the sustainable area of Naseby visitors activities, as the cost of overshoot energy is zero, its GDPs is equal to $100 \%$ of the GDP of Naseby visitor activities (\$NZ744,446, see Table 6.64).

\section{d.2. Naseby Visitor Activities (First Scenario)}

The present CF of Naseby visitor activities is the same as the first scenario shown in Figure 6.33. In the first scenario the total number of visitor activities $(13,088)$ comprises 8,650 visitors doing other activities, 4,350 visitors curling, 60 visitors playing golf, and 28 visitors walking (see Table 6.65). (Note: the number of activities is greater than the number of visitors to Naseby, as each visitor is assumed to participate in more than one activity).

The total EF of Naseby visitors' activities (EF1) in the first scenario is 3.02 gha (see Table 6.65) and this is used as the $100 \%$ benchmark when comparing this with other scenarios and the Ideal model (see Figure 6.24). As shown in Figure 6.33 and Table 6.64 in the first scenario GDPs1 (present situation, Table 6.64) is equal to $99.33 \%$ $(\mathrm{NZ} \$ 739,498)$ of the total GDP of Naseby visitor activities $(\mathrm{NZ} \$ 744,446)$.

\section{d.3. OCRT Visitor Activities (Second Scenario)}

The second scenario is proposed based on the assumption that the 60 visitors who play golf in the first scenario choose walking instead (see Table 6.65). In the second scenario, walking is assumed to have an EF of 0.00006gha/visitor, see Table 5.82). 


\begin{tabular}{|c|c|c|c|c|c|c|c|}
\hline \multicolumn{4}{|c|}{ First scenario } & \multicolumn{4}{|c|}{ Second scenario } \\
\hline Category & $\mathrm{NV}^{4}$ & EF/visitor & EF1 & Category & NV & $\begin{array}{c}\text { EF } \\
\text { (gha/visitor) }\end{array}$ & EF2 (gha) \\
\hline Golf & 60 & $0.003^{1}$ & 0.18 & - & - & & - \\
\hline Curling & 4,350 & $0.00016^{2}$ & 0.7 & Curling & 4,350 & $0.00016^{2}$ & 0.7 \\
\hline Walking & 28 & $0.00005^{3}$ & 0.0014 & Walking & 88 & $0.00005^{3}$ & 0.0044 \\
\hline Other & 8,650 & 0.00025 & 2.13 & Other & 8,650 & 0.00025 & 2.13 \\
\hline Total & 13,088 & - & 3.02 & Total & 13,088 & - & 2.83 \\
\hline $\begin{array}{l}\text { 1. See Tabl } \\
\text { 2. See Tabl } \\
\text { 3. See Table } \\
\text { 4. NV: Nun }\end{array}$ & $\begin{array}{l}58 \\
.35 \\
.56 \\
\text { r of vis }\end{array}$ & ities & .64) & & & & \\
\hline
\end{tabular}

As shown in Table 6.65 and Figure 6.33 in comparison with the first scenario, in the second scenario the EF2 is reduced by 0.19 gha compared to EF1 (3.02 gha), a $6.3 \%$ reduction just from 60 visitors changing their activity from golfing to walking. In addition, the overshoot portion of the EF of Naseby activities that in the first scenario is 2.55 gha (see Table 6.64) in the second scenario is reduced to (2.55 gha - 0.19 gha) 2.36 gha. As a result the overshoot energy of Naseby activities in the second scenario is 2.36 gha $\times 100$ (carrying capacity of land) $=236$ GJ. Moreover, the total cost to generate the overshoot energy of Naseby visitor activities in the second scenario is:

236 GJ (overshoot portion of energy) $\times \mathrm{NZ} \$ 19.8$ (cost to generate 1GJ energy using wind-solar systems) = NZ\$4,672.

Since the total GDP of Naseby visitor activities is NZ\$744,445 (Table 6.64), the GDPs of the second scenario is $\mathrm{NZ} \$ 744,446$ (GDP) - NZ\$4,672 (the total cost to generate overshoot energy in the second scenario) $=$ NZ\$739,773 = GDPs2. As shown in Figure 6.33, GDPs2 is $99.37 \%$ of the total GDP of Naseby visitor activities $(\$ N Z 744,445)$ and

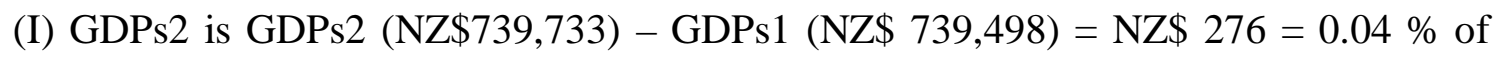
GDP $(\mathrm{NZ} \$ 744,446)$.

\section{d.4. Comparison between Ideal Model and First and Second Scenarios}

In comparison with the EFs of the two scenarios (EF1 and EF2), the EF of the Ideal model (0.47gha, see section 6.13.3.4-a) is $15.56 \%$ of EF1 (3.02gha) and $16.60 \%$ of EF2 (2.83 gha, see Table 6.65) (Figure 6.33). The proposed CF model in Figure 6.33 determines that for changing EF1 (3.02 gha) to the ideal EF (0.47gha), $0.66 \%$ (\$NZ 4984) of the total GDP of Naseby visitor activities must be spent to generate the overshoot portion of its energy. Likewise the model (Figure 6.33) indicates that for changing the EF of Naseby visitor activities in the second scenario (EF2) to a sustainable EF, 
0.62\% (NZ\$ 4,672) of the total GDP of Naseby visitor activities $(\mathrm{NZ} \$ 744,446)$ must be spent to reduce the environmental impact.

\section{d.5. Naseby Visitor Activities (Third Scenario)}

The third scenario is proposed based on the assumption that 60 visitors who play golf and 4,350 visitors who play curling in the first scenario (see Table 6.65) choose walking as their activity instead (see Table 6.66).

As shown in Table 6.66 and Figure 6.33, in comparison with the first scenario, in the third scenario, the EF3 is reduced by $77.81 \%$ (2.35 gha) compared to EF1. Furthermore, the difference between EF2 and EF3 (2.83 - 2.35=0.48gha) that is equal to $15.9 \%$ of EF1 (100\% of EF) determines the reduced portion of EF2 influenced by changing curling and golfing for walking at Naseby. This portion (15.9\%) is shown as (R) EF2 in Figure 6.33. In addition, the overshoot portion of the EF of Naseby activities that in the first scenario is 2.55 gha (see Table 6.63) in the third scenario is reduced to (2.55 gha 0.48 gha) 2.07 gha. As a result the overshoot energy use of Naseby activities in third scenario is equal to 2.07 gha $\times 100$ (carrying capacity of land) $=207$ GJ. In addition, the total cost to generate the overshoot energy used in third scenario is equal to:

207 GJ (overshoot portion of energy use) × NZ\$19.8 (the cost to generate 1GJ energy using wind-solar systems) $=$ NZ\$ 4,098.

Since the total GDP of Naseby visitor activities is NZ\$744,446 (see Table 6.63) consequently, the GDPs of the third scenario is NZ\$744,446 (GDP) - NZ\$4,098 (the total cost to generate overshoot energy in the third scenario) $=$ NZ\$740,348 $=$ GDPs3. As shown in Figure 6.33, GDPs3 is $99.45 \%$ of the total GDP of Naseby visitors activities and (I) GDPs3 is GDPs3 - GDPs2 $=0.08 \%$ of GDP $(\mathrm{NZ \$ 744,445)}=\mathrm{NZ \$} 615$.

\section{d.6. Comparison between the Ideal Model and the Third Scenario}

As Figure 6.33 illustrates, the EF of the Ideal model (1.04 gha, see section 6.13.3.4-a) is equal to $44.8 \%$ of EF3 (2.32 gha, see Table 6.66). The proposed CF model in Figure 6.33 determines that for changing EF3 (2.35gha) to the ideal EF (0.47 gha), $0.55 \%$ $(\$ N Z 4,098)$ of the total GDP of Naseby visitor activities must be spent to generate the overshoot portion of its energy. 
Table 6.66: The CF of Naseby visitor activities - first and third scenarios

\begin{tabular}{l|l|c|c|c|c|c|c|}
\hline \multicolumn{4}{|c|}{ First scenario } \\
\hline Category & NV $^{4}$ & EF/visitor & EF1 & Category & NV & $\begin{array}{c}\text { EF } \\
\text { (gha/visitor) }\end{array}$ & EF3 (gha) \\
\hline Golf & 60 & $0.04^{1}$ & 0.18 & - & - & - & - \\
\hline Curling & 4,350 & $0.00016^{2}$ & 0.7 & - & - & - & - \\
\hline Walking & 28 & $0.00005^{3}$ & 0.0014 & Walking & 4,438 & $0.00005^{3}$ & 0.22 \\
\hline Other & 8,650 & 0.00025 & 2.13 & Other & 8,650 & 0.00025 & 2.13 \\
\hline Total & 13,088 & - & 3.02 & Total & 13,088 & - & 2.35 \\
\hline $\begin{array}{l}\text { 1. See Table 5.58 } \\
\text { 2. See Table 6.35 }\end{array}$ \\
$\begin{array}{l}\text { 3. See Table 5.56 } \\
\text { 4. NV: Number of visitor activities (see Table 6.62) }\end{array}$
\end{tabular}

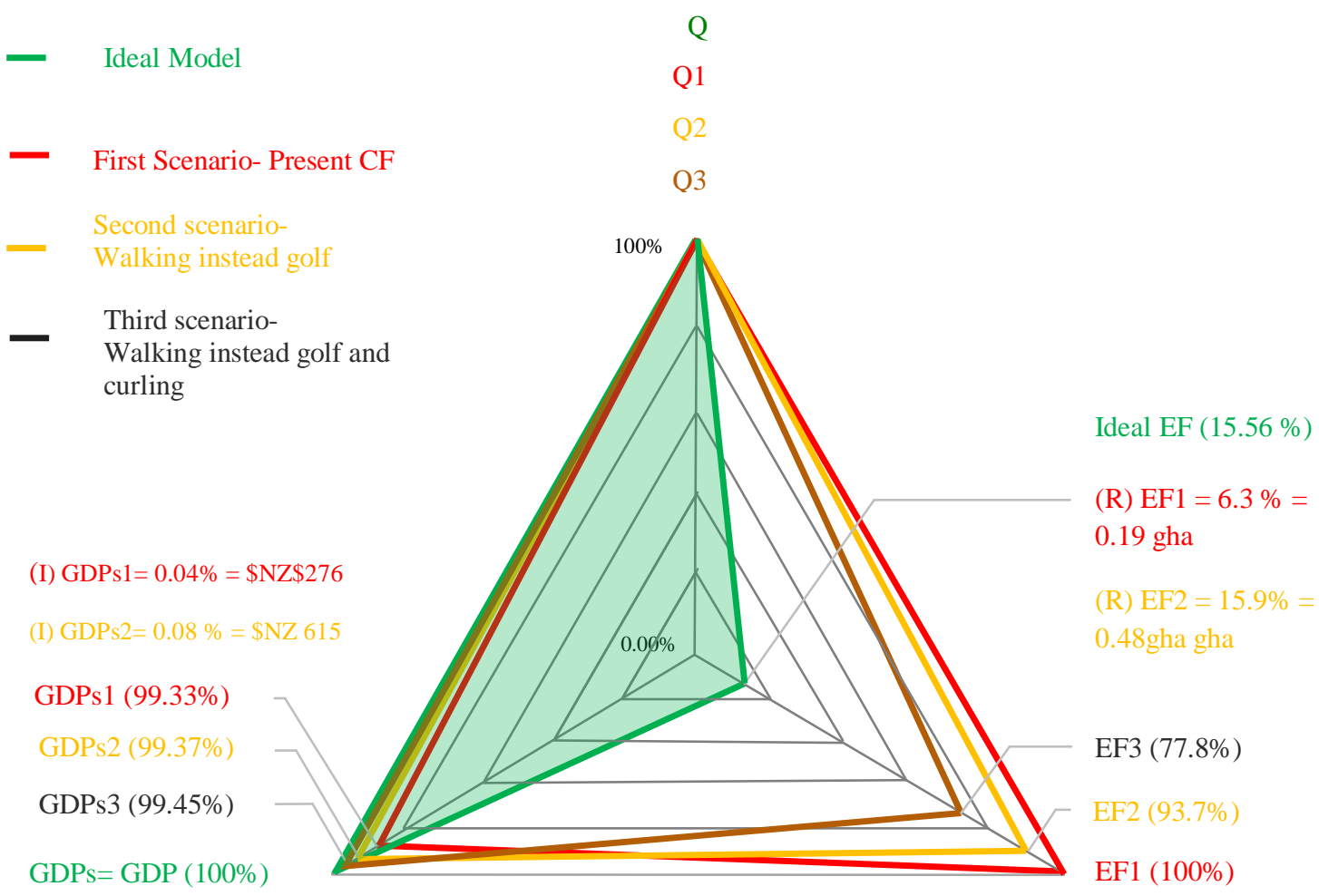

Figure 6.33: The CF of Naseby visitor activities

- $\quad$ Q: $100 \%$ of Naseby visitor activities in Ideal model

- $\quad$ Q1: $100 \%$ of Naseby visitor activities first scenario (present)

- Q2: $100 \%$ of Naseby visitor activities second scenario (Walking instead golfing)

- Q3: $100 \%$ of Naseby visitor activities Third scenario (Walking instead golfing and curling)

- $\quad$ EF1: The EF of Naseby visitor activities first scenario (present EF)

- $\quad$ EF2: The EF of Naseby visitor activities second scenario

- $\quad$ EF3: The EF of Naseby visitor activities third scenario

- (R) EF1: Reduced portion of EF1 influenced by changing golf for walking

- (R) EF2: Reduced portion of EF2 influenced by changing golfing and curling for walking

- GDP: The GDP of Naseby visitor activities

- $\quad$ GDPs: The GDPs of Ideal model for visitor activities = GDP

- GDPs1: The GDPs of Naseby visitor activities first scenario

- $\quad$ GDPs2: The GDPs of Naseby visitor activities second scenario

- $\quad$ GDPs3: The GDPs of Naseby visitor activities third scenario

- (I) GDPs 1: Increased portion of GDPs1 influenced by changing golf for walking

- (I) GDPs 2: Increased portion of GDPs2 influenced by changing golf and curling for walking 


\subsection{Case Study 3: Cromwell}

\subsubsection{Introduction}

This thesis is based on Cromwell as a host destination for OCRT visitors with the intention of comparing the results of the analysis with those of Naseby. As discussed in Chapter 3 (section 3.3.2.3) one of the main reasons for choosing Cromwell as a case study is that new buildings form $100 \%$ of accommodation services in this place. Furthermore, due to the existence of environmental attractions and tourism activities in Cromwell, in 2011 22\% of 11,788 OCRT people visited this place. These characteristics of Cromwell and its differences from Naseby show how the proposed comprehensive framework can be used to compare the influences exerted by ecotourism and its architecture on different destinations in terms of being sustainable.

This section aims to investigate the influences exerted by ecotourism on the sociocultural behaviours of visitors and host communities that in turn could change their environmental and economic footprint to being sustainable. The investigation of the influences of the OCRT and its architecture on Cromwell is conducted through using the method and holistic framework explained and used in the first and the second case studies.

\subsection{2: Background to Cromwell}

Cromwell is a town located at an elevation of $199 \mathrm{~m}, 45^{\circ} \mathrm{S}$ and $169^{\circ} \mathrm{E}$. The town is strategically placed as a holiday centre for the whole of Central Otago. On the shores of Lake Dunstan, Cromwell's centrality to Wanaka (55km), Queenstown (62km) and the remainder of Central Otago makes it a great location for easy day trips to all corners of Central Otago (see Figures 6.34 and 6.35). 


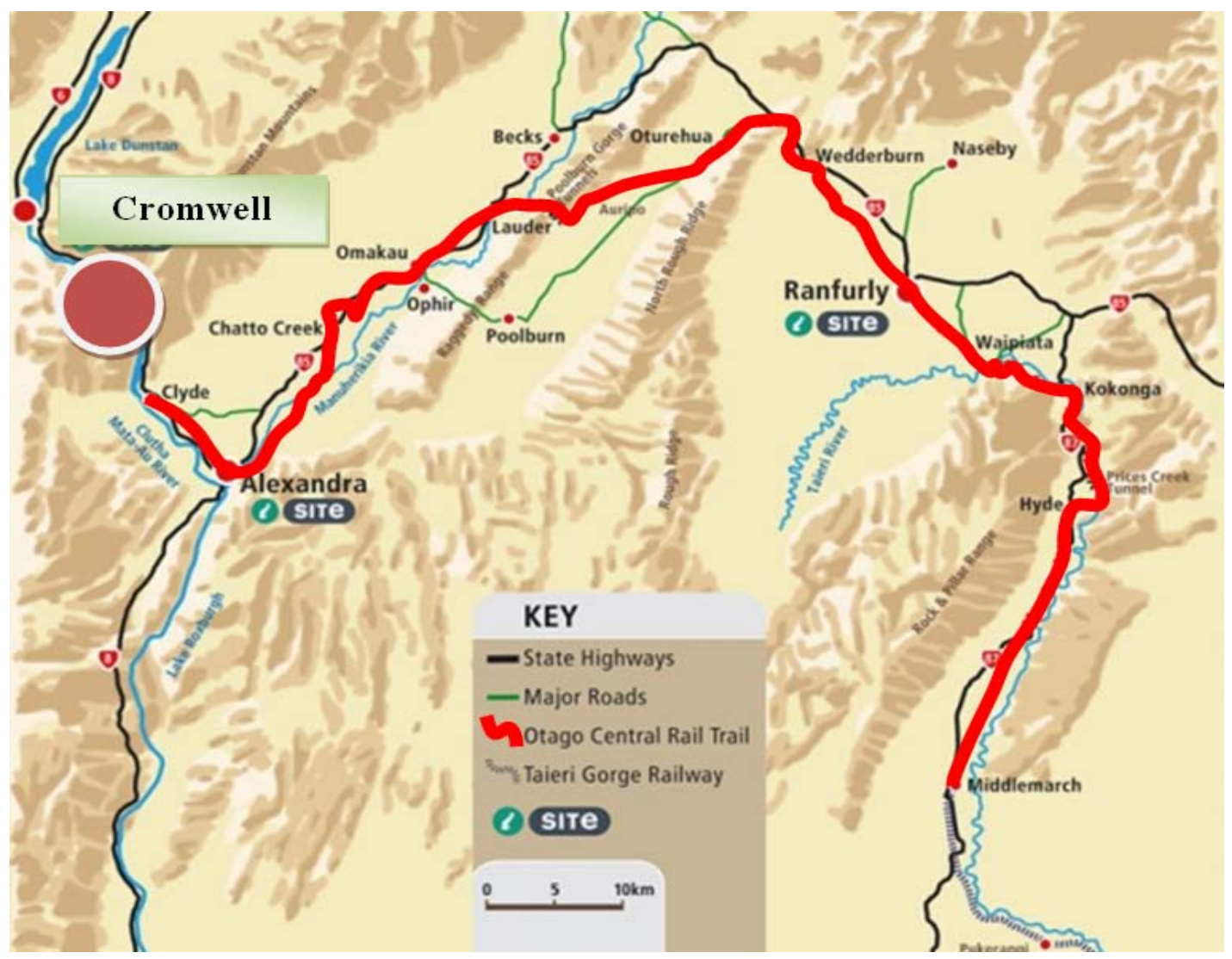

Figure 6.34: Cromwell, Central Otago, New Zealand http://www.centralotagonz.com/PicsHotel/CentralOtagoRTO/Images/Discovery/RailTrail/Hotsp otMap.jpg viewed August 2012

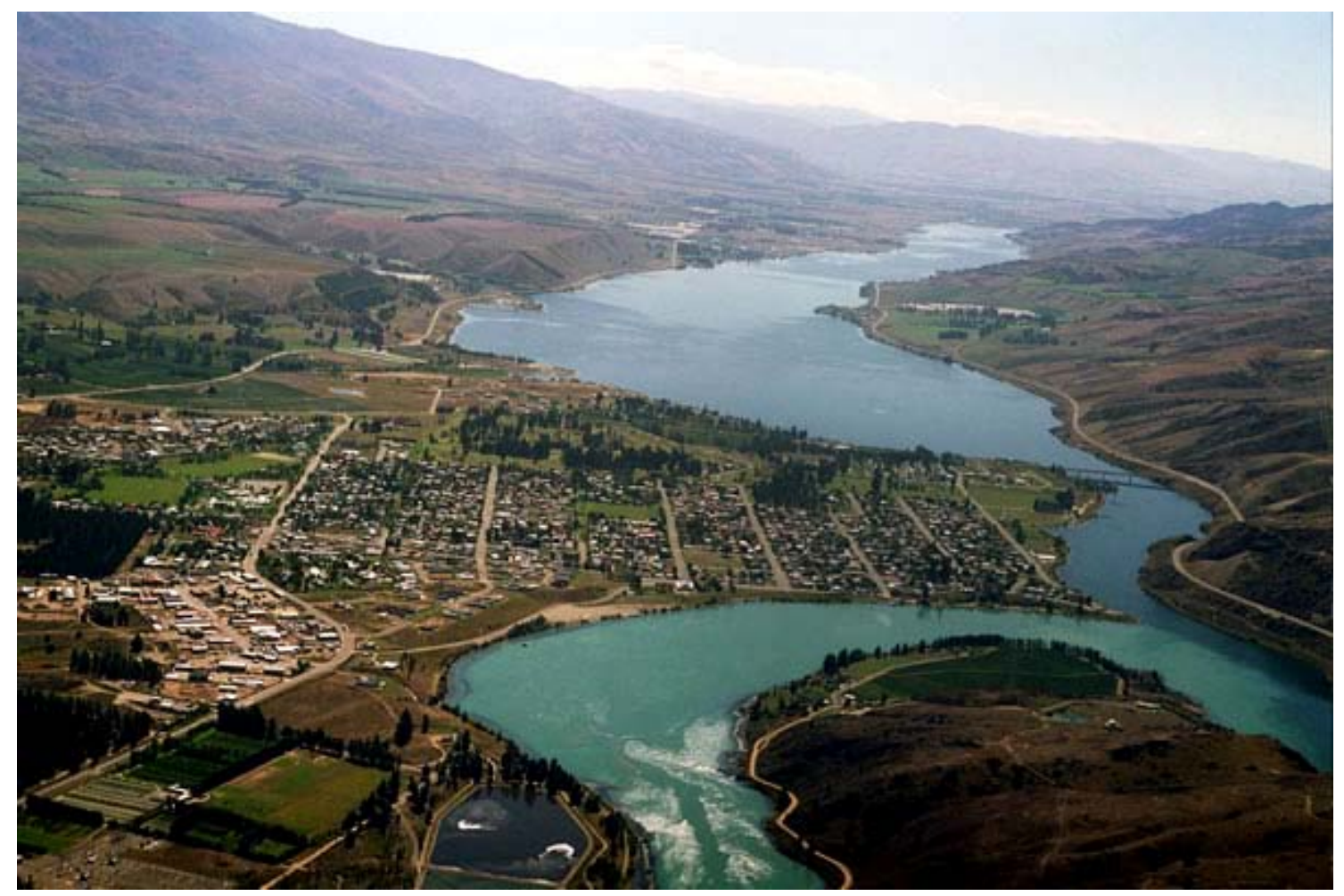

Figure 6.35: A view of Cromwell http://cromwellsports.files.wordpress.com/2010/05/cromwell.jpg viewed August 2012 
As pointed out in the Cromwell Community Plan (2008:8), Cromwell had its beginnings in 1862 when two miners, the American Horatio Hartley and the Irishman Christopher Reilly, took to Dunedin 87lbs (about 40kg) of gold they had recovered from a spot about a mile below the junction of the Kawarau and Clutha Rivers.

Furthermore, Cromwell was the host destination for Chinese miners who began to arrive in 1866 (see Figure 6.36).Emmitt (2010:1) describes how the Chinese miners who landed in Dunedin between 1868 and 1900 were engaged by the Otago Provincial Council. The latter body had made overtures in the Victorian gold fields for people to come and work in the Otago gold fields. Emmitt (2010:1) points out that Cromwell's Chinatown was continuously occupied for more than fifty years and more than 400 Chinese lived in the settlement between 1866 and 1920, when it was finally abandoned.

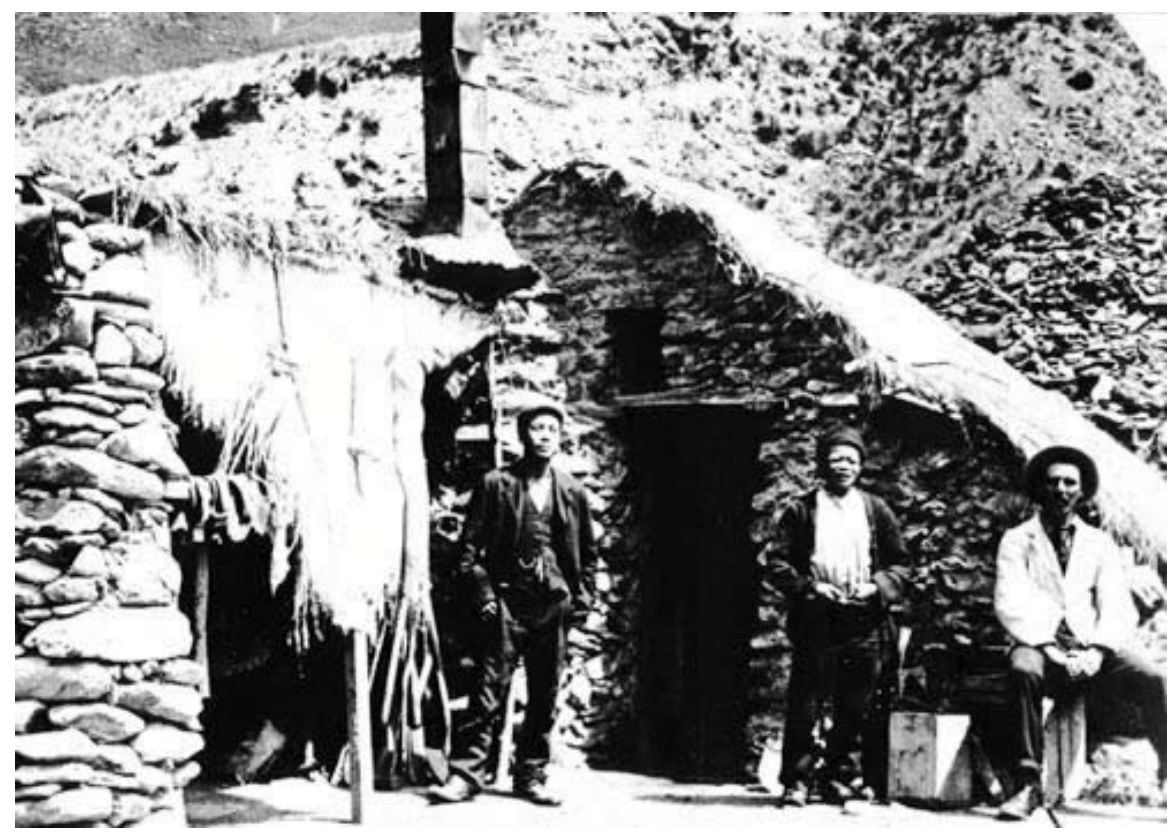

Figure 6.36: Cromwell Chinese miners http://www.cromwell.org.nz/gfx/popup/chinese.jpg viewed August 2012

The construction of the Clyde Dam (see Figure 6.37) and subsequent filling of Lake Dunstan in the 1980s (Cromwell Community Plan, 2008:8) caused major changes to Cromwell. Many of its historic places like its gold mining heritage (built between 1866 and 1920) and acres of orchards disappeared under water. 


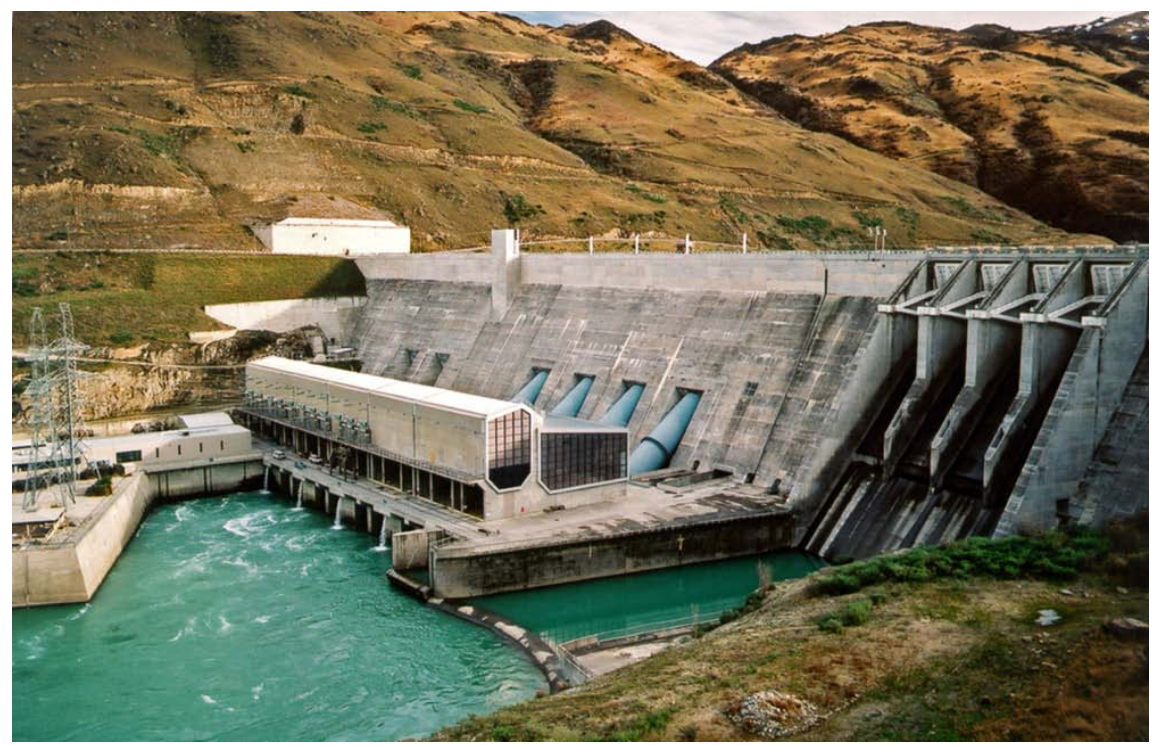

Figure 6.37: The Clyde Dam http://www.niwa.co.nz/sites/default/files/imported/attachments/92967/Clyde-Dam.jpg viewed August 2012

Since the Clyde Dam development polices involved the destruction of Cromwell historic heritage, by early 1985 a group of concerned residents came together to try and save its valuable heritage. As a result, 'Save Old Cromwell' was born and an autonomous voluntary Incorporated Society formed, with its board managing the activities (see www.cromwell.org.nz). Initially, eight buildings were chosen from the old commercial area due to be submerged by the advent of the new hydro lake. These buildings were to be relocated further up the main street to a site above the new lake level. This was the origin of the area today known as ‘Old Cromwell Town’ (see Figures 6.38 and 6.39).

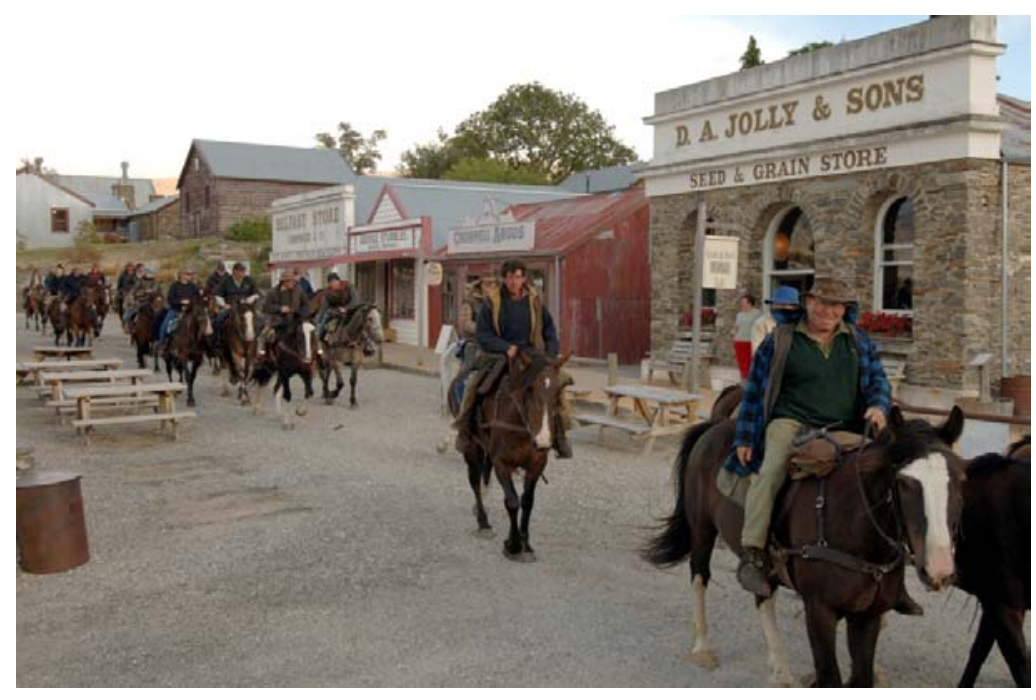

Figure 6.38: Old Cromwell Town http://oldcromwelltown.files.wordpress.com/2012/02/old-cromwell-cavalcade.jpg viewed August 2012 


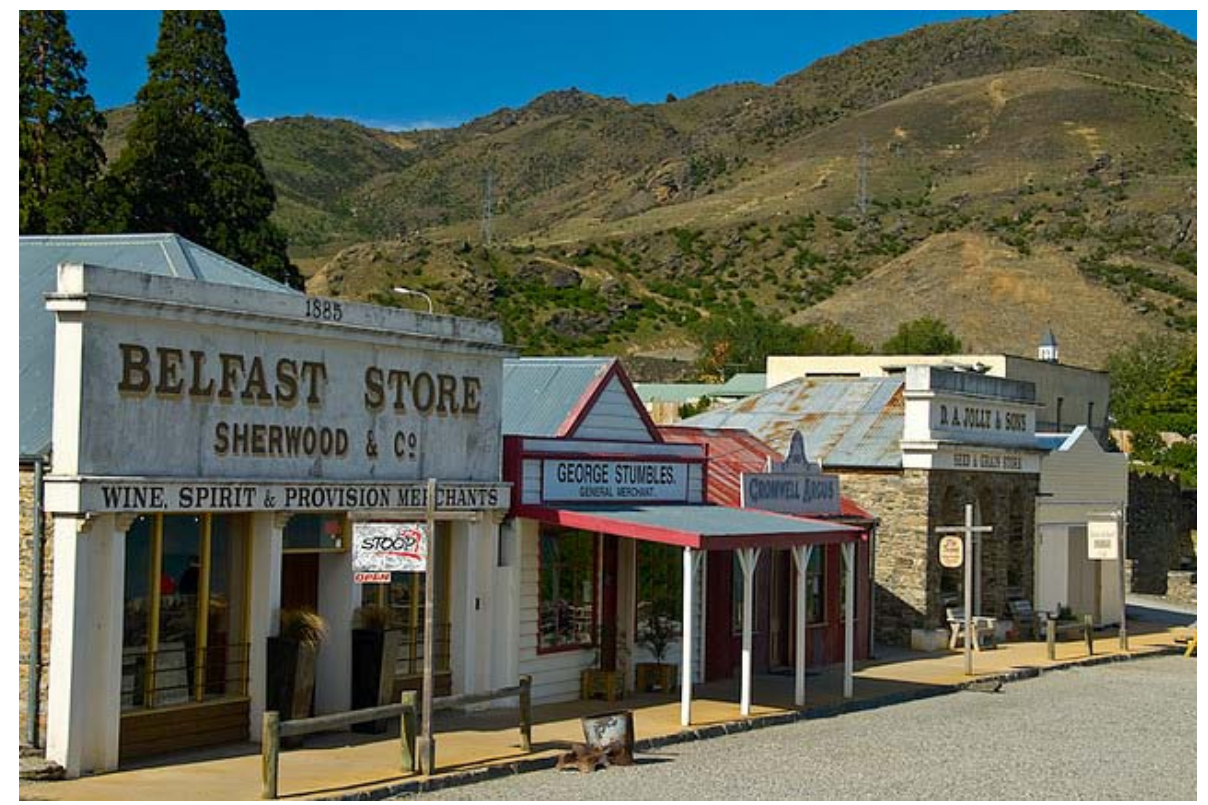

Figure 6.39: Old Cromwell Town

http://farm3.staticflickr.com/2449/4065341439_ce1516df36_z.jpg?zz=1

viewed August 2012

Although the construction of the Clyde dam damaged many of the Cromwell's natural and cultural heritages, since the 1980s the town has entered a new era of vitality and growth. The shorelines of the Clyde Dam and its two rivers make a destination for holiday makers. In addition, Cromwell has since become the fruit and wine centre of the South Island (see Figure 6.40).

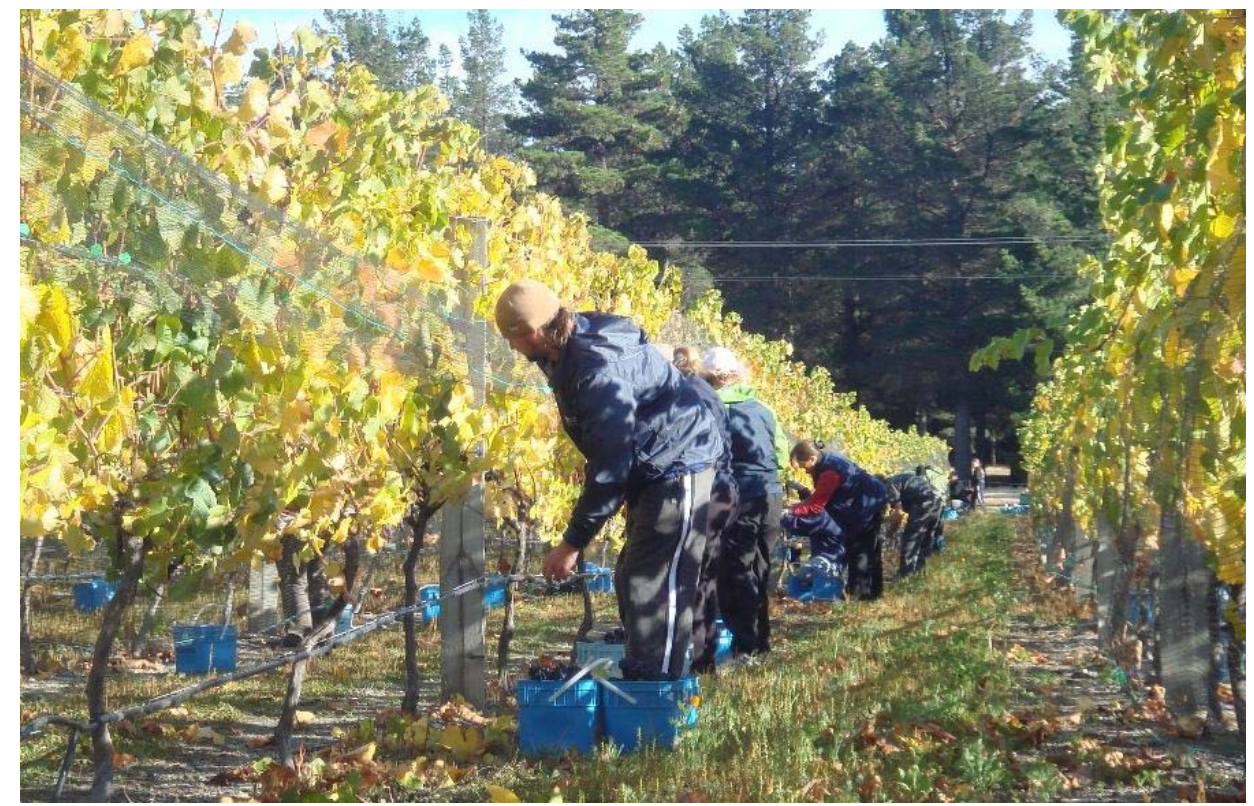

Figure 6.40: Cromwell - fruit picking http://img.scoop.co.nz/stories/images/0903/a3c9ec856fb8af54f8b9.jpeg viewed August 2012 
Cromwell with its historic and environmental heritage and capitals is considered one of the attractive destinations for a holiday as offered by the OCRT to its visitors. The following section, therefore, explores the environmental, cultural and economic influences of the development of the OCRT on Cromwell, through using the holistic framework proposed in Chapter 5 for the development of ecotourism and its architecture.

\subsection{3: Visitors to Cromwell}

In this chapter Cromwell's visitors are classified into international and domestic visitors. This section presents information related to each group, including the number of visitors, visitor nights and their home places.

\subsubsection{1: Visitor Numbers}

As shown in Table 5.49 (Chapter 5, 5.7.4) of 11,788 total OCRT visitors, $22.5 \%(2,652)$ choose Cromwell as their destination. This chapter therefore uses the 2,652 OCRT visitors to Cromwell as its total number of visitors in 2011, as shown in Table 6.67. Moreover, as shown in Figure 5.7 (Chapter 5), in 2011 the average stay of OCRT visitors is 3.6 nights. This section calculates total visitor nights at Cromwell based on its 2,652 visitors and 3.6 average visitor nights (2,562 visitors $\times 3.6$ visitor nights) to be 9,547 (Table 6.67).

\begin{tabular}{|c|c|c|c|c|}
\hline \multicolumn{5}{|c|}{ Table 6.67: Cromwell visitors based on CODC survey (2011) } \\
\hline $\begin{array}{l}\text { Total numbers of } \\
\text { OCRT visitors }{ }^{1}\end{array}$ & $\begin{array}{c}\% \text { of OCRT } \\
\text { visitors who } \\
\text { visit Cromwell }\end{array}$ & $\begin{array}{l}\text { Numbers of OCRT } \\
\text { visitors who visit } \\
\text { Cromwell }^{2}\end{array}$ & $\begin{array}{l}\text { Average visitor } \\
\text { nights }^{3}\end{array}$ & $\begin{array}{c}\text { Total visitor } \\
\text { nights }\end{array}$ \\
\hline 11,788 & 22.5 & 2,652 & 3.6 & 9,547 \\
\hline $\begin{array}{l}\text { 1. See Figure } 5.2 \\
\text { 2. See Table } 5.49 \\
\text { 3. See Figure } 5.3\end{array}$ & & & & \\
\hline
\end{tabular}

\subsubsection{2: Visitors Home Place}

As illustrated in Figure 5.13 (Chapter 5, 5.3.3) 22\% of the total 11,788 OCRT visitors are international and $78 \%$ are domestic. This thesis uses these proportions to calculate the number of visitors in each category to Cromwell as shown in Table 6.68. Table 6.68 determines that the total for domestic visitors to Cromwell is $78 \%$ of 2,652 or 2,069 visitors. Likewise, Table 6.68 indicates that 22\% (583) of Cromwell's 2,652 visitors are international in 2011. 
Table 6.68: Cromwell international and domestic visitors (2011)

Domestic visitors International visitors

\begin{tabular}{|l|l|l|l} 
Number of visitors & $\%$ of total number of visi- & Number of visitors & $\%$ of total number of visi-
\end{tabular} tors tors

\begin{tabular}{|l|l|l|}
\hline 2,069 & 78 & 583 \\
\hline
\end{tabular}

- Cromwell total visitors (2011) = 2,652 (see Table 6.68)

\section{a. Cromwell - International Visitors}

This thesis uses the percentage break down of OCRT international visitors cited in Table 5.1 (Chapter 5, section 5.3.3.1) and the right hand column in Table 6.69 as a base to calculate from where in 2011 Cromwell international visitors originated. As presented in Table 6.69, of the 583 Cromwell international visitors, the majority or 249 visitors came from Europe, followed by 192 (32.9\%) Australia, and 73 (12.6\%) UK. Other Cromwell international visitors from North America, Middle East and Africa are in the range of $8(1.4 \%)-53(9 \%)$ (Table 6.69).

\begin{tabular}{|l|c|c|}
\hline \multicolumn{3}{|c|}{ Table 6.69: Cromwell international visitors 2011 } \\
\hline Home place & $\mathrm{NV}^{1}$ & $\%$ \\
\hline Europe & 249 & 42.7 \\
\hline Australia & 192 & 32.9 \\
\hline UK & 73 & 12.6 \\
\hline North America & 53 & 9 \\
\hline Middle East & 8 & 1.4 \\
\hline South Africa & 8 & 1.4 \\
\hline Total & 583 & 100 \\
\hline $\begin{array}{l}\text { 3. NV: Number of Visitors. } \\
\text { • Total visitor numbers (2011) = 2,652 (See Table 6.68) } \\
\text { - Percentage of international visitors (2011) = 22\% (See Table 6.68) } \\
\text { • Total number of Cromwell international visitors = 583 (See Table 6.68) }\end{array}$ \\
\hline
\end{tabular}

\section{b. Cromwell - Domestic Visitors}

This chapter uses the percentage break down of OCRT domestic visitors in Table 5.3 (Chapter 5, section 5.3.3.2) and Table 6.70 (right hand column) to calculate the home places of Cromwell domestic visitors in 2011. Table 6.70 shows that of 2,069 Cromwell domestic visitors, the majority or 741 (35.8\%) were from upper NI followed by 470 (22.75\%) from central SI, 439 (21.2\%) upper SI, 308 (14.9\%) lower NI, and 111(5.35\%) lower SI. 
Table 6.70: Cromwell domestic visitors by home place

\begin{tabular}{|l|c|c|}
\hline Region/ city & Number of visitors & $\%$ \\
\hline Upper NI & 741 & 35.8 \\
\hline Lower NI & 308 & 14.9 \\
\hline Upper SI & 439 & 21.2 \\
\hline Central SI (Otago) & 470 & 22.75 \\
\hline Lower SI & 111 & 5.35 \\
\hline Total & 2,069 & 100 \\
\hline
\end{tabular}

\subsubsection{Transportation}

This section explores the types of international and domestic transportation used by Cromwell's visitors and calculates their EFs. The total EF of Cromwell visitor transportation is calculated through integration of the EFs of international and domestic travel.

\subsubsection{International Transportation}

\section{a. Types of International Transportation}

As discussed in the Section 6.1.4.1, in this study air travel is the only type of international transportation considered.

\section{b. Cromwell - International Tourism-kilometres (T-km)}

Table 6.71 shows that 583 Cromwell visitors (22\%) are international tourists and the total distance between their home places and Auckland's international airport is 7,198,999 T-km.

\begin{tabular}{|c|c|c|c|c|c|c|c|}
\hline \multicolumn{8}{|c|}{ Table 6.71: Cromwell international Tourism-km } \\
\hline \multirow{2}{*}{$\begin{array}{l}\text { Home } \\
\text { place }\end{array}$} & \multirow[b]{2}{*}{$\begin{array}{l}\text { Assumed } \\
\text { city of } \\
\text { origin } \\
\text { (central in } \\
\text { country of } \\
\text { origin) }\end{array}$} & \multirow[b]{2}{*}{$\begin{array}{c}\text { Distance } \\
\text { between } \\
\text { central } \\
\text { cities and } \\
\text { Auckland } \\
\text { (km). }\end{array}$} & \multicolumn{2}{|c|}{ Visitors } & \multirow{2}{*}{$\begin{array}{c}\text { Total In- } \\
\text { ternational } \\
\text { T-km }\end{array}$} & \multirow{2}{*}{$\begin{array}{l}\text { Auckland to } \\
\text { Dunedin } \\
\text { (km, return) }\end{array}$} & \multirow[b]{2}{*}{$\begin{array}{l}\text { Air domestic } \\
\text { International } \\
\text { visitors T-km } \\
\text { from Auckland } \\
\text { to Dunedin (re- } \\
\text { turn) }\end{array}$} \\
\hline & & & No & $\%$ & & & \\
\hline Australia & $\begin{array}{l}\text { Sydney } \\
\text { and Mel- } \\
\text { bourne }\end{array}$ & 2,512 & 192 & 32.9 & 482,304 & 2,156 & 413,952 \\
\hline Europe & Frankfurt & 18,180 & 249 & 42.7 & $4,526,820$ & 2,156 & 536,844 \\
\hline UK & London & 18,334 & 73 & 12.6 & 1338309 & 2,156 & 157,388 \\
\hline $\begin{array}{c}\text { North } \\
\text { America }\end{array}$ & $\begin{array}{l}\text { Montana } \\
\text { Billings }\end{array}$ & 11,950 & 53 & 9 & 633,350 & 2,156 & 114,268 \\
\hline $\begin{array}{c}\text { Middle } \\
\text { East }\end{array}$ & Tehran & 15,005 & 8 & 1.4 & 120,040 & 2,156 & 17,248 \\
\hline $\begin{array}{l}\text { South } \\
\text { Africa }\end{array}$ & Pretoria & 12,272 & 8 & 1.4 & 98,176 & 2,156 & 17,428 \\
\hline Total & - & 78,253 & 583 & 100 & 7,198,999 & - & 1,257128 \\
\hline
\end{tabular}


As discussed in Section 6.1.4.1-b, in this case study international travel distances are calculated as a one way trip. Because international tourists have to travel from Auckland to Dunedin and back, this part of the journey is a domestic flight and is equivalent to 1,257128 T-km, shown in Table 6.71 as Air domestic international visitors.

\subsubsection{Types of Domestic Transportation}

The types and percentages of transport use for holidays in New Zealand cited in Chapter 5 and shown in Table 5.5 (Matthews, 2009:13) are used as a pattern for transport used to access Cromwell.

\section{a. Domestic Tourism-Kilometres (T-km)}

This chapter uses Auckland as the central city for the upper NI and Wellington as the equivalent for the lower NI. In the same way, Nelson/Marlborough are used for the upper SI), Otago for the central SI, and Southland for the lower SI to calculate the distance between domestic visitors' home places and Cromwell (Table 6.72). In addition, to measure the distance between home places of visitors from the upper SI, the average distance between Nelson and Cromwell and Marlborough and Cromwell is calculated and used (Table 6.72).Visitors to Cromwell who come by car/van from the upper NI (Auckland) and lower NI (Wellington), shown as the grey areas in Table 6.72, need to use the ferry to cross to the South Island. Distances between these places and Cromwell are separated into three parts; home to Wellington (except Wellington); Wellington to Picton by ferry; and Picton to Cromwell (Table 6.72).

\begin{tabular}{|c|c|c|c|c|c|c|c|}
\hline \multicolumn{8}{|c|}{ Table 6.72: Cromwell domestic T-km } \\
\hline Region & Central city & \multicolumn{4}{|c|}{ Distance to Cromwell (km-return) } & Number & Domestic \\
\hline \multirow[t]{2}{*}{$\begin{array}{l}\text { Upper } \\
\text { NI }\end{array}$} & \multirow[t]{2}{*}{ Auckland } & $\begin{array}{c}\text { Auckland } \\
\text { to Wel- } \\
\text { lington }\end{array}$ & $\begin{array}{l}\text { Wellington } \\
\text { to Picton }\end{array}$ & $\begin{array}{l}\text { Picton to } \\
\text { Cromwell }\end{array}$ & Total & \multirow{2}{*}{741} & \multirow[t]{2}{*}{$2,224,482$} \\
\hline & & 1,318 & 204 & 1,480 & 3,002 & & \\
\hline $\begin{array}{l}\text { Lower } \\
\text { NI }\end{array}$ & Wellington & - & 204 & 1,480 & 1,684 & 308 & 518,672 \\
\hline $\begin{array}{l}\text { Upper } \\
\text { SI }^{1}\end{array}$ & $\begin{array}{c}\text { Nelson/ } \\
\text { Marlborough }\end{array}$ & & 1,6861 & & 1,686 & 439 & 740,154 \\
\hline $\begin{array}{l}\text { Central } \\
\text { SI }\end{array}$ & Otago & & $1,252^{2}$ & & 125 & 470 & 58,750 \\
\hline $\begin{array}{l}\text { Lower } \\
\text { SI }\end{array}$ & $\begin{array}{l}\text { Invercargill, } \\
\text { Southland }\end{array}$ & & 488 & & 488 & 111 & 258,288 \\
\hline Total & - & & - & & - & 2,069 & $3,800,346$ \\
\hline
\end{tabular}


According to Matthews (2009:13), in New Zealand 1\% of people going on holiday are pedestrians but in this study these have been ignored as Cromwell is far from the places of origin of visitors. The remaining 3\% of visitors are assumed to be domestic air travel. In addition, the ferry T-km of 228,021 (Table 6.73) are subtracted from the total car/van $\mathrm{T}-\mathrm{km}$ of 3,610,329 to give the road T-km.

\begin{tabular}{c|c|c|c|c|}
\hline \multicolumn{3}{|c|}{ Table 6.73: Types of transport used by Cromwell domestic visitors (based on Matthews, 2009:13) } \\
\hline Type of Transport & Car/van driver/passenger & Bus & Car-Ferry & Air domestic \\
\hline \% of total & 89 & 2 & 6 & 3 \\
\hline Tourist-km & $3,382,308$ & 76,007 & 228,021 & 114,010 \\
\hline Total domestic T-km & & $3,800,346$ & \\
\hline
\end{tabular}

\subsubsection{The Ecological Footprint (EF) of Cromwell Transportation}

Table 6.74 shows that total transportation energy of Cromwell visitors (2011) is $26,946,557 \mathrm{MJ}(26,946.6 \mathrm{GJ})$. The land to energy conversion capacity used in this thesis is the global average of $100 \mathrm{GJ} / \mathrm{gha}$. As demonstrated in Table 6.74 the total EF of Cromwell transportation is $26,946.6 \mathrm{GJ} \div 100 \mathrm{GJ} / \mathrm{gha}=269.5$ gha.

\begin{tabular}{|c|c|c|c|}
\hline \multicolumn{4}{|c|}{ Table 6.74: EF of Cromwell transportation (2011) } \\
\hline Transport Mode & Tourist-km & $\begin{array}{c}\text { Energy use } \\
\text { MJ/passenger-km }\end{array}$ & $\begin{array}{c}\text { Total annual energy } \\
\text { use by OCRT visitors } \\
(\mathrm{MJ})\end{array}$ \\
\hline Air International & 7,198,999 & 1.25 (Boeing 747) & $8,998,749$ \\
\hline $\begin{array}{c}\text { Air domestic } \\
\text { (International visitors from } \\
\text { Auckland to Dunedin) }\end{array}$ & $1,257,128$ & 3.88 & $4,877,657$ \\
\hline Air domestic & 114,010 & 3.88 & 442,359 \\
\hline $\begin{array}{l}\text { Car/van driver and passen- } \\
\text { ger }\end{array}$ & $3,382,308$ & 3.1 (average) & $10,485,155$ \\
\hline Bus & 76,007 & 1.01 & 76,767 \\
\hline Car-ferry & 228,021 & 9.06 & $2,065,870$ \\
\hline Total & & $\begin{array}{l}\text { Reference: Vale \& } \\
\text { Vale, } 2009 .\end{array}$ & $26,946,557$ \\
\hline \multicolumn{3}{|c|}{ EF for all types of transportation used by Cromwell visitors } & 269.5 \\
\hline
\end{tabular}

Table 6.74 gives the total energy use of Cromwell transportation (26,946,557 MJ) made up of $13,876 \mathrm{GJ}$ for air international and domestic travel by international visitors and 13,070 GJ for domestic visitor transportation. As a result, of total EF of Cromwell 
transportation (269.5gha) is 51.5\% (138.8 gha) for international travelers and $48.5 \%$ (130.7 gha) for domestic travelers.

\subsubsection{Food}

As shown in Table 6.67, 2,652 visitors went to Cromwell in 2011 and the mean number of nights they stayed was 3.6. Thus annual total visitor nights for tourists coming to Cromwell equal 9,547. Due to the lack of local information, the food consumption data presented by Collins et al (2005) are used to calculate the EF of food consumption for Cromwell visitors.

This chapter uses the same two scenarios to calculate the EF of food that were used for Naseby. In the first scenario, the EF of food is calculated based on the assumption $100 \%$ of food is conventional. In the second scenario, the total amount of food eaten by Cromwell visitors is separated into the two areas of local (home prepared) food (2.5\% of total food) and conventional food (97.5\% of total food).Comparison between the results of the two scenarios will indicate the influence exerted by the use of home prepared food (as a cultural product) on the EF and the Economic Footprint (ECF) of Cromwell.

\subsubsection{The EF of Cromwell Food (First Scenario)}

In the first scenario, the total amount of food eaten by Cromwell visitors has been assumed to have a footprint equivalent to that of food consumed outside the home by Cardiff residents. This totals $67.04 \mathrm{~kg}$ per resident/yr (Collins et al, 2005:25) with a footprint of 0.429gha/resident (Collins et al, 2005:32). This means $1 \mathrm{~kg}$ of food eaten out has an EF of $0.429 / 67.04 \mathrm{gha} / \mathrm{kg}=0.006 \mathrm{gha} / \mathrm{kg}$. The average amount of food eaten by a Cardiff resident each day is $1.85 \mathrm{~kg}$, and this value has been used for visitors to Cromwell. The results are shown in Table 6.75. The EF of tourist food is equivalent to $1.85 \times 0.006=0.01$ gha/visitor night (Table 6.75). The total EF of food consumed by Cromwell's visitors is $9,547 \times 0.01=95.47$ gha and the EF of this food/visitor is 95.47gha/2,652 (total visitors) $=0.036$ gha (Table 6.75). 
Table 6.75: EF of food consumed by Cromwell visitors (2011)

\begin{tabular}{|l|c|c|}
\hline \multicolumn{1}{|c|}{ Item } & Data & Reference \\
\hline Cromwell visitor numbers (2011) & 2,652 (See Table 6.68) & \\
\hline Average visitor nights/visitor & 3.6 & Collins et al , 2005:32 \\
\hline Total visitor nights & 9,547 & \\
\hline EF of food eaten out gha/cap & 0.429 & \\
\hline $\begin{array}{l}\text { EF of tourist food gha/visitor } \\
\text { night }\end{array}$ & 0.01 & \\
\hline $\begin{array}{l}\text { Total EF of food consumed by } \\
\text { Cromwell visitors (gha) }\end{array}$ & 95.47 \\
\hline $\begin{array}{l}\text { EF of food consumed by Crom- } \\
\text { well visitors/visitor (gha/visitor) }\end{array}$ & 0.036 \\
\hline
\end{tabular}

\subsubsection{The EF of Cromwell Food (Second Scenario)}

In Cromwell local products (such as food, beverage and wine) are offered to visitors by 492 separate accommodation services with 3,170 available bed spaces (Appendix 5Rows188-200- Cromwell). The information related to Cromwell products arising from Appendix 5, are summarised and set out in Table 6.76.

Table 6.76 demonstrates that in 2011, of the 19 (3.8\% of the total 492 accommodation services with 267 bed spaces) that offered local produce, 4 (0.8\% of total with 78 bed spaces) offered home baking and local food and beverages to their visitors and 2 (2\% of total with 189 bed spaces) offered locally produced wine. Since the total visitor nights for Cromwell are 9,547 and total Cromwell bed spaces are 3,170, the average visitor nights per bed space is $9,547 \div 3,170=3.01$ visitor nights/bed space. Consequently as shown in Table 6.76, the total visitor nights that Cromwell visitors consume local food is $78 \times 3.01=235$ visitor nights, equal to $2.5 \%$ of total 9,547 Cromwell visitor nights (Table 6.76).

\begin{tabular}{|c|c|c|c|c|c|}
\hline \multicolumn{7}{|c|}{ Table 6.76. Cromwell local produce (summary) } \\
\hline Produce & F & $\%$ & $\begin{array}{c}\text { \% of } 492 \text { accommo- } \\
\text { dations services }\end{array}$ & Bed spaces & Visitor nights $^{1}$ \\
\hline Local food and beverage & 4 & 21 & 0.8 & 78 & $235^{2}$ \\
\hline Wine & 15 & 79 & 3 & 189 & 569 \\
\hline Total & 19 & 100 & 3.8 & 267 & \\
\hline $\begin{array}{l}\text { 1. Average visitor nights/bed space }=9,547 \text { (total visitor nights)/3,170 (total bed spaces) }=3.01 \\
\text { 2. 78 (bed spaces) } \times 3.01 \text { (average visitor nights per bed space) }=235 \text { visitor nights }\end{array}$
\end{tabular}

This chapter uses the EFs of home cooked and conventional foods (per capita, per kg and per visitor night) as discussed in Chapter 5 (section.5.5.2 and Table s 5.11 and 5.12) to calculate the EFs of home cooked and conventional foods consumed by Cromwell's visitors, as in the following table. 
Table 6.77: Ecological footprint of home prepared and conventional foods consumed by Cromwell visitors (2011)

\begin{tabular}{|l|c|l|c|}
\hline \multicolumn{1}{|c|}{ Home prepared and local food } & EF & \multicolumn{1}{|c|}{ Conventional food } & EF \\
\hline $\begin{array}{l}\text { EF of local food gha/cap } \\
\text { EF of } 1 \mathrm{~kg} \text { local food gha/kg }\end{array}$ & 0.254 & $\begin{array}{l}\text { EF of 1 kg conventional food eaten out } \\
\text { (gha/cap) }\end{array}$ & 0.429 \\
\hline $\begin{array}{l}\text { EF of local food gha/visitor night } \\
\text { EF of local food of 235 visitor } \\
\text { nights (gha) }\end{array}$ & 0.0068 & EF of tourist food (gha/visitor night) & 0.01 \\
\hline $\begin{array}{l}\text { Total EF of home cooked and conventional foods (gha) } \\
\text { 1. 9,547 (total visitor nights) - 253 (visitor nights that tourists consume local food) }=9,312\end{array}$ & $\begin{array}{l}\text { Total EF of conventional food con- } \\
\text { sumed by Cromwell visitors/9,312 } \\
\text { visitor nights (gha) }\end{array}$ & 93.12 \\
\hline
\end{tabular}

Table 6.77 indicates that the EF of local food consumed by Cromwell visitors in 2011 is 235 visitor nights $\times 0.0068$ gha $=1.59$ gha. As the total visitor nights are 9,547 (see Table 6.67) and the number of visitor nights that visitors consume home cooked food is 235, consequently the total visitor nights that visitors to Cromwell consume conventional food is 9,547 - 235=9,312 visitor nights (see Table 6.77). Likewise the EF of conventional food consumed by Cromwell's visitors in 2011 is 9,312 visitor nights $\times$ 0.01 (EF of conventional tourist food, gha/visitor night) =93.12gha (Table 6.77). The total EF of consumed food in the second scenario (present circumstance) including the EFs of conventional and home prepared food is 93.12gha +1.59 gha $=94.71$ gha (Table 6.77).

\subsubsection{Environmental Effects of using local Food}

Comparing the results of the two scenarios shows that producing $2.5 \%$ (435kg) of $(17,662 \mathrm{~kg})$ food locally reduces the total EF of food by 0.76 gha ( $0.8 \%$ of $95.47 \mathrm{gha})$ (Table 6.78).

\begin{tabular}{|c|c|c|}
\hline & $\begin{array}{l}100 \%(17,662 \mathrm{~kg}) \text { con- } \\
\text { ventional eaten out food }\end{array}$ & $\begin{array}{c}97.5 \% \text { (17,227 kg) conventional eaten } \\
\text { out food and } 2.5 \% \text { (435 kg) home } \\
\text { cooked (organic) food }\end{array}$ \\
\hline Cromwell EF of food & 95.47 & 94.71 \\
\hline $\begin{array}{l}\text { Reduction in EF of food con- } \\
\text { sumed by Cromwell visitors } \\
\text { (gha) }\end{array}$ & & 0.76 \\
\hline
\end{tabular}




\subsubsection{Accommodation Services}

\subsubsection{Types of Accommodation Services}

This thesis places the 492 Cromwell accommodation services into the four categories of self-contained (SC), bed and breakfast (B\&B), motel, and powered and non-powered camping sites based on the information collected through using 12 official websites related to OCRT Cromwell accommodation services (Appendix 5 and 6-rows: 188-200). Table 6.79 summarises the parts of Appendix 5 that address available bed spaces by types of accommodation service. As shown in Table 6.79 and Figure 6.41, in 2011, of the total 492 Cromwell accommodation services, the majority or 95.7\% are camping sites (including 58.5\% powered and 37.2\% non-powered sites) followed by $2.9 \%$ motels, $1 \%$ SC and $0.4 \%$ B\&B. Looking at available bed spaces, the majority or $89.1 \%$ still occur in camping sites (including 54.5\% powered and 34.6\% non-powered sites), followed by $7.2 \%$ in motels, $3.2 \%$ in B\&Bs and $0.5 \%$ SC.

\begin{tabular}{|l|l|l|l|l|l|}
\hline \multicolumn{7}{c}{ Table 6.79: Cromwell accommodation services (2011) } \\
\hline \multicolumn{1}{|c|}{ Type } & $\mathrm{Q}^{1}$ & Number & $\%$ & Available bed spaces & $\%$ \\
\hline SC & NB & 5 & 1 & 15 & 0.5 \\
\hline B\&B & NB & 2 & 0.4 & 100 & 3.2 \\
\hline Motel & NB & 14 & 2.9 & 229 & 7.2 \\
\hline Camping ( powered sites) & NB & 288 & 58.5 & 1,728 & 54.5 \\
\hline Camping (non-powered sites) & NB & 183 & 37.2 & 1,098 & 34.6 \\
\hline Total & & & & & 100 \\
\hline 1. Quality of buildings and sites used as accommodation services at Cromwell & \\
\hline
\end{tabular}

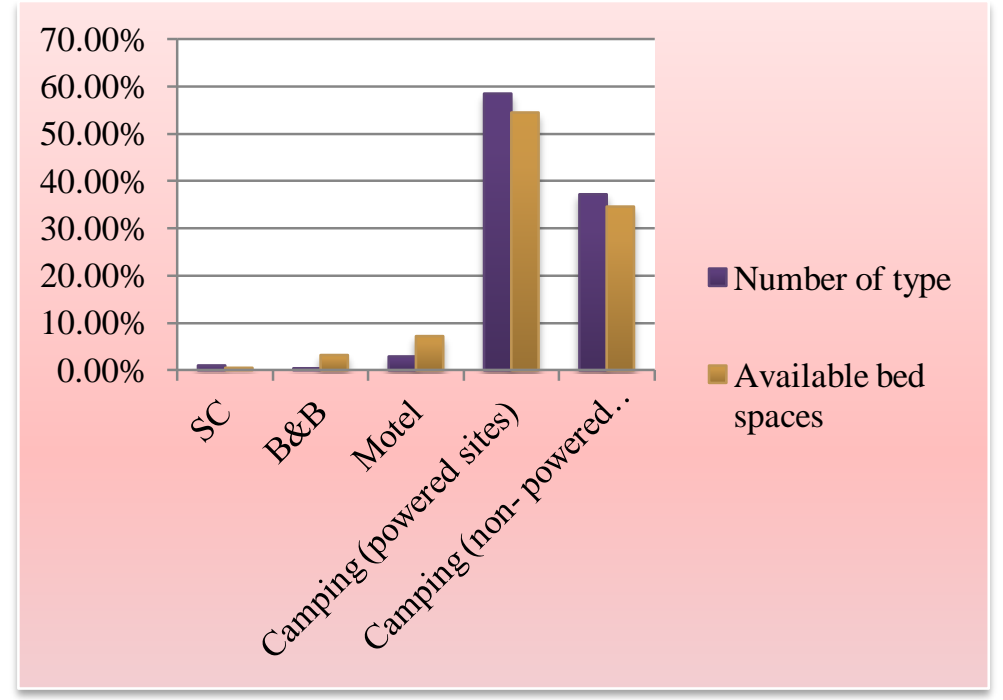

Figure 6.41: Percentage and available bed spaces by type of accommodation service (2011) 


\subsubsection{Cromwell - Quality of Accommodation Services}

This thesis reveals that $100 \%$ of Cromwell accommodation services that are offered through the OCRT websites are new buildings (see Appendix 5 and Table 6.79).

\subsubsection{Cromwell Accommodation Services - Materials}

The main materials for constructing of type of accommodation service (except camping sites) are set out in Appendix5 and the summarised information is shown in Table 6.80, Figures 6.41 and 6.42. Only two types of materials are used in Cromwell, timber and mixed timber (Table 6.80). As shown in Table 6.80 and Figure 6.42, of the total 344 accommodation bed spaces the majority or $54.4 \%$ (187) are timber and $45.6 \%$ (157) are timber mixed with other materials.

\begin{tabular}{|c|c|c|c|c|c|c|c|c|}
\hline \multirow[t]{3}{*}{ Material(s) } & \multirow[t]{3}{*}{ Total bed spaces } & \multirow[t]{3}{*}{$\%$} & \multicolumn{6}{|c|}{ Type of accommodation service } \\
\hline & & & \multicolumn{2}{|c|}{ SC } & \multicolumn{2}{|c|}{$\mathrm{B} \& \mathrm{~B}$} & \multicolumn{2}{|c|}{ Motel } \\
\hline & & & No & $\mathrm{C}$ & No & $\mathrm{C}$ & No & $\mathrm{C}$ \\
\hline Timber & 187 & 54.4 & 4 & 11 & 1 & 4 & 13 & 172 \\
\hline $\begin{array}{l}\text { Timber (mixed with other } \\
\text { materials) }\end{array}$ & 157 & 45.6 & 1 & 4 & 1 & 96 & 1 & 57 \\
\hline Total bed spaces & 344 & 100 & 5 & 15 & 2 & 100 & 14 & 229 \\
\hline
\end{tabular}

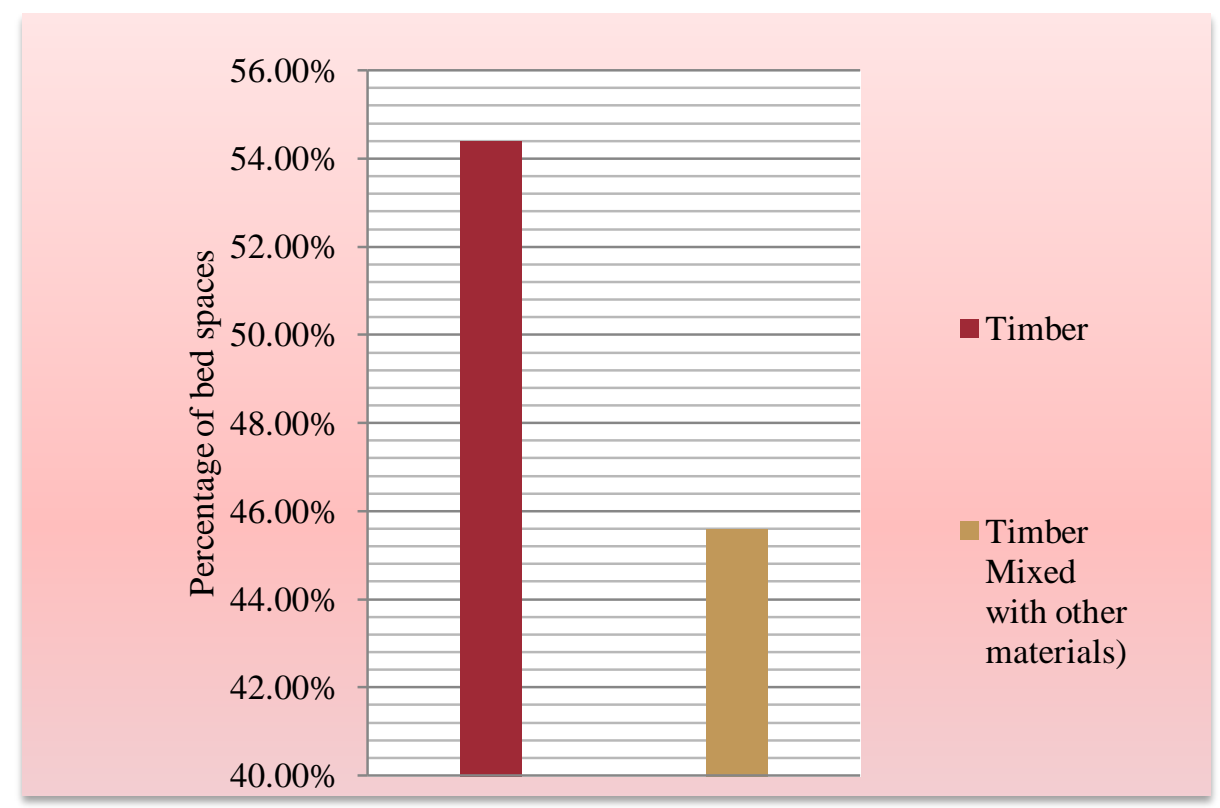

Figure 6.42: Cromwell - bed spaces by type of materials used (2011) 
Table 6.80 and Figure 6.43 indicate that 15 SC bed spaces comprise 11 (73\%) are timber as the main construction material and 4 (26.90\%) are timber mixed with other materials. In the same way, out of the $100 \mathrm{~B} \& \mathrm{~B}$ bed spaces available in Cromwell, 96 use timber as the main material and only 4 are timber mixed with other materials. Similarly, 172 (75.1\%) out of 229 Cromwell's motel bed spaces are timber and 57 (24.9\%) are timber mixed with other materials.

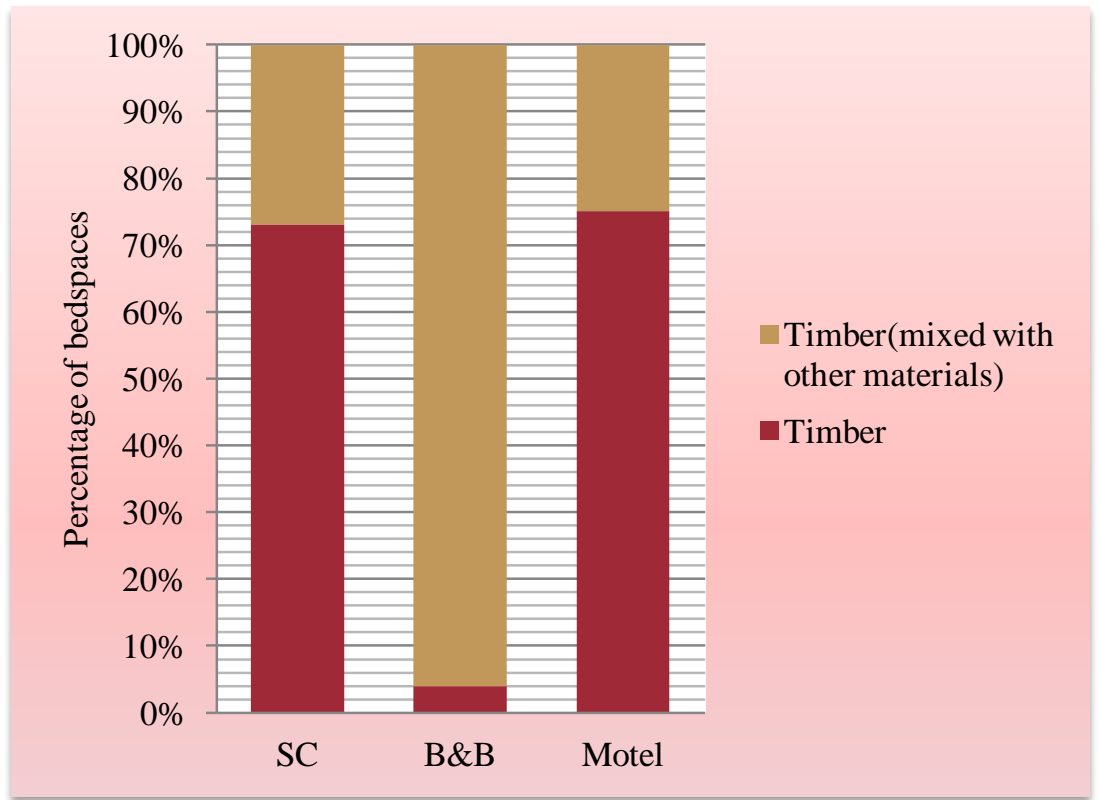

Figure 6.43: Cromwell - materials used by type of accommodation services (2011)

\subsubsection{Accommodation Services - Energy Sources, Facilities and Spaces}

As shown in Appendix 5 (rows 188-200) this research explores the energy sources and facilities used in the 492 Cromwell accommodation services through using related information and documents published in these accommodation services' websites. This chapter classifies information related to the energy sources used, facilities and spaces, demonstrated in Appendix 5 and summarised in Appendices 29-33, by type of accommodation service. All heating systems found (EL, log, gas and solar) are investigated here. For facilities and equipment, TV and internet are chosen to be explored as using these facilities can be considered an indicator of a modern lifestyle (Appendices 29-33). Since using open air areas has been introduced earlier as one of the environmental and cultural indicators for evaluating architecture as being sustainable, verandas, balconies and outdoor seating areas are also investigated in this study. The following section presents data classified by type of accommodation service shown in Appendix33, and summarises Appendices 29- 32. 


\section{a. Cromwell SC Accommodation Services - Energy Sources, Facilities and Open}

\section{Air Spaces (2011)}

Figure 6.44, and Appendices 29 and 33 demonstrate that for the 15 SC bed spaces, 100\% use electricity (for heating), have a TV and have access to the internet. Furthermore, 4 (26.6\%) of Cromwell's SC bed spaces have a private V/B and 11 (73.30\%) have access to a shared outdoor sitting area (Figure 6.44 and Appendix 33).

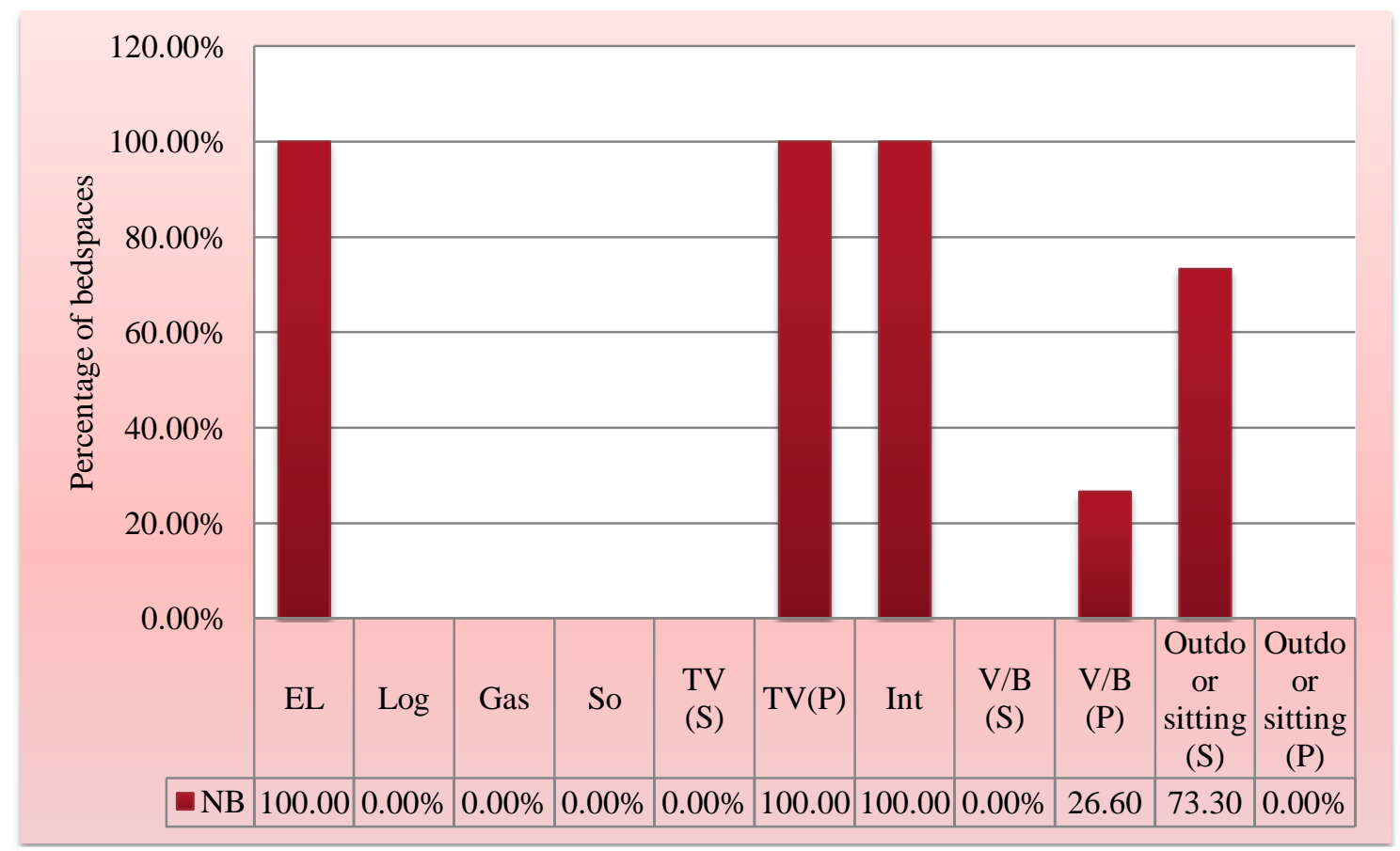

Figure 6.44: Cromwell SC accommodation services - energy sources, facilities and spaces (2011)

El: Electricity

So: Solar system

TV (S): Share TV

TV (P): Private TV

Int: Internet

V/B (S): Share veranda/ balcony

V/B (P): Private veranda/balcony

Outdoor sitting (S): Share outdoor sitting

Outdoor sitting $(\mathrm{P})$ : Private outdoor sitting

\section{b. Cromwell B\&B Accommodation Services - Energy Sources, Facilities and Open} Air Spaces (2011)

Figure 6.45, and Appendices 30 and 33 demonstrate that of Cromwell's 100 B\&B bed spaces, 96 use electricity and 4 use gas for heating. In addition, 100\% of B\&B bed spaces have both TV and access to internet, and 96 of them have access to 47 private outdoor sitting areas (Figure 6.45 and Appendix 33). 


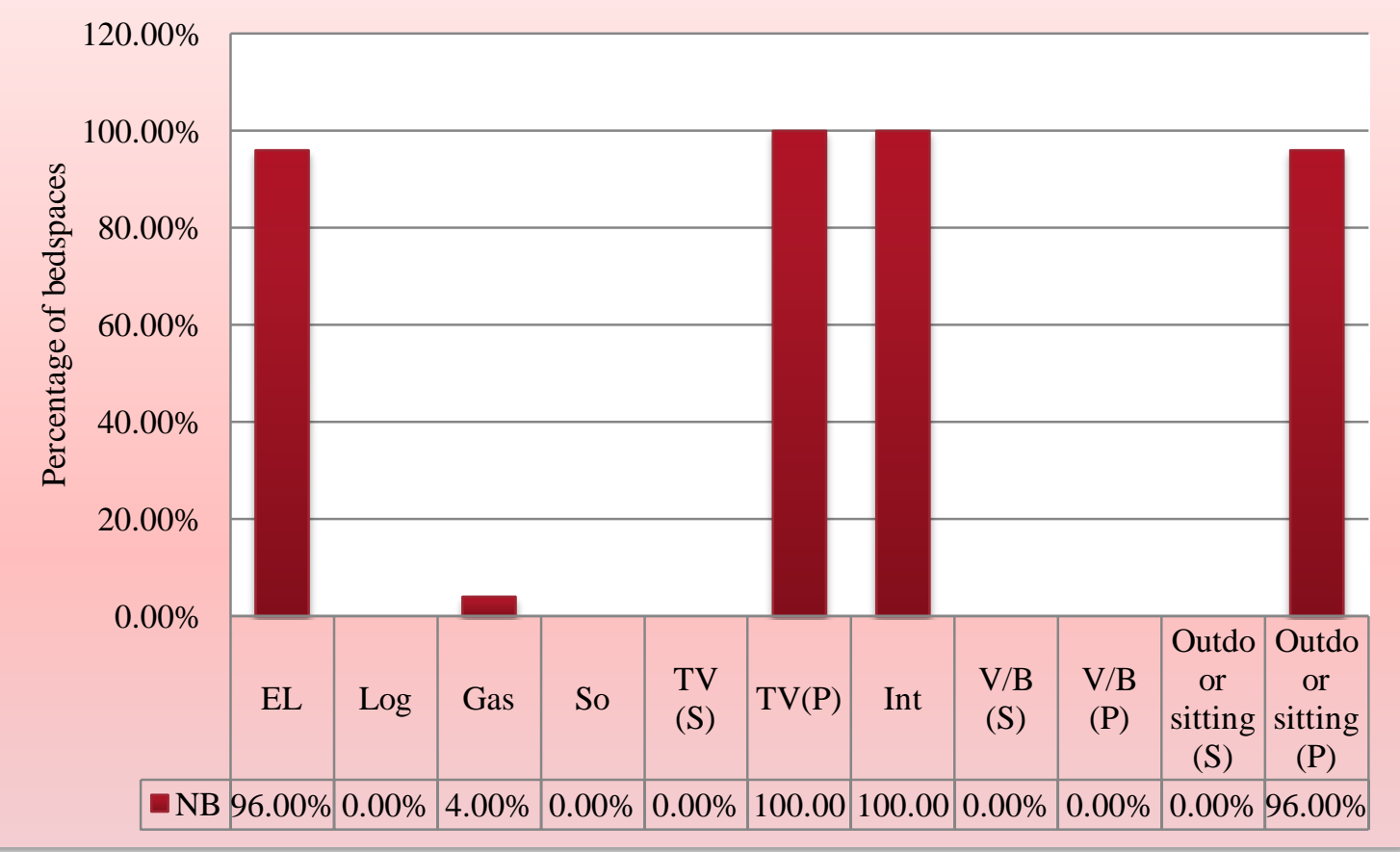

Figure 6.45: Cromwell B\&B accommodation services - energy source, facilities and spaces (2011)

\section{c. Cromwell's Motel Accommodation Services - Energy Sources, Facilities and}

\section{Open Air Spaces (2011)}

As illustrated in Figure 6.46 and shown in Appendices.31 and 33, 213 (96\%) of Cromwell’s 229 motel accommodation services use electricity as the main source for heating.

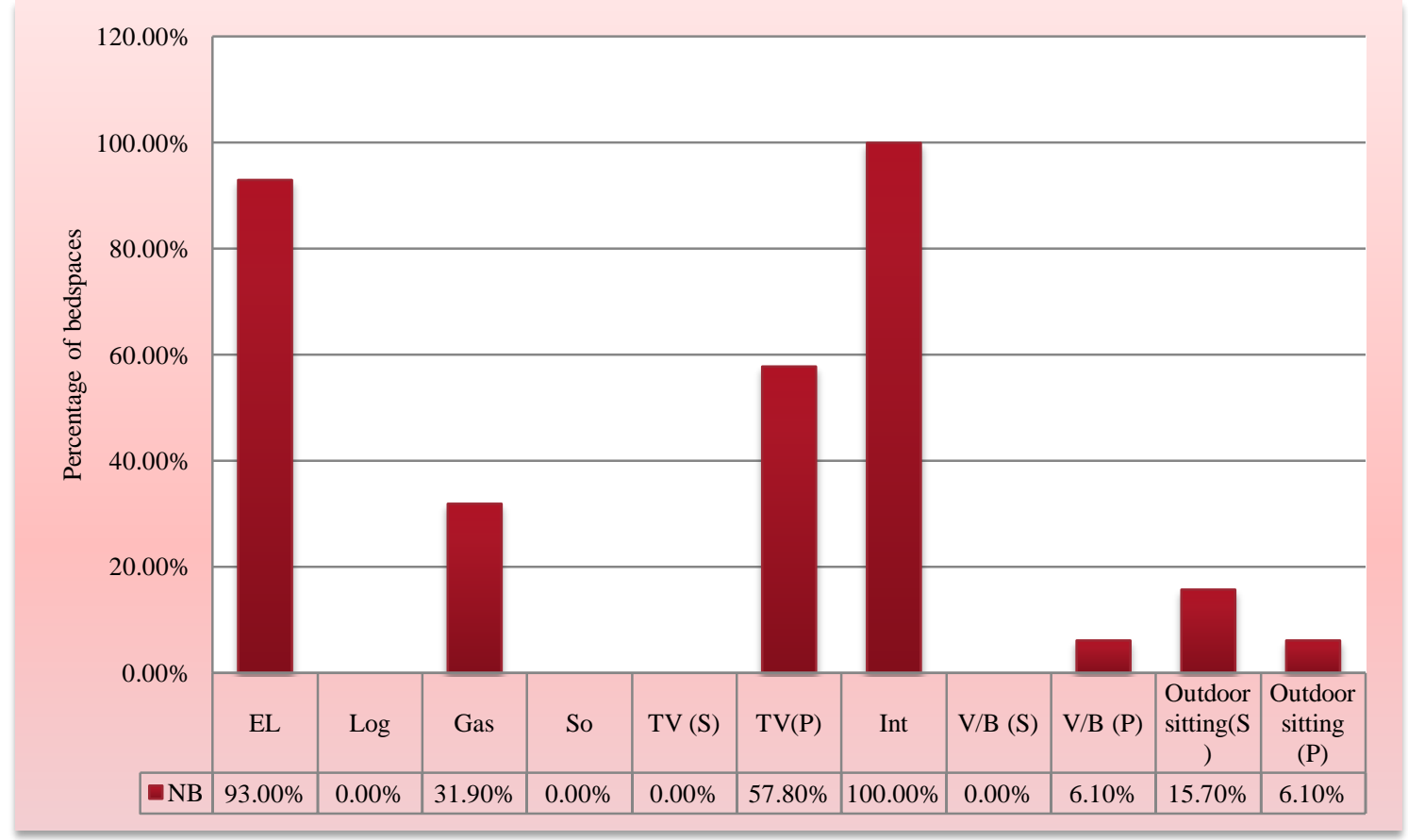

Figure 6.46: Cromwell’s Motel accommodation services - energy sources, facilities and spaces (2011) 
Likewise, 73 (31.9\%) of these bed spaces use gas (Figure 6.46 and Appendix 33). Furthermore, 130 (57.8 \%) of the motel bed spaces have a private TV and 100\% of them have access to the Internet. Also as demonstrated in Figure 6.46 and Appendix33, 14 motel bed spaces have private $\mathrm{V} / \mathrm{Bs}$ and same number have access to private outdoor sitting areas. Furthermore, of the 229 motel bed spaces, 36 (15.70\%) can use a shared outdoor sitting area (Figure 6.46 and Appendix 33).

\section{d. Camping Accommodation Services - Energy Sources, Facilities and Open air Spaces (2011)}

Figure 6.47 and Appendix 33 show that of Cromwell's 2,826 camping sites (powered and non-powered), 1,728 (61.1\%) of the powered sites use electricity (for heating cabins and caravans, lighting and other services such as the BBQ) and 100\% of them have access to the Internet.

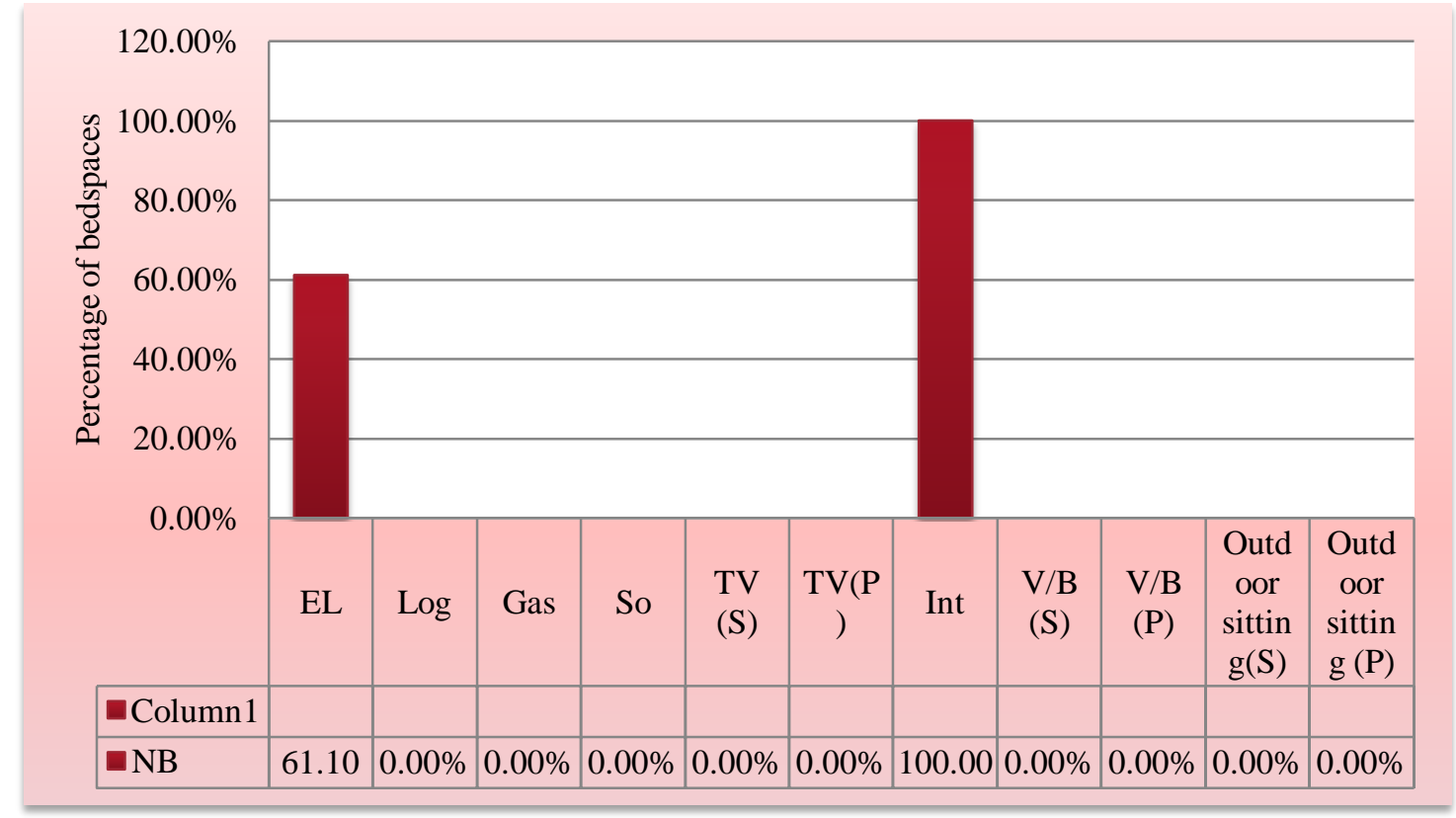

Figure 6.47: Cromwell’s camping sites - energy sources, facilities and spaces (2011)

\section{e. All Types of Cromwell Accommodation Services - Energy sources, Facilities and Open air spaces (2011)}

Figure 6.48, Appendix33 and Table 6.81 indicate the energy sources, facilities and open air spaces in all types of Cromwell accommodation services. As shown, of the 3170 bed spaces $62 \%(1,965)$ use electricity and 2.3\% (73) use gas for heating. In addition, of the same total 3,170 bed spaces, $7.7 \%$ (245) have private TVs and $100 \%$ access to the Internet (Figure 6.48 and Table 6.81). Furthermore, 1.5\% (47) of Cromwell's bed 
spaces have access to private open air spaces (including V/B and outdoor sitting areas) and $4 \%$ (128) can use shared open spaces.

\begin{tabular}{|c|c|c|c|c|c|c|c|c|c|c|c|c|c|c|c|c|c|}
\hline \multicolumn{6}{|c|}{ Heating } & \multirow[t]{2}{*}{ So } & \multirow[t]{2}{*}{$\%$} & \multicolumn{4}{|c|}{ TV } & \multirow[b]{2}{*}{ Int } & \multirow[t]{2}{*}{$\%$} & \multicolumn{4}{|c|}{ Outdoor sitting ${ }^{1}$} \\
\hline El & $\%$ & $\begin{array}{c}\text { Lo } \\
\text { g }\end{array}$ & $\%$ & $\begin{array}{c}\mathrm{Ga} \\
\mathrm{s}\end{array}$ & $\%$ & & & $S$ & $\%$ & $\mathrm{P}$ & $\%$ & & & S & $\%$ & $\mathrm{P}$ & $\%$ \\
\hline 1,965 & 62 & 0 & 0 & 73 & 2.3 & 0 & 0 & 0 & 0 & 245 & 7.7 & 3,170 & 100 & 47 & 1.5 & 128 & 4 \\
\hline
\end{tabular}

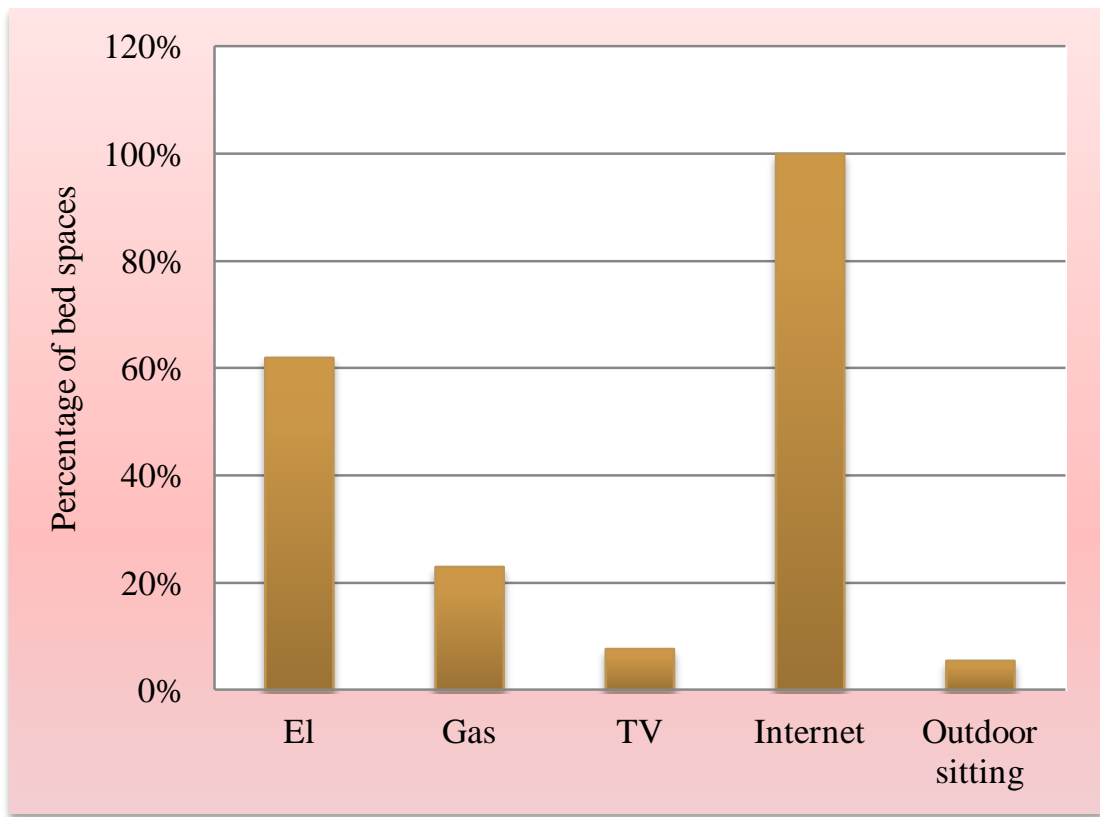

Figure 6.48: Cromwell accommodation services - energy sources, facilities and spaces (2011)

\subsubsection{Cromwell Accommodation Services - Occupancy Share}

This chapter calculates the occupancy shares of Cromwell's accommodation services based on the occupancy shares of the OCRT accommodation services cited in Table 5.19 (Chapter 5-section 5.6.5). Since two types of accommodation service (Bp and homestead) are not found at Cromwell, $50 \%$ of the sum of their occupancy shares (cited in Table 5.19) has been included in the occupancy share of Cromwell SCs and another $50 \%$ has been included in B\&B accommodation. Consequently, the occupancy of Cromwell SC accommodation is $13.1 \%$ (occupancy share of OCRT SC accommodation services in Table 5.19) $+8.5 \%$ (50\% of total occupancy shares of OCRT Bp and homestead accommodation in Table 5.19) $=21.6 \%$ (see Table 6.82). In addition the occupancy share of Cromwell's B\&B accommodation is $13.9 \%$ (occupancy share of OCRT 
B\&B accommodation in Table 5.19) + 8.5\% (50\% of total occupancy of OCRT Bps and homesteads in Table 5.19) $=22.4 \%$ (See Table 6.82) .

Since there are no hotel bed spaces in Cromwell, the hotel occupancy rate cited in Table 5.19 has been included in the occupancy share of motels. As a result, the occupancy rate of Cromwell motels is $21.1 \%$ (occupancy share of OCRT motels in Table 5.19) $+10.9 \%$ (occupancy share of OCRT hotels in Table 5.19) = 32\% (occupancy share of Cromwell’s motels, see Table 6.82).

Table 6.82 comprises the visitor nights for each type of accommodation service found in Cromwell, which are calculated as: occupancy share of each type $\times$ total Cromwellvisitor nights (9,547, see Table 6.67). As shown in Table 6.82, Cromwell's motels have the majority or 3,055 visitor nights, followed by camping sites $(2,291)$, B\&Bs $(2,139)$ and SCs (2,062).

Table 6.82: Cromwell accommodation services - occupancy shares (2011)

\begin{tabular}{|c|c|c|}
\hline Type of accommodation service & \% of visitor nights (2005) & Visitor nights (2011) \\
\hline SC & $21.6^{1}$ & 2,062 \\
\hline $\mathrm{B} \& \mathrm{~B}$ & $22.4^{2}$ & 2,139 \\
\hline Motel & $32^{3}$ & 3,055 \\
\hline Camping & 24 & 2,291 \\
\hline Total & 100 & $9,547^{4}$ \\
\hline \multicolumn{3}{|c|}{$\begin{array}{l}\text { 1. SC } \% \text { of visitor nights }=8.5 \%(50 \% \text { of total } \mathrm{Bp} \text { and homestead })+13.1 \% \text { ( } \% \text { of visitor nights of SC in } 2005) \\
\text { (see Table } 5.19) \text {. } \\
\text { 2. B\&B } \% \text { of visitor nights }=8.5 \%(50 \% \text { of total Bp and homestead })+13.9 \% \text { ( } \% \text { of visitor nights of B\&B in 2005) } \\
\text { (see Table } 5.19) \text {. } \\
\text { 3. Motel } \% \text { of visitor nights }=10.9 \%+21.1 \% \text { (see Table } 5.19) \text {. } \\
\text { 4. See Table } 6.67\end{array}$} \\
\hline
\end{tabular}

\subsubsection{Cromwell Accommodation Services - Area}

This section uses the average area per bed of OCRT accommodation services (cited in Table 5.20) as the average area per bed of Cromwell's accommodation services to calculate the total area of each type. Table 6.83 indicates that the total area of 492 Cromwell accommodation services is 25,178 $\mathrm{m}^{2}$, including $443 \mathrm{~m}^{2} \mathrm{SC}, 4000 \mathrm{~m}^{2} \mathrm{~B} \& \mathrm{~B}, 3,779$ $\mathrm{m}^{2}$ motel, and 16,956 $\mathrm{m}^{2}$ camping sites.

As shown in Table 6.83, the occupied area of each type of accommodation service is calculated as its total area multiplied by its percentage occupancy rate. Table 6.83 demonstrates that the total occupied area of Cromwell accommodation is $6,270 \mathrm{~m}^{2}$, including $96 \mathrm{~m}^{2} \mathrm{SC}, 896 \mathrm{~m}^{2} \mathrm{~B} \& \mathrm{~B}, 1,209 \mathrm{~m}^{2}$ motel and 4,069 $\mathrm{m}^{2}$ camping sites. 


\begin{tabular}{|c|c|c|c|c|c|c|c|}
\hline \multicolumn{6}{|c|}{ Table 6.83: Cromwell Accommodation services - Total area and Occupied area (2011) } \\
\hline Type & No. & C & $\begin{array}{c}\text { Area per } \\
\text { bed }\left(\mathrm{m}^{2}\right)^{1}\end{array}$ & $\begin{array}{c}\text { Total } \\
\text { Area }\end{array}$ & $\begin{array}{c}\text { Occupancy } \\
(\%)^{2}\end{array}$ & $\begin{array}{c}\text { Visitor } \\
\text { nights }\end{array}$ & $\begin{array}{c}\text { Occupied Area } \\
\left(\mathrm{m}^{2}\right)\end{array}$ \\
\hline SC & 5 & 15 & 29.5 & 443 & 21.6 & 2,062 & 96 \\
\hline B\&B & 2 & 100 & 40 & 4,000 & 22.4 & 2,139 & 896 \\
\hline Motel & 14 & 229 & 16.9 & 3,779 & 32 & 3,055 & 1,209 \\
\hline Camping & 471 & 2,826 & 6 & 16,956 & 24 & 2,291 & 4,069 \\
\hline Total & 492 & 3,170 & - & 25,178 & 100 & 9,547 & 6,270 \\
\hline $\begin{array}{l}\text { 1. See Table 5.20 } \\
\text { 2. See Table 6.82 }\end{array}$ & & & & & & \\
\hline
\end{tabular}

\subsubsection{The EF of Cromwell's Accommodation Services}

In the section of Cromwell accommodation services, two scenarios are used. In the first scenario, all areas of buildings are assumed to be indoor spaces. In the second scenario that reflects the current position of Cromwell's accommodation services, the buildings include both indoor and open air (veranda and balcony) areas.

Comparison between the EFs of the two scenarios can indicate the influence exerted on the EF of Cromwell's accommodation services by using open air spaces as part of the buildings used by tourists.

\section{a. First Scenario: All Buildings are assumed to be Indoor Spaces}

\section{a.1 . The EF of Cromwell SC Accommodation Services}

In Table 6.84, the total area of SC timber construction is calculated through the following equation:

Total area of SC timber construction $=$ the number of SC timber bed spaces $(11$, see Table 6.80) $\times$ SC area/bed space $\left(29.5 \mathrm{~m}^{2}\right.$, see Table 6.83$)=325 \mathrm{~m}^{2}$

As the embodied energy of timber construction over a 50 year life cycle is $1.1 \mathrm{GJ} / \mathrm{m}^{2}$, the embodied energy of $325 \mathrm{~m}^{2}$ of SC accommodation per a year is $\left(325 \mathrm{~m}^{2} \times 1.1 \mathrm{GJ}\right) \div$ $50=7.15$ GJ/year (Table 6.84).

The operational energy of SC accommodation as indicated in Table 6.84 is 0.3 $\mathrm{GJ} / \mathrm{m}^{2} /$ year. Since the occupied area of SC timber construction in Table 6.84 is $70 \mathrm{~m}^{2}$, the total operating energy use of SC timber structures is $70 \mathrm{~m}^{2} \times 0.3 \mathrm{GJ} / \mathrm{m}^{2} /$ year $=28$ GJ/year (see Table 6.84). As shown in Table 6.84, the total life cycle energy use of SC timber structures is $7.15 \mathrm{GJ} /$ year (total embodied energy) + $21 \mathrm{GJ} /$ year (total operating 
energy) $=28.15 \mathrm{GJ} /$ year. Also the total EF of SC timber construction is equivalent to 28.15 GJ/year (life cycle energy used) $\div 100$ (average global carrying capacity) $=0.3$ gha/year (Table 6.84).

This chapter uses the same method as above to calculate the EF of Cromwell's SC accommodation services made of timber mixed with other materials (Table 6.84). As demonstrated in Table 6.84, this type of accommodation accounts for 123GJ/year life cycle energy and the EF is 0.1 gha/year. Consequently the total EF of Cromwell's SC accommodation services is $(0.3+0.1$ gha $)=0.4$ gha $($ Table 6.84$)$.

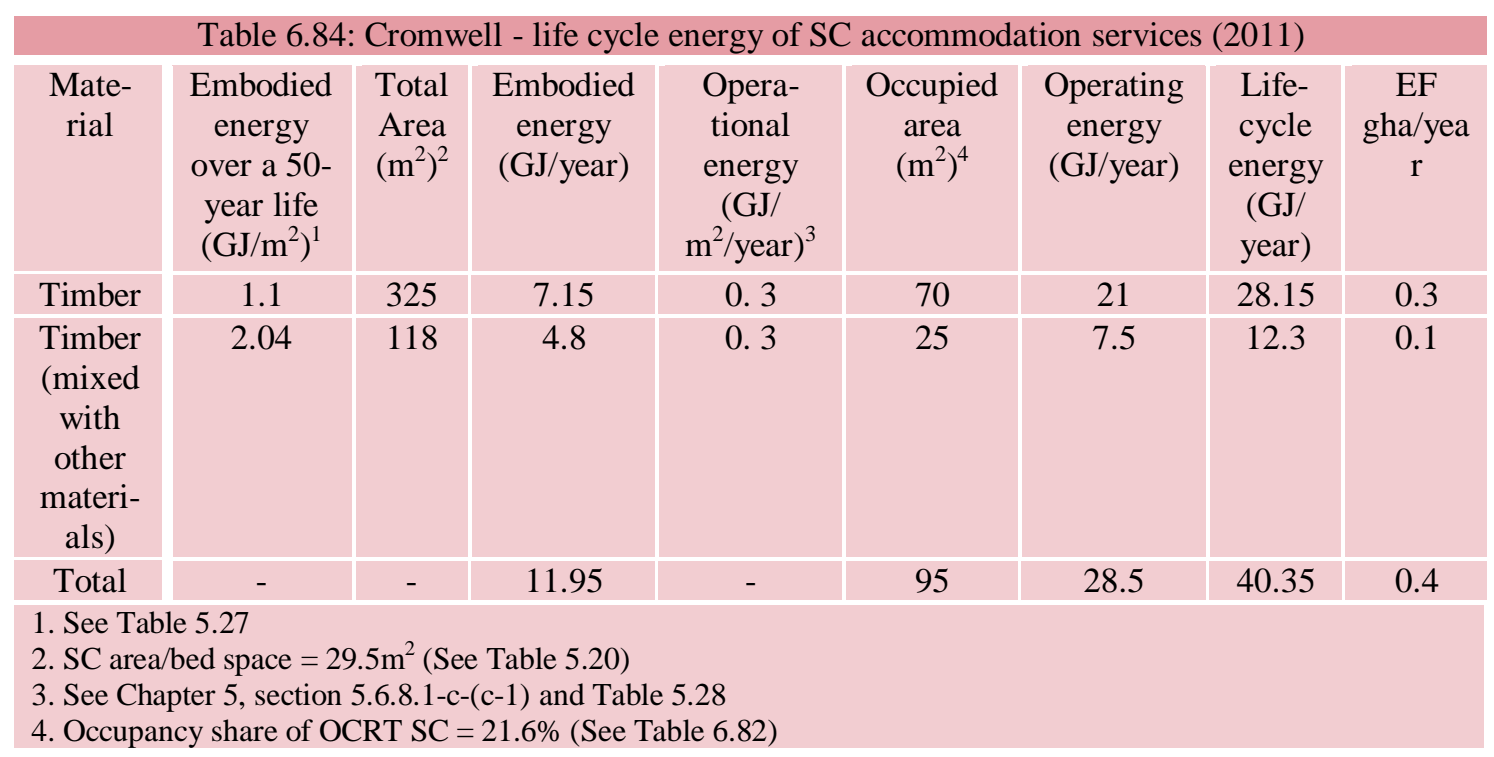

\section{a.2 . The EF of Cromwell B\&B Accommodation Services}

Table 6.85 demonstrates that $160 \mathrm{~m}^{2}$ of Cromwell B\&B accommodation services of timber constructions have an embodied energy of $3.52 \mathrm{GJ} /$ year and 3,840 $\mathrm{m}^{2}$ B\&Bs of mixed timber construction have an embodied energy of $156.6 \mathrm{GJ} /$ year.

In Table 6.85, B\&B operating energy is assumed to be $0.3 \mathrm{GJ} / \mathrm{m}^{2} /$ year, equal to the B\&B operating energy in Table 5.28. As determined in Table 6.85, Cromwell B\&B accommodation services of timber construction use $10.8 \mathrm{GJ} /$ year operating energy and those of mixed timber construction use $258 \mathrm{GJ} /$ year. In addition the table shows that in Cromwell, timber B\&B accommodation has a life cycle energy of $14.3 \mathrm{GJ} /$ year and that of mixed timber construction has a life cycle energy of $414 \mathrm{GJ} /$ year 
The total EF of Cromwell B\&B accommodation services as shown in Table 6.85 is 4.29 gha, made up of 0.14 gha of $\mathrm{B} \& \mathrm{~B}$ of timber construction and 4.15 gha of mixed timber construction B\&Bs.

\begin{tabular}{|c|c|c|c|c|c|c|c|c|}
\hline \multicolumn{9}{|c|}{ Table 6.85:Cromwell - life cycle energy of B\&B accommodation services (2011) } \\
\hline Material & $\begin{array}{l}\text { Embod- } \\
\text { ied en- } \\
\text { ergy over } \\
\text { a } 50 \text {-year } \\
\text { life cycle } \\
\left(\mathrm{GJ} / \mathrm{m}^{2}\right)^{1}\end{array}$ & $\begin{array}{l}\text { Total } \\
\text { Area } \\
\left(\mathrm{m}^{2}\right)^{2}\end{array}$ & $\begin{array}{l}\text { Embod- } \\
\text { ied en- } \\
\text { ergy } \\
\text { (GJ/year) }\end{array}$ & $\begin{array}{l}\text { Opera- } \\
\text { tion en- } \\
\text { ergy (GJ/ } \\
\mathrm{m}^{2} / \text { year) }\end{array}$ & $\begin{array}{l}\text { Occu- } \\
\text { pied area } \\
\left(\mathrm{m}^{2}\right)^{4}\end{array}$ & $\begin{array}{l}\text { Operat- } \\
\text { ing en- } \\
\text { ergy } \\
\text { (GJ/year) }\end{array}$ & $\begin{array}{l}\text { Life- } \\
\text { cycle } \\
\text { en- } \\
\text { ergy } \\
\text { use } \\
\text { (GJ/ } \\
\text { year) }\end{array}$ & $\begin{array}{l}\text { EF } \\
\text { gha/ } \\
\text { year }\end{array}$ \\
\hline Timber & 1.1 & 160 & 3.52 & 0.3 & 36 & 10.8 & 14.3 & 0.14 \\
\hline $\begin{array}{c}\text { Timber } \\
\text { (mixed } \\
\text { with other } \\
\text { materi- } \\
\text { als). }\end{array}$ & 2.04 & 3,840 & 156.6 & 0.3 & 860 & 258 & 414.6 & 4.15 \\
\hline Total & & 4,000 & 160.12 & & 896 & 268.8 & 428.9 & 4.29 \\
\hline $\begin{array}{l}\text { 1. See Table } \\
\text { 2. B\&B area } \\
\text { 3. See Chapt } \\
\text { 4. Occupanc }\end{array}$ & of & & 20) & & & & & \\
\hline
\end{tabular}

\section{a.3. The EF of Cromwell Motel Accommodation Services}

Cromwell's motel accommodation services comprise 2,907 $\mathrm{m}^{2}$ timber and $963 \mathrm{~m}^{2}$ mixed timber constructions that use 64 and $39.3 \mathrm{GJ} /$ year respectively of embodied energy (Table 6.86).

The motel operating energy use is shown in Table 6.86 and is assumed to be the same OCRT motel operational energy use, cited in Table 5.28 (0.57 GJ/m²/year). In Cromwell, motels of timber construction use 153.2 GJ/year and those with mixed timber construction $50.8 \mathrm{GJ} /$ year for their operating energies (Table 6.86). Moreover, total lifecycle energy use of Cromwell motels is $307.1 \mathrm{GJ} / \mathrm{year}$, the sum of the life cycle energy use of timber (217 GJ/ year) and mixed timber (90.1 GJ/year) constructions (Table 6.86).

As shown in Table 6.86, the total EF of the motel accommodation services of Cromwell is 3.07 gha, made up of the EFs of motels of timber (2.17gha) and mixed timber (0.9 gha) constructions. 


\begin{tabular}{|c|c|c|c|c|c|c|c|c|}
\hline Material & $\begin{array}{l}\text { Embodied } \\
\text { energy } \\
\text { over a 50- } \\
\text { year life } \\
\text { cycle } \\
\left(\mathrm{GJ} / \mathrm{m}^{2}\right)^{1}\end{array}$ & $\begin{array}{l}\text { Total } \\
\text { Area } \\
\left(\mathrm{m}^{2}\right)^{2}\end{array}$ & $\begin{array}{l}\text { Embodied } \\
\text { energy } \\
\text { (GJ/year) }\end{array}$ & $\begin{array}{l}\text { Opera- } \\
\text { tional } \\
\text { energy } \\
(\mathrm{GJ} / \\
\left.\mathrm{m}^{2} / \text { year }\right)^{3}\end{array}$ & $\begin{array}{l}\text { Occupied } \\
\text { area }\left(\mathrm{m}^{2}\right)^{4}\end{array}$ & $\begin{array}{l}\text { Operating } \\
\text { energy } \\
\text { (GJ/year) }\end{array}$ & $\begin{array}{l}\text { Life- } \\
\text { cycle } \\
\text { energy } \\
\text { use (GJ/ } \\
\text { year) }\end{array}$ & $\begin{array}{l}\text { EF } \\
\text { gha/yea } \\
r\end{array}$ \\
\hline Timber & 1.1 & 2,907 & 64 & 0.25 & 613 & 153.2 & 217 & 2.17 \\
\hline $\begin{array}{l}\text { Timber } \\
\text { and ma- } \\
\text { sonry }\end{array}$ & 2.04 & 963 & 39.3 & 0.25 & 203 & 50.8 & 90.1 & 0.9 \\
\hline Total & - & 3,870 & 102 & - & 816 & 204 & 307.1 & 3.07 \\
\hline \multicolumn{9}{|c|}{$\begin{array}{l}\text { 1. See Table } 5.27 \\
\left.\text { 2. Motel area/bed space }=40 \mathrm{~m}^{2} \text { (see Table } 5.20\right) \\
\text { 3. See Chapter 5-section 5.6.8.1-c-(c-1) and Table } 5.28 \\
\text { 4. Occupancy rate of OCRT Motels = } 32 \% \text { (see Table 6.82) }\end{array}$} \\
\hline
\end{tabular}

\section{a.4. The EF of Cromwell Camping Accommodation Services}

The total available bed spaces of camping sites at Cromwell are 2,826 bed spaces including 1,728 (61.1\%) in powered sites and 1,098 in non-powered sites (Table 6.87). This section uses these proportions to divide the total 2,291 camping visitor nights into 1,400 (61.1\%) that use powered sites in Cromwell and 891 (38.9\%) that stay at nonpowered sites (Table 87).

\begin{tabular}{|c|c|c|c|c|c|}
\hline \multicolumn{4}{|c|}{ Table 6.87: Cromwell camping sites - available bed spaces and visitor nights } \\
\hline Type & No. & Available bed spaces & $\%$ & Visitor nights \\
\hline Powered sites & 288 & 1,728 & 61.1 & 1,400 \\
\hline $\begin{array}{c}\text { Non- powered } \\
\text { sites }\end{array}$ & 183 & 1,098 & 38.9 & 891 \\
\hline Total & 471 & 2,826 & 100 & $2,291^{2}$ \\
\hline $\begin{array}{l}\text { 1. See Table 6.80 } \\
\text { 2. See Table 6.83 }\end{array}$ & & & \\
\hline
\end{tabular}

As explained in Table 6.88 the embodied energy of Cromwell camping sites is not available and this study considers the operational energy use of this type of accommodation to be the same as its life cycle energy use. Table 6.88 indicates that the total EF of 288 Cromwell powered sites (see Table 6.87) with 1400 visitor nights and 0.025 GJ/visitor night operational energy use is 0.35 gha/year.

\begin{tabular}{|c|c|c|c|c|c|c|c|}
\hline Material & $\begin{array}{c}\text { Embodied } \\
\text { energy over a } \\
50 \text { year life } \\
\text { cycle } \\
\left(\mathrm{GJ} / \mathrm{m}^{2}\right)\end{array}$ & $\begin{array}{l}\text { Total } \\
\text { visitor } \\
\text { nights }\end{array}$ & $\begin{array}{l}\text { Embodied } \\
\text { energy } \\
\text { (GJ/year) }\end{array}$ & $\begin{array}{c}\text { Operation } \\
\text { energy (GJ/ } \\
\text { visitor night) }\end{array}$ & $\begin{array}{l}\text { Operating } \\
\text { energy } \\
\text { (GJ/year) }\end{array}$ & $\begin{array}{l}\text { Life-cycle } \\
\text { energy use } \\
\text { (GJ/year) }\end{array}$ & $\begin{array}{c}\text { EF } \\
\text { gha/year }\end{array}$ \\
\hline $\begin{array}{l}\text { Assumed } \\
\text { negligible }\end{array}$ & N/A & $1400^{1}$ & N/A & 0.025 & 35 & 35 & 0.35 \\
\hline \multicolumn{8}{|c|}{$\begin{array}{l}\text { 1. See Table } 6.87 \\
\text { 2. See Table } 5.28\end{array}$} \\
\hline
\end{tabular}




\section{a.5. Total EF of Cromwell Accommodation Services (First Scenario)}

The total EF of Cromwell accommodation services in the first scenario in which all associated areas are assumed to be indoor spaces is 8.11gha /year (Table 6.89). As demonstrated in this table, Cromwell B\&B accommodation accounts for the largest share of the EF at 4.29 gha, followed by motel (3.07gha), SC (0.4gha) and camping sites (0.35gha).

Table 6.89: Cromwell - EF of accommodation services (2011)

\begin{tabular}{|l|c|}
\hline Type of accommodation & EF (gha/year) \\
\hline SC & 0.4 \\
\hline B\&B & 4.29 \\
\hline Motel & 3.07 \\
\hline Camping & 0.35 \\
\hline Total & 8.11 \\
\hline
\end{tabular}

\section{b. The EF of Cromwell Accommodation Services (Second Scenario)}

This section attempts to determine the influence of using open air spaces including verandas and balconies (V/B) on the EF of Cromwell's accommodation buildings. In this scenario, the accommodation services contain both indoor and outdoor spaces and their EFs comprise the EFs of both types of space. The difference between the results of the first and second scenario indicates the influence of using open air areas on the EF of Cromwell accommodation services.

Table 6.90 contains summarised information about the numbers and types of V/Bs and outdoor sitting areas by type of accommodation, which are explained in Appendix 5 (rows-188-200). Table 6.90 shows that 4 Cromwell SC bed spaces have access to 2 private V/Bs. Furthermore, as shown in Table 6.90, 14 Cromwell's motel bed spaces have access to 2 shared outdoor sitting areas. Since the outdoor sitting areas are considered to be part of the landscaping, in this thesis their EF is not included

Table 6.90: Cromwell - outdoor sitting space used as part of accommodation services

\begin{tabular}{|c|c|c|c|c|c|c|c|c|}
\hline \multirow[t]{2}{*}{ Туре } & \multicolumn{4}{|c|}{$\mathrm{V}^{1} / \mathrm{B}^{2}$} & \multicolumn{4}{|c|}{ Outdoor sitting } \\
\hline & $\mathrm{S}^{3}$ & $\mathrm{NV}^{4}$ & $\mathrm{P}^{5}$ & NV & $\mathrm{S}$ & $\mathrm{No}^{6}$ & $\mathrm{P}$ & No \\
\hline $\mathrm{SC}$ & 0 & 0 & 4 & 2 & 11 & 2 & 0 & 0 \\
\hline $\mathrm{B} \& \mathrm{~B}$ & 0 & 0 & 0 & 0 & 0 & 0 & 96 & 46 \\
\hline Motel & 0 & 0 & 14 & 2 & 36 & 1 & 14 & 2 \\
\hline $\begin{array}{l}\text { 1. V: V } \\
\text { 2. B: B } \\
\text { 3. S: Nu } \\
\text { 4. NV: } \\
\text { 5. P: Nu } \\
\text { 6. No: I }\end{array}$ & $\begin{array}{l}\text { droo } \\
\text { vera } \\
\text { droo } \\
\text { outd }\end{array}$ & $\begin{array}{l}\text { ing ou } \\
\text { lconie } \\
\text { g priv } \\
\text { ig spa }\end{array}$ & & & & & & \\
\hline
\end{tabular}


Table 6.91 indicates that the embodied energy intensity of $57.2 \mathrm{~m}^{2}$ of Cromwell V/B is 0.7 GJ/year. Since V/Bs are open areas and do not use operational energy, in particular for heating, their operational energy is assumed to be zero. As a result, the life cycle energy use of $57.2 \mathrm{~m}^{2} \mathrm{~V} / \mathrm{B}$ is equal to its embodied energy (0.7 GJ/ year) and its EF is 0.7 (life cycle energy use) $\div 100$ (global average carrying capacity of land) $=0.007$ gha (Table 6.91).

\begin{tabular}{|c|c|c|c|c|c|c|}
\hline Type & $\begin{array}{l}\text { Embodied } \\
\text { energy }\left(\mathrm{GJ} / \mathrm{m}^{2}\right. \\
\text { per year })\end{array}$ & $\begin{array}{l}\text { Area } \\
\left(\mathrm{m}^{2}\right)^{1-}\end{array}$ & $\begin{array}{l}\text { Embodied } \\
\text { energy } \\
(\mathrm{GJ} / \text { year })^{3}\end{array}$ & $\begin{array}{c}\text { Operating } \\
\text { energy }\end{array}$ & $\begin{array}{l}\text { Life-cycle } \\
\text { energy } \\
\text { (GJ/year) }\end{array}$ & $\begin{array}{c}\text { EF } \\
\text { (gha) }\end{array}$ \\
\hline Veranda/balcony & 0.012 & 57.2 & 0.7 & 0 & 0.7 & 0.007 \\
\hline \multicolumn{7}{|c|}{$\begin{array}{l}\text { 1. Total numbers of verandas/balconies }=4 \text { (see Table } 6.91) \\
\text { 2. Average area }\left(\mathrm{m}^{2}\right) \text { of veranda/balcony }=14.3(\text { Table } 5.40) \\
\text { 3. Embodied energy of veranda/ balcony }\left(\mathrm{GJ} / \mathrm{m}^{2} / \text { year }\right)=0.012(\text { Table } 5.42)\end{array}$} \\
\hline
\end{tabular}

In Table 6.29, the total 25,178 $\mathrm{m}^{2}$ of Cromwell accommodation services (see Table $6.92)$ is separated into the two areas of $25,120.8 \mathrm{~m}^{2}$ indoor spaces and $57.2 \mathrm{~m}^{2} \mathrm{~V} / \mathrm{B}$. The EF of indoor spaces is 0.00032 gha $/ \mathrm{m}^{2}\left(8.11\right.$ gha $\left.\div 25,178 \mathrm{~m}^{2}\right)$. Thus the EF of 25,120 $\mathrm{m}^{2}$ indoor spaces is $25,121 \mathrm{~m}^{2} \times 0.00032 \mathrm{gha} / \mathrm{m}^{2}=8.039$ gha (Table 6.92). Likewise the EF (gha) of $57.2 \mathrm{~m}^{2} \mathrm{~V} / \mathrm{B}$ is equivalent to 0.007 gha (see Table 6.91). As a result the total EF of 25,178 $\mathrm{m}^{2}$ accommodation services in Cromwell composed of 25,120.8 $\mathrm{m}^{2}$ indoor spaces and $57.2 \mathrm{~m}^{2} \mathrm{~V} / \mathrm{B}$ is the sum of 8.039 and $0.007 \mathrm{gha}=8.046$ gha (Table $6.92)$.

As shown in Table 6.89, in the first scenario in which all Cromwell accommodation services are assumed to be indoor spaces, the total EF (EF1) is 8.11 gha. In the second scenario where both indoor and outdoor spaces are considered, the total EF (EF2) is 8.046 gha. Thus the 0.064 gha difference between the two EFs (EF1 and EF2) can be considered the reduction in Cromwell accommodation EF influenced by the use of 57.2 $\mathrm{m}^{2}$ veranda and balcony (Table 6.92). On the other hand, as 0.064 gha $\left(640 \mathrm{~m}^{2}\right) \div 57.2$ $\mathrm{m}^{2}=11.2 \mathrm{~m}^{2}$, it can be considered that using $1 \mathrm{~m}^{2}$ of V/B reduces the EF of Cromwell accommodation by $11.2 \mathrm{~m}^{2}$. 


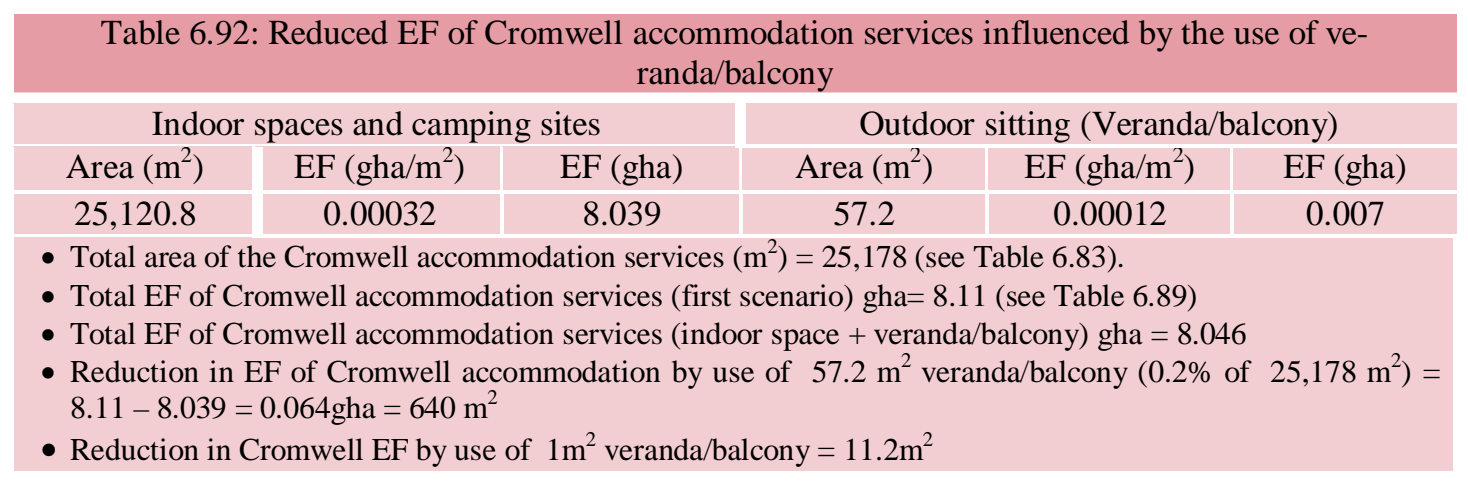

\subsubsection{Tourism Products and Activities (Frequency)}

This section investigates the environmental impacts of Cromwell visitor activities (as a part of their social-cultural behaviours) through calculation of their EF. The first step is to make assumptions about the frequency of activities offered by Cromwell accommodation services to visitors through using the official websites of the OCRT and 12 websites related to Cromwell accommodation services. The activities offered are explained in Appendix 5 (rows 188-200) and are classified into the two categorises of indoor and outdoor activities in Appendix 34.

In addition the frequency of the offered activates as shown in Appendix 34 and summarised in Tables 6.94 and 6.95 can be considered as an indicator of the attitude of Cromwell OCRT participants (in this case accommodation owners) to types of tourism activity, and consequently to sustainability. Comparison between the EFs of the activities and their frequency also can indicate from a social-ecological perspective the attitude of tourists to being sustainable.

\subsubsection{Local Produce}

The frequency of local products offered by the Cromwell's accommodation services and the environmental effects of using home prepared foods have been discussed above in section 6.2.5.2 and demonstrated in Table 6.76.

\subsubsection{Tourism Activities}

The activities offered by Cromwell accommodation services are explained in Appendix 5 (rows 188-200) and classified into indoor and outdoor activates in Appendix 34. The 
following section of this chapter investigates the frequency of these offered activities and their environmental impacts through calculation of their ecological footprints.

\section{a. Indoor Visitor Activities (Frequency)}

Table 6.93 presents the frequencies of reading materials as only indoor activity offered by Cromwell accommodation services to their visitors (Appendices 5 and 34). The table shows that only 2 of 12 websites related to 492 Cromwell accommodation services offer an indoor activity to their visitors.

\begin{tabular}{|l|c|c|c|}
\hline \multicolumn{2}{|c|}{ Table 93: Cromwell - indoor activities } & & \\
\hline Activity & & F & $\%$ \\
\hline Reading materials & & 2 & 100 \\
\hline
\end{tabular}

\section{b. Cromwell - Outdoor Visitor Activities (Frequency)}

This study found 20 types of outdoor activity offered by Cromwell accommodation services to visitors in 2011, as shown in Appendices 5, 34 and 35. These are classified into the 8 types of activities shown in Table 6.94.

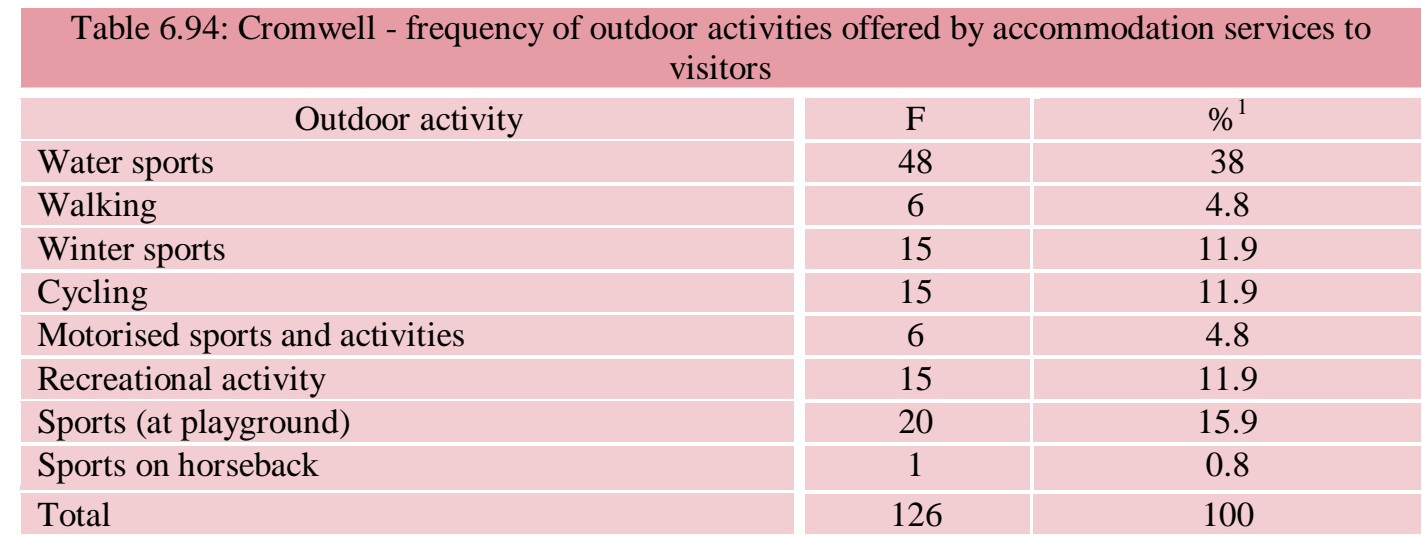

Figure 6.49 and Table 6.94 illustrate that the largest occurrence of an offered activity is water sports at 48 (38\%), followed by sports at a play ground 20 (15.9\%), winter sports, cycling and recreational activities all at 15 (11.9\%). Likewise other outdoor activities including motorised sports and walking have equal frequencies of occurrence of 6 (4.8) (Figure 6.49 and Table 6.94). 


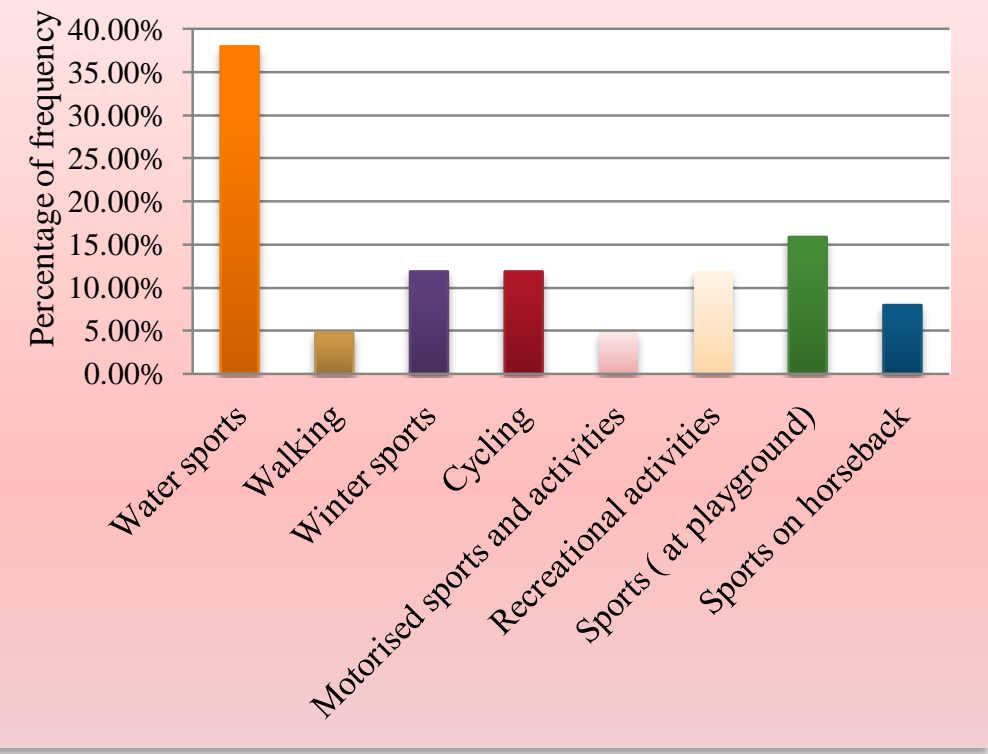

Figure 6.49: The frequency of outdoor activities offered by Cromwell accommodation services to its visitors

\section{c. Cromwell -Frequency of the Subjects Offered to Visit}

As shown in Appendix 5 this chapter looks at subjects to visit offered by Cromwell accommodation services to visitors in 2011. Appendix 35 contains summarised information about Cromwell visiting activities and their frequency. Table 6.95 and Figure 6.50 classify the 10 categories of subjects offered to visit cited in Appendices 5 and 35 into five types: cities and villages, historical sites and buildings, scenery, museum/art gallery, and gardens.

\begin{tabular}{|l|c|c|}
\hline \multicolumn{2}{|c|}{ Table 6.95: Cromwell - frequency of subjects to visit offered by accommodation services } \\
to visitors (2011)
\end{tabular}

As illustrated in Figure 6.50 and Table 6.95, gardens are offered the most at 25 (48\%) mentions, followed by cities and villages 17 (32.7\%), historic sites and buildings 5 (9.5\%), scenery 4 (7.7\%), and museum/art gallery 1 (2\%). 


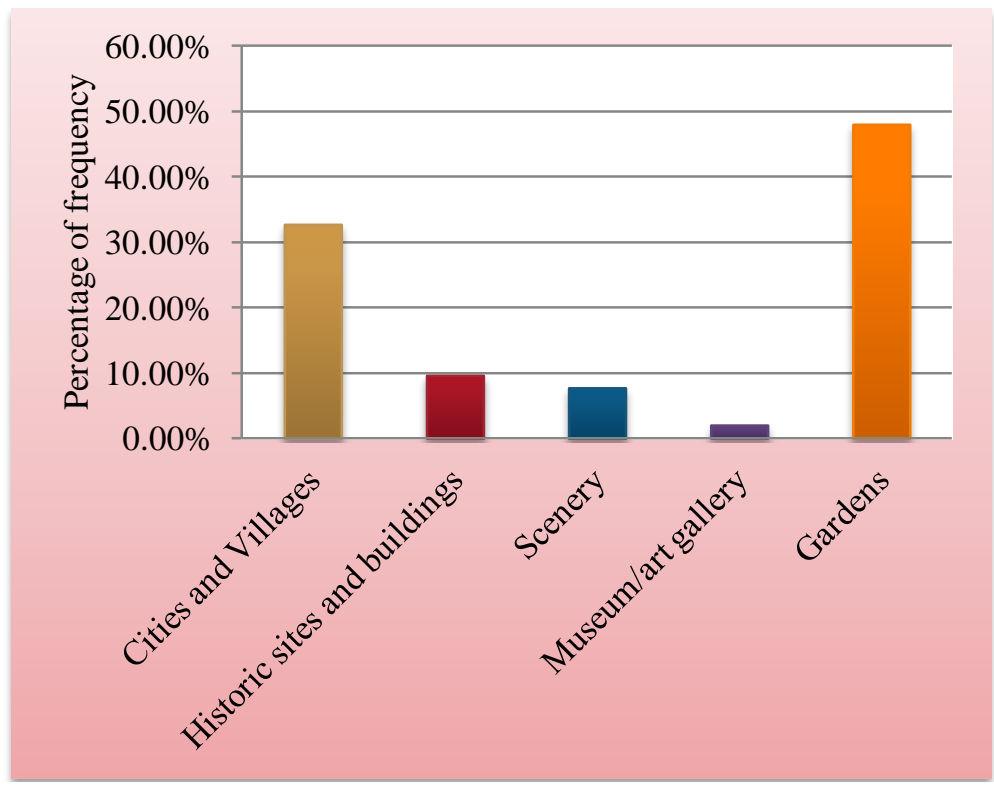

Figure 6.50: The frequency of subjects offered by Cromwell accommodation services to visit (2011)

\section{d. Activities per Visitor}

This section calculates the number of Cromwell visitors per activity (except visiting old Cromwell town) through using the percentage split of 160 visitors (Table 5.48) and applying this to the 2,652 Cromwell visitors in 2011 (Table 6.96).

\begin{tabular}{|c|c|c|}
\hline \multicolumn{3}{|c|}{ Table 6.96: Cromwell activities conducted by 2,652¹ visitors (2011) } \\
\hline Activity & $\%$ of 2,652 visitors ${ }^{2}$ & Number of Visitors \\
\hline Curling (at Naseby) & 36.9 & 979 \\
\hline Taieri Gorge Railway & 46.3 & 1,228 \\
\hline Gold mining towns & 35 & 928 \\
\hline Sightseeing tours & 30.6 & 812 \\
\hline Old Cromwell town & 100 & 2,652 \\
\hline Wineries & 16.9 & 448 \\
\hline Visiting friends and relatives & 15 & 398 \\
\hline Hayes engineering & 8.1 & 215 \\
\hline Mountain biking at Naseby & 2.5 & 66 \\
\hline Fishing & 1.9 & 50 \\
\hline Golf & 1.3 & 34 \\
\hline Swim at Ophir and Alexandra & 0.6 & 16 \\
\hline Clyde Dam Tour & 0.6 & 16 \\
\hline Golden Progress mine & 0.6 & 16 \\
\hline Walking & 0.6 & 16 \\
\hline Ophir high country farm & 0.6 & 16 \\
\hline Biking & 0.6 & 16 \\
\hline Fruit picking along trail & 0.6 & 16 \\
\hline Total & - & 7,922 \\
\hline $\begin{array}{l}\text { 1. See Table } 6.67 \\
\text { 2. \% of } 2,652 \text { visitor assumed san } \\
\text { Cromwell town. } \\
\text { - Reference: CODC, } 2011: 18\end{array}$ & of 160 visitors cited i & 5 except for visitors to \\
\hline
\end{tabular}


The assumption is that the visitors who come to Cromwell will go to the old town but will also do other activities. The number of OCRT visitors who visit Cromwell is already known (see Table 4.45). Table 6.96 shows that in comparison with other Cromwell activities, the majority of visitor activities is visiting old Cromwell town $(2,652)$, followed by Taieri Gorge Railway (1,228), curling at Naseby (979), visiting gold mining towns (928), and sightseeing tours (812). The numbers of Cromwell visitors who do other types of activity fall between 16 and 448 .

\subsubsection{EF of Visitor Activities}

\subsubsection{EF of Indoor Activities (Curling at Naseby)}

As indicated in Table 6.97, 979, 7.5\% of the total 13,000 visitors who went curling at Naseby stay at Cromwell. Since the total EF of 13,000 visitors is 2.07gha, the EF of Cromwell visitors equals $7.5 \%$ of $2.07=0.16$ gha (Table 6.97).

\begin{tabular}{|c|c|c|c|c|}
\hline \multicolumn{5}{|c|}{ Table 6.97: Cromwell visitors - curling EF (gha) (2011) } \\
\hline $\begin{array}{l}\text { Total num- } \\
\text { ber of } \\
\text { Cromwell } \\
\text { visitors }\end{array}$ & $\begin{array}{l}\% \text { of Cromwell } \\
\text { visitors who play } \\
\text { indoor curling at } \\
\text { Naseby }^{1}\end{array}$ & $\begin{array}{l}\text { Total number of } \\
\text { Cromwell visi- } \\
\text { tors who play } \\
\text { curling at } \\
\text { Naseby }{ }^{1}\end{array}$ & $\begin{array}{l}\text { EF of } 13,000 \text { visi- } \\
\text { tors of curling cen- } \\
\text { tre (gha) }\end{array}$ & $\begin{array}{l}\text { EF of } 979 \text { Cromwell } \\
\text { visitors ( } 7.5 \% \text { of } 13,000 \\
\text { indoor curling centre } \\
\text { visitors) gha }\end{array}$ \\
\hline 2,652 & 36.9 & 979 & $2.07^{3}$ & 0.16 \\
\hline \multicolumn{5}{|c|}{$\begin{array}{l}\text { 1. See Table } 6.34 \\
\text { 2. EF of Cromwell curling (indoor curling) per visitor per } 3 \text { hours playing/ year }=(0.15 \text { gha } \div 979 \text { visitors }) 0.00016 \\
\text { (gha) } \\
\text { 3. See Table } 5.56\end{array}$} \\
\hline
\end{tabular}

\subsubsection{EF of Outdoor Activities}

\section{a. EF of Walking}

Tables 6.96 and 6.98 show that 16 Cromwell visitors chose walking as a tourist activity. As shown in Chapter 5 (Table 5.58) the EF of a visitor who walks 3 hours is 0.00005 gha. Thus the EF of 16 Cromwell visitors who walk 3 hours is equivalent to: 0.00005 $($ gha/visitor $) \times 16$ (number of visitors $)=0.0008$ (gha) $($ Table 6.98) .

Table 6.98: Ecological footprint of Cromwell visitors (walking).

Number of Cromwell visitors who walk.

EF (gha) of Cromwell visitor who walks 3 hours

EF of 16 Cromwell visitors who walk 3 hours (gha)

1. See Table 6.96

2. See Table 5.58

\section{$16^{1}$}

$0.00005^{2}$

0.0008 


\section{b. The EF of Golf}

Tables 6.96 and 6.99 show 34 Cromwell visitors played golf 2011. Table s.5.60 and 6.37 indicate that the EF of a visitor who plays golf is 0.003 gha/ visitor. Consequently the EF of the 34 Cromwell visitors playing golf is $34 \times 0.003$ gha/visitor $=0.102$ gha (Table 6.99).

Table 6.99: Ecological footprint of Cromwell visitors (golf)

Number of Cromwell visitors who play golf

$34^{1}$

EF (gha) of Cromwell visitor who plays golf

EF of 34 Cromwell visitors who play golf (gha)

1. See Table 6.96

2. See Table 5.60

\section{c. EF of Mountain Biking}

As shown in Tables 6.96 and 6.100, 66 Cromwell visitors went mountain biking in 2011. Tables 5.67 and 6.100 indicate that the EF of a visitor who goes mountain biking is 0.0009 gha/visitor. As a result the EF of 66 mountain biking Cromwell visitors is 66 $\times 0.0009$ gha/visitor $=0.06$ gha $($ Table 6.100$)$.

Table 6.100: Ecological footprint of Cromwell visitors (mountain biking)

Number of Cromwell visitors who go mountain biking EF (gha) of Cromwell visitors who goes mountain biking

EF of 66 Cromwell visitors who go mountain biking (gha)

1. See Table 6.96

2. See Table 5.67

\section{d. EF of Fishing}

In 2011, 50 Cromwell visitors chose fishing as a leisure activity (Tables 6.96 and 6.101). Since the EF of a visitor fishing is 0.0009 gha (see Tables 5.68 and 6.101), the EF of 50 Cromwell visitors fishing is 0.0009 (gha/visitor) $\times 50$ (visitors) $=0.045$ gha (Table 6.101).

Table 6.101: Ecological footprint of Cromwell visitors (fishing)

Number of Cromwell visitors who fish

EF (gha) of Cromwell visitors who fish

EF of 50 Cromwell visitors fishing (gha)

1. See Table 6.96

2. See Table 5.68
$50^{1}$

$0.0009^{2}$

0.045
$66^{1}$

$0.0009^{2}$

0.06 (1) 


\title{
e. EF of Swimming at Ophir and Alexandra
}

Tables 6.96 and 6.102 show 16 Cromwell visitors went swimming at Ophir and Alexandra in 2011. Tables 5.69 and 6.102 determine that the EF of a visitor who swims is 0.0009 gha. As a result the EF of 16 Cromwell visitors going swimming is $16 \times 0.0009$ gha/visitor $=0.014$ gha $($ Table 6.102) .

\begin{tabular}{|l|c|}
\hline \multicolumn{2}{|c|}{ Table 6.102: Ecological footprint of Cromwell visitors (swimming) } \\
\hline Number of Cromwell visitors who swim & $16^{1}$ \\
\hline EF (gha) of Cromwell visitors swimming & $0.0009^{2}$ \\
\hline EF of 16 Cromwell visitors swimming (gha) & 0.014 \\
\hline 1. See Table 6.96 & \\
2. See Table 5.69 & \\
\hline
\end{tabular}

\section{f. EF of Wineries}

Tables 6.96 and 6.103 show that 448 people who came to Cromwell visited wineries. Table s.5.70 and 6.103 determine that the EF of a visitor going to a winery is 0.00006 gha/ visitor. As a result the EF of 448 Cromwell visitors who go to wineries is $448 \times$ 0.00006 gha/visitor $=0.027$ gha $($ Table 6.103) .

\begin{tabular}{l|c|}
\hline \multicolumn{2}{|c|}{ Table 6.103: Ecological footprint of Cromwell visitors (wineries) } \\
\hline Number of Cromwell visitors who visit wineries & $448^{1}$ \\
\hline EF (gha) of Cromwell visitor to winery & $0.00006^{2}$ \\
\hline EF of 448 Cromwell visitors to wineries (gha) & 0.027 \\
\hline 1. See Table 6.96 & \\
2. See Table 5.70 & \\
\hline
\end{tabular}

\section{g. EF of Sightseeing}

Tables 6.96 and 6.104 indicate that 812 visitors chose sightseeing as one of their activities at Cromwell. As explained in Table 5.71 (Chapter 5) the EF of a visitor sightseeing is 0.0009 gha. Consequently, the EF of 812 Cromwell visitors is $812 \times 0.0009$ gha/visitor $=0.73$ gha $($ Table 6.104) .

Table 6.104: Ecological footprint of Cromwell visitors (sightseeing)

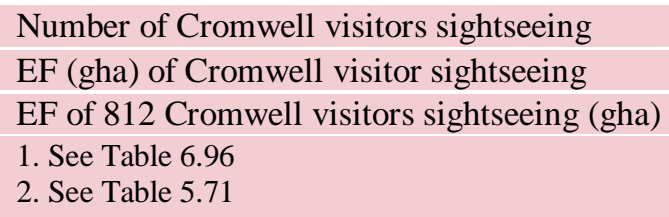

\author{
$812^{1}$ \\ $0.0009^{2}$ \\ 0.73
}

\section{h. EF of Hayes Engineering}

Hayes Engineering heritage was visited by 215 Cromwell visitors as shown in Tables 6.96 and 6.105. Since the EF of a visitor in this case is 0.00006 gha (see Table 5.72) the 
EF of the latter visitors is equivalent to 215 (number of visitors) $\times 0.00006$ gha/visitor $=$ 0.013 gha (Table 6.105).

Table 6.105: Ecological footprint of Cromwell visitors (Hayes Engineering)

Number of Cromwell visitors to Hayes Engineering $215^{1}$

EF (gha) of a Cromwell visitor to Hayes Engineering EF of 215 Cromwell visitors to Hayes Engineering (gha)

1. See Table 6.96

2. See Table 5.72

\section{i. EF of Ophir High Country Farm}

The total EF of 16 Cromwell visitors who go to Ophir High Country Farm Tour is calculated as for the examples above. As shown in Table 6.106 it is 0.001gha.

Table 6.106: Ecological footprint of Cromwell visitors (Ophir High Country Farm)

Number of Cromwell visitors to Ophir High Country Farm

EF (gha) of Cromwell visitor to Ophir High Country Farm

$16^{1}$

EF of 16 Cromwell visitors to Ophir High Country Farm (gha)

$0.00006^{2}$

1. See Table 6.96

2. See Table 5.73

\section{j. EF of Visitors to Taieri Gorge Railway}

Using the same method as before the total EF of 1,228 Cromwell visitors to the Taieri Gorge Railway is 0.074 gha (Table 6.107).

Table 6.107: Ecological footprint of Cromwell visitors (Taieri)

Number of Cromwell visitors to Taieri GR

1. See Table 6.96

2. See Table 5.74

\section{k. EF of Visitors to Gold Mining Towns}

The total EF of 928 Cromwell visitors who visit gold mining towns is 0.06 gha (Table 6.108).

Table 6.108: Ecological footprint of Cromwell visitors (gold mining towns)

Number of Cromwell visitors to Gold mining towns $928^{1}$

EF (gha) of Cromwell visitor to Gold mining towns $0.00006^{2}$

EF of 928 Cromwell visitors to Gold mining towns 0.06

1. See Table 6.96

2. See Table 5.75 


\section{EF of Visitors to Old Cromwell Town}

The EF of 2,652 Cromwell visitors to this town is 0.16 gha (Table 6.109).

Table 6.109: Ecological footprint of Cromwell visitors (Old Cromwell town)

\begin{tabular}{l|c|}
\hline Number of Cromwell visitors to old Cromwell town & $2,652^{1}$ \\
\hline EF (gha) of Cromwell visitor to old Cromwell town & $0.00006^{2}$ \\
\hline EF of 2,652 Cromwell visitors to old Cromwell \\
town
\end{tabular}

\section{m. EF of Visitors to Friends and Relatives}

The EF of 398 Cromwell visitors who visit friends and relatives is 0.08 gha (Table 6.110).

Table 6.110: Ecological footprint of Cromwell visitors (visiting friends and relatives)

Number of Cromwell visitors to friends and relatives

EF (gha) of Cromwell visitor to friends and relatives

$398^{1}$

EF of 398 Cromwell visitors to friends and relatives

$0.0002^{2}$

0.08

1. See Table 6.96

2. See Table 5.78

\section{n. EF of Visitors on Clyde Dam Tour}

The 16 Cromwell visitors who participate in a Clyde Dam Tour have an EF of 0.014 gha (Table 6.111).

Table 6.111: Ecological footprint of Cromwell visitors (Clyde Dam Tour)

Number of Cromwell visitors on Clyde Dam Tour

EF (gha) of Cromwell visitor on Clyde Dam Tour

$16^{1}$

EF of 16 Cromwell visitors on Clyde Dam Tour

$0.0009^{2}$

1. See Table 6.96

2. See Table 5.79

\section{o. EF of Visitors to Golden Progress Mine}

The EF of visitors to the Golden Progress Mine is 0.001 gha (Table 6.112).

Table 6.112: Ecological footprint of Cromwell visitors (Golden Progress Mine)

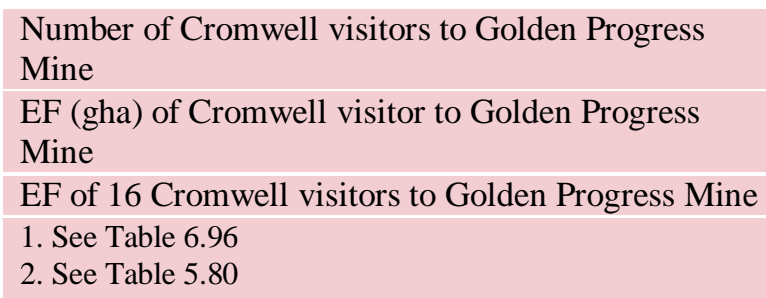

$16^{1}$

$0.00006^{2}$

0.001 


\section{p. EF of Visitors Fruit Picking Along Trail}

The EF of 16 visitors fruit picking along the trail is 0.001 gha (Table 6.113).

Table 6.113: Ecological footprint of Cromwell visitors (fruit picking along trail)

\begin{tabular}{|l|c|}
\hline Number of Cromwell visitors fruit picking along trail & $16^{1}$ \\
\hline EF (gha) of Cromwell visitor fruit picking along trail & $0.00006^{2}$ \\
\hline EF of16 Cromwell visitors fruit picking along trail & 0.001 \\
\hline 1. See Table 6.96 & \\
\hline 2. See Table 5.81 & \\
\hline
\end{tabular}

\section{q. EF of Biking}

The EF of 16 visitors biking as a tourist activity is 0.014 gha (Table 6.114).

Table 6.114: Ecological footprint of Cromwell visitors (biking)

Number of Cromwell visitors biking
EF (gha) of Cromwell visitor biking
EF of 16 Cromwell visitors biking
1. See Table 6.96
2. See Table 5.66

\subsubsection{Total EF of Cromwell Visitor Activities}

The total EF of Cromwell visitor activities as shown in Table 6.115 is 1.55 gha. Table 6.115 and Figure 6.51 demonstrate that the three largest EFs are the EF of sightseeing (0.73 gha), curling at Naseby and visiting old Cromwell town (0.16 gha) each and golf (0.1gha). Likewise, walking as a Cromwell visitor activity has the smallest EF of 0.0008 gha (Table 6.115 and Figure 6.51). 


\begin{tabular}{|c|c|c|c|c|c|}
\hline \multicolumn{6}{|c|}{ Table 6.115: Total EF of Cromwell visitor activities } \\
\hline $\begin{array}{l}\text { Type of activ- } \\
\text { ity }\end{array}$ & Activities & $\begin{array}{l}\text { Numbers } \\
\text { of visi- } \\
\text { tors per } \\
\text { activity }\end{array}$ & $\begin{array}{l}\% \text { of total } \\
2,652 \text { Crom- } \\
\text { well visitors }\end{array}$ & $\begin{array}{c}\text { EF } \\
\text { (gha/visitor) }\end{array}$ & EF (gha) \\
\hline Indoor activity & Curling at Naseby & 979 & 36.9 & 0.00016 & 0.16 \\
\hline \multirow{18}{*}{$\begin{array}{c}\text { Outdoor activi- } \\
\text { ties }\end{array}$} & Walking & 16 & 0.6 & 0.00005 & 0.0008 \\
\hline & Golf & 34 & 1.3 & 0.04 & 0.1 \\
\hline & Taieri & 1,228 & 46.3 & 0.00006 & 0.074 \\
\hline & Gold mining towns & 928 & 35 & 0.00006 & 0.06 \\
\hline & Sightseeing & 812 & 30.6 & 0.0009 & 0.73 \\
\hline & Old Cromwell town & 2,652 & 100 & 0.00006 & 0.16 \\
\hline & Wineries & 448 & 16.9 & 0.00006 & 0.027 \\
\hline & $\begin{array}{l}\text { Visiting friends and } \\
\text { relatives }\end{array}$ & 398 & 15 & 0.0002 & 0.08 \\
\hline & Hayes engineering & 215 & 8.1 & 0.00006 & 0.013 \\
\hline & $\begin{array}{l}\text { Mountain biking at } \\
\text { Naseby }\end{array}$ & 66 & 2.5 & 0.0009 & 0.06 \\
\hline & Fishing & 50 & 1.9 & 0.0009 & 0.045 \\
\hline & $\begin{array}{l}\text { Swim at Ophir and Al- } \\
\text { exandra }\end{array}$ & 16 & 0.6 & 0.0009 & 0.014 \\
\hline & Clyde Dam Tour & 16 & 0.6 & 0.0009 & 0.014 \\
\hline & Golden progress mine & 16 & 0.6 & 0.00006 & 0.001 \\
\hline & Ophir high country farm & 16 & 0.6 & 0.00006 & 0.001 \\
\hline & Biking & 16 & 0.6 & 0.0009 & 0.014 \\
\hline & Fruit picking along trail & 16 & 0.6 & 0.00006 & 0.001 \\
\hline & Total & 7,922 & - & - & 1.55 \\
\hline
\end{tabular}

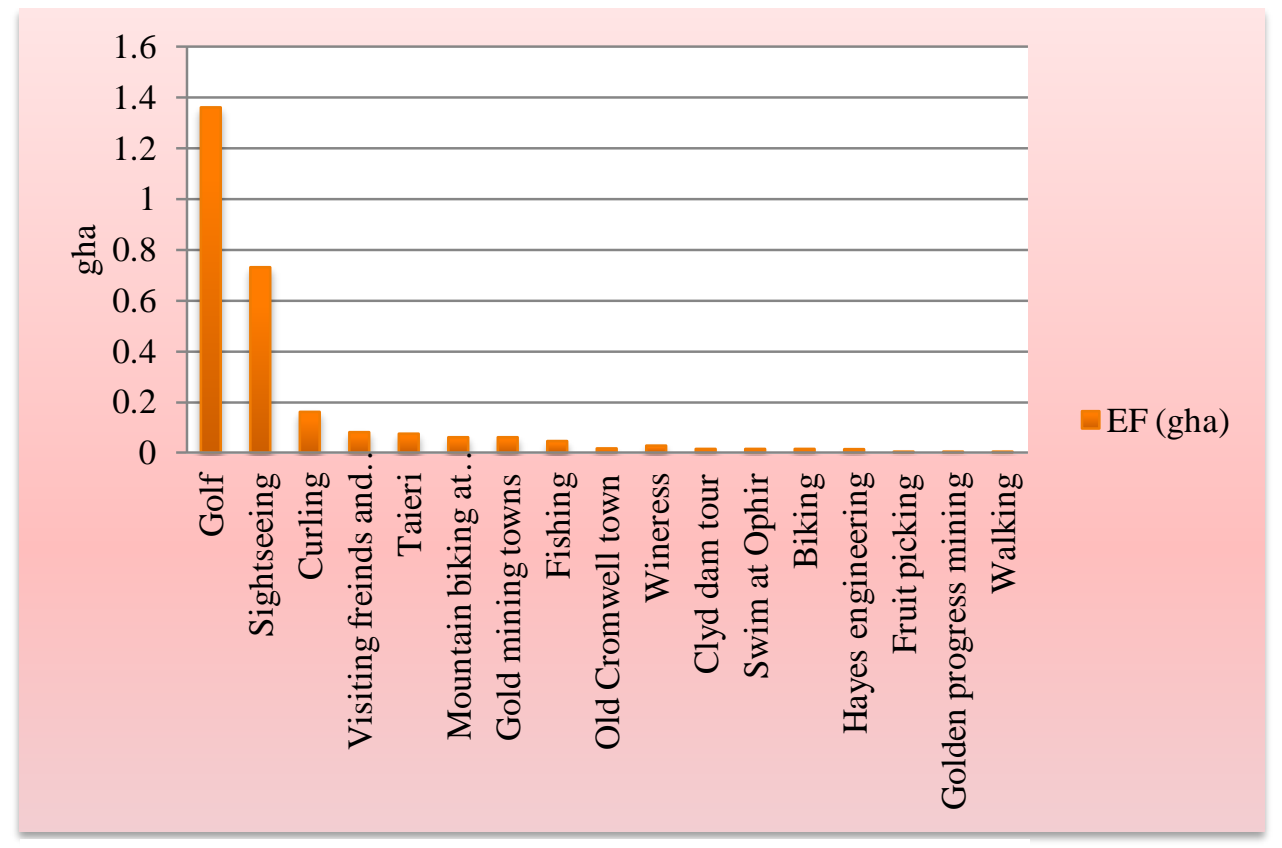

Figure 6.51: Cromwell - EF of visitor activities (2011) 


\subsubsection{Comparison between the EFs of Indoor and Outdoor Activities}

Table 6.115 shows that 2,652 Cromwell visitors participated in 7,922 visitor activities. As a result the average number of activities per visitor is 2.98, so each Cromwell visitor engages in 2.98 activities proposed by the OCRT.

As shown in Table 6.116 and Figure 6.52, the EF of 979 Cromwell visitors (12.35\% of 7,992 visitors activities) who participate in an indoor activity (curling at Naseby) is 0.16 gha and this accounts for $10.3 \%$ of the total EF of Cromwell activities (indoor and outdoor). Furthermore, Table 6.116 and Figure 6.52 indicate that the total EF of 7,013 Cromwell outdoor visitor activities (87.75\% of 7,992 Cromwell visitor activities) is 1.39 gha, or $89.7 \%$ of the total EF of Cromwell visitor activities (1.55 gha).

\begin{tabular}{|c|c|c|c|c|c|c|c|c|c|}
\hline \multicolumn{5}{|c|}{ Indoor activity } & \multicolumn{5}{|c|}{ Outdoor activities } \\
\hline $\begin{array}{l}\text { Numbers } \\
\text { of visitor } \\
\text { activities }\end{array}$ & $\begin{array}{l}\% \text { of } 7,922 \\
\text { Cromwell } \\
\text { visitor } \\
\text { activities }\end{array}$ & $\begin{array}{c}\text { EF } \\
\text { (gha) }\end{array}$ & $\begin{array}{l}\% \\
\text { total } \\
\mathrm{EF}^{1}\end{array}$ & $\begin{array}{l}\text { EF } \\
\text { (gha/visi } \\
\text { tor) }\end{array}$ & $\begin{array}{l}\text { Numbers } \\
\text { of visitor } \\
\text { activities }\end{array}$ & $\begin{array}{l}\% \text { of total } \\
\text { Cromwell } \\
\text { visitor } \\
\text { activities }\end{array}$ & $\begin{array}{c}\text { EF } \\
\text { (gha) }\end{array}$ & $\begin{array}{c}\% \text { total } \\
\mathrm{EF}^{1}\end{array}$ & $\begin{array}{l}\text { EF } \\
\text { (gha/vi } \\
\text { sitor) }\end{array}$ \\
\hline 979 & 12.35 & 0.16 & 5.7 & 0.00016 & 7,013 & 87.75 & 1.39 & 89.7 & 0.0004 \\
\hline \multicolumn{10}{|c|}{$\begin{array}{l}\text { 1. Percentage of the total 2.81gha EF of 7,992Cromwell visitor activities } \\
\text { - Total number of Cromwell visitors }=2,652 \text { (see Table } 6.67 \text { ) } \\
\text { - Total number of Cromwell visitor activities }=7,992 \text { (see Table } 6.51 \text { ) } \\
\text { - Average number of activities per visitor }=7,992 \div 2,652=2.98\end{array}$} \\
\hline
\end{tabular}

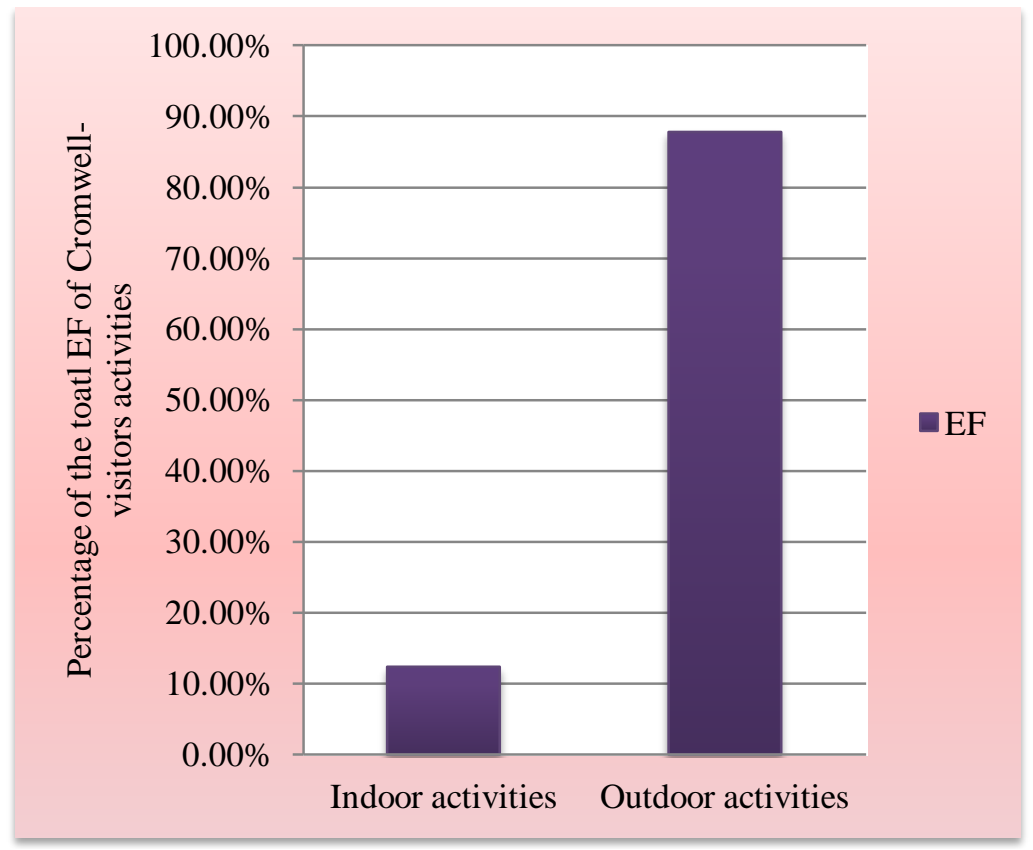

Figure 6.52: Comparison between EFs of Cromwell indoor and outdoor activities 
This chapter selects curling as an indoor activity and golf as an outdoor activity with the biggest EF/visitor in their categories to be compared with walking as the outdoor activity with the smallest EF (Figure 6.53).

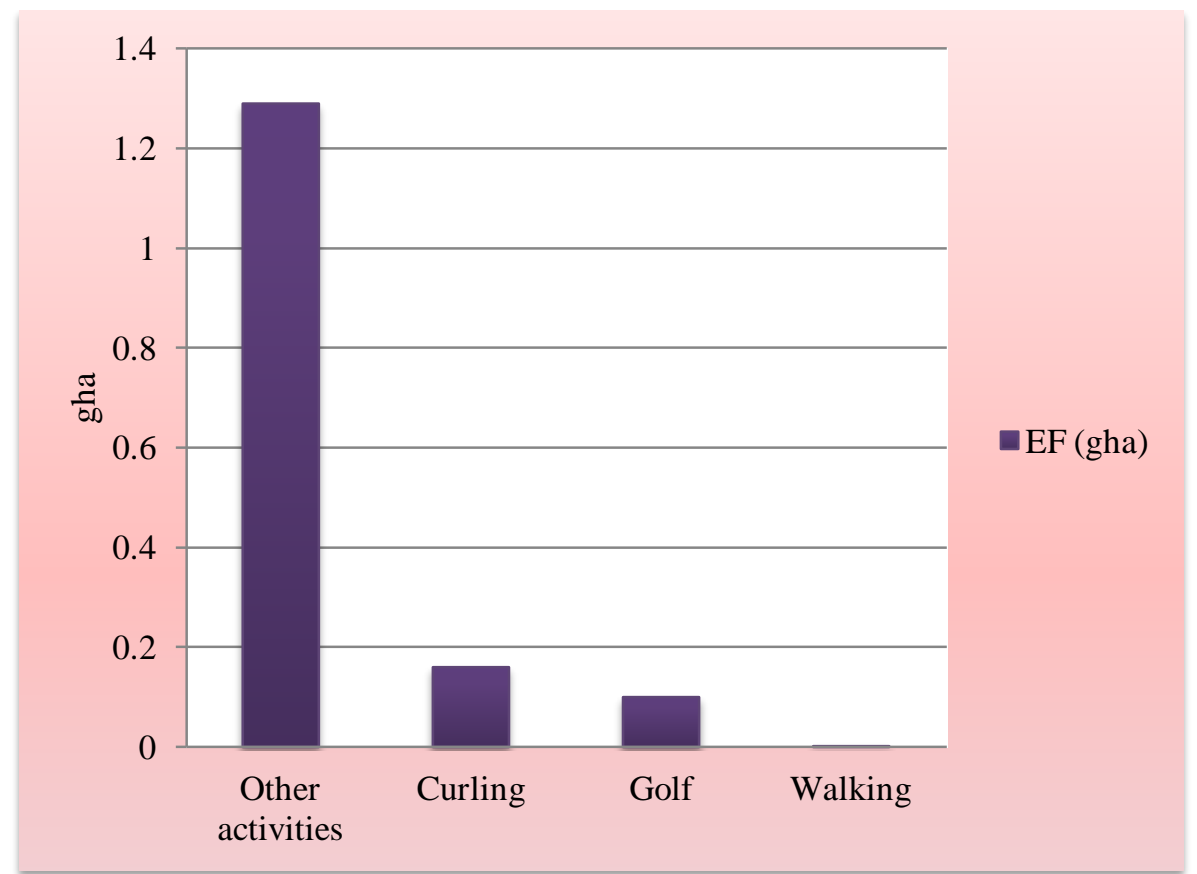

Figure 6.53: Cromwell - Comparison between EFs of golf, curling, walking, and other activities excluding these three

Figure 6.54 determines that the majority of 6,893 Cromwell visitor activities are within the ‘other activity' category (excluding golfing, curling and walking) followed by curling (979), golfing (34) and walking (16).

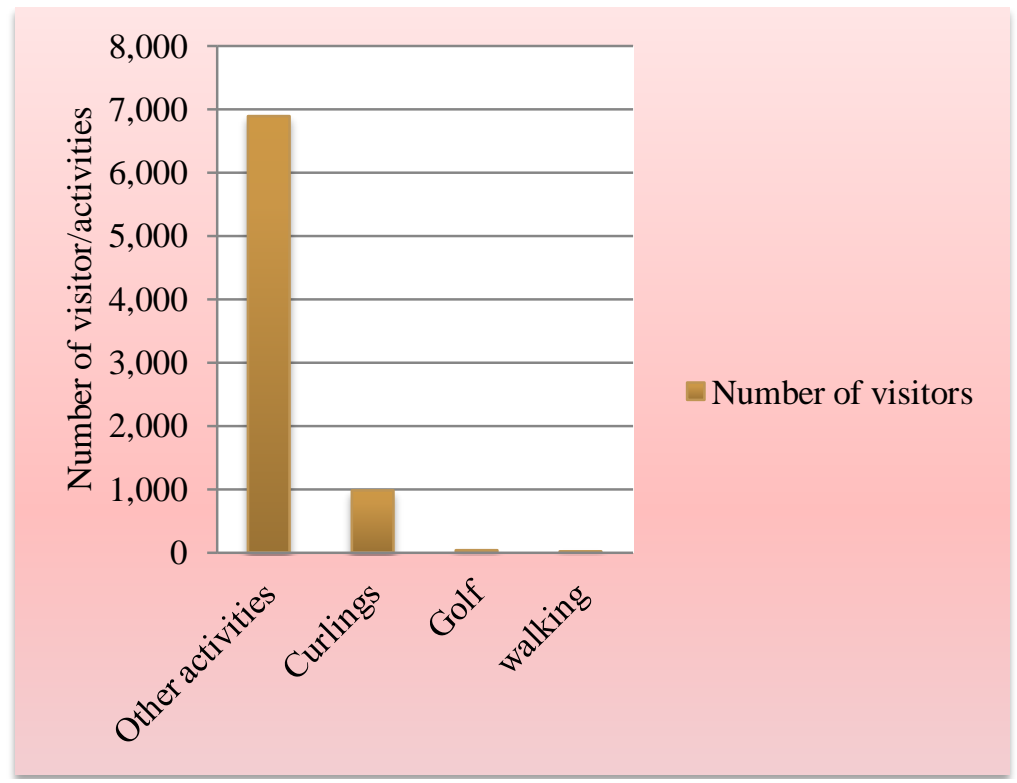

Figure 6.54: Cromwell - comparison between number of visitor activities for golf, curling, walking, and other activities 
Since this study uses the same EF/visitor activity as explained in Chapter 5, comparison between the EF/visitor of the four categories in Figure 6.39 can be conducted through using Figure 5.36 (Chapter 5- section 5.7.5.4) with the same results.

\subsubsection{The Total EF of Cromwell (2011)}

Table 6.117 demonstrates that in 2011 the total EF of Cromwell visitors was 373.8gha. As shown in Table 6.117 and Figure 6.55 transportation accounts for the largest share of the total EF at 269.5gha (72.1\%), followed by food at 94.71 gha (25.3\%), accommodation services at 8.05 gha (2.2 \%) and visitor activities at 1.55 gha (0.4\%). In addition, the transportation EF that forms $72.1 \%$ of the total EF of Cromwell can be divided into $51.5 \%$ of international transportation and $49.5 \%$ domestic transportation (see Table 6.74).

\begin{tabular}{l|c|c|}
\hline \multicolumn{3}{|c|}{ Table 6.117: Total EF of Cromwell (2011) } \\
\hline Category & EF (gha) & $\%$ \\
\hline Transportation & $269.5^{1}$ & 72.1 \\
\hline Food (second scenario) & $94.71^{2}$ & 25.3 \\
\hline Accommodation (second scenario) & $8.05^{3}$ & 2.2 \\
\hline Activities & $1.55^{4}$ & 0.4 \\
\hline Total & 373.8 & 100 \\
\hline 1. See Table 6.74 & & \\
2. See Table 6.77 & \\
3. See Table 6.92 & & \\
4. See Table 6.115 \\
- Total number of Cromwell visitors (2011) = 2,652 \\
- EF (gha/visitor) of Cromwell = 373.8 (gha) / 2,652 (visitors) = 0.14 \\
\hline
\end{tabular}

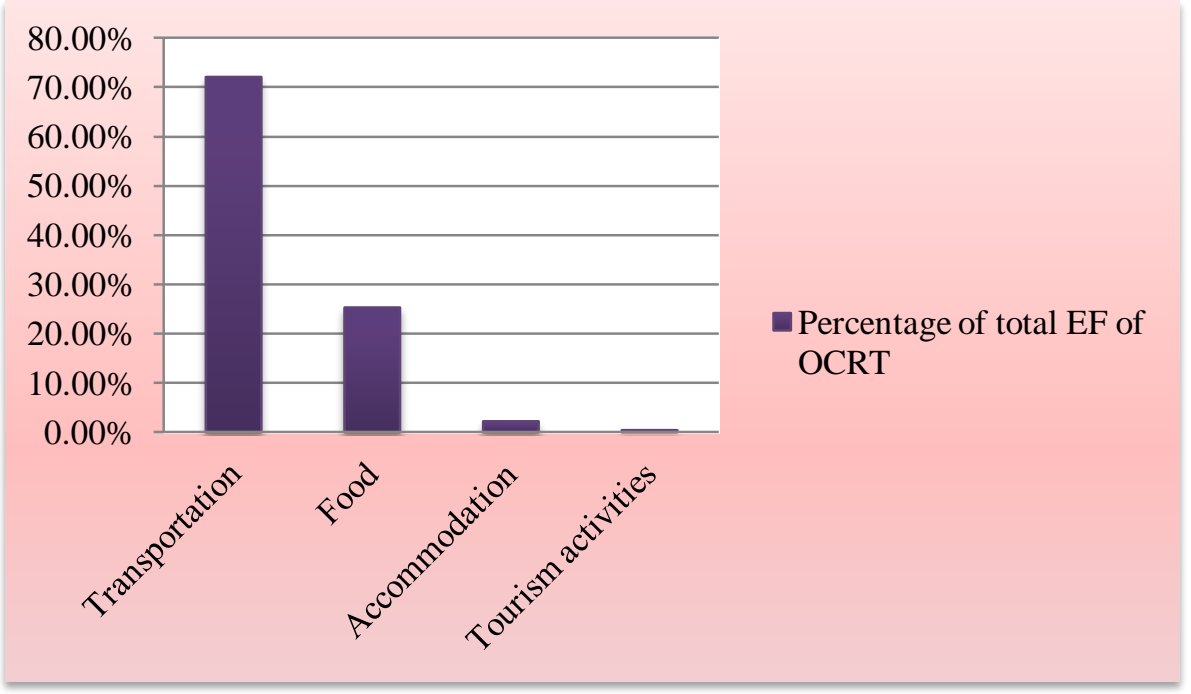

Figure 6.55: Comparison between EFs of Cromwell for transportation, food, accommodation services, and visitor activities (2011) 


\subsubsection{Cromwell Overshoot Portion of EF}

Table 6.118 compares the present EF of Cromwell per visitor and the target EF (0.03gha) investigated in Chapter 5- section 5.9.1. As shown in this table the present EF of Cromwell at $0.14 \mathrm{gha} /$ visitor (see Table 6.118 ) is $0.11 \mathrm{gha} /$ visitor more than the target EF for sustainable living. Furthermore, the overshoot portion of the total EF of 2,652 Cromwell visitors as shown in Table 6.118 is $(2,652 \times 0.11)=291.72$ gha .

Table 6.118: Overshoot portion of Cromwell visitors EF (gha/visitor/year) (2011)

\begin{tabular}{|c|c|c}
\hline Now & Goal & Overshoot EF (gha/visitor/year) \\
\hline $0.14^{1}$ & $0.03^{2}$ & 0.11 \\
\hline
\end{tabular}

1. See Table 6.117

2. See Chapter 5-section.5.9.1 and Table s.5.85 - 5.86

- Total overshoot EF (gha) of 2,652 Cromwell visitors $=2,652 \times 0.11=291.72$ gha

\subsubsection{Overshoot portion as Energy}

\subsubsection{Overshoot Portion of Energy Use per Visitor}

This chapter calculates the overshoot portion of Cromwell visitors through using the method explained in Chapter 5- section 5.9.2. As shown in Table 6.120 the EF of Cromwell is $0.14 \mathrm{gha} /$ visitor and as a result energy use is equivalent to:

0.14 (gha/visitor) $\times 100$ (carrying capacity of land $)=14(\mathrm{GJ} /$ visitor $)$

In addition sustainable energy use per visitor is:

0.03 (gha/visitor) $\times 100$ (carrying capacity of land $)=3(\mathrm{GJ} /$ visitor $)$

The overshoot portion of Cromwell energy use is:

$14 \mathrm{GJ} /$ visitor $-3 \mathrm{GJ} /$ visitor $=11 \mathrm{GJ} /$ visitor $($ Table 6.119).

Table 6.119: Overshoot portion of Cromwell visitor energy use (GJ/visitor/year) (2011)

\begin{tabular}{l|l|l} 
Now & Goal Overshoot energy use
\end{tabular}

14

3

(GJ/visitor/year)

11

\subsubsection{Total Overshoot Portion of Energy Used}

The total overshoot energy of Cromwell visitors can be calculated as the total number of visitors (2,652) multiplied by the overshoot energy use per visitor (11 GJ/visitor/year), which is 29,172 GJ/year (Table 6.120). 


\section{Table 6.120. Total overshoot portion of Cromwell visitor energy use (GJ/year) (2011)}

Number of visitors

2,652
Overshoot energy use

(GJ/visitor/year)

11
Total overshoot energy use

(GJ/year)

29,172

\subsubsection{Overshoot Portion of Energy Use by Categories}

In this section, the overshoot portion of each category (transportation, food, accommodation and visitor activities) is calculated through using the equation explained in Chapter 5-section 5.9.3. As shown in Table 6.121, transportation uses the majority share of 21,033GJ/year overshoot energy, followed by food (7,380.5 GJ/year), accommodation services (641.8 GJ/year) and Cromwell visitor activities (116.7 GJ/year).

\begin{tabular}{|l|c|c|c|}
\hline \multicolumn{4}{|c|}{ Table 6.121: Cromwell overshoot portion of energy used by categories (2011) } \\
\hline Category & EF (gha) & $\begin{array}{c}1 \\
\text { \% of total } \\
\text { EF }^{1}\end{array}$ & Overshoot portion of energy use (GJ/year) \\
\hline Transportation & 269.5 & 72.1 & 21,033 \\
\hline Food & 94.71 & 25.3 & $7,380.5$ \\
\hline Accommodation & 8.05 & 2.2 & 641.8 \\
\hline Activities & 1.55 & 0.4 & 116.7 \\
\hline Total & 373.8 & 100 & $29,172^{2}$ \\
\hline 1. See Table 6.118 & & & \\
\hline 2. See Table 6.120 & & & \\
\hline
\end{tabular}

\subsubsection{Economic Footprint of Cromwell Rail Trail Visitors}

In 2011, the total GDP of the OCRT was NZ\$6,245,289 (see Table 5.90) and GDP per visitor was NZ\$530 (NZ\$6,245,289 $\div$ 11,788 OCRT visitors) (Table 6.122). This study uses this visitor GDP figure to calculate the GDP of Cromwell visitors in 2011. Table 6.122 determines that the total GDP of Cromwell rail trail visitors is NZ\$530 $(\mathrm{GDP} /$ visitor $) \times 2,652$ (total number of Cromwell visitors) $=\mathrm{NZ} \$ 1,405,560$

\begin{tabular}{|c|c|c|c|c|}
\hline \multicolumn{5}{|c|}{ Table 6.122: Cromwell GDP (2011) } \\
\hline $\begin{array}{l}\text { OCRT total GDP } \\
(\mathrm{NZ \$ )}\end{array}$ & $\begin{array}{l}\text { OCRT number of } \\
\text { visitors }\end{array}$ & $\begin{array}{l}\text { OCRT GDP } \\
\text { (NZ\$/visitor) }\end{array}$ & $\begin{array}{l}\text { Cromwell number of } \\
\text { visitors }\end{array}$ & $\begin{array}{l}\text { Cromwell total } \\
\text { GDP }(\mathrm{NZ} \$)^{3}\end{array}$ \\
\hline $6,245,289^{1}$ & $11,788^{2}$ & 530 & 2,652 & $1,405,560$ \\
\hline $\begin{array}{l}\text { 4. See Table } 5.90 \\
\text { 5. See Figure } 5.2 \\
6.2,652 \times N Z \$ 53\end{array}$ & 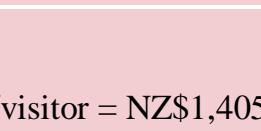 & & & \\
\hline
\end{tabular}

The following table shows that the total overshoot energy of Cromwell visitors is $29,172 \mathrm{GJ} /$ year. Since the cost to generate $1 \mathrm{GJ}$ through using renewable resources is NZ\$19.8 (see Table 5.94), then the total cost that must be paid to generate 29,172 GJ/year is NZ\$577,605.6 (29,172 × NZ\$19.8) (Table 6.123). This means the sustainable 
portion of Cromwell GDP is NZ\$1,405,560 (GDP) - NZ\$ 577,605.6 = NZ\$827,954.4 = 58.9\% of total Cromwell GDP (Table 6.123 and Figure 6.56).

On the other hand the GDPs of Cromwell, as an ecological-economic indicator, shows that $41.1 \%$ (100\%-58.9\%) of Cromwell GDP must be paid to restore its environmental impacts to being ecologically sustainable. Furthermore, GDPs as a social-economic indicator evaluates the contribution of the Rail Trail to local economic development, through engagement of local participants and organizations (in this case accommodation services) in the development process.

Table 6.123: Cromwell sustainable portion of GDP (GDPs)

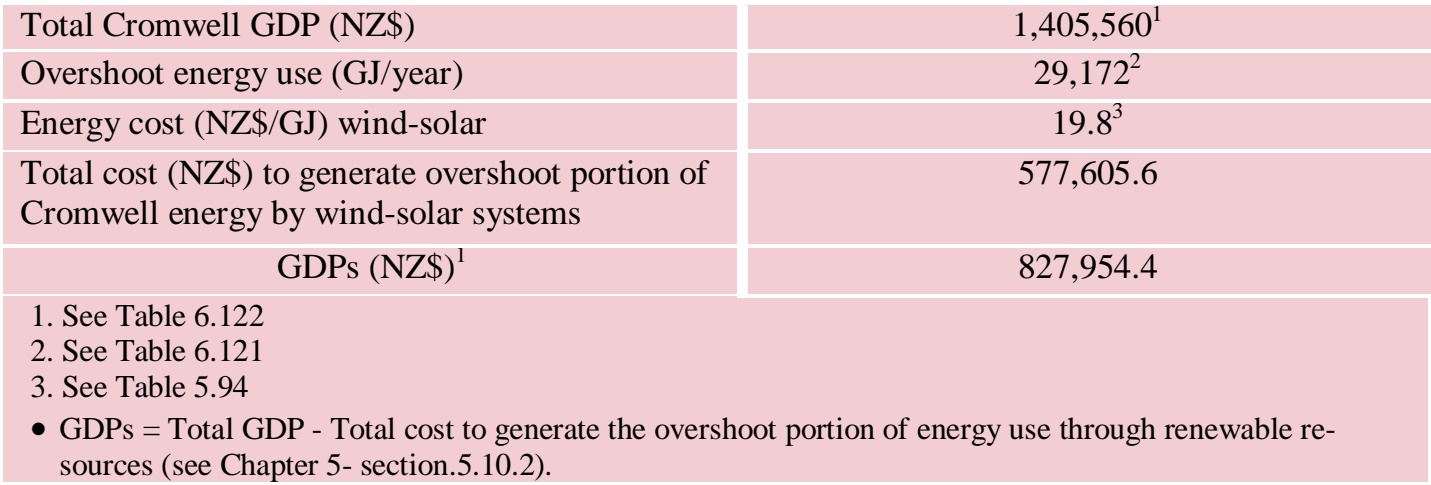

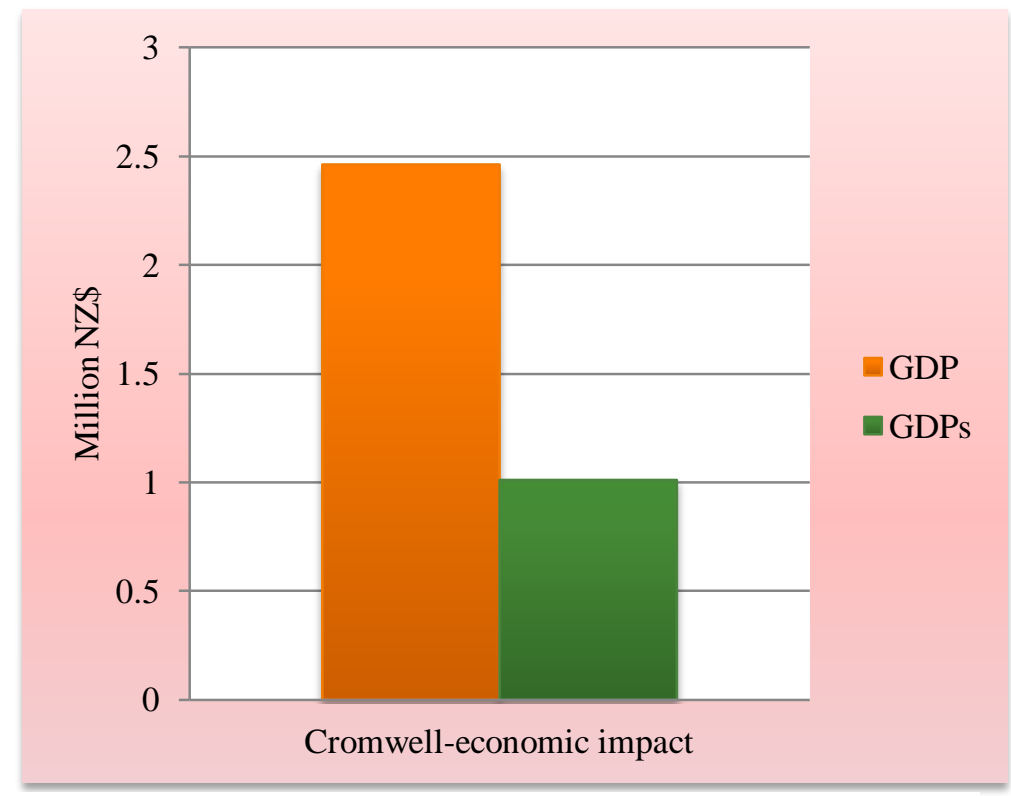

Figure 6.56: Cromwell - comparison between GDP and GDPs 


\subsubsection{Cromwell Cultural Footprint (CF)}

This section uses the CF model explained in Chapter 4 to explore the cultural footprint of the OCRT on Cromwell as one of its host destinations. In this case study the CF of the Rail Trail in Cromwell is categorised into the three areas of food, accommodation and visitor activities and, as explained in Chapter 5, the CF of transportation is not included in this research.

\subsubsection{Cromwell CF -Food}

Table 6.124 contains the summarised results of two proposed scenarios for Cromwell food (sections 6.2.5.1 and 6.2.5.2). As shown in Table 6.124, the total conventional eaten out food in the first scenario is $17,662 \mathrm{~kg}$ ( $100 \%$ of food consumed) and in the second scenario it is 17,662 made up of $17,222 \mathrm{~kg}$ (97.5\%) conventional food and 435 $\mathrm{kg}(2.5 \%)$ home prepared (organic food). As a result, the total EF of Cromwell food in the first scenario is 95.47 gha/year and in the second scenario this reduces to 94.71 gha/year (Table 6.124).

Table 6.124 indicates that in the second scenario the overshoot portion of the energy value of the food consumed is equivalent to 7,380.5 GJ/year. Since the EF of food in the first scenario (EF1) is 0.76 gha larger than that of the second scenario (EF2), the overshoot energy value of food consumed in the first scenario is 76 GJ $(0.76$ gha $\times 100-$ global average carrying capacity of land). This is bigger than the overshoot portion of the energy value of food in the second scenario (7,380.5 GJ/year). Consequently the overshoot energy value of food in the first scenario is 7,380.5 GJ + 76 GJ = 7,456.5 GJ/year (Table 6.124).

In this section, the overshoot portions of EF1 of 74.56 gha (see Table 6.124) and EF2 of 73.80 gha (see Table 6.124) is calculated using the method explained in Chapter 5- section.5.11.1. As the cost to generate 1GJ energy using wind-solar systems is NZ $\$ 19.8$, the cost to generate 7,456.5 GJ/year (overshoot portion of energy value of food in the first scenario) is 7,456.5 GJ $\times$ NZ\$19.8 $=$ NZ\$147,638.7 (see Table 6.124). Likewise the total cost to generate the overshoot energy using wind-solar systems in the second scenario is $\mathrm{NZ} \$ 19.8 \times 7,380.5$ /year = NZ\$146,133.9 (Table 6.124). 
The total GDP of Cromwell food is calculated using the GDP/kg food of OCRT multiplied by total Cromwell food consumed $(\mathrm{kg})$. As the total GDP for food related to the OCRT is NZ\$782,795 (see Table 5.91) and total produced/consumed food is 78,508.45 $\mathrm{kg}$, the GDP of food related to OCRT per kilogram is equal NZ\$782,795 $\div 78,508.45 \mathrm{~kg}$ $=9.97 \sim \mathrm{NZ} \$ 10 / \mathrm{kg}$.

In Cromwell the total amount food consumed is $17,662 \mathrm{~kg}$ (see Table 6.125). As a result the GDP of Cromwell food is equivalent to $17,662(\mathrm{~kg}) \times \mathrm{NZ} \$ 10 / \mathrm{kg}$ of food $=$ NZ\$176,620 (Table 6.124). Table 6.124 shows the GDP associated with food for both scenarios is $\mathrm{NZ} \$ 176,620$. As a result:

GDPs1= NZ\$176,620 (GDP) - NZ\$147,638.7 (renewable energy cost) = NZ\$28,981; GDPs2 = NZ\$176,620 (GDP) - NZ\$146,133.9 (renewable energy cost) $=$ NZ\$ 30,486. The difference between GDPs1 and GDPs2 shows the NZ\$1,505contribution to GDPs from producing $435 \mathrm{~kg}$ home prepared (organic) food by Cromwell accommodation services. In addition, it can be considered that producing $1 \mathrm{~kg}$ home prepared (organic) food contributes NZ\$3.46 to GDPs (Table 6.124).

\begin{tabular}{|c|c|c|c|}
\hline \multicolumn{4}{|c|}{ Table 6.124: Cromwell - local product (food) contribution to GDPs } \\
\hline \multicolumn{2}{|c|}{ First scenario (100\% conventional food) } & \multicolumn{2}{|c|}{$\begin{array}{l}\text { Second scenario (97.5\% conventional food and } \\
2.5 \% \text { home prepared (organic) food }\end{array}$} \\
\hline $\begin{array}{l}\text { Total conventional eaten out } \\
\text { food }(\mathrm{kg})\end{array}$ & $17,662^{1}$ & $\begin{array}{l}\text { Total conventional eaten out } \\
\text { food }(\mathrm{kg})\end{array}$ & $17,222^{1}$ \\
\hline $\begin{array}{l}\text { Total home prepared food } \\
\text { (kg) }\end{array}$ & 0.00 & $\begin{array}{l}\text { Total home prepared food } \\
\text { (kg) }\end{array}$ & $435^{1}$ \\
\hline $\begin{array}{l}\text { Total EF of consumed food } \\
\text { gha/year - (EF1) }\end{array}$ & $95.47^{1}$ & $\begin{array}{l}\text { Total EF of consumed food } \\
\text { gha - (EF2) }\end{array}$ & $94.71^{1}$ \\
\hline $\begin{array}{l}\text { Overshoot portion of energy } \\
\text { related to food (GJ/year) }\end{array}$ & $7,456.5$ & $\begin{array}{l}\text { Overshoot portion of energy } \\
\text { related to food (GJ/year) }\end{array}$ & $7,380.5^{2}$ \\
\hline $\begin{array}{l}\text { Overshoot portion of EF1 } \\
\text { (gha/year) }\end{array}$ & 74.56 & $\begin{array}{l}\text { Overshoot portion of EF2 } \\
\text { (gha/year) }\end{array}$ & 73.80 \\
\hline $\begin{array}{l}\text { Cost to generate } 1 \mathrm{GJ} \text { energy } \\
\text { using wind-solar systems } \\
(\mathrm{NZ \$ )}\end{array}$ & 19.8 & $\begin{array}{l}\text { Cost to generate } 1 \mathrm{GJ} \text { energy } \\
\text { using wind-solar systems } \\
\text { (NZ\$) }\end{array}$ & 19.8 \\
\hline $\begin{array}{l}\text { Total cost to generate over- } \\
\text { shoot energy using wind-solar } \\
\text { systems (NZ\$) }\end{array}$ & $147,638.7$ & $\begin{array}{l}\text { Total cost to generate over- } \\
\text { shoot energy using wind- } \\
\text { solar systems (NZ\$) }\end{array}$ & $146,133.9$ \\
\hline Total food GDP (NZ\$) & 176,620 & Total food GDP (NZ\$) & 176,620 \\
\hline Total food GDPs1 & 28,981 & Total food GDPs2 & 30,486 \\
\hline $\begin{array}{l}\text { 1. See Table } 6.78 \\
\text { 2. See Table } 6.121 \\
\text { - Increased portion }\end{array}$ & & & \\
\hline
\end{tabular}




\section{a. Ideal Sustainable Life Model: Food}

Table 6.124 shows that the present EF2 of Cromwell food (second scenario) is 94.71 gha and the overshoot portion of EF2 is 73.80 gha. As a result the sustainable EF of Cromwell food can be determined through the following equation:

94.71 gha (EF2, present EF) - 73.80 gha (overshoot portion of EF2) = 20.91 gha.

In the ideal model for Cromwell tourist related food, since the overshoot portion of energy use is zero, the GDPs is equal to the total GDP of Cromwell food (NZ\$176,620, see Table 6.124).This section uses the ideal model of Cromwell food and compares it with the two scenarios shown in Table 6.124.

In Figure 6.57, the area of 'Q1, EF1, and GDPs1' illustrates ordinary life; this is the area in which all Cromwell visitors consume 100\% (17,662 kg) conventional eaten out food (first scenario). In this area, development of the Rail Trail (OCRT) does not exert any influence on Cromwell (as one of the host destinations) and its visitors to produce and consume local foods. In Figure 6.57 the EF of using 100\% conventional food (EF1) is $95.47 \mathrm{gha} /$ year and this represents the total food EF. In the ordinary life area, GDPs1 is equal to NZ\$28,981 (see Table 6.124).

Comparison between the first scenario and the ideal model of Cromwell food consumption indicates that in the first scenario EF1 (95.47 gha) is $78.09 \%$ (74.56 gha) bigger than the ideal EF (20.91gha) (Figure 6.57 and Table 6.124). As shown in Figure 6.57, in the first scenario, GDPs1 is equal to $16.4 \%$ of the total GDP of Cromwell food. On the other hand, $83.6 \%$ (100 - 16.4\%) of total GDP related to food must be spent to change EF1 to the ideal EF through generating the overshoot portion of food energy by using wind-solar systems.

In Figure 6.42, the area of 'Q2, EF2, and GDPs2' determines the CF area of Cromwell for producing and consuming home prepared (organic) foods. As demonstrated in Figure 6.57 total Q2 in the CF for food consumed by Cromwell visitors $(17,662 \mathrm{~kg})$ is divided into the two portions of 2.5\% (435kg) home prepared food (Q3) and 97.5\% $(17,222 \mathrm{~kg})$ conventional eaten out food (Q 4). The EF in the CF (EF2) contains the EF of using 2.5\% (435kg) home cooked and 97.5\% (17,222 kg) conventional eaten out foods. The GDPs2 in the CF is equal to NZ\$30,486 which is NZ\$1,505 (.085\% of the total GDP) more than GDPs1 (NZ\$28,981) (Table 6.124). 
Figure 6.57 and Table 6.124 indicate that using 2.5\% home prepared (organic) food reduces total Cromwell food EF by $0.79 \%$ (shown in Figure 6.57 as R-EF1). As demonstrated in this figure, producing 2.5\% local food increases GDPs1 by $0.85 \%$, shown in the figure as (I) GDPs 1.

Comparison between the ideal model and present pattern of Cromwell food consumption demonstrates that EF2 (94.7 gha) is 73.79 gha (77.3 \% of EF1 as benchmark) more than ideal EF (20.91gha), and $83.6 \%$ (NZ\$147,638.7) of the GDP (NZ\$176,620) of food must be spent to reduce this overshoot portion of EF2 to the ideal EF (Table 6.124 and Figure 6.57).

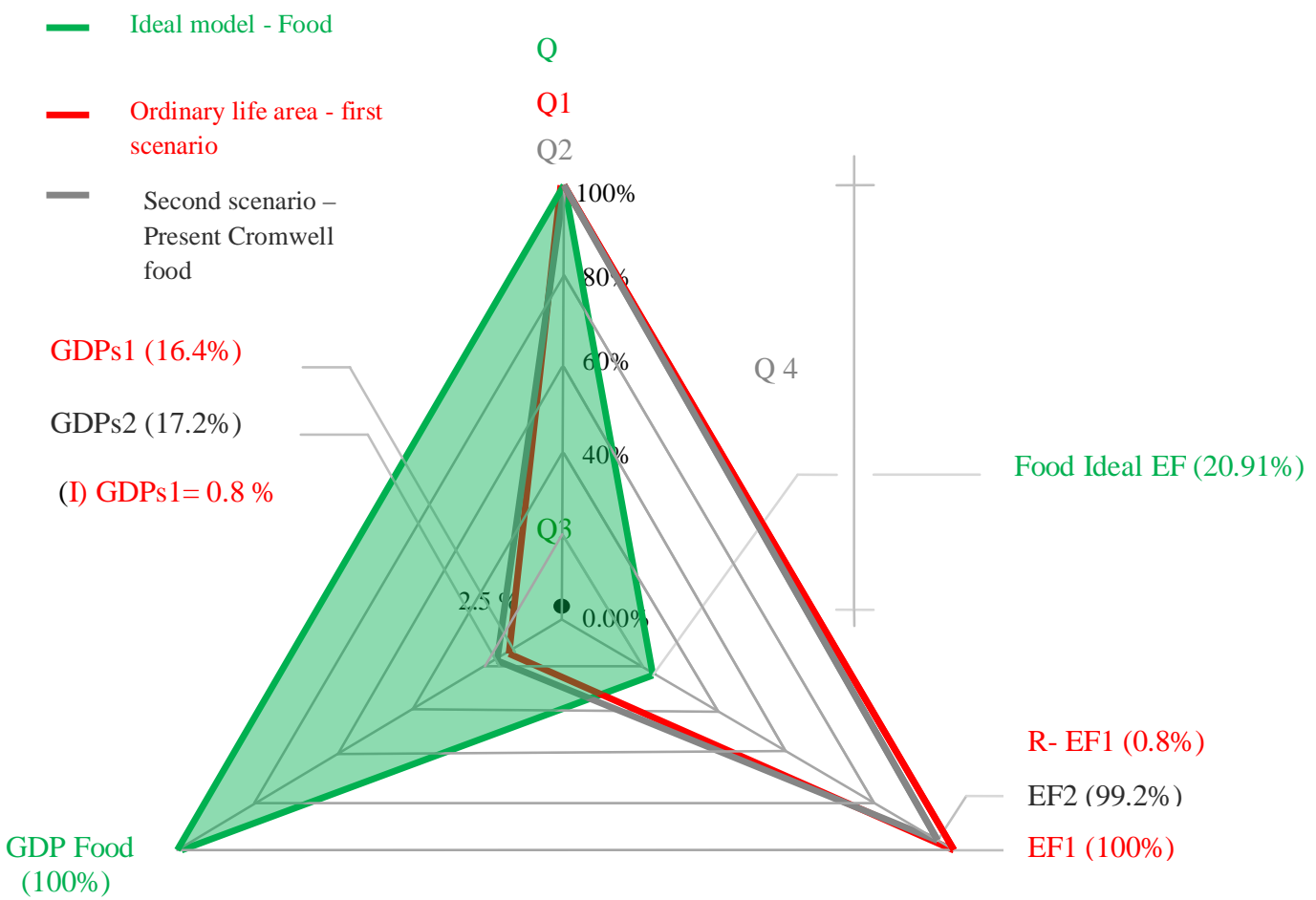

Figure 6.57: The cultural footprint (CF) of Cromwell visitor food

- Q: $100 \%$ food in Ideal model

- Q1: $100 \%$ conventional eaten out food - first scenario

- $\quad$ Q2: $97.5 \%$ conventional eaten out food $+2.5 \%$ home prepared food - second scenario

- $\quad$ Q3: 2.5\% home prepared food (second scenario)

- Q.4: 97.5\% conventional eaten out food (second scenario)

- GDPs1: sustainable portion of GDP - first scenario

- GDPs2: sustainable portion of GDP - second scenario

- (I) GDPs1: Increased portion of GDPs1

- EF1: ecological footprint of consumed food - first scenario

- EF2: ecological footprint of consumed food - second scenario

- R- EF1: reduced portion of total EF1 of consumed food 


\subsubsection{The CF of Cromwell Accommodation Services}

This section explores the CF of 492 Cromwell accommodation services (all NB) through using two scenarios contain different quantities, EFs and GDPs of the accommodation services used. In the first scenario, all buildings are assumed not to have open air areas (veranda/balcony). In the second scenario $57.2 \mathrm{~m}^{2}(0.2 \%)$ of a total area of $25178 \mathrm{~m}^{2}$ accommodation services area is outdoor sitting space (V/B) (Table 6.91).

Table 6.125 shows that in the first scenario the total EF of using $100 \%$ buildings as accommodation services is 8.11 gha. In this Chapter, the GDP of Cromwell's accommodation services with its total 9,547 visitor nights is calculated using the same method explained in section 6.1.13.2.

Since the overshoot portion of energy use in the second scenario has already been calculated as 627.2 GJ (see Tables 6.125 and 6.121), it is used here as a base to calculate the overshoot energy of the first scenario through the method explained in Chapter 5section-5.11.2. Table 6.125 shows that the overshoot portions of energy for accommodation services in the first and the second scenarios are $633.6 \mathrm{GJ}$ and $627.2 \mathrm{GJ}$ respectively.

Table 6.125 contains the total costs to generate the overshoot energy related to each of the two scenarios. These costs are calculated through using the method explained in Chapter 5-section.5.11.2. As shown in Table 6.125, the costs to generate the overshoot energy of the first and second scenarios in an environmentally sustainable way are NZ\$12,545, and NZ\$12,418.56 respectively.

In this section, the GDPs of the two scenarios cited in Table 6.125 are calculated based on the method used in Chapter 5-section.5.11.2 for the GDPs of OCRT accommodation services. Table 6.125 determines that the GDPs of the second scenario (GDPs2) is the larger at NZ\$1,625,893 compared to NZ\$625,893.86 (GDPs1). 
Table 6.125: Cromwell accommodation services - CF (2011)

\begin{tabular}{|c|c|c|c|}
\hline \multirow{2}{*}{\multicolumn{2}{|c|}{$\begin{array}{c}\text { First scenario } \\
\text { All Cromwell accommodation assumed as indoor } \\
\text { area }\end{array}$}} & \multicolumn{2}{|c|}{ Second scenario } \\
\hline & & \multicolumn{2}{|c|}{ Accommodation areas contain V/B } \\
\hline $\begin{array}{l}\text { Total number of accommodation } \\
\text { services }\end{array}$ & $492^{1}$ & $\begin{array}{l}\text { Total number of accommoda- } \\
\text { tion services }\end{array}$ & $492^{1}$ \\
\hline $\begin{array}{l}\text { Accommodation area (indoor } \\
\text { spaces) }\left(\mathrm{m}^{2}\right)\end{array}$ & $25,178^{1}$ & $\begin{array}{l}\text { Accommodation area (indoor } \\
\text { spaces) }\end{array}$ & $25,120.8^{1}$ \\
\hline Accommodation area (V/B) $\left(\mathrm{m}^{2}\right)$ & 0.00 & Accommodation area (V/B) & $57.2^{2}$ \\
\hline Total EF (EF1) (gha) & $8.11^{2}$ & Total EF (EF2) (gha) & $8.046^{3}$ \\
\hline GDP (NZ\$) & $638,312.42^{4}$ & GDP (NZ\$) & $638,312.42^{4}$ \\
\hline Overshoot energy use (GJ/year) & 633.6 & Overshoot energy use (GJ/year) & $627.2^{5}$ \\
\hline $\begin{array}{l}\text { Cost to generate } 1 \text { GJ energy using } \\
\text { wind-solar systems (NZ\$) }\end{array}$ & $19.8^{6}$ & $\begin{array}{l}\text { Cost to generate } 1 \text { GJ energy } \\
\text { using wind-solar systems (NZ\$) }\end{array}$ & $19.8^{6}$ \\
\hline $\begin{array}{l}\text { Cost to generate } 625.8 \text { GJ energy } \\
\text { using wind-solar systems (NZ\$) }\end{array}$ & $12,545.28$ & $\begin{array}{l}\text { Cost to generate } 575.8 \mathrm{GJ} \text { en- } \\
\text { ergy using wind-solar systems } \\
(\mathrm{NZ} \$)\end{array}$ & $12,418.56$ \\
\hline GDPs1 & $625,767.14$ & GDPs2 & $625,893.86$ \\
\hline \multicolumn{4}{|l|}{$\begin{array}{l}\text { 1. See Table } 6.83 \\
\text { 2. See Table } 6.89 \\
\text { 3. See Table } 6.92 \\
\text { 4. See Table } 5.37 \\
\text { 5. See Table } 6.121 \\
\text { 6. See Table } 5.94\end{array}$} \\
\hline
\end{tabular}

\section{a. Sustainable Living Cromwell Accommodation Services (Ideal Model)}

This section determines an ideal model for Cromwell accommodation services in which its EF is considered environmentally sustainable and its GDPs is equal to the total GDP of Cromwell accommodation services. This model is then used to compare each of the two scenarios for Cromwell accommodation services (cited in Table 6.125) with an environmentally and economically sustainable pattern of accommodation.

\section{a. 1. Sustainable EF}

As shown in Table 6.125 the total overshoot energy of Cromwell accommodation services at present is $627.2 \mathrm{GJ} /$ year. As a result the overshoot portion of its EF is $627.2 / 100=6.27$ gha. As indicated in Table 6.125, the present EF of Cromwell is 8.046 gha (EF2). Consequently the environmentally sustainable EF can be considered as 8.046 gha $-6.27=1.79$ gha.

\section{a. 2. Sustainable Living: GDPs}

As explained in section 6.1.13.2, a.2, the GDPs of Cromwell accommodation in sustainable living is equal with its total GDP. 


\section{b. Comparison between the Ideal Model, First and Second Scenarios}

Figure 6.58 comprises the quantities, EFs and GDPs(s) of Cromwell accommodation services arising from the Ideal model, first, and second scenarios discussed above. This figure makes this an opportunity to explore the ecological and economic influences exerted by using $57.2 \mathrm{~m}^{2}\left(0.2 \%\right.$ of the total $25,178 \mathrm{~m}^{2}$, see Table 6.125$)$ of accommodation area as open air areas (V/B).

As shown in Figure 6.58, in the Ideal model of accommodation services, Q is the $100 \%$ Cromwell accommodation services area $\left(25,178 \mathrm{~m}^{2}\right)$ with an EF of 1.79 gha and GDPs of NZ\$638,312.42 (equal to total accommodation GDP). In the ideal model as discussed above, the overshoot portion of the EF and energy use are zero. In first scenario, Q1 is the total area of Cromwell accommodation services $\left(25,178 \mathrm{~m}^{2}\right)$ where $100 \%$ of accommodation buildings are assumed to be indoor spaces (Figure 6.58). In the second scenario as illustrated in Figure 6.58, Q2 represents the Cromwell accommodation area $\left(25,178 \mathrm{~m}^{2}\right)$ divided into two portions of $99.8 \%\left(25,120.8 \mathrm{~m}^{2}\right.$, see Table 6.126$)$ indoor spaces and $0.2 \%\left(57.2 \mathrm{~m}^{2}\right.$, see Table 6.125) V/B.

In Figure 6.58, EF1 is the biggest EF in comparison with the EFs of the other scenarios and is here considered to be the $100 \%$ benchmark. Likewise, in this figure, EF2 and Ideal-EF are compared with EF1 as the $100 \%$ benchmark.

As shown in Figure 6.58, in the ideal model of Cromwell accommodation services, the acceptable EF of these services is $22.07 \%$ of EF1 (8.11gha, see Table 6.125). On the other hand, in the first scenario in which all buildings are assumed as indoor space, the EF (EF1 = 8.11gha) is $77.93 \%$ (6.32 gha) larger than the sustainable EF (1.79 gha) (Figure 6.58).

As illustrated in Figure 6.58, the EF of Cromwell accommodation services in the second scenario $(\mathrm{EF} 2=8.046$ gha, see Table 6.126$)$ is $99.2 \%$ of EF1 (8.11gha, see Table 6.125). Consequently (R) EF1 is equal to $0.8 \%$ of EF1= 0.065 gha (Figure 6.58).

GDPs1 (GDPs of the first scenario $=\mathrm{NZ} \$ 625,767.14$ ) is equal to $98.03 \%$ of total GDP of Cromwell accommodation services (NZ\$638,312.42) (Figure 6.58). On the other hand, in the first scenario 1.97\% (NZ\$12,545.28) of the total GDP of Cromwell ac- 
commodation services must be spent to generate the overshoot portion of accommodation energy use through wind-solar systems to change EF1 to the ideal EF.

As shown in Figure 6.58, GDPs 2 (NZ\$625,893.86, the GDPs of the second scenario) is 98.05\% of the total GDP of Cromwell accommodation (NZ\$638,312.42) and 1.95\% (NZ\$12,418.56) of the total GDP of Cromwell accommodation services must be spent to generate the overshoot portion of associated energy use through using wind-solar systems to reduce EF2 to the ideal EF. Likewise, as determined in Figure 6.43, (I) GDPs1 is equal to $0.02 \%$ of the total GDP of Cromwell accommodation services. This means using V/B (open air areas) as $0.2 \%$ of accommodation area contributes to an increase of $0.02 \%$ in GDPs1.

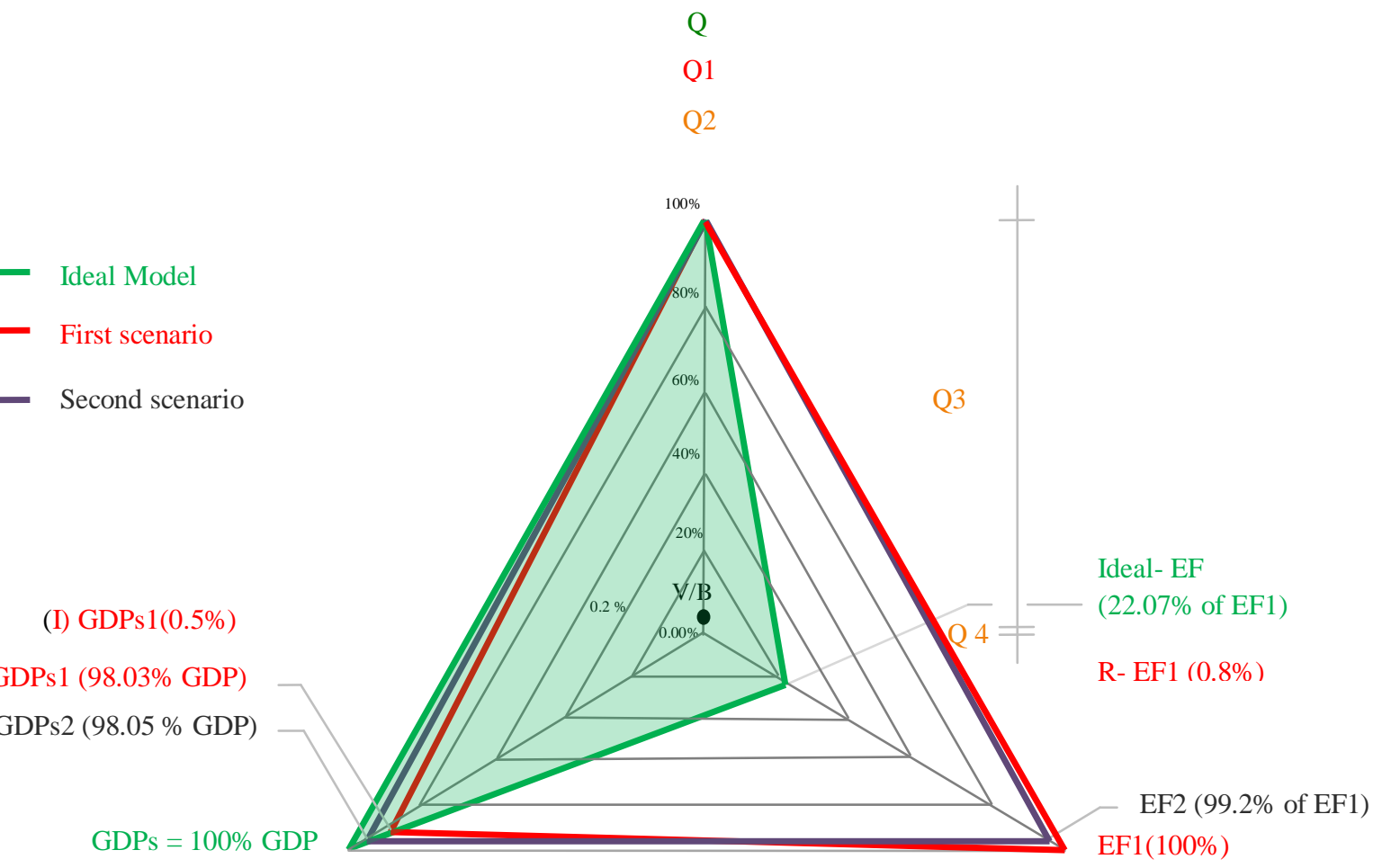

Figure 6.58: The CF of Cromwell accommodation services with V/B as part of the used spaces

Q: $(100 \%)$ area of 492 Cromwell accommodation services $\left(25,178 \mathrm{~m}^{2}\right)$ - Ideal model GDP: GDP of Cromwell accommodation services NZ\$1,638,312.42 (see Table 6.126) GDPs: Total sustainable portion of Cromwell accommodation services - Ideal model Q1: Total (100\%) area of Cromwell accommodation services $\left(25,178 \mathrm{~m}^{2}\right)$ - all buildings area assumed as indoor spaces (first scenario)

EF1: Total EF of Cromwell accommodation services (first scenario)

(R) EF1: Reduced portion of Cromwell EF1 influenced by using V/B as part of buildings

GDPs1: Total GDPs of Cromwell accommodation services (first scenario)

(I) GDPs1: Increased portion of GDPs1 influenced by using V/B as part of buildings

Q2: Area Cromwell accommodation services $\left(25,178 \mathrm{~m}^{2}\right)$ including; $99.08 \%\left(25,120.8 \mathrm{~m}^{2}\right)$ indoor spaces and $0.2 \%\left(57.1 \mathrm{~m}^{2}\right) \mathrm{V} / \mathrm{B}$ (second scenario)

Q3: $99.08 \%\left(25,120.8 \mathrm{~m}^{2}\right)$ total indoor area of 492 Cromwell accommodation services (second scenario) 
Q 4 (V/B area): $0.2 \%\left(57.2 \mathrm{~m}^{2}\right)$ of total Cromwell accommodation area (second scenario)

EF2: EF of 25,178m² Cromwell accommodation services (Q2) including EFs of Q3 and Q 4 (present EF)

(R) EF1: Reduced portion of Cromwell EF1 influenced by using V/B

GDPs2: Total GDPs of Cromwell accommodation services (second scenario)

\subsubsection{The CF of Cromwell Visitor Activities}

\section{a. Overshoot EF of Cromwell Visitor Activities}

As shown in Table 6.126, the category of other activities accounts for the largest portion at $83.2 \%$ (1.29 gha) of the total EF of Cromwell visitor activities (1.55 gha) followed by curling (10.3\%), golf (6.45\%) and walking (0.05\%). Table 6.126 shows that the total overshoot portion of the EF of Cromwell activities is 1.167 gha (see also Table 6.121). The overshoot portion of the EFs of the four activity groups above are calculated through using same method explained in section 6.1.13.3- a. As shown in Table 6.126, in comparison with other categories the category of other activities has the largest overshoot EF of $0.97 \mathrm{GJ}$, followed by 0.12 GJ curling, golf 0.075 and $0.0006 \mathrm{GJ}$ walking.

\section{b. Cromwell Activities GDP}

The GDP of Cromwell activities are calculated through using the method explained in section 6.1.13.3- b. Table 6.126 demonstrates that of the NZ\$450,603 GDP of Cromwell activities (7,922 visitor activities $\times$ NZ\$56.88 GDP/visitor activity), the largest portion of NZ\$392,074 is the GDP of other visitor activities, followed by NZ\$55,686 (curling), NZ\$1,934 (golf) and NZ\$910 walking.

\section{c. Cromwell Activities GDPs}

Table 6.126 comprises the overshoot EFs of the four categories of curling, golf, walking and other activities that are calculated though using the equation explained in section6.1.13.3- b.

In Table 6.126, the overshoot energy use of each category is calculated as its overshoot EF multiplied by $100 \mathrm{GJ} / \mathrm{gha}$ (global average carrying capacity of land). Also as shown, other activities has the largest overshoot of $97 \mathrm{GJ}$, followed by curling (12GJ), golf (7.5) and walking (0.06GJ ).

This section calculates the cost to generate the overshoot portion of the energy of each category of Cromwell activities through using the method explained in Chapter 5- section 5.10.2. 
Table 6.126 contains the total cost that must be paid to generate the overshoot energy of each type of Cromwell activity through using wind-solar systems. As presented in Table 6.126, other activities (excepted curling, golf and walking) has the largest cost of NZ\$1,921 and walking the minimum cost (\$NZ1 0.00) to generate the overshoot energy in a sustainable way. The total cost of Cromwell activities overshoot energy as shown in Table 6.126 is \$NZ 2,309.

The GDPs of each category of Cromwell visitor activities (GDP - cost of overshoot energy used) are set out in Table 6.126. As determined in Table 6.126 other activities has the largest GDPs of NZ\$390,153 and the GDPs of other categories including curling, golf and walking are in a range of NZ\$909 - NZ\$55,448. In addition, the total GDPs of Cromwell's visitor activities is NZ\$448, 296, equivalent to $99.48 \%$ of its total GDP (\$NZ450, 603) (Table 6.126).

\begin{tabular}{|c|c|c|c|c|c|c|c|c|}
\hline \multicolumn{9}{|c|}{ Table 6.126: Cromwell visitor activities - EF and GDPs } \\
\hline Category & $\begin{array}{l}\text { Number } \\
\text { of visi- } \\
\text { tors/acti } \\
\text { vity }\end{array}$ & $\begin{array}{l}\mathrm{EF}^{1} \\
\text { (gha) }\end{array}$ & $\%$ & $\begin{array}{l}\text { Overshoot } \\
\text { EF (gha) }\end{array}$ & $\begin{array}{c}\text { Overshoot } \\
\text { energy }(G J)^{2}\end{array}$ & $\begin{array}{l}\text { GDP } \\
(\mathrm{NZ \$})\end{array}$ & $\begin{array}{l}\text { Cost of } \\
\text { overshoot } \\
\text { energy } \\
\text { (NZ\$) }\end{array}$ & $\begin{array}{l}\text { GDPs } \\
\text { (NZ\$) }\end{array}$ \\
\hline Golf & 34 & 0.1 & 6.45 & 0.075 & 7.5 & 1,934 & 148.5 & 1,786 \\
\hline Curling & 979 & 0.16 & 10.3 & 0.12 & 12 & 55,686 & 238 & 55,448 \\
\hline Walking & 16 & 0.0008 & 0.05 & 0.0006 & 0.06 & 910 & 1 & 909 \\
\hline Other & 6,893 & 1.29 & 83.2 & 0.97 & 97 & 392,074 & 1,921 & 390,153 \\
\hline Total & 7,922 & 1.55 & 100 & $1.167^{3}$ & 116.7 & 450,603 & 2,309 & 448,296 \\
\hline \multicolumn{9}{|c|}{$\begin{array}{l}\text { 1. See Figure } 5.34 \text { and Table } 5.82 \\
\text { 2. Total overshoot energy use of activities = } 116.7 \text { GJ/year (see Table } 6.121 \text { ) } \\
\text { - Average GDP of OCRT visitor activity = Total GDP of OCRT activities/visitor activity }=1483,180 \div 26,077= \\
\text { NZ\$56.88 (see Chapter 5: Table 5.98) }\end{array}$} \\
\hline
\end{tabular}

\section{d. CF: Ideal Model for Sustainable Activities and the Three Scenarios}

This chapter proposes three scenarios for the CF of Cromwell visitor activities (illustrated in Figure 6.59) and compares the areas generated with each other and the sustainable area (Ideal model) of these activities (shown as the green area in Figure 6.59).

\section{d.1. Cromwell Visitor Activities (Ideal model)}

As shown in Table 6.126, the present EF of Cromwell visitor activities is 1.55 gha and its overshoot EF is 1.167 gha. The EF of the Ideal model for Cromwell visitors activities (zero overshoot EF) is 1.55 gha -1.167 gha $=0.38$ gha. This means the ecologically sustainable EF of Cromwell visitors activities (0.38 gha) is $24.5 \%$ of its present EF (EF1= 1.55gha). 
In addition, in the sustainable area of Cromwell visitors' activities, as the cost of overshoot energy is zero, its GDPs is equal to $100 \%$ of the GDP of Cromwell visitor activities (\$NZ450,603, see Table 6.126).

\section{d.2. Cromwell Visitor Activities (First Scenario)}

The present CF of Cromwell visitor activities is the same as the first scenario shown in Figure 6.59. In the first scenario the total number of visitor activities $(7,922)$ comprises 6,893 visitors doing other activities, 979 visitors curling, 34 visitors playing golf, and 16 visitors walking (see Table 6.127).

The total EF of Cromwell visitors activities (EF1) in the first scenario is 1.55 gha (see Table 6.126) and this is used as the 100\% benchmark when comparing this with the second scenario and the Ideal model (see Figure 6.59). As shown in Figure 6.59 and Table 6.126 in the first scenario GDPs1 (present situation, Table 6.126) is equal to $99.48 \%(\mathrm{NZ} \$ 448,296)$ of the total GDP of Cromwell visitor activities (NZ\$ 450,603).

\section{d.3. Cromwell Visitor Activities (Second Scenario)}

The second scenario is proposed based on the assumption that the 34 visitors who play golf in the first scenario choose walking instead (see Table 6.127). In the second scenario, walking is assumed to have an EF of 0.00005gha/visitor, see Table 5.82).

\begin{tabular}{|c|c|c|c|c|c|c|c|}
\hline \multicolumn{4}{|c|}{ First scenario } & \multicolumn{4}{|c|}{ Second scenario } \\
\hline Category & $\mathrm{NV}^{4}$ & EF/visitor & EF1 & Category & NV & $\begin{array}{c}\mathrm{EF} \\
\text { (gha/visitor) }\end{array}$ & EF2 (gha) \\
\hline Golf & 34 & $0.003^{1}$ & 0.1 & - & - & 10 & - \\
\hline Curling & 979 & $0.00016^{2}$ & 0.16 & Curling & 979 & $0.00016^{2}$ & 0.16 \\
\hline Walking & 16 & $0.00005^{3}$ & 0.0008 & Walking & 50 & $0.00005^{3}$ & 0.0025 \\
\hline Other & 6,893 & 0.00025 & 1.29 & Other & 6,893 & 0.00025 & 1.29 \\
\hline Total & 7,922 & - & 1.55 & Total & 7,922 & - & 1.45 \\
\hline $\begin{array}{l}\text { 1. See Tabl } \\
\text { 2. See Table } \\
\text { 3. See Table } \\
\text { 4. NV: Nun }\end{array}$ & $\begin{array}{l}88 \\
35 \\
56 \\
\text { of visit }\end{array}$ & vitie & $6.96)$ & & & & \\
\hline
\end{tabular}

As shown in Table 6.127 and Figure 6.59 in comparison with the first scenario, in the second scenario EF2 is reduced by 1.45gha compared to EF1 (1.55 gha), a $6.45 \%$ (0.1gha) reduction just from 34 visitors changing their activity from golfing to walking. In addition, the overshoot portion of the EF of Cromwell activities that in the first scenario is 1.167 gha (see Table 6.126) in the second scenario is reduced to (1.167 gha - 
0.1 gha) 1.067 gha. As a result the overshoot energy of Cromwell activities in the second scenario is 1.067 gha $\times 100$ (carrying capacity of land) $=106.7$ GJ. Likewise, the total cost to generate the overshoot energy of Cromwell visitor activities in the second scenario is:

106.7 GJ (overshoot portion of energy) $\times$ NZ\$19.8 (cost to generate 1GJ energy using wind-solar systems) $=$ NZ $\$ 2,113$.

Since the total GDP of Cromwell visitor activities is NZ\$450,603 (Table 6.126), the GDPs of the second scenario is NZ\$450,603 (GDP) - NZ\$2,113 (the total cost to generate overshoot energy in the second scenario) $=$ NZ\$448,490 = GDPs2. As shown in Figure6.59, GDPs2 is $99.53 \%$ of the total GDP of Cromwell visitor activities (\$NZ450,603) and (I) GDPs2 is GDPs2 (99.53\% of GDP) - GDPs1 (99.48 \% of GDP) $=0.05 \%$ of GDP $(\mathrm{NZ} \$ 450,603)=\mathrm{NZ} \$ 225$.

\section{d. 4. Comparison between Ideal Model and First and Second Scenarios}

In comparison with the EFs of the two scenarios (EF1 and EF2), the EF of the Ideal model (0.38 gha, see section 6.2.13.3.4-a) is $24.5 \%$ of EF1 (1.55 gha) and $26.2 \%$ of EF2 (1.45 gha, see Table 6.127) (Figure 6.59). The proposed CF model in Figure 6.59 determines that for changing EF1 (1.55 gha) to the ideal EF (0.38), 0.45\% (\$NZ2,309) of the total GDP of Cromwell visitor activities must be spent to generate the overshoot portion of its energy. Also, the model (Figure 6.59) indicates that for changing the EF of Cromwell visitor activities in the second scenario (EF2) to a sustainable EF, $0.49 \%$ (NZ\$2,218) of the total GDP of Cromwell visitor activities (NZ\$450,603) must be spent to reduce the environmental impact.

\section{d. 5. Cromwell Visitor Activities (Third Scenario)}

The third scenario is proposed based on the assumption that 34 visitors who play golf and 979 visitors who play curling in the first scenario (see Table 6.126) choose walking as their activity instead (see Table 6.128).

As shown in Table 6.128 and Figure 6.59, in comparison with the first scenario, in the third scenario, the EF3 is reduced by $86.45 \%$ (1.34 gha) compared to EF1 (1.55gha). Furthermore, the difference between EF2 and EF3 $(1.45-1.34=0.11$ gha $)$ that is equal to $7.09 \%$ of EF1 (100\% of EF) determines the reduced portion of EF2 influenced by 
changing curling and golfing for walking at Cromwell. This portion (7.09 \%) is shown as (R) EF2 in Figure 6.59.

As shown in Table 128, EF3 (1.34) compared with EF1 (1.55) is reduced 0.21 gha (1.55gha- 1.34gha). In addition, the overshoot portion of the EF of Cromwell activities that in the first scenario is 1.167 gha (see Table 6.126) in the third scenario is reduced to (1.167gha - 0.21 gha) 0.96 gha. As a result the overshoot energy use of Cromwell activities in the third scenario is equal to 0.96 gha $\times 100$ (carrying capacity of land) $=96$ GJ. Likewise, the total cost to generate the overshoot energy used in third scenario is equal to:

96 GJ (overshoot portion of energy use) $\times$ NZ\$19.8 (the cost to generate 1GJ energy using wind-solar systems) $=\mathrm{NZ} \$ 1,900$.

Since the total GDP of Cromwell visitor activities is NZ\$450,603 (see Table 6.126) consequently, the GDPs of the third scenario is NZ\$450,603 (GDP) - NZ\$1,900 (the total cost to generate overshoot energy in the third scenario) $=$ NZ\$448,703= GDPs3. As shown in Figure 6.59, GDPs3 is 99.58 \% of the total GDP of Cromwell visitors activities and (I) GDPs2 is GDPs3 - GDPs2 $=0.05 \%$ of GDP $(\mathrm{NZ} \$ 450,603)=\mathrm{NZ} \$ 225$.

\section{d. 6. Comparison between the Ideal Model and the Third Scenario}

As Figure 6.59 illustrates, the EF of the Ideal model (0.38 gha, see section 6.2.13.3.4-a) is $28.4 \%$ of EF3 (1.34 gha, see Table 6.128). The proposed CF model in Figure 6.59 determines that for changing EF3 (1.34gha) to the ideal EF $(0.38), 0.4 \%$ (\$NZ1,900) of the total GDP of Cromwell visitor activities must be spent to generate the overshoot portion of its energy.

\begin{tabular}{|c|c|c|c|c|c|c|c|}
\hline \multicolumn{8}{|c|}{ Table 6.128: The CF of Cromwell visitor activities - first and third scenarios } \\
\hline \multicolumn{4}{|c|}{ First scenario } & \multicolumn{4}{|c|}{ Third scenario } \\
\hline Category & $\mathrm{NV}^{4}$ & $\begin{array}{c}\text { EF } \\
\text { (gha/visitor) }\end{array}$ & EF1 (gha) & Category & NV & $\begin{array}{c}\mathrm{EF} \\
\text { (gha/visitor) }\end{array}$ & EF3 (gha) \\
\hline Golf & 34 & $0.003^{1}$ & 0.1 & - & - & - & - \\
\hline Curling & 979 & $0.00016^{2}$ & 0.16 & - & - & - & - \\
\hline Walking & 16 & $0.00005^{3}$ & 0.0008 & Walking & 1,029 & $0.00005^{3}$ & 0.051 \\
\hline Other & 6,893 & 0.00025 & 1.29 & Other & 6,893 & 0.00025 & 1.29 \\
\hline Total & 7,922 & - & 1.55 & Total & 7,922 & - & 1.34 \\
\hline $\begin{array}{l}\text { 1. See Tab } \\
\text { 2. See Tab } \\
\text { 3. See Tab } \\
\text { 4. NV: Nul }\end{array}$ & $\begin{array}{l}58 \\
35 \\
56 \\
\text { of vi }\end{array}$ & tivities (s & ble 6.96) & & & & \\
\hline
\end{tabular}




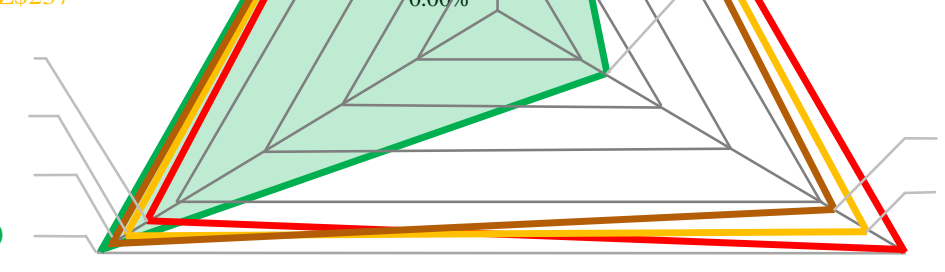

Figure 6.59: The CF of Cromwell visitor activities
Ideal EF (24.5\%)

(R) $\mathrm{EF} 1=6.45 \%$ $=1.36 \mathrm{gha}$

(R) $\mathrm{EF} 2=7.09 \%$ $=0.11 \mathrm{gha}$

EF3 (86.45\%) EF2 (93.55\%) EF1(100\%)

- $\quad$ Q: $100 \%$ of Cromwell visitor activities in Ideal model

- $\quad$ Q1: $100 \%$ of Cromwell visitor activities first scenario (present)

- Q2: $100 \%$ of Cromwell visitor activities second scenario (walking instead golfing)

- Q3: $100 \%$ of Cromwell visitor activities third scenario (walking instead golfing and curling)

- $\quad$ EF1: The EF of Cromwell visitor activities first scenario (present EF)

- EF2: The EF of Cromwell visitor activities second scenario

- $\quad$ EF3: The EF of Cromwell visitor activities third scenario

- $\quad$ (R) EF1: Reduced portion of EF1 influenced by changing golf for walking

- (R) EF2: Reduced portion of EF2 influenced by changing golfing and curling for walking

- GDP: The GDP of Cromwell visitor activities

- $\quad$ GDPs: The GDPs of Ideal model for visitor activities = GDP

- GDPs1: The GDPs of Cromwell visitor activities first scenario

- GDPs2: The GDPs of Cromwell visitor activities second scenario

- GDPs3: The GDPs of Cromwell visitor activities third scenario

- $\quad$ GDPs2: Increased portion of GDPs2 (compared with GDPs1) influenced by walking instead golfing

- $\quad$ GDPs3: Increased portion of GDPs3 (compared with GDPs2) influenced by walking instead golfing and curling.

\subsubsection{Comparison between the CFs of Naseby and Cromwell}

\subsubsection{Food}

Figure 6.60 compares the CFs of food consumed in Naseby and Cromwell by their visitors. In this figure the quantities of food consumed in Naseby $(30,936 \mathrm{~kg})$ in both Ideal (Q) and present (Q1) models are used as the $100 \%$ benchmark of food to compare the Ideal and present model of Cromwell with Naseby (see Figure 6.60). 
In Figure 6.60, the present ecological footprint of food in Naseby (160.9gha) shown in the figure as EF-Na is used as the $100 \%$ benchmark for comparison between the present EFs of food in Naseby and Cromwell. In addition in Figure 6.60 the Ideal EFs of Cromwell and Naseby are compared with EF-Na as the $100 \%$ benchmark.

In Figure 6.60, the total GDP of Naseby $(\mathrm{NZ} \$ 309,360)$ is used as the $100 \%$ benchmark for comparison of the economic footprints of food in Naseby and Cromwell. In 2011, in Naseby 30,936kg (100\%) and in Cromwell 17,662kg (57.09\% compared with Naseby) of food was consumed by visitors. Figure 6.60 indicates that $11.8 \%(3,650 \mathrm{~kg})$ of food in Naseby and 1.4\% (435kg) of the total 17,662kg (57.09\% compared with Q1) in Cromwell were home prepared food.

Figure 6.60 demonstrates that the total EF of food consumed by Cromwell visitors (94.71gha) is $58.86 \%$ of the EF of food consumed by Naseby visitors (160.9gha as $100 \%)$. On the other hand the EF of food consumed by Cromwell visitors (94.71gha) is 66.19gha (160.9 - 94.71gha) smaller than the EF of Naseby food (160.9gha).

Furthermore as shown in Figure 6.60 the ideal EF of 30,936kg food consumed by Naseby visitors (30.61 gha) is 9.7gha (30.61- 20.91 gha) bigger than the ideal EF of 17,662 kg food consumed by Cromwell visitors (20.91 gha). In Figure 6.60, the over shoot portion of the EF of food in Naseby is 160.9gha (EF of Naseby) - 30.61 gha (Ideal EF of Naseby) $=130.29$ gha. In addition, the overshoot portion of the consumed food in Cromwell is 73.8gha (94.71 - 20.91gha). Consequently, the overshoot portion of the EF of food consumed in Naseby is 56.49 gha (130.29 - 73.8gha) bigger than the overshoot portion of the EF of food consumed in Cromwell.

The total GDP of Naseby related to consumed food (NZ\$309,360) as shown in Figure 6.60 is $42.91 \%(309,360-176,620=\mathrm{NZ} \$ 132,740)$ more than total GDP of Cromwell’s visitor food. Furthermore the GDPs of the visitor food in Naseby (16.6\% of its GDPSee Figure 6.60) is NZ\$20,900 $(51,386$ - 30,486) more than the GDPs of the food consumed in Cromwell.

As a conclusion for this section, it can be determined that if in Naseby the overshoot portion of its EF related to food is to be changed to its ideal EF through using a portion of its GDP, it has more GDPs than Cromwell. In addition, since Naseby takes the op- 
portunity for those engaged in OCRT accommodation services to produce $10.4 \%$ $(3,215 \mathrm{~kg})$ more home prepared food as a part of their cultural products, it can be said the CF of Naseby related to food is bigger than the CF of food in Cromwell. Likewise as shown in Table s 6.12 and 6.78, in both Naseby and Cromwell case studies, producing home prepared food that in this thesis is considered as a cultural indicator, contributes to reducing the EF of consumed food by $0.0017 \mathrm{gha} / \mathrm{kg}$. This means producing home prepared food is not only culturally appropriate but is also more environmentally sustainable than providing conventional food.

As explored in both case studies consuming home prepared food contributes to reducing the EF of food, consequently it contributes to increasing the sustainable portion of GDP. This means producing home prepared food is also more economically viable than providing conventional foods.

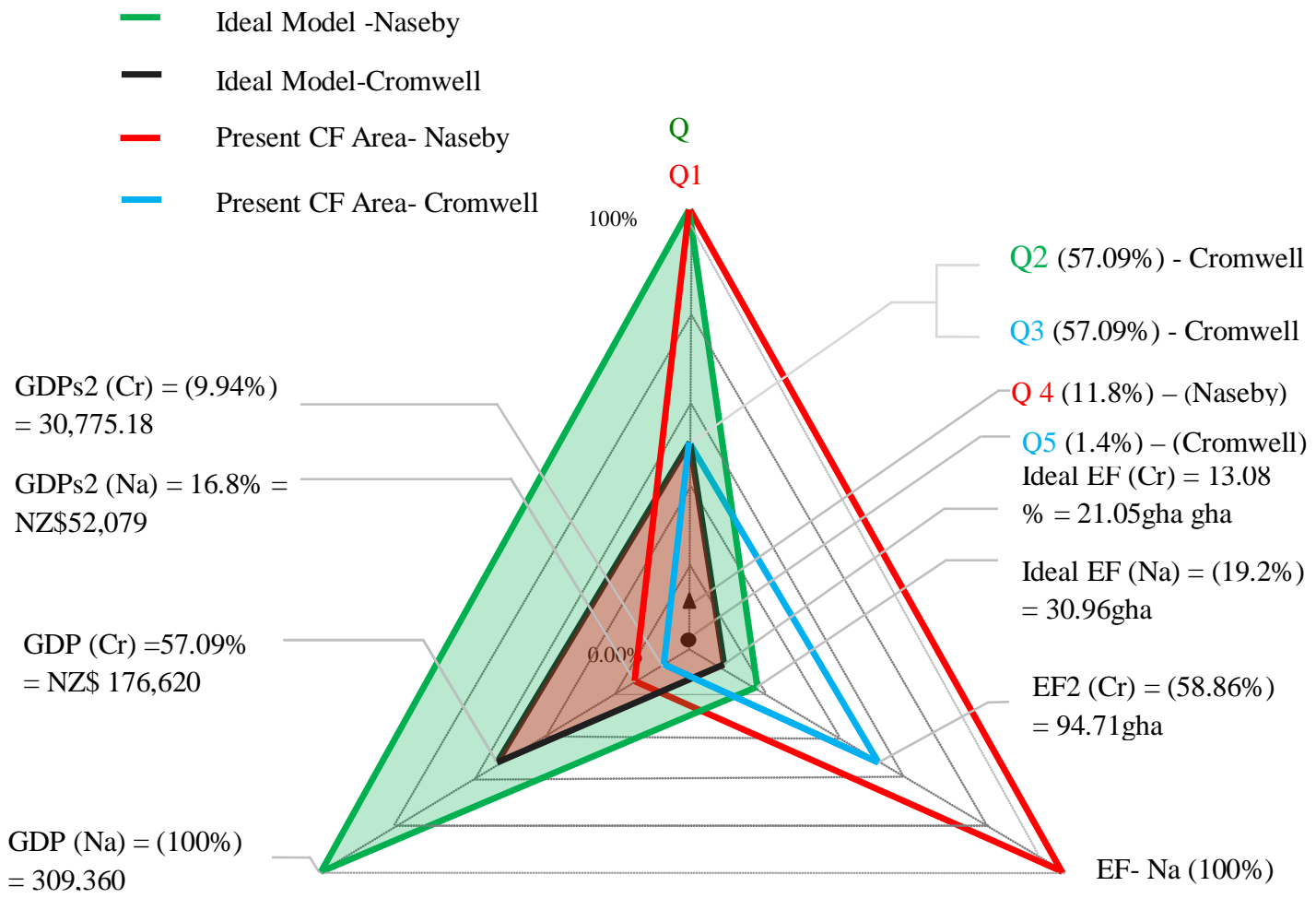

Figure 6.60: The CF of Naseby and Cromwell related to Food

- $\quad$ Q: $100 \%(30,936 \mathrm{~kg})$ food in Naseby - Ideal model

- $\quad$ Q1: $100 \%$ (30,936kg) food in Naseby including 98.2\% (27,286 kg) conventional food + $11.8 \%$ (3650kg) home prepared (organic) food (used as benchmark)

- $\quad$ Q2: $100 \%(17,662 \mathrm{~kg})$ food in Cromwell - Ideal model that is equal to $57.9 \%$ of Q (30,936kg)

- $\quad$ Q3: $100 \%(17,662 \mathrm{~kg})$ food in Cromwell that is equal to $57.9 \%$ of Q1 (30,936kg) including $1.4 \%$ (435kg) home prepared food and 56.5\% $(17,227 \mathrm{~kg})$ conventional food

- $\quad$ Q 4: $11.8 \%(3,650 \mathrm{~kg})$ home prepared food in Naseby

- $\quad$ Q5: 1.4\% (435kg) home prepared food in Cromwell (compared with Q1)

- $\quad$ GDP (Na): $100 \%$ of GDP related to food in Naseby (Used as benchmark) 
- GDP (Cr): $100 \%$ of GDP related to food in Cromwell that is equal to $57.09 \%$ of GDP (Na)

- GDPs2 (Na): GDPs Naseby related to food that is equal with $16.8 \%$ (NZ\$52,079) of GDP(Na)

- GDPs (Cr): GDPs of Cromwell related to food that is equal to 9.94\% (NZ\$30,775.18) of GDP (Na)

- EF-Na: Present EF of Naseby related to food (160.9gha) that is used as $100 \%$ EF benchmark

- EF2 (Cr): 100\% (94.71gha) EF of consumed food in Cromwell that is equal to $58.86 \%$ of E-Na (160.9gha)

- Ideal EF (Na): Ideal EF of consumed food in Naseby that is equal to 19.2\% (30.96gha) of EF-Na

- Ideal EF (Cr): Ideal EF of consumed food in Cromwell that is equal to 13.08\% (30.96gha) of EF-Na

\subsubsection{Accommodation Services}

Figure 6.61 compares the CFs of accommodation services used in Naseby and Cromwell in terms of the quantities, qualities, EFs and economic impacts of each. In Figure $6.61100 \%\left(25,178 \mathrm{~m}^{2}\right)$ of the area of Cromwell's accommodation services is used as a benchmark to compare the areas of Naseby and Cromwell accommodation services with each other.

Figure 6.61 shows that total area of Cromwell accommodation services is $25,178 \mathrm{~m}^{2}$ shown in the figure as Q1. In addition in Figure 6.61, Q is the total area of Cromwell accommodation services $\left(25,178 \mathrm{~m}^{2}\right)$ in its Ideal model that quantitatively is equal to $100 \%$ of Q1.

As illustrated in Figure 6.61, the total area of Naseby's accommodation services in both its ideal model (Q2) and present (Q3) is $8,144 \mathrm{~m}^{2}$ and this is $32.3 \%$ of the total area of Cromwell accommodation services $\left(\mathrm{Q} 1=25,178 \mathrm{~m}^{2}\right)$. Figure 6.61 shows that of the $8,144 \mathrm{~m}^{2}$ of Naseby accommodation, $2,592 \mathrm{~m}^{2}(\mathrm{Q} 4)$ is refurbished (RB) buildings, and this is equal to $10.33 \%$ of Q1 (the total area of Cromwell accommodation). As discussed in section.6.2.13.2, all Cromwell accommodation buildings are new (NB).

Figure 6.61 determines that $0.2 \%\left(57.2 \mathrm{~m}^{2}\right)$ of the total $\left(25,178 \mathrm{~m}^{2}\right)$ area of Cromwell accommodation services (Q6) is open air spaces (V/B) and $100.1 \mathrm{~m}^{2}(0.4 \%$ of Q1) V/B are used as part of Naseby's accommodation area. In comparison with Cromwell accommodation, Naseby accommodation services contain $42.9 \mathrm{~m}^{2}\left(100.1-57.2 \mathrm{~m}^{2}\right)$ more open air spaces (V/B).

In Figure 6.61, 100\% of the present EF of Cromwell accommodation services (8.046 gha) is used as a benchmark for comparisons between the EFs of the two case studies. This benchmark is shown as EF (Cr) in Figure 6.61. The figure shows that the total EF of $8,144 \mathrm{~m}^{2}$ of Naseby accommodation services (7.012 gha) is equal to $87.1 \%$ of the 
total EF (8.046 gha) of the 25,178 $\mathrm{m}^{2}$ that make up Cromwell's accommodation buildings.

As shown in Figure 6.61, Ideal-EF (Cr) (the ideal EF of Cromwell accommodation services) is $22.24 \%$ (1.79gha) of its present EF (8.046gha). On the other hand the overshoot portion of EF (Cr) is 6.25 gha (8.046 - 1.79gha). Figure 6.61 indicates that the Ideal EF of Naseby accommodation services (1.34gha) is $16.65 \%$ of EF (Cr). Consequently, the Ideal EF (Na) is 5.75\% (22.24\% - 16.25\%) smaller than Ideal EF (Cr). Indeed, Ideal EF (Cr) is (1.79 gha - 1.34gha) 0.45gha bigger that ideal EF (Na) (Figure 6.61).

Since the GDP of Naseby related to its accommodation services $(\mathrm{NZ} \$ 1,118,033)$ is more than the GDP of the Cromwell accommodation (NZ\$ 638,312.42), as shown in Figure 6.61 , this study uses $100 \%$ of the GDP of Naseby (NZ\$1,118,033) as a benchmark to make a comparison between the economic footprints of accommodation services in the two case studies. As shown in Figure 6.61, the total GDP of Cromwell related to accommodation services (NZ\$638,312.42) that is shown in the figure as GDP $(\mathrm{Cr})$ is $57.1 \%$ of GDP (Na). In fact, GDP $(\mathrm{Na})$ is $(\mathrm{NZ} \$ 1,118,033-\mathrm{NZ} \$ 638,312.42)$ NZ\$479,720 more than GDP (Cr). Using GDP (Na) as an economic benchmark, the GDPs of Naseby accommodation services (NZ\$1,106,806) is 98.99\% of $(\mathrm{NZ} \$ 1,118,033)$ GDP $(\mathrm{Na})$, and the GDPs of Cromwell accommodation services (NZ\$625,893) is 55.98\% of this benchmark (NZ\$1,118,033). On the other hand, the difference between GDPs (Na) and GDPs (Cr) is equivalent to 98.99\% of GDP (Na) $55.98 \%$ of GDP $(\mathrm{Na})=43.01 \%$ of $(\mathrm{NZ} \$ 1,118,033)$ GDP $(\mathrm{Na})=\mathrm{NZ} \$ 480,913$. Likewise, the GDPs of Naseby accommodation services (NZ\$1,106,806) shown as GDPs $(\mathrm{Na})$ is $\mathrm{NZ} \$ 480,913(\mathrm{NZ} \$ 1,106,806$ - NZ\$625,893) more than the GDPs of Cromwell accommodation services.

Figure 6.61 gives the result that Naseby accommodation services use less area, have a smaller EF and a larger economic footprint than Cromwell accommodation services. In comparison with Cromwell, Naseby's accommodation buildings use more open air spaces (V/B) and have conserved more old buildings than Cromwell. As a result, Naseby accommodation services as shown in Figure 6.61 have a larger CF area than Cromwell accommodation. Likewise, the CF of the OCRT in Naseby in terms of the accommodation services is larger than its equivalent in Cromwell. 


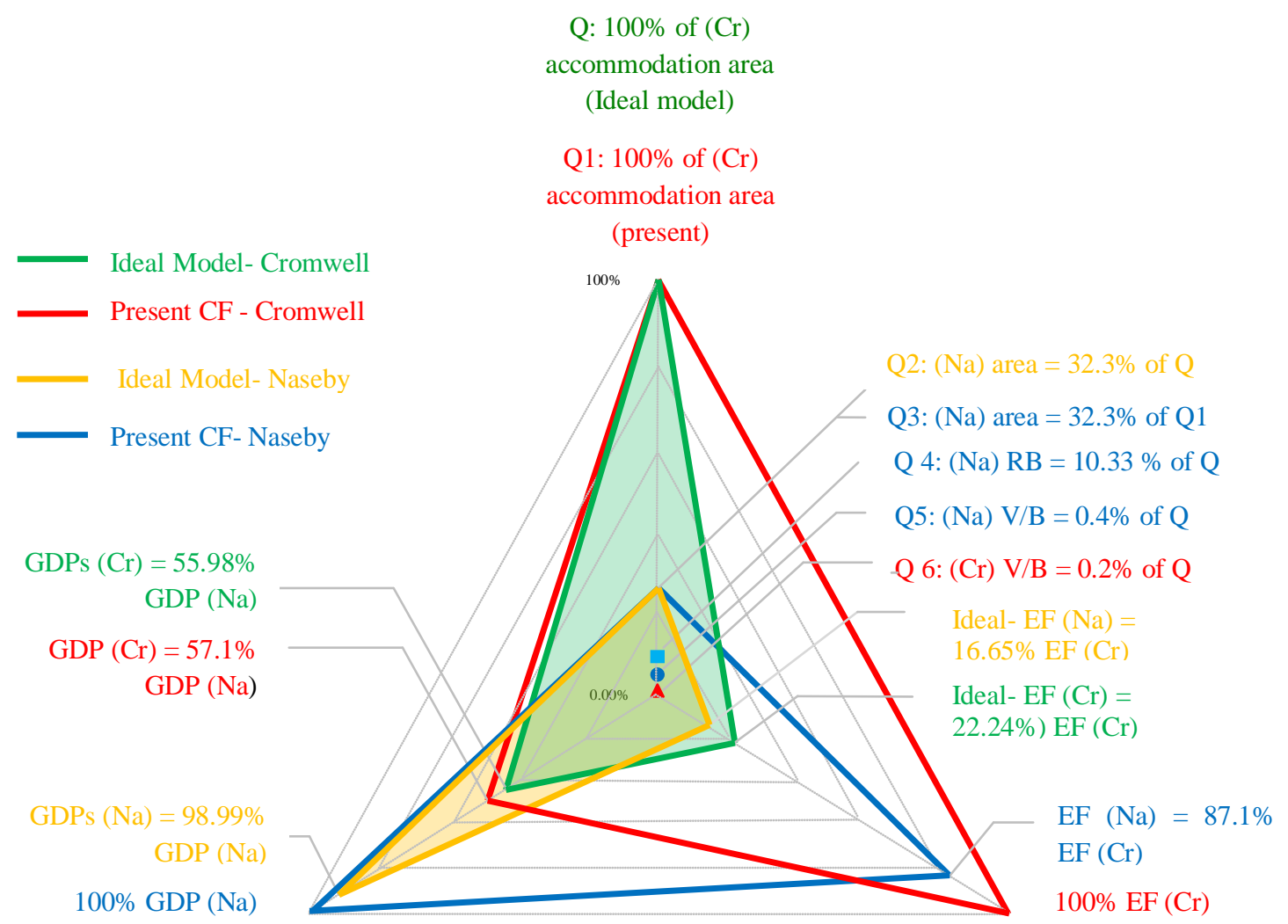

Figure 6.61: The CFs of Naseby and Cromwell related to accommodation services

- Cr: Cromwell

- Na: Naseby

- $\quad$ Q: $100 \%\left(25,178 \mathrm{~m}^{2}\right)$ of the area of Cromwell accommodation services in ideal model

- Q1: $100 \%\left(25,178 \mathrm{~m}^{2}\right)$ of the area of Cromwell accommodation services (present) used as benchmark

- $\quad$ Q2: $100 \%\left(8,144 \mathrm{~m}^{2}\right)$ of the area of Naseby accommodation services in ideal model that is equal to $32.3 \%$ of Q1 (benchmark)

- Q3: $100 \%\left(8,144 \mathrm{~m}^{2}\right)$ of the area of Naseby accommodation services (present) that is equal to $32.3 \%$ of Q1 (benchmark)

- $\quad$ Q 4: $31.8 \%\left(2,592 \mathrm{~m}^{2}\right)$ of Naseby buildings (RB) that is equal to $10.33 \%$ of Q1 (benchmark)

- $\quad$ Q5: 6.1\% $\left(100.1 \mathrm{~m}^{2}\right)$ of Naseby buildings used as V/B that is equal to $0.4 \%$ of Q1 (benchmark)

- $\quad$ Q6: $0.2 \%\left(57.2 \mathrm{~m}^{2}\right)$ of Cromwell accommodation services $\left(\mathrm{Q} 1=25,178 \mathrm{~m}^{2}\right)$ used as V/B

- $\quad$ EF (Cr): 100\% (8.046gha) of the present EF of Cromwell accommodation services used as benchmark

- $\quad$ E (Na): 100\% present EF of Naseby accommodation services (7.012gha) that is equal to $87.1 \%$ of $\mathrm{EF}(\mathrm{Cr})$

- Ideal- EF (Cr): The Ideal EF of Cromwell accommodation services (1.79gha) that is equal to $22.24 \%$ of the EF (Cr)

- Ideal EF (Na): The Ideal EF of Naseby accommodation services (1.34gha) that is equal to $16.65 \%$ of the EF (Cr)

- $\quad$ GDP (Na): $100 \%$ of Naseby GDP related to accommodation services $(\mathrm{NZ} \$ 1,118,033)$ that is used as benchmark

- $\quad$ GDPs (Na): GDPs of Naseby accommodation services (NZ\$1,106,806) that is equal to $98.99 \%$ of GDP $(\mathrm{Na})$

- $\quad$ GDP (Cr): $100 \%$ of the Cromwell GDP $(\mathrm{NZ} \$ 638,312)$ related to accommodation services that is equal to $57.1 \%$ of GDP (Na)

- $\quad$ GDPs (Cr): $100 \%$ of GDPs of Cromwell (NZ\$ 625,893.86) that is equal to $55.98 \%$ of GDP (Na) 


\subsubsection{Visitor Activities}

Figure 6.62 compares the CF areas of Naseby and Cromwell related to their visitor activities. In Figure 6.62, Q1 represents $100 \%$ of total Naseby visitor activities (13,088 see Table 6.53) and this is used as a benchmark and compared with Cromwell visitor activities. As shown in Figure 6.62, the total number of Cromwell visitor activities (7,922 - see Table 6.115) is 60.53\% of Q1 (13,088 visitor activities). As shown in Figure 6.62, in both case studies the number of visitors activities at present and in the ideal model are equal $(\mathrm{Q} 1=\mathrm{Q}$ and $\mathrm{Q} 2=\mathrm{Q} 3)$.

In Figure $6.62,100 \%$ of the EF and $100 \%$ of the GDP of Naseby are used as respective benchmarks for comparing both case studies with each other. Figure 6.62 illustrates that $100 \%$ of the EF of Cromwell related to its visitor activities (1.55gha - see Table 6.115) is equal to $51.3 \%$ of the total EF of Naseby visitor activities (3.02 gha- see Table 6.53). As shown in Figure 6.62, the Ideal EFs of Naseby and Cromwell related to their visitor activities are equal to $15.6 \%$ (0.47gha) and $11.6 \%$ (0.38 gha) of EF (Na) respectively.

As demonstrated in Figure 6.62, the GDP of Cromwell related to its visitor activities (NZ\$450,603 - see Table 6.125) is $60.33 \%$ of (NZ\$744,446 - see Table 6.64) GDP (Na). In addition as shown in Figure 6.62, GDPs (Na) and GDPs (Cr) are equal to 99.33\% (NZ\$739,498 - see Table 6.65) and 60.2\% (NZ\$448,296) of GDP (Na) respectively.

Figure 6.62 shows that the visitor numbers, EF, GDP and GDPs related to visitor activities in Naseby are more than in Cromwell. If the EFs of Naseby and Cromwell related to visitors activities are to be changed to their ideal EF through using part of their GDP to generate their overshoot portion of energy used in a sustainable way, then the visitor activities in both case studies can be considered to be environmentally sustainable activities.

However it should be remembered that in both case studies golfing represents the biggest EF/ visitor (0.003 gha). Although at present just few people choose golfing as one of their outdoor activities, its total EF is one of the four top EFs. This indicates that more development of golfing as a social activity can exert negative environmental impacts on the host destinations and it needs to be ignored in future policy for sustainable development of OCRT. 
As discussed above, curling at Naseby is considered one of the main social-cultural activities that has been developed at the regional, national and international scales in this town, and that attracts many OCRT visitors to it. This makes an opportunity for local and indigenous people who are engaged with the OCRT to present other social-cultural products to visitors that in turn can contribute to conservation of their cultural heritage. However, reducing the EF of indoor curling can be achieved, for example by using more seasonal outdoor curling and spending a part of GDP to generate curling's overshoot portion of energy use in a sustainable way.

In this chapter Cromwell is introduced as one the main destinations offered by the OCRT to its visitors. On the other hand like curling in Naseby, visiting old Cromwell town could be considered as an activity with local potential that could be developed at regional, national and international scales. Like Naseby, through reducing the EF of visitor activities at Cromwell it can become a more environmentally friendly host for its visitors with a rich social cultural heritage and sustainable economic gross domestic product.

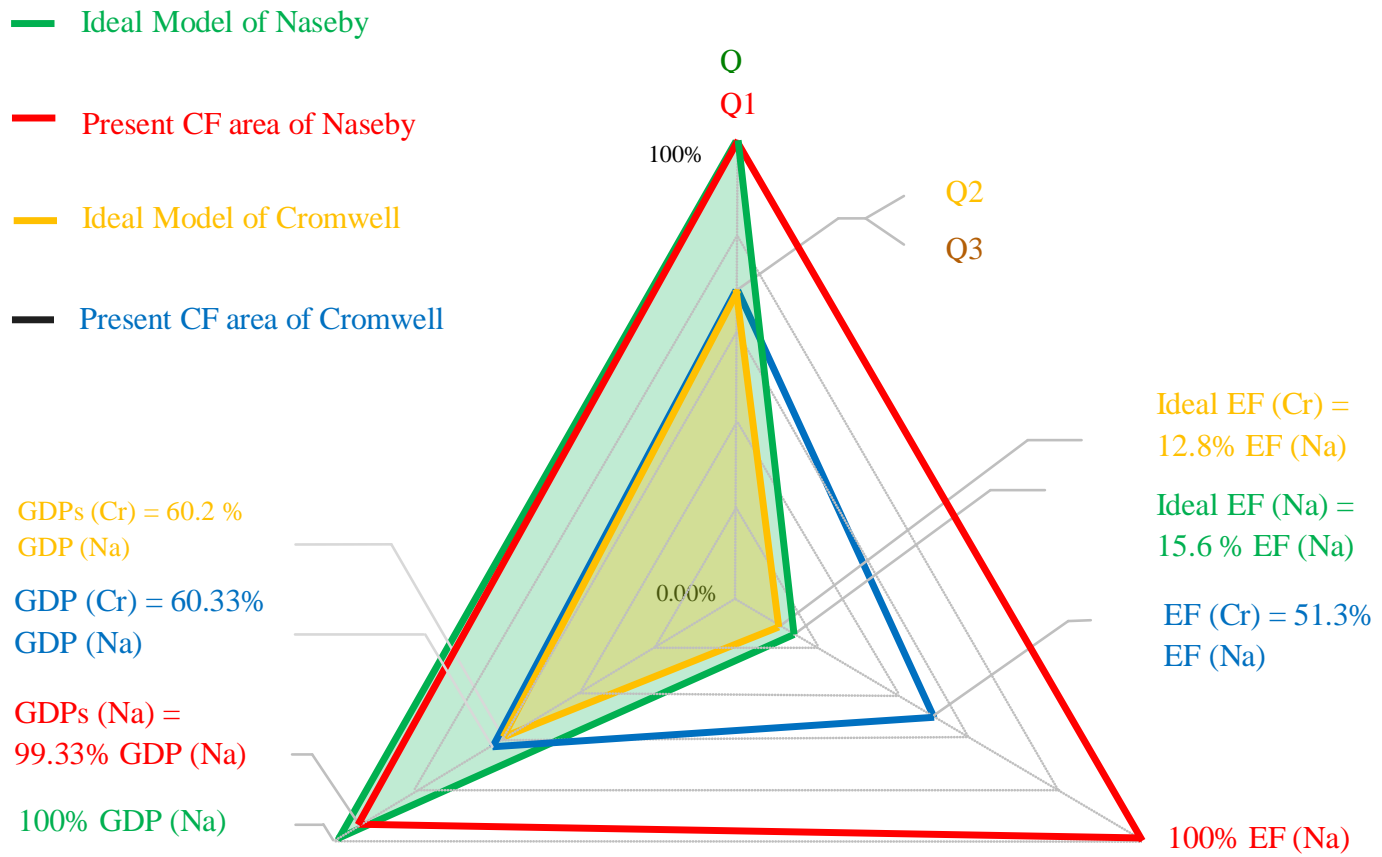

Figure 6.47: The CFs of Naseby and Cromwell related to visitor activities

- Q: $100 \%$ Naseby visitor activities (Ideal Model)

- $\quad$ Q1: $100 \%$ Naseby visitor activities (present) used as benchmark

- $\quad$ Q2: $100 \%$ Cromwell visitor activities (Ideal model) that is equal to 60.53\% of Q1 (benchmark)

- Q3: $100 \%$ Cromwell visitor activities (present) that is equal to 60.53\% of Q1 (benchmark)

- $\quad$ EF (Na): $100 \%$ of the EF of Naseby visitor activities used as benchmark

- $\quad$ EF (Cr): $100 \%$ of the EF of Cromwell visitor's activities that is equal to $53.62 \%$ of EF (Na) 
- Ideal EF (Na): Ideal EF of Naseby visitor activities that is equal to $19.84 \%$ of EF (Na)

- Ideal EF (Cr): Ideal EF of Cromwell visitor activities that is equal to $11.8 \%$ of EF (Na)

- GDP (Na): $100 \%$ of the GDP of Naseby related to its visitor activities used as benchmark

- GDP (Cr): $100 \%$ of the GDP of Cromwell related to its visitor activities that is equal to $60.33 \%$ of GDP (Na)

- GDPs (Na): GDPs of Naseby related to its visitor activities that is equal to 99.88\% of GDP (Na)

- GDPs (Cr): $100 \%$ of the GDPs of Cromwell related to its visitor activities that is equal to $59.95 \%$ of GDP (Na)

\section{Chapter 6: Summary}

Naseby and Cromwell form two case studies with different environmental, cultural and economic characteristics (see section 3.3.2). Using these case studies allows the thesis to determine how the ecological, cultural and economic influences of ecotourism and its architecture on given host destinations can differ from one destination to another. Also in this chapter the main reasons for these differences are clarified. For example as (see Figure 6.47) the EF of visitor activities at Cromwell is 51.3\% of the EF of visitor activities at Naseby. One of the main reasons for this difference are the curling clubs at Naseby (in particular indoor curling) accessed by 4,350 visitors in 2011.

As complementary answers to the research questions answers in Chapter 5, comparison between the different cultural footprints of the OCRT in these two case studies indicates that the environmental, social and economic influences of OCRT have different causes. Therefore sustainable development of the OCRT requires different strategies to reduce the impacts on its various destinations. On the other hand the framework and model demonstrate that even though achieving sustainability through ecotourism and its products has global goals, these rely on local and regional strategies for sustainable development. For instance the main environmental outcomes for the sustainable development of ecotourism is conservation of environmental resources, but, in the case of Naseby reducing the EF of indoor curling and in Cromwell reducing the EF of other tourism activities are two different priorities needed to achieve the environmental goal of sustainable development.

In response to questions 3.3.1.2 and 3.3.1.2 it can be stated that a strategy for the sustainable development of ecotourism and its architecture should determine the main environmental, cultural, and economic outcomes for the sustainable development of ecotourism and its architecture. Likewise a strategy should indicate the priorities that contribute 
to achieving these outcomes. But, as shown in this chapter, at the site scale, these priorities change from one destination to another. This means all such priorities should be proposed in relation to the environmental, social and economic characteristics of the destinations. For instance in the case of Cromwell development of visiting historical sites and in Naseby development of outdoor curling can be considered as priorities for the sustainable development of the OCRT in these destinations. 



\section{Chapter 7: Conclusion}

This thesis contributes to knowledge by setting the environmental, cultural and economic characteristics of ecotourism and its related architecture into a holistic model to explore the linkage between these profiles and their sustainability. To achieve this goal the thesis develops a quantitative methodology that comprises the comprehensive framework for the sustainable development of ecotourism and its architecture. This holistic cultural footprint model (CF-model) summarises a comprehensive framework proposed for sustainable development of ecotourism and its products including the architecture used as accommodation services. The CF- model contains an area that is introduced as the cultural footprint area and which is determined by the quantity of the social- cultural products and activities offered by the host destination of ecotourism and consumed/conducted by eco-tourists at one apex, and the ecological and economic footprints of these social-cultural behaviours on the two other apexes.

As sustainability is a multi-dimensional phenomenon that covers all human activities and products, this thesis uses its principles and strategies to determine a sustainable living area for those engaged in ecotourism development. Then the present cultural footprint of ecotourism, its products and activities can be quantitatively compared with the proposed sustainable living area. This quantitative comparison between the cultural footprints of ecotourism and its products and activities can show how far ecotourism is environmentally, culturally and economically from the sustainable model and what could be done to change the present cultural footprint to sustainable living circumstances.

Since environmental conservation is considered as the main environmental outcome for sustainable development of ecotourism and its related products and activities then their ecological impacts must be at a point of ecologically sustainable life. This study uses the ecological footprint (EF) of products and activities related to ecotourism as the main ecological indicator for evaluation of ecotourism and architecture as being sustainable. Comparison between the present EFs of ecotourism and its architecture with the EF of sustainable life related to these categories indicate how far is its environmental characteristic from the sustainable EF. 
Awareness of the members of a society about their cultural capitals and heritage are introduced as one of the main anticipated cultural outcomes for sustainable development of ecotourism. The main productive activity that contributes to achieving the mentioned outcomes is conservation of the cultural heritage in the host society. In addition making this opportunity for local and endogenous participants to present their cultural capitals and products is viewed as a social-economic strategy for culturally sustainable development of ecotourism. This thesis uses the quantity of the cultural heritage that is preserved and cultural products that are produced through ecotourism as the cultural indicators to be used as tools to evaluate it and its products (including architecture) as being culturally sustainable.

This thesis uses a social- ecological lens to view the ecological and social impacts of economic development on the host society of ecotourism. Since the use of the current economic indicators such as GDP and GNP ignores the cost of environmental decline caused through the development process, for the first time, this study introduces GDPs (sustainable portion of GDP) as a social- economic indicator that is environmentally sensitive in relation to the development of ecotourism and its environmental impacts. The difference between the GDPs and the total GDP of ecotourism indicates the cost that must be spent to change the present EF of an ecotourism project to its sustainable EF.

The GDPs is not just a conventional economic indicator. As producing local products and development of local activities are considered as cultural indicators for sustainable development, the GDPs that determines the gross domestic products created through an activity as ecotourism also can be viewed as a social indicator. Since the cost of environmental conservation is subtracted from the total GDP to create GDPs, it can be introduced as an economic indicator that is environmentally sensitive.

In this thesis, the three cultural, environmental and economic indicators determine the three apexes of the cultural footprint area of ecotourism, its products and activities in the CF-model. Indeed the CF can be introduced as a holistic and multi dimensional indicator that contains all other ecological, cultural and economic indicators. For the first time this thesis proposes the CF model in which ecological, socio-cultural and economic aspects of a phenomenon such as ecotourism are linked together. Using the CF model can contribute to its users being able to measure and understand how their behaviours change their surrounding environment and their economic system. Likewise, using the 
CF-model can contribute to making decisions about future development. It can indicate which parts of the current social-cultural behaviours should be developed, justified or must be completely ignored in strategies for future sustainable development.

The above-mentioned comprehensive framework that is summarised as the CF-model is tested through using three case studies of the Otago Central Rail Trail (OCRT), Naseby and Cromwell located along the rail trail.

This thesis investigates the cultural, environmental and economic impacts of the OCRT at the regional scale on its host destinations. The thesis finds that many historical sites and buildings have been refurbished through development of this project. This using of historical sites and buildings as OCRT attractions contributes to awareness of the engaged communities and components about their cultural identity. In addition the communities and their components are engaged in conservation of their cultural heritage through development of OCRT as an ecotourism project. On the other hand development of OCRT changes the attitude of people engaged in this project to their cultural heritage.

In terms of architecture used as accommodation services in the OCRT, using 78 refurbished buildings as accommodation services can be considered as one of the positive influences of OCRT on its host destination that contributes to preservation of their cultural identity.

Furthermore, the OCRT make the opportunity for local people to present their cultural products such as food, artefacts, crafts, activities and historic architecture to their visitors. The OCRT should not be considered as a merely recreational project, but also is an educational project that contributes to its visitors being able to know more about the cultural and environmental heritage and capitals of their hosts.

This thesis indicates that about $78.2 \%$ of the total EF of OCRT (1,617gha) is its current overshoot portion of the EF. As shown in Chapter 5 about $71.1 \%$ of the total EF is caused by transportation. Moreover, this $71.1 \%$ of the total EF of OCRT is made up of 38.1\% which is the EF of international transportation and 33.8\% which is the EF of domestic transportation. It should be remembered at the same time that international visitors comprise just $22 \%$ of the total numbers of OCRT visitors, although their transport forms $38.1 \%$ of the OCRT's total EF. 
Through the use of the GDPs as an economic indicator it has been determined that because of the high overshoot portion of the EF of OCRT, 43.6\% of its total GDP must be spent to change its present EF to the sustainable living EF with zero overshoot portion of ecological footprint.

This thesis determines that producing local products instead of using conventional products contributes to reducing the total EF of OCRT. As investigated in Chapter 5, in 2011, producing locally $17 \%$ of the total $65165.05 \mathrm{~kg}$ of food consumed by OCRT visitors, can reduce by 1.5\% (23.2gha) its total EF (1,617 gha).Looked at in another way,, producing $1 \mathrm{~kg}$ of home cooked food instead of conventional food can reduce the total EF of OCRT by143 $\mathrm{m}^{2}$. In addition, as determined in Chapter 5 producing home cooked food (as a cultural product) contributes to increasing income by NZ\$ $3.80 / \mathrm{kg}$ of home cooked food. This thesis has made it possible to demonstrate clearly that using local products in ecotourism development not only is culturally appropriate, but also is environmentally friendly and economically viable.

In 2011 the OCRT visitors were involved with 18 types of activities that are categorised into the two categories of indoor (curling at Naseby) and outdoor activities. The curling at Naseby can be viewed as one of the important attractions of OCRT that attracts its visitors at the regional, National and international scales. This activity is considered as one of the distinctive potentials of OCRT for development of tourism. However its EF is one of the three top EFs of OCRT activities. The EF of indoor curling at Naseby can be reduced through making it a seasonal activity that is conducted during winter. Since the curling at Naseby attracts 4,350 of 11,788 OCRT visitors, this would make an opportunity for other OCRT components to develop their other cultural products.

If the EF of curling will be changed to the sustainable EF related to this activity through spending a portion of OCRT GDP to generate its overshoot portion of energy use, curling at Naseby can be considered as one of the culturally appropriate activities.

Most of the outdoor activities such as sightseeing, visiting historic sites and buildings, wineries, art galleries offered by OCRT and conducted by its visitors make the opportunity for its visitors to educate themselves about the environmental and cultural heritage of their host destination. Also the host people have the opportunity to present their cultural products to their visitors as a cultural economic activity. As demonstrated in Chapter 5 the total EF of OCRT activities covers $0.4 \%$ (6.57 gha) of the total EF of OCRT 
(1,617gha). In comparison with the EF of other categories including transportation (72.2\%), food (24.8\%) and accommodation services (2.6\%) this is a small area of EF. However in comparison with other categories of activities, golfing has the highest EF/ visitor (0.003 gha) and the smallest EF (0.00006 gha /visitors) belongs to activities such as walking , fruit picking and visiting heritage (e.g. Old Cromwell town).

This thesis determines that if an OCRT visitor changes his or her preferred activity from golfing to outdoor activities with small EF (e.g. walking) it can reduce his/her EF related to the activities by 0.00294 gha /visitor ( $29 \mathrm{~m}^{2} /$ visitor). As shown in Chapter 5 just a few visitors (153) of total 11,788 OCRT visitors choose golfing as one of their activities but the EF of them related to golfing is one of the three top EFs related to OCRT activities. The number of visitors that play golf indicates that golfing is not a significant cultural activity because just a few people are interested to do that. Likewise in comparison with other activities its EF/visitor is the highest EF area. Consequently its GDPs /visitor is the lowest amount of GDPs/ visitor. This thesis suggested that the development of activities such as golf with high EF and low GDPs and small number of participants should be completely ignored in the future strategy for development of the OCRT.

Using renovated historic buildings for 78 of 226 OCRT accommodation buildings shows the changing of the attitudes of OCRT components to their cultural heritage caused by OCRT development. This indicates that the architecture used in OCRT is culturally sensitive and contributes to the visitors being able to know more about the cultural background of their host destination. In addition as determined in this study using refurbished buildings as accommodation services not only is culturally sustainable but also can decrease the total EF of OCRT and increase its GDPs. The thesis demonstrates that using refurbished buildings to provide $21,378 \mathrm{~m}^{2}$ of the total of OCRT accommodation services $\left(80,356 \mathrm{~m}^{2}\right)$ can reduce by 0.8 gha its total EF. However it should be remembered that both types of refurbished (RB) and new (NB) buildings used as accommodation services use electricity as the main source of energy.

Using renewable sources of energy can be considered as one necessity that should be applied in OCRT accommodation services to make this sector more environmentally sustainable. 
The thesis shows that using open air spaces as parts of the buildings is advertised by most of the accommodation owners as a positive potential of their services. This means using open air spaces can be viewed as one of the appropriate cultural behaviours in OCRT.

Furthermore as has been indicated in Chapter 5, using $378 \mathrm{~m}^{2}$ of the total 80,356 $\mathrm{m}^{2}$ accommodation area as veranda and balcony contributes to reducing 0.8 gha of the total EF of OCRT accommodation services. This means using $1 \mathrm{~m}^{2}$ of veranda/ balcony can reduce the EF of accommodation services by $21.1 \mathrm{~m}^{2}$. In turn it can contribute to development of the GDPs. Consequently using verandas and balconies in OCRT accommodation services is a culturally appropriate policy for development of architecture which also is an environmentally friendly and economically sustainable strategy.

Using the comprehensive framework and its conceptualised model (CF-model) to investigate the cultural footprint of OCRT on Naseby and Cromwell indicates that using local products has the same ecological and economic outcomes in both case studies as in the OCRT as a whole.

This thesis determines that in Naseby local products (home prepared food) are used more than in Cromwell. In addition the GDPs of Naseby related to the local products is more than for Cromwell. If the overshoot portions of the EF of both Naseby and Cromwell will be changed to the Ideal sustainable EF, as Naseby has more economic outcome and more quantity of the local products than Cromwell, its cultural footprint is more positive than Cromwell in terms of the local products.

Since the accommodation services in Naseby contain refurbished buildings, use less indoor areas, comprise more area of veranda and balcony, have more GDPs and smaller EF than Cromwell's accommodation services, Naseby also has more sustainable cultural footprint than Cromwell in terms of accommodation services.

Chapter 6 shows that Naseby activities have more potential than Cromwell to attract OCRT visitors. Also Naseby has more GDPs than Cromwell, and the same EF/visitor activity. If the overshoot portions of the EFs of both case studies will be changed to the Ideal EF of sustainable activities, then Naseby's activities will have more positive CF than Cromwell visitors' activities. 
This thesis concludes that the proposed comprehensive framework and the CF-model can be used as an holistic tool to make the link between ecological, cultural and economic characteristics of ecotourism and its related architecture as being sustainable.

This thesis results in the finding that the main characteristics of an architecture that will contribute to sustainable development through ecotourism can be conceptualised as one characteristic of its cultural footprint. As the main finding of the thesis, in response to the key question it is concluded that the main characteristic of an architecture that will contribute to sustainable development through ecotourism is having a sustainable cultural footprint on the host destination.

The proposed methodology and methodological framework have advantages that can be conceptualised as:

- Using a holistic approach to the development of ecotourism and its architecture through sustainability and its principles.

- Using an existing information system for research that contributes to testing the system as being appropriate for the sustainable development of ecotourism.

Conversely, the main shortcoming of the methodology and the methodological formwork is the lack of an efficient information system for creation and management of the necessary data.

Arising from the above limitation, the accuracy of the results is another potential shortcoming of the thesis. However, using a comparative method to evaluate ecotourism and architecture helps to ameliorate these limitations. Furthermore, the thesis shows the necessity of having an efficient information system as a fundamental for the sustainable development of ecotourism and its architecture.

Goals for future research in terms of the CF-model are using it to determine the different cultural footprints of ecotourism and its architecture at different times in the same place. This would contribute to clarifying how tourism and its architecture exert influence on a society through the evolution of other factors such as technology, facilities, materials, and social behaviours at different times in the same place. The results could be used in the sustainable restoration of a part of the cultural identity of a given society previously impacted by the unsustainable development of tourism and its architecture arising from modernism and capitalism. 
Moreover using the CF-model could contribute to defining a new structure of urban spaces for use as host destinations for soft ecotourism, based on introducing social cultural boundaries for each category of urban component related to soft ecotourism. The CF boundaries could be used to determine the way in which the interactions between different urban spaces with each other and their surrounding environment can be assessed as being more or less sustainable. 


\section{Bibliography}

Ancient Briton Hotel. (2011). "Naseby”. www.ancientbriton.co.nz. Viewed July 2010

Appleby, P. (2011). Integrated sustainable design of buildings, Published by Earth Scan, London ; Washington, DC.

Ashley, C \& Garland, E. (1994). "Promoting community-based tourism development why, what and how?” Directorate of Environmental Affairs Ministry of Environment and Tourism, Private Bag 13306 Windhoek, Namibia.

Australia's guide to environmentally sustainable homes. www.yourhome.gov.au/technical/fs52.html. Viewed February 2010

Becken ,S ., Frampton, C ., Simmons, D.( 2001). "Energy consumption pattern in the accommodation sector-the New Zealand case”. Ecological Economics, No.39:371-386.

Bernard, F., Groot, R.S.D. and Campos, J.J. (2009). "Valuation of tropical forest services and mechanism to finance their conservation and sustainable use: A case study of Tapanti National park, Costa Rica”, Forest policy and Economics, No.11: 174-183.

Blangy, S. and Mehta, H. (2006). "Ecotourism and ecological restoration”, Journal for Nature Conservation, No.14: 233-236.

Breis, G.C (1992). Towards A Framework of Socio-Cultural Indicators for the Development Process, Published by UNESCO, Paris.

Browning, R. C., Baker, E.A., Herron, J.A and Kram, R. (2005). "Effects of obesity and sex on the energetic cost and preferred speed of walking”. J Appl Physiol No.100 : 390- 398.

Buckley, R. (2009). Ecotourism, principles \& practices, Wallingford, UK; Cambridge, MA: CABI.

Burns, G. \& Corbet, R. (2007). “New Zealand Tourism Strategy 2015”. New Zealand Ministry of Tourism. http://www.nztourismstrategy.com

Catley, A. (1999). Methods On The Move, International Institute for Environment and Development, London

Central Otago District Council (CODC). (2011). “OCRT user survey gross results”. CODC, Otago, New Zealand.

Central Otago District Council. (2009). “Otago Central Rail Trail”. http://www.codc.govt.nz 
Chapman, A.R.(1999) Indicators and Standards for Monitoring, published by United Nations Development Programme (UNDP): New York [US].

Chel, A and Tiwari, G.N. (2009). "Thermal performance and embodied energy analysis of a passive house - Case study of Vault roof mud- house in India”. Applied Energy No.86: 1956-1969.

Choi, H.C., Sirakaya, E. (2006). "Sustainability Indicators for managing community tourism”. Tourism Management No. 27: 1274-1289.

Christian, A., Holmberg, J and Lindgren, K. (1996). Social- ecological indicators for sustainability”. Journal of Ecological Economic No.18: 89-112.

Cochrane, P.(2006). "Exploring cultural capital and its importance in sustainable development”. Ecological Economics No. 57: 318-330.

Cohen, E. (1988). “Authenticity and Commoditization in Tourism”. Annals of Tourism No.15: 371-381.

Collins, A and Fairchild, R. (2007). "Sustainable food consumption at a subnational level: an EF, nutritional and economic analysis”. Journal of Environmental Policy and Planning (Special Issue: Sustainable Food Supply Chains), Vol. 9.No.10: 5-30.

Collins, A., Flynn, A., Netherwood, A. (2005). Reducing Cardiff's Ecological Footprint. WWF, Cardiff.

Coppock, J.T. (1982). “Tourism and Conservation”, Tourism Management, 3 (4): 270-276.

Costanza, R. (2009). “Toward a new sustainable economy”, Real World Economics Review No.49: 19-21

Courvisandos, J. and Jain, A. (2006). "A framework for sustainable Ecotourism: Application to Costa Rica”, Tourism and Hospitality planning \& Development. No.2, 131-142.

Cromwell Community.(2008). "Cromwell Community Plan”. http://www.centralotagonz.com/Central-Otago-New-Zealand/CromwellCommunity-Plan_IDL=22_IDT =294_ID=1495_html

Delucchi, M.A and Jacobson, M.Z. (2011). "Providing all global energy with wind, water, and solar power, part 2: Reliability, system and transmission costs, and policies”. Energy Policy, No.39:1170- 1190.

Duxbury, N (2003).”Cultural Indicators and Benchmarks in Community Indicator Projects: Performance Measures for Cultural Investment?” Accounting for Culture Colloquium.13-15. 
Ding, G.K.C.(2008). "Sustainable construction- The role of environmental assessment tools”. Journal of Environmental Management No. 451-464.

EECA (http://www.energywise.govt.nz/how-to-be-energy-efficient/generatingrenewable-energy-at-home/solar-electricity-generation\#costs

Esther, T; Matthijs, H and Herman, E.(2005).

"Ecological indicators: Between the two fires of science and policy". Ecological Indicators No.7: 215-228.

Fallon, L.D. and Kriwoken, L.K.(2003).” Community involvement in Tourism infrastructure-the case of the Strahan visitor Center, Tasmania”. Tourism Management No.24: 289-308.

Farrell, H and Runyan, D. (1991). "Ecology and Tourism”, Annals of Tourism Research No.18: 26-40.

Field, C. (2011). “The Ecological Footprint of Wellington in the 1950s”. Victoria University of Wellington, New Zealand.

Filho, J.A.F.D., Prado, P.G and Santos, I.D.L.(2011). "Green Economy Challenges and Opportunities”. Political Ambiental, No.8: 16.

Frederking, L.C ( 2002).” Is there an endogenous relationship between culture and economic development?”. Journal of Economic Behavior \& Organization No.48: 105- 126.

Fennell, D.(1999). Ecotourism: An introduction, Published by Routledge, London.

Gerring, J., Barresi, P.A.(2003).”Putting Ordinary Language to Work”. Journal of Theoretical Politics No. 15(2): 201-232.

Getz, D. (1986).”Models in Tourism Planning; Towards integration of Research on Practices”, Tourism Management No.17: 21-32

GlOBE 90. (1990). An Action Strategy for Sustainable Development. Tourism Stream, Action Strategy Committee, Glob 90. Vancouver, British Colombia.

Gössling, S. (1999). "Ecotourism: a means to safeguard biodiversity and ecosystem functions?”, Ecological Economics No.29, 303- 320.

Graaf, H.J.de., Musters, C.J.M. and Keurs, W.J.T. (1996). "Sustainable development: looking for new strategies”, Ecological Economics, No.16:205- 216.

Graham, O. J. (2009). “The Otago Central Rail Trail: Preservation of Heritage Sites Through development for Visitor Use”. $3^{\text {rd }}$ Australasian Engineering Heritage Conference. http://www.ipenz.org.nz

Herdeg, K.(1990) . Formal Structure in Islamic Architecture of Iran and Turkistan, Published by Rizzoli International Publications, INC. New York, USA. 
Horn, R.V ( 1983). “Cultural Statistics and Indicators”. Journal of Cultural Economics, USA No.7 (2): 25-40.

Hueting, R. and Reijnders, L. (2004). "Broad sustainability contra sustainability: the proper construction of sustainability indicators”. Ecological Economics, No.50, 249-260. WWW.elsevier.com/locate/ecolecon

International Federation of Arts Councils and Culture Agencies (IFACCA).(2005) . Statistical Indicators for Art Policy. IFACCA, Sydney. www.ifacca.org/ifacca2/en/orgnisation/page09_BrowseDart.asp.

Jafari, J., Ritchie, J.R.B (1981). "Toward a Framework for Tourism Education, Problems and Prospects”. Annals of Tourism Research. No.3 (1): 13-34.

Jellum, C \& Reis, A. (2008). “Otago Central Rail Trail economic impact survey”. Otago Central Rail Trail Trust. http://www.otagocentralrailtrail.co.nz.

Kazimee, B.A.(2008). "Learning from Vernacular Architecture; Sustainability and Cultural Conformity" published in the conference proceedings of the second International Conference on Eco-Architecture .

Kermani, A.A and Luiten, E.(2010).” Preservation and Transformation of Historic Urban Cores in Iran, the Case of Kerman”. Journal of WSEAS Transactions on Environment and Development, : 53-62.

Khan, M. (2003). 'Ecoserve Eco-tourists' Quality Expectations, Annals of Tourism Research, No. 30(1):109-124

Kim, J.J and Rigdon, B. (1998). Introduction to Sustainable Design, Published by National Pollution Centre for Higher Education, Michigan, USA, 1998.

Kohlmann, B., Mitsch, W.J. and Hansen, D.O. (2008). "Ecological management and sustainable development in the humid tropics of Costa Rica”, Ecological Engineering No.34, 254-266.

Lawrence, R.J.(2003). "Human ecology and its application”. Journal of Landscape and Urban Planning:31-40.

Madden, C. (2005). Indicators for Arts and Cultural policy”. Journal of Cultural Trends:217-248.

Madden , C.(2005). Statistical Indicators for Arts Policy, Published by International Federation of Arts Councils and Culture Agencies, www. ifacca.org.

Mahravan, A. and Vale, B. ( 2010), “Community- Based Tourism Development as a Strategy Towards Sustainable Tourism Planning and Development”, Published by $4^{\text {th }}$ International Conference on Built Environment in Developing CountriesPenang, Malaysia: 1382-1394. 
Maniototo Curling International, Indoor Curling Rink - Naseby. (2011). http://www.curling.co.nz/PicsHotel/Curling/CustomPages/NormalPres.aspx?ID= 19051

Marcouiller, D.W.( 2004), “ Natural Amenities, Tourism and Income Distribution”, Annals of Tourism Research No.31 (4): 1031-1050.

Matthews, M. (2009)."How New Zealanders Travel”. New Zealand Ministry of Transprot.www.transport.govt.nz/research/travelsutvey/

Matvienko, O. (2010). “Bottled Water, know the facts”. Iowa State University: 1-4

May, V. (1991). “Tourism, environment and development”, Tourism Management No.12 (2): 112-118.

McDonald, G \& Patterson, M.(2003). "Ecological Footprints of New Zealand and its Regions”. Ministry for Environment. http://www.mfe.govt.nz

McKercher, B., Ho, P.S.Y., Cros, H. d. (2005). "Relationship between tourism and cultural heritage management: evidence from Hong Kong”. Tourism Management No.26: 539-548.

MDF-Training \& Consultancy (2005). “Indicators”. www.mdf.nl

Minnaert, L., Maitland, R., Miller, G .(2009). Tourism and Social Policy: The Value of Social Tourism. Annals of Tourism Research, No.36 (2): 316-334.

Ministry of Culture, Madrid, Spain. (1985)."Cultural Indicators Project”. Presented in Division of statistics on culture and communication office of statistics UNESCO, Paris.

Mithraratne, N \& Vale, R. (2007). “Rain Tanks Or Reticulated Water Supply?”. Landcare Research, Private Bag 92170, Auckland, New Zealand.

Mog, J.M. (2004). "Struggling with Sustainability- A Comparative Framework for Evaluating Sustainable Development Programs”. World Development No. 12, 2139- 2160.

Munda, G. (2006). "Social multi- criteria evaluation for sustainability policies". Land Use Policy No.23: 86-94. WWW.elsevier.com/locate/landusepol

Murphy, P.E. (1983), “Tourism as a community industry”, Tourism Management No. 4(3): 180-193.

Sorrel, p. (1999). "Naseby”.The Cyclopaedia of Otago and Southland No 1. http://www.nasebyvision.org.nz/2000/12/naseby.html

Naseby community. (2010). Naseby community plan. www.codc.govt.nz/assets/pdfs/.../naseby cp 2010 final oct.pdf 
Ness, B., Piirsalu, E. U., Anderberg, S. and Olsson, L. (2007). "Categorising tools for sustainability assessment”. Ecological Economics No.60, 498-508.

New Zealand Wind Energy Association. (2011). www. windenergy.org.nz/windenergy/costs.

New Zealand Council of Trade Unions (NZCTU) . (2010). http://union.org.nz

Niemi, G.J and MacDonald, M.E. (2004). “Application of Ecological Indicators”. Annual Review of Ecology, Evolution, and Systematics, No.35:89-11.

O’ Connor, H.D. (2010). “New Zealand Tourism Strategy 2015”. Published by New Zealand Minister of Tourism. http://nztourismstrategy.com/download.html

Odum, E.P. (1975). Ecology, the link between the natural and the social science, Holt, Rinehart and Winston, New York.

O’Mahony, C., Ferreira, M., Fernandez- Palacios, Y., Cummins, V., Haroun, R (2009). "Data availability and accessibility for sustainable tourism: An assessment involving different European coastal tourism destination". Journal of Coastal Research, SI 56 (Proceedings of the $10^{\text {th }}$ International Coastal Symposium): 1134-1139.

Omer, A. M. (2008). "Energy, environment and sustainable development". Renewable and Sustainable Energy Review, No.12: 2265-2300. WWW.elsevier.com/locate/resre

Organisation For Economic Development and Co-operation. (2008). Key Environmental Indicators. OECD Environmental Director, Paris, France.

Otago Central Rail Trail Trust. (2005).”The Otago Central Rail Trail Means Business". http://www.otagocentralrailtrail.co.nz

Pattanaik, P. (1997). "Cultural Indicators of Well-being: Some Conceptualised Issues”. Published by UNESCO and UNIRSD.

Pereira, J. (1994). Islamic Sacred Architecture a Stylistic History, New Delhi

Pigram, J.J. (1980). “Environmental Implications of Tourism Development”, Annals of Tourism Research No.4: 554-583.

Pipithvanichtham, P. (1997). "Issues and challenges of ecotourism in the National parks of Thailand", Proceedings of international Conference on advances in Ecotourism for Conservation and Community Development, Bangkok, Thailand, January.

Poria, Y., Butler, R., Airey, D.( 2003). “The Core of Heritage Tourism”. Annals of Tourism Research No.30(1): 238-254.

Quran, 8:41. 
Rapoport, A. (1969). House, Form and Culture, Published by Prentice-Hall, Inc. Englewood Cliffs, N.J. USA.

Reid, D.G., Mair, H., George, W. (2004). "Community Tourism Planning: A SelfAssessment Instrument”. Annals of Tourism Research No.31(3): 623-639.

Reis, A., Jellum, C. and Lovelock, B. (2010). "Linking the Taieri Gorge Railway and the Otago Central Rail Trail: A survey of user demands”. Center for Recreation, School of Business, University of Otago, Otago, New Zealand.

Renewable Energy Magazine.(2000). "Geothermal Ice Plant Efficiently Replaced Again Ammonia System - Oliver Club, Oliver, B.C.”. Renewable Energy Magazine, British Columbia, Canada, No. 1, Issue 3. www.publications.gc.ca/collections/Collection/M143-4-1-3E.pdf

Rizvi. S.M. (1991). "Khums an Islamic Tax”. http://www.shiamultimedia.com/books/english/Sayyid\%20Muhammad\%20Rizvi \%20-\%20Khums.pdf

Robin, C.P.Y. and Poon, C .S.(2009). "Cultural shift towards sustainability in the construction industry of Hong Kong”. Journal of Environmental Management No.90, 3616- 3628. WWW.elsvier.com/locate/jenvman

Schianetz, K., Kavanagh, L., Lockington, D (2007). "The learning Tourism Destination: The potential of a Learning Organisation approach for improving the sustainability of tourism destinations”. Tourism Management No.28: 1485-1496.

Secretariat of the Pacific Community (2010). "Culture Statistics and Indicators". Twenty-third Meeting of the Council of Pacific Arts, Noumea, New Caledonia : $1-4$.

Simpson, M. C. (2008). “Community Benefit Tourism Initiative-A conceptual oxymoron?” Tourism Management No. 29: 1-18.

Statistics New Zealand and Ministry for culture and heritage (2006). "Cultural Indicators for New Zealand”. Published by Statistics New Zealand and Ministry for culture and heritage. Wellington, New Zealand: Catalogue Number: 16.001

Statistics New Zealand (SNZ). (2006). "Cultural Indicators for New Zealand. Ministry for Culture and Heritage, Wellington, New Zealand.

Statistics New Zealand (SNZ). (2009). “Cultural Indicators for New Zealand”. Ministry for Culture and Heritage, Wellington, New Zealand.

Steele, J. (2005). Ecological architecture: a critical history, Thames \& Hudson, London.

Symmetric. Co.UK. http://www.simetric.co.uk/si_materials.htm 
The institute for Statistics of the United Nations Education Scientific and Cultural Organization (UIS) (2010). "The 2009 UNESCO Framework for Cultural Statistics (FCS)”. Statistical Commission Forty-first session: 23-26.

The International Federation of Arts Councils and Culture Agencies (IFACCA). (2005). “Cultural Trends”. Published by Routledge, Vol. 20

Torres, S and Sakamoto. (2004). "Subjective factors in sustainable building". Published in the conference proceedings of the $21^{\text {th }}$ conference on Passive and Low Energy Architecture. Eindhoven, the Netherlands:19-22.

Tourism Satellite Account. (2009). Published in October 2009 by Statistics New Zealand, Wellington, New Zealand.

Travis, S (1982). "Managing the environmental and cultural impacts of tourism and leisure development”. Tourism Management: 256-262.

Tsaur, S. H., Lin, Y.C. and Lin, J.H. (2006). "Evaluation ecotourism sustainability from the integrated perspective of resource, community and tourism”. Tourism management, No.27, 640-653.

Twyman, C. (2000). "Participatory Conservation? Community based natural resource management in Botswana”. The Geographical Journal No.166: 323-335.

UNEP. (2011). “Towards a Green Economy”. 100 Watt, France. http://www.unep.org/greeneconomy/Portals/88/documents/ger/GER_synthesis_e n.pdf

UNESCO (2003). "Convention for the safeguarding of the Cultural Heritage”. Published in by UNESCO Conference, 32nd session, Paris, France.

US Department of Energy (2011) 2010 "Solar Technologies Market Report D DOE/GO-102011-3318 NREL November p60)”.

Vale, B \& Vale, R. (2009). Time to eat the dog? Thames \& Hudson Ltd, London.

Veron, R. (2001).” The New Kerala Model: Lesson for Sustainable Development”. World Development No.4:601-617. WWW.elsevier.com/locate/worlddev

Wall, G. (1997). “Is Ecotourism sustainable?”, Environmental Management No.21, 483-491.

Wang, J. P. (2004). "Community- based Ecotourism development in Northern Thailand", Available online at: http://www.asianscholarship.org/asf/ejourn/articles/jianping_w.pdf.

Wearing, S. and Neil, J. (2009). Ecotourism, Impacts, Potentials and possibility, Oxford; Boston: Butterworth-Heinemann.

Weaver, D.B. (2009). Ecotourism, impacts, potentials and possibilities, Amsterdam; Boston; London: Butterworth-Heinemann. 
Weaver, D.B and Lawton, L .J. (2007). "Twenty years on: The state of contemporary ecotourism research”, Tourism Management No.28, 1168- 1179.

Weaver, D. and Lawton, L. (1999). "Sustainable Tourism: A critical Analysis”, Cooperative Research Center for Sustainable Tourism, Research report series.

Weaver, D.B. (1999).” Magnitude of Ecotourism in Costa Rica and Kenya”, Annals of tourism research.No.4: 792-816.

Wester, L. and Youngvanit, S. (2005).” Farmers, foresters and forest temples: Conservation in the Dong Mun uplands, Northeast Thailand” Geo forum No.36: 735749 .

Williams, D.E. (2007). Sustainable Design, Published by John Wiley \& Sons, Inc., Hoboken, New Jersey.

Wolf, A.T. (2011). "Sustainability Trends and Innovation in Glass and Glazing". http://ebookbrowse.com/sustainability-driven-trends-and-innovation-in-glassand-glazing-pdf-d142015576

Wood, E.M. (2002). "Ecotourism: principles, practices \& policies for sustainability”. UNDP, Production and consumption Unit Tour Mirabeau: 39-43.

World Bank (2010). "Safer Homes, Stronger Communities: A handbook for Reconstructing after Natural disaster: Chapter 11, Cultural Heritage conservation”. www.housingreconstruction.org

World Commission on Environment and Development (WCED). (1987). "Brundtland Report”. Brundtland Commission.

World Commission on Culture and Development (1996). “Our Creative Diversity”. WCOCAD, Paris. http://unesdoc.unesco.org. 


\begin{tabular}{|c|c|}
\hline $\begin{array}{c}\text { Priority } \\
\text { No. }\end{array}$ & Action needs to do to achieve priorities \\
\hline \multirow{4}{*}{1} & $\begin{array}{l}\text { Invest in strengthening New Zealand's global marketing campaign with more funding } \\
\text { for } \\
\text { Tourism New Zealand }\end{array}$ \\
\hline & $\begin{array}{l}\text { Develop marketing initiatives that focus on changing consumer attitudes and } \\
\text { expectations } \\
\text { in China and other new markets }\end{array}$ \\
\hline & $\begin{array}{l}\text { Coordinate the investment in overseas marketing (public and private) to improve the } \\
\text { conversion of preference to travel to actual travel. }\end{array}$ \\
\hline & $\begin{array}{l}\text { Make sure that the unique elements of Maori culture are reflected in the way New } \\
\text { Zealand's } \\
\text { brand is positioned internationally, and in national and regional product development }\end{array}$ \\
\hline \multirow[t]{3}{*}{2} & $\begin{array}{l}\text { Monitor how and where international and domestic visitors make their bookings, so } \\
\text { that we } \\
\text { invest in the most effective distribution channels, and educate tourism operators about } \\
\text { how } \\
\text { to get the most out of those channels }\end{array}$ \\
\hline & $\begin{array}{l}\text { Make sure that tourism operators invest in the technology they need to best manage } \\
\text { their } \\
\text { bookings and inventory }\end{array}$ \\
\hline & $\begin{array}{l}\text { Make sure that quality Maori tourism products and services are better integrated } \\
\text { throughout } \\
\text { the full range of wholesale and retail distribution channels }\end{array}$ \\
\hline \multirow{2}{*}{3} & $\begin{array}{l}\text { Build on the common business and information systems in the i-SITE network to } \\
\text { make sure } \\
\text { that i-SITEs across the country offer a consistent experience }\end{array}$ \\
\hline & $\begin{array}{l}\text { Carry out research into ways in which the I-Site network could be used to help } \\
\text { improve } \\
\text { visitor experiences. This would build on previous research into the information needs } \\
\text { of I-Site users }\end{array}$ \\
\hline \multirow{3}{*}{4} & $\begin{array}{l}\text { Broaden Qualmark's coverage by adding new categories into the existing range of } \\
\text { gradings } \\
\text { and endorsements, and increasing the uptake of Qualmark by tourism operators }\end{array}$ \\
\hline & $\begin{array}{l}\text { Make sure that Qualmark's assessment systems are consistently applied, and are } \\
\text { recognised } \\
\text { and supported by the tourism sector }\end{array}$ \\
\hline & $\begin{array}{l}\text { Help domestic and international consumers get a better understanding of the Qualmark } \\
\text { grading system, so that they feel more confident about selecting Qualmark-endorsed } \\
\text { products, and they know what these services will deliver }\end{array}$ \\
\hline \multirow{3}{*}{5} & $\begin{array}{l}\text { Enhance the quality of the accuracy of the International Visitor Survey and Domestic } \\
\text { Tourism Survey }\end{array}$ \\
\hline & $\begin{array}{l}\text { Integrate all the research about visitor satisfaction into one common platform or } \\
\text { database }\end{array}$ \\
\hline & $\begin{array}{l}\text { Extend the Regional Visitor Monitor (RVM) into more regions, and expand the focus } \\
\text { of both } \\
\text { the RVM and the Domestic Tourism Survey to provide information about buying } \\
\text { behaviour } \\
\text { and what drives travel trends }\end{array}$ \\
\hline \multirow[t]{2}{*}{6} & $\begin{array}{l}\text { Make sure that there is enough investment in the infrastructure needed to meet } \\
\text { growing } \\
\text { visitor demand. This includes roads, the availability of broadband, especially in more } \\
\text { rural } \\
\text { and remote areas, water supplies, managing waste water, public toilets, signage, and } \\
\text { car parks }\end{array}$ \\
\hline & $\begin{array}{l}\text { Make sure that airport authorities and border control agencies have the information } \\
\text { they } \\
\text { need to plan for tourism growth so that they have the staff and resources they need to } \\
\text { cope }\end{array}$ \\
\hline
\end{tabular}




\begin{tabular}{|c|c|}
\hline & at peak times \\
\hline & $\begin{array}{l}\text { Investigate the case for developing national facilities such as a national convention } \\
\text { centre } \\
\text { and cruise-ship facilities }\end{array}$ \\
\hline \multirow[b]{2}{*}{7} & Establish a domestic tourism working group to develop a domestic tourism plan \\
\hline & $\begin{array}{l}\text { Encourage and support Regional Tourism Organisations to communicate with } \\
\text { stakeholders } \\
\text { the importance of regional campaigns in driving the domestic market and creating } \\
\text { opportunities to increase domestic travel }\end{array}$ \\
\hline \multirow{5}{*}{8} & Keep building business capability through the Maori Business Facilitation Service \\
\hline & $\begin{array}{l}\text { Increase the number of Maori tourism businesses involved in quality accreditation } \\
\text { schemes } \\
\text { such as Qualmark and Toi tho }\end{array}$ \\
\hline & $\begin{array}{l}\text { Help tourism businesses to incorporate a Maori dimension into the products and } \\
\text { services } \\
\text { they provide }\end{array}$ \\
\hline & $\begin{array}{l}\text { Increase the range of Maori products and services being offered to the international } \\
\text { and } \\
\text { domestic markets }\end{array}$ \\
\hline & $\begin{array}{l}\text { Strengthen relationships between Regional Tourism Organisations and Maori Regional } \\
\text { Tourism Organisations to maximise the development of a Maori tourism product that } \\
\text { reinforces regional differentiation and meets visitor demand }\end{array}$ \\
\hline
\end{tabular}

\begin{tabular}{|c|c|}
\hline \multicolumn{2}{|c|}{$\begin{array}{l}\text { Apendix2: NZST 2015- forecasted activity need to do to achieve the anticipated priorities (cited in } \\
\text { Table 2. 3). }\end{array}$} \\
\hline $\begin{array}{l}\text { Priority } \\
\text { NO. }\end{array}$ & Action needs to do to achieve priorities \\
\hline \multirow{5}{*}{1} & $\begin{array}{l}\text { Improve resource efficiencies, workforce management, and profitability } \\
\text { opportunities for all businesses through more clustering, joint ventures, and other } \\
\text { industry cooperation initiatives. }\end{array}$ \\
\hline & $\begin{array}{l}\text { Encourage the hotel industry, inbound tour operators, and airlines to work together } \\
\text { on things such as pricing, seasonal campaigns, and the way products are packaged } \\
\text { to increase occupancy rates and profitability. }\end{array}$ \\
\hline & $\begin{array}{l}\text { Streamline and improve the focus of existing business-assistance programmes to } \\
\text { help operators increase their return on investment and develop quality products. }\end{array}$ \\
\hline & $\begin{array}{l}\text { Build management capability by identifying and developing staff to undertake } \\
\text { effective management training programmes. }\end{array}$ \\
\hline & $\begin{array}{l}\text { Make sure that tourism operators and destinations get better access to broadband } \\
\text { services when the New Zealand Digital Strategy is being implemented. }\end{array}$ \\
\hline \multirow{4}{*}{2} & Target those markets that are likely to spend more than average. \\
\hline & $\begin{array}{l}\text { Develop existing products and services so that they increase the value of the visitor } \\
\text { experience and encourage higher levels of spending. }\end{array}$ \\
\hline & $\begin{array}{l}\text { Provide better service delivery and interpretation, including on-site interpretation at } \\
\text { visitor attractions. }\end{array}$ \\
\hline & $\begin{array}{l}\text { Ensure businesses have access to research and other market intelligence to assist } \\
\text { them in their product development. }\end{array}$ \\
\hline \multirow{3}{*}{3} & $\begin{array}{l}\text { Implement the Tourism and Hospitality Workforce Strategy by: } \\
\text { Making sure the tourism sector has a strong voice with labour, education and } \\
\text { training policy makers. } \\
\text { Identifying and promoting opportunities for the sector to increase its productivity. }\end{array}$ \\
\hline & $\begin{array}{l}\text { Get more benefits from education and training through an active partnership with } \\
\text { the education and training sector. This should be underpinned by closer links } \\
\text { between businesses, providers of training, and industry-training organisations. The } \\
\text { industry also needs to coordinate its input to the Tertiary Education Commission } \\
\text { reviews. }\end{array}$ \\
\hline & $\begin{array}{l}\text { Identify opportunities to make the best use of people, including: } \\
\text { • investing in technology that improves business efficiency and means that } \\
\text { employees are engaged in the most productive activities. } \\
\text { - developing visitor experiences that are less labour-intensive but do not } \\
\text { compromise quality. }\end{array}$ \\
\hline
\end{tabular}




\begin{tabular}{|c|c|}
\hline & $\begin{array}{l}\text { Seek targeted immigration to fill critical skill gaps and provide workers during the } \\
\text { peak period. This will require coordination within the industry, and cooperation } \\
\text { with the } \\
\text { Department of Labour. }\end{array}$ \\
\hline & $\begin{array}{l}\text { Develop ways for tourism businesses to monitor labour productivity and improve } \\
\text { the contribution that their people make to the performance of their business. }\end{array}$ \\
\hline \multirow{4}{*}{4} & Bid for, and secure, major international events, which will help reduce seasonality. \\
\hline & $\begin{array}{l}\text { Coordinate existing conference and incentive marketing activities to increase the } \\
\text { performance in this market. }\end{array}$ \\
\hline & $\begin{array}{l}\text { Invest in marketing initiatives that target those domestic and international visitors } \\
\text { who are more likely to travel in the shoulder and off-peak seasons. }\end{array}$ \\
\hline & $\begin{array}{l}\text { Identify and develop products that are likely to increase travel during the shoulder } \\
\text { and off- peak seasons. Examples include Matariki and the Pacifica Festival. } \\
\text { Products with all-weather options are another possibility. }\end{array}$ \\
\hline \multirow{4}{*}{5} & $\begin{array}{l}\text { Develop and fund an industry-government partnership model to advance sector } \\
\text { research, including determining priority research and then directing, funding, and } \\
\text { managing the delivery of this research. }\end{array}$ \\
\hline & $\begin{array}{l}\text { Establish quality measures for the Core Tourism Data Set and develop and } \\
\text { implement ways of achieving the agreed quality standards. }\end{array}$ \\
\hline & $\begin{array}{l}\text { Make more sectoral and regional data available to the sector through: } \\
\text { - further analysis of existing data sources } \\
\text { • collecting additional data through industry partnerships. }\end{array}$ \\
\hline & $\begin{array}{l}\text { Establish a set of measures to track performance in the sector, including financial } \\
\text { performance, managing seasonality, and increasing visitor satisfaction. }\end{array}$ \\
\hline
\end{tabular}

\begin{tabular}{|c|c|}
\hline \multicolumn{2}{|r|}{$\begin{array}{c}\text { Apendix3: NZST 2015- forecasted activity need to do to achieve anticipated priorities (cited in } \\
\text { Table } 2.4 \text { ). }\end{array}$} \\
\hline Priority & Action needs to do to achieve priorities \\
\hline NO. & \\
\hline \multirow{5}{*}{1} & $\begin{array}{l}\text { Seek the completion of a national environmental management plan to clearly articulate } \\
\text { priorities for all of New Zealand by December } 2008 \text {. }\end{array}$ \\
\hline & $\begin{array}{l}\text { Understand and use the value of kaitiakitanga (guardianship) as the basis for the tourism } \\
\text { sector's actions to enhance the environment. }\end{array}$ \\
\hline & $\begin{array}{l}\text { Participate in and influence global forums and agreements on environmental issues that } \\
\text { affect New Zealand tourism, and implement their requirements. }\end{array}$ \\
\hline & $\begin{array}{l}\text { Both advocate for and actively support stronger environmental policies, standards and } \\
\text { regulations throughout New Zealand. }\end{array}$ \\
\hline & $\begin{array}{l}\text { Develop indicators for the sector (at both a national and operator level) to measure, } \\
\text { manage, and monitor environmental impacts, and use these to evaluate performance } \\
\text { over time. }\end{array}$ \\
\hline \multirow{3}{*}{2} & $\begin{array}{l}\text { Understand the environmental aspirations of our current and future international visitors, } \\
\text { and how they view New Zealand's environmental management. }\end{array}$ \\
\hline & $\begin{array}{l}\text { Use this information in marketing and to develop tourism products, targeting visitors } \\
\text { who support New Zealand's environmental values and whose ethics, behaviour, and } \\
\text { impacts align with those values. }\end{array}$ \\
\hline & $\begin{array}{l}\text { Help consumers to make informed product choices that align with their environmental } \\
\text { values by using environmental ratings and labelling, and making consumers aware of } \\
\text { environmental accreditation schemes. }\end{array}$ \\
\hline \multirow[b]{2}{*}{3} & $\begin{array}{l}\text { Make sure that tourism vehicle, aircraft, and boat fleets are New Zealand's most } \\
\text { fuel-efficient and produce the lowest emissions possible by, for example: } \\
\text { - using transport fuels that contain the highest available proportion of bio-fuels, } \\
\text { and encouraging visitors to do the same } \\
\text { - buying vehicles for commercial and rental fleets that are the most fuel-efficient for } \\
\text { their } \\
\text { intended use, such as diesel or hybrid vehicles } \\
\text { - buying Euro IV standard engines for diesel transport fleets, and Euro V standard } \\
\text { engines once compatible fuel is introduced on } 1 \text { January } 2009 \text {. }\end{array}$ \\
\hline & $\begin{array}{l}\text { Improve the fuel efficiency of operators and visitors by, for example: } \\
\text { - adopting best-practice standards for fuel-efficient fleet management. This includes } \\
\text { engine maintenance, tyre pressure, and reducing visible smoke emissions }\end{array}$ \\
\hline
\end{tabular}




\begin{tabular}{|c|c|}
\hline & $\begin{array}{l}\text { - recommending the most fuel-efficient transport to visitors to meet their travel needs } \\
\text { - educating operators and visitors about driving to improve fuel efficiency. }\end{array}$ \\
\hline & $\begin{array}{l}\text { Work with Air New Zealand and other airlines to develop options that allow tourists to } \\
\text { reduce and/or offset the carbon emissions they generate by flying to New Zealand. }\end{array}$ \\
\hline & $\begin{array}{l}\text { Support government initiatives to adopt and enforce emissions testing and minimum } \\
\text { emissions standards for importing vehicles into New Zealand. }\end{array}$ \\
\hline & $\begin{array}{l}\text { Promote the use of public transport, rail, coaches, cycling, and walking as } \\
\text { environmentally friendly modes of transport. Advocate for and support the development } \\
\text { of the services and infrastructure needed to make these forms of transport possible. }\end{array}$ \\
\hline \multirow{3}{*}{4} & $\begin{array}{l}\text { Work with organisations such as the Energy Efficiency and Conservation Authority on } \\
\text { initiatives to improve environmental management in tourism and to implement the goals } \\
\text { of the New Zealand Energy Efficiency and Conservation Strategy. }\end{array}$ \\
\hline & $\begin{array}{l}\text { Make sure new buildings and retrofits use the highest possible energy-efficient building } \\
\text { standards, and that eco-verified procurement criteria are used when buying products and } \\
\text { appliances. }\end{array}$ \\
\hline & $\begin{array}{l}\text { Adopt energy-management practices and new technologies to reduce overall energy } \\
\text { consumption. This includes using timers, thermostats, occupancy sensors, and } \\
\text { renewable and local sources of energy wherever possible. }\end{array}$ \\
\hline \multirow[b]{2}{*}{5} & $\begin{array}{l}\text { Tourism operators must regularly audit the amount of waste they produce, take steps to } \\
\text { reduce, reuse, and recycle waste, and provide facilities that encourage visitors to do the } \\
\text { same. }\end{array}$ \\
\hline & $\begin{array}{l}\text { Work with local government to develop appropriate facilities, standards, and planning } \\
\text { for waste management. Examples include public recycling in all main centres, } \\
\text { developing policies on freedom camping, and preparing regional waste strategies. }\end{array}$ \\
\hline \multirow[t]{2}{*}{6} & $\begin{array}{l}\text { Encourage tourism operators to use the environmental elements in Qualmark to lift their } \\
\text { environmental performance, and look at ways of increasing the uptake of these elements } \\
\text { across the sector. }\end{array}$ \\
\hline & $\begin{array}{l}\text { Work with Qualmark or other service providers to build operator capability in delivering } \\
\text { environmentally sustainable products and services. }\end{array}$ \\
\hline \multirow{5}{*}{7} & $\begin{array}{l}\text { Take an early interest in the development of national park management plans, } \\
\text { conservation management strategies, reserve management plans, marine plans, and other } \\
\text { related processes, to make sure that they are developed in a collaborative, proactive style } \\
\text { and address the interests and needs of operators, visitors, and local communities. }\end{array}$ \\
\hline & $\begin{array}{l}\text { Make sure that all DOC }{ }^{1} \text { visitor monitoring and evaluation is carried out in a consistent } \\
\text { manner, and that the data is used to establish appropriate management objectives and } \\
\text { shared with the relevant tourism agencies. }\end{array}$ \\
\hline & $\begin{array}{l}\text { Look at developing visitor services and facilities, such as walking and mountain biking } \\
\text { tracks and interpretation centres, in places where they can provide social, cultural, } \\
\text { economic, and conservation benefits. }\end{array}$ \\
\hline & $\begin{array}{l}\text { Investigate potential funding mechanisms for visitors to contribute towards adding value } \\
\text { to the visitor experience and the provision of facilities and services on lands and waters } \\
\text { managed for the benefit of the public. }\end{array}$ \\
\hline & $\begin{array}{l}\text { Manage issues at key sites where important values (environmental, social, or cultural) } \\
\text { are perceived to be under pressure environmentally and/or socially. }\end{array}$ \\
\hline \multirow{3}{*}{8} & $\begin{array}{l}\text { Develop products that make it possible for visitors to reduce their environmental } \\
\text { footprint and leave New Zealand 'better than they found it'. Possibilities include } \\
\text { conservation projects that encourage visitor interaction, such as ecological restoration, } \\
\text { pest eradication, or investment in renewable energy. }\end{array}$ \\
\hline & $\begin{array}{l}\text { Make sure all visitors and tourism operators have the opportunity to participate in } \\
\text { verified carbon offsetting programmes for any remaining emissions. }\end{array}$ \\
\hline & $\begin{array}{l}\text { Develop national guidelines for running major events within New Zealand that } \\
\text { incorporate } \\
\text { environmental requirements into any government-funded events. }\end{array}$ \\
\hline & OC: Department Of Conservation \\
\hline
\end{tabular}




\begin{tabular}{|c|c|}
\hline \multicolumn{2}{|r|}{$\begin{array}{l}\text { Apendix4: NZST 2015- forecasted activity need to do to achieve anticipated priorities (cited in } \\
\text { Table5). }\end{array}$} \\
\hline $\begin{array}{l}\text { Priority } \\
\text { NO. }\end{array}$ & Action needs to do to achieve priorities \\
\hline \multirow{4}{*}{1} & $\begin{array}{l}\text { Engage with communities at a strategic level through increased engagement at the } \\
\text { governance level — for example, through community boards and councils. }\end{array}$ \\
\hline & $\begin{array}{l}\text { Contribute to local government processes by providing coordinated comments and } \\
\text { submissions on, for example: } \\
\text { - district plans } \\
\text { - long-term council community plans } \\
\text { - annual business plans } \\
\text { - regional tourism } \\
\text { - destination-management or economic development strategies } \\
\text { - Resource Management Act processes. }\end{array}$ \\
\hline & $\begin{array}{l}\text { Use the best available advice and information, such as Have Your Say, published by the } \\
\text { Tourism Industry Association, to participate in local government processes. }\end{array}$ \\
\hline & $\begin{array}{l}\text { Work with local authorities to increase the value that tourism brings to their } \\
\text { communities and to manage and mitigate any undesirable effects. }\end{array}$ \\
\hline \multirow{6}{*}{2} & $\begin{array}{l}\text { Refine and promote the tools and statistical resources that now exist to help local } \\
\text { authorities to plan for, invest in, and manage tourism. }\end{array}$ \\
\hline & $\begin{array}{l}\text { Prepare tourism strategies or destination-management plans that establish what strategic } \\
\text { directions, management, infrastructure, and product development are needed and what } \\
\text { the community outcomes of tourism will be. }\end{array}$ \\
\hline & Review 'Postcards From Home’, the Local Government New Zealand Tourism Strategy. \\
\hline & $\begin{array}{l}\text { Take part in national decision-making on tourism issues, such as strategy development, } \\
\text { policy, and funding. }\end{array}$ \\
\hline & $\begin{array}{l}\text { Keep strengthening the governance, management, and operations of Regional Tourism } \\
\text { Organisations through wider uptake of the Roles and Guidelines for Tourism } \\
\text { Organisations advice produced as a result of the New Zealand Tourism Strategy } 2010 .\end{array}$ \\
\hline & $\begin{array}{l}\text { Provide certainty of funding to Regional Tourism Organisations and i-SITEs through a } \\
\text { more proactive use of the range of funding tools currently available. }\end{array}$ \\
\hline \multirow{3}{*}{3} & $\begin{array}{l}\text { Make sure that high-quality research is available to local communities to inform } \\
\text { decision-making by local government and the tourism sector, including information on } \\
\text { the social, economic, environmental, and cultural benefits of tourism to communities. }\end{array}$ \\
\hline & $\begin{array}{l}\text { Research affordability issues for domestic tourism, including expectations, price } \\
\text { sensitivities, and pricing mitigation options, to inform business positioning, product } \\
\text { differentiation, and market segmentation decisions. }\end{array}$ \\
\hline & $\begin{array}{l}\text { Research the perceptions and experiences of crowding at times of peak capacity from a } \\
\text { community perspective. This includes assessment of impacts on communities and visitor } \\
\text { satisfaction, case studies, management options, and tools for local authorities to use. } \\
\text { This should sit alongside work previously undertaken by DOC in relation to capacity } \\
\text { issues on the conservation estate. }\end{array}$ \\
\hline \multirow{3}{*}{4} & $\begin{array}{l}\text { Strengthen existing events and set up new events and products that promote regional } \\
\text { identity and differentiation. }\end{array}$ \\
\hline & $\begin{array}{l}\text { Build the capability of the i-SITE network to promote local culture, and build } \\
\text { connections with the local tangata whenua and with the iconic landscape of each region. }\end{array}$ \\
\hline & $\begin{array}{l}\text { Communicate and promote the concept of manaakitanga and its importance in hosting } \\
\text { both domestic and international visitors. }\end{array}$ \\
\hline 5 & $\begin{array}{l}\text { Assess core needs, and investigate and consider funding models to improve the standard } \\
\text { and provision of appropriate infrastructure. }\end{array}$ \\
\hline
\end{tabular}




\begin{tabular}{|c|c|c|c|c|c|c|c|c|c|}
\hline \multicolumn{10}{|c|}{ Appendix.5: OCRT-Accommodation Facilities, Area and the Used Materials (2011) } \\
\hline \multicolumn{10}{|c|}{$\begin{array}{l}\text { 1. } \mathrm{RB}=\text { Restored and Historic } \\
\text { 2. } \mathrm{SC}=\text { Self-Contained } \\
\text { 3. } \mathrm{Bp}=\text { Backpacker } \\
\text { 4. } \mathrm{B} \& \mathrm{~B}=\text { Bed and breakfast } \\
\text { 5. N/A information not available }\end{array}$} \\
\hline \multirow[b]{2}{*}{ NU } & \multirow[b]{2}{*}{ Location } & \multirow[b]{2}{*}{ Accommodation } & \multirow[b]{2}{*}{ Type } & \multirow[b]{2}{*}{ Quality } & \multicolumn{2}{|l|}{ Facility } & \multirow[b]{2}{*}{ Products and activities } & \multirow[b]{2}{*}{ Area } & \multirow[b]{2}{*}{ Materials } \\
\hline & & & & & Common area & Bedrooms & & & \\
\hline 1 & Middlemarch & Annandale & $1 \times B \& B$ & New & $\begin{array}{l}\text { Writing area, guest living } \\
\text { room, TV, a Victorian } \\
\text { fireplace, a heat pump, log } \\
\text { burner and HRV } \\
\text { ventilation on cooler days. }\end{array}$ & $\begin{array}{l}\text { All bedrooms with bathroom } \\
\text { with shower and bath, tea and } \\
\text { coffee making facilities, heaters, } \\
\text { electric blankets and lighting. }\end{array}$ & $\begin{array}{l}\text { Breakfast, homemade } \\
\text { preserves and jams, } \\
\text { OCRT, Middlemarch } \\
\text { city, views of the Rock } \\
\text { and Pillar Range, } \\
\text { productive garden, } \\
\text { reading materials. }\end{array}$ & & Timber \\
\hline 2 & Middlemarch & $\begin{array}{l}\text { Blind Billy’s Holiday } \\
\text { Camp (3motel units) }\end{array}$ & $3 \times$ Motel & New & $\begin{array}{l}\text { Each of } 3 \text { units has full } \\
\text { equipped kitchen, } \\
\text { bathroom and toilet, tea } \\
\text { and coffee making } \\
\text { facilities. }\end{array}$ & $\begin{array}{l}\text { All bedrooms with heating and } \\
\text { lighting. }\end{array}$ & \multirow{4}{*}{$\begin{array}{l}\text { OCRT, Middlemarch } \\
\text { city and its products } \\
\text { and attractions, Sutton } \\
\text { Salt Lake, a tour of the } \\
\text { nearby Oceania Gold } \\
\text { open-cast mine at } \\
\text { Macraes, walks up the } \\
\text { Rock \& Pillar Range, } \\
\text { landscape views, } \\
\text { historical sites and } \\
\text { buildings, bike hire, } 18 \\
\text { cultural events listed } \\
\text { in table 1, golf course, } \\
\text { horse riding, fishing, } \\
\text { curling at Naseby, } \\
\text { gold mining. }\end{array}$} & & Timber \\
\hline 3 & Middlemarch & $\begin{array}{l}\text { Blind Billy's Holiday } \\
\text { Camp (2 studio units) }\end{array}$ & $2 \times \mathrm{SC}$ & New & $\begin{array}{l}\text { Each of } 3 \text { units has full } \\
\text { equipped kitchen, } \\
\text { bathroom and toilet, tea } \\
\text { and coffee making } \\
\text { facilities. }\end{array}$ & $\begin{array}{l}\text { All bedrooms with heating and } \\
\text { lighting. }\end{array}$ & & & Timber \\
\hline 4 & Middlemarch & $\begin{array}{l}\text { Blind Billy's Holiday } \\
\text { Camp (7 tourist } \\
\text { cabins) }\end{array}$ & $7 \times \mathrm{SC}$ & New & $\begin{array}{l}\text { Each cabin has bedding, } \\
\text { tea and coffee making } \\
\text { facilities. }\end{array}$ & $\begin{array}{l}\text { Each cabin has heater and } \\
\text { lighting. }\end{array}$ & & & Timber \\
\hline 5 & Middlemarch & $\begin{array}{l}\text { Blind Billy’s Holiday } \\
\text { Camp ( } 3 \text { backpacker } \\
\text { cabins) }\end{array}$ & $3 \times B p$ & New & $\begin{array}{l}\text { Using communal services } \\
\text { in the camp including } \\
\text { children's playground, } \\
\text { free gas BBQ, free } \\
\text { laundry facilities (staff use } \\
\text { only), toilet block and }\end{array}$ & $\begin{array}{l}\text { Each cabin has heater and } \\
\text { lighting. }\end{array}$ & & & Timber \\
\hline
\end{tabular}




\begin{tabular}{|c|c|c|c|c|c|c|c|c|}
\hline & & & & & shower facilities. & & & \\
\hline 6 & Middlemarch & $\begin{array}{l}\text { Blind Billy’s Holiday } \\
\text { Camp( On-site } \\
\text { caravans) }\end{array}$ & $1 \times$ Caravan/camping & New & $\begin{array}{l}\text { Fully equipped rail } \\
\text { carriage kitchen, sun deck } \\
\text { dining, children’s } \\
\text { playground, free gas } \\
\text { BBQ, free laundry } \\
\text { facilities (staff use only), } \\
\text { toilet block and shower } \\
\text { facilities. }\end{array}$ & $\begin{array}{l}\text { Caravans with [heating] and } \\
\text { lighting. }\end{array}$ & & - \\
\hline 8 & Middlemarch & Cottesbrook & $2 \times$ SC-cottages & $\mathrm{RB}$ & $\begin{array}{l}\text { Each of } 3 \text { bedrooms } \\
\text { cottage has its own gas- } \\
\text { heated, shower and toilet, } \\
\text { full equipped kitchen, } \\
\text { lounge, laundry facility, } \\
\text { verandah and log burner. } \\
\text { One cottage has a shared } \\
\text { TV. }\end{array}$ & All bedrooms with lighting. & $\begin{array}{l}\text { Continental breakfast, } \\
\text { surrounding private } \\
\text { farm, mature gardens, } \\
\text { OCRT, Taieri river, } \\
\text { walkway up to the } \\
\text { nearby Rock and Pillar } \\
\text { range and walk to } \\
\text { Sutton Salt lake, } \\
\text { wildlife watching, } \\
\text { fishing, canoeing, } \\
\text { swimming, golf } \\
\text { course, farm tracks, } \\
\text { local shuttle service. }\end{array}$ & Timber \\
\hline 9 & Middlemarch & Gladbrook & $1 \times \mathrm{B} \& \mathrm{~B}$ & $\mathrm{RB}$ & $\begin{array}{l}2 \text { shared bathrooms, } \\
\text { sitting room, dining room, } \\
\text { verandah and open fire. }\end{array}$ & $\begin{array}{l}\text { All } 5 \text { bedrooms have electric } \\
\text { blankets, hairdryer and lighting. }\end{array}$ & $\begin{array}{l}\text { Breakfast, OCRT, } \\
\text { Middlemarch city, } \\
\text { historic area, garden, } \\
\text { walk up the Rock and } \\
\text { Pillar range and the } \\
\text { Sutton Salt lake, sheep } \\
\text { mustering activity, }\end{array}$ & Timber \\
\hline
\end{tabular}




\begin{tabular}{|c|c|c|c|c|c|c|c|c|}
\hline & & & & & & & $\begin{array}{l}\text { vistas of Strath Taieri, } \\
\text { reading materials, } \\
\text { tennis, croquet. }\end{array}$ & \\
\hline 10 & Middlemarch & Gladbrook (cottage) & $1 \times \mathrm{SC}-$ & $\mathrm{RB}$ & \multirow{2}{*}{$\begin{array}{l}\text { Both cottage and hut have } \\
\text { bathroom comprising } \\
\text { toilet and shower, } \\
\text { equipped kitchen } \\
\text { including fridge/freezer, } \\
\text { separate freezer, stove and } \\
\text { oven, tea and coffee } \\
\text { supplied, toaster, TV, } \\
\text { radio, laundry tub, } \\
\text { washing machine, ironing } \\
\text { equipment, BBQ and } \\
\text { fireplace. }\end{array}$} & \multirow{2}{*}{$\begin{array}{l}\text { Each room has electric blankets } \\
\text { (in cottage), hot water bottle (in } \\
\text { hut), heater and lighting. }\end{array}$} & \multirow{2}{*}{$\begin{array}{l}\text { Food (free range eggs } \\
\text { from owners’ hens, } \\
\text { bread and butter and } \\
\text { milk), continental } \\
\text { breakfast, OCRT, } \\
\text { Middlemarch city, } \\
\text { historic area, garden, } \\
\text { walk up the Rock and } \\
\text { Pillar range and the } \\
\text { Sutton Salt lake, sheep } \\
\text { mustering activity, } \\
\text { vistas of Strath Taieri, } \\
\text { books/library, small } \\
\text { conferences, wedding } \\
\text { and Christmas party. }\end{array}$} & Timber \\
\hline 11 & Middlemarch & Gladbrook (hut) & $1 \times \mathrm{SC}-$ & $\mathrm{RB}$ & & & & Timber \\
\hline 12 & Middlemarch & Jack’s Stone Cottage & $1 \times \mathrm{SC}$ & New & $\begin{array}{l}\text { Bathroom and toilet, fully } \\
\text { equipped kitchen, lounge, } \\
\text { TV, DVD player, laundry } \\
\text { facilities, log burner, on } \\
\text { cooler nights. }\end{array}$ & $\begin{array}{l}\text { Both bedrooms with electric } \\
\text { blankets and lighting. }\end{array}$ & $\begin{array}{l}\text { Continental breakfast, } \\
\text { OCRT, Middlemarch } \\
\text { city, planted garden, } \\
\text { fishing rods and golf } \\
\text { clubs (left and right } \\
\text { handed). }\end{array}$ & Schist stone \\
\hline 13 & Middlemarch & Middlemarch B\&B & $1 \times B \& B$ & New & $\begin{array}{l}\text { Shared bath room, } \\
\text { outdoor-indoor living, } \\
\text { garden room, log burner, } \\
\text { TV, }\end{array}$ & $\begin{array}{l}\text { All } 3 \text { rooms have electric } \\
\text { blankets, wireless internet, and } \\
\text { lighting. }\end{array}$ & $\begin{array}{l}\text { Home baking, } \\
\text { continental breakfast, } \\
\text { wine, OCRT, } \\
\text { Middlemarch city, } \\
\text { views of the Rock and } \\
\text { Pillar Range, } \\
\text { surrounding private } \\
\text { garden (1.2 acre). }\end{array}$ & $\begin{array}{l}\text { Timber, stone } \\
\text { and concrete }\end{array}$ \\
\hline 14 & Middlemarch & $\begin{array}{l}\text { Pukenangi Country } \\
\text { Retreat }\end{array}$ & $1 \times \mathrm{SC}$ & $\mathrm{RB}$ & $\begin{array}{l}\text { Kitchen, parlour, } \\
\text { fireplace. }\end{array}$ & $\begin{array}{l}\text { Each room has bathroom, TV, } \\
\text { hair dryer, under floor heating, } \\
\text { wall heater, electric blankets, } \\
\text { and heated towel rail. }\end{array}$ & $\begin{array}{l}\text { Packaged foods, } \\
\text { cooked or continental } \\
\text { breakfast, wine and } \\
\text { beer, OCRT, } \\
\text { Middlemarch city, tour } \\
6000 \text { hectare Bare }\end{array}$ & Stone- timber \\
\hline
\end{tabular}




\begin{tabular}{|c|c|c|c|c|c|c|c|c|}
\hline & & & & & & & $\begin{array}{l}\text { Wood Station, watch } \\
\text { farming demonstration } \\
\text { appropriate to the } \\
\text { season, visit Sutton } \\
\text { Salt lake, local } \\
\text { transport service along } \\
\text { the OCRT way, } \\
\text { booking services } \\
\text { including shuttles, } \\
\text { bike hire, } \\
\text { accommodation, bag } \\
\text { transfer, Taieri Gorge } \\
\text { Railway. }\end{array}$ & \\
\hline 15 & Middlemarch & Rose Cottage B\&B & $1 \times \mathrm{B} \& \mathrm{~B}$ & New & $\begin{array}{l}\text { A shared bathroom with } \\
\text { gas heated shower, } \\
\text { outdoor seating, TV, BBQ } \\
\text { facilities, a fridge with } \\
\text { chilled rainwater, tea and } \\
\text { coffee making facilities. }\end{array}$ & $\begin{array}{l}\text { An individual bathroom with } \\
\text { gas heated shower, a private } \\
\text { verandah, hair dryer, heating, } \\
\text { electric blankets, lighting. }\end{array}$ & $\begin{array}{l}\text { Food and beverages, } \\
\text { OCRT, Middlemarch } \\
\text { city, Middlemarch } \\
\text { museum, visit } \\
\text { historical heritage, } \\
\text { rural garden, books } \\
\text { and magazine, in } \\
\text { conjunction with Mt } \\
\text { Ida adventure tour, } \\
\text { tennis racquets and } \\
\text { balls, petanque, golf } \\
\text { clubs and mountain } \\
\text { bikes, fishing, } \\
\text { walking, visiting wild } \\
\text { life watching, } \\
\text { landscape painting and } \\
\text { photography, cafe } \\
\text { culture, local } \\
\text { transportation and bag } \\
\text { transfers between } \\
\text { accommodation } \\
\text { places. }\end{array}$ & Timber \\
\hline 16 & Middlemarch & Strathmore B\&B & $1 \times \mathrm{B} \& \mathrm{~B}$ & $\mathrm{RB}$ & $\begin{array}{l}\text { A shared bathroom with } \\
\text { under floor heating, BBQ, } \\
\text { outdoor sitting, in ground }\end{array}$ & $\begin{array}{l}\text { TV, coffee tea making } \\
\text { facilities, heating and lighting. }\end{array}$ & $\begin{array}{l}\text { Country style cooked } \\
\text { breakfast, OCRT, } \\
\text { Middlemarch city, }\end{array}$ & Stone \\
\hline
\end{tabular}




\begin{tabular}{|c|c|c|c|c|c|c|c|c|}
\hline & & & & & swimming pool. & & $\begin{array}{l}\text { visit historical } \\
\text { heritage, local } \\
\text { transportation along } \\
\text { the OCRT. }\end{array}$ & \\
\hline 17 & Middlemarch & Strath Taieri Hotel & $1 \times$ Hotel & $\mathrm{RB}$ & $\begin{array}{l}3 \text { shared bathrooms, } 2 \\
\text { showers, laundry, guest } \\
\text { lounge, } 2 \text { bars with } \\
\text { outdoor sitting ATM cash } \\
\text { machine. }\end{array}$ & $\begin{array}{l}\text { Each room has tea making } \\
\text { facilities, heater and lighting. }\end{array}$ & $\begin{array}{l}\text { Food and beverages, } \\
\text { OCRT, Middlemarch } \\
\text { city, historic sites, } \\
\text { landscape views. }\end{array}$ & Schist stone \\
\hline 18 & Middlemarch & The Farm Homestay & $1 \times$ Homestay & $\mathrm{RB}$ & $\begin{array}{l}\text { Guest lounge with fire } \\
\text { place, shared bathroom, } \\
\text { shower, disabled access, } \\
\text { washing machine, dryer, } \\
\text { spa pool, VCR, Sky TV, } \\
\text { DVD player, internet. }\end{array}$ & $\begin{array}{l}2 \text { bedrooms with lighting and } \\
\text { [heating]. }\end{array}$ & $\begin{array}{l}\text { Breakfast, OCRT, } \\
\text { Middlemarch city, } \\
\text { farm sheep, cattle and } \\
\text { horse, landscape } \\
\text { views, historic sites } \\
\text { and buildings, local } \\
\text { transportation. }\end{array}$ & Stone \\
\hline 19 & Middlemarch & The Lodge & $1 \times \mathrm{B} \& \mathrm{~B}$ & $\mathrm{RB}$ & $\begin{array}{l}\text { A shared bathroom ( used } \\
\text { by } 2 \text { bedrooms),fully } \\
\text { equipped kitchen with } \\
\text { micro-wave oven, } \\
\text { dishwasher, toaster, coffee } \\
\text { percolator, fridge/freezer, } \\
\text { dining room, lounge, } \\
\text { TV,DVD player, stereo, } \\
\text { laundry facilities, } 2 \\
\text { recreational area with gas } \\
\text { BBQs. }\end{array}$ & $\begin{array}{l}2 \text { bedrooms with ensuites (one } \\
\text { with disabled access), heating, } \\
\text { lighting. }\end{array}$ & $\begin{array}{l}\text { Continental breakfast, } \\
\text { OCRT, Middlemarch } \\
\text { city, historic sites, } \\
\text { landscape views, } \\
\text { books and magazines. }\end{array}$ & Timber \\
\hline 20 & Middlemarch & $\begin{array}{l}\text { The Rocks luxury } \\
\text { Accommodation / } \\
\text { Longford Retreat }\end{array}$ & $1 \times \mathrm{SC}$ & $\mathrm{RB}$ & $\begin{array}{l}\text { Shared bathroom, kitchen, } \\
\text { sitting room, TV, veranda, } \\
\text { outdoor sitting room, } \\
\text { dining room. }\end{array}$ & $\begin{array}{l}\text { All bedrooms have heating and } \\
\text { lighting. }\end{array}$ & $\begin{array}{l}\text { Food and beverages, } \\
\text { continental breakfast, } \\
\text { wine, OCRT, } \\
\text { Middlemarch city, } \\
\text { historic sites, Sutton } \\
\text { Salt lake, Taieri Gorge } \\
\text { Train, landscape } \\
\text { views, 7000acre Fine } \\
\text { Wool Merino Station, } \\
\text { farm tour, fishing, } \\
\text { walk up to the Rock }\end{array}$ & Timber \\
\hline
\end{tabular}




\begin{tabular}{|c|c|c|c|c|c|c|c|c|}
\hline & & & & & & & $\begin{array}{l}\text { and Pillar } \\
\text { range, Smooth Cone } \\
\text { hill, riding mountain } \\
\text { bikes, photography. }\end{array}$ & \\
\hline 21 & Middlemarch & $\begin{array}{l}\text { The Rocks luxury } \\
\text { Accommodation / } \\
\text { Braeside Cottage }\end{array}$ & $1 \times B \& B$ & New & $\begin{array}{l}\text { Shared bathroom, kitchen, } \\
\text { fireplace, TV, self- } \\
\text { catering setup. }\end{array}$ & $\begin{array}{l}\text { All bedrooms have heating and } \\
\text { lighting. }\end{array}$ & $\begin{array}{l}\text { Food and beverages, } \\
\text { continental breakfast, } \\
\text { wine, OCRT, } \\
\text { Middlemarch city, } \\
\text { historic sites, Sutton } \\
\text { Salt lake, Taieri Gorge } \\
\text { Train, landscape } \\
\text { views, 7000acre Fine } \\
\text { Wool Merino Station, } \\
\text { farm tour, fishing, } \\
\text { walking up to the } \\
\text { Rock and Pillar } \\
\text { range, Smooth Cone } \\
\text { hill, riding mountain } \\
\text { bikes, photography. }\end{array}$ & Schist stone \\
\hline 22 & Middlemarch & Trails End & $2 \times \mathrm{SC}$ & New & $\begin{array}{l}\text { Kitchenette, ensuite } \\
\text { bathroom, BBQ facilities, } \\
\text { a lounge with TV, spa } \\
\text { pool. }\end{array}$ & $\begin{array}{l}\text { Each bedroom has TV, tea and } \\
\text { coffee making, heater and } \\
\text { lighting. }\end{array}$ & $\begin{array}{l}\text { Food and beverages, } \\
\text { OCRT, Middlemarch, } \\
\text { city, historic heritage, } \\
\text { fishing, Macraes } \\
\text { goldmine tour, Sutton } \\
\text { Salt lake, Taieri Gorge } \\
\text { Train. }\end{array}$ & Timber \\
\hline 23 & Ngapuna & Off The Trax & $1 \times \mathrm{SC}$ & $\mathrm{RB}$ & $\begin{array}{l}\text { Bathroom and toilet, } \\
\text { kitchen with gas cooking, } \\
\text { microwave and fridge, } \\
\text { outdoor sitting area, BBQ } \\
\text { area, sitting room with } \\
\text { TV, fire place, washing } \\
\text { machine and dryer. }\end{array}$ & $\begin{array}{l}\text { Bedrooms have heating and } \\
\text { lighting. }\end{array}$ & $\begin{array}{l}\text { Food and beverages, } \\
\text { fresh vegetables, farm } \\
\text { fresh eggs, OCRT,20 } \\
\text { hectares surrounding } \\
\text { farm land, landscape } \\
\text { views, wildlife } \\
\text { watching, fishing, } \\
\text { swimming, horse } \\
\text { riding, local } \\
\text { transportation, luggage } \\
\text { transfer. }\end{array}$ & Stone \\
\hline
\end{tabular}




\begin{tabular}{|c|c|c|c|c|c|c|c|c|}
\hline 24 & Ngapuna & Big Hut & $1 \times \mathrm{Bp}$ & $\mathrm{RB}$ & $\begin{array}{l}\text { Small kitchen, dining } \\
\text { table wash room, (all with } \\
\text { solar powered lighting), } \\
\text { tank water, outside toilet } \\
\text { (no artificial heating in the } \\
\text { hub), tennis table. }\end{array}$ & $\begin{array}{l}\text { No individual services, lighting } \\
\text { through the use of solar } \\
\text { system. Big hut can be subzero } \\
\text { in winter. }\end{array}$ & $\begin{array}{l}\text { OCRT, the Rock and } \\
\text { Pillar conservation } \\
\text { area, recreational } \\
\text { activities- tramping, } \\
\text { mountain biking, rock } \\
\text { climbing, landscape } \\
\text { photography, cross- } \\
\text { country skiing. }\end{array}$ & $\begin{array}{l}\text { Timber, } \\
\text { corrugated } \\
\text { iron }\end{array}$ \\
\hline 25 & Macraes Flat & Stanley's Hotel & $1 \times$ Hotel & $\mathrm{RB}$ & $\begin{array}{l}\text { Shared bathroom and } \\
\text { toilets, kitchen (used by } \\
\text { hotel to prepare food for } \\
\text { visitors), dining with fire } \\
\text { place and TV. }\end{array}$ & $\begin{array}{l}\text { All rooms have heating and } \\
\text { lighting, one unit with own } \\
\text { bathroom. }\end{array}$ & $\begin{array}{l}\text { Fresh food, beverages, } \\
\text { wine and beer, } \\
\text { continental breakfast, } \\
\text { OCRT, hotel's garden, } \\
\text { visit Macraes Flat gold } \\
\text { mining heritage tour, } \\
\text { visit historic village, } \\
\text { scenery, walking, } \\
\text { photography, local } \\
\text { transportation. }\end{array}$ & Schist stone \\
\hline 26 & Hyde & $\begin{array}{l}\text { Otago Central Hotel } \\
\text { B\&B \& Hyde café } \\
\text { (B\&B) }\end{array}$ & $1 \times \mathrm{B} \& \mathrm{~B}$ & $\mathrm{RB}$ & $\begin{array}{l}4 \text { bedrooms with shared } \\
\text { bathrooms and toilets, } \\
\text { lounge room, TV, } \\
\text { camping area with shared } \\
\text { shower. }\end{array}$ & $\begin{array}{l}4 \text { double rooms with ensuites, } 3 \\
\text { bedrooms with disabled access. } \\
2 \text { bedrooms have coffee and tea } \\
\text { making facilities, all rooms with } \\
\text { heating and lighting. }\end{array}$ & \multirow{2}{*}{$\begin{array}{l}\text { Food and refreshments } \\
\text { OCRT, the historic } \\
\text { Macraes Flat gold } \\
\text { mining town, the old } \\
\text { Golden Point mine in } \\
\text { Deepdell Creek. }\end{array}$} & \multirow[t]{2}{*}{ Timber } \\
\hline 27 & Hyde & $\begin{array}{l}\text { Otago Central Hotel } \\
\text { B\&B \& Hyde café } \\
\text { (camping) }\end{array}$ & $1 \times$ Camping & & & & & \\
\hline 28 & Hyde & $\begin{array}{l}\text { Emerald Hills } \\
\text { Country (guesthouse) }\end{array}$ & $1 \times \mathrm{B} \& \mathrm{~B}$ & New & $\begin{array}{l}3 \text { bedrooms with shared } \\
\text { bathrooms and separate } \\
\text { toilet, kitchen with oven, } \\
\text { microwave, fridge freezer, } \\
\text { living room, washing } \\
\text { machine, BBQ and TV. }\end{array}$ & $\begin{array}{l}\text { All rooms have heater, electric } \\
\text { blanket, hairdryer and toiletries } \\
\text { and lighting, }\end{array}$ & $\begin{array}{l}\text { Food-wine and beer, } \\
\text { continental breakfast, } \\
\text { oven fresh baking, } \\
\text { OCRT,4 acres private } \\
\text { country garden, } \\
\text { magazines, farm tours, }\end{array}$ & $\begin{array}{l}\text { Stone and } \\
\text { timber }\end{array}$ \\
\hline
\end{tabular}




\begin{tabular}{|c|c|c|c|c|c|c|c|c|}
\hline 29 & Hyde & $\begin{array}{l}\text { Emerald Hills } \\
\text { Country (cottage) }\end{array}$ & $1 \times B \& B$ & New & $\begin{array}{l}\text { Kitchens with oven, } \\
\text { microwave, fridge freezer, } \\
\text { living room, washing } \\
\text { machine, BBQ and TV. }\end{array}$ & $\begin{array}{l}2 \text { bedrooms with ensuite, } 1 \\
\text { bedroom with private bathroom } \\
\text { and toilet, all rooms have } \\
\text { heater, electric blanket, } \\
\text { hairdryer and toiletries and } \\
\text { lighting. }\end{array}$ & $\begin{array}{l}\text { Macraes Flat gold } \\
\text { mining town, historic } \\
\text { sites tour, landscape } \\
\text { views, walking, horse } \\
\text { riding, }\end{array}$ & \\
\hline 30 & Hyde & Pine Grove & $1 \times \mathrm{SC}$ & $\mathrm{RB}$ & $\begin{array}{l}\text { Kitchen with oven, } \\
\text { microwave, fridge and } \\
\text { freezer, claw foot bath, } \\
\text { living room, log burner } \\
\text { with wood supplied, BBQ, } \\
\text { laundry with washing } \\
\text { machine. }\end{array}$ & $\begin{array}{l}3 \text { furnished bedrooms with } \\
\text { heating and lighting. }\end{array}$ & $\begin{array}{l}\text { Food and beverages, } \\
\text { beer and wine, OCRT, } \\
\text { surrounding farm } \\
\text { tours, verandah, } \\
\text { garden, visiting } \\
\text { historic sites and } \\
\text { photography, fishing } \\
\text { and swimming, tours } \\
\text { of goldmine, walking, } \\
\text { local transportation. }\end{array}$ & Timber \\
\hline 31 & Tiroiti & $\begin{array}{l}\text { Tiroiti- Hyde and } \\
\text { Tiroiti- Kokonga }\end{array}$ & $2 \times$ Camp & $\mathrm{RB}$ & - & - & $\begin{array}{l}\text { OCRT, visiting } \\
\text { historic buildings and } \\
\text { sites, tunnels and } \\
\text { bridges, landscape } \\
\text { views, walking and } \\
\text { picnic. }\end{array}$ & $\begin{array}{l}\text { Restored } \\
\text { buildings and } \\
\text { infrastructures } \\
\text { made from } \\
\text { local } \\
\text { materials } \\
\end{array}$ \\
\hline 32 & Kokonga & Kokonga Lodge & $1 \times \mathrm{B} \& \mathrm{~B}$ & New & $\begin{array}{l}\text { Dining room, full laundry } \\
\text { facilities, tea, coffee } \\
\text { making area, TV, internet } \\
\text { access. }\end{array}$ & $\begin{array}{l}\text { All rooms with bathroom, } \\
\text { hairdryer, toiletries, heater and } \\
\text { lighting. }\end{array}$ & $\begin{array}{l}\text { Local food, wine, } \\
\text { beer, non-alcoholic } \\
\text { drinks, OCRT, rural } \\
\text { art deco town of } \\
\text { Ranfurly, gold mining } \\
\text { heritage exploring, } \\
\text { rural and garden } \\
\text { views, biking, fishing, } \\
\text { 4WD tour, painting } \\
\text { and photography, } \\
\text { curling and winter } \\
\text { sports in Naseby. }\end{array}$ & Timber \\
\hline 33 & Kokonga & Kokonga Cottage & $1 \times \mathrm{SC}$ & New & $\begin{array}{l}\text { Fully equipped kitchen, } \\
\text { two bedrooms share a } \\
\text { bathroom with bath and } \\
\text { shower, laundry, dining }\end{array}$ & $\begin{array}{l}\text { Two bed rooms with heating } \\
\text { and lighting. }\end{array}$ & $\begin{array}{l}\text { Local food, wine, } \\
\text { beer, non-alcoholic } \\
\text { drinks, OCRT, rural } \\
\text { art deco town of }\end{array}$ & Timber \\
\hline
\end{tabular}




\begin{tabular}{|c|c|c|c|c|c|c|c|c|}
\hline & & & & & room, TV. & & $\begin{array}{l}\text { Ranfurly, gold mining } \\
\text { heritage exploring, } \\
\text { rural and garden } \\
\text { views, biking, fishing, } \\
\text { 4WD tour, painting } \\
\text { and photography, } \\
\text { curling and winter } \\
\text { sports at Naseby. }\end{array}$ & \\
\hline 34 & Kokonga & 2Wheel Backpackers & $1 \times \mathrm{Bp}$ & New & $\begin{array}{l}\text { Full kitchen facilities, } \\
\text { shared bathroom and toilet } \\
\text { facilities, dining room, } \\
\text { lounge, outdoor furniture, } \\
\text { TV, BBQ. }\end{array}$ & $\begin{array}{l}\text { Bunkroom ( } 4 \times \text { single bed) and } 1 \\
\text { private room with heating and } \\
\text { lighting. }\end{array}$ & $\begin{array}{l}\text { Continental breakfast, } \\
\text { OCRT, rural art deco } \\
\text { town of Ranfurly, gold } \\
\text { mining heritage } \\
\text { exploring, Taieri river, } \\
\text { landscape views, } \\
\text { fishing, waking, } \\
\text { painting and } \\
\text { photography quiet and } \\
\text { peaceful place. }\end{array}$ & Timber \\
\hline 35 & Pareau/Styx & Styx Hotel & $\begin{array}{l}\text { Privet holiday- } \\
\text { home }\end{array}$ & $\mathrm{RB}$ & NA & NA & $\begin{array}{l}\text { Open for public visit, } \\
\text { New Zealand's only } \\
\text { example of a scroll } \\
\text { plain wetland, a } \\
\text { common system in } \\
\text { Australia, known there } \\
\text { as billabongs. Privet } \\
\text { holiday-home does } \\
\text { not function as OCRT } \\
\text { accommodation } \\
\text { service. }\end{array}$ & $\begin{array}{l}\text { Stone and } \\
\text { timber }\end{array}$ \\
\hline 36 & Patearoa & $\begin{array}{l}\text { Patearoa Hotel + } 7 \\
\text { Chalets }\end{array}$ & $1 \times$ Hotel & $\mathrm{RB}$ & $\begin{array}{l}\text { Shared bathrooms and } \\
\text { toilets, dining room, } 7 \\
\text { historic chalets. }\end{array}$ & $\begin{array}{l}1 \text { bedroom has en-suite } \\
\text { bathroom, all } 4 \text { rooms with } \\
\text { lighting and [heating]. }\end{array}$ & $\begin{array}{l}\text { Food and breakfast, } \\
\text { OCRT, exploring } \\
\text { historic sites and } \\
\text { buildings, walking. }\end{array}$ & $\begin{array}{l}\text { Red brick and } \\
\text { timber }\end{array}$ \\
\hline 37 & Waipiata & $\begin{array}{l}\text { Historic Waipiata } \\
\text { Country Hotel }\end{array}$ & $1 \times$ Hotel & $\mathrm{RB}$ & $\begin{array}{l}\text { Restaurant, country style } \\
\text { bar, guests lounge, } \\
\text { disabled facilities, BBQ, }\end{array}$ & $\begin{array}{l}\text { All rooms with either en-suites } \\
\text { or private bathrooms, heating } \\
\text { and lighting. }\end{array}$ & $\begin{array}{l}\text { Food and beverages, } \\
\text { wine and beer, OCRT, }\end{array}$ & $\begin{array}{l}\text { Mud brick } \\
\text { walls and }\end{array}$ \\
\hline
\end{tabular}




\begin{tabular}{|c|c|c|c|c|c|c|c|c|}
\hline & & & & & TV. & & $\begin{array}{l}\text { newspapers and } \\
\text { magazines, chat with } \\
\text { locals, discover local } \\
\text { sights which include } \\
\text { the historic Hamilton's } \\
\text { goldfields cemetery }\end{array}$ & timber \\
\hline 38 & Waipiata & $\begin{array}{l}\text { Komako Peonies \& } \\
\text { Cottage Garden (the } \\
\text { station building) }\end{array}$ & $1 \times B \& B$ & New & $\begin{array}{l}\text { Fully equipped kitchen, } \\
\text { laundry facilities, BBQ. }\end{array}$ & $\begin{array}{l}\text { All bedrooms with separate } \\
\text { shower, private toilet, electric } \\
\text { blankets, heating and lighting. }\end{array}$ & $\begin{array}{l}\text { Food, continental } \\
\text { breakfast, OCRT, } \\
\text { historic Hamilton's } \\
\text { goldfield and } \\
\text { cemetery, the old } \\
\text { Waipiata sanatorium, } \\
\text { garden views, curling } \\
\text { at Naseby, fishing and } \\
\text { hiking. }\end{array}$ & Timber \\
\hline 39 & Waipiata & $\begin{array}{l}\text { Komako Peonies \& } \\
\text { Cottage Garden (the } \\
\text { bank) }\end{array}$ & $1 \times B \& B$ & New & $\begin{array}{l}\text { Fully equipped kitchen, } \\
\text { laundry facilities, BBQ. }\end{array}$ & $\begin{array}{l}\text { All bedrooms with separate } \\
\text { shower, private toilet, electric } \\
\text { blankets, heating and lighting. }\end{array}$ & $\begin{array}{l}\text { Food, continental } \\
\text { breakfast, OCRT, } \\
\text { historic Hamilton's } \\
\text { goldfield and } \\
\text { cemetery, the old } \\
\text { Waipiata sanatorium, } \\
\text { garden views, curling } \\
\text { at Naseby, fishing and } \\
\text { hiking. }\end{array}$ & Concrete \\
\hline 40 & Waipiata & $\begin{array}{l}\text { Komako Peonies \& } \\
\text { Cottage Garden (the } \\
\text { tool shed) }\end{array}$ & $1 \times B \& B$ & New & $\begin{array}{l}\text { Fully equipped kitchen, } \\
\text { laundry facilities, BBQ. }\end{array}$ & $\begin{array}{l}\text { All bedrooms with separate } \\
\text { shower, private toilet, electric } \\
\text { blankets, heating and lighting. }\end{array}$ & $\begin{array}{l}\text { Food, continental } \\
\text { breakfast, OCRT, } \\
\text { historic Hamilton's } \\
\text { goldfield and } \\
\text { cemetery, the old } \\
\text { Waipiata sanatorium, } \\
\text { garden views, curling } \\
\text { at Naseby, fishing and } \\
\text { hiking. }\end{array}$ & Timber \\
\hline 41 & Waipiata & $\begin{array}{l}\text { Peter's Farm Lodge - } \\
\text { the homestead }\end{array}$ & $1 \times$ Homestead & $\mathrm{RB}$ & $\begin{array}{l}\text { Full kitchen facilities, } 2 \\
\text { outdoor bathrooms and } \\
\text { toilets, laundry, washing }\end{array}$ & $\begin{array}{l}\text { All bed rooms with heater and } \\
\text { lighting. }\end{array}$ & $\begin{array}{l}\text { Food, continental } \\
\text { breakfast, OCRT, }\end{array}$ & $\begin{array}{l}\text { Rock (from } \\
\text { local quarry- }\end{array}$ \\
\hline
\end{tabular}




\begin{tabular}{|c|c|c|c|c|c|c|c|c|}
\hline & & & & & machine and dryer, BBQ. & & $\begin{array}{l}\text { exploring historical } \\
\text { areas, free kayaks, } \\
\text { gold pans, fishing rod, } \\
\text { exploring historical } \\
\text { area. }\end{array}$ & $\begin{array}{l}\text { mud brick- } \\
\text { limited milled } \\
\text { timber }\end{array}$ \\
\hline 42 & Waipiata & $\begin{array}{l}\text { Peter's Farm lodge - } \\
\text { cabins }\end{array}$ & $1 \times$ Homestead & New & $\begin{array}{l}2 \text { outdoor bathrooms, } \\
\text { shared homestead } \\
\text { facilities, private garden } \\
\text { setting. }\end{array}$ & $\begin{array}{l}\text { All bed rooms with heater and } \\
\text { lighting. }\end{array}$ & $\begin{array}{l}\text { Food, continental } \\
\text { breakfast, OCRT, } \\
\text { exploring historical } \\
\text { areas, free kayaks, } \\
\text { gold pans, fishing rod, } \\
\text { exploring historical } \\
\text { area. }\end{array}$ & Timber \\
\hline 43 & Waipiata & Tregonning Cottage & $1 \times \mathrm{SC}$ & $\mathrm{RB}$ & $\begin{array}{l}\text { Country size-kitchen, } \\
\text { [shared bathroom and } \\
\text { toilet], dining room, metro } \\
\text { log fire, guests lounge, } \\
\text { TV, BBQ and picnic area } \\
\text { outside or under the } \\
\text { sheltered verandah. }\end{array}$ & $\begin{array}{l}\text { All bedrooms with electric } \\
\text { blankets, heaters, and lighting. }\end{array}$ & $\begin{array}{l}\text { OCRT, the Waipiata } \\
\text { township, the Taieri } \\
\text { river, surrounding } \\
\text { sheep and beef farm, } \\
\text { farm tour, gold } \\
\text { panning, kayaking, } \\
\text { fishing local } \\
\text { transportation along } \\
\text { the rail trail. }\end{array}$ & $\begin{array}{l}\text { Mud brick } \\
\text { and schist } \\
\text { stone }\end{array}$ \\
\hline 44 & Waipiata & Waipiata Cottage & $1 \times B \& B$ & $\mathrm{RB}$ & $\begin{array}{l}\text { Equipped kitchen, } \\
\text { bathroom and toilet, } \\
\text { lounge, fire place. }\end{array}$ & $\begin{array}{l}\text { All bedrooms have heating and } \\
\text { lighting. }\end{array}$ & $\begin{array}{l}\text { Wine, OCRT, the } \\
\text { Waipiata township, } \\
\text { the Taieri river, } \\
\text { scenery, walking. }\end{array}$ & Timber \\
\hline 45 & Waipiata & Waipiata Motel & $1 \times$ Motel & New & - & $\begin{array}{l}\text { All bedrooms have private } \\
\text { bathroom with heater and hair } \\
\text { dryer, wall heater and lighting, } \\
\text { small kitchenette with } \\
\text { microwave and coffee and tea } \\
\text { making, TV. }\end{array}$ & $\begin{array}{l}\text { Continental breakfast, } \\
\text { wine and beer, OCRT, } \\
\text { scenery, visiting } \\
\text { historic places, } \\
\text { walking and } \\
\text { photography. }\end{array}$ & $\begin{array}{l}\text { Timber and } \\
\text { glass }\end{array}$ \\
\hline 46 & Ranfurly & $\begin{array}{l}\text { Plains Retreat, } \\
\text { Ranfurly Hospital }\end{array}$ & $1 \times$ Hotel & $\mathrm{RB}$ & $\begin{array}{l}\text { Two bathrooms, living } \\
\text { room. }\end{array}$ & $\begin{array}{l}\text { All bedrooms have heater and } \\
\text { lighting. }\end{array}$ & $\begin{array}{l}\text { Continental breakfast, } \\
\text { wine and beer, OCRT, } \\
\text { surrounding garden, } \\
\text { art deco town of }\end{array}$ & Red brick \\
\hline
\end{tabular}




\begin{tabular}{|c|c|c|c|c|c|c|c|c|}
\hline & & & & & & & $\begin{array}{l}\text { Ranfurly, historic } \\
\text { villages such as } \\
\text { Wedderburn, Naseby, } \\
\text { St Bathans, tour to } \\
\text { visit historical places } \\
\text { and landscape, } \\
\text { viewing scenery, local } \\
\text { transportation along } \\
\text { the OCRT. }\end{array}$ & \\
\hline 47 & Ranfurly & Railway Cottage & $1 \times \mathrm{SC}$ & $\mathrm{RB}$ & $\begin{array}{l}\text { Kitchen, dining room, } \\
\text { guest lounge, fireplace, } \\
\text { outdoor sitting with BBQ } \\
\text { facilities. }\end{array}$ & $\begin{array}{l}1 \text { ensuite and } 1 \text { private } \\
\text { bathroom, all bedrooms have } \\
\text { heater and lighting. }\end{array}$ & $\begin{array}{l}\text { Continental breakfast, } \\
\text { wine and beer, OCRT, } \\
\text { surrounding garden, } \\
\text { art deco town of } \\
\text { Ranfurly, historic } \\
\text { villages such as } \\
\text { Wedderburn, Naseby, } \\
\text { St Bathans, tour to } \\
\text { visit historical places } \\
\text { and landscape, } \\
\text { viewing scenery, local } \\
\text { transportation along } \\
\text { the OCRT. }\end{array}$ & Timber \\
\hline 48 & Ranfurly & Annerly House & $1 \times B \& B$ & New & $\begin{array}{l}\text { Kitchenette with } \\
\text { refrigerator, coffee and tea } \\
\text { making, guest bathroom, } \\
\text { children’s playground, } \\
\text { laundry with washing } \\
\text { machine, courtyard } \\
\text { garden, guest lounge with } \\
\text { balcony, verandah, CD } \\
\text { player, TV and video } \\
\text { cassette player. }\end{array}$ & $\begin{array}{l}\text { All bedrooms with heating and } \\
\text { lighting. }\end{array}$ & $\begin{array}{l}\text { Food and continental } \\
\text { breakfast, home } \\
\text { baking, OCRT, art deco } \\
\text { town of Ranfurly, } \\
\text { historic villages such } \\
\text { as Wedderburn, } \\
\text { Naseby, St Bathans, } \\
\text { landscape views, } \\
\text { books and magazines, } \\
\text { walking, ideal stop for } \\
\text { horse trekkers. }\end{array}$ & Timber \\
\hline 49 & Ranfurly & Hawkdun Lodge & $1 \times$ Motel style & New & $\begin{array}{l}\text { Equipped kitchen, gas } \\
\text { fire, outdoor BBQ for } \\
\text { dining and socializing }\end{array}$ & $\begin{array}{l}\text { Each suite has bathroom and } \\
\text { toilet, cooling, coffee and tea } \\
\text { making, microwave, TV, }\end{array}$ & $\begin{array}{l}\text { OCRT, Ranfurly, } \\
\text { historic villages such }\end{array}$ & $\begin{array}{l}\text { Assumed } \\
\text { concrete, }\end{array}$ \\
\hline
\end{tabular}




\begin{tabular}{|c|c|c|c|c|c|c|c|c|}
\hline & & & & & $\begin{array}{l}\text { with other guests, indoor } \\
\text { spa room with a large } \\
\text { (8person) spa pool, } \\
\text { laundry with a tub, two } \\
\text { washing machine, two } \\
\text { dryers, internet access, } \\
\text { heat pump for air } \\
\text { conditioning for summer } \\
\text { and heating for winter, } \\
\text { 45” TV (used for } \\
\text { conference). }\end{array}$ & lighting. & $\begin{array}{l}\text { as Wedderburn, } \\
\text { Naseby, St Bathans } \\
\text { conference facilities } \\
\text {,tour around the } \\
\text { historic gold mining } \\
\text { sites and lunch in one } \\
\text { of historic pubs, } \\
\text { garden tour } \\
\text { (Clachanburn country } \\
\text { garden), high country } \\
\text { experience (full day } \\
\text { and half day) ,4 wheel } \\
\text { drive excursion. }\end{array}$ & wood \\
\hline 50 & Ranfurly & $\begin{array}{l}\text { Moyola Art Deco } \\
\text { Guest House }\end{array}$ & $1 \times \mathrm{SC}$ & $\mathrm{RB}$ & $\begin{array}{l}\text { Equipped kitchen, coffee } \\
\text { machine, } 1 \text { spa bathroom } \\
\text { with shower and } 1 \\
\text { standard bathroom, gas } \\
\text { fire, laundry with washing } \\
\text { machine and dryer, } \\
\text { covered outdoor spa pool } \\
\text { (up to } 8 \text { persons), outdoor } \\
\text { BBQ area, guest } \\
\text { computer, a communal } \\
\text { large plasma TV. }\end{array}$ & $\begin{array}{l}\text { All rooms with private sky TV, } \\
\text { heating and lighting. }\end{array}$ & $\begin{array}{l}\text { Food and beverages, } \\
\text { continental breakfast, } \\
\text { OCRT, art deco town } \\
\text { of Ranfurly, art deco } \\
\text { museum, art deco } \\
\text { weekend, guided art } \\
\text { deco walks, historic } \\
\text { tour in the art deco } \\
\text { car ( } 1949 \text { Chevy), 4wd } \\
\text { high country tours, } \\
\text { garden tour horse } \\
\text { treks, mustering } \\
\text { experiences, golf, } \\
\text { fishing, mountain } \\
\text { biking, curling and ice } \\
\text { sport (at Naseby), } \\
\text { railway station } \\
\text { museum, early } \\
\text { settlers museum. }\end{array}$ & Red brick \\
\hline 51 & Ranfurly & $\begin{array}{l}\text { Old Post Office } \\
\text { (B\&B) }\end{array}$ & $1 \times B \& B$ & $\mathrm{RB}$ & $\begin{array}{l}\text { Full kitchen facilities for } \\
\text { self catering, showers, log } \\
\text { fire, laundry/ launderette } \\
\text { on site, tea and coffee }\end{array}$ & $\begin{array}{l}\text { All rooms are on a shared } \\
\text { facilities basis, heated by } \\
\text { communal log fire, lighting. }\end{array}$ & $\begin{array}{l}\text { OCRT, art deco town } \\
\text { of Ranfurly, Naseby } \\
\text { (curling, ice luge and }\end{array}$ & $\begin{array}{l}\text { Red brick and } \\
\text { concrete }\end{array}$ \\
\hline
\end{tabular}




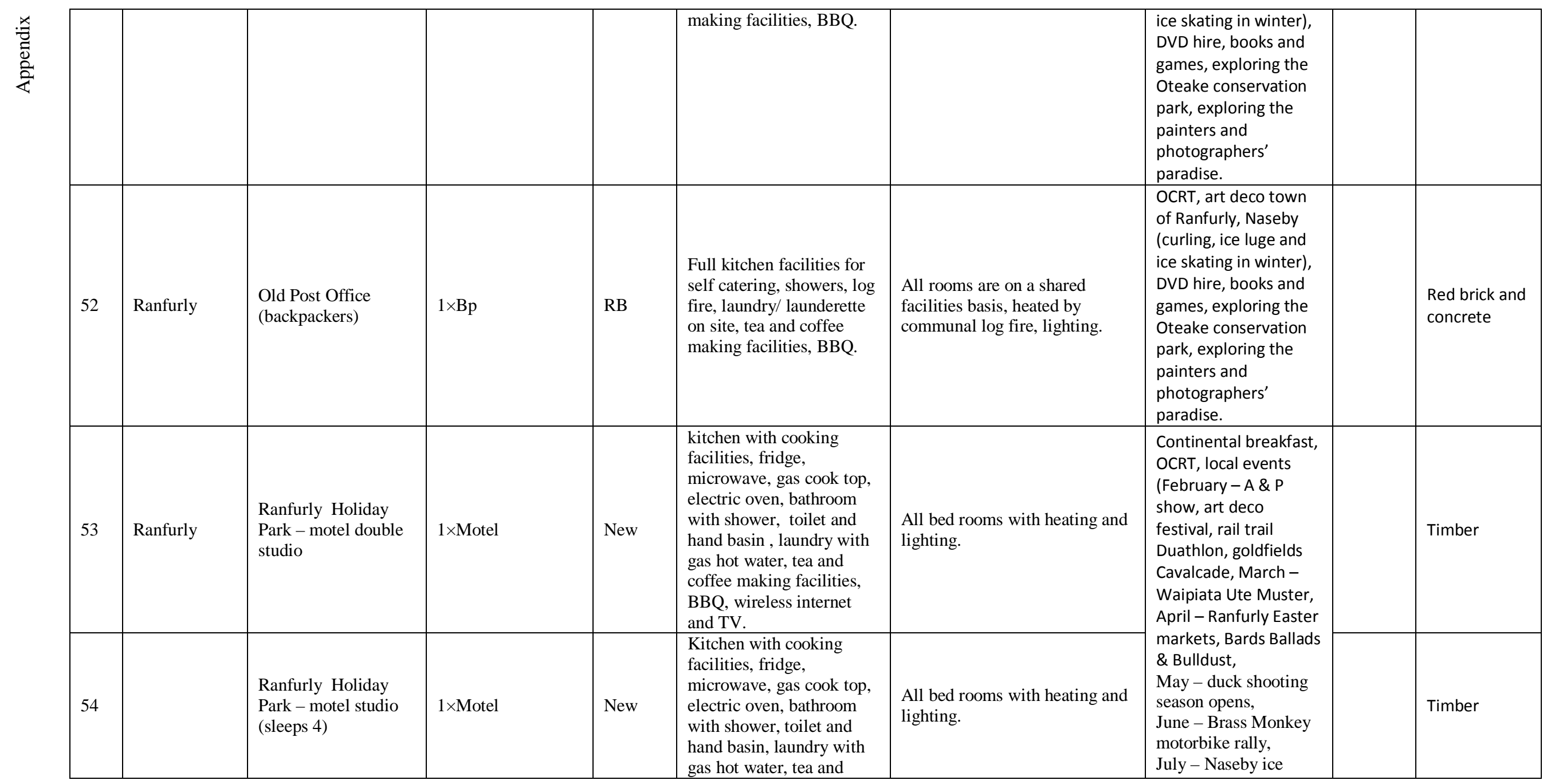




\begin{tabular}{|c|c|c|c|c|c|c|c|c|c|}
\hline & & & & & $\begin{array}{l}\text { coffee making facilities, } \\
\text { BBQ, wireless internet } \\
\text { and TV. }\end{array}$ & & \multirow{8}{*}{$\begin{array}{l}\text { festival, Highland Ball } \\
\text { August - 100\% pure } \\
\text { winter games, } \\
\text { October - labour } \\
\text { weekend markets, } \\
\text { vintage car swap meet) } \\
\text {, Hayes engineering } \\
\text { works, rail trail } \\
\text { adventure tours, Taieri } \\
\text { Gorge railway, Otago } \\
\text { fish \& game, Otago } \\
\text { goldfields, bike-car } \\
\text { and coach hire. }\end{array}$} & & \\
\hline 55 & Ranfurly & $\begin{array}{l}\text { Ranfurly Holiday } \\
\text { Park - motel studio } \\
\text { (sleeps 5) }\end{array}$ & $1 \times$ Motel & New & $\begin{array}{l}\text { Kitchen with cooking } \\
\text { facilities, fridge, } \\
\text { microwave, gas cook top, } \\
\text { electric oven, bathroom } \\
\text { with shower, toilet and } \\
\text { hand basin, laundry with } \\
\text { gas hot water, tea and } \\
\text { coffee making facilities, } \\
\text { BBQ, wireless internet } \\
\text { and TV. }\end{array}$ & $\begin{array}{l}\text { All bed rooms with heating and } \\
\text { lighting. }\end{array}$ & & & Timber \\
\hline 56 & Ranfurly & $\begin{array}{l}\text { Ranfurly Holiday } \\
\text { Park - one bedroom } \\
\text { motel unit }\end{array}$ & $1 \times$ Motel & New & $\begin{array}{l}\text { A kitchen with cooking } \\
\text { facilities, fridge, } \\
\text { microwave, bathroom } \\
\text { with shower, toilet and } \\
\text { hand basin, TV. }\end{array}$ & $\begin{array}{l}\text { All bed rooms with heating and } \\
\text { lighting. }\end{array}$ & & & Timber \\
\hline 57 & Ranfurly & $\begin{array}{l}\text { Ranfurly Holiday } \\
\text { Park - double cabin }\end{array}$ & $1 \times \mathrm{SC}$ & New & $\begin{array}{l}\text { Communal kitchen and } \\
\text { bathroom with gas hot } \\
\text { water, wireless internet. }\end{array}$ & $\begin{array}{l}\text { All bed rooms with heating and } \\
\text { lighting. }\end{array}$ & & & Timber \\
\hline 59 & Ranfurly & $\begin{array}{l}\text { Ranfurly Holiday } \\
\text { Park - cabin (sleeps } \\
\text { 5) }\end{array}$ & $1 \times \mathrm{SC}$ & New & $\begin{array}{l}\text { Communal kitchen and } \\
\text { bathroom with gas hot } \\
\text { water, wireless internet. }\end{array}$ & $\begin{array}{l}\text { All bed rooms with heating and } \\
\text { lighting. }\end{array}$ & & & Timber \\
\hline 60 & Ranfurly & $\begin{array}{l}\text { Ranfurly Holiday } \\
\text { Park - two bedroom } \\
\text { cabin(sleeps 8) }\end{array}$ & $1 \times \mathrm{SC}$ & New & $\begin{array}{l}\text { Communal kitchen and } \\
\text { bathroom with gas hot } \\
\text { water, wireless internet. }\end{array}$ & $\begin{array}{l}\text { All bed rooms with heating and } \\
\text { lighting. }\end{array}$ & & & Timber \\
\hline 61 & Ranfurly & $\begin{array}{l}\text { Ranfurly Holiday } \\
\text { Park - powered sites } \\
\text { (8 person) }\end{array}$ & $1 \times$ Camping & - & $\begin{array}{l}\text { Sites are sheltered, with } \\
\text { level ground and heaps of } \\
\text { room to spread out, use of } \\
\text { the communal cooking, } \\
\text { bathing and laundry } \\
\text { facilities. }\end{array}$ & - & & - & - \\
\hline 62 & Ranfurly & Ranfurly Holiday & $1 \times$ Camping & - & Sites are well sheltered, & - & & - & - \\
\hline
\end{tabular}




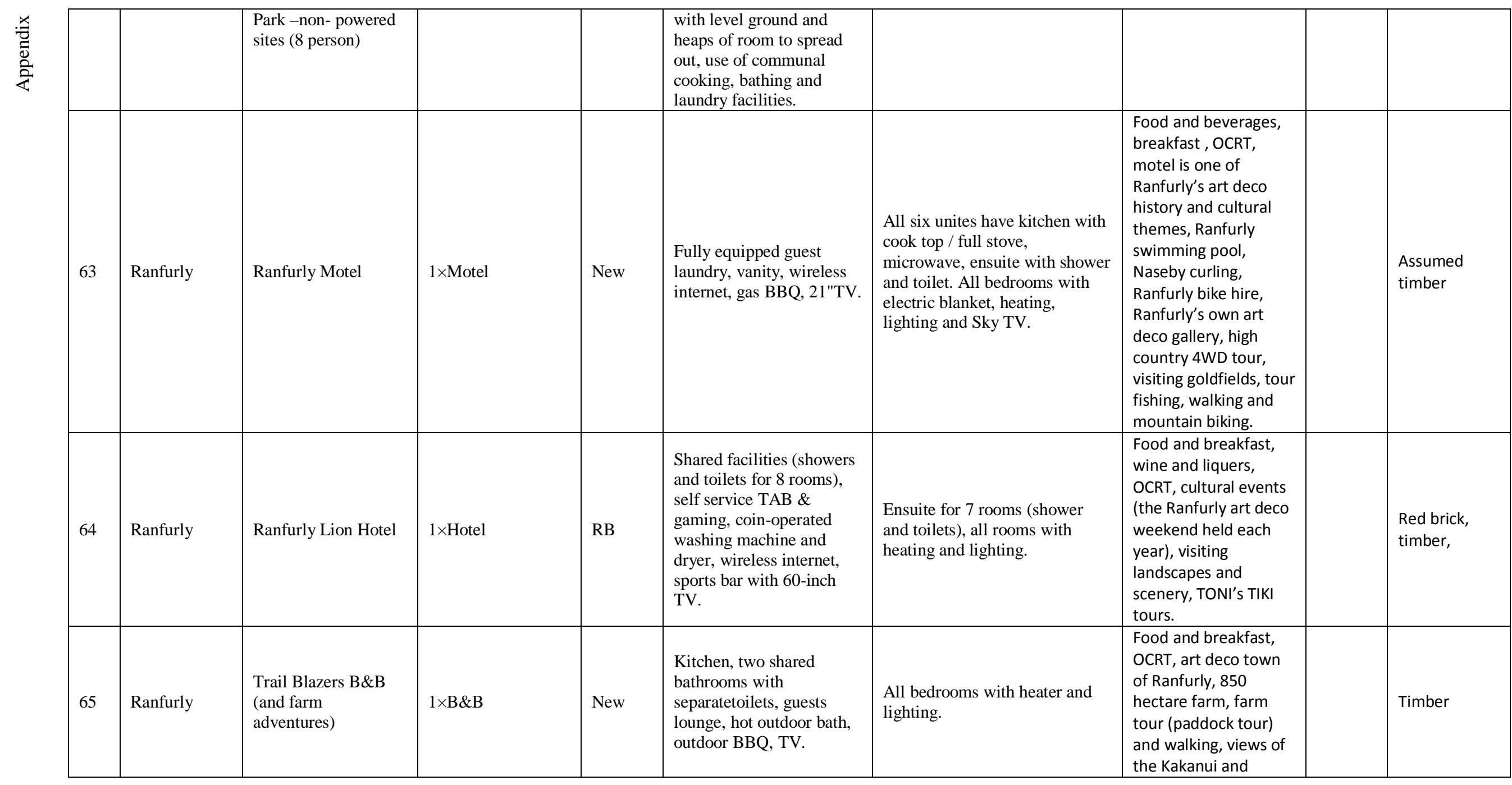




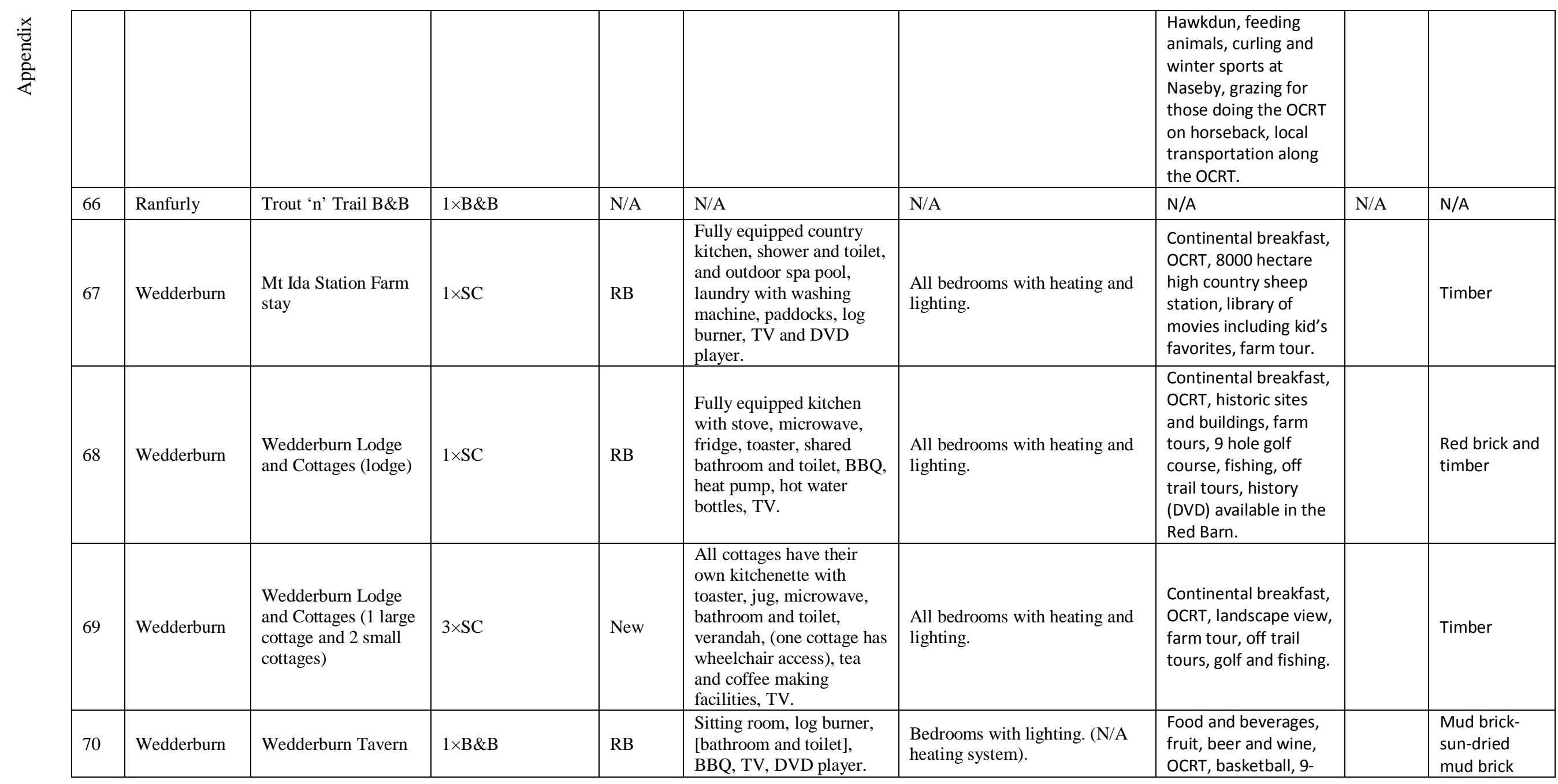




\begin{tabular}{|c|c|c|c|c|c|c|c|c|c|}
\hline 71 & Wedderburn & Wedderburn Tavern & $1 \times$ Camping(Tent) & - & Tent sleeps 6 guests. & - & \multirow{2}{*}{$\begin{array}{l}\text { hole golf (clubs for } \\
\text { hire), historic sites and } \\
\text { outstanding } \\
\text { landscapes, DVDs. }\end{array}$} & - & - \\
\hline 72 & Wedderburn & Wedderburn Tavern & $1 \times$ Camping & - & \multicolumn{2}{|l|}{ Freedom camping site } & & - & - \\
\hline 73 & Danseys Pass & Danseys Pass Hotel & $1 \times$ Hotel & $\mathrm{RB}$ & $\begin{array}{l}\text { Dining room and } \\
\text { verandah, Log fire. }\end{array}$ & $\begin{array}{l}\text { Ensuite and double rooms with } \\
\text { bathroom and toilet, heating } \\
\text { and lighting. }\end{array}$ & $\begin{array}{l}\text { Food and beverages, } \\
\text { breakfast, OCRT, } \\
\text { books, conference } \\
\text { facilities (a } \\
\text { whiteboard, overhead } \\
\text { projector, fax, email } \\
\text { [internet]), clay target } \\
\text { shooting, curling, } \\
\text { mountain biking, gold } \\
\text { panning, trekking and } \\
\text { horse riding, fishing } \\
\text { (world famous), } \\
\text { tennis, } \\
\text { swimming,4WD tours } \\
\text { to surrounding } \\
\text { mountainous tussock } \\
\text { country. }\end{array}$ & & Stone \\
\hline 74 & Danseys Pass & $\begin{array}{l}\text { Short Lands Station } \\
\text { Farm stay }\end{array}$ & $1 \times B \& B$ & New & $\begin{array}{l}3 \text { separate bathrooms, log } \\
\text { fire, sitting room. }\end{array}$ & $\begin{array}{l}\text { All bed rooms with lighting, } \\
\text { (N/A heating system for } \\
\text { bedrooms). }\end{array}$ & $\begin{array}{l}\text { Food and beverages, } \\
\text { OCRT, curling (in } \\
\text { Naseby), mountain } \\
\text { scenery and } \\
\text { wilderness. }\end{array}$ & & $\begin{array}{l}\text { Red brick and } \\
\text { timber. }\end{array}$ \\
\hline 75 & Naseby & Ancient Briton Hotel & $1 \times$ Hotel & $\mathrm{RB}$ & $\begin{array}{l}\text { Fully equipped kitchen, } 4 \\
\text { rooms with shared } \\
\text { bathrooms, sitting room } \\
\text { with fireplace. }\end{array}$ & $\begin{array}{l}12 \text { bedrooms with ensuite, all } \\
\text { rooms have tea and coffee } \\
\text { making facilities, [heating] and } \\
\text { lighting. }\end{array}$ & $\begin{array}{l}\text { Food and beverages, } \\
\text { breakfast, OCRT, } \\
\text { Bards Ballads \& } \\
\text { Bullust (annual } \\
\text { festival), heritage } \\
\text { museum, forest walks, } \\
\text { mountain biking, } \\
\text { curling, annual events, } \\
\text { ice skating, swimming }\end{array}$ & & $\begin{array}{l}\text { Brick and } \\
\text { timber }\end{array}$ \\
\hline
\end{tabular}




\begin{tabular}{|c|c|c|c|c|c|c|c|c|}
\hline & & & & & & & $\begin{array}{l}\text { and tennis, Art, } \\
\text { Sculpture \& Gardens } \\
\text { (studio gallery), } \\
\text { Ballarat cottage (art } \\
\text { museum), Naseby } \\
\text { town garden, local } \\
\text { café, fishing and } \\
\text { hunting, Otago tour } \\
\text { fishing, gold panning, } \\
\text { golf, 4WD \& horse } \\
\text { trekking. }\end{array}$ & \\
\hline 76 & Naseby & $\begin{array}{l}\text { Larchview Holiday } \\
\text { Park (tourist flat) }\end{array}$ & $1 \times \mathrm{SC}$ & New & \multirow{6}{*}{$\begin{array}{l}\text { Adventure playground, } \\
\text { adjacent swimming dam, } \\
\text { coin operated shower, } \\
\text { coin operated washing } \\
\text { machine and dryer, dump } \\
\text { point for van waste, } \\
\text { caravan storage, bike } \\
\text { hire, toboggan hire, wheat } \\
\text { bag hire, hot water heated } \\
\text { by solar energy, BBQ, } \\
\text { wireless and kiosk internet } \\
\text { access, free view TV in } \\
\text { the main amenity block. }\end{array}$} & $\begin{array}{l}\text { Fully equipped kitchen, shower } \\
\text { and toilet, heated by log burner. }\end{array}$ & \multirow{6}{*}{$\begin{array}{l}\text { Food and beverages, } \\
\text { OCRT Naseby, historic } \\
\text { sites and buildings, } \\
\text { mountain biking, } \\
\text { swimming, curling, } \\
\text { lugeing, ice skating, } 4 \\
\text { wheel driving, gold } \\
\text { panning, forests } \\
\text { walks, scenery. }\end{array}$} & Timber \\
\hline 77 & Naseby & $\begin{array}{l}\text { Larchview Holiday } \\
\text { Park (miners } \\
\text { cottage })^{1}\end{array}$ & $2 \times \mathrm{SC}$ & $\mathrm{RB}$ & & $\begin{array}{l}\text { Fully equipped kitchen, modern } \\
\text { shower and toilet, log burner, } \\
\text { heater and lighting. }\end{array}$ & & Timber \\
\hline 78 & Naseby & $\begin{array}{l}\text { Larchview Holiday } \\
\text { Park (10 chalets) }\end{array}$ & $10 \times \mathrm{SC}$ & New & & $\begin{array}{l}\text { Each chalet has heater, jug, } \\
\text { toaster, crockery lighting. }\end{array}$ & & $\begin{array}{l}\text { Assumed } \\
\text { timber }\end{array}$ \\
\hline 79 & Naseby & $\begin{array}{l}\text { Larchview Holiday } \\
\text { Park (3 cabins) }\end{array}$ & $1 \times B \& B$ & New & & $\begin{array}{l}\text { Heaters, occupants supply } \\
\text { crockery, utensils. }\end{array}$ & & - \\
\hline 80 & Naseby & $\begin{array}{l}\text { Larchview Holiday } \\
\text { Park ( } 48 \text { powered } \\
\text { sites) }\end{array}$ & $48 \times$ camping & - & & These sites include 4 caravans. & & - \\
\hline 81 & Naseby & $\begin{array}{l}\text { Larchview Holiday } \\
\text { Park ( } 24 \text { non- } \\
\text { powered sites) }\end{array}$ & $24 \times$ Camping & - & & 24 available & & - \\
\hline 82 & Naseby & $\begin{array}{l}\text { Mountain View } \\
\text { (cottage) }\end{array}$ & $1 \times \mathrm{SC}$ & New & $\begin{array}{l}\text { Equipped kitchen, living } \\
\text { area with log fire, laundry } \\
\text { facilities. }\end{array}$ & $\begin{array}{l}\text { Each bedroom has ensuite } \\
\text { bathroom, electric blanket, } \\
\text { heater and lighting. }\end{array}$ & \multirow{3}{*}{$\begin{array}{l}\text { Continental breakfast, } \\
\text { OCRT, Naseby, } \\
\text { historical sites and } \\
\text { buildings, indoor } \\
\text { curling all year, } \\
\text { outdoor curling and } \\
\text { luge from June to } \\
\text { September, mountain }\end{array}$} & Timber \\
\hline 83 & Naseby & $\begin{array}{l}\text { Mountain View ( } 2 \\
\text { ensuite units) }\end{array}$ & $1 \times \mathrm{B} \& \mathrm{~B}$ & New & $\begin{array}{l}\text { Each unit with tea and } \\
\text { coffee making, } \\
\text { microwave, fridge, BBQ } \\
\text { and TV. }\end{array}$ & $\begin{array}{l}\text { Ensuite bathroom, each } \\
\text { bedroom with electric blanket, } \\
\text { heater and lighting. }\end{array}$ & & Timber \\
\hline 84 & Naseby & $\begin{array}{l}\text { Mountain View } \\
\text { (tourist flat) }\end{array}$ & $1 \times \mathrm{B} \& \mathrm{~B}$ & New & $\begin{array}{l}\text { Limited cooking } \\
\text { facilities. }\end{array}$ & $\begin{array}{l}\text {, Ensuite bathroom, each } \\
\text { bedroom with hairdryer heater }\end{array}$ & & Timber \\
\hline
\end{tabular}




\begin{tabular}{|c|c|c|c|c|c|c|c|c|}
\hline & & & & & & and lighting. & \multirow{2}{*}{$\begin{array}{l}\text { biking, hiking, forest } \\
\text { walks. }\end{array}$} & \\
\hline 85 & Naseby & $\begin{array}{l}\text { Mountain View (the } \\
\text { house with } 3 \text { bed } \\
\text { rooms) }\end{array}$ & $1 \times \mathrm{SC}$ & New & $\begin{array}{l}\text { Fully equipped kitchen, } \\
\text { laundry, verandah, heat } \\
\text { pump. }\end{array}$ & $\begin{array}{l}\text { Ensuite bathroom, separate } \\
\text { toilet equipped with disabled } \\
\text { facilities, each bedroom with } \\
\text { hairdryer, heater and lighting. }\end{array}$ & & Timber \\
\hline 86 & Naseby & $\begin{array}{l}\text { Naseby Trail Lodge } \\
\text { ( } 6 \text { one bedroom } \\
\text { units) }\end{array}$ & $1 \times \mathrm{SC}$ & New & $\begin{array}{l}\text { Each one unit has private } \\
\text { timber deck verandah, } \\
\text { Italian leather sofa, } \\
\text { European style kitchen, } \\
\text { central heating, iron and } \\
\text { ironing board, internet } \\
\text { access, microwave, } \\
\text { toaster, heated towel rail, } \\
\text { TV, telephone }\end{array}$ & $\begin{array}{l}6 \text { ensuite bathrooms with under } \\
\text { floor heating, wheelchair access } \\
\text { bathroom (unit 1), each } \\
\text { bedroom has reading lamps, } \\
\text { radio alarm clock, and hair } \\
\text { dryer and lighting. }\end{array}$ & $\begin{array}{l}\text { Continental and/or } \\
\text { cooked breakfast, } \\
\text { OCRT, Naseby, year- } \\
\text { round, curling, winter } \\
\text { lugeing, winter ice } \\
\text { skating, year round } \\
\text { mountain biking, golf, } \\
\text { river and lake fishing, } \\
\text { 4WD adventures, } \\
\text { horse treks. }\end{array}$ & $\begin{array}{l}\text { Timber- } \\
\text { aluminum } \\
\text { façade, } \\
\text { wooden } \\
\text { joinery }\end{array}$ \\
\hline 87 & Naseby & $\begin{array}{l}\text { Naseby Trail Lodge } \\
\text { ( } 2 \text { two bedroom } \\
\text { units) }\end{array}$ & $1 \times \mathrm{SC}$ & New & $\begin{array}{l}\text { Each units has kitchen } \\
\text { with microwave, cooking } \\
\text { hob, dishwasher, toaster, } \\
\text { kettle, fridge, central } \\
\text { heating, iron and ironing } \\
\text { board, separate lounge } \\
\text { with sofa (coverts to } \\
\text { innersprung double bed), } \\
\text { TV, internet access. }\end{array}$ & $\begin{array}{l}\text { Each unit has separate bathroom } \\
\text { with under floor heating, wet- } \\
\text { area shower, heated towel rail, } \\
\text { hair dryer, toiletries. Each } \\
\text { bedroom has reading lamps, } \\
\text { telephone, radio alarm clock } \\
\text { and lighting }\end{array}$ & $\begin{array}{l}\text { Continental and/or } \\
\text { cooked breakfast, } \\
\text { OCRT, Naseby, year- } \\
\text { round, curling, winter } \\
\text { lugging, winter ice } \\
\text { skating, year round } \\
\text { mountain biking, golf, } \\
\text { river and lake fishing, } \\
4 W D \text { adventures, } \\
\text { horse treks. }\end{array}$ & $\begin{array}{l}\text { Locally made } \\
\text { mud-brick }\end{array}$ \\
\hline 88 & Naseby & $\begin{array}{l}\text { Old Doctor's } \\
\text { Residence }\end{array}$ & $1 \times B \& B$ & $\mathrm{RB}$ & $\begin{array}{l}\text { Guest lounge with tea and } \\
\text { coffee making facilities, } \\
\text { TV, DVD, broadband } \\
\text { access. }\end{array}$ & $\begin{array}{l}\text { Private bathroom, hair dryer, } \\
\text { ironing, heated towel rail, } \\
\text { heating and lighting. }\end{array}$ & $\begin{array}{l}\text { Food, special } \\
\text { breakfast, homemade } \\
\text { baking, wine, OCRT, } \\
\text { the gold fields, } \\
\text { magazines, a library, } \\
\text { music and games, } \\
\text { curling,4WD tour, } \\
\text { mountain biking, } \\
\text { fishing, golf, day walks } \\
\text { in the Manitoto, }\end{array}$ & $\begin{array}{l}\text { Clay brick, } \\
\text { timber }\end{array}$ \\
\hline
\end{tabular}




\begin{tabular}{|c|c|c|c|c|c|c|c|c|}
\hline & & & & & & & $\begin{array}{l}\text { wildflower walks, } \\
\text { horse riding. }\end{array}$ & \\
\hline 89 & Naseby & Royal Hotel (hotel) & $1 \times$ Hotel & $\mathrm{RB}$ & $\begin{array}{l}\text { Bar and cafe, large open } \\
\text { fire, Sky TV, outdoor } \\
\text { patio, banquet room (used } \\
\text { for meetings and } \\
\text { conferences). }\end{array}$ & $\begin{array}{l}\text { Each bedroom has bathroom } \\
\text { and toilet (one suitable for } \\
\text { disabled), heating and lighting. }\end{array}$ & \multirow{2}{*}{$\begin{array}{l}\text { Food, wine and beer, } \\
\text { beverages, } \\
\text { homemade biscuits } \\
\text { and cakes, OCRT, } \\
\text { mountain biking, } \\
\text { curling, goldfield } \\
\text { heritage, museum, } \\
\text { golfing, fishing, } \\
\text { hunting, 4WD, horse } \\
\text { riding, walking, } \\
\text { regular events, } \\
\text { upcoming events. }\end{array}$} & Timber \\
\hline 90 & Naseby & $\begin{array}{l}\text { Royal Hotel (self } \\
\text { contained) }\end{array}$ & $1 \times \mathrm{SC}$ & $\mathrm{RB}$ & $\begin{array}{l}\text { Self contained uses shared } \\
\text { facilities with hotel. }\end{array}$ & $\begin{array}{l}\text { Each bedroom with ensuite } \\
\text { bathroom, heating and lighting. }\end{array}$ & & Timber \\
\hline 91 & Oturehua & $\begin{array}{l}\text { Crowsnest } \\
\text { Accommodation } \\
\text { (bunkrooms/lodge) }\end{array}$ & $1 \times \mathrm{Bp}$ & New & $\begin{array}{l}\text { Kitchen, toilet, bathroom, } \\
\text { outdoor cooking and } \\
\text { eating area. }\end{array}$ & $\begin{array}{l}\text { Each bedroom heating and } \\
\text { lighting. }\end{array}$ & \multirow{3}{*}{$\begin{array}{l}\text { Food and beverages, } \\
\text { OCRT, curling on the } \\
\text { Ida Dam, Hayes } \\
\text { Engineering, the Brass } \\
\text { Monkey motorcycle } \\
\text { rally, farm tours, gold } \\
\text { fields heritage, } \\
\text { hunting and fishing, } \\
\text { garden tours. }\end{array}$} & Timber \\
\hline 92 & Oturehua & $\begin{array}{l}\text { Crowsnest } \\
\text { Accommodation (2 } \\
\text { cabins) }\end{array}$ & $2 \times \mathrm{SC}$ & New & $\begin{array}{l}\text { Both cabins have small } \\
\text { kitchen, toilet and } \\
\text { bathroom. }\end{array}$ & $\begin{array}{l}\text { Bothe cabins with heating and } \\
\text { lighting. }\end{array}$ & & Timber \\
\hline 94 & Oturehua & $\begin{array}{l}\text { Crowsnest } \\
\text { Accommodation (tent } \\
\text { sites and powered } \\
\text { sites) }\end{array}$ & $1 \times$ Camping & - & $\begin{array}{l}\text { Guest toilet, bathroom, } \\
\text { laundry facilities, outdoor } \\
\text { bath, hot tub (does not use } \\
\text { electricity have a pump or } \\
\text { need chemicals), BBQ } \\
\text { area. }\end{array}$ & - & & - \\
\hline 95 & Oturehua & Hawk Rock Cottage & $1 \times \mathrm{B} \& \mathrm{~B}$ & New & $\begin{array}{l}\text { Full equipped kitchen } \\
\text { with freezer, fridge, } \\
\text { dishwasher, microwave } \\
\text { and toaster, fireplace, } \\
\text { shower, fire wood supply, } \\
\text { washing machine, iron, } \\
\text { BBQ, TV\& DVD player, } \\
\text { cell phone coverage. }\end{array}$ & $\begin{array}{l}3 \text { bedrooms have hair dryers, } \\
\text { heating and lighting. }\end{array}$ & $\begin{array}{l}\text { Continental breakfast, } \\
\text { OCRT, natural views } \\
\text { and landscape, } \\
\text { (children welcome), } \\
\text { fishing, Oturehua local } \\
\text { village, historic } \\
\text { buildings, historic }\end{array}$ & Stone \\
\hline
\end{tabular}




\begin{tabular}{|c|c|c|c|c|c|c|c|c|}
\hline & & & & & & & $\begin{array}{l}\text { Hayes Engineering, } \\
\text { Golden progress mine } \\
\text { (heritage), 4WD tour. }\end{array}$ & \\
\hline 96 & Oturehua & Hill Creek Hideaway & $1 \times \mathrm{B} \& \mathrm{~B}$ & New & $\begin{array}{l}\text { Shared bathroom with } \\
\text { separate shower. }\end{array}$ & $\begin{array}{l}\text { Bedrooms with lighting and } \\
\text { heating. }\end{array}$ & $\begin{array}{l}\text { Continental breakfast, } \\
\text { food, OCRT, Oturehua } \\
\text { local village, historic } \\
\text { sites and buildings, } \\
\text { views of Hawkdun and } \\
\text { Rough Ridges ranges, } \\
\text { local transportation. }\end{array}$ & $\begin{array}{l}\text { Assumed } \\
\text { concrete }\end{array}$ \\
\hline 97 & Oturehua & Inver Lair lodge & $1 \times \mathrm{B} \& \mathrm{~B}$ & New & $\begin{array}{l}\text { Full kitchen, wheelchair } \\
\text { friendly facilities, outdoor } \\
\text { spa pool, fire place, BBQ, } \\
\text { TV and DVD player. }\end{array}$ & $\begin{array}{l}6 \text { stylish ensuite rooms with } \\
\text { bathroom, toilet, heating and } \\
\text { lighting. }\end{array}$ & $\begin{array}{l}\text { BBQ packed lunch } \\
\text { (meats, bread \& } \\
\text { salad), OCRT, book } \\
\text { and DVDs, curling, ice } \\
\text { luge, 4WD tour, } \\
\text { hunting and fishing, } \\
\text { golf. }\end{array}$ & Stone \\
\hline 98 & Oturehua & $\begin{array}{l}\text { Oturehua Lodge \& } \\
\text { Cottage (1Queen, } \\
\text { twin rooms cottage) }\end{array}$ & $1 \times B \& B$ & New & \multirow{3}{*}{$\begin{array}{l}\text { Indoor heated pool, spa, } \\
\text { sunroom/ reading room, } \\
\text { games room. Each cottage } \\
\text { has kitchen, fire place and } \\
\text { TV. }\end{array}$} & $\begin{array}{l}\text { Each cottage with private } \\
\text { bathroom and toilet, heating, } \\
\text { lighting. }\end{array}$ & \multirow{3}{*}{$\begin{array}{l}\text { OCRT, side trips to } \\
\text { Naseby and St } \\
\text { Bathans, access to } \\
\text { nearby places, } \\
\text { activities and } \\
\text { attractions books and } \\
\text { magazines, touring, } \\
\text { trekking, fishing, } \\
\text { hunting. }\end{array}$} & Timber \\
\hline 99 & Oturehua & $\begin{array}{l}\text { Oturehua Lodge } \& \\
\text { Cottage }(1 \times 2 \\
\text { bedroom cottage })\end{array}$ & $1 \times \mathrm{SC}$ & New & & $\begin{array}{l}\text { Each cottage with private } \\
\text { bathroom and toilet, heating, } \\
\text { lighting. }\end{array}$ & & Timber \\
\hline 100 & Oturehua & $\begin{array}{l}\text { Oturehua Lodge \& } \\
\text { Cottage }(2 \times \text { studio } \\
\text { cottage })\end{array}$ & $1 \times \mathrm{SC}$ & New & & $\begin{array}{l}\text { Each cottage with private } \\
\text { bathroom and toilet, heating, } \\
\text { lighting. }\end{array}$ & & Timber \\
\hline 101 & Oturehua & $\begin{array}{l}\text { Oturehua Tavern and } \\
\text { Self Contained }\end{array}$ & $1 \times \mathrm{SC}$ & $\begin{array}{l}\text { RB } \\
\text { (tavern) } \\
\text { New SC }\end{array}$ & $\begin{array}{l}\text { Kitchen sink and } \\
\text { crockery, shower, BBQ. }\end{array}$ & Heating and lighting & $\begin{array}{l}\text { Home cooked meals, } \\
\text { continental breakfast, } \\
\text { vegetable, beverages } \\
\text { (alcoholic and non- } \\
\text { alcoholic), beer } \\
\text { garden, OCRT, Hayes } \\
\text { engineering, curling, } \\
\text { fishing, historic gold } \\
\text { mine site. }\end{array}$ & $\begin{array}{l}\text { Container- } \\
\text { cottage }\end{array}$ \\
\hline
\end{tabular}




\begin{tabular}{|c|c|c|c|c|c|c|c|c|}
\hline 102 & Oturehua & The Mill B\&B & $1 \times B \& B$ & $\mathrm{RB}$ & $\begin{array}{l}\text { Separate lounge with Sky } \\
\text { TV, twin room with } \\
\text { shared facilities. }\end{array}$ & $\begin{array}{l}\text { Two bedrooms with claw foot } \\
\text { bath and a shower, all bedrooms } \\
\text { with heating and lighting. }\end{array}$ & $\begin{array}{l}\text { Full country breakfast, } \\
\text { OCRT, [using } \\
\text { Oturehua attractions]. }\end{array}$ & $\begin{array}{l}\text { Quarried } \\
\text { stone }\end{array}$ \\
\hline 103 & Oturehua & The Old Shop B\&B & $1 \times \mathrm{B} \& \mathrm{~B}$ & $\mathrm{RB}$ & $\begin{array}{l}\text { Full bathroom, sitting } \\
\text { room with tea and coffee } \\
\text { making facilities. }\end{array}$ & $\begin{array}{l}\text { Bedrooms with heating and } \\
\text { lighting. }\end{array}$ & $\begin{array}{l}\text { OCRT sheltered } \\
\text { garden, central } \\
\text { Otago's summer skies, } \\
\text { Hayes Engineering } \\
\text { works, gold mine } \\
\text { sites. }\end{array}$ & $\begin{array}{l}\text { Assumed } \\
\text { timber }\end{array}$ \\
\hline 104 & St Bathans & Cambrian Station & $1 \times \mathrm{SC}$ & $\mathrm{RB}$ & $\begin{array}{l}\text { Fully equipped kitchen } \\
\text { including fridge/freezer, } \\
\text { microwave, stove/oven, } \\
\text { fully facility bathroom, } \\
\text { heaters and hair dryer, } \\
\text { TV, radio, stereo, washing } \\
\text { machine, BBQ, (firewood } \\
\text { provided), separate } \\
\text { laundry area with extra } \\
\text { toilets and showers. }\end{array}$ & $\begin{array}{l}\text { Bedrooms with heating and } \\
\text { lighting. }\end{array}$ & $\begin{array}{l}\text { OCRT, Otago gold } \\
\text { fields, St Bathans } \\
\text { historic mining } \\
\text { township, Blue lake, } \\
\text { games and books. }\end{array}$ & Mud brick \\
\hline 105 & St Bathans & $\begin{array}{l}\text { Constable Cottage \& } \\
\text { Gaol (cottage) }\end{array}$ & $1 \times \mathrm{Sc}$ & $\mathrm{RB}$ & $\begin{array}{l}\text { Kitchen, lounge, bathroom } \\
\text { and toilet, TV. }\end{array}$ & $\begin{array}{l}\text { Bedrooms with heating, } \\
\text { lighting. }\end{array}$ & \multirow[b]{2}{*}{$\begin{array}{l}\text { Continental breakfast, } \\
\text { meals are available at } \\
\text { Vulcan hotel, OCRT, } \\
\text { explore the township } \\
\text { and its buildings, the } \\
\text { remnants of } 19 \\
\text { century gold mining, } \\
\text { Oteake conservation } \\
\text { park, neighbouring } \\
\text { historic towns, ice } \\
\text { skating and curling at } \\
\text { Naseby or Alexandra, } \\
\text { art deco architecture } \\
\text { at Ranfurly, visit Ophir } \\
\text { and Daniel O'Connell } \\
\text { memorial bridge, Blue } \\
\text { lake. }\end{array}$} & Timber \\
\hline 106 & St Bathans & $\begin{array}{l}\text { Constable Cottage \& } \\
\text { Gaol (gaol) }\end{array}$ & $1 \times \mathrm{SC}$ & $\mathrm{RB}$ & Small kitchen, TV. & Heating and lighting. & & Timber \\
\hline
\end{tabular}




\begin{tabular}{|c|c|c|c|c|c|c|c|c|}
\hline 107 & St Bathans & Lombardy Cottage & $1 \times \mathrm{SC}$ & $\mathrm{RB}$ & $\begin{array}{l}\text { Kitchen- sitting room , } \\
\text { two bathrooms, under } \\
\text { floor heating, an open fire, } \\
\text { coal range and oil-fired } \\
\text { heater, a laundry facilities, } \\
\text { gas BBQ, an outdoor hot } \\
\text { tub, stocked woodshed, } \\
\text { high speed internet. }\end{array}$ & $\begin{array}{l}\text { Bedrooms with heating and } \\
\text { lighting. }\end{array}$ & $\begin{array}{l}\text { OCRT, wine and beer, } \\
\text { an in-season } \\
\text { vegetable garden, a } \\
\text { book-lined study, } \\
\text { scenery, historic sites } \\
\text { and buildings, } \\
\text { kayaking, mountain } \\
\text { biking. }\end{array}$ & Stone \\
\hline 108 & St Bathans & $\begin{array}{l}\text { Lombardy Cottage } \\
\text { (caravan) }\end{array}$ & $1 \times \mathrm{SC}$ & New & - & $\begin{array}{l}\text { 4.5m , } 1977 \text { Zephyr caravan } \\
\text { which sleeps four (two singles } \\
\text { and a double berth), with an } \\
\text { additional queen bed }\end{array}$ & $\begin{array}{l}\text { Using shared products } \\
\text { and attractions which } \\
\text { are available at the } \\
\text { cottage. }\end{array}$ & - \\
\hline 109 & St Bathans & St Bathans B\&B & $1 \times B \& B$ & New & $\begin{array}{l}\text { Sitting room with lounge, } \\
\text { wood burner. }\end{array}$ & $\begin{array}{l}\text { All bedrooms have bathroom, } \\
\text { toiletries, tea/coffee making } \\
\text { facilities, free DVDs, heating } \\
\text { and lighting. } 2 \text { rooms have } \\
\text { private balcony. }\end{array}$ & $\begin{array}{l}\text { Food and full cooked } \\
\text { breakfast, OCRT, the } \\
\text { village of St Bathans, } \\
\text { Otago goldfields' park, } \\
\text { historic precinct with } \\
\text { the original buildings, } \\
\text { seasonal vistas, art } \\
\text { works for sale, } \\
\text { kayaking, horse riding, } \\
\text { boating, fishing, rabbit } \\
\text { shooting, swimming, } \\
\text { Triathlon track, } \\
\text { photography, ice } \\
\text { skating, falls dam. }\end{array}$ & Timber \\
\hline 110 & St Bathans & Vulcan Hotel & $1 \times$ Hotel & $\mathrm{RB}$ & $\begin{array}{l}\text { Self-catering kitchen, } \\
\text { shared bathroom facilities, } \\
\text { a deep bath, and veranda, } \\
\text { TV / CD player. }\end{array}$ & $\begin{array}{l}\text { All bedrooms with heating and } \\
\text { lighting. }\end{array}$ & $\begin{array}{l}\text { Food and breakfast, } \\
\text { OCRT, Blue lake, lunar } \\
\text { landscape, historical } \\
\text { buildings and sites, } \\
\text { walking, photography } \\
\text { share, using products } \\
\text { and attraction which } \\
\text { are available at } \\
\text { adjacent towns and }\end{array}$ & Mud brick \\
\hline
\end{tabular}




\begin{tabular}{|c|c|c|c|c|c|c|c|c|}
\hline & & & & & & & villages. & \\
\hline 111 & Ida Valley & Parkside Farm Stay & $1 \times B \& B$ & New & $\begin{array}{l}\text { Shared bathroom facility, } \\
\text { sleep-out with a double } \\
\text { and single bed. }\end{array}$ & $\begin{array}{l}\text { All bedrooms with heating and } \\
\text { lighting. }\end{array}$ & $\begin{array}{l}\text { Farm meals, OCRT, } \\
\text { historic site and } \\
\text { buildings, country } \\
\text { garden, scenery, Ida } \\
\text { valley, } 816 \text { ha farm, } \\
\text { farming activities, } \\
\text { farm tour, walking, } \\
\text { fishing curling (at } \\
\text { Naseby), mountain } \\
\text { bike, golf, } \\
\text { photography. }\end{array}$ & $\begin{array}{l}\text { Assumed } \\
\text { timber }\end{array}$ \\
\hline 112 & Auripo & Poolburn Hotel & $1 \times$ Hotel & $\mathrm{RB}$ & $\begin{array}{l}\text { Dining room, shared } \\
\text { garden bar, } 3 \text { shared } \\
\text { bathrooms, outdoor sitting } \\
\text { with BBQ area. }\end{array}$ & $\begin{array}{l}\text { Bedrooms with lighting. } \\
\text { (Heating information N/A). }\end{array}$ & $\begin{array}{l}\text { Food, wine and beer, } \\
\text { OCRT, Poolburn } \\
\text { Gorge, historic sites } \\
\text { and buildings, } \\
\text { photography, } \\
\text { landscape view, using } \\
\text { products and activities } \\
\text { at adjacent towns and } \\
\text { villages such as } \\
\text { Omakau and Ophir } \\
\text { and Naseby. }\end{array}$ & $\begin{array}{l}\text { Mud brick } \\
\text { and timber }\end{array}$ \\
\hline 113 & Auripo & Taradale Homestead & $1 \times$ Homestead & $\mathrm{RB}$ & $\begin{array}{l}\text { Shared sun-room, dining } \\
\text { room. }\end{array}$ & $\begin{array}{l}\text { Ensuite (one room), private } \\
\text { shower and toilet, heating and } \\
\text { lighting. }\end{array}$ & $\begin{array}{l}\text { Continental breakfast, } \\
\text { home cooked dinner, } \\
\text { OCRT, fishing, curling, } \\
\text { skating, tour Hayes } \\
\text { historic engineering } \\
\text { works, visit gold mine, } \\
\text { historic store and } \\
\text { museum (Oturehua) } \\
\text { Naseby and Ophir. }\end{array}$ & Mud brick \\
\hline
\end{tabular}




\begin{tabular}{|c|c|c|c|c|c|c|c|c|}
\hline 114 & Becks & White Horse Hotel & $1 \times$ Hotel & $\mathrm{RB}$ & $\begin{array}{l}7 \text { rooms with shared } \\
\text { facilities including } \\
\text { bathrooms and toilets, } 2 \\
\text { sitting rooms, dining } \\
\text { room. }\end{array}$ & $\begin{array}{l}\text { All bedrooms with heating and } \\
\text { lighting. }\end{array}$ & $\begin{array}{l}\text { Food, continental } \\
\text { breakfast, wines, } \\
\text { OCRT, visiting } \\
\text { historical sites and } \\
\text { buildings, adjacent } \\
\text { villages and town, } \\
\text { fishing, curling, horse } \\
\text { riding, ice skating, } \\
\text { scenery and } \\
\text { photography, local } \\
\text { transportation along } \\
\text { the OCRT. }\end{array}$ & $\begin{array}{l}\text { A mixture of } \\
\text { timber and } \\
\text { stacked stone } \\
\text { schist slabs } \\
\text { with } \\
\text { corrugated } \\
\text { iron roof. }\end{array}$ \\
\hline 115 & Lauder & Big Sky Cottage & $1 \times \mathrm{SC}$ & $\mathrm{RB}$ & $\begin{array}{l}\text { Equipped kitchen (electric } \\
\text { cooker and oven, fridge } \\
\text { freezer, microwave), } \\
\text { bathroom with shower, } \\
\text { heated towel rail and } \\
\text { heater, hair dryer, log } \\
\text { burner, washing machine } \\
\text { and ironing facility, } \\
\text { veranda, sheltered sun- } \\
\text { trap garden, TV, DVD } \\
\text { player, CD/radio, tape } \\
\text { player. }\end{array}$ & $\begin{array}{l}\text { All bedrooms with electric } \\
\text { blanket, oil filled electric heater } \\
\text { and lighting. }\end{array}$ & $\begin{array}{l}\text { Continental breakfast, } \\
\text { OCRT, wine, books, } \\
\text { maps and local } \\
\text { information, selection } \\
\text { of CDs, DVDs and } \\
\text { tapes, historical } \\
\text { bridges, historic sites } \\
\text { and buildings, walking, } \\
\text { fishing, ice skating and } \\
\text { curling at Naseby, } \\
\text { landscape view and } \\
\text { photography. }\end{array}$ & Mud brick \\
\hline 116 & Lauder & $\begin{array}{l}\text { Lauder Rail Trail } \\
\text { School and Milmor } \\
\text { Cottage (3 school } \\
\text { ensuite rooms) }\end{array}$ & $1 \times \mathrm{B} \& \mathrm{~B}$ & $\mathrm{RB}$ & $\begin{array}{l}\text { Kitchen, spa pool and } \\
\text { school garden (share with } \\
\text { other Lauder Rail Trail } \\
\text { School and Milmor } \\
\text { accommodation options), } \\
\text { TV and wireless internet. }\end{array}$ & $\begin{array}{l}\text { Each of } 3 \text { ensuite rooms with } \\
\text { bathroom and toilet, hairdryer, } \\
\text { electric blankets, heating and } \\
\text { lighting. }\end{array}$ & \multirow{2}{*}{$\begin{array}{l}\text { Continental breakfast, } \\
\text { home baking, wine } \\
\text { and beer, OCRT, } \\
\text { library, art room, } \\
\text { historic bridges and } \\
\text { tunnels, historic } \\
\text { adjacent sites, garden } \\
\text { tour, villages and } \\
\text { towns, horse riding, } \\
\text { goldfield mining, } \\
\text { fishing, golf, curling }\end{array}$} & Timber \\
\hline 117 & Lauder & $\begin{array}{l}\text { Lauder Rail Trail } \\
\text { School and Milmor } \\
\text { Cottage (studio } \\
\text { cottages) }\end{array}$ & $1 \times \mathrm{SC}$ & New & $\begin{array}{l}\text { Kitchen, spa pool and } \\
\text { school garden (shared } \\
\text { with other Lauder Rail } \\
\text { Trail School and Milmor } \\
\text { accommodation options), } \\
\text { TV and wireless internet. }\end{array}$ & $\begin{array}{l}\text { All cottages with private } \\
\text { bathroom and toilet, all } \\
\text { bedrooms with heater, electric } \\
\text { blankets, hair dryer and } \\
\text { lighting. }\end{array}$ & & Timber \\
\hline
\end{tabular}




\begin{tabular}{|c|c|c|c|c|c|c|c|c|}
\hline 118 & Lauder & $\begin{array}{l}\text { Lauder Rail Trail } \\
\text { School and Milmor } \\
\text { Cottage (standard } \\
\text { rooms) }\end{array}$ & $1 \times \mathrm{SC}$ & New & $\begin{array}{l}\text { Kitchen, spa pool and } \\
\text { school garden (share with } \\
\text { other Lauder Rail Trail } \\
\text { School and Milmor } \\
\text { accommodation options), } \\
\text { TV and wireless internet. }\end{array}$ & $\begin{array}{l}\text { The cottage with private } \\
\text { bathroom and toilet, all } \\
\text { bedrooms with heater, electric } \\
\text { blankets, hair dryer and } \\
\text { lighting. }\end{array}$ & \multirow[t]{3}{*}{$\begin{array}{l}\text { and ice skating at } \\
\text { Naseby, landscape } \\
\text { views and } \\
\text { photography, local } \\
\text { transportation along } \\
\text { the OCRT. }\end{array}$} & Timber \\
\hline 119 & Lauder & $\begin{array}{l}\text { Lauder Rail Trail } \\
\text { School and Milmor } \\
\text { Cottage (cabin) }\end{array}$ & $1 \times \mathrm{SC}$ & New & $\begin{array}{l}\text { Small kitchen, use shared } \\
\text { bathroom and toilet, using } \\
\text { shared facilities with other } \\
\text { Lauder Rail Trail School } \\
\text { and Milmor } \\
\text { accommodation options, } \\
\text { TV and wireless internet. }\end{array}$ & $\begin{array}{l}\text { The cabin with heater, electric } \\
\text { blankets, and lighting. }\end{array}$ & & Timber \\
\hline 120 & Lauder & $\begin{array}{l}\text { Lauder Rail Trail } \\
\text { School and Milmor } \\
\text { Cottage (Milmor } \\
\text { cottage) }\end{array}$ & $1 \times \mathrm{SC}$ & New & $\begin{array}{l}\text { Equipped kitchen, shared } \\
\text { bathroom, complimented } \\
\text { with a second toilet, } \\
\text { hairdryers, TV and } \\
\text { wireless internet. }\end{array}$ & $\begin{array}{l}3 \text { bedrooms with heating, } \\
\text { electric blankets and lighting. }\end{array}$ & & Timber \\
\hline 121 & Lauder & $\begin{array}{l}\text { Lauder Store B\&B } \\
\text { (the old store) }\end{array}$ & $1 \times \mathrm{SC}$ & $\mathrm{RB}$ & $\begin{array}{l}\text { A kitchen, a shared fully } \\
\text { equipped bathroom, guest } \\
\text { lounge, outdoor } \\
\text { entertaining area with } \\
\text { BBQ, multi fuel log } \\
\text { burner, electric heater } \\
\text { (used for guest lounge), } \\
\text { disabled facilities, play } \\
\text { ground for children, } \\
\text { laundry facilities. }\end{array}$ & $\begin{array}{l}\text { All bedrooms have electric } \\
\text { blankets, bedside lights, electric } \\
\text { wall heater and lighting. }\end{array}$ & \multirow{2}{*}{$\begin{array}{l}\text { Food and beverages, } \\
\text { OCRT, tours to various } \\
\text { historical gold mining } \\
\text { sites including } \\
\text { Matakanui, Cambrian, } \\
\text { St Bathans Naseby } \\
\text { and Falls Dam, historic } \\
\text { bridges and tunnels, } \\
\text { horse riding, fishing, } \\
\text { golf course, hiking, } \\
\text { mountain biking, } \\
\text { winter sports and } \\
\text { curling at Naseby, } \\
\text { scenery a } \\
\text { photography, 4WD } \\
\text { tour. }\end{array}$} & $\begin{array}{l}\text { Mix stone and } \\
\text { timber }\end{array}$ \\
\hline 122 & Lauder & $\begin{array}{l}\text { Lauder Store B\&B } \\
\text { (Storekeeper house) }\end{array}$ & $1 \times \mathrm{SC}$ & New & $\begin{array}{l}\text { A kitchen, a shared fully } \\
\text { equipped bathroom, guest } \\
\text { lounge, outdoor } \\
\text { entertaining area with } \\
\text { BBQ, multi fuel log } \\
\text { burner, electric heater } \\
\text { (used for guest lounge), } \\
\text { disabled facilities, play } \\
\text { ground for children, } \\
\text { laundry facilities. }\end{array}$ & $\begin{array}{l}\text { All bedrooms have electric } \\
\text { blankets, bedside lights, electric } \\
\text { wall heater and lighting. }\end{array}$ & & Timber \\
\hline
\end{tabular}




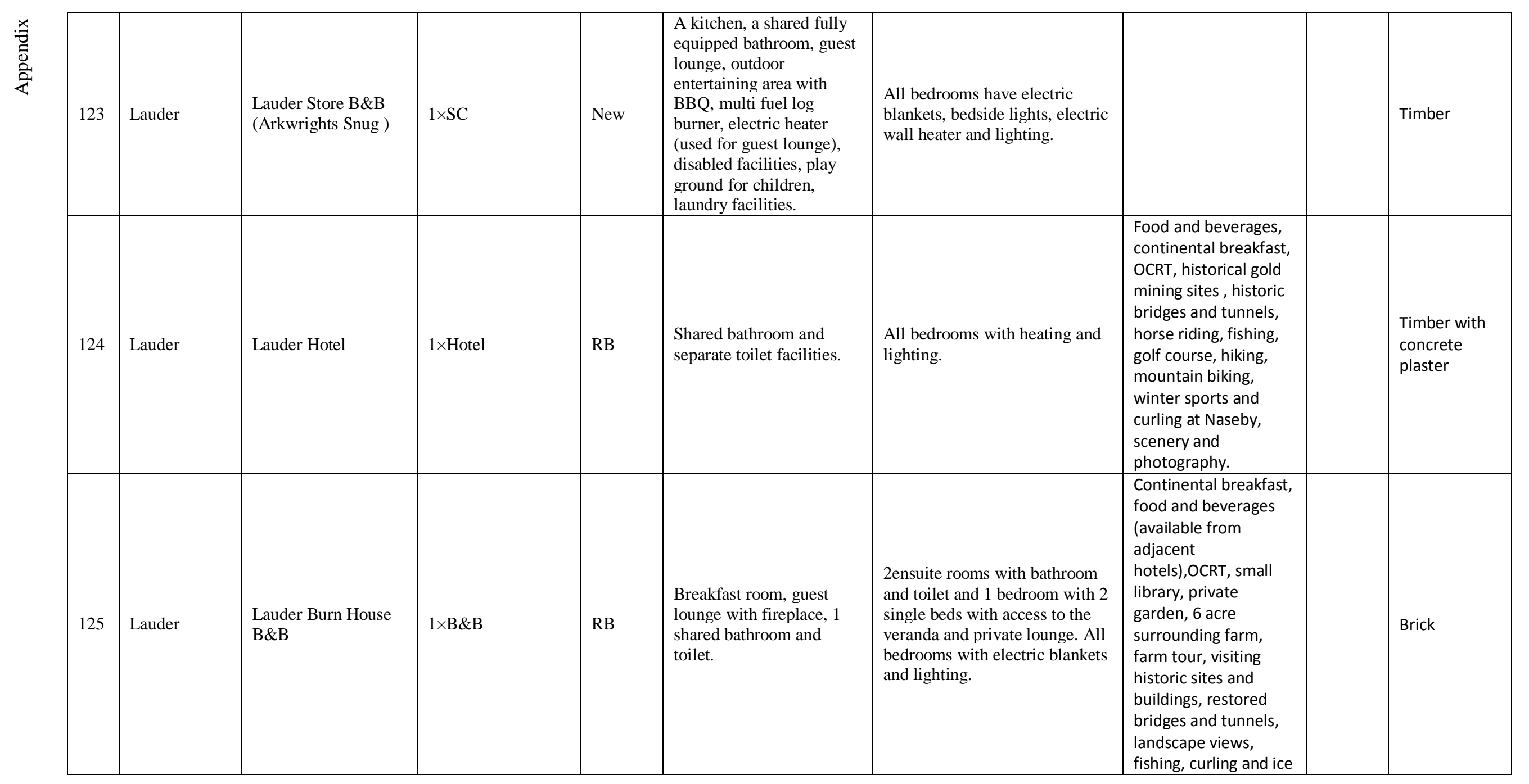




\begin{tabular}{|c|c|c|c|c|c|c|c|c|}
\hline & & & & & & & skating at Naseby. & \\
\hline 126 & Lauder & $\begin{array}{l}\text { Lauderdale Estate } \\
\text { (cottages) }\end{array}$ & $1 \times \mathrm{B} \& \mathrm{~B}$ & $\mathrm{RB}$ & $\begin{array}{l}4 \text { double with shared } \\
\text { bathrooms, } 1 \text { twin with } \\
\text { shared bathroom, living } \\
\text { room, commercial kitchen } \\
\text { used for events. }\end{array}$ & $\begin{array}{l}2 \text { double en suite, } 1 \text { twin en } \\
\text { suite with bathroom and toilet. } \\
\text { All bedrooms with heating and } \\
\text { lighting. }\end{array}$ & $\begin{array}{l}\text { OCRT, food and } \\
\text { beverages, wine, beer, } \\
\text { surrounded by } \\
\text { farmland, separate } 20 \\
\text { acres of park used as } \\
\text { sitting area, farm tour, } \\
\text { exploring historic sites } \\
\text { and buildings, fishing, } \\
\text { mountain biking, } \\
\text { curling and winter } \\
\text { sports at Naseby, } \\
\text { landscape views and } \\
\text { photography, a centre } \\
\text { for wedding } \\
\text { celebration and } \\
\text { parties. }\end{array}$ & $\begin{array}{l}\text { Stone and } \\
\text { mud brick } \\
\text { (original } \\
\text { buildings)- } \\
100 \text { year old } \\
\text { wooden } \\
\text { chalets } \\
\text { moved from } \\
\text { Pleasant } \\
\text { valley }\end{array}$ \\
\hline 127 & Lauder & Muddy Creek & $1 \times \mathrm{SC}$ & $\mathrm{RB}$ & $\begin{array}{l}5 \text { bed rooms, full kitchen } \\
\text { with gas stove, electric } \\
\text { wok, microwave and } \\
\text { fridge, several resting/ } \\
\text { lounging spaces, two } \\
\text { shared toilets /bathroom } \\
\text { with under floor heating, } \\
5 \text {-person sauna, an open } \\
\text { fire and a garden room, a } \\
\text { piano, TV and landline } \\
\text { phone. }\end{array}$ & $\begin{array}{l}\text { All bed rooms with heating and } \\
\text { lighting. }\end{array}$ & $\begin{array}{l}\text { Farm made food and } \\
\text { vegetables, OCRT, } \\
\text { farm tour, exploring } \\
\text { historic sites and } \\
\text { buildings, fishing, } \\
\text { mountain biking, } \\
\text { curling and winter } \\
\text { sports at Naseby, } \\
\text { walking, landscape } \\
\text { views and } \\
\text { photography. }\end{array}$ & Mud brick \\
\hline 128 & Lauder & Pedal Inn & $1 \times \mathrm{SC}$ & $\mathrm{RB}$ & $\begin{array}{l}\text { Two cottages each has } \\
\text { kitchenette that has } \\
\text { microwave, fridge and } \\
\text { sink, coffee and tea } \\
\text { making facilities. }\end{array}$ & $\begin{array}{l}\text { Two cottages each has ensuite } \\
\text { bathroom, one with disabled } \\
\text { access. All bed rooms with } \\
\text { heating and lighting. }\end{array}$ & $\begin{array}{l}\text { Continental breakfast } \\
\text { and/or farm fresh } \\
\text { eggs, OCRT, } 485 \text { ha } \\
\text { farm, farm tour, } \\
\text { exploring historic sites } \\
\text { and buildings, fishing, }\end{array}$ & $\begin{array}{l}\text { Assumed } \\
\text { timber }\end{array}$ \\
\hline
\end{tabular}




\begin{tabular}{|c|c|c|c|c|c|c|c|c|c|}
\hline & & & & & & & $\begin{array}{l}\text { mountain biking, } \\
\text { curling and winter } \\
\text { sports at Naseby, } \\
\text { landscape views and } \\
\text { photography. }\end{array}$ & & \\
\hline 129 & Moa Creek & $\begin{array}{l}\text { Bonspiel Station \& } \\
\text { Old Moa Creek hotel }\end{array}$ & $1 \times$ Hotel & New & $\begin{array}{l}4 \text { rooms plus sleep out, } \\
\text { Spa and sauna, two } \\
\text { bathrooms, a lounge and a } \\
\text { separate bar area are all } \\
\text { fully furnished, using } 2 \\
\text { open fire places for } \\
\text { warming the rooms. }\end{array}$ & Sleeping bags required. & $\begin{array}{l}\text { OCRT, heritage } \\
\text { exploring, walking, } \\
\text { mountain biking, } \\
\text { riding the Dunstan } \\
\text { Trail, gold panning, } \\
\text { river swimming, } \\
\text { canoeing, fishing, } \\
\text { hunting, 4WD driving. } \\
\text { Chinese miner's hut } \\
\text { (as accommodation } \\
\text { without electricity and } \\
\text { running water). }\end{array}$ & & Stone \\
\hline 130 & Poolburn & Poolburn Hotel & $1 \times$ Hotel & $\mathrm{RB}$ & $\begin{array}{l}\text { Three shared bathrooms, } \\
\text { dining room, garden bar, } \\
\text { BBQ. }\end{array}$ & $\begin{array}{l}8 \text { rooms with heating and } \\
\text { lighting. }\end{array}$ & $\begin{array}{l}\text { Food, wine and beer, } \\
\text { OCRT, exploring } \\
\text { historic sites and } \\
\text { buildings, visiting } \\
\text { Poolburn dam (film } \\
\text { site of Rohan), } \\
\text { mountain biking, road } \\
\text { biking, kayaking, } \\
\text { fishing, curling and ice } \\
\text { skating at Naseby. }\end{array}$ & & Timber \\
\hline 131 & Poolburn & $\begin{array}{l}\text { Solandra Lodge } \\
\text { country style B\&B }\end{array}$ & $1 \times \mathrm{B} \& \mathrm{~B}$ & New & $\begin{array}{l}\text { Self contained kitchen } \\
\text { with microwave, oven, } \\
\text { full size fridge freezer, } \\
\text { dishwasher, } 1 \text { shared } \\
\text { bathroom and a separate } \\
\text { toilet with under floor } \\
\text { heating, } 1 \text { kitchenette, two } \\
\text { lounges, } 2 \text { heat pumps, } \\
\text { TV/ DVD, projector, } \\
\end{array}$ & $\begin{array}{l}\text { All bedrooms with under floor } \\
\text { heating and lighting. }\end{array}$ & $\begin{array}{l}\text { Food, wine and beer, } \\
\text { OCRT, exploring } \\
\text { historic sites and } \\
\text { buildings, visiting } \\
\text { Poolburn dam (film } \\
\text { site of Rohan), } \\
\text { mountain biking, road } \\
\text { biking, kayaking, }\end{array}$ & 120 sqm & Timber \\
\hline
\end{tabular}




\begin{tabular}{|c|c|c|c|c|c|c|c|c|}
\hline & & & & & surround sound. & & $\begin{array}{l}\text { fishing, curling and ice } \\
\text { skating at Naseby, } \\
\text { bike hire. }\end{array}$ & \\
\hline 132 & Ophir & Blacks Hotel & $1 \times$ Hotel & $\mathrm{RB}$ & $\begin{array}{l}\text { Bar, } 100 \text { square metre } \\
\text { deck, dining room. }\end{array}$ & $\begin{array}{l}\text { All } 10 \text { rooms with ensuite } \\
\text { (bathroom and toilet), heating, } \\
\text { lighting, two rooms have } \\
\text { disabled facilities. }\end{array}$ & $\begin{array}{l}\text { Food and bar meals, } \\
\text { wine, beer, OCRT, } \\
\text { beer garden, Naseby, } \\
\text { Ranfurly and St. } \\
\text { Bathans, visit the } \\
\text { Daniel O'Connell } \\
\text { bridge, Manuherikia } \\
\text { river, fish for trout, } \\
\text { Ophir walk, 4WD } \\
\text { tracks, golf and tennis, } \\
\text { local transportation } \\
\text { along the OCRT. }\end{array}$ & Timber \\
\hline 133 & Ophir & Flannery Lodge & $1 \times B \& B$ & New & $\begin{array}{l}\text { Full kitchen, shared } \\
\text { bathroom and toilet (for } \\
\text { bunkrooms), BBQ, } \\
\text { wireless internet, laundry, } \\
\text { TV. }\end{array}$ & $\begin{array}{l}\text { Double rooms, both with } \\
\text { ensuites, bunkrooms, four or } \\
\text { five to a room, backpacker } \\
\text { style, all rooms with heating, } \\
\text { lighting. }\end{array}$ & $\begin{array}{l}\text { Food, vegetables, } \\
\text { wine and beer, OCRT, } \\
\text { books, Naseby, } \\
\text { Ranfurly and St. } \\
\text { Bathans, visit the } \\
\text { Daniel O'Connell } \\
\text { bridge, Manuherikia } \\
\text { river, fish for trout, } \\
\text { Ophir walk, golf and } \\
\text { tennis, local painting, } \\
\text { photography } \\
\text { workshop, tour (The } \\
\text { Best Bits tour), fishing. }\end{array}$ & $\begin{array}{l}\text { Concrete } \\
\text { blocks }\end{array}$ \\
\hline 134 & Ophir & $\begin{array}{l}\text { Flannery Lodge (tent } \\
\text { site) }\end{array}$ & 10×Camping & - & - & 25 person & $\begin{array}{l}\text { Food, vegetables, } \\
\text { wine and beer, OCRT, } \\
\text { books, Naseby, } \\
\text { Ranfurly and St. } \\
\text { Bathans, visit the } \\
\text { Daniel O'Connell } \\
\text { bridge, Manuherikia }\end{array}$ & - \\
\hline
\end{tabular}




\begin{tabular}{|c|c|c|c|c|c|c|c|c|}
\hline & & & & & & & $\begin{array}{l}\text { river, fish for trout, } \\
\text { Ophir walk, golf and } \\
\text { tennis, local painting, } \\
\text { photography } \\
\text { workshop, tour (The } \\
\text { Best Bits tour), fishing. }\end{array}$ & \\
\hline 135 & Ophir & Millfield Cottage & $1 \times \mathrm{SC}$ & New & $\begin{array}{l}\text { Fully furnished kitchen, } \\
\text { fridge/freezer, dishwasher, } \\
\text { shower, toilet facilities, } \\
\text { laundry, a log burner, } \\
\text { lounge and dining area, } \\
\text { BBQ on the deck outside, } \\
\text { Sky TV, DVD player. }\end{array}$ & $\begin{array}{l}\text { All bedrooms with heating and } \\
\text { lighting. }\end{array}$ & $\begin{array}{l}\text { Continental breakfast, } \\
\text { OCRT, rural views, } 20 \\
\text { minutes from } \\
\text { Alexandra, and } \\
\text { walking distance from } \\
\text { historic Ophir and } \\
\text { Omakau township } \\
\text { with shops, pub, } \\
\text { swimming pool and a } \\
\text { golf course, fishing } \\
\text { and swimming in } \\
\text { Manuherikia river. }\end{array}$ & Timber \\
\hline 136 & Ophir & Ophir Bridge B\&B & $1 \times \mathrm{B} \& \mathrm{~B}$ & New & $\begin{array}{l}\text { A shared bathroom with } \\
\text { under floor heating, } \\
\text { complimentary Nellie Tier } \\
\text { toiletries, hairdryer. }\end{array}$ & $\begin{array}{l}2 \text { bedrooms each with access to } \\
\text { its own outdoor area,, heating } \\
\text { and lighting. }\end{array}$ & $\begin{array}{l}\text { Continental breakfast } \\
\text { with homemade } \\
\text { products (yoghurt, } \\
\text { fresh fruits, muesli } \\
\text { etc), beverages, OCRT, } \\
\text { Ophir Village walk, } \\
\text { Matakanui and } \\
\text { Drybread, St Bathans } \\
\text { and Cambrian, golf at } \\
\text { Omakau, Poolburn } \\
\text { dam, Thompsons } \\
\text { Gorge, Clyde, } \\
\text { Alexandra Basin } \\
\text { Vineyards, Hayes } \\
\text { Engineering, 4WD } \\
\text { trips, word class } \\
\text { gardens, curling and }\end{array}$ & $\begin{array}{l}\text { Assumed } \\
\text { concrete }\end{array}$ \\
\hline
\end{tabular}




\begin{tabular}{|c|c|c|c|c|c|c|c|c|}
\hline & & & & & & & $\begin{array}{l}\text { luge at Naseby, } \\
\text { historic sites and } \\
\text { landscape. }\end{array}$ & \\
\hline 137 & Ophir & $\begin{array}{l}\text { The Old Bakery ( } \\
\text { Stables building) }\end{array}$ & $1 \times \mathrm{B} \& \mathrm{~B}$ & $\mathrm{RB}$ & $\begin{array}{l}\text { Full kitchen, bathroom } \\
\text { facilities, washing } \\
\text { machine, log burner, } \\
\text { BBQ, outdoors area ,TV, } \\
\text { a selection of DVDs. }\end{array}$ & $\begin{array}{l}\text { All bedrooms with heating and } \\
\text { lighting. }\end{array}$ & $\begin{array}{l}\text { Homemade breakfast, } \\
\text { farm fruits and eggs, } \\
\text { OCRT, walking, } \\
\text { historic Ophir, visiting } \\
\text { historic buildings and } \\
\text { sites, landscape views } \\
\text { and photography, } \\
\text { fishing, swimming, } \\
\text { golf course. }\end{array}$ & Schist stone \\
\hline 139 & Omakau & Church Hill B\&B & $1 \times \mathrm{B} \& \mathrm{~B}$ & $\mathrm{RB}$ & $\begin{array}{l}\text { Twin room with shared } \\
\text { facilities (bathroom and } \\
\text { toilet), lounge and dining } \\
\text { area, open fire, tea and } \\
\text { coffee making facilities, } \\
\text { outdoor areas, grazing for } \\
\text { horse trekkers, TV, DVD } \\
\text { player, stereo. }\end{array}$ & $\begin{array}{l}\text { Queen room with ensuite, } \\
\text { bathroom and toilet, all } \\
\text { bedrooms with heating, lighting. }\end{array}$ & $\begin{array}{l}\text { Breakfast, food (from } \\
\text { other restaurants and } \\
\text { hotels), wine, OCRT, } \\
\text { historic Ophir, } \\
\text { adjacent historic } \\
\text { Omakau Catholic } \\
\text { Church. }\end{array}$ & Red brick \\
\hline 140 & Omakau & Hawksview B\&B & $1 \times \mathrm{B} \& \mathrm{~B}$ & $\mathrm{RB}$ & $\begin{array}{l}\text { Fully equipped kitchen, } \\
\text { bathroom, washing and } \\
\text { drying facilities, outdoor } \\
\text { spa pool, garden sitting, } \\
\text { BBQ, lounge with TV, } \\
\text { free email access. }\end{array}$ & $\begin{array}{l}\text { All bedrooms with electric } \\
\text { heaters electric blankets and } \\
\text { lighting. }\end{array}$ & $\begin{array}{l}\text { Continental breakfast, } \\
\text { OCRT, historical sites } \\
\text { and heritage, } \\
\text { landscape views, } \\
\text { photography, } \\
\text { Omakau, books, } \\
\text { (swimming pool, golf } \\
\text { course and astro } \\
\text { tennis courts available } \\
\text { from other nearby } \\
\text { places and } \\
\text { accommodation } \\
\text { services). }\end{array}$ & $\begin{array}{l}\text { Red brick, } \\
\text { timber }\end{array}$ \\
\hline 141 & Omakau & Killarney Cottage & $1 \times \mathrm{SC}$ & RB & Kitchen, two bedrooms & 1 bedroom with ensuite. All & OCRT, historical sites & Timber, \\
\hline
\end{tabular}




\begin{tabular}{|c|c|c|c|c|c|c|c|c|}
\hline & & & & & $\begin{array}{l}\text { with a separate toilet and } \\
\text { shared bathroom and } \\
\text { shower, dining area, } \\
\text { laundry with machine } \\
\text { washing, sheltered } \\
\text { veranda. }\end{array}$ & $\begin{array}{l}\text { bedrooms with heating and } \\
\text { lighting. }\end{array}$ & $\begin{array}{l}\text { and heritage, } \\
\text { landscape views, } \\
\text { photography, } \\
\text { Omakau, books, } \\
\text { (swimming pool, golf } \\
\text { course and astro } \\
\text { tennis courts available } \\
\text { from other nearby } \\
\text { places and } \\
\text { accommodation } \\
\text { services). }\end{array}$ & $\begin{array}{l}\text { stucco finish } \\
\text { walls }\end{array}$ \\
\hline 142 & Omakau & $\begin{array}{l}\text { Omakau } \\
\text { Accommodation } \\
\text { Cottage \& Ensuite } \\
\text { Studio Units (units) }\end{array}$ & $1 \times \mathrm{SC}$ & New & laundry block & $\begin{array}{l}5 \text { Studio units each has: ensuite, } \\
\text { heating and lighting, a private } \\
\text { courtyard that includes outdoor } \\
\text { dining and bike stand, TV , each } \\
\text { bedroom has radio alarm clock } \\
\text { and electric blanket and hair } \\
\text { dryer. } 1 \text { unit with disabled } \\
\text { facilities. }\end{array}$ & $\begin{array}{l}\text { Continental breakfast, } \\
\text { wine, OCRT, view of } \\
\text { the Dunstan Ranges, } \\
\text { Manuherikia river, } \\
\text { landscape garden, } \\
\text { sports equipments, } \\
\text { tennis, golf course, } \\
\text { swimming, fishing, } \\
\text { walking, local } \\
\text { transportation along } \\
\text { the OCRT. }\end{array}$ & Timber \\
\hline 143 & Omakau & $\begin{array}{l}\text { Omakau } \\
\text { Accommodation } \\
\text { Cottage \& Ensuite } \\
\text { Studio Units } \\
\text { (cottage) }\end{array}$ & $1 \times \mathrm{SC}$ & New & $\begin{array}{l}\text { Full kitchen facilities with } \\
\text { fridge, oven, microwave, } \\
\text { dishwasher, one bathroom } \\
\text { and toilet, open plan } \\
\text { dining and living area } \\
\text { with TV and stereo, } \\
\text { landscape garden area. }\end{array}$ & $\begin{array}{l}\text { All bedrooms with heating and } \\
\text { lighting. }\end{array}$ & $\begin{array}{l}\text { Continental breakfast, } \\
\text { wine, OCRT, view of } \\
\text { the Dunstan Ranges, } \\
\text { Manuherikia river, } \\
\text { landscape garden, } \\
\text { sports equipment, } \\
\text { tennis, golf course, } \\
\text { swimming, fishing, } \\
\text { walking, local } \\
\text { transportation along } \\
\text { the OCRT. }\end{array}$ & Timber \\
\hline 144 & Omakau & $\begin{array}{l}\text { Omaka bed post } \\
\text { (Post Master's House } \\
\text { \& Post Office), (Post }\end{array}$ & $1 \times B \& B$ & New & $\begin{array}{l}2 \text { bathrooms, } 3 \text { toilets, } \\
\text { separate lounge with log } \\
\text { burner, separate living }\end{array}$ & $\begin{array}{l}\text { All bedrooms with heating and } \\
\text { lighting. }\end{array}$ & $\begin{array}{l}\text { Continental breakfast, } \\
\text { wine and beer, OCRT, }\end{array}$ & Red brick \\
\hline
\end{tabular}




\begin{tabular}{|c|c|c|c|c|c|c|c|c|}
\hline & & Master's House) & & & room. & & $\begin{array}{l}\text { historical sites and } \\
\text { buildings, wine and } \\
\text { beer, garden, } \\
\text { landscape views, } \\
\text { walking, photography. }\end{array}$ & \\
\hline 145 & Omakau & $\begin{array}{l}\text { Omaka Bed Post } \\
\text { (Post Master's House } \\
\& \text { post office), (post } \\
\text { office) }\end{array}$ & $1 \times B \& B$ & $\mathrm{RB}$ & $\begin{array}{l}\text { A fully equipped kitchen, } \\
\text { lounge, log burner, } \\
\text { bathroom and toilet. }\end{array}$ & $\begin{array}{l}\text { All bedrooms with heating and } \\
\text { lighting }\end{array}$ & $\begin{array}{l}\text { Continental breakfast, } \\
\text { wine and beer, OCRT, } \\
\text { historical sites and } \\
\text { buildings, wine and } \\
\text { beer, garden, } \\
\text { landscape views, } \\
\text { walking, photography. }\end{array}$ & $\begin{array}{l}\text { Triple brick } \\
\text { walls }\end{array}$ \\
\hline 146 & Omakau & $\begin{array}{l}\text { Omakau Commercial } \\
\text { Hotel }\end{array}$ & $1 \times$ Hotel & $\mathrm{RB}$ & $\begin{array}{l}5 \text { shared facilities rooms, } \\
\text { bar and cafe with Sky TV, } \\
\text { fire place, tea and coffee } \\
\text { facilities, laundry services, } \\
\text { irons and ironing boards, } \\
\text { disabled access facilities } \\
\text { in cafe, guests TV lounge, } \\
\text { internet and wireless } \\
\text { broadband. }\end{array}$ & $\begin{array}{l}4 \text { ensuite rooms each with } \\
\text { bathroom and toilet, } 2 \text { bedroom } \\
\text { cabin and bunkroom with } \\
\text { adjacent private ensuite. All } \\
\text { bedrooms with hairdryer, } \\
\text { electric blankets, heaters, } \\
\text { lighting and alarm clock. }\end{array}$ & $\begin{array}{l}\text { Food, breakfast, wine, } \\
\text { OCRT, garden area, } \\
\text { visiting historical sites } \\
\text { and buildings, } \\
\text { landscape views, } \\
\text { conference and } \\
\text { function facilities, golf, } \\
\text { tennis, swimming, } \\
\text { playground, historic } \\
\text { Ophir, guided tours. }\end{array}$ & Schist stone \\
\hline 147 & Omakau & Peddl'n Away B\&B & $1 \times \mathrm{B} \& \mathrm{~B}$ & New & $\begin{array}{l}\text { Fridge and freezer, dining } \\
\text { room, dishwasher, } \\
\text { cafe/restaurant, guest } \\
\text { lounge, laundry facilities, } \\
\text { clothes dryer, iron/ironing } \\
\text { board, lounge area with } \\
\text { fire place, BBQ, TV } \\
\text { room, DVD player, } \\
\text { internet access. }\end{array}$ & $\begin{array}{l}\text { All beds have electric blanket, } \\
\text { shower facilities, toilet } \\
\text { facilities. }\end{array}$ & $\begin{array}{l}\text { Continental breakfast, } \\
\text { food, wine, OCRT, golf } \\
\text { course, tennis courts } \\
\text { ( } 5 \text { minutes away). }\end{array}$ & Red brick \\
\hline 148 & Omakau & $\begin{array}{l}\text { Tiger Hill Lodge and } \\
\text { Wilson’s (Tiger Hill } \\
\text { Lodge) }\end{array}$ & $1 \times \mathrm{SC}$ & New & $\begin{array}{l}\text { Fully equipped kitchen, } 2 \\
\text { lounges, one is used as TV } \\
\text { room. }\end{array}$ & $\begin{array}{l}4 \text { ensuite bedrooms (bathroom } \\
\text { and toilet), heating and lighting, } \\
1 \text { ensuite includes disabled } \\
\text { facilities. }\end{array}$ & $\begin{array}{l}\text { Continental breakfast, } \\
\text { food, wine, OCRT, golf } \\
\text { course, visiting } \\
\text { historic buildings sites, } \\
\text { scenery. }\end{array}$ & $\begin{array}{l}\text { Schist stone } \\
\text { and timber }\end{array}$ \\
\hline
\end{tabular}




\begin{tabular}{|c|c|c|c|c|c|c|c|c|}
\hline 149 & Omakau & $\begin{array}{l}\text { Tiger Hill Lodge and } \\
\text { Wilson's (Wilson's) }\end{array}$ & $1 \times \mathrm{SC}$ & New & $\begin{array}{l}\text { Kitchen, communal } \\
\text { lounge, TV viewing room, } \\
\text { hot tub. }\end{array}$ & $\begin{array}{l}3 \text { bedrooms with three } \\
\text { bathrooms and toilets, heating, } \\
\text { lighting. }\end{array}$ & $\begin{array}{l}\text { Continental breakfast, } \\
\text { food, wine, OCRT, golf } \\
\text { course, visiting } \\
\text { historic buildings sites, } \\
\text { scenery feeding cattle. }\end{array}$ & $\begin{array}{l}\text { Assumed } \\
\text { stone, } \\
\text { concrete and } \\
\text { timber }\end{array}$ \\
\hline 150 & Chatto Creek & $\begin{array}{l}\text { Chatto Creek Tavern } \\
\text { Budget } \\
\text { Accommodation }\end{array}$ & $1 \times \mathrm{Bp}$ & $\mathrm{RB}$ & $\begin{array}{l}2 \text { double rooms and } 6 \text { bed } \\
\text { bunk room with shared } \\
\text { bathroom, dining room, } \\
\text { bar, beer garden. }\end{array}$ & $\begin{array}{l}\text { All bedrooms with heating and } \\
\text { lighting. }\end{array}$ & $\begin{array}{l}\text { Continental breakfast, } \\
\text { food, wine, beer } \\
\text { garden, OCRT, historic } \\
\text { artefacts, walking, } \\
\text { visiting historical sites } \\
\text { and buildings. }\end{array}$ & $\begin{array}{l}\text { Schist stone } \\
\text { and mud } \\
\text { walls }\end{array}$ \\
\hline 151 & Chatto Creek & The Magdelans B\&B & $1 \times B \& B$ & New & $\begin{array}{l}\text { Lounge with Sky TV, } \\
\text { selections of DVDs, } \\
\text { swimming pool. }\end{array}$ & $\begin{array}{l}\text { Two bedrooms with private } \\
\text { bathrooms, heating and lighting. }\end{array}$ & $\begin{array}{l}\text { Continental breakfast } \\
\text { and full Irish } \\
\text { breakfast, OCRT, } \\
\text { beverage, wine and } \\
\text { beer, landscape views, } \\
\text { guided walks, local } \\
\text { transportation along } \\
\text { the OCRT. }\end{array}$ & $\begin{array}{l}\text { Assumed } \\
\text { timber }\end{array}$ \\
\hline 152 & Chatto Creek & Rockdale B\&B & $1 \times B \& B$ & $\mathrm{RB}$ & $\begin{array}{l}\text { Country style kitchen, } \\
\text { wood panelled lounge, } \\
\text { two modern bathrooms } \\
\text { with claw foot bath. }\end{array}$ & $\begin{array}{l}2 \text { double rooms and } 1 \text { bunk } \\
\text { room with heating and lighting, } \\
\text { one bedroom with fire place. }\end{array}$ & $\begin{array}{l}\text { Food, continental } \\
\text { breakfast, OCRT, } \\
\text { scenery and landscape } \\
\text { views, local } \\
\text { transportation to dine } \\
\text { at adjacent hotels and } \\
\text { restaurant. }\end{array}$ & Timber \\
\hline 153 & Alexander & Avenue Motel & $1 \times$ Motel & New & $\begin{array}{l}\text { Fully equipped kitchen } \\
\text { with microwave, guest } \\
\text { laundry, BBQ. }\end{array}$ & $\begin{array}{l}\text { All } 13 \text { spacious units with } \\
\text { bathroom and toilet, heating and } \\
\text { lighting, internet access, Sky } \\
\text { TV. } 3 \text { units with spa baths. }\end{array}$ & $\begin{array}{l}\text { Continental breakfast, } \\
\text { OCRT, golf, wine, } \\
\text { historic heritage and } \\
\text { sites, scenery. }\end{array}$ & Concrete \\
\hline 154 & Alexander & $\begin{array}{l}\text { Alexander's } \\
\text { Riverview B\&B }\end{array}$ & $1 \times B \& B$ & New & $\begin{array}{l}2 \text { bedrooms with shared } \\
\text { tiled bathroom, lounge, } \\
\text { TV, patio with natural } \\
\text { views. }\end{array}$ & $\begin{array}{l}\text { All bedrooms with heating and } \\
\text { lighting, } 1 \text { bedroom with tiled } \\
\text { ensuite. }\end{array}$ & $\begin{array}{l}\text { Continental breakfast, } \\
\text { OCRT, historical sites } \\
\text { and heritage at } \\
\text { Alexandra, iconic steel } \\
\text { lattice bridge, the }\end{array}$ & $\begin{array}{l}\text { Assumed } \\
\text { timber }\end{array}$ \\
\hline
\end{tabular}




\begin{tabular}{|c|c|c|c|c|c|c|c|c|}
\hline & & & & & & & $\begin{array}{l}\text { historic Doctors Point } \\
\text { Gold Diggings Clutha } \\
\text { River, Alexandra } \\
\text { walkway, fishing. }\end{array}$ & \\
\hline 155 & Alexander & $\begin{array}{l}\text { Alexandra Garden } \\
\text { Court Motel }\end{array}$ & $1 \times$ Motel & New & $\begin{array}{l}\text { Swimming pool, BBQ } \\
\text { area, children's } \\
\text { playground. }\end{array}$ & $\begin{array}{l}6 \text { self contained units all with } \\
\text { electric blankets, dual-heating, } \\
\text { oven, microwave, TV, lighting, } \\
\text { bathroom and toilet. }\end{array}$ & $\begin{array}{l}\text { Food and beverages, } \\
\text { breakfast, OCRT, } \\
\text { landscape gardens, } \\
\text { 4WD tours, golf, } \\
\text { boating, mountain } \\
\text { biking, fishing, } \\
\text { kayaking, walking } \\
\text { tracks, walk to the } \\
\text { clock on the hill, ice } \\
\text { skating, curling, gun } \\
\text { club, bowling greens, } \\
\text { new swimming } \\
\text { complex, blossom } \\
\text { festival, thyme } \\
\text { festival, wine tours, } \\
\text { vineyard tours. }\end{array}$ & Timber \\
\hline 157 & Alexandra & $\begin{array}{l}\text { All Seasons Tourists } \\
\text { Flats }\end{array}$ & $1 \times \mathrm{SC}$ & New & Laundry facilities, & $\begin{array}{l}\text { All flats with private bathroom, } \\
\text { separate lounge with TV and } \\
\text { DVD, self catering kitchen with } \\
\text { small oven, microwave, fridge, } \\
\text { heating and lighting. }\end{array}$ & $\begin{array}{l}\text { OCRT, wine industry, } \\
\text { historic and natural } \\
\text { heritage, mountain } \\
\text { view, close to the } \\
\text { historic Shaky Bridge, } \\
\text { Alexandra festivals } \\
\text { and events (listed in } \\
\text { table 2), skiing, fishing }\end{array}$ & Timber \\
\hline
\end{tabular}




\begin{tabular}{|c|c|c|c|c|c|c|c|c|}
\hline & & & & & & & $\begin{array}{l}\text { (famous for salmon } \\
\text { and trout fishing),golf, } \\
\text { swimming, tramping, } \\
\text { mountain biking. }\end{array}$ & \\
\hline 158 & Alexander & $\begin{array}{l}\text { Alexandra Almond } \\
\text { Court Motel }\end{array}$ & $1 \times$ Motel & New & $\begin{array}{l}\text { Swimming pool, guest } \\
\text { laundry. }\end{array}$ & $\begin{array}{l}8 \text { units all with kitchen, } \\
\text { bathroom and toilet (three of the } \\
\text { units have spa bath ), one of two } \\
\text { bedrooms units has disabled } \\
\text { facilities, central heating, } \\
\text { lighting, glare reducing tinted } \\
\text { glass, thermal drapes, TV, } \\
\text { radio, phone, DVD player, } \\
\text { wireless internet. }\end{array}$ & $\begin{array}{l}\text { Cooked or continental } \\
\text { breakfast, OCRT, daily } \\
\text { paper delivered, } 33 \\
\text { annual cultural events } \\
\text { and activities (listed in } \\
\text { table 2), skiing, fishing } \\
\text { (famous for salmon } \\
\text { and trout fishing), } \\
\text { golf, swimming, } \\
\text { tramping, mountain } \\
\text { biking. }\end{array}$ & Timber \\
\hline 159 & Alexander & $\begin{array}{l}\text { Central Court Motor } \\
\text { Inn }\end{array}$ & $1 \times$ Motel & New & $\begin{array}{l}\text { Restaurant, house bar, } \\
\text { BBQ for hire, conference } \\
\text { centre. }\end{array}$ & $\begin{array}{l}33 \text { units all with ensuite or } \\
\text { private bathroom, kitchen or } \\
\text { kitchenette with fridge and } \\
\text { coffee making facilities, } \\
\text { wireless internet, Sky TV, } \\
\text { heater, electric blankets and } \\
\text { lighting. }\end{array}$ & $\begin{array}{l}\text { Kiwi dishes, } \\
\text { continental breakfast, } \\
\text { OCRT, local wines, } \\
\text { scenery, gold mining } \\
\text { heritage, } 33 \text { annual } \\
\text { cultural events and } \\
\text { activities (listed in } \\
\text { table 2), conferences } \\
\text { and seminars. }\end{array}$ & $\begin{array}{l}\text { Timber, } \\
\text { concrete, red } \\
\text { brick (used } \\
\text { for the } \\
\text { conference } \\
\text { center } \\
\text { building) }\end{array}$ \\
\hline 160 & Alexandra & $\begin{array}{l}\text { Criterion Club Hotel } \\
\text { (Bp) }\end{array}$ & $1 \times \mathrm{Bp}$ & New & $\begin{array}{l}\text { All 7bedrooms with } \\
\text { shared bathroom facilities, } \\
\text { beer garden, restaurant. }\end{array}$ & $\begin{array}{l}\text { All bedrooms with heating and } \\
\text { lighting. }\end{array}$ & $\begin{array}{l}\text { Food, breakfast, beer, } \\
\text { wine, OCRT, } 33 \text { annual } \\
\text { cultural events and } \\
\text { activities (listed in } \\
\text { table 2). }\end{array}$ & $\begin{array}{l}\text { Assumed } \\
\text { timber and } \\
\text { brick }\end{array}$ \\
\hline 161 & Alexandra & $\begin{array}{l}\text { Criterion Club Hotel } \\
\text { (Bp) }\end{array}$ & $1 \times \mathrm{Bp}$ & New & $\begin{array}{l}\text { A fully equipped kitchen, } \\
\text { dishwasher, lounge, beer } \\
\text { garden, restaurant. }\end{array}$ & $\begin{array}{l}\text { Ensuite bathrooms, TV (some } \\
\text { of rooms), lighting and heating. }\end{array}$ & $\begin{array}{l}\text { Food, breakfast, beer, } \\
\text { wine, OCRT, } 33 \text { annual } \\
\text { cultural events and } \\
\text { activities (listed in } \\
\text { table 2). }\end{array}$ & $\begin{array}{l}\text { Assumed } \\
\text { timber and } \\
\text { brick }\end{array}$ \\
\hline 162 & Alexandra & Hawkdun Rise & $1 \times B \& B$ & New & Dining room. & 3 bedrooms with 3 separate & Breakfast, local fresh & Timber \\
\hline
\end{tabular}




\begin{tabular}{|c|c|c|c|c|c|c|c|c|}
\hline & & Vineyard Homestay & & & & $\begin{array}{l}\text { ensuite bathrooms and } \\
\text { verandas, heating and lighting. }\end{array}$ & $\begin{array}{l}\text { fruits, food, Hawkdun } \\
\text { Rise wine, OCRT, } \\
\text { vineyard, using local } \\
\text { grown produce, } 33 \\
\text { annual cultural events } \\
\text { and activities (listed in } \\
\text { table 2), skiing, fishing } \\
\text {,golf, swimming, } \\
\text { tramping, mountain } \\
\text { biking. }\end{array}$ & \\
\hline 163 & Alexandra & Hillview Park B\&B & $1 \times \mathrm{SC}$ & New & $\begin{array}{l}\text { Fully equipped and } \\
\text { disabled accessible } \\
\text { kitchen, a shared } \\
\text { bathroom, BBQ, lounge } \\
\text { /dining, large trampoline } \\
\text { for children wireless } \\
\text { internet. }\end{array}$ & $\begin{array}{l}\text { All bedrooms with heating and } \\
\text { lighting. }\end{array}$ & $\begin{array}{l}\text { Continental breakfast, } \\
\text { food, off - road } \\
\text { motorcycle or } 4 \\
\text { Wheel drive tour, } \\
\text { OCRT, } 33 \text { annual } \\
\text { cultural events and } \\
\text { activities (listed in } \\
\text { table 2). }\end{array}$ & Timber \\
\hline 164 & Alexandra & Log House Retreat & $1 \times B \& B$ & New & $\begin{array}{l}\text { Dining room, 1shared } \\
\text { bathroom, guest reading } \\
\text { lounge, outdoor living } \\
\text { area. }\end{array}$ & $\begin{array}{l}3 \text { bedrooms with electric } \\
\text { blankets, hairdryer, clock radio, } \\
\text { stereo/CD player, tea \& coffee } \\
\text { making facilities. A queen size } \\
\text { suite with ensuite and private } \\
\text { lounge. }\end{array}$ & $\begin{array}{l}\text { Breakfast, wine, Pinot } \\
\text { Noirs and wine tours, } \\
\text { OCRT, guest reading } \\
\text { lounge, wedding } \\
\text { services, historical } \\
\text { countryside, stone } \\
\text { fruit, hydro electric } \\
\text { dam (related } \\
\text { recreational } \\
\text { activities), } 33 \text { annual } \\
\text { cultural events and } \\
\text { activities (listed in } \\
\text { table 2). }\end{array}$ & Timber (log) \\
\hline 165 & Alexandra & $\begin{array}{l}\text { Marj’s Place (Home } \\
\text { stay) }\end{array}$ & $1 \times \mathrm{SC}$ & New & $\begin{array}{l}\text { Kitchen, lounge with Sky } \\
\text { TV , central heating, } 3 \\
\text { person sauna, double spa } \\
\text { bath, internet. }\end{array}$ & All bedrooms with lighting. & $\begin{array}{l}\text { Food, wine, Pinot } \\
\text { Noirs and wine tours, } \\
\text { OCRT, guest reading } \\
\text { lounge, wedding }\end{array}$ & Timber \\
\hline
\end{tabular}




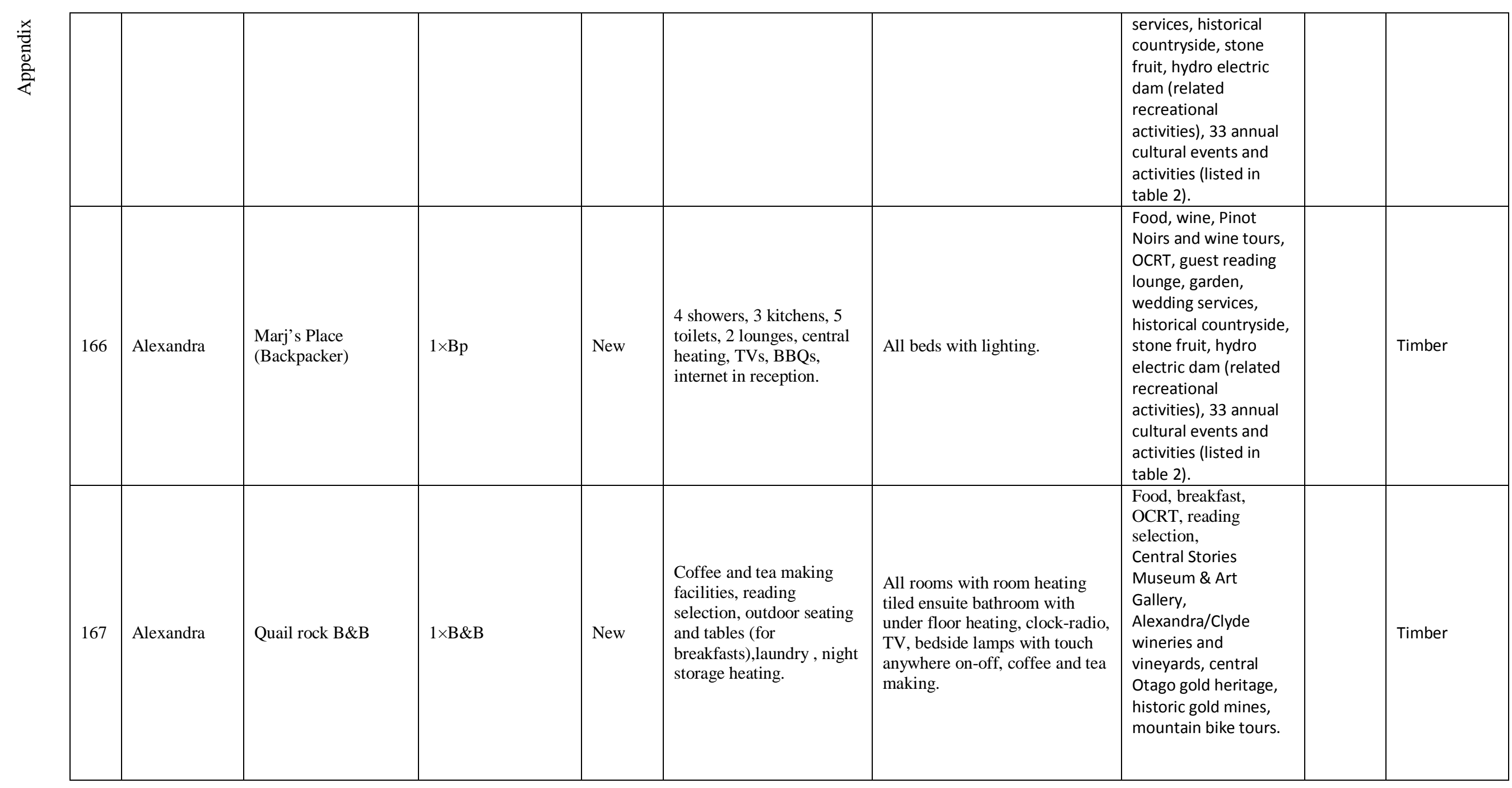




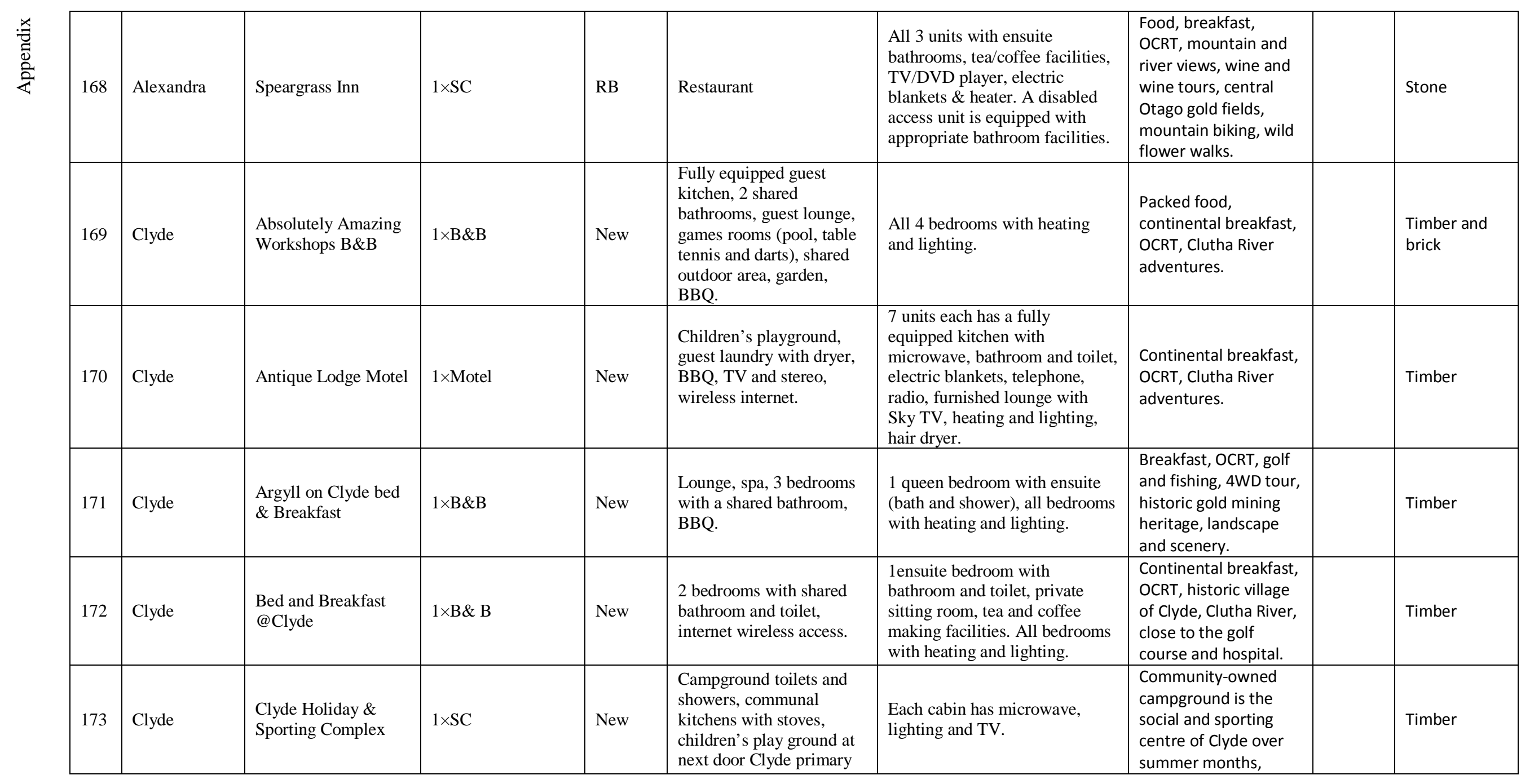




\begin{tabular}{|c|c|c|c|c|c|c|c|c|}
\hline & & & & & school. & & $\begin{array}{l}\text { indoor and lawn bowls } \\
\text { touch rugby, soccer, } \\
\text { golf, fishing and } \\
\text { boating on Lake } \\
\text { Dunstan, adjacent } \\
\text { towns and places and } \\
\text { their attractions such } \\
\text { as Manitoto Plains, St } \\
\text { Bathans, Ranfurly, } \\
\text { Alexandra, } 9 \text { hole } \\
\text { Dunstan golf course, } \\
\text { ice sports and curling } \\
\text { at Naseby, Clyde dam, } \\
\text { gold mining, the Clyde } \\
\text { museum, the Herb } \\
\text { museum and the } \\
\text { Station museum, } \\
\text { Cromwell Gorge. }\end{array}$ & \\
\hline 174 & Clyde & Clyde Motel & $1 \times$ Motel & New & $\begin{array}{l}\text { Guest laundry equipped } \\
\text { with washer and dryer, } \\
\text { outdoor BBQ, Sky TV. }\end{array}$ & $\begin{array}{l}9 \text { units all have bathroom with } \\
\text { shower, vanity, hair dryer, fully } \\
\text { equipped kitchen with oven, } \\
\text { microwave and fridge, } \\
\text { Telephone, broadband internet, } \\
\text { heating and lighting. }\end{array}$ & $\begin{array}{l}\text { Food, wine, OCRT, } \\
\text { adjacent towns and } \\
\text { places and their } \\
\text { attractions such as } \\
\text { Manitoto Plains, St } \\
\text { Bathans, Ranfurly, } \\
\text { Alexandra, } 9 \text { hole } \\
\text { Dunstan golf course, } \\
\text { garden and its views, } \\
\text { historic sites and } \\
\text { buildings, ice sports } \\
\text { and curling at Naseby, } \\
\text { Clyde dam, gold } \\
\text { mining, the Clyde } \\
\text { museum, the Herb } \\
\text { museum and the } \\
\text { Station museum, }\end{array}$ & $\begin{array}{l}\text { Assumed } \\
\text { timber }\end{array}$ \\
\hline
\end{tabular}




\begin{tabular}{|c|c|c|c|c|c|c|c|c|}
\hline & & & & & & & Cromwell Gorge. & \\
\hline 175 & Clyde & $\begin{array}{l}\text { Clyde's Plum Tree } \\
\text { Cottage }\end{array}$ & $1 \times \mathrm{SC}$ & $\mathrm{RB}$ & $\begin{array}{l}\text { Fully equipped kitchen, } \\
\text { bathroom and toilet, } \\
\text { lounge with stove, } \\
\text { veranda views of the } \\
\text { garden, heat pump, BBQ, } \\
\text { TV and stereo. }\end{array}$ & $\begin{array}{l}\text { Both bedrooms with electric } \\
\text { blanket and lighting, private } \\
\text { courtyard with open air dining } \\
\text { area. }\end{array}$ & $\begin{array}{l}\text { Continental breakfast, } \\
\text { OCRT, library, plum } \\
\text { tree garden, adjacent } \\
\text { towns and places and } \\
\text { their attractions such } \\
\text { as Manitoto Plains, St } \\
\text { Bathans, Ranfurly, } \\
\text { Alexandra, } 9 \text { hole } \\
\text { Dunstan golf course, } \\
\text { garden and its views, } \\
\text { historic sites and } \\
\text { buildings, ice sports } \\
\text { and curling at Naseby, } \\
\text { Clyde dam, gold } \\
\text { mining, the Clyde } \\
\text { museum, the Herb } \\
\text { museum and the } \\
\text { Station museum, } \\
\text { Cromwell Gorge. }\end{array}$ & Timber \\
\hline 176 & Clyde & Cottage Wheatsheaf & $1 \times \mathrm{B} \& \mathrm{~B}$ & $\mathrm{RB}$ & $\begin{array}{l}\text { A fully equipped kitchen, } \\
\text { dining room, lounge, } \\
\text { bathroom and a fully } \\
\text { fenced private courtyard, } \\
\text { wood burner. }\end{array}$ & $\begin{array}{l}\text { Two bedrooms with electric } \\
\text { blankets, heating and lighting. }\end{array}$ & $\begin{array}{l}\text { OCRT, surrounding } \\
\text { orchards and } \\
\text { vineyard, Clutha } \\
\text { Bridge, adjacent } \\
\text { towns and places and } \\
\text { their attractions such } \\
\text { as Manitoto Plains, St } \\
\text { Bathans, Ranfurly, } \\
\text { Alexandra, } 9 \text { hole } \\
\text { Dunstan golf course, } \\
\text { garden and its views, } \\
\text { historic sites and } \\
\text { buildings, ice sports } \\
\text { and curling at Naseby, } \\
\text { Clyde dam, gold }\end{array}$ & $\begin{array}{l}\text { Schist and } \\
\text { concrete, and } \\
\text { timber }\end{array}$ \\
\hline
\end{tabular}




\begin{tabular}{|c|c|c|c|c|c|c|c|c|}
\hline & & & & & & & $\begin{array}{l}\text { mining, the Clyde } \\
\text { museum, the Herb } \\
\text { museum and the } \\
\text { Station museum, } \\
\text { Cromwell Gorge. }\end{array}$ & \\
\hline 177 & Clyde & Dunstan Hotel & $1 \times$ Hotel & $\mathrm{RB}$ & $\begin{array}{l}10 \text { rooms of which } 9 \text { have } \\
\text { shared bathroom facilities, } \\
\text { shared TV lounge with tea } \\
\text { and coffee facilities, } \\
\text { restaurant, lounge bar, } \\
\text { public bar, } \\
\text { TAB, gaming machines, } \\
\text { pool tables }\end{array}$ & $\begin{array}{l}\text { All bedrooms with heating and } \\
\text { lighting. }\end{array}$ & $\begin{array}{l}\text { Food and beverages, } \\
\text { breakfast, wine and } \\
\text { beer, OCRT, adjacent } \\
\text { towns and places and } \\
\text { their attractions such } \\
\text { as Manitoto Plains, St } \\
\text { Bathans, Ranfurly, } \\
\text { Alexandra, } 9 \text { hole } \\
\text { Dunstan golf course, } \\
\text { garden and its views, } \\
\text { historic sites and } \\
\text { buildings, ice sports } \\
\text { and curling at Naseby, } \\
\text { Clyde dam, gold } \\
\text { mining, the Clyde } \\
\text { museum, the Herb } \\
\text { museum and the } \\
\text { Station museum, } \\
\text { Cromwell Gorge. }\end{array}$ & Schist stone \\
\hline 178 & Clyde & Dunstan House & $1 \times$ Hotel & $\mathrm{RB}$ & $\begin{array}{l}6 \text { rooms have shared } \\
\text { bathroom, laundry } \\
\text { facilities, a separate TV } \\
\text { lounge, colonial veranda, } \\
\text { central heating, internet } \\
\text { facilities - broadband and } \\
\text { WiFi. }\end{array}$ & $\begin{array}{l}13 \text { guest rooms, } 6 \text { rooms have } \\
\text { own ensuite bathroom facilities, } \\
\text { tea and coffee making, toaster, } \\
\text { fridge and microwave, private } \\
\text { courtyard and hill view, one } \\
\text { room has self contained kitchen } \\
\text { / sitting room, shower and } \\
\text { separate toilet. }\end{array}$ & $\begin{array}{l}\text { Packed lunches, } \\
\text { OCRT, walking to visit } \\
\text { historical and natural } \\
\text { heritage, golf, tennis, } \\
\text { bowls, vineyards, } \\
\text { fishing, kayaking, } \\
\text { 4WD, skiing, curling } \\
\text { and ice skating (in } \\
\text { Alexandra or Naseby). }\end{array}$ & $\begin{array}{l}\text { Stone and } \\
\text { timber }\end{array}$ \\
\hline 179 & Clyde & $\begin{array}{l}\text { Hartly Arms } \\
\text { Backpackers / B\&B }\end{array}$ & $1 \times \mathrm{B} \& \mathrm{~B} / 1 \times \mathrm{Bp}$ & $\mathrm{RB}$ & $\begin{array}{l}\text { Shared shower facilities } \\
\text { and toilet, an equipped } \\
\text { kitchen. }\end{array}$ & $\begin{array}{l}\text { Bedrooms with heating and } \\
\text { lighting. }\end{array}$ & $\begin{array}{l}\text { Continental breakfast, } \\
\text { OCRT, Clyde historical }\end{array}$ & Stacked-stone \\
\hline
\end{tabular}




\begin{tabular}{|c|c|c|c|c|c|c|c|c|}
\hline & & & & & & & $\begin{array}{l}\text { and natural heritage } \\
\text { and activities, } \\
\text { adjacent towns and } \\
\text { places and their } \\
\text { attractions such as } \\
\text { Manitoto Plains, St } \\
\text { Bathans, Ranfurly, } \\
\text { Alexandra, } 9 \text { hole } \\
\text { Dunstan golf course, } \\
\text { garden and its views, } \\
\text { historic sites and } \\
\text { buildings, ice sports } \\
\text { and curling at Naseby, } \\
\text { Clyde dam, gold } \\
\text { mining, the Clyde } \\
\text { museum, the Herb } \\
\text { museum and the } \\
\text { Station museum, } \\
\text { Cromwell Gorge. }\end{array}$ & \\
\hline 180 & Clyde & $\begin{array}{l}\text { Hartley Homestead } \\
\text { Boutique B\&B }\end{array}$ & $1 \times B \& B$ & $\mathrm{RB}$ & $\begin{array}{l}\text { Guest lounge with TV, } \\
\text { internet access. }\end{array}$ & $\begin{array}{l}3 \text { rooms each has bathroom, } \\
\text { heating and lighting. }\end{array}$ & $\begin{array}{l}\text { Continental breakfast, } \\
\text { including our own } \\
\text { preserved fresh fruits } \\
\text { and jam, hot and cold } \\
\text { beverages, wine, } \\
\text { OCRT, library, top } \\
\text { quality New Zealand } \\
\text { made beds, historic } \\
\text { city of Clyde, Clutha } \\
\text { River, walking, }\end{array}$ & Mud brick \\
\hline 181 & Clyde & $\begin{array}{l}\text { Hill view Park B \& B } \\
\text { and SC }\end{array}$ & $1 \times \mathrm{B} \& \mathrm{~B} / 1 \times \mathrm{SC}$ & New & $\begin{array}{l}\text { Spa, BBQ, internet } \\
\text { broadband. }\end{array}$ & $\begin{array}{l}2 \text { bedrooms with bathroom and } \\
\text { toilet with disabled access, } \\
\text { heating, lighting. }\end{array}$ & $\begin{array}{l}\text { Continental breakfast, } \\
\text { food, OCRT, gardens, } \\
\text { landscape views, } \\
\text { Clyde historic gold } \\
\text { mining heritage, } \\
\text { walking, motorcycle or }\end{array}$ & Timber \\
\hline
\end{tabular}




\begin{tabular}{|c|c|c|c|c|c|c|c|c|}
\hline & & & & & & & 4-wheel drives tours. & \\
\hline 182 & Clyde & Kahu Stone Cottage & $1 \times \mathrm{SC}$ & $\mathrm{RB}$ & $\begin{array}{l}\text { Full kitchen and dining } \\
\text { facilities, lounge, log } \\
\text { burner, bathroom and } \\
\text { separate toilet. }\end{array}$ & Two bedrooms with lighting. & $\begin{array}{l}\text { Food, local wine, } \\
\text { OCRT, Clyde city and } \\
\text { its attractions, } \\
\text { production of } \\
\text { electricity via solar } \\
\text { panels and } \\
\text { batteries, water supply } \\
\text { is from a spring } \\
\text { running from high in } \\
\text { the mountains behind, } \\
\text { garden, reading room, } \\
\text { fishing, hunting, 4x4 } \\
\text { trips, mountain biking, } \\
\text { river trips, skiing in } \\
\text { the winter, vineyard } \\
\text { tours, water skiing and } \\
\text { sightseeing. }\end{array}$ & $\begin{array}{l}\text { Stone and } \\
\text { timber }\end{array}$ \\
\hline 183 & Clyde & $\begin{array}{l}\text { Lavender Drive Bed } \\
\text { and Breakfast }\end{array}$ & $1 \times \mathrm{B} \& \mathrm{~B}$ & New & $\begin{array}{l}2 \text { bedrooms with shared } \\
\text { bathroom, spa, laundry } \\
\text { facilities. }\end{array}$ & $\begin{array}{l}\text { One bedroom with ensuite, all } \\
\text { bedrooms with heating and } \\
\text { lighting. }\end{array}$ & $\begin{array}{l}\text { Continental breakfast, } \\
\text { local and homemade } \\
\text { fruits and jams, } \\
\text { OCRT, views of } \\
\text { Dunstan Mountains, } \\
\text { historic heritage of } \\
\text { Clyde city, play house } \\
\text { and sandpit for } \\
\text { children, vineyard, } \\
\text { Clyde dam, walks, } \\
\text { golf course. }\end{array}$ & $\begin{array}{l}\text { Schist stone } \\
\text { and timber }\end{array}$ \\
\hline 184 & Clyde & Mirabell Chalets & $1 \times \mathrm{SC}$ & New & $\begin{array}{l}\text { BBQ area, swimming } \\
\text { pool, hot tub heated by } \\
\text { gas. }\end{array}$ & $\begin{array}{l}3 \text { chalets with ensuite facilities } \\
\text { including fully equipped } \\
\text { kitchen, bathroom, toilet, } \\
\text { laundry facilities and private } \\
\text { lounge, heating and lighting. }\end{array}$ & $\begin{array}{l}\text { Continental breakfast, } \\
\text { and fresh local eggs, } \\
\text { OCRT, historic } \\
\text { heritage of Clyde city, } \\
\text { four hectares fenced } \\
\text { ground for play, }\end{array}$ & Timber \\
\hline
\end{tabular}




\begin{tabular}{|c|c|c|c|c|c|c|c|c|}
\hline & & & & & & & $\begin{array}{l}\text { feeding hens, adjacent } \\
\text { towns and places and } \\
\text { their attractions such } \\
\text { as Manitoto Plains, St } \\
\text { Bathans, Ranfurly, } \\
\text { Alexandra, } 9 \text { hole } \\
\text { Dunstan golf course, } \\
\text { garden and its views, } \\
\text { ice sports and curling } \\
\text { at Naseby, Clyde dam, } \\
\text { gold mining, the Clyde } \\
\text { museum, the Herb } \\
\text { museum and the } \\
\text { Station museum, } \\
\text { Cromwell Gorge. }\end{array}$ & \\
\hline 185 & Clyde & $\begin{array}{l}\text { Olivers of Clyde ( } \\
\text { lodge and stable) }\end{array}$ & $1 \times$ Motel & $\mathrm{RB}$ & Dining room, courtyard. & $\begin{array}{l}6 \text { of } 11 \text { double bedrooms all } \\
\text { have en suites with claw-foot } \\
\text { baths, } 5 \text { of } 11 \text { bedrooms have } \\
\text { private en suite bathrooms, all } \\
\text { bedrooms with heating and } \\
\text { lighting. }\end{array}$ & $\begin{array}{l}\text { Breakfast, OCRT, the } \\
\text { Clutha river cruises, } \\
\text { heritage tour, wine } \\
\text { tours, adjacent towns } \\
\text { and places and their } \\
\text { attractions such as } \\
\text { Manitoto Plains, St } \\
\text { Bathans, Ranfurly, } \\
\text { Alexandra, } 9 \text { hole } \\
\text { Dunstan golf course, } \\
\text { ice sports and curling } \\
\text { at Naseby, Clyde dam, } \\
\text { gold mining, the Clyde } \\
\text { museum, the Herb } \\
\text { museum and the } \\
\text { Station museum, } \\
\text { Cromwell Gorge. }\end{array}$ & Schist stone \\
\hline 186 & Clyde & $\begin{array}{l}\text { Picnic Creek Bed \& } \\
\text { Breakfast }\end{array}$ & $1 \times \mathrm{B} \& \mathrm{~B}$ & New & $\begin{array}{l}\text { Communal lounge, } \\
\text { outdoor courtyard. }\end{array}$ & $\begin{array}{l}\text { All three bedrooms have private } \\
\text { ensuite, TV, tea and coffee } \\
\text { making facilities, individually } \\
\text { controlled central heating. }\end{array}$ & $\begin{array}{l}\text { Continental breakfast, } \\
\text { OCRT, historic } \\
\text { heritage of Clyde city, }\end{array}$ & Timber \\
\hline
\end{tabular}




\begin{tabular}{|c|c|c|c|c|c|c|c|c|}
\hline & & & & & & & $\begin{array}{l}\text { Clyde dam, landscape } \\
\text { and scenery. }\end{array}$ & \\
\hline 187 & Clyde & Postmaster’s House & $1 \times B \& B$ & New & $\begin{array}{l}2 \text { bedrooms with shared } \\
\text { bathroom and toilet, } \\
\text { sitting room, cooking } \\
\text { facilities. }\end{array}$ & $\begin{array}{l}1 \text { bedroom with ensuite, private } \\
\text { lounge, all bedrooms with } \\
\text { lighting, heating. }\end{array}$ & $\begin{array}{l}\text { Continental breakfast, } \\
\text { OCRT, Clutha river } \\
\text { cruises, scenery of the } \\
\text { Roxburgh Gorge, } \\
\text { historical gold mining } \\
\text { towns. }\end{array}$ & $\begin{array}{l}\text { Stone and } \\
\text { timber }\end{array}$ \\
\hline 188 & Cromwell & $\begin{array}{l}\text { Anderson Park } \\
\text { Motels }\end{array}$ & $1 \times$ Motel & New & $\begin{array}{l}\text { Spa pool, children's } \\
\text { playground, guest laundry, } \\
\text { hot tub, outdoor living, } \\
\text { BBQ. }\end{array}$ & $\begin{array}{l}\text { All } 11 \text { ground floor units with } \\
\text { ensuite bathroom, equipped } \\
\text { kitchen, Sky TV, DVD, } \\
\text { broadband, heating and lighting. }\end{array}$ & $\begin{array}{l}\text { Breakfast, OCRT, } \\
\text { sports equipment and } \\
\text { bikes, Clyde city and } \\
\text { its attractions, } \\
\text { historic Cromwell } \\
\text { Anderson park, Lake } \\
\text { Dunstan and } \\
\text { Bannockburn for } \\
\text { water sports, } \\
\text { vineyards and } \\
\text { wineries, mountain } \\
\text { bike, walking, horse } \\
\text { and car racing, skiing. }\end{array}$ & Timber \\
\hline 189 & Cromwell & Carrick Lodge Motel & $1 \times$ Motel & New & Outdoor sitting. & $\begin{array}{l}\text { Four studios all with en-suite } \\
\text { bathroom, heated towel rail, } \\
\text { hair dryer, Sky TV, kitchen } \\
\text { (cooking facilities), broadband } \\
\text { and wireless internet, air } \\
\text { conditioning and lighting. }\end{array}$ & $\begin{array}{l}\text { OCRT, wineries and } \\
\text { vineyards, golf course, } \\
\text { biking, goldfield jet } \\
\text { thrill, historic heritage } \\
\text { and buildings of } \\
\text { Cromwell city, policies } \\
\text { for being sustainable } \\
\text { such as replacing } \\
\text { electric bulbs and } \\
\text { appliances with low } \\
\text { energy environment- } \\
\text { friendly bulbs whilst } \\
\text { recognizing the need } \\
\text { of guests to be able to }\end{array}$ & $\begin{array}{l}\text { Assumed } \\
\text { timber }\end{array}$ \\
\hline
\end{tabular}




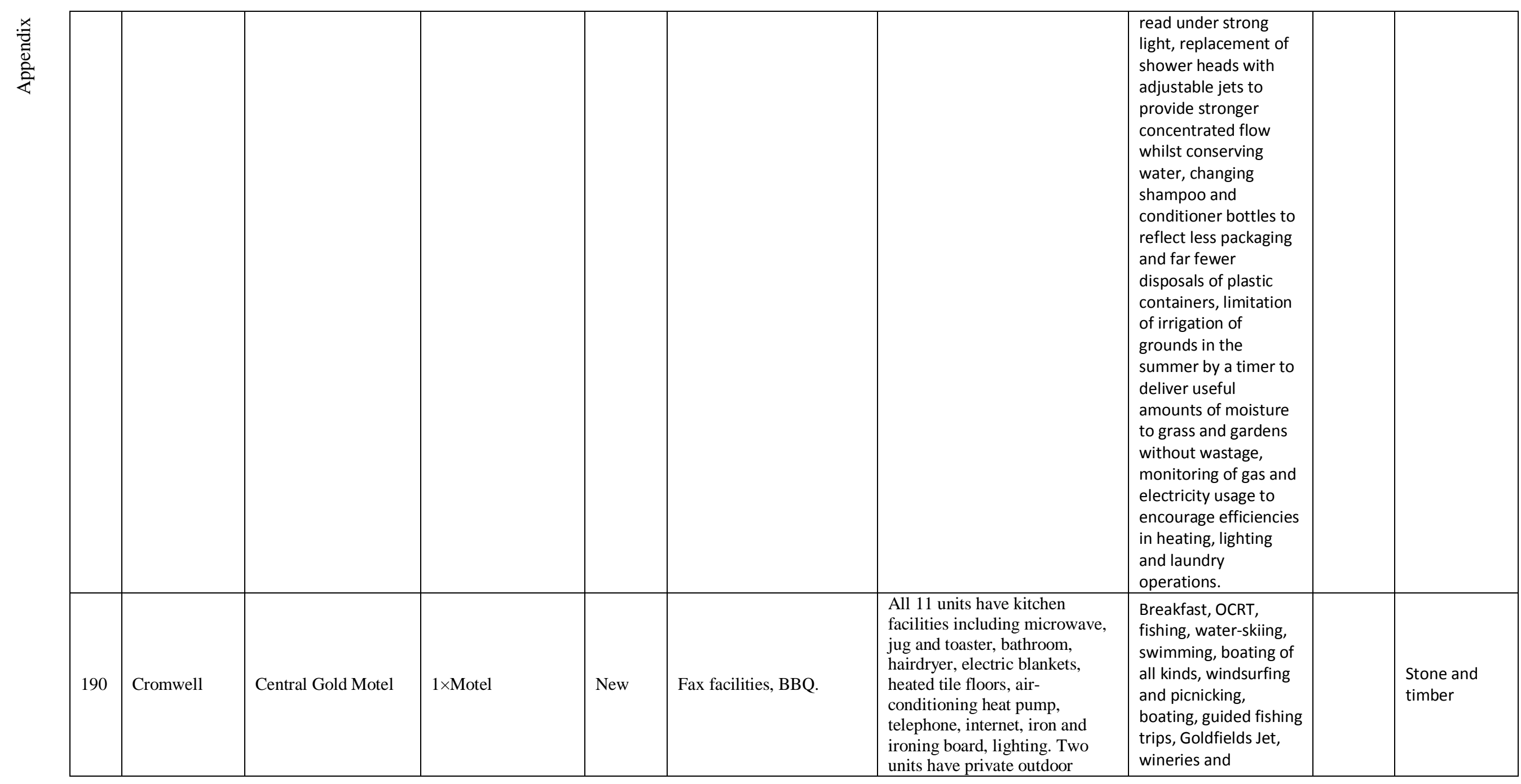




\begin{tabular}{|c|c|c|c|c|c|c|c|c|}
\hline & & & & & & sitting. & $\begin{array}{l}\text { vineyards, gold mining } \\
\text { heritage, walks, } 4 \mathrm{WD} \\
\text { trips. }\end{array}$ & \\
\hline 191 & Cromwell & $\begin{array}{l}\text { Colonial Manor } \\
\text { Motel }\end{array}$ & $1 \times$ Motel & New & $\begin{array}{l}\text { Selection of DVD movies, } \\
\text { gas BBQ, recycling } \\
\text { collection area. }\end{array}$ & $\begin{array}{l}\text { All units have wireless internet, } \\
\text { room heaters and electric } \\
\text { blankets, double glazing, } \\
\text { bathroom and toilet, equipped } \\
\text { kitchen, two units have TV, one } \\
\text { unit has DVD player, two } \\
\text { upstairs units have own } \\
\text { balcony. Each ground floor unit } \\
\text { has private outdoor seating area. }\end{array}$ & $\begin{array}{l}\text { OCRT, daily } \\
\text { newspaper, vineyards } \\
\text { and wineries, golf } \\
\text { course, trout and } \\
\text { salmon fishing, scenic } \\
\text { walks, nature, historic } \\
\text { goldfield, sites and } \\
\text { trails, water sports on } \\
\text { Lakes Dunstan, } \\
\text { Hawea, Wanaka and } \\
\text { Whakatipu, winter ski } \\
\text { fields. }\end{array}$ & Timber \\
\hline 192 & Cromwell & Cromwell Motel & $1 \times$ Motel & New & $\begin{array}{l}\text { Children’s playground, } \\
\text { outdoor swimming pool, } \\
\text { BBQ area. }\end{array}$ & $\begin{array}{l}11 \text { family units with kitchen, } \\
\text { bathroom, wireless internet, } \\
\text { heating and lighting. }\end{array}$ & $\begin{array}{l}\text { Continental breakfast, } \\
\text { OCRT, vineyards and } \\
\text { wineries, wine, private } \\
\text { gardens, bike hire, } \\
\text { walks, Cromwell town, } \\
\text { Lake Dunstan, } \\
\text { museum. }\end{array}$ & Timber \\
\hline 193 & Cromwell & $\begin{array}{l}\text { Cromwell Top } 10 \\
\text { Holiday Park(Park } \\
\text { Motel } 2 \text { rooms and } \\
\text { Park Motel } 1 \text { room) } \\
\end{array}$ & $2 \times$ Motel & New & $\begin{array}{l}\text { Laundry room, play } \\
\text { ground for kids, internet } \\
\text { kiosk with wireless } \\
\text { available, BBQ area. }\end{array}$ & $\begin{array}{l}\text { Both park motels have fully } \\
\text { equipped kitchen, TV, private } \\
\text { bathroom, lounge, heating and } \\
\text { lighting. }\end{array}$ & \multirow{3}{*}{$\begin{array}{l}\text { Food and beverages, } \\
\text { wine and beer, OCRT, } \\
\text { fishing, swimming, } \\
\text { boating, motorcycle } \\
\text { hire, skiing and scenic } \\
\text { flights, cycling, } \\
\text { kayaking, golf, } \\
\text { vineyards and } \\
\text { wineries, wine, } \\
\text { Cromwell town, and } \\
\text { its attractions. }\end{array}$} & Timber \\
\hline 194 & Cromwell & $\begin{array}{l}\text { Cromwell Top } 10 \\
\text { Holiday Park ( Self } \\
\text { Contained Unit } 1 \text { and } \\
\text { 2) }\end{array}$ & $2 \times \mathrm{SC}$ & New & $\begin{array}{l}\text { Laundry room, play } \\
\text { ground for kids, internet } \\
\text { kiosk with wireless } \\
\text { available, BBQ area. }\end{array}$ & $\begin{array}{l}\text { Both self contained units have } \\
\text { fully equipped kitchen, shower } \\
\text { and toilet, lounge, TV, heating } \\
\text { and lighting. }\end{array}$ & & Timber \\
\hline 195 & Cromwell & $\begin{array}{l}\text { Cromwell Top } 10 \\
\text { Holiday Park(ensuite } \\
\text { units, deluxe units, } \\
\text { standard units) }\end{array}$ & $1 \times \mathrm{SC}$ & New & $\begin{array}{l}\text { Communal kitchen, } \\
\text { shared bathroom and toilet } \\
\text { (communal services for } \\
\text { deluxe and standard } \\
\text { units), play ground for } \\
\text { kids, laundry room, }\end{array}$ & $\begin{array}{l}\text { All units have TV, jug and } \\
\text { toaster, heating and lighting. } \\
\text { Ensuite units have their own } \\
\text { shower and toilet. }\end{array}$ & & Timber \\
\hline
\end{tabular}




\begin{tabular}{|c|c|c|c|c|c|c|c|c|}
\hline & & & & & $\begin{array}{l}\text { internet kiosk with } \\
\text { wireless available, } \\
\text { outdoor BBQ. }\end{array}$ & & & \\
\hline 196 & Cromwell & $\begin{array}{l}\text { Cromwell Top } 10 \\
\text { Holiday } \\
\text { Park(powered and } \\
\text { non-powered sites) }\end{array}$ & $\begin{array}{l}288 \times \text { powered sites } \\
\text { and } 183 \text { non- } \\
\text { powered sites. }\end{array}$ & - & $\begin{array}{l}\text { Carwash facilities, using } \\
\text { communal kitchen and } \\
\text { laundry facilities of the } \\
\text { close self contained units, } \\
\text { play ground for kids, } \\
\text { internet kiosk with } \\
\text { wireless available, BBQ } \\
\text { area. }\end{array}$ & - & & - \\
\hline 197 & Cromwell & Golden Gate Lodge & $1 \times \mathrm{B} \& \mathrm{~B}$ & New & $\begin{array}{l}\text { Fully equipped guest } \\
\text { laundry, gym, restaurant, } \\
\text { cafe and bar, fitness } \\
\text { centre, wholesale liquor } \\
\text { outlet. }\end{array}$ & $\begin{array}{l}\text { All } 47 \text { studio units have their } \\
\text { own courtyard, coffee and tea } \\
\text { making facilities, Sky TV, mini } \\
\text { bar, fridge, bathroom and toilet } \\
\text { (some units with spa bath), } \\
\text { hairdryer, ironing facilities, } \\
\text { broadband internet (wireless), } \\
\text { telephone, electric blanket, } \\
\text { heating and lighting. }\end{array}$ & $\begin{array}{l}\text { Food and beverage, } \\
\text { wine and beer, OCRT, } \\
\text { equipped conference } \\
\text { centre with its own } \\
\text { courtyard (used for } \\
\text { weddings as well), } 42 \\
\text { annual cultural events } \\
\text { ( listed in table 3), } \\
\text { historic Cromwell city, } \\
\text { vineyard, wineries, } \\
\text { gold field mining } \\
\text { centre, golf, jet ski, } \\
\text { landscape view, } \\
\text { gardens. }\end{array}$ & $\begin{array}{l}\text { Timber and } \\
\text { schist rock }\end{array}$ \\
\hline 198 & Cromwell & Dunstan Motel & $1 \times$ Motel & New & $\begin{array}{l}\text { Guest laundry, children's } \\
\text { playground, gas BBQ. }\end{array}$ & $\begin{array}{l}\text { All units have fully equipped } \\
\text { kitchen, bathroom and toilet, } \\
\text { telephone, electric blankets, Sky } \\
\text { TV, DVD player, wireless } \\
\text { internet. }\end{array}$ & $\begin{array}{l}\text { Continental breakfast, } \\
\text { food and beverages, } \\
\text { OCRT, DVD library, } \\
\text { scenery, tour activities } \\
\text { booking service Lake } \\
\text { Dunstan, vineyards, } \\
\text { orchards, gold mining } \\
\text { trails, 4WD, five ski } \\
\text { fields, golf course, } \\
\text { bowling, squash, } \\
\text { swimming, tennis, }\end{array}$ & Timber \\
\hline
\end{tabular}




\begin{tabular}{|c|c|c|c|c|c|c|c|c|}
\hline & & & & & & & $\begin{array}{l}\text { netball, mountain } \\
\text { biking, walking tracks } \\
\text { and trail, arts and } \\
\text { crafts, Cromwell } \\
\text { heated swimming } \\
\text { pool, } 42 \text { annual } \\
\text { cultural events (listed } \\
\text { in table3). }\end{array}$ & \\
\hline 199 & Cromwell & Orchard House & $1 \times \mathrm{SC}$ & New & $\begin{array}{l}\text { Dining room, laundry } \\
\text { facilities, BBQ. }\end{array}$ & $\begin{array}{l}\text { Both suites have ensuite } \\
\text { bathroom, balcony, TV and } \\
\text { DVD player, heated towel rail, } \\
\text { hairdryer, wireless internet, } \\
\text { private guest area with coffee } \\
\text { and tea making facilities. }\end{array}$ & $\begin{array}{l}\text { Continental breakfast, } \\
\text { eggs from the house } \\
\text { hens, fresh fruits, } \\
\text { wine, OCRT, garden } \\
\text { walking tracks, water } \\
\text { skiing, Kawarau river } \\
\text { jet boating, skiing, } \\
\text { fishing, heritage sites, } \\
\text { orchard tours, golf } \\
\text { tours. }\end{array}$ & $\begin{array}{l}\text { Brick and } \\
\text { timber }\end{array}$ \\
\hline 200 & Cromwell & $\begin{array}{l}\text { River Rock Estate } \\
\text { Vineyard } \\
\text { Accommodation }\end{array}$ & $1 \times \mathrm{B} \& \mathrm{~B}$ & New & $\begin{array}{l}\text { Bar, BBQ, heat pump/ air } \\
\text { conditioning. }\end{array}$ & $\begin{array}{l}\text { Both units have ensuite } \\
\text { bathroom, toilet, tea and coffee } \\
\text { making facilities, microwave } \\
\text { and fridge, TV, DVD player, } \\
\text { wireless internet, hair dryer, } \\
\text { lighting. }\end{array}$ & $\begin{array}{l}\text { Breakfast, OCRT, } \\
\text { historic Cromwell city, } \\
\text { scenery and landscape } \\
\text { views, golf course, } \\
\text { swimming pool, } \\
\text { vineyards, } 42 \text { cultural } \\
\text { events (listed in } \\
\text { table3), wine. This } \\
\text { accommodation is not } \\
\text { suitable for children } \\
\text { under } 14 \text { years. }\end{array}$ & $\begin{array}{l}\text { Timber, walls } \\
\text { and roof are } \\
\text { covered by } \\
\text { corrugated } \\
\text { iron }\end{array}$ \\
\hline 201 & Queenstown & Four Seasons Motel & $1 \times$ Motel & New & $\begin{array}{l}\text { An outdoor swimming } \\
\text { pool, heated spa pool, play } \\
\text { area, drying room for skis, } \\
\text { a guest computer kiosk, } \\
\text { central heating, guest } \\
\text { laundry room, BBQ. }\end{array}$ & $\begin{array}{l}\text { All } 15 \text { units are equipped with } \\
\text { kitchen facilities, bathroom, } \\
\text { electric blanket, hair dryer, iron } \\
\text { and ironing board, DVD, stereo } \\
\text { and Sky TV, WiFi internet. }\end{array}$ & $\begin{array}{l}\text { Continental breakfast, } \\
\text { OCRT, DVD Library, } \\
\text { Queenstown Bay, } \\
\text { skiing, scenery, } \\
\text { surrounding national } \\
\text { parks. }\end{array}$ & Concrete \\
\hline
\end{tabular}




\begin{tabular}{|c|c|c|c|c|c|c|c|c|}
\hline \multicolumn{9}{|c|}{ Appendix.6: OCRT- Types of Accommodation services (2011) } \\
\hline $\mathrm{Nu}$ & Location & Accommodation Service & C & Facilities & $\begin{array}{l}\text { Cost } \\
\text { Single-double/night }\end{array}$ & Type & Nua & Email \\
\hline 1 & Middlemarch & Annandale & 6 & 3 queens rooms & $\$ 90-130$ & $B \& B$ & 1 & info@annandalebnb.co.nz \\
\hline 2 & Middlemarch & $\begin{array}{l}\text { Blind Billy's Holiday Camp } \\
\text { (3motel units) }\end{array}$ & 13 & $\begin{array}{l}3 \text { motel units, } 3 \text { double and } 6 \\
\text { single beds }+1 \text { double sofa bed. }\end{array}$ & $\begin{array}{l}\$ 115 \text { for two people+ } 45 \\
\text { for extra person }\end{array}$ & Motel & 3 & info@middlemarch-motels.co.nz \\
\hline 3 & Middlemarch & $\begin{array}{l}\text { Blind Billy's Holiday Camp ( } 2 \\
\text { studio units) }\end{array}$ & 7 & $\begin{array}{l}2 \text { studio units, } 2 \text { double and } 3 \\
\text { single beds. }\end{array}$ & $\begin{array}{l}\$ 105 \text { for two+ } 45 \text { for } \\
\text { extra people }\end{array}$ & SC & 2 & info@middlemarch-motels.co.nz \\
\hline 4 & Middlemarch & $\begin{array}{l}\text { Blind Billy's Holiday Camp ( } 7 \\
\text { tourist cabins) }\end{array}$ & 42 & 7 tourist cabins. & \$35/person & SC & 7 & info@middlemarch-motels.co.nz \\
\hline 5 & Middlemarch & $\begin{array}{l}\text { Blind Billy's Holiday Camp ( } 3 \\
\text { backpacker cabins) }\end{array}$ & 18 & 3 backpacker cabins. & $\begin{array}{l}\text { \$22/person excluded } \\
\text { bedding }\end{array}$ & Вp & 3 & info@middlemarch-motels.co.nz \\
\hline 6 & Middlemarch & $\begin{array}{l}\text { Blind Billy's Holiday Camp( On- } \\
\text { site caravans) }\end{array}$ & N/A & On-site caravans & $\begin{array}{l}\$ 11 \text { per person (plus } \$ 5 \\
\text { per site if power } \\
\text { required). }\end{array}$ & \multirow{2}{*}{$\begin{array}{l}\text { Caravan/c } \\
\text { amping }\end{array}$} & \multirow{2}{*}{ N/A } & nfo@middlemarch-motels.co.nz \\
\hline 7 & Middlemarch & $\begin{array}{l}\text { Blind Billy's Holiday Camp( } \\
\text { Caravan, campervan points and } \\
\text { tent site) }\end{array}$ & N/A & $\begin{array}{l}\text { Caravan, campervan points \& } \\
\text { tent sites. }\end{array}$ & $\$ 11$ per person & & & nfo@middlemarch-motels.co.nz \\
\hline 8 & Middlemarch & Cottesbrook & 13 & $\begin{array}{l}\text { Cottage 1- } 1 \text { double, } 2 \text { queens, } 2 \\
\text { singles, } 1 \text { double sofa bed. } \\
\text { Cottage 2- } 2 \text { queen beds, } 2 \\
\text { singles. }\end{array}$ & \$250-300/ cottage/ night & SC & 2 & www.cottesbrook.co.nz \\
\hline 9 & Middlemarch & Gladbrook (B\&B) & 10 & 3 queens and two twin bedroom. & $\$ 85-\$ 210 /$ room & $\mathrm{B} \& \mathrm{~B}$ & 1 & enquiries@ gladbrook Station.co.nz \\
\hline 10 & Middlemarch & Gladbrook(cottage) & 5 & 3 bed room up to 5 guests. & $\begin{array}{l}\text { \$60/person - For two } \\
\text { nights or more } \\
\$ 180 / \text { night / group. }\end{array}$ & SC & 1 & enquiries@ gladbrook Station.co.nz \\
\hline 11 & Middlemarch & Gladbrook (hut) & 5 & 2 bed rooms. & $\begin{array}{l}\text { \$60/person- for two } \\
\text { nights or more } \\
\text { \$250/night/group. }\end{array}$ & SC & 1 & enquiries@ gladbrook Station.co.nz \\
\hline 12 & Middlemarch & Jack’s Stone Cotta & 4 & Cottage, 2 bedroom & Double $\$ 115$, single $\$ 8$ & & & janice.harvie@gmail.com \\
\hline
\end{tabular}




\begin{tabular}{|c|c|c|c|c|c|c|c|c|}
\hline & & & & & $\begin{array}{l}\text { each extra person } \$ 40 \\
\text { (whole house } \$ 195\end{array}$ & & 1 & \\
\hline 13 & Middlemarch & Middlemarch B\&B & 7 & $\begin{array}{l}\text { 3queen rooms, one with an } \\
\text { additional single bed. }\end{array}$ & $\$ 80-\$ 150$ & $\mathrm{~B} \& \mathrm{~B}$ & 1 & equiies@middlemarchbnb.cnz \\
\hline 14 & Middlemarch & Pukenangi Country Retreat & 8 & $\begin{array}{l}\text { Renewed farm house( } 1908), 1 \\
\text { king bedroom, } 3 \text { queens } \\
\text { bedrooms. }\end{array}$ & $\begin{array}{l}\text { Double rooms (all with } \\
\text { ensuite) } \$ 180 \text { - } \$ 240 \text { per } \\
\text { night, single occupancy } \\
\text { deduct } \$ 20 \text {, } \\
\text { whole house: } \$ 600\end{array}$ & SC & 1 & enquiries@pukerangihomestead.co.nz \\
\hline 15 & Middlemarch & Rose Cottage B\&B & 6 & $\begin{array}{l}\text { Cottage studio including } \\
\text { two queen rooms, up to } 4 \text { guests. } \\
\text { Rose bedroom, } 1 \text { queen bedroom, } \\
\text { up to } 2 \text { guests. }\end{array}$ & $\begin{array}{l}\text { Queen suite } \$ 130.00 \text { per } \\
\text { night per couple. } \$ 70.00 \\
\text { single, double suite } \\
\$ 120.00 \text { per night per } \\
\text { couple. } \$ 70.00 \text { single }\end{array}$ & $\mathrm{B} \& \mathrm{~B}$ & 1 & inquiries@xtra.co.nz \\
\hline 16 & Middlemarch & Strathmore B\&B & 5 & $\begin{array}{l}\text { Renewed bakery- 1880- two } \\
\text { bedrooms. }\end{array}$ & $\$ 120$ couple, $\$ 80$ single & $\mathrm{B} \& \mathrm{~B}$ & 1 & mjwilson@actrix.co.nz \\
\hline 17 & Middlemarch & Strath Taieri Hotel & 12 & $\begin{array}{l}\text { Historic hotel (1890), plus B\&B } \\
\text { (renewed bakery), } 2 \text { double } \\
\text { bedrooms, } 5 \text { single bedrooms, } 1 \\
\text { hut ( } 1 \text { double and } 1 \text { single). }\end{array}$ & $\begin{array}{l}2 \text { double bedroom } \$ 90 \\
\text { per night, } 5 \text { single } \\
\text { bedroom } \$ 40 \text { each share } \\
\text { twin or } \$ 50 \text { for single } \\
\text { room, 'the hut' } 1 x \text { double, } \\
1 \text { single } \$ 90 \text { per night. }\end{array}$ & Hotel & 1 & strathtaierihotel@xtra.co.nz \\
\hline 18 & Middlemarch & The Farm Homestay & 4 & $\begin{array}{l}1 \text { king, } 1 \text { twin , total } 2 \text { bedrooms, } \\
\text { can be altered to } 4 \text { singles }\end{array}$ & $\begin{array}{l}\text { \$ } 75 \text { pppn } \\
\text { \$110 -2people in 1,pn }\end{array}$ & Home stay & 1 & http://www.thefarmhomestay.co.nz \\
\hline 19 & Middlemarch & The Lodge & 10 & $\begin{array}{l}5 \text { rooms lodge, } 2 \text { queens, } 1 \\
\text { double, } 2 \text { x twin rooms. }\end{array}$ & $\begin{array}{l}\text { Queen } \$ 140 \\
\text { double } \$ 100 \\
\text { Single } \$ 50\end{array}$ & $\mathrm{~B} \& \mathrm{~B}$ & 1 & enquiries@thelodge-middlemarch.co.nz \\
\hline 20 & Middlemarch & $\begin{array}{l}\text { The Rocks luxury } \\
\text { Accommodation ( Longford } \\
\text { Retreat) }\end{array}$ & 5 & 2 bedrooms & $\begin{array}{l}\$ 210 \text { for } 2 \text { people and } \\
\$ 50 \text { for each additional } \\
\text { person }\end{array}$ & SC & 1 & info@therocks.co.nz \\
\hline 21 & Middlemarch & $\begin{array}{l}\text { The Rocks luxury } \\
\text { Accommodation ( Braeside } \\
\text { cottage) }\end{array}$ & 5 & 1 queen bed an 2 king singles. & $\begin{array}{l}\$ 180 \text { for } 2 \text { people and } \\
\$ 50 \text { for each additional } \\
\text { person }\end{array}$ & $\mathrm{B} \& \mathrm{~B}$ & 1 & info@therocks.co.nz \\
\hline
\end{tabular}




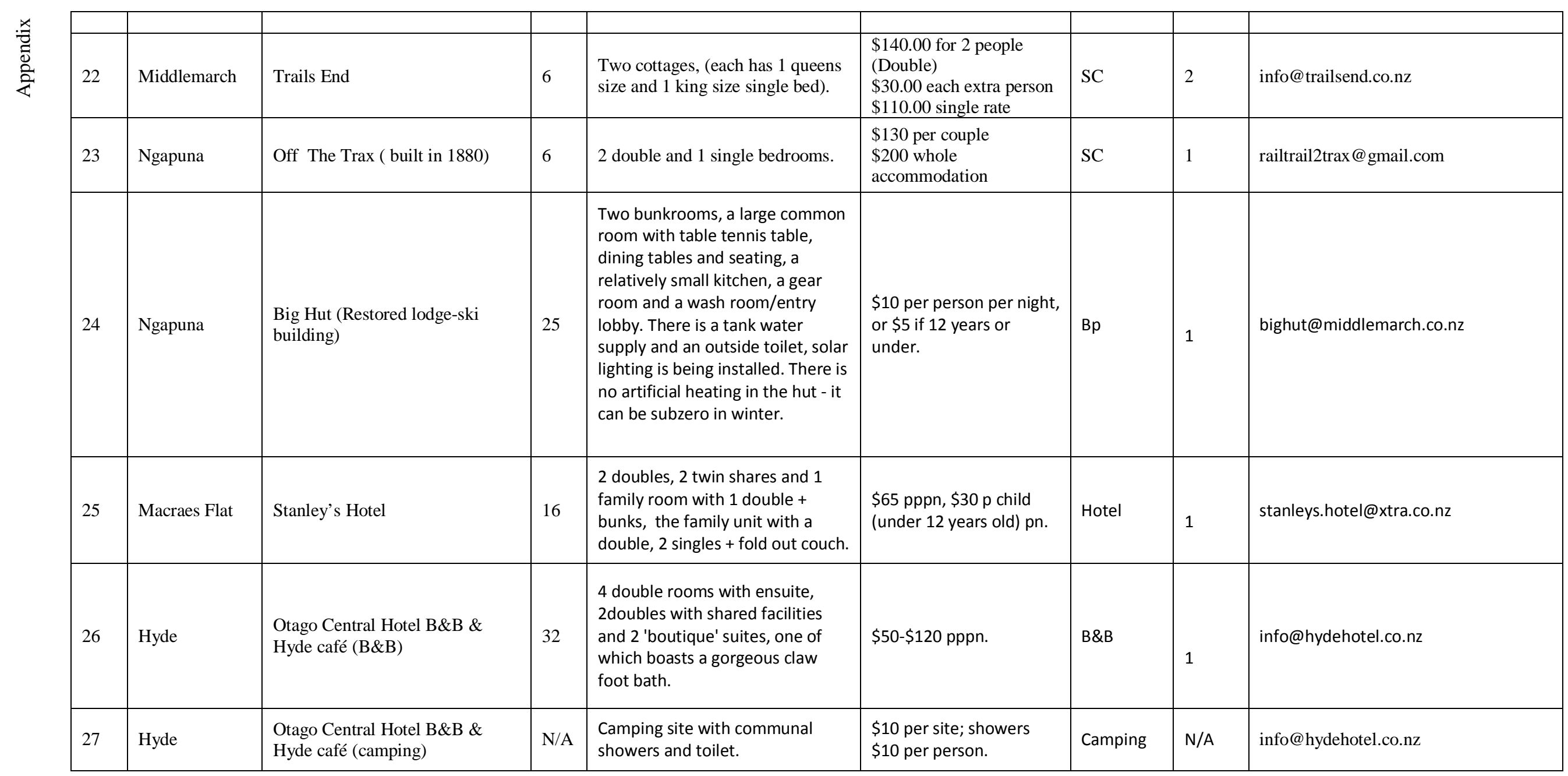




\begin{tabular}{|c|c|c|c|c|c|c|c|c|}
\hline 28 & Hyde & $\begin{array}{l}\text { Emerald Hills Country } \\
\text { (guesthouse) }\end{array}$ & 6 & 3 bed rooms. & $\begin{array}{l}\$ 65 / \text { person/night (price } \\
\text { includes continental } \\
\text { breakfast) queen, twin, } \\
\text { super king or } 2 \text { single } \\
\text { combination. }\end{array}$ & $\mathrm{B} \& \mathrm{~B}$ & 1 & emeraldhills@clear.net.nz \\
\hline 29 & Hyde & Emerald Hills Country (cottage) & 6 & 3 bed rooms. & $\$ 65$ pppn & $\mathrm{B} \& \mathrm{~B}$ & 1 & emeraldhills@clear.net.nz \\
\hline 30 & Hyde & Pine Grove (1869 historic cottage & 6 & 3 bed rooms. & $\begin{array}{l}\$ 65 / \text { person/night (price } \\
\text { includes continental } \\
\text { breakfast) double, twin, } \\
\text { and super king/ } 2 \text { single } \\
\text { combination. }\end{array}$ & SC & 1 & matandro@slingshot.co.nz \\
\hline 31 & Tiroiti & $\begin{array}{l}\text { Tiroiti- Hyde and Tiroiti- } \\
\text { Kokonga }\end{array}$ & - & $\begin{array}{l}\text { Historic site for visit ( no } \\
\text { accommodation). }\end{array}$ & - & - & - & - \\
\hline 32 & Kokonga & Kokonga Lodge & 12 & 6 double bedrooms. & $\begin{array}{l}\text { Bed and full breakfast } \\
\$ 285 / \$ 260 \text { (double/twin) } \\
\text { per room. } \$ 235 / \$ 210 \text { sole } \\
\text { occupancy. }\end{array}$ & $\mathrm{B} \& \mathrm{~B}$ & 1 & info@kokongalodge.co.nz \\
\hline 33 & Kokonga & Kokonga Cottage & 4 & $\begin{array}{l}1 \text { double bed room, } 1 \text { twin bed } \\
\text { room. }\end{array}$ & $\begin{array}{l}\$ 250 \text { for } 2 \text { guests, } \\
\text { maximum sleeps } 4 \text {. }\end{array}$ & SC & 1 & info@kokongalodge.co.nz \\
\hline 34 & Kokonga & 2Wheel Backpackers & 6 & $\begin{array}{l}1 \text { private room with queen size } \\
\text { bed and } 1 \text { bunk with } 4 \text { single } \\
\text { beds. }\end{array}$ & $\begin{array}{l}\text { Private room } \$ 110 \text { pppn, } \\
\text { bunk } \$ 40 \text { pppn }\end{array}$ & Bp & 1 & frednewman@xtra.co.nz \\
\hline 35 & Pareau/Styx & Styx Hotel & \multicolumn{6}{|c|}{ The jail, Styx Hotel - now is a holiday home - and hotel stables are privately owned but the public is welcome to look around. } \\
\hline 36 & Patearoa & Patearoa Hotel +7 Chalets & 36 & $\begin{array}{l}4 \text { twin bed room, up to } 22 \text { guests } \\
+14 \text { (chalets). }\end{array}$ & $\begin{array}{l}\text { Hotel \$35 per person } \\
\text { (ensuite twin bedroom } \\
\$ 37.50 \text { pp), } \\
\text { chalet \$25 pp. }\end{array}$ & Hotel & 1 & $\begin{array}{lr}\text { Fax: } & 034447865 \\
\text { Phone: } 034447865\end{array}$ \\
\hline 37 & Waipiata & $\begin{array}{l}\text { Waipiata Country Hotel (built in } \\
\text { 1899) }\end{array}$ & N/A & N/A & $\begin{array}{l}\text { From } \$ 70 \text { pppn (includes } \\
\text { a hearty continental } \\
\text { breakfast). }\end{array}$ & Hotel & 1 & enquiries@waipiatahotel.co.nz \\
\hline 38 & Waipiata & $\begin{array}{l}\text { Komako Peonies \& Cottage } \\
\text { Garden (the station building) }\end{array}$ & 4 & 1 queen size and 2 single bed. & \multirow{2}{*}{$\begin{array}{l}\text { \$60 per person including } \\
\text { continental breakfast. } \\
\$ 100 \text { per person }\end{array}$} & $B \& B$ & 1 & enquiries@waipiatahotel.co.nz \\
\hline 39 & Waipiata & Komako Peonies \& Cottage & 2 & 1 queen size bed accommodation. & & & & \\
\hline
\end{tabular}




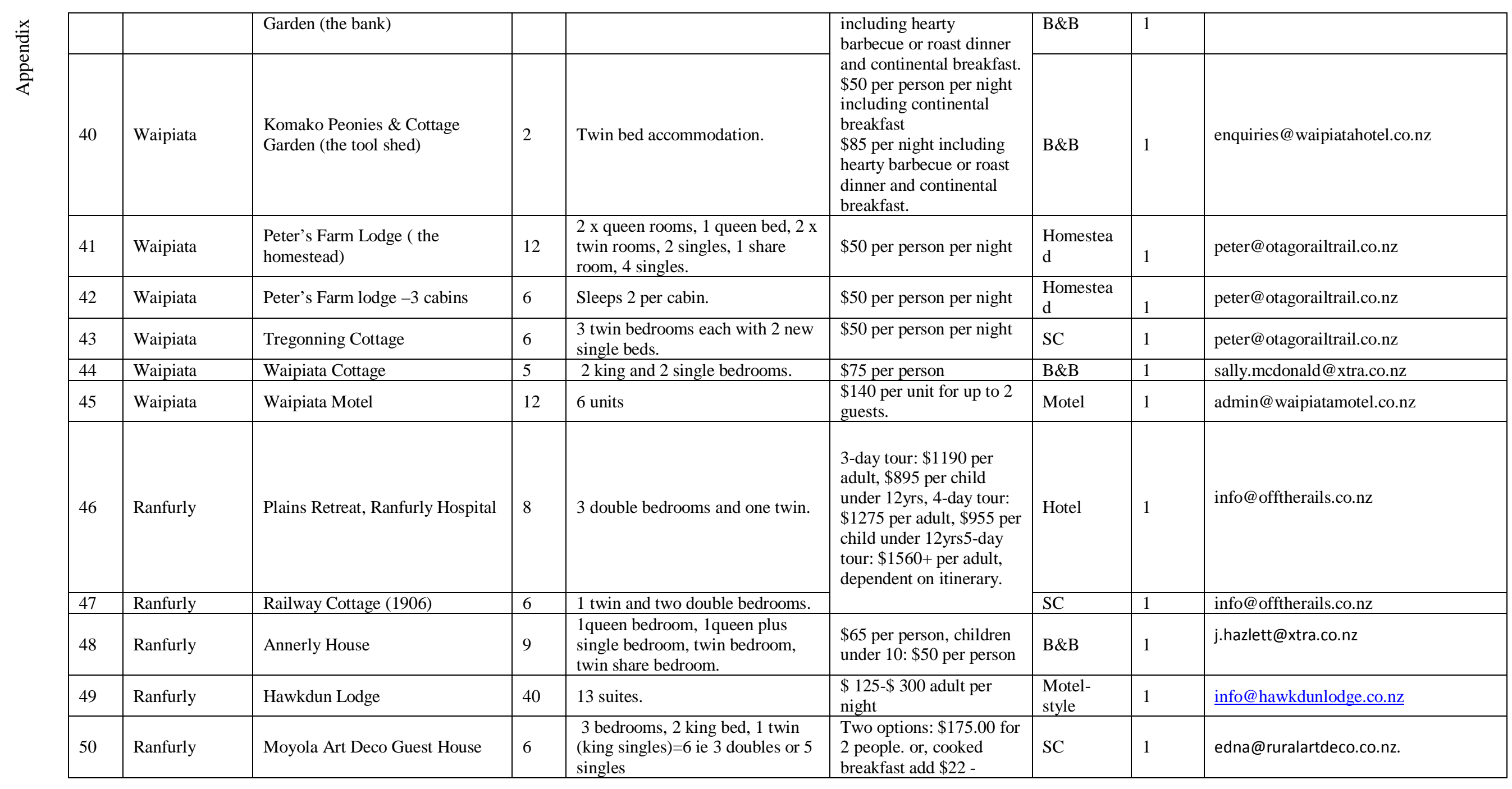




\begin{tabular}{|c|c|c|c|c|c|c|c|c|}
\hline 51 & Ranfurly & Old Post Office (B\&B) & 11 & $\begin{array}{l}\text { 1double (bed) room, } 2 \text { twin room } \\
\text { (bunk), } 1 \text { twin room (bunk), + } \\
\text { additional small single bed if } \\
\text { required, } 1 \text { family room = double } \\
\text { bed + bunks. }\end{array}$ & $\begin{array}{l}\$ 65 \text { / } \$ 50 \text { single } \\
\text { occupancy, } \$ 95 \text { for the } \\
\text { family room (sleeps up to } \\
\text { 4) }\end{array}$ & $\mathrm{B} \& \mathrm{~B}$ & 1 & oldpobackpackers@gmail.com \\
\hline 52 & Ranfurly & Old Post Office (backpackers) & 18 & $\begin{array}{l}\text { Dormitory (two interconnecting } \\
\text { rooms of } 5+4 \text { with } 4 \text { sets bunks } \\
+1 \text { single bed). }\end{array}$ & \$28 per adult, & Bp & 1 & oldpobackpackers@gmail.com \\
\hline 53 & Ranfurly & $\begin{array}{l}\text { Ranfurly Holiday Park ( motel } \\
\text { double studio) }\end{array}$ & 2 & 1double bed. & $\begin{array}{l}1 \text { bedroom motel } \$ 90 \text { per } \\
\text { night for two people. }\end{array}$ & Motel & 1 & booking@ranfurlyholidaypark.co.nz \\
\hline 55 & & $\begin{array}{l}\text { Ranfurly Holiday Park(motel } \\
\text { studio ) }\end{array}$ & 4 & Double bed \& set of bunks & $\begin{array}{l}\text { Rates for two people } \$ 90 \\
\text { per night, extra adults } \\
\$ 20 \text { each, extra children } \\
\$ 15 \text { each. }\end{array}$ & Motel & 1 & booking@ranfurlyholidaypark.co.nz \\
\hline 55 & Ranfurly & $\begin{array}{l}\text { Ranfurly Holiday Park (motel } \\
\text { studio) }\end{array}$ & 5 & $\begin{array}{l}\text { Double bed set of bunks \& single } \\
\text { bed. }\end{array}$ & $\begin{array}{l}\text { Rates for two people } \$ 90 \\
\text { per night, extra adults } \\
\$ 20 \text { each, extra children } \\
\$ 15 \text { each. }\end{array}$ & Motel & 1 & booking@ranfurlyholidaypark.co.nz \\
\hline 56 & Ranfurly & $\begin{array}{l}\text { Ranfurly Holiday Park ( } 1 \\
\text { bedroom motel unit) }\end{array}$ & 6 & $\begin{array}{l}\text { Queen size bed lounge including } \\
\text { set of bunks and a double pull out } \\
\text { divan bed. }\end{array}$ & $\begin{array}{l}\text { Price from: } \$ 100.00 \text { per } \\
\text { night }\end{array}$ & Motel & 1 & booking@ranfurlyholidaypark.co.nz \\
\hline 57 & Ranfurly & $\begin{array}{l}\text { Ranfurly Holiday Park (double } \\
\text { cabin) }\end{array}$ & 2 & $\begin{array}{l}\text { Bunk bed with double on the } \\
\text { bottom . }\end{array}$ & $\begin{array}{l}\text { Cabin } \$ 40 \text { per night for } \\
\text { two people maximum } 8 \\
\text { people, extra adults } \$ 15 \text {, } \\
\text { extra children } \$ 12 \text {. }\end{array}$ & SC & 1 & booking@ranfurlyholidaypark.co.nz \\
\hline 58 & Ranfurly & $\begin{array}{l}\text { Ranfurly Holiday Park( cabin - } \\
\text { sleeps 4) }\end{array}$ & 4 & Two sets of single bunks. & $\begin{array}{l}\text { Rates for two people } \$ 40 \\
\text { per night, extra adults } \\
\$ 15 \text { each, extra children } \\
\$ 12 \text { each. }\end{array}$ & SC & 1 & booking@ranfurlyholidaypark.co.nz \\
\hline 59 & Ranfurly & $\begin{array}{l}\text { Ranfurly Holiday Park( cabin - } \\
\text { sleeps 5) }\end{array}$ & 5 & $\begin{array}{l}\text { Double bunk (double bed below } \\
\text { and single bed on top) and a set } \\
\text { of single bunks. }\end{array}$ & $\begin{array}{l}\text { Rates for two people } \$ 40 \\
\text { per night, extra adults } \\
\$ 15 \text { each, extra children } \\
\$ 12 \text { each. }\end{array}$ & $\mathrm{SC}$ & 1 & booking@ranfurlyholidaypark.co.nz \\
\hline 60 & Ranfurly & $\begin{array}{l}\text { Ranfurly Holiday Park - two } \\
\text { bedroom cabin(sleeps 8) }\end{array}$ & 8 & $\begin{array}{l}2 \text { rooms, } 2 \text { sets of single bunks in } \\
\text { each room. }\end{array}$ & $\begin{array}{l}\text { Rates for two people } \$ 40 \\
\text { per night, extra adults }\end{array}$ & $\mathrm{SC}$ & 1 & booking@ranfurlyholidaypark.co.nz \\
\hline
\end{tabular}




\begin{tabular}{|c|c|c|c|c|c|c|c|c|}
\hline & & & & & $\begin{array}{l}\text { \$15 each, extra children } \\
\text { \$12 each. }\end{array}$ & & & \\
\hline 61 & Ranfurly & $\begin{array}{l}\text { Ranfurly Holiday Park (powered } \\
\text { sites-8 person) }\end{array}$ & 8 & Camp site & $\begin{array}{l}\$ 28 \text { per night for two } \\
\text { people, extra adults } \$ 12 \text {, } \\
\text { extra children } \$ 8 \text {. }\end{array}$ & Camping & 1 & booking@ranfurlyholidaypark.co.nz \\
\hline 62 & Ranfurly & $\begin{array}{l}\text { Ranfurly Holiday Park (non- } \\
\text { powered sites -8 person) }\end{array}$ & 8 & Camp site & $\begin{array}{l}\$ 12 \text { per adult, } \$ 8 \text { per } \\
\text { child. }\end{array}$ & Camping & 1 & booking@ranfurlyholidaypark.co.nz \\
\hline 63 & Ranfurly & Ranfurly Motel & 16 & $\begin{array}{l}2 \text { studio units (one with a queen, } \\
\text { the other a super king), ensuite, up } \\
\text { to } 4 \text { guests, } 4 \text { family or group units } \\
\text { with } 2 \text { bedrooms ( } 3 \text { with two } \\
\text { singles in each bedroom and } \\
\text { queen in the lounge, the } 4 \text { th has a } \\
\text { queen in the bedroom and two } \\
\text { singles. }\end{array}$ & $\begin{array}{l}\text { Studio 1-2 people - } \$ 100 \text {, } \\
2 \text { bedroom units } 2 \text { people } \\
\text { - } \$ 115 .\end{array}$ & Motel & 1 & ranfurlymotels@xtra.co.nz \\
\hline 64 & Ranfurly & Ranfurly Lion Hotel & 30 & Twin and single rooms. & $\begin{array}{l}\text { From } \$ 50 \text { single } \\
\text { to } \$ 85 \text { double room }\end{array}$ & Hotel & 1 & Ranfurly.hotel@xtra.co.nz \\
\hline 65 & Ranfurly & $\begin{array}{l}\text { Trail Blazers B\&B (and farm } \\
\text { adventures) }\end{array}$ & 8 & $\begin{array}{l}3 \text { queen bedrooms and } 1 \text { twin } \\
\text { bedroom. }\end{array}$ & $\begin{array}{l}\text { \$55 per person (including } \\
\text { continental breakfast). }\end{array}$ & $\mathrm{B} \& \mathrm{~B}$ & 1 & Trail_blazers@live.com \\
\hline 66 & Ranfurly & Trout 'n' Trail B\&B & 8 & 1 queen and 1 king studio. & $\begin{array}{l}\text { Queen studio (two } \\
\text { people) \$110,queen } \\
\text { studio (single } \\
\text { occupancy)-\$60, king } \\
\text { studio (two people)\$110 } \\
\text {, king studio, extra adult } \\
\text { \$40, king studio family } \\
\text { rate (2 adults, } 2 \text { children } \\
\text { double sofa) \$165. }\end{array}$ & $\mathrm{B} \& \mathrm{~B}$ & 1 & booking@troutntrailbnb.co.nz \\
\hline 67 & Wedderburn & Mt Ida Station Farm stay & 5 & 3 bedrooms. & N/A & SC & 1 & mt.ida@farmside.co.nz \\
\hline 68 & Wedderburn & $\begin{array}{l}\text { Wedderburn Lodge and Cottages } \\
\text { (lodge) }\end{array}$ & 9 & $\begin{array}{l}\text { Lodg-1 double bedroom } \\
1 \text { bedroom with } 1 \text { double + } 2 \\
\text { singles } \\
1 \text { bedroom with } 3 \text { singles. }\end{array}$ & $\begin{array}{l}\$ 50.00 \text { per person, } \\
\text { children under } 10 \text { half } \\
\text { adult price. }\end{array}$ & SC & 1 & enquiries@wedderburn.net.nz \\
\hline 69 & Wedderburn & $\begin{array}{l}\text { Wedderburn Lodge and Cottages } \\
\text { (1 large cottage and } 2 \text { small }\end{array}$ & 8 & $\begin{array}{l}1 \text { large cottages, bedroom with } 2 \\
\text { singles } 1 \text { loungette with } 1 \text { king }\end{array}$ & $\$ 75.00$ per person & SC & 1 & ienquiries@wedderburn.net.nz \\
\hline
\end{tabular}




\begin{tabular}{|c|c|c|c|c|c|c|c|c|}
\hline & & cottages) & & $\begin{array}{l}\text { single, up to } 4 \text {, 2smaller cottages, } \\
\text { each has } 2 \text { singles or } 1 \text { super king } \\
\text { ensuite, up to } 4 \text { guests. }\end{array}$ & & & & \\
\hline 70 & Wedderburn & $\begin{array}{l}\text { Wedderburn Tavern (mud brick } \\
\text { bunkhouse) }\end{array}$ & 6 & $\begin{array}{l}1 \text { double bed and two sets of } \\
\text { bunks. }\end{array}$ & $\$ 45-\$ 50$ per person & $\mathrm{B} \& \mathrm{~B}$ & 1 & info@wedderburntavern.co.nz \\
\hline 71 & Wedderburn & Wedderburn Tavern (tavern) & 14 & $\begin{array}{l}\text { Tavern, two sets of bunks, } 3 \text { bed } \\
\text { rooms, } 1 \text { family room up to } 14 \\
\text { guests }\end{array}$ & $\$ 50-\$ 110$ & SC & 1 & info@wedderburntavern.co.nz \\
\hline 72 & Wedderburn & $\begin{array}{l}\text { Wedderburn Tavern ( tent and } \\
\text { camp) }\end{array}$ & 12 & 1 tent sleeps 6 and 1 campsite. & N/A & Camping & 1 & info@wedderburntavern.co.nz \\
\hline 73 & Danseys Pass & Danseys Pass Hotel & 6 & $\begin{array}{l}1 \text { ensuite double twin and } 2 \\
\text { double twins. }\end{array}$ & $\begin{array}{l}\text { Ensuite double/twin } \\
\$ 160 \text {, double twin } \$ 140\end{array}$ & Hotel & 1 & booking@danseyspass.co.nz \\
\hline 74 & Danseys Pass & Short Lands Station Farm Stay & 6 & $\begin{array}{l}\text { Double storey homestead } \\
3 \text { double rooms convert to twin or } \\
\text { single.. }\end{array}$ & Single $\$ 60$, double $\$ 110$ & $\mathrm{~B} \& \mathrm{~B}$ & 1 & shortlands@scorch.co.nz \\
\hline 75 & Naseby & Ancient Briton Hotel & 40 & $\begin{array}{l}\text { Historical } 16 \text { rooms hotel } 3 \\
\text { double bedroom with ensuite, } 4 \\
\text { double \& one single bedroom } \\
\text { with ensuite ,1twin bedroom with } \\
\text { bunk set and ensuite ,2triple } \\
\text { single bedroom with ensuite), } \\
\text { 1single bedroom [not ensuite), } 1 \\
\text { bunk room [not ensuite] connects } \\
\text { to family room ,1 studio unit with } \\
\text { twin beds and ensuite, some } \\
\text { cooking facilities ,1 family unit } \\
\text { with double bed, single bed, and } \\
\text { ensuite, day bed in living area and } \\
\text { cooking facilities . }\end{array}$ & $\begin{array}{l}\$ 105 \text { for twin share } \\
\$ 75 \text { for single room. }\end{array}$ & Hotel & 1 & ancientbriton@xtra.co.nz \\
\hline 76 & Naseby & $\begin{array}{l}\text { Larchview Holiday Park (tourist } \\
\text { flat) }\end{array}$ & 9 & 3 separate bedrooms. & \$81per 1 and 2 persons. & $\mathrm{SC}$ & 1 & bookings@larchviewholidaypark.co.nz \\
\hline 77 & Naseby & $\begin{array}{l}\text { Larchview Holiday Park (miners } \\
\text { cottage) }\end{array}$ & 6 & $\begin{array}{l}2 \text { separate bedrooms with log } \\
\text { burner, heater, fully equipped } \\
\text { kitchen, shower and toilet. }\end{array}$ & \$81per 1 and 2 persons- & SC & 2 & bookings@larchviewholidaypark.co.nz \\
\hline
\end{tabular}




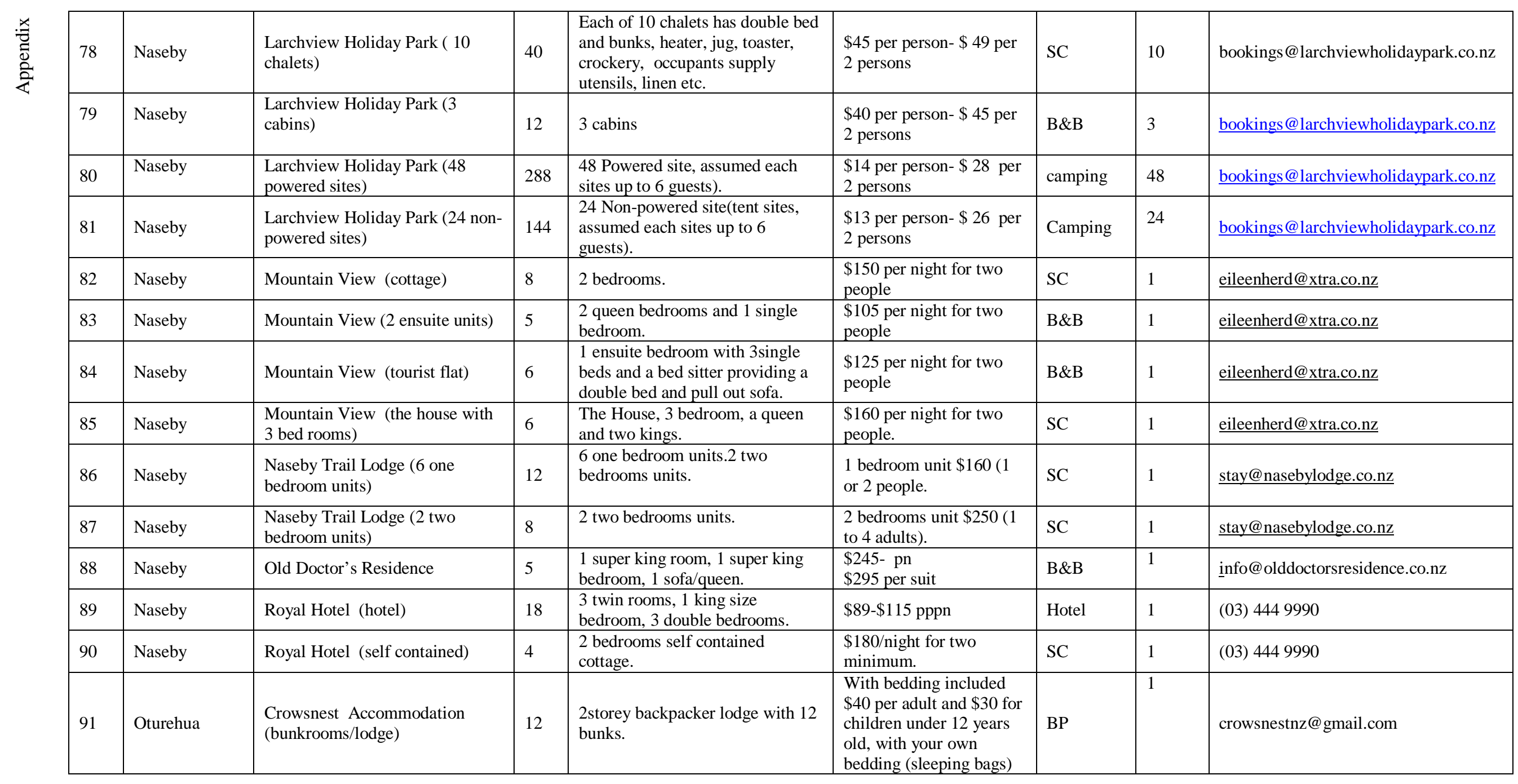




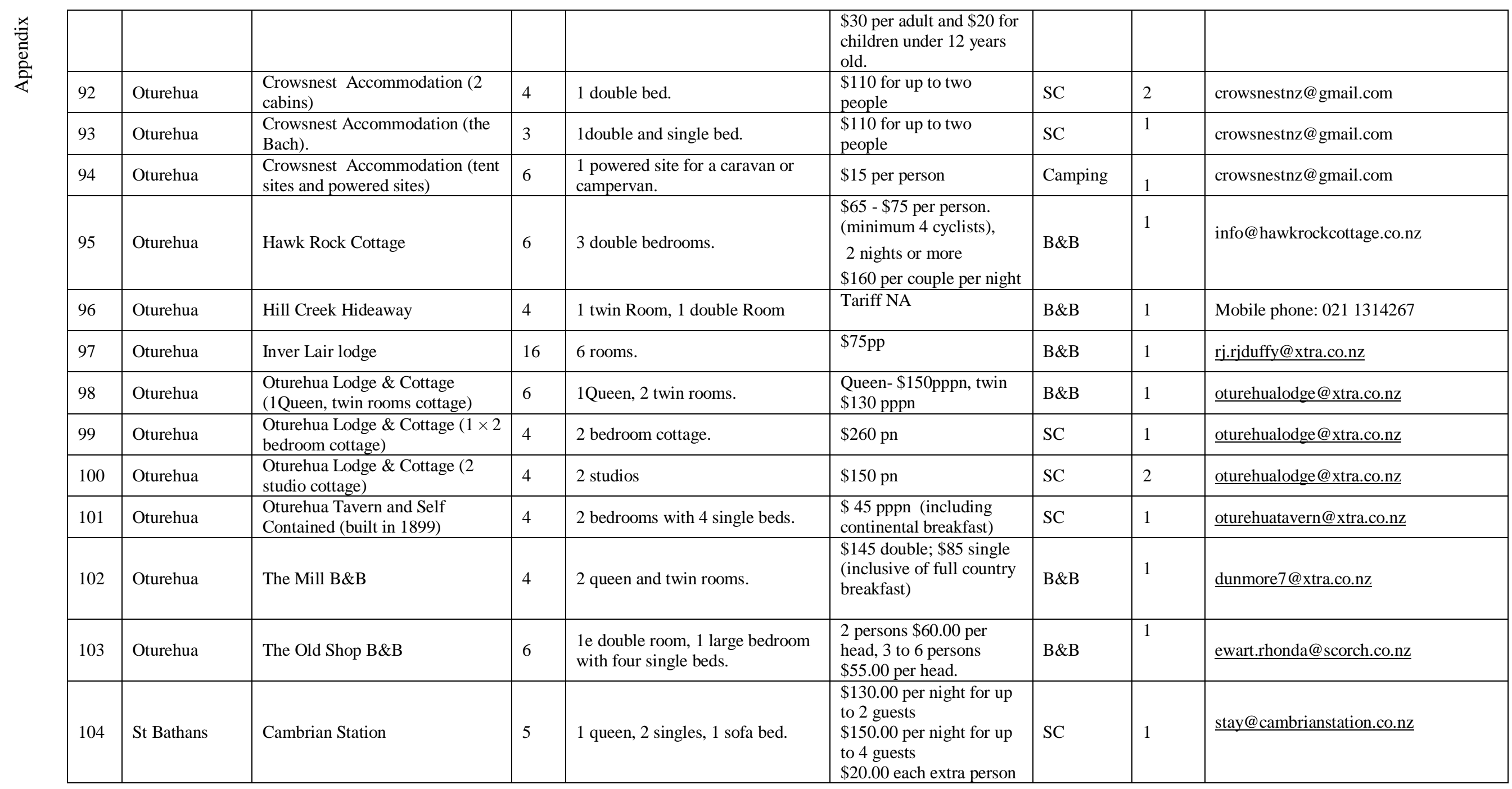




\begin{tabular}{|c|c|c|c|c|c|c|c|c|}
\hline & & & & & thereafter. & & & \\
\hline 105 & St Bathans & $\begin{array}{l}\text { Constable Cottage \& Goal } \\
\text { (cottage) }\end{array}$ & 6 & 3 bedrooms . & \$220 pnp couple & SC & 1 & info@stbathansnz.co.nz \\
\hline 106 & St Bathans & Constable Cottage \& goal(goal) & 2 & The Jail (Gaol): 1 queen size bed. & \$125 pnp couple & $\mathrm{SC}$ & 1 & info@stbathansnz.co.nz \\
\hline 107 & St Bathans & Lombardy Cottage ( cottage) & 4 & 1 king and 1 queen bedroom. & $\begin{array}{l}\$ 250 \text { a night for the } \\
\text { cottage - } 2 \text { night } \\
\text { minimum for the first } \\
\text { couple then } \$ 50 \text { per } \\
\text { person after }\end{array}$ & SC & 1 & www.lombardycottage.co.nz \\
\hline 108 & St Bathans & Lombardy Cottage (caravan) & 4 & $\begin{array}{l}2 \text { singles and a double berth, with } \\
\text { an additional queen bed in the } \\
\text { awning if required }\end{array}$ & NA & $\mathrm{SC}$ & 1 & www.lombardycottage.co.nz \\
\hline 109 & St Bathans & St Bathans B\&B & 5 & $\begin{array}{l}2 \text { king size single beds, } 1 \text { queen } \\
\text { room and } 1 \text { family room. }\end{array}$ & $\begin{array}{l}\text { Single } \$ 80 \text { - Double } \$ \\
130 \text {, family room: } \$ 50 \\
\text { per guest }\end{array}$ & $\mathrm{B} \& \mathrm{~B}$ & 1 & enquiries@stbathansbnb.co.nz \\
\hline 110 & St Bathans & Vulcan Hotel (built in 1882) & 8 & .2 double and 2 twin bed rooms. & $\begin{array}{l}2 \text { double rooms at } \$ 45 \\
\text { pp. } 2 \text { Twin at } \$ 35 \text { pp. }\end{array}$ & Hotel & 1 & stbathans.vulcanhotel@scorch.co.nz \\
\hline 111 & Ida Valley & Parkside Farm Stay & 9 & $\begin{array}{l}1 \text { Queen Room } \\
1 \text { Twin Bedroom } \\
1 \text { Double Bedroom } \\
\text { sleep-out with a double and single } \\
\text { bed. Up to } 9 \text { guests. }\end{array}$ & Web site is NA & $\mathrm{B} \& \mathrm{~B}$ & 1 & jdkewhyte@xtra.co.nz \\
\hline 112 & Auripo & Poolburn Hotel & 16 & 8 rooms. & $\begin{array}{l}\$ 50 \text { a double room with } \\
\text { breakfast }\end{array}$ & Hotel & 1 & dmshearing@xtra.co.nz \\
\hline 113 & Auripo & $\begin{array}{l}\text { Taradale Homestead (built in } \\
\text { 1880) }\end{array}$ & 6 & $\begin{array}{l}2 \text { large queen size and } 2 \text { single } \\
\text { bed rooms. }\end{array}$ & \$90-\$110 per night & $\begin{array}{l}\text { Homestea } \\
\mathrm{d}\end{array}$ & 1 & davitook@xtra.co.nz \\
\hline 114 & Becks & White Horse Hotel(1864) & 18 & 7 room & $\begin{array}{l}\text { Room rates start from } \\
\$ 40.00 \text { single and } \\
\text { includes a continental } \\
\text { breakfast. }\end{array}$ & Hotel & 1 & becks_hotel@hotmail.com \\
\hline 115 & Lauder & Big Sky Cottage & 4 & 2 bedrooms. & $\begin{array}{l}\text { \$135 for } 2 \text { people per } \\
\text { night, \$174 for } 3 \text { people } \\
\text { per night, \$195 for } 4 \\
\text { people per night. }\end{array}$ & SC & 1 & enquiries@bigskycottage.co.nz \\
\hline
\end{tabular}




\begin{tabular}{|c|c|c|c|c|c|c|c|c|}
\hline & & & & & & & & \\
\hline & & & & & & & & \\
\hline 116 & Lauder & $\begin{array}{l}\text { Lauder Rail Trail School and } \\
\text { Milmor Cottage ( } 3 \text { school ensuite } \\
\text { rooms) }\end{array}$ & 6 & 3 school ensuite rooms. & $\$ 80$ per person & $\mathrm{B} \& \mathrm{~B}$ & 1 & lauderschool@xtra.co.nz \\
\hline 117 & Lauder & $\begin{array}{l}\text { Lauder Rail Trail School and } \\
\text { Milmor Cottage (studio cottages) }\end{array}$ & N/A & 2 cottage studios. & $\$ 80$ per person & SC & 1 & auderschool@xtra.co.nz \\
\hline 118 & Lauder & $\begin{array}{l}\text { Lauder Rail Trail School and } \\
\text { Milmor Cottage (standard rooms) }\end{array}$ & 6 & $\begin{array}{l}1 \text { queen room, } 1 \text { twin Room, } 1 \\
\text { double room. }\end{array}$ & $\$ 60$ per person & SC & 1 & auderschool@xtra.co.nz \\
\hline 119 & Lauder & $\begin{array}{l}\text { Lauder Rail Trail School and } \\
\text { Milmor Cottage (cabin) }\end{array}$ & 2 & 1queen/twin room. & $\$ 60$ per person & SC & 1 & auderschool@xtra.co.nz \\
\hline 120 & Lauder & $\begin{array}{l}\text { Lauder Rail Trail School and } \\
\text { Milmor Cottage (Milmor cottage) }\end{array}$ & 8 & $\begin{array}{l}1 \text { double, } 2 \text { twin , } 2 \text { more singles } \\
\text { available. }\end{array}$ & $\$ 60$ per person & SC & 1 & auderschool@xtra.co.nz \\
\hline 121 & Lauder & Lauder Store B\&B (the old store) & 4 & 1 double bed, 2 single beds. & $\begin{array}{l}\text { \$135 per night-2 people } \\
\$ 220 \text { - } 3 \text { people } \$ 260-4 \\
\text { people } \$ 300 .\end{array}$ & SC & 1 & info@lauderstore.co.nz \\
\hline 122 & Lauder & $\begin{array}{l}\text { Lauder Store B\&B (Storekeeper } \\
\text { house) }\end{array}$ & 8 & $\begin{array}{l}2 \text { queens, } 1 \text { twin and } 1 \text { bunk } \\
\text { rooms }\end{array}$ & \$60-\$135 per night & SC & 1 & info@lauderstore.co.nz \\
\hline 123 & Lauder & $\begin{array}{l}\text { Lauder Store B\&B (Arkwrights } \\
\text { Snug ) }\end{array}$ & 4 & 1ensuite, 1super king/zipped. & $\$ 90-\$ 150$ per night & $\mathrm{SC}$ & 1 & info@lauderstore.co.nz \\
\hline 124 & Lauder & Lauder Hotel & 14 & 7 rooms. & $\begin{array}{l}\text { \$55 per person per night } \\
\text { including continental } \\
\text { breakfast. }\end{array}$ & Hotel & 1 & gerald@lauderhotel.co.nz \\
\hline 125 & Lauder & Lauder Burn House B\&B & 8 & 4 bedrooms. & $\begin{array}{l}\$ 75-\$ 100 \text { single } \\
\$ 150-\$ 160 \text { couple }\end{array}$ & $\mathrm{B} \& \mathrm{~B}$ & 1 & lauderburn@farmside.co.nz \\
\hline 126 & Lauder & $\begin{array}{l}\text { Lauderdale Estate( built in 1921- } \\
\text { cottages) }\end{array}$ & 6 & 3 bed rooms. & $\begin{array}{l}\$ 75-\$ 100 \text { single } \\
\$ 150-\$ 160 \text { couple }\end{array}$ & $\mathrm{B} \& \mathrm{~B}$ & 1 & info@lauderdaleestate.co.nz \\
\hline 127 & Lauder & Muddy Creek (built in 1930) & 10 & 5 bedrooms. & $\begin{array}{l}\$ 70 \text { pppn, children (2- } \\
11 \text { years) \$30 pppn. }\end{array}$ & SC & 1 & enquiries@muddycreekcutting.co.nz \\
\hline 128 & Lauder & Pedal Inn & 6 & 2 suits include 6 single beds. & $\begin{array}{l}\text { \$80 pppn, trundle bed } \\
\$ 50 \text { pppn }\end{array}$ & $\mathrm{SC}$ & 1 & benandcatherine@farmside.co.nz \\
\hline
\end{tabular}




\begin{tabular}{|c|c|c|c|c|c|c|c|c|}
\hline 129 & Moa Creek & $\begin{array}{l}\text { Bonspiel Station \& Old Moa } \\
\text { Creek hotel }\end{array}$ & 6 & 2 studio six beds. & $\begin{array}{l}\text { N/A. Advertised as } \\
\text { (negotiable). }\end{array}$ & Hotel & 1 & ben.falconer@farmside.co.nz \\
\hline 130 & Poolburn & Poolburn Hotel & 12 & $\begin{array}{l}\text { Stacked stone huts built by } \\
\text { Chinese gold miners in the } 1860 \text { s. } \\
\text { No electricity no running water. } 4 \\
\text { rooms up to } 12 \text { plus sleep out. }\end{array}$ & $\begin{array}{l}\$ 50 \text { a double room with } \\
\text { breakfast }\end{array}$ & Hotel & 1 & dmshearing@xtra.co.nz \\
\hline 131 & Poolburn & $\begin{array}{l}\text { Solandra Lodge country style } \\
\text { B\&B( built in 1930) }\end{array}$ & 8 & 8 bedrooms. & $\begin{array}{l}1 \text { to } 2 \text { adults }=\$ 150 \text { per } \\
\text { night, additional adults } \\
\text { up to total of } 6=\$ 50 \\
\text { pppn. }\end{array}$ & $\mathrm{B} \& \mathrm{~B}$ & 1 & bookings@solandralodge.co.nz \\
\hline 132 & Ophir & Blacks Hotel & 20 & 10 double rooms. & $\begin{array}{l}\text { \$60 per person (includes } \\
\text { continental breakfast) }\end{array}$ & Hotel & 1 & steven.chapman@clear.net.nz \\
\hline 133 & Ophir & Flannery Lodge & 10 & $\begin{array}{l}2 \text { double room, } \\
\text { four or five to a room Flash } \\
\text { packer style. }\end{array}$ & $\begin{array}{l}2 \text { double room, } \$ 125 \text { per } \\
\text { room, room flash packer } \\
\text { style, } \$ 40 \text { per person. }\end{array}$ & $\mathrm{B} \& \mathrm{~B}$ & 1 & flannerys@scorch.co.nz \\
\hline 134 & Ophir & Flannery Lodge (tent site) & 25 & Tent Sites & N/A ( $\$ 5$ for BBQ) & Camping & 10 & flannerys@scorch.co.nz \\
\hline 135 & Ophir & Millfield Cottage & 6 & 3 bedrooms. & $\begin{array}{l}\text { Peak (Nov-Apr): } \\
\$ 60 \text { pppn for } 2 \text { adults, } \$ 50 \\
\text { per additional adult, } \\
\text { peak-off, } \$ 50 \text { pppn, } \\
\text { children } \$ 40 \text { pppn. }\end{array}$ & SC & 1 & Bm.waller@xnet.co.nz \\
\hline 136 & Ophir & Ophir Bridge B\&B & 4 & 2 bedrooms. & $\begin{array}{l}\$ 200 \text { per night when } 1 \\
\text { room booked (total use of } \\
\text { B \& B), } \$ 180 \text { per room } \\
\text { per night when } 2 \text { rooms } \\
\text { booked (shared } \\
\text { bathroom), } \$ 150 \text { per } \\
\text { night single rate. }\end{array}$ & $\mathrm{B} \& \mathrm{~B}$ & 1 & $\begin{array}{l}\text { http://www.ophirbridgebandb.co.nz/ind } \\
\underline{\text { ex.html }}\end{array}$ \\
\hline 137 & Ophir & $\begin{array}{l}\text { The Old Bakery ( built in 1880- } \\
\text { Stables building) }\end{array}$ & 4 & 1 double and 1queen bedroom. & $\begin{array}{l}\$ 65 \text { per person, } \$ 100 \text { for } \\
\text { two guests. }\end{array}$ & $\mathrm{B} \& \mathrm{~B}$ & 1 & lauderschool@xtra.co.nz \\
\hline 138 & Ophir & $\begin{array}{l}\text { The Old Bakery (built in 1880- } \\
\text { Bakery) }\end{array}$ & 3 & 1 queen and 1 single bed. & $\$ 50$ per person & $\mathrm{B} \& \mathrm{~B}$ & 1 & lauderschool@xtra.co.nz \\
\hline 139 & Omakau & Church Hill B\&B & 6 & 1 queen and 1 twin room. & $\begin{array}{l}1 \text { queen with ensuite - } \\
\text { Single \$110 Double } \\
\text { \$140,1 twin Room, }\end{array}$ & $\mathrm{B} \& \mathrm{~B}$ & 1 & Church_hill-bandb@xtra.co.nz \\
\hline
\end{tabular}




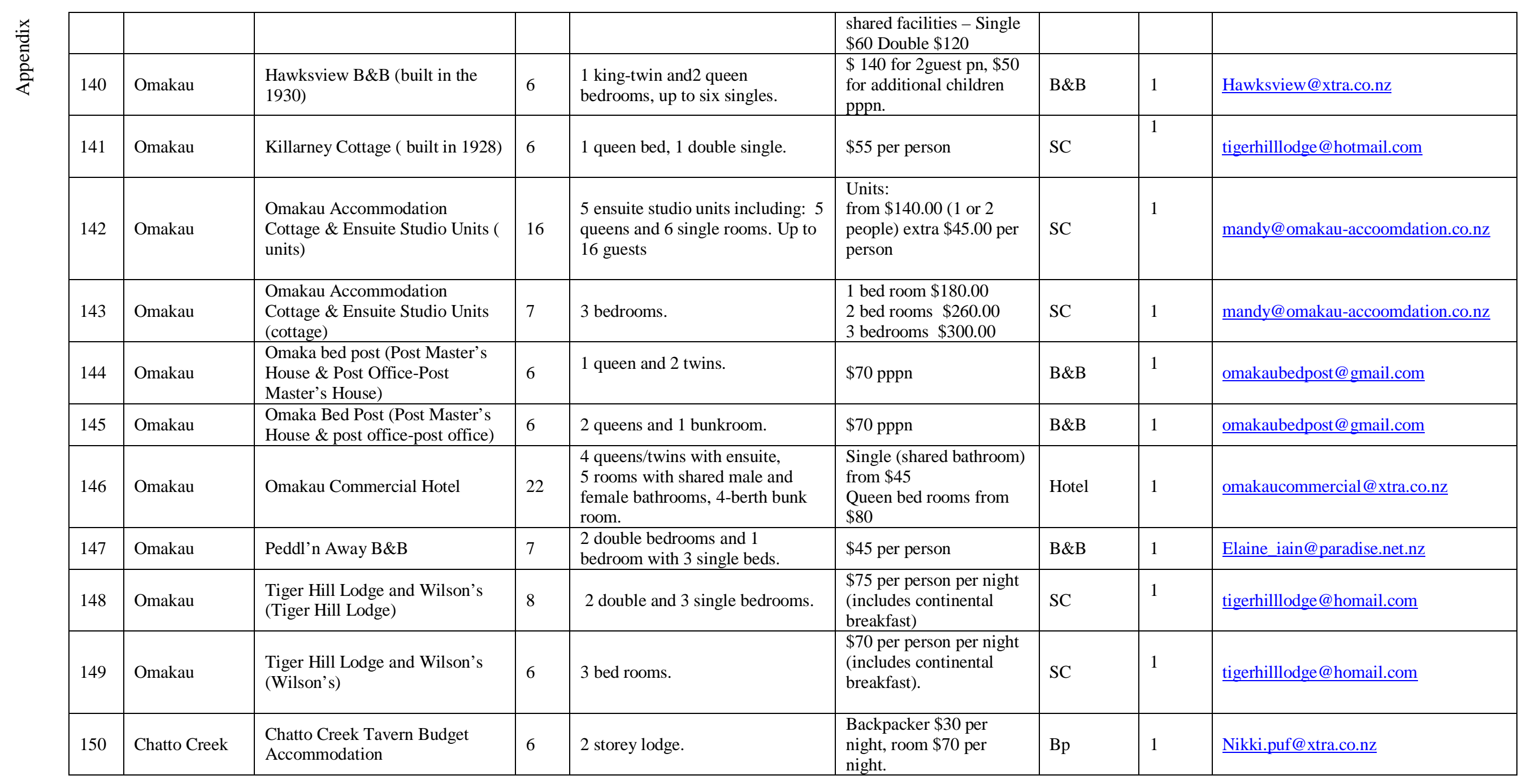




\begin{tabular}{|c|c|c|c|c|c|c|c|c|}
\hline 151 & Chatto Creek & The Magdelans B\&B & 24 & $\begin{array}{l}4 \text { bedrooms- } 4 \text { superbly ensuits- } 4 \\
\text { super king size beds. }\end{array}$ & & $\mathrm{B} \& \mathrm{~B}$ & 1 & dgandbw@homail.com \\
\hline 152 & Chatto Creek & Rockdale B\&B & 6 & 2 double room- 1 bunk room. & $\begin{array}{l}\text { \$70 per person per night } \\
\text { (includes continental } \\
\text { breakfast), } \$ 60 \text { per } \\
\text { person per night for } \\
\text { groups of } 5 \text { or more } \\
\text { (includes continental } \\
\text { breakfast). }\end{array}$ & $\mathrm{B} \& \mathrm{~B}$ & 1 & $\underline{\text { Rockdale.farm@xtra.co.nz }}$ \\
\hline 153 & Alexander & Avenue Motel & 26 & 13 units. & \$125-\$145pppn & Motel & 1 & Avenue.motel@xtra.co.nz \\
\hline 154 & Alexander & Alexander’s Riverview B\&B & 6 & 2 queen bedrooms up to 6 & $\begin{array}{l}\$ 150 \text { per bedroom or } 3 \\
\text { bedroom } \$ 390 \text { (6 guests } \\
\$ 65 \text { per person) }\end{array}$ & $\mathrm{B} \& \mathrm{~B}$ & 1 & ji-instone@clear.net.nz \\
\hline 155 & Alexander & Alexandra Garden Court Motel & 30 & 6 fully self contained units. & $\begin{array}{l}\$ 85-\$ 115 \text { per }(1-2 \\
\text { person). }\end{array}$ & Motel & 1 & alex.gardencourt@xtra.co.nz \\
\hline 156 & Alexander & Alexandra Heights Motel & 44 & $\begin{array}{l}4 \text { two bedrooms (queen } \& \text { twin), } 3 \\
\text { studio apartments with spa bath } \\
\text { (queen } \& \text { single),3 studio units } \\
\text { (queen } \& \text { single), up to } 34 \text { guests } \\
+10 \text { extra person. }\end{array}$ & $\begin{array}{l}\$ 120-\$ 140 \text { two adults, } \$ \\
20 \text { extra person. }\end{array}$ & Motel & 1 & alexandraheightsmotel@xtra.co.nz \\
\hline 157 & Alexandra & All Seasons Tourists Flats & 2 & 1 queen size bedroom. & $\begin{array}{l}\text { \$70 single - } \$ 90 \text { double } \\
\text { pppn }\end{array}$ & SC & 1 & allseasons.tf@clear.net.nz \\
\hline 158 & Alexander & Alexandra Almond Court Motel & 48 & 8 self contained units. & $\begin{array}{l}\$ 115.00-\$ 140.00 \text { and } \\
\$ 20.00 \text { extra }\end{array}$ & Motel & 1 & info@almondcourtmotel.co.nz \\
\hline 159 & Alexander & Central Court Motor Inn & 66 & $\begin{array}{l}33 \text { units including studios and } 1 \& \\
2 \text { bedroom suites. }\end{array}$ & $\$ 70-\$ 149$ pppn & Motel & 1 & $\underline{\text { www.centennialcourt.co.n }}$ \\
\hline 160 & Alexandra & $\begin{array}{l}\text { Criterion Club Hotel (Bp- } \\
\text { downstairs) }\end{array}$ & 7 & 1 bunk with 7 beds. & $\begin{array}{l}\text { \$25 per person (linen } \\
\text { provided), \$20 per person } \\
\text { (own bedding) }\end{array}$ & Bp & 1 & middle.pub@xtra.co.nz \\
\hline 161 & Alexandra & $\begin{array}{l}\text { Criterion Club Hotel (Bp- } \\
\text { upstairs) }\end{array}$ & 22 & 11 guest rooms. & $\begin{array}{l}\$ 50 \text { to } \$ 60 \text { per person } \\
\$ 80-\$ 90 \text { two persons }\end{array}$ & $\mathrm{Bp}$ & 1 & middle.pub@xtra.co.nz \\
\hline 162 & Alexandra & $\begin{array}{l}\text { Hawkdun Rise Vineyard } \\
\text { Homestay }\end{array}$ & 6 & $\begin{array}{l}2 \text { queen size bedrooms. a double } \\
\text { bedroom. }\end{array}$ & $\$ 180-\$ 235$ pn. & $\mathrm{B} \& \mathrm{~B}$ & 1 & hillviewpark@xtra.co.nz \\
\hline
\end{tabular}




\begin{tabular}{|c|c|c|c|c|c|c|c|c|}
\hline 163 & Alexandra & Hillview Park B\&B & 6 & $\begin{array}{l}1 \text { queen, two super singles and } \\
\text { pull-out sofa (double). }\end{array}$ & $\begin{array}{l}\text { \$90 single } \\
\$ 110 \text { double }\end{array}$ & SC & 1 & marg@loghouseretreat.co.nz \\
\hline 164 & Alexandra & Log House Retreat & 6 & 3 queen size bedrooms. & \$220-\$265 pppn & B\&B & 1 & marg@loghouseretreat.co.nz \\
\hline 165 & Alexandra & Marj’s Place (Home stay) & 5 & $\begin{array}{l}\text { 1double bedroom and } 1 \text { three } \\
\text { bedded room. }\end{array}$ & $\begin{array}{l}\$ 70.00 \text { double - } \$ 35.00 \\
\text { single }\end{array}$ & SC & 1 & http://www.quailrock.co.nz/index.html \\
\hline 166 & Alexandra & Marj’s Place (Backpacker) & 18 & 3 separate units with 18 beds. & $\$ 25 \mathrm{pn}$ & Bp & 1 & http://www.quailrock.co.nz/index.html \\
\hline 167 & Alexandra & Quail rock B\&B & 4 & $\begin{array}{l}1 \text { master bedroom with ensuite, } 1 \\
\text { king/twin bedroom. }\end{array}$ & $\$ 100-\$ 150$ pn & $\mathrm{B} \& \mathrm{~B}$ & 1 & info@speargrassinn.co.nz \\
\hline 168 & Alexandra & Speargrass Inn & 6 & 3 units include 3 queen size beds. & \$155-\$170 (1-2 people). & SC & 1 & info@theworkshopsclyde.co.nz \\
\hline 169 & Clyde & $\begin{array}{l}\text { Absolutely Amazing Workshops } \\
\text { B\&B }\end{array}$ & 10 & 4 bedrooms. & $\begin{array}{l}\text { Beds } \$ 50 \text { per person, } \\
\text { single occupancy of } \\
\text { Queen } \$ 75\end{array}$ & $B \& B$ & 1 & info@theworkshopsclyde.co.nz \\
\hline 170 & Clyde & Antique Lodge Motel & 24 & $\begin{array}{l}7 \text { units- } 6 \text { of } 7 \text { having two } \\
\text { bedrooms (double bed/2 or } 3 \\
\text { single). }\end{array}$ & $\begin{array}{l}\text { Single from NZ\$95 } \\
\text { Double from NZ\$105 }\end{array}$ & Motel & 1 & stay@antiquelodgemotel.co.nz \\
\hline 171 & Clyde & Argyll on Clyde bed \& Breakfast & 6 & $\begin{array}{l}2 \text { queen bedrooms and } 1 \text { twin } \\
\text { bedrooms. }\end{array}$ & $\$ 120$ - \$180 per room & $\mathrm{B} \& \mathrm{~B}$ & 1 & argyllonclyde@yahoo.co.nz \\
\hline 172 & Clyde & Bed and Breakfast @Clyde & 8 & $\begin{array}{l}2 \text { queen rooms, } 1 \text { ensuite, and } 1 \\
\text { twin bedroom. }\end{array}$ & $\$ 100-\$ 140$ pn & $\mathrm{B} \& \mathrm{~B}$ & 1 & info@bedandbreakfastatclyde.co.nz \\
\hline 174 & Clyde & Clyde Motel & N/A & $\begin{array}{l}2 \text { bedroom units and one bedroom } \\
\text { units. } 9 \text { one bedroom and } 2 \\
\text { bedrooms units. }\end{array}$ & Units from $\$ 95$ to $\$ 140$ & Motel & 1 & clyde.motels@xtra.co.nz \\
\hline 175 & Clyde & Clyde's Plum Tree Cottage & 4 & 2 bedrooms. & $\begin{array}{l}\$ 140.00 \text { a night for two } \\
\text { people \& } \$ 20.00 \text { each } \\
\text { additional person }\end{array}$ & SC & 1 & cmgrieve@farmside.co.nz \\
\hline 176 & Clyde & $\begin{array}{l}\text { Cottage Wheatsheaf (built over } \\
100 \text { years ago). }\end{array}$ & 4 & $\begin{array}{l}2 \text { bedrooms ( } 1 \text { queen and } 1 \text { two } \\
\text { singles). }\end{array}$ & $\begin{array}{l}\$ 130 \text { a night for two } \\
\text { people; } \$ 25 \text { each } \\
\text { additional person }\end{array}$ & $B \& B$ & 1 & pip.mark.wellstead@xtra.co.nz \\
\hline 177 & Clyde & Dunstan Hotel ( built in 1940). & 17 & $\begin{array}{l}10 \text { rooms including: } 3 \text { single } \\
\text { rooms, } 3 \text { twin rooms, } 3 \text { double } \\
\text { rooms, } 1 \text { double room with } \\
\text { ensuite. }\end{array}$ & Single $\$ 45$ - double $\$ 80$ & Hotel & 1 & tonokajo@xtra.co.nz \\
\hline 178 & Clyde & Dunstan House ( built in 1900) & 29 & 13 bedrooms. & $\$ 120-\$ 220$ & Hotel & 1 & davidson@dunstanhouse.co.nz \\
\hline 179 & Clyde & Hartly Arms Backpackers / B\&B & 8 & 1 double room and two bedrooms & NZ\$40 per night & B\&B- & 1 & hartlyarms@xtra.co.nz \\
\hline
\end{tabular}




\begin{tabular}{|c|c|c|c|c|c|c|c|c|}
\hline & & & & with four beds in each. & & $\mathrm{Bp}$ & $\begin{array}{l}\mathrm{B} \& \mathrm{~B}- \\
1 \mathrm{Bp}\end{array}$ & \\
\hline 180 & Clyde & $\begin{array}{l}\text { Hartley Homestead Boutique } \\
\text { B\&B }\end{array}$ & 6 & 3 bedrooms. & $\$ 150-\$ 220$ pn & $\mathrm{B} \& \mathrm{~B}$ & 1 & info@hartlyhomestead.co.nz \\
\hline 181 & Clyde & Hill view Park B \& B and SC & 6 & 2- bedrooms. & $\begin{array}{l}\$ 90 \text { single } \\
\$ 110 \text { double }\end{array}$ & $\mathrm{B} \& \mathrm{~B} / \mathrm{SC}$ & $\begin{array}{l}1 \mathrm{~B} \& \mathrm{~B} \\
-1 \mathrm{SC}\end{array}$ & hillviewpark@xtra.co.nz \\
\hline 182 & Clyde & $\begin{array}{l}\text { Kahu Stone Cottage (built in } \\
\text { 1865). }\end{array}$ & 4 & $\begin{array}{l}1 \text { super king and } 1 \text { queen bed } \\
\text { rooms. }\end{array}$ & $\begin{array}{l}1 \text { night stay } \$ 350 \\
2 \text { nights stay } \$ 600\end{array}$ & SC & 1 & stay@kahuvalley.co.nz \\
\hline 183 & Clyde & $\begin{array}{l}\text { Lavender Drive Bed and } \\
\text { Breakfast }\end{array}$ & 6 & $\begin{array}{l}1 \text { king room with ensuite, } 2 \text { queen } \\
\text { bedrooms. }\end{array}$ & $\$ 140-\$ 160$ pn & $\mathrm{B} \& \mathrm{~B}$ & 1 & lavenderdrivebandb@yahoo.co.nz \\
\hline 184 & Clyde & Mirabell Chalets & 13 & 3 chalets. & $\$ 115-\$ 125$ two adults & SC & 1 & andy.family@xtra.co.nz \\
\hline 185 & Clyde & $\begin{array}{l}\text { Olivers of Clyde ( lodge and } \\
\text { stable- Built in 1860). }\end{array}$ & 22 & 11 double bedrooms. & $\begin{array}{l}\text { 1-2 guests: } \$ 185-\$ 315 \\
\text { family suit: } 1-4 \text { guests } \\
\$ 385-\$ 405\end{array}$ & Motel & 1 & enquiries@oliverscentralotago.co.nz \\
\hline 186 & Clyde & Picnic Creek Bed \& Breakfast & 6 & 3 bedrooms. & $\$ 180$ per room & $\mathrm{B} \& \mathrm{~B}$ & 1 & wendy.picniccreek@xtra.co.nz \\
\hline 187 & Clyde & Postmaster’s House & 6 & 3 bedrooms. & $\begin{array}{l}\$ 95 \text { room rate for queen } \\
\text { and twin, } \$ 125 \text { room rate } \\
\text { for queen ensuite. }\end{array}$ & B\&B & 1 & postofficecafeclyde@xtra.co.nz \\
\hline 189 & Cromwell & Carrick Lodge Motel & 16 & 4 studio- 1 bedroom family unit. & \$ 96- \$156 pppn. & Motel & 1 & carricklodge@xtra.co.nz \\
\hline 190 & Cromwell & Central Gold Motel & 57 & $\begin{array}{l}11 \text { mainly ground floor units, } \\
\text { (each unit up to } 2-5 \text { guests), plus } 1 \\
\text { private executive suite with super } \\
\text { king size. }\end{array}$ & \$110-\$160 (1-2 persons). & Motel & 1 & info@centralgoldmotel.co.nz \\
\hline 191 & Cromwell & Colonial Manor Motel & 14 & $\begin{array}{l}1 \text { upstairs studio, 1studio, } 1 \\
\text { deluxe studio, 1executive/spa unit } \\
\text { and } 1 \text { family unit. }\end{array}$ & $\begin{array}{l}\$ 115-\$ 165 \text { pppn } \\
\text { family unit, from } \$ 180 \\
\text { for } 4 \text { people. }\end{array}$ & Motel & 1 & \\
\hline 192 & Cromwell & Cromwell Motel & 42 & 11 spacious ground floor units. & $\begin{array}{l}\$ 75 \text { to } \$ 130 \text { for } 1-2 \\
\text { persons. }\end{array}$ & Motel & 1 & info@cromwellmotel.co.nz \\
\hline 193 & Cromwell & $\begin{array}{l}\text { Cromwell Top } 10 \text { Holiday } \\
\text { Park(park motel } 2 \text { rooms and } \\
\text { park motel } 1 \text { room) }\end{array}$ & 36 & 8 park motels. & $\begin{array}{l}\$ 130 \text { motel1- } \$ 145 \text { motel } \\
2 \text { per unit. }\end{array}$ & Motel & 8 & Tel: +64 (3) 4450164 \\
\hline
\end{tabular}




\begin{tabular}{|c|c|c|c|c|c|c|c|c|}
\hline 194 & Cromwell & $\begin{array}{l}\text { Cromwell Top } 10 \text { Holiday Park ( } \\
\text { Self Contained Unit } 1 \text { and 2) }\end{array}$ & 5 & 3units & $\$ 110$ unit 1, \$130 unit 2. & SC & 2 & Tel: +64 (3) 4450164 \\
\hline 195 & Cromwell & $\begin{array}{l}\text { Cromwell Top } 10 \text { Holiday } \\
\text { Park(ensuite units, deluxe units, } \\
\text { standard units) }\end{array}$ & 6 & 2 ensuite units. & $\begin{array}{l}\$ 98 \text { en-suit unit-\$80 } \\
\text { deluxe. }\end{array}$ & SC & 2 & Tel: +64 (3) 4450164 \\
\hline 196 & Cromwell & $\begin{array}{l}\text { Cromwell Top } 10 \text { Holiday } \\
\text { Park(powered and non-powered } \\
\text { sites) }\end{array}$ & $\begin{array}{l}282 \\
6\end{array}$ & $\begin{array}{l}\text { Powered site (288) - non-powered } \\
\text { site (183) }\end{array}$ & Price per unit/site= $\$ 38$ & Camping & 471 & Tel: +64 (3) 4450164 \\
\hline 197 & Cromwell & Golden Gate Lodge & 96 & $\begin{array}{l}47 \text { bedrooms rooms, a mix of } \\
\text { twin, double and triple studio } \\
\text { units, two luxury suites, two } \\
\text { interconnecting units, three units } \\
\text { for disabled guests. }\end{array}$ & $\begin{array}{l}\text { Single from NZ\$125, } \\
\text { double/Twin from } \\
\text { NZ\$125. }\end{array}$ & $\mathrm{B} \& \mathrm{~B}$ & 1 & stay@goldengate.co.nz \\
\hline 198 & Cromwell & Lake Dunstan Motel & 28 & 15 modern, spacious units. & \$120 pppn & Motel & 1 & info@lakedunstanmotel.co.nz \\
\hline 199 & Cromwell & Orchard House & 4 & 2 king suites. & $\begin{array}{l}\text { single } \$ 230 \\
\text { Double } \$ 250 \\
\text {, tariffs include breakfast. }\end{array}$ & $\mathrm{SC}$ & 1 & booking@orchardhouse.co.nz \\
\hline 200 & Cromwell & $\begin{array}{l}\text { River Rock Estate Vineyard } \\
\text { Accommodation }\end{array}$ & 4 & 2 kings double unite. & $1-2$ adult $\$ 155$ pn & $\mathrm{B} \& \mathrm{~B}$ & 1 & info@riverrockestate.com \\
\hline 201 & Queenstown & Four season Motel & 53 & $\begin{array}{l}4 \text { one-bedroom units, up to } 16 \\
\text { guests, } 1 \text { family unit up to six } \\
\text { guests, nine studios unites up to } \\
27 \text { guests, directors suit, up to } 4 \\
\text { guests. }\end{array}$ & $\$ 125-\$ 190$ pppn & Motel & 1 & stay@queenstownmotel.com \\
\hline
\end{tabular}

Appendix.7: OCRT Cultural Products and Activities (2011)

\begin{tabular}{|c|c|c|c|c|c|c|c|c|c|c|c|}
\hline \multicolumn{12}{|c|}{ Local produce } \\
\hline 1 & Continental Breakfast. & 80 & 5 & Home baking and local food. & 20 & 9 & Beer. & 30 & 13 & Crafts. & 1 \\
\hline 2 & Country style cooked breakfast. & 6 & 6 & Local vegetables. & 8 & 10 & Non-alcoholic drinks. & 5 & - & - & - \\
\hline 3 & Irish breakfast. & 1 & 7 & Fruit. & 9 & 11 & $\begin{array}{l}\text { Grazing for those doing OCRT } \\
\text { on horseback. }\end{array}$ & 8 & - & - & - \\
\hline
\end{tabular}




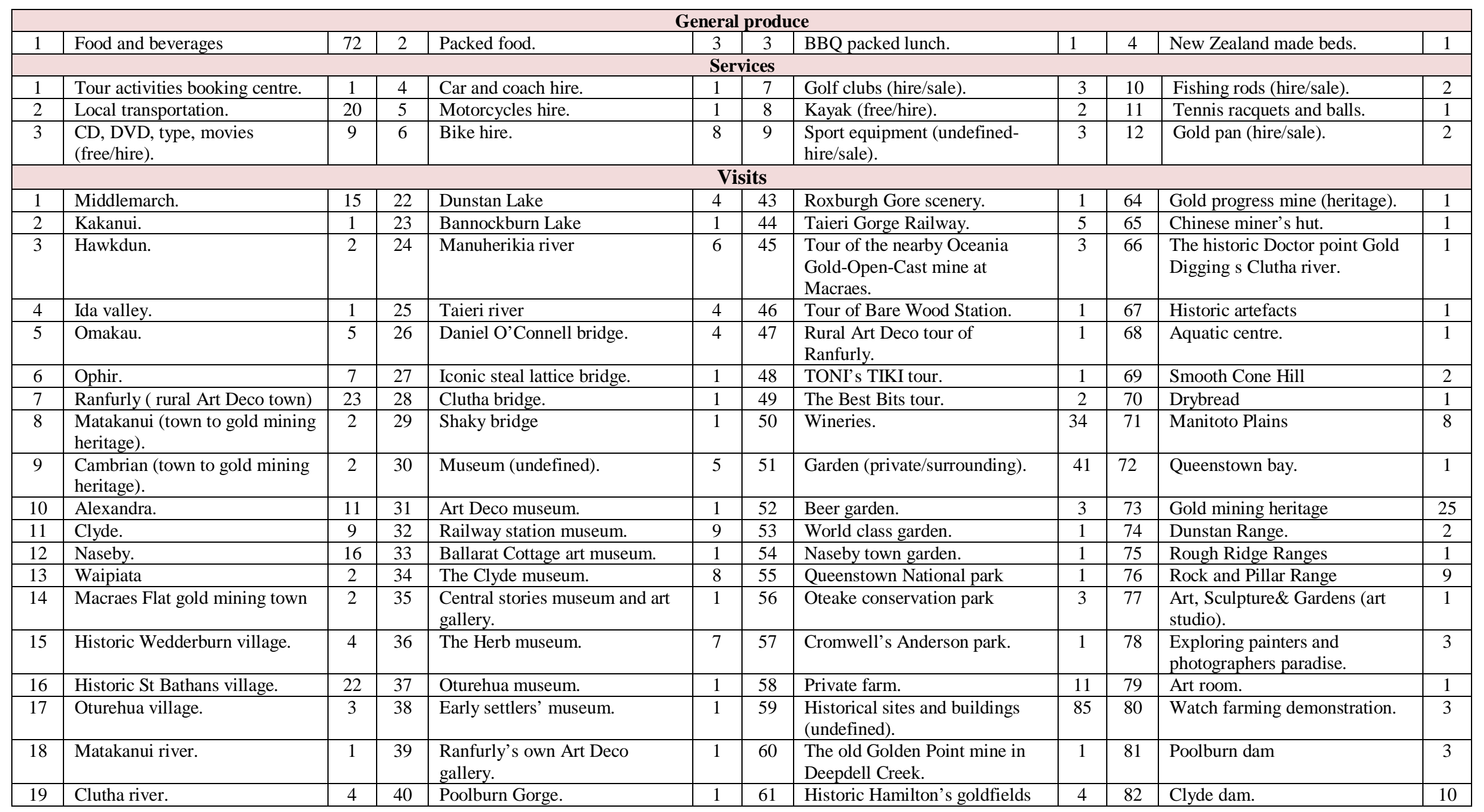




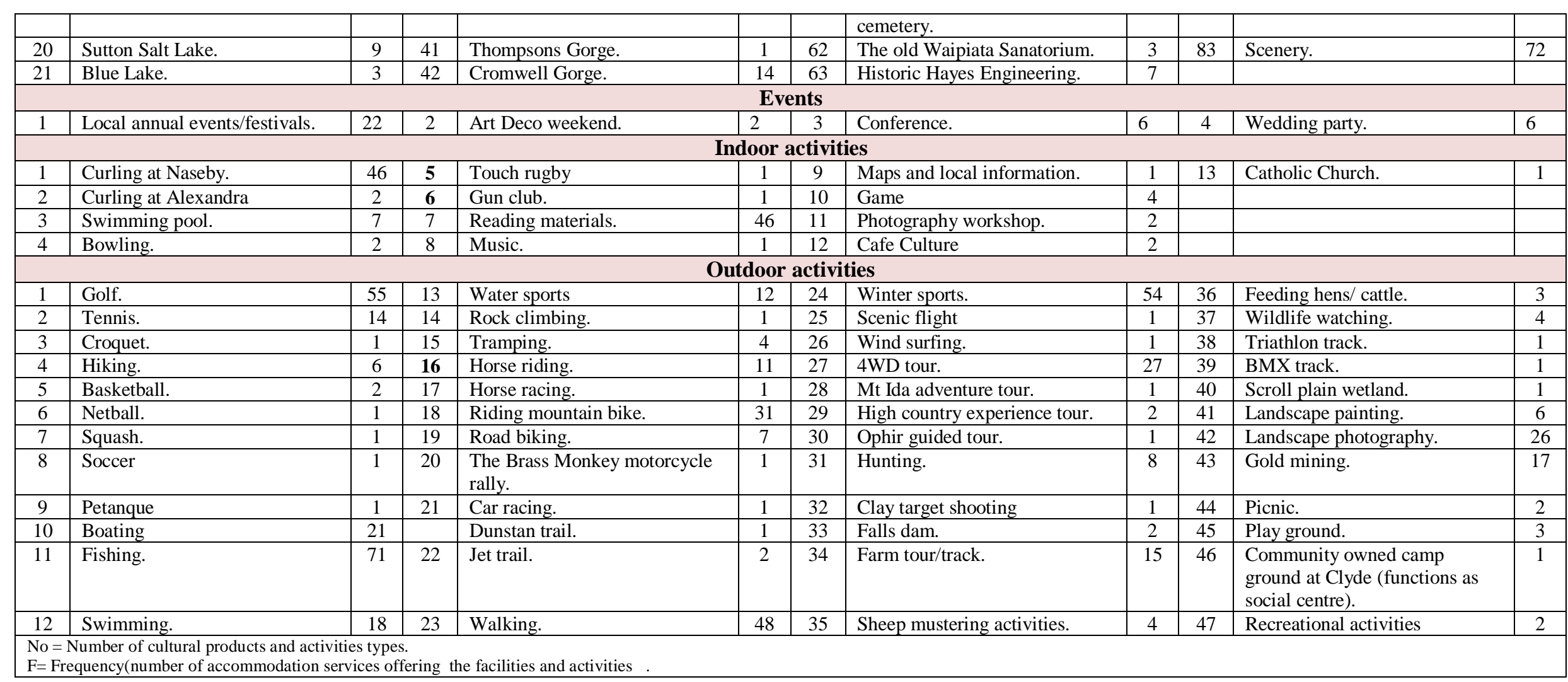


Appentix.8: OCRT- accommodation services- Used materials by types of accommodation (2011)

\begin{tabular}{|c|c|c|c|c|c|c|c|c|c|c|c|c|c|c|}
\hline \multirow[t]{3}{*}{ Used material(S) } & \multirow{3}{*}{$\begin{array}{l}\text { Total } \\
\text { bed } \\
\text { spaces }\end{array}$} & \multirow[t]{3}{*}{$\%$} & \multicolumn{12}{|c|}{ Types of accommodation services } \\
\hline & & & \multicolumn{2}{|c|}{ SC } & \multicolumn{2}{|c|}{$\mathrm{B} \& \mathrm{~B}$} & \multicolumn{2}{|c|}{ Bp } & \multicolumn{2}{|c|}{ Motel } & \multicolumn{2}{|c|}{ Homestead } & \multicolumn{2}{|c|}{ Hotel } \\
\hline & & & $\mathrm{N}$ & $\mathrm{C}$ & $\mathrm{N}$ & $\mathrm{C}$ & $\mathrm{N}$ & $\mathrm{C}$ & $\mathrm{N}$ & $\mathrm{C}$ & $\mathrm{N}$ & $\mathrm{C}$ & $\mathrm{N}$ & $\mathrm{C}$ \\
\hline Timber & 1032 & 48.5 & 71 & 368 & 33 & 242 & 3 & 36 & 20 & 332 & - & - & 4 & 54 \\
\hline Concrete & 99 & 4.6 & - & - & 4 & 20 & - & - & 2 & 79 & - & - & - & - \\
\hline${ }^{1}$ stone & 172 & 8 & 4 & 20 & 7 & 43 & 1 & 4 & 1 & 22 & 1 & 4 & 6 & 79 \\
\hline${ }^{2}$ brick & 47 & 2.2 & 1 & 6 & 5 & 33 & - & - & - & - & - & - & 1 & 8 \\
\hline Mud brick & 47 & 2.2 & 4 & 27 & 1 & 6 & - & - & - & - & 1 & 6 & 1 & 8 \\
\hline Container ( iron) & 4 & 0.2 & 1 & 4 & - & - & - & - & - & - & - & - & - & - \\
\hline Cabins (N/A material) & 12 & 0.5 & - & - & 3 & 12 & - & - & - & - & - & - & - & - \\
\hline $\begin{array}{l}\text { Mixed timber and } \\
\text { corrugated iron }\end{array}$ & 29 & 1.3 & - & - & 1 & 4 & 1 & 25 & - & - & - & - & - & - \\
\hline $\begin{array}{l}\text { Mixed red brick or brick } \\
\text { and timber }\end{array}$ & 174 & 8.1 & 2 & 13 & 3 & 26 & 2 & 29 & - & - & - & - & 3 & 106 \\
\hline Mixed stone and timber & 248 & 11.7 & 4 & 24 & 5 & 120 & - & - & 1 & 57 & - & - & 2 & 47 \\
\hline $\begin{array}{l}\text { Mixed mud brick and } \\
\text { timber }\end{array}$ & 16 & 0.7 & - & - & - & - & - & - & - & - & - & - & 2 & ${ }^{3} 16$ \\
\hline $\begin{array}{l}\text { Mixed mud brick and } \\
\text { stone }\end{array}$ & 18 & 0.8 & 1 & 6 & 1 & 6 & 1 & 6 & - & - & - & - & - & - \\
\hline $\begin{array}{l}\text { Mixed concrete and } \\
\text { timber }\end{array}$ & 40 & 1.9 & - & - & - & - & - & - & 1 & 40 & - & - & - & - \\
\hline $\begin{array}{l}\text { Mixed brick and } \\
\text { concrete }\end{array}$ & 73 & 3.4 & - & - & 1 & 11 & 1 & 18 & 1 & 44 & - & - & - & - \\
\hline $\begin{array}{l}\text { Mixed mud brick and } \\
\text { sundried brick }\end{array}$ & 6 & 0.3 & - & - & 1 & 6 & - & - & - & - & - & - & - & - \\
\hline Mixed clay brick and & 5 & 0.2 & - & - & 1 & 5 & - & - & - & - & - & - & - & - \\
\hline
\end{tabular}




\begin{tabular}{|c|c|c|c|c|c|c|c|c|c|c|c|c|c|c|}
\hline timber & & & & & & & & & & & & & & \\
\hline $\begin{array}{l}\text { Mixed stone and mud } \\
\text { brick }\end{array}$ & 18 & 0.8 & 1 & 6 & 1 & 6 & 1 & 6 & - & - & - & - & - & - \\
\hline $\begin{array}{l}\text { Mixed timber, stone and } \\
\text { concrete }\end{array}$ & 17 & 0.8 & 1 & 6 & 2 & 11 & - & - & - & - & - & - & - & - \\
\hline $\begin{array}{l}\text { Mixed stone, mud brick } \\
\text { and timber }\end{array}$ & 12 & 0.5 & - & - & - & - & - & - & - & - & - & - & 1 & 12 \\
\hline $\begin{array}{l}\text { Mixed timber, } \\
\text { aluminium facade and } \\
\text { wood joinery }\end{array}$ & 12 & 0.5 & 1 & 12 & - & - & - & - & - & - & - & - & - & - \\
\hline $\begin{array}{l}\text { Mixed timber, concrete } \\
\text { and brick }\end{array}$ & 44 & 2 & - & - & - & - & - & - & 1 & 44 & - & - & - & - \\
\hline Total bed spaces & 2125 & 100 & & & & & & & & & & & & \\
\hline \multicolumn{15}{|c|}{$\begin{array}{l}\text { Stone includes: schist stone, stone and rock. } \\
\text { Brick includes: red brick and brick. } \\
\text { One of two hotels with N/A capacity. } \\
\text { N= Number of accommodation services. } \\
\text { C= Capacity (number of bed space). }\end{array}$} \\
\hline
\end{tabular}




\begin{tabular}{|c|c|c|c|c|c|c|c|c|c|c|c|c|c|}
\hline \multirow{3}{*}{ No. $^{1}$} & \multirow{3}{*}{$\frac{\mathrm{AppeI}^{2}}{\mathrm{Q}^{2}}$} & \multirow{3}{*}{$\mathrm{C}^{3}$} & \multirow{2}{*}{\multicolumn{3}{|c|}{$\begin{array}{l}\text { - SC (self con } \\
\text { Heating }\end{array}$}} & \multirow{3}{*}{$\begin{array}{l}\text { ed) acc } \\
\text { So }\end{array}$} & \multirow{2}{*}{\multicolumn{2}{|c|}{$\frac{\text { nmoc }}{\text { TV }}$}} & \multirow{3}{*}{$\begin{array}{l}\text { vices, } \\
\text { Int }^{8}\end{array}$} & \multirow{3}{*}{$\frac{\text { energy }}{\text { V/B }}$} & \multirow{3}{*}{$\begin{array}{l}\text { urces } \\
\mathrm{S} / \mathrm{P}\end{array}$} & \multirow{3}{*}{$\begin{array}{l}\text { nd faciliti } \\
\text { Outdoor } \\
\text { sitting }\end{array}$} & \multirow{3}{*}{$\mathrm{S} / \mathrm{P}$} \\
\hline & & & & & & & & & & & & & \\
\hline & & & $\mathrm{El}^{4}$ & Log & $\mathrm{Gas}^{10}$ & & $S^{6}$ & $\mathrm{P}^{7}$ & & & & & \\
\hline 2 & NB & 7 & $\cdot$ & - & - & - & - & - & - & - & - & - & - \\
\hline 7 & NB & 42 & $\cdot$ & - & - & - & - & - & - & - & - & - & - \\
\hline 2 & $\mathrm{RB}$ & 13 & - & 2 & $\cdot$ & - & 2 & - & - & 2 & $\mathrm{~S}$ & - & - \\
\hline 1 & RB & 5 & $\cdot$ & 1 & - & - & 1 & - & - & - & - & - & - \\
\hline 1 & $\mathrm{RB}$ & 5 & $\cdot$ & 1 & - & - & 1 & - & - & - & - & - & - \\
\hline 1 & NB & 4 & $\cdot$ & 1 & - & - & 1 & - & - & - & - & - & - \\
\hline 1 & RB & 8 & $\bullet$ & 1 & - & - & - & 3 & - & - & - & - & - \\
\hline 1 & RB & 5 & • & - & - & - & 1 & - & - & 1 & $\mathrm{~S}$ & 1 & $\mathrm{~S}$ \\
\hline 2 & NB & 6 & $\cdot$ & - & - & - & 1 & 4 & - & - & - & - & - \\
\hline 1 & $\mathrm{RB}$ & 6 & $\cdot$ & 1 & - & - & 1 & - & - & - & - & 1 & $\mathrm{~S}$ \\
\hline 1 & $\mathrm{RB}$ & 6 & $\cdot$ & 1 & - & - & - & - & - & - & - & - & - \\
\hline 1 & $\mathrm{NB}$ & 4 & $\cdot$ & - & - & - & 1 & - & - & - & - & - & - \\
\hline 1 & $\mathrm{RB}$ & 6 & $\cdot$ & 1 & - & - & 1 & - & - & 1 & $\mathrm{~S}$ & 1 & $\mathrm{~S}$ \\
\hline 1 & $\mathrm{RB}$ & 6 & $\cdot$ & 1 & - & - & - & - & - & - & - & 1 & $\mathrm{~S}$ \\
\hline 1 & $\mathrm{RB}$ & 6 & $\cdot$ & - & $\cdot$ & - & 1 & 3 & $\cdot$ & - & - & 1 & $\mathrm{~S}$ \\
\hline 1 & NB & 2 & $\cdot$ & - & - & - & - & - & $\cdot$ & - & - & - & - \\
\hline 1 & $\mathrm{NB}$ & 4 & $\cdot$ & - & - & - & - & - & $\cdot$ & - & - & - & - \\
\hline 1 & NB & 5 & $\cdot$ & - & - & - & - & - & $\cdot$ & - & - & - & - \\
\hline 1 & NB & 8 & $\cdot$ & - & - & - & - & - & $\cdot$ & - & - & - & - \\
\hline 1 & $\mathrm{RB}$ & 5 & $\cdot$ & 1 & - & - & 1 & - & - & - & - & 1 & $\mathrm{~S}$ \\
\hline 1 & $\mathrm{RB}$ & 9 & - & - & $\cdot$ & - & 1 & - & - & - & - & - & - \\
\hline 3 & NB & 8 & $\cdot$ & - & - & - & - & 3 & - & - & - & 3 & $\mathrm{P}$ \\
\hline 1 & RB & 14 & • & 1 & - & - & 1 & - & - & - & - & - & - \\
\hline 1 & NB & 9 & - & 1 & - & $\cdot$ & 1 & - & $\cdot$ & - & - & - & - \\
\hline 2 & $\mathrm{RB}$ & 6 & • & 1 & - & • & 1 & - & $\cdot$ & - & - & - & - \\
\hline 10 & NB & 40 & • & - & - & • & - & - & $\cdot$ & - & - & - & - \\
\hline 1 & NB & 8 & $\cdot$ & 1 & - & - & - & - & - & - & - & - & - \\
\hline 1 & NB & 6 & - & - & • & - & - & - & - & 1 & $\mathrm{~S}$ & - & - \\
\hline 1 & NB & 12 & - & - & $\cdot$ & - & 2 & - & $\cdot$ & 6 & $P$ & - & - \\
\hline 1 & NB & 8 & - & - & • & - & 2 & - & $\cdot$ & - & - & - & - \\
\hline 1 & $\mathrm{RB}$ & 4 & - & - & $\cdot$ & - & - & - & $\cdot$ & - & - & - & - \\
\hline 2 & NB & 4 & $\bullet$ & - & - & - & - & - & - & - & - & - & - \\
\hline 1 & NB & 3 & $\cdot$ & - & - & - & - & - & - & - & - & - & - \\
\hline 1 & NB & 4 & $\bullet$ & 1 & - & - & 2 & - & - & - & - & - & - \\
\hline 1 & NB & 4 & $\bullet$ & 1 & - & - & 2 & - & - & - & - & - & - \\
\hline 1 & $\mathrm{RB}$ & 4 & $\cdot$ & - & - & - & - & - & - & - & - & - & - \\
\hline 1 & $\mathrm{RB}$ & 5 & $\bullet$ & 1 & - & - & 1 & - & - & - & - & - & - \\
\hline 1 & $\mathrm{RB}$ & 6 & $\cdot$ & - & - & - & 1 & - & - & - & - & - & - \\
\hline 1 & $\mathrm{RB}$ & 2 & $\bullet$ & - & - & - & - & 1 & - & - & - & - & - \\
\hline 1 & $\mathrm{RB}$ & 4 & - & 1 & • & - & - & - & $\cdot$ & - & - & 1 & $\mathrm{~S}$ \\
\hline 1 & NB & 4 & - & - & - & - & - & - & - & - & - & - & \\
\hline 1 & RB & 4 & $\cdot$ & 1 & $\bullet$ & - & - & 1 & - & 1 & $\mathrm{~S}$ & 1 & $\mathrm{~S}$ \\
\hline 1 & NB & 6 & $\cdot$ & - & - & - & - & 1 & $\cdot$ & - & - & - & - \\
\hline 1 & NB & 6 & $\cdot$ & - & - & - & 1 & - & $\cdot$ & - & - & - & - \\
\hline 1 & NB & 2 & $\bullet$ & - & - & - & - & 1 & $\cdot$ & - & - & - & - \\
\hline 1 & NB & 8 & $\cdot$ & - & - & - & - & 1 & $\cdot$ & - & - & - & - \\
\hline 1 & $\mathrm{RB}$ & 4 & $\cdot$ & - & $\cdot$ & $\cdot$ & - & - & - & - & - & 1 & $\mathrm{~S}$ \\
\hline 1 & NB & 8 & $\cdot$ & - & $\cdot$ & - & - & - & - & - & - & 1 & $\mathrm{~S}$ \\
\hline 1 & NB & 4 & $\cdot$ & 1 & - & - & - & - & - & - & - & 1 & $\mathrm{~S}$ \\
\hline 1 & RB & 10 & - & 1 & $\bullet$ & - & 1 & - & - & - & - & - & - \\
\hline 1 & $\mathrm{RB}$ & 6 & $\cdot$ & - & - & - & - & - & - & - & - & - & - \\
\hline 1 & NB & 6 & $\bullet$ & 1 & - & - & 1 & - & - & - & - & 1 & $\mathrm{~S}$ \\
\hline 1 & RB & 6 & $\cdot$ & - & - & - & - & - & - & 1 & $\mathrm{~S}$ & - & - \\
\hline 1 & NB & 16 & $\bullet$ & - & - & - & - & 5 & - & - & - & 5 & $\mathrm{P}$ \\
\hline 1 & NB & 7 & $\bullet$ & - & - & - & 1 & - & - & - & - & 1 & $\mathrm{~S}$ \\
\hline 1 & NB & 8 & $\cdot$ & - & - & - & 1 & - & - & - & - & - & - \\
\hline 1 & NB & 6 & $\cdot$ & - & - & - & 1 & - & - & - & - & - & - \\
\hline 1 & NB & 2 & $\cdot$ & - & - & - & 1 & - & - & - & - & - & - \\
\hline 1 & NB & 6 & $\bullet$ & - & - & - & - & - & $\cdot$ & - & - & - & - \\
\hline 1 & NB & 5 & - & - & $\cdot$ & - & 1 & - & $\cdot$ & - & - & - & - \\
\hline
\end{tabular}




\begin{tabular}{|c|c|c|c|c|c|c|c|c|c|c|c|c|c|}
\hline 1 & $\mathrm{RB}$ & 6 & $\bullet$ & - & - & - & - & 3 & - & - & - & - & - \\
\hline 1 & NB & 28 & • & - & - & - & - & 8 & - & - & - & - & - \\
\hline 1 & $\mathrm{RB}$ & 4 & $\cdot$ & - & - & - & 1 & - & - & 1 & $\mathrm{~S}$ & 2 & $\mathrm{P}$ \\
\hline 1 & NB & 3 & • & - & - & - & - & - & • & - & - & - & - \\
\hline 1 & $\mathrm{RB}$ & 4 & - & 1 & - & $\bullet$ & - & - & - & - & - & - & - \\
\hline 1 & NB & 13 & • & - & • & - & - & - & - & - & - & 1 & $\mathrm{~S}$ \\
\hline 2 & NB & 5 & $\cdot$ & - & - & - & - & 2 & • & - & - & 1 & $\mathrm{~S}$ \\
\hline 1 & NB & 6 & $\cdot$ & - & - & - & - & 2 & • & - & - & 1 & $\mathrm{~S}$ \\
\hline 1 & NB & 4 & • & - & - & - & - & 2 & • & 2 & $\mathrm{P}$ & - & - \\
\hline $\begin{array}{l}1 . \\
2 . \\
3 . \\
4 . \\
5 . \\
6 . \\
7 . \\
8 . \\
9 . \\
10 . \\
11 .\end{array}$ & $\begin{array}{l}\text { No: Nun } \\
\text { Q: Type } \\
\text { C: Capa } \\
\text { El: Elect } \\
\text { So: Solar } \\
\text { S: Share } \\
\text { P: Privat } \\
\text { Int: Inter } \\
\text { V/B: Ve } \\
\text { Gas incl } \\
\text { Solar sys }\end{array}$ & $\begin{array}{l}\text { of of a } \\
\text { build } \\
\text { (nur } \\
\text { ity. } \\
\text { ystem } \\
\text { acilit } \\
\text { acilit } \\
\text { t. } \\
\text { da/ba } \\
\text { ng ce } \\
\text { ms us }\end{array}$ & $\begin{array}{l}\mathrm{mn} \\
\text { use } \\
\text { r of }\end{array}$ & $\begin{array}{l}\text { on } \\
\text { icco } \\
\text { pac }\end{array}$ & es. & ng & & & & & & & \\
\hline
\end{tabular}

\begin{tabular}{|c|c|c|c|c|c|c|c|c|c|c|c|c|c|}
\hline \multicolumn{14}{|c|}{ Appendix.10: OCRT-B\&B accommodation services, energy sources and facilities } \\
\hline \multirow[t]{2}{*}{ No. ${ }^{1}$} & \multirow[t]{2}{*}{$\mathrm{Q}^{2}$} & \multirow[t]{2}{*}{$\mathrm{C}^{3}$} & \multicolumn{3}{|c|}{ Heating } & \multirow[t]{2}{*}{ So $0^{5 \& 11}$} & \multicolumn{2}{|c|}{ TV } & \multirow[t]{2}{*}{$\mathrm{Int}^{8}$} & \multirow[t]{2}{*}{$\mathrm{V} / \mathrm{B}^{9}$} & \multirow[t]{2}{*}{$\mathrm{S} / \mathrm{P}$} & \multirow{2}{*}{$\begin{array}{l}\text { Outdoor } \\
\text { sitting }\end{array}$} & \multirow[t]{2}{*}{$\mathrm{S} / \mathrm{H}$} \\
\hline & & & $\mathrm{El}^{4}$ & $\log$ & Gas $^{10}$ & & $\mathrm{~S}^{6}$ & $\mathrm{P}^{7}$ & & & & & \\
\hline 1 & NB & 6 & • & 2 & - & - & 1 & - & - & - & - & - & - \\
\hline 1 & $\mathrm{RB}$ & 10 & • & 1 & - & - & - & - & - & 1 & $\mathrm{~S}$ & - & - \\
\hline 1 & NB & 7 & • & 1 & - & - & 1 & - & • & - & - & 1 & $\mathrm{~S}$ \\
\hline 1 & NB & 6 & • & - & • & - & 1 & - & - & 1 & $\mathrm{P}$ & - & - \\
\hline 1 & RB & 5 & - & - & • & - & - & 2 & - & - & - & 1 & S \\
\hline 1 & RB & \begin{tabular}{|l|}
10 \\
\end{tabular} & • & - & - & - & 1 & - & - & - & - & 2 & $\mathrm{~S}$ \\
\hline 1 & NB & 5 & • & 1 & - & - & 1 & - & - & - & - & - & - \\
\hline 1 & $\mathrm{RB}$ & 32 & - & - & - & - & 1 & - & - & - & - & 1 & $\mathrm{~S}$ \\
\hline 1 & NB & \begin{tabular}{|l}
6 \\
\end{tabular} & $\bullet$ & - & - & - & 1 & - & - & - & - & - & - \\
\hline 1 & NB & 6 & • & - & - & - & 1 & - & - & - & - & - & - \\
\hline 1 & NB & 12 & • & - & - & - & 1 & - & • & - & - & - & - \\
\hline 1 & NB & 4 & - & - & - & - & - & - & - & - & - & - & - \\
\hline 1 & NB & 2 & $\bullet$ & - & - & - & - & - & - & - & - & - & - \\
\hline 1 & NB & 2 & • & - & - & - & - & - & - & - & - & - & - \\
\hline 1 & $\mathrm{RB}$ & 5 & $\bullet$ & 1 & - & - & - & - & - & - & - & - & - \\
\hline 1 & NB & 9 & • & - & - & - & 1 & - & - & 1 & $\mathrm{~S}$ & 1 & $\mathrm{~S}$ \\
\hline 1 & $\mathrm{RB}$ & 11 & - & 1 & - & - & - & - & - & - & - & - & - \\
\hline 1 & $\mathrm{NB}$ & 8 & • & - & - & - & 1 & - & - & - & - & 1 & $\mathrm{~S}$ \\
\hline 1 & NB & 8 & • & - & - & - & 1 & - & - & - & - & - & - \\
\hline 1 & $\mathrm{RB}$ & 6 & $\bullet$ & 1 & - & - & - & - & - & - & - & - & - \\
\hline 1 & $\mathrm{NB}$ & 6 & • & 1 & - & - & - & - & - & - & - & - & - \\
\hline 3 & NB & \begin{tabular}{|l|}
12 \\
\end{tabular} & $\bullet$ & - & - & $\cdot$ & 2 & - & 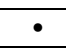 & - & - & - & - \\
\hline 1 & $\mathrm{NB}$ & 5 & $\bullet$ & - & - & - & - & - & - & - & - & - & - \\
\hline 1 & $\mathrm{NB}$ & 6 & $\bullet$ & - & - & - & 1 & - & - & - & - & - & - \\
\hline 1 & RB & 5 & • & - & - & - & 1 & - & • & - & - & - & - \\
\hline 1 & NB & 6 & • & 1 & - & - & - & - & - & - & - & - & - \\
\hline 1 & NB & 4 & • & - & - & - & 1 & - & - & - & - & - & - \\
\hline 1 & NB & 16 & • & 1 & - & - & 1 & - & - & - & - & 1 & S \\
\hline 1 & NB & 6 & $\bullet$ & 1 & - & - & 1 & - & - & - & - & - & - \\
\hline 1 & $\mathrm{RB}$ & 4 & • & - & - & - & - & - & - & - & - & - & - \\
\hline 1 & $\mathrm{RB}$ & 6 & • & - & - & - & - & - & - & - & - & - & - \\
\hline 1 & NB & 5 & • & 1 & - & - & - & 2 & - & 2 & $\mathrm{P}$ & - & - \\
\hline 1 & NB & 9 & • & - & - & - & 1 & - & - & - & - & 3 & $\mathrm{P}$ \\
\hline 1 & $\mathrm{RB}$ & 6 & $\bullet$ & - & - & - & - & - & • & - & - & 1 & $\mathrm{~S}$ \\
\hline 1 & $\mathrm{RB}$ & 8 & $\bullet$ & 1 & - & - & - & - & - & 1 & $\mathrm{P}$ & - & - \\
\hline 1 & $\mathrm{RB}$ & 6 & $\cdot$ & - & - & - & 1 & - & - & - & - & - & - \\
\hline 1 & $\mathrm{NB}$ & 8 & - & - & • & - & 1 & - & - & - & - & - & - \\
\hline 1 & $\mathrm{NB}$ & 10 & - & - & - & - & - & - & • & - & - & - & - \\
\hline 1 & NB & 4 & - & - & • & - & 1 & - & - & - & - & 2 & $\mathrm{P}$ \\
\hline 1 & $\mathrm{RB}$ & 4 & • & 1 & - & - & - & - & - & - & - & 1 & $\mathrm{~S}$ \\
\hline
\end{tabular}




\begin{tabular}{|c|c|c|c|c|c|c|c|c|c|c|c|c|c|}
\hline 1 & RB & 3 & - & - & - & - & 1 & - & - & - & - & - & \\
\hline 1 & $\mathrm{RB}$ & 6 & • & 1 & - & - & 1 & - & - & - & - & 1 & $\mathrm{~S}$ \\
\hline 1 & $\mathrm{RB}$ & 6 & $\bullet$ & - & - & - & - & - & • & - & - & 1 & $\mathrm{~S}$ \\
\hline 1 & NB & 6 & $\bullet$ & 1 & - & - & - & - & - & - & - & - & - \\
\hline 1 & $\mathrm{RB}$ & 6 & - & 1 & - & - & 1 & - & - & - & - & - & - \\
\hline 1 & NB & 7 & - & 1 & - & - & 1 & - & • & - & - & - & - \\
\hline 1 & NB & 24 & - & - & - & - & - & - & - & - & - & - & - \\
\hline 1 & $\mathrm{RB}$ & 6 & $\bullet$ & 1 & - & - & 1 & - & - & - & - & - & - \\
\hline 1 & NB & 6 & $\bullet$ & - & - & - & - & - & - & - & - & - & - \\
\hline 1 & NB & 6 & $\bullet$ & - & - & - & 3 & - & - & - & - & - & - \\
\hline 1 & NB & 6 & $\bullet$ & - & - & - & 2 & - & - & - & - & 1 & $\mathrm{~S}$ \\
\hline 1 & NB & 4 & - & - & • & - & - & - & - & - & - & 1 & $\mathrm{~S}$ \\
\hline 1 & NB & 10 & $\bullet$ & - & - & - & - & - & - & - & - & 1 & $\mathrm{~S}$ \\
\hline 1 & NB & 6 & $\bullet$ & - & - & - & - & - & - & - & - & - & - \\
\hline 1 & NB & 8 & $\bullet$ & - & - & - & - & - & $\bullet$ & - & - & - & - \\
\hline 1 & $\mathrm{RB}$ & 4 & $\bullet$ & 1 & - & - & - & - & - & - & - & 1 & $\mathrm{~S}$ \\
\hline 1 & $\mathrm{RB}$ & 4 & $\bullet$ & - & - & - & - & - & - & - & - & - & - \\
\hline 1 & $\mathrm{RB}$ & 6 & - & - & - & - & 1 & - & • & - & - & - & - \\
\hline 1 & NB & 3 & $\bullet$ & - & - & - & - & - & • & - & - & - & - \\
\hline 1 & NB & 6 & • & - & - & - & - & - & - & - & - & - & - \\
\hline 1 & NB & 6 & - & - & • & - & 3 & - & - & - & - & 1 & $\mathrm{~S}$ \\
\hline 1 & NB & 6 & - & - & - & - & - & - & - & - & - & - & - \\
\hline 1 & NB & 96 & $\bullet$ & - & - & - & - & 47 & • & - & - & 47 & $\mathrm{P}$ \\
\hline 1 & $\mathrm{NB}$ & 4 & - & - & $\bullet$ & - & - & 2 & $\bullet$ & - & - & - & - \\
\hline \multicolumn{14}{|c|}{$\begin{array}{ll}\text { 1. } & \text { No: Number of accommodation services. } \\
\text { 2. } & \text { Q: Type of building used as accommodation services. } \\
\text { 3. } & \text { C: Capacity (number of bed spaces). } \\
\text { 4. } & \text { El: Electricity. } \\
\text { 5. } & \text { So: Solar system. } \\
\text { 6. } & \text { S: Shared facilities. } \\
\text { 7. } & \text { P: Private facilities. } \\
\text { 8. } & \text { Int: Internet. } \\
\text { 9. } & \text { V/B: Veranda/balcony. } \\
\text { 10. } & \text { Gas including central heating and under floor heating systems. } \\
\text { 11. } & \text { Solar systems used for hot water and lighting (PVs). }\end{array}$} \\
\hline
\end{tabular}

\begin{tabular}{|c|c|c|c|c|c|c|c|c|c|c|c|c|c|}
\hline \multicolumn{14}{|c|}{ Appendix.11: OCRT- Bp accommodation services, energy sources and facilities } \\
\hline \multirow[t]{2}{*}{ No. ${ }^{1}$} & \multirow[t]{2}{*}{$\mathrm{Q}^{2}$} & \multirow[t]{2}{*}{$\mathrm{C}^{3}$} & \multicolumn{3}{|c|}{ Heating } & \multirow[t]{2}{*}{$\mathrm{So}^{5 \& 11}$} & \multicolumn{2}{|c|}{ TV } & \multirow[t]{2}{*}{$\operatorname{Int}^{8}$} & \multirow[t]{2}{*}{$\mathrm{V} / \mathrm{B}^{9}$} & \multirow[t]{2}{*}{$\mathrm{S} / \mathrm{P}$} & \multirow{2}{*}{$\begin{array}{l}\text { Outdoor } \\
\text { sitting }\end{array}$} & \multirow[t]{2}{*}{$\mathrm{S} / \mathrm{F}$} \\
\hline & & & $\mathrm{El}^{4}$ & Log & Gas $^{10}$ & & $S^{6}$ & $\mathrm{P}^{7}$ & & & & & \\
\hline 3 & NB & 18 & • & - & - & - & - & - & - & - & - & - & - \\
\hline 1 & $\mathrm{RB}$ & 25 & - & - & - & - & 1 & - & - & - & - & - & - \\
\hline 1 & NB & 6 & • & 1 & - & - & - & - & - & - & - & - & - \\
\hline 1 & $\mathrm{RB}$ & 18 & - & - & - & - & - & - & - & - & - & - & - \\
\hline 1 & NB & 12 & $\bullet$ & - & - & - & - & - & - & - & - & - & - \\
\hline 1 & $\mathrm{RB}$ & 6 & $\bullet$ & - & - & - & - & - & - & - & - & - & - \\
\hline 1 & NB & 7 & $\bullet$ & - & - & - & - & - & - & - & - & - & - \\
\hline 1 & NB & 22 & $\bullet$ & - & - & - & - & 5 & - & - & - & - & - \\
\hline 1 & NB & 18 & - & - & $\bullet$ & - & - & 3 & $\bullet$ & - & - & - & - \\
\hline 1 & $\mathrm{RB}$ & 4 & • & - & - & - & - & - & - & - & - & - & - \\
\hline $\begin{array}{ll}\text { 1. } & \text { I } \\
2 . & \\
\text { 3. } & \\
4 . & \\
5 . & \\
6 . & \\
7 . & \mathrm{P} \\
8 . & \mathrm{I} \\
9 . & \\
10 . & \\
11 . & \end{array}$ & $\begin{array}{l}\text { Numb } \\
\text { Гype of } \\
\text { Capacit } \\
\text { Electric } \\
\text { Solar s } \\
\text { hared f } \\
\text { rivate f } \\
\text { Interne } \\
\text { : Verar } \\
\text { includ } \\
\text { r syste }\end{array}$ & $\begin{array}{l}\text { of ac } \\
\text { uildir } \\
\text { (num } \\
\text { y. } \\
\text { tem. } \\
\text { cilitie } \\
\text { cilitie } \\
\text { a/bal } \\
\text { g cen } \\
\text { s use }\end{array}$ & $\begin{array}{l}\text { ony. } \\
\text { for hea } \\
\text { for ho }\end{array}$ & $\begin{array}{l}\text { ion ser } \\
\text { accom } \\
\text { spaces }\end{array}$ & $\begin{array}{l}\text { ler floor } \\
\text { lighting }\end{array}$ & $\begin{array}{l}\text { ting syste } \\
\text { (s). }\end{array}$ & & & & & & & \\
\hline
\end{tabular}




\begin{tabular}{|c|c|c|c|c|c|c|c|c|c|c|c|c|c|}
\hline \multicolumn{14}{|c|}{ Appendix.12: OCRT - Motel accommodation services, energy sources and facilities. } \\
\hline \multirow[t]{2}{*}{ No. $^{1}$} & \multirow[t]{2}{*}{$\mathrm{Q}^{2}$} & \multirow[t]{2}{*}{$\mathrm{C}^{3}$} & \multicolumn{3}{|c|}{ Heating } & \multirow[t]{2}{*}{$\mathrm{So}^{5}$} & \multicolumn{2}{|c|}{ TV } & \multirow[t]{2}{*}{$\operatorname{Int}^{8}$} & \multirow[t]{2}{*}{$\mathrm{V} / \mathrm{B}^{9}$} & \multirow[t]{2}{*}{$\mathrm{S} / \mathrm{P}$} & \multirow{2}{*}{$\begin{array}{c}\text { Outdoor } \\
\text { sitting }\end{array}$} & \multirow[t]{2}{*}{$\mathrm{S} / \mathrm{P}$} \\
\hline & & & $\mathrm{El}^{4}$ & Log & $\mathrm{Gas}^{10}$ & & $S^{6}$ & $\mathrm{P}^{7}$ & & & & & \\
\hline 3 & NB & 13 & $\cdot$ & - & - & - & - & - & - & - & - & - & - \\
\hline 1 & NB & 12 & • & - & - & - & - & 6 & - & - & - & - & - \\
\hline 1 & NB & 40 & - & - & $\bullet$ & - & 1 & 13 & $\cdot$ & - & - & - & - \\
\hline 1 & NB & 2 & • & - & • & - & 1 & - & • & - & - & - & - \\
\hline 1 & NB & 4 & $\cdot$ & - & $\cdot$ & - & 1 & - & $\cdot$ & - & - & - & - \\
\hline 1 & NB & 5 & $\cdot$ & - & - & - & 1 & - & - & - & - & - & - \\
\hline 1 & NB & 6 & $\cdot$ & - & - & - & 1 & - & - & - & - & - & - \\
\hline 1 & NB & 16 & - & - & - & - & 1 & 6 & - & - & - & - & - \\
\hline 1 & NB & 26 & • & - & - & - & - & 13 & • & - & - & - & - \\
\hline 1 & NB & 30 & $\cdot$ & - & - & - & - & 6 & - & - & - & 1 & $S$ \\
\hline 1 & NB & 44 & - & - & - & - & - & 10 & - & - & - & 1 & $S$ \\
\hline 1 & NB & 48 & - & - & • & - & - & 8 & - & - & - & - & - \\
\hline 1 & NB & 66 & • & - & - & - & 1 & - & - & - & - & - & - \\
\hline 1 & NB & 24 & • & - & - & - & 1 & 7 & • & - & - & - & - \\
\hline 1 & NB & $\begin{array}{l}25 \\
1\end{array}$ & • & - & - & - & 1 & - & • & - & - & - & - \\
\hline 1 & $\mathrm{RB}$ & 22 & $\cdot$ & - & - & - & - & - & - & - & - & 1 & $S$ \\
\hline 1 & NB & 36 & $\cdot$ & - & - & - & - & 11 & - & - & - & 1 & $S$ \\
\hline 1 & NB & 16 & - & - & $\bullet$ & - & - & 4 & $\bullet$ & - & - & 1 & $S$ \\
\hline 1 & NB & 57 & • & - & • & - & - & - & - & - & - & 2 & $\mathrm{P}$ \\
\hline 1 & NB & 14 & • & - & - & - & - & 2 & - & 2 & $\mathrm{P}$ & 2 & $\mathrm{P}$ \\
\hline 1 & NB & 42 & • & - & - & - & - & - & • & - & - & - & - \\
\hline 8 & NB & 36 & • & - & - & - & - & 1 & • & - & - & 1 & $S$ \\
\hline 1 & NB & 28 & • & - & - & - & - & 15 & • & - & - & - & - \\
\hline 1 & NB & 53 & - & - & • & - & - & 15 & • & - & - & - & - \\
\hline $\begin{array}{ll}1 . & \\
2 . & \\
3 . & \\
4 . & \mathrm{E} \\
5 . & \mathrm{S} \\
6 . & \mathrm{S} \\
7 . & \mathrm{P} \\
8 . & \mathrm{I} \\
9 . & \mathrm{S} \\
10 . & \mathrm{S}\end{array}$ & $\begin{array}{l}\text { Numb } \\
\text { Type of } \\
\text { Capacit } \\
\text { Electric } \\
\text { Solar s } \\
\text { hared f } \\
\text { rivate } \\
\text { Interne } \\
\text { : Vera } \\
\text { ar syste }\end{array}$ & $\begin{array}{l}\text { r of a } \\
\text { buildi } \\
\text { (num } \\
\text { ty. } \\
\text { stem. } \\
\text { cilitie } \\
\text { cilitie } \\
\\
\text { ta/bal } \\
\text { ns use }\end{array}$ & $\begin{array}{l}\text { comm } \\
\text { g used } \\
\text { er of }\end{array}$ & $\begin{array}{l}\text { ation } \mathrm{s} \\
\text { accon } \\
\text { space }\end{array}$ & $\begin{array}{l}\text { fices. } \\
\text { odation }\end{array}$ & ices. & & & & & & & \\
\hline
\end{tabular}

Appendix.13: OCRT- Homestead accommodation services, energy sources and facilities

\begin{tabular}{|c|c|c|c|c|c|c|c|c|c|c|c|c|c|}
\hline \multirow[t]{2}{*}{ No. $^{1}$} & \multirow[t]{2}{*}{$\mathrm{Q}^{2}$} & \multirow[t]{2}{*}{$\mathrm{C}^{3}$} & \multicolumn{3}{|c|}{ Heating } & \multirow[t]{2}{*}{$\mathrm{So}^{5}$} & \multicolumn{2}{|c|}{ TV } & \multirow[t]{2}{*}{$\mathrm{Int}^{8}$} & \multirow{2}{*}{$\begin{array}{l}\mathrm{V} / \mathrm{B} \\
\end{array}$} & \multirow[t]{2}{*}{$\mathrm{S} / \mathrm{P}$} & \multirow{2}{*}{$\begin{array}{l}\text { Outdoor } \\
\text { sitting }\end{array}$} & \multirow[t]{2}{*}{$\mathrm{S} / \mathrm{P}$} \\
\hline & & & $\mathrm{El}^{4}$ & Log & Gas & & $S^{6}$ & $\mathrm{P}^{7}$ & & & & & \\
\hline 1 & RB & 4 & • & - & - & - & 1 & - & - & - & - & - & - \\
\hline 1 & $\mathrm{RB}$ & 12 & - & - & - & - & - & - & - & - & - & - & - \\
\hline 1 & NB & 6 & • & - & - & - & - & - & - & - & - & 1 & $\mathrm{P}$ \\
\hline 1 & RB & 6 & - & - & - & - & - & - & - & - & - & - & - \\
\hline $\begin{array}{ll}1 . & \mathrm{N} \\
2 . & \mathrm{C} \\
3 . & \mathrm{C} \\
4 . & \mathrm{B} \\
5 . & \mathrm{S} \\
6 . & \mathrm{S} \\
7 . & \mathrm{P} \\
8 . & \mathrm{I} \\
9 . & \mathrm{V} \\
10 . & \mathrm{C} \\
11 . & \mathrm{S}\end{array}$ & $\begin{array}{l}\text { Numb } \\
\text { Cype o } \\
\text { Capaci } \\
\text { Electri } \\
\text { Solar } \\
\text { hared } \\
\text { rivate } \\
\text { Intern } \\
\text { Vera } \\
\text { inclu } \\
\text { r syst }\end{array}$ & $\begin{array}{l}\text { of ac } \\
\text { ouildi } \\
\text { (num } \\
\text { ty. } \\
\text { stem. } \\
\text { cilitie } \\
\text { cilitie } \\
\text { da/bal } \\
\text { ig cen } \\
\text { s use }\end{array}$ & $\begin{array}{l}\text { ony. } \\
\text { ral he } \\
\text { for h }\end{array}$ & $\begin{array}{l}\text { tion se } \\
\text { accon } \\
\text { space }\end{array}$ & $\begin{array}{l}\text { ices. } \\
\text { iodation }\end{array}$ & ervice & ste & & & & & & \\
\hline
\end{tabular}




\begin{tabular}{|c|c|c|c|c|c|c|c|c|c|c|c|c|c|}
\hline \multicolumn{14}{|c|}{ Appendix.14: OCRT- Hotel accommodation services, energy sources and facilities } \\
\hline \multirow[t]{2}{*}{ No. ${ }^{1}$} & \multirow[t]{2}{*}{$\mathrm{Q}^{2}$} & \multirow[t]{2}{*}{$\mathrm{C}^{3}$} & \multicolumn{3}{|c|}{ Heating } & \multirow[t]{2}{*}{$\mathrm{So}^{5}$} & \multicolumn{2}{|c|}{ TV } & \multirow[t]{2}{*}{$\mathrm{Int}^{8}$} & \multirow[t]{2}{*}{$\mathrm{V} / \mathrm{B}^{9}$} & \multirow[t]{2}{*}{$\mathrm{S} / \mathrm{P}$} & \multirow{2}{*}{$\begin{array}{l}\text { Outdoor } \\
\text { sitting }\end{array}$} & \multirow[t]{2}{*}{$\mathrm{S} / \mathrm{H}$} \\
\hline & & & $\mathrm{El}^{4}$ & Log & Gas $^{10}$ & & $S^{6}$ & $\mathrm{P}^{7}$ & & & & & \\
\hline 1 & RB & 12 & $\cdot$ & - & - & - & - & - & - & - & - & 1 & $\mathrm{~S}$ \\
\hline 1 & RB & 16 & $\bullet$ & 1 & - & - & 1 & - & - & - & - & - & - \\
\hline 1 & RB & 36 & - & - & - & - & - & - & - & - & - & - & - \\
\hline 1 & $\mathrm{RB}$ & $\begin{array}{c}18 \\
1\end{array}$ & $\cdot$ & - & - & - & 1 & - & - & - & - & - & - \\
\hline 1 & RB & 8 & • & - & - & - & - & - & - & - & - & - & - \\
\hline 1 & RB & 30 & - & - & - & - & 1 & - & $\cdot$ & - & - & - & - \\
\hline 1 & $\mathrm{RB}$ & 6 & - & 1 & - & - & - & - & - & 1 & $S$ & - & - \\
\hline 1 & RB & 40 & • & 1 & - & - & - & - & - & - & - & - & - \\
\hline 1 & RB & 18 & $\cdot$ & - & - & - & 1 & - & - & - & - & 1 & $\mathrm{~S}$ \\
\hline 1 & $\mathrm{RB}$ & 8 & - & - & - & - & 1 & - & - & 1 & S & - & - \\
\hline 1 & $\mathrm{RB}$ & 16 & • & - & - & - & - & - & - & - & - & 1 & $\mathrm{~S}$ \\
\hline 1 & $\mathrm{RB}$ & 18 & - & - & - & - & - & - & - & - & - & - & - \\
\hline 1 & $\mathrm{RB}$ & 14 & $\bullet$ & - & - & - & - & - & - & - & - & - & - \\
\hline 1 & NB & 6 & - & 2 & - & - & - & - & - & - & - & - & - \\
\hline 1 & $\mathrm{RB}$ & 12 & $\cdot$ & - & - & - & - & - & - & - & - & - & - \\
\hline 1 & $\mathrm{RB}$ & 20 & • & - & - & - & - & - & - & - & - & - & - \\
\hline 1 & $\mathrm{RB}$ & 22 & - & 1 & - & - & 1 & - & • & - & - & - & - \\
\hline 1 & $\mathrm{RB}$ & 17 & • & - & - & - & 1 & - & - & - & - & - & - \\
\hline 1 & RB & 29 & - & - & $\bullet$ & - & 1 & - & - & 1 & $\mathrm{~S}$ & 6 & $\mathrm{P}$ \\
\hline $\begin{array}{ll}1 . & \mathrm{N} \\
2 . & \mathrm{C} \\
3 . & \mathrm{C} \\
4 . & \mathrm{E} \\
5 . & \mathrm{S} \\
6 . & \mathrm{S} \\
7 . & \mathrm{P} \\
8 . & \mathrm{I} \\
9 . & \\
10 . & \\
11 . & \mathrm{S}\end{array}$ & $\begin{array}{l}\text { Numb } \\
\text { ype of } \\
\text { apacit } \\
\text { Electric } \\
\text { Solar } \\
\text { hared } \\
\text { rivate } \\
\text { Interne } \\
\text { : Vera } \\
\text { includ } \\
\text { r syste }\end{array}$ & $\begin{array}{l}\text { of ac } \\
\text { ouildi } \\
\text { (num } \\
\text { ty. } \\
\text { stem. } \\
\text { cilitie } \\
\text { cilitie } \\
\text { ta/bal } \\
\text { ig cen } \\
\text { is use }\end{array}$ & $\begin{array}{l}\text { ony. } \\
\text { cal he } \\
\text { for h }\end{array}$ & $\begin{array}{l}\text { tion se } \\
\text { accom } \\
\text { space }\end{array}$ & $\begin{array}{l}\text { ices. } \\
\text { odation }\end{array}$ & vices. & ems. & & & & & & \\
\hline
\end{tabular}




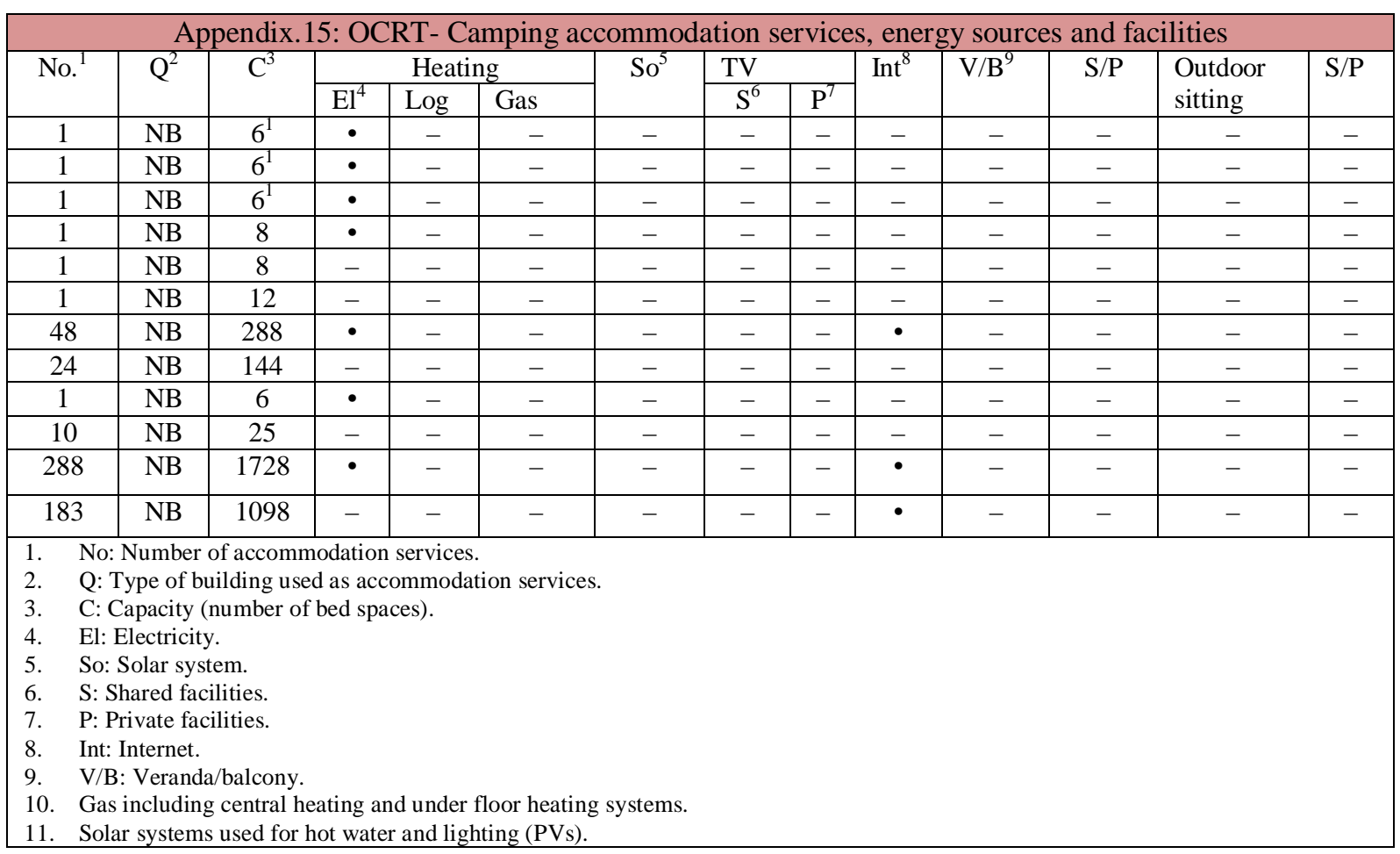




\begin{tabular}{|c|c|c|c|c|c|c|c|c|c|c|c|c|c|c|c|c|c|c|}
\hline \multicolumn{19}{|c|}{ Appendix.16: OCRT- All types of accommodation service- energy sources and facilities } \\
\hline \multirow[t]{2}{*}{ Type } & \multirow[t]{2}{*}{ Q } & \multicolumn{3}{|c|}{ Heating } & \multirow[t]{2}{*}{ So } & \multicolumn{4}{|c|}{ TV } & \multirow[t]{2}{*}{ Int } & \multicolumn{4}{|c|}{$\mathrm{V} / \mathrm{B}$} & \multicolumn{4}{|c|}{ Outdoor sitting } \\
\hline & & El & Log & Gas & & $\mathrm{S}$ & $\begin{array}{l}\mathrm{N} \\
\mathrm{T}\end{array}$ & $\mathrm{P}$ & NT & & $\mathrm{S}$ & $\begin{array}{l}\mathrm{N} \\
\mathrm{V}\end{array}$ & $\mathrm{P}$ & $\begin{array}{l}\mathrm{N} \\
\mathrm{V}\end{array}$ & $\mathrm{S}$ & $\begin{array}{l}\mathrm{N} \\
\mathrm{O}\end{array}$ & $\mathrm{P}$ & $\begin{array}{l}\mathrm{N} \\
\mathrm{O}\end{array}$ \\
\hline \multirow[t]{3}{*}{ SC } & NB & 297 & 39 & 52 & 49 & 91 & 18 & 89 & 29 & 143 & 6 & 1 & 16 & 8 & 49 & 7 & 24 & 8 \\
\hline & $\mathrm{RB}$ & 125 & 107 & 54 & 14 & 105 & 15 & 26 & 11 & 16 & 38 & 7 & 0 & 0 & 46 & 9 & 4 & 2 \\
\hline & Total & 422 & 146 & 106 & 63 & 196 & 33 & 115 & 40 & 159 & 44 & 8 & 16 & 8 & 95 & 16 & 28 & 10 \\
\hline \multirow[t]{3}{*}{$\mathrm{B} \& \mathrm{~B}$} & NB & 356 & 70 & 32 & 12 & 163 & 28 & 105 & 51 & 159 & 9 & 1 & 11 & 3 & 66 & 8 & 109 & 52 \\
\hline & RB & 143 & 66 & 5 & 0 & 80 & 9 & 5 & 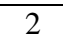 & 23 & 10 & 1 & 8 & 1 & 73 & 9 & 0 & 0 \\
\hline & Total & 499 & 136 & 37 & 12 & 243 & 37 & 110 & 53 & 182 & 19 & 2 & 19 & 4 & 139 & 17 & 109 & 52 \\
\hline \multirow[t]{3}{*}{$\mathrm{Bp}$} & NB & 65 & 6 & 18 & 18 & 0 & 0 & 8 & 8 & 18 & 0 & 0 & 0 & 0 & 0 & 0 & 0 & 0 \\
\hline & $\mathrm{RB}$ & 10 & 0 & 0 & 0 & 25 & 1 & 0 & 0 & 0 & 0 & 0 & 0 & 0 & 0 & 0 & 0 & 0 \\
\hline & Total & 75 & 6 & 18 & 18 & 25 & 1 & 8 & 8 & 18 & 0 & 0 & 0 & 0 & 0 & 0 & 0 & 0 \\
\hline \multirow[t]{3}{*}{ Motel } & NB & 486 & 0 & 225 & 0 & 188 & 9 & 423 & 117 & 582 & 0 & 0 & 14 & 2 & 162 & 5 & 71 & 4 \\
\hline & $\mathrm{RB}$ & 22 & 0 & 0 & 0 & 0 & 0 & 0 & 0 & 0 & 0 & 0 & 0 & 0 & 22 & 1 & 0 & 0 \\
\hline & Total & 508 & 0 & 225 & 0 & 188 & 9 & 423 & 117 & 582 & 0 & 0 & 14 & 2 & 184 & 6 & 71 & 4 \\
\hline \multirow{3}{*}{$\begin{array}{l}\text { Homest } \\
\text { ead }\end{array}$} & NB & 6 & 0 & 0 & 0 & 0 & 0 & 0 & 0 & 0 & 0 & 0 & 0 & 0 & 0 & 0 & 6 & 1 \\
\hline & RB & 22 & 4 & 0 & 0 & 4 & 1 & 0 & 0 & 4 & 0 & 0 & 0 & 0 & 0 & 0 & 0 & 0 \\
\hline & Total & 28 & 4 & 0 & 0 & 4 & 1 & 0 & 0 & 4 & 0 & 0 & 0 & 0 & 0 & 0 & 6 & 1 \\
\hline \multirow[t]{3}{*}{ Hotel } & NB & 0 & 6 & 0 & 0 & 0 & 0 & 0 & 0 & 0 & 0 & 0 & 0 & 0 & 0 & 0 & 0 & 0 \\
\hline & $\mathrm{RB}$ & 303 & 84 & 29 & 0 & 158 & 8 & 0 & 0 & 81 & 43 & 3 & 0 & 0 & 44 & 3 & 29 & 6 \\
\hline & Total & 303 & 90 & 29 & 0 & 158 & 8 & 0 & 0 & 81 & 43 & 3 & 0 & 0 & 44 & 3 & 29 & 6 \\
\hline \multirow{3}{*}{$\begin{array}{l}\text { Campi } \\
\text { ng }\end{array}$} & NB & 2048 & 0 & 0 & 0 & 0 & 0 & 0 & 0 & 3114 & 0 & 0 & 0 & 0 & 0 & 0 & 0 & 0 \\
\hline & $\mathrm{RB}$ & 0 & 0 & 0 & 0 & 0 & 0 & 0 & 0 & 0 & 0 & 0 & 0 & 0 & 0 & 0 & 0 & 0 \\
\hline & Total & 2048 & 0 & 0 & 0 & 0 & 0 & 0 & 0 & 3114 & 0 & 0 & 0 & 0 & 0 & 0 & 0 & 0 \\
\hline
\end{tabular}


Appendix.17: OCRT- All accommodation services by type - energy sources and facilities

\begin{tabular}{|c|c|c|c|c|c|c|c|c|c|c|c|c|c|c|c|c|c|}
\hline \multirow[t]{2}{*}{$\mathrm{Q}$} & \multicolumn{3}{|c|}{ Heating } & \multirow[t]{2}{*}{ So } & \multicolumn{4}{|c|}{ TV } & \multirow[t]{2}{*}{ Int } & \multicolumn{4}{|c|}{$\mathrm{V} / \mathrm{B}$} & \multicolumn{4}{|c|}{ Outdoor sitting } \\
\hline & El & Log & Gas & & $\mathrm{S}$ & NT & $\mathrm{P}$ & NT & & $\mathrm{S}$ & $\begin{array}{l}\mathrm{N} \\
\mathrm{V}\end{array}$ & $\mathrm{P}$ & $\begin{array}{l}\mathrm{N} \\
\mathrm{V}\end{array}$ & $\mathrm{S}$ & $\begin{array}{l}\mathrm{N} \\
\mathrm{O}\end{array}$ & $\mathrm{P}$ & $\mathrm{NO}$ \\
\hline NB & 3258 & 121 & 327 & 79 & 442 & 55 & 625 & 205 & 4016 & 15 & 2 & 41 & 13 & 277 & 20 & 210 & 65 \\
\hline $\mathrm{RB}$ & 626 & 261 & 88 & 14 & 372 & 34 & 34 & 13 & 124 & 91 & 11 & 8 & 1 & 185 & 22 & 33 & 8 \\
\hline Total & 3884 & 382 & 415 & 93 & 814 & 89 & 659 & 218 & 4140 & 106 & 13 & 49 & 14 & 462 & 42 & 243 & 73 \\
\hline
\end{tabular}

\begin{tabular}{|c|c|c|c|c|c|c|}
\hline \multicolumn{7}{|c|}{ Appendix.18: OCRT- accommodation services- Area per bed space (2011) } \\
\hline Location & Accommodation & Type & $\mathrm{Q}$ & Area $\left(\mathrm{m}^{2)}\right.$ & $\mathrm{C}$ & $\begin{array}{l}\text { Area per Bed } \\
\left(\mathrm{m}^{2}\right)\end{array}$ \\
\hline \multicolumn{7}{|c|}{ SC } \\
\hline Middlemarch & Gladbrook & SC & $\mathrm{RB}$ & 371 & 13 & 28.5 \\
\hline Middlemarch & Pukenangi Country Retreat & SC & $\mathrm{RB}$ & 314 & 8 & 39 \\
\hline Middlemarch & $\begin{array}{l}\text { The Rocks Luxury } \\
\text { Accommodation/ Longford } \\
\text { Retreat }\end{array}$ & SC & $\mathrm{RB}$ & 178 & 5 & 37 \\
\hline Ranfurly & Moyola Art Deco Guest House & SC & $\mathrm{RB}$ & 200 & 6 & 33 \\
\hline Naseby & Royal Hotel (SC) & SC & $\mathrm{RB}$ & 88 & 4 & 22 \\
\hline Oturehua & $\begin{array}{l}\text { Crowsnest Accommodation (2 } \\
\text { cabins) }\end{array}$ & SC & NB & 22 & 4 & 5.5 \\
\hline Lauder & Big Shy Cottage & SC & $\mathrm{RB}$ & 233 & 4 & 58 \\
\hline Lauder & Pedal Inn & SC & $\mathrm{RB}$ & 142 & 6 & 24 \\
\hline Omakau & Hawksview B\&B & SC & $\mathrm{RB}$ & 190 & 6 & 32 \\
\hline Alexandra & Marj’s Place (Homestead) & SC & NB & 79 & 5 & 16 \\
\hline \multicolumn{7}{|l|}{$\mathrm{B} \& \mathrm{~B}$} \\
\hline Middlemarch & Annandale & $\mathrm{B} \& \mathrm{~B}$ & NB & 244 & 6 & 41 \\
\hline Middlemarch & Middlemarch B\&B & $\mathrm{B} \& \mathrm{~B}$ & NB & 260 & 7 & 37 \\
\hline Middlemarch & Rose cottage & $\mathrm{B} \& \mathrm{~B}$ & NB & 142 & 6 & 24 \\
\hline Middlemarch & Strathmore B\&B & $\mathrm{B} \& \mathrm{~B}$ & $\mathrm{RB}$ & 287 & 5 & 56 \\
\hline Middlemarch & The Lodge & $\mathrm{B} \& \mathrm{~B}$ & $\mathrm{RB}$ & 282 & 10 & 28 \\
\hline Middlemarch & The Rocks Luxury & $\mathrm{B} \& \mathrm{~B}$ & NB & 408 & 5 & 82 \\
\hline
\end{tabular}




\begin{tabular}{|c|c|c|c|c|c|c|}
\hline & $\begin{array}{l}\text { Accommodation/ Braeside } \\
\text { Cottage }\end{array}$ & & & & & \\
\hline Ranfurly & Annerly House & $\mathrm{B} \& \mathrm{~B}$ & $\mathrm{NB}$ & 338 & 9 & 37.5 \\
\hline Wedderburn & Wedderburn Tavern & $\mathrm{B} \& \mathrm{~B}$ & $\mathrm{RB}$ & 309 & 14 & 22 \\
\hline Naseby & Old Doctor’s Residence & $\mathrm{B} \& \mathrm{~B}$ & $\mathrm{RB}$ & 254 & 5 & 51 \\
\hline Poolburn & $\begin{array}{l}\text { Solandra Lodge Country style } \\
\text { B\&B }\end{array}$ & $\mathrm{B} \& \mathrm{~B}$ & NB & 120 & 8 & 15 \\
\hline Omakau & Church Hill B\&B & $\mathrm{B} \& \mathrm{~B}$ & $\mathrm{RB}$ & 224 & 6 & 37 \\
\hline Alexandra & $\begin{array}{l}\text { Hawkdun Rise Vineyard } \\
\text { Homestead }\end{array}$ & $\mathrm{B} \& \mathrm{~B}$ & NB & 433 & 6 & 72 \\
\hline Alexandra & Log House retreat & $\mathrm{B} \& \mathrm{~B}$ & NB & 135 & 6 & 22.5 \\
\hline \multicolumn{7}{|c|}{ Motel } \\
\hline Waipiata & Waipiata Motel & Motel & NB & 137 & 12 & 11.5 \\
\hline Ranfurly & Ranfurly Motel & Motel & NB & 195 & 16 & 12.2 \\
\hline Alexandra & Alexandra Almond Court Motel & Motel & NB & 411 & 48 & 8.6 \\
\hline Alexandra & Central Court Motel Inn & Motel & $\mathrm{NB}$ & 1530 & 66 & 23 \\
\hline Clyde & Antique Lodge Motel & Motel & NB & 539 & 24 & 22.5 \\
\hline \multicolumn{7}{|c|}{ Bp } \\
\hline Kokonga & 2 Wheel Backpacker & $\mathrm{Bp}$ & NB & 144 & 6 & 24 \\
\hline Oturehua & $\begin{array}{l}\text { Crowsnest Accommodation } \\
\text { (Bunkrooms/lodge) }\end{array}$ & Bp & NB & 60 & 12 & 5 \\
\hline Alexandra & Criterion Club Hotel (BP) & $\mathrm{Bp}$ & NB & 378 & 22 & 17 \\
\hline Alexandra & Marj's Place (Bp) & Bp & NB & 115 & 18 & 6 \\
\hline \multicolumn{7}{|c|}{ Hotel } \\
\hline Middlemarch & Strath Taieri Hotel & Hotel & $\mathrm{RB}$ & 473 & 12 & 39.4 \\
\hline Macraes Flat & Stanley's Hotel & Hotel & $\mathrm{RB}$ & 466 & 16 & 29.1 \\
\hline Ranfurly & Ranfurly Lion Hotel & Hotel & $\mathrm{RB}$ & 1698 & 30 & 56.6 \\
\hline Naseby & Ancient Briton Hotel & Hotel & RB & 360 & 40 & 9 \\
\hline Naseby & Royal Hotel(hotel) & Hotel & $\mathrm{RB}$ & 421 & 18 & 23.4 \\
\hline Becks & White House Hotel & Hotel & RB & 478 & 18 & 26.5 \\
\hline Omakau & Omaka Commercial Hotel & Hotel & $\mathrm{RB}$ & 642 & 22 & 29.2 \\
\hline \multicolumn{7}{|c|}{ Homestead } \\
\hline Waipiata & Peter's Farm Lodge- the homestead & $\begin{array}{c}\text { Homes } \\
\text { tead }\end{array}$ & NB & 253 & 6 & 42 \\
\hline Auripo & Taradale Homestead & $\begin{array}{c}\text { Homes } \\
\text { tead }\end{array}$ & $\mathrm{RB}$ & 100 & 6 & 16.6 \\
\hline
\end{tabular}




\begin{tabular}{|c|c|c|c|c|c|}
\hline \multicolumn{6}{|c|}{ Appendix.19: OCRT accommodation services - Average area of outdoor sitting spaces } \\
\hline Location & No. & Accommodation & Type & Area $\left(\mathrm{m}^{2}\right)$ & Type \\
\hline \multicolumn{6}{|c|}{ Veranda/ Balcony } \\
\hline Middlemarch & 1 & Cottesbrook & SC & 14 & Veranda \\
\hline Middlemarch & 2 & Gladbrook & $B \& B$ & 30 & Veranda \\
\hline Middlemarch & 3 & Gladbrook & SC & 30 & Veranda \\
\hline Hyde & 4 & Pine Grove & SC & 18 & Veranda \\
\hline Hyde & 5 & Pine Grove & SC & 18 & Veranda \\
\hline Kokonga & 6 & 2 Wheel Backpackers & Bp & 5 & Veranda \\
\hline Waipiata & 7 & Peter's farm Lodge & Homestead & 12 & $\begin{array}{l}\text { Part of } \\
\text { veranda used } \\
\text { as sitting }\end{array}$ \\
\hline Lauder & 8 & Big Sky Cottage & SC & 15 & Veranda \\
\hline Lauder & 9 & Lauder Burn House & B\&B & 18 & Veranda \\
\hline Lauder & 10 & Lauder Burn House & $\mathrm{B} \& \mathrm{~B}$ & 6 & Balcony \\
\hline Cromwell & 11 & Colonial Manor Motel & Motel & 3 & Balcony \\
\hline Cromwell & 12 & Golden Gate lodge & B\&B & 3 & Balcony \\
\hline Total & - & - & - & 172 & - \\
\hline \multicolumn{6}{|c|}{ Average area $\left(\mathrm{m}^{2}\right)$ per veranda/ balcony $=14.3$} \\
\hline \multicolumn{6}{|c|}{ Outdoor sitting } \\
\hline Middlemarch & 1 & Middlemarch B\&B & $\mathrm{B} \& \mathrm{~B}$ & 30 & $\begin{array}{c}\text { Sheltered } \\
\text { outdoor sitting }\end{array}$ \\
\hline Kokonga & 2 & 2 Wheel Backpackers & Bp & 15 & $\begin{array}{c}\text { Outdoor } \\
\text { sitting }\end{array}$ \\
\hline Waipiata & 3 & Peter's farm Lodge & Homestead & 35 & Garden sitting \\
\hline Cromwell & 4 & Central gold Motel & Motel & 9 & $\begin{array}{l}\text { Outdoor } \\
\text { sitting }\end{array}$ \\
\hline Cromwell & 5 & Golden Gate lodge & $\mathrm{B} \& \mathrm{~B}$ & 9 & $\begin{array}{c}\text { Outdoor } \\
\text { sitting }\end{array}$ \\
\hline Total & 5 & - & - & 98 & - \\
\hline
\end{tabular}




\begin{tabular}{|c|c|c|c|c|c|c|c|c|c|c|c|c|c|c|c|c|c|c|c|c|c|}
\hline \multicolumn{22}{|c|}{ Appendix.20: OCRT- Types of outdoor activities and their frequency (2011) } \\
\hline $\begin{array}{l}\text { Sports ( at } \\
\text { playground } \\
\text { ) }\end{array}$ & $\mathbf{F}$ & $\begin{array}{l}\text { Motorise } \\
\text { d sports } \\
\text { and } \\
\text { activities }\end{array}$ & $\mathbf{F}$ & $\begin{array}{l}\text { Water } \\
\text { sports }\end{array}$ & $\mathbf{F}$ & $\begin{array}{l}\text { Winter } \\
\text { sports }\end{array}$ & $\mathbf{F}$ & Cycling & $\mathbf{F}$ & $\begin{array}{l}\text { Sports } \\
\text { on } \\
\text { horseba } \\
\text { ck }\end{array}$ & $\mathbf{F}$ & $\begin{array}{l}\text { Recreatio } \\
\text { nal } \\
\text { activities }\end{array}$ & $\mathbf{F}$ & $\begin{array}{l}\text { Art } \\
\text { activities }\end{array}$ & $\mathbf{F}$ & $\begin{array}{l}\text { Farm } \\
\text { activities }\end{array}$ & $\mathbf{F}$ & Walking & $\mathbf{F}$ & $\begin{array}{l}\text { Climb } \\
\text { ing. }\end{array}$ & $\mathbf{F}$ \\
\hline Golf & 55 & $\begin{array}{l}\text { The Brass } \\
\text { Monkey } \\
\text { motorcycl } \\
\text { e rally }\end{array}$ & 1 & Boating & 21 & $\begin{array}{l}\text { Winter } \\
\text { sports }\end{array}$ & 54 & $\begin{array}{l}\text { Riding } \\
\text { mountain } \\
\text { bike }\end{array}$ & 31 & $\begin{array}{l}\text { Horse } \\
\text { riding }\end{array}$ & 11 & $\begin{array}{l}\text { Scenic } \\
\text { flight }\end{array}$ & 1 & $\begin{array}{l}\text { Landscape } \\
\text { painting }\end{array}$ & 6 & $\begin{array}{l}\text { Farm } \\
\text { tour/track }\end{array}$ & 15 & Tramping & 4 & $\begin{array}{l}\text { Rock } \\
\text { climbi } \\
\text { ng }\end{array}$ & 1 \\
\hline Tennis & 14 & Car racing & 1 & Fishing & 71 & - & - & $\begin{array}{l}\text { Road } \\
\text { biking }\end{array}$ & 7 & $\begin{array}{l}\text { Horse } \\
\text { racing }\end{array}$ & 1 & $\begin{array}{l}\text { Wind } \\
\text { surfing }\end{array}$ & 1 & $\begin{array}{l}\text { Landscape } \\
\text { photograp } \\
\text { hy }\end{array}$ & 26 & $\begin{array}{l}\text { Sheep } \\
\text { mustering } \\
\text { activities }\end{array}$ & 4 & Walking & 48 & - & - \\
\hline Croquet & 1 & $\begin{array}{l}4 \mathrm{WD} \\
\text { track/tour }\end{array}$ & 27 & $\begin{array}{l}\text { Swimmin } \\
\mathrm{g}\end{array}$ & 18 & - & - & $\begin{array}{l}\text { Dunstan } \\
\text { trail }\end{array}$ & 1 & - & - & $\begin{array}{l}\text { Recreatio } \\
\text { nal } \\
\text { activities }\end{array}$ & 2 & - & - & $\begin{array}{l}\text { Feeding } \\
\text { hens/ } \\
\text { cattle }\end{array}$ & 3 & - & - & - & - \\
\hline Hiking & 6 & Jet trail & 2 & $\begin{array}{l}\text { Water } \\
\text { sports } \\
\text { (undefine } \\
\text { d) }\end{array}$ & 12 & - & - & $\begin{array}{l}\text { Triathlon } \\
\text { track. }\end{array}$ & 1 & - & - & $\begin{array}{l}\text { Gold } \\
\text { mining }\end{array}$ & 17 & - & - & - & - & - & - & - & - \\
\hline Basketball & 2 & - & - & - & - & - & - & $\begin{array}{l}\text { BMX } \\
\text { track }\end{array}$ & 1 & - & - & Picnic & 2 & - & - & - & - & - & - & - & - \\
\hline Netball & 1 & - & & - & - & - & - & $\begin{array}{l}\text { Scroll } \\
\text { plain } \\
\text { wetland }\end{array}$ & 1 & - & - & Hunting & 8 & - & - & - & - & - & - & - & - \\
\hline Squash & 1 & - & & - & - & - & - & - & - & - & - & $\begin{array}{l}\text { Clay } \\
\text { target } \\
\text { shooting }\end{array}$ & 1 & - & - & - & - & - & - & - & - \\
\hline Soccer & 1 & - & & - & - & - & - & - & - & - & - & Falls dam & 2 & - & - & - & - & - & - & - & - \\
\hline $\begin{array}{l}\text { Touch } \\
\text { rugby }\end{array}$ & 1 & - & & - & - & - & - & - & - & - & - & $\begin{array}{l}\text { Wildlife } \\
\text { watching }\end{array}$ & 4 & - & - & - & - & - & - & - & - \\
\hline Petanque & 1 & - & & - & - & - & - & - & - & - & - & - & - & - & - & - & - & - & - & - & - \\
\hline $\begin{array}{l}\text { Play ground } \\
\text { (undefined } \\
\text { sports) }\end{array}$ & 3 & - & & - & - & - & - & - & - & & - & - & - & - & - & - & - & - & - & - & - \\
\hline Total & 86 & & 31 & & 122 & & 54 & & 42 & & 12 & & 38 & & 32 & & 22 & & 52 & & 1 \\
\hline
\end{tabular}




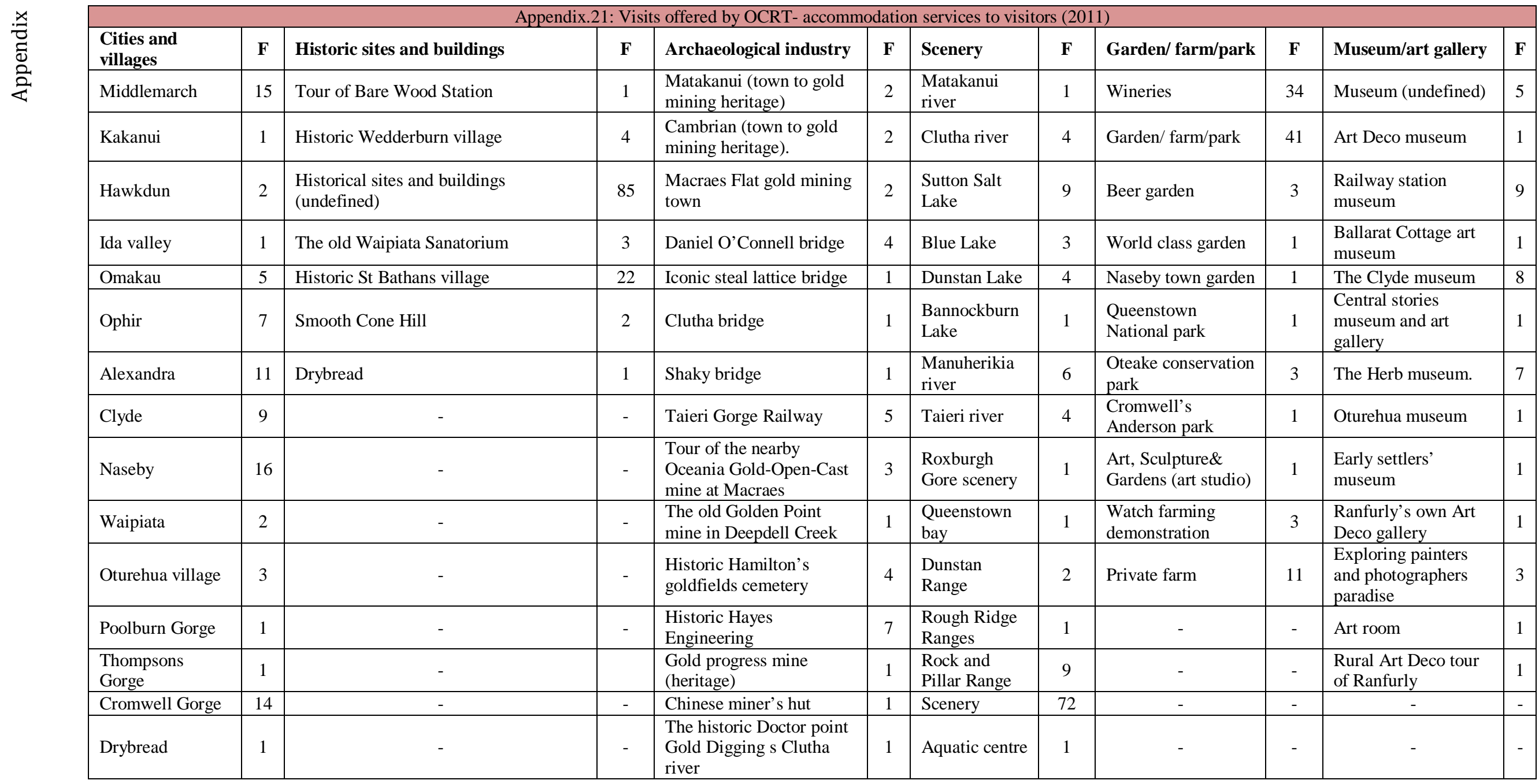




\begin{tabular}{|c|c|c|c|c|c|c|c|c|c|c|c|}
\hline Manitoto Plains & 8 & - & - & Gold mining heritage & 25 & Poolburn dam & 3 & - & - & - & - \\
\hline- & - & - & - & - & & Clyde dam & 10 & - & - & - & - \\
\hline Total & 97 & - & 118 & - & 61 & - & 132 & - & 100 & - & 4 \\
\hline
\end{tabular}


Appendix.22: Naseby: All types of accommodation service - energy sources and facilities

\begin{tabular}{|c|c|c|c|c|c|c|c|c|c|c|c|c|c|c|c|c|c|c|}
\hline \multirow[t]{2}{*}{ Type } & \multirow[t]{2}{*}{$\mathbf{Q}$} & \multicolumn{3}{|c|}{ Heating } & \multirow[t]{2}{*}{ So } & \multicolumn{4}{|c|}{ TV } & \multirow[t]{2}{*}{ Int } & \multicolumn{4}{|c|}{ V/B } & \multicolumn{4}{|c|}{ Outdoor sitting } \\
\hline & & El & Log & $\begin{array}{l}\text { G } \\
\text { as }\end{array}$ & & $\mathrm{S}$ & $\begin{array}{l}\mathbf{N} \\
\mathbf{T}\end{array}$ & $\mathbf{P}$ & NT & & $\mathrm{S}$ & $\begin{array}{l}\mathbf{N} \\
\mathbf{V}\end{array}$ & $\mathbf{P}$ & $\begin{array}{l}\mathbf{N} \\
\mathbf{V}\end{array}$ & $\mathbf{S}$ & $\begin{array}{l}\mathbf{N} \\
\mathbf{O}\end{array}$ & $\mathbf{P}$ & $\begin{array}{l}\mathbf{N} \\
\mathbf{O}\end{array}$ \\
\hline \multirow[t]{3}{*}{ SC } & $\mathrm{NB}$ & 48 & 17 & 26 & 49 & 29 & & 0 & 0 & 69 & 6 & 1 & $\begin{array}{l}1 \\
2\end{array}$ & 6 & 0 & 0 & 0 & 0 \\
\hline & $\mathrm{RB}$ & 6 & 6 & 4 & 0 & 6 & & 0 & 0 & 10 & 0 & 0 & 0 & 0 & 0 & 0 & 0 & 0 \\
\hline & Total & 54 & 23 & 30 & 49 & 35 & & 0 & 0 & 79 & 6 & 1 & $\begin{array}{l}1 \\
2\end{array}$ & 6 & 0 & 0 & 0 & 0 \\
\hline \multirow[t]{3}{*}{$\mathrm{B} \& \mathrm{~B}$} & NB & 23 & 0 & 0 & 12 & 18 & 3 & 0 & 0 & 12 & 0 & 0 & 0 & 0 & 0 & 0 & 0 & 0 \\
\hline & $\mathrm{RB}$ & 5 & 0 & 0 & 0 & 5 & 1 & 0 & 0 & 5 & 0 & 0 & 0 & 0 & 0 & 0 & 0 & 0 \\
\hline & Total & 28 & 0 & 0 & 12 & 23 & 4 & 0 & 0 & 17 & 0 & 0 & 0 & 0 & 0 & 0 & 0 & 0 \\
\hline \multirow[t]{3}{*}{ Hotel } & $\mathrm{NB}$ & 0 & 0 & 0 & 0 & 0 & 0 & 0 & 0 & 0 & 0 & 0 & 0 & 0 & 0 & 0 & 0 & 0 \\
\hline & $\mathrm{RB}$ & 58 & 40 & 0 & 0 & 18 & 1 & 0 & 0 & 0 & 0 & 0 & 0 & 0 & 18 & 1 & 0 & 0 \\
\hline & Total & 58 & 40 & 0 & 0 & 18 & 1 & 0 & 0 & 0 & 0 & 0 & 0 & 0 & 18 & 1 & 0 & 0 \\
\hline \multirow{3}{*}{$\begin{array}{c}\text { Campi } \\
\text { ng }\end{array}$} & NB & 288 & 0 & 0 & 0 & 0 & 0 & 0 & 0 & 288 & 0 & 0 & 0 & 0 & 0 & 0 & 0 & 0 \\
\hline & $\mathrm{RB}$ & 0 & 0 & 0 & 0 & 0 & 0 & 0 & 0 & 0 & 0 & 0 & 0 & 0 & 0 & 0 & 0 & 0 \\
\hline & Total & 288 & 0 & 0 & 0 & 0 & 0 & 0 & 0 & 288 & 0 & 0 & 0 & 0 & 0 & 0 & 0 & 0 \\
\hline
\end{tabular}

\begin{tabular}{|c|c|c|c|c|c|c|c|c|c|c|c|c|c|}
\hline \multicolumn{14}{|c|}{ Appendix.23: Naseby: Self contained accommodation services, energy sources and facilities. } \\
\hline \multirow{2}{*}{$\begin{array}{c}\text { No. } \\
1\end{array}$} & \multirow[t]{2}{*}{$\mathrm{Q}^{2}$} & \multirow[t]{2}{*}{$\mathrm{C}^{3}$} & \multicolumn{3}{|c|}{ Heating } & \multirow[t]{2}{*}{$\mathrm{So}^{5,11}$} & \multicolumn{2}{|c|}{ TV } & \multirow[t]{2}{*}{$\operatorname{Int}^{8}$} & \multirow[t]{2}{*}{$\mathrm{V} / \mathrm{B}^{9}$} & \multirow[t]{2}{*}{$\mathrm{S} / \mathrm{P}$} & \multirow{2}{*}{$\begin{array}{l}\text { Outdoor } \\
\text { sitting }\end{array}$} & \multirow[t]{2}{*}{$\mathrm{S} / \mathrm{P}$} \\
\hline & & & $\mathrm{El}^{4}$ & Log & $\mathrm{Gas}^{10}$ & & $\mathrm{~S}^{6}$ & $\mathrm{P}^{7}$ & & & & & \\
\hline 1 & NB & 9 & - & 1 & - & 1 & 1 & - & 1 & - & - & - & - \\
\hline 2 & RB & 6 & $\cdot$ & 1 & - & - & 1 & - & 1 & - & - & - & - \\
\hline 10 & NB & 40 & • & - & - & • & - & - & • & - & - & - & - \\
\hline 1 & NB & 8 & $\cdot$ & 1 & - & - & - & - & - & - & - & - & - \\
\hline 1 & NB & 6 & - & - & $\bullet$ & - & - & - & - & 1 & $\mathrm{~S}$ & - & - \\
\hline 1 & NB & 12 & - & - & $\bullet$ & - & 2 & - & $\cdot$ & 6 & $\mathrm{P}$ & - & - \\
\hline 1 & NB & 8 & - & - & $\bullet$ & - & 2 & - & • & - & - & - & - \\
\hline 1 & $\mathrm{RB}$ & 4 & - & - & $\cdot$ & - & - & - & • & - & - & - & - \\
\hline $\begin{array}{l}\text { No: I } \\
\text { Q: T } \\
\text { C: Ca } \\
\text { El: E } \\
\text { So: S } \\
\text { S: Sh } \\
\text { P: Pr } \\
\text { Int: I } \\
\text { V/B: } \\
\text { Gas i } \\
\text { Solar }\end{array}$ & $\begin{array}{l}\text { Imber } \\
\text { e of } \\
\text { acity } \\
\text { ctrici } \\
\text { lar sy } \\
\text { ced fa } \\
\text { ate fa } \\
\text { ernet. } \\
\text { eran } \\
\text { cludir }\end{array}$ & $\begin{array}{l}\text { ff acc } \\
\text { ildin } \\
\text { numb } \\
\text { em. } \\
\text { lities } \\
\text { lities }\end{array}$ & $\begin{array}{l}\text { ummod } \\
\text { used of }\end{array}$ & $\begin{array}{l}\text { tion s } \\
\text { accol } \\
\text { space }\end{array}$ & $\begin{array}{l}\text { vices. } \\
\text { modatio } \\
\text {. }\end{array}$ & ervice. & yster & & & & & & \\
\hline
\end{tabular}




\begin{tabular}{|c|c|c|}
\hline \multicolumn{3}{|c|}{ Appendix.28: Naseby: Cultural Products and Activities } \\
\hline \multicolumn{3}{|c|}{ Local produce } \\
\hline No. & & F \\
\hline 1 & Continental Breakfast & 7 \\
\hline 2 & Home baking and local food & 3 \\
\hline 3 & Wine & 2 \\
\hline \multicolumn{3}{|c|}{ General produce } \\
\hline 1 & Food and beverages & 10 \\
\hline \multicolumn{3}{|c|}{ Services } \\
\hline \multicolumn{3}{|c|}{ No services } \\
\hline \multicolumn{3}{|c|}{ Visits } \\
\hline 1 & Naseby & 13 \\
\hline 2 & Historical sites and buildings (undefined) & 10 \\
\hline 3 & Scenery & 6 \\
\hline 4 & Museum (undefined) & 5 \\
\hline 5 & Gold mining heritage & 3 \\
\hline 6 & Naseby town garden & 1 \\
\hline 7 & Art, Sculpture\& Gardens (art studio) & 1 \\
\hline \multicolumn{3}{|c|}{ Events } \\
\hline 1 & Local annual events/festivals & 4 \\
\hline \multicolumn{3}{|c|}{ Indoor activities } \\
\hline 1 & Curling at Naseby & 16 \\
\hline 2 & Reading materials & 1 \\
\hline 3 & Music & 1 \\
\hline 4 & Cafe Culture & 1 \\
\hline \multicolumn{3}{|c|}{ Outdoor activities } \\
\hline 1 & Walking & 15 \\
\hline 2 & Riding mountain bike & 14 \\
\hline 3 & Winter sports & 14 \\
\hline 4 & 4WD track/tour & 13 \\
\hline 5 & Fishing & 11 \\
\hline 6 & Horse riding & 7 \\
\hline 7 & Gold mining & 7 \\
\hline 8 & Swimming & 7 \\
\hline 9 & Golf & 5 \\
\hline 10 & Hiking & 4 \\
\hline 11 & Hunting & 4 \\
\hline
\end{tabular}


Appendix.29: Cromwell - Self contained accommodation services, energy sources and facilities.

\begin{tabular}{|c|c|c|c|c|c|c|c|c|c|c|c|c|c|}
\hline \multirow[t]{2}{*}{ No. ${ }^{1}$} & \multirow[t]{2}{*}{$\mathrm{Q}^{2}$} & \multirow[t]{2}{*}{$\mathrm{C}^{3}$} & \multicolumn{3}{|c|}{ Heating } & \multirow[t]{2}{*}{$\mathrm{So}^{5}$} & \multicolumn{2}{|l|}{ TV } & \multirow[t]{2}{*}{$\mathrm{Int}^{8}$} & \multirow[t]{2}{*}{$\mathrm{V} / \mathrm{B}^{9}$} & \multirow[t]{2}{*}{$\mathrm{S} / \mathrm{P}$} & \multirow{2}{*}{$\begin{array}{l}\text { Outdoor } \\
\text { sitting }\end{array}$} & \multirow[t]{2}{*}{$\mathrm{S} / \mathrm{P}$} \\
\hline & & & $\mathrm{El}^{4}$ & Log & $\mathrm{Gas}^{10}$ & & $\mathrm{~S}^{6}$ & $\mathrm{P}^{7}$ & & & & & \\
\hline 2 & NB & 5 & • & - & - & - & - & 2 & $\bullet$ & - & - & 1 & $S$ \\
\hline 2 & NB & 6 & $\cdot$ & - & - & - & - & 2 & $\cdot$ & - & - & 1 & $S$ \\
\hline 1 & NB & 4 & $\cdot$ & - & - & - & - & 2 & $\cdot$ & 2 & $P$ & - & - \\
\hline
\end{tabular}

1. No: Number of accommodation services.

2. Q: Type of building used as accommodation service.

3. C: Capacity (number of bed spaces).

4. El: Electricity.

5. So: Solar system used for water heating.

6. S: Shared facilities.

7. P: Private facilities.

8. Int: Internet.

9. V/B: Veranda/balcony.

10. Gas including central heating and under floor heating systems.

\begin{tabular}{|c|c|c|c|c|c|c|c|c|c|c|c|c|c|}
\hline \multirow{3}{*}{ No. ${ }^{1}$} & \multirow{3}{*}{$\frac{\text { Appen }}{\mathrm{Q}^{2}}$} & $\operatorname{dix.3}$ & $\mathrm{Crc}$ & iwell & $\mathrm{B} \& \mathrm{~B}$ a & ommc & tion & erv & es, en & gy sol & es an & facilities & \\
\hline & & \multirow[t]{2}{*}{$\mathrm{C}^{3}$} & \multicolumn{3}{|c|}{ Heating } & \multirow[t]{2}{*}{$\mathrm{So}^{51}$} & \multicolumn{2}{|c|}{ TV } & \multirow[t]{2}{*}{$\operatorname{Int}^{8}$} & \multirow[t]{2}{*}{$\mathrm{V} / \mathrm{B}^{9}$} & \multirow[t]{2}{*}{$\mathrm{S} / \mathrm{P}$} & \multirow{2}{*}{$\begin{array}{l}\text { Outdoo } \\
\text { r sitting }\end{array}$} & \multirow[t]{2}{*}{$\mathrm{S} / \mathrm{P}$} \\
\hline & & & $\mathrm{El}^{4}$ & Log & $\mathrm{Gas}^{10}$ & & $\mathrm{~S}^{6}$ & $\overline{\mathrm{P}^{7}}$ & & & & & \\
\hline 1 & NB & 96 & $\cdot$ & - & - & - & - & 47 & $\cdot$ & - & - & 47 & $\mathrm{P}$ \\
\hline 1 & NB & 4 & - & - & - & - & - & 2 & $\cdot$ & - & - & - & - \\
\hline
\end{tabular}

1. No: Number of accommodation services.

2. Q: Type of building used as accommodation services.

3. C: Capacity (number of bed spaces).

4. El: Electricity.

5. So: Solar system used for water heating.

6. S: Shared facilities.

7. P: Private facilities.

8. Int: Internet.

9. V/B: Veranda/balcony.

10. Gas including central heating and under floor heating systems. 
Appendix.31: Cromwell - Motel accommodation services, energy sources and facilities

\begin{tabular}{|c|c|c|c|c|c|c|c|c|c|c|c|c|c|}
\hline \multirow[t]{2}{*}{ No. ${ }^{1}$} & \multirow[t]{2}{*}{$\mathrm{Q}^{2}$} & \multirow[t]{2}{*}{$\mathrm{C}^{3}$} & \multicolumn{3}{|c|}{ Heating } & \multirow[t]{2}{*}{$\mathrm{So}^{51}$} & \multicolumn{2}{|c|}{ TV } & \multirow[t]{2}{*}{$\operatorname{Int}^{8}$} & \multirow[t]{2}{*}{$\mathrm{V} / \mathrm{B}^{9}$} & \multirow[t]{2}{*}{$\mathrm{S} / \mathrm{P}$} & \multirow{2}{*}{$\begin{array}{l}\text { Outdoo } \\
\text { r sitting }\end{array}$} & \multirow[t]{2}{*}{$\mathrm{S} / \mathrm{P}$} \\
\hline & & & $\mathrm{El}^{4}$ & $\log$ & $\operatorname{Gas}^{10}$ & & $\mathrm{~S}^{6}$ & $\mathrm{P}^{7}$ & & & & & \\
\hline 1 & NB & 36 & • & - & - & - & - & 11 & • & - & - & 1 & $\mathrm{~S}$ \\
\hline 1 & NB & 16 & - & - & • & - & - & 4 & • & - & - & 1 & $\mathrm{~S}$ \\
\hline 1 & NB & 57 & • & - & • & - & - & - & • & - & - & 2 & P \\
\hline 1 & NB & 14 & • & - & - & - & - & 2 & • & 2 & P & 2 & P \\
\hline 1 & $\mathrm{NB}$ & 42 & $\bullet$ & - & - & - & - & - & $\bullet$ & - & - & - & - \\
\hline 8 & NB & 36 & • & - & - & - & - & 1 & - & - & - & 1 & S \\
\hline 1 & NB & 28 & • & - & - & - & - & 15 & • & - & - & - & - \\
\hline
\end{tabular}

1. No: Number of accommodation services.

2. Q: Type of building used as accommodation services.

3. C: Capacity (number of bed spaces).

4. El: Electricity.

5. So: Solar system.

6. S: Shared facilities.

7. P: Private facilities.

8. Int: Internet.

9. V/B: Veranda/balcony.

10. Gas including central heating and under floor heating systems.

11. Solar systems used for hot water and lighting (PVs).

\begin{tabular}{|c|c|c|c|c|c|c|c|c|c|c|c|c|c|}
\hline \multicolumn{14}{|c|}{ Appendix.32: Cromwell - Camping accommodation services, energy sources and facilities } \\
\hline \multirow[t]{2}{*}{ No. ${ }^{1}$} & \multirow[t]{2}{*}{$\mathrm{Q}^{2}$} & \multirow[t]{2}{*}{$\mathrm{C}^{3}$} & \multicolumn{3}{|c|}{ Heating } & \multirow{2}{*}{$\mathrm{So}_{1}^{5 \& 1}$} & \multicolumn{2}{|c|}{ TV } & \multirow[t]{2}{*}{ Int $^{8}$} & \multirow{2}{*}{$\mathrm{V} / \mathrm{B}$} & \multirow[t]{2}{*}{$\mathrm{S} / \mathrm{P}$} & \multirow{2}{*}{$\begin{array}{l}\text { Outdoo } \\
\text { r sitting }\end{array}$} & \multirow{2}{*}{$\begin{array}{l}\mathrm{S} / \\
\mathrm{P}\end{array}$} \\
\hline & & & $\mathrm{El}^{4}$ & $\log$ & $\begin{array}{l}\text { Gas } \\
10\end{array}$ & & $\mathrm{~S}^{6}$ & $\mathrm{P}^{7}$ & & & & & \\
\hline 288 & $\begin{array}{c}\text { NB- } \\
\text { powered } \\
\text { sites } \\
\end{array}$ & $\begin{array}{c}172 \\
8\end{array}$ & $\cdot$ & - & - & - & - & - & • & - & - & - & - \\
\hline 183 & $\begin{array}{c}\text { NB- } \\
\text { non- } \\
\text { powered } \\
\text { sites } \\
\end{array}$ & $\begin{array}{c}109 \\
8\end{array}$ & - & - & - & - & - & - & • & - & - & - & - \\
\hline $\begin{array}{ll}1 . & \mathrm{N} \\
2 . & \mathrm{C} \\
3 . & \mathrm{C} \\
4 . & \mathrm{E} \\
5 . & \mathrm{S} \\
6 . & \mathrm{S} \\
7 . & \mathrm{P} \\
8 . & \mathrm{I} \\
9 . & \mathrm{V} \\
10 . & \mathrm{C} \\
11 . & \mathrm{S}\end{array}$ & $\begin{array}{l}\text { : Number } \\
\text { Type of bu } \\
\text { Capacity ( } \\
\text { : Electricity } \\
\text { : Solar syst } \\
\text { Shared fac } \\
\text { Private fac } \\
\text { : Internet. } \\
\text { B: Veranda } \\
\text { is including } \\
\text { lar systems }\end{array}$ & $\begin{array}{l}\text { accol } \\
\text { ding } \\
\text { amber } \\
\text { m. } \\
\text { ties. } \\
\text { ities. } \\
\text { alcon } \\
\text { entra }\end{array}$ & $\begin{array}{l}\text { moda } \\
\text { ed as } \\
\mathrm{f} \text { bed }\end{array}$ & $\begin{array}{l}\text { n servi } \\
\text { comme } \\
\text { aces). }\end{array}$ & $\begin{array}{l}\text { es. } \\
\text { dation }\end{array}$ & $\begin{array}{l}\text { eating s } \\
\text { Vs). }\end{array}$ & stem & & & & & & \\
\hline
\end{tabular}




\begin{tabular}{|c|c|c|c|c|c|c|c|c|c|c|c|c|c|c|c|c|c|}
\hline \multicolumn{18}{|c|}{ Appendix.33: Cromwell - All types of accommodation service, energy sources and facilities } \\
\hline \multirow[t]{2}{*}{ Type } & \multicolumn{3}{|c|}{ Heating } & \multirow[t]{2}{*}{ So } & \multicolumn{4}{|c|}{ TV } & \multirow[t]{2}{*}{ Int } & \multicolumn{4}{|c|}{$\mathrm{V} / \mathrm{B}$} & \multicolumn{4}{|c|}{ Outdoor sitting } \\
\hline & El & Log & $\begin{array}{c}\mathrm{Ga} \\
\mathrm{s}\end{array}$ & & $\mathrm{S}$ & $\begin{array}{l}\mathrm{N} \\
\mathrm{T}\end{array}$ & $\mathrm{P}$ & $\begin{array}{l}\mathrm{N} \\
\mathrm{T}\end{array}$ & & $\mathrm{S}$ & \begin{tabular}{l|}
$\mathrm{N}$ \\
$\mathrm{V}$
\end{tabular} & $\mathrm{P}$ & NV & $\mathrm{S}$ & No & $\mathrm{P}$ & No \\
\hline $\mathrm{SC}$ & 15 & 0 & 0 & 0 & 0 & 0 & 15 & 6 & 15 & 0 & 0 & 4 & 2 & 11 & 2 & 0 & 0 \\
\hline $\mathrm{B} \& \mathrm{~B}$ & 96 & 0 & 4 & 0 & 0 & 0 & 100 & 49 & 100 & 0 & 0 & 0 & 0 & 0 & 0 & 96 & 47 \\
\hline Motel & $\begin{array}{c}21 \\
3\end{array}$ & 0 & 73 & 0 & 0 & 0 & 130 & 33 & 229 & 0 & 0 & 14 & 2 & 36 & 1 & 14 & 2 \\
\hline $\begin{array}{l}\text { Camping } \\
*\end{array}$ & $\begin{array}{l}1,7 \\
28\end{array}$ & 0 & 0 & 0 & 0 & 0 & 0 & 0 & $\begin{array}{c}2,82 \\
6\end{array}$ & 0 & \begin{tabular}{l|l}
0 \\
\end{tabular} & 0 & 0 & 0 & 0 & 0 & 0 \\
\hline$\bullet$ & , & . & & & & & 11 & & & & & & & & & & \\
\hline
\end{tabular}




\section{Appendix.34: Cromwell - Cultural Products and Activities}

\begin{tabular}{|c|c|c|}
\hline No. & Local produce & $\mathrm{F}$ \\
\hline 1 & Wine. & 15 \\
\hline 2 & Continental Breakfast. & 3 \\
\hline 3 & Home baking and local food. & 1 \\
\hline \multicolumn{3}{|c|}{ General produce } \\
\hline 1 & Food and beverages & 17 \\
\hline \multicolumn{3}{|c|}{ Services } \\
\hline 1 & Motorcycle hire & 12 \\
\hline 2 & Tour activities booking centre & 1 \\
\hline 3 & Bike hire & 1 \\
\hline 4 & Sport equipments (undefined- hire/sale) & 1 \\
\hline \multicolumn{3}{|c|}{ Visits } \\
\hline 1 & Wineries & 20 \\
\hline 2 & Cromwell town & 16 \\
\hline 3 & Garden ( private/ surrounding) & 4 \\
\hline 4 & Gold mining heritage & 3 \\
\hline 5 & Scenery & 3 \\
\hline 6 & Historic sites and buildings (undefined) & 2 \\
\hline 7 & Clyde & 1 \\
\hline 8 & Lake Dunstan & 1 \\
\hline 9 & Museum (undefined) & 1 \\
\hline 10 & Cromwell Anderson park & 1 \\
\hline \multicolumn{3}{|c|}{ Events } \\
\hline 1 & Local annual events/festivals. & 3 \\
\hline 2 & Conference & 1 \\
\hline \multicolumn{3}{|c|}{ Indoor activities } \\
\hline 1 & Reading materials. & 2 \\
\hline \multicolumn{3}{|c|}{ Outdoor activities } \\
\hline 1 & Golf & 17 \\
\hline 2 & Swimming & 16 \\
\hline 3 & Winter sports & 15 \\
\hline 4 & Fishing & 14 \\
\hline 5 & Boating & 13 \\
\hline 6 & Biking & 13 \\
\hline 7 & Scenic flights & 12 \\
\hline 8 & Walking & 6 \\
\hline 9 & Water sports & 5 \\
\hline 10 & Jet trail & 3 \\
\hline 11 & Mountain biking & 2 \\
\hline 12 & 4WD track/tour & 2 \\
\hline 13 & Tennis & 1 \\
\hline 14 & Netball & 1 \\
\hline 15 & Squash & 1 \\
\hline 16 & Horse racing & 1 \\
\hline 17 & Car racing & 1 \\
\hline 18 & Wind surfing & 1 \\
\hline 19 & Gold mining & 1 \\
\hline 20 & Picnic & 1 \\
\hline
\end{tabular}




\begin{tabular}{|l|c|c|}
\hline \multicolumn{3}{|c|}{ Apendix.35: Cromwell - outdoor activities } \\
\hline Activity & $\mathrm{F}$ & $\%$ \\
\hline Golf & 17 & 13.5 \\
\hline Swimming & 16 & 12.7 \\
\hline Winter sports & 15 & 11.9 \\
\hline Fishing & 14 & 11.1 \\
\hline Boating & 13 & 10.3 \\
\hline Biking & 13 & 10.3 \\
\hline Scenic flights & 12 & 9.5 \\
\hline Walking & 6 & 4.8 \\
\hline Water sports & 5 & 3.9 \\
\hline Jet trail & 3 & 2.4 \\
\hline Mountain biking & 2 & 1.6 \\
\hline 4WD track/tour & 2 & 1.6 \\
\hline Tennis & 1 & 0.8 \\
\hline Netball & 1 & 0.8 \\
\hline Squash & 1 & 0.8 \\
\hline Horse racing & 1 & 0.8 \\
\hline Car racing & 1 & 0.8 \\
\hline Wind surfing & 1 & 0.8 \\
\hline Gold mining & 1 & 0.8 \\
\hline Picnic & 1 & 0.8 \\
\hline Total & 126 & 100 \\
\hline
\end{tabular}

\begin{tabular}{|l|c|c|}
\hline \multicolumn{3}{|c|}{ Appendix.36: Cromwell - Subjects offered to visit and their frequency } \\
\hline Wineries & 20 & 38.5 \\
\hline Cromwell town & 16 & 30.8 \\
\hline Garden (private/ surrounding) & 4 & 7.7 \\
\hline Gold mining heritage & 3 & 5.8 \\
\hline Scenery & 3 & 5.8 \\
\hline Historic sites and buildings (undefined) & 2 & 3.8 \\
\hline Clyde & 1 & 1.9 \\
\hline Lake Dunstan & 1 & 1.9 \\
\hline Museum (undefined) & 1 & 1.9 \\
\hline Cromwell Anderson park & 1 & 1.9 \\
\hline Total & 52 & 100 \\
\hline
\end{tabular}

